\title{
Bildung auf einen Blick
}

OECD-Indikatoren 2002
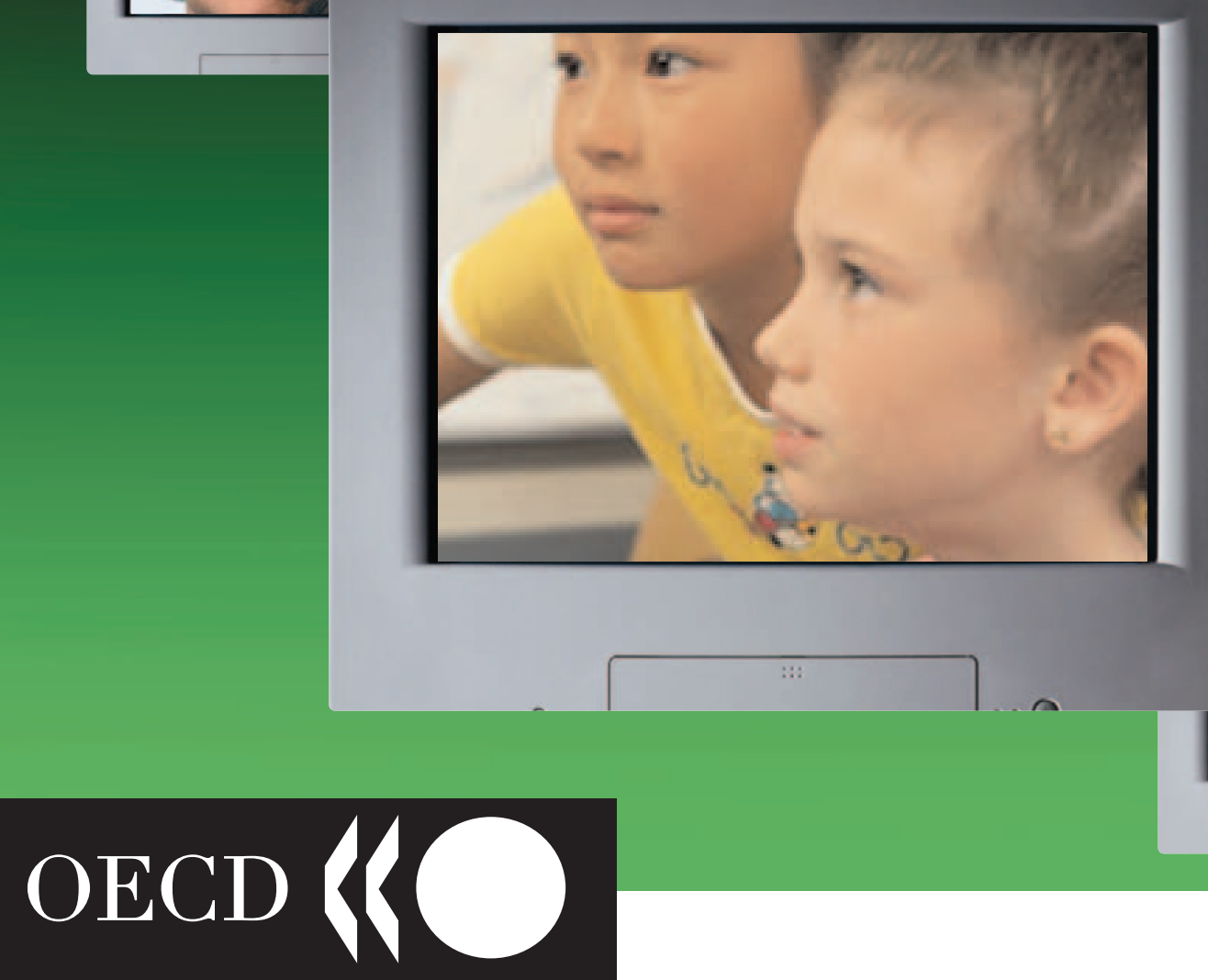


\section{Bildung auf einen Blick}

\section{OECD-INDIKATOREN 2002}

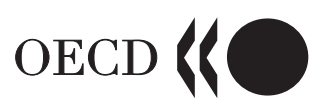

ORGANISATION FÜR WIRTSCHAFTLICHE ZUSAMMENARBEIT UND ENTWICKLUNG 


\section{ORGANISATION FÜR WIRTSCHAFTLICHE ZUSAMMENARBEIT UND ENTWICKLUNG}

Gemäß Artikel 1 des am 14. Dezember 1960 in Paris unterzeichneten und am 30. September 1961 in Kraft getretenen Übereinkommens fördert die Organisation für wirtschaftliche Zusammenarbeit und Entwicklung (OECD) eine Politik, die darauf gerichtet ist:

- in den Mitgliedstaaten unter Wahrung der finanziellen Stabilität eine optimale Wirtschaftsentwicklung und Beschäftigung sowie einen steigenden Lebensstandard zu erreichen und dadurch zur Entwicklung der Weltwirtschaft beizutragen;

- in den Mitglied- und Nichtmitgliedstaaten, die in wirtschaftlicher Entwicklung begriffen sind, zu einem gesunden wirtschaftlichen Wachstum beizutragen; und

- im Einklang mit internationalen Verpflichtungen auf multilateraler und nichtdiskriminierender Grundlage zur Ausweitung des Welthandels beizutragen.

Die Gründungsmitglieder der OECD sind: Belgien, Dänemark, Deutschland, Frankreich, Griechenland, Irland, Island, Italien, Kanada, Luxemburg, Niederlande, Norwegen, Österreich, Portugal, Schweden, Schweiz, Spanien, Türkei, Vereinigtes Königreich und Vereinigte Staaten. Folgende Staaten wurden zu den nachstehend genannten Daten Mitglieder der OECD: Japan (28. April 1964), Finnland (28. Januar 1969), Australien (7. Juni 1971), Neuseeland (29. Mai 1973), Mexiko (18. Mai 1994), die Tschechische Republik (21. Dezember 1995), Ungarn (7. Mai 1996), Polen (22. November 1996), Korea (12. Dezember 1996) und die Slowakische Republik (14. Dezember 2000). Die Kommission der Europäischen Gemeinschaften nimmt an den Tätigkeiten der OECD teil (Artikel 13 des Übereinkommens über die OECD).

Das Zentrum für Forschung und Innovation im Bildungswesen (CERI) wurde im Juni 1968 vom Rat der Organisation für wirtschaftliche Zusammenarbeit und Entwicklung ins Leben gerufen. Alle OECD-Mitgliedsländer nehmen daran teil.

Die Hauptziele dieses Zentrums sind:

- Förderung und Unterstützung der Entwicklung von Forschungsaktivitäten im Bildungsbereich und gegebenenfalls die Durchführung solcher Forschungsaktivitäten;

- Förderung und Unterstützung von Pilotversuchen im Hinblick auf die Einführung und Erprobung von Innovationen im Bildungssystem;

- Förderung der Entwicklung einer Zusammenarbeit zwischen den Mitgliedstaaten auf dem Gebiet der pädagogischen Forschung und Innovation.

Das Zentrum arbeitet innerhalb der Organisation für wirtschaftliche Zusammenarbeit und Entwicklung gemäß den Entscheidungen des Rats der Organisation und im Auftrag des Generalsekretärs sowie unter der direkten Kontrolle eines Lenkungsausschusses, in den jeder am Arbeitsprogramm des CERI beteiligte Mitgliedstaat einen nationalen Experten für den Aufgabenbereich des Zentrums entsendet.

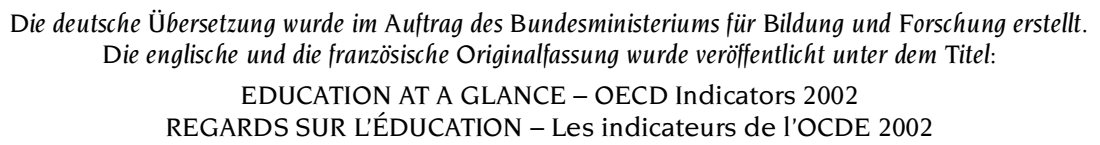

(C) OECD 2002

Genehmigungen zum Nachdruck von Teilen dieses Werks für nichtkommerzielle Zwecke oder zur Verwendung im Unterricht sind einzuholen beim Centre français d'exploitation du droit de copie (CFC), 20, rue des GrandsAugustins, 76006 Paris, Frankreich, tel: (33-1) 4407 47 70, fax: (33-1) 463467 19. Dies gilt für alle Länder mit Ausnahme der Vereinigten Staaten, wo das Copyright Clearance Center Inc. (CCC), Customer Service, tel: (508) 750-8400, 222 Rosewood Drive, Danvers, MA 01923, USA oder CCC online: www.copyright.com die entsprechenden Genehmigungen erteilt. Alle sonstigen Anträge auf Überlassung von Nachdruck- oder Übersetzungsrechten für das gesamte Dokument oder Teile davon sind zu richten an: OECD Publications, 2, rue André-Pascal, 75775 Paris Cedex 16, Frankreich. 


\section{VORWORT}

Überzeugende Anreize für den Einzelnen, die Volkswirtschaften und die Gesellschaft in den OECD-Ländern insgesamt, das Bildungsniveau weiter anzuheben, sind in den letzten Jahren die treibende Kraft hinter der verstärkten Beteiligung von Menschen aller Altersstufen - von der frühen Kindheit bis zum späten Erwachsenenleben - an einem immer größer werdenden Spektrum von Lernangeboten. In Zeiten einer zunehmenden und immer vielfältigeren Nachfrage nach Lernen über das ganze Leben hinweg besteht die große Aufgabe für die OECD-Länder darin, sicherzustellen, dass die verschiedenen Arten und Formen des Lernens dem sich ständig ändernden Bedarf auf möglichst kosteneffektive Art und Weise entsprechen.

Bei der Suche nach einer effektiven Bildungspolitik, die die sozialen und wirtschaftlichen Aussichten des Einzelnen verbessert, Anreize für eine größere Effizienz bei der Bildungsvermittlung bietet und dazu beiträgt, Ressourcen zur Bewältigung der steigenden Bildungsnachfrage zu mobilisieren, lenken die Regierungen ihre Aufmerksamkeit in verstärktem Maße auf internationale Vergleiche. Im Rahmen ihrer Zusammenarbeit innerhalb der OECD streben die Länder an, voneinander zu lernen, wie die Hürden bei der Finanzierung der Bildung überwunden und die Vorteile von Bildung für alle sichergestellt werden können, wie man die für eine Wissensgesellschaft notwendigen Kompetenzen fördern kann, und wie Lehren und Lernen so gestaltet werden können, dass das lebensbegleitende Lernen tatsächlich zur Realität wird.

Als Teil der Bemühungen, die Arbeit der OECD in diesem Bereich weiter zu verbessern und den Bedürfnissen der Bürger und der Regierungen besser gerecht werden zu können, hat die OECD den Bildungsbereich im OECD-Sekretariat in eine unabhängige Direktion erweitert. Zentraler Bestandteil der strategischen Ausrichtung der Direktion für Bildungswesen ist die Entwicklung und Analyse quantitativer Indikatoren, die jedem einzelnen Land die Möglichkeit bieten, sein eigenes Bildungssystem im Licht der Leistungsfähigkeit anderer Länder zu betrachten. Zusammen mit den länderspezifischen Untersuchungen der OECD sind die Indikatoren darauf ausgelegt, die Regierungen in ihren Bemühungen um Reformen in der Bildungspolitik zu unterstützen.

Die Veröffentlichung Bildung auf einen Blick - OECD-Indikatoren 2002 ist ein zentrales Instrument zur Verbreitung der Indikatoren an ein breit gestreutes Publikum - von den Regierungen, die von den bildungspolitischen Erfahrungen anderer Länder lernen wollen, über Wissenschaftler, die Daten für weitergehende Analysen benötigen, bis zur allgemeinen Öffentlichkeit, die einen Überblick darüber gewinnen möchte, welche Fortschritte das Bildungssystem des eigenen Landes dabei macht, Schüler und Studierende von Weltklasseformat auszubilden. Bildung auf einen Blick enthält ein umfangreiches aktuelles Spektrum an Indikatoren, die den Konsens der Fachwelt zur Erfassung und Messung des gegenwärtigen Standes der Bildung im internationalen Vergleich widerspiegeln.

Die Ausgabe des Jahres 2002 von Bildung auf einen Blick ist gegenüber den Ausgaben früherer Jahre in drei wesentlichen Punkten verbessert worden. Erstens liefert die von der OECD durchgeführte Internationale Schulleistungsstudie PISA, die von den Regierungen initiiert wurde, um die an den Schülerleistungen gemessenen Ergebnisse ihrer Bildungssysteme in regelmäßigen Abständen und innerhalb eines gemeinsamen, international vereinbarten Rahmens zu bewerten, vergleichbare Informationen zu den Bildungs- und Lernergebnissen sowie zu den wichtigsten Faktoren, die die Lernergebnisse beeinflussen. Das Fehlen solcher Informationen stellte lange Zeit eine wesentliche Lücke bei den untersuchten Indikatoren dar. Zweitens behandelt ein immer größerer Teil der Indikatoren inzwischen nicht mehr nur die zusammengefassten Ergebnisse der einzelnen Länder sondern auch Unterschiede innerhalb der Länder, so dass jetzt auch Fragen der Chancengleichheit beim Bildungsangebot und den Bildungsergebnissen untersucht werden können. Dies betrifft u.a. Aspekte wie Geschlecht, Alter, sozioökonomischer Hintergrund, Art der Bildungseinrichtung und Ausrichtung der Bildungsgänge. Drittens wird die Arbeit in ein neues Rahmenwerk eingestellt, in dem die Indikatoren je nach Schwerpunkt ihrer Analyse in 
Gruppen zusammenfasst werden. Zum einen die Indikatoren, die sich mit den Lernergebnissen von Einzelnen und ganzen Ländern beschäftigen, dann die Indikatoren, deren Fokus auf den diese Ergebnisse beeinflussenden Bedingungen bzw. politischen Ansatzpunkten liegt sowie eine dritte Gruppe von Indikatoren, die die bei politischen Entscheidungen zu berücksichtigenden Gegebenheiten bzw. Einschränkungen untersuchen.

Diese Veröffentlichung ist das Ergebnis langjähriger gemeinsamer Bemühungen der Regierungen der OECDLänder, der Experten und Institutionen, die im Rahmen des OECD Education Indicators Programme (INES) zusammenarbeiten, sowie dem Sekretariat der OECD. Sie wurde erstellt von der Abteilung für Indikatoren und Analysen unter der Leitung von Andreas Schleicher in Zusammenarbeit mit Eric Charbonnier, Hannah Cocks, Jean-Luc Heller, Judit Kadar-Fülop, Karine Tremblay und Claire Shewbridge. Die Entwicklung der Veröffentlichung wurde von den Nationalen INES-Koordinatoren in den Mitgliedsländern gesteuert und durch die drei Länder, die für die Koordination der INES-Netzwerke verantwortlich zeichnen - die Niederlande, Schweden und die Vereinigten Staaten - wesentlich unterstützt. Außerdem wurde die Arbeit an dieser Veröffentlichung durch das National Center for Education Statistics (NCES) in den Vereinigten Staaten finanziell gefördert. Die Publikation der deutschen Fassung wurde ermöglicht durch das Bundesministerium für Bildung und Forschung. Die Übersetzung ins Deutsche wurde vom Hochschul-Informations-System (HIS) angefertigt. Der Anhang enthält eine Liste der Mitglieder der verschiedenen Organe sowie der einzelnen Fachleute, die an diesem Bericht und den OECD-Indikatoren generell mitgewirkt haben.

In den letzten Jahren ist viel erreicht worden, aber es sind noch umfangreiche Bemühungen notwendig, um für eine ganze Reihe von bildungspolitischen Fragen die besten verfügbaren Daten zusammenstellen zu können. Auch in Zukunft wird sich die Arbeit weiterhin mit den verschiedenen Herausforderungen, auch im jeweiligen nationalen Kontext, beschäftigen müssen. Erstens müssen die Indikatoren die bildungspolitischen Fragen ansprechen, die in den einzelnen Ländern von großer bildungspolitischer Bedeutung sind, und bei denen eine international vergleichende Perspektive gegenüber nationalen Analysen und Bewertungen einen wirklichen Mehrwert liefern kann. Zweitens müssen die Indikatoren zwar so vergleichbar wie möglich sein, gleichzeitig aber auch länderspezifisch genug, um historische, systembedingte und kulturelle Unterschiede zwischen den einzelnen Ländern berücksichtigen zu können. Drittens muss die Darstellung in den Indikatoren so klar wie möglich sein, gleichzeitig aber auch der facettenreichen Realität von Bildungssystemen in der heutigen Welt gerecht werden. Viertens besteht der allgemeine Wunsch, die Zahl der Indikatoren so niedrig wie möglich zu halten, während gleichzeitig ihre Anzahl aber groß genug sein muss, um den politischen Entscheidungsträgern in den einzelnen Ländern, die sich teilweise ganz unterschiedlichen bildungspolitischen Herausforderungen gegenüber sehen, wirklich von Nutzen zu sein.

Die neue organisatorische Struktur bei der OECD liefert den Rahmen, um diese Herausforderungen noch energischer anzugehen und die Entwicklung von Indikatoren nicht nur in den Bereichen voranzutreiben, in denen die Entwicklung von Daten vielversprechend ist, sondern auch in jene Bereiche vorzudringen, in denen noch sehr viel grundlegende konzeptionelle Arbeit vonnöten ist.

Für diesen Bericht zeichnet der Generalsekretär der OECD verantwortlich.

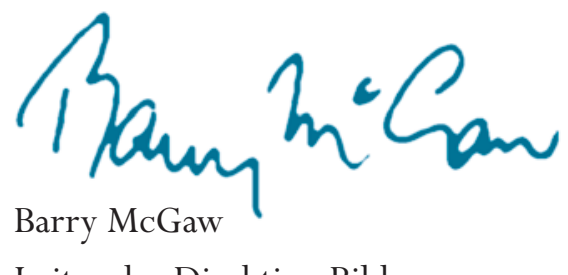

Leiter der Direktion Bildungswesen

OECD 


\section{INHALTSVERZEICHNIS}

Bezeichung des Indikators in der Ausgabe von 2001

Einleitung 7

Bildung auf einen Bilck 2002 ................................................ 7

Inhalt und interessante Einzelergebnisse ................................... 9

Weitere Quellen .......................................................... 24

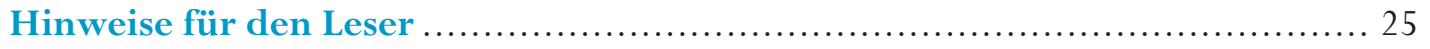

Kapitel A: Bildungs- und Lernergebnisse .................................. 29

A1 Aktuelle Abschlussquoten im Sekundarbereich II und Bildungsstand der

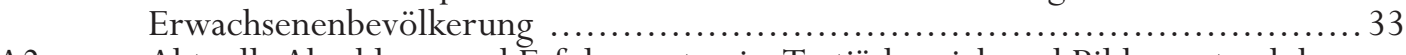

A2 Aktuelle Abschluss- und Erfolgsquoten im Tertiärbereich und Bildungsstand der

Erwachsenenbevölkerung................................................ 42

A3 Bildungsstand der Erwerbsbevölkerung und der Erwachsenenbevölkerung insgesamt. 53

A4

Absolventen nach Studienbereichen

A5

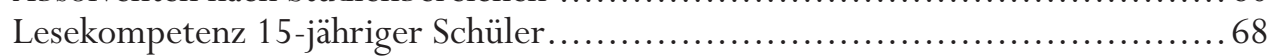

A6 Mathematische und naturwissenschaftliche Grundbildung 15-Jähriger............80

A7 Leistungsunterschiede zwischen den Schulen .............................. 90

A8 Politische Bildung und politisches Engagement ............................ 100

A9 Beruflicher Status der Eltern und Leistungen der Schüler ..................... 107

A10 Geburtsland, zu Hause gesprochene Sprache und Lesekompetenz 15-Jähriger .. 113

A11 Erwerbsquoten nach Bildungsstand .................................... 122

A12 Zu erwartende Jahre in Ausbildung und Beschäftigung im Alter zwischen 15

und 29 Jahren ..................................................... 132

A13 Ertragsraten von Bildung: Individuelle und gesellschaftliche Ertragsraten von

Bildung und deren bestimmende Faktoren ................................ 137

A14 Ertragsraten von Bildung: Zusammenhang zwischen Humankapital und

Wirtschaftswachstum ................................................. 151

Kapitel B: Finanz- und Humanressourcen - Investitionen in die Bildung ...... 157

B1 Bildungsausgaben pro Schüler/Studierenden ........................... 161

B2 Ausgaben für Bildungseinrichtungen im Verhältnis zum Bruttoinlandsprodukt... 179

B3 Die öffentlichen Bildungsausgaben ....................................... 194

B4 Relative Anteile öffentlicher und privater Ausgaben für Bildungseinrichtungen.. 200

B5 Unterstützung für Schüler/Studierende und private Haushalte durch öffentliche

Subventionen ...................................................... 215

B6 Ausgaben für Bildungseinrichtungen nach Art der erbrachten Leistung und

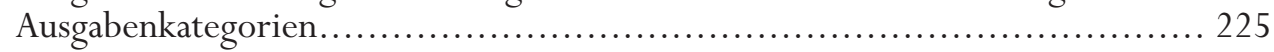

Kapitel C: Bildungszugang, Bildungsbeteiligung und Bildungserwartung .... 237

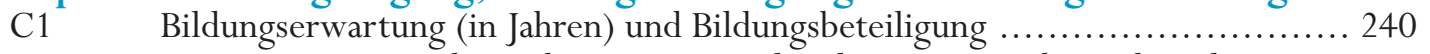

C2 Zugang zum Tertiärbereich, zu erwartende Jahre im Tertiärbereich und Bildungsbeteiligung im Sekundarbereich.................................... 248

C3 Ausländische Studierende im Tertiärbereich .................................... 263

C4 Beteiligung der Erwachsenenbevölkerung an Fort- und Weiterbildung........... 276

C5 Bildung und Beschäftigungsstatus junger Menschen........................... 281

C6 Die Situation junger Menschen mit einem niedrigen Bildungsniveau ............. 293

Kapitel D: Das Lernumfeld und die Organisation von Schulen ................... 299

D1 Insgesamt vorgesehene Unterrichtszeit für 9- bis 14-jährige Schüler ............ 303

D2 Klassengrößen und zahlenmäßiges Schüler/Lehrer-Verhältnis .................. 318

D3 Nutzung und Verfügbarkeit von Computern in der Schule und zu Hause ......... 326

D4 Einstellungen und Erfahrungen von Schülerinnen und Schülern bei der Nutzung von

Computern .......................................................... 340
C1, C3

C5 (2000)

C6

E3

D4

D5

D7 
D5 Unterrichts- und Schulklima ............................................. 349

D6 Lehrergehälter an öffentlichen Schulen des Primar- und Sekundarbereichs ...... 366

D7 Unterrichtszeit und Arbeitszeit der Lehrer ................................ 379

Anhang 1: Typische Abschlussalter ...................................... 389

Anhang 2: Grundlegende statistische Bezugsdaten .......................... 395

Anhang 3: Quellen, Methoden und technische Hinweise.................... 401

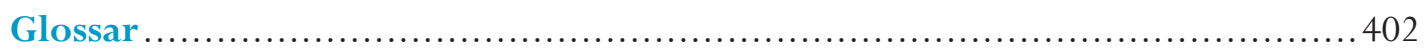

Mitwirkende an dieser Publikation .......................................... 415

Zugehörige OECD-Publikationen ........................................... 419 


\section{EINLEITUNG}

\section{BILDUNG AUF EINEN BLICK 2002}

\section{Die OECD-Indikatoren geben den Konsens der Fachwelt über die international vergleichende Erfassung und Messung der gegenwärtigen Situation der Bildung wieder.}

Bildung auf einen Blick - OECD-Indikatoren 2002 bietet ein umfangreiches aktuelles Spektrum an vergleichbaren Indikatoren, die den Konsens der Fachwelt zur Erfassung und Messung der gegenwärtigen Situation der Bildung im internationalen Vergleich darstellen. Sie enthalten Informationen zu den in Bildung investierten personellen und finanziellen Ressourcen, zur Funktionsweise und Weiterentwicklung von Bildungssystemen und zu den Erträgen der Investitionen in die Bildung. Die Indikatoren sind thematisch gegliedert und jeweils von entsprechenden Hintergrundinformationen begleitet. Bildung auf einen Blick 2002 ist gegenüber früheren Ausgaben durch die Erweiterung um drei wesentliche Aspekte verbessert worden:

Vergleichbare Daten über die Lernergebnisse erweitern die OECD-Indikatoren um eine weitere Dimension, ...

Die von der OECD durchgeführte Internationale Schulleistungsstudie PISA, die von den Regierungen initiiert wurde, um die an den Schülerleistungen gemessenen Ergebnisse ihrer Bildungssysteme in regelmäßigen Abständen und innerhalb eines gemeinsamen, international vereinbarten Rahmens zu bewerten, liefert vergleichbare Informationen zu den Bildungs- und Lernergebnissen sowie zu den wichtigsten Faktoren, die diese beeinflussen. Mit PISA soll eine neue Basis für den bildungspolitischen Dialog geschaffen werden, die eine Zusammenarbeit bei der Definition von Bildungszielen erlaubt, die innovativ und realistisch sind, und die Kompetenzen berücksichtigen, die für das spätere Leben relevant sind. PISA ist Teil einer Verlagerung des Schwerpunkts in der Bildungspolitik vom Input auf die Ergebnisse des Lernens. Diese Verlagerung soll dazu dienen, die Länder bei ihren Bemühungen um Verbesserung ihrer Schulsysteme zu unterstützen und so dazu beitragen, junge Menschen besser auf ein Erwachsenenleben in einer Welt des raschen Wandels und der immer stärker werdenden globalen Interdependenz vorzubereiten.

\section{... bessere Informationen zu den Unterschieden zwischen Schülern und Schulen sind hilfreich bei der Untersuchung der Chancengleich heit sowohl beim Bildungsangebot als auch bei den Bildungsergebnissen,...}

Ein immer größerer Teil der Indikatoren behandelt inzwischen nicht mehr nur die zusammengefassten Ergebnisse der einzelnen Länder sondern auch Unterschiede innerhalb der Länder, so dass jetzt auch Fragen der Chancengleichheit beim Bildungsangebot und den Bildungsergebnissen untersucht werden können. Dies betrifft u.a. Aspekte wie Geschlecht, Alter, sozio-ökonomischer Hintergrund, Art der Bildungseinrichtung und Ausrichtung der Bildungsgänge.

\section{...und ein neues Rahmenwerk für die Indikatoren erleichtert deren Nutzung.}

Die OECD-Indikatoren werden nach und nach in ein neues Rahmenwerk eingestellt, das

- zwischen den Akteuren im Bildungssystem unterscheidet - einzelne Schüler, Anbieter von Bildungsleistungen, Arten des Unterrichts und Lernumgebungen, sowie das Bildungssystem als Ganzes; 
- die Indikatoren in Gruppen zusammenfasst, je nachdem, womit sie sich beschäftigen - die Lernergebnisse von Einzelnen und ganzen Ländern, die politischen Ansatzpunkte oder Bedingungen, die diese Ergebnisse beeinflussen, oder die Gegebenheiten oder Einschränkungen, die bei politischen Entscheidungen zu berücksichtigen sind;

- die politischen Fragen identifiziert, auf die sich die Indikatoren beziehen, und die in drei Kategorien eingeteilt sind - die Qualität der Bildungserfolge und des Bildungsangebots, Fragen der Chancengleichheit beim Bildungsangebot und den Bildungsergebnissen sowie die Angemessenheit und Effektivität des Managements von Ressourcen.

Die folgende Matrix veranschaulicht die ersten beiden Dimensionen:

\begin{tabular}{|c|c|c|c|}
\hline & $\begin{array}{l}\text { Bildungs- und } \\
\text { Lernergebnisse }\end{array}$ & $\begin{array}{l}\text { Politische Ansatzpunkte } \\
\text { und Bedingungen, die } \\
\text { die Bildungsergebnisse } \\
\text { beeinflussen }\end{array}$ & $\begin{array}{l}\text { Gegebenheiten und } \\
\text { Einschränkungen, } \\
\text { die von der Politik zu } \\
\text { berücksichtigen sind }\end{array}$ \\
\hline $\begin{array}{l}\text { Einzelne } \\
\text { Bildungsteilnehmer } \\
\text { und Lernende }\end{array}$ & $\begin{array}{l}\text { 1. Die Qualität und Vertei- } \\
\text { lung der individuellen } \\
\text { Bildungsergebnisse }\end{array}$ & $\begin{array}{l}\text { 5. Einstellungen, } \\
\text { persönlicher Bezug und } \\
\text { Verhalten des Einzelnen }\end{array}$ & $\begin{array}{l}\text { 9. Persönlicher Hintergrund } \\
\text { des einzelnen Lernenden }\end{array}$ \\
\hline $\begin{array}{l}\text { Arten des } \\
\text { Unterrichts }\end{array}$ & $\begin{array}{l}\text { 2. Die Qualität des } \\
\text { Unterricht }\end{array}$ & $\begin{array}{l}\text { 6. Pädagogische Praktiken } \\
\text { und Lern-Praktiken sowie } \\
\text { das Unterrichtsklima }\end{array}$ & $\begin{array}{l}\text { 10. Unterrichts- und Lernbe- } \\
\text { dingungen der Lernenden } \\
\text { und Arbeitsbedingungen } \\
\text { der Lehrenden }\end{array}$ \\
\hline $\begin{array}{l}\text { Anbieter von } \\
\text { Bildung }\end{array}$ & $\begin{array}{l}\text { 3. Die Abschlussquoten der } \\
\text { Bildungseinrichtungen } \\
\text { und die Leistungen der } \\
\text { Bildungseinrichtungen }\end{array}$ & $\begin{array}{l}\text { 7. Organisation der Schulen } \\
\text { und ihr Umfeld }\end{array}$ & $\begin{array}{l}\text { 11. Merkmale der Anbieter von } \\
\text { Bildungsdienstleistungen } \\
\text { und ihres Umfelds }\end{array}$ \\
\hline $\begin{array}{l}\text { Das Bildungssystem } \\
\text { als Ganzes }\end{array}$ & $\begin{array}{l}\text { 4. Die Gesamtleistung der } \\
\text { Bildungssysteme }\end{array}$ & $\begin{array}{l}\text { 8. Institutionelle Gegeben- } \\
\text { heiten des Bildungs- } \\
\text { systems, Zuweisung von } \\
\text { Mitteln und politische } \\
\text { Maß-nahmen }\end{array}$ & $\begin{array}{l}\text { 12. Der jeweilige nationale, } \\
\text { bildungspolitische, soziale, } \\
\text { wirtschaftliche und } \\
\text { demographische Kontext }\end{array}$ \\
\hline
\end{tabular}




\title{
INHALT UND INTERESSANTE EINZELERGEBNISSE
}

\author{
Bildung auf einen Blick 2002 teilt sich in vier Kapitel auf.
}

Kapitel A untersucht die Bildungs- und Lernergebnisse, und zwar in Form...

... der aktuellen Abschlussquoten der Bildungseinrichtungen und des Bildungsstands der Erwachsenenbevölkerung, ...

In Kapitel A werden zunächst die Abschlussquoten im Sekundarbereich II und im Tertiärbereich untersucht (Indikatoren A1 und A2). Diese Indikatoren beschäftigen sich sowohl mit den Ergebnissen der Bildungssysteme auf institutioneller als auch auf nationaler Ebene. Um die Fortschritte bei den Bildungsergebnissen zu messen, werden dann die aktuellen Abschlussquoten mit dem Bildungsstand von älteren Personen verglichen, die das Bildungssystem zu unterschiedlichen Zeitpunkten verlassen haben.

Es werden auch die Fortschritte der einzelnen Länder bei der Überwindung der Unterschiede zwischen Männern und Frauen beim Bildungsstand und den Abschlussquoten, sowohl insgesamt als auch über die einzelnen Bildungsbereiche hinweg, untersucht (Indikatoren A1, A2 und A4).

Abschluss- und Abbruchquoten (Indikator A2) geben in gewissem Maß Aufschluss über die interne Effizienz von tertiären Bildungssystemen. Es gibt viele Gründe, warum Studierende ihr Studium abbrechen: man merkt, dass man das falsche Fach oder den falschen Studiengang gewählt hat; dass man den Mindestanforderungen der Bildungseinrichtungen nicht gerecht wird; oder es bietet sich bereits vor Abschluss des Studiums eine attraktive Beschäftigung an. Hohe Studienabbruchquoten weisen jedoch darauf hin, dass das Bildungssystem den Bedürfnissen seiner Nutzer nicht gerecht wird. Studierende sind vielleicht der Ansicht, dass die angebotenen Studiengänge ihren Erwartungen bzw. den Arbeitsmarktanforderungen nicht entsprechen. Und es kann sein, dass aus Sicht der Studierenden das lange Studium nicht die entgangene Zeit im Arbeitsmarkt rechtfertigt.

- In fast allen OECD-Ländern ist der Anteil von Personen, die nicht über einen Abschluss im Sekundarbereich II verfügen, zurückgegangen, in einigen Ländern sogar recht schnell. Mit Ausnahme von fünf OECDLändern liegt das Verhältnis von Absolventen des Sekundarbereich II zur Bevölkerung im typischen Abschlussalter inzwischen bei über 70 Prozent und in vielen Ländern sogar bei über 90 Prozent (S. 34).

- Durchschnittlich erzielen gegenwärtig 26 Prozent der Personen im typischen Abschlussalter einen Abschluss im Tertiärbereich A. Hierbei reicht die Bandbreite von einem Drittel und mehr in Australien, Finnland, Island, Polen, dem Vereinigten Königreich und den Vereinigten Staaten bis zu weniger als 20 Prozent in Dänemark, Deutschland, Italien, Österreich, der Schweiz und der Tschechischen Republik (S. 44).

- Bei den älteren Altersgruppen sind die Abschlussquoten im Sekundarbereich II bei den Frauen niedriger als bei den Männern, doch bei den jüngeren Menschen hat sich dieses Verhältnis umgekehrt. Heute übersteigen in den meisten Ländern die Abschlussquoten der Frauen die der Männer (S. 35).

- In den Bereichen Geisteswissenschaften, Kunst, Pädagogik, Gesundheit und Soziales sind im Durchschnitt der OECD-Länder mehr als zwei Drittel der Absolventen des Tertiärbereich A Frauen, wohingegen ihr Anteil in Mathematik und den Naturwissenschaften weniger als ein Drittel ausmacht und weniger als ein Viertel in den Bereichen Ingenieurwesen, Fertigung und Bauwesen (S. 62).

- Die Erwachsenenbevölkerung verfügt heute über einen größeren Bestand an Kenntnissen und Fähigkeiten auf Hochschulniveau. Der größte Teil dieser Zunahme ist jedoch auf einen signifikanten Anstieg der tertiären Abschlussquoten in einer vergleichsweise geringen Zahl von Ländern zurückzuführen (S. 45).

- Im Durchschnitt bricht ein Drittel der Studierenden in den OECD-Ländern ihr Studium vor einem ersten Abschluss ab (S. 46). 
Das reine Zählen der Absolventen liefert noch keine Hinweise auf die Qualität der Lernergebnisse. Um diese Frage zu untersuchen, beschäftigen sich die Indikatoren A5 and A6 mit der Lesekompetenz und der mathematischen und naturwissenschaftlichen Grundbildung von 15-jährigen Schülern und zwar sowohl im Hinblick auf die relativen Leistungen der einzelnen Länder als auch auf die Unterschiede bei den Lernergebnissen innerhalb der einzelnen Länder. Lesekompetenzen sowie eine mathematische und naturwissenschaftliche Grundbildung gelten in allen OECD-Ländern als grundlegende Fertigkeiten, und die Leistungsniveaus in diesen Bereichen sind daher wesentliche Anhaltspunkte zur Messung der Qualität der Lernergebnisse. Man ist sich allerdings auch in zunehmendem Maße der Tatsache bewusst, dass es einer ganzen Reihe weiterer Kompetenzen bedarf, um als Einzelner und auch als Gesellschaft erfolgreich zu sein. Indikator A8 beschäftigt sich mit einer vergleichenden Untersuchung zur politischen Bildung und Einstellung von 14-Jährigen und unternimmt damit einen ersten Schritt zur Untersuchung solcher Kompetenzen.

- Im Durchschnitt aller OECD-Länder entsprechen 10 Prozent der 15-jährigen Schüler den Anforderungen der Stufe 5 im Bereich Lesekompetenz, bei der es um die Bewertung von Informationen und die Entwicklung von Hypothesen geht, die Nutzung von Fachwissen und die Verarbeitung von gedanklichen Konzepten, die unter Umständen den eigenen Erwartungen widersprechen. Dieser Prozentsatz variiert jedoch von 19 Prozent in Finnland und Neuseeland bis zu weniger als 1 Prozent in Mexiko. Im Durchschnitt erfüllen 12 Prozent der 15-jährigen Schüler nur die Anforderungen der grundlegendsten Kompetenzstufe 1 und weitere 6 Prozent liegen sogar noch darunter (S. 70).

- 15-Jährige in Japan erzielen die höchsten Mittelwerte bei der mathematischen Grundbildung, allerdings unterscheiden sich ihre Punktwerte statistisch nicht von denen der Schüler in den beiden anderen Ländern an der Spitze (Korea und Neuseeland). Bei der naturwissenschaftlichen Grundbildung erzielen die Schüler in Korea und Japan die höchsten Durchschnittsleistungen (S. 84).

- Während es bereits große Unterschiede bei den mittleren Leistungen zwischen den einzelnen Ländern gibt, sind die Unterschiede der Leistungen 15-Jähriger innerhalb der einzelnen Länder um ein Vielfaches größer. Große Disparitäten bei den Leistungen sind offensichtlich keine Voraussetzung dafür, dass ein Land ein insgesamt hohes Leistungsniveau erzielt. Die Leistungen von fünf der Länder mit der kleinsten Varianz der Leistungen auf der Skala mathematische Grundbildung, nämlich Finnland, Island, Japan, Kanada und Korea liegen signifikant über dem OECD-Durchschnitt (S. 86).

- 14-Jährige sehen es im Allgemeinen als sehr wichtige Aufgabe Erwachsener an, die Gesetze zu respektieren und zur Wahl zu gehen. Für sie sind auch Aktivitäten in den Bereichen Menschenrechte und Umweltschutz wichtig, sowie Aktivitäten zum Wohl ihres sozialen Umfelds. Die Teilnahme an politischen Diskussionen und die Mitgliedschaft in einer politischen Partei sind für sie dagegen weniger wichtig (S. 103).

\section{... und der Unterschiede dieser Ergebnisse zwischen Schülern und Schulen,...}

Die Indikatoren A5 und A6 zeigen, dass in den meisten Ländern innerhalb der jeweiligen Bildungssysteme erhebliche Leistungsunterschiede bestehen. Diese Unterschiede können durch den speziellen Hintergrund der einzelnen Schulen und der sie besuchenden Schüler, den Umfang der den Schulen zur Verfügung stehenden personellen und finanziellen Mittel, unterschiedliche Lehrpläne, vorhandene Selektionsmechanismen und durch die Art und Weise, wie der Unterricht organisiert und durchgeführt wird, bedingt sein.

Einige Länder haben nicht-selektive Schulsysteme, die allen Schülern die gleichen Lernmöglichkeiten bieten sollen und allen Schulen die Möglichkeit geben, die gesamte Palette der Schülerleistungen abzudecken. 
Andere Länder versuchen derVerschiedenheit der Schüler gerecht zu werden, indem sie sie bewusst (durch Selektion zwischen oder innerhalb der Schulen) in Gruppen mit ähnlichem Leistungsniveau einteilen, mit dem Ziel, sie entsprechend ihrem besonderen Bildungsbedarf bestmöglich zu fördern. In wieder anderen Ländern werden diese beiden Ansätze kombiniert. Selbst in Gesamtschulsystemen können große Unterschiede zwischen den einzelnen Schulen bestehen, bedingt z.B. durch die sozio-ökonomischen und kulturellen Merkmale des von ihnen versorgten Einzugsbereichs oder durch geographische Unterschiede. Der Indikator A7 betrachtet daher die Leistungsunterschiede zwischen den Schulen und untersucht, auf welche Faktoren diese Unterschiede zurückzuführen sind.

- Im Durchschnitt tragen bei den 15-Jährigen die Leistungsunterschiede zwischen den Schulen zu 36 Prozent zu der durchschnittlichen Gesamtvarianz der Schülerleistungen innerhalb der OECD bei, dieser Anteil variiert jedoch von 10 Prozent in Finnland und Schweden bis zu mehr als 50 Prozent in Belgien, Deutschland, Griechenland, Italien, Österreich, Polen, der Tschechischen Republik und Ungarn (S. 93).

- Ein Teil der Unterschiede zwischen den Schulen lässt sich auf geographische oder institutionelle Faktoren zurückführen, oder auf die Auswahl von Schülern nach ihren Fähigkeiten. Zu diesen Faktoren kommt oft auch noch der familiäre Hintergrund hinzu, insbesondere in Ländern mit einem differenzierten Schulsystem, da die Schülerleistungen nicht nur vom eigenen familiären Hintergrund, sondern auch - und zwar in größerem Maße - vom Hintergrund der Mitschüler an der Schule beeinflusst werden (S. 95).

\section{... der Chancengleich heit und der Bildungserfolge ...}

Schüler kommen aus ganz unterschiedlichen sozio-ökonomischen und kulturellen Verhältnissen. Daher müssen die Schulen sich bemühen, dieser vielfältig zusammengesetzten Schülerschaft adäquate und gleiche Bildungschancen zur Verfügung zu stellen. Das Lernumfeld der Schulen kann durch den unterschiedlichen Hintergrund und die vielfältigen Interessen der Schüler zwar angeregt werden, gleichzeitig vergrößern jedoch die Heterogenität der Fähigkeiten und die unterschiedliche Art der Schulvorbereitung die Herausforderung an die Schulen, den Anforderungen von Schülern mit ganz unterschiedlichem sozioökonomischen Hintergrund gerecht zu werden.

Um dieser Frage nachzugehen, untersuchen die Indikatoren A9 und A10 die Beziehungen zwischen den Schülerleistungen im Bereich Lesekompetenz und dem beruflichen Status ihrer Eltern, ihrem Geburtsort und der zu Hause gesprochenen Sprache. Auch wenn diese Merkmale keine direkten Ansatzpunkte für bildungspolitische Maßnahmen bieten, kann die Bestimmung der spezifischen Merkmale von Schülern, die mit großer Wahrscheinlichkeit niedrige Leistungen erzielen werden, Pädagogen und politischen Entscheidungsträgern dabei helfen, diejenigen Bereiche zu identifizieren, in denen Handlungsbedarf besteht. Wenn gezeigt werden kann, dass einige Länder weniger Probleme dabei haben, Schülern mit unterschiedlichem Hintergrund gerecht zu werden, könnten hieraus wichtige bildungspolitische Schlüsse gezogen werden, die auch in anderen Ländern zum Tragen kommen könnten.

- 15-Jährige, deren Eltern einen hohen beruflichen Status aufweisen, erbringen im Durchschnitt in allen drei Grundbildungsbereichen bessere Leistungen. In einigen Ländern ist der Vorteil der betreffenden Schüler jedoch viel größer als in anderen, was insbesondere auf Belgien, Deutschland, Luxemburg und die Schweiz zutrifft (S. 109).

- Zwar übt der sozio-ökonomische Hintergrund einen sehr starken Einfluss auf die Schülerleistungen aus, aber einige Länder - insbesondere Finnland, Island, Irland, Japan, Kanada und Korea - zeigen, dass hohe Durchschnittsleistungen und vom sozialen Hintergrund unbeeinflusste Lernerfolge durchaus Hand in Hand gehen können (S. 110). 
- In den meisten Ländern mit einer großen Migrantenpopulation liegt die Lesekompetenz von im Erhebungsland geborenen Schülern mit im Ausland geborenen Eltern weit unter der von im Erhebungsland geborenen Schülern mit mindestens einem im Inland geborenen Elternteil. Diese Unterschiede sind jedoch je nach Land unterschiedlich stark ausgeprägt (S. 115).

- Es überrascht nicht, das Schüler ohne einen majoritätssprachlichen Hintergrund wesentlich schlechter abschneiden als Schüler mit einem solchen. In allen Ländern gehören diese Schüler mit einer wesentlich größeren Wahrscheinlichkeit zu den leistungsschwächsten Schülern im betreffenden Land, wobei auch hier die Unterschiede zwischen den einzelnen Ländern unterschiedlich stark ausgeprägt sind (S. 116).

\section{... sowie der individuellen und gesellschaftlichen Bildungserträge.}

Da das Niveau an Kenntnissen und Fähigkeiten in der Regel mit zunehmendem Bildungsstand wächst, steigen auch die gesellschaftlichen Kosten, wenn die besser Ausgebildeten nicht arbeiten. Da gleichzeitig die Bevölkerung in den OECD-Ländern zunehmend älter wird, kann eine stärkere und längere Erwerbsbeteiligung zu einer Senkung der Abhängigkeitsquoten und der Entlastung der staatlichen Rentenkassen beitragen. Die Indikatoren A11 und A12 untersuchen die Beziehung zwischen Bildungsstand und Erwerbsquote, wobei zuerst die Erwerbsquoten und anschließend die Arbeitslosenquoten verglichen werden.

Die Märkte bieten auch durch Einkommensunterschiede Anreize für den Einzelnen, angemessene Fähigkeiten und Kenntnisse zu entwickeln und zu erhalten, und zwar insbesondere durch höhere Verdienstmöglichkeiten für diejenigen, die sich weiterbilden und zusätzliche Abschlüsse erzielen. Das Streben nach einem höheren Bildungsstand kann auch als Investition in das Humankapital gesehen werden. Das Humankapital ist der Bestand an Fähigkeiten und Kenntnissen, die der Einzelne besitzt oder - normalerweise durch Bildung oder Ausbildung - (weiter-)entwickelt und sodann als Gegenleistung für ein Einkommen auf dem Arbeitsmarkt anbietet. Je höher die Einkommen sind, die sich aus einer Vermehrung des Humankapitals ergeben, umso höher ist der Ertrag dieser Investition und der Einkommenszuschlag für bessere Fähigkeiten und Kenntnisse und/oder eine höhere Produktivität. Die Indikatoren A13 und A14 versuchen, die Erträge von Bildung zu messen, für den Einzelnen in Form der höheren Einkommen, für den Steuerzahler in Form eines höheren Steuereinkommens aufgrund besser ausgebildeter Arbeitskräfte, und für die Gesellschaften allgemein durch den Zusammenhang zwischen Bildung und wirtschaftlichem Wachstum. So beschreiben diese beiden Indikatoren die langfristigen Auswirkungen von Bildung für den Einzelnen und die Gesellschaft.

- In den meisten OECD-Ländern steigen die Erwerbsquoten mit zunehmendem Bildungsstand.Von sehr wenigen Ausnahmen abgesehen, ist die Erwerbsquote von Absolventen des Tertiärbereichs wesentlich höher als die von Absolventen des Sekundarbereich II. Besonders groß ist der Abstand zwischen den Erwerbsquoten von Männern mit einem Abschluss des Sekundarbereich II und ohne einen Abschluss im Sekundarbereich (S. 124).

- Obwohl auch bei den höchsten Bildungsabschlüssen noch eine Kluft zwischen der Erwerbsbeteiligung von Männern und Frauen besteht, ist sie hier doch weit geringer als bei den niedrigeren Bildungsabschlüssen (S. 125).

- Ein Fünfzehnjähriger kann damit rechnen, für 6,5 Jahre der kommenden 15 Jahre erwerbstätig, für insgesamt 0,8 Jahre arbeitslos und für 1,4 Jahre nicht auf dem Arbeitsmarkt zu sein. Am stärksten unterscheiden sich die Länder in der durchschnittlichen Länge der Phasen der Arbeitslosigkeit, was Unterschiede in den Beschäftigungsquoten junger Menschen widerspiegelt (S. 133).

- Zwischen Bildungsstand und Einkommen besteht eine positiver Zusammenhang. Insbesondere der Abschluss im Sekundarbereich II stellt in vielen Ländern einen Wendepunkt dar. Ab hier bringt jede zusätzliche Ausbildung einen besonders hohen Einkommenszuschlag mit sich. Frauen verdienen zwar im Vergleich zu Männern mit einem ähnlichen Bildungsstand nach wie vor weniger, aber bei einem höheren Bildungsstand sind die Unterschiede geringer (S. 140). 
- In allen Ländern liegt die individuelle Ertragsrate für die Investitionen in Bildung über den realen Zinssätzen, häufig sogar erheblich. Zwar liegt auch die gesellschaftliche Ertragsrate weit über den risikofreien Realzinsen, jedoch meistens unter der individuellen Ertragsrate, da Bildung erhebliche gesellschaftliche Kosten verursacht (S. 141).

- Tendenziell sind das Einkommensdifferential und die Dauer der Ausbildung die ausschlaggebenden Faktoren für die Erträge, aber auch andere Faktoren spielen eine Rolle, so z.B. Steuern, die sich ertragsmindernd auswirken, ein geringeres Arbeitslosigkeitsrisiko, das sich ertragssteigernd auswirkt, Studiengebühren, die sich ertragsmindernd auswirken und staatliche Beihilfen oder Darlehen, die sich wiederum ertragssteigernd auswirken (S. 146).

- Die Verbesserungen des Humankapitals sind ein bedeutender Faktor der Wachstumsprozesse der letzten Jahrzehnte gewesen. In den neunziger Jahren war in einigen Ländern mehr als ein halber Prozentpunkt Wachstum auf sie zurückzuführen (S. 153).

\section{Kapitel B untersucht die in Bildung investierten Finanz- und Humanressourcen und zwar in Form ...}

Die Finanzressourcen sind ein zentraler politischer Ansatzpunkt, wenn es darum geht, die Bildungsergebnisse zu verbessern. Bildung ist eine Investition in die Kenntnisse und Fähigkeiten von Menschen. Daher kann sie dazu beitragen, das Wirtschaftswachstum zu stärken, die Produktivität zu steigern, die persönliche und gesellschaftliche Entwicklung zu sichern und soziale Unterschiede zu verringern. Wie jede Investition verursacht Bildung ebenso Kosten wie sie zu Erträgen führt. Nachdem in Kapitel A die Erträge von Bildung untersucht werden, enthält Kapitel B eine vergleichende Untersuchung der Bildungsausgaben in den OECD-Ländern. Durch die stärkere Betonung von tendenziellen Entwicklungen bei den Bildungsausgaben wird in Bildung auf einen Blick 2002 untersucht, wie verschiedene Angebots- und Nachfragefaktoren sich gegenseitig beeinflussen, und wie sich die Ausgaben für Bildung im Verhältnis zu den Ausgaben in anderen Bereichen von großer gesellschaftlicher Bedeutung verändert haben.

\section{... der von jedem einzelnen Land in Bildung investierten Ressourcen in Relation zu der Zahl der Schüler/ Studierenden,...}

Leistungsfähige Bildungseinrichtungen brauchen die richtige Mischung aus gut ausgebildetem und begabtem Personal, modern ausstatteten Einrichtungen sowie motivierten und lernwilligen Schülern oder Studierenden. Allerdings muss die Forderung nach hochwertiger Bildung, die höhere Kosten pro Schüler/ Studierenden mit sich bringen kann, gegen die zumutbare Belastung für den Steuerzahler abgewogen werden. Es gibt keine absolut gültigen Richtwerte für die pro Schüler/Studierenden erforderlichen Ressourcen, um optimale Erträge für den einzelnen Schüler/Studierenden bzw. die Gesellschaft insgesamt zu erreichen. Internationale Vergleiche können jedoch als Ausgangspunkt für die Diskussion dienen, indem sie die Unterschiede bei den Bildungsinvestitionen unter den OECD-Ländern untersuchen. Indikator B1 beschreibt die direkten öffentlichen und privaten Ausgaben für Bildungseinrichtungen im Verhältnis zur Anzahl der vollzeitäquivalenten Schüler/Studierenden dieser Bildungseinrichtungen. Untersucht wird ferner, wie die einzelnen OECD-Länder ihre Ausgaben pro Schüler/Studierenden zwischen den verschiedenen Bildungsbereichen aufteilen.

- Im OECD-Raum insgesamt beziffern sich die Ausgaben der OECD-Länder auf 4.229 US-Dollar pro Schüler im Primarbereich, 5.174 US-Dollar im Sekundarbereich und 11.422 US-Dollar pro Studierenden im Tertiärbereich. Hinter diesen Durchschnittswerten stehen jedoch sehr unterschiedliche Ausgaben in den einzelnen Ländern (S. 164).

- Im Durchschnitt der OECD-Länder sind die Ausgaben pro Studierenden im Tertiärbereich 2,3-mal so hoch wie die Ausgaben pro Schüler im Primarbereich (S. 172). 
- Geringe jährliche Ausgaben pro Studierenden im Tertiärbereich führen in einigen OECD-Ländern aufgrund der langen Studienzeiten dennoch zu hohen Gesamtkosten im tertiären Bildungsbereich (S. 173).

- Im Tertiärbereich haben die Bildungsausgaben nicht immer mit dem raschen Anstieg der Studierendenzahlen Schritt gehalten (S. 167).

- Geringere Ausgaben pro Schüler/Studierenden können nicht automatisch mit einer geringeren Qualität der Bildungsdienstleistungen gleichgesetzt werden. So gehören beispielsweise Australien, Finnland, Korea und das Vereinigte Königreich, Länder mit moderaten Bildungsausgaben pro Schüler im Primar- und Sekundarbereich I, zu den OECD-Ländern mit den besten Leistungen 15-Jähriger in mathematischer Grundbildung (S. 168).

...zum Volkseinkommen und zum BIP pro Kopf,

Indikator B2 untersucht den Anteil nationaler Ressourcen, der für Bildungseinrichtungen bereitgestellt wird und die Bildungsbereiche, in die er fließt. Die Entscheidung, welcher Anteil der insgesamt zur Verfügung stehenden Finanzmittel für das Bildungswesen bereitgestellt werden soll, ist in jedem OECDLand von zentraler Bedeutung. An dieser Entscheidung sind Regierungen und Unternehmensleitungen ebenso wie der einzelne Schüler/Studierende und seine Familie beteiligt. Indikator B2 zeigt auch auf, wie sich im Verlauf der Jahre der Umfang der Bildungsausgaben in Relation zum Bruttoinlandsprodukt und in absoluten Zahlen verändert hat.

Indikator B3 vervollständigt das Bild der in Bildung investierten Ressourcen, indem die Veränderungen bei den öffentlichen Ausgaben für Bildung, sowohl absolut als auch im Verhältnis zu dem sich ändernden Umfang der öffentlichen Gesamtausgaben, untersucht werden. Bildung ist ein Bereich, in dem alle Staaten intervenieren, indem sie das Leistungsangebot finanzieren oder lenken. Da keine Garantie dafür besteht, dass die Märkte allen den gleichen Zugang zu Bildungschancen bieten, soll durch die staatliche Finanzierung der Bildung sichergestellt werden, dass sie der gesamten Gesellschaft zugänglich ist. Die öffentlichen Ausgaben für Bildung in Prozent der gesamten öffentlichen Ausgaben sind ein Indiz für den Stellenwert der Bildung im Vergleich zu anderen öffentlichen Aufgaben wie Gesundheitswesen, soziale Sicherung, Verteidigung und innere Sicherheit.

- Die OECD-Länder geben 5,8 Prozent der Gesamtsumme ihrer Bruttoinlandsprodukte für Bildungseinrichtungen aus (S. 182).

- In 14 von 18 OECD-Ländern stiegen zwischen 1995 und 1999 die privaten und öffentlichen Ausgaben für Bildung um mehr als 5 Prozent (S. 182).

- Im Durchschnitt wenden die OECD-Länder 12,7 Prozent ihrer gesamten öffentlichen Ausgaben für Bildungseinrichtungen auf (S. 196).

- In vier von fünf OECD-Ländern stiegen die öffentlichen Bildungsausgaben zwischen 1995 und 1999 real um mehr als 5 Prozent (S. 197).

- Die öffentlichen Ausgaben für Bildung stiegen eher schneller als die gesamten öffentlichen Ausgaben, jedoch langsamer als das BIP. In Italien, den Niederlanden, Schweden und dem Vereinigten Königreich stiegen die öffentlichen Bildungsausgaben zwischen 1995 und 1999 trotz real sich verringernder öffentlicher Haushalte (S. 197). 


\section{... der Finanzierung der Bildungssysteme sowie der Herkunft der investierten Mittel, ...}

Die Lastenverteilung bei den Kosten zwischen den Teilnehmern der Bildungsangebote einerseits und der Gesellschaft als Ganzes andererseits ist in vielen OECD-Ländern ein Gegenstand intensiver Debatten. Die Frage nach der Aufteilung der Kosten stellt sich insbesondere zu Beginn und Ende des Bildungsweges, also für den Elementar- und Tertiärbereich, wo eine staatliche Vollfinanzierung oder nahezu vollständige Finanzierung durch den Staat weniger üblich ist. Angesichts einer wachsenden Beteiligung neuer Gruppen von Bildungsinteressenten und eines immer breiteren Angebots an Bildungsmöglichkeiten, Bildungsgängen und Bildungsanbietern gehen die Regierungen neue Partnerschaften für die Mobilisierung der für die Bildungsfinanzierung erforderlichen Ressourcen ein. Die öffentlichen Bildungsausgaben werden heute immer mehr als nur ein - wenn auch sehr wichtiger - Teil der Investitionen in die Bildung gesehen. Die privaten Finanzierungsquellen gewinnen zunehmend an Bedeutung. Um hier zu einem besseren Verständnis zu gelangen, untersucht Indikator B4 die relativen Anteile öffentlicher und privater Investitionen in Bildungseinrichtungen und deren Veränderung gegenüber 1995.

Neue Finanzierungsstrategien zielen nicht nur darauf ab, die Finanzierung durch öffentliche und private Mittel auf eine breitere Basis zu stellen, sondern auch darauf, das Bildungsangebot auszuweiten und die Wirtschaftlichkeit der Schulen zu verbessern. Zwar wird in den meisten OECD-Ländern die Bildung im öffentlich finanzierten Primar- und Sekundarbereich auch von öffentlichen Einrichtungen organisiert und bereitgestellt, es gibt aber auch eine nennenswerte Anzahl von OECD-Ländern, in denen die öffentlichen Mittel im Endeffekt an private Einrichtungen oder direkt an die privaten Haushalte gehen, damit diese die betreffenden Mittel an die Bildungseinrichtungen ihrer Wahl weiterleiten. Im erstgenannten Fall kann die letztendliche Finanzierung und Bereitstellung von Bildungsangeboten als eine Art Auftragsvergabe durch die öffentliche Hand an nichtöffentliche Einrichtungen betrachtet werden, während im letztgenannten Fall den Schülern und ihren Familien die Entscheidung überlassen wird, welche Art von Einrichtung ihren Bedürfnissen am besten entspricht. Indikator B4 untersucht auch die Aufteilung der Mittel auf öffentliche und private Bildungseinrichtungen.

- Der private Anteil an den Gesamtausgaben für Bildungseinrichtungen reicht von etwa 3 Prozent und weniger in Finnland, Norwegen, Portugal, Schweden, der Slowakischen Republik und der Türkei bis zu 40 Prozent in Korea (S. 203).

- In einigen OECD-Ländern zahlt der Staat den größten Teil der Kosten für den Primar-, Sekundar- und post-sekundären, nicht-tertiären Bereich, überlässt jedoch die Leitung der Bildungseinrichtungen dem privaten Sektor, um so ein breiteres Lernangebot ohne Zugangsbeschränkungen für Schüler/Studierende aus einkommensschwachen Familien bereitzustellen. In Belgien und den Niederlanden besucht die Mehrzahl der Schüler im Primar- und Sekundarbereich solche staatlich-subventionierten privaten Bildungseinrichtungen. In Australien, Frankreich, Korea, Spanien und dem Vereinigten Königreich beträgt ihr Anteil immer noch mehr als 20 Prozent (S. 204).

- Im Primar- und Sekundarbereich sind private Einrichtungen, die überwiegend von privaten Haushalten finanziert werden, sehr viel seltener als staatlich finanzierte Einrichtungen (S. 205).

- Tendenziell beziehen tertiäre Bildungseinrichtungen einen weitaus größeren Teil ihrer Finanzierung aus privaten Quellen als Bildungseinrichtungen des Primar-, Sekundar- und des post-sekundären, nicht-tertiären Bereichs. Der Anteil der privaten Finanzierung reicht von 3 Prozent und weniger in Belgien (fläm.), Dänemark, Finnland, Griechenland, Österreich und der Schweiz bis zu 78 Prozent in Korea (S. 207).

- In 10 von 19 OECD-Ländern sind zwischen 1995 und 1999 die privaten Ausgaben für den Tertiärbereich um mehr als 30 Prozent gestiegen. In den meisten Ländern hatte dies jedoch keine Verringerung der öffentlichen Ausgaben für den Tertiärbereich zur Folge (S. 209). 


\section{... der unterschiedlichen Finanzierungsinstrumente ...}

In den meisten OECD-Ländern besteht der Hauptfinanzierungsmechanismus für Bildung immer noch in direkten Ausgaben für Bildungseinrichtungen. Die Staaten bemühen sich jedoch verstärkt um eine größere Diversifizierung der Finanzierungsinstrumente. Ein Vergleich solcher Instrumente kann hilfreich dabei sein, politische Alternativen auszumachen. Subventionen an Schüler/Studierende und ihre Familien, der Schwerpunkt von Indikator B5, stellen eine solche Alternative zu den Direktausgaben für Bildungseinrichtungen dar. Regierungen tragen mittels Subventionen zur Deckung der direkten und indirekten Bildungskosten bei, um den Bildungszugang zu erweitern und soziale Ungleichheiten abzubauen. Darüber hinaus spielen öffentliche Subventionen auch bei der indirekten Finanzierung von Bildungseinrichtungen eine nicht unerhebliche Rolle. Indem Finanzierungsmittel für Bildungseinrichtungen über Schüler/Studierende gelenkt werden, kann eine größere Effizienz der Bildungsfinanzierung erreicht werden. Da Zuschüsse zu den Lebenshaltungskosten der Schüler/Studierenden eine Erwerbstätigkeit zur Finanzierung von Bildung ersetzen können, tragen öffentliche Subventionen vermutlich auch dazu bei, den Bildungsstand anzuheben, indem Schülern/Studierenden die Möglichkeit eines Vollzeitschulbesuchs bzw. Vollzeitstudiums gegeben wird, auch wenn sie durch ihr Studium nur vermindert oder überhaupt keiner bezahlten Tätigkeit nachgehen können.

Öffentliche Subventionen können in vielfältiger Weise bereitgestellt werden: als einkommensabhängige Zuschüsse, als Familienbeihilfen für alle Schüler/Studierenden, als Steuerfreibeträge für Schüler/ Studierende oder ihre Eltern oder in Form sonstiger Transferleistungen an private Haushalte. Sollen Finanzhilfen an private Haushalte in Form von Zuschüssen oder Darlehen gewährt werden? Können Finanzhilfen in Form von Darlehen dazu beitragen, dieWirksamkeit der in Bildung investierten Ressourcen zu erhöhen und einen Teil der Bildungskosten auf die Nutznießer der Bildungsinvestitionen zu verlagern? Oder sind Studiendarlehen weniger wirksam als Zuschüsse, wenn es darum geht, einkommensschwache Schüler/Studierende zu mehr Bildung zu ermutigen? Dieser Indikator kann diese Fragen zwar nicht beantworten, er kann jedoch die von den einzelnen OECD-Ländern in diesem Bereich verfolgte Subventionspolitik aufzeigen.

- Durchschnittlich 16 Prozent der öffentlichen Ausgaben für den Tertiärbereich betreffen finanzielle Unterstützungsleistungen an Studierende, private Haushalte und andere private Einheiten. In Australien, Dänemark und dem Vereinigten Königreich machen die öffentlichen Subventionen ungefähr ein Drittel und mehr der öffentlichen Bildungsetats für den Tertiärbereich aus (S. 219).

- Subventionen sind besonders dort von Bedeutung, wo von den Schülern/Studierenden erwartet wird, dass sie wenigstens einen Teil der Kosten für ihre Bildung selbst tragen (S. 219).

- In den meisten OECD-Ländern genießen die Empfänger von Subventionen einen erheblichen Ermessensspielraum hinsichtlich der Verwendung der erhaltenen Subventionen. In allen OECD-Ländern, über die Zahlenmaterial vorliegt, werden die Zuschüsse hauptsächlich außerhalb der Bildungseinrichtungen ausgegeben, und in jedem dritten Land ist dies ausschließlich der Fall (S. 219).

\section{... und der Aufteilung der Mittel auf die unterschiedlichen Ausgabenkategorien.}

Kapitel B schließt mit einer Untersuchung der Aufteilung der Finanzressourcen auf die unterschiedlichen Ausgabenkategorien (Indikator B6). Die Zuweisung der Finanzmittel kann sich auf die Qualität des Unterrichts auswirken (z.B. durch die relativen Ausgaben für Lehrergehälter), auf den Zustand der Bildungseinrichtungen (z.B. durch die Instandhaltungsausgaben für Schulgebäude) sowie auf die Anpassungsfähigkeit des Bildungssystems an sich ändernde Entwicklungstendenzen der Bevölkerung und der Bildungsbeteiligung. OECD-Ländervergleiche über die Verteilung der Bildungsausgaben auf 
die verschiedenen Ressourcenkategorien können Einblick in das Ausmaß der Unterschiede im Bereich der Organisationsstrukturen und der Leitung von Bildungseinrichtungen gewähren. Die auf der jeweils zuständigen Ebene getroffenen Verteilungsentscheidungen - sowohl zu Fragen des gesamten Bildungsetats als auch seiner Aufteilung - finden letztendlich ihren Niederschlag im Klassenzimmer und beeinflussen sowohl die Art des Unterrichts als auch die Bedingungen, unter denen er stattfindet.

- Im Durchschnitt entfällt ein Viertel der Ausgaben für den Tertiärbereich auf Forschung und Entwicklung an tertiären Bildungseinrichtungen. Der Umfang von Forschung und Entwicklung an tertiären Einrichtungen unterscheidet sich erheblich zwischen den einzelnen OECD-Ländern, was teilweise die großen Unterschiede bei den Ausgaben pro Studierenden an tertiären Einrichtungen erklären kann (S. 227).

- Die Ausgaben für zusätzliche Dienstleistungen im Primar-, Sekundar- und post-sekundären, nicht-tertiären Bildungsbereich machen durchschnittlich 5 Prozent der Gesamtausgaben für Bildungseinrichtungen aus. Dies ist in der Regel mehr als die OECD-Länder für Subventionen an private Haushalte aufwenden (S. 227).

- Primar-, Sekundar- und post-sekundären, nicht-tertiären Bildungsbereich zusammengenommen machen die laufenden Ausgaben im Durchschnitt aller OECD-Länder 92 Prozent der Gesamtausgaben aus. Mit Ausnahme von vier OECD-Ländern machen die Personalkosten mindestens 70 Prozent der laufenden Ausgaben aus (S. 228).

- Im Tertiärbereich verwenden die OECD-Länder tendenziell einen höheren Teil der laufenden Ausgaben für Dienstleistungen, die per Auftrag vergeben oder zugekauft werden (S. 230).

\section{In Kapitel $C$ werden der Bildungszugang, die Bildungsbeteiligung und die Bildungserwartung untersucht, und zwar in Form ...}

Eine gut ausgebildete Bevölkerung ist zu einem bestimmenden Merkmal für eine moderne Gesellschaft geworden. Bildung dient der Vermittlung gesellschaftlicher Werte und ist ein Mittel zur Entwicklung des Leistungsvermögens und der sozialen Fähigkeiten jedes Einzelnen. Programme im Elementarbereich bereiten die Kinder auf den Primarbereich vor. Primar- und Sekundarbereich statten junge Menschen mit grundlegenden Kompetenzen aus und bereiten sie auf lebenslanges Lernen und die Entwicklung hin zu produktiven Mitgliedern der Gesellschaft vor. Der Tertiärbereich bietet dem Einzelnen eine Vielzahl von Möglichkeiten, entweder direkt nach der Schulbildung oder später im Leben erweiterte und spezielle Kenntnisse und Fähigkeiten zu erlangen. Viele Arbeitgeber ermutigen und unterstützen es, wenn Mitarbeiter ihre Fähigkeiten und Kenntnisse auffrischen, sich weiterbilden und neu orientieren, um den Anforderungen sich ständig ändernder Technologien gerecht zu werden. Kapitel C zeichnet ein vergleichendes Bild des Bildungszugangs, der Bildungsbeteiligung und des Fortschreitens auf dem Bildungsweg in den OECD-Ländern.

\section{... der Bildungserwartung in Jahren, sowohl insgesamt als auch in den einzelnen Bildungsbereichen,...}

Informationen über die zu erwartende Ausbildungsdauer und die Bildungsbeteiligung in den verschiedenen Bildungsbereichen (Indikator C1) vermitteln einen Einblick in die Struktur der verschiedenen Bildungssysteme sowie den Zugang zum Bildungsangebot dieser Systeme. Die Entwicklungstendenzen der Bildungsbeteiligung in den verschiedenen Bildungsbereichen und Bildungseinrichtungen dienen ebenfalls als Indikatoren dafür, wie Angebot und Nachfrage nach Bildungsressourcen in den einzelnen Ländern gegeneinander abgewogen werden. 
- In 25 von 27 OECD-Ländern nimmt der Einzelne im Durchschnitt zwischen 15 und 20 Jahren an formellen Bildungsmaßnahmen teil, wobei der Großteil der Unterschiede auf eine unterschiedliche Bildungsbeteiligung im Sekundarbereich II zurückzuführen ist (S. 241).

- In 18 von 20 OECD-Ländern stieg die Bildungserwartung (in Jahren) zwischen 1995 und 2000. In Australien, Finnland, Griechenland, Korea, Polen, der Tschechischen Republik, Ungarn und dem Vereinigten Königreich betrug der Anstieg über diesen relativ kurzen Zeitraum hinweg mehr als ein Jahr (S. 242).

- In zwei Fünftel der OECD-Länder werden mehr als 70 Prozent der 3- bis 4-Jährigen im Elementar- oder Primarbereich unterrichtet. Am anderen Ende des Spektrums kann ein heute 17-Jähriger davon ausgehen, dass er voraussichtlich im Durchschnitt 2,5 Jahre im tertiären Bildungsbereich verbringen wird (S. 243).

- In der Mehrheit der OECD-Länder können Frauen erwarten, länger in Ausbildung zu verbleiben als Männer - im Durchschnitt zusätzliche 0,5 Jahre. In Korea, der Schweiz und der Türkei können jedoch Männer erwarten, zwischen 0,7 und 2,8 Jahren länger als Frauen in Ausbildung zu verbleiben (S. 242).

\section{...des Zugangs zu und der Bildungsbeteiligung an verschiedenen Bildungsgängen und Bildungseinrichtungen, ...}

Praktisch alle jungen Menschen in den OECD-Ländern können erwarten, mindestens 11 Jahre lang an formeller Bildung teilzunehmen. Wer jedoch wann, wie und für wie lange an welchen Bildungsmaßnahmen teilnimmt, das variiert während der gesamten Lebensspanne erheblich. Die Bildungsbeteiligung während der Vorschuljahre und nach Beendigung der Schulpflicht weicht in den einzelnen Ländern erheblich voneinander ab. In einigen Ländern wurde die Bildungsbeteiligung zum Beispiel dadurch erhöht, dass eine nahezu vollständige Vorschulbildung ab dem Alter von drei Jahren eingeführt wurde, dass man die Mehrheit der Jugendlichen bis zum Alter von 18 oder 19 Jahren im Bildungssystem behält, oder dadurch, dass in den Altersgruppen bis Ende 20 eine Bildungsbeteiligung von 10 bis 20 Prozent erzielt wurde. Hohe Studienanfängerquoten und eine hohe Bildungsbeteiligung im Tertiärbereich tragen dazu bei, die Entwicklung und den Erhalt einer hochqualifizierten Bevölkerung und Erwerbsbevölkerung sicherzustellen. Die Studienanfängerquoten für beide Tertiärbereiche (Indikator C2) sind zum Teil auch ein Anzeichen dafür, inwieweit die Bevölkerung hochqualifizierte Fähigkeiten und Kenntnisse anstrebt, die auf dem Arbeitsmarkt einer Wissensgesellschaft von Bedeutung sind.

Während ein erfolgreicher Abschluss des Sekundarbereich II in den meisten OECD-Ländern zunehmend zum Normalfall wird, gestalten sich die Wege zu diesem Bildungsziel immer vielfältiger. Die Bildungsgänge im Sekundarbereich II können sich beispielsweise hinsichtlich der Lerninhalte unterscheiden, die oftmals davon abhängen, auf welche weiteren Bildungsgänge oder welchen Beruf die Schüler vorbereitet werden sollen. Die meisten Bildungsgänge im Sekundarbereich II in den OECD-Ländern zielen vornehmlich auf die Vorbereitung zum weiterführenden Studium im Tertiärbereich ab. Diese Bildungsgänge können allgemeinbildend, berufsvorbereitend oder berufsbildend ausgerichtet sein. Neben Bildungsgängen, die die Schüler hauptsächlich auf eine weiterführende Bildung vorbereiten, gibt es in den meisten Ländern auch Bildungsgänge im Sekundarbereich II, die zurVorbereitung auf den direkten Eintritt in den Arbeitsmarkt ausgelegt sind. Indikator C2 untersucht auch die Schülerzahlen in diesen unterschiedlichen Bildungsgängen.

- Heutzutage ist davon auszugehen, dass vier von zehn Schulabgängern an tertiären Bildungsgängen teilnehmen, die auf das Äquivalent eines Bachelor-Abschlusses oder eines höheren Abschlusses im Tertiärbereich A hinführen. In einigen OECD-Ländern liegt dieser Anteil sogar bei der Hälfte aller Schulabgänger (S. 249).

- Mit Ausnahme von Deutschland, Frankreich und der Türkei hat die Bildungsbeteiligung im Tertiärbereich zwischen 1995 und 2000 in allen OECD-Ländern zugenommen, in den meisten OECD-Ländern um mehr als 15 Prozent und in Korea, Polen, der Tschechischen Republik und Ungarn um mehr als 50 Prozent (S. 251). 
- Die Mehrheit der Schüler im Primar- und Sekundarbereich besucht öffentliche Bildungseinrichtungen, inzwischen besuchen jedoch auch durchschnittlich 11 Prozent der Schüler im Primarbereich, 14 Prozent der Schüler im Sekundarbereich I und 19 Prozent der Schüler im Sekundarbereich II privat geleitete Bildungseinrichtungen. In Belgien und den Niederlanden ist es sogar in allen Bildungsbereichen die Mehrheit der Schüler und in Korea und dem Vereinigten Königreich die Mehrheit der Schüler im Sekundarbereich II (S. 251).

- Während die Mehrzahl der Studierenden an öffentlichen Bildungseinrichtungen eingeschrieben ist, besuchen in OECD-Ländern wie Belgien, Japan, Korea, den Niederlanden und dem Vereinigten Königreich die meisten Studierenden privat geleitete Bildungseinrichtungen (S. 251).

\section{... des Lernens über die Erstausbildung hinaus...}

Es gibt zahlreiche Anzeichen dafür, dass ein größeres Ausmaß an sekundärer und tertiärer Bildung für junge Menschen deren wirtschaftliche und gesellschaftliche Aussichten verbessert. Es gibt auch immer mehr, wenn auch weniger direkte, Anzeichen dafür, dass sich eine Anhebung des allgemeinen Bildungsstands auch für die Gesellschaft insgesamt auszahlt (Indikatoren A13 and A14). Da jedoch technologischer Fortschritt und zunehmende Globalisierung die Art der Nachfrage nach qualifizierten Arbeitnehmern in der ganzen Welt verändern, kann die Erhöhung des Anteils junger Menschen in Bildungsgängen des Sekundarbereich II und des Tertiärbereichs aus einer Reihe von Gründen nur ein Teil der Lösung sein. Erstens wird der Zuwachs an besser ausgebildeten jungen Menschen den allgemeinen Bildungsstand der bestehenden Erwerbsbevölkerung nur allmählich ändern. Zweitens ist der Bildungsstand nur ein Element des Humankapitals. Menschen erwerben im Laufe ihres Lebens ständig neue Kenntnisse und Fähigkeiten, nicht nur im Rahmen formeller Bildung, sondern auch durch Erfahrungen in der Familie, im Gemeinwesen und am Arbeitsplatz. Entwicklungsstrategien für Möglichkeiten des lebenslangen Lernens dürfen sich daher nicht nur auf die herkömmlichen Bildungseinrichtungen konzentrieren, sondern müssen darüber hinaus gehen, um optimale Investitionen in das Humankapital sicherzustellen. Indikator C4 fasst die Ergebnisse der Internationalen Untersuchung der Lesefähigkeit von Erwachsenen (International Adult Literacy Survey-IALS, 1994 und 1998) und von nationalen Haushaltsumfragen zur Fort- und Weiterbildung von Erwachsenen zusammen und ermöglicht damit ein besseres Verständnis der Beteiligung an berufsbezogener Fort- und Weiterbildung aller Beschäftigten.

- In der Hälfte der OECD-Länder, die Daten zur Verfügung gestellt haben, nahmen über 40 Prozent der Erwachsenenbevölkerung im Zeitraum von zwölf Monaten an irgendeiner Form von Fort- oder Weiterbildungsmaßnahme teil (S. 277).

- Häufigkeit und Intensität der Fort- und Weiterbildungsmaßnahmen schwanken stark zwischen den einzelnen OECD-Ländern. Die Beteiligungsquoten liegen zwischen maximal 18 Prozent in Polen, Portugal und Ungarn und über 50 Prozent in Dänemark, Finnland, Schweden und den Vereinigten Staaten (S. 277).

- In 11 von 19 OECD-Ländern ist die Wahrscheinlichkeit der Teilnahme von Erwachsenen mit einem tertiären Abschluss an berufsbezogener Fort- und Weiterbildung zwei- bis dreimal so hoch wie von Erwachsenen ohne einen Abschluss des Sekundarbereich II, so dass der Bildungsstand in Kombination mit anderen Einflussfaktoren dazu beiträgt, dass Weiterbildung für Erwachsene innerhalb der größten Risikogruppe am wenigsten verbreitet ist (S. 277).

- Frauen mit einem niedrigeren Bildungsstand erhalten meist weniger berufsbezogene Fort- und Weiterbildung, doch ist dieser Unterschied bei denjenigen mit einem Abschluss des Sekundarbereich II bzw. einem Abschluss im Tertiärbereich weniger stark ausgeprägt (S. 278). 


\section{... und der grenzüberschreitenden Mobilität Studierender.}

Die internationale Dimension der Hochschulausbildung findet immer mehr Beachtung. Der generelle Trend zur Freizügigkeit von Kapital, Waren und Arbeitskräften zusammen mit der zunehmenden Öffnung der Arbeitsmärkte hat in den OECD-Ländern die Nachfrage nach modernen Kenntnissen und Fähigkeiten verstärkt. Die Regierungen erwarten zunehmend von den Hochschulen, dass sie den Horizont junger Menschen erweitern und ihnen ein umfassenderes Verständnis fremder Sprachen, Kulturen und wirtschaftswissenschaftlicher Methoden vermitteln.

Eine Möglichkeit für Studierende, ihre Kenntnisse über andere Kulturen und Gesellschaften zu erweitern, besteht darin, tertiäre Bildungseinrichtungen anderer Länder zu besuchen. Die internationale Mobilität von Studierenden (Indikator C3) impliziert für die Studierenden ebenso wie für die Bildungseinrichtungen Kosten und Nutzen, und zwar sowohl in den 'Sender' - als auch den 'Empfänger'-Ländern. Während sich die kurzfristigen monetären Kosten und der Nutzen dieser Mobilität relativ leicht erfassen lassen, sind die längerfristigen sozialen und wirtschaftlichen Vorteile für die Studierenden, die Bildungseinrichtungen und auch die beteiligten Länder selbst schwer zu quantifizieren. Zahlen im Ausland studierender junger Menschen vermitteln jedoch einen ersten Eindruck von dem Ausmaß dieser grenzüberschreitenden Mobilität Studierender.

- Sieben von zehn ausländischen Studierenden im OECD-Raum konzentrieren sich auf 5 Gastländer: Australien, Frankreich, Deutschland, das Vereinigte Königreich und die Vereinigten Staaten (S. 264).

- Absolut gesehen entsenden Korea, Japan und Griechenland die meisten der im Ausland Studierenden aus OECDLändern, von außerhalb des OECD-Raums kommen die meisten Studierenden aus China und Südostasien (S. 266).

- Relativ gesehen schwankt der Anteil der ausländischen Studierenden in den OECD-Ländern von weniger als 1 Prozent bis zu fast 17 Prozent. Proportional zu ihrer Größe weisen Australien, Belgien, Österreich, die Schweiz und das Vereinigte Königreich den größten Nettozugang ausländischer Studierender auf (gemessen in Prozent der Studierendenzahlen in diesen Ländern) (S. 267).

In Kapitel D wird das Lernumfeld und die Organisation der Schulen untersucht, und zwar in Form ...

Wärend in den Kapiteln A, B und C die in Bildung investierten finanziellen Mittel, die Beteiligungsmuster an Bildung und die Ergebnisse von Bildung in Form von Schülerleistungen und der Auswirkungen von Qualifikationen für das spätere Erwerbsleben untersucht wurden, befasst sich Kapitel D mit den Lehrund Lernbedingungen im öffentlichen Bildungswesen. Das Lernen in der Schule erfolgt meist in Form von Unterricht im Klassenzimmer, wobei den Lehrkräften eine Schlüsselrolle dabei zukommt, das Lernen der Schüler zu planen und zu steuern. Die ersten fünf Indikatoren untersuchen die schulischen Bedingungen aus Sicht der Lernenden, während sich die letzten beiden Indikatoren dieses Kapitels mit Informationen zu den Arbeitsbedingungen der Lehrkräfte beschäftigen.

$$
\text { ... der Unterrichts- und Lernbedingungen der Schüler, ... }
$$

Wie effektiv die zum Lernen vorhandene Zeit genutzt wird, hängt zum einen davon ab, wie angemessen die einzelnen Bildungsgänge sind und zum anderen, wie viel Unterricht der einzelne Schüler enthält. Indikator D1 untersucht die in verschiedenen Fachbereichen vorgesehene Unterrichtszeit für 9- bis 14-jährige Schüler. Unterschiedliche Klassengrößen und die damit verbundene Frage, wie sehr sich der einzelne Schüler die Aufmerksamkeit des Lehrers mit anderen teilen muss, sind eine weitere Variable, die es zu berücksichtigen gilt, wenn untersucht wird, wie die Unterrichtszeit genutzt wird. Indikator D2 betrachtet die Bandbreite der durchschnittlichen Klassengrößen sowie des zahlenmäßigen Schüler/Lehrer-Verhätnisses in den OECDLändern, um die Humanressourcen zu erfassen, die letztlich dem einzelnen Schüler zur Verfügung stehen. 
- Im Durchschnitt verbringen 9- bis 11-jährige Schüler 841 Stunden im Jahr im Klassenzimmer, die 1214-Jährigen fast einhundert Stunden mehr. Es gibt hierbei jedoch erhebliche Unterschiede zwischen den einzelnen Ländern (S. 310).

- Im Durchschnitt entfällt bei den 9- bis 11-Jährigen ungefähr die Hälfte des Pflichtunterrichts auf das Lesen und Schreiben in der Unterrichtssprache, Mathematik und die Naturwissenschaften, bei den 12- bis 14Jährigen liegt der Anteil dieser Fächer bei 40 Prozent (S. 309).

- Im Durchschnitt verwenden 15-jährige Schüler zusätzlich zum Unterricht im Klassenzimmer pro Woche 4,6 Stunden auf Hausaufgaben und das Lernen in der Unterrichtssprache, Mathematik und den Naturwissenschaften (S. 311).

- Im Durchschnitt erhält jeder dritte 15-Jährige, zumindest gelegentlich, Nachhilfe oder Privatunterricht (S. 312).

- Die durchschnittliche Klassengröße liegt im Primarbereich bei 22 Schülern, sie reicht jedoch von 36 Schülern pro Klasse in Korea bis zu weniger als der Hälfte dieser Zahl in Griechenland, Island und Luxemburg (S. 319).

\section{... der Verfügbarkeit und Nutzung von Computern in der Schule und zu Hause,...}

Abgesehen von der Zeit im Klassenzimmer und den Humanressourcen gewinnen die modernen Technologien in der Bildung immer mehr an Bedeutung. Sie statten die Schüler nicht nur mit den notwendigen Fähigkeiten und Fertigkeiten aus, um effektiv an der modernen Welt teilzunehmen, sondern fördern auch die Entwicklung von selbstgesteuerten Lernstrategien und Fähigkeiten, essentiellen Bestandteilen einer soliden Grundlage für lebenslanges Lernen. Die Präsenz moderner Informationsund Telekommunikationstechnologien in der Schule alleine garantiert zwar keineswegs deren sinnvolle Nutzung, aber ihre Verfügbarkeit ist eine entscheidende Voraussetzung für die Verbesserung der Lehrund Lernbedingungen an den Schulen und für die Chancengleichheit im Bildungswesen. Indikator D3 betrachtet die Verfügbarkeit von Computern zu Hause und in der Schule und den Einsatz von Computern beim Lehren und Lernen. Indikator D4 geht darüber hinaus und untersucht die Einstellungen und Erfahrungen der Schülerinnen und Schüler hinsichtlich der Nutzung von Computern.

- Im OECD-Durchschnitt besucht ein typischer 15-jähriger Schüler eine Schule, an der sich 13 Schüler einen Computer teilen. Diese Zahl variiert jedoch stark von Land zu Land, in einigen Ländern sogar zwischen einzelnen Regionen und Schulen (S. 328).

- Im OECD-Durchschnitt gaben etwa ein Drittel der 15-Jährigen an, in der Schule täglich oder wenigstens mehrmals pro Woche einen Computer zu benutzen, zu Hause jedoch wird fast doppelt so häufig ein Computer genutzt. Der Anteil der Schüler, die angeben, zu Hause nie Zugang zu einem Computer zu haben ist jedoch 10 Prozentpunkte höher als der Anteil derjenigen, die dasselbe für die Schule sagten. Das legt die Vermutung nahe, dass die Schule eine wichtige Rolle dabei spielt, die Bildungslücke zwischen Schülern, die einen unterschiedlich ausgeprägten Zugang zu Computern haben, zu schließen (S. 329).

- Im OECD-Durchschnitt sind die 15-jährigen Jungen deutlich vertrauter mit Computern und schätzen ihre Fähigkeiten im Umgang mit Computern signifikant höher ein als die Mädchen. Die geschlechtsspezifischen Unterschiede sind in Ländern wie Dänemark, Finnland und Schweden am größten und in Australien, Neuseeland, Schottland und den Vereinigten Staaten am geringsten (S. 341).

- In allen OECD-Ländern, mit Ausnahme von Irland, Mexiko und den Vereinigten Staaten, gaben die 15jährigen Jungen ein signifikant höheres Interesse an Computern an als die Mädchen (S. 343). 
Lehrer haben ein relativ hohes $\mathrm{Ma} ß$ an Freiheit bei der Organisation der Lernaktivitäen ihrer Schüler sowie bei der Bewertung ihrer Fortschritte. Ihr Fachwissen, ihre pädagogischen Fähigkeiten, ihre Disziplin, Begeisterung und Einsatzfreude sind wichtige Determinanten des Lernklimas im Unterricht und der Schule insgesamt. Darüber hinaus beeinflussen jedoch Faktoren wie die Disziplin der Schüler, die Verfügbarkeit von Bildungsressourcen und die Autonomie der Schule das Arbeitsklima an der Schule, was wiederum einen großen Einfluss auf die Lernerfolge hat. Indikator D5 untersucht zunächst die Aspekte des Unterrichtsklimas, die als förderlich für das Lernen von 15-Jährigen gelten, sowie die länderspezifischen Unterschiede bei diesen Faktoren. Außerdem liefert der Indikator Indizes zum Arbeitsklima an den Schulen, wobei gewisse Unterschiede zwischen den einzelnen Ländern hinsichtlich der für das Schulklima relevanten Faktoren ersichtlich werden.

- Im Vergleich zum OECD-Mittel erhielten 15-jährige Schüler nach eigenen Angaben in Australien, Kanada, Neuseeland, Portugal, Schweden, dem Vereinigten Königreich und den Vereinigten Staaten mehr Unterstützung durch die Lehrkräfte als die Schüler in Belgien, Deutschland, Italien, Korea, Luxemburg, Österreich, Polen und der Tschechischen Republik (S. 353).

- Im Durchschnitt gab jeder dritte 15-Jährige an, dass zu Beginn einer Schulstunde mehr als fünf Minuten vergingen, in denen nichts passiere und mehr als jeder vierte beklagte sich darüber, dass es laut sei und alles durcheinander gehe (S. 353).

- Über die Hälfte der 15-Jährigen in Australien, Dänemark, Irland, Neuseeland, Norwegen, Schweden und dem Vereinigten Königreich, aber weniger als zehn Prozent der Schüler in Finnland und Ungarn gaben an, regelmäßig ein Biologie-, Physik- oder Chemielabor zu benutzen (S. 355).

- In leistungsstarken Ländern werden die Schulressourcen tendenziell häufiger genutzt, die Schulen haben einen höheren Grad der Autonomie, die Stimmung und Arbeitshaltung der Lehrkräfte ist besser und die Beziehungen zwischen Schülern und Lehrern sind in der Regel besser, dagegen sind in den leistungsschwachen Länder verstärkt negative Indizes beim Schulklima zu beobachten, und die Indizes der Nutzung der Schulressourcen, der Stimmung und Arbeitshaltung der Lehrkräfte, der Autonomie der Schule und der Lehrer-Schüler-Beziehung liegen in der Regel unter dem OECD-Durchschnitt (S. 357).

\section{... und der Arbeitsbedingungen der Lehrer.}

Kapitel D schließt mit einer vergleichenden Übersicht der Arbeitsbedingungen der Lehrer. Im Bildungssystem ist eine große Anzahl von qualifizierten Kräften unter zunehmend wettbewerbsorientierten Marktbedingungen beschäftigt und allen OECD-Ländern ist es ein großes Anliegen, dafür Sorge zu tragen, dass es genug qualifizierte Lehrer gibt. Das Angebot an qualifizierten Lehrkräften wird hauptsächlich bestimmt durch die Gehälter und Arbeitsbedingungen von Lehrern, einschließlich dem Anfangsgehalt und dem System der Besoldungs- und Vergütungsgruppen, sowie die dem Einzelnen während der Ausbildung zum Lehrer entstehenden Kosten im Vergleich zu den Gehältern und Kosten für andere hochqualifizierte Berufe. All dies beeinflusst die beruflichen Entscheidungen potentieller Lehrer und derjenigen, die sich für den Lehrerberuf interessieren. Gleichzeitig sind die Lehrergehälter der größte Einzelposten, wenn es um die Kosten der Bildung geht. Die Gehälter der Lehrer sind daher für die politischen Entscheidungsträger, die die Qualität des Unterrichts und einen ausgeglichenen Bildungsetat aufrechterhalten wollen, ein entscheidender Faktor. Die Höhe der Bildungsetats spiegelt das Ausbalancieren einer ganzen Reihe miteinander in Zusammenhang stehender Faktoren wider. Hierzu gehören die Lehrergehälter, das zahlenmäßige Schüler/Lehrer-Verhältnis, der Umfang der geplanten Unterrichtszeit für Schüler und die vorgesehene Anzahl der Unterrichtsstunden der Lehrer. Um dies näher zu beleuchten, untersucht Indikator D6 das Anfangs-, das mittlere und das Höchstgehalt von Lehrern an öffentlichen Schulen des 
Primar- und Sekundarbereichs und die zusätzlich zu den Grundvergütungen und -besoldungen gewährten Zulagen und Anreize.

Zusammen mit Faktoren wie der Klassengröße und dem zahlenmäßigen Schüler/Lehrer-Verhältnis (Indikator D2), der Unterrichtszeit der Schüler (Indikator D1) und den Lehrergehältern (Indikator D6) beeinflusst die Zeit, die ein Lehrer tatsächlich unterrichtet, die für Bildung aufzuwendenden Finanzmittel. Die Unterrichtszeit und die nicht-unterrichtsbezogenen Aktivitäten sind ein entscheidender Bestandteil der Arbeitsbedingungen der Lehrer und beeinflussen dadurch auch die Attraktivität des Lehrerberufs an sich. Indikator D7 untersucht die gesetzliche, bzw. vertraglich festgelegte Arbeitszeit der Lehrer in den verschiedenen Bildungsbereichen sowie die Zahl der abzuleistenden Unterrichtsstunden, d.h. die Zeit, die Vollzeitlehrer auf das Unterrichten der Schüler verwenden müssen. Obwohl die Arbeits- und Unterrichtszeit die tatsächliche Arbeitsbelastung der Lehrer nur zu einem Teil bestimmen, gewähren sie doch einen Einblick in die Unterschiede zwischen den Ländern hinsichtlich der Anforderungen an die Lehrer.

- Die Gehälter von Lehrern des Sekundarbereich I nach 15 Jahren Berufserfahrung reichen von weniger als 10.000 US-Dollar in Ungarn und der Tschechischen Republik bis zu 40.000 US-Dollar und mehr in Deutschland, Japan, Korea, der Schweiz und den Vereinigten Staaten. Einige Länder investieren trotz eines geringeren Volkseinkommens massiv in die Humanressourcen (S. 368).

- Das Gehalt je Unterrichtsstunde eines Lehrers im Sekundarbereich II liegt im Durchschnitt um 42 Prozent über dem Gehalt eines Lehrers im Primarbereich, aber die Differenz bei den Gehältern je Unterrichtsstunde zwischen diesen beiden Bildungsbereichen liegt in Australien, Neuseeland, Schottland und den Vereinigten Staaten bei unter 10 Prozent, während sie in Spanien und der Schweiz bei über 80 Prozent liegt (S. 370).

- Lehrer in Australien, Dänemark, England, Neuseeland und Schottland erreichen die oberste Besoldungsbzw. Vergütungsstufe in höchstens 11 Jahren, während Lehrer in Frankreich, Griechenland, Italien, Japan, Korea, Österreich, Spanien, der Tschechischen Republik und Ungarn hierfür mehr als 30 Dienstjahre benötigen (S. 371).

- In etwa der Hälfte der OECD-Länder sind die Schulen zumindest in gewissem Ausmaß für Entscheidungen über Höhe und Ausmaß der Zulagen für zusätzlich übernommene Aufgaben und Überstunden zuständig (S. 371).

- Die durchschnittliche Zahl der Unterrichtsstunden an öffentlichen Schulen des Primarbereichs beträgt 792 Stunden, reicht aber von 583 bis zu 1.139 Stunden (S. 380).

- Im Sekundarbereich I liegt die Zahl der Unterrichtsstunden bei durchschnittlich 720 Stunden, reicht aber von 555 bis zu 1.182 Stunden. Die Vorgaben für die Arbeitszeit der Lehrer unterscheiden sich in den einzelnen Ländern. In den meisten Ländern müssen die Lehrer formell eine bestimmte Stundenzahl arbeiten, in einigen Ländern ist auch die Zahl der wöchentlichen Unterrichtsstunden geregelt, einige Länder sehen Zeit für Tätigkeiten außerhalb des Unterrichts vor und einige Länder legen fest, wie viel Zeit die Lehrer in der Schule verbringen müssen (S. 380). 


\section{WEITERE QUELLEN}

Auf der Website www.oecd.org/els/education/eag2002 finden sich umfangreiche Informationen zu den für die Indikatoren verwendeten Erhebungs- und Berechnungsmethoden, der Interpretation der Indikatoren im jeweiligen nationalen Kontext und den benutzten Datenquellen. Die Website bietet auch Zugang zu den Daten, die den Indikatoren zugrunde liegen.

Auf der Website Www.pisa.oecd.org finden sich Informationen zu der Internationalen Schulleistungsstudie PISA, die für viele der Indikatoren von Bildung auf einen Blick 2002 herangezogen wurde.

Die Veröffentlichung Bildungspolitische Analyse ist ein Begleitband zu Bildung auf einen Blick und greift ausgewählte Themen von besonderer Relevanz für die Regierungen auf. Die fünf Kapitel der diesjährigen Ausgabe der Bildungspolitischen Analyse beschäftigen sich mit folgenden Themenbereichen: Identifizierung von acht Schlüsselstrategien zur Verbesserung des Zugangs zu einer qualitativ hochwertigen Erziehung und Bildung imVorschulalter, Analyse der spezifischen Merkmale jener Länder, die eine sehr gute Lesekompetenz bei 15-jährigen Schülern jeglichen sozialen Hintergrunds erzielt haben; mögliche politische Ansatzpunkte, um gute Lehrer anzuziehen, zu fördern und zu binden; Darstellung der grenzüberschreitenden Bildung und deren Herausforderung an die nationale Bildungspolitik. Außerdem wird ein umfassenderes Konzept des 'Humankapitals' entwickelt, das dazu beitragen soll, die vermeintliche Kluft zwischen der wirtschaftlichen Funktion von Bildung und ihrem weiterreichenden sozialen und persönlichen Nutzen zu überbrücken. 


\section{HINWEISE FÜR DEN LESER}

\section{Statistische Erfassung}

Zwar ist die Gültigkeit der Indikatoren in vielen Ländern nach wie vor durch unvollständige Daten eingeschränkt, prinzipiell wird jedoch das gesamte nationale Bildungssystem erfasst, unabhängig davon, wer Eigentümer oder Geldgeber der betreffenden Bildungseinrichtungen ist und in welchen Strukturen das Bildungsangebot vermittelt wird. Abgesehen von einer Ausnahme, die nachstehend näher erläutert ist, werden sämtliche Schüler und Studierende sowie alle Altersgruppen berücksichtigt: Kinder (einschließlich derjenigen, die als Kinder mit besonderen pädagogischen Bedürfnissen eingestuft sind), Erwachsene, Inländer, Ausländer sowie Schüler und Studierende, die an Fernkursen, in Sonderschulmaßnahmen oder an Ausbildungsgängen teilnehmen, die von anderen Ministerien als dem Bildungsministerium angeboten werden, sofern das Hauptziel der betreffenden Ausbildung die bildungsmäßige Förderung des Einzelnen ist. Die berufliche und technische Ausbildung am Arbeitsplatz bleibt jedoch - mit Ausnahme der dualen Ausbildung, d.h. einer kombinierten schulischen und betrieblichen Ausbildung, die ausdrücklich als Bestandteil des Bildungssystems gilt - bei den Angaben zu den Ausgaben für die Ausbildung und zur Bildungsbeteiligung unberücksichtigt.

Bildungsaktivitäten, die als „Erwachsenenbildung“ oder „nicht-reguläre Bildung“ eingestuft sind, werden berücksichtigt, sofern diese Aktivitäten Kurse und Studiengänge umfassen oder fachliche Inhalte vermitteln, die mit „regulären“ Bildungsgängen vergleichbar sind, oder sofern die zugrundeliegenden Bildungsgänge zu ähnlichen Abschlüssen führen wie die entsprechenden regulären Bildungsgänge. Kurse für Erwachsene, die in erster Linie aus allgemeinem Interesse, zur persönlichen ideellen Bereicherung, als Freizeitvergnügen oder zur Erholung belegt werden, sind hierbei ausgeschlossen.

\section{Berechnung von internationalen Mittelwerten}

Für viele Indikatoren ist ein Ländermittel und für manche ein OECD-Gesamtwert angegeben.

Das Ländermittel wird als der ungewichtete Mittelwert der Datenwerte aller Länder berechnet, für die entsprechende Daten vorliegen oder geschätzt werden können. Das Ländermittel bezieht sich somit auf einen Durchschnitt von Datenwerten auf der Ebene des nationalen Bildungssystems und dient als Antwort auf die Frage, wie ein Indikatorwert für ein bestimmtes Land im Vergleich zum Wert eines typischen Landes oder dem OECD-Mittel abschneidet. Dabei bleibt die absolute Größe des Bildungssystems der einzelnen Länder unberücksichtigt.

Der OECD-Gesamtwert wird als der gewichtete Mittelwert der Datenwerte aller Länder berechnet, für die entsprechende Daten vorliegen oder geschätzt werden können. Er spiegelt den Wert eines bestimmten Indikators für die OECD-Länder in ihrer Gesamtheit wider. Die Ermittlung dieses Wertes dient zu Vergleichszwecken, wenn beispielsweise die Ausgabenzahlen für einzelne Länder mit denen aller OECD-Länder insgesamt verglichen werden sollen, für die jeweils relevante Daten vorliegen, wobei diese OECD-Länder als eine Einheit betrachtet werden. Es sei darauf hingewiesen, daß sowohl das Ländermittel als auch der OECDGesamtwert durch fehlende Daten für verschiedene Länder erheblich verfälscht werden können. Aufgrund der relativ kleinen Zahl der untersuchten Länder wird dies jedoch nicht durch statistische Verfahren ausgeglichen. In den Fällen, in denen eine Kategorie für ein Land nicht zutrifft (angezeigt durch ein "a”) oder der Datenwert für die entsprechende Berechnung vernachlässigbar gering ist (angezeigt durch ein “n”), wird hier zur Berechnung von Mittelwerten der Wert Null angesetzt. In den Fällen, in denen ein Datenpunkt das 
Verhältnis von zwei Werten angibt, die beide auf ein bestimmtes Land nicht zutreffen (angezeigt durch ein "a"), wird das betreffende Land bei der Mittelwertberechnung nicht berücksichtigt.In den Tabellen zur Finanzstatistik, die das Jahr 1995 berücksichtigen, wurde für die Gruppe der Länder, die Daten für die Jahre 1995 und 1999 berichten, sowohl das Ländermittel als auch der OECD-Gesamtwert extra berechnet. Dies erlaubt einen Vergleich zwischen den Jahren, der nicht durch fehlende Daten für eines der Jahre beeinträchtigt wird.

\section{Einstufung der Bildungsbereiche nach dem ISCED-System}

Die Einstufung der Bildungsbereiche beruht auf der überarbeiteten Internationalen Standardklassifikation des Bildungswesens (International Standard Classification of Education - ISCED-97). Der größte Unterschied zwischen der alten (ISCD-76) und der überarbeiteten Fassung der ISCED-Klassifikation ist die Einführung eines mehrdimensionalen Systems für die Klassifizierung, das die Bildungsinhalte von Bildungsprogrammen mittels multipler Kriterien abschätzt und vergleicht.

Die ISCED-Klassifikation ist ein Instrument zur Erstellung von internationalen Bildungsstatistiken und unterscheidet zwischen sechs Bildungsbereichen. Im Glossar und in den Anmerkungen in Anhang 3 werden die ISCED-Stufen beschrieben, und in Anhang 1 sind die typischen Abschlussalter für die wichtigsten Bildungsgänge nach ISCED-Stufen aufgeführt.

\section{Symbole für fehlende Daten}

In den Tabellen und Grafiken werden zur Kennzeichnung fehlender Daten die folgenden vier Symbole verwendet:

a Daten nicht zutreffend, da die Kategorie nicht zutrifft.

c Zu wenige Beobachtungen, um verlässliche Werte anzugeben (d.h. eine Zelle ist mit weniger als fünf Schulen oder weniger als 30 Schülern besetzt).

m Keine Daten verfügbar.

$n \quad$ Die Größenordnung ist entweder vernachlässigbar oder Null.

$x \quad$ Die Daten sind in einer anderen Kategorie oder Spalte der Tabelle enthalten. (z.B. bedeutet x(2), dass die Daten in Spalte 2 enthalten sind). 


\section{Ländercodes}

OECD-Mitgliedsländer

$\begin{array}{llll}\text { Australien } & \text { AUS } & \text { Neuseeland } & \text { NZL } \\ \text { Belgien } & \text { BEL } & \text { Niederlande } & \text { NLD } \\ \text { Belgien (fläm. Gem.) } & \text { BFL } & \text { Norwegen } & \text { NOR } \\ \text { Dänemark } & \text { DNK } & \text { Österreich } & \text { AUT } \\ \text { Deutschland } & \text { DEU } & \text { Polen } & \text { POL } \\ \text { Finnland } & \text { FIN } & \text { Portugal } & \text { PRT } \\ \text { Frankreich } & \text { FRA } & \text { Schweden } & \text { SWE } \\ \text { Griechenland } & \text { GRC } & \text { Schweiz } & \text { CHE } \\ \text { Irland } & \text { IRL } & \text { Slowakische Republik } & \text { SVK } \\ \text { Island } & \text { ISL } & \text { Spanien } & \text { ESP } \\ \text { Italien } & \text { ITA } & \text { Tschechische Republik } & \text { CZE } \\ \text { Japan } & \text { JPN } & \text { Türkei } & \text { TUR } \\ \text { Kanada } & \text { CAN } & \text { Ungarn } & \text { HUN } \\ \text { Korea } & \text { KOR } & \text { Vereinigtes Königreich } & \text { UKM } \\ \text { Luxemburg } & \text { LUX } & \text { Vereinigte Staaten } & \text { USA } \\ \text { Mexiko } & \text { MEX } & & \end{array}$

\section{Länder, die am UNESCO/OECD World Education Indicators (WEI) Programm teilnehmen}

Ägypten, Argentinien, Brasilien, Chile, China, Indien, Indonesien, Jamaika, Jordanien, Malaysia, Paraguay, Peru, Philippinen, die Russische Föderation, Thailand, Tunesien, Uruguay und Zimbabwe nehmen am UNESCO/OECD World Education Indicators (WEI) Programm teil. Die Daten für diese Länder wurden auf Grundlage der selben Standards erhoben, die auch für die OECD-Länder gelten. Die WEI-Teilnehmerstaaten wurden daher in diese Publikation mit aufgenommen. Israel beteiligt sich an den OECD-Aktivitäten im Bereich Bildung als Beobachter und war an der Entwicklung der OECDIndikatoren zur Finanzierung des Bildungswesens beteiligt. 

Kapitel

A

BILDUNGS- UND LERNERGEBNISSE

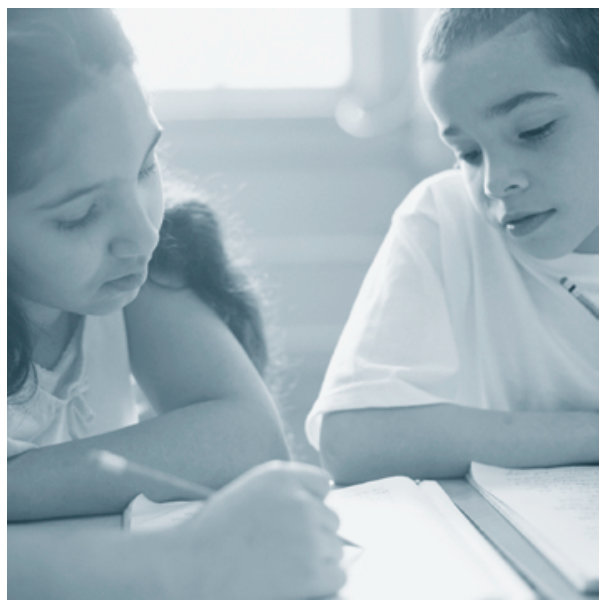





\section{ÜBERBLICK}

Indikator A1: Aktuelle Abschlussquoten im Sekundarbereich II und Bildungsstand der Erwachsenenbevölkerung

Tabelle A1.1. Abschlussquoten im Sekundarbereich II (2000)

Tabelle A1.2. Anteil der Bevölkerung mit mindestens einem Abschluss des Sekundarbereich II (2001)

Tabelle A1.3. Abschlussquoten im post-sekundären, nicht-tertiären Bereich (2000)

Indikator A2: Aktuelle Abschluss- und Erfolgsquoten im Tertiärbereich und Bildungsstand der Erwachsenenbevölkerung

Tabelle A2.1. Abschlussquoten im Tertiärbereich (2000)

Tabelle A2.2. Erfolgsquoten im Tertiärbereich (2000)

Tabelle A2.3. Anteil der Bevölkerung mit einem Abschluss im Tertiärbereich (2001)

\section{Indikator A3: Bildungsstand der Erwerbsbevölkerung und der Erwachsenenbevölkerung insgesamt}

Tabelle A3.1a. Bildungsstand der Erwachsenenbevölkerung (2001)

Tabelle A3.1b. Bildungsstand der Erwerbsbevölkerung (2001)

Tabelle A3.1c. Bildungsstand der Bevölkerung, nach Geschlecht (2001)

\section{Indikator A4: Absolventen nach Studienbereichen}

Tabelle A4.1. Absolventen des Tertiärbereichs, nach Studienbereichen und Bildungsbereichen (2000)

Tabelle A4.2. Prozentsatz der von Frauen im Tertiärbereich erworbenen Abschlüsse, nach Art des Tertiärbereichs und Studienbereichen (2000)

\section{Indikator A5: Lesekompetenz 15-jähriger Schüler}

Tabelle A5.1. Lesekompetenz 15-jähriger Schüler (2000)

Tabelle A5.2. Verteilung der Leistung 15-jähriger Schüler auf der PISA-Skala für Lesekompetenz (2000)

\section{Indikator A6: Mathematische und naturwissenschaftliche Grundbildung 15-Jähriger}

Tabelle A6.1. Verteilung der Leistung 15-jähriger Schüler auf der PISA-Skala für mathematische Grundbildung (2000)

Tabelle A6.2. Verteilung der Leistung 15-jähriger Schüler auf der PISA-Skala für naturwissenschaftliche Grundbildung (2000)

\section{Indikator A7: Leistungsunterschiede zwischen den Schulen}

Tabelle A7.1. Zusammensetzung der Leistungsvarianz im Bereich Lesekompetenz 15-jähriger Schüler (2000)

\section{Indikator A8: Politische Bildung und politisches Engagement}

Tabelle A8.1. Einstellung 14-jähriger Schüler zu Politik und Gesellschaft (1999)
Kapitel A untersucht die Bildungs-und

Lernergebnisse und zwar

in Form der aktuellen

Abschlussquoten der

Bildungseinrichtungen

und des Bildungsstands

der Erwachsenen-

bevölkerung, ...
... der Qualität der
Lernergebnisse und
der Unterschiede dieser
Ergebnisse zwischen
Schülern und Schulen, ... 
der Chancengleichheit und der Bildungserfolge... sowie den individuellen und gesellschaftlichen Bildungserträgen.

\section{Indikator A9: Beruflicher Status der Eltern und Leistungen der Schüler}

Tabelle A9.1. Schülerleistungen und sozio-ökonomischer Status der Eltern (2000)

Indikator A1 0: Geburtsland, zu Hause gesprochene Sprache und Lesekompetenz 15-Jähriger

Tabelle A10.1. Leistung im Bereich Lesekompetenz und Geburtsland 15jähriger Schüler und Geburtsland ihrer Eltern (2000)

Tabelle A10.2. Leistung im Bereich Lesekompetenz nach der bei 15-jährigen Schülern zu Hause gesprochenen Sprache (2000)

\section{Indikator A1 1 : Erwerbsquoten nach Bildungsstand}

Tabelle A11.1. Erwerbsquoten (2001)

Tabelle A11.2. Arbeitslosenquoten (2001)

Indikator A1 2: Zu erwartende Jahre in Ausbildung und Beschäftigung im Alter zwischen 15 und 29 Jahren

Tabelle A12.1. Zu erwartende Jahre in Ausbildung und nicht in Ausbildung für 15- bis 29-Jährige, nach Geschlecht und Beschäftigungsstatus (2001)

\section{Indikator A1 3: Ertragsraten von Bildung: Individuelle und gesellschaftliche Ertragsraten von Bildung und deren bestimmende Faktoren}

Tabelle A13.1. Relative Einkommen der Bevölkerung mit Erwerbseinkommen Tabelle A13.2. Einkommensunterschiede zwischen Frauen und Männern Tabelle A13.3. Individuelle Ertragsraten aus Bildungsinvestitionen (1999-2000) Tabelle A13.4. Gesellschaftliche Ertragsraten aus Bildungsinvestitionen (1999-2000)

\section{Indikator A1 4: Ertragsraten von Bildung: Zusammenhang zwischen Humankapital und Wirtschaftswachstum}

Tabelle A14.1. Aufschlüsselung der Veränderungen in den durchschnittlichen jährlichen Wachstumsraten des BIP pro Kopf (1980-1997)

Die Indikatoren A5, A6, A7, A9 und A10 beruhen auf Daten der Internationalen Schulleistungsstudie PISA. Ausführlichere Informationen zu dieser Studie finden sich auf der Website www.pisa.oecd.org. 


\section{AKTUELLE ABSCHLUSSQUOTEN IM SEKUNDARBEREICH II UND BILDUNGSSTAND DER ERWACHSENENBEVÖLKERUNG}

- In der Mehrheit der OECD-Länder, für die Daten verfügbar sind, beträgt der Anteil an Absolventen des Sekundarbereich II an der Bevölkerung im typischen Abschlussalter mehr als 70 Prozent. In Deutschland, Japan, Polen, der Slowakischen Republik und Ungarn liegt dieser Anteil bei 90 Prozent oder mehr. Jetzt muss sichergestellt werden, dass der übrige Teil den Anschluss nicht verliert und sich dem möglichen Risiko sozialer Ausgrenzung gegenübersieht.

- Ein Vergleich des Bildungsstands der 25- bis 34-Jährigen mit dem der Altersgruppe zwischen 45 und 54 Jahren zeigt, dass in fast allen OECD-Ländern der Anteil von Personen, die über keinen Abschluss im Sekundarbereich II verfügen, zurückgegangen ist, in einigen Ländern sogar recht schnell.

- Bei den älteren Altersgruppen sind die Abschlussquoten im Sekundarbereich II bei den Frauen niedriger als bei den Männern, doch bei den jüngeren Menschen kehrt sich dieses Verhältnis gegenwärtig um. Heute übersteigen die Abschlussquoten der Frauen in den meisten Ländern die der Männer.

\section{Abbildung A1.1.}

Abschlussquoten im Sekundarbereich II (2000)

Anteil aller Absolventen des Sekundarbereich II (ohne Doppelzählungen) an der Population im typischen Abschlussalter

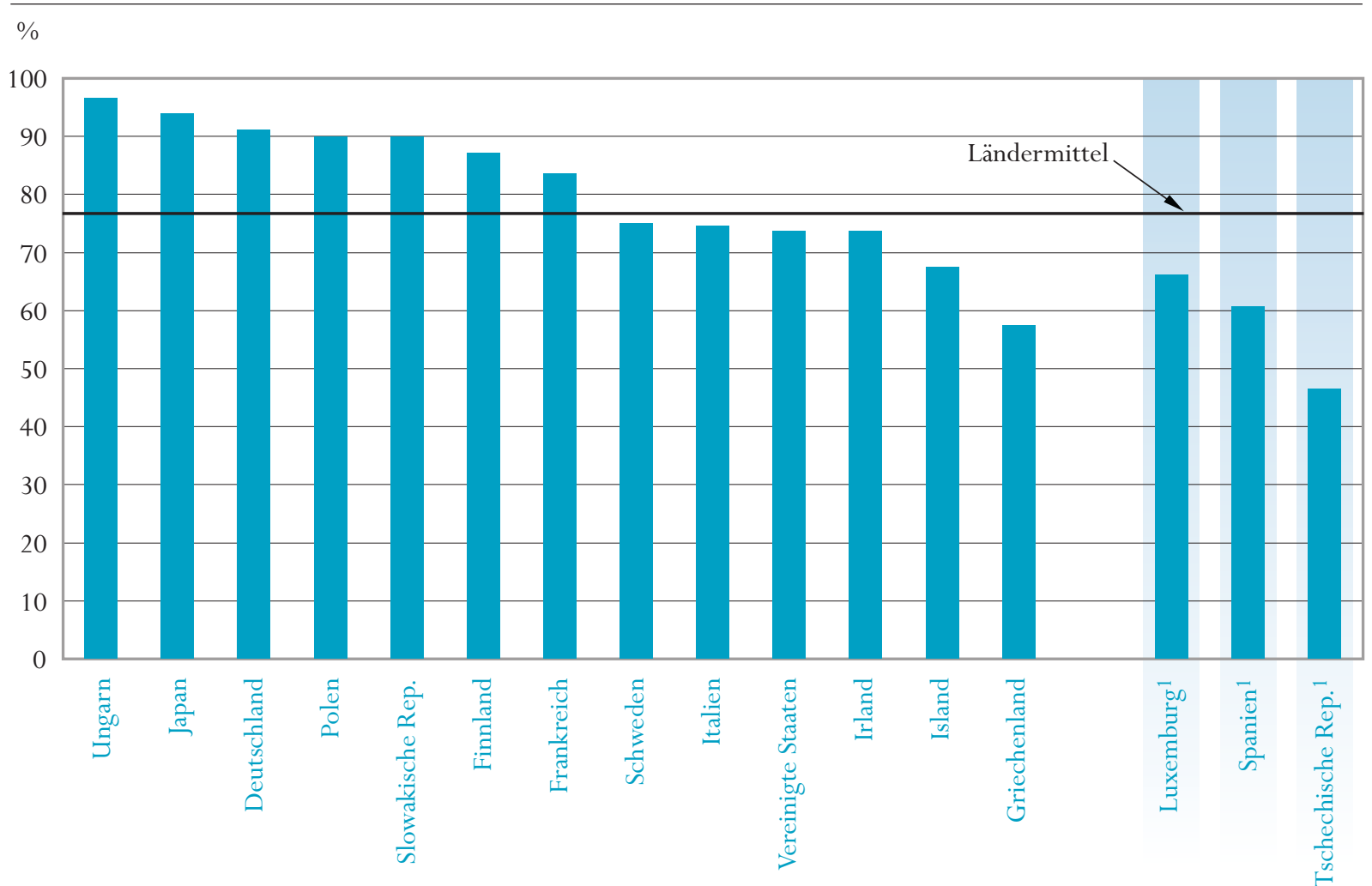

1. Ein größererTeil der Kohorte von Jugendlichen fehlt.

Anordnung der Länder in absteigender Reihenfolge der Gesamtabschlussquoten im Sekundarbereich II.

Quelle: OECD. Tabelle A1.1. Hinweise s. Anhang 3 (www.oecd.org/els/education/eag2002). 


\section{$\mathbf{A}_{1}$}

Um den Anteil der

Bevölkerung zu

bemessen, der über die

Mindestvoraussetzungen

für einen erfolgreichen

Eintritt in den

Arbeitsmarkt verfügt,...

... zeigt dieser

Indikator die aktuellen Abschlussquoten der

Bildungseinrichtungen

im Sekundarbereich II

ebenso auf...

.. wie die in der

Vergangenheit

vorherrschenden

Abschlussquoten im

Sekundarbereich II.

In 11 von 13

OECD-Ländern

mit vergleichbaren

Daten liegen die

Abschlussquoten im

Sekundarbereichll bei

über 70 Prozent...

... und in Deutschland, Japan, Polen, der Slowakischen Republik und Ungarn bei 90 Prozent und darüber.

\section{Politischer Hintergrund}

Aufgrund der gestiegenen Anforderungen an Kenntnissen und Fähigkeiten in den OECD-Ländern ist ein Abschluss des Sekundarbereich II zur Mindestvoraussetzung für einen erfolgreichen Eintritt in den Arbeitsmarkt geworden. Er bildet die Grundlage für weiterführendes Lernen sowie Fort- und Weiterbild ungsmöglichkeiten und dient auch der Vorbereitung auf den direkten Eintritt in den Arbeitsmarkt. Obwohl es die Bildungssysteme vieler Länder ermöglichen, dass Schüler die Schule nach dem Ende des Sekundarbereich I verlassen können, treffen in den OECD-Ländern Jugendliche, die ohne einen Abschluss des Sekundarbereich II abgehen, in der Regel auf große Schwierigkeiten bei ihrem Eintritt in den Arbeitsmarkt (s. Indikatoren A11 bis A14).

Die Abschlussquote des Sekundarbereich II zeigt den gegenwärtigen ,Output' der Bildungssysteme, d.h. den Prozentsatz der Bevölkerung im typischen Alter für den Sekundarbereich II, der einen Bildungsgang des Sekundarbereich II erfolgreich abschließt. Obwohl hohe Abschlussquoten im Sekundarbereich II nicht garantieren, dass ein Bildungssystem seine Schulabgänger ausreichend mit dem notwendigen Wissen und den grundlegenden Fertigkeiten und Fähigkeiten ausgerüstet hat, die für den Eintritt in den Arbeitsmarkt erforderlich sind, weil hierbei nicht die Qualität der Ausbildung erfasst wird, sind diese Abschlussquoten für den Sekundarbereich II doch ein Indikator dafür, inwieweit es den Bildungssystemen gelingt, den Mindestanforderungen des Arbeitsmarktes zu entsprechen.

Um den Bestand an ,Humankapital' in der Gesamtbevölkerung zu bemessen, ist es hilfreich, den Bildungsstand der Bevölkerung genauer zu untersuchen. Vor allem durch den Vergleich des Bildungsstands zwischen verschiedenen Generationen lässt sich die Entwicklung des Bildungsstands in der Bevölkerung nachverfolgen, die sowohl die sich verändernden Anforderungen des Arbeitsmarktes als auch bildungspolitische Veränderungen widerspiegelt.

\section{Ergebnisse und Erläuterungen}

Die Abschlussquoten im Sekundarbereich II werden geschätzt als die Zahl jener Personen, die, unabhängig vom ihrem Alter, einen Erstabschluss im Sekundarbereich II erzielen, bezogen auf 100 Personen in dem Alter, in dem Schüler in der Regel Bildungsgänge des Sekundarbereich II abschließen (s. Anhang 1). Diese Quoten beinhalten also sowohl Schüler, die den Sekundarbereich II im üblichen oder typischen Alter abschließen, als auch ältere Absolventen (z.B. über den zweiten Bildungsweg). In 11 von 13 OECD-Ländern mit vergleichbaren Daten liegen die Abschlussquoten für den Sekundarbereich II bei über 70 Prozent (Abb. A1.1).

In fünf der 13 Länder, für die vergleichbare Absolventenzahlen verfügbar sind, liegen die Abschlussquoten bei 90 Prozent und darüber (Deutschland, Japan, Polen, die Slowakische Republik und Ungarn). Die in Abbildung A1.1 dargestellten Abschlussquoten sollten hinsichtlich einiger Länder jedoch mit 
Vorsicht interpretiert werden. So wurde vor kurzem in der Tschechischen Republik und in Spanien die Dauer der Bildungsgänge im Sekundarbereich verlängert, was zu einer Unterschätzung der Abschlussquoten führt, und viele luxemburger Staatsbürger gehen in einem der Nachbarländer zur Schule.

Einige Länder bieten anstelle von speziellen Bildungsgängen für Erwachsene im Sekundarbereich II die Möglichkeit einer separaten Abschlussprüfung an. In den Vereinigten Staaten nutzen Schüler, die das letzte Jahr der Ausbildung im Sekundarbereich II nicht erfolgreich absolvieren - ein relativ großer Anteil - häufig die Möglichkeit, zu einem späteren Zeitpunkt den General Educational Development (GED) Test abzulegen und sind dabei auch erfolgreich. Diese Qualifikation wird formell als Äquivalent zu einem Abschluss im Sekundarbereich II angesehen.

Ein Vergleich der Abschlussquoten mit dem Bildungsstand älterer Jahrgangsgruppen deutet auf einen starken Anstieg des Prozentsatzes derjenigen Personen hin, die einen Abschluss im Sekundarbereich II erzielen (Abb. A1.2). Im Durchschnitt verfügen nur 60 Prozent der 45- bis 54-Jährigen über einen Abschluss im Sekundarbereich II, im Gegensatz zu 74 Prozent bei den 25- bis 34-Jährigen.

Besonders auffällig ist dies in Ländern, in denen die Erwachsenenbevölkerung in der Regel über einen geringeren Bildungsstand verfügt. Bei den jüngeren Altersgruppen sind die Unterschiede im Bildungsstand zwischen den einzelnen Ländern weniger ausgeprägt. Bei vielen Ländern, die gegenwärtig einen geringen Bildungsstand in der Erwachsenenbevölkerung aufweisen, ist zu erwarten, dass sie sich an die Länder mit einem höherem Bildungsstand annähern werden. In Korea, Portugal und Spanien liegt der Anteil der 25- bis 34-Jährigen, die mindestens über einen Abschluss des Sekundarbereich II verfügen, rund zweimal so hoch wie in der Altersgruppe der 45- bis 54-Jährigen.

\section{Geschlechtsspezifische Unterschiede bei den Abschlussquoten}

Das Verhältnis zwischen dem Bildungsstand von erwachsenen Männern und Frauen ist in den meisten OECD-Ländern unausgeglichen. In der Vergangenheit hatten die Frauen nicht genügend Möglichkeiten und/oder Anreize, den gleichen Bildungsstand wie die Männer zu erreichen. Frauen sind in der Regel überrepräsentiert in der Gruppe derjenigen, die gar nicht erst den Sekundarbereich II besuchten, und unterrepräsentiert bei den höheren Bildungsniveaus (s. auch Indikator A3).

Allerdings sind diese Unterschiede hauptsächlich auf die großen geschlechtsspezifischen Unterschiede beim Bildungsstand der älteren Altersgruppen zurückzuführen und haben sich in den jüngeren Altersgruppen beträchtlich reduziert oder sogar umgekehrt.
In fast allen Ländern sind die Abschlussquoten im Sekundarbereich II gestiegen ...

...und viele Länder mit einem in der Vergangenheit geringen Bildungsstand holen nun auf.

Beiden älteren Altersgruppen haben Frauen einen geringeren Bildungsstand als Männer,...

... doch bei den jüngeren Menschen kehrt sich dieses verhältnis gegenwärtig um. 


\section{Abbildung A1.2.}

Prozentsatz der Bevölkerung mit mindestens einem Abschluss im Sekundarbereich $\mathrm{II}^{1}$, nach Altersgruppen (2001)

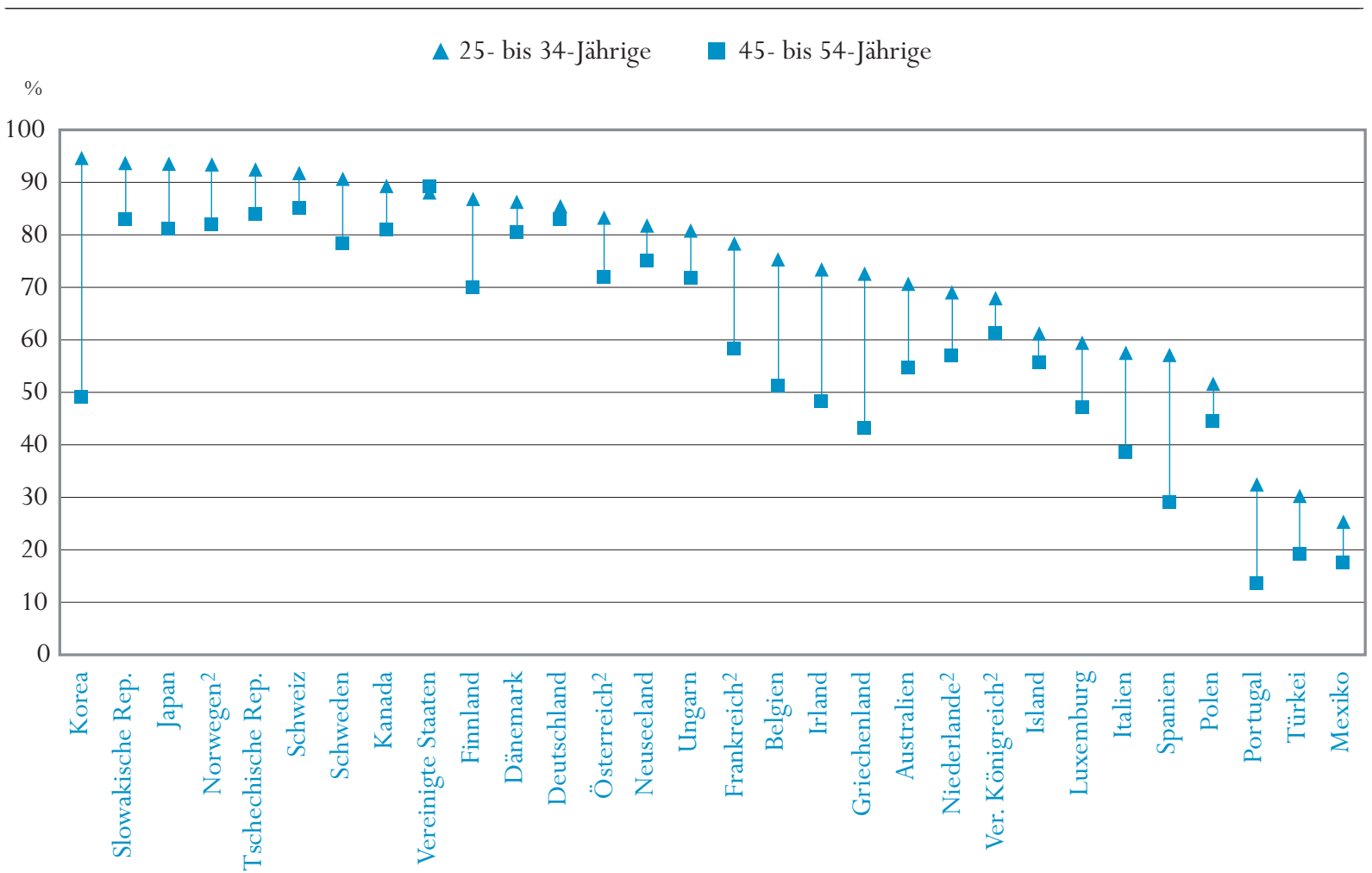

Hinweis: Nicht alle ISCED 3 Bildungsgänge erfüllen die Mindestanforderungen für ISCED 3C lange Bildungsgänge. Hinweise s. Anhang 3 (www.oecd.org/els/education/eag2002).

1. Ohne ISCED 3C kurze Bildungsgänge.

2. Referenzjahr 2000.

Anordnung der Länder in absteigender Reihenfolge des Prozentsatzes 25- bis 34-Jähriger, die mindestens einen Abschluss im Sekundarbereich II haben.

Quelle: OECD. Tabelle A1.2. Beschreibung der ISCED-97-Stufen, Zuordnung der länderspezifischen Bildungsgänge zu ISCED 97 und länderspezifische Datenquellen s. Anhang 3 (www.oecd.org/els/education/eag2002).

Heutzutage übersteigen die Abschlussquoten der

Frauen in den meisten Ländern die der Männer.
Heute zeigen sich in der Hälfte der Länder mit verfügbaren Daten bei den Abschlussquoten keine signifikanten Unterschiede mehr zwischen Frauen und Männern (Tabelle A1.1). In 14 von 16 OECD-Ländern, für die nach Geschlecht aufgeschlüsselte Abschlussquoten des Sekundarbereich II verglichen werden können, übertreffen die Abschlussquoten für Frauen die der Männer, und in Finnland, Griechenland, Irland, Island, Italien und Spanien beträgt dieser Unterschied 10 Prozentpunkte und mehr. In der Mehrheit der OECD-Länder liegt der Frauenanteil bei den Bildungsgängen im Sekundarbereich II, die auf weiterführende Bildungsgänge im Tertiärbereich A hinführen sollen (ISCED 3A), deutlich höher, nur in Korea und der Türkei liegen die Abschlussquoten der Männer höher als die der Frauen. 


\section{Abschlussquoten bei post-sekundären, nicht tertiären Bildungsgängen}

Post-sekundäre, nicht-tertiäre Bildungsgänge befinden sich aus international vergleichender Sicht im Grenzbereich zwischen Sekundarbereich II und postsekundärem Bereich, auch wenn sie im nationalen Zusammenhang eindeutig als zum Sekundarbereich II oder zum post-sekundären Bereich gehörig angesehen werden können. Selbst wenn der Inhalt dieser Bildungsgänge nicht wesentlich anspruchsvoller ist als der des Sekundarbereich II, können sie doch den Kenntnisstand derjenigen, die schon einen Abschluss im Sekundarbereich II erworben haben, erweitern. Die Teilnehmer der betreffenden Bildungsgänge sind in der Regel älter als im Sekundarbereich II.

Typische Beispiele für solche post-sekundären, nicht tertiären Bildungsgänge sind die Trade and Vocational Certificates in Kanada und den Vereinigten Staaten, die Kindergärtnerausbildung in Österreich und der Schweiz oder die Berufsausbildung von Absolventen des allgemeinbildenden Sekundarbereich II im dualen System in Deutschland. In den meisten Ländern sind diese postsekundären, nicht tertiären Bildungsgänge beruflich orientiert.

In der Hälfte der OECD-Länder, in denen post-sekundäre, nicht-tertiäre Bildungsgänge angeboten werden, erzielt ein beachtlicher Teil der Absolventen des Sekundarbereich II auch einen post-sekundären, nicht-tertiären Abschluss, entweder als Alternative oder zusätzlich zu einem Abschluss im Tertiärbereich. Der OECD-Durchschnitt liegt bei 9 Prozent. In Irland, Kanada und Ungarn schließen über 28 Prozent der jeweils typischen Altersgruppe einen postsekundären, nicht tertiären Bildungsgang ab (Tabelle A1.3).

In fast zwei Drittel der OECD-Länder mit verfügbaren Daten sind die meisten Abschlüsse im post-sekundären, nicht-tertiären Bereich Abschlüsse in ISCED 4C-Bildungsgängen, die hauptsächlich der Vorbereitung auf den direkten Einstieg in den Arbeitsmarkt dienen sollen. Auch Lehrlingsausbildungen für Schüler, die bereits einen Abschluss im Sekundarbereich II erzielt haben, fallen in diese Kategorie. Allerdings kommt in Belgien, Deutschland, Spanien, der Slowakischen Republik und der Tschechischen Republik die Mehrheit der Abgänger im post-sekundären, nicht-tertiären Bereich aus ISCED 4ABildungsgängen, die einen direkten Zugang zu Bildungsgängen im Tertiärbereich A gewähren sollen.

\section{Definitionen und angewandte Methodik}

Als Absolventen des Sekundarbereich II gelten diejenigen Schulabgänger, die das letzte Jahr der Ausbildung im Sekundarbereich II erfolgreich abschließen und zwar unabhängig von ihrem Alter. In einigen aber nicht allen Ländern gehört zum erfolgreichen Abschluss eine Abschlussprüfung.

Die Brutto-Abschlussquoten für die Bildungsgänge der ISCED-Kategorien 3A, 3B und 3C können nicht einfach aufaddiert werden, da die Absolventen von mehr als einem dieser Bildungsgänge im Sekundarbereich II doppelt
In einigen Ländern erweitert ein beträchtlicher Anteil von Schülern nach einem ersten Abschluss des Sekundarbereich II sein Wissen durch einen weiteren Verbleib im sekundarbereich II.

In Irland, Kanada und Ungarn erwerben über 28 Prozent der jeweils typischen Altersgruppe einen Abschluss in einem post-sekundüren, nicht tertiären Bildungsgang.

Die Absolventendaten beziehen sich auf das Schuljahr 1999-2000 und beruhen auf der alljährlich von der OECD aufgelegten VOE-Datenerhebung zur Bildungsstatistik. 
gezählt würden. Das gleiche gilt für Abschlussquoten nach Ausrichtung des Bildungsgangs, d.h. allgemeinbildend oder berufsbildend. Die Anzahl der Absolventen ohne Doppelzählungen wird ermittelt, indem man die Anzahl der Absolventen abzieht, die in einem vorherigen Jahr einen anderen Bildungsgang im Sekundarbereich II abgeschlossen haben.

Für einige Länder stehen keine Angaben zur Zahl der Absolventen postsekundärer, nicht-tertiärer Bildungsgänge ohne Doppelzählung zur Verfügung und die Abschlussquoten könnten wegen der Absolventen, die mehrere Bildungsgänge im gleichen Bildungsbereich abschließen, etwas zu hoch angesetzt sein. In Tabelle A1.3 sind die betreffenden Länder gekennzeichnet.

Berufsvorbereitende und berufsbildende Bildungsgänge umfassen sowohl schulische als auch kombinierte schulische und betriebliche Ausbildungen, die als Bestandteil des Bildungssystems gelten. Ausschließlich in Betrieben durchgeführte Ausbildungen, die keiner formellen Aufsicht durch eine Bildungsbehörde unterstehen, bleiben unberücksichtigt.

Die Daten zum Die Daten zu Bevölkerung und Bildungsstand stammen aus Datenbanken Bildungsstand stammen aus den nationalen Arbeitskräfteerhebungen und beruhen auf der Internationalen Standardklassifikation des Bildungswesens (ISCED-97). der OECD und EUROSTAT, die aus nationalen Arbeitskräfteerhebungen zusammengetragen sind. Länderspezifische Datenquellen s. Anhang 3 unter www.oecd.oeg/els/education/eag2002).

Die Unterschiede im Bildungstand basieren auf dem Prozentsatz der Bevölkerung im Alter von 25 bis 64 Jahren, der einen bestimmten Bildungsstand erreicht hat. Die Festlegung der einzelnen Bildungsbereiche erfolgt auf Grundlage der Internationalen Standardklassifikation des Bildungswesens (ISCED-97). Zur Beschreibung der einzelnen Bildungsbereiche laut ISCED-97 und der entsprechenden Zuordnung der jeweiligen landespezifischen Bildungsgänge s. Anhang 3 unter www.oecd.org/els/education/eag2002. 
Tabelle A1.1.

Abschlussquoten im Sekundarbereich II (2000)

Anteil der Absolventen des Sekundarbereich II an der Gesamtpopulation im typischen Abschlussalter (x 100) in öffentlichen und privaten Bildungseinrichtungen, nach Ausrichtung und Ziel des Bildungsgangs sowie Geschlecht

\begin{tabular}{|c|c|c|c|c|c|c|c|c|c|c|c|c|c|c|c|}
\hline \multirow[b]{4}{*}{ Australien } & \multicolumn{3}{|c|}{$\begin{array}{c}\text { Gesamt } \\
\text { (keine Doppelzählung) }\end{array}$} & \multicolumn{2}{|c|}{\begin{tabular}{|c} 
ISCED 3A \\
(Bildungsgänge \\
sollen direkten \\
Zugang zum \\
Tertiärbereich A \\
eröffnen) \\
\end{tabular}} & \multicolumn{2}{|c|}{\begin{tabular}{|c} 
ISCED 3B \\
(Bildungsgänge \\
sollen direkten \\
Zugang zum \\
Tertiärbereich B \\
eröffnen)
\end{tabular}} & \multicolumn{2}{|c|}{$\begin{array}{c}\text { ISCED 3C } \\
\text { (lang) ähnlich } \\
\text { lang wie typische } \\
\text { 3A oder 3B } \\
\text { Bildungsgänge }\end{array}$} & \multicolumn{2}{|c|}{$\begin{array}{c}\text { ISCED 3C (kurz) } \\
\text { kürzere Dauer als } \\
\text { typische 3A oder } \\
\text { 3B Bildungsgänge }\end{array}$} & \multicolumn{2}{|c|}{$\begin{array}{l}\text { allgemein- } \\
\text { bildende } \\
\text { Ausbildungs- } \\
\text { gänge }\end{array}$} & \multicolumn{2}{|c|}{$\begin{array}{l}\text { berufsvorberei- } \\
\text { tende / } \\
\text { berufsbildende } \\
\text { Ausbildungs- } \\
\text { gänge }\end{array}$} \\
\hline & $M+F$ & Männer & Frauen & $M+F$ & Frauen & $M+F$ & Frauen & $M+F$ & Frauen & $M+F$ & Frauen & $M+F$ & Frauen & $M+F$ & Frauen \\
\hline & (1) & (2) & (3) & (4) & (5) & (6) & (7) & $(8)$ & (9) & $(10)$ & (11) & (12) & (13) & (14) & (15) \\
\hline & $\mathrm{m}$ & $\mathrm{m}$ & $\mathrm{m}$ & 67 & 73 & $\mathrm{~m}$ & $\mathrm{~m}$ & $\mathrm{~m}$ & $\mathrm{~m}$ & $\mathrm{~m}$ & $\mathrm{~m}$ & $\mathrm{~m}$ & $\mathrm{~m}$ & $\mathrm{~m}$ & $\mathrm{~m}$ \\
\hline Österreich & $\mathrm{m}$ & $\mathrm{m}$ & $\mathrm{m}$ & $\mathrm{m}$ & $\mathrm{m}$ & $\mathrm{m}$ & $\mathrm{m}$ & $\mathrm{m}$ & $\mathrm{m}$ & $\mathrm{m}$ & $\mathrm{m}$ & $\mathrm{m}$ & $\mathrm{m}$ & $\mathrm{m}$ & $\mathrm{m}$ \\
\hline Belgien & $\mathrm{m}$ & $\mathrm{m}$ & $\mathrm{m}$ & 60 & 64 & $\mathrm{a}$ & $\mathrm{a}$ & 19 & 19 & 11 & 15 & 36 & 40 & 54 & 57 \\
\hline Kanada & $\mathrm{m}$ & $\mathrm{m}$ & $\mathrm{m}$ & $\mathrm{m}$ & $\mathrm{m}$ & $\mathrm{m}$ & $\mathrm{m}$ & $\mathrm{m}$ & $\mathrm{m}$ & $\mathrm{m}$ & $\mathrm{m}$ & $\mathrm{m}$ & $\mathrm{m}$ & $\mathrm{m}$ & $\mathrm{m}$ \\
\hline Tschechische Rep. ${ }^{1 *}$ & 47 & 50 & 42 & 18 & 21 & $\mathrm{n}$ & $\mathrm{n}$ & $\mathrm{a}$ & $\mathrm{a}$ & 31 & 23 & 8 & 10 & 41 & 35 \\
\hline Dänemark & $\mathrm{m}$ & $\mathrm{m}$ & $\mathrm{m}$ & 52 & 64 & $\mathrm{a}$ & $\mathrm{a}$ & 54 & 64 & $\mathrm{a}$ & $\mathrm{a}$ & 52 & 64 & 54 & 64 \\
\hline Finnland & 87 & 81 & 94 & 87 & 94 & $\mathrm{a}$ & $\mathrm{a}$ & $\mathrm{a}$ & $\mathrm{a}$ & $\mathrm{a}$ & a & 53 & 64 & 72 & 77 \\
\hline Frankreich & 84 & 81 & 86 & 49 & 57 & 10 & 8 & 2 & 2 & 37 & 32 & 31 & 37 & 67 & 62 \\
\hline Deutschland & 91 & 89 & 94 & 33 & 36 & 58 & 57 & $\mathrm{a}$ & $\mathrm{a}$ & $\mathrm{a}$ & $\mathrm{a}$ & 33 & 36 & 58 & 57 \\
\hline Griechenland & 58 & 50 & 66 & 56 & 64 & $\mathrm{~m}$ & $\mathrm{~m}$ & 26 & 22 & $\mathrm{~m}$ & $\mathrm{~m}$ & 56 & 64 & 26 & 22 \\
\hline Ungarn & 97 & 98 & 95 & 58 & 65 & 1 & 2 & $\mathrm{x}(10)$ & $\mathrm{x}(11)$ & 37 & 28 & 26 & 32 & 70 & 62 \\
\hline Island & 67 & 60 & 76 & 47 & 58 & $\mathrm{n}$ & $\mathrm{n}$ & 22 & 14 & 14 & 16 & 47 & 58 & 36 & 30 \\
\hline Irland & 74 & 67 & 80 & 74 & 80 & a & $\mathrm{a}$ & 5 & 5 & a & $\mathrm{a}$ & 59 & 63 & 20 & 23 \\
\hline Italien & 75 & 68 & 81 & 74 & 80 & 1 & 1 & a & a & 19 & 18 & 29 & 39 & 64 & 60 \\
\hline Japan & 94 & 92 & 96 & 69 & 73 & 1 & $\mathrm{n}$ & 24 & 23 & $\mathrm{x}(8)$ & $\mathrm{x}(9)$ & 69 & 73 & 26 & 24 \\
\hline Korea & $\mathrm{m}$ & $\mathrm{m}$ & $\mathrm{m}$ & 60 & 58 & $\mathrm{a}$ & $\mathrm{a}$ & 37 & 38 & a & a & 60 & 58 & 37 & 38 \\
\hline Luxemburg $^{1 *}$ & 66 & 63 & 69 & 39 & 47 & 6 & 5 & 20 & 17 & $\mathrm{a}$ & $\mathrm{a}$ & 26 & 29 & 40 & 40 \\
\hline Mexiko & $\mathrm{m}$ & $\mathrm{m}$ & $\mathrm{m}$ & 28 & 30 & $\mathrm{a}$ & $\mathrm{a}$ & 4 & 5 & $\mathrm{x}(8)$ & $\mathrm{x}(9)$ & 28 & 30 & 4 & 5 \\
\hline Niederlande & $\mathrm{m}$ & $\mathrm{m}$ & $\mathrm{m}$ & 63 & 68 & $\mathrm{a}$ & $\mathrm{a}$ & 32 & 29 & $\mathrm{x}$ & $\mathrm{x}$ & 37 & 41 & 57 & 56 \\
\hline Neuseeland & $\mathrm{m}$ & $\mathrm{m}$ & $\mathrm{m}$ & 65 & 70 & 45 & 52 & 12 & 14 & $\mathrm{x}(8)$ & $\mathrm{x}(9)$ & $\mathrm{m}$ & $\mathrm{m}$ & $\mathrm{m}$ & $\mathrm{m}$ \\
\hline Norwegen & $\mathrm{m}$ & $\mathrm{m}$ & $\mathrm{m}$ & 64 & 79 & $\mathrm{a}$ & $\mathrm{a}$ & 52 & 44 & $\mathrm{~m}$ & $\mathrm{~m}$ & 64 & 79 & 52 & 44 \\
\hline Polen & 90 & 87 & 94 & 70 & 78 & $\mathrm{a}$ & $\mathrm{a}$ & $\mathrm{a}$ & $\mathrm{a}$ & 29 & 21 & 32 & 41 & 67 & 58 \\
\hline Portugal & $\mathrm{m}$ & $\mathrm{m}$ & $\mathrm{m}$ & $\mathrm{m}$ & $\mathrm{m}$ & $\mathrm{m}$ & $\mathrm{m}$ & $\mathrm{m}$ & $\mathrm{m}$ & $\mathrm{m}$ & $\mathrm{m}$ & $\mathrm{m}$ & $\mathrm{m}$ & $\mathrm{m}$ & $\mathrm{m}$ \\
\hline Slowakische Rep. & 90 & 90 & 90 & 72 & 80 & $\mathrm{n}$ & $\mathrm{n}$ & 1 & 1 & 24 & 17 & 18 & 21 & 79 & 77 \\
\hline Spanien ${ }^{1 *}$ & 61 & 54 & 67 & 46 & 53 & $\mathrm{n}$ & $\mathrm{n}$ & 9 & 9 & 13 & 15 & 46 & 53 & 22 & 24 \\
\hline Schweden & 75 & 72 & 78 & 74 & 77 & $\mathrm{a}$ & $\mathrm{a}$ & 1 & $\mathrm{n}$ & a & $\mathrm{a}$ & 42 & 46 & 32 & 31 \\
\hline Schweiz & $\mathrm{m}$ & $\mathrm{m}$ & $\mathrm{m}$ & 19 & 22 & 50 & 42 & 13 & 19 & $\mathrm{n}$ & $\mathrm{n}$ & $\mathrm{m}$ & $\mathrm{m}$ & $\mathrm{m}$ & $\mathrm{m}$ \\
\hline Türkei* $^{*}$ & $\mathrm{~m}$ & $\mathrm{~m}$ & $\mathrm{~m}$ & 37 & 31 & $\mathrm{a}$ & $\mathrm{a}$ & $\mathrm{m}$ & $\mathrm{m}$ & a & $\mathrm{a}$ & 20 & 19 & 16 & 13 \\
\hline Ver. Königreich & $\mathrm{m}$ & $\mathrm{m}$ & $\mathrm{m}$ & $\mathrm{m}$ & $\mathrm{m}$ & $\mathrm{m}$ & $\mathrm{m}$ & $\mathrm{m}$ & $\mathrm{m}$ & $\mathrm{m}$ & $\mathrm{m}$ & $\mathrm{m}$ & $\mathrm{m}$ & $\mathrm{m}$ & $\mathrm{m}$ \\
\hline Vereinigte Staaten & 74 & 73 & 74 & $\mathrm{~m}$ & $\mathrm{~m}$ & $\mathrm{~m}$ & $\mathrm{~m}$ & $\mathrm{~m}$ & $\mathrm{~m}$ & $\mathrm{~m}$ & $\mathrm{~m}$ & $\mathrm{~m}$ & $\mathrm{~m}$ & $\mathrm{~m}$ & $\mathrm{~m}$ \\
\hline Ländermittel & 77 & 74 & 80 & 55 & 61 & 8 & 7 & 15 & 15 & 12 & 10 & 40 & 45 & 45 & 44 \\
\hline Argentinien ${ }^{2}$ & 48 & 40 & 55 & 48 & 55 & $\mathrm{a}$ & $\mathrm{a}$ & $\mathrm{a}$ & $\mathrm{a}$ & a & $\mathrm{a}$ & 26 & 34 & 21 & 21 \\
\hline Brasilien $^{2}$ & $\mathrm{a}$ & $\mathrm{a}$ & $\mathrm{a}$ & 62 & 70 & $\mathrm{~m}$ & $\mathrm{~m}$ & $\mathrm{a}$ & $\mathrm{a}$ & $\mathrm{a}$ & $\mathrm{a}$ & $\mathrm{m}$ & $\mathrm{m}$ & $\mathrm{m}$ & $\mathrm{m}$ \\
\hline Chile $^{2}$ & a & a & $\mathrm{a}$ & 34 & 39 & 28 & 28 & $\mathrm{a}$ & a & $\mathrm{a}$ & a & 34 & 39 & 28 & 28 \\
\hline China $^{2}$ & a & a & a & 17 & 15 & a & a & 20 & 21 & 4 & $\mathrm{~m}$ & $\mathrm{~m}$ & $\mathrm{~m}$ & $\mathrm{~m}$ & $\mathrm{~m}$ \\
\hline Indien & 34 & 40 & 28 & 34 & 28 & $\mathrm{a}$ & $\mathrm{a}$ & $\mathrm{m}$ & $\mathrm{m}$ & $\mathrm{m}$ & $\mathrm{m}$ & $\mathrm{m}$ & $\mathrm{m}$ & $\mathrm{m}$ & $\mathrm{m}$ \\
\hline Indonesien $^{3}$ & $\mathrm{a}$ & $\mathrm{a}$ & a & 19 & 20 & 13 & 11 & $\mathrm{a}$ & $\mathrm{a}$ & $\mathrm{a}$ & $\mathrm{a}$ & 19 & 20 & 13 & 11 \\
\hline Israel & $\mathrm{m}$ & $\mathrm{m}$ & $\mathrm{m}$ & 59 & 67 & 26 & 23 & 3 & 1 & $\mathrm{a}$ & $\mathrm{a}$ & 59 & 67 & 26 & 23 \\
\hline Jamaika & a & $\mathrm{a}$ & $\mathrm{a}$ & 65 & 67 & $\mathrm{n}$ & $\mathrm{n}$ & $\mathrm{a}$ & $\mathrm{a}$ & a & a & 65 & 67 & $\mathrm{n}$ & $\mathrm{n}$ \\
\hline Jordanien & a & $\mathrm{a}$ & $\mathrm{a}$ & 68 & 75 & $\mathrm{a}$ & $\mathrm{a}$ & 3 & $\mathrm{n}$ & a & $\mathrm{a}$ & 55 & 63 & 13 & 13 \\
\hline Malaysia $^{2}$ & $\mathrm{~m}$ & $\mathrm{~m}$ & $\mathrm{~m}$ & 14 & 19 & a & a & 53 & 63 & a & a & 65 & 81 & 2 & 1 \\
\hline Paraguay $^{2}$ & a & $\mathrm{a}$ & $\mathrm{a}$ & 35 & 38 & $\mathrm{a}$ & $\mathrm{a}$ & $\mathrm{m}$ & $\mathrm{m}$ & a & a & 28 & 31 & 8 & 8 \\
\hline Peru $^{2}$ & a & a & a & 50 & 50 & $\mathrm{x}(4)$ & $\mathrm{x}(5)$ & $\mathrm{a}$ & $\mathrm{a}$ & a & a & 41 & 42 & 9 & 8 \\
\hline Philippinen ${ }^{2}$ & $\mathrm{a}$ & a & a & 66 & 72 & a & a & a & $\mathrm{a}$ & $\mathrm{a}$ & a & 66 & 72 & a & $\mathrm{a}$ \\
\hline Russische Föd. ${ }^{3}$ & a & a & a & 53 & $\mathrm{~m}$ & $\mathrm{a}$ & $\mathrm{a}$ & $\mathrm{m}$ & $\mathrm{m}$ & $\mathrm{m}$ & $\mathrm{m}$ & $\mathrm{m}$ & $\mathrm{m}$ & $\mathrm{m}$ & $\mathrm{m}$ \\
\hline Thailand & $\mathrm{a}$ & a & $\mathrm{a}$ & 27 & 30 & 18 & 18 & $\mathrm{a}$ & $\mathrm{a}$ & $\mathrm{a}$ & $\mathrm{a}$ & 27 & 30 & 18 & 18 \\
\hline Tunesien & a & a & a & 26 & 29 & 2 & 1 & 2 & 1 & $\mathrm{a}$ & $\mathrm{a}$ & 26 & 29 & 4 & 2 \\
\hline Simbabwe $^{3}$ & a & a & a & 3 & 3 & 1 & 1 & $\mathrm{~m}$ & $\mathrm{~m}$ & $\mathrm{~m}$ & $\mathrm{~m}$ & $\mathrm{~m}$ & $\mathrm{~m}$ & $\mathrm{~m}$ & $\mathrm{~m}$ \\
\hline
\end{tabular}

Hinweis: $\mathrm{x}$ bedeutet, dass die Daten in einer anderen Spalte enthalten sind, deren Referenz in runden Klammern nach dem ' $\mathrm{x}$ ' angegeben ist. So bedeutet z.B. $x(2)$, dass die Daten in Spalte 2 enthalten sind.

1. Größerer Anteil der Kohorte von Jugendlichen fehlt.

2. Referenzjahr 1999.

3. Referenzjahr 2001 .

* Hinweise siehe Anhang 3 (www.oecd.org/els/education/eag2002).

Quelle: OECD. 
KAPITEL A Bildungs-und Lernergebnisse

Tabelle A1.2.

Anteil der Bevölkerung mit mindestens einem Abschluss des Sekundarbereich II (2001)

Prozentsatz der Bevölkerung mit mindestens einem Abschluss des Sekundarbereich II ${ }^{1}$, nach Altersgruppen

\begin{tabular}{|c|c|c|c|c|c|}
\hline \multirow[b]{3}{*}{ Australien } & \multicolumn{5}{|c|}{ Altersgruppen } \\
\hline & $25-64$ & $25-34$ & $35-44$ & $45-54$ & $55-64$ \\
\hline & 59 & 71 & 60 & 55 & 44 \\
\hline Österreich $^{2}$ & 76 & 83 & 80 & 72 & 63 \\
\hline Belgien $^{2}$ & 59 & 75 & 63 & 51 & 38 \\
\hline Kanada & 82 & 89 & 85 & 81 & 67 \\
\hline Tschechische Rep. & 86 & 92 & 90 & 84 & 76 \\
\hline Dänemark & 80 & 86 & 80 & 80 & 72 \\
\hline Finnland & 74 & 87 & 84 & 70 & 51 \\
\hline Frankreich $^{3}$ & 64 & 78 & 67 & 58 & 46 \\
\hline Deutschland & 83 & 85 & 86 & 83 & 76 \\
\hline Griechenland & 51 & 73 & 60 & 43 & 28 \\
\hline Ungarn & 70 & 81 & 79 & 72 & 44 \\
\hline Island & 57 & 61 & 60 & 56 & 46 \\
\hline Italien & 43 & 57 & 49 & 39 & 22 \\
\hline Japan & 83 & 94 & 94 & 81 & 63 \\
\hline Korea & 68 & 95 & 77 & 49 & 30 \\
\hline Luxemburg & 53 & 59 & 57 & 47 & 42 \\
\hline Mexiko & 22 & 25 & 25 & 17 & 11 \\
\hline Niederlande $e^{2,3}$ & 65 & 74 & 69 & 60 & 51 \\
\hline Neuseeland & 76 & 82 & 80 & 75 & 60 \\
\hline Norwegen $^{2}$ & 85 & 93 & 90 & 82 & 70 \\
\hline Polen & 46 & 52 & 48 & 44 & 36 \\
\hline Portugal & 20 & 32 & 20 & 14 & 9 \\
\hline Slowakische Rep. & 85 & 94 & 90 & 83 & 66 \\
\hline Spanien & 40 & 57 & 45 & 29 & 17 \\
\hline Schweden & 81 & 91 & 86 & 78 & 65 \\
\hline Schweiz & 87 & 92 & 90 & 85 & 81 \\
\hline Vereinigte Staaten & 88 & 88 & 89 & 89 & 83 \\
\hline Ländermittel & 64 & 74 & 68 & 60 & 49 \\
\hline
\end{tabular}

1. Ohne ISCED 3C kurze Bildungsgänge.

2. Referenzjahr 2000.

3. Nicht alle ISCED 3 Bildungsgänge erfüllen die Mindestanforderungen für ISCED 3C lange Bildungsgänge. Hinweise s. Anhang 3

(www.oecd.org/els/education/eag2002).

Quelle: OECD. Beschreibung der ISCED-97-Stufen, Zuordnung der länderspezifischen Bildungsgänge zu ISCED 97 und länderspezifische Datenquellen s.

Anhang 3 (www.oecd.org/els/education/eag2002). 
Tabelle A1.3.

Abschlussquoten im post-sekundären, nicht-tertiären Bereich (2000)

Anteil der Absolventen im post-sekundären, nicht-tertiären Bereich an der Gesamtpopulation im typischen Abschlussalter (x 100) in öffentlichen und privaten Bildungseinrichtungen, nach Ziel des Bildungsgangs und Geschlecht

\begin{tabular}{|c|c|c|c|c|c|c|c|c|c|}
\hline & \multicolumn{3}{|c|}{$\begin{array}{c}\text { Gesamt } \\
\text { (keine Doppelzählungen) }\end{array}$} & \multicolumn{2}{|c|}{\begin{tabular}{|} 
ISCED 4A (Bildungsgänge \\
sollen direkten Zugang zum \\
Tertiärbereich A eröffnen) \\
\end{tabular}} & \multicolumn{2}{|c|}{\begin{tabular}{|} 
ISCED 4B (Bildungsgänge \\
sollen direkten Zugang zum \\
Tertiärbereich B eröffnen)
\end{tabular}} & \multicolumn{2}{|c|}{ ISCED 4C } \\
\hline & $M+F$ & Männer & Frauen & $M+F$ & Frauen & $M+F$ & Frauen & $M+F$ & Frauen \\
\hline & $(1)$ & (2) & (3) & (4) & $(5)$ & (6) & (7) & $(8)$ & (9) \\
\hline Australien & $\mathrm{m}$ & $\mathrm{m}$ & $\mathrm{m}$ & $\mathrm{m}$ & $\mathrm{m}$ & $\mathrm{m}$ & $\mathrm{m}$ & $\mathrm{m}$ & $\mathrm{m}$ \\
\hline Österreich & $\mathrm{m}$ & $\mathrm{m}$ & $\mathrm{m}$ & $\mathrm{m}$ & $\mathrm{m}$ & $\mathrm{m}$ & $\mathrm{m}$ & $\mathrm{m}$ & $\mathrm{m}$ \\
\hline Belgien $^{1}$ & 17.8 & 16.1 & 19.6 & 10.2 & 10.3 & $\mathrm{a}$ & $\mathrm{a}$ & 7.6 & 9.2 \\
\hline Kanada $^{\prime}$ & 28.1 & 31.5 & 24.7 & $\mathrm{n}$ & $\mathrm{n}$ & $\mathrm{n}$ & $\mathrm{n}$ & 28.1 & 24.7 \\
\hline Tschechische Rep. ${ }^{1}$ & 9.0 & 9.7 & 8.2 & 9.0 & 8.2 & a & a & $\mathrm{n}$ & $\mathrm{n}$ \\
\hline Dänemark' & 1.7 & 2.9 & 0.4 & 0.1 & $\mathrm{n}$ & a & a & 1.6 & 0.4 \\
\hline Finnland & 1.5 & 1.6 & 1.4 & $\mathrm{a}$ & a & a & a & 1.9 & 1.9 \\
\hline Frankreich $^{1}$ & 1.2 & 0.8 & 1.7 & 0.7 & 0.8 & a & a & 0.6 & 0.9 \\
\hline Deutschland & 14.8 & 16.0 & 13.5 & 9.3 & 8.7 & 5.5 & 4.8 & $\mathrm{a}$ & a \\
\hline Griechenland $^{1}$ & 15.3 & 11.6 & 19.2 & $\mathrm{a}$ & $\mathrm{a}$ & a & $\mathrm{a}$ & 15.3 & 19.2 \\
\hline Ungarn ${ }^{1}$ & 31.2 & 29.1 & 33.5 & 5.8 & 6.1 & a & a & 25.3 & 27.2 \\
\hline Island & 6.1 & 8.3 & 3.9 & $\mathrm{a}$ & $\mathrm{a}$ & a & a & 6.2 & 4.0 \\
\hline Irland & 28.9 & 15.1 & 43.4 & a & a & a & a & 28.9 & 43.4 \\
\hline Italien & 3.1 & 2.3 & 4.0 & a & a & a & a & 3.1 & 4.0 \\
\hline Japan & $\mathrm{m}$ & $\mathrm{m}$ & $\mathrm{m}$ & $\mathrm{m}$ & $\mathrm{m}$ & $\mathrm{m}$ & $\mathrm{m}$ & $\mathrm{m}$ & $\mathrm{m}$ \\
\hline Korea & $\mathrm{a}$ & $\mathrm{a}$ & a & $\mathrm{a}$ & $\mathrm{a}$ & a & a & $\mathrm{a}$ & $\mathrm{a}$ \\
\hline Luxemburg $^{1}$ & 3.1 & 4.5 & 1.8 & a & a & a & a & 3.1 & 1.6 \\
\hline Mexiko & $\mathrm{a}$ & $\mathrm{a}$ & $\mathrm{a}$ & a & a & a & a & a & a \\
\hline Niederlande $^{1}$ & 1.0 & 1.5 & 0.4 & a & a & a & a & 1.0 & 0.4 \\
\hline Neuseeland $^{1}$ & 2.6 & 1.7 & 3.6 & $\mathrm{n}$ & 0.1 & 0.2 & 0.2 & 2.3 & 3.3 \\
\hline Norwegen $^{1}$ & 11.4 & 16.4 & 6.2 & 4.8 & 3.2 & a & a & 6.6 & 3.0 \\
\hline Polen ${ }^{1}$ & 12.6 & 8.4 & 16.9 & $\mathrm{a}$ & $\mathrm{a}$ & 12.6 & 16.9 & a & a \\
\hline Portugal & $\mathrm{m}$ & $\mathrm{m}$ & $\mathrm{m}$ & $\mathrm{m}$ & $\mathrm{m}$ & $\mathrm{m}$ & $\mathrm{m}$ & $\mathrm{m}$ & $\mathrm{m}$ \\
\hline Slowakische Rep. ${ }^{1}$ & 2.2 & 1.3 & 3.1 & 2.2 & 3.1 & a & a & a & a \\
\hline Spanien & 9.8 & 9.2 & 10.5 & 9.5 & 10.1 & 0.3 & 0.4 & $\mathrm{n}$ & $\mathrm{n}$ \\
\hline Schweden & $\mathrm{m}$ & $\mathrm{m}$ & $\mathrm{m}$ & $\mathrm{m}$ & $\mathrm{m}$ & $\mathrm{m}$ & $\mathrm{m}$ & 0.5 & 0.3 \\
\hline Schweiz ${ }^{1}$ & 17.6 & 16.1 & 19.1 & 3.0 & 2.0 & 14.6 & 17.2 & $\mathrm{n}$ & $\mathrm{n}$ \\
\hline Türkei & a & a & a & a & a & a & a & a & a \\
\hline Ver. Königreich & $\mathrm{m}$ & $\mathrm{m}$ & $\mathrm{m}$ & $\mathrm{m}$ & $\mathrm{m}$ & $\mathrm{m}$ & $\mathrm{m}$ & $\mathrm{m}$ & $\mathrm{m}$ \\
\hline Vereinigte Staaten & 6.6 & 5.8 & 7.3 & $\mathrm{a}$ & $\mathrm{a}$ & $\mathrm{a}$ & a & 6.6 & 7.3 \\
\hline Ländermittel & 9.4 & 8.7 & 10.1 & 2.3 & 2.2 & 1.4 & 1.7 & 5.5 & 6.0 \\
\hline Argentinien ${ }^{2}$ & a & a & a & a & a & a & a & a & a \\
\hline Brasilien $^{2}$ & a & a & a & a & a & $\mathrm{m}$ & $\mathrm{m}$ & a & a \\
\hline China $^{2}$ & a & a & a & a & a & a & a & 2.0 & 2.0 \\
\hline Indonesien $^{3}$ & a & a & a & a & a & a & a & a & a \\
\hline Jordanien & a & a & a & $\mathrm{a}$ & $\mathrm{a}$ & a & a & a & a \\
\hline Malaysia $^{2}$ & $\mathrm{~m}$ & $\mathrm{~m}$ & $\mathrm{~m}$ & 0.6 & 0.6 & 0.7 & 0.2 & 0.3 & 0.3 \\
\hline Paraguay $^{2}$ & $\mathrm{a}$ & $\mathrm{a}$ & a & a & a & a & a & a & a \\
\hline Peru $^{2}$ & a & a & a & $\mathrm{a}$ & a & a & a & $\mathrm{m}$ & $\mathrm{m}$ \\
\hline Philippinen ${ }^{2}$ & a & a & a & 6.0 & $\mathrm{~m}$ & $x(5)$ & $\mathrm{m}$ & $\mathrm{x}(5)$ & $\mathrm{m}$ \\
\hline Russische Föd. ${ }^{3}$ & a & a & a & $\mathrm{a}$ & $\mathrm{a}$ & a & a & 32.5 & 22.7 \\
\hline Thailand & a & a & a & a & a & a & a & $\mathrm{m}$ & $\mathrm{m}$ \\
\hline Tunesien & a & a & a & a & a & $\mathrm{n}$ & $\mathrm{n}$ & a & a \\
\hline
\end{tabular}

Hinweis: $\mathrm{x}$ bedeutet, dass die Daten in einer anderen Spalte enthalten sind, deren Referenz in runden Klammern nach dem ' $\mathrm{x}$ ' angegeben ist. So bedeutet $\mathrm{z} . \mathrm{B}$. $\mathrm{x}(2)$, dass die Daten in Spalte 2 enthalten sind.

1. Bruttoabschlussquote kann einige Doppelzählungen enthalten.

2. Referenzjahr 1999.

3. Referenzjahr 2001

Quelle: $O E C D$. 


\section{AKTUELLE ABSCHLUSS- UND ERFOLGSOUOTEN IM TERTIÄRBEREICH UND BILDUNGSSTAND DER ERWACHSENENBEVÖLKERUNG}

- Im Durchschnitt aller OECD-Länder erzielen gegenwärtig 26 Prozent der Personen im typischen Abschlussalter einen Abschluss im Tertiärbereich A.

- Im Durchschnitt bricht ein Drittel der Studierenden der OECD ihr Studium vor einem ersten Abschluss ab, unabhängig davon, ob sie Studiengänge des Tertiärbereich A oder B belegen.

- In der Erwachsenenbevölkerung ist der Bestand an Kenntnissen und Fähigkeiten auf Hochschulniveau angestiegen. Der größte Teil dieses Anstiegs ist jedoch auf einen bedeutenden Anstieg der tertiären Abschlussquoten in einer vergleichsweise geringen Zahl von Ländern zurückzuführen.

\section{Abbildung A2.1}

Abschlussquoten im Tertiärbereich A, nach Dauer des Studiengangs (2000)

Anteil der Absolventen imTertiärbereich A an der Population im typischen Abschlussalter (x 100)

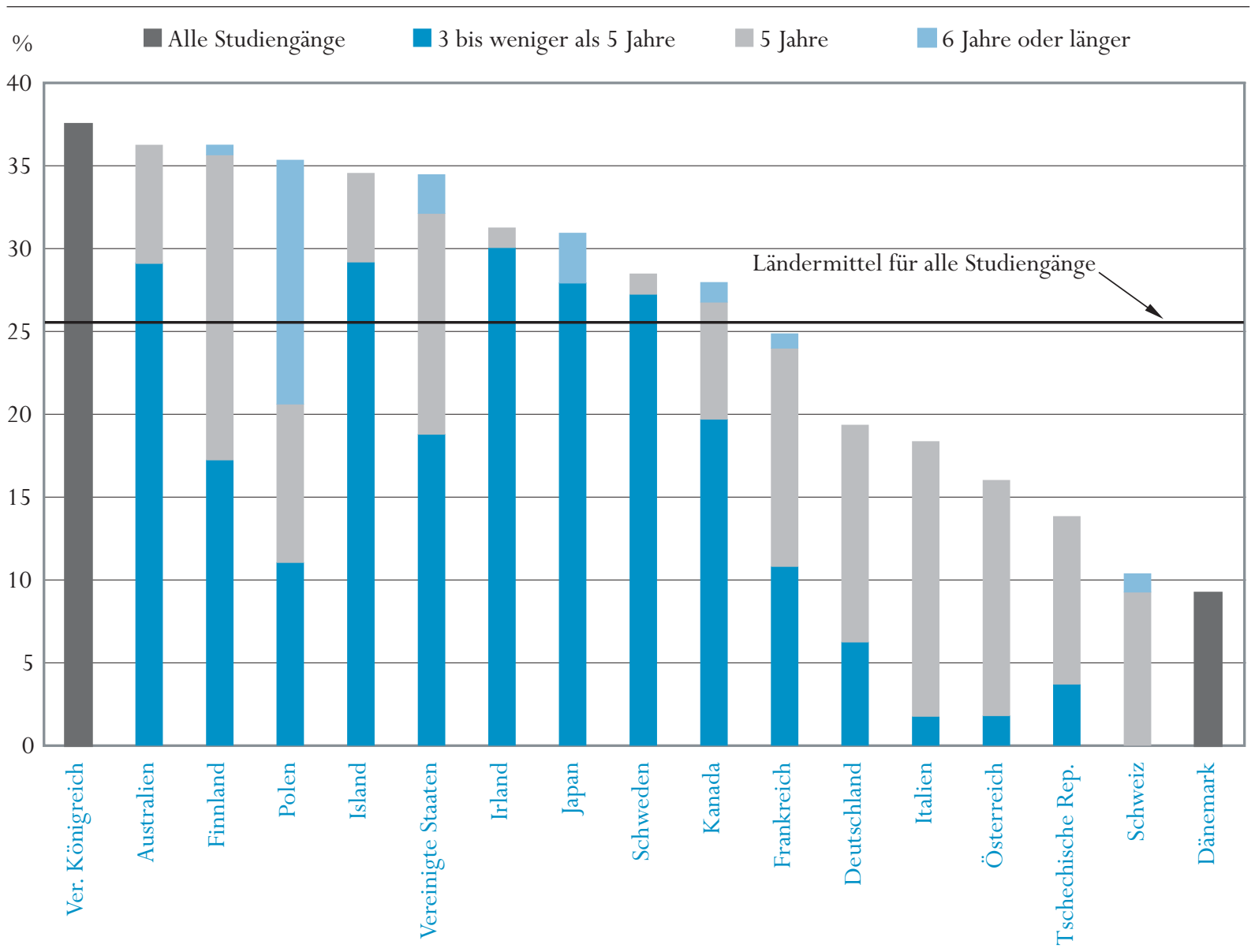

Anordnung der Länder in absteigender Reihenfolge der Gesamtabschlussquoten im Tertiärbereich A.

Quelle: OECD. Tabelle A2.1. Hinweise s. Anhang 3 (www.oecd.org/els/education/eag2002). 


\section{Politischer Hintergrund}

Abschlussquoten im Tertiärbereich sind ein Indikator dafür, in welchem Umfang die Bildungssysteme der einzelnen Länder zum gegenwärtigen Zeitpunkt höhere Kenntnisse und Fähigkeiten vermitteln. Länder mit hohen Abschlussquoten im Tertiärbereich haben bzw. entwickeln derzeit aller Wahrscheinlichkeit nach eine hochqualifizierte Erwerbsbevölkerung. Kennzahlen zum Bildungsstand zeigen auf, wie sich der Wissensbestand in der Bevölkerung entwickelt.

Sowohl Abschluss- als auch Abbruchquoten können hilfreiche Indikatoren für die Effizienz von tertiären Bildungssystemen sein. Es gibt jedoch viele Gründe, warum Studierende ihr Studium abbrechen: man merkt, dass man das falsche Fachgebiet oder den falschen Studiengang gewählt hat; die von den Bildungseinrichtungen vorgegebenen Mindestleistungen werden nicht erreicht; oder es bietet sich bereits vor Abschluss des Studiums eine attraktive Beschäftigung an. Ein Studienabbruch ist nicht unbedingt ein Anzeichen für ein persönliches Versagen des einzelnen Studierenden. Vielmehr können hohe Studienabbruchquoten darauf hinweisen, dass das Bildungssystem den Bedürfnissen seiner Nutzer nicht gerecht wird. Die Studierenden sind vielleicht der Meinung, dass die angebotenen Studiengänge ihren Erwartungen bzw. den Arbeitsmarktanforderungen nicht entsprechen. Und es kann sein, dass die Studiengänge aus Sicht der Studierenden länger dauern, als in ihren Augen die Zeit außerhalb des Arbeitsmarktes zu rechtfertigen ist.

\section{Ergebnisse und Erläuterungen}

\section{Abschlussquoten im Tertiärbereich}

Die Abschlussquoten im Tertiärbereich werden sowohl von den Zugangsmöglichkeiten zu Studiengängen im Tertiärbereich als auch von der Nachfrage nach entsprechenden Kenntnissen und Fähigkeiten auf dem Arbeitsmarkt beeinflusst. Sie werden außerdem auch durch Aufbau und Organisation der Hochschulabschlüsse und der akademischen Grade in den einzelnen Ländern beeinflusst.

Dieser Indikator unterscheidet zwischen verschiedenen Kategorien von Abschlüssen im tertiären Bereich: 1.) Abschlüsse im Tertiärbereich B (ISCED 5B); 2.) Abschlüsse im Tertiärbereich A (ISCED 5A) und 3.) Abschlüsse in weiterführenden Forschungsprogrammen auf dem Niveau der Promotion (ISCED 6).

Studiengänge im Tertiärbereich A sind weitgehend theoretisch orientiert und sollen hinreichende Qualifikationen für den Zugang zu weiterführenden Forschungsprogrammen und Berufen mit hohen Qualifikationsanforderungen vermitteln. Der Aufbau der Studiengänge im Tertiärbereich A ist von Land zu Land unterschiedlich, sowohl an den Hochschulen als auch in anderen Bildungseinrichtungen. Die Dauer von Studiengängen im Tertiärbereich A, die zu einem Erstabschluss führen, variiert zwischen drei Jahren (z.B. der BachelorAbschluss an den meisten Colleges in Irland und dem Vereinigten Königreich und
Dieser Indikator zeigt

die gegenwärtigen

Abschlussquoten im

Tertiärbereich ebenso wie

die Entwicklungsmuster

des erreichten

Bildungsstands und

gibt Auskunft über die

Effizienz von tertiären

Bildungssystemen.
Aufbau und Umfang

der Bildungsgänge

im Tertiärbereich

unterscheiden sich in

den einzelnen Ländern erheblich.

Studiengänge im

Tertiärbereich A werden nach ihrer theoretischen Gesamtdauer unterteilt, um sie unabhängig von unterschiedlichen nationalen Abschlussstrukturen vergleichen zu können. 
die Licence in Frankreich) und bis zu fünf Jahren und länger (z.B. das Diplom in Deutschland und die Laurea in Italien).

Während in vielen Ländern klar zwischen erstem und zweitem akademischem Abschluss, d.h. dem Abschluss von Undergraduate- und Graduate-Studiengängen, unterschieden wird, machen einige Länder diese Unterscheidung nicht. In dieser zweiten Ländergruppe erwirbt man einen Grad, der international mit dem Niveau eines Master-Abschlusses vergleichbar ist, am Ende eines einzigen, langen Studiengangs. Um die internationale Vergleichbarkeit zu gewährleisten, müssen deshalb zu akademischen Abschlüssen führende Studiengänge mit ähnlicher Gesamtdauer sowie die Abschlussquoten der zum ersten akademischen Abschluss führenden Studiengänge verglichen werden.

Abschlüsse im Tertiärbereich A werden gemäß der theoretischen Gesamtdauer tertiärer Studiengänge untergliedert, um einen von den unterschiedlichen nationalen Abschlussstrukturen unabhängigen Vergleich zu ermöglichen. Zum Zwecke dieses Indikators werden Abschlüsse von Studiengängen mittlerer (3 bis weniger als 5 Jahre), langer ( 5 bis weniger als 6 Jahre) und sehr langer Studiendauer (6 Jahre und länger) unterschieden. Abschlüsse, die nach kurzen Studiengängen mit einer Dauer von weniger als 3 Jahren erworben werden, gelten hier als nicht als gleichwertig mit einem Abschluss des Tertiärbereich A und werden somit bei diesem Indikator nicht berücksichtigt. Zu einem zweiten Abschluss führende Studiengänge werden nach der kumulierten Dauer der zum ersten und zum zweitem Abschluss führenden Studiengänge klassifiziert, und Absolventen, die bereits über einen Erstabschluss verfügen, werden hiervon abgezogen.

Im Durchschnitt $\operatorname{der} O E C D$-Länder machen 26 Prozent der Personen im typischen Abschlussalter einen Abschluss in einem Studiengang des Tertiärbereich A, ...

... während die Abschlussquote im Tertiärbereich B bei 11 Prozent liegt...
Im Durchschnitt der OECD-Länder erwerben 26 Prozent der Personen im typischen Abschlussalter einen Abschluss in einem Studiengang des Tertiärbereich A. Die Bandbreite reicht von mehr als einem Drittel in Australien, Finnland, Island, Polen, dem Vereinigten Königreich und den Vereinigten Staaten bis zu weniger als 15 Prozent in Dänemark, der Schweiz und der Tschechischen Republik (Abb. A2.1). Allgemein absolvieren in den Ländern mit höheren Abschlussquoten die Studierenden überwiegend Bildungsgänge mittlerer Dauer (3 bis weniger als 5 Jahre). Bemerkenswerte Ausnahmen von dieser Regel sind Finnland und Polen, wo die Mehrheit der Studierenden einen Abschluss in Studiengängen längerer Dauer erwirbt. Spezifischer sind die Verhältnisse in Ländern mit geringeren Abschlussquoten im Tertiärbereich A: In Deutschland, Italien, Österreich, der Schweiz und der Tschechischen Republik wird die Mehrheit der Abschlüsse in den längeren Studiengängen (mit einer Studiendauer von mindestens 5 Jahren) erzielt. Die Abschlussquoten liegen dort bei unter 20 Prozent.

Studiengänge im Tertiärbereich B sind stärker berufsorientiert und führen zum direkten Eintritt in den Arbeitsmarkt. Üblicherweise sind diese Studiengänge kürzer als die des Tertiärbereich A (in der Regel 2 bis 3 Jahre), und im 


\section{Abbildung A2.2.}

Abschlussquoten bei weiterführenden Forschungsprogrammen (2000)

Summe der Abschlussquoten der einzelnen Altersjahrgänge (x 100)

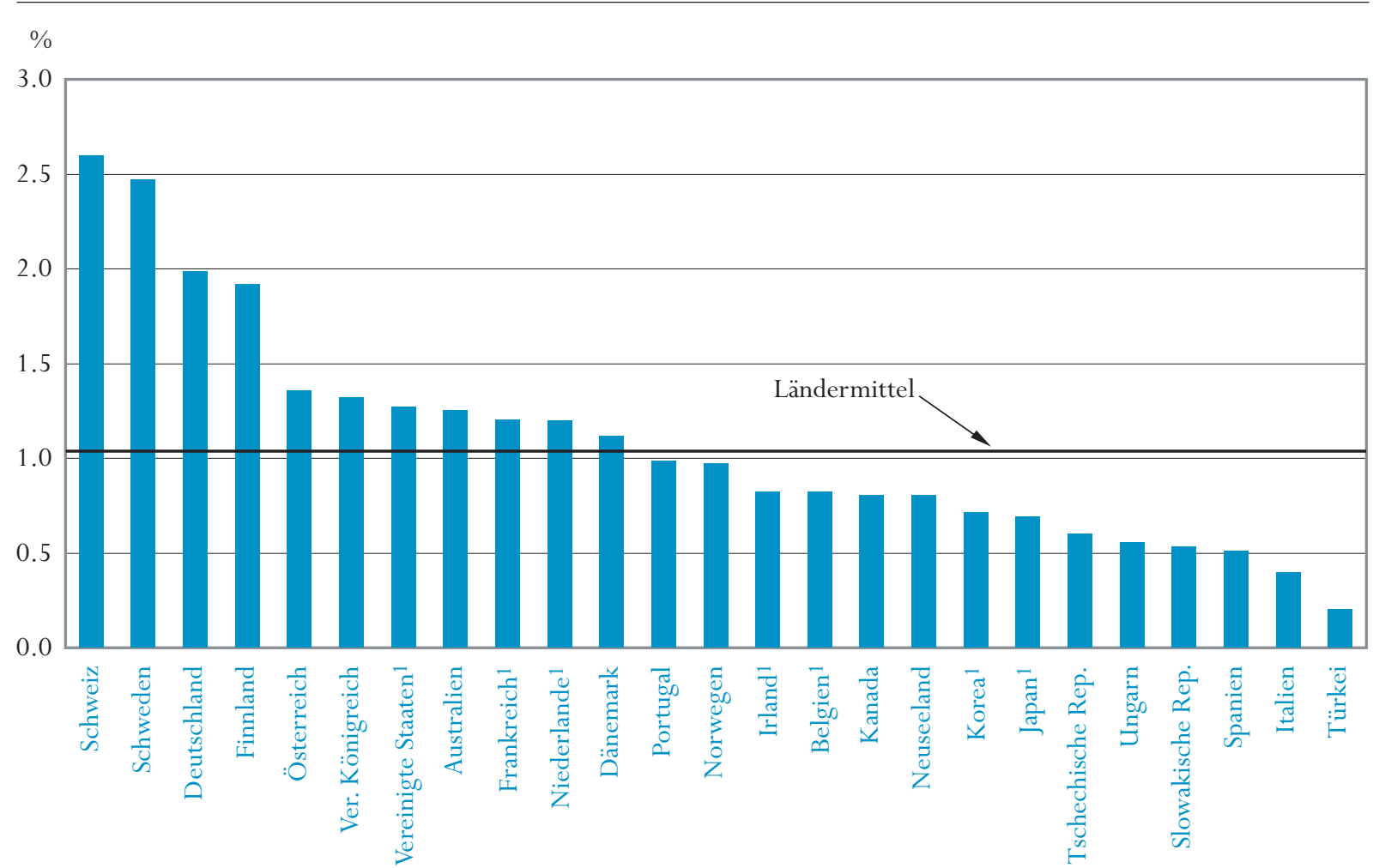

1. Bei diesen Ländern wurden die Brutto-Abschlussquoten verwendet, die sich aus dem Verhältnis der Zahl der Absolventen zur Population im typischen Abschlussalter (x 100) berechnen.

Anordnung der Länder in absteigender Reihenfolge der Abschlussquoten für weiterführende Forschungsprogramme.

Quelle: OECD. Tabelle A2.1. Hinweise s. Anhang 3 (www.oecd.org/els/education/eag2002).

Allgemeinen gelten sie nicht als auf einen akademischen Abschluss hinführend. Die Abschlussquoten für Bildungsgänge im Tertiärbereich B betragen im Durchschnitt der OECD-Länder 11 Prozent der jeweiligen Altersgruppe (Tabelle A2.1). In Dänemark und Japan erwerben rund 25 Prozent der Bevölkerung im typischen Abschlussalter einen Abschluss des Tertiärbereich B, und für Deutschland, Finnland und Irland liegt diese Zahl zwischen 11 und 15 Prozent.

Im Durchschnitt erwirbt in den OECD-Ländern rund 1 Prozent der Bevölkerung einen Abschluss in einem weiterführenden Forschungsprogramm wie den Doktortitel. In Schweden und der Schweiz beträgt der Anteil 2,5 Prozent und in Deutschland und Finnland knapp 2 Prozent (Abb. A2.4).

Die gestiegenen Anforderungen des Arbeitsmarktes, die Zunahme der Arbeitslosigkeit in den letzten Jahren und die höheren Erwartungen des

....und ein Prozent der Bevölkerung einen Abschluss in einem weiterführenden Forschungsprogramm erwirbt. 
KAPITEL A Bildungs-und Lernergebnisse

Die Zahljunger Einzelnen und der Gesellschaft haben den Anteil junger Menschen, die Menschen mit einem mindestens einen Abschluss im Tertiärbereich erwerben, beeinflusst. Der

Abschluss, der einem Abschluss des

Tertiärbereich A oder Anteil der Erwachsenenbevölkerung mit hochausgebildeten Kenntnissen und Fähigkeiten hat im Allgemeinen zugenommen. Im Durchschnitt aller OECDLänder haben nur 14 Prozent der 45- bis 54-Jährigen einen Abschluss im einem weiterführenden Forschungsprogramm gleichwertig ist, hat zugenommen.

Die Erfolgsquoten im Tertiärbereich A oder einem weiterführenden Forschungsprogramm, bei den 25- bis 34-Jährigen sind es jedoch 18 Prozent (Abb. A2.3). In einigen Ländern war dieser Anstieg sehr ausgeprägt: in Korea und Spanien beispielsweise haben nur 16 bzw. 13 Prozent der 46- bis 54-Jährigen einen Abschluss im Tertiärbereich A, während es bei den 25- bis 34-Jährigen 40 bzw. 36 Prozent sind.

\section{Erfolgsquoten im Tertiärbereich}

Tertiärbereich A sind in der Regel in Ländern mit

flexibleren Abschluss-

strukturen höher, ...

Die Erfolgsquoten im Tertiärbereich A schwanken stark zwischen den einzelnen OECD-Ländern und liegen zwischen über 80 Prozent in Irland, Japan, der Türkei und dem Vereinigten Königreich und unter 60 Prozent in Frankreich, Italien, Österreich und Schweden (Abb. A2.2). Sowohl in Italien als auch in Österreich absolviert die Mehrheit der Studierenden, die einen Erstabschluss im Tertiärbereich A erwerben, Studiengänge mit langer Studiendauer von 5 bis

\section{Abbildung A2.3.}

Prozentsatz der Bevölkerung mit mindestens einem Abschluss im Tertiärbereich, nach Altersgruppen (2001)

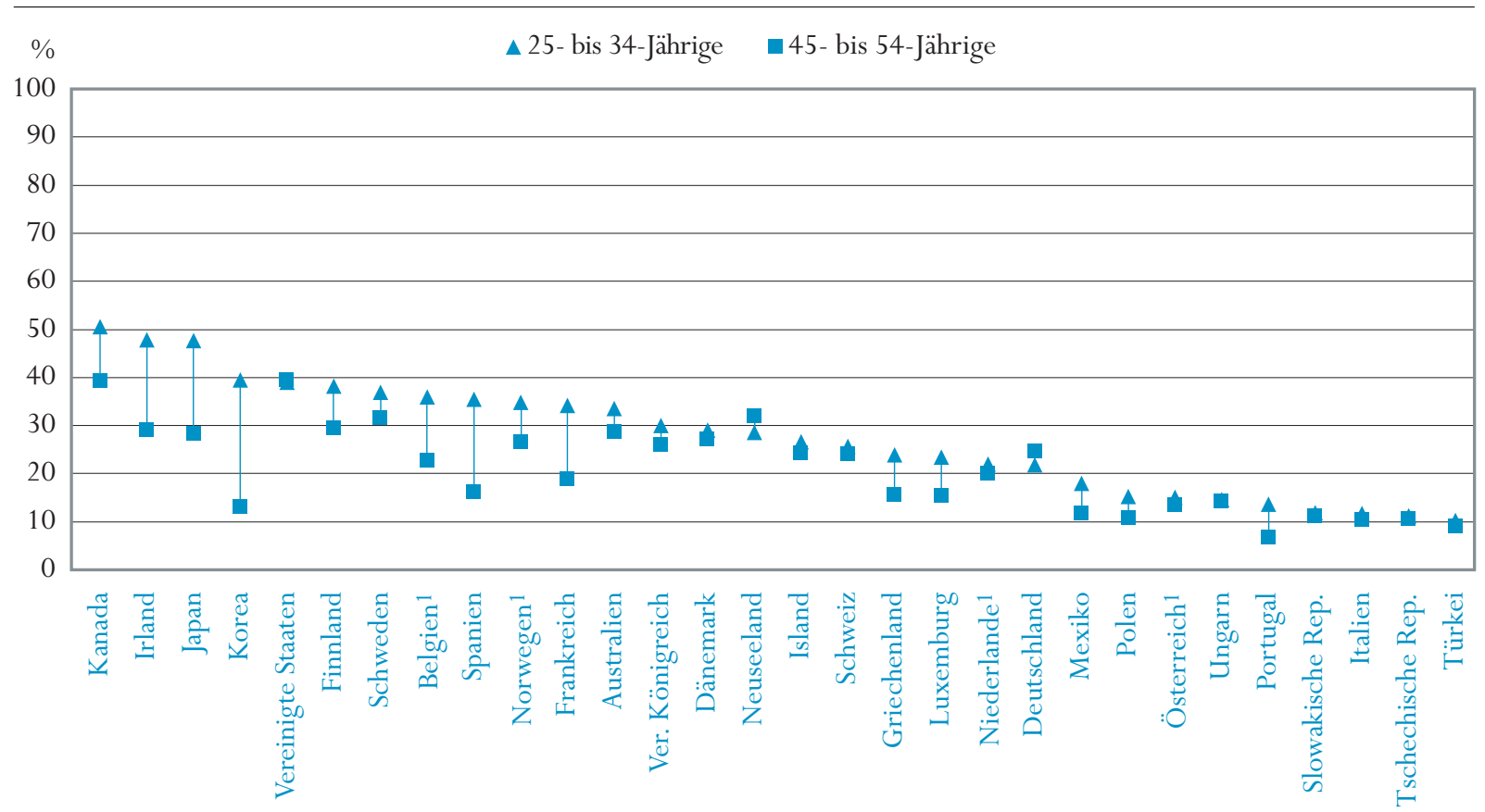

1. Referenzjahr 2000.

Anordnung der Länder in absteigender Reihenfolge des Prozentsatzes 25-bis 34-Jähriger mit mindestens einem Abschluss im Tertiärbereich. Quelle: OECD. Tabelle A2.3. Beschreibung der ISCED-97-Stufen, Zuordnung der länderspezifischen Bildungsgänge zu ISCED 97 und länderspezifische Datenquellen s. Anhang 3 (www.oecd.org/els/education/eag2002). 
6 Jahren. Im Gegensatz dazu erwirbt die Mehrheit der Studierenden in Irland, Japan, Korea, derTürkei und dem Vereinigten Königreich, wo die Erfolgsquoten bei rund 80 Prozent liegen, einen Erstabschluss in einem Studiengang des Tertiärbereich A von mittlerer Dauer (3 bis 5 Jahre) (s. Tabelle A2.2).

Die Erfolgsquoten im Tertiärbereich B liegen zwischen mehr als 80 Prozent in Belgien (fläm.), Dänemark, Japan, Mexiko, Polen und Schweden und ungefähr 50 Prozent in Irland und Italien. Allgemein sind die Studiengänge im Tertiärbereich B von kürzerer Dauer als im Tertiärbereich A. In der Mehrheit der Länder, für die Daten verfügbar sind, erwerben die meisten, wenn nicht alle, Studierenden einen Abschluss in einem kurzen Studiengang (2 bis 3 Jahre). Interessanterweise absolvieren jedoch sowohl in Dänemark als auch in Belgien (fläm.) die Mehrheit der Studierenden Studiengänge mittlerer Dauer (in der flämischen Sprachgemeinschaft Belgiens die einzige Möglichkeit für einen Studiengang im Tertiärbereich B). Gleichfalls sind dort die Erfolgsquoten im Tertiärbereich B am höchsten (Tabelle A2.2).

\section{...was sich im \\ Tertiärbereich B nicht \\ ganz so deutlich \\ feststellen lässt.}

\section{Abbildung A2.4.}

Erfolgsquoten in Studiengängen des Tertiärbereich A, nach Dauer des Studiengangs (2000) Absolventenzahlen geteilt durch Anzahl an Studienanfängern im typischen Eintrittsalter des entsprechenden Studiengangs

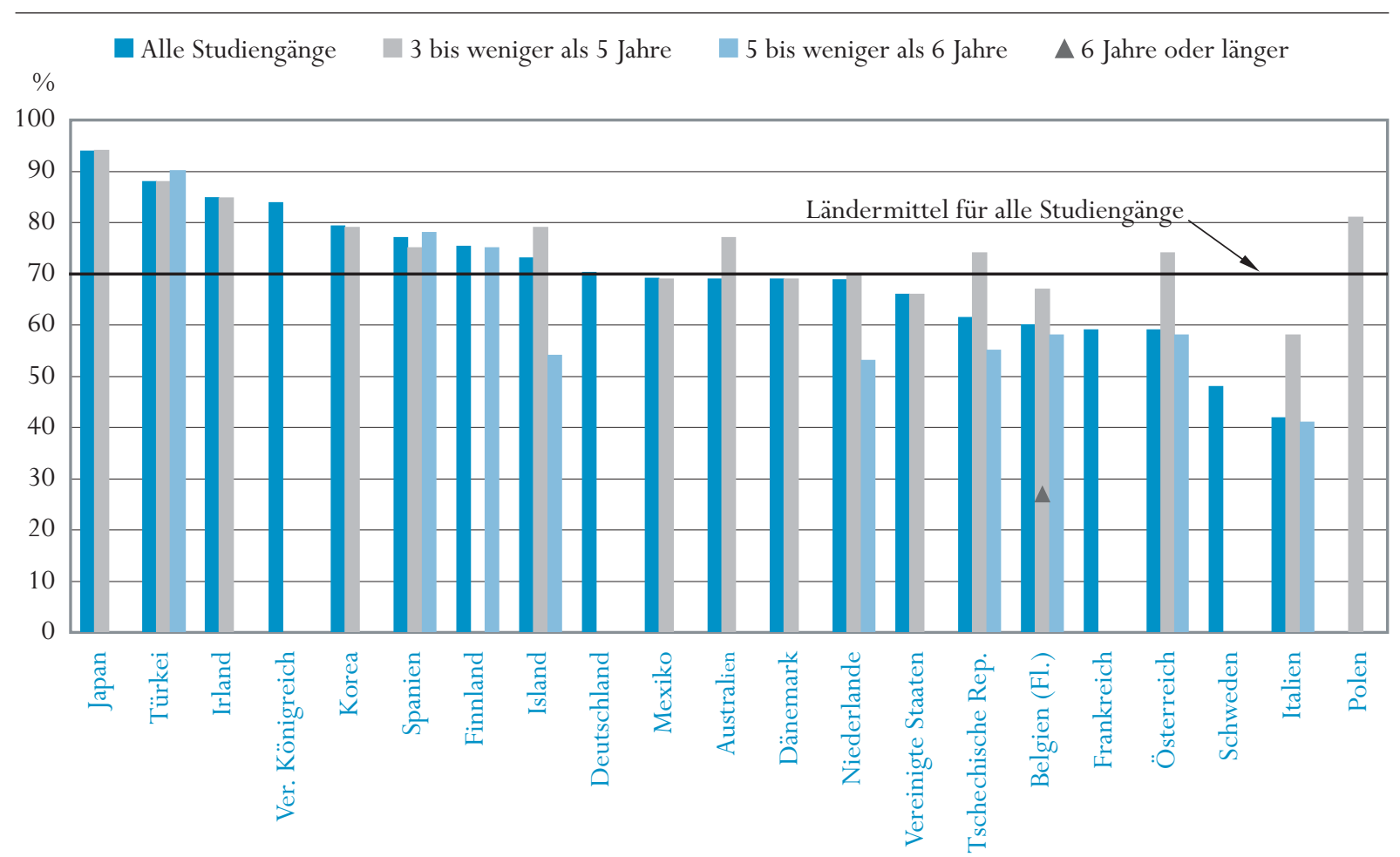

Anordnung der Länder in absteigender Reihenfolge der Erfolgsquote für alle Studiengänge im Tertiärbereich A.

Quelle: OECD. Tabelle A2.2. Hinweise s. Anhang 3 (www.oecd.org/els/education/eag2002). 
Bei den weiterführenden Forschungsprogrammen weisen Italien, Japan und Korea hohe Erfolgsquoten auf.

Die Absolventendaten beziehen sich auf das Studienjahr 19992000 und beruhen auf der alljährlich von der OECD aufgelegten UOE-Datenerhebung zur Bildungsstatistik.
In Italien, Japan und Korea liegen die Erfolgsquoten für Studierende in weiterführenden Forschungsprogrammen bei 85 Prozent und darüber. Umgekehrt liegt die Abbruchwahrscheinlichkeit für solche Studiengänge in Frankreich und Island beträchtlich höher (Erfolgsquote entspricht dort 36 bzw. 50 Prozent).

\section{Definitionen und angewandte Methodik}

Als Absolventen im Tertiärbereich gelten alle Personen, die in einem bestimmten Referenzjahr einen Abschluss im Tertiärbereich A oder B erworben haben. Der Indikator unterscheidet zwischen verschiedenen Kategorien von Abschlüssen: 1.) Abschluss im Tertiärbereich B (ISCED 5B); 2.) Abschluss im Tertiärbereich A (ISCED 5A) und 3.) Abschluss in einem weiterführenden Forschungsprogramm auf dem Niveau der Promotion (ISCED 6). Für einige Länder sind Daten für die entsprechenden Kategorien nicht verfügbar. In diesen Fällen wurden die Absolventen von der OECD der passendsten Kategorie zugeordnet. Abschlüsse im Tertiärbereich A wurden außerdem gemäß der theoretischen Gesamtstudiendauer im Bereich ISCED 5A untergliedert, um Vergleiche unabhängig von der jeweils landespezifischen Abschlussstruktur zu ermöglichen.

Die Abschlussquoten für die Studiengänge, die zu einem Erstabschluss führen (Tertiärbereich A und B), werden als Bruttoabschlussquoten berechnet. Zur Berechnung der Brutto-Abschlussquoten gab jedes Land das typische Abschlussalter an (s. Anhang 1). Die Absolventen können jedoch jeden Alters sein. Die Zahl der Absolventen wurde dann durch die Bevölkerung im üblichen Abschlussalter geteilt. In vielen Ländern ist es jedoch schwierig, ein typisches Abschlussalter anzugeben, weil die Altersverteilung der Absolventen sehr weit gestreut ist.

Eine Nettoabschlussquote wird für Studiengänge, die zu einem Zweit- oder weiteren Abschluss führen, als Summe der altersspezifischen Abschlussquoten ermittelt (hier stellt die Doppelzählung von Abschlüssen kein Problem dar). Die Netto-Abschlussquoten können interpretiert werden als prozentualer Anteil einer fiktiven Altersgruppe, der einen Abschluss im Tertiärbereich erwirbt. Damit sind die Netto-Abschlussquoten unbeeinflusst von Änderungen des Umfangs der entsprechenden Bevölkerungsgruppe oder des typischen Abschlussalters. Für diejenigen Länder, die keine ausführlichen Daten bereitstellen konnten, werden die Brutto-Abschlussquoten dargestellt.

Erfolgsquoten im Tertiärbereich werden definiert als Prozentsatz derjenigen Studienanfänger im jeweiligen Bildungsbereich, die diesen mit einem ersten Abschluss beenden. Studienabbrecher werden dementsprechend definiert als Studierende, die den jeweiligen Bildungsbereich ohne einen ersten Abschluss verlassen. Als erster Abschluss im Tertiärbereich gilt unabhängig von der Studiendauer jeder Abschluss, der am Ende eines Studiengangs erworben wird und nicht einen vorherigen erfolgreichen Abschluss im gleichen Bildungsbereich 
voraussetzt. Die Erfolgsquote wird berechnet als das Verhältnis der Anzahl der Studierenden, die einen ersten Abschluss erwerben, zur Anzahl der Studienanfänger im entsprechenden Bildungsbereich vor $n$ Jahren, wobei $n$ der Anzahl an Jahren entspricht, die zum Erwerb des Abschlusses in einem Vollzeitstudium erforderlich sind.

Die Daten zu Bevölkerung und Bildungsstand stammen aus Datenbanken der OECD und EUROSTAT, die aus nationalen Arbeitskräfteerhebungen zusammengetragen sind. Länderspezifische Datenquellen s. Anhang 3 unter www.oecd.org/els/education/eag2002).

Die Unterschiede im Bildungstand basieren auf dem Prozentsatz der Bevölkerung im Alter von 25 bis 64 Jahren, der einen bestimmten Bildungsstand erreicht hat. Die Festlegung der einzelnen Bildungsbereiche erfolgt auf Grundlage der Internationalen Standardklassifikation des Bildungswesens (ISCED-97). Zur Beschreibung der Bildungsbereiche laut ISCED-97 und der entsprechenden Zuordnung der landesspezifischen Bildungsgänge s. Anhang 3 unter www.oecd.org/els/education/eag2002).
Die Daten zum

Bildungsstand stammen aus nationalen

Arbeitskräfteerhebungen und beruhen auf der Internationalen Standardklassifikation des Bildungswesens (ISCED -97). 
Tabelle A2.1.

Abschlussquoten im Tertiärbereich (2000)

Österreich

Belgien

Kanada

Tschechische Rep. *

Dänemark

Finnland ${ }^{*}$

Frankreich

Deutschland

Griechenland

Ungarn

Island ${ }^{*}$

Irland

Italien

Japan

Korea

Luxemburg

Mexiko

Niederlande

Neuseeland

Norwegen

Polen

Portugal

Slowakische Rep

Spanien

Schweden

Schweiz

Türkei

Ver. Königreich

Vereinigte Staaten

Ländermittel

\begin{tabular}{|c|c|c|c|c|c|}
\hline \multirow[b]{2}{*}{$\begin{array}{c}\text { Studiengänge des } \\
\text { Tertiärbereich B } \\
\text { (Erstabschluss) }\end{array}$} & \multicolumn{4}{|c|}{ Studiengänge des Tertiärbereich A (Erstabschluss) } & \multirow[b]{2}{*}{$\begin{array}{l}\text { Weiterführende } \\
\text { Forschungspro- } \\
\text { gramme }^{1}\end{array}$} \\
\hline & Alle Studiengänge & $\begin{array}{c}3 \text { bis weniger als } \\
5 \text { Jahre (ohne } \\
\text { Studierende mit } \\
\text { anschließendem } \\
\text { Abschluss in einem } \\
\text { längeren Studiengang) }\end{array}$ & 5 Jahre & 6 Jahre und länger & \\
\hline (1) & $(2)$ & (3) & $(4)$ & $(5)$ & (6) \\
\hline $\mathrm{m}$ & 36.3 & 29.1 & 7.1 & $\mathrm{n}$ & 1.3 \\
\hline $\mathrm{m}$ & 16.0 & 1.8 & 14.2 & $\mathrm{n}$ & 1.4 \\
\hline $\mathrm{m}$ & $\mathrm{m}$ & $\mathrm{m}$ & $\mathrm{m}$ & $\mathrm{m}$ & 0.8 \\
\hline $\mathrm{m}$ & 27.9 & 19.7 & 7.1 & 1.2 & 0.8 \\
\hline 4.8 & 13.6 & 3.7 & 10.1 & $\mathrm{a}$ & 0.6 \\
\hline 24.5 & 9.2 & $\mathrm{~m}$ & $\mathrm{~m}$ & $\mathrm{~m}$ & 1.1 \\
\hline 14.3 & 36.3 & 17.2 & 18.4 & 0.6 & 1.9 \\
\hline 18.3 & 24.6 & 10.8 & 13.2 & 0.9 & 1.2 \\
\hline 10.7 & 19.3 & 6.2 & 13.1 & $\mathrm{a}$ & 2.0 \\
\hline $\mathrm{m}$ & $\mathrm{m}$ & $\mathrm{m}$ & $\mathrm{m}$ & $\mathrm{m}$ & $\mathrm{m}$ \\
\hline $\mathrm{m}$ & $\mathrm{m}$ & $\mathrm{m}$ & $\mathrm{m}$ & $\mathrm{m}$ & 0.6 \\
\hline 5.5 & 33.2 & 29.2 & 5.4 & $\mathrm{n}$ & $\mathrm{n}$ \\
\hline 15.2 & 31.2 & 30.0 & 1.2 & $x(4)$ & 0.8 \\
\hline 0.6 & 18.1 & 1.8 & 16.6 & $\mathrm{n}$ & 0.4 \\
\hline 28.8 & 30.9 & 27.2 & $x(3)$ & 3.3 & 0.7 \\
\hline $\mathrm{m}$ & $\mathrm{m}$ & $\mathrm{m}$ & $\mathrm{m}$ & $\mathrm{m}$ & 0.7 \\
\hline $\mathrm{m}$ & $\mathrm{m}$ & $\mathrm{m}$ & $\mathrm{m}$ & $\mathrm{m}$ & $\mathrm{m}$ \\
\hline $\mathrm{m}$ & $\mathrm{m}$ & $\mathrm{m}$ & $\mathrm{m}$ & $\mathrm{m}$ & $\mathrm{m}$ \\
\hline $\mathrm{m}$ & $\mathrm{m}$ & $\mathrm{m}$ & $\mathrm{m}$ & $\mathrm{m}$ & 1.2 \\
\hline $\mathrm{m}$ & $\mathrm{m}$ & $\mathrm{m}$ & $\mathrm{m}$ & $\mathrm{m}$ & 0.8 \\
\hline $\mathrm{m}$ & $\mathrm{m}$ & $\mathrm{m}$ & $\mathrm{m}$ & $\mathrm{m}$ & 1.0 \\
\hline $\mathrm{m}$ & 34.4 & 11.0 & 9.6 & 14.7 & $\mathrm{~m}$ \\
\hline $\mathrm{m}$ & $\mathrm{m}$ & $\mathrm{m}$ & $\mathrm{m}$ & $\mathrm{m}$ & 1.0 \\
\hline 2.2 & $\mathrm{~m}$ & $\mathrm{~m}$ & $\mathrm{~m}$ & $\mathrm{~m}$ & 0.5 \\
\hline 7.8 & $\mathrm{~m}$ & $\mathrm{~m}$ & $\mathrm{~m}$ & $\mathrm{~m}$ & 0.5 \\
\hline 4.2 & 28.1 & 27.2 & 1.2 & $\mathrm{a}$ & 2.5 \\
\hline $\mathrm{m}$ & 10.4 & $\mathrm{n}$ & 9.3 & 1.1 & 2.6 \\
\hline $\mathrm{m}$ & $\mathrm{m}$ & $\mathrm{m}$ & $\mathrm{m}$ & $\mathrm{m}$ & 0.2 \\
\hline $\mathrm{m}$ & 37.5 & $\mathrm{~m}$ & $\mathrm{~m}$ & $\mathrm{~m}$ & 1.3 \\
\hline 8.3 & 33.2 & 18.8 & 13.3 & 2.3 & 1.3 \\
\hline 11.2 & 25.9 & 15.6 & 10.0 & 1.7 & 1.0 \\
\hline
\end{tabular}

Hinweis: $\mathrm{x}$ bedeutet, dass die Daten in einer anderen Spalte enthalten sind, deren Referenz in runden Klammern nach dem ' $\mathrm{x}$ ' angegeben ist. So bedeutet z.B. $\mathrm{x}(2)$, dass die Daten in Spalte 2 enthalten sind.

1. Berechnung der Netto-Abschlussquote durch Addieren der Beteiligungsquoten der einzelnen Altersjahrgänge außer bei Belgien, Frankreich, Irland, Japan, Korea, Niederlande und den Vereinigten Staaten.

* Hinweise s. Anhang 3 (www.oecd.org/els/education/eag2002).

Quelle: OECD. 
Tabelle A2.2.

Erfolgsquoten im Tertiärbereich (2000)

Anzahl der Absolventen geteilt durch die Anzahl von Studienanfängern im typischen Studienanfangsjahr, nach Ziel des Studiengangs und Verteilung der Absolventen nach Dauer des Studiengangs

\begin{tabular}{|c|c|c|c|c|c|c|c|c|c|}
\hline & \multicolumn{4}{|c|}{ Studiengänge im Tertiärbereich A } & \multicolumn{4}{|c|}{ Studiengänge im Tertiärbereich B } & \multirow{3}{*}{$\begin{array}{l}\text { Weiter- } \\
\text { führende } \\
\text { Forschungs } \\
\text { programme }\end{array}$} \\
\hline & \multirow{2}{*}{$\begin{array}{l}\text { Erfolgsquote } \\
\text { für alle } \\
\text { Studiengänge } \\
\text { im Tertiärbe- } \\
\text { reich A } \\
\end{array}$} & \multicolumn{3}{|c|}{$\begin{array}{l}\text { Verteilung der Absolventen } \\
\text { nach Dauer des Studiengangs }\end{array}$} & \multirow{2}{*}{\begin{tabular}{|c|} 
Erfolgsquote \\
für alle \\
Studiengänge \\
im Tertiärbe- \\
reich B
\end{tabular}} & \multicolumn{3}{|c|}{$\begin{array}{l}\text { Verteilung der Absolventen } \\
\text { nach Dauer des Studiengangs }\end{array}$} & \\
\hline & & $\begin{array}{l}3 \text { bis weniger } \\
\text { als } 5 \text { Jahre }\end{array}$ & $\begin{array}{l}5 \text { bis weniger } \\
\text { als } 6 \text { Jahre }\end{array}$ & $\begin{array}{l}6 \text { Jahre und } \\
\text { länger }\end{array}$ & & $\begin{array}{l}2 \text { bis weniger } \\
\text { als } 3 \text { Jahre }\end{array}$ & $\begin{array}{l}3 \text { bis weniger } \\
\text { als } 5 \text { Jahre }\end{array}$ & $\begin{array}{l}5 \text { Jahre und } \\
\text { länger }\end{array}$ & \\
\hline & (1) & (2) & (3) & (4) & (5) & (6) & (7) & $(8)$ & (9) \\
\hline Australien ${ }^{*}$ & 69 & 77 & $\mathrm{~m}$ & $\mathrm{n}$ & $\mathrm{m}$ & $\mathrm{m}$ & $\mathrm{a}$ & $\mathrm{a}$ & $\mathrm{m}$ \\
\hline Österreich & 59 & 74 & 58 & $\mathrm{n}$ & $\mathrm{m}$ & $\mathrm{m}$ & $\mathrm{m}$ & $\mathrm{m}$ & $\mathrm{m}$ \\
\hline Belgien $(\mathrm{Fl} .)^{*}$ & 60 & 67 & 58 & 27 & 88 & a & 88 & a & $\mathrm{m}$ \\
\hline Tschechische Rep. & 61 & 74 & 55 & a & 77 & 75 & 78 & a & $\mathrm{m}$ \\
\hline Dänemark & 69 & 69 & a & a & 84 & 65 & 90 & a & $\mathrm{m}$ \\
\hline Finnland & 75 & $\mathrm{~m}$ & 75 & a & $\mathrm{m}$ & $\mathrm{m}$ & $\mathrm{m}$ & $\mathrm{m}$ & $\mathrm{m}$ \\
\hline Frankreich $^{*}$ & 59 & $\mathrm{~m}$ & $\mathrm{~m}$ & $\mathrm{~m}$ & 72 & 72 & $\mathrm{n}$ & a & 36 \\
\hline Deutschland & 70 & $\mathrm{a}$ & a & $\mathrm{a}$ & 75 & $\mathrm{a}$ & a & a & $\mathrm{m}$ \\
\hline Island & 73 & 79 & 54 & $\mathrm{n}$ & 55 & 73 & 31 & $\mathrm{n}$ & 50 \\
\hline Irland & 85 & 85 & $\mathrm{x}(2)$ & $\mathrm{x}(2)$ & 50 & 50 & $x(6)$ & a & $\mathrm{m}$ \\
\hline Italien & 42 & 58 & 41 & a & 51 & $\mathrm{a}$ & 51 & a & 89 \\
\hline Japan & 94 & 94 & $\mathrm{x}(2)$ & $\mathrm{x}(2)$ & 86 & 86 & $\mathrm{x}(6)$ & $\mathrm{x}(6)$ & 85 \\
\hline Korea & 79 & 79 & $\mathrm{x}(2)$ & a & 74 & 73 & 78 & a & 95 \\
\hline Mexiko & 69 & 69 & $x(2)$ & a & 81 & 81 & $\mathrm{x}(6)$ & a & 54 \\
\hline Niederlande & 69 & 70 & 53 & a & 58 & 59 & 50 & a & $\mathrm{m}$ \\
\hline Polen & $\mathrm{m}$ & 81 & $\mathrm{~m}$ & a & 84 & 84 & a & a & $\mathrm{m}$ \\
\hline Spanien & 77 & 75 & 78 & $\mathrm{n}$ & 74 & 74 & $\mathrm{n}$ & $\mathrm{n}$ & $\mathrm{m}$ \\
\hline Schweden & 48 & $\mathrm{~m}$ & $\mathrm{~m}$ & $\mathrm{a}$ & 85 & $\mathrm{~m}$ & $\mathrm{~m}$ & a & $\mathrm{m}$ \\
\hline Türkei & 88 & 88 & 90 & a & 77 & 77 & a & $\mathrm{a}$ & a \\
\hline Ver. Königreich ${ }^{*}$ & 83 & $\mathrm{~m}$ & $\mathrm{~m}$ & $\mathrm{~m}$ & $\mathrm{~m}$ & $\mathrm{~m}$ & $\mathrm{~m}$ & $\mathrm{~m}$ & $\mathrm{~m}$ \\
\hline Vereinigte Staaten ${ }^{*}$ & 66 & 66 & $\mathrm{a}$ & $\mathrm{a}$ & 62 & 62 & $\mathrm{x}(6)$ & $x(6)$ & $\mathrm{m}$ \\
\hline Ländermittel & 70 & 76 & 62 & 2 & 73 & 72 & 67 & $n$ & 58 \\
\hline Israel & 70 & $\mathrm{~m}$ & $\mathrm{~m}$ & $\mathrm{~m}$ & 91 & $\mathrm{~m}$ & $\mathrm{~m}$ & $\mathrm{~m}$ & $\mathrm{~m}$ \\
\hline
\end{tabular}


KAPITEL A Bildungs-und Lernergebnisse

Tabelle A2.3.

Anteil der Bevölkerung mit einem Abschluss im Tertiärbereich (2001)

Prozentsatz der Bevölkerung mit einem Abschluss in einem Studiengang des Tertiärbereich B, des Tertiärbereich A oder einem weiterfuihrenden Forschungsprogramm, nach Altersgruppen

\begin{tabular}{|c|c|c|c|c|c|c|c|c|c|c|}
\hline & \multicolumn{5}{|c|}{ Tertiärbereich B } & \multicolumn{5}{|c|}{ Tertiärbereich A oder weiterführende Forschungsprogramme } \\
\hline & $25-64$ & $25-34$ & $35-44$ & $45-54$ & $55-64$ & $25-64$ & $25-34$ & $35-44$ & $45-54$ & $55-64$ \\
\hline & $(1)$ & $(2)$ & (3) & (4) & $(5)$ & (6) & (7) & $(8)$ & (9) & $(10)$ \\
\hline Australien & 10 & 10 & 10 & 10 & 9 & 19 & 24 & 19 & 19 & 12 \\
\hline Österreich $^{1}$ & 7 & 8 & 8 & 7 & 5 & 7 & 7 & 8 & 6 & 4 \\
\hline Belgien' & 15 & 19 & 16 & 13 & 9 & 12 & 17 & 13 & 10 & 8 \\
\hline Kanada & 21 & 25 & 23 & 20 & 15 & 20 & 25 & 20 & 20 & 15 \\
\hline Tschechische Rep. & $x(6)$ & $x(7)$ & $\mathrm{x}(8)$ & $\mathrm{x}(9)$ & $\mathrm{x}(10)$ & 11 & 11 & 13 & 11 & 9 \\
\hline Dänemark & 19 & 18 & 20 & 21 & 16 & 8 & 11 & 8 & 6 & 4 \\
\hline Finnland & 17 & 20 & 21 & 16 & 12 & 15 & 18 & 16 & 13 & 11 \\
\hline Frankreich & 11 & 17 & 12 & 9 & 6 & 12 & 18 & 11 & 10 & 8 \\
\hline Deutschland & 10 & 8 & 11 & 10 & 10 & 13 & 14 & 15 & 15 & 10 \\
\hline Griechenland & 5 & 7 & 7 & 4 & 3 & 12 & 17 & 14 & 12 & 6 \\
\hline Ungarn & $\mathrm{x}(6)$ & $\mathrm{x}(7)$ & $\mathrm{x}(8)$ & $\mathrm{x}(9)$ & $\mathrm{x}(10)$ & 14 & 15 & 15 & 14 & 12 \\
\hline Island & 6 & 6 & 8 & 6 & 4 & 19 & 21 & 21 & 19 & 11 \\
\hline Irland & 22 & 28 & 23 & 18 & 13 & 14 & 20 & 14 & 11 & 8 \\
\hline Italien & $\mathrm{x}(6)$ & $\mathrm{x}(7)$ & $\mathrm{x}(8)$ & $\mathrm{x}(9)$ & $\mathrm{x}(10)$ & 10 & 12 & 11 & 10 & 6 \\
\hline Japan & 15 & 23 & 19 & 11 & 5 & 19 & 24 & 25 & 17 & 10 \\
\hline Korea & 7 & 15 & 6 & 2 & 1 & 17 & 25 & 20 & 11 & 8 \\
\hline Luxemburg & 7 & 8 & 6 & 6 & 5 & 11 & 15 & 11 & 10 & 8 \\
\hline Mexiko & 2 & 3 & 2 & 1 & 0 & 13 & 15 & 15 & 11 & 7 \\
\hline Niederlande $^{1}$ & 3 & 2 & 3 & 3 & 2 & 21 & 24 & 22 & 20 & 15 \\
\hline Neuseeland & 15 & 12 & 16 & 18 & 17 & 14 & 17 & 15 & 14 & 7 \\
\hline Norwegen $^{1}$ & 3 & 3 & 3 & 3 & 2 & 26 & 32 & 26 & 23 & 19 \\
\hline Polen & $x(6)$ & $\mathrm{x}(7)$ & $\mathrm{x}(8)$ & $\mathrm{x}(9)$ & $\mathrm{x}(10)$ & 12 & 15 & 11 & 11 & 10 \\
\hline Portugal & 2 & 3 & 3 & 2 & 2 & 7 & 11 & 7 & 5 & 3 \\
\hline Slowakische Rep. & 1 & 1 & 1 & 1 & 0 & 10 & 11 & 11 & 10 & 8 \\
\hline Spanien & 7 & 12 & 7 & 3 & 2 & 17 & 24 & 18 & 13 & 8 \\
\hline Schweden & 15 & 17 & 17 & 14 & 10 & 17 & 20 & 16 & 17 & 15 \\
\hline Schweiz & 10 & 10 & 11 & 9 & 8 & 16 & 16 & 18 & 15 & 13 \\
\hline Türkei & $x(6)$ & $x(7)$ & $\mathrm{x}(8)$ & $\mathrm{x}(9)$ & $\mathrm{x}(10)$ & 9 & 10 & 8 & 9 & 6 \\
\hline Ver. Königreich & 8 & 9 & 9 & 8 & 7 & 18 & 21 & 18 & 18 & 12 \\
\hline Vereinigte Staaten & 9 & 9 & 10 & 10 & 7 & 28 & 30 & 28 & 30 & 24 \\
\hline Ländermittel & 8 & 10 & 9 & 7 & 6 & 15 & 18 & 16 & 14 & 10 \\
\hline
\end{tabular}

Hinweis: $\mathrm{x}$ bedeutet, dass die Daten in einer anderen Spalte enthalten sind, deren Referenz in runden Klammern nach dem ' $\mathrm{x}$ ' angegeben ist. So bedeutet z.B. x(2), dass die Daten in Spalte 2 enthalten sind.

1. Referenzjahr 2000.

Quelle: OECD. Beschreibung der ISCED-97-Stufen, Zuordnung der länderspezifischen Bildungsgänge zu ISCED 97 und länderspezifische Datenquellen s.

Anhang 3 (www.oecd.org/els/education/eag2002). 


\section{BILDUNGSSTAND DER ERWERBSBEVÖLKERUNG UND DER ERWACHSENENBEVÖLKERUNG INSGESAMT}

- Der Bildungsstand ist bei der Erwerbsbevölkerung in der Regel höher als bei den Erwachsenen im erwerbsfähigen Alter, die nicht zur Erwerbsbevölkerung gehören.

- In Mexiko, Portugal, und der Türkei haben mehr als zwei Drittel der Erwerbsbevölkerung zwischen 25 und 64 Jahren keinen Abschluss im Sekundarbereich II, und in Italien und Spanien beträgt ihr Anteil ungefähr 50 Prozent. Der Anteil der Erwerbsbevölkerung im Alter von 25 bis 64 Jahren mit einem Abschluss im Sekundarbereich II liegt in Deutschland, Kanada, Japan, Norwegen, Polen, Schweden, der Schweiz, der Slowakischen Republik, der Tschechischen Republik, dem Vereinigten Königreich und den Vereinigten Staaten bei 85 Prozent und mehr.

\section{Abbildung A3.1.}

Bildungsstand der Erwachsenenbevölkerung (2001)

Verteilung der Bevölkerung im Alter von 25 bis 64 Jahren, nach Bildungsstand

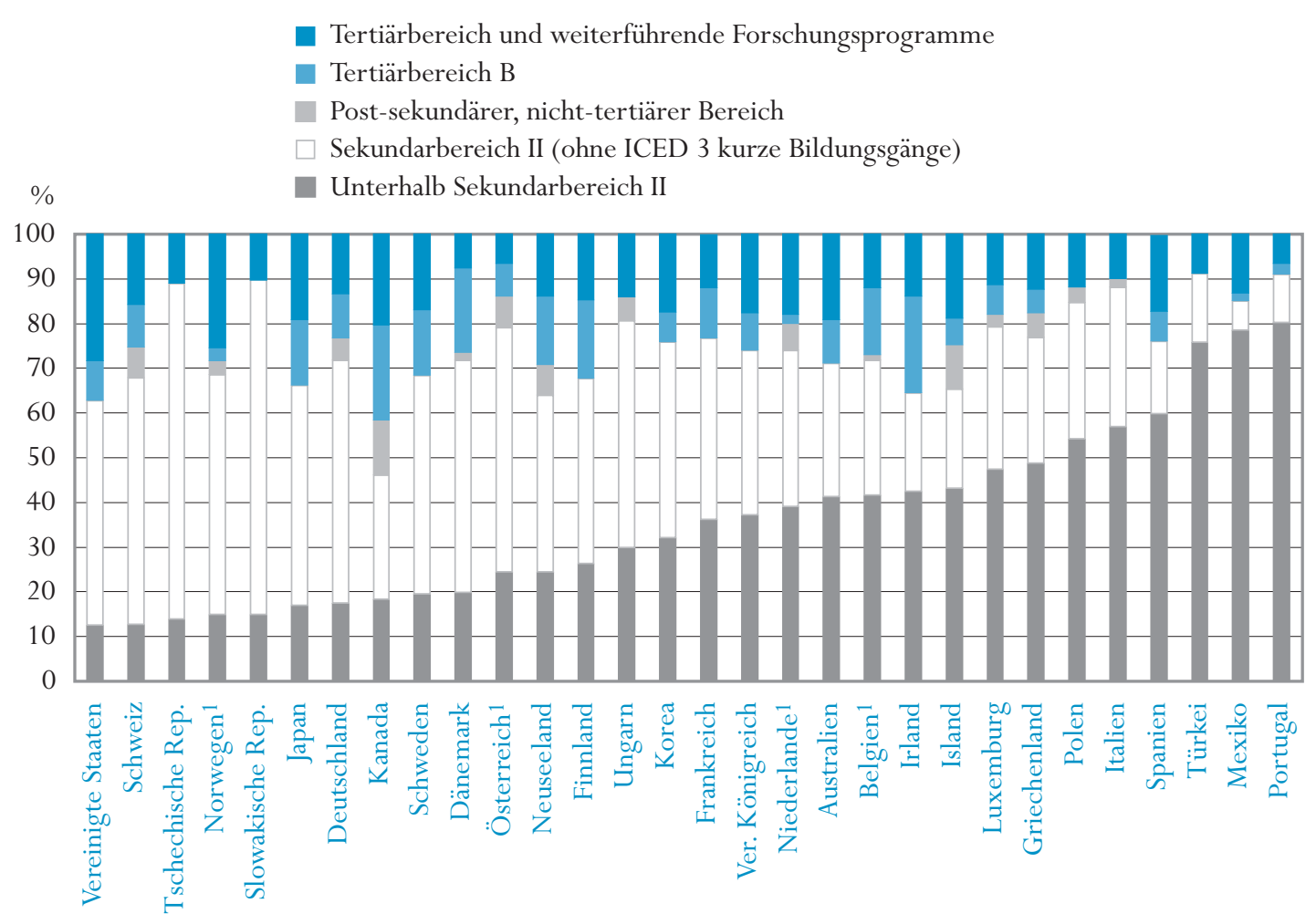

Hinweis: Nicht alle ISCED 3 Bildungsgänge erfüllen die Mindestanforderungen für ISCED 3C lange Bildungsgänge.

Hinweise s. Anhang 3 (www.oecd.org/els/education/eag2002).

1. Referenzjahr 2000

Anordnung der Länder in aufsteigender Reihenfolge des Prozentsatzes 25- bis 64-Jähriger mit einem Abschluss unterhalb des Sekundarbereich II Quelle: OECD. Tabelle A3.1a. Beschreibung der ISCED-97-Stufen, Zuordnung der länderspezifischen Bildungsgänge zu ISCED 97 und länderspezifische Datenquellen s. Anhang 3 (www.oecd.org/els/education/eag2002). 
Dieser Indikator zeigt den unterschiedlichen Bildungsstand der Erwachsenenbevölkerung insgesamt und der Erwerbsbevölkerung als indirekte Kennzahl hinsichtlich der in Wirtschaft und Gesellschaft vorhandenen Kenntnisse und Fähigkeiten auf.

Die Länder unterscheiden sich hinsichtlich der Verteilung des Bildungsstands ihrer Erwerbsbevölkerung erheblich.

Der Bildungsstand ist bei der Erwerbsbevölkerung in der Regel höher als bei Erwachsenen im erwerbsfähigen Alter, die nicht zur Erwerbsbevölkerung gehören.

\section{Politischer Hintergrund}

Eine gut ausgebildete und qualifizierte Erwerbsbevölkerung ist für das soziale und wirtschaftliche Wohl eines Landes und des Einzelnen von großer Bedeutung. Bildung ist ein entscheidender Faktor, damit der Einzelne über Wissen, Kenntnisse, Fähigkeiten und Kompetenzen verfügt, um gleichfalls besser am gesellschaftlichen und wirtschaftlichen Leben teilnehmen zu können. Bildung trägt auch zu einer Erweiterung des wissenschaftlichen und kulturellen Wissens bei. Dieser Indikator zeigt den unterschiedlichen Bildungsstand der Erwachsenenbevölkerung insgesamt und der Erwerbsbevölkerung auf.

\section{Ergebnisse und Erläuterungen}

In 20 von 30 OECD-Ländern haben mindestens 60 Prozent der Erwerbsbevölkerung zwischen 25 und 64 Jahren mindestens einen Abschluss im Sekundarbereich II (Abb. A3.1b). Dies bezieht sich auf Absolventen von Bildungsgängen auf Stufe ISCED 3A oder 3B sowie von langen Bildungsgängen auf Stufe ISCED 3C gemäß ISCED-97. Ihr Anteil beträgt in 13 OECD-Ländern mindestens 80 Prozent, und zwar in Dänemark, Deutschland, Japan, Kanada, Neuseeland, Norwegen, Österreich, Schweden, der Schweiz, der Slowakischen Republik, der Tschechischen Republik, Ungarn und den Vereinigten Staaten. In anderen Ländern, insbesondere - aber nicht nur - in Südeuropa, sieht die Aufteilung der Bevölkerung nach Bildungsabschlüssen ganz anders aus. In Italien, Mexiko, Portugal, Spanien und der Türkei hat mehr als die Hälfte der Erwerbsbevölkerung im Alter von 25 bis 64 Jahren keinen Abschluss im Sekundarbereich II.

Ein Vergleich der Verteilung des Bildungsstands unter der Erwerbsbevölkerung im Alter zwischen 25 und 64 Jahren mit der Verteilung des Bildungsstands unter der Gesamtbevölkerung derselben Altersgruppe zeigt, dass der Prozentsatz der Erwerbsbevölkerung, der einen Abschluss des Sekundarbereich II oder des Tertiärbereichs erlangt hat, höher ist (s. Tabelle A3.1b). Während über alle OECD-Länder hinweg durchschnittlich 66 Prozent der Erwachsenenbevölkerung mindestens einen Abschluss im Sekundarbereich II aufweisen, sind es bei den erwachsenen Erwerbspersonen 71 Prozent. Der Anteil der Gesamtbevölkerung im Erwachsenenalter mit einem Abschluss im Sekundarbereich II oder im Tertiärbereich ist in Belgien, Italien und Ungarn um mehr als 9 Prozentpunkte geringer als in der Erwerbsbevölkerung, wohingegen dieser Unterschied in Island, Japan, Korea und der Schweiz weniger als 2 Prozentpunkte beträgt. 


\section{Abbildung A3.2.}

Geschlechtspezifische Unterschiede beim Bildungsstand, nach Altersgruppen (2001)

Unterschied zwischen 25- bis 34-jährigen und 45- bis 54-jährigen Frauen und Männern im Prozentsatz der Bevölkerung mit mindestens einem Abschluss im Sekundarbereich II oder im Tertiärbereich

25- bis 34-Jährige 45 - bis 54-Jährige

Mindestens ein Abschluss im Sekundarbereich II

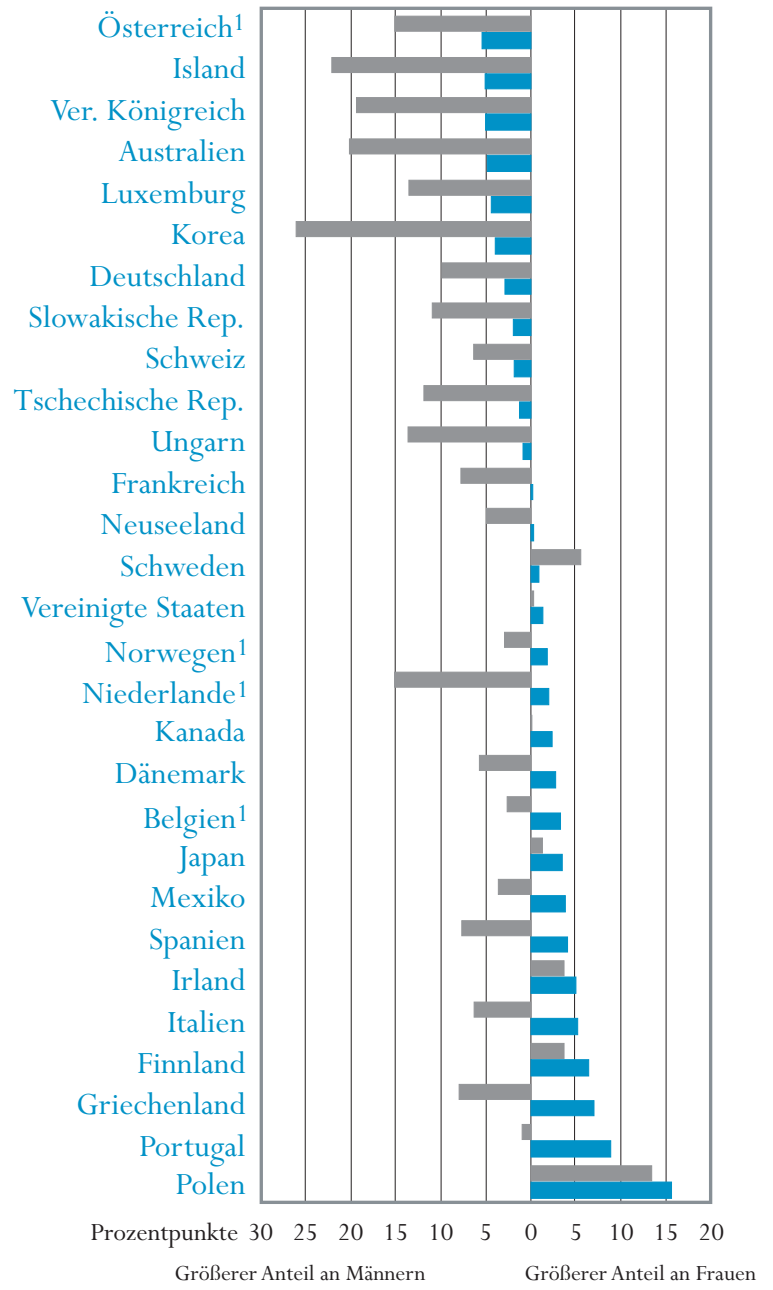

25-bis 34-Jährige 45-bis 54-Jährige

Mindestens ein Abschluss im Tertiärbereich

Schweiz

Korea

Mexiko

Deutschland

Luxemburg

Türkei

Österreich 1

Ver. Königreich

Tschechische Rep.

Slowakische Rep.

Niederlande ${ }^{1}$

Italien

Japan

Ungarn

Island

Neuseeland

Frankreich

Irland

Schweden

Griechenland

Vereinigte Staaten

Belgien $^{1}$

Polen

Spanien

Portugal

Dänemark

Australien

Norwegen ${ }^{1}$

Kanada

Finnland

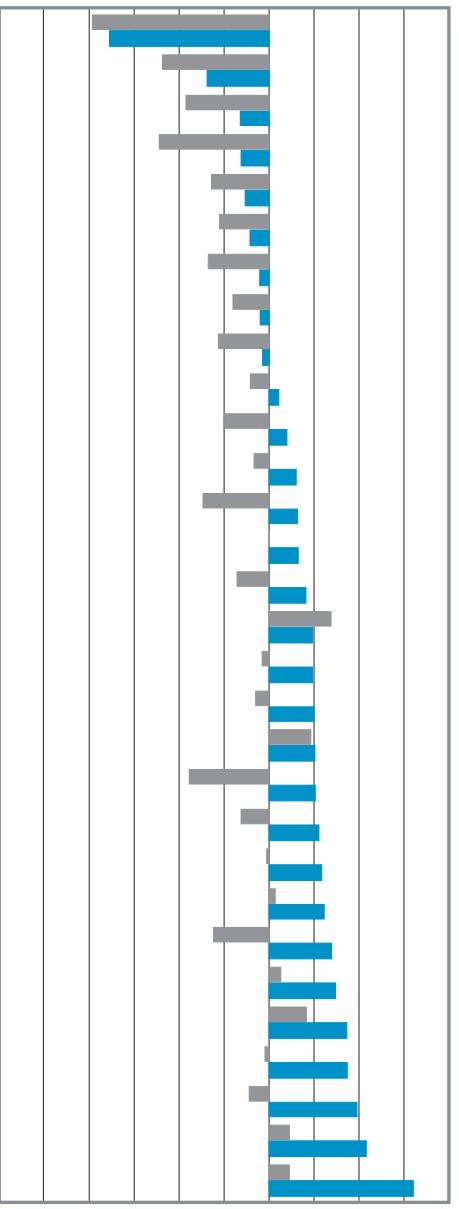

Prozentpunkte $\begin{array}{llllllllllll}30 & 25 & 20 & 15 & 10 & 5 & 0 & 5 & 10 & 15 & 20\end{array}$

Größerer Anteil an Männern

Größerer Anteil an Frauen

Hinweis: Nicht alle ISCED 3 Bildungsgänge erfüllen die Mindestanforderungen an ISCED 3C lange Bildungsgänge. Hinweise s. Anhang 3. (www.oecd.org/els/education / eag2002).

1. Referenzjahr 2000.

Anordnung der Länder in aufsteigender Reihenfolge des Unterschieds zwischen Frauen und Männern als Prozentsatz der 25- bis 34-Jährigen mit mindestens einem Abschluss im Sekundarbereich II bzw. im Tertiärbereich.

Quelle: OECD. Tabelle A3.1c. Beschreibung der ISCED-97-Stufen, Zuordnung der länderspezifischen Bildungsgänge zu

ISCED 97 und länderspezifische Datenquellen s. Anhang 3 (www.oecd.org/els/education/eag2002). 
Die Daten stammen aus nationalen

Arbeitskräfteerhebungen und beruhen auf der Internationalen Standardklassifikation des Bildungswesens (ISCED -97).

\section{Definitionen und angewandte Methodik}

Die hier aufgezeigten Unterschiede im Bildungsstand basieren auf dem Anteil der Bevölkerung bzw. der Erwerbsbevölkerung im Alter von 25 bis 64 Jahren, der einen bestimmten Bildungsstand erreicht hat. Die Festlegung der einzelnen Bildungsbereiche erfolgt auf Grundlage der Internationalen Standardklassifikation des Bildungswesens (ISCED-97). Der post-sekundäre, nicht-tertiäre Bereich (ISCED 4) deckt Bildungsgänge ab, die sich im Grenzbereich zwischen Sekundarbereich II und Tertiärbereich bewegen.

Der Tertiärbereich umfasst zwei Stufen (ISCED 5 und ISCED 6). ISCED 5 umfasst Bildungsgänge, die nicht direkt zu einem Abschluss in einem weiterführenden Forschungsprogramm führen, während ISCED 6 für solche Bildungsgänge (mit beispielsweise einem Doktortitel als Abschluss) reserviert ist. Der Tertiärbereich ISCED 5 ist darüber hinaus in zwei Unterbereiche aufgegliedert, ISCED 5A und 5B. ISCED 5A, der Tertiärbereich A, deckt die mehr theoretisch ausgerichteten Ausbildungsgänge ab, die den Weg zu Abschlüssen in weiterführenden Forschungsprogrammen und Berufen mit sehr hohen, allgemeinen Anforderungen öffnen sollen, während ISCED 5B, der Tertiärbereich B, die mehr praktisch orientierten bzw. speziell berufsspezifischen Ausbildungsgänge abdeckt, die den Teilnehmern einen für den Arbeitsmarkt unmittelbar relevanten Abschluss ermöglichen sollen.

Die Daten zu Bevölkerung und Bildungsstand stammen aus Datenbanken der OECD und EUROSTAT, die aus nationalen Arbeitskräfteerhebungen zusammengetragen sind. Länderspefizische Datenquellen s. Anhang 3 unter www.oecd.org/els/education/eag2002). 
Tabelle A3.1a.

Bildungsstand der Bevölkerung (2001)

Verteilung der Bevölkerung im Alter von 25 bis 64 Jahren, nach dem höchsten erreichten Bildungsstand

Australien
Österreich ${ }^{1}$
Belgien ${ }^{1}$
Kanada

Tschechische Rep.

Dänemark

Finnland

Frankreich

Griechenland

Ungarn

Island

Irland

Italien

Japan

Korea

Luxemburg

Mexiko

Niederlande

Neuseeland

Norwegen ${ }^{1}$

Polen

Portugal

Slowakische Rep.

Spanien

Schweden

Schweiz

Türkei

Ver. Königreich

Vereinigte Staaten

Ländermittel
Deutschland

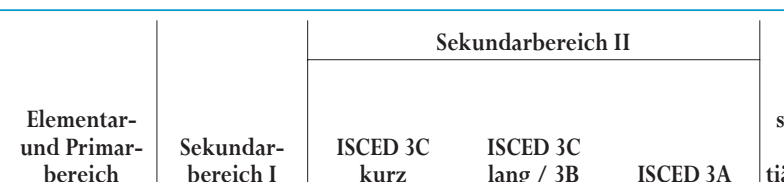

\begin{tabular}{|c|c|c|c}
$\begin{array}{c}\text { Post- } \\
\text { sekundärer, } \\
\text { nicht-ter- } \\
\text { tiärer Bereich }\end{array}$ & $\begin{array}{c}\text { Tertiärbe- } \\
\text { reich A und } \\
\text { weiter- } \\
\text { führende } \\
\text { reich B }\end{array}$ & $\begin{array}{c}\text { Forschungs- } \\
\text { programme }\end{array}$ & $\begin{array}{c}\text { Alle } \\
\text { Bildungs- } \\
\text { bereiche }\end{array}$ \\
\hline$(6)$ & $(7)$ & $(8)$ & $(9)$
\end{tabular}

\begin{tabular}{c|cccc|c|r|r|r}
\hline$(1)$ & $(2)$ & $(3)$ & $(4)$ & $(5)$ & $(6)$ & $(7)$ & programme & bereiche \\
\hline $\mathrm{x}(2)$ & 41 & $\mathrm{a}$ & 11 & 19 & $\mathrm{x}(5)$ & 10 & 19 & 100 \\
$\mathrm{x}(2)$ & 24 & $\mathrm{a}$ & 48 & 7 & 7 & 7 & 7 & 100
\end{tabular}

Hinweis: $\mathrm{x}$ bedeutet, dass die Daten in einer anderen Spalte enthalten sind, deren Referenz in runden Klammern nach dem 'x' angegeben ist. So bedeutet z. B. $\mathrm{x}(2)$, dass die Daten in Spalte 2 enthalten sind.

1. Referenzjahr 2000 .

Quelle: OECD. Beschreibung der ISCED-97-Stufen, Zuordnung der länderspezifischen Bildungsgänge zu ISCED 97 und länderspezifische Datenquellen s. Anhang 3 (www.oecd.org/els/education/eag2002). 
Tabelle A3.1b.

Bildungsstand der Erwerbsbevölkerung (2001)

Verteilung der Erwerbsbevölkerung im Alter von 25 bis 64 Jahren, nach dem höchsten erreichten Bildungsstand

$$
\text { 䚓 }
$$

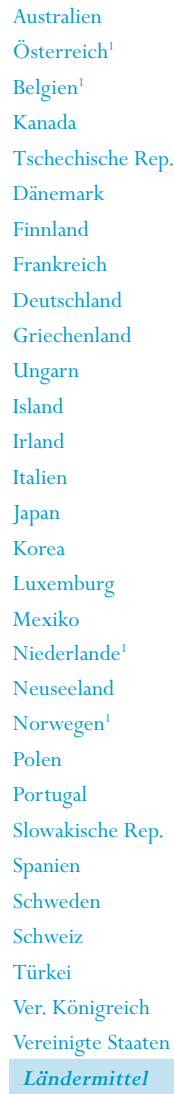

Tertiärbereich

A und weiter-

führende

Forschungsprogramme ildungsbereiche

\begin{tabular}{c|cccc|c} 
bereich & bereich I & kurz & lang / 3B & ISCED 3A & ti \\
\hline$(1)$ & $(2)$ & $(3)$ & $(4)$ & $(5)$ & \\
\hline $\mathrm{x}(2)$ & 35 & a & 12 & 20 &
\end{tabular}
tiärer Bereich bereich B (8)

(6)

(7)

19
21

21$$
4
$$$$
\mathrm{x}(2)
$$$$
\text { n }
$$$$
\mathrm{x}(2)
$$$$
\begin{array}{r}
13 \\
1
\end{array}
$$$$
\begin{array}{r}
1 \\
32
\end{array}
$$$$
\begin{array}{r}
32 \\
1 \\
2
\end{array}
$$$$
\begin{array}{r}
1 \\
2 \\
18
\end{array}
$$$$
\begin{aligned}
& 18 \\
& 12
\end{aligned}
$$$$
\mathrm{x}(2)
$$$$
15
$$$$
\begin{aligned}
& 15 \\
& 23 \\
& 50
\end{aligned}
$$$$
50
$$$$
8
$$$$
\mathrm{x}(2)
$$$$
\text { n }
$$$$
\mathrm{x}(2)
$$$$
64
$$$$
\begin{array}{r}
64 \\
\mathrm{n}
\end{array}
$$$$
\begin{array}{r}
26 \\
7
\end{array}
$$$$
\begin{aligned}
& 7 \\
& 3
\end{aligned}
$$$$
\begin{array}{r}
3 \\
59
\end{array}
$$$$
59
$$$$
\mathrm{x}(2)
$$$$
3
$$

3
12

Hinweis: $\mathrm{x}$ bedeutet, dass die Daten in einer anderen Spalte enthalten sind, deren Referenz in runden Klammern nach dem ' $\mathrm{x}$ ' angegeben ist. So bedeutet z. B. x(2), dass die Daten in Spalte 2 enthalten sind.

1. Referenzjahr 2000.

Quelle: OECD. Beschreibung der ISCED-97-Stufen, Zuordnung der länderspezifischen Bildungsgänge zu ISCED 97 und länderspezifische Datenquellen s. Anhang 3 (www.oecd.org/els/education/eag2002). 
Tabelle A3.1c.

Bildungsstand der Bevölkerung, nach Geschlecht (2001)

Prozentsatz der Bevölkerung mit mindestens einem Abschluss des Sekundarbereich II oder des Tertiärbereichs, nach Altersgruppen und Geschlecht

\begin{tabular}{|c|c|c|c|c|c|c|c|c|c|c|c|}
\hline & & \multicolumn{5}{|c|}{ Mindestens Abschluss im Sekundarbereich II ${ }^{1}$} & \multicolumn{5}{|c|}{\begin{tabular}{|c} 
Mindestens Abschluss im Tertiärbereich (Tertiärbereich A, \\
Tertiärbereich B oder weiterführende Forschungsprogramme)
\end{tabular}} \\
\hline & & $25-64$ & $25-34$ & $35-44$ & $45-54$ & $55-64$ & $25-64$ & $25-34$ & $35-44$ & $45-54$ & $55-64$ \\
\hline \multirow[t]{2}{*}{ Australien } & Männer & 66 & 73 & 67 & 65 & 54 & 27 & 29 & 27 & 29 & 22 \\
\hline & Frauen & 52 & 68 & 54 & 45 & 34 & 31 & 38 & 32 & 29 & 21 \\
\hline \multirow[t]{2}{*}{ Österreich ${ }^{2}$} & Männer & 82 & 86 & 85 & 79 & 73 & 17 & 16 & 19 & 17 & 15 \\
\hline & Frauen & 69 & 81 & 75 & 64 & 52 & 11 & 14 & 14 & 10 & 5 \\
\hline \multirow[t]{2}{*}{ Belgien $^{2}$} & Männer & 59 & 74 & 61 & 53 & 42 & 27 & 33 & 28 & 23 & 20 \\
\hline & Frauen & 58 & 77 & 65 & 50 & 35 & 28 & 39 & 31 & 23 & 14 \\
\hline \multirow[t]{2}{*}{ Kanada } & Männer & 81 & 88 & 83 & 81 & 68 & 39 & 45 & 39 & 38 & 30 \\
\hline & Frauen & 82 & 91 & 86 & 81 & 65 & 44 & 56 & 46 & 40 & 30 \\
\hline \multirow[t]{2}{*}{ Tschechische Rep. } & Männer & 91 & 93 & 93 & 90 & 86 & 13 & 12 & 14 & 14 & 12 \\
\hline & Frauen & 82 & 92 & 87 & 78 & 68 & 9 & 11 & 12 & 8 & 7 \\
\hline \multirow[t]{2}{*}{ Dänemark } & Männer & 82 & 85 & 82 & 83 & 75 & 24 & 25 & 24 & 25 & 21 \\
\hline & Frauen & 79 & 88 & 79 & 78 & 69 & 29 & 34 & 32 & 29 & 19 \\
\hline \multirow[t]{2}{*}{ Finnland } & Männer & 72 & 84 & 81 & 68 & 51 & 29 & 30 & 32 & 28 & 25 \\
\hline & Frauen & 76 & 90 & 87 & 72 & 51 & 36 & 46 & 42 & 31 & 22 \\
\hline \multirow[t]{2}{*}{ Frankreich $^{3}$} & Männer & 67 & 78 & 69 & 62 & 52 & 22 & 32 & 21 & 19 & 16 \\
\hline & Frauen & 61 & 78 & 66 & 55 & 40 & 24 & 37 & 24 & 18 & 13 \\
\hline \multirow[t]{2}{*}{ Deutschland } & Männer & 87 & 87 & 88 & 88 & 85 & 28 & 23 & 30 & 31 & 28 \\
\hline & Frauen & 78 & 84 & 83 & 78 & 67 & 18 & 20 & 21 & 18 & 12 \\
\hline Griechenland & Männer & 54 & 69 & 62 & 47 & 33 & 20 & 21 & 24 & 20 & 13 \\
\hline & Frauen & 49 & 76 & 58 & 40 & 23 & 16 & 27 & 19 & 11 & 6 \\
\hline Ungarn & Männer & 75 & 81 & 82 & 79 & 49 & 14 & 13 & 13 & 14 & 14 \\
\hline & Frauen & 66 & 80 & 75 & 65 & 40 & 15 & 16 & 18 & 14 & 10 \\
\hline Island & Männer & 64 & 64 & 67 & 66 & 58 & 24 & 25 & 27 & 26 & 16 \\
\hline & Frauen & 49 & 59 & 54 & 44 & 33 & 25 & 29 & 31 & 22 & 15 \\
\hline Irland & Männer & 55 & 71 & 59 & 46 & 35 & 35 & 45 & 37 & 30 & 22 \\
\hline & Frauen & 60 & 76 & 66 & 50 & 36 & 36 & 50 & 36 & 28 & 20 \\
\hline Italien & Männer & 44 & 55 & 48 & 42 & 26 & 10 & 10 & 11 & 11 & 8 \\
\hline & Frauen & 43 & 60 & 51 & 35 & 18 & 10 & 13 & 11 & 10 & 5 \\
\hline Japan & Männer & 83 & 92 & 93 & 80 & 65 & 36 & 46 & 46 & 32 & 20 \\
\hline & Frauen & 83 & 95 & 95 & 82 & 61 & 32 & 49 & 41 & 25 & 11 \\
\hline Korea & Männer & 76 & 95 & 84 & 61 & 45 & 30 & 42 & 34 & 19 & 15 \\
\hline & Frauen & 59 & 91 & 68 & 35 & 16 & 18 & 35 & 17 & 7 & 3 \\
\hline Luxemburg & Männer & 58 & 62 & 61 & 54 & 53 & 21 & 25 & 20 & 19 & 20 \\
\hline & Frauen & 47 & 57 & 53 & 40 & 31 & 15 & 22 & 15 & 12 & 8 \\
\hline Mexiko & Männer & 22 & 23 & 25 & 19 & 12 & 18 & 20 & 22 & 17 & 10 \\
\hline & Frauen & 22 & 27 & 24 & 16 & 10 & 12 & 16 & 13 & 7 & 4 \\
\hline Niederlande $^{2,3}$ & Männer & 63 & 73 & 71 & 67 & 62 & 26 & 27 & 27 & 27 & 22 \\
\hline & Frauen & 61 & 75 & 67 & 53 & 41 & 21 & 26 & 22 & 18 & 13 \\
\hline Neuseeland & Männer & 77 & 82 & 80 & 78 & 65 & 26 & 26 & 27 & 29 & 23 \\
\hline & Frauen & 74 & 82 & 79 & 73 & 55 & 32 & 31 & 34 & 35 & 26 \\
\hline Norwegen $^{2}$ & Männer & 86 & 93 & 90 & 83 & 73 & 28 & 30 & 28 & 28 & 23 \\
\hline & Frauen & 84 & 94 & 91 & 80 & 66 & 29 & 40 & 30 & 25 & 18 \\
\hline Polen & Männer & 39 & 44 & 39 & 38 & 34 & 11 & 12 & 9 & 10 & 11 \\
\hline & Frauen & 52 & 60 & 56 & 51 & 38 & 13 & 18 & 13 & 11 & 10 \\
\hline Portugal & Männer & 19 & 28 & 19 & 14 & 10 & 7 & 10 & 7 & 6 & 5 \\
\hline & Frauen & 21 & 37 & 21 & 13 & 7 & 11 & 17 & 11 & 7 & 4 \\
\hline Slowakische Rep. & Männer & 90 & 95 & 92 & 89 & 79 & 11 & 11 & 11 & 12 & 10 \\
\hline & Frauen & 81 & 93 & 88 & 78 & 56 & 11 & 12 & 12 & 10 & 7 \\
\hline Spanien & Männer & 42 & 55 & 46 & 34 & 22 & 24 & 32 & 25 & 19 & 14 \\
\hline & Frauen & 40 & 59 & 46 & 26 & 14 & 23 & 39 & 25 & 13 & 7 \\
\hline Schweden & Männer & 79 & 90 & 84 & 76 & 63 & 30 & 34 & 31 & 29 & 24 \\
\hline & Frauen & 82 & 91 & 88 & 81 & 67 & 34 & 39 & 35 & 34 & 25 \\
\hline Schweiz & Männer & 90 & 93 & 92 & 88 & 87 & 35 & 35 & 37 & 34 & 33 \\
\hline & Frauen & 85 & 91 & 88 & 82 & 75 & 16 & 17 & 21 & 15 & 8 \\
\hline Türkei & Männer & 28 & 35 & 28 & 23 & 15 & 10 & 11 & 9 & 11 & 8 \\
\hline & Frauen & 19 & 25 & 18 & 13 & 10 & 7 & 9 & 7 & 6 & 4 \\
\hline Ver. Königreich ${ }^{3}$ & Männer & 69 & 70 & 70 & 71 & 63 & 27 & 30 & 28 & 28 & 20 \\
\hline & Frauen & 57 & 65 & 59 & 52 & 42 & 25 & 29 & 26 & 24 & 17 \\
\hline Vereinigte Staaten & Männer & 87 & 87 & 88 & 89 & 83 & 37 & 36 & 37 & 41 & 35 \\
\hline & Frauen & 88 & 89 & 89 & 89 & 82 & 37 & 42 & 38 & 38 & 27 \\
\hline Ländermittel & Männer & 66 & 73 & 70 & 64 & 54 & 24 & 26 & 25 & 23 & 18 \\
\hline & Frauen & 62 & 74 & 67 & 57 & 43 & 22 & 29 & 24 & 19 & 13 \\
\hline
\end{tabular}

1. Daten ohne ISCED 3C kurze Bildungsgänge.

2. Referenzjahr 2000.

3. Nicht alle ISCED 3 Bildungsgänge erfüllen die Mindestanforderungen für ISCED 3C lange Bildungsgänge. Hinweise s. Anhang 3 (www.oecd.org/els/ education/eag2002)

Quelle: OECD. Beschreibung der ISCED-97-Stufen, Zuordnung der länderspezifischen Bildungsgänge zu ISCED 97 und länderspezifische Datenquellen s. Anhang 3 (www.oecd.org/els/education/eag2002). 


\section{ABSOLVENTEN NACH STUDIENBEREICHEN}

- Im Durchschnitt aller OECD-Länder erzielt jeder dritte Absolvent des Tertiärbereich A einen Abschluss in Sozial-, Rechts- oder Wirtschaftswissenschaften. Als zweitbeliebtester Bereich folgen die Geisteswissenschaften, Kunst und Pädagogik.

- In den Bereichen Geisteswissenschaften, Kunst, Pädagogik, Gesundheit und Soziales sind im Durchschnitt der OECD-Länder mehr als zwei Drittel der Absolventen des Tertiärbereich A Frauen, wohingegen ihr Anteil in Mathematik und Informatik weniger als ein Drittel ausmacht und weniger als ein Viertel in den Bereichen Ingenieurwesen, Fertigung und Bauwesen.

- In den OECD-Ländern nehmen immer noch eher Männer als Frauen an weiterführenden Forschungsprogrammen (z.B. Promotion) teil.

- Die meisten Absolventen im Tertiärbereich B konzentrieren sich in den Sozial-, Rechts- und Wirtschafts-wissenschaften (zusammen 26 Prozent). 


\section{Abbildung A4.1.}

Absolventen im Tertiärbereich, nach Studienbereichen (2000)

Absolventen mit einem Abschluss im Tertiärbereich A oder einem weiterführenden

Forschungsprogramm, nach Studienbereichen

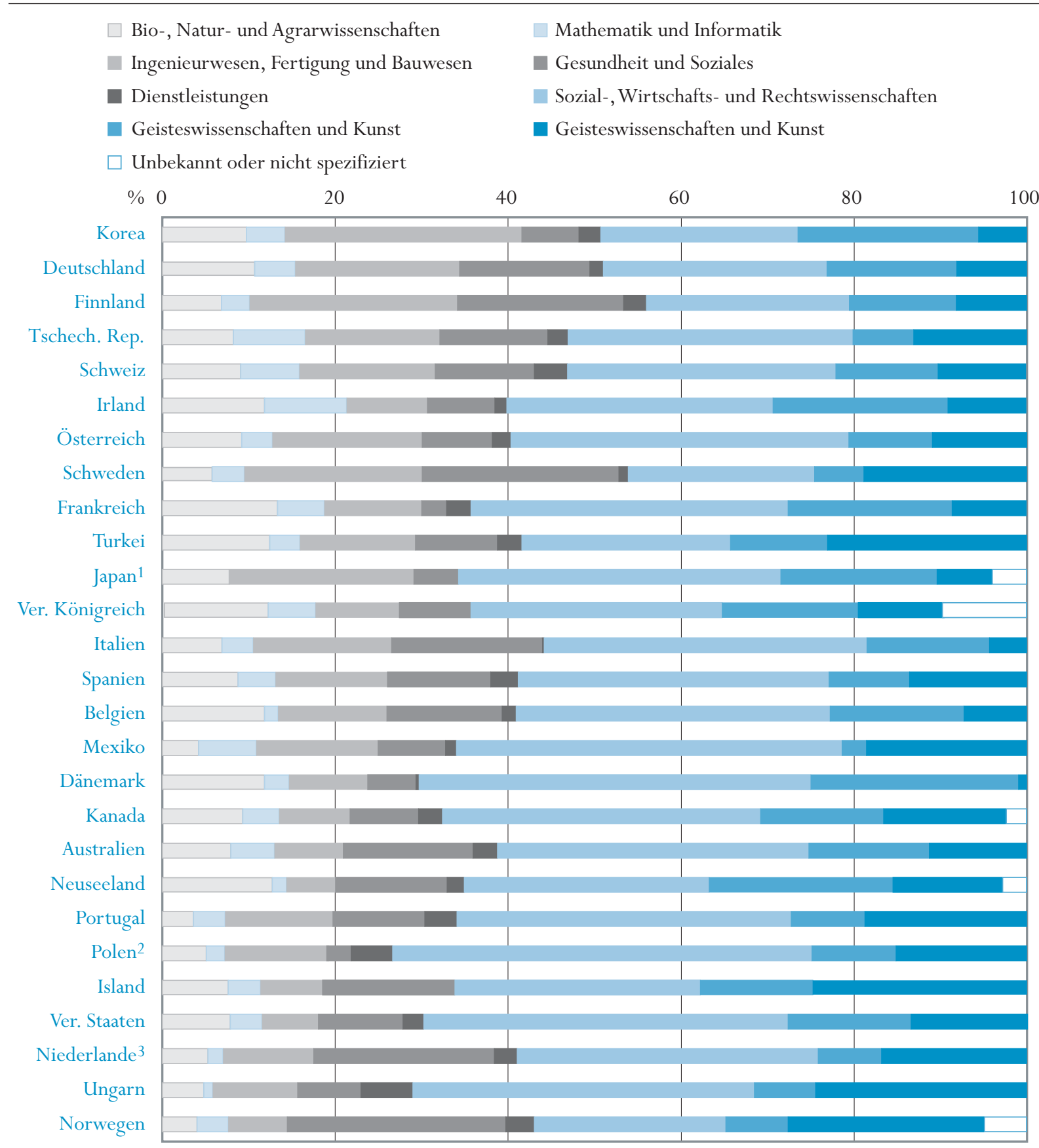

Anordnung der Länder in absteigender Reihenfolge des Anteils der Abschlüsse in Bio-, Natur- und Agrarwissenschaften, Mathematik und Informatik sowie Ingenieurwesen, Fertigung und Bauwesen.

1. Mathematik und Informatik sind in der Kategorie "Bio-, Natur- und Agrarwissenschaften" enthalten.

2. Ohne Studiengänge, die zu einem Zweitabschluss im Tertiärbereich A führen und weiterführende Forschungsprogramme.

3. Ohne weiterführende Forschungsprogramme.

Quelle: OECD. Tabelle A4.1. Hinweise s. Anhang 3 (www.oecd.org/els/education/eag2002). 
Dieser Indikator zeigt die Aufteilung der Absolventen des Tertiärbereichs nach Studienbereichen.

Im Durchschnitt der OECD-Länder erwirbt jeder dritte Absolvent des

Tertiärbereich A einen Abschluss in den Sozial-, Rechts- und Wirtschaftswissenschaften.

\section{Als zweitbeliebtester \\ Bereich folgen die Geisteswissenschaften, Kunst und Pädagogik.}

Individuelle Präferenzen, die jeweilige Zulassungspolitik und die Struktur der Abschlüsse beeinflussen die Verteilung der Abschlussquoten auf die einzelnen Studienbereiche.

\section{Politischer Hintergrund}

Die sich ändernden Gegebenheiten des Arbeitsmarkts, die relativen Einkommensaussichten in den verschiedenen Berufen und Wirtschaftszweigen, sowie die Zulassungspolitik und -praxis der tertiären Bildungsreinrichtungen haben Auswirkungen auf die Studienfächer, die von den Studierenden gewählt werden. Die jeweilige Beliebtheit der verschiedenen Studienfächer bzw. Studienbereiche beeinflusst wiederum die Nachfrage nach Studiengängen und Lehrpersonal sowie das Angebot an neuen Studienabsolventen. Dieser Indikator gibt Auskunft über die Verteilung der Absolventen des Tertiärbereichs nach Studienbereichen, sowie über den relativen Anteil von Frauen an den Abschlussquoten für die verschiedenen Studienbereiche.

\section{Ergebnisse und Erläuterungen}

\section{Abschlussquoten nach Studienbereichen}

In 24 der 28 Länder, die Daten zurVerfügung gestellt haben, werden die meisten Abschlüsse im Tertiärbereich A und in weiterführenden Forschungsprogrammen in den Sozial-, Rechts- und Wirtschaftswissenschaften erworben (Tabelle A4.1). Im Durchschnitt der OECD-Länder erwirbt jeder dritte Absolvent des Tertiärbereich A einen Abschluss in den Sozial-, Rechts- und Wirtschaftswissenschaften. Der Anteil der Sozial-, Rechts- und Wirtschaftswissenschaften an den Abschlüssen im Tertiärbereich A liegt zwischen weniger als 25 Prozent in Finnland, Korea, Norwegen, Schweden und der Türkei und mehr als 40 Prozent in Dänemark, Mexiko, Polen und den Vereinigten Staaten. In Finnland und Korea findet sich die größte Konzentration von Abschlüssen im Tertiärbereich A und in weiterführenden Forschungsprogrammen in den Bereichen Ingenieurwesen, Fertigung und Bauwesen und in Norwegen und Schweden in den Bereichen Gesundheit und Soziales.

Üblicherweise erwirbt jeder dritte oder vierte Absolvent einen Abschluss in Geisteswissenschaften, Kunst oder Pädagogik. Für den Anteil der Studierenden in den naturwissenschaftlich ausgerichteten Fächern (Ingenieurwesen, Fertigung und Bauwesen, Biowissenschaften, Physik und Agrarwissenschaft, Mathematik und Informatik, jedoch ohne Gesundheit und Soziales) reicht die Bandbreite von weniger als 19 Prozent in Island, den Niederlanden, Norwegen, Ungarn und den Vereinigten Staaten über 34 Prozent in Deutschland und Finnland bis hin zu 42 Prozent in Korea.

Die Verteilung der erworbenen Abschlüsse auf die Studienbereiche richtet sich nach deren relativer Beliebtheit bei den Studierenden, der relativen Anzahl der in den betreffenden Studienbereichen an den Hochschulen und entsprechenden Bildungseinrichtungen zugelassenen Studierenden und der Struktur der in diesen Studienfächern angebotenen Abschlüsse in dem jeweiligen Land.

Die unterschiedlichen Abschlussquoten der einzelnen Länder (Tabelle A2.1) lassen sich teilweise durch die Unterschiede in der Anzahl der Abschlüsse in Studiengängen des Tertiärbereich A in den Fächern Pädagogik und Geistes- 
wissenschaften erklären. Länder mit hohen Abschlussquoten haben im Durchschnitt einen höheren Anteil von Absolventen der Erziehungs- und Geisteswissenschaften und einen geringeren Anteil von Absolventen in den naturwissenschaftlich ausgerichteten Fächern. Mit anderen Worten schwankt die Anzahl der Absolventen der naturwissenschaftlich ausgerichteten Fächer zwischen den Ländern nicht so stark wie die Abschlussquoten insgesamt.

Im Tertiärbereich B, in dem die Studiengänge stärker berufsorientiert sind, ergibt sich ein ähnliches Bild. Die meisten Absolventen konzentrieren sich in den Sozial-, Rechts- undWirtschaftswissenschaften (zusammen 26 Prozent), gefolgt von den Geisteswissenschaften, Kunst und Pädagogik (zusammen 21 Prozent). Es finden sich in diesem Bereich jedoch mehr Absolventen von Studiengängen in Gesundheit und Soziales als von Studiengängen in Ingenieurwesen, Fertigung und Bauwesen (zusammen 19 bzw. 15 Prozent) (s. Tabelle A4.1).

Die Auswahl eines bestimmten Studienfachs auf diesem Niveau hängt sehr stark davon ab, ob man auch im post-sekundären, nicht-tertiären Bereich oder auf Ebene der Studiengänge im Tertiärbereich A ähnliche Fachgebiete studieren bzw. sich auf ähnliche Berufe vorbereiten kann. Wenn z.B. Krankenschwestern in einem bestimmten Land vor allem in Bildungsgängen des Tertiärbereich B ausgebildet werden, wird der Anteil der Absolventen mit einem Abschluss in medizinischen Fächern im Tertiärbereich B höher sein, als wenn sie vor allem im Sekundarbereich II oder in Studiengängen des Tertiärbereich A ausgebildet werden.

\section{Geschlechtsspezifische Unterschiede bei den Abschlüssen im Tertiärbereich}

Die Erstabschlussquoten von Frauen in Studiengängen des Tertiärbereich A entsprechen oder übersteigen in 21 von 27 OECD-Ländern diejenigen der Männer. Im Durchschnitt aller OECD-Länder sind 54 Prozent aller Absolventen mit einem ersten Abschluss im Tertiärbereich A Frauen. Allerdings gibt es hier weiterhin beträchtliche Unterschiede zwischen den einzelnen Studienfächern. In den Bereichen Geisteswissenschaften, Kunst, Pädagogik, Gesundheit und Soziales sind im OECD-Durchschnitt mehr als zwei Drittel der Absolventen des Tertiärbereich A Frauen, wohingegen ihr Anteil in Mathematik und Informatik weniger als ein Drittel ausmacht und weniger als ein Fünftel in den Bereichen Ingenieurwesen, Fertigung und Bauwesen.

In Island, Neuseeland, Norwegen und Portugal macht der Anteil der Frauen mit einem ersten Abschluss im Tertiärbereich A über 60 Prozent aus, während er in Deutschland, Japan, Korea, Österreich, der Schweiz und der Türkei bei 48 Prozent und darunter liegt (Tabellen A4.2 und A3.1.c).

In den OECD-Ländern erwerben immer noch eher Männer als Frauen einen Abschluss in einem weiterführenden Forschungsprogramm (Tabelle A4.2). Die Abschlussquoten für weiterführende Forschungsprogramme, z.B. Promotionsstudiengänge, sind in allen Ländern außer Italien für Frauen niedriger als für
Sozial-, Rechts-, Wirtschaftswissenschaften und Pädagogik sind auch im Tertiärbereich B beliebt.

In den meisten

Ländern entsprechen oder übersteigen die Abschlussquoten der Frauen im Tertiärbereich A diejenigen der Männer, ... 


\section{Abbildung A4.2.}

Anteil der von Frauen erworbenen Abschlüsse im Tertiärbereich (2000)

Für alle Studienbereiche für Frauen mit einem Abschluss im Tertiärbereich A oder einem weiterführenden Forschungsprogramm

Erstabschluss im Tertiärbereich A Zweitabschluss im Tertiärbereich A $\square$ Weiterführende Forschungsprogramme $\%$

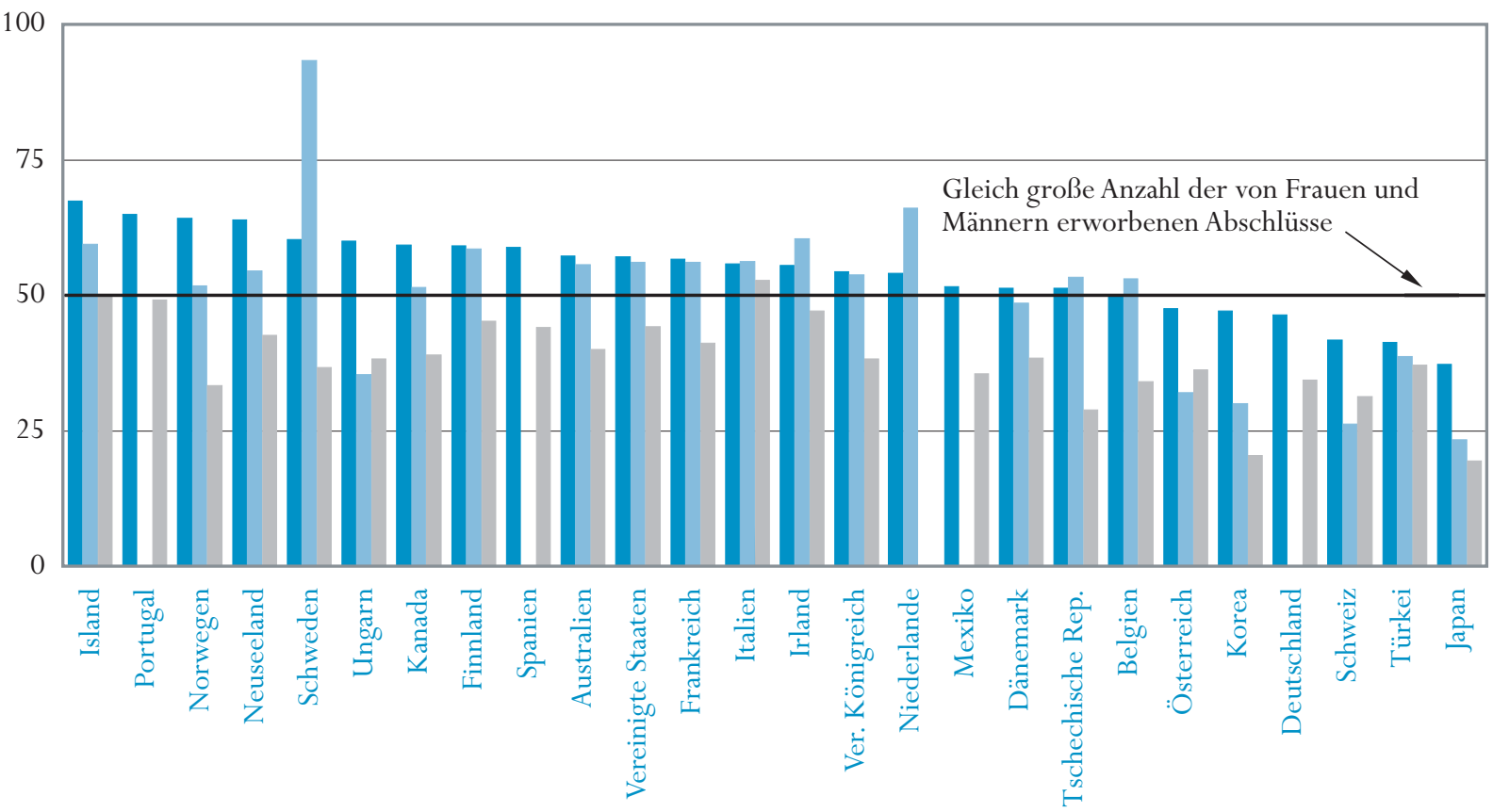

Anordnung der Länder in absteigender Reihenfolge des Prozentsatzes der von Frauen erworbenen Erstabschlüsse im Tertiärbereich A.

Source: OECD. Table A4.2. Hinweise s. Anhang 3 (www.oecd.org/els/education/eag2002).

Die Absolventendaten

beziehen sich auf das akademische Jahr 1999-

2000 und beruhen auf der alljährlich von der OECD aufgelegten UOE-Datenerhebung zur Bildungsstatistik.
Männer. Im Durchschnitt aller OECD-Länder sind fast zwei Drittel aller Absolventen auf diesem Niveau männlich. In Japan und Korea werden rund 80 Prozent der Abschlüsse in weiterführenden Forschungsprogrammen von Männern erworben.

\section{Definitionen und angewandte Methodik}

Als Absolventen im Tertiärbereich gelten alle Personen, die in einem bestimmten Referenzjahr einen Abschluss im Tertiärbereich A oder B oder einen gleichwertigen Abschluss erworben haben. Der Indikator unterscheidet zwischen verschiedenen Kategorien von Abschlüssen: 1.) Abschluss im Tertiärbereich B (ISCED 5B); 2.) Abschluss im Tertiärbereich A (ISCED 5A), und 3.) Abschluss in einem weiterführenden Forschungsprogramm (ISCED 6). Für einige Länder sind Daten für die entsprechenden Kategorien nicht verfügbar. In diesen Fällen wurden die Absolventen von dem jeweiligen Land der passendsten Kategorie zugeordnet. 
Tabelle A4.2 zeigt die prozentuale Verteilung von Abschlüssen bei den Frauen nach Studienbereichen. Absolventinnen des Tertiärbereichs, die ihren Abschluss innerhalb des Referenzjahres erwerben, werden entsprechend ihrer Hauptfachausrichtung in die Kategorien aufgeteilt. Die Zahlen berücksichtigen Absolventinnen aller tertiären Abschlüsse aus Tabelle A2.1. 
Tabelle A4.1.

Absolventen desTertiärbereichs, nach Studienbereichen und Bildungsbereichen (2000)

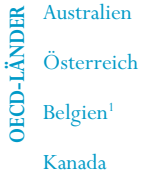

Tschechische Rep.

Dänemark

Finnland ${ }^{*}$

Frankreich

Deutschland

Griechenland

Ungarn $^{1}$

Island

Italien

Japan

Korea

Luxemburg

Mexiko

Niederlande ${ }^{2}$

Neuseeland

Norwegen

Polen ${ }^{3}$

Portugal $^{1}$

Slowakische Rep.

Spanien

Schweden

Schweiz

Türkei

Ver. Königreich

Vereinigte Staaten

ereinigte Staate
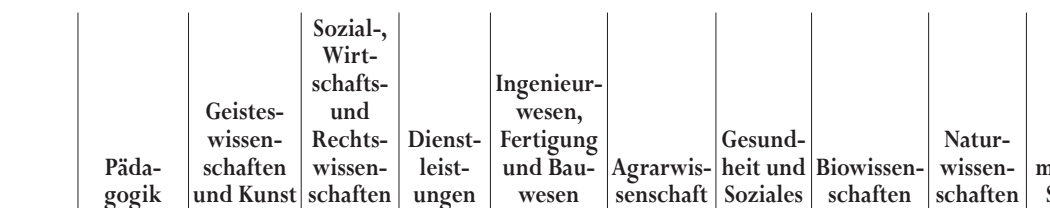

Math

wissen- matik und Infor- nicht

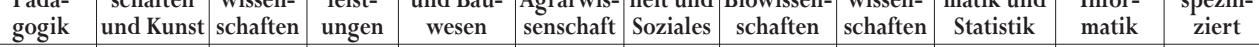

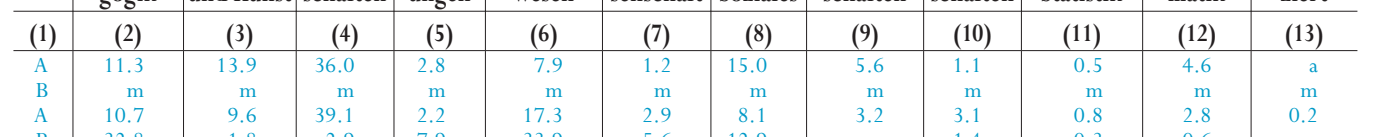

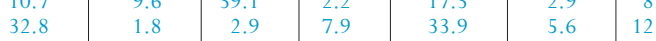

\begin{tabular}{l|l|l|l|l}
22.6 & 6.9 & 25.2 & 2.3 & 10.8
\end{tabular}

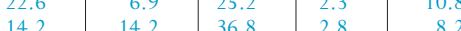

\begin{tabular}{l|r|r|r|r}
14.2 & 14.2 & 36.8 & 2.8 & 8.2
\end{tabular}

\begin{tabular}{r|r|r|r|r}
4.5 & 7.8 & 29.0 & 12.8 & 16.9 \\
13.1 & 7.1 & 32.9 & 2.3 & 15.5
\end{tabular}

\begin{tabular}{l|r|r|r|r}
\hline a & 8.1 & 35.4 & 8.0 & 6.1 \\
\hline
\end{tabular}

\begin{tabular}{l|l|l|l|l}
1.0 & 23.6 & 44.7 & 0.3 & 8.9
\end{tabular}

\begin{tabular}{l|r|r|r|r}
1.2 .2 & 2.2 & 7.9 & 5.4 & 12.4 \\
\hline
\end{tabular}

\begin{tabular}{l|r|r|r|r}
8.2 & 12.4 & 23.5 & 2.6 & 24.0 \\
0.3 & 4.2 & 22.1 & 16.9 & 19.5
\end{tabular}

\begin{tabular}{l|r|r|r|r}
8.3 & 4.2 & 22.1 & 16.9 & 19.5 \\
8.3 & 19.0 & 36.6 & 2.8 & 11.2
\end{tabular}

\begin{tabular}{l|r|l|l|l} 
a & 1.5 & 39.5 & 5.6 & 11.2 \\
\hline & 15.0 & 25.9 & 1.6 & 19.0
\end{tabular}

\begin{tabular}{r|r|r|r|r}
8.1 & 15.0 & 25.9 & 1.6 & 19.0 \\
10.9 & 1.2 & 9.6 & 9.6 & 13.7
\end{tabular}

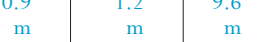

\begin{tabular}{ll|l|l|l}
$\mathrm{m}$ & $\mathrm{m}$ & $\mathrm{m}$ & $\mathrm{m}$ & $\mathrm{m}$ \\
$\mathrm{m}$ & $\mathrm{m}$ & $\mathrm{m}$
\end{tabular}

\begin{tabular}{r|r|r|r|r} 
& & $\mathrm{m}$ & $\mathrm{m}$ & $\mathrm{m}$ \\
24.4 & 7.1 & 39.5 & 6.0 & 9.8
\end{tabular}

\begin{tabular}{l|l|l|l}
$\mathrm{n}$ & $\mathrm{n}$ & 38.9 & 53.5
\end{tabular}

\begin{tabular}{l|l|l}
24.8 & 13.0 & 28.4 \\
6.4 & 14.0
\end{tabular}

\begin{tabular}{l|l|l}
6.4 & 14.0 & 47.5 \\
9.0 & 20.2 & 30.8
\end{tabular}

\begin{tabular}{r|r|r}
9.0 & 20.2 & 30.8 \\
0.9 & 6.9 & 31.5
\end{tabular}

\begin{tabular}{r|r|r}
0.9 & 6.9 & 31.5 \\
4.3 & 14.2 & 37.3
\end{tabular}

\begin{tabular}{l|l}
38.7 & 61.3
\end{tabular}

\begin{tabular}{l|r|r}
6.3 & 18.1 & 37.2
\end{tabular}

\begin{tabular}{l|l|l|l}
8.1 & 17.9 & 9.6 & 22.7
\end{tabular}

\begin{tabular}{l|l|l|l|l}
\hline 5.6 & 20.9 & 22.8 & 2.5 & 16.9
\end{tabular}

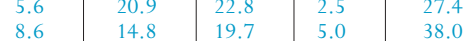

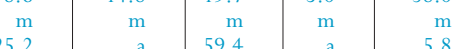

\begin{tabular}{l|r|r|r|r}
18.6 & 2.8 & 44.6 & 1.3 & 5.8 \\
& 2.8 & 34.4 & 3.8 & 37.7
\end{tabular}

\begin{tabular}{l|l|l|l|l}
2.8 & 44.6 & 1.3 & 14.0 \\
\hline & 0.7 & 34.4 & 3.8 & 37.7
\end{tabular}

\begin{tabular}{l|l|l|l|l}
16.8 & 7.3 & 34.8 & 2.8 & 37.7 \\
& & 39.7 & 11.1
\end{tabular}

\begin{tabular}{r|r|r|r|r}
$\mathrm{a}$ & $\mathrm{a}$ & 39.7 & 11.1 & 2.3 \\
12.6 & 21.3 & 28.3 & 2.0 & 5.6
\end{tabular}

\begin{tabular}{l|l|l|r|r}
27.8 & 13.2 & 22.4 & 18.1 & 3.4
\end{tabular}

\begin{tabular}{l|l|l|l|l}
22.7 & 7.2 & 22.1 & 3.3 & 6.8
\end{tabular}

\begin{tabular}{r|r|r|r|r}
$\mathrm{a}$ & 5.5 & 51.0 & 5.2 & 14.9 \\
15.1 & 9.7 & 48.5 & 4.8 & 12.0
\end{tabular}

\begin{tabular}{r|r}
100.0 & a \\
18.7 & a
\end{tabular}

\begin{tabular}{l|l|l|l|r|}
18.7 & 8.5 & 38.6 & 3.7 & 12.4
\end{tabular}

\begin{tabular}{l|l|l|l|l}
18.7 & 8.5 & 38.6 & 3.7 & 12.4 \\
21.0 & 5.5 & 30.1 & 8.7 & 12.4 \\
3.2 & 12.5 & 5.0 & 7.1 & 15.9
\end{tabular}

\begin{tabular}{l|l|l}
30.1 & 8.3 & 15.4
\end{tabular}

\begin{tabular}{l|r|r|r|r}
13.6 & 9.3 & 36.0 & 3.2 & 12.9 \\
\hline 4.4 & 6.7 & 30.9 & 12.8 & 23.6
\end{tabular}

\begin{tabular}{l|l|l|l|l}
\hline 4.4 & 6.7 & 30.9 & 12.8 & 23.6
\end{tabular}

\begin{tabular}{r|r|r|r|r}
18.8 & 5.7 & 30.9 & 12.8 & 23.6 \\
\hline & 5.7 & 21.6 & 1.0 & 20.5
\end{tabular}

\begin{tabular}{l|l|l|l|l}
4.9 & 6.3 & 14.6 & 14.3 & 23.3
\end{tabular}

\begin{tabular}{l|l|l|l|l}
9.9 & 11.8 & 31.1 & 3.8 & 15.7 \\
\hline
\end{tabular}

\begin{tabular}{l|l|l|l|l}
14.4 & 2.7 & 39.3 & 10.5 & 12.6
\end{tabular}

\begin{tabular}{r|r|r|r|r}
23.0 & 11.2 & 24.2 & 2.8 & 13.3
\end{tabular}

\begin{tabular}{r|r|r|r|r}
\hline $\mathrm{a}$ & 3.7 & 34.8 & 6.8 & 37.6 \\
\hline 0.0 & 15.7 & 28.8 & & 9.9
\end{tabular}

\begin{tabular}{r|r|r|r|r}
0.0 & 15.7 & 28.8 & $\mathrm{n}$ & 9.9 \\
6.1 & 7.6 & 22.6 & $\mathrm{n}$ & 9.2
\end{tabular}

\begin{tabular}{l|l|l|l|l}
13.1 & 14.2 & 42.2 & 2.4 & 6.5
\end{tabular}

\begin{tabular}{r|r|r|r|r}
13.1 & 14.2 & 42.2 & 2.4 & 6.5 \\
2.5 & 0.2 & 33.4 & 8.6 & 18.6
\end{tabular}

至 Israel

\begin{tabular}{r|r|r}
13.2 & 12.6 & 33.5 \\
13.0 & 7.6 & 25.8 \\
\hline 18.2 & 13.6 & 43.1
\end{tabular}

\begin{tabular}{r|r|r}
13.0 & 7.6 & 25.8 \\
18.2 & 13.6 & 43.1 \\
17.7 & 7.5 & 18.2
\end{tabular}

\begin{tabular}{r|r}
8.6 & 18.6 \\
9.0 & 13.2 \\
$\mathrm{~m}$ & 14.7 \\
\hline
\end{tabular}

\begin{tabular}{l|l}
3.5 & 13.3 \\
0.5 & 26.6
\end{tabular}

\begin{tabular}{l|l}
0.5 & 26.6
\end{tabular}

\begin{tabular}{l|r}
1.3 & 7.9 \\
3.3 & 18.6
\end{tabular}

\begin{tabular}{l|l}
3.3 & 18.6 \\
3.8 & 12.5
\end{tabular}

\begin{tabular}{l|l}
3.8 & 12.5 \\
2.6 & 35.1
\end{tabular}

\begin{tabular}{l|r}
3.2 & 5.5
\end{tabular}

\begin{tabular}{l|l}
1.1 & 49.2 \\
2.3 & 19.3
\end{tabular}

\begin{tabular}{l|l}
2.3 & 19.3 \\
1.5 & 31.5
\end{tabular}

\begin{tabular}{l|r}
1.5 & 31.5 \\
0.8 & 2.9
\end{tabular}

$\begin{array}{ll}\mathrm{n} & 20.2 \\ \end{array}$

\begin{tabular}{l|l}
1.9 & 15.0
\end{tabular}

\begin{tabular}{r|r}
3.4 & 50.3 \\
$\mathrm{~m}$ & $\mathrm{~m}$
\end{tabular}

$\mathrm{m}$

$3.6 \quad \mathrm{~m}$

n n

\begin{tabular}{l|l}
0.7 & 15.3
\end{tabular}

\begin{tabular}{r|r|r}
0.7 & 15.3 & 4.9 \\
$\mathrm{n}$ & $\mathrm{n}$ & $\mathrm{n}$ \\
1.7 & 7.8 & 6.9 \\
0.7 & 8.9 & 2.7 \\
\hline & 17.3 & 3.0
\end{tabular}

\begin{tabular}{r|r}
2.1 & 17.3 \\
$\mathrm{a}$ & $\mathrm{a}$ \\
3.4 & 5.2
\end{tabular}

\begin{tabular}{l|r}
3.4 & 5.2 \\
0.7 & 18.1
\end{tabular}

\begin{tabular}{r|r}
0.7 & 18.1 \\
3.2 & 6.6
\end{tabular}

3.2

\begin{tabular}{r|r}
1.3 & 8 \\
$\mathrm{~m}$ &
\end{tabular}

\begin{tabular}{r|r} 
& $\mathrm{m}$ \\
$\mathrm{a}$ & 9.6
\end{tabular}

\begin{tabular}{l|l}
2.0 & 7.8 \\
1.8 & 7.2
\end{tabular}

\begin{tabular}{l|r}
1.8 & 7.2 \\
2.3 & 20.9
\end{tabular}

\begin{tabular}{r|r}
2.3 & 20.9 \\
a & 37.7
\end{tabular}

\begin{tabular}{r|r}
1.4 & 12.9
\end{tabular}

\begin{tabular}{l|l}
2.4 & 7.7
\end{tabular}

\begin{tabular}{l|r}
1.4 & 25.3
\end{tabular}

\begin{tabular}{l|r}
0.1 & 1.0 \\
2.4 & 2.8
\end{tabular}

\begin{tabular}{r|r}
2.4 & 2.8 \\
$\mathrm{a}$ & $\mathrm{a}$ \\
1.7 & 10.6
\end{tabular}

\begin{tabular}{r|r}
$\mathrm{a}$ & $\mathrm{a}$ \\
1.7 & 10.6 \\
1.7 & 10.6
\end{tabular}

\begin{tabular}{l|r}
1.7 & 10.6 \\
4.4 & 8.5
\end{tabular}

\begin{tabular}{l|r}
4.4 & 8.5 \\
1.5 & 63.7
\end{tabular}

\begin{tabular}{l|l}
3.0 & 11.9
\end{tabular}

\begin{tabular}{l|l}
3.0 & 11.9 \\
0.5 & 10.6
\end{tabular}

\begin{tabular}{l|l}
0.5 & 10.6 \\
1.0 & 22.8 \\
\hline
\end{tabular}

\begin{tabular}{r|r}
7.1 & 8.9 \\
1.4 & 11.4
\end{tabular}

\begin{tabular}{l|l}
1.4 & 11.4 \\
1.4 & 12.3
\end{tabular}

\begin{tabular}{l|r}
1.4 & 12.3 \\
5.1 & 9.5
\end{tabular}

\begin{tabular}{l|l}
5.1 & 9.5 \\
6.3 & 5.4 \\
1.1 & 8.3
\end{tabular}

\begin{tabular}{l|r}
6.3 & 5.4 \\
1.1 & 8.3 \\
1.6 & 28.4
\end{tabular}

\begin{tabular}{r|r}
1.6 & 28.4 \\
2.3 & 9.8 \\
1.9 & 27.9
\end{tabular}

\begin{tabular}{r|l}
$\mathrm{n}$ & 1.4 \\
6.3 & 2.0 \\
\hline
\end{tabular}

\begin{tabular}{l|l}
6.3 & 2.0 \\
0.5 & 0.3
\end{tabular}

5.92

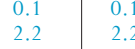

\begin{tabular}{r|r}
$\mathrm{a}$ \\
4.2
\end{tabular}

\begin{tabular}{r|r}
$\mathrm{n}$ & \\
1.9 & 2.7
\end{tabular}

\begin{tabular}{r|r}
$\mathrm{a}$ & \\
6.7 & 5.8
\end{tabular}

$3.0 \quad 5.8$

$\begin{array}{cc}\mathrm{a} & \mathrm{n} \\ \mathrm{m} & \mathrm{m}\end{array}$

$\mathrm{m} \quad \mathrm{m}$

\begin{tabular}{r|r|r}
$\mathrm{m}$ & $\mathrm{m}$ & $\mathrm{m}$ \\
0.5 & 0.7 & 0.1 \\
$\mathrm{n}$ & $\mathrm{n}$ & 3.4 \\
4.9 & 2.1 & 0.5
\end{tabular}

\begin{tabular}{l|l}
2.7 & 4.5
\end{tabular}

\begin{tabular}{rr|r|r|r}
$\mathrm{a}$ & $\mathrm{a}$ & $\mathrm{a}$ & $\mathrm{a}$ & $\mathrm{n}$ \\
4.4 & $\mathrm{x}(9)$ & $\mathrm{x}(9)$ & $\mathrm{x}(9)$ & 4.0
\end{tabular}

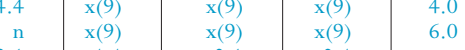

$2.1 \quad 4.4$

\begin{tabular}{r|r}
$\mathrm{m}$ \\
$\mathrm{a}$ \\
0.8
\end{tabular}

\begin{tabular}{l}
0.6 \\
1.1 \\
\hline
\end{tabular}

\begin{tabular}{r|r|r|r|r}
$\mathrm{a}$ & $\mathrm{a}$ & $\mathrm{a}$ & 9.2 & $\mathrm{a}$ \\
$\mathrm{n}$ & 11.3 & 0.1 & 1.6 & 2.8 \\
& 0.3 & &
\end{tabular}

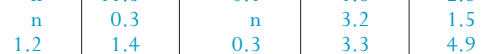

\begin{tabular}{r|r|r|r|r}
$\mathrm{n}$ & $\mathrm{a}$ & $\mathrm{a}$ & 21.6 & 0.7 \\
1.6 & 1.1 & 1.0 & 0.9 & $\mathrm{a}$ \\
$\mathrm{a}$ & $\mathrm{a}$ & $\mathrm{a}$ & $\mathrm{a}$ & $\mathrm{a}$
\end{tabular}

\begin{tabular}{l|l}
0.9 & 1.0 \\
0.9 & 1.0
\end{tabular}

\begin{tabular}{r|r}
1.0 \\
$\mathrm{n}$ \\
2.5
\end{tabular}

\begin{tabular}{r|r}
$\mathrm{n}$ & \\
2.3 & 2.4 \\
0.1 & 0.1 \\
3.3
\end{tabular}

\begin{tabular}{l|r|r} 
& 0.6 & 3.1 \\
0.1 & 0.2 & 20.5 \\
\hline 4.3 & 1.1 & 5.8
\end{tabular}

2.1

\begin{tabular}{r|r}
2.1 & 5.3 \\
$\mathrm{a}$ & $\mathrm{n}$ \\
6.0 & 5.0 \\
1.6 & 1.5 \\
4.1 & 1.5
\end{tabular}

\begin{tabular}{l|l}
\hline .3 & 11.5 \\
2.4 & 18.8 \\
\hline
\end{tabular}

\begin{tabular}{r|r}
$\mathrm{a}$ & \\
3.1 & 3.0 \\
$n$ & \\
2.7 &
\end{tabular}

\begin{tabular}{r|r|r}
$n$ & 6.8 \\
$-\mathrm{a}$ & 5.9 & $\mathrm{x}(11)$ \\
$\mathrm{n}$ & $\mathrm{x}(11)$
\end{tabular}

Hinweis: In Spalte 1 ist der Bildungsbereich angegeben. A entspricht Studiengängen im Tertiärbereich A und weiterführenden Forschungsprogrammen und B Studiengängen im Tertiärbereich B.

Hinweis: $\mathrm{x}$ bedeutet, dass die Daten in einer anderen Spalte enthalten sind, deren Referenz in runden Klammern nach dem ' $\mathrm{x}$ ' angegeben ist. So

bedeutet z.B. x(2), dass die Daten in Spalte 2 enthalten sind.

1. Ohne Studiengänge im Tertiärbereich B, die zu einem Zweitabschluss führen.

2. Ohne weiterführende Forschungsprogramme.

3. Ohne Studiengänge im Tertiärbereich A, die zu einem Zweitabschluss führen und weiterführende Forschungsprogramme.

* Hinweise s. Anhang 3 (www.oecd.org/els/education/eag2002).

Quelle: OECD. 
Tabelle A4.2.

Prozentsatz der von Frauen im Tertiärbereich erworbenen Abschlüsse, nach Art desTertiärbereichs und Studienbereichen (2000)

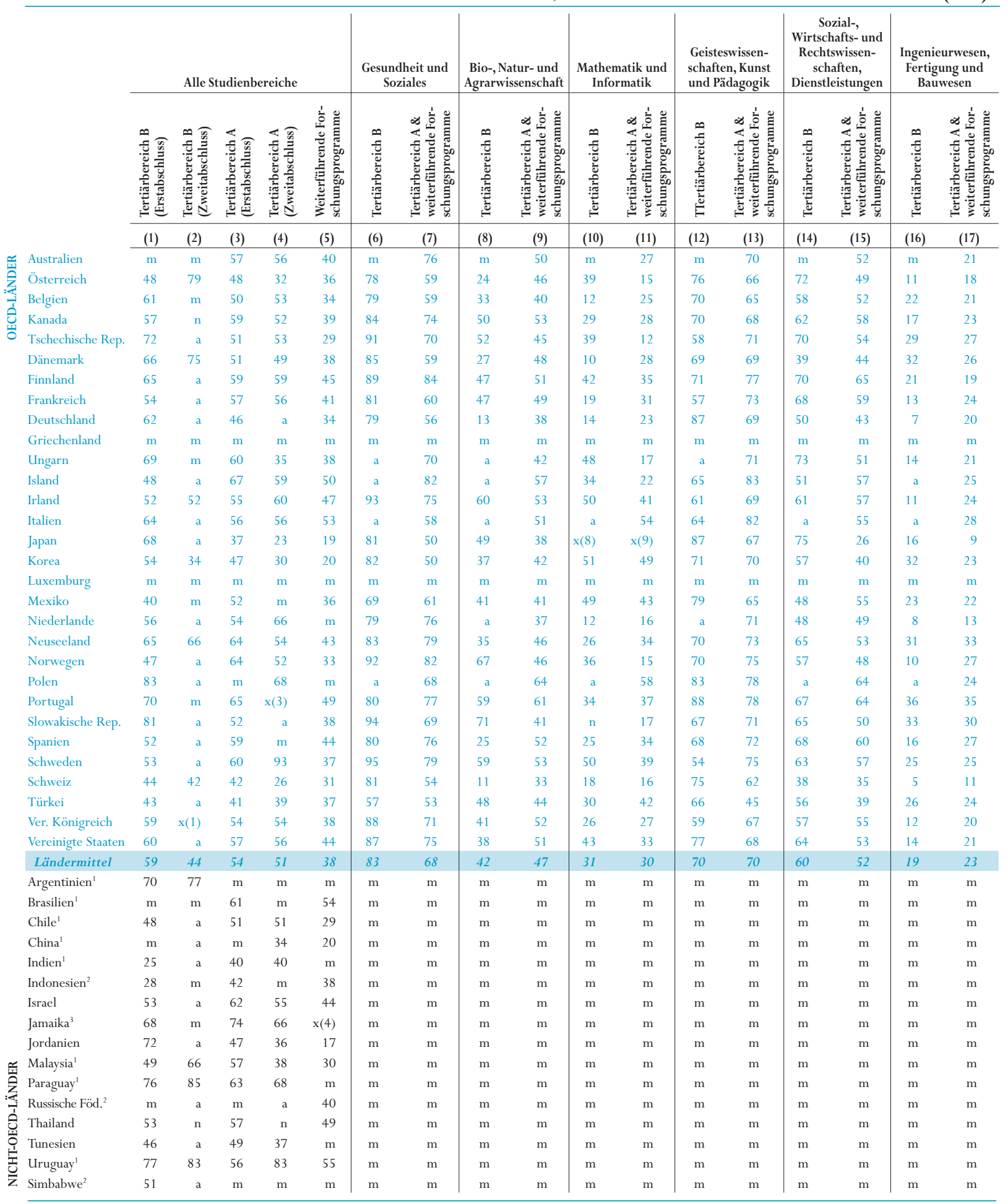

Hinweis: $\mathrm{x}$ bedeutet, dass die Daten in einer anderen Spalte enthalten sind, deren Referenz in runden Klammern nach dem ' $\mathrm{x}$ ' angegeben ist. So bedeutet z.B. $x(2)$, dass die Daten in Spalte 2 enthalten sind.

1. Referenzjahr 1999.

2. Referenzjahr 2001

3. Nur öffentliche Bildungseinrichtungen.

Quelle: OECD. 


\section{LESEKOMPETENZ 15-JÄHRIGER SCHÜLER}

- Im Durchschnitt aller OECD-Länder entsprechen 10 Prozent der 15-jährigen Schüler den Anforderungen der Stufe 5 im Bereich Lesekompetenz, bei der es ebenso um die Bewertung von Informationen und die Entwicklung von Hypothesen geht, wie auch um die Nutzung von Fachwissen und die Verarbeitung von gedanklichen Konzepten, die unter Umständen den eigenen Erwartungen widersprechen.

- 12 Prozent der 15-jährigen Schüler erfüllen nur die Anforderungen der grundlegendsten Kompetenzstufe 1 und weitere 6 Prozent liegen sogar noch darunter.

- Einige Länder, insbesondere Finnland, Japan und Korea haben sowohl hohe Durchschnittsergebnisse erreicht als auch nur eine geringe Spanne zwischen den einzelnen Schülerleistungen.

\section{Abbildung A5.1.}

Lesekompetenz 15-jähriger Schüler (2000)

Prozentsatz 15-jähriger Schüler auf den einzelnen Kompetenzstufen der PISA-Skala für Lesekompetenz

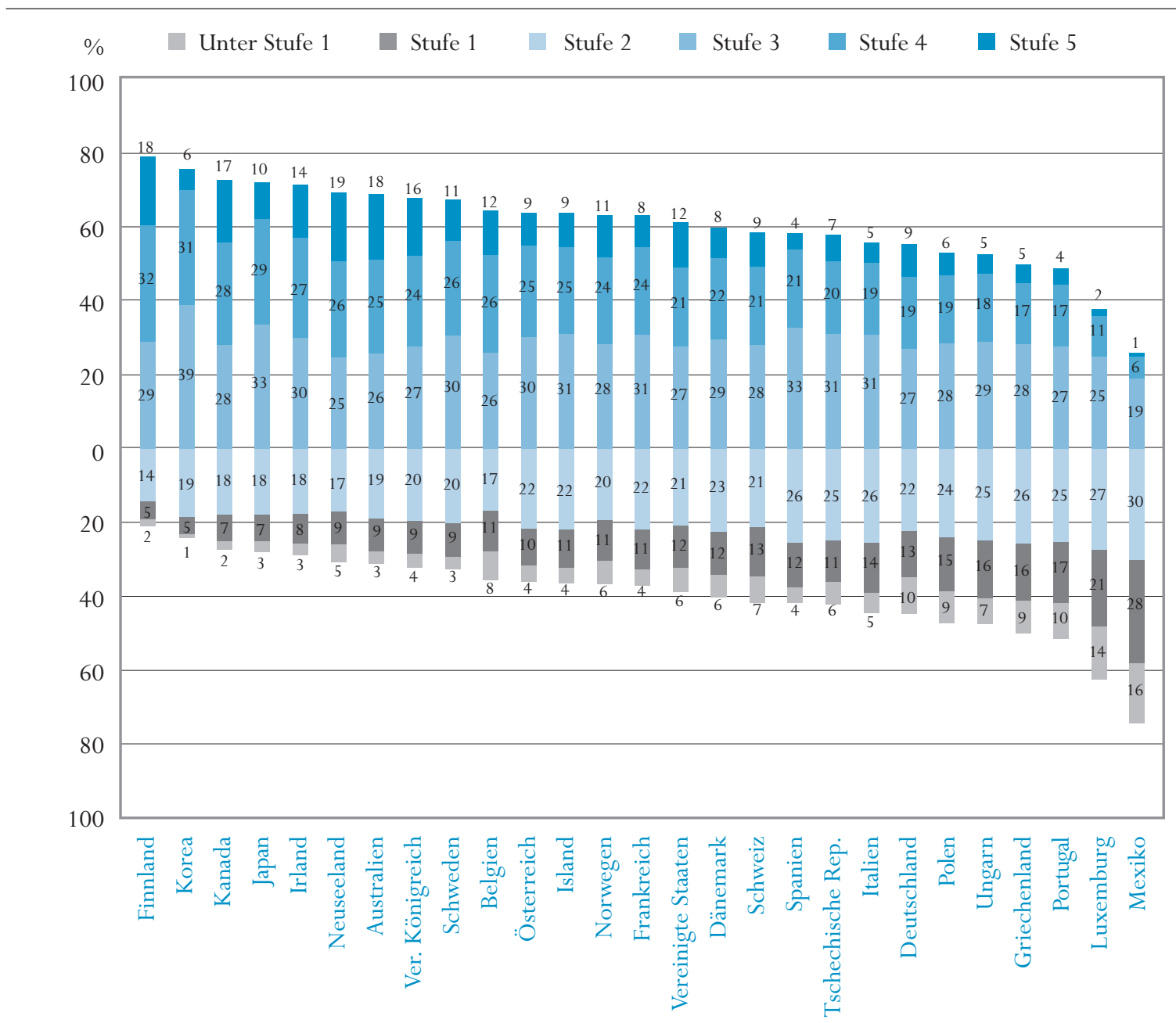

Anordnung der Länder in absteigender Reihenfolge des Prozentsatzes der Schüler auf den Stufen 3, 4 und 5 der PISA-Skala für Lesekompetenz. Quelle: OECD PISA-Datenbank, 2001. Tabelle A5.1. Hinweise zur Methodik s. Anhang 3 (www.oecd.org/els/education/eag2002) und www.pisa.oecd.org. 


\section{Politischer Hintergrund}

Die Fähigkeit zu lesen, das Gelesene zu verstehen und die gewonnenen Informationen zu nutzen sind Kernpunkte des Lernens, nicht nur in der Schule sondern während des gesamten Lebens. Dieser Indikator zeigt die Leistungen 15-jähriger Schüler bei Aufgaben, die auf einem Konzept der Lesekompetenz beruhen, das über die Vorstellung hinausgeht, wonach Lesekompetenz in dem Entschlüsseln und wörtlichen Verständnis von Texten besteht. Zur Lesekompetenz im Rahmen von PISA gehört auch das Verstehen von Texten und das Nachdenken darüber. Lesekompetenz beinhaltet die Fähigkeit, schriftliche Informationen so zu nutzen, dass diese den jeweiligen Zielen dienen, sowie die entsprechende Fähigkeit komplexer moderner Gesellschaften, schriftliche Informationen effektiv zu nutzen.

\section{Ergebnisse und Erläuterungen}

\section{Prozentsatz 15-Jähriger auf den einzelnen Stufen der Lesekompetenz}

Bei diesem Indikator wird die Lesekompetenz auf verschiedene Art und Weise untersucht (s. Kasten A5.1 zur Erläuterung der Lesekompetenz in der PISAStudie). Zuerst werden die Leistungen in Form der Bandbreite der von den 15-Jährigen in den einzelnen Ländern erzielten Punktzahlen beschrieben. Die Lesekompetenz wird auf fünf Leistungsstufen untersucht, wobei die Aufgaben von Stufe zu Stufe komplexer werden, Stufe 5 ist die höchste Stufe. Zweitens beschreibt dieser Indikator Leistungen in Form der von den 15-Jährigen erreichten mittleren Punktwerte und wie sich die Punktwerte auf die einzelnen

Dieser Indikator zeigt die Leistungen 15-jähriger Schüler im Bereich Lesekompetenz auf.

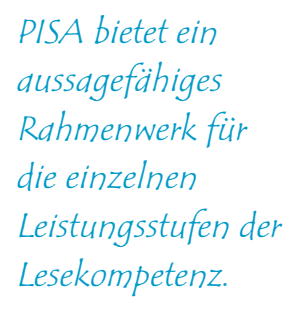

PISA bietet ein aussagefähiges Rahmenwerk für die einzelnen Leistungsstufen der Lesekompetenz.

\section{Kasten A5.1. Was bedeutet Lesekompetenz im Rahmen von PISA?}

Lesekompetenz (reading literacy) ist die Fähigkeit, geschriebene Texte zu verstehen, zu nutzen und über sie zu reflektieren, um eigene Ziele zu erreichen, das eigene Wissen und Potential weiterzuentwickeln und aktiv am gesellschaftlichen Leben teilzunehmen. Diese Definition geht über die Vorstellung hinaus, wonach Lesekompetenz in dem Entschlüsseln und wörtlichen Verständnis von Texten besteht. Zum Lesen gehört auch das Verstehen von Texten und das Nachdenken darüber, aus einer Reihe von Gründen und in den unterschiedlichsten Kontexten. Die Bewertung der Lesekompetenz im Rahmen von PISA berücksichtigt drei Dimensionen: die Leseaufgabe an sich, die Art des Lesestoffs und die Art des Gebrauchs, für den der Text geschrieben wurde.

Was für Skalen werden benutzt? Die Bewertung der Lesekompetenz im Rahmen von PISA erfolgt mittels drei Skalen. Die Skala „Informationen ermitteln“ gibt die Fähigkeit der Schüler wieder, Informationen in einem Text zu lokalisieren. Die Skala „Textbezogenes Interpretieren“ umfasst die Fähigkeit, der schriftlichen Information Bedeutung zu verleihen und Schlüsse daraus zu ziehen. Die Skala „Reflektieren und Bewerten“ bezieht sich auf die Fähigkeit der Schüler, 
Verbindungen zwischen einem Text und eigenen Kenntnissen, Vorstellungen und Erfahrungen herzustellen. Außerdem fasst eine Gesamtskala die Ergebnisse der drei Leseskalen zusammen. Indikator A5 konzentriert sich nur auf diese Gesamtskala, die als „PISA-Skala für Lesekompetenz“ bezeichnet wird.

Was bedeuten die Punktzahlen auf diesen Skalen? Die Punktzahlen auf diesen Skalen stehen für ein unterschiedliches Ausmaß an Kompetenz bezüglich jeder der Dimensionen oder Aspekte der Lesekompetenz (hier der Gesamtskala). So bedeutet beispielsweise eine niedrige Punktzahl auf einer Skala, dass ein Schüler in diesem Bereich nur über begrenzte Kompetenzen verfügt, während ein hoher Punktwert bedeutet, dass ein Schüler in diesem Bereich über größere Kompetenzen verfügt.

Was sind die einzelnen Kompetenzstufen? In einem Versuch, diese zunehmende Schwierigkeit der Aufgaben zu erfassen, ist jede der Lesekompetenzskalen in fünf Stufen unterteilt, basierend auf den Kenntnissen und Fähigkeiten, über die die Schüler auf einer bestimmten Stufe verfügen müssen. Schüler auf einer bestimmten Kompetenzstufe verfügen nicht nur über die Kenntnisse und Fähigkeiten, die dieser Stufe zugeordnet sind, sondern sie erbringen auch die auf den darunter liegenden Stufen geforderten Leistungen. So erbringen alle Schüler auf der Kompetenzstufe 3 auch die für die Kompetenzstufen 1 und 2 erforderlichen Leistungen.

10 Prozent der 15-Jährigen in den OECD Ländern verfügen über eine Lesekompetenz der Stufe 5, sie können Informationen bewerten und Hypothesen entwickeln, Fachwissen nutzen und gedankliche Konzepte verarbeiten, die unter Umständen den eigenen Erwartungen widersprechen, aber dieser Prozentsatz variiert zwischen den einzelnen Ländern von 19 bis weniger als 1 Prozent.
Abbildung A5.1 zeigt einen Überblick über die Leistungen auf der PISA-Skala für Lesekompetenz, wobei die Länge der einzelnen farbigen Abschnitte der Balken den prozentualen Anteil der 15-Jährigen angibt, die die Anforderungen der jeweiligen Kompetenzstufe erfüllen (s. Kasten A5.2). Wie aus der Abbildung ersichtlich ist, unterscheidet sich der Prozentsatz der Schüler auf den einzelnen Stufen der Lesekompetenz und dieVerteilung über alle Kompetenzstufen hinweg von Land zu Land. Über alle Länder hinweg, erreichen durchschnittlich 10 Prozent der Schüler Kompetenzstufe 5, 32 Prozent zumindest Kompetenzstufe 4 (d.h. Stufen 4 oder 5), 61 Prozent erreichen zumindest Kompetenzstufe 3, 82 Prozent zumindest Kompetenzstufe 2, und 94 Prozent zumindest Stufe 1.

Betrachtet man die in einem einzelnen Land erbrachten Leistungen Stufe für Stufe so zeigt sich, dass in fünf Ländern (Australien, Finnland, Kanada, Neuseeland und dem Vereinigten Königreich) 15 Prozent der Schüler und mehr die höchste Stufe bei der Lesekompetenz erreichen. In weiteren drei Ländern (Belgien, Irland, und den Vereinigten Staaten), erreichen zwischen 12 und 15 Prozent der Schüler diese Stufe. Aber nur 5 Prozent der Schüler und weniger in Brasilien, Griechenland, Lettland, Luxemburg, Mexiko, Portugal, Spanien und der Russischen Föderation erreichen diese höchste Kompetenzstufe. 


\section{Kasten A5.2. Was leisten Schüler auf den einzelnen Kompetenzstufen und welche Punktzahlen sind mit diesen Kompetenzstufen verbunden?}

Schüler, deren Leistungen Stufe 5 (über 625 Punkte) entsprechen, sind in der Lage, anspruchsvolle Leseaufgaben zu lösen, d.h. mit Informationen umzugehen, die in ungewohnten Texten nur schwer zu finden sind, ein genaues Verständnis dieser Texte nachzuweisen und herauszufinden, welche der im Text enthaltenen Informationen für die Aufgabe von Belang sind, sowie einen Text kritisch $\mathrm{zu}$ bewerten und Hypothesen aufzustellen, dabei Fachwissen heranzuziehen und Konzepte zu begreifen, die u.U. im Widerspruch zu den eigenen Erwartungen stehen.

Schüler, deren Leistungen Stufe 4 (553 bis 625 Punkte) entsprechen, sind in der Lage, schwierige Leseaufgaben zu lösen, beispielsweise implizite Informationen zu finden, den Sinn sprachlicher Nuancen zu verstehen und einen Text kritisch zu bewerten.

Schüler, deren Leistungen Stufe 3 (481 bis 552 Punkte) entsprechen, sind in der Lage, Leseaufgaben mittlerer Komplexität zu lösen, z.B. mehrere Informationen aufzufinden, Verbindungen zwischen verschiedenen Abschnitten eines Textes herzustellen und den Text zu ihrem Alltagswissen in Beziehung zu setzen.

Schüler, deren Leistungen Stufe 2 (408 bis 480 Punkte) entsprechen, sind in der Lage, grundlegende Leseaufgaben zu lösen, z. B. eindeutige Informationen zu finden, wenig anspruchsvolle Schlussfolgerungen verschiedener Art zu ziehen, die Bedeutung eines genau definierten Textteils zu erkennen und gewisse externe Kenntnisse zu dessen Verständnis heranzuziehen.

Schüler, deren Leistungen Stufe 1 (335 bis 407 Punkte) entsprechen, können nur die einfachsten der für PISA ausgearbeiteten Leseaufgaben lösen, z.B. eine Einzelinformation finden, das Hauptthema eines Textes erkennen oder eine einfache Verbindung zu Alltagskenntnissen ziehen.

Schüler, deren Leistungen unterhalb der Stufe 1 liegen (weniger als 335 Punkte), sind nicht in der Lage, die elementarsten Lesekompetenzen nachzuweisen, die in PISA gemessen werden. Solchen Schülern bereitet es große Schwierigkeiten, Lesekompetenzen als ein wirksames Mittel zur Förderung und Erweiterung ihrer Kenntnisse und Fähigkeiten in anderen Bereichen einzusetzen.

Obwohl normalerweise die Länder mit einem hohen Anteil an leistungsstarken 15-Jährigen weniger Schüler unterhalb der Stufe 1 haben (beispielsweise Finnland), ist dies nicht immer der Fall. So fallen beispielsweise Belgien und die Vereinigten Staaten aus diesem Muster, da sie einen überdurchschnittlich großen Anteil 15-Jähriger auf der höchsten Kompetenzstufe aufweisen, während gleichzeitig der Anteil der Schüler unterhalb von Stufe 1 auch überdurchschnittlich groß ist.
Ein hoher Anteil

leistungsstarker Schüler bedeutet normalerweise weniger leistungsschwache schüler, aber in einigen Ländern gibt es große Disparitäten. 


\section{A5}

In einem Drittel der $O E C D$-Länder erreichen mehr als zwei Drittel der 15-Jährigen wenigstens Stufe 3 .

Die einfachsten Aufgaben der PISAStudie verlangen von den Schülern mehr als nur flüssig zu lesen, ...
Die Hälfte aller 15-Jährigen in Finnland und mindestens 40 Prozent der Schüler in fünf anderen Ländern erreichen mindestens Stufe 4 auf der PISA-Skala für Lesekompetenz. Mit Ausnahme von Luxemburg und Mexiko erreicht in jedem OECD-Land wenigstens jeder fünfte 15-Jährige die Kompetenzstufe 4. In Brasilien, dem Land mit dem niedrigsten Gesamtergebnis im Bereich Lesekompetenz erreichen nur 4 Prozent der Schüler zumindest Stufe 4.

In einem Drittel der OECD-Länder erreichen zwischen 67 und 80 Prozent der 15-Jährigen wenigstens Stufe 3 auf der PISA-Skala für Lesekompetenz (Australien, Finnland, Irland, Japan, Kanada, Korea, Neuseeland, Schweden und das Vereinigte Königreich). Nimmt man diese neun Länder als Grundlage zur Beantwortung der Frage, ob sich die Leistungsprofile in den verschiedenen Ländern ähneln, lassen sich verschiedene Kategorien erkennen. In Finnland und Kanada beispielsweise erreichen relativ hohe Anteile der Schüler Stufe 5, und mindestens 90 Prozent der Schüler erreichen zumindest Stufe 2. Es sind also Länder, die gute Ergebnisse auf der PISA-Skala für Lesekompetenz aufweisen. In Australien, Irland, Neuseeland und dem Vereinigten Königreich gibt es sehr viele Schüler auf der obersten Stufe, aber mehr als 10 Prozent liegen dort auf Stufe 1 oder darunter. Diesen Ländern ist es zwar gelungen, die Schüler auf höhere Leistungsniveaus anzuheben, gleichzeitig ist es ihnen jedoch im Vergleich zu Finnland oder Kanada in nur geringerem Maße gelungen, den Anteil der leistungsschwachen Schüler zu verringern. Das Gegenteil ist bei Korea der Fall, wo weniger als 6 Prozent der Schüler auf Stufe 1 oder darunter liegen, wo aber gleichzeitig nur ein unterdurchschnittlich niedriger Anteil (6 Prozent) Stufe 5 erreicht.

In jedem OECD-Land erbringt wenigstens die Hälfte aller Schüler Leistungen, die Stufe 2 oder einer höheren Stufe entsprechen. Interessanterweise erreichen in Spanien, wo nur 4 Prozent aller Schüler auf Stufe 5 liegen, überdurchschnittliche 84 Prozent zumindest Stufe 2. Allerdings erreichen auch über 40 Prozent der Schüler in Spanien überhaupt nur Stufe 2.

Beim PISA-Konzept der Lesekompetenz liegt der Schwerpunkt auf den Kenntnissen und Fähigkeiten, die für das „Lesen, um zu lernen“ notwendig sind. Das PISA-Konzept geht damit über die rein technischen Fertigkeiten des „Lesenlernens" hinaus. Da in den OECD-Ländern nur vergleichsweise wenige junge Erwachsene die rein „technischen“ Lesefertigkeiten nicht erworben haben, soll mit PISA daher nicht gemessen werden, inwieweit 15-Jährige fließend lesen können, oder Wörter richtig buchstabieren oder erkennen können. In Einklang mit den meisten gegenwärtig anerkannten Definitionen der Lesekompetenz zielt PISA darauf ab festzustellen, inwieweit die Schüler in der Lage sind, die Bedeutung eines breiten Spektrums von Texten aus allgemeinen schulischen wie außerschulischen Kontexten zu erfassen, zu erweitern und darüber zu reflektieren. Die einfachsten Aufgaben, die diesem Konzept der Lesekompetenz gerecht werden, sind jene auf Stufe 1. Schüler, deren Leistungen nur den Anforderungen dieser Stufe entsprechen, können 
nur die einfachsten der für PISA ausgearbeiteten Leseaufgaben lösen, z.B. eine Einzelinformation finden, das Hauptthema eines Textes erkennen oder eine einfache Verbindung zu Alltagskenntnissen ziehen.

Schüler, die weniger als 335 Punkte erzielt haben, Stufe 1 also nicht erreichen, sind nicht in der Lage, die elementarsten Lesekompetenzen nachzuweisen, die in PISA gemessen werden. Dies soll nicht heißen, dass die fraglichen Schüler über keinerlei Grundqualifikationen verfügen. Die meisten dieser Schüler dürften wohl im „technischen“ Sinne durchaus lesen können, und die Mehrzahl von ihnen (54 Prozent im OECD-Durchschnitt) sind in der Lage, mindestens 10 Prozent der in PISA 2000 gestellten offenen Fragen, d.h. jenen, bei denen keine Multiple-Choice-Antworten vorgegeben sind, erfolgreich zu lösen (und 6 Prozent gelang es, ein Viertel dieser Aufgaben richtig zu lösen). Das Muster ihrer Antworten bei den Testaufgaben lässt jedoch darauf schließen, dass sie weniger als die Hälfte der Fragen eines Tests mit ausschließlich Stufe 1 entsprechenden Aufgaben beantworten könnten und den Anforderungen von Stufe 1 folglich nicht gerecht würden. Diesen Schülern bereitet es große Schwierigkeiten, Lesekompetenzen als ein wirksames Mittel zur Förderung und Erweiterung ihrer Kenntnisse und Fähigkeiten in anderen Bereichen einzusetzen. Bei Schülern, deren Grundqualifikationen unter Stufe 1 liegen, kann daher nicht nur die Gefahr bestehen, dass sie beim Übergang vom Schulins Arbeitsleben großen Problemen gegenüberstehen werden, sondern auch, dass sie in ihrem weiteren Leben Möglichkeiten zur Fort- und Weiterbildung nicht wirklich werden nutzen können.

Wenn in einem Bildungssystem die Leistung eines hohen Prozentsatzes der Schüler unter oder gerade noch bei Stufe 1 liegt, steht zu befürchten, dass ein großer Teil der Schüler u.U. nicht die erforderlichen Grundkenntnisse und -fähigkeiten erwirbt, um aus den gebotenen Bildungsmöglichkeiten wirklichen Nutzen zu ziehen. Diese Situation ist umso besorgniserregender, als zahlreiche Anzeichen dafür sprechen, dass es äußerst schwierig ist, in der Schulzeit entstandene Bildungslücken im späteren Leben zu schließen. Zwischen dem Grundqualifikationsniveau der Erwachsenen und deren Beteiligung an Fortund Weiterbildung bestehen enge Zusammenhänge, selbst wenn man auch andere Merkmale kontrolliert, die Einfluss auf die Teilnahme an derartigen Maßnahmen haben.

In allen OECD-Ländern zusammen liegen die Leistungen von insgesamt 12 Prozent der Schüler bei Stufe 1 und von 6 Prozent unter Stufe 1, es bestehen jedoch große Unterschiede zwischen den einzelnen Ländern. In Finnland und Korea liegt die Leistung von nur ca. 5 Prozent der Schüler auf Stufe 1, und weniger als 2 Prozent liegen darunter. Diese beiden Länder bilden jedoch Ausnahmen. In allen anderen OECD-Ländern liegen zwischen 9 und 44 Prozent der Schüler auf Stufe 1 oder darunter. Von ihnen erfüllen über 2 Prozent - in der Hälfte der OECD-Länder sogar über 5 Prozent - nicht einmal die Anforderungen von Stufe 1 .

Der Prozentsatz von Schülern auf Stufe 1 oder darunter variiert stark zwischen den einzelnen Ländern, von wenigen Prozent bis zu fast der Hälfte, ... 


\section{A5}

... und in einigen

Ländern erreicht

ein nicht zu

vernachlässigender

Anteil nicht die Stufe 1.

Durchschnittsergebnisse

können nützlich sein, um ein Gesamtbild der schülerleistungen eines Landes zu vermitteln, ...

... sie täuschen jedoch über die großen Leistungsunterschiede zwischen den Ländern hinweg.

Finnland weist einzigartige

Gesamtergebnisse auf. Diese liegen fast

zwei Drittel einer

Kompetenzstufe oberhalb des OECD-

Durchschnitts.
Die Länder mit 20 Prozent der Schüler auf Stufe 1 oder darunter sind (in dieser Reihenfolge): Brasilien, Mexiko, Luxemburg, Lettland, die Russische Föderation, Portugal, Griechenland, Polen, Ungarn, Deutschland, Liechtenstein und die Schweiz. In Brasilien, Mexiko, Luxemburg, Lettland, Portugal und Deutschland erreichen zwischen knapp 10 und 23 Prozent der Schüler nicht Stufe 1, d.h. sie sind nicht in der Lage, die elementarsten Kenntnisse und Fähigkeiten, die in PISA 2000 gemessen wurden, routinemäßig nachzuweisen. Dies ist besonders in Deutschland bemerkenswert, wo ein relativ hoher Prozentsatz von 9 Prozent der Schüler den Anforderungen von Stufe 5 entspricht.

\section{Länderspezifische Mittelwerte und Leistungsverteilung bei der Lesekompetenz}

Eine weitere Möglichkeit, in PISA 2000 die Schülerleistungen im Bereich Lesekompetenz zusammenfassend darzustellen und die jeweilige Position der Länder zueinander zu vergleichen, ist die Darstellung der Ländermittelwerte. Soweit eine hohe Durchschnittsleistung der 15-Jährigen eine für die Zukunft hoch qualifizierte Erwerbsbevölkerung andeutet, werden Länder mit hohen Durchschnittsergebnissen einen erheblichen wirtschaftlichen und sozialen Vorteil haben. Es muss jedoch berücksichtigt werden, dass sich hinter Durchschnittsergebnissen oft erhebliche Unterschiede innerhalb ein und desselben Landes verbergen, in denen sich die unterschiedlichen Leistungen zahlreicher verschiedener Schülergruppen widerspiegeln.

Wie bereits bei früheren internationalen Schulleistungsstudien, wie der Dritten Internationalen Mathematik- und Naturwissenschaftsstudie (TIMSS), entfällt auch bei PISA nur etwa ein Zehntel der erfassten Gesamtvarianz auf Leistungsunterschiede zwischen den einzelnen Ländern, so dass auch nur ein Zehntel davon in einem Vergleich der Durchschnittswerte der einzelnen Länder dargestellt werden kann. Die restliche Varianz tritt innerhalb der einzelnen Länder auf, zwischen unterschiedlichen Bildungsgängen, zwischen den einzelnen Schulen und zwischen den Schülern innerhalb einer Schule. Daher bietet dieser Indikator auch Informationen $\mathrm{zu}$ der Verteilung der erreichten Punktzahlen im Bereich Lesekompetenz, indem auch die Bandbreite der Leistungen zwischen dem obersten und dem untersten Quartil der Schüler innerhalb eines Landes untersucht wird.

Die Schüler Finnlands erzielen auf der PISA-Skala für Lesekompetenz höhere Durchschnittsergebnisse als die Schüler aller anderen Länder, die an der Studie teilgenommen haben (s. Abb. A5.2). Ihr Mittelwert liegt mit 546 Punkten fast zwei Drittel einer Kompetenzstufe über dem OECD-Mittelwert von 500 Punkten (bzw. statistisch ausgedrückt übertrifft er den OECD-Mittelwert um mehr als eine halbe internationale Standardabweichung). 12 weitere Länder, Australien, Belgien, Island, Irland, Japan, Kanada, Korea, Neuseeland, die Niederlande, Österreich, Schweden und das Vereinigte Königreich liegen über dem OECD-Durchschnitt. In fünf Ländern entspricht der Mittelwert in etwa 


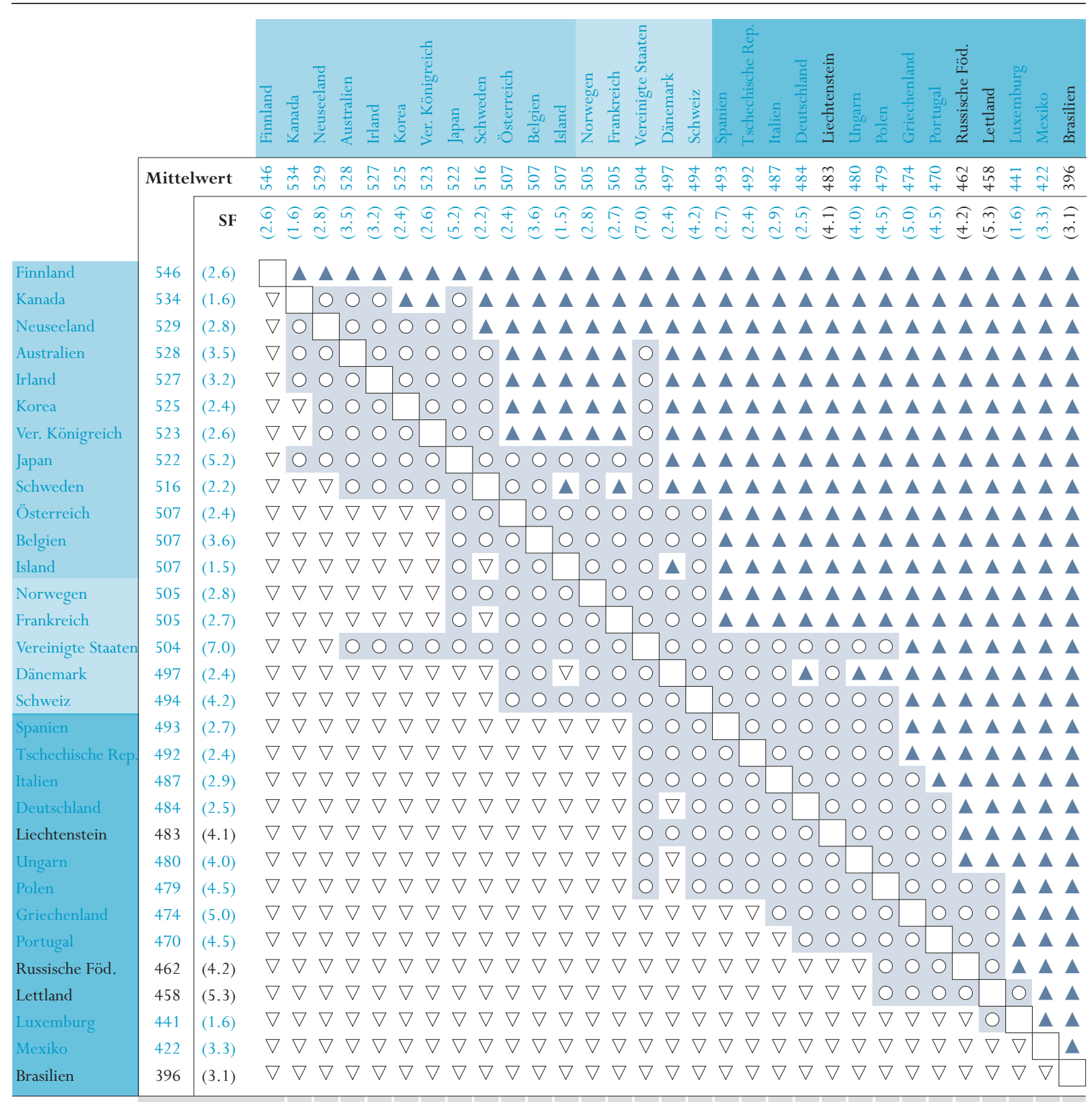

\begin{tabular}{llllllllllllllllll|l|l|l|l|l|l|l|l|l|l|l|l|l|l|l} 
Oberer Rang* & 1 & 2 & 2 & 2 & 3 & 4 & 5 & 3 & 9 & 11 & 11 & 11 & 11 & 11 & 10 & 16 & 16 & 17 & 17 & 19 & 21 & 20 & 21 & 21 & 23 & 24 & 27 & 27 & 30 & 31 & 32
\end{tabular}

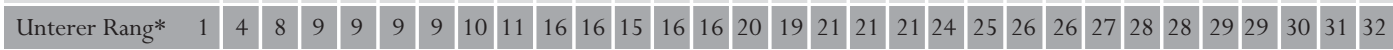

* Hinweis: Da die Daten auf Stichprobenerhebungen basieren, ist es nicht möglich, den genauen Rangplatz eines Landes zu bestimmen. Es können jedoch der jeweils obere und untere Rangplatz angegeben werden, zwischen denen das Land mit seinen mittleren Schülerleistungen mit 95\%-igerWahrscheinlichkeit liegt.

Erläuterungen

Zum Vergleich der Schülerleistungen eines Landes mit denen der Länder im Tabellenkopf ist die Zeile des betreffenden Landes zu lesen. Die Symbole zeigen, ob die mittleren Schülerleistungen des Landes in der jeweiligen Zeile statistisch signifikant über oder unter denen des Vergleichslandes liegen oder ob zwischen den mittleren Schülerleistungen beider Länder kein statistisch signifikanter Unterschied besteht.

Anmerkung: Anordnung der Länder in absteigender Reihenfolge der mittleren Schülerleistungen auf der PISA-Skala für Lesekompetenz. Aufgrund der geringen Beteiligungsquote sind die Niederlande nicht enthalten. Ausgehend von einem zu vernachlässigenden bis geringfügigen statistischen Fehler aufgrund der Nichtbeteiligung ist zu erwarten, dass die Niederlande mit 95\%-iger Wahrscheinlichkeit zwischen Rangplatz 2 und 14 liegen würden.
A Mittlere Schülerleistungen statistisch signifikant höher als im Vergleichsland

Kein statistisch signifikanter Unterschied gegenüber dem Vergleichsland

$\nabla$ Mittlere Schülerleistungen signifikant niedriger als im Vergleichsland

Statistisch signifikant über dem OECD-Durchschnitt

Kein statistisch signifikanter Unterschied zum OECD-Durchschnitt

Statistisch signifikant unter dem OECD-Durchschnitt 


\section{A5}

Hohe Durchschnittsergebnisse allein sind nicht ausreichend - die

Länder sind auch bemüht, das Niveau der leistungsschwachen schüler anzuheben.

Sind diese zu beobachtenden Disparitäten unvermeidlich? Schwer zu sagen,...

....aber einige Länder

begrenzen sie auf eine wesentlich engere Bandbreite als andere, ...

... und einigen Ländern gelingt es, hohe Durchschnittsergebnisse mit geringen Disparitäten zu verbinden. dem OECD-Durchschnitt und in 14 Ländern, einschließlich 4 Nicht-OECDLändern, liegt er signifikant unter dem OECD-Durchschnitt.

Ein Blick auf die Verteilung der Schülerleistungen (Tabelle A 5.2) zeigt, dass die Unterschiede der Schülerleistungen auf der PISA-Skala für Lesekompetenz innerhalb der einzelnen Länder sehr groß sind. Die Unterschiede innerhalb der einzelnen Länder sind deutlich größer als die Unterschiede zwischen den Ländern. Der Unterschied zwischen dem 75. und dem 25. Perzentil, der die mittlere Hälfte der Verteilung der nationalen Leistungen abdeckt, übersteigt in allen Ländern die Größenordnung einer ganzen Kompetenzstufe (72 Punkte), und ist in Australien, Belgien, Deutschland und Neuseeland so groß wie zwei ganze Kompetenzstufen. (Der OECD-Durchschnitt bei dieser Kenngröße beläuft sich auf das 1,8-fache einer Kompetenzstufe).

Zusammengenommen deuten diese Ergebnisse darauf hin, dass sich die Bildungssysteme in vielen Ländern schwierigen Herausforderungen dabei gegenüber sehen, dem Bildungsbedarf aller Schüler gerecht zu werden, sowohl derer, die den größten Bedarf haben als auch derer, die sehr leistungsstark sind.

Es zeigt sich außerdem, dass Länder mit ähnlichen Durchschnittsergebnissen beträchtliche Unterschiede bei den Varianzen der Schülerleistungen aufweisen. So erzielten beispielsweise sowohl Korea als auch das Vereinigte Königreich mit ungefähr 525 Punkten überdurchnittliche mittlere Leistungen auf der PISASkala für Lesekompetenz. Der Unterschied zwischen dem 75. und 25. Perzentil in Korea beträgt 92 Punkte, signifikant unter dem OECD-Durchschnitt, im Vereinigten Königreich jedoch beläuft er sich auf 137 Punkte, mehr oder minder auf dem Niveau des OECD-Durchschnitts. Ähnliches lässt sich auch für Länder mit unterdurchschnittlichen Durchschnittsergebnissen beobachten. Sowohl in Deutschland als auch Italien beläuft sich der Mittelwert auf ungefähr 485 Punkte, signifikant unter dem OECD-Durchschnitt. In Italien beläuft sich der Unterschied zwischen dem 75. und 25. Perzentil auf 124 Punkte, in Deutschland jedoch auf 146 Punkte. Das unterste Quartil der Schüler näher an den Mittelwert heranzubringen, ist für Länder mit großen internen Unterschieden eine der Möglichkeiten, die Gesamtleistungen zu steigern.

Schließlich zeigt ein Vergleich der Bandbreite der Leistungen innerhalb eines Landes mit seinem Mittelwert, dass es einigen Ländern gelingt, sowohl relativ geringe Unterschiede zwischen den leistungsstarken und den leistungsschwachen Schülern als auch relativ hohe Gesamtleistungen zu erreichen. Leistungsstarke Länder weisen tendenziell relativ geringe Unterschiede innerhalb des Landes auf. So gehören beispielsweise die drei Länder mit dem kleinsten Unterschied zwischen dem 75. und 25. Perzentil, Finnland, Japan und Korea, auch zu den Ländern mit den besten Leistungen im Bereich Lesekompetenz. Im Gegensatz dazu erzielt eins der drei Länder mit den größten Leistungsunterschieden, Deutschland, Punktwerte, die beträchtlich unter dem OECD-Durchschnitt liegen. 


\section{Definitionen und angewandte Methodik}

Die für diesen Indikator untersuchte Zielpopulation waren 15-jährige Schüler. In der Praxis bezieht sich dies auf Schüler, die zu Beginn der Testperiode im Alter von 15 Jahren und 3 (abgeschlossenen) Monaten bis zu 16 Jahren und 2 (abgeschlossenen) Monaten waren und eine Bildungseinrichtung besuchten, ungeachtet der Klassenstufe und der Art der von ihnen besuchten Bildungseinrichtung und unabhängig davon, ob es sich um eine Ganztags- oder Halbtagsschule handelte.

Um die Interpretation der von den Schülern erzielten Punktwerte in der PISAStudie zu erleichtern, wurde die PISA-Skala für Lesekompetenz so konstruiert, dass der Mittelwert bei 500 Punkten liegt und die Standardabweichung bei 100, wobei die Daten gewichtet wurden, so dass jedes Land gleichermaßen hierzu beitrug. Diese Bezugspunkte bilden gewissermaßen den „Anker“ der im Rahmen von PISA durchgeführten Messung der Schülerleistungen.

Hinweise zu Standardfehlern, Signifikanztests und multiplen Vergleichen s. Anhang 3 unter www.oecd.org/els/education/eag2002.
Die erzielten

Punktzahlen basieren

auf Leistungsmessungen,

die Teil der im Jahr

2000 von der OECD

durchgeführten

Internationalen

Schulleistungsstudie

PISA waren. 
Tabelle A5.1.

Lesekompetenz 15-jähriger Schüler (2000)

Prozentsatz 15-jähriger Schüler auf den einzelnen Kompetenzstufen der PISA-Skala für Lesekompetenz

Australien
Österreich
Belgien
Kanada

Tschechische Rep.

Dänemark

Finnland

Frankreich

Deutschland

Griechenland

Ungarn

Island

Irland

Italien

Japan

Korea

Luxemburg

Mexiko

Neuseeland

Norwegen

Polen

Portugal

Spanien

Schweden

Schweiz

Ver. Königreich

Vereinigte Staaten OECD Insgesamt

Ländermittel

Brasilien

Rusiche

ssische Föd.

\section{Kompetenzstufen}

\begin{tabular}{|c|c|c|c|c|c|c|c|c|c|c|c|}
\hline \multicolumn{2}{|c|}{$\begin{array}{c}\text { Unter Stufe } 1 \\
\text { (weniger als } 335 \\
\text { Punkte) }\end{array}$} & \multicolumn{2}{|c|}{$\begin{array}{c}\text { Stufe } 1 \\
\text { (335 bis } 407 \text { Punkte) }\end{array}$} & \multicolumn{2}{|c|}{$\begin{array}{c}\text { Stufe } 2 \\
\text { (408 bis } 480 \text { Punkte) }\end{array}$} & \multicolumn{2}{|c|}{$\begin{array}{c}\text { Stufe } 3 \\
\text { (481 bis } 552 \text { Punkte) }\end{array}$} & \multicolumn{2}{|c|}{$\begin{array}{c}\text { Stufe } 4 \\
\text { (553 bis } 625 \text { Punkte) }\end{array}$} & \multicolumn{2}{|c|}{$\begin{array}{c}\text { Stufe } 5 \\
\text { (über } 625 \text { Punkte) }\end{array}$} \\
\hline$\%$ & SF & $\%$ & SF & $\%$ & SF & $\%$ & SF & $\%$ & SF & $\%$ & SF \\
\hline
\end{tabular}

7.7

2.4

6.1

$(0.4)$

$(1.0)$

10.2

10.2
11.3

(0.3) $\quad 7.2$

\begin{tabular}{l|l}
$(0.6)$ & 11.4 \\
$(0.6)$ & 12.0
\end{tabular}

7

$(0.5)$

12.0
5.2

0.6) 11.0

(0.7) $\quad 12.7$

(1.2) $\quad 15.7$

$0.7) \quad 15.8$

(0.3) $\quad 10.5$

(0.5) $\quad 7.9$

(0.9) $\quad 13.5$

0.6) 7.3

(0.2) $\quad 4.8$

\begin{tabular}{l|r}
$(0.2)$ & 4.8 \\
$(0.7)$ & 20.9
\end{tabular}

(1.2) 28.1

(0.5) $\quad 8.9$

\begin{tabular}{l|r}
$(0.5)$ & 8.9 \\
$(0.6)$ & 11.2
\end{tabular}

(1.0) $\quad 14.6$

(1.0) $\quad 16.7$

(0.5) $\quad 12.2$

(0.4) 9.3

(0.7) $\quad 13.3$

(0.4) $\quad 9.2$

(1.2)

\begin{tabular}{l|l}
$(1.2)$ & 11.5 \\
$(0.4)$ & 12.1
\end{tabular}

\begin{tabular}{l}
$(0.6) \quad 21.7$ \\
\hline
\end{tabular}

(0.7) 16.8

(0.3) 18.0

(0.7) $\quad 24.8$

(0.7) 22.5

(0.4) $\quad 14.3$

(0.8) 22.0

(0.6) 22.3

(1.4) 25.9

(1.2) 25.0

(0.6) 22.0

(0.8) $\quad 17.9$

(0.9) 25.6

(1.1) 18.0

(0.6) $\quad 18.6$

(0.8) 27.5

(1.4) 30.3

(0.5) 17.2

(0.8) $\quad 19.5$

(1.0) 24.1

(1.2) 25.3

(0.9) 25.7

(0.6) 20.3

(0.9) 21.4

(0.5) 19.6

(1.2)

\begin{tabular}{l|l}
$(1.2)$ & 21.0 \\
$(0.4)$ & 21.8
\end{tabular}

(0.9) 29.9

(0.7) $\quad 25.8$

(0.4) 28.0

(1.2) $\quad 30.9$

(0.9) 29.5

$\begin{array}{ll}(0.7) & 28.7\end{array}$

(0.8) $\quad 30.6$

(0.8) 26.8

(1.4) 28.1

(1.1) 28.8

$\begin{array}{ll}(0.8) & 30.8\end{array}$

$\begin{array}{ll}(0.9) & 29.7\end{array}$

(1.0) 30.6

(1.3) 33.3

$\begin{array}{ll}(0.9) & 38.8\end{array}$

(1.3) 24.6

(1.1) 18.8

(0.9) 24.6

(0.8) 28.1

(1.4) 28.2

(1.0) 27.5

$\begin{array}{ll}(0.7) & 32.8\end{array}$

(0.7) $\quad 30.4$

(1.0) 28.0

(0.7) 27.5

\begin{tabular}{l|l}
$(1.2)$ & 27.4 \\
$(0.4)$ & 28.6
\end{tabular}

(1.2) 24.9

(0.9) 26.3

(0.5) $\quad 27.7$

(1.1) 19.8

(1.0) 22.0

(0.8) $\quad 31.6$

(1.0) 23.7

(1.0) $\quad 19.4$

(1.7) $\quad 16.7$

(1.3) 18.5

(0.9) 23.6

(1.1) 27.1

(1.0) 19.5

(1.3) 28.8

(1.1) $\quad 31.1$

(1.1) $\quad 11.2$

(1.2) 6.0

(1.1) 25.8

(0.8) $\quad 23.7$

(1.3) 18.6

(1.2) 16.8

(1.0) 21.1

(1.0) 25.6

(1.0) 21.0

(0.9) 24.4

(1.3) 21.5

\begin{tabular}{l|l}
$(1.3)$ & 21.5 \\
$(0.4)$ & 21.8
\end{tabular}

(0.2) 21.7

(1.2) 27.7

(0.2) $\quad 28.7$

(0.2) 22.3

$\begin{array}{rrr}(1.0) & 8.8 & (0.8)\end{array}$

$\begin{array}{lll}(1.0) & 8.8 & (0.8)\end{array}$

$\begin{array}{ll}(0.6) & 16.8\end{array}$

(0.8) $\quad 7.0$

(0.9) $\quad 8.1$

(0.9) $\quad 18.5$

(0.9) 8.5

$\begin{array}{ll}8.5 & (0.6)\end{array}$

\begin{tabular}{l|l}
$(0.1)$ & 11.9 \\
$(1.4)$ & 32.5
\end{tabular}

(1.4) 32.5

(1.3) 26.3

(1.3) 12.9

(1.1) 3.1

(1.3) 13.8

(3.4) 19.5

(2.9) 30.1

(1.1) 13.3

(1.1) 29.2

(0.8) 26.9

(1.0) 18.5

$26.9 \quad(1.1)$

$13.3 \quad(1.0)$

$\begin{array}{lll}(1.0) & 8.8 & (0.5) \\ (1.4) & 5.0 & (0.7)\end{array}$

$\begin{array}{lll}(1.4) & 5.0 & (0.7)\end{array}$

$\begin{array}{lll}(1.1) & 5.1 & (0.8) \\ (1.1) & 9.1 & (0.7)\end{array}$

(1.1) $\quad 14.2 \quad(0.8)$

(1.1) $5.3 \quad(0.5)$

$\begin{array}{lll}(1.7) & 9.9 & (1.1)\end{array}$

$\begin{array}{lll}(1.2) & 5.7 & (0.6)\end{array}$

$\begin{array}{lll}(0.5) & 1.7 & (0.3)\end{array}$

$\begin{array}{lll}(0.7) & 0.9 & (0.2)\end{array}$

(1.1) $18.7 \quad(1.0)$

$\begin{array}{lll}(0.9) & 11.2 \quad(0.7)\end{array}$

(1.3) $5.9 \quad(1.0)$

(1.1) $\quad 4.2 \quad(0.5)$

$\begin{array}{lll}(0.9) & 4.2 & (0.5)\end{array}$

(1.0) $11.2 \quad(0.7)$

$1.0) \quad 9.2$
$(0.9)$

(0.9) $\quad 15.6 \quad(1.0)$

\begin{tabular}{r|rr}
$(1.4)$ & 12.2 & $(1.4)$ \\
$(0.4)$ & 9.4 & $(0.4)$
\end{tabular}

$\begin{array}{lll}(0.2) & 9.5 & (0.1)\end{array}$

$\begin{array}{lll}(0.5) & 0.6 & (0.2)\end{array}$

$\begin{array}{lll}(1.1) & 4.1 \quad(0.6)\end{array}$

$\begin{array}{lll}(1.0) & 5.1 & (1.6)\end{array}$

Quelle: OECD PISA-Datenbank, 2001. Hinweise zur Methodik s. Anhang 3 (www.oecd.org/els/education/eag2002) und www.pisa.oecd.org. 
Tabelle A5.2.

Verteilung der Leistung 15-jähriger Schüler auf der PISA-Skala für Lesekompetenz (2000) Leistung 15-jähriger Schüler auf der PISA-Skala für Lesekompetenz, nach Perzentilen

\begin{tabular}{|c|c|c|c|c|c|c|c|c|c|c|c|c|c|c|c|c|}
\hline & & & & & & & & & & Perze & ntile & & & & & \\
\hline & Mittel & vert & & & 5 & & 10 & & 25 & & 7 & & 90 & & 95 & \\
\hline & $\begin{array}{c}\text { mittlere } \\
\text { Punkt- } \\
\text { zahl }\end{array}$ & SF & SA & SF & $\begin{array}{c}\text { Punkt- } \\
\text { zahl }\end{array}$ & SF & $\begin{array}{c}\text { Punkt- } \\
\text { zahl }\end{array}$ & SF & $\begin{array}{c}\text { Punkt- } \\
\text { zahl }\end{array}$ & SF & $\begin{array}{c}\text { Punkt- } \\
\text { zahl }\end{array}$ & SF & $\begin{array}{c}\text { Punkt- } \\
\text { zahl }\end{array}$ & SF & $\begin{array}{c}\text { Punkt- } \\
\text { zahl }\end{array}$ & SF \\
\hline Australien & 528 & $(3.5)$ & 102 & $(1.6)$ & 354 & $(4.8)$ & 394 & $(4.4)$ & 458 & $(4.4)$ & 602 & $(4.6)$ & 656 & $(4.2)$ & 685 & $(4.5)$ \\
\hline Österreich & 507 & $(2.4)$ & 93 & (1.6) & 341 & (5.4) & 383 & $(4.2)$ & 447 & $(2.8)$ & 573 & $(3.0)$ & 621 & $(3.2)$ & 648 & $(3.7)$ \\
\hline Belgien & 507 & (3.6) & 107 & $(2.4)$ & 308 & $(10.3)$ & 354 & $(8.9)$ & 437 & $(6.6)$ & 587 & $(2.3)$ & 634 & $(2.5)$ & 659 & $(2.4)$ \\
\hline Kanada & 534 & $(1.6)$ & 95 & $(1.1)$ & 371 & (3.8) & 410 & $(2.4)$ & 472 & $(2.0)$ & 600 & $(1.5)$ & 652 & $(1.9)$ & 681 & (2.7) \\
\hline Tschechische Rep. & 492 & $(2.4)$ & 96 & (1.9) & 320 & (7.9) & 368 & $(4.9)$ & 433 & $(2.8)$ & 557 & $(2.9)$ & 610 & $(3.2)$ & 638 & $(3.6)$ \\
\hline Dänemark & 497 & $(2.4)$ & 98 & (1.8) & 326 & $(6.2)$ & 367 & $(5.0)$ & 434 & $(3.3)$ & 566 & $(2.7)$ & 617 & $(2.9)$ & 645 & $(3.6)$ \\
\hline Finnland & 546 & $(2.6)$ & 89 & (2.6) & 390 & $(5.8)$ & 429 & (5.1) & 492 & $(2.9)$ & 608 & $(2.6)$ & 654 & $(2.8)$ & 681 & $(3.4)$ \\
\hline Frankreich & 505 & $(2.7)$ & 92 & (1.7) & 344 & $(6.2)$ & 381 & $(5.2)$ & 444 & $(4.5)$ & 570 & $(2.4)$ & 619 & $(2.9)$ & 645 & $(3.7)$ \\
\hline Deutschland & 484 & $(2.5)$ & 111 & (1.9) & 284 & (9.4) & 335 & (6.3) & 417 & $(4.6)$ & 563 & $(3.1)$ & 619 & $(2.8)$ & 650 & (3.2) \\
\hline Griechenland & 474 & $(5.0)$ & 97 & $(2.7)$ & 305 & $(8.2)$ & 342 & $(8.4)$ & 409 & $(7.4)$ & 543 & $(4.5)$ & 595 & $(5.1)$ & 625 & $(6.0)$ \\
\hline Ungarn & 480 & $(4.0)$ & 94 & (2.1) & 320 & (5.6) & 354 & (5.5) & 414 & $(5.3)$ & 549 & $(4.5)$ & 598 & $(4.4)$ & 626 & $(5.5)$ \\
\hline Island & 507 & $(1.5)$ & 92 & (1.4) & 345 & (5.0) & 383 & (3.6) & 447 & $(3.1)$ & 573 & $(2.2)$ & 621 & $(3.5)$ & 647 & (3.7) \\
\hline Irland & 527 & (3.2) & 94 & (1.7) & 360 & (6.3) & 401 & (6.4) & 468 & $(4.3)$ & 593 & $(3.6)$ & 641 & $(4.0)$ & 669 & (3.4) \\
\hline Italien & 487 & (2.9) & 91 & $(2.7)$ & 331 & (8.5) & 368 & (5.8) & 429 & $(4.1)$ & 552 & $(3.2)$ & 601 & $(2.7)$ & 627 & (3.1) \\
\hline Japan & 522 & $(5.2)$ & 86 & $(3.0)$ & 366 & (11.4) & 407 & $(9.8)$ & 471 & $(7.0)$ & 582 & $(4.4)$ & 625 & $(4.6)$ & 650 & (4.3) \\
\hline Korea & 525 & $(2.4)$ & 70 & (1.6) & 402 & $(5.2)$ & 433 & (4.4) & 481 & $(2.9)$ & 574 & $(2.6)$ & 608 & $(2.9)$ & 629 & $(3.2)$ \\
\hline Luxemburg & 441 & (1.6) & 100 & $(1.5)$ & 267 & (5.1) & 311 & (4.4) & 378 & $(2.8)$ & 513 & $(2.0)$ & 564 & $(2.8)$ & 592 & $(3.5)$ \\
\hline Mexiko & 422 & (3.3) & 86 & (2.1) & 284 & (4.4) & 311 & (3.4) & 360 & $(3.6)$ & 482 & $(4.8)$ & 535 & $(5.5)$ & 565 & (6.3) \\
\hline Neuseeland & 529 & (2.8) & 108 & $(2.0)$ & 337 & (7.4) & 382 & $(5.2)$ & 459 & $(4.1)$ & 606 & $(3.0)$ & 661 & (4.4) & 693 & (6.1) \\
\hline Norwegen & 505 & (2.8) & 104 & (1.7) & 320 & (5.9) & 364 & (5.5) & 440 & $(4.5)$ & 579 & $(2.7)$ & 631 & (3.1) & 660 & (4.6) \\
\hline Polen & 479 & $(4.5)$ & 100 & (3.1) & 304 & (8.7) & 343 & (6.8) & 414 & $(5.8)$ & 551 & $(6.0)$ & 603 & $(6.6)$ & 631 & $(6.0)$ \\
\hline Portugal & 470 & (4.5) & 97 & (1.8) & 300 & $(6.2)$ & 337 & $(6.2)$ & 403 & $(6.4)$ & 541 & $(4.5)$ & 592 & $(4.2)$ & 620 & (3.9) \\
\hline Spanien & 493 & (2.7) & 85 & $(1.2)$ & 344 & (5.8) & 379 & $(5.0)$ & 436 & $(4.6)$ & 553 & $(2.6)$ & 597 & $(2.6)$ & 620 & (2.9) \\
\hline Schweden & 516 & $(2.2)$ & 92 & $(1.2)$ & 354 & $(4.5)$ & 392 & $(4.0)$ & 456 & $(3.1)$ & 581 & $(3.1)$ & 630 & $(2.9)$ & 658 & (3.1) \\
\hline Schweiz & 494 & $(4.2)$ & 102 & $(2.0)$ & 316 & (5.5) & 355 & (5.8) & 426 & $(5.5)$ & 567 & $(4.7)$ & 621 & $(5.5)$ & 651 & $(5.3)$ \\
\hline Ver. Königreich & 523 & $(2.6)$ & 100 & $(1.5)$ & 352 & $(4.9)$ & 391 & $(4.1)$ & 458 & $(2.8)$ & 595 & $(3.5)$ & 651 & $(4.3)$ & 682 & $(4.9)$ \\
\hline Vereinigte Staaten & 504 & $(7.1)$ & 105 & $(2.7)$ & 320 & $(11.7)$ & 363 & $(11.4)$ & 436 & $(8.8)$ & 577 & $(6.8)$ & 636 & $(6.5)$ & 669 & $(6.8)$ \\
\hline OECD Insgesamt & 499 & $(2.0)$ & 100 & $(0.8)$ & 322 & (3.4) & 363 & (3.3) & 433 & $(2.5)$ & 569 & $(1.6)$ & 622 & $(2.0)$ & 653 & (2.1) \\
\hline Ländermittel & 500 & $(0.6)$ & 100 & $(0.4)$ & 324 & (1.3) & 366 & (1.1) & 435 & $(1.0)$ & 571 & $(0.7)$ & 623 & $(0.8)$ & 652 & $(0.8)$ \\
\hline Brasilien & 396 & $(3.1)$ & 86 & (1.9) & 255 & (5.0) & 288 & $(4.5)$ & 339 & $(3.4)$ & 452 & $(3.4)$ & 507 & $(4.2)$ & 539 & $(5.5)$ \\
\hline Lettland & 458 & (5.3) & 102 & (2.3) & 283 & $(9.7)$ & 322 & $(8.2)$ & 390 & $(6.9)$ & 530 & $(5.3)$ & 586 & $(5.8)$ & 617 & $(6.6)$ \\
\hline Liechtenstein & 483 & $(4.1)$ & 96 & (3.9) & 310 & (15.9) & 350 & $(11.8)$ & 419 & $(9.4)$ & 551 & $(5.8)$ & 601 & $(7.1)$ & 626 & $(8.2)$ \\
\hline Russische Föd. & 462 & $(4.2)$ & 92 & (1.8) & 306 & (6.9) & 340 & (5.4) & 400 & $(5.1)$ & 526 & $(4.5)$ & 579 & $(4.4)$ & 608 & $(5.3)$ \\
\hline
\end{tabular}

Quelle: OECD PISA-Datenbank, 2001. Hinweise zur Methodik s. Anhang 3 (www.oecd.org/els/education/eag2002) und www.pisa.oecd.org. 


\section{MATHEMATISCHE UND NATURWISSENSCHAFTLICHE GRUNDBILDUNG 15-JÄHRIGER}

- 15-Jährige in Japan erzielen die höchsten Mittelwerte bei der mathematischen Grundbildung, obwohl sich ihre Punktwerte nicht statistisch von denen der Schüler in den beiden anderen Ländern an der Spitze - Korea und Neuseeland - unterscheiden. Bei der naturwissenschaftlichen Grundbildung erzielen die Schüler in Korea und Japan die höchsten Durchschnittsleistungen.

- Während es bereits große Unterschiede bei den mittleren Leistungen zwischen den einzelnen Ländern gibt, sind die Unterschiede der Leistungen 15-Jähriger innerhalb der einzelnen Länder um ein Vielfaches größer. Große Disparitäten bei den Leistungen sind jedoch offensichtlich keine Voraussetzung, damit ein Land ein insgesamt hohes Leistungsniveau erzielt. Ganz im Gegenteil liegen die Leistungen von fünf der Länder mit der kleinsten Varianz der Leistungen auf der PISA-Skala für mathematische Grundbildung, nämlich Finnland, Island, Japan, Kanada und Korea, signifikant über dem OECD-Durchschnitt und vier von ihnen, Finnland, Japan, Kanada und Korea, gehören zu den sechs Ländern mit den besten Leistungen bei der mathematischen Grundbildung. 


\section{Abbildung A6.1.}

Vergleich der mittleren Schülerleistungen der Länder auf der PISA-Skala für mathematische Grundbildung (2000)

\begin{tabular}{|c|c|c|c|c|c|c|c|c|c|c|c|c|c|c|c|c|c|c|c|c|c|c|c|c|c|c|c|c|c|c|c|c|c|}
\hline & & & $\cong$ & & & 胥 & $\begin{array}{ll} \\
0 \\
0\end{array}$ & & & 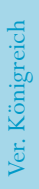 & $\frac{\vec{J}}{\vec{D}}$ & & $\begin{array}{l}\frac{\pi}{0} \\
0 \\
0 \\
0 \\
0 \\
0 \\
0\end{array}$ & 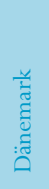 & ฮี & है & 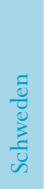 & & 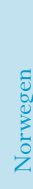 & & 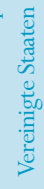 & 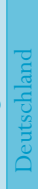 & & & & & & & 5 & E. & 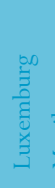 & $\sum^{\frac{9}{2}}$ & 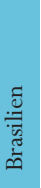 \\
\hline & \multicolumn{2}{|c|}{ Mittelwert } & 点 & 孚 & 命 & ڤn? & in & लै & ते & ते & in & $\frac{N}{5}$ & $\stackrel{\llcorner n}{i n}$ & $\frac{t}{n}$ & $\frac{t}{i n}$ & $\frac{\Delta}{\operatorname{tn}}$ & 음 & ô & के & $\stackrel{\circ}{q}$ & nू & $\frac{9}{q}$ & $\stackrel{\infty}{\infty}$ & & & & & $\begin{array}{ll}3 & 1 \\
+ & 1 \\
+ & 4\end{array}$ & 拿莩 & 字 & f & & $\dot{m}$ \\
\hline & & SF & $\begin{array}{l}\text { 昏 } \\
\dot{e n}\end{array}$ & $\begin{array}{l}a \\
\dot{d} \\
\dot{a}\end{array}$ & $\stackrel{\vec{n}}{\stackrel{c}{c}}$ & $\stackrel{\vec{d}}{c}$ & 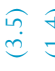 & $\stackrel{\overparen{f}}{\stackrel{f}{\rightleftarrows}}$ & $\stackrel{\stackrel{\Im}{ \pm}}{ \pm}$ & I? & $\hat{\sigma}$ & $\mathrm{S}$ & 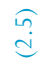 & 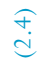 & 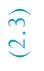 & $\stackrel{0}{\stackrel{0}{c}}$ & 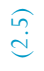 & $\stackrel{i}{i}$ & $\infty$ & $\stackrel{\infty}{d}$ & $\stackrel{6}{0}$ & 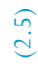 & อ & & & & & & 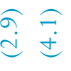 & $\begin{array}{l}6 \\
\dot{0}\end{array}$ & $\stackrel{o}{i}$ & & $\begin{array}{l}\hat{E} \\
\dot{n}\end{array}$ \\
\hline Japan & 557 & (5.5) & & 0 & 0 & $\Delta$ & $\Delta$ & $\mathbf{\Delta}$ & $\Delta$ & $\boldsymbol{\Delta}$ & $\Delta$ & $\Delta$ & $\boldsymbol{\Delta}$ & $\Delta$ & $\Delta$ & $\Delta$ & $\Delta$ & $\boldsymbol{\Delta}$ & $\Delta$ & $\Delta$ & $\Delta$ & $\mathbf{\Delta}$ & $\Delta$ & & 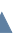 & 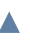 & $\Delta$ & $\mathbf{\Delta}$ & $\Delta \boldsymbol{\Delta}$ & $\Delta$ & $\boldsymbol{\Delta}$ & $\mathbf{\Delta}$ & $\Delta$ \\
\hline Korea & 547 & (2.8) & $O$ & & 0 & 0 & 02 & $\boldsymbol{\Delta}$ & $\boldsymbol{\Delta}$ & $\boldsymbol{\Delta}$ & $\boldsymbol{\Delta}$ & $\Delta$ & $\boldsymbol{\Delta}$ & $\Delta$ & $\Delta$ & $\boldsymbol{\Delta}$ & $\boldsymbol{\Delta}$ & $\boldsymbol{\Delta}$ & $\mathbf{A}$ & $\Delta$ & $\boldsymbol{\Delta}$ & $\Delta$ & $\Delta$ & & a & $\Delta$ & $\boldsymbol{\Delta}$ & $\Delta$ & $\boldsymbol{\Delta} \mathbf{\Delta}$ & $\boldsymbol{\Delta}$ & $\boldsymbol{\Delta}$ & $\boldsymbol{\Delta}$ & $\boldsymbol{\Delta}$ \\
\hline Neuseeland & 537 & (3.1) & 0 & 0 & & 0 & $0 c$ & 0 & 0 & 0 & $\boldsymbol{\Delta}$ & $\Delta$ & $\boldsymbol{\Delta}$ & $\boldsymbol{\Delta}$ & $\Delta$ & $\Delta$ & $\boldsymbol{\Delta}$ & $\boldsymbol{\Delta}$ & $\Delta$ & $\Delta$ & $\Delta$ & $\Delta$ & $\Delta$ & & $\mathbf{A}$ & $\boldsymbol{\Delta}$ & $\Delta$ & $\Delta$ & $\Delta \boldsymbol{\Delta}$ & $\Delta$ & $\boldsymbol{\Delta}$ & $\Delta$ & $\Delta$ \\
\hline Finnland & 536 & (2.1) & $\nabla$ & 0 & $O$ & & 0 & O & 0 & 0 & $\boldsymbol{\Delta}$ & $\Delta$ & $\boldsymbol{\Delta}$ & $\Delta$ & $\Delta$ & O & $\boldsymbol{\Delta}$ & $\boldsymbol{\Delta}$ & $\Delta$ & $\Delta$ & $\Delta$ & $\Delta$ & 4 & & $\Lambda$ & $\Delta$ & $\Delta$ & $\Delta$ & $\Delta \boldsymbol{\Delta}$ & $\boldsymbol{\Delta}$ & $\Delta$ & $\Delta$ & $\boldsymbol{\Delta}$ \\
\hline Australien & 533 & (3.5) & $\nabla$ & 0 & 0 & 0 & & 0 & 0 & 0 & 0 & $\Delta$ & $\boldsymbol{\Delta}$ & $\Delta$ & $\boldsymbol{\Delta}$ & 0 & $\boldsymbol{\Delta}$ & $\Delta$ & $\Delta$ & $\Delta$ & $\boldsymbol{\Delta}$ & $\Delta$ & $\Delta$ & & A & $\boldsymbol{A}$ & $\Delta$ & $\Delta$ & $\Delta \boldsymbol{\Delta}$ & $\boldsymbol{\Delta}$ & $\Delta$ & $\Delta$ & $\boldsymbol{\Delta}$ \\
\hline Kanada & 533 & (1.4) & $\nabla$ & $\nabla$ & 0 & 0 & 0 & & 0 & 0 & $\boldsymbol{\Delta}$ & $\Delta$ & $\boldsymbol{\Delta}$ & $\Delta$ & $\Delta$ & 0 & $\Delta$ & $\boldsymbol{\Delta}$ & $\Delta$ & $\Delta$ & $\Delta$ & $\Delta$ & $\Delta$ & & $\mathbf{\Delta}$ & $\boldsymbol{\Delta}$ & $\Delta$ & $\Delta$ & $\Delta \boldsymbol{\Delta}$ & $\Delta$ & $\boldsymbol{\Delta}$ & $\Delta$ & $\boldsymbol{\Delta}$ \\
\hline Schweiz & 529 & (4.4) & $\nabla$ & $\nabla$ & 0 & 0 & $0 c$ & O & & 0 & 0 & C & 0 & 0 & 0 & O & $\boldsymbol{\Delta}$ & $\Delta$ & $\Delta$ & $\Delta$ & $\boldsymbol{\Delta}$ & $\Delta$ & 4 & & $\Delta$ & $\boldsymbol{\Delta}$ & $\Delta$ & $\Delta$ & $\Delta \boldsymbol{\Delta}$ & $\boldsymbol{\Delta}$ & $\boldsymbol{\Delta}$ & $\Delta$ & $\boldsymbol{\Delta}$ \\
\hline Ver. Königreich & 529 & (2.5) & $\nabla$ & $\nabla$ & 0 & 0 & $0 c$ & 0 & $O$ & & 0 & $\Delta$ & $\boldsymbol{\Delta}$ & $\Delta$ & $\Delta$ & 0 & $\Delta$ & $\Delta$ & $\Delta$ & $\Delta$ & $\boldsymbol{\Delta}$ & $\Delta$ & 4 & & $\mathbf{\Delta}$ & $\boldsymbol{\Delta}$ & $\Delta$ & $\Delta$ & $\Delta \boldsymbol{\Delta}$ & $\Delta$ & $\boldsymbol{\Delta}$ & $\boldsymbol{\Delta}$ & $\boldsymbol{\Delta}$ \\
\hline Belgien & 520 & (3.9) & $\nabla$ & $\nabla$ & $\nabla$ & $\nabla$ & 0 & $\nabla$ & 0 & 0 & & c & 0 & 0 & 0 & 0 & 0 & $\boldsymbol{\Delta}$ & $\Delta$ & $\Delta$ & $\Delta$ & $\Delta$ & 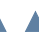 & & D & $\boldsymbol{\Delta}$ & $\Delta$ & $\Delta$ & $\Delta \boldsymbol{\Delta}$ & $\boldsymbol{\Delta}$ & $\boldsymbol{\Delta}$ & $\Delta$ & $\boldsymbol{\Delta}$ \\
\hline Frankreich & 517 & (2.7) & $\nabla$ & $\nabla$ & $\nabla$ & $\nabla$ & $\nabla$ & $\nabla$ & 0 & $\nabla$ & 0 & & 0 & 0 & 0 & 0 & 0 & $\boldsymbol{\Delta}$ & $\Delta$ & $\boldsymbol{\Delta}$ & 0 & $\Delta$ & $\Delta$ & & $\mathbf{\Delta}$ & $\Delta$ & $\Delta$ & $\Delta$ & $\Delta \boldsymbol{\Delta}$ & $\boldsymbol{\Delta}$ & $\boldsymbol{\Delta}$ & $\boldsymbol{\Delta}$ & $\boldsymbol{\Delta}$ \\
\hline Österreich & 515 & (2.5) & $\nabla$ & $\nabla$ & $\nabla$ & $\nabla$ & $\nabla$ & $\nabla$ & 0 & $\nabla$ & 0 & c & & 0 & 0 & 0 & 0 & $\boldsymbol{\Delta}$ & $\Delta$ & $\Delta$ & 0 & $\Delta$ & 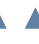 & & | & $\boldsymbol{\Delta}$ & $\Delta$ & $\Delta$ & $\Delta \boldsymbol{\Delta}$ & $\boldsymbol{\Delta}$ & $\Delta$ & $\Delta$ & $\boldsymbol{\Delta}$ \\
\hline Dänemark & 514 & (2.4) & $\nabla$ & $\nabla$ & $\nabla$ & $\nabla$ & $\nabla$ & $\nabla$ & 0 & $\nabla$ & 0 & c & 0 & & 0 & 0 & 0 & $\boldsymbol{\Delta}$ & $\Delta$ & $\Delta$ & 0 & $\Delta$ & $\Delta$ & & A & $\Delta$ & $\Delta$ & $\Delta$ & $\Delta \boldsymbol{\Delta}$ & $\boldsymbol{\Delta}$ & $\boldsymbol{\Delta}$ & $\Delta$ & $\boldsymbol{\Delta}$ \\
\hline Island & 514 & (2.3) & $\nabla$ & $\nabla$ & $\nabla$ & $\nabla$ & $\nabla$ & $\nabla$ & 0 & $\nabla$ & 0 & c & 0 & 0 & & 0 & 0 & $\boldsymbol{\Delta}$ & $\Delta$ & $\Delta$ & 0 & $\Delta$ & 4 & & 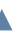 & $\Delta$ & $\Delta$ & $\Delta$ & $\boldsymbol{\Delta} \boldsymbol{\Delta}$ & $\boldsymbol{\Delta}$ & $\boldsymbol{\Delta}$ & $\Delta$ & $\Delta$ \\
\hline Liechtenstein & 514 & (7.0) & $\nabla$ & $\nabla$ & 0 & 0 & $0 c$ & 0 & 0 & 0 & 0 & c & 0 & 0 & 0 & & 0 & 0 & o & o & 0 & $\Delta$ & 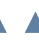 & & $\mathbf{A}$ & $\boldsymbol{\Delta}$ & $\Delta$ & $\Delta$ & $\boldsymbol{\Delta} \Delta$ & $\boldsymbol{\Delta}$ & $\boldsymbol{\Delta}$ & $\Delta$ & $\Delta$ \\
\hline Schweden & 510 & (2.5) & $\nabla$ & $\nabla$ & $\nabla$ & $\nabla$ & $\nabla$ & $\nabla$ & $\nabla$ & $\nabla$ & 0 & C & 0 & 0 & 0 & 0 & 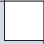 & 0 & c & $\Delta$ & 0 & $\Delta$ & 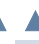 & & | & $\Delta$ & $\Delta$ & $\Delta$ & $\boldsymbol{\Delta} \Delta$ & $\boldsymbol{\Delta}$ & $\boldsymbol{\Delta}$ & $\Delta$ & $\Delta$ \\
\hline Irland & 503 & (2.7) & $\nabla$ & $\nabla$ & $\nabla$ & $\nabla$ & $\nabla$ & $\nabla$ & $\nabla$ & $\nabla$ & $\nabla$ & $\nabla$ & $\nabla$ & $\nabla$ & $\nabla$ & 0 & $\mathrm{O}$ & & c & o & 0 & $\Delta$ & c & & & $\mathbf{A}$ & $\boldsymbol{\Delta}$ & $\Delta$ & $\Delta \boldsymbol{\Delta}$ & $\boldsymbol{\Delta}$ & $\boldsymbol{\Delta}$ & $\Delta$ & $\boldsymbol{\Delta}$ \\
\hline Norwegen & 499 & (2.8) & $\nabla$ & $\nabla$ & $\nabla$ & $\nabla$ & $\nabla$ & $\nabla$ & $\nabla$ & $\nabla$ & $\nabla$ & $\nabla$ & $\nabla$ & $\nabla$ & $\nabla$ & 0 & 0 & 0 & & O & 0 & 0 & $\mathrm{C}$ & & $\boldsymbol{\Lambda}$ & $\boldsymbol{\Delta}$ & $\Delta$ & $\Delta$ & $\Delta \boldsymbol{\Delta}$ & $\boldsymbol{\Delta}$ & $\boldsymbol{\Delta}$ & $\Delta$ & $\Delta$ \\
\hline Tschechische Rep & 498 & (2.8) & $\nabla$ & $\nabla$ & $\nabla$ & $\nabla$ & $\nabla$ & $\nabla$ & $\nabla$ & $\nabla$ & $\nabla$ & $\nabla$ & $\nabla$ & $\nabla$ & $\nabla$ & 0 & $\nabla$ & 0 & 0 & & 0 & 0 & $\mathrm{c}$ & & $J$ & $\Delta$ & $\Delta$ & $\Delta$ & $\Delta \boldsymbol{\Delta}$ & $\boldsymbol{\Delta}$ & $\boldsymbol{\Delta}$ & $\Delta$ & $\Delta$ \\
\hline Vereinigte Staater & 493 & (7.6) & $\nabla$ & $\nabla$ & $\nabla$ & $\nabla$ & $\nabla\rangle$ & $\nabla$ & $\nabla$ & $\nabla$ & 0 & c & 0 & 0 & 0 & 0 & 0 & 0 & o & $\mathrm{O}$ & & 0 & $\mathrm{c}$ & & $J$ & O & 0 & $\Delta$ & $\boldsymbol{\Delta} \boldsymbol{\Delta}$ & $\boldsymbol{\Delta}$ & $\boldsymbol{\Delta}$ & $\boldsymbol{\Delta}$ & $\boldsymbol{\Delta}$ \\
\hline Deutschland & 490 & (2.5) & $\nabla$ & $\nabla$ & $\nabla$ & $\nabla$ & $\nabla$ & $\nabla$ & $\nabla$ & $\nabla$ & $\nabla$ & $\nabla$ & $\nabla$ & $\nabla$ & $\nabla$ & $\nabla$ & $\nabla$ & $\nabla$ & c & o & 0 & & C & & $J$ & $\Delta$ & $\boldsymbol{\Delta}$ & $\Delta$ & $\boldsymbol{\Delta} \boldsymbol{\Delta}$ & $\boldsymbol{\Delta}$ & $\boldsymbol{\Delta}$ & $\boldsymbol{\Delta}$ & $\Delta$ \\
\hline Ungarn & 488 & (4.0) & $\nabla$ & $\nabla$ & $\nabla$ & $\nabla$ & $\nabla$ & $\nabla$ & $\nabla$ & $\nabla$ & $\nabla$ & $\nabla$ & $\nabla$ & $\nabla$ & $\nabla$ & $\nabla$ & $\nabla$ & 0 & o & o & 0 & 0 & & & & 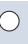 & 0. & $\Delta$ & $\Delta \boldsymbol{\Delta}$ & $\boldsymbol{\Delta}$ & $\boldsymbol{\Delta}$ & $\Delta$ & $\Delta$ \\
\hline Russische Föd. & 478 & (5.5) & $\nabla$ & $\nabla$ & $\nabla$ & $\nabla$ & $\nabla$ & $\nabla$ & $\nabla$ & $\nabla$ & $\nabla$ & $\nabla$ & $\nabla$ & $\nabla$ & $\nabla$ & $\nabla$ & $\nabla$ & $\nabla$ & $\nabla$ & 0 & 0 & 0 & $\mathrm{C}$ & & & & 0 & 0 & $\Delta \boldsymbol{\Delta}$ & $\boldsymbol{\Delta}$ & $\boldsymbol{\Delta}$ & $\boldsymbol{\Delta}$ & $\boldsymbol{\Delta}$ \\
\hline Spanien & 476 & (3.1) & $\nabla$ & $\nabla$ & $\nabla$ & $\nabla$ & $\nabla$ & $\nabla$ & $\nabla$ & $\nabla$ & $\nabla$ & $\nabla$ & $\nabla$ & $\nabla$ & $\nabla$ & $\nabla$ & $\nabla$ & $\nabla$ & $\nabla$ & $\nabla$ & 70 & $\nabla$ & $7 \mathrm{c}$ & & D & & 0 & 0 & $\Delta \boldsymbol{\Delta}$ & $\Delta$ & $\boldsymbol{\Delta}$ & $\Delta$ & $\Delta$ \\
\hline Polen & 470 & (5.5) & $\nabla$ & $\nabla$ & $\nabla$ & $\nabla$ & $\nabla$ & $\nabla$ & $\nabla$ & $\nabla$ & $\nabla$ & $\nabla$ & $\nabla$ & $\nabla$ & $\nabla$ & $\nabla$ & $\nabla$ & $\nabla$ & $\nabla$ & $\nabla$ & 70 & $\nabla$ & 70 & & J & $U$ & 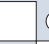 & 0 & 00 & 0 & $\Delta$ & $\Delta$ & $\Delta$ \\
\hline Lettland & 463 & (4.5) & $\nabla$ & $\nabla$ & $\nabla$ & $\nabla$ & $\nabla$ & $\nabla$ & $\nabla$ & $\nabla$ & $\nabla$ & $\nabla$ & $\nabla$ & $\nabla$ & $\nabla$ & $\nabla$ & $\nabla$ & $\nabla$ & $\nabla$ & $\nabla$ & $\nabla$ & $7 \nabla$ & 78 & & J & & 0 & & 00 & 0 & $\Delta$ & $\Delta$ & $\boldsymbol{\Delta}$ \\
\hline Italien & 457 & (2.9) & $\nabla$ & $\nabla$ & $\nabla$ & $\nabla$ & $\nabla$ & $\nabla$ & $\nabla$ & $\nabla$ & $\nabla$ & $\nabla$ & $\nabla$ & $\nabla$ & $\nabla$ & $\nabla$ & $\nabla$ & $\nabla$ & $\nabla$ & $\nabla$ & $\nabla$ & $\nabla \nabla$ & 78 & & $\sqrt{ }$ & $\nabla$ & $0^{2}$ & O & 0 & 0 & $\Delta$ & $\Delta$ & \\
\hline Portugal & 454 & (4.1) & $\nabla$ & $\nabla$ & $\nabla$ & $\nabla$ & $\nabla$ & $\nabla$ & $\nabla$ & $\nabla$ & $\nabla$ & $\nabla$ & $\nabla$ & $\nabla$ & $\nabla$ & $\nabla$ & $\nabla$ & $\nabla$ & $\nabla$ & $\nabla$ & $\nabla$ & $\nabla \nabla$ & 7 & & $V$ & $\nabla$ & 0 & 0 & $O$ & 0 & 0 & $\Delta$ & \\
\hline Griechenland & 447 & (5.6) & $\nabla$ & $\nabla$ & $\nabla$ & $\nabla$ & $\nabla$ & $\nabla$ & $\nabla$ & $\nabla$ & $\nabla$ & $\nabla$ & $\nabla$ & $\nabla$ & $\nabla$ & $\nabla$ & $\nabla$ & $\nabla$ & $\nabla$ & $\nabla$ & $\nabla$ & $\nabla$ & 78 & & $V$ & & 0 & 0 & 00 & & 0 & $\Delta$ & $\Delta$ \\
\hline Luxemburg & 446 & (2.0) & $\nabla$ & $\nabla$ & $\nabla$ & $\nabla$ & $\nabla$ & $\nabla$ & $\nabla$ & $\nabla$ & $\nabla$ & $\nabla$ & $\nabla$ & $\nabla$ & $\nabla$ & $\nabla$ & $\nabla$ & $\nabla$ & $\nabla$ & $\nabla$ & $\nabla$ & $7 \nabla$ & 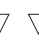 & & $\sqrt{ }$ & & $\nabla$ & $\nabla$ & $\nabla 0$ & 0 & & $\Delta$ & $\Delta$ \\
\hline Mexiko & 387 & (3.4) & $\nabla$ & $\nabla$ & $\nabla$ & $\nabla$ & $\nabla$ & $\nabla$ & $\nabla$ & $\nabla$ & $\nabla$ & $\nabla$ & $\nabla$ & $\nabla$ & $\nabla$ & $\nabla$ & $\nabla$ & $\nabla$ & $\nabla$ & $\nabla$ & $\nabla$ & $\nabla \nabla$ & 7 & & $\nabla$ & & $\nabla$ & $\nabla$ & $\nabla \nabla$ & $\nabla$ & $\nabla$ & & $\Delta$ \\
\hline Brasilien & 334 & (3.7) & $\nabla$ & $\nabla$ & $\nabla$ & $\nabla$ & $\nabla$ & $\nabla$ & $\nabla$ & $\nabla$ & $\nabla$ & 8 & $\nabla$ & $\nabla$ & $\nabla$ & $\nabla$ & $\nabla$ & $\nabla$ & $\nabla$ & $\nabla$ & $\nabla$ & 78 & 7 & & & & & $\nabla$ & $\nabla \nabla$ & $\nabla$ & $\nabla$ & $\nabla$ & \\
\hline & Ude & ang* & 1 & 2 & 4 & 4 & 4 & 5 & 4 & 6 & , & & 10 & 10 & 11 & 9 & 13 & 16 & 1 & 717 & 716 & 62 & 02 & & & & 23 & 25 & 2626 & 27 & 729 & 31 & 32 \\
\hline & ite & ang* & 3 & & 8 & 7 & 9 & 8 & 10 & & & & & & 16 & & 17 & 19 & & & & & & & & & & 28 & \begin{tabular}{|l|l}
28 & 29
\end{tabular} & 30 & & 31 & \\
\hline
\end{tabular}

* Hinweis: Da die Daten auf Stichprobenerhebungen basieren, ist es nicht möglich, den genauen Rangplatz eines Landes zu bestimmen. Es können jedoch der jeweils obere und untere Rangplatz angegeben werden, zwischen denen das Land mit seinen mittleren Schülerleistungen mit 95\%-igerWahrscheinlichkeit liegt.

\section{Erläuterungen}

Anordnung der Länder in absteigender Reihenfolge der mittleren Schülerleistungen auf der PISA-Skala für mathematische Grundbildung. Aufgrund der geringen Beteiligungsquote sind die Niederlande nicht enthalten. Ausgehend von einem zu vernachlässigenden bis geringfügigen statistischen Fehler aufgrund der Nichtbeteiligung ist zu erwarten, dass die Niederlande mit 95\%-iger Wahrscheinlichkeit zwischen Rangplatz 1 und 4 liegen würden.

* Hinweis: Da die Daten auf Stichprobenerhebungen basieren, ist es nicht möglich, den genauen Rangplatz eines Landes zu bestimmen. Es können jedoch der jeweils obere und untere Rangplatz angegeben werden, zwischen denen das Land mit seinen mittleren Schülerleistungen mit 95\%-iger Wahrscheinlichkeit liegt.
A Mittlere Schülerleistungen statistisch signifikant höher als im Vergleichsland

Kein statistisch signifikanter Unterschied gegenüber dem Vergleichsland $\nabla$ Mittlere Schülerleistungen signifikant niedriger als im Vergleichsland

Statistisch signifikant über dem OECD-Durchschnitt Kein statistisch signifikanter Unterschied zum OECD-Durchschnitt Statistisch signifikant unter dem OECD-Durchschnitt 


\section{$\mathbf{A}_{6}$}

Mathematik und

Naturwissenschaften

müssen heute von

vielen und nicht nur

von wenigen genutzt

werden, ...

wenn die Menschen

die moderne Welt von

heute verstehen und an

ihr aktiv teilnehmen

sollen.

Dieser Indikator zeigt

die Leistungen von

15-Jährigen auf den

PISA-Skalen für

mathematische und naturwissenschaftliche

Grundbildung auf.

\section{Politischer Hintergrund}

Im vergangenen Jahrhundert standen die Curricula der Schulmathematik und der naturwissenschaftlichen Fächer meist im Zeichen der Notwendigkeit, eine solide Grundlage für die berufliche Ausbildung einer kleinen Zahl von Mathematikern, Wissenschaftlern und Ingenieuren zu liefern. Mit der zunehmenden Bedeutung von Naturwissenschaften, Mathematik und Technologie für das moderne Leben erfordern Ziele wie Selbstverwirklichung, Berufstätigkeit und aktive Teilnahme am gesellschaftlichen Leben allerdings, dass alle Erwachsenen über mathematische, naturwissenschaftliche und technische Grundkompetenzen verfügen.

Defizite in der mathematischen und naturwissenschaftlichen Grundbildung können nicht nur sehr negative Auswirkungen auf die Arbeitsmarktund Einkommensaussichten des Einzelnen, sondern auch auf die Wettbewerbsfähigkeit der einzelnen Länder haben. Umgekehrt können die Leistungen der besten Schüler eines Landes in Mathematik und naturwissenschaftlichen Fächern Auswirkungen auf die Rolle haben, die dieses Land innerhalb der Spitzentechnologien von morgen einnehmen wird. Abgesehen von den Anforderungen im Berufsleben sind mathematische und naturwissenschaftliche Grundkompetenzen für das Verständnis ökologischer, medizinischer, ökonomischer und sonstiger Probleme von Bedeutung, denen sich die modernen Gesellschaften heute gegenübersehen, und deren Lösung sehr stark vom technologischen und naturwissenschaftlichen Fortschritt abhängt.

Infolgedessen messen politische Entscheidungsträger und Pädagogen gleichermaßen der mathematischen und naturwissenschaftlichen Bildung große Bedeutung bei. Um der wachsenden Nachfrage nach mathematischen und naturwissenschaftlichen Kompetenzen gerecht zu werden, bedarf es eines in jeder Hinsicht hervorragenden Bildungssystems, und es ist hilfreich zu verfolgen, wie gut die Länder junge Erwachsene mit den Grundkompetenzen in diesen Bereichen ausstatten. Die internationale Schulleistungsstudie PISA liefert Informationen darüber, wie gut 15-Jährige in diesen Bereichen abschneiden, wobei der Schwerpunkt auf der Bewertung derjenigen Kenntnisse und Fertigkeiten liegt, die die Schüler auf das Leben und lebenslanges Lernen vorbereiten (s. Kasten A6.1).

\section{Ergebnisse und Erläuterungen}

In den Abbildungen A6.1 und A6.2 sind die Länder gemäß der mittleren Leistungen ihrer Schüler auf den PISA-Skalen für mathematische und naturwissenschaftliche Grundbildung angeordnet. Aus den Abbildungen ist auch ersichtlich, welche Länder Schülerleistungen oberhalb, unterhalb oder auf der Höhe des OECD-Durchschnitts aufweisen, und wie die Leistungen ihrer Schüler im Vergleich zu den Schülern in jedem andern Land sind. 
Kasten A6.1. Was bedeuten mathematische und naturwissenschaftliche Grundbildung im Rahmen von PISA?

Was bedeutet mathematische Grundbildung? Mathematische Grundbildung im Rahmen von PISA bezieht sich auf die Fähigkeit der Schüler, mathematische Probleme zu erkennen und zu interpretieren, denen sie in ihrem Umfeld begegnen, diese Probleme in mathematische Strukturen umzusetzen, mathematische Kenntnisse und Verfahren zur Lösung von Problemen innerhalb dieses mathematischen Kontexts anzuwenden, die Lösung im Hinblick auf das Ausgangsproblem zu interpretieren, über die angewandte Methode zu reflektieren und die Ergebnisse zu formulieren und zu kommunizieren.

Was bedeuten die unterschiedlichen Punktwerte auf der PISA-Skala für mathematische Grundbildung? Die Punktwerte spiegeln die Unterschiede in den nachzuweisenden Kenntnissen und Fähigkeiten im Bereich mathematische Grundbildung wider:

- Am oberen Ende der Skala, bei rund 750 Punkten, nehmen die Schüler bei der Lösung mathematischer Probleme eine kreative und aktive Rolle ein.

- Bei rund 570 Punkten auf der Skala sind die Schüler generell in der Lage, verschiedene Darstellungen eines mathematischen Problems oder Informationen aus verschiedenen Quellen zu interpretieren, zu verknüpfen und zu integrieren und/oder ein bestimmtes Modell anzuwenden oder zu handhaben, das in vielen Fällen algebraische Formeln oder sonstige symbolische Darstellungen enthält, und/oder gegebene Lösungsvorschläge bzw. Modelle zu überprüfen oder zu testen.

- Am unteren Ende der Skala, bei rund 380 Punkten, sind die Schüler in der Regel nur in der Lage, einen einzigen Rechenschritt auszuführen, der darin besteht, grundlegende mathematische Fakten oder Prozesse wiederzugeben bzw. einfache Rechenfertigkeiten anzuwenden.

Was bedeutet naturwissenschaftliche Grundbildung? Naturwissenschaftliche Grundbildung steht für die Fähigkeit der Schüler, naturwissenschaftliches Wissen anzuwenden, naturwissenschaftliche Fragen zu erkennen bzw. zu identifizieren, worum es bei naturwissenschaftlichen Untersuchungen geht, Behauptungen und Schlussfolgerungen mit naturwissenschaftlichen Daten zu verbinden und diese Aspekte der Naturwissenschaften zu kommunizieren.

Was bedeuten die unterschiedlichen Punktwerte auf der PISA-Skala für naturwissenschaftliche Grundbildung? Die Skala lässt sich anhand der zunehmenden Schwierigkeit der von den Schülern zu lösenden Aufgaben, bzw. der zu ihrer Lösung notwendigen Kenntnisse und Fähigkeiten, beschreiben:

- Am oberen Ende der Skala, bei rund 690 Punkten, sind die Schüler generell in der Lage, konzeptuelle Modelle zu entwickeln und zu nutzen, um Vorhersagen zu machen und Erklärungen zu geben; naturwissenschaftliche Untersuchungen z.B. hinsichtlich der verwendeten Versuchsanordnung oder der in der Untersuchung geprüften Idee zu analysieren; auf der 
Basis des Vergleichs von Daten alternative Standpunkte oder unterschiedliche Perspektiven zu evaluieren; und wissenschaftliche Argumente und/oder Darlegungen detailliert und präzise zu kommunizieren.

- Bei rund 550 Punkten sind die Schüler generell in der Lage, naturwissenschaftliche Konzepte zu benutzen, um Vorhersagen zu machen oder Erklärungen zu geben; Fragen zu erkennen, die durch naturwissenschaftliche Untersuchungen beantwortet werden können, und/oder Details über den Gegenstand einer naturwissenschaftlichen Untersuchung zu identifizieren; ferner können sie beim Ziehen von Schlussfolgerungen, bzw. bei der kritischen Bewertung derselben relevante Informationen aus konkurrierenden Daten oder Argumentationsketten auswählen.

- Am unteren Ende der Skala, bei rund 400 Punkten (die von mindestens drei Viertel aller Schülern in fast allen Ländern erreicht werden), sind die Schüler in der Lage, einfaches naturwissenschaftliches Faktenwissen abzurufen (d.h. Namen, Fakten, Begriffe, einfache Regeln und Gesetze) und naturwissenschaftliches Allgemeinwissen beim Ziehen oder Bewerten von Schlussfolgerungen zu verwenden.

Japan hat den höchsten

Mittelwert bei der mathematischen Grundbildung ...

... und Korea bei der naturwissenschaftlichen Grundbildung.
Die Schüler in Japan erreichen bei der mathematischen Grundbildung die höchste mittlere Punktzahl, allerdings besteht zwischen dem Durchschnittsergebnis von Japan und den Durchschnittsergebnissen von Korea, Neuseeland und den Niederlanden kein statistisch signifikanter Unterschied. Die anderen Länder, deren Punktzahlen ebenfalls signifikant über dem OECD-Durchschnitt liegen, sind Australien, Belgien, Dänemark, Finnland, Frankreich, Island, Kanada, Liechtenstein, Österreich, Schweden, die Schweiz und das Vereinigte Königreich

Japan und Korea weisen auf der PISA-Skala für naturwissenschaftliche Grundbildung im Vergleich zu den anderen OECD-Ländern die höchsten Ergebnisse auf. Zu den anderen Ländern, deren Ergebnisse ebenfalls statistisch signifikant über dem OECD-Durchschnitt liegen, gehören Australien, Finnland, Irland, Kanada, Neuseeland, Österreich, Schweden, die Tschechische Republik und das Vereinigte Königreich.

Bei einem Blick auf die in den vorherigen Absätzen angeführten Auflistungen der Länder mit überdurchschnittlichen Ergebnissen zeigt sich, dass ganz allgemein gesprochen Länder mit guten Ergebnissen in einem Grundbildungsbereich auch in den anderen Bereichen gut abschneiden (d.h. es besteht eine enge Korrelation zwischen den Mittelwerten auf der PISA-Skala für mathematische Grundbildung und der PISA-Skala für naturwissenschaftliche Grundbildung). Es gibt jedoch einige Ausnahmen. So liegen beispielsweise die Ergebnisse bei der mathematischen Grundbildung in Irland und der Tschechischen Republik mehr oder minder auf dem OECD-Durchschnitt, bei der PISA-Skala für 
Abbildung A6.2.

Vergleich der mittleren Schülerleistungen der Länder auf der PISA-Skala für naturwissenschafltiche Grundbildung (2000)

\begin{tabular}{|c|c|c|c|c|c|c|c|c|c|c|c|c|c|c|c|c|c|c|c|c|c|c|c|c|c|c|c|c|c|c|c|}
\hline & & & \begin{tabular}{l}
$\widetilde{J}$ \\
$\stackrel{0}{0}$ \\
\hdashline
\end{tabular} & & & & 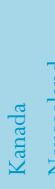 & 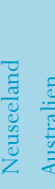 & 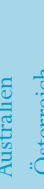 & ț & & 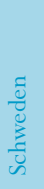 & 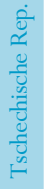 & है & $\begin{array}{l}\tilde{0} \\
0 \\
0 \\
\sum_{0} \\
\text { z }\end{array}$ & 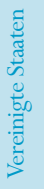 & & & 袍 & & & & & & 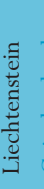 & & 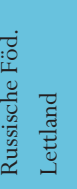 & $\stackrel{2}{0}$ & & 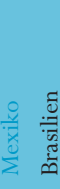 & ڤ్ \\
\hline & \multicolumn{2}{|c|}{ Mittelwert } & กิ & $\begin{array}{l}\circ \\
\text { in } \\
\text { in }\end{array}$ & $\tilde{N}_{n}^{\infty}$ & సิ & ते & î & $\begin{array}{ll}0 \\
n \\
n & 1\end{array}$ & & $\frac{m}{n}$ & $\stackrel{2}{i n}$ & $\bar{E}$ & 8 & 8 & gे & $\begin{array}{l}\circ \\
+ \\
+\end{array}$ & 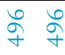 & 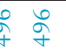 & $\bar{\sigma}$ & $\stackrel{\Gamma}{\infty}$ & $\begin{array}{l}\infty \\
\infty \\
+\end{array}$ & 文 & $\stackrel{\infty}{\stackrel{\infty}{+}}$ & $\frac{6}{2}$ & & $\begin{array}{ll}8 \\
8\end{array}$ & 字 & $\stackrel{\Re}{\ddagger}$ & & $\stackrel{n}{n}$ \\
\hline & & SF & $\widehat{E}$ & (5) & ก? & 8 & $\begin{array}{l}\stackrel{6}{=} \\
\stackrel{=}{0}\end{array}$ & 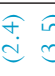 & 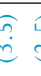 & & ণิ & 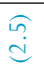 & $\underset{d}{\stackrel{d}{d}}$ & $\begin{array}{l}\text { ț } \\
\text { ñ }\end{array}$ & $\stackrel{\text { a }}{\mathrm{i}}$ & & $\begin{array}{l}\text { I } \\
\text { ¿ }\end{array}$ & $\stackrel{\overbrace{}}{\mathrm{d}} \stackrel{\overbrace{}}{ \pm}$ & 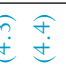 & $\begin{array}{c}\hat{\sigma} \\
\dot{n}\end{array}$ & $\underset{d}{d}$ & કे & & $\overrightarrow{\vec{n}}$ & $\stackrel{\overbrace{}}{\stackrel{5}{8}}$ & & $\begin{array}{l}\widehat{G} \\
\dot{I} \\
\dot{ \pm}\end{array}$ & $\begin{array}{l}\partial \hat{\partial} \\
\dot{ \pm}\end{array}$ & $\stackrel{\widetilde{m}}{\mathrm{~d}} \widehat{\mathrm{g}}$ & $\begin{array}{ll}\hat{\imath} & \hat{m} \\
& \stackrel{n}{n}\end{array}$ & $\widehat{\tilde{n}}$ \\
\hline Korea & 552 & $(2.7)$ & & 0 & $\Delta$ & $\Delta$ & $\Delta$ & $\Delta$ & $\boldsymbol{\Delta}$ & $\boldsymbol{\Delta}$ & $\Delta$ & $\Delta$ & $\Delta$ & $\boldsymbol{\Delta}$ & $\Delta$ & $\Delta$ & $\Delta$ & $\Delta \Delta$ & $\Delta \Delta$ & $\Delta$ & $\Delta$ & $\boldsymbol{\Delta}$ & $\Delta$ & $\boldsymbol{\Delta}$ & $\Delta$ & $\Delta$ & $\Delta \Delta$ & $\Delta$ & $\Delta$ & $\Delta \Delta$ & $\boldsymbol{\Delta}$ \\
\hline apan & 550 & $(5.5)$ & $O$ & & 0 & 0 & $\Delta$ & $\Delta$ & $\Delta$ & $\boldsymbol{\Delta}$ & $\boldsymbol{\Delta}$ & $\Delta$ & $\Delta$ & $\boldsymbol{\Delta}$ & $\Delta$ & $\Delta$ & $\boldsymbol{\Delta}$ & $\Delta \mathbf{\Delta}$ & $\Delta \boldsymbol{\Delta}$ & $\Delta$ & $\boldsymbol{\Delta}$ & $\boldsymbol{\Delta}$ & $\boldsymbol{\Delta}$ & $\boldsymbol{\Delta}$ & $\boldsymbol{\Delta}$ & $\Delta$ & $\Delta \boldsymbol{\Delta}$ & $\Delta$ & $\Delta$ & $\Delta$ & $\boldsymbol{\Delta}$ \\
\hline Finnland & 538 & $(2.5)$ & $\nabla$ & 0 & & 0 & 0 & $O C$ & 0 & $\boldsymbol{\Delta}$ & $\boldsymbol{\Delta}$ & $\Delta$ & $\boldsymbol{\Delta}$ & $\boldsymbol{\Delta}$ & $\Delta$ & $\Delta$ & $\boldsymbol{\Delta}$ & $\Delta \mathbf{\Delta}$ & $\boldsymbol{\Delta} \boldsymbol{\Delta}$ & $\boldsymbol{\Delta}$ & $\boldsymbol{\Delta}$ & $\boldsymbol{\Delta}$ & $\boldsymbol{\Delta}$ & $\boldsymbol{\Delta}$ & $\boldsymbol{\Delta}$ & $\Delta$ & $\Delta \boldsymbol{\Delta}$ & $\Delta$ & $\Delta$ & $\Delta \Delta$ & $\boldsymbol{\Delta}$ \\
\hline Ver. Königreich & 532 & $(2.7)$ & $\nabla$ & 0 & 0 & & 0 & 00 & 0 & $\boldsymbol{\Delta}$ & $\Delta$ & $\Delta$ & $\Delta$ & $\boldsymbol{\Delta}$ & $\Delta$ & $\Delta$ & $\boldsymbol{\Delta}$ & $\Delta \Delta$ & $\Delta \boldsymbol{\Delta}$ & $\Delta$ & $\Delta$ & $\boldsymbol{\Delta}$ & $\Delta$ & $\Delta$ & $\Delta$ & $\Delta$ & $\Delta \boldsymbol{\Delta}$ & $\Delta$ & $\Delta$ & $\Delta \Delta$ & $\boldsymbol{\Delta}$ \\
\hline Kanada & 529 & $(1.6)$ & $\nabla$ & $\nabla$ & 0 & O & & $0 c$ & 0 & $\boldsymbol{\Delta}$ & $\Delta$ & $\Delta$ & $\Delta$ & $\Delta$ & $\Delta$ & $\Delta$ & $\Delta$ & $\Delta \Delta$ & $\Delta \Delta$ & $\Delta$ & $\Delta$ & $\Delta$ & $\Delta$ & $\Delta$ & $\boldsymbol{\Delta}$ & $\Delta$ & $\Delta \Delta$ & $\Delta$ & $\Delta$ & $\Delta$ & $\boldsymbol{\Delta}$ \\
\hline Neuseeland & 528 & $(2.4)$ & $\nabla$ & $\nabla$ & 0 & 0 & 0 & c & 0 & 0 & $\Delta$ & $\Delta$ & $\Delta$ & $\boldsymbol{\Delta}$ & $\Delta$ & $\Delta$ & $\Delta$ & $\Delta \Delta$ & $\Delta \Delta$ & $\Delta$ & $\boldsymbol{\Delta}$ & $\Delta$ & $\Delta$ & $\Delta$ & $\Delta$ & $\Delta$ & $\Delta \Delta$ & $\Delta$ & $\Delta$ & $\Delta$ & $\boldsymbol{\Delta}$ \\
\hline Australien & 528 & (3.5) & $\nabla$ & $\nabla$ & 0 & 0 & 0 & O & & 0 & 0 & $\Delta$ & $\Delta$ & $\boldsymbol{\Delta}$ & $\Delta$ & $\Delta$ & $\Delta$ & $\Delta \Delta$ & $\Delta \boldsymbol{\Delta}$ & $\Delta$ & $\boldsymbol{\Delta}$ & $\boldsymbol{\Delta}$ & $\Delta$ & $\boldsymbol{\Delta}$ & $\boldsymbol{\Delta}$ & $\Delta$ & $\Delta \boldsymbol{\Delta}$ & $\Delta$ & $\Delta$ & $\Delta \Delta$ & $\boldsymbol{\Delta}$ \\
\hline Österreich & 519 & $(2.5)$ & $\nabla$ & $\nabla$ & $\nabla$ & $\nabla$ & $\nabla$ & $0 c$ & $\mathrm{O}$ & & 0 & 0 & 0 & $\Delta$ & $\Delta$ & 0 & $\Delta$ & $\Delta \Delta$ & $\Delta \boldsymbol{\Delta}$ & $\Delta$ & $\Delta$ & $\boldsymbol{\Delta}$ & $\Delta$ & $\boldsymbol{\Delta}$ & $\Delta$ & $\Delta$ & $\Delta \boldsymbol{\Delta}$ & $\Delta$ & $\Delta$ & $\Delta$ & $\boldsymbol{\Delta}$ \\
\hline Irland & 513 & $(3.2)$ & $\nabla$ & $\nabla$ & $\nabla$ & $\nabla$ & $\nabla$ & $\nabla c$ & 0 & 0 & & 0 & 0 & 0 & 0 & 0 & $\boldsymbol{\Delta}$ & $\Delta \Delta$ & $\boldsymbol{\Delta} \boldsymbol{\Delta}$ & $\boldsymbol{\Delta}$ & $\boldsymbol{\Delta}$ & $\boldsymbol{\Delta}$ & $\boldsymbol{\Delta}$ & $\Delta$ & $\Delta$ & $\Delta$ & $\Delta \boldsymbol{\Delta}$ & $\Delta$ & $\Delta$ & $\Delta \mathbf{\Delta}$ & \\
\hline Schweden & 512 & $(2.5)$ & $\nabla$ & $\nabla$ & $\nabla$ & $\nabla$ & $\nabla$ & $\nabla \nabla$ & $\nabla$ & 0 & 0 & & 0 & 0 & $\boldsymbol{\Delta}$ & 0 & $\Delta$ & $\Delta \Delta$ & $\boldsymbol{\Delta} \Delta$ & $\Delta$ & $\boldsymbol{\Delta}$ & $\boldsymbol{\Delta}$ & $\boldsymbol{\Delta}$ & $\Delta$ & $\boldsymbol{\Delta}$ & $\Delta$ & $\Delta \boldsymbol{\Delta}$ & $\Delta$ & $\Delta$ & $\Delta \Delta$ & $\boldsymbol{\Delta}$ \\
\hline Tschechische Rep. & 511 & $(2.4)$ & $\nabla$ & $\nabla$ & $\nabla$ & $\nabla$ & $\nabla$ & $\nabla \nabla$ & $\nabla$ & 0 & 0 & 0 & & 0 & 0 & 0 & $\boldsymbol{\Delta}$ & $\Delta \boldsymbol{\Delta}$ & $\Delta$ & $\Delta$ & $\boldsymbol{\Delta}$ & $\boldsymbol{\Delta}$ & $\boldsymbol{\Delta}$ & $\boldsymbol{\Delta}$ & $\Delta$ & $\Delta$ & $\Delta \boldsymbol{\Delta}$ & $\Delta$ & $\Delta$ & $\Delta \Delta$ & \\
\hline Fran & 5 & $(3.2)$ & $\nabla$ & $\nabla$ & $\nabla$ & $\nabla$ & $\nabla$ & $\nabla \nabla$ & $\nabla$ & $\nabla$ & 0 & 0 & $O$ & & 0 & 0 & 0 & 00 & 00 & 0 & $\boldsymbol{\Delta}$ & 0 & $\Delta$ & $\boldsymbol{\Delta}$ & 0 & $\Delta$ & $\Delta \boldsymbol{\Delta}$ & $\Delta$ & $\Delta$ & $\Delta \Delta$ & \\
\hline Norwegen & 500 & $(2.7)$ & $\nabla$ & $\nabla$ & $\nabla$ & $\nabla$ & $\nabla$ & $\nabla \nabla$ & $\nabla$ & $\nabla$ & 0 & $\nabla$ & 0 & 0 & & 0 & 0 & 00 & 00 & 0 & $\boldsymbol{\Delta}$ & O & $\Delta$ & $\Delta$ & $\Delta$ & $\Delta$ & $\Delta \boldsymbol{\Delta}$ & $\Delta$ & $\Delta$ & $\Delta \mathbf{\Delta}$ & $\boldsymbol{\Delta}$ \\
\hline Vereinigte Staaten & 499 & $(7.3)$ & $\nabla$ & $\nabla$ & $\nabla$ & $\nabla$ & $\nabla$ & $\nabla \nabla$ & $\nabla$ & 0 & 0 & 0 & 0 & 0 & 0 & & 0 & 00 & 00 & 0 & 0 & 0 & 0 & 0 & 0 & $\Delta$ & $\Delta \Delta$ & $\Delta$ & $\Delta$ & $\Delta$ & \\
\hline Ungarn & 496 & $(4.2)$ & $\nabla$ & $\nabla$ & $\nabla$ & $\nabla$ & $\nabla$ & $\nabla \nabla$ & $\nabla$ & $\nabla$ & $\nabla$ & $\nabla$ & $\nabla$ & 0 & 0 & $O$ & L & 100 & 00 & 0 & 0 & 0 & 0 & $\Delta$ & 0 & $\Delta$ & $\Delta \Delta$ & $\Delta$ & $\Delta$ & $\Delta$ & \\
\hline Island & 496 & $(2.2)$ & $\nabla$ & $\nabla$ & $\nabla$ & $\nabla$ & $\nabla$ & $\nabla \nabla$ & $\nabla$ & $\nabla$ & $\nabla$ & $\nabla$ & $\nabla$ & 0 & 0 & 0 & 0 & O & 00 & 0 & 0 & 0 & $\Delta$ & $\Delta$ & 0 & $\Delta$ & $\Delta \boldsymbol{\Delta}$ & $\Delta$ & $\Delta$ & $\Delta \mathbf{\Delta}$ & $\boldsymbol{\Delta}$ \\
\hline Belgien & 496 & $(4.3)$ & $\nabla$ & $\nabla$ & $\nabla$ & $\nabla$ & $\nabla$ & $\nabla \nabla$ & $\nabla$ & $\nabla$ & $\nabla$ & $\nabla$ & $\nabla$ & 0 & 0 & 0 & 0 & 0 & 0 & 0 & 0 & 0 & 0 & $\Delta$ & 0 & $\Delta$ & $\Delta \boldsymbol{\Delta}$ & $\Delta$ & $\Delta$ & $\Delta$ & \\
\hline Schweiz & 496 & $(4.4)$ & $\nabla$ & $\nabla$ & $\nabla$ & $\nabla$ & $\nabla$ & $\nabla \nabla$ & $\nabla$ & $\nabla$ & $\nabla$ & $\nabla$ & 0 & 0 & 0 & 0 & 0 & 00 & 0 & 0 & 0 & O & 0 & $\Delta$ & 0 & $\Delta$ & $\Delta \boldsymbol{\Delta}$ & $\Delta$ & $\Delta$ & $\Delta \Delta$ & \\
\hline Spanien & 491 & (3.0) & $\nabla$ & $\nabla$ & $\nabla$ & $\nabla$ & $\nabla$ & $\nabla \nabla$ & $\nabla$ & $\nabla$ & $\nabla$ & $\nabla$ & $\nabla$ & 0 & 0 & 0 & 0 & 00 & 00 & & 0 & 0 & 0 & 0 & 0 & $\Delta$ & $\Delta \Delta$ & $\Delta$ & $\Delta$ & $\Delta \Delta$ & $\Delta$ \\
\hline Deutschland & 487 & $(2.4)$ & $\nabla$ & $\nabla$ & $\nabla$ & $\nabla$ & $\nabla$ & $\nabla \nabla$ & $\nabla$ & $\nabla$ & $\nabla$ & $\nabla$ & $\nabla$ & $\nabla$ & $\nabla$ & 0 & 0 & 00 & 00 & $O$ & & 0 & 0 & 0 & 0 & $\Delta$ & $\Delta \Delta$ & $\Delta$ & $\Delta$ & $\Delta \Delta$ & \\
\hline Polen & 483 & $(5.1)$ & $\nabla$ & $\nabla$ & $\nabla$ & $\nabla$ & $\nabla$ & $\nabla \nabla$ & $\nabla$ & $\nabla$ & $\nabla$ & $\nabla$ & $\nabla$ & 0 & 0 & 0 & 0 & 00 & 00 & 0 & $O$ & & 0 & 0 & 0 & $\Delta$ & $\Delta O$ & $\Delta$ & $\Delta$ & $\Delta \boldsymbol{\Delta}$ & \\
\hline Dänemark & 481 & (2.8) & $\nabla$ & $\nabla$ & $\nabla$ & $\nabla$ & $\nabla$ & $\nabla \nabla$ & $\nabla$ & $\nabla$ & $\nabla$ & $\nabla$ & $\nabla$ & $\nabla$ & $\nabla$ & 0 & 0 & $\nabla 0$ & 00 & 0 & 0 & 0 & & 0 & 0 & $\Delta$ & $\Delta \Delta$ & $\Delta$ & $\Delta$ & $\Delta$ & $\Delta$ \\
\hline Italien & 478 & $(3.1)$ & $\nabla$ & $\nabla$ & $\nabla$ & $\nabla$ & $\nabla$ & $\nabla \nabla$ & $\nabla$ & $\nabla$ & $\nabla$ & $\nabla$ & $\nabla$ & $\nabla$ & $\nabla$ & 0 & $\nabla$ & $\nabla \nabla$ & $\nabla \nabla$ & 0 & 0 & 0 & $O$ & & 0 & 0 & 00 & $\Delta$ & $\Delta$ & $\Delta \Delta$ & \\
\hline Liechtenstein & 476 & $(7.1)$ & $\nabla$ & $\nabla$ & $\nabla$ & $\nabla$ & $\nabla$ & $\nabla \nabla$ & $\nabla$ & $\nabla$ & $\nabla$ & $\nabla$ & $\nabla$ & 0 & $\nabla$ & 0 & 0 & 00 & 00 & 0 & 0 & 0 & 0 & $O$ & & 0 & 00 & 0 & $\Delta$ & $\Delta \Delta$ & \\
\hline Griechenland & 461 & $(4.9)$ & $\nabla$ & $\nabla$ & $\nabla$ & $\nabla$ & $\nabla$ & $\nabla \nabla$ & $\nabla$ & $\nabla$ & $\nabla$ & $\nabla$ & $\nabla$ & $\nabla$ & $\nabla$ & $\nabla$ & $\nabla$ & $\nabla \nabla$ & $\nabla \nabla$ & $\nabla$ & $\nabla$ & $\nabla$ & $\nabla$ & 0 & 0 & & 00 & 0 & $\Delta$ & $\Delta \Delta$ & \\
\hline Russische Föd. & 460 & $(4.7)$ & $\nabla$ & $\nabla$ & $\nabla$ & $\nabla$ & $\nabla$ & $\nabla \nabla$ & $\nabla$ & $\nabla$ & $\nabla$ & $\nabla$ & $\nabla$ & $\nabla$ & $\nabla$ & $\nabla$ & $\nabla$ & $\nabla \nabla$ & $\nabla \nabla$ & $\nabla$ & $\nabla$ & $\nabla$ & $\nabla$ & 0 & 0 & 0 & 0 & 0 & $\Delta$ & $\Delta$ & \\
\hline Lettland & 460 & $(5.6)$ & $\nabla$ & $\nabla$ & $\nabla$ & $\nabla$ & $\nabla$ & $\nabla \nabla$ & $\nabla$ & $\nabla$ & $\nabla$ & $\nabla$ & $\nabla$ & $\nabla$ & $\nabla$ & $\nabla$ & $\nabla$ & $\nabla \nabla$ & $\nabla \nabla$ & $\nabla$ & $\nabla$ & 0 & $v$ & 0 & 0 & & $O L$ & 10 & 0 & $\Delta \Delta$ & \\
\hline Portugal & 459 & $(4.0)$ & $\nabla$ & $\nabla$ & $\nabla$ & $\nabla$ & $\nabla$ & $\nabla \nabla$ & $\nabla$ & $\nabla$ & $\nabla$ & $\nabla$ & $\nabla$ & $\nabla$ & $\nabla$ & $\nabla$ & $\nabla$ & $\nabla \nabla$ & $\nabla \nabla$ & $\nabla$ & $\nabla$ & $\nabla$ & $\nabla$ & $\nabla$ & 0 & $O c$ & 00 & & $\Delta$ & $\Delta$ & \\
\hline Luxemburg & 443 & $(2.3)$ & $\nabla$ & $\nabla$ & $\nabla$ & $\nabla$ & $\nabla$ & $\nabla \nabla$ & $\nabla$ & $\nabla$ & $\nabla$ & $\nabla$ & $\nabla$ & $\nabla$ & $\nabla$ & $\nabla$ & $\nabla$ & $\nabla \nabla$ & $\nabla \nabla$ & $\nabla$ & $\nabla$ & $\nabla$ & $v$ & $\nabla$ & $\nabla$ & $\nabla$ & $\nabla \circ$ & $\nabla$ & & $\Delta \Delta$ & \\
\hline Mexiko & 422 & $(3.2)$ & $\nabla$ & $\nabla$ & $\nabla$ & $\nabla$ & $\nabla$ & $\nabla \nabla$ & $\nabla$ & $\nabla$ & $\nabla$ & $\nabla$ & $\nabla$ & $\nabla$ & $\nabla$ & $\nabla$ & $\nabla$ & $\nabla \nabla$ & $\nabla \nabla$ & $\nabla$ & $\nabla$ & $\nabla$ & V & $\nabla$ & $\nabla$ & $\nabla$ & $\nabla \nabla$ & $\nabla$ & $\nabla$ & & $\Delta$ \\
\hline Brasilien & 375 & $(3.3)$ & $\nabla$ & $\nabla$ & $\nabla$ & $\nabla$ & $\nabla$ & $\nabla \nabla$ & $\nabla$ & $\nabla$ & $\nabla$ & $\nabla$ & $\nabla$ & $\nabla$ & $\nabla$ & $\nabla$ & $\nabla$ & $\nabla \nabla$ & $\nabla \nabla$ & $\nabla$ & $\nabla$ & $\nabla$ & & $\nabla$ & 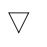 & $\nabla$ & $\nabla \nabla$ & $\nabla$ & $\nabla$ & $\nabla$ & \\
\hline
\end{tabular}

\begin{tabular}{lllllllllllllllllllllllllllll|l|l|l} 
Oberer Rang* & 1 & 1 & 3 & 3 & 4 & 4 & 4 & 8 & 9 & 9 & 10 & 13 & 13 & 11 & 13 & 14 & 13 & 13 & 16 & 19 & 19 & 21 & 22 & 20 & 25 & 26 & 25 & 26 & 30 & 31 & 32
\end{tabular}

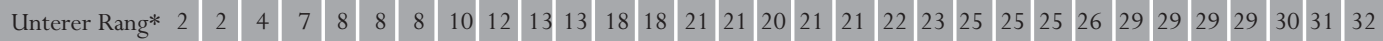

* Hinweis: Da die Daten auf Stichprobenerhebungen basieren, ist es nicht möglich, den genauen Rangplatz eines Landes zu bestimmen. Es können jedoch der jeweils obere und untere Rangplatz angegeben werden, zwischen denen das Land mit seinen mittleren Schülerleistungen mit 95\%-igerWahrscheinlichkeit liegt.

Erläuterungen

Zum Vergleich der Schülerleistungen eines Landes mit denen der Länder im Tabellenkopf ist die Zeile des betreffenden Landes zu lesen. Die Symbole zeigen, ob die mittleren Schülerleistungen des Landes in der jeweiligen Zeile statistisch signifikant über oder unter denen des Vergleichslandes liegen oder ob zwischen den mittleren Schülerleistungen beider Länder kein statistisch signifikanter Unterschied besteht.

Anmerkung: Anordnung der Länder in absteigender Reihenfolge der mittleren Schülerleistungen auf der PISA-Skala für naturwissenschaftliche Grundbildung. Aufgrund der geringen Beteiligungsquote sind die Niederlande nicht enthalten. Ausgehend von einem zu vernachlässigenden bis geringfügigen statistischen Fehler aufgrund der Nichtbeteiligung ist zu erwarten, dass die Niederlande mit 95\%-iger Wahrscheinlichkeit zwischen Rangplatz 3 und 14 liegen würden.

Quelle: OECD PISA-Datenbank, 2001. Hinweise zur Methodik s. Anhang 3 (www.oecd.org/els/education/eag2002) und www.pisa.oecd.org.

BILDUNG AUF EINEN BLICK (C) OECD 2002
A Mittlere Schülerleistungen statistisch signifikant höher als im Vergleichsland

Kein statistisch signifikanter Unterschied gegenüber dem Vergleichsland $\nabla$ Mittlere Schülerleistungen signifikant niedriger als im Vergleichsland

Statistisch signifikant über dem OECD-Durchschnitt Kein statistisch signifikanter Unterschied zum OECD-Durchschnitt Statistisch signifikant unter dem OECD-Durchschnitt 


\section{$\mathbf{A}_{6}$}

Es gibt großse

Unterschiede zwischen

den Ländermittelwerten,

wobei die Unterschiede

der Schülerleistungen

innerhalb der einzelnen

Länder jedoch wesentlich

größser sind.

Disparitäten bei den

Leistungen führen

nicht zu einem

insgesamt höheren

Leistungsniveau.
Lesekompetenz liegen ihre Schüler jedoch signifikant über dem OECDDurchschnitt. Umgekehrt erbringen Schüler in Belgien, Frankreich, Island und der Schweiz bei der mathematischen Grundbildung Leistungen, die signifikant über dem OECD-Durchschnitt liegen, während sich ihre Leistungen bei der naturwissenschaftlichen Grundbildung nicht signifikant vom OECDDurchschnitt unterscheiden. Schüler in Dänemark und Liechtenstein liegen zwar bei der mathematischen Grundbildung über dem OECD-Durchschnitt, bei der naturwissenschaftlichen Grundbildung jedoch unter dem OECDDurchschnitt.

Auch wenn die Durchschnittsergebnisse der einzelnen Länder stark voneinander abweichen, sind die Leistungsunterschiede zwischen den Schülern innerhalb der einzelnen Länder um ein Vielfaches größer. In den Tabellen A6.1 und A6.2 sind die Schülerleistungen beim 5., 25., 75., und 95. Perzentil für jedes einzelne Land angegeben. Die Verteilung der Schülerleistungen auf der PISA-Skala für mathematische Grundbildung für Belgien, Deutschland, Griechenland, Neuseeland, Polen, die Schweiz, Ungarn und die Vereinigten Staaten, zeigen eine relativ große Spannweite zwischen dem 75. und 25. Perzentil - zwischen 135 und 149 Punkten. Finnland, Irland, Island, Japan und Korea, weisen mit maximal 113 Punkten zwischen dem 75. und 25. Perzentil vergleichsweise kleinere Unterschiede auf.

Bei der wissenschaftlichen Grundbildung in Belgien, Dänemark, Deutschland, Frankreich, Neuseeland, der Schweiz, Ungarn und den Vereinigten Staaten bestehen relativ große Unterschiede zwischen den Schülerleistungen beim 75 . und 25. Perzentil, jeweils zwischen 141 und 154 Punkten, während in Finnland, Japan, Korea und Mexiko die Unterschiede zwischen den unterschiedlichen Schülergruppen relativ gering sind, mit Unterschieden von weniger als 118 Punkten.

Es ist sehr hilfreich, die Bandbreite der Leistungen mit den Durchschnittsergebnissen in Relation zu setzen. Ein solcher Vergleich zeigt, dass große Disparitäten der Schülerleistungen nicht zu einem insgesamt höheren Leistungsniveau führen. Augenfällig ist ganz im Gegenteil, dass die Durchschnittsergebnisse in fünf der Länder mit der geringsten Varianz zwischen dem 75. und 25. Perzentil auf der PISA-Skala für mathematische Grundbildung, nämlich Finnland, Island, Japan, Kanada und Korea, alle signifikant über dem OECD-Durchschnitt liegen (Tabelle A6.1). Außerdem gehören vier dieser Länder, nämlich Finnland, Japan, Kanada und Korea zu den sechs leistungsstärksten OECD-Ländern in mathematischer Grundbildung. Ähnlich sieht es bei der naturwissenschaftlichen Grundbildung aus. Auch hier gehören Finnland, Japan, Kanada und Korea sowohl zu den sechs Ländern mit dem geringsten Unterschied zwischen dem 75. und 25. Perzentil als auch zu den sechs leistungsstärksten Ländern. 
Umgekehrt liegen die Ergebnisse der Länder mit den größten Unterschieden innerhalb des Landes in der Regel unterhalb des OECD-Mittelwertes. So liegen beispielsweise bei der mathematischen Grundbildung von den sechs Ländern mit dem größten Unterschied zwischen dem 75. und dem 25. Perzentil (Belgien, Deutschland Griechenland, Polen, Ungarn und die Vereinigten Staaten) nur zwei (Belgien und die Vereinigten Staaten) nicht signifikant unter dem OECD-Durchschnitt.

\section{Definitionen und angewandte Methodik}

Die für diesen Indikator untersuchte Zielpopulation waren 15-jährige Schüler. In der Praxis bezieht sich dies auf Schüler, die zu Beginn der Testperiode im Alter von 15 Jahren und 3 (abgeschlossenen) Monaten bis zu 16 Jahren und 2 (abgeschlossenen) Monaten waren und eine Bildungseinrichtung besuchten, ungeachtet der Klassenstufe und der Art der von ihnen besuchten Bildungseinrichtung und unabhängig davon, ob es sich um eine Ganztags- oder Halbtagsschule handelte.

Um die Interpretation der von den Schülern erzielten Punktwerte in der PISA-Studie zu erleichtern, wurden die Skalen für mathematische und naturwissenschaftliche Grundbildung so konstruiert, dass der Mittelwert bei 500 Punkten liegt und die Standardabweichung bei 100, wobei die Daten gewichtet wurden, so dass jedes Land gleichermaßen hierzu beitrug.

Hinweise zu Standardfehlern, Signifikanztests und multiplen Vergleichen s. Anhang 3 unter www.oecd.org/els/education/eag2002.
Die erzielten

Punktzahlen basieren

auf Leistungsmessungen,

die Teil der im Jahr

2000 von der OECD

durchgeführten

Internationalen

Schulleistungsstudie

PISA waren. 
Tabelle A6.1.

Verteilung der Leistung 15-jähriger Schüler auf der PISA-Skala für mathematische Grundbildung (2000) Leistung 15-jähriger Schüler auf der PISA-Skala für mathematische Grundbildung, nach Perzentilen

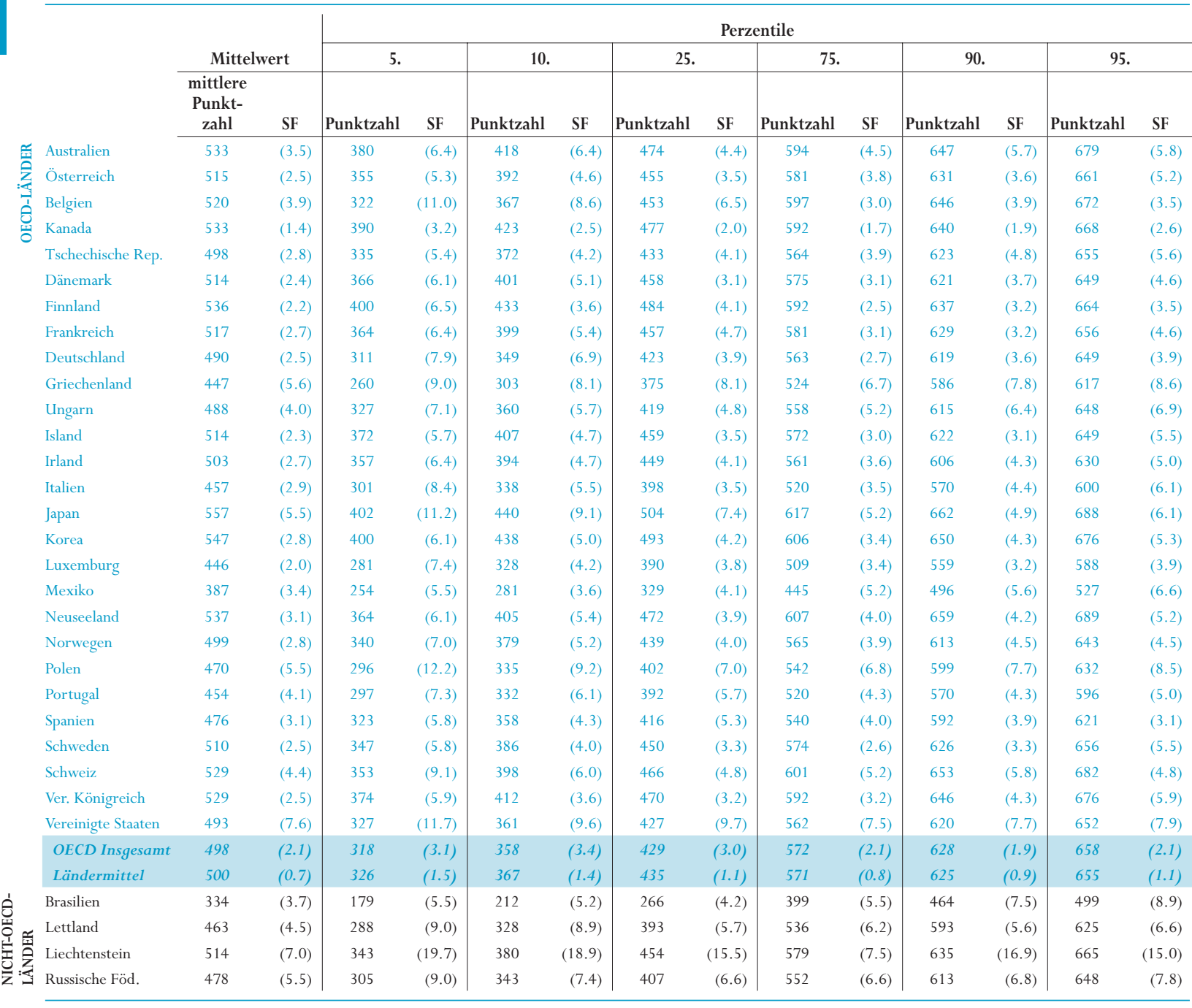

Quelle: OECD PISA-Datenbank, 2001. Hinweise zur Methodik s. Anhang 3 (www.oecd.org/els/education/eag2002) und www.pisa.oecd.org. 
Tabelle A6.2.

Verteilung der Leistung 15-jähriger Schüler auf der PISA-Skala für naturwissenschaftliche Grundbildung (2000) Leistung 15-jähriger Schüler auf der PISA-Skala für naturwissenschaftliche Grundbildung, nach Perzentilen

\begin{tabular}{|c|c|c|c|c|c|c|c|c|c|c|c|c|c|c|}
\hline & & & \multicolumn{12}{|c|}{ Perzentile } \\
\hline & \multicolumn{2}{|c|}{ Mittelwert } & \multicolumn{2}{|c|}{5.} & \multicolumn{2}{|c|}{10.} & \multicolumn{2}{|c|}{25.} & \multicolumn{2}{|c|}{75.} & \multicolumn{2}{|c|}{90.} & \multicolumn{2}{|c|}{95.} \\
\hline & $\begin{array}{c}\text { mittlere } \\
\text { Punkt- } \\
\text { zahl } \\
\end{array}$ & SF & $\begin{array}{c}\text { Punkt- } \\
\text { zahl }\end{array}$ & SF & $\begin{array}{c}\text { Punkt- } \\
\text { zahl }\end{array}$ & SF & $\begin{array}{c}\text { Punkt- } \\
\text { zahl }\end{array}$ & SF & $\begin{array}{c}\text { Punkt- } \\
\text { zahl }\end{array}$ & SF & $\begin{array}{c}\text { Punkt- } \\
\text { zahl }\end{array}$ & SF & $\begin{array}{c}\text { Punkt- } \\
\text { zahl }\end{array}$ & SF \\
\hline Australien & 528 & $(3.5)$ & 368 & $(5.1)$ & 402 & $(4.7)$ & 463 & $(4.6)$ & 596 & $(4.8)$ & 646 & $(5.1)$ & 675 & (4.8) \\
\hline Österreich & 519 & $(2.6)$ & 363 & $(5.7)$ & 398 & $(4.0)$ & 456 & $(3.8)$ & 584 & $(3.5)$ & 633 & $(4.1)$ & 659 & $(4.3)$ \\
\hline Belgien & 496 & $(4.3)$ & 292 & $(13.5)$ & 346 & $(10.2)$ & 424 & $(6.6)$ & 577 & $(3.5)$ & 630 & $(2.6)$ & 656 & $(3.0)$ \\
\hline Kanada & 529 & $(1.6)$ & 380 & $(3.7)$ & 412 & $(3.4)$ & 469 & $(2.2)$ & 592 & $(1.8)$ & 641 & $(2.2)$ & 670 & $(3.0)$ \\
\hline Tschechische Rep. & 511 & $(2.4)$ & 355 & $(5.6)$ & 389 & $(4.0)$ & 449 & $(3.6)$ & 577 & $(3.8)$ & 632 & $(4.1)$ & 663 & $(4.9)$ \\
\hline Dänemark & 481 & $(2.8)$ & 310 & $(6.0)$ & 347 & $(5.3)$ & 410 & $(4.8)$ & 554 & $(3.5)$ & 613 & $(4.4)$ & 645 & $(4.7)$ \\
\hline Finnland & 538 & $(2.5)$ & 391 & $(5.2)$ & 425 & $(4.2)$ & 481 & $(3.5)$ & 598 & $(3.0)$ & 645 & $(4.3)$ & 674 & $(4.3)$ \\
\hline Frankreich & 500 & $(3.2)$ & 329 & $(6.1)$ & 363 & $(5.4)$ & 429 & $(5.3)$ & 575 & $(4.0)$ & 631 & $(4.2)$ & 663 & $(4.9)$ \\
\hline Deutschland & 487 & $(2.4)$ & 314 & $(9.5)$ & 350 & $(6.0)$ & 417 & $(4.9)$ & 560 & $(3.3)$ & 618 & (3.5) & 649 & $(4.7)$ \\
\hline Griechenland & 461 & $(4.9)$ & 300 & $(9.3)$ & 334 & $(8.3)$ & 393 & $(7.0)$ & 530 & $(5.3)$ & 585 & $(5.3)$ & 616 & $(5.8)$ \\
\hline Ungarn & 496 & $(4.2)$ & 328 & $(7.5)$ & 361 & $(4.9)$ & 423 & $(5.5)$ & 570 & $(4.8)$ & 629 & $(5.1)$ & 659 & $(8.5)$ \\
\hline Island & 496 & $(2.2)$ & 351 & $(7.0)$ & 381 & $(4.3)$ & 436 & $(3.7)$ & 558 & (3.1) & 607 & $(4.1)$ & 635 & (4.8) \\
\hline Irland & 513 & $(3.2)$ & 361 & $(6.5)$ & 394 & $(5.7)$ & 450 & $(4.4)$ & 578 & $(3.4)$ & 630 & $(4.6)$ & 661 & (5.4) \\
\hline Italien & 478 & $(3.1)$ & 315 & (7.1) & 349 & $(6.2)$ & 411 & $(4.4)$ & 547 & $(3.5)$ & 602 & $(4.0)$ & 633 & $(4.4)$ \\
\hline Japan & 550 & $(5.5)$ & 391 & (11.3) & 430 & $(9.9)$ & 495 & $(7.2)$ & 612 & $(5.0)$ & 659 & $(4.7)$ & 688 & (5.7) \\
\hline Korea & 552 & $(2.7)$ & 411 & (5.3) & 442 & $(5.3)$ & 499 & $(4.0)$ & 610 & $(3.4)$ & 652 & (3.9) & 674 & (5.7) \\
\hline Luxemburg & 443 & $(2.3)$ & 278 & $(7.2)$ & 320 & $(6.8)$ & 382 & $(3.4)$ & 510 & $(2.8)$ & 563 & $(4.4)$ & 593 & $(4.0)$ \\
\hline Mexiko & 422 & $(3.2)$ & 303 & $(4.8)$ & 325 & $(4.6)$ & 368 & $(3.1)$ & 472 & $(4.7)$ & 525 & $(5.5)$ & 554 & (7.0) \\
\hline Neuseeland & 528 & $(2.4)$ & 357 & $(5.6)$ & 392 & $(5.2)$ & 459 & $(3.8)$ & 600 & $(3.4)$ & 653 & $(5.0)$ & 683 & (5.1) \\
\hline Norwegen & 500 & $(2.8)$ & 338 & $(7.3)$ & 377 & $(6.6)$ & 437 & $(4.0)$ & 569 & $(3.5)$ & 619 & (3.9) & 649 & $(6.2)$ \\
\hline Polen & 483 & (5.1) & 326 & $(9.2)$ & 359 & $(5.8)$ & 415 & $(5.5)$ & 553 & $(7.3)$ & 610 & $(7.6)$ & 639 & (7.5) \\
\hline Portugal & 459 & $(4.0)$ & 317 & $(5.0)$ & 343 & $(5.1)$ & 397 & $(5.2)$ & 521 & $(4.7)$ & 575 & $(5.0)$ & 604 & (5.3) \\
\hline Spanien & 491 & $(3.0)$ & 333 & $(5.1)$ & 367 & $(4.3)$ & 425 & $(4.4)$ & 558 & $(3.5)$ & 613 & (3.9) & 643 & (5.5) \\
\hline Schweden & 512 & $(2.5)$ & 357 & $(5.7)$ & 390 & $(4.6)$ & 446 & $(4.1)$ & 578 & $(3.0)$ & 630 & $(3.4)$ & 660 & $(4.5)$ \\
\hline Schweiz & 496 & $(4.4)$ & 332 & $(5.8)$ & 366 & $(5.4)$ & 427 & $(5.1)$ & 567 & $(6.4)$ & 626 & $(6.4)$ & 656 & $(9.0)$ \\
\hline Ver. Königreich & 532 & $(2.7)$ & 366 & $(6.8)$ & 401 & $(6.0)$ & 466 & $(3.8)$ & 602 & $(3.9)$ & 656 & $(4.7)$ & 687 & $(5.0)$ \\
\hline Vereinigte Staaten & 499 & $(7.3)$ & 330 & $(11.7)$ & 368 & $(10.0)$ & 430 & $(9.6)$ & 571 & $(8.0)$ & 628 & $(7.0)$ & 658 & $(8.4)$ \\
\hline OECD Insgesamt & 502 & $(2.0)$ & 332 & (3.3) & 368 & (3.1) & 431 & $(2.8)$ & 576 & (2.1) & 631 & (1.9) & 662 & (2.3) \\
\hline Ländermittel & 500 & $(0.7)$ & 332 & (1.5) & 368 & (1.0) & 431 & (1.0) & 572 & $(0.8)$ & 627 & $(0.8)$ & 657 & (1.2) \\
\hline Brasilien & 375 & $(3.3)$ & 230 & $(5.5)$ & 262 & $(5.9)$ & 315 & $(3.7)$ & 432 & $(4.9)$ & 492 & $(7.8)$ & 531 & $(8.2)$ \\
\hline Lettland & 460 & $(5.6)$ & 299 & $(10.1)$ & 334 & $(8.8)$ & 393 & $(7.7)$ & 528 & $(5.7)$ & 585 & $(7.2)$ & 620 & $(8.0)$ \\
\hline Liechtenstein & 476 & $(7.1)$ & 314 & $(23.5)$ & 357 & $(20.0)$ & 409 & $(12.3)$ & 543 & $(12.7)$ & 595 & $(12.4)$ & 629 & $(24.0)$ \\
\hline Russische Föd. & 460 & $(4.7)$ & 298 & $(6.5)$ & 333 & $(5.4)$ & 392 & $(6.2)$ & 529 & $(5.8)$ & 591 & $(5.9)$ & 625 & $(5.7)$ \\
\hline
\end{tabular}

Quelle: OECD PISA-Datenbank, 2001. Hinweise zur Methodik s. Anhang 3 (www.oecd.org/els/education/eag2002) und www.pisa.oecd.org. 


\section{LEISTUNGSUNTERSCHIEDE ZWISCHEN DEN SCHULEN}

- Im Durchschnitt tragen bei den 15-Jährigen die Leistungsunterschiede zwischen den Schulen zu 36 Prozent zu der durchschnittlichen Leistungsvarianz der Schülerleistungen innerhalb der OECD bei, dieser Anteil variiert jedoch von 10 Prozent in Finnland und Schweden bis zu mehr als 50 Prozent in Belgien, Deutschland, Griechenland, Italien, Österreich, Polen, der Tschechischen Republik und Ungarn.

- Ein Teil der Varianz zwischen den Schulen lässt sich auf geographische oder institutionelle Faktoren zurückführen, oder auf die Auswahl von Schülern nach ihren Fähigkeiten. Zu diesen Faktoren kommt oft auch noch der soziale Hintergrund dazu, insbesondere in Ländern mit einem differenzierten Schulsystem, da die Schülerleistungen nicht nur vom eigenen familiären Hintergrund, sondern auch - und zwar in größerem Maße - vom Hintergrund der Mitschüler an der Schule beeinflusst werden.

- Eine hohe Gesamtvarianz kann auf erhebliche Leistungsunterschiede innerhalb der Schulen zurückzuführen sein, auf große Unterschiede zwischen den Schulen oder auf eine Kombination dieser beiden Faktoren.

- In Bildungssystemen, die eine Einteilung in verschiedene Schultypen vorsehen, treffen Schüler mit ähnlichen sozio-ökonomischen Merkmalen häufiger in denselben Schulen zusammen als in Systemen, in denen sich der Lehrplan der verschiedenen Schulen nicht wesentlich unterscheidet. In Belgien, Deutschland, Italien, den Niederlanden, Österreich und der Tschechischen Republik beispielsweise wird der mit der Verteilung der Schüler auf unterschiedliche Schultypen zusammenhängende Leistungsabstand zwischen den Schulen durch Differenzen in Bezug auf den sozialen und familiären Hintergrund zusätzlich verstärkt. 


\section{Abbildung A7.1.}

Leistungsunterschiede zwischen den Schulen und innerhalb der Schulen auf der PISA-Skala für Lesekompetenz (2000)

Ausgedrückt als Prozentsatz der durchschnittlichen Gesamtvarianz der Schülerleistungen in den OECD-Ländern

Varianz zwischen Schulen

- Varianz zwischen Schulen, erklärt durch

Varianz innerhalb von Schulen geographische und institutionelle Faktoren

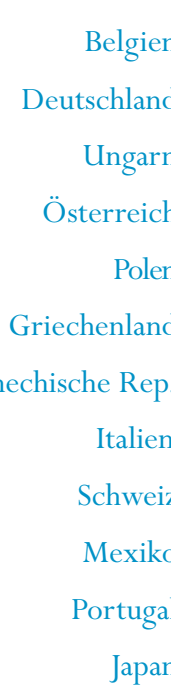

Vereinigte Staaten

Luxemburg

Ver. Königreich

Australien

Neuseeland

Korea

Dänemark

Irland

Kanada

Spanien

Norwegen

Finnland

Schweden

Island
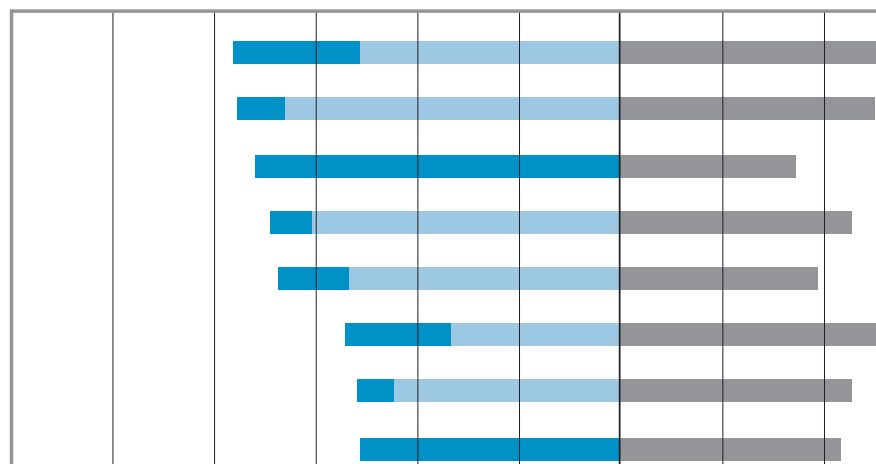

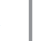




\section{$\mathbf{A}_{7}$}

Zu den bei PISA

beobachteten

Leistungsunterschieden tragen viele Faktoren

bei...

. und die Organisation

des Bildungssystems

kann hierbei eine sehr wichtige Rolle spielen.

Um dies genauer darzustellen, werden in diesem Indikator die Unterschiede zwischen den Schulen untersucht.

Abbildung A 7.1 vergleicht die innerhalb der einzelnen Länder beobachtete Varianz der Schülerleistungen, ...

\section{Politischer Hintergrund}

Die Indikatoren A 5 und A6 haben gezeigt, dass in den meisten Ländern innerhalb der jeweiligen Bildungssysteme erhebliche Leistungsunterschiede bestehen. Diese Unterschiede können durch den speziellen Hintergrund der einzelnen Schulen und der sie besuchenden Schüler, den Umfang der den Schulen zur Verfügung stehenden personellen und finanziellen Mittel, unterschiedliche Lehrpläne, vorhandene Selektionsmechanismen und durch die Art und Weise, wie der Unterricht organisiert und durchgeführt wird, bedingt sein.

Einige Länder haben nicht-selektive Schulsysteme, die allen Schülern die gleichen Lernmöglichkeiten bieten sollen und allen Schulen die Möglichkeit geben, die gesamte Palette der Schülerleistungen abzudecken. Andere Länder versuchen der Verschiedenheit der Schüler gerecht zu werden, indem sie sie bewusst (durch Selektion zwischen oder innerhalb der Schulen) in Gruppen mit ähnlichem Leistungsniveau einteilen, mit dem Ziel, sie entsprechend ihrem besonderen Bildungsbedarf bestmöglich zu fördern. In wieder anderen Ländern werden diese beiden Ansätze kombiniert. Auch in Gesamtschulsystemen können große Unterschiede zwischen den einzelnen Schulen bestehen, bedingt z.B. durch die sozio-ökonomischen und kulturellen Merkmale des von ihnen versorgten Einzugsbereichs oder durch geographische Unterschiede (zwischen einzelnen Regionen, Provinzen oder Bundesstaaten in föderativen Regierungsformen oder zwischen ländlichen und städtischen Gebieten). Zudem können auch zwischen den einzelnen Schulen beträchtliche Unterschiede bestehen, die sich nicht ohne weiteres erklären lassen und die teilweise auf Differenzen in der Qualität und der Effektivität des von ihnen angebotenen Unterrichts zurückzuführen sein könnten.

Um die Auswirkungen solch unterschiedlicher Strategien und Praktiken $\mathrm{zu}$ untersuchen, analysiert dieser Indikator die Leistungsunterschiede im Bereich Lesekompetenz zwischen den Schulen. Die Ergebnisse im Bereich der mathematischen und naturwissenschaftlichen Grundbildung sind im Großen und Ganzen ähnlich und werden daher in diesem Indikator nicht aufgeführt.

\section{Ergebnisse und Erläuterungen}

Abbildung A7.1 und Tabelle A 7.1 zeigen, in welchem Ausmaß die in den einzelnen Ländern ermittelten Leistungsunterschiede auf die verschiedenen Faktoren zurückzuführen sind. Die Länge der Balken verdeutlicht die beobachtete Varianz der Schülerleistungen auf der PISA-Skala für Lesekompetenz. Es ist zu beachten, dass die Werte als Prozentsätze der durchschnittlichen Gesamtvarianz der Schülerleistungen zwischen den OECD-Ländern auf der PISA-Skala für Lesekompetenz ausgedrückt sind. Wenn die Summe zweier Balken für ein Land größer als 100 ist, bedeutet dies, dass die Varianz der Schülerleistungen in dem entsprechenden Land größer ist als in einem typischen OECD-Land. Werte unter 100 bedeuten dementsprechend, dass die Varianz der Schülerleistungen kleiner ist als im Durchschnitt. 
Die Balken der einzelnen Länder sind so angeordnet, dass die Varianz zwischen den Schulen durch die Länge des Balkenabschnitts links der vertikalen Linie in der Mitte der Abbildung und die Varianz innerhalb der Schulen durch die Länge des Balkenabschnitts rechts dieser Linie dargestellt ist. Längere Balkensegmente links der Vertikalen bedeuten, dass die mittleren Ergebnisse der Schulen weiter auseinander liegen. Längere Balkenabschnitte rechts der Vertikalen bedeuten größere Leistungsunterschiede zwischen den Schülern derselben Schule.

Wie aus Abbildung A7.1 ersichtlich, ist in den meisten Ländern ein großer Teil der Varianz der Schülerleistungen auf Unterschiede zwischen den Schulen zurückzuführen. In den 26 in diesen Vergleich einbezogenen OECD-Ländern lassen sich 36 Prozent der im OECD-Durchschnitt beobachteten Varianz der Schülerleistungen auf Unterschiede zwischen den Schulen zurückführen. In Belgien, Deutschland, Griechenland, Italien, Österreich, Polen, der Tschechischen Republik und Ungarn entfallen sogar über 50 Prozent der Gesamtvarianz auf Unterschiede zwischen den Schulen (s. Spalte 3 in Tabelle A7.1). Wo große Leistungsdifferenzen zwischen den Schulen bestehen, die Leistungsunterschiede zwischen den Schülern der einzelnen Schulen aber vergleichsweise gering sind, werden die Schüler in der Regel Schulen besuchen, in denen das Niveau aller Schüler in etwa gleich ist. Diese Aufteilung kann auf bewusste Entscheidungen der Familien in Bezug auf die Schuloder Wohnortwahl, die Aufnahmebestimmungen der Schulen, die von den Bildungsbehörden verfolgte Politik hinsichtlich der Verteilung der Schüler oder aber auf die Gestaltung der Lehrpläne zurückzuführen sein.

In Korea beträgt die Gesamtvarianz der Schülerleistungen auf der PISA-Skala für Lesekompetenz nur rund die Hälfte des OECD-Durchschnitts, die Varianz zwischen den Schulen beläuft sich auf nur 20 Prozent der durchschnittlichen Gesamtvarianz über alle OECD-Länder hinweg. Korea erzielt somit im Bereich der Lesekompetenz nicht nur hohe Durchschnittsleistungen mit einer geringen Gesamtvarianz der Schülerleistungen, sondern ist auch in der Lage, die Unterschiede zwischen den Durchschnittsleistungen der Schüler verschiedener Schulen niedrig zu halten. Auch in Spanien sind sowohl die Gesamtvarianz (die sich auf rund drei Viertel des OECD-Durchschnitts beläuft) als auch die Unterschiede zwischen den Schulen gering (16 Prozent der durchschnittlichen Gesamtvarianz der Schülerleistungen in den OECD-Ländern), doch liegt der spanische Mittelwert, im Gegensatz zum koreanischen, deutlich unter dem OECD-Durchschnitt.

Im Bereich Lesekompetenz sind die geringsten Leistungsdifferenzen zwischen den Schulen in Finnland, Island und Schweden zu beobachten, wo diese Unterschiede nur zwischen 7 Prozent und 11 Prozent der durchschnittlichen Gesamtvarianz der Schülerleistungen ausmachen. In diesen Ländern besteht im Großen und Ganzen kein Zusammenhang zwischen den Leistungen der Schüler und den Schulen, die sie besuchen. Die Schüler treffen dort mit großer Wahrscheinlichkeit überall auf ein ähnliches Lernumfeld, was das Spektrum
... aufgegliedert in

die Varianz zwischen

Schulen und die Varianz innerhalb von Schulen.

In manchen Ländern sind die Leistungsunterschiede sowohl zwischen als auch innerhalb der schulen gering, ... 


\section{A7}

Eine hohe

Gesamtvarianz

kann auf erhebliche

Leistungsunterschiede

innerhalb der Schulen zurückzuführen sein, ...

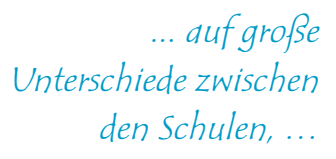

... oder aufeine

Kombination dieser

beiden Faktoren. der Fähigkeiten ihrer Mitschüler anbelangt. Es ist zu erwähnen, dass in diesen Ländern auch die Gesamtvarianz der Schülerleistungen unter dem OECDDurchschnitt liegt. Den Bildungssystemen dieser Länder gelingt es also, im Bereich der Lesekompetenz sowohl die Unterschiede zwischen den Schulen als auch die Leistungsunterschiede zwischen den Schülern insgesamt in engem Rahmen zu halten.

Australien, Neuseeland und Norwegen gehören (mit 112, 126 bzw. 116 Prozent der durchschnittlichen Gesamtvarianz der Schülerleistungen in den OECD-Ländern) zu den Ländern mit der größten Gesamtvarianz im Bereich Lesekompetenz, doch nur ein geringer Teil dieser Unterschiede (21, 20 bzw. 13 Prozent der durchschnittlichen Gesamtvarianz) geht auf Unterschiede zwischen den Schulen zurück. In diesen Ländern ist die Varianz der Schülerleistungen innerhalb der einzelnen Schulen am größten, was darauf hindeutet, dass die Schulen in diesen Ländern ganz unterschiedlichen Schülern gerecht werden müssen.

Weitere Länder mit vergleichsweise hohen Varianzen der Schülerleistungen sind Belgien, Deutschland und die Schweiz (124, 133 und 112 Prozent der durchschnittlichen Gesamtvarianz), doch resultiert in diesen Ländern ein großer Teil der Varianz (76, 75 bzw. 49 Prozent der durchschnittlichen Gesamtvarianz) aus Leistungsunterschieden zwischen den Schulen.

Die Vereinigten Staaten, ein weiteres Land mit einer vergleichsweise hohen Gesamtvarianz der Schülerleistungen (118 Prozent der durchschnittlichen Gesamtvarianz), liegen in etwa im mittleren Bereich: Im Vergleich zur durchschnittlichen Gesamtvarianz der Schülerleistungen in den OECDLändern entfallen dort 35 Prozent auf Unterschiede zwischen den Schulen.

\section{Kasten A7.1. Mögliche Faktoren der Leistungsunterschiede zwischen den Schulen}

Die Unterschiede zwischen den durchschnittlichen Leistungen der Schüler verschiedener Schulen werden durch vielerlei Faktoren beeinflusst. Hierzu gehören u.a.:

- Unterschiede zwischen verschiedenen Verwaltungseinheiten: In mehreren Ländern sind die Schulsysteme dezentral organisiert und werden beispielsweise auf Ebene von verschiedenen Sprachgemeinschaften wie in Belgien, von Provinzen und Territorien wie in Kanada, von Bundesländern wie in Deutschland, oder von Bundesstaaten wie in Australien oder den Vereinigten Staaten verwaltet. In anderen Ländern unterscheiden sich die Schulsysteme durch die Kombination verschiedener Kantone und Sprachgruppen (z.B. in der Schweiz).

- Stadt und Land: Oft unterscheiden sich die Unterrichtsformen und die Lehrpläne in städtischen und ländlichen Regionen. 
- Staatliche oder private Schulen: In vielen Ländern stehen öffentlich und privat verwaltete Schulen miteinander im Wettbewerb. In einigen Ländern haben die Privatschulen gewöhnlich strengere Aufnahmebestimmungen. Zudem können bei privat finanzierten Schulen Zugangsbarrieren für Schüler bestehen, die aus sozio-ökonomisch benachteiligten Verhältnissen kommen.

- Art der Bildungsgänge: In einigen Bildungssystemen gibt es unterschiedlich ausgerichtete Schulen, die sich in dem von ihnen angebotenen Lehrplan substantiell unterscheiden (z.B. bereiten sie die Schüler entweder für eine Ausbildung im Tertiärbereich vor oder für den direkten Eintritt in den Arbeitsmarkt.) Selbst in den Bildungssystemen, bei denen innerhalb der Schulen differenziert wird, kann es unterschiedliche Züge geben, z.B. berufsbildende und allgemeinbildende.

- Bildungsbereiche: In einigen wenigen Ländern besucht ein Teil der 15-Jährigen Schulen des Sekundarbereich II, während ein anderer Teil Schulen des Sekundarbereich I besucht, abhängig vom Geburtsmonat, der Versetzungspraxis oder wie im Falle der Schweiz vom jeweiligen Kanton. In anderen Ländern gibt es innerhalb einer Schule Bildungsgänge verschiedener Bildungsbereiche. Das bedeutet, dass die Varianz der Schülerleistungen, die auf Unterschiede im Lehrplan zwischen Sekundarbereich I und II zurückzuführen sind, im ersteren Fall in der Varianz der Leistungen zwischen den Schulen enthalten ist, während sie im letzteren Fall in der Varianz der Schülerleistungen innerhalb der Schulen enthalten ist.

- Sozio-ökonomischer Hintergrund der Schüler: Die sozio-ökonomischen Merkmale der Einzugsbereiche der Schulen sind häufig unterschiedlich, wenngleich sich das Ausmaß dieser Unterschiede sehr von Land zu Land unterscheiden kann. Der unterschiedliche sozio-ökonomische Hintergrund der Schüler einer Schule kann Einfluss auf die Schülerleistungen haben.

Woher kommt die Varianz der Schülerleistungen im Bereich Lesekompetenz? Die Antwort kann je nach Land unterschiedlich ausfallen (s. auch Kasten A7.1). Viele teilnehmende Länder haben Angaben zu den in PISA erfassten geographischen oder institutionellen Aspekten ihrer Bildungssysteme gemacht, denen sie im Hinblick auf die Leistungsunterschiede zwischen den Schulen die größte Bedeutung beimessen. Der Anteil der Varianz der Schülerleistungen, der sich aus diesen Variablen erklären lässt, ist in Abbildung A7.1 im linken Teil der Balken dargestellt:

- In Australien reduzieren sich die Leistungsdifferenzen zwischen den Schulen nach Berücksichtigung der zwischen den verschiedenen Bundesstaaten und Territorien bestehenden Unterschiede von 21 auf 19 Prozent der durchschnittlichen Gesamtvarianz der Schülerleistungen.

- In Österreich reduzieren sich die Leistungsdifferenzen zwischen den Schulen nach Berücksichtigung der Aufteilung der Schüler auf die unterschiedlichen Züge sechs verschiedener Schultypen von 68 auf 8 Prozent der durchschnittlichen Gesamtvarianz. In Belgien verringern sich die Leistungsdifferenzen zwischen den Schulen nach Berücksichtigung
Ein Teil der Unterschiede zwischen den Schulen ist durch geographische oder institutionelle Faktoren oder auch die Auswahl der Schülerschaft entsprechend ihrer Fähigkeiten bedingt, ... 
der Unterschiede zwischen den Sprachgruppen sowie den verschiedenen Schultypen von 76 auf 25 Prozent. Nach Berücksichtigung der Unterschiede zwischen den verschiedenen Schultypen und Bildungsgängen reduzieren sich die Leistungsdifferenzen zwischen den Schulen in Deutschland von 75 auf 10 Prozent, in Ungarn von 71 auf 19 Prozent, in Polen von 67 auf 14 Prozent und in Korea von 20 auf 9 Prozent der Varianz der Schülerleistungen im OECD-Durchschnitt.

- Die Berücksichtigung der Unterschiede zwischen allgemein- und berufsbildenden Schulen sowie zwischen Bildungsgängen des Sekundarbereich I und II hat in der Tschechischen Republik eine Verringerung der Varianz der Schülerleistungen zwischen den Schulen von 52 auf 7 Prozent der Gesamtvarianz und in Griechenland von 54 auf 21 Prozent zur Folge.

- In Irland reduzieren sich die Leistungsdifferenzen zwischen den Schulen nach Berücksichtigung der unterschiedlichen Schultypen, der Unterschiede zwischen regulären Schulen und sogenannten ,benachteiligten' Schulen sowie der Unterscheidung zwischen städtischen und ländlichen Gebieten von 17 auf 7 Prozent der Gesamtvarianz.

- In Italien verringern sich die Differenzen zwischen den Schulen nach Berücksichtigung der Unterschiede zwischen den Bildungsbereichen und den Schultypen (Licei gegenüber berufsbildenden Schulen und istituti tecnichi) von 51 auf 23 Prozent und in Mexiko von 43 auf 16 Prozent der Gesamtvarianz.

- In Kanada reduziert sich die Leistungsdifferenz zwischen den Schulen nach Berücksichtigung der Unterschiede zwischen den Provinzen von 17 auf 16 Prozent der Gesamtvarianz.

- In Island hat die Berücksichtigung der Schulgröße und des Urbanisierungsgrads eine Verringerung der Leistungsdifferenzen zwischen den Schulen von 7 auf 6 Prozent der Gesamtvarianz zur Folge.

- In Neuseeland reduzieren sich die Leistungsdifferenzen zwischen den Schulen nach Berücksichtigung des sozialen Hintergrunds der Schüler (Einstufung nach dem sozio-ökonomischen Index sowie Anteil der MaoriSchüler und Schüler aus anderen Pazifikregionen) von 20 auf 7 Prozent der Gesamtvarianz.

- In Norwegen schrumpfen die Leistungsunterschiede zwischen den Schulen nach Abzug der auf Migrantenkinder entfallenden Leistungsdifferenz von 13 auf 12 Prozent und in Schweden von 9 Prozent auf 6 Prozent der Gesamtvarianz.

- In Spanien verringern sich die Leistungsdifferenzen zwischen den Schulen nach Berücksichtigung der Unterschiede zwischen öffentlich und privat verwalteten Schulen von 16 auf 10 Prozent der Gesamtvarianz. 
- In der Schweiz reduzieren sich die Leistungsunterschiede zwischen den Schulen nach Berücksichtigung der Differenzen zwischen den verschiedenen Bildungsgängen, den Bildungsbereichen und den Sprachregionen, in denen die Schulen angesiedelt sind, von 49 auf 27 Prozent der Gesamtvarianz.

- Im Vereinigten Königreich schrumpft der Abstand zwischen den Schulen nach Berücksichtigung der Unterschiede zwischen den von nachgeordneten Gebietskörperschaften oder von anderen Organen - beispielsweise eigenständigen Einrichtungen oder kirchlichen Stiftungen - verwalteten Schulen, zwischen den gemischten Schulen und den getrennten Mädchenund Jungenschulen sowie zwischen den verschiedenen Regionen von 22 auf 15 Prozent der Gesamtvarianz.

Die vorliegenden Daten legen insgesamt auch den Schluss nahe, dass in Bildungssystemen, die eine Einteilung in verschiedene Schultypen vorsehen, Schüler mit ähnlichen sozio-ökonomischen Merkmalen häufiger in denselben Schulen zusammentreffen als in Systemen, in denen sich der Lehrplan der verschiedenen Schulen nicht wesentlich unterscheidet. In Belgien, Deutschland, Italien, den Niederlanden, Österreich und der Tschechischen Republik beispielsweise wird der mit der Verteilung der Schüler auf unterschiedliche Schultypen zusammenhängende Leistungsabstand zwischen den Schulen durch Differenzen in Bezug auf den sozialen und familiären Hintergrund zusätzlich verstärkt. Dies könnte die Folge eines Selektionsbzw. Autoselektionsphänomens sein: Wenn der Bildungsmarkt eine gewisse Differenzierung vorsieht, besteht die Tendenz, dass Schüler aus unteren sozialen Schichten in weniger anspruchsvolle Bildungsgänge gelenkt werden bzw. sich selbst für solche Bildungsgänge entscheiden oder beschließen, nicht an den im Bildungssystem vorgesehenen Auswahlverfahren teilzunehmen.

Die ausführlichere Analyse in Leben für das Lernen, Erste Ergebnisse von PISA 2000 (OECD, 2001) deutet darauf hin, dass der soziale Hintergrund der Gesamtheit der Schüler einer Schule in der Regel größere Auswirkungen hat als der soziale Hintergrund des einzelnen Schülers. Schüler aus schlechter gestellten sozioökonomischen Verhältnissen erzielen in der Regel wesentlich bessere Ergebnisse, wenn sie Schulen besuchen, deren Schülerschaft im Durchschnitt gehobenen Verhältnissen entstammt, als wenn sie in Schulen gehen, in denen der sozioökonomische Hintergrund der Schülerschaft unter dem Durchschnitt liegt. Das Gegenteil ist der Fall für Schüler aus besser gestellten Verhältnissen in Schulen, deren sozio-ökonomischer Hintergrund weniger günstig ist. Daraus lässt sich schließen, dass die institutionelle Differenzierung von Bildungssystemen - häufig verstärkt durch Unterschiede beim sozialen Hintergrund der Schüler, die eigenen Entscheidungen der Schüler bzw. ihrer Eltern sowie die Beurteilung früherer Leistungen - großen Einfluss auf den schulischen Erfolg der einzelnen Schüler haben kann.
... wobei die Anhäufung
sozial besser gestellter
Schüler, vor allem
in Ländern mit
unterschiedlichen
Schultypen im
Sekundarbereich, als
verstärkender Faktor
hinzukommen kann,...

... da Schülerleistungen nicht nur vom eigenen familiären Hintergrund, sondern auch - und zwar in größserem Maßse - vom Hintergrund der Mitschüler an der Schule beeinflusst werden. 
Die erzielten

Punktzahlen basieren

auf Leistungsmessungen,

die Teil der im Jahr

2000 von der OECD

durchgeführten

Internationalen

Schulleistungsstudie

PISA waren.

\section{Definitionen und angewandte Methodik}

Die in diesem Indikator untersuchte Zielpopulation waren 15-jährige Schüler. In der Praxis bezieht sich dies auf Schüler, die zu Beginn der Testperiode im Alter von 15 Jahren und 3 (abgeschlossenen) Monaten bis zu 16 Jahren und 2 (abgeschlossenen) Monaten waren und eine Bildungseinrichtung besuchten, ungeachtet der Klassenstufe und der Art der von ihnen besuchten Bildungseinrichtung und unabhängig davon, ob es sich um eine Ganztags- oder Halbtagsschule handelte.

Um die Interpretation der von den Schülern erzielten Punktwerte in der PISAStudie zu erleichtern, wurde die PISA-Skala für Lesekompetenz so konstruiert, dass der Mittelwert bei 500 Punkten liegt und die Standardabweichung bei 100, wobei die Daten gewichtet wurden, so dass jedes Land gleichermaßen hierzu beitrug. Diese Bezugspunkte bilden gewissermaßen den „Anker“ der im Rahmen von PISA durchgeführten Messung der Schülerleistungen.

In Tabelle A7.1 werden die Unterschiede in Form der statistischen Varianz ausgedrückt. Sie ergibt sich, wenn man die an früherer Stelle in diesem Kapitel angeführte Standardabweichung quadriert. Dieser Vergleich wird mittels der statistischen Varianz und nicht der Standardabweichung durchgeführt, damit eine Aufgliederung in die einzelnen Komponenten der Unterschiede in den Schülerleistungen möglich ist. Der Durchschnitt wird aus den in der Tabelle erfassten OECD-Ländern berechnet. Aufgrund der Methode der Stichprobenziehung in Japan schließt dort die Varianz zwischen den Schulen die Varianz zwischen Klassen innerhalb der Schulen ein.

Hinweise zu Standardfehlern, Signifikanztests und multiplen Vergleichen s. Anhang 3 unter www.oecd.org/els/education/eag2002. 
Tabelle A7.1.

Zusammensetzung der Leistungsvarianz im Bereich Lesekompetenz 15-jähriger Schüler (2000) Varianz der Schülerleistungen auf der PISA-Skala für Lesekompetenz zwischen Schulen und innerhalb von Schulen

\begin{tabular}{|c|c|c|c|c|c|c|c|c|c|c|c|c|c|}
\hline & \multirow[b]{3}{*}{$\begin{array}{c}\text { Gesamt- } \\
\text { varianz der } \\
\text { Schülerleis- } \\
\text { tungen' }^{1}\end{array}$} & \multicolumn{11}{|c|}{ Varianz, ausgedrückt in Prozent der durchschnittlichen Gesamtvarianz der Schülerleistungen in den OECD-Ländern } & \multirow{3}{*}{$\begin{array}{c}\text { Gesamt- } \\
\text { varianz } \\
\text { zwischen } \\
\text { Schulen, } \\
\text { ausge- } \\
\text { drückt als } \\
\text { Prozent } \\
\text { der } \\
\text { Gesamt- } \\
\text { varianz } \\
\text { innerhalb } \\
\text { des } \\
\text { jeweiligen } \\
\text { Landes }^{2}\end{array}$} \\
\hline & & \multirow{2}{*}{$\begin{array}{l}\text { Gesamt- } \\
\text { varianz der } \\
\text { Schüler- } \\
\text { leistungen, } \\
\text { ausgedrückt } \\
\text { in Prozent } \\
\text { der durch- } \\
\text { schnittlichen } \\
\text { Gesamt- } \\
\text { varianz der } \\
\text { Schülerleis- } \\
\text { tungen in } \\
\text { den OECD- } \\
\text { Ländern }\end{array}$} & \multirow[b]{2}{*}{$\begin{array}{l}\text { Gesamt- } \\
\text { varianz der } \\
\text { Schülerleis- } \\
\text { tungen } \\
\text { zwischen } \\
\text { Schulen }\end{array}$} & \multirow{2}{*}{$\begin{array}{c}\text { Gesamt- } \\
\text { varianz } \\
\text { der Schü- } \\
\text { lerleis- } \\
\text { tungen } \\
\text { innerhalb } \\
\text { von } \\
\text { Schulen }\end{array}$} & \multicolumn{2}{|c|}{$\begin{array}{c}\text { Varianz, erklärt } \\
\text { durch den interna- } \\
\text { tionalen sozio-öko- } \\
\text { nomischen Index der } \\
\text { beruflichen Stellung } \\
\text { der Schülereltern }\end{array}$} & \multicolumn{2}{|c|}{$\begin{array}{c}\text { Varianz, } \\
\text { erklärt durch den } \\
\text { durchschnittlichen } \\
\text { internationalen } \\
\text { sozio-ökonomischen } \\
\text { Index der berufli- } \\
\text { chen Stellung der } \\
\text { Schülereltern } \\
\text { pro Schule }\end{array}$} & \multicolumn{2}{|c|}{$\begin{array}{l}\text { Varianz, } \\
\text { erklärt durch } \\
\text { geographische/ } \\
\text { institutionelle } \\
\text { Faktoren }\end{array}$} & \multicolumn{2}{|c|}{\begin{tabular}{|c|} 
Varianz, erlärt durch \\
geographische/ \\
institutionelle \\
Faktoren und den \\
durchschnittlichen \\
internationalen \\
sozio-ökonomischen \\
Index der beruflichen \\
Stellung der Schüler- \\
eltern pro Schule
\end{tabular}} & \\
\hline & & & & & \begin{tabular}{|l} 
erklärte \\
Varianz \\
zwischen \\
Schulen
\end{tabular} & $\begin{array}{c}\text { erklärte } \\
\text { Varianz } \\
\text { innerhalb } \\
\text { von } \\
\text { Schulen }\end{array}$ & $\begin{array}{c}\text { erklärte } \\
\text { Varianz } \\
\text { zwischen } \\
\text { Schulen }\end{array}$ & $\begin{array}{c}\text { erklärte } \\
\text { Varianz } \\
\text { innerhalb } \\
\text { von } \\
\text { Schulen }\end{array}$ & \begin{tabular}{|l} 
erklärte \\
Varianz \\
zwischen \\
Schulen
\end{tabular} & $\begin{array}{c}\text { erklärte } \\
\text { Varianz } \\
\text { innerhalb } \\
\text { von } \\
\text { Schulen }\end{array}$ & $\begin{array}{l}\text { erklärte } \\
\text { Varianz } \\
\text { zwischen } \\
\text { Schulen }\end{array}$ & $\begin{array}{c}\text { erklärte } \\
\text { Varianz } \\
\text { innerhalb } \\
\text { von } \\
\text { Schulen }\end{array}$ & \\
\hline Australien & 10357 & 111.6 & 20.9 & 90.6 & 8.3 & 6.7 & 14.2 & 6.9 & 1.8 & 0.1 & 15.0 & 7.0 & 18.8 \\
\hline Österreich & 8649 & 93.2 & 68.6 & 45.7 & 10.4 & 0.4 & 42.6 & 0.3 & 60.4 & 0.0 & 61.6 & 0.5 & 60.0 \\
\hline Belgien & 11455 & 123.5 & 76.0 & 50.9 & 11.0 & 1.8 & 44.2 & 1.9 & 50.7 & 0.0 & 61.9 & 1.9 & 59.9 \\
\hline Kanada & 8955 & 96.5 & 17.1 & 80.1 & 4.6 & 5.0 & 7.8 & 5.1 & 1.1 & 0.0 & 8.4 & 5.1 & 17.6 \\
\hline Tschechische Rep. & 9278 & 100.0 & 51.9 & 45.3 & 8.8 & 1.8 & 34.4 & 1.8 & 44.5 & 0.0 & 46.8 & 1.8 & 53.4 \\
\hline Dänemark & 9614 & 103.6 & 19.6 & 85.9 & 10.2 & 8.0 & 11.6 & 8.1 & $\mathrm{~m}$ & $\mathrm{~m}$ & $\mathrm{~m}$ & $\mathrm{~m}$ & 18.6 \\
\hline Finnland & 7994 & 86.2 & 10.7 & 76.5 & 1.5 & 4.6 & 1.7 & 4.6 & $\mathrm{~m}$ & $\mathrm{~m}$ & $\mathrm{~m}$ & $\mathrm{~m}$ & 12.3 \\
\hline Frankreich & $\mathrm{m}$ & $\mathrm{m}$ & $\mathrm{m}$ & $\mathrm{m}$ & $\mathrm{m}$ & $\mathrm{m}$ & $\mathrm{m}$ & $\mathrm{m}$ & $\mathrm{m}$ & $\mathrm{m}$ & $\mathrm{m}$ & $\mathrm{m}$ & $\mathrm{m}$ \\
\hline Deutschland & 12368 & 133.3 & 74.8 & 50.2 & 11.7 & 2.3 & 51.5 & 2.3 & 65.2 & 0.0 & 66.9 & 2.3 & 59.8 \\
\hline Griechenland & 9436 & 101.7 & 53.8 & 52.9 & 7.0 & 1.1 & 25.0 & 1.1 & 33.3 & 0.0 & 40.1 & 0.4 & 50.4 \\
\hline Ungarn & 8810 & 95.0 & 71.2 & 34.8 & 8.3 & 0.3 & 49.4 & 0.2 & 52.5 & 0.0 & 58.7 & 0.1 & 67.2 \\
\hline Island & 8529 & 91.9 & 7.0 & 85.0 & 1.6 & 5.0 & 1.7 & 5.0 & 0.9 & 0.0 & 2.3 & 5.0 & 7.6 \\
\hline Irland & 8755 & 94.4 & 17.1 & 79.2 & 5.5 & 5.7 & 10.1 & 5.7 & 9.7 & 0.0 & 12.7 & 5.5 & 17.8 \\
\hline Italien & 8356 & 90.1 & 50.9 & 43.4 & 3.4 & 0.5 & 23.8 & 0.5 & 27.6 & 0.0 & 30.1 & 0.5 & 54.0 \\
\hline$J^{J} a n^{3}$ & 7358 & 79.3 & 36.5 & 43.9 & $\mathrm{~m}$ & $\mathrm{~m}$ & $\mathrm{~m}$ & $\mathrm{~m}$ & $\mathrm{~m}$ & $\mathrm{~m}$ & $\mathrm{~m}$ & $\mathrm{~m}$ & 45.4 \\
\hline Korea & 4833 & 52.1 & 19.7 & 33.0 & 1.0 & 0.2 & 7.1 & 0.2 & 10.9 & 0.0 & 12.0 & 0.2 & 37.4 \\
\hline Luxemburg & 10088 & 108.7 & 33.4 & 74.9 & 11.1 & 8.3 & 26.7 & 8.2 & $\mathrm{~m}$ & $\mathrm{~m}$ & $\mathrm{~m}$ & $\mathrm{~m}$ & 30.8 \\
\hline Mexiko & 7370 & 79.4 & 42.9 & 37.4 & 5.2 & 0.1 & 25.7 & 0.1 & 26.5 & 0.0 & 35.3 & 0.1 & 53.4 \\
\hline Neuseeland & 11701 & 126.1 & 20.1 & 103.9 & 7.3 & 10.9 & 11.6 & 11.0 & 12.9 & 0.0 & 14.8 & 11.0 & 16.2 \\
\hline Norwegen & 10743 & 115.8 & 12.6 & 102.4 & 3.7 & 8.7 & 4.9 & 8.7 & 0.5 & 3.8 & 5.2 & 10.1 & 10.9 \\
\hline Polen & 9958 & 107.3 & 67.0 & 38.9 & 6.3 & 1.1 & 42.4 & 1.1 & 53.0 & 0.0 & 55.9 & 1.1 & 63.2 \\
\hline Portugal & 9436 & 101.7 & 37.5 & 64.3 & 10.6 & 4.6 & 23.8 & 4.6 & $\mathrm{~m}$ & $\mathrm{~m}$ & $\mathrm{~m}$ & $\mathrm{~m}$ & 36.8 \\
\hline Spanien & 7181 & 77.4 & 15.9 & 60.9 & 5.4 & 3.0 & 9.1 & 3.1 & 6.2 & 0.0 & 10.9 & 3.1 & 20.7 \\
\hline Schweden & 8495 & 91.6 & 8.9 & 83.0 & 4.5 & 6.9 & 5.8 & 6.9 & 2.7 & 2.6 & 6.9 & 8.1 & 9.7 \\
\hline Schweiz & 10408 & 112.2 & 48.7 & 63.7 & 12.7 & 4.0 & 24.3 & 3.9 & 22.1 & 0.0 & 29.7 & 4.1 & 43.4 \\
\hline Ver. Königreich & 10098 & 108.9 & 22.4 & 82.3 & 9.6 & 8.4 & 16.0 & 8.7 & 7.3 & 0.0 & 17.1 & 6.7 & 21.4 \\
\hline Vereinigte Staaten & 10979 & 118.3 & 35.1 & 83.6 & 12.0 & 5.6 & 25.5 & 5.8 & $\mathrm{~m}$ & $\mathrm{~m}$ & $\mathrm{~m}$ & $\mathrm{~m}$ & 29.6 \\
\hline Brasilien & 7427 & 80.1 & 35.8 & 47.1 & 6.5 & 1.9 & 19.7 & 2.1 & 5.3 & 0.0 & 21.7 & 2.1 & 43.1 \\
\hline Lettland & 10435 & 112.5 & 35.1 & 77.5 & 4.9 & 4.4 & 16.7 & 4.5 & $\mathrm{~m}$ & $\mathrm{~m}$ & $\mathrm{~m}$ & $\mathrm{~m}$ & 31.2 \\
\hline Liechtenstein & $\mathrm{m}$ & $\mathrm{m}$ & $\mathrm{m}$ & $\mathrm{m}$ & $\mathrm{m}$ & $\mathrm{m}$ & $\mathrm{m}$ & $\mathrm{m}$ & $\mathrm{m}$ & $\mathrm{m}$ & $\mathrm{m}$ & $\mathrm{m}$ & 43.9 \\
\hline Russische Föd. & 8466 & 91.3 & 33.6 & 57.1 & 4.8 & 2.4 & 15.4 & 2.3 & 16.6 & 0.0 & 21.0 & 2.3 & 37.1 \\
\hline
\end{tabular}

1. Die Gesamtvarianz der Schülerleistungen ergibt sich aus dem Quadrat der in Tabelle A5.2 angegebenen Standardabweichung (SA). Für diesen Vergleich wird die statistische Varianz und nicht die Standardabweichung verwendet, um die einzelnen Komponenten der Varianz der Schülerleistungen bestimmen zu können. Aus Gründen, die im PISA-2000 Technical Report erläutert werden, kann sich die Summe der Varianzkomponenten zwischen und innerhalb der Schulen für einige Länder von dem Quadrat der in Tabelle A5.2 angegebenen Standardabweichung etwas unterscheiden.

2. Dieser Index wird häufig als Intra-Klassen-Korrelation bezeichnet.

3. Bedingt durch die in Japan verwendete Methode der Stichprobenziehung umfasst die Varianz zwischen Schulen in Japan auch Unterschiede zwischen einzelnen Klassen innerhalb von Schulen.

Quelle: OECD PISA-Datenbank, 2001. Hinweise zur Methodik s. Anhang 3 (www.oecd.org/els/education/eag2002) und www.pisa.oecd.org.. 


\section{POLITISCHE BILDUNG UND POLITISCHES ENGAGEMENT}

- Im Rahmen der IEA-Studie zur politischen Bildung wiesen die 14-Jährigen in den meisten OECDLändern ein solides Verständnis der grundlegenden demokratischen Werte und Institutionen auf, sowie die Fähigkeit, entsprechendes schriftliches Material wie politische Cartoons oder eine fiktive Wahlkampfbroschüre zu interpretieren und zwischen Meinungsäußerungen undTatsachendarstellungen zu unterscheiden.

- 14-Jährige sehen es im Allgemeinen als sehr wichtige Aufgabe Erwachsener an, die Gesetze zu respektieren und zur Wahl zu gehen. Für sie sind Aktivitäten in den Bereichen Menschenrechte und Umweltschutz wichtig, sowie Aktivitäten zum Wohl ihres sozialen Umfelds. Die Teilnahme an politischen Diskussionen und die Mitgliedschaft in einer politischen Partei sind für sie weniger wichtig.

\section{Abbildung A8.1.}

Politische Bildung 14-Jähriger (1999)

Mittelwert und Signifikanz des Mittelwerts imVergleich zum Ländermittelwert auf den Teilskalen

"InhaltlichesWissen" und "Interpretationsfähigkeiten" sowie der Gesamtskala

"Politische Bildung" der IEA-Studie zur politischen Bildung

\begin{tabular}{|c|c|c|c|c|c|c|c|}
\hline \multicolumn{3}{|c|}{$\begin{array}{l}\text { Mittelwert statistisch signifikant höher als } \\
\text { Ländermittelwert }{ }^{4}\end{array}$} & \multicolumn{2}{|c|}{$\begin{array}{l}\text { Kein statistisch signifikanter Unterschied } \\
\text { zum Ländermittelwert }{ }^{4}\end{array}$} & \multirow{2}{*}{\multicolumn{3}{|c|}{$\begin{array}{l}\text { Mittelwert statistisch signifikant niedriger } \\
\text { als Ländemittelwert }{ }^{4} \\
\text { Skalenwert }\end{array}$}} \\
\hline \multirow[t]{2}{*}{ Land } & \multicolumn{3}{|c|}{ Mittlerer Skalenwert } & \multirow[b]{2}{*}{80} & & & \\
\hline & $\begin{array}{l}\text { Inhaltliches } \\
\text { Wissen }\end{array}$ & $\begin{array}{l}\text { Interpretations- } \\
\text { fähigkeiten }\end{array}$ & $\begin{array}{c}\text { Gesamtskala } \\
\text { Politische Bildung }\end{array}$ & & 100 & 120 & 140 \\
\hline Polen & $112(1.3)$ & $106(1.7)$ & $111(1.7)$ & & & & \\
\hline Finnland & $108(0.7)$ & $110(0.6)$ & $109(0.7)$ & & & & \\
\hline Griechenland & $109(0.7)$ & $105(0.7)$ & $108(0.8)$ & & & & \\
\hline Vereinigte Staaten $^{1}$ & $102(1.1)$ & $114(1.0)$ & $106(1.2)$ & & & & \\
\hline Italien & $105(0.8)$ & $105(0.7)$ & $105(0.8)$ & & & & \\
\hline Slowakische Rep. & $107(0.7)$ & $103(0.7)$ & $105(0.7)$ & & & & \\
\hline Norwegen $^{2}$ & $103(0.5)$ & $103(0.4)$ & $103(0.5)$ & & & & \\
\hline Tschechische Rep. & $103(0.8)$ & $102(0.8)$ & $103(0.8)$ & & - & & \\
\hline Australien & $99(0.7)$ & $107(0.8)$ & $102(0.8)$ & & & & \\
\hline Ungarn & $102(0.6)$ & $101(0.7)$ & $102(0.6)$ & & & & \\
\hline Dänemark² & $100(0.5)$ & $100(0.5)$ & $100(0.5)$ & & & & \\
\hline Deutschland ${ }^{3}$ & $99(0.5)$ & $101(0.5)$ & $100(0.5)$ & & & & \\
\hline England $^{1}$ & $96(0.6)$ & $105(0.7)$ & $99(0.6)$ & & & & \\
\hline Schweden $^{1}$ & $97(0.8)$ & $102(0.7)$ & $99(0.8)$ & & - & & \\
\hline Schweiz & $96(0.8)$ & $102(0.8)$ & $98(0.8)$ & & & & \\
\hline Portugal & $97(0.7)$ & $95(0.7)$ & $96(0.7)$ & & - & & \\
\hline Belgien (Frz.) ${ }^{2}$ & $94(0.9)$ & $96(0.9)$ & $95(0.9)$ & & 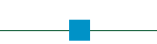 & & \\
\hline
\end{tabular}

1. Erhebungsdatum am Beginn des Schuljahres.

2. Beteiligungsrate unter Berücksichtigung der im Nachrückverfahren einbezogenen Schulen unter 85 Prozent.

3. Grundgesamtheit auf Länderebene entspricht nicht den angestrebten Standards.

4. Der Ländermittelwert basiert auf allen an der IEA-Studie zur politischen Bildung teilnehmenden Ländern und beschränkt sich nicht auf die in der Tabelle aufgeführten Länder.

Anordnung der Länder in absteigender Reihenfolge des Mittelwertes auf der Gesamtskala "Politische Bildung" der IEA-Studie zur politischen Bildung. Quelle: IEA-Studie zur politischen Bildung (2001). 


\section{Politischer Hintergrund}

Demokratische Gesellschaften hängen nicht nur von soliden, grundlegenden Kenntnissen und Fähigkeiten in Sachfächern wie Lesen, Mathematik und Naturwissenschaften ab, sondern auch von der permanenten Heranbildung informierter Bürger, die das notwendige Verständnis und die notwendigen Fähigkeiten haben, grundlegende Formen der politischen Meinungsbildung zu verstehen. Sie gründen sich auch darauf, dass sich der Einzelne als Staatsbürger aktiv engagiert.

Wie können die Schulen das Verständnis junger Menschen über die zivile Gesellschaft und den Staat erweitern und ihr Engagement fördern? Um Näheres über das Verständnis und die Vorstellungen von Schülern in den einzelnen Ländern hinsichtlich Staatsbürgerschaft, Staat und Gesetzen herauszufinden, wurde von der International Association for the Evaluation of Educational Achievement (IEA) eine internationale Vergleichsstudie zur politischen Bildung durchgeführt, die IEA-Studie zur politischen Bildung. Man wollte herauszufinden, wie junge Menschen - innerhalb und außerhalb der Schule auf ihre Rolle als Staatsbürger in einer Demokratie vorbereitet werden. Im Rahmen der Studie nahmen 14-Jährige in 28 Ländern, einschließlich 17 OECDLändern, an Tests zu ihren Kenntnissen im Bereich des politischen Geschehens, zu ihren Fähigkeiten des Veständnisses politischer Meinungsbildung und zu ihren Vorstellungen und Einstellungen zu staatsbürgerlichen Sachverhalten teil.

\section{Ergebnisse und Erläuterungen}

\section{Politische Bildung und Fähigkeiten}

Abbildung A8.1 zeigt die Mittelwerte der 14-Jährigen im Bereich der politischen Bildung auf. Die Gesamtpunktzahl setzt sich aus zwei Einzelwerten zusammen dem „inhaltlichen Wissen“ (Kenntnis der grundlegenden demokratischen Prinzipien) und den „Interpretationsfähigkeiten“ (Fähigkeiten der Auslegung von Informationen in diesem Bereich, wie z.B. politischen Cartoons, Wahlkampfbroschüren oder Zeitungsartikeln). Die Gesamtskala für alle 28 Länder, die an der IEA-Studie zur politischen Bildung teilnahmen, sowie die zwei Einzelskalen wurden so konstruiert, dass der Gesamtmittelwert bei 100 lag. Die Standardabweichung für die 28 Länder zusammen lag bei 20.

Die Ergebnisse weisen darauf hin, dass im Durchschnitt aller teilnehmenden Länder die Schüler innerhalb des von der IEA-Studie zur politischen Bildung vorgegebenen Referenzrahmens ein solides Verständnis demokratischer Werte und Institutionen aufweisen. Die Ergebnisse lassen erkennen, dass international gesehen die Mehrheit der Schüler die grundlegenden Funktionen von Gesetzen, nicht-staatlichen gesellschaftlichen Organisationen und politischen Parteien kennt. (Von den 38 im Test verwendeten Fragen wurden 25 von wenigstens 60 Prozent der gesamten Schülerpopulation aller teilnehmenden Länder richtig beantwortet, 13 Fragen wurden von mehr als 70 Prozent richtig beantwortet.)
Demokratische

Gesellschaften hängen

von politischer Bildung

und politischem

Engagement ab.

Dieser Indikator

beschäftigt sich mit

den Kenntnissen 14-

Jähriger im Bereich des

politischen Geschehens, ihren Fähigkeiten zum verständnis politischer Meinungsbildung und ihren Einstellungen zum Stadt.

Beim Vergleich der politischen Bildung über verschiedene Länder hinweg wird in der IEAStudie zur politischen Bildung zwischen inhaltlichem Wissen und Interpretationsfähigkeiten unterschieden.

Die Ergebnisse lassen daraufschließen, dass im Durchschnitt der OECD 14-Jährige ein solides verständnis bezüglich der grundlegenden demokratischen Werte und Institutionen haben,... 
sowie grundlegende Fähigkeiten bei der Auslegung entsprechenden schriftlichen Materials aufweisen. Auch können sie zwischen subjektiven Meinungsäußerungen und Tatsachendarstellungen unterscheiden.

Zwischen den einzelnen Ländern gibt es große Unterschiede, für die es keine einfachen Erklärungen gibt, ...

\section{.. sie könnten jedoch zum Teil auf unterschiedliche schwerpunkte in den Lehrplänen zurückzuführen sein.}

Die Schüler wurden auch zu ihrem Vertrauen in den Statat befragt und was in ihren Augen für einen Erwachsenen wichtig ist, damit er ein guter Staatsbürger ist.
Die Schüler zeigten im Durchschnitt solide Fähigkeiten bei der Auslegung von entsprechendem schriftlichen Material, wie z.B. politischen Cartoons oder fiktiven Wahlkampfbroschüren, und der Unterscheidung von Meinungsäußerungen und Tatsachendarstellungen. Unter den 14-Jährigen fanden sich hohe durchschnittliche Fähigkeiten bei der Auslegung von staatsbürgerlichen und politischen Informationen hauptsächlich in den Ländern, in denen die Demokratie schon seit mehr als 40 Jahren die Staatsform ist. Australien, England, Griechenland, Finnland, Italien, Norwegen, Polen, die Slowakische Republik, Schweden und die Vereinigten Staaten erzielten allesamt Punktzahlen oberhalb des internationalen Mittelwerts auf der Teilskala „Interpr etationsfähigkeiten“ der IEA-Studie zur politischen Bildung.

Es gibt keine einfachen Erklärungen für die Unterschiede zwischen den einzelnen Ländern beim inhaltlichen Wissen und den Interpretationsfähigkeit en. Zu den Ländern mit sehr guten Ergebnissen gehören nicht nur Länder mit einer langen demokratischen Tradition, sondern auch Länder, die während der letzten 10 bis 15 Jahre massive politische Veränderungen erlebt haben (z.B. Polen, die Slowakische Republik und die Tschechische Republik). Finnland, Griechenland, Italien, Norwegen, Polen, die Slowakische Republik, die Tschechische Republik und Ungarn lagen alle über dem Ländermittel (aller teilnehmenden Länder, nicht nur der OECD-Länder) auf der Teilskala “inhaltliches Wissen” der IEA-Studie.

Bei einem Vergleich der auf den zwei Teilskalen erzielten Punktzahlen ergeben sich interessante Unterschiede. Die Schüler in Australien, England, Schweden, der Schweiz und den Vereinigten Staaten erzielten bei den Aufgaben, bei denen es um das Verständnis von entsprechendem schriftlichen Material ging, bessere Leistungen als bei den Aufgaben, bei denen es um die Kenntnisse grundlegender demokratischer Prinzipien ging. Im Gegensatz hierzu erbrachten Schüler in der Tschechischen Republik und Ungarn bessere Leistungen bei den Aufgaben zum inhaltlichen Wissen als bei den Aufgaben zur Interpretationsfähigkeit.

\section{Vertrauen in den Staat und Einstellungen zu Politik und Gesellschaft}

In der Studie zur politischen Bildung wurden die Schüler auch darüber befragt, in welchem Ausmaß man bestimmten Arten von staatlichen Einrichtungen nationalen Regierungen, kommunalen Verwaltungen und lokalen Einrichtungen in der Stadt, in der sie leben, Gerichten, der Polizei, politischen Parteien und dem nationalen Parlament - trauen kann. In Abbildung A8.2 sind die Ergebnisse tabellarisch zusammengefasst. Die Skala hat über alle 28 Länder der Studie hinweg einen Mittelwert von 10 und eine Standardabweichung von 2. Australien, Dänemark, Griechenland, Norwegen, die Slowakische Republik, die Schweiz und die Vereinigten Staaten liegen alle über dem internationalen Mittelwert, während Portugal und die Tschechische Republik unter ihm liegen. Einige Länder mit geringen Punktzahlen hinsichtlich des Vertrauens in den Staat und staatliche Organisationen waren nicht OECD-Länder, sie sind in der Abbildung nicht aufgenommen. 
Den Schülern wurden auch Fragen dahingehend gestellt, was in ihren Augen zu den Pflichten von Erwachsenen als guten Staatsbürgern gehört. Tabelle A8.1 zeigt ausgewählte Antworten (andere Antworten s. Citizenship and Education in Twenty-Eight Countries, IEA 2001). In den Augen der 14-Jährigen ist es generell eine sehr wichtige Verpflichtung, als erwachsener Staatsbürger die Gesetze zu respektieren, auch zu Wahlen zu gehen ist in ihren Augen wichtig.

Die Schüler glauben auch, dass es zu den Pflichten erwachsener Staatsbürger gehört, sich an Aktivitäten zur Förderung der Menschenrechte und im Rahmen des Umweltschutzes zu engagieren und zum Wohle des sozialen Umfeldes tätig zu sein. In einigen Ländern wird auch das Verfolgen von politischen Fragen in den Medien als wichtig erachtet.
14-Jährige sind im Allgemeinen der Ansicht, dass es zu den wichtigen Pflichten von Erwachsenen gehört, die Gesetze zu respektieren und zu Wahlen zu gehen. Für sie sind auch Aktivitäten in den Bereichen Menschenrechte und Umweltschutz wichtig, ebenso wie Aktivitäten zum Wohle ihres sozialen Umfelds,...

\section{Abbildung A8.2.}

Vertrauen 14-Jähriger in politische Institutionen (1999)

Mittelwert und Signifikanz des Mittelwertes im Vergleich zum Ländermittelwert auf der IEA-Skala "Vertrauen in politische Institutionen"

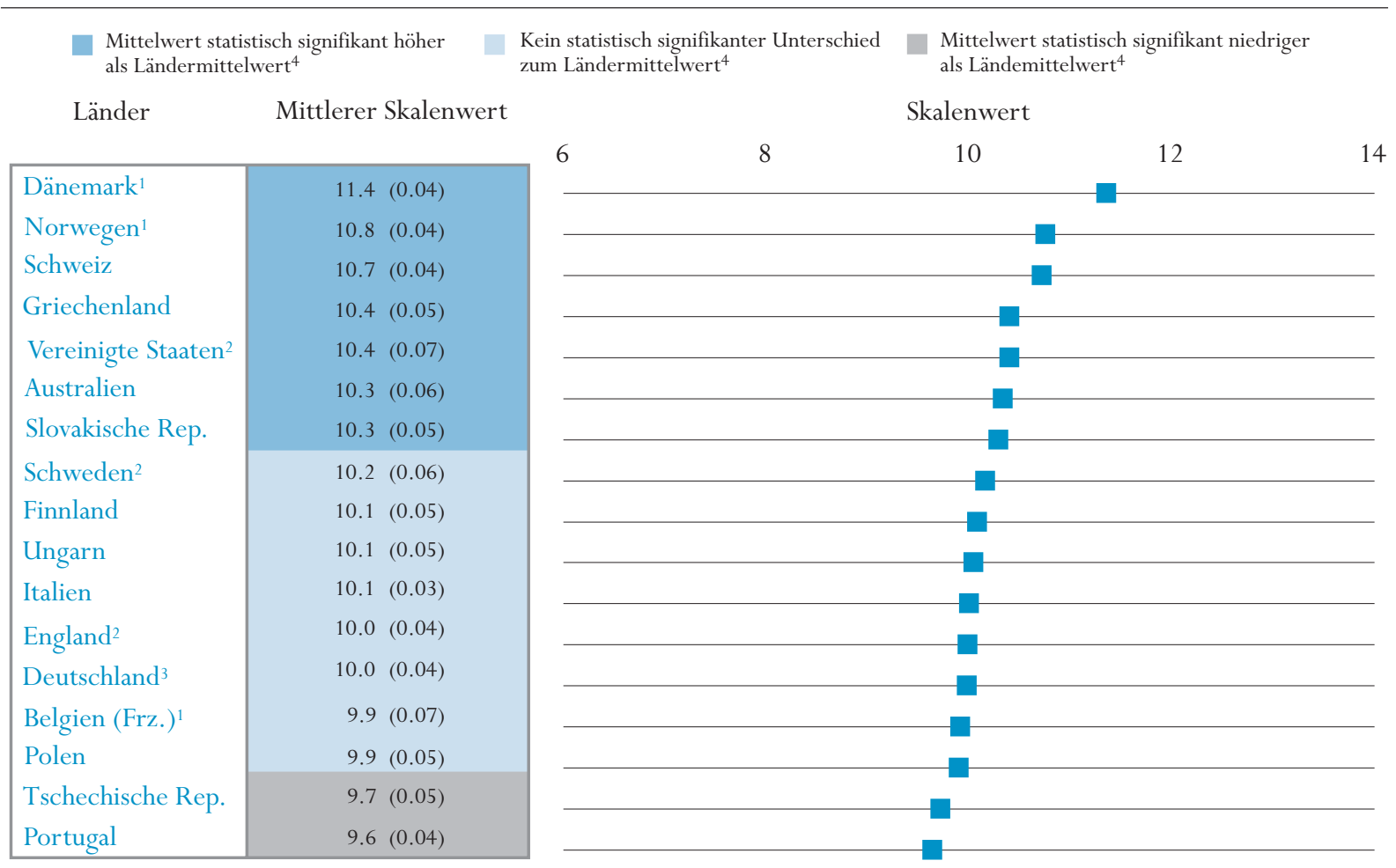

1. Beteiligungsrate unter Berücksichtigung der im Nachrückverfahren einbezogenen Schulen unter 85 Prozent.

2. Erhebungsdatum am Beginn des Schuljahres.

3. Grundgesamtheit auf Länderebene entspricht nicht den angestrebten Standards.

4. Der Ländermittelwert basiert auf allen an der IEA-Studie zur politischen Bildung teilnehmenden Ländern und beschränkt sich nicht auf die in der Tabelle aufgeführten Länder.

Anordnung der Länder in absteigender Reihenfolge des Mittelwertes auf der IEA-Skala "Vertrauen in politische Institutionen".

Quelle: IEA-Studie zur politischen Bildung (2001). 
... während es für sie wesentlich weniger

wichtig ist, an

politischen Diskussionen

teilzunehmen oder

einer politischen Partei

beizutreten.
Schließlich wurden die Schüler gebeten, anzugeben, an welcher Art von politischer Beteiligung sie glauben, als Erwachsene teilnehmen zu werden. Nur 20 Prozent der Befragten über die Länder hinweg gaben an, dass sie beabsichtigen, an den Aktivitäten teilzunehmen, die normalerweise im Zusammenhang mit politischer Beteiligung genannt werden, wie z.B. einer politischen Partei beizutreten, Leserbriefe zu sozialen und politischen Fragen zu schreiben oder für ein lokales oder kommunales Amt zu kandidieren. Wesentliche Anteile der 14-Jährigen gaben jedoch an, dass sie davon ausgehen, dass sie zu Wahlen gehen werden und dass sie es für wichtig erachten, dass erwachsene Staatsbürger zur Wahl gehen. In einigen Ländern ist dieser Anteil wesentlich höher als der Anteil derjenigen jungen Erwachsenen, die wirklich zur Wahl gehen (Abb. A8.3).

\section{Abbildung A8.3.}

Wahrscheinlichkeit der Wahlbeteiligung von 14-Jährigen (1999)

Prozentsatz 14-Jähriger, die angaben, bestimmt oder wahrscheinlich als Erwachsene zu nationalen Wahlen zu gehen

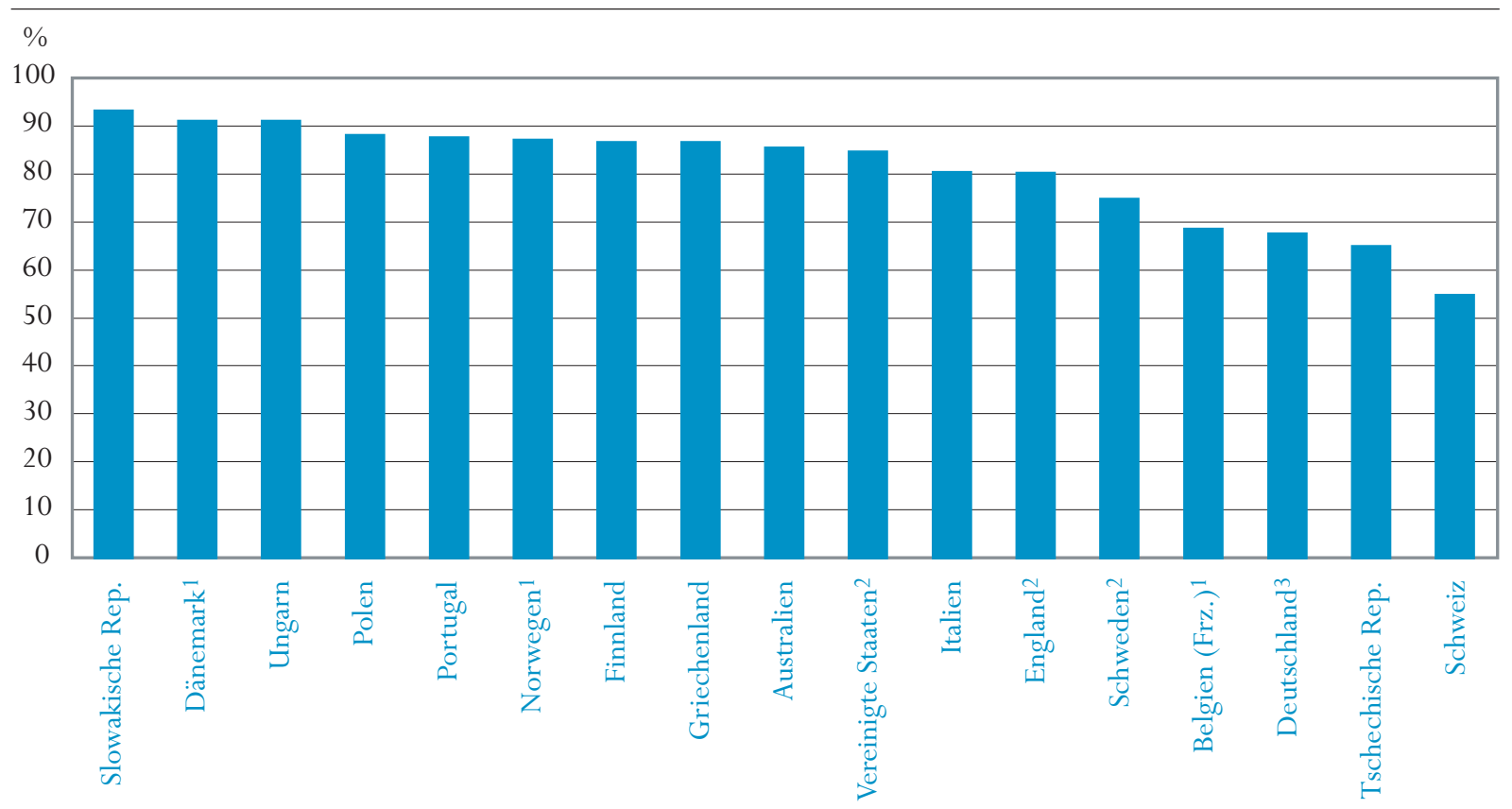

1. Beteiligungsrate unter Berücksichtigung der im Nachrückverfahren einbezogenen Schulen unter 85 Prozent.

2. Erhebungsdatum am Beginn des Schuljahres.

3. Grundgesamtheit auf Länderebene entspricht nicht den angestrebten Standards.

Anordnung der Länder in absteigender Reihenfolge des Prozentsatzes 14-jähriger Schüler, die angaben, bestimmt oder wahrscheinlich als Erwachsene zu nationalen Wahlen zu gehen.

Quelle: IEA-Studie zur politischen Bildung (2001). Tabelle A8.1. 


\section{Definitionen und angewandte Methodik}

Dieser Indikator wurde aus der Studie der IEA (Intenational Association for the Evaluation of Educational Achievements) zur Politischen Bildung abgeleitet, in der 1999 national repräsentative Stichproben von 90.000 Schülern in 28 Ländern untersucht wurden. Zielpopulation waren die Schüler der Klassen, in der sich die Mehrheit der 14-jährigen Schüler befindet (8. oder 9. Klasse).

In einem Teil der Erhebung wurden die Schüler gefragt, wie groß die Wahrscheinlichkeit wäre, dass sie an Wahlen teilnehmen würden und wie wichtig es in ihren Augen für gute Staatsbürger ist, an einer Reihe von politischen und staatsbürgerlichen Aktivitäten teilzunehmen. Die entsprechenden Prozentangaben werden zusammen mit dem der Methode der Stichprobenziehung entsprechenden Standardfehler angegeben.

Weitere Informationen s. Citizenship and Education across Countries: Civic Knowledge and Engagement at Age Fourteen (Torney-Purta, Lehmann, Oswald, and Schulz, Hrsg. IEA Amsterdam).
Dieser Indikator basiert auf der IEA-Studie zur politischen Bildung mit einer Datenerhebung durch die IEA im Jahr 1999. 
KAPITEL A Bildungs-und Lernergebnisse

Tabelle A8.1.

Einstellungen zu Politik und Gesellechaft 14-jähriger Schüler (1999)

Prozentsatz der Schüler, die wahrscheinlich oder bestimmt als Erwachsener zu nationalenWahlen gehen wollen und Prozentsatz der Schüler, die es für sehr wichtig oder wichtig erachten, dass sich Staatsbürger in ausgewählten Bereichen politisch betätigen

\begin{tabular}{|c|c|c|c|c|c|c|c|c|c|c|c|c|}
\hline & \multirow{2}{*}{\multicolumn{2}{|c|}{$\begin{array}{l}\text { Prozentsatz der } \\
\text { Schüler, die wahr- } \\
\text { scheinlich oder } \\
\text { bestimmt als Erwach- } \\
\text { sene zu nationalen } \\
\text { Wahlengehen wollen }\end{array}$}} & \multicolumn{10}{|c|}{ Prozentsatz der Schüler, die es für sehr wichtig oder wichtig erachten, dass Staatsbürger .... } \\
\hline & & & \multicolumn{2}{|c|}{$\begin{array}{c}\text { sich an jeder } \\
\text { Wahl beteiligen }\end{array}$} & \multicolumn{2}{|c|}{ einer Partei beitreten } & \multicolumn{2}{|c|}{$\begin{array}{l}\text { an politischen Diskus- } \\
\text { sionen teilnehmen }\end{array}$} & \multicolumn{2}{|c|}{$\begin{array}{l}\text { sich zum Wohl der Mit- } \\
\text { menschen im sozialen } \\
\text { Umfeld engagieren }\end{array}$} & \multicolumn{2}{|c|}{$\begin{array}{c}\text { sich für den Schutz } \\
\text { der Umwelt } \\
\text { engagieren }\end{array}$} \\
\hline & $\begin{array}{c}\text { Mittel- } \\
\text { wert }\end{array}$ & SF & $\begin{array}{l}\text { Mittel- } \\
\text { wert }\end{array}$ & SF & $\begin{array}{c}\text { Mittel- } \\
\text { wert }\end{array}$ & SF & $\begin{array}{c}\text { Mittel- } \\
\text { wert }\end{array}$ & SF & $\begin{array}{c}\text { Mittel- } \\
\text { wert }\end{array}$ & SF & $\begin{array}{c}\text { Mittel- } \\
\text { wert }\end{array}$ & SF \\
\hline Australien & 85 & $(1.0)$ & 89 & $(0.8)$ & 17 & $(1.0)$ & 34 & $(1.1)$ & 80 & $(1.0)$ & 74 & $(1.1)$ \\
\hline Belgien (Frz.) $)^{1}$ & 69 & $(2.0)$ & 82 & $(1.4)$ & 24 & $(1.0)$ & 39 & $(1.4)$ & 54 & $(2.0)$ & 71 & $(1.7)$ \\
\hline Tschechische Rep. & 65 & $(1.7)$ & 66 & $(1.1)$ & 18 & $(1.0)$ & 29 & $(1.4)$ & 78 & $(0.9)$ & 84 & $(1.0)$ \\
\hline Dänemark ${ }^{1}$ & 91 & $(0.7)$ & 60 & $(1.0)$ & 17 & $(0.8)$ & 44 & $(0.9)$ & 86 & $(0.8)$ & 83 & $(0.7)$ \\
\hline England $^{2}$ & 80 & $(1.0)$ & 76 & $(1.1)$ & 20 & $(0.9)$ & 42 & $(1.3)$ & 78 & $(1.0)$ & 76 & $(1.1)$ \\
\hline Finnland & 87 & $(0.7)$ & 59 & $(1.2)$ & 13 & $(0.8)$ & 23 & $(1.1)$ & 60 & $(1.0)$ & 74 & $(1.0)$ \\
\hline Deutschland $^{3}$ & 67 & $(1.1)$ & 69 & $(0.9)$ & 18 & $(0.7)$ & 43 & $(1.2)$ & 85 & $(0.9)$ & 72 & $(1.2)$ \\
\hline Griechenland & 86 & $(0.9)$ & 94 & $(0.6)$ & 49 & $(1.0)$ & 59 & $(1.0)$ & 90 & $(0.7)$ & 89 & $(0.7)$ \\
\hline Ungarn & 91 & $(0.7)$ & 81 & $(0.9)$ & 29 & $(0.9)$ & 21 & $(0.8)$ & 89 & $(0.6)$ & 77 & $(1.0)$ \\
\hline Italien & 80 & $(1.1)$ & 84 & $(0.7)$ & 32 & $(1.0)$ & 49 & $(1.0)$ & 82 & $(0.7)$ & 79 & $(0.8)$ \\
\hline Norwegen ${ }^{1}$ & 87 & $(0.7)$ & 71 & $(0.9)$ & 21 & $(0.8)$ & 37 & $(1.0)$ & 83 & $(0.8)$ & 91 & $(0.6)$ \\
\hline Polen & 88 & $(1.2)$ & 91 & $(0.8)$ & 35 & $(1.2)$ & 53 & $(1.4)$ & 90 & $(0.8)$ & 77 & $(0.8)$ \\
\hline Portugal & 88 & $(0.8)$ & 71 & $(0.9)$ & 36 & $(1.1)$ & 40 & $(1.0)$ & 94 & $(0.6)$ & 92 & $(0.6)$ \\
\hline Slowakische Rep. & 93 & $(0.6)$ & 91 & $(0.7)$ & 23 & $(1.3)$ & 51 & $(1.0)$ & 87 & $(0.8)$ & 87 & $(0.8)$ \\
\hline Schweden $^{2}$ & 75 & $(1.4)$ & 78 & $(1.0)$ & 21 & $(0.9)$ & 37 & $(1.5)$ & 83 & $(1.2)$ & 81 & $(1.3)$ \\
\hline Schweiz & 55 & $(1.3)$ & 68 & $(1.2)$ & 23 & $(1.1)$ & 42 & $(1.2)$ & 76 & $(0.9)$ & 70 & $(1.2)$ \\
\hline Vereinigte Staaten ${ }^{2}$ & 85 & $(1.0)$ & 83 & $(0.9)$ & 48 & $(1.4)$ & 58 & $(1.1)$ & 88 & $(0.8)$ & 83 & $(0.8)$ \\
\hline
\end{tabular}

1. Gesamtbeteiligungsrate unter Berücksichtigung der im Nachrückverfahren einbezogenen Schulen unter 85 Prozent.

2. Grundgesamtheit auf Länderebene entspricht nicht den angestrebten Standards.

3. Erhebungsdatum am Beginn des Schuljahres.

Quelle: IEA-Studie zur politischen Bildung (2001). 


\section{BERUFLICHER STATUS DER ELTERN UND LEISTUNGEN DER SCHÜLER}

- 15-Jährige, deren Eltern einen hohen beruflichen Status aufweisen, erbringen im Durchschnitt bessere Leistungen in allen drei Grundbildungsbereichen. In einigen Ländern ist der Vorteil der betreffenden Schüler jedoch viel größer als in anderen, was insbesondere auf Belgien, Deutschland, Luxemburg und die Schweiz zutrifft.

- Zwar übt der sozio-ökonomische Hintergrund mit den stärksten Einfluss auf die Schülerleistungen aus, aber die Ergebnisse einiger Länder zeigen, dass hohe Durchschnittsleistungen und vom sozialen Hintergrund unbeeinflusste Lernerfolge durchaus Hand in Hand gehen können.

\section{Abbildung A9.1.}

Schülerleistungen und Chancengleichheit (2000)

Zusammenhang zwischen durchschnittlicher Schülerleistung auf der PISA-Skala für Lesekompetenz und Differenz der Schülerleistungen zwischen dem untersten und obersten Quartil der Schüler auf dem internationalen sozio-ökonomischen Index der beruflichen Stellung der Eltern (ISEI)

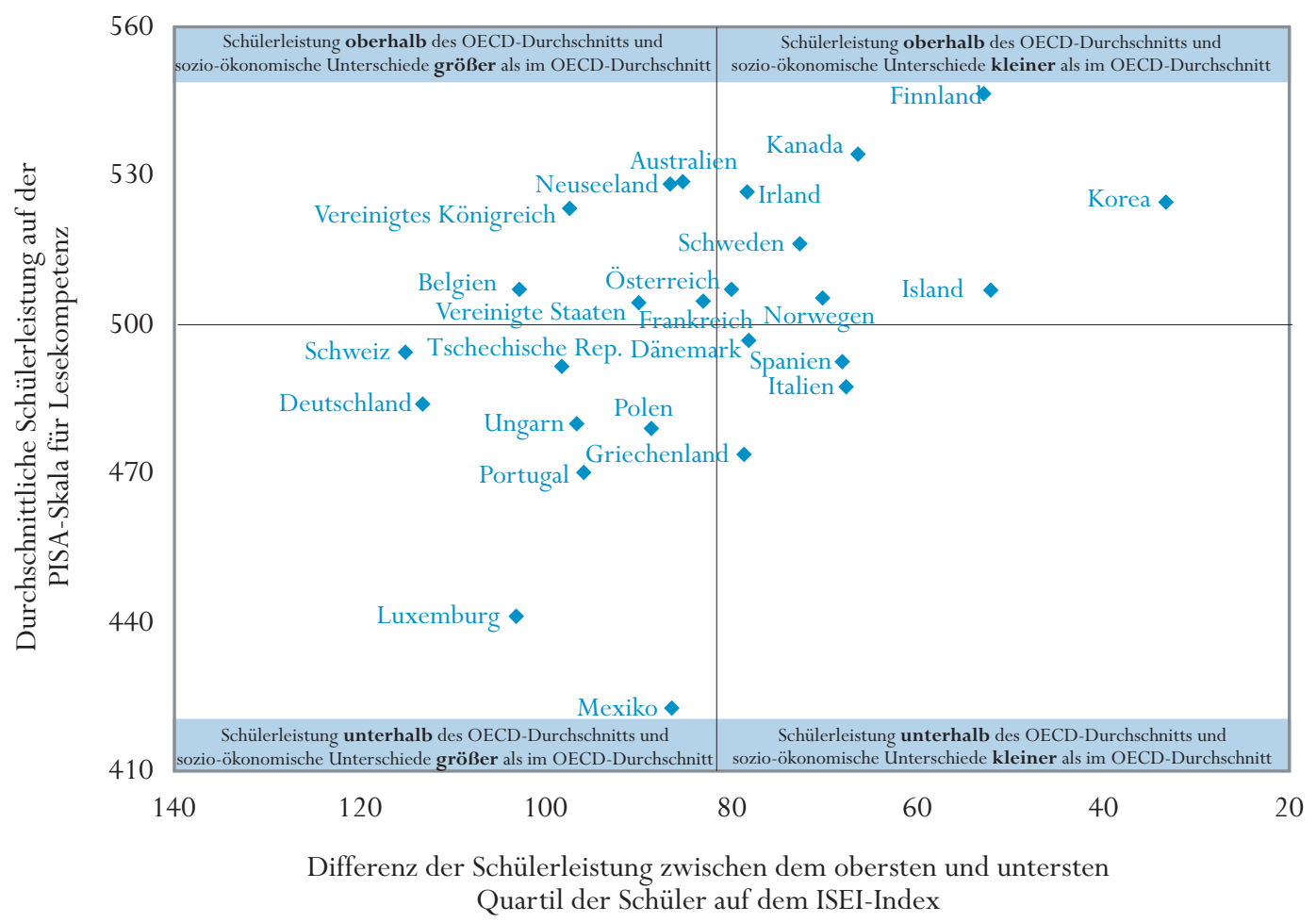

Quelle: OECD PISA-Datenbank, 2001. Tabelle A9.1. Hinweise zur Methodik s. Anhang 3 (www.oecd.org/els/education/eag2002) und www.pisa.oecd.org. 
Die Schulen müssen

Kindern jeglichen

familiären, sozio-

ökonomischen und

kulturellen Hintergunds

gerecht werden, ...

... und die Untersuchung

der Zusammenhänge

zwischen Hintergrund

und Leistungen kann

Pädagogen dabei helfen,

dies effektiver zu tun.

Um dies näher zu

betrachten, untersucht

dieser Indikator die

Beziehung zwischen

den Leistungen 15

Jähriger und ihrem

sozio-ökonomischen

Hintergrund.

Die berufliche Stellung

der Eltern ist ein

Maßs für den sozio-

ökonomischen Status

und kann die Ziele

und Einstellungen der

schüler beeinflussen.

\section{Politischer Hintergrund}

Schüler kommen aus ganz unterschiedlichen familiären, sozio-ökonomischen und kulturellen Verhältnissen. Daher müssen die Schulen sich bemühen, dieser vielfältig zusammengesetzten Schülerschaft adäquate und gleiche Bildungschancen zur Verfügung zu stellen. Das Lernumfeld der Schulen kann durch den unterschiedlichen Hintergrund und die vielfältigen Interessen der Schüler zwar angeregt werden, gleichzeitig erschweren jedoch die Heterogenität der Fähigkeiten und die unterschiedliche Art der Schulvorbereitung die Aufgabe der Schulen, den Anforderungen von Schülern mit ganz unterschiedlichem sozio-ökonomischen Hintergrund gerecht zu werden.

Die Bestimmung der spezifischen Merkmale von Schülern, die mit großer Wahrscheinlichkeit niedrige Leistungen erzielen werden, kann Pädagogen und politischen Entscheidungsträgern helfen, diejenigen Bereiche zu identifizieren, in denen Handlungsbedarf besteht. Ebenso kann die Identifizierung der spezifischen Merkmale von Schülern, die vielleicht hervorragende schulische Leistungen erbringen werden, den politischen Entscheidungsträgern dabei helfen, die Bedingungen für hohe Leistungsniveaus zu fördern. Wenn gezeigt werden kann, dass einige Länder weniger Probleme dabei haben, beiden Gruppen gerecht zu werden, würde dies darauf hindeuten, dass es möglich ist, sowohl Chancengleichheit als auch eine hohe Qualität zu fördern.

Um dieser Frage nachzugehen, untersucht dieser Indikator die Beziehungen zwischen den Schülerleistungen im Bereich Lesekompetenz und einem wichtigen Faktor ihres familiären Hintergrunds, nämlich dem beruflichen Status ihrer Eltern. Die Beziehungen zwischen den Schülerleistungen in den Bereichen mathematischer und naturwissenschaftlicher Grundbildung und dem jeweiligen sozio-ökonomischen Hintergrund sind ähnlich und werden daher in diesem Indikator nicht gesondert aufgeführt.

\section{Ergebnisse und Erläuterungen}

Eine höhere berufliche Stellung der Eltern kann den Berufswunsch sowie die beruflichen Zielsetzungen und Erwartungen der Schüler und damit auch ihren Lerneifer als Mittel zur Erreichung dieser Ziele beeinflussen. Eine hohe berufliche Stellung der Eltern kann auch das Spektrum der im Bewusstsein der Kinder existierenden Optionen erweitern. PISA erfasst diesen Aspekt des familiären Hintergrunds der Schüler durch international vergleichbare Informationen über die Berufe der Eltern sowie die damit in Zusammenhang stehenden Aktivitäten. Der entsprechende sozio-ökonomische Index der beruflichen Stellung, der Werte zwischen 0 und 90 aufweist, misst diejenigen Merkmale eines Berufs, durch die sich die Ausbildung einer Person in ihrem Einkommen niederschlägt. Je höher der Indexwert, umso höher die berufliche Stellung der Schülereltern. Im Durchschnitt der OECD-Länder beträgt der Indexwert 49 und seine Standardabweichung 16. Typische berufliche Tätigkeiten der Eltern von 15-Jährigen mit 16 bis 35 Indexpunkten sind z.B. Kleinbauern, Metallarbeiter, Kfz-Mechaniker, Taxi- und Lkw-Fahrer sowie Kellner. Die 
häufigsten beruflichen Tätigkeiten mit 35 bis 53 Indexpunkten sind Buchhalter, Verkaufspersonal, Manager von Kleinbetrieben oder Pflegepersonal. Je höher die für eine Tätigkeit geforderten Kenntnisse und Fähigkeiten sind, umso höher ist auch der damit verbundene berufliche Status. Zwischen 54 und 70 Indexpunkten liegen z.B. Tätigkeiten wie Marketingmanager, Lehrer, Hoch- und Tiefbauingenieur und Wirtschaftsprüfer. Im oberen internationalen Indexquartil liegen mit Punkten zwischen 71 und 90 berufliche Tätigkeiten wie Mediziner, Hochschullehrer und Juristen.

Wie aus Tabelle A9.1 ersichtlich wird, gehen Unterschiede im sozioökonomischen Index der beruflichen Stellung (der Eltern) mit großen Unterschieden in den Schülerleistungen im Bereich Lesekompetenz innerhalb der einzelnen Länder einher. Bei den Schülern aus den OECD-Ländern, die in ihrem jeweiligen Land nach dem sozio-ökonomischen Index dem obersten Quartil angehören, liegt die mittlere Punktzahl auf der PISA-Skala für Lesekompetenz bei 545 Punkten, also 45 Punkte über dem OECD-Durchschnitt für alle Schüler. Hingegen beträgt das Durchschnittsergebnis für das jeweils unterste Quartil der Schüler nach dem sozio-ökonomischen Index lediglich 463 Punkte. Die Durchschnittsdifferenz zwischen den beiden Gruppen im Bereich Lesekompetenz beträgt mehr als eine ganze Kompetenzstufe.

Die größten Unterschiede, nämlich 100 Punkte und mehr in allen drei Grundbildungsbereichen weisen Belgien, Deutschland, Luxemburg und die Schweiz auf. In Deutschland ist der Unterschied besonders auffallend. Schüler, deren Eltern die höchsten beruflichen Stellungen innehaben (das oberste Quartil auf dem berufsbezogenen Index), erzielen im Durchschnitt ungefähr die gleichen Leistungen wie auf dem Durchschnitt liegende Schüler in Finnland, dem nach PISA leistungsstärksten Land; die Schüler, deren Eltern den niedrigsten beruflichen Status aufweisen, erzielen in etwa die gleichen Leistungen wie die Schüler in Mexiko, dem leistungsschwächsten Land der OECD.

Auch in der Tschechischen Republik, Ungarn, dem Vereinigten Königreich und den Vereinigten Staaten betragen die Unterschiede für Schüler im jeweils obersten und untersten nationalen Quartil des sozio-ökonomischen Index in allen drei Grundbildungsbereichen über 90 Punkte, was weit mehr als eine Kompetenzstufe ausmacht. Wie in Belgien, Deutschland und der Schweiz, so ist auch in diesen Ländern für die Schüler im untersten Quartil des berufsbezogenen Index im Vergleich zu anderen Schülern die Wahrscheinlichkeit, ebenfalls zu den leistungsschwächsten 25 Prozent ihres Landes auf der PISA-Skala für Lesekompetenz zu gehören, mehr als doppelt so groß.

Auch wenn PISA zeigt, dass schlechte schulische Leistungen keine automatische Folge benachteiligter sozio-ökonomischen Verhältnisse sind, scheinen sie doch mit den stärksten Einfluss auf die Leistungen der Schüler auf der PISASkala Lesekompetenz auszuüben. Dies bedeutet eine große Aufgabe für die Politik, die allen Schülern unabhängig von ihrem familiären Hintergrund
Schüler, deren Eltern angesehenere Berufe ausüben, erreichen im Durchschnitt höhere Leistungen, ...

... aber in einigen Ländern ist der Vorteil der betreffenden Schüler ausgeprägter als in anderen. 


\section{A9}

... aber die Ergebnisse

einiger Länder

zeigen, dass hohe

Durchschnittsleistungen

und vom sozialen

Hintergrund

unbeeinflusste

Lernerfolge durchaus

Hand in Hand gehen

können.

Die erzielten Punktzahlen

basieren auf Leistungsmes-

sungen, die Teil der im Jahr

2000 von der OECD durchge-

führten Internationalen Schul-

leistungsstudie PISA waren.
Lernmöglichkeiten bieten will. Die Ergebnisse nationaler Untersuchungen in den einzelnen Ländern waren diesbezüglich im Allgemeinen entmutigend. Die Schulen haben hier offensichtlich wenig erreicht. Sei es, weil Familien aus privilegierten Verhältnissen eher in der Lage sind, den Effekt der Schulen zu verstärken und zu verbessern, oder weil es den Schulen besser gelingt, junge Menschen zu erziehen und zu fördern, die aus privilegierten Verhältnissen kommen. Es zeigte sich auf jeden Fall häufig, dass die Schulen die bereits existierenden Privilegien reproduzieren, anstatt allen gleiche Möglichkeiten zu bieten und auf diese Weise eine ausgewogenere Verteilung der Bildungserträge zu gewährleisten.

Die Ergebnisse von PISA sind ermutigender. Zwar besteht in allen Ländern ein klarer Zusammenhang zwischen dem familiären Hintergrund der Schüler und den Bildungserfolgen, einige Länder zeigten jedoch, dass eine hohe durchschnittliche Qualität mit einer ausgewogenen Verteilung der Bildungserfolge einhergehen kann. In Finnland, Island, Kanada, Korea und Schweden liegt das Leistungsniveau der Schüler auf der PISA-Skala für Lesekompetenz über dem Durchschnitt, während gleichzeitig die Leistungsunterschiede zwischen in sozio-ökonomischer Hinsicht privilegierten und benachteiligten Schülern unterdurchschnittlich stark ausgeprägt sind (Abb. 9.1). Umgekehrt liegen die durchschnittlichen Ergebnisse der Schüler auf der PISA-Skala für Lesekompetenz in Deutschland, Luxemburg, Polen, Portugal, der Tschechischen Republik und Ungarn deutlich unter dem OECD-Durchschnitt, während die Leistungsunterschiede zwischen in sozio-ökonomischer Hinsicht privilegierten und benachteiligten Schülern dort überdurchschnittlich groß sind.

Es darf jedoch nicht davon ausgegangen werden, dass alle diese Differenzen ein unmittelbares Ergebnis der auf dem familiären Hintergrund beruhenden Vorteile und der durch Eltern mit angesehenen beruflichen Positionen vermittelten Erwartungen sind. Die Leistungen der Schüler in den drei Grundbildungsbereichen werden von vielen Faktoren beeinflusst. Der sozioökonomische Status kann z.B. mit dem Ort zusammenhängen, an dem die Schüler leben, und mit der Qualität der Schulen, zu denen sie Zugang haben (dies würde in Schulsystemen von Bedeutung sein, die von lokalen Steuern abhängig sind), mit der Wahrscheinlichkeit des Besuchs von Privatschulen, dem Grad der Unterstützung durch die Eltern und deren Engagement, usw.

\section{Definitionen und angewandte Methodik}

Die für diesen Indikator untersuchte Zielpopulation waren 15-jährige Schüler. In der Praxis bezieht sich dies auf Schüler, die zu Beginn der Testperiode im Alter von 15 Jahren und 3 (abgeschlossenen) Monaten bis zu 16 Jahren und 2 (abgeschlossenen) Monaten waren und eine Bildungseinrichtung besuchten, ungeachtet der Klassenstufe und der Art der von ihnen besuchten Bildungseinrichtung und unabhängig davon, ob es sich um eine Ganztags- oder Halbtagsschule handelte. 
Der in der PISA-Studie verwendete Internationale sozio-ökonomische Index der beruflichen Stellung (ISEI) wurde von den Schülerantworten auf die Frage nach den Berufen der Eltern abgeleitet. Der Index erfasst die Merkmale von Berufen, durch die sich die Bildungsabschlüsse der Eltern in deren Einkommen niederschlagen. Zur Konstruktion dieses Index wurden die Berufsgruppen auf einer linearen Skala so angeordnet, dass der indirekte Einfluss der Bildung auf das Einkommen mittels der Bildungsabschlüsse maximiert und der direkte Einfluss der Bildung auf das Einkommen, unabhängig vom Beruf, minimiert wurde (bei beiden Effekten wurde dem Alter nicht Rechnung getragen). Der in PISA verwendete Internationale sozio-ökonomische Index der beruflichen Stellung basiert auf dem Beruf des Vaters oder der Mutter, je nachdem wer die höhere Stellung hat. Die Werte auf dem Index reichen von 0 bis 90, niedrige Werte repräsentieren einen niedrigen und hohe Werte einen hohen sozioökonomischen Status. Weitere Informationen über die Methodik s. PISA 2000 Technical Report (OECD, 2002).

Hinweise zu Standardfehlern, Signifikanztests und multiplen Vergleichen s. Anhang 3 unter www.oecd.org/els/education/eag2002. 
Tabelle A9.1.

Schülerleistungen und sozio-ökonomischer Status der Eltern (2000)

Internationaler sozio-ökonomischer Index der beruflichen Stellung der Eltern (ISEI) und Leistungen auf der PISA-Skala für Lesekompetenz, nach Indexquartilen für das jeweilige Land. Die Ergebnisse basieren auf Schülerangaben.

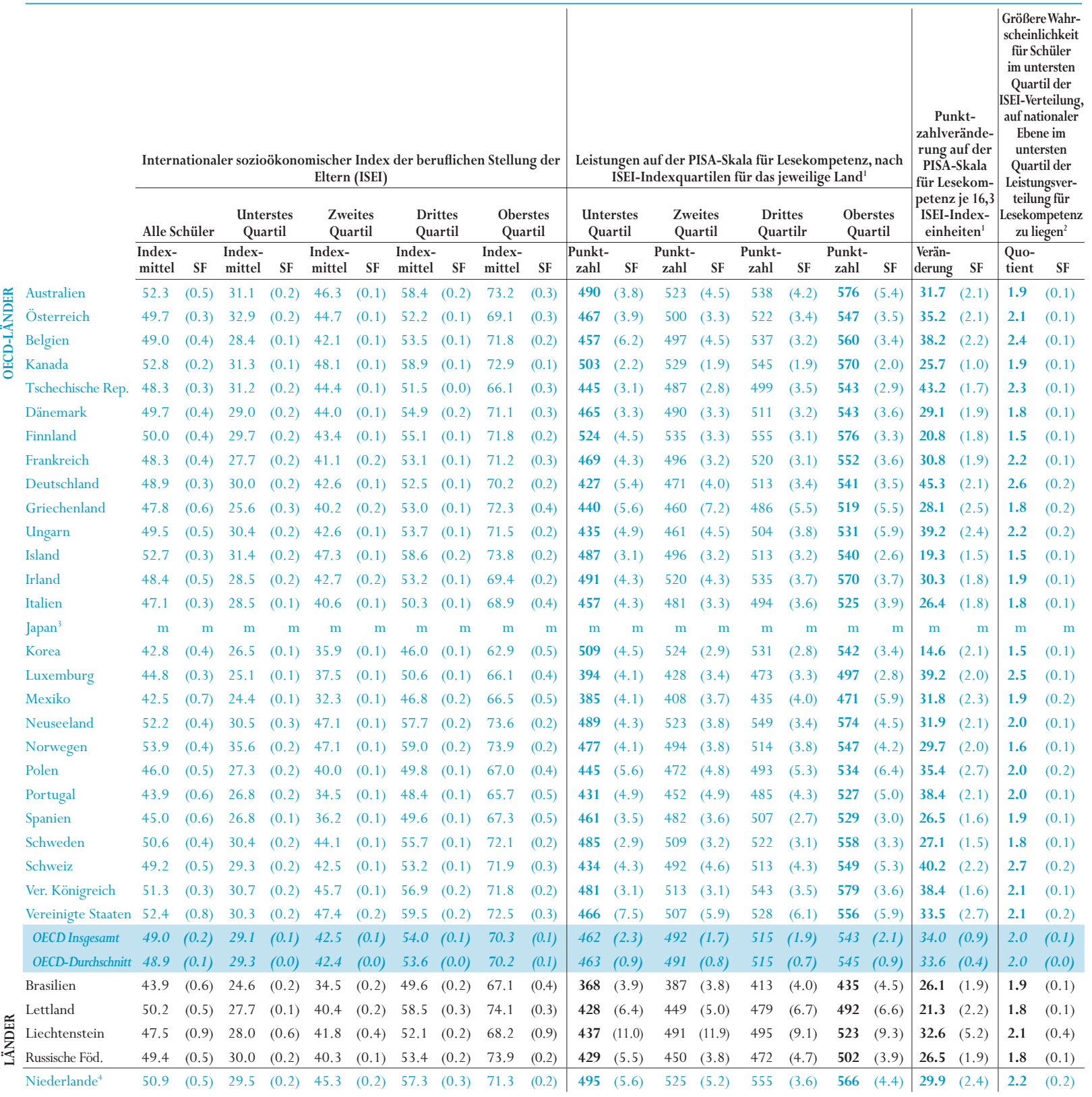

1. Fettgedruckte Veränderungen der Indexeinheiten sind statistisch signifikant. Wenn die untersten und obersten Quartile fettgedruckt sind, weist dies auf eine statistisch signifikante Differenz zwischen diesen Quartilen hin. 16,3 Indexeinheiten entsprechen einer internationalen Standardabweichung.

2. Statistisch signifikante Quotienten von mehr als 1 durch Fettdruck gekennzeichnet.

3. Japan bei diesem Vergleich wegen großteils fehlender Daten nicht berücksichtigt.

4. Die Beteiligungsquote ist zu niedrig, um die Vergleichbarkeit zu gewährleisten (s. Anhang 3, www.oecd.org/els/education/eag2002).

Quelle: OECD PISA-Datenbank, 2001. Hinweise zur Methodiks. Anhang 3 (www.oecd.org/els/education/eag2002) und www.pisa.oecd.org. 


\section{GEBURTSLAND, ZU HAUSE GESPROCHENE SPRACHE UND LESEKOMPETENZ 15-JÄHRIGER}

- In den meisten Ländern mit einer großen Migrantenpopulation liegt die Lesekompetenz von im Erhebungsland geborenen Schülern mit im Ausland geborenen Eltern weit unter der von im Erhebungsland geborenen Schülern mit mindestens einem im Inland geborenen Elternteil.

- Nicht im Erhebungsland geborene Schüler liegen im Vergleich dazu noch weiter zurück, wenn auch in den einzelnen Ländern in unterschiedlichem Ausmaß

- Es überrascht nicht, dass Schüler ohne einen majoriätssprachlichen Hintergrund wesentlich schlechter abschneiden als Schüler mit einem solchen und mit wesentlich größerer Wahrscheinlichkeit zu den leistungsschwächsten Schülern im betreffenden Land gehören.

- Es gibt jedoch einige Länder, in denen Schüler, in deren Elternhaus normalerweise die Unterrichtssprache nicht gesprochen wird, doch relative gute Leistungen im Bereich Lesekompetenz erbringen. So erzielen beispielsweise Schüler in Australien und Kanada Punktzahlen, die um den OECD-Durchschnitt herum liegen und ähnlich dem Durchschnitt vieler Länder sind, die nur wenige Schüler mit minoritätssprachlichem Hintergrund haben. 
Geburtsort, zu Hause gesprochene Sprache und Schülerleistung auf der PISA-Skala für Lesekompetenz (2000)

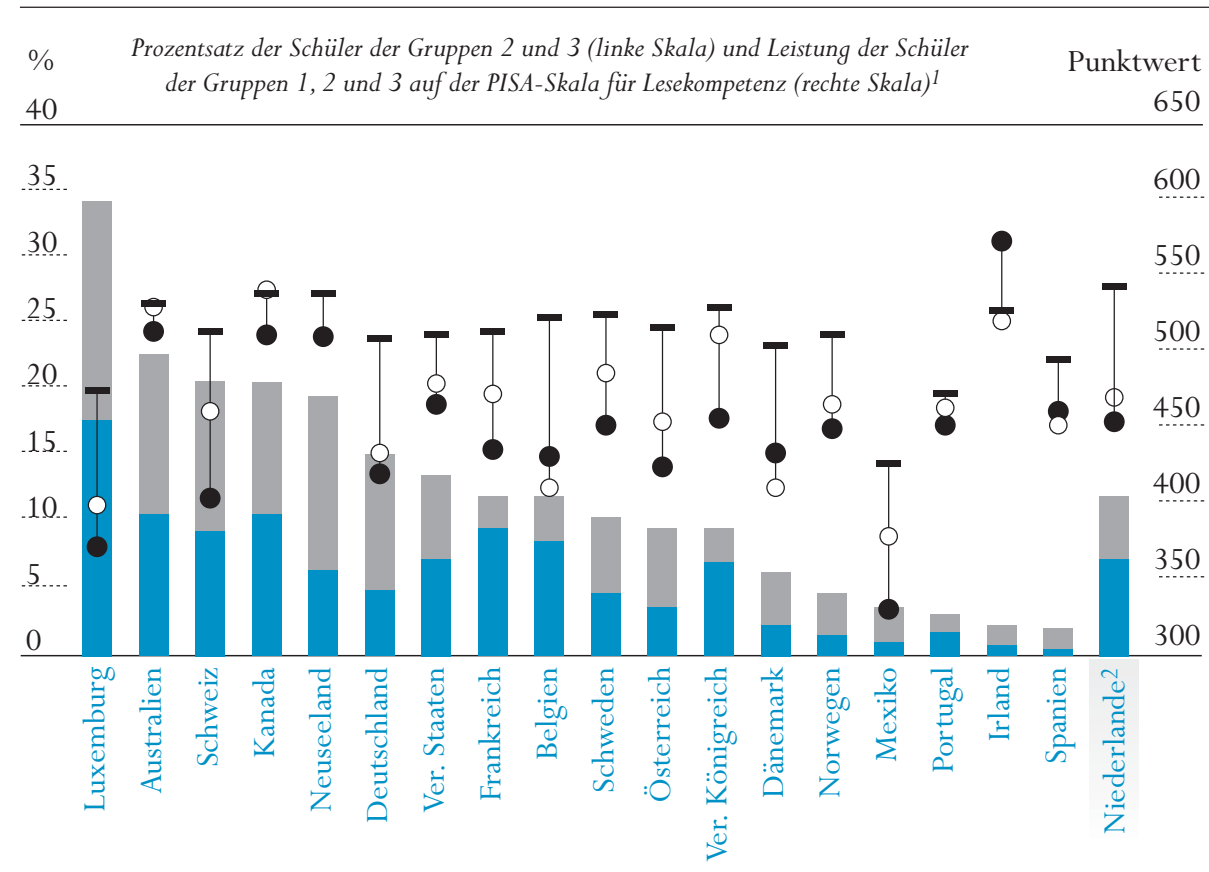

Prozentsatz der Schüler, die zu Hause normalerweise eine andere Sprache als die Unterrichtssprache,

$\% \quad$ eine andere offizielle Landessprache oder andere nationale Dialekte sprechen (linke Skala) und Punktwert 20 Leistung der Schüler auf der PISA-Skala für Lesekompetenz nach Sprachgruppen (rechte Skala) ${ }^{1}$

600

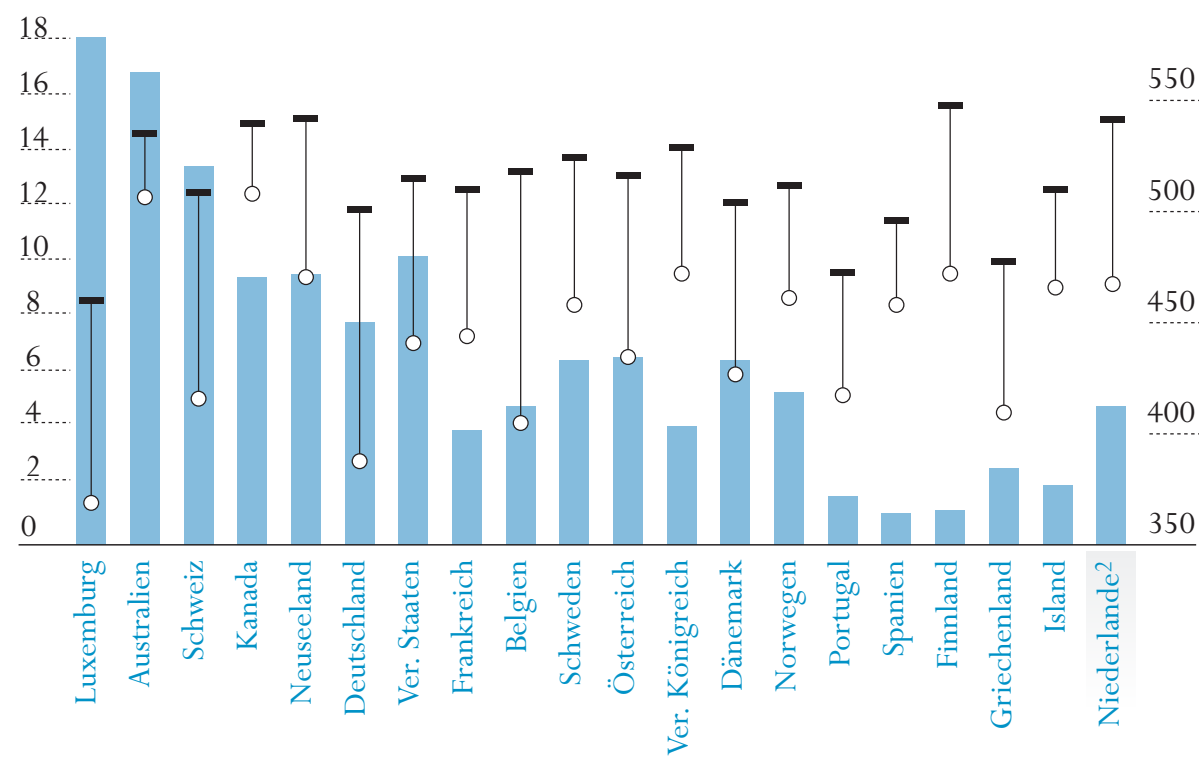

1. Abbildung enthält nur Länder, deren Anteil an Schülern der Gruppe 2 drei Prozent übersteigt.

2. Die Beteiligungsquote ist zu niedrig, um die Vergleichbarkeit zu gewährleisten (s. Anhang 3).

3. Abbildung enthält nur Länder, deren Anteil an Schülern, die zu Hause normalerweise eine andere Sprache als die Unterrichtssprache, eine andere offizielle Landessprache oder andere nationale Dialekte sprechen, drei Prozent übersteigt.

Anordnung der Länder in absteigender Reihenfolge des Prozentsatzes der Schüler der Gruppen 2 und 3 zusammen. Quelle: OECD PISA-Datenbank, 2001. Tabellen A10.1 und A10.2. Hinweise zur Methodik

s. Anhang 3 (www.oecd.org/els/education/eag2002) und www.pisa.oecd.org.
Linke Skala

Prozentsatz im Ausland geborener Schüler mit im Ausland geborenen Eltern (Gruppe 3)

Prozentsatz im Inland geborener Schüler mit im Ausland geborenen Eltern (Gruppe 2)

Rechte Skala

- Mittlere Leistungen auf der PISA-Skala für

Lesekompetenz von im Inland geborenen Schülern mit mindestens einem im Inland geborenen Elternteil (Gruppe 1)

$\bigcirc$ Mittlere Leistungen auf der PISA-Skala für

Lesekompetenz von im Inland geborenen Schülern mit im Ausland geborenen Eltern (Gruppe 2)

- Mittlere Leistungen auf der PISA-Skala für Lesekompetenz von im Ausland geborenen Schülern mit im Ausland geborenen Eltern (Gruppe 3)

\section{Linke Skala}

Prozentsatz der Schüler, die zu Hause normalerweise eine andere Sprache als die Unterrichtssprache, eine andere offizielle Landessprache oder andere nationale

Dialekte sprechen

Rechte Skala

- Mittlere Leistungen auf der PISA-Skala für

Lesekompetenz von

Schülern, die zu

Hause normalerweise eine andere Sprache als die

Unterrichtssprache, eine andere offizielle Landessprache oder andere nationale

Dialekte sprechen

Mittlere Leistungen auf der PISA-Skala für

Lesekompetenz von Schülern, die zu Hause normalerweise die gleiche Sprache wie die Unterrichtssprache, eine andere offizielle Landessprache oder andere nationale Dialekte sprechen 


\section{Politischer Hintergrund}

Migration von einem Land in ein anderes wird immer mehr zur Normalität, da die internationalen Handelsbeziehungen sich ausweiten, Beschäftigungsmöglichkeiten die Menschen dorthin ziehen, wo ihnen bessere oder andere Existenzgrundlagen geboten werden, und Staaten Flüchtlingen Zuflucht gewähren, die vor politischen Unruhen oder wirtschaftlicher Not geflohen sind. Unabhängig von den Gründen, die Menschen zur Migration veranlassen, sehen sich ihre schulpflichtigen Kinder häufig einem neuen Umfeld gegenüber, dessen Unterrichtssprache ihnen vielleicht auch noch fremd ist. Gezwungen, in einer Sprache, die nicht ihre Muttersprache ist, zu lernen, und sich vielleicht auch noch einer neuen sozio-ökonomischen Umgebung anzupassen, kann man bei einigen dieser Migrantenkinder davon ausgehen, dass sie wahrscheinlich leistungsmäßig hinter denjenigen ihrer Altersgenossen zurückbleiben werden, bei denen die Muttersprache auch die Unterrichtssprache ist.

Eine länderübergreifende Analyse kann gewisse Erkenntnisse darüber liefern, wie einige Länder diesen Ungleichheiten erfolgreicher begegnen als andere.

\section{Ergebnisse und Erläuterungen}

Um die mit dem Migrationsstatus und der gesprochenen Sprache verbundenen Auswirkungen auf die Leistungen im Bereich Lesekompetenz untersuchen zu können, wurden die Schüler in dem PISA-Kontextfragebogen aufgefordert anzugeben, ob sie selbst und jedes ihrer beiden Elternteile in dem Land geboren wurden, in dem sie leben, oder in einem anderen Land. Sie wurden ebenfalls gefragt, welche Sprache sie normalerweise zu Hause sprechen.

Es ist wichtig, den begrenzten Charakter der vorhandenen Daten zu erkennen. PISA stellte den Schülern nicht die Frage, wie lange sie schon in dem Land leben, in dem die Erhebung durchgeführt wurde. Einige, wenn nicht sogar viele der Schüler, die nicht in dem Land geboren wurden, in dem die Erhebung durchgeführt wurde, hatten vielleicht schon den größten Teil ihres Lebens in diesem Land zugebracht und sprachen wahrscheinlich die Unterrichtssprache fließend. Andere waren zweifellos erst vor kurzer Zeit zugewandert und besuchten die Schule in ihrem „neuen“ Land lediglich im zweiten Jahr. Bei der Interpretation der Daten ist auch zu berücksichtigen, dass Schüler, die nicht in der Lage waren, die Unterrichtssprache zu lesen bzw. zu sprechen, da sie bis zum Zeitpunkt der Erhebung weniger als ein Jahr Unterricht in dieser Sprache hatten, in der Erhebung nicht erfasst sind. Es liegen ebenfalls keine Informationen darüber vor, wie ähnlich oder verschieden die Muttersprache eines Schülers im Vergleich zur Unterrichtssprache ist, was natürlich Auswirkungen auf die sprachlichen Fähigkeiten in der Zweitsprache haben könnte. Schließlich kann sich auch die sozio-ökonomische Zusammensetzung der Migranten-Population von Land zu Land unterscheiden.
Verstärkte

Migrationsbewegungen

stellen die

Bildungssysteme vor

große Aufgaben. Ein

vergleichender Blick,

wie in diesem Indikator.

auf Länder, die diese

Aufgaben erfolgreich

angehen, kann dafür

wichtige politische

Ansatzpunkte liefern.

PISA ermöglicht

es, Leistungen von

schülern mit ihrem

Migrationsstatus und

der im Elternhaus

gesprochenen Sprache

in Relation zu

setzen, es gibt jedoch

Einschränkungen

hinsichtlich der

Interpretierbarkeit dieser

Daten. 
Die Analyse vergleicht ...

...Schüler der Gruppe 1 ...

... der Gruppe 2...

... und der Gruppe 3.

Für viele Schüler, die

im Ausland geboren

wurden oder deren

Eltern aus dem Ausland

stammen, ist die Sprache

ein Schlüsselfaktor.

In den meisten Ländern mit einem bedeutsamen

Anteil an Migranten

liegen die Schüler

der Gruppe 2 in der

Lesekompetenz weit

unter dem Niveau der schüler der Gruppe 1, ...

... und das

beunruhigenderweise, obwohl sie selbst im

Erhebungsland geboren wurden.

\section{Geburtsland}

Um den Einfluss des Geburtslandes auf die Leistungen zu bewerten, wurden drei Schülerkategorien miteinander verglichen:

- Schüler, die im Erhebungsland geboren wurden und bei denen zumindest ein Elternteil ebenfalls in diesem Land geboren wurde (nachfolgend als „Gruppe 1" bezeichnet);

- Schüler, die im Erhebungsland geboren wurden, deren Eltern jedoch in einem anderen Land geboren wurden (nachfolgend als „Gruppe 2“ bezeichnet); und

- Schüler, die außerhalb des Erhebungslandes geboren wurden und deren Eltern ebenfalls in einem anderen Land geboren wurden (nachfolgend als „Gruppe 3“ bezeichnet).

Für viele Schüler der Gruppe 3 ist die Unterrichtssprache eine Zweitsprache (s. den zweitenTeil dieses Indikators, in dem es um die im Elternhaus gesprochene Sprache geht), und einige von ihnen haben noch keine langjährigen Erfahrungen mit dem Bildungssystem des Landes, in dem der Test stattfand. Die Schüler der Gruppe 2 leben zudem vielleicht in Familien, in denen die Erstsprache bzw. die zu Hause gesprochene Sprache nicht die Unterrichtssprache ist. Die zu Gruppe 2 und 3 gehörenden Schüler müssen unabhängig von ihrem Geburtsland, bzw. dem ihrer Eltern, Kenntnisse und Fähigkeiten erwerben, die von denen der Gruppe 1 erwartet werden, je näher sie dem Abschluss ihrer regulären Bildung kommen.

Bei einem Vergleich der Lesekompetenz von Schülern der Gruppe 2 mit der von Schülern der Gruppe 1 in den 14 Ländern, in denen Schüler der Gruppe 2 mindestens 3 Prozent der in PISA 2000 erfassten Schüler ausmachen, zeigen sich in 10 dieser 14 Länder verhältnismäßig große und statistisch signifikante Unterschiede zu Gunsten der Schüler der Gruppe 1 (s. Abb. A10.1). Auf der PISA-Skala für Lesekompetenz betragen die Leistungsunterschiede in Frankreich, Neuseeland, Schweden und den Vereinigten Staaten, zwischen 31 und 41 Punkten, in Liechtenstein, Österreich und der Schweiz ungefähr 53 bis 62 Punkte, und in Deutschland, Luxemburg und den Niederlanden mehr als 70 Punkte, d.h. ungefähr eine volle Kompetenzstufe. In Belgien, dem Land mit den größten Unterschieden, betragen sie 112 Punkte.

Diese Unterschiede sind beunruhigend, da beide Schülergruppen im Erhebungsland geboren wurden und anzunehmen ist, dass für sie die gleichen Lehrpläne und auch Vorteile galten, die im Rahmen des jeweiligen nationalen Bildungssystems allen Schülern geboten werden. Trotz möglicher Ähnlichkeiten des individuellen schulischen "Werdegangs“ ist die Tatsache, zur Gruppe 2 zu gehören, in diesen Ländern in irgendeiner Form mit einem relativen Nachteil hinsichtlich der Lesekompetenz verbunden. Besorgnis über derartige 
Differenzen mag besonders in den Ländern angebracht sein, in denen Schüler der Gruppe 2 signifikant schlechter als Schüler der Gruppe 1 abschneiden und in denen es einen verhältnismäßig großen Anteil an Schülern der Gruppe 2 gibt. Hierzu gehören Luxemburg (ungefähr 18 Prozent), Liechtenstein (ungefähr 10 Prozent) und die Schweiz (ungefähr 9 Prozent).

Ein weiterer Vergleich, den man durchführen kann, ist der zwischen Schülern der Gruppen 3 und 1. Angesichts der Unterschiede in vielen Ländern zwischen Schülern der Gruppen 1 und 2 und der Unterschiede in einigen Ländern zwischen Schülern der Gruppen 2 und 3 wäre zu erwarten, dass sich der größte Gesamtunterschied zwischen Schülenr der Gruppen 3 und 1 findet. Bei 13 von 14 Ländern unterstützen die gefundenen Daten diese Annahme. Im Durchschnitt übertreffen Schüler der Gruppe 1 ihre Mitschüler der Gruppe 3 in diesen 14 Ländern bei der Lesekompetenz um 73 Punkte, d.h. um eine volle Kompetenzstufe. Die Unterschiede reichen von 103 bis 112 Punkten in Liechtenstein, Luxemburg und der Schweiz und von 72 bis 93 Punkten in Belgien, Deutschland, Frankreich, den Niederlanden, Österreich, Schweden und im Vereinigten Königreich. Die geringsten signifikanten Unterschiede finden sich in Kanada (27 Punkte), Neuseeland (30 Punkte) und den Vereinigten Staaten (45 Punkte). Australien ist mit einem Unterschied von 19 Punkten das einzige Land, in dem die Leistungsunterschiede zwischen den Schülern dieser beiden Gruppen nicht signifikant sind.

Bei einem Vergleich der Leistungen im Bereich Lesekompetenz zwischen den Schülern der Gruppen 2 und 3 in denselben 14 Ländern ergibt sich bei sechs der Länder kein statistisch signifikanter Unterschied (Australien, Belgien, Deutschland, die Niederlande, Neuseeland und die Vereinigten Staaten). Mit anderen Worten finden sich in PISA keine mit dem Geburtsort (d.h. im Erhebungsland oder im Ausland geboren) verbundenen Nachteile bei der Lesekompetenz von Schülern, deren Eltern nicht im Erhebungsland geboren sind. Zu den übrigen 8 Ländern, in denen die Leistungsunterschiede zwischen Schülern der Gruppe 2 und 3 statistisch signifikant sind, zählen Frankreich, Kanada, Liechtenstein, Luxemburg, Österreich, Schweden, die Schweiz und das Vereinigte Königreich. In diesen Ländern erzielen Schüler der Gruppe 3 zwischen 28 und 58 Punkte weniger bei der Lesekompetenz als Schüler der Gruppe 2, obwohl sie absolut gesehen im Vergleich mit Schülern der Gruppe 3 in anderen Ländern immer noch gut abschneiden. Schüler der Gruppe 3 stellten ungefähr 2 Prozent der an PISA 2000 teilnehmenden Schüler in Frankreich und dem Vereinigten Königreich, etwas weniger als 6 Prozent in Österreich und Schweden, und zwischen 9 und 16 Prozent in den anderen vier Ländern, in denen die Unterschiede zwischen diesen beiden Gruppen signifikant sind.

\section{Im Elternhaus gesprochene Sprache}

Eine andere Möglichkeit, Fragen des Zusammenhangs von Migrantenstatus und erzielten Leistungen zu untersuchen, ist die Beschäftigung mit der im Elternhaus gesprochenen Sprache. Um deren Auswirkung auf die von den
Nicht im Erhebungsland geborene Schüler liegen noch weiter zurück, wenn auch in den einzelnen Ländern in unterschiedlichem Ausmafs.

Die Unterschiede zwischen Schülern der Gruppen 2 und 3 sind in der Regel geringer.

Es überrascht nicht, dass Schüler ohne einen majoritätssprachlichen 
Hintergrund wesentlich schlechter abschneiden als diejenigen mit einem solchen,...

... und mit größserer Wahrscheinlichkeit in jedem Land zu den leistungsschwächsten 25 Prozent der Schüler gehören, ...

...mit Ausnahme der englischsprachigen Länder, in denen diese Unterschiede tendenziell geringer sind.
Schülern im Bereich Lesekompetenz erbrachten Leistungen zu untersuchen, wurden zwei Gruppen von Schülern miteinander verglichen:

- Schüler, die im Elternhaus normalerweise die Unterrichtssprache oder eine andere Landessprache bzw. einen nationalen Dialekt sprechen („Schüler mit majoritätssprachlichem Hintergrund“), und

- Schüler, die mit ihren Eltern und Geschwistern normalerweise in einer anderen Sprache kommunizieren („Schüler mit minoritätssprachlichem Hintergrund").

Im Durchschnitt der 17 Länder, in denen mindestens 3 Prozent der an PISA teilnehmenden Schüler der letzteren Gruppe angehörten, übertrafen die Schüler mit majoritätssprachlichem Hintergrund diejenigen mit minoritätssprachlichem Hintergrund (s. Abb. A10.1). In den OECD-Ländern beträgt die durchschnittliche Differenz bei der Lesekompetenz zwischen den beiden Gruppen 66 Punkte. Die Bandbreite der Unterschiede reicht von 30 bis 34 Punkten in Australien, Kanada und der Russischen Föderation bis zu ungefähr 114 Punkten in Belgien und Deutschland.

Aus diesen Unterschieden ergibt sich u.a., dass für diejenigen 15-jährigen Schüler in Belgien, Dänemark, Deutschland, Luxemburg und der Schweiz, die zu Hause nicht die Unterrichtssprache oder eine andere offizielle Landessprache bzw. einen nationalen Dialekt sprechen, die Wahrscheinlichkeit wenigstens zweieinhalbmal so hoch ist, zu den leistungsschwächsten 25 Prozent der Schüler im Bereich Lesekompetenz zu gehören, wie für Schüler, die normalerweise die Unterrichtssprache sprechen. In Frankreich, Griechenland, den Niederlanden, Neuseeland, Österreich, Schweden und den Vereinigten Staaten ist für Schüler mit minoritätssprachlichem Hintergrund die Wahrscheinlichkeit mehr als zweimal so hoch, in diesem Bereich im untersten Quartil zu liegen, wie für Schüler mit majoritätssprachlichem Hintergrund.

Es gibt jedoch einige Länder, in denen Schüler, in deren Elternhaus normalerweise die Unterrichtssprache nicht gesprochen wird, doch relative gute Leistungen im Bereich Lesekompetenz erbringen. So erzielen beispielsweise Schüler in Australien und Kanada Punktzahlen, die um den OECD-Durchschnitt herum liegen und ähnlich dem Durchschnitt vieler Länder sind, die nur wenige Schüler mit minoritätssprachlichem Hintergrund haben.

Ein interessantes Ergebnis ist, dass Schüler mit minoritätssprachlichem Hintergrund in den englischsprachigen Ländern relativ gut abschneiden. Der durchschnittliche Unterschied im Bereich Lesekompetenz zwischen Schülern mit majoritäts- und minoritätssprachlichem Hintergrund beträgt in den fünf hauptsächlich englischsprachigen Ländern (Australien, Kanada, Neuseeland, Vereinigtes Königreich und Vereinigte Staaten) 54 Punkte. Schüler mit minoritätsprachlichem Hintergrund kommen auch in Dänemark, den Niederlanden, Norwegen und Schweden, in denen sie große Anteile der 
Schülerschaft stellen, mit einem mittleren Unterschied bei der Lesekompetenz von 66 Punkten relativ gut zurecht. Im Gegensatz dazu ergaben sich bei den Schüler mit minoritätssprachlichem Hintergund in den deutschsprachigen OECD-Ländern mit einem durchschnittlichen Unterschied von 95 Punkten die größten Unterschiede.

\section{Definitionen und angewandte Methodik}

Die für diesen Indikator untersuchte Zielpopulation waren 15-jährige Schüler. In der Praxis bezieht sich dies auf Schüler, die zu Beginn der Testperiode im Alter von 15 Jahren und 3 (abgeschlossenen) Monaten bis zu 16 Jahren und 2 (abgeschlossenen) Monaten waren und eine Bildungseinrichtung besuchten, ungeachtet der Klassenstufe und der Art der von ihnen besuchten Bildungseinrichtung und unabhängig davon, ob es sich um eine Ganztags- oder Halbtagsschule handelte.

Hinsichtlich der im Elternhaus gesprochenen Sprache wurden die Schüler im PISA-Kontextfragebogen gefragt: „Welche Sprache sprecht ihr normalerweise zu Hause?“. Die Schüler konnten antworten, dass sie die Sprache, in der der Test vorgenommen wurde, eine „andere offizielle Landessprache“, ,andere nationale Dialekte “ oder „andere Sprachen“ sprechen. Die in diesem Zusammenhang aufgeführten Daten vergleichen Schüler der letzten Gruppe (d.h. „andere Sprachen“) mit Schülern in den ersten drei Gruppen.

Tabelle A10.2 enthält eine Angabe der erhöhten Wahrscheinlichkeit, mit der Schüler mit einem bestimmten Merkmal zu dem untersten Quartil der Verteilung der Schülerleistungen auf der PISA-Skala für Lesekompetenz gehören werden. Dies ist ein Maß für die relative Wahrscheinlichkeit. So bedeutet beispielsweise der Wert "2" für die erhöhte Wahrscheinlichkeit eines Schülers, der im Elternhaus normalerweise nicht die Unterrichtssprache spricht, Leistungen im untersten Quartil der Leistungsverteilung zu erreichen, dass Schüler, in deren Elternhaus normalerweise nicht die Unterrichtssprache gesprochen wird, eine zweimal so hohe Wahrscheinlichkeit haben, mit ihren Leistungen im untersten Quartil eingestuft zu werden als Schüler, die normalerweise im Elternhaus die Unterrichtssprache sprechen.

Hinweise zu Standardfehlern, Signifikanztests und multiplen Vergleichen s. Anhang 3 unter www.oecd.org/els/education/eag2002.
Die erzielten Punktzahlen basieren auf Leistungsmessungen, die Teil der im Jahr 2000 von der OECD durchgeführten Internationalen Schulleistungsstudie PISA waren. 
Tabelle A10.1.

Leistung im Bereich Lesekompetenz und Geburtsland 15-jähriger Schüler und Geburtsland ihrer Eltern (2000) Aufteilung der Schüler nach ihrem Geburtsland und dem ihrer Eltern (laut Schülerangaben) sowie ihre Leistungen auf der PISA-Skala für Lesekompetenz

\begin{tabular}{|c|c|c|c|c|c|c|c|c|c|c|c|c|}
\hline & \multicolumn{4}{|c|}{$\begin{array}{l}\text { Gruppe } 1 \\
\text { (im Inland geborene Schüler mit mindestens } \\
\text { einem im Inland geborenen Elternteil) }\end{array}$} & \multicolumn{4}{|c|}{$\begin{array}{c}\text { Gruppe } 2 \\
\text { (im Inland geborene Schüler mit im Ausland } \\
\text { geborenen Eltern) }\end{array}$} & \multicolumn{4}{|c|}{$\begin{array}{c}\text { Gruppe } 3 \\
\text { (im Ausland geborene Schüler mit im Ausland } \\
\text { geborenen Eltern) }\end{array}$} \\
\hline & \multirow[b]{2}{*}{$\begin{array}{c}\text { Prozentsatz der } \\
\text { Schüler }^{1}\end{array}$} & \multirow[b]{2}{*}{ SF } & \multicolumn{2}{|c|}{$\begin{array}{l}\text { Ergebnis auf der } \\
\text { PISA-Skala für } \\
\text { Lesekompetenz }^{2}\end{array}$} & \multirow[b]{2}{*}{$\begin{array}{c}\text { Prozentsatz der } \\
\text { Schüler }^{1}\end{array}$} & \multirow[b]{2}{*}{ SF } & \multicolumn{2}{|c|}{$\begin{array}{l}\text { Ergebnis auf der } \\
\text { PISA-Skala für } \\
\text { Lesekompetenz }^{2}\end{array}$} & \multirow[b]{2}{*}{$\begin{array}{c}\text { Prozentsatz der } \\
\text { Schüler }^{1}\end{array}$} & \multirow[b]{2}{*}{ SF } & \multicolumn{2}{|c|}{$\begin{array}{l}\text { Ergebnis auf der } \\
\text { PISA-Skala für } \\
\text { Lesekompetenz }^{2}\end{array}$} \\
\hline & & & $\begin{array}{c}\text { Punkt- } \\
\text { zahl }\end{array}$ & $\mathrm{SF}$ & & & $\begin{array}{c}\text { Punkt- } \\
\text { zahl }\end{array}$ & SF & & & $\begin{array}{c}\text { Punkt- } \\
\text { zahl }\end{array}$ & SF \\
\hline Australien & 77.4 & $(1.8)$ & 532 & $(3.6)$ & 10.7 & $(1.1)$ & 528 & $(7.1)$ & 11.9 & $(1.2)$ & 513 & $(9.3)$ \\
\hline Österreich & 90.4 & $(0.9)$ & 515 & $(2.4)$ & 3.7 & $(0.4)$ & 453 & $(9.4)$ & 5.9 & $(0.6)$ & 422 & $(8.2)$ \\
\hline Belgien & 88.0 & $(1.1)$ & 522 & $(3.8)$ & 8.6 & $(0.9)$ & 411 & $(8.7)$ & 3.4 & $(0.4)$ & 431 & $(9.5)$ \\
\hline Kanada & 79.5 & $(1.0)$ & 538 & $(1.5)$ & 10.8 & $(0.5)$ & 539 & $(3.1)$ & 9.8 & $(0.6)$ & 511 & $(4.9)$ \\
\hline Tschechische Rep. & 98.9 & $(0.2)$ & 501 & $(2.1)$ & 0.6 & $(0.1)$ & c & c & 0.5 & $(0.1)$ & c & $\mathrm{c}$ \\
\hline Dänemark & 93.8 & $(0.6)$ & 504 & $(2.2)$ & 2.4 & $(0.4)$ & 409 & (13.9) & 3.8 & $(0.4)$ & 433 & $(7.6)$ \\
\hline Finnland & 98.7 & $(0.2)$ & 548 & $(2.6)$ & 0.2 & $(0.1)$ & c & c & 1.0 & $(0.2)$ & 468 & (12.9) \\
\hline Frankreich & 88.0 & $(0.9)$ & 512 & $(2.8)$ & 9.8 & $(0.7)$ & 471 & $(6.2)$ & 2.2 & $(0.3)$ & 434 & (11.5) \\
\hline Deutschland & 84.8 & $(0.8)$ & 507 & $(2.3)$ & 5.1 & $(0.5)$ & 432 & $(9.0)$ & 10.1 & $(0.6)$ & 419 & $(7.5)$ \\
\hline Griechenland & 95.2 & $(0.9)$ & 478 & $(4.7)$ & 0.5 & $(0.1)$ & C & $\mathrm{C}$ & 4.3 & $(0.9)$ & 403 & $(17.5)$ \\
\hline Ungarn & 98.3 & $(0.2)$ & 482 & $(4.0)$ & 0.1 & $(0.0)$ & C & C & 1.6 & $(0.2)$ & 486 & $(11.6)$ \\
\hline Island & 99.2 & $(0.2)$ & 509 & $(1.5)$ & 0.2 & $(0.1)$ & c & c & 0.6 & $(0.1)$ & c & c \\
\hline Irland & 97.7 & $(0.3)$ & 528 & $(3.2)$ & 0.9 & $(0.2)$ & 519 & $(20.2)$ & 1.4 & $(0.3)$ & 573 & $(9.2)$ \\
\hline Italien & 99.1 & $(0.2)$ & 489 & (2.9) & 0.2 & $(0.1)$ & c & c & 0.8 & $(0.2)$ & 445 & $(15.1)$ \\
\hline Japan & 99.9 & $(0.1)$ & 525 & $(5.1)$ & 0.0 & $(0.0)$ & c & c & 0.1 & $(0.1)$ & c & c \\
\hline Korea $^{3}$ & a & $\mathrm{a}$ & $\mathrm{a}$ & $\mathrm{a}$ & $\mathrm{a}$ & $\mathrm{a}$ & $\mathrm{a}$ & a & $\mathrm{a}$ & a & a & a \\
\hline Luxemburg & 65.8 & $(0.7)$ & 474 & $(1.7)$ & 17.8 & $(0.7)$ & 399 & $(4.6)$ & 16.4 & $(0.6)$ & 370 & $(4.7)$ \\
\hline Mexiko & 96.4 & $(0.4)$ & 427 & $(3.3)$ & 1.1 & $(0.2)$ & 378 & $(15.3)$ & 2.5 & $(0.3)$ & 329 & $(8.2)$ \\
\hline Neuseeland & 80.4 & $(1.1)$ & 538 & $(2.7)$ & 6.4 & $(0.5)$ & 507 & $(10.3)$ & 13.2 & $(0.8)$ & 507 & $(7.6)$ \\
\hline Norwegen & 95.4 & $(0.4)$ & 510 & $(2.7)$ & 1.5 & $(0.2)$ & 464 & $(10.6)$ & 3.1 & $(0.3)$ & 449 & $(8.5)$ \\
\hline Polen & 99.7 & $(0.1)$ & 482 & $(4.4)$ & 0.0 & $(0.0)$ & c & c & 0.2 & $(0.1)$ & $\mathrm{c}$ & c \\
\hline Portugal & 96.9 & $(0.3)$ & 472 & $(4.5)$ & 1.8 & $(0.2)$ & 463 & $(14.3)$ & 1.4 & $(0.2)$ & 450 & $(15.8)$ \\
\hline Spanien & 98.0 & $(0.4)$ & 494 & $(2.6)$ & 0.6 & $(0.1)$ & 450 & $(15.9)$ & 1.4 & $(0.3)$ & 460 & $(17.8)$ \\
\hline Schweden & 89.5 & $(0.9)$ & 523 & $(2.1)$ & 4.7 & $(0.6)$ & 485 & $(7.3)$ & 5.9 & $(0.6)$ & 450 & $(7.2)$ \\
\hline Schweiz & 79.3 & $(0.9)$ & 514 & $(4.0)$ & 9.3 & $(0.6)$ & 460 & $(6.8)$ & 11.4 & $(0.7)$ & 402 & $(6.1)$ \\
\hline Ver. Königreich & 90.4 & $(1.2)$ & 528 & $(2.6)$ & 7.0 & $(0.9)$ & 510 & $(9.4)$ & 2.6 & $(0.4)$ & 456 & $(15.1)$ \\
\hline Vereinigte Staaten & 86.4 & $(2.1)$ & 511 & $(6.5)$ & 7.4 & $(1.4)$ & 478 & (19.4) & 6.1 & $(0.9)$ & 466 & $(10.0)$ \\
\hline OECD Insgesamt & 91.3 & $(0.6)$ & 503 & $(1.9)$ & 4.6 & $(0.4)$ & 479 & $(9.1)$ & 4.1 & $(0.3)$ & 452 & $(4.9)$ \\
\hline Ländermittel & 91.0 & $(0.2)$ & 506 & $(0.6)$ & 4.3 & $(0.1)$ & 467 & $(2.8)$ & 4.7 & $(0.1)$ & 446 & $(2.5)$ \\
\hline Brasilien & 99.6 & $(0.1)$ & 398 & $(3.0)$ & 0.3 & $(0.1)$ & C & c & 0.1 & $(0.1)$ & c & $\mathrm{C}$ \\
\hline Lettland & 77.9 & $(2.4)$ & 462 & $(6.0)$ & 1.5 & $(0.3)$ & 423 & $(15.1)$ & 20.6 & $(2.4)$ & 454 & $(7.3)$ \\
\hline Liechtenstein & 79.4 & $(2.1)$ & 500 & $(5.0)$ & 10.2 & $(1.8)$ & 446 & $(14.8)$ & 10.4 & $(1.6)$ & 392 & $(21.4)$ \\
\hline Russische Föd. & 95.4 & $(0.6)$ & 463 & $(4.3)$ & 1.8 & $(0.3)$ & 452 & $(9.9)$ & 2.8 & $(0.4)$ & 458 & $(9.6)$ \\
\hline Niederlande $^{4}$ & 88.1 & $(1.8)$ & 542 & $(3.0)$ & 7.4 & $(1.2)$ & 470 & (14.2) & 4.5 & $(0.8)$ & 453 & $(15.6)$ \\
\hline
\end{tabular}

1. Prozentsatz der am Test zur Lesekompetenz teilnehmenden Schüler der entsprechenden Kategorie.

2. Fettgedruckte Mittelwerte bedeuten statistisch signifikante Leistungsunterschiede zwischen Schülern der Gruppe 1 und Schülern der Gruppe 2.

3. Frage in Korea nicht gestellt.

4. Die Beteiligungsquote ist zu niedrig, um die Vergleichbarkeit zu gewährleisten (s. Anhang 3, www.oecd.org/els/education/eag2002).

Quelle: OECD PISA-Datenbank, 2001. Hinweise zur Methodik s. Anhang 3 (www.oecd.org/els/education/eag2002) und www.pisa.oecd.org. 
Tabelle A10.2.

Leistung im Bereich Lesekompetenz nach der bei 15-jährigen Schülern zu Hause gesprochenen Sprache (2000) Schülerleistung auf der PISA-Skala für Lesekompetenz nach der (laut Schülerangaben) zu Hause gesprochenen Sprache sowie die größereWahrscheinlichkeit, mit der Schüler, die zu Hause nicht die Unterrichtssprache sprechen, auf nationaler Ebene im untersten Quartil der Leistungsverteilung für Lesekompetenz liegen

\begin{tabular}{|c|c|c|c|c|c|c|c|c|c|c|}
\hline & \multicolumn{4}{|c|}{$\begin{array}{l}\text { Die zu Hause normalerweise gesprochene } \\
\text { Sprache UNTERSCHEIDET SICH von der } \\
\text { Unterrichtssprache, anderen offiziellen } \\
\text { Landessprachen oder nationalen Dialekten }\end{array}$} & \multicolumn{4}{|c|}{$\begin{array}{l}\text { Die zu Hause normalerweise gesprochene } \\
\text { Sprache IST IDENTISCH mit der Unterrichts- } \\
\text { sprache, anderen offiziellen Landessprachen } \\
\text { oder nationalen Dialekten }\end{array}$} & \multirow{2}{*}{\multicolumn{2}{|c|}{$\begin{array}{l}\text { Größere Wahrscheinlickeit mit der } \\
\text { Schüler, die zu Hause nicht die } \\
\text { Unterrichtssprache sprechen, auf } \\
\text { nationaler Ebene im untersten } \\
\text { Quartil der Leistungsverteilung für } \\
\text { Lesekompetenz liegen }\end{array}$}} \\
\hline & \multirow{2}{*}{$\begin{array}{l}\text { Prozentsatz } \\
\text { der Schüler }\end{array}$} & \multirow[b]{2}{*}{ SF } & \multicolumn{2}{|c|}{$\begin{array}{l}\text { Ergebnis auf der } \\
\text { PISA-Skala für } \\
\text { Lesekompetenz }^{2}\end{array}$} & \multirow{2}{*}{$\begin{array}{l}\text { Prozentsatz } \\
\text { der Schüler }\end{array}$} & \multirow[b]{2}{*}{ SF } & \multicolumn{2}{|c|}{$\begin{array}{l}\text { Ergebnis auf der } \\
\text { PISA-Skala für } \\
\text { Lesekompetenz }^{2}\end{array}$} & & \\
\hline & & & Mittelwert & SF & & & Mittelwert & SF & Quotient & SF \\
\hline Australien & 17.0 & (1.6) & 504 & (7.6) & 83.0 & $(1.6)$ & 534 & (3.6) & 1.6 & $(0.1)$ \\
\hline Österreich & 6.7 & $(0.7)$ & 434 & (7.2) & 93.3 & $(0.7)$ & 515 & (2.4) & 2.3 & $(0.2)$ \\
\hline Belgien & 4.9 & $(0.6)$ & 403 & (8.6) & 95.2 & $(0.6)$ & 518 & (3.7) & 2.8 & $(0.2)$ \\
\hline Kanada & 9.4 & $(0.6)$ & 506 & (3.8) & 90.6 & $(0.6)$ & 540 & $(1.5)$ & 1.6 & $(0.1)$ \\
\hline Tschechische Rep. & 0.8 & $(0.2)$ & c & c & 99.2 & $(0.2)$ & 494 & $(2.2)$ & c & c \\
\hline Dänemark & 6.7 & $(0.4)$ & 425 & $(8.1)$ & 93.3 & $(0.4)$ & 503 & $(2.2)$ & 2.5 & $(0.2)$ \\
\hline Finnland & 1.3 & $(0.2)$ & 470 & (12.5) & 98.7 & $(0.2)$ & 548 & (2.6) & c & $\mathrm{c}$ \\
\hline Frankreich & 4.0 & $(0.5)$ & 442 & (7.7) & 96.0 & $(0.5)$ & 510 & (2.6) & 2.3 & $(0.2)$ \\
\hline Deutschland & 7.9 & $(0.8)$ & 386 & $(13.9)$ & 92.1 & $(0.8)$ & 500 & (2.9) & 2.9 & $(0.3)$ \\
\hline Griechenland & 2.8 & (0.6) & 407 & (18.3) & 97.2 & $(0.6)$ & 477 & $(4.8)$ & 2.3 & $(0.4)$ \\
\hline Ungarn & $\mathrm{m}$ & $\mathrm{m}$ & $\mathrm{m}$ & $\mathrm{m}$ & $\mathrm{m}$ & $\mathrm{m}$ & $\mathrm{m}$ & $\mathrm{m}$ & $\mathrm{m}$ & $\mathrm{m}$ \\
\hline Island & 1.9 & $(0.3)$ & 463 & (13.4) & 98.1 & $(0.3)$ & 509 & (1.5) & c & c \\
\hline Irland & 0.9 & $(0.2)$ & c & c & 99.1 & $(0.2)$ & 527 & (3.2) & c & c \\
\hline Italien & 0.7 & $(0.2)$ & c & c & 99.3 & $(0.2)$ & 491 & (3.0) & c & c \\
\hline Japan & 0.3 & $(0.1)$ & c & c & 99.7 & $(0.1)$ & 525 & $(5.2)$ & c & c \\
\hline Korea $^{4}$ & a & $\mathrm{a}$ & a & a & $\mathrm{a}$ & a & $\mathrm{a}$ & a & $\mathrm{a}$ & $\mathrm{a}$ \\
\hline Luxemburg & 18.3 & $(0.7)$ & 367 & (4.1) & 81.7 & $(0.7)$ & 460 & (1.6) & 2.8 & $(0.1)$ \\
\hline Mexiko & 0.2 & $(0.1)$ & c & c & 99.8 & $(0.1)$ & 422 & (3.4) & c & c \\
\hline Neuseeland & 9.6 & $(0.6)$ & 469 & (9.6) & 90.4 & $(0.6)$ & 541 & (2.6) & 2.1 & $(0.2)$ \\
\hline Norwegen & 5.3 & $(0.4)$ & 459 & (8.4) & 94.7 & (0.4) & 510 & $(2.8)$ & 1.8 & $(0.1)$ \\
\hline Polen & 0.5 & $(0.2)$ & c & c & 99.5 & $(0.2)$ & 482 & (4.4) & c & c \\
\hline Portugal & 1.5 & $(0.2)$ & 416 & (13.8) & 98.5 & $(0.2)$ & 471 & (4.6) & c & c \\
\hline Spanien & 1.2 & $(0.2)$ & 456 & $(16.0)$ & 98.8 & $(0.2)$ & 495 & (2.6) & c & c \\
\hline Schweden & 6.7 & (0.6) & 456 & $(7.1)$ & 93.3 & $(0.6)$ & 523 & $(2.0)$ & 2.1 & $(0.2)$ \\
\hline Schweiz & 13.6 & $(0.6)$ & 414 & (6.1) & 86.4 & $(0.6)$ & 509 & $(4.1)$ & 2.8 & $(0.2)$ \\
\hline Ver. Königreich & 4.1 & $(0.7)$ & 470 & (12.8) & 95.9 & $(0.7)$ & 528 & $(2.5)$ & 1.9 & $(0.2)$ \\
\hline Vereinigte Staaten & 10.8 & (2.4) & 438 & (13.1) & 89.2 & (2.4) & 514 & (5.8) & 2.1 & $(0.2)$ \\
\hline OECD Insgesamt & 5.5 & $(0.7)$ & 443 & $(8.2)$ & 94.5 & $(0.7)$ & 503 & $(1.8)$ & 2.0 & $(0.1)$ \\
\hline Ländermittel & 5.5 & $(0.2)$ & 440 & (2.6) & 94.5 & $(0.2)$ & 506 & $(0.6)$ & 2.1 & $(0.0)$ \\
\hline Brasilien & 0.8 & $(0.2)$ & c & c & 99.2 & $(0.2)$ & 397 & (3.0) & c & c \\
\hline Lettland & 0.0 & $(0.0)$ & a & a & 100.0 & $(0.0)$ & 460 & $(5.2)$ & a & a \\
\hline Liechtenstein & 20.7 & $(2.2)$ & 441 & (14.3) & 79.3 & $(2.2)$ & 494 & $(5.1)$ & c & c \\
\hline Russische Föd. & 7.3 & $(2.1)$ & 432 & $(9.3)$ & 92.7 & $(2.1)$ & 465 & $(4.3)$ & 1.5 & $(0.2)$ \\
\hline Niederlande $^{5}$ & 6.3 & $(1.1)$ & 466 & $(13.1)$ & 93.7 & (1.1) & 539 & $(2.7)$ & 2.2 & $(0.3)$ \\
\hline
\end{tabular}

1. Prozentsatz der am Test zur Lesekompetenz teilnehmenden Schüler der entsprechenden Kategorie.

2. Fettgedruckte Mittelwerte bedeuten statistisch signifikante Leistungsunterschiede zwischen Schülern, die zu Hause nicht die Unterrichtssprache sprechen, und Schülern, die zu Hause die Unterrichtssprache sprechen.

3. Statistisch signifikante Quotienten von mehr als 1 durch Fettdruck gekennzeichnet.

4. Frage in Korea nicht gestellt.

5. Die Beteiligungsquote ist zu niedrig, um die Vergleichbarkeit zu gewährleisten (Hinweise s. Anhang 3, unter www.oecd.org/els/education/eag2002).

Quelle: OECD PISA-Datenbank, 2001. Hinweise zur Methodik s. Anhang 3 (www.oecd.org/els/education/eag2002) und www.pisa.oecd.org. 


\section{ERWERBSQUOTEN NACH BILDUNGSSTAND}

- In den meisten OECD-Ländern steigen die Erwerbsquoten mit zunehmendem Bildungsstand. Von sehr wenigen Ausnahmen abgesehen, ist die Erwerbsquote von Absolventen des Tertiärbereichs wesentlich höher als die von Absolventen des Sekundarbereich II. Besonders groß ist der Abstand zwischen den Erwerbsquoten von Männern mit einem Abschluss des Sekundarbereich II und ohne einen solchen Abschluss.

- Die Erwerbsquoten von Frauen mit einem Abschluss unterhalb des Sekundarbereich II sind besonders gering. Die Quoten für Frauen mit einem tertiärem Abschluss liegen, mit Ausnahme von vier Ländern, überall bei 80 Prozent oder darüber. Sie sind jedoch bis auf eine Ausnahme in allen Ländern geringer als die der Männer.

- Mit zunehmendem Bildungsstand nimmt der geschlechtsspezifische Unterschied in der Erwerbsbeteiligung ab. Obwohl auch bei den höchsten Bildungsabschlüssen noch eine Kluft zwischen der Erwerbsbeteiligung von Männern und Frauen besteht, ist sie hier weit geringer als bei den niedrigeren Bildungsabschlüssen. 


\section{Abbildung A11.1.}

Unterschiede in den Erwerbsquoten von Frauen und Männern, nach Bildungsstand, für 25- bis 64- Jährige (2001)

Unterhalb Sekundarbereich II

Sekundarbereich II und post-sekundärer, nicht-tertiärer Bereich

- Tertiärbereich A und weiterführende Forschungsprogramme

Prozentpunkte

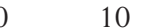

20

30

40

50

$60 \quad 70$

\section{Türkei \\ Mexiko \\ Korea \\ Japan}

Griechenland

Irland

Luxemburg

Spanien

Australien

Italien

Schweiz

Neuseeland

Belgien ${ }^{1}$

Österreich 1

Tschechische Rep.
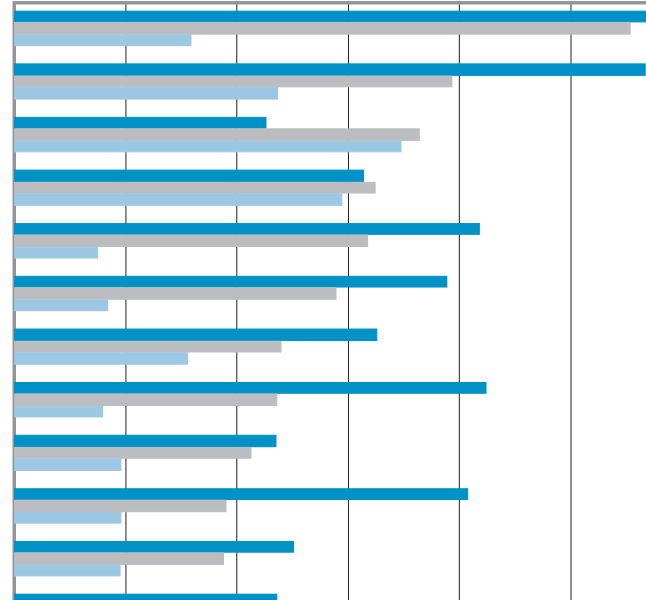

-

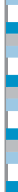

Ungarn

Niederlande ${ }^{1}$

Kanada

Deutschland

Vereinigte Staaten

Slowakische Rep.

Polen

Frankreich

Ver. Königreich

Island

Norwegen ${ }^{1}$

Dänemark

Finnland

Schweden

Portugal

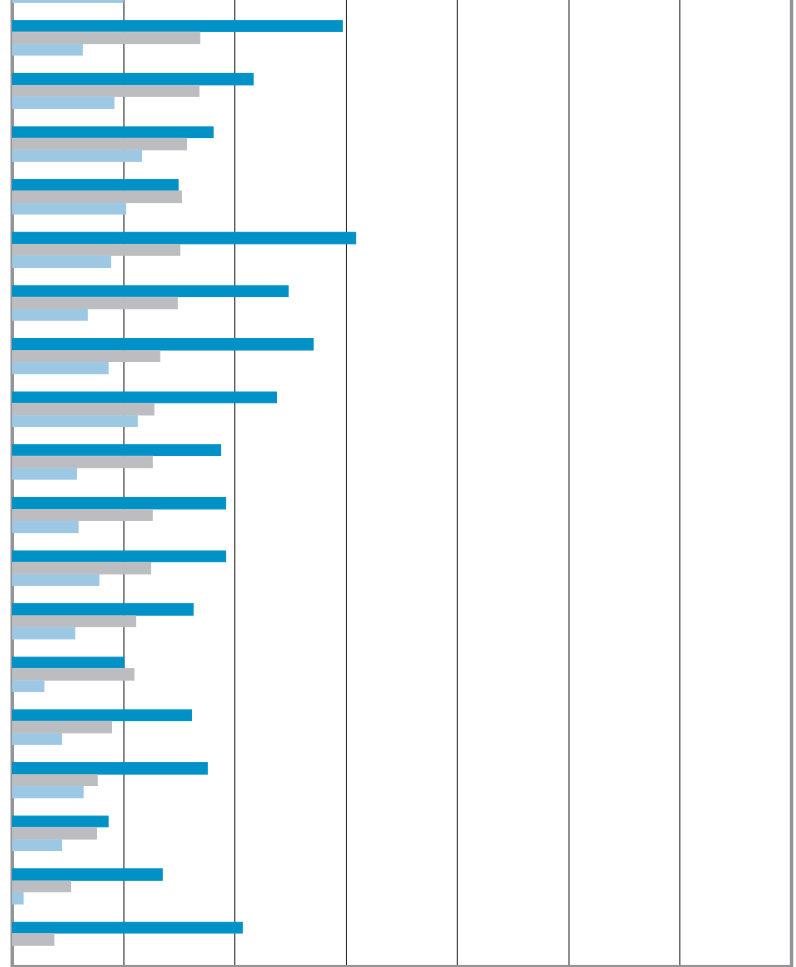

1. Referenzjahr 2000.

Anordnung der Länder in absteigender Reihenfolge des Unterschieds der Erwerbsquote von Frauen und Männern mit einem Abschluss im Sekundarbereich II oder im post-sekundären, nicht-tertiären Bereich.

Quelle: OECD. Tabelle 11.1. Beschreibung der ISCED-97-Stufen, Zuordnung der länderspezifischen Bildungsgänge zu ISCED 97 und länderspezifische Datenquellen s. Anhang 3 (www.oecd.org/els/education/eag2002). 
Dieser Indikator untersucht die Beziehung zwischen Bildungsstand und der Stellung im Arbeitsmarkt.

Die länderspezifischen Unterschiede bei den Erwerbsquoten der Männer sind geringer als bei den Frauen.

In den meisten OECDLändern steigen die Erwerbsquoten der Männer mit zunehmendem Bildungsstand.

\section{Politischer Hintergrund}

Um ihre wirtschaftliche Entwicklung voranzutreiben und ihre Wettbewerbsfähigkeit aufrecht zu erhalten, hängen die Volkswirtschaften und Arbeitsmärkte der OECD-Länder in zunehmendem Maße von einem stabilen Angebot gut ausgebildeter Arbeitskräfte ab. Da das Niveau an Kenntnissen und Fähigkeiten in der Regel mit zunehmendem Bildungsstand wächst, steigen auch die Kosten, wenn die besser Ausgebildeten nicht arbeiten; und da die Bevölkerung in den OECD-Ländern zunehmend älter wird, kann eine stärkere und längere Erwerbsbeteiligung zu einer Senkung der Abhängigkeitsquoten und der Entlastung der staatlichen Rentenkassen beitragen.

Dieser Indikator untersucht die Beziehung zwischen Bildungsstand und Erwerbsquote, wobei zuerst die Erwerbsquoten und anschließend die Arbeitslosenquoten verglichen werden. Für die politischen Entscheidungsträger ist es von großer Bedeutung, inwieweit die Kenntnisse und Fähigkeiten der Arbeitnehmer angemessen sind und der Arbeitsmarkt in der Lage ist, die passenden Arbeitsplätze für diese Kenntnisse und Fähigkeiten bereitzustellen.

\section{Ergebnisse und Erläuterungen}

\section{Erwerbsbeteiligung}

Die länderspezifischen Schwankungen zwischen den OECD-Ländern in der Gesamterwerbsbeteiligung sind hauptsächlich auf die unterschiedliche Erwerbsbeteiligung der Frauen zurückzuführen. Die Gesamterwerbsquoten für 25- bis 64-jährige Männer liegen zwischen 81 Prozent und darunter in Italien und Ungarn und 94 Prozent und darüber in Island, Japan, Mexiko und der Schweiz (Abb. A11.1). Im Gegensatz dazu variiert die Erwerbsbeteiligung der Frauen zwischen 55 Prozent und darunter in Griechenland, Italien, Mexiko, Spanien und der Türkei und über 77 Prozent in den nordischen Ländern. Zwei Faktoren, die zu diesen starken Unterschieden beitragen, sind längere Zeiten der Ausbildung und der Nicht-Beschäftigung, beide erhöhen sie die Zahl der Personen, die nicht im Arbeitsmarkt sind.

Im Allgemeinen sind die Erwerbsquoten von Männern mit höheren Bildungsabschlüssen höher. Mit Ausnahme von Mexiko, Spanien und der Türkei, wo dieser Trend weniger stark ausgeprägt ist, lässt sich beobachten, dass die Erwerbsquoten von Absolventen des Tertiärbereichs wesentlich höher sind als die von Absolventen des Sekundarbereich II. Dieser Unterschied reicht von wenigen bis zu 8 bis 10 Prozentpunkten in Dänemark, Deutschland, Österreich und Polen. Er ist sehr gering für die Altersgruppe der 35- bis 44Jährigen, von denen die meisten erwerbstätig sind, und ist hauptsächlich auf die Tatsache zurückzuführen, dass weniger gut ausgebildete Arbeitskräfte den Arbeitsmarkt früher verlassen. In der Altersgruppe über 55 Jahre verbleiben die besser ausgebildeten Erwerbstätigen in der Regel länger als andere in einem Beschäftigungsverhältnis (Tabelle A11.1). 
Der Abstand zwischen den Erwerbsquoten 25- bis 64-jähriger Männer mit einem Abschluss des Sekundarbereich II und ohne einen solchen ist besonders groß. In 14 von 29 OECD-Ländern übersteigt der Unterschied in den Erwerbsquoten derjenigen mit einem Abschluss des Sekundarbereich II und derjenigen ohne einen solchen Abschluss 10 Prozentpunkte. Der Extremfall ist Ungarn, wo nur die Hälfte der Männer mit einem Bildungsabschluss unterhalb des Sekundarbereich II aber über 80 Prozent der Männer mit einem Abschluss des Sekundarbereich II im Erwerbsleben stehen. Der Unterschied in den Erwerbsquoten zwischen Männern mit niedrigen und Männern mit höheren Bildungsabschlüssen ist in Island, Korea, Mexiko, Portugal, der Schweiz und der Türkei (wo die Erwerbsbeteiligung von Männern auf allen Ebenen generell sehr hoch ist) gering.

Die Erwerbsquoten von Frauen im Alter zwischen 25 und 64 Jahren weisen noch deutlichere Unterschiede auf, und zwar nicht nur zwischen denen ohne einen Abschluss des Sekundarbereich II und denen, die mindestens einen solchen Abschluss besitzen (ca. 20 Prozentpunkte oder mehr in 15 von 30 OECD-Ländern), sondern auch zwischen denen mit einem Abschluss im Sekundarbereich II und und denen mit einem Abschluss im Tertiärbereich (ca. 10 Prozentpunkte oder mehr in 22 Ländern). Ausnahmen bilden hier Japan, Korea, und Schweden, wo die Erwerbsquoten von Frauen mit einem Abschluss des Sekundarbereich II den Erwerbsquoten von Frauen mit einem Abschluss des Tertiärbereichs vergleichbar sind (Unterschiede von ca. 5 bis 7 Prozentpunkten).

Die Erwerbsquoten von Frauen mit einem Abschluss unterhalb des Sekundarbereich II sind besonders niedrig, im Durchschnitt aller OECDLänder ungefähr 50 Prozent und ca. ein Drittel oder weniger in Italien, der Türkei und Ungarn. Die Quoten von Frauen mit einem Abschluss des Tertiärbereichs liegen überall nahe 80 Prozent oder darüber, außer in Japan, Korea, Luxemburg, Mexiko, der Türkei und Ungarn. Sie sind jedoch in allen Ländern geringer als die der Männer (Abb. A11.1).

Obwohl auch bei den höchsten Bildungsabschlüssen noch eine Kluft zwischen der Erwerbsbeteiligung von Männern und Frauen besteht, ist sie hier weit geringer als bei niedrigeren Bildungsabschlüssen. So nimmt für alle OECDLänder der geschlechtsspezifische Abstand zwischen der Erwerbsbeteiligung der Männer und der Frauen mit jeder nächsthöheren Bildungsstufe durchschnittlich um 10 Prozentpunkte ab: von ca. 30 Prozentpunkten im Bereich unterhalb des Sekundarbereich II auf 20 Prozentpunkte im Sekundarbereich II und 10 Prozentpunkte im tertiären Bereich.

Ein Großteil der Unterschiede zwischen den Erwerbsquoten der Männer mit unterschiedlichem Bildungsstand ist auf die größeren Unterschiede bei der älteren Bevölkerung, insbesondere in der Altersgruppe zwischen 55 und 64 Jahren, zurückzuführen (Tabelle A11.1). In 20 von 29 Ländern sind über 70
Besonders großs ist der

Abstand zwischen

den Erwerbsquoten

von Männern mit

einem Abschluss des

Sekundarbereich II und

ohne einen solchen.

Bei den Frauen weist die Erwerbsbeteiligung nach dem Bildungsstand sogar noch stärkere unterschiede auf.

Die Erwerbsbeteiligung von Frauen mit einem Abschluss unterhalb des sekundarbereich // ist besonders gering,...

...jedoch nimmt mit zunehmendem Bildungsstand der geschlechtsspezifische Unterschied in der Erwerbsbeteiligung ab.

Der bildungsbedingte Unterschied in der Erwerbsbeteiligung der Männer wird erheblich 
durch die Unterschiede

bei der älteren

Bevölkerung beeinflusst.
Prozent der 55- bis 64-Jährigen mit einem Abschluss des Tertiärbereichs erwerbstätig. Die Quoten derjenigen ohne einen Abschluss im Sekundarbereich II sind nur in Griechenland, Korea, Mexiko und der Türkei genauso hoch. Der bildungsbedingte Unterschied bei der Erwerbsbeteiligung von Frauen ist dagegen in allen Altersgruppen relativ groß.

Die hier beobachteten Sachverhalte lassen sich auf eine Reihe von Ursachen zurückführen. Da das Einkommen normalerweise mit höherem Bildungsstand wächst, besteht für Personen mit einem höheren Bildungsabschluss ein größerer finanzieller Anreiz für eine Erwerbstätigkeit. Daneben haben diese Personen in der Regel interessantere und reizvollere Aufgaben und tragen mehr Verantwortung, wodurch ihre Motivation, erwerbstätig zu bleiben, steigt. Im Gegensatz dazu kann schwere körperliche Arbeit, die in der Regel mit einem eher niedrigen Bildungsstand in Verbindung steht, zum Bedürfnis nach einem vorzeitigen Ruhestand führen. Darüber hinaus hat die Umstrukturierung der Industrie in vielen Ländern zu einer Reduzierung der Arbeitsplätze für ungelernte Arbeitskräfte und für solche, die in technisch mittlerweile überholten Berufen ausgebildet wurden, geführt. Viele von ihnen sind aufgrund von Vorruhestandsregelungen oder nur begrenzten Beschäftigungsmöglichkeiten aus dem Arbeitsmarkt ausgeschieden. Der Bildungsstand von Frauen und ihre Erwerbsbeteiligung haben seit jeher unterhalb des Bildungsstands und der Erwerbsquote von Männern gelegen, und trotz beträchtlicher Fortschritte im Laufe der letzten Jahrzehnte belegen die derzeitigen Erwerbsquoten auch weiterhin den Einfluss dieser historischen Faktoren.

\section{Arbeitslosenquoten nach Bildungsstand}

Personen mit

niedrigerem

Bildungsstand sind

sowohl häufiger

überhaupt nicht im

Arbeitsmarkt als auch

häufiger arbeitslos.

Mit höherem

Bildungsstand gehen die Arbeitslosenquoten

zurück.
Anhand der Arbeitslosenquote lässt sich die Fähigkeit einer Volkswirtschaft bemessen, jeden Arbeitswilligen mit einem Arbeitsplatz zu versorgen. Soweit man den Bildungsstand als Indikator für vorhandene Kenntnisse und Fähigkeiten ansieht, kann er dem Arbeitgeber als Anhaltspunkt dafür dienen, welche Kenntnisse, Fähigkeiten und Leistungen am Arbeitsplatz von einem Stellenbewerber zu erwarten sind. Bei unterschiedlichem Bildungsstand werden die Beschäftigungsaussichten des Einzelnen sowohl von den Erfordernissen des Arbeitsmarktes abhängen als auch von dem Angebot an Arbeitskräften mit unterschiedlichen Kenntnissen und Fähigkeiten. Besonders diejenigen mit niedrigem Bildungsstand laufen Gefahr, wirtschaftlich ausgegrenzt zu werden, da sie sich einerseits eher nicht im Arbeitsmarkt befinden und andererseits auch eher ohne Arbeit bleiben, wenn sie sich aktiv um eine Arbeitsstelle bemühen.

In 19 von 30 OECD-Ländern ist die Wahrscheinlichkeit, arbeitslos zu sein, für männliche Erwerbstätige im Alter von 25 bis 64 Jahren mit einem Abschluss unterhalb des Sekundarbereich II mehr als 1,5 Mal höher als für diejenigen mit einem Abschluss des Sekundarbereich II (Abb. A11.2). In 17 Ländern ist die Arbeitslosenquote für männliche Absolventen des Sekundarbereich II mindestens 1,5 Mal höher als die für Absolventen des Tertiärbereichs. Im tertiären Bereich sind in ungefähr der Hälfte der Länder 


\section{Abbildung A11.2.}

Unterschiede in den Arbeitslosenquoten von Frauen und Männern, nach Bildungsstand, für 30- bis 44-Jährige (2001)

Unterhalb Sekundarbereich II $\square$ Sekundarbereich II und post-sekundärer, —Tertiärbereich nicht-tertiärer Bereich

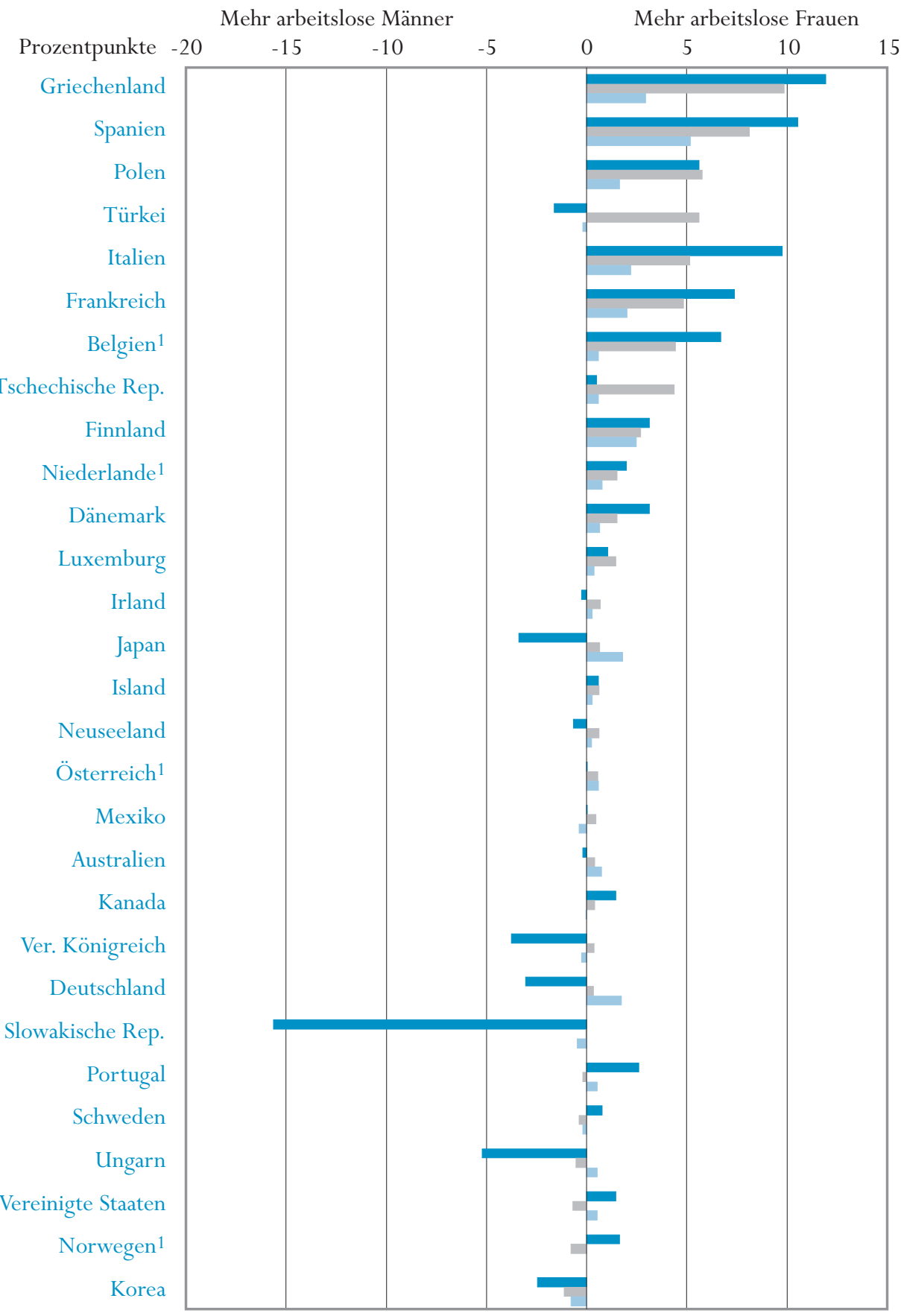

1. Referenzjahr 2000 .

Anordnung der Länder in absteigender Reihenfolge des Unterschieds der Arbeitslosenquoten von Frauen und Männern mit einem Abschluss im Sekundarbereich II oder im post-sekundären nicht-tertiären Bereich.

Quelle: OECD. Tabelle A11.2. Beschreibung der ISCED-97-Stufen, Zuordnung der länderspezifischen Bildungsgänge zu ISCED 97 und länderspezifische Datenquellen s. Anhang 3 (www.oecd.org/els/education/eag2002). 


\section{$\mathbf{A}_{11}$}

Die länderspezifischen

Unterschiede im

Zusammenhang

zwischen

Arbeitslosenquote und

Bildungsstand sind auf

eine Reihe von Faktoren

zurückzuführen.

Die Daten stammen aus nationalen Arbeitskräfteerhebungen. die Arbeitslosenquoten der erwachsenen Bevölkerung mit einem Abschluss von kürzeren berufsorientierten Bildungsgängen (ISCED 5B) höher als die der Absolventen der eher theoretisch orientierten und längeren Studiengängen der Stufe ISCED 5A (Tabelle A11.2), während sie in der anderen Hälfte der Länder signifikant geringer sind.

In den meisten Ländern ist die Diskrepanz der Arbeitslosenquoten zwischen den einzelnen Bildungsniveaus bei den 30- bis 44-jährigen Männern besonders ausgeprägt. Der Zusammenhang zwischen Arbeitslosenquote und Bildungsstand ist bei den Frauen ähnlich, obwohl in vielen Ländern der Abstand zwischen denjenigen mit einem Abschluss des Sekundarbereich II und denjenigen mit einem Abschluss des Tertiärbereichs noch größer ist. In einem Drittel der Länder sind die Nachteile für die Frauen offensichtlich, in den anderen Ländern sind die Arbeitslosenquoten jedoch unabhängig vom Bildungsstand gleich. Nur in wenigen Ländern zeigen sich signifikante Unterschiede zugunsten der Frauen mit dem geringsten Bildungsstand - in Deutschland, Japan, Korea, der Slowakischen Republik, Türkei, Ungarn und dem Vereinigten Königreich. Auf Ebene der Tertiärabschlüsse ist dieser Abstand wesentlich weniger deutlich, auch in den Ländern, in denen er sonst allgemein vorhanden ist (Abb. A11.2).

Die großen länderspezifischen Unterschiede hinsichtlich der Arbeitslosenquote von Personen mit einem niedrigen Bildungsstand sind auf eine Reihe von Faktoren zurückzuführen. In einigen Ländern (insbesondere solchen, die sich in einem Übergangsprozess befinden wie Polen, die Slowakische Republik, die Tschechische Republik und Ungarn) sind die hohen Arbeitslosenquoten von Personen mit einem niedrigen Bildungsstand Ausdruck der allgemein schwierigen Arbeitsmarktlage, die sich auf sie besonders negativ auswirkt. In geringerem $\mathrm{Ma}$ ist das auch in Deutschland, Finnland und Frankreich der Fall. Die Arbeitslosenquote von Personen ohne einen Abschluss im Sekundarbereich II ist auch in einigen Ländern mit weniger stark regulierten Arbeitsmärkten relativ hoch (Kanada, Vereinigte Staaten und das Vereinigte Königreich). Andererseits sind die Arbeitslosenquoten von Personen ohne einen Abschluss im Sekundarbereich II in Ländern, in denen die Landwirtschaft noch ein bedeutender Beschäftigungsfaktor ist (Mexiko und Portugal), eher niedrig. Schließlich scheint es dort, wo die gesamte Arbeitsmarktsituation besonders günstig ist (Island, Luxemburg, die Niederlande, Norwegen, Österreich und die Schweiz) Arbeitsplätze für Arbeitskräfte sowohl mit niedrigem als auch mit hohem Bildungsstand zu geben (Tabelle A11.2).

\section{Definitionen und angewandte Methodik}

Die Erwerbsquote für eine bestimmte Altersgruppe entspricht dem Prozentsatz der Personen in der Bevölkerung der gleichen Altersgruppe, die entweder beschäftigt oder arbeitslos sind, wobei diese Begriffe gemäß den Richtlinien der Internationalen Arbeitsorganisation (ILO) definiert sind. 
Arbeitslose werden definiert als Personen, die keinen Arbeitsplatz haben, aktiv einen Arbeitsplatz suchen und dem Arbeitsmarkt zur Verfügung stehen. Beschäftigte werden definiert als diejenigen, die während der untersuchten Bezugswoche: 1. mindestens eine Stunde für ein Gehalt (Arbeitnehmer) oder für einen Gewinn (Selbständige und unentgeltlich mithelfende Familienangehörige) arbeiten oder 2. einen Arbeitsplatz haben, aber vorübergehend nicht zur Arbeit gehen (aufgrund von Verletzung, Krankheit, Urlaub, Streik oder Aussperrung, Bildungs- oder Schulungsurlaub, Mutterschafts- oder Erziehungsurlaub, usw.) und eine formelle Bindung an ihren Arbeitsplatz haben.

Die Arbeitslosenquote ist die Anzahl der Arbeitslosen dividiert durch die Anzahl der Erwerbspersonen (x 100), die Angabe erfolgt in Prozent. Die Angabe des Bildungsstandes richtet sich nach den Definitionen laut ISCED-97. 
Tabelle A11.1.

Erwerbsquoten (2001)

25- bis 64-Jährige und 55-bis 64-Jährige, nach Bildungsstand und Geschlecht

\begin{tabular}{|c|c|c|c|c|c|c|c|c|c|c|}
\hline & & \multicolumn{5}{|c|}{ 25- bis 64-Jährige } & \multicolumn{4}{|c|}{ 55- bis 64-Jährige } \\
\hline & & $\begin{array}{l}\text { Unterhalb } \\
\text { Sekundar- } \\
\text { bereich II }\end{array}$ & $\begin{array}{l}\text { Sekundar- } \\
\text { bereich II } \\
\text { und post- } \\
\text { sekundärer, } \\
\text { nicht-ter- } \\
\text { tiärer Bereich } \\
\end{array}$ & $\begin{array}{c}\text { Tertiärbe- } \\
\text { reich B }\end{array}$ & $\begin{array}{l}\text { Tertiärbe- } \\
\text { reich A und } \\
\text { weiterf. } \\
\text { Forschungs- } \\
\text { programme }\end{array}$ & $\begin{array}{c}\text { Alle } \\
\text { Bildungs- } \\
\text { bereiche }\end{array}$ & $\begin{array}{l}\text { Unterhalb } \\
\text { Sekundar- } \\
\text { bereich II }\end{array}$ & $\begin{array}{l}\text { Sekundar- } \\
\text { bereich II } \\
\text { und post- } \\
\text { sekundärer, } \\
\text { nicht-ter- } \\
\text { tiärer Bereich } \\
\end{array}$ & $\begin{array}{l}\text { Tertiär- } \\
\text { bereich }\end{array}$ & $\begin{array}{c}\text { Alle } \\
\text { Bildungs- } \\
\text { bereiche }\end{array}$ \\
\hline & & (1) & (2) & (3) & (4) & (5) & (6) & (7) & (8) & (9) \\
\hline \multirow[t]{2}{*}{ Australien } & Männer & 79 & 89 & 89 & 92 & 86 & 54 & 67 & 74 & 62 \\
\hline & Frauen & 55 & 68 & 77 & 83 & 66 & 30 & 42 & 61 & 38 \\
\hline \multirow{2}{*}{ Österreich ${ }^{1}$} & Männer & 71 & 85 & 87 & 95 & 83 & 32 & 41 & 65 & 42 \\
\hline & Frauen & 49 & 68 & 83 & 86 & 64 & 15 & 18 & 43 & 17 \\
\hline \multirow[t]{2}{*}{ Belgien ${ }^{1}$} & Männer & 71 & 87 & 92 & 92 & 82 & 29 & 42 & 58 & 38 \\
\hline & Frauen & 41 & 71 & 82 & 86 & 62 & 13 & 22 & 31 & 17 \\
\hline \multirow{2}{*}{ Kanada } & Männer & 73 & 88 & 91 & 90 & 86 & 52 & 64 & 66 & 61 \\
\hline & Frauen & 48 & 73 & 81 & 83 & 72 & 28 & 46 & 51 & 41 \\
\hline \multirow{2}{*}{ Tschechische Rep. } & Männer & 70 & 88 & $\mathrm{x}(4)$ & 94 & 87 & 35 & 55 & 79 & 55 \\
\hline & Frauen & 52 & 73 & $\mathrm{x}(4)$ & 83 & 70 & 13 & 27 & 61 & 25 \\
\hline \multirow[t]{2}{*}{ Dänemark } & Männer & 75 & 87 & 91 & 96 & 86 & 55 & 65 & 81 & 66 \\
\hline & Frauen & 57 & 79 & 88 & 90 & 77 & 31 & 60 & 67 & 52 \\
\hline \multirow[t]{2}{*}{ Finnland } & Männer & 70 & 86 & 90 & 93 & 83 & 43 & 54 & 65 & 51 \\
\hline & Frauen & 61 & 79 & 86 & 88 & 77 & 40 & 53 & 67 & 49 \\
\hline \multirow[t]{2}{*}{ Frankreich } & Männer & 76 & 88 & 92 & 92 & 85 & 36 & 44 & 66 & 44 \\
\hline & Frauen & 57 & 76 & 85 & 84 & 70 & 29 & 36 & 51 & 34 \\
\hline \multirow[t]{2}{*}{ Deutschland } & Männer & 77 & 84 & 88 & 92 & 84 & 44 & 49 & 67 & 53 \\
\hline & Frauen & 50 & 70 & 81 & 83 & 68 & 26 & 35 & 53 & 34 \\
\hline Griechenland & Männer & 82 & 88 & 85 & 90 & 85 & 60 & 48 & 57 & 57 \\
\hline & Frauen & 40 & 57 & 79 & 83 & 52 & 25 & 16 & 30 & 24 \\
\hline Ungarn & Männer & 50 & 83 & $\mathrm{x}(4)$ & 89 & 75 & 22 & 46 & 64 & 36 \\
\hline & Frauen & 35 & 67 & $\mathrm{x}(4)$ & 79 & 58 & 8 & 21 & 43 & 16 \\
\hline Island & Männer & 95 & 95 & 97 & 98 & 96 & 91 & 92 & 99 & 93 \\
\hline & Frauen & 85 & 84 & 91 & 95 & 87 & 81 & 83 & 82 & 82 \\
\hline Irland & Männer & 79 & 93 & 95 & 94 & 87 & 61 & 72 & 80 & 66 \\
\hline & Frauen & 40 & 64 & 74 & 85 & 60 & 21 & 35 & 50 & 29 \\
\hline Italien & Männer & 74 & 86 & $\mathrm{x}(4)$ & 91 & 80 & 36 & 49 & 71 & 41 \\
\hline & Frauen & 34 & 67 & $x(4)$ & 81 & 50 & 12 & 29 & 41 & 16 \\
\hline Japan & Männer & 87 & 95 & 98 & 97 & 95 & 80 & 86 & 86 & 84 \\
\hline & Frauen & 56 & 63 & 66 & 68 & 63 & 48 & 49 & 47 & 49 \\
\hline Korea & Männer & 84 & 89 & 94 & 91 & 88 & 74 & 67 & 70 & 71 \\
\hline & Frauen & 61 & 53 & 58 & 56 & 57 & 51 & 25 & 42 & 48 \\
\hline Luxemburg & Männer & 79 & 87 & 92 & 92 & 85 & 22 & 35 & 73 & 36 \\
\hline & Frauen & 46 & 63 & 80 & 77 & 56 & 9 & 20 & 48 & 14 \\
\hline Mexiko & Männer & 94 & 96 & 97 & 94 & 94 & 81 & 78 & 79 & 80 \\
\hline & Frauen & 37 & 56 & 61 & 70 & 43 & 27 & 37 & 37 & 28 \\
\hline Niederlande $^{1}$ & Männer & 77 & 89 & 90 & 92 & 86 & 42 & 53 & 64 & 51 \\
\hline & Frauen & 46 & 73 & 80 & 83 & 64 & 19 & 32 & 45 & 26 \\
\hline Neuseeland & Männer & 80 & 91 & 89 & 93 & 89 & 66 & 79 & 80 & 75 \\
\hline & Frauen & 56 & 74 & 77 & 83 & 71 & 41 & 58 & 65 & 52 \\
\hline Norwegen $^{1}$ & Männer & 75 & 89 & 95 & 94 & 89 & 62 & 75 & 87 & 74 \\
\hline & Frauen & 59 & 80 & 88 & 89 & 80 & 47 & 63 & 86 & 62 \\
\hline Polen & Männer & 64 & 83 & $\mathrm{x}(4)$ & 92 & 81 & 35 & 41 & 68 & 41 \\
\hline & Frauen & 45 & 71 & $\mathrm{x}(4)$ & 86 & 67 & 20 & 24 & 45 & 24 \\
\hline Portugal & Männer & 87 & 87 & 94 & 94 & 87 & 63 & 57 & 78 & 64 \\
\hline & Frauen & 66 & 84 & 88 & 95 & 71 & 41 & 32 & 60 & 42 \\
\hline Slowakische Rep. & Männer & 62 & 88 & 89 & 93 & 86 & 25 & 46 & 64 & 43 \\
\hline & Frauen & 43 & 76 & 90 & 88 & 71 & 3 & 12 & 52 & 11 \\
\hline Spanien & Männer & 83 & 90 & 93 & 91 & 86 & 59 & 62 & 73 & 61 \\
\hline & Frauen & 41 & 66 & 77 & 83 & 54 & 20 & 38 & 58 & 24 \\
\hline Schweden & Männer & 79 & 88 & 89 & 91 & 87 & 68 & 74 & 82 & 74 \\
\hline & Frauen & 66 & 83 & 86 & 90 & 82 & 56 & 69 & 82 & 68 \\
\hline Schweiz & Männer & 87 & 93 & 96 & 96 & 94 & 78 & 82 & 85 & 83 \\
\hline & Frauen & 62 & 74 & 85 & 86 & 74 & 41 & 58 & 68 & 54 \\
\hline Türkei & Männer & 82 & 87 & $\mathrm{x}(4)$ & 87 & 84 & 52 & 25 & 43 & 49 \\
\hline & Frauen & 22 & 32 & $\mathrm{x}(4)$ & 71 & 27 & 14 & 5 & 15 & 14 \\
\hline Ver. Königreich & Männer & 67 & 88 & 93 & 93 & 86 & 51 & 67 & 73 & 64 \\
\hline & Frauen & 51 & 77 & 85 & 87 & 74 & 44 & 65 & 69 & 58 \\
\hline Vereinigte Staaten & Männer & 75 & 86 & 90 & 92 & 87 & 55 & 66 & 77 & 68 \\
\hline & Frauen & 52 & 73 & 80 & 81 & 73 & 33 & 54 & 66 & 54 \\
\hline Ländermittel & Männer & 77 & 88 & 92 & 93 & 86 & 52 & 59 & 72 & 60 \\
\hline & Frauen & 50 & 70 & 80 & 83 & 65 & 30 & 39 & 54 & 37 \\
\hline
\end{tabular}

Hinweis: $\mathrm{x}$ bedeutet, dass die Daten in einer anderen Spalte enthalten sind, deren Referenz in runden Klammern nach dem ' $\mathrm{x}$ ' angegeben ist. So bedeutet $\mathrm{z} . \mathrm{B}$. $\mathrm{x}(2)$, dass die Daten in Spalte 2 enthalten sind.

1. Referenzjahr 2000.

Quelle: OECD. Beschreibung der ISCED-97-Stufen, Zuordnung der länderspezifischen Bildungsgänge zu ISCED 97 und länderspezifische Datenquellen

s. Anhang 3 (www.oecd.org/els/education/eag2002). 
Tabelle A11.2.

Arbeitslosenquoten (2001)

25- bis 64-Jährige und 30- bis 44-Jährige, nach Bildungsstand und Geschlecht

\begin{tabular}{|c|c|c|c|c|c|c|c|c|c|c|}
\hline & & \multicolumn{5}{|c|}{ 25- bis 64-Jährige } & \multicolumn{4}{|c|}{ 30- bis 44-Jährige } \\
\hline & & $\begin{array}{c}\text { Unterhalb } \\
\text { Sekundar- } \\
\text { bereich II }\end{array}$ & $\begin{array}{c}\text { Sekundar- } \\
\text { bereich II } \\
\text { und post- } \\
\text { sekundärer, } \\
\text { nicht-ter- } \\
\text { tiärer Bereich }\end{array}$ & $\begin{array}{c}\text { Tertiär- } \\
\text { bereich B }\end{array}$ & $\begin{array}{c}\text { Tertiärbereich } \\
\text { A und weiterf. } \\
\text { Forschungs- } \\
\text { programme }\end{array}$ & $\begin{array}{l}\text { Alle } \\
\text { Bildungs- } \\
\text { bereiche }\end{array}$ & $\begin{array}{l}\text { Unterhalb } \\
\text { Sekundar- } \\
\text { bereich II }\end{array}$ & $\begin{array}{c}\text { Sekundar- } \\
\text { bereich II } \\
\text { und post- } \\
\text { sekundärer, } \\
\text { nicht-ter- } \\
\text { tiärer Bereich }\end{array}$ & $\begin{array}{l}\text { Tertiär- } \\
\text { bereich }\end{array}$ & $\begin{array}{l}\text { Alle } \\
\text { Bildungs- } \\
\text { bereiche }\end{array}$ \\
\hline \multirow{3}{*}{ Australien } & & (1) & $(2)$ & (3) & (4) & (5) & (6) & (7) & (8) & (9) \\
\hline & Männer & 8.1 & 4.5 & 4.5 & 2.5 & 5.2 & 8.6 & 4.6 & 2.8 & 5.3 \\
\hline & Frauen & 7.0 & 5.2 & 3.9 & 2.6 & 5.1 & 8.4 & 5.0 & 3.5 & 5.7 \\
\hline \multirow[t]{2}{*}{ Österreich ${ }^{1}$} & Männer & 6.9 & 2.8 & 1.1 & 1.8 & 3.2 & 6.2 & 2.3 & 1.2 & 2.6 \\
\hline & Frauen & 5.9 & 3.2 & 1.3 & 2.5 & 3.6 & 6.2 & 2.8 & 1.8 & 3.3 \\
\hline \multirow[t]{2}{*}{ Belgien ${ }^{1}$} & Männer & 7.7 & 3.9 & 2.2 & 2.4 & 4.8 & 8.1 & 3.2 & 2.2 & 4.5 \\
\hline & Frauen & 13.5 & 7.0 & 3.0 & 3.3 & 7.4 & 14.8 & 7.6 & 2.7 & 7.6 \\
\hline \multirow{2}{*}{ Kanada } & Männer & 10.2 & 6.2 & 4.8 & 4.4 & 6.2 & 10.8 & 6.3 & 4.8 & 6.3 \\
\hline & Frauen & 10.2 & 6.2 & 4.5 & 4.4 & 5.8 & 12.3 & 6.7 & 4.8 & 6.2 \\
\hline \multirow[t]{2}{*}{ Tschechische Rep. } & Männer & 19.3 & 4.7 & $x(4)$ & 1.9 & 5.4 & 23.4 & 4.5 & 1.8 & 5.3 \\
\hline & Frauen & 19.1 & 8.0 & $\mathrm{x}(4)$ & 2.2 & 8.9 & 24.0 & 8.9 & 2.4 & 9.7 \\
\hline \multirow[t]{2}{*}{ Dänemark } & Männer & 4.0 & 2.7 & 3.3 & 3.5 & 3.1 & 4.0 & 2.3 & 3.2 & 2.8 \\
\hline & Frauen & 6.2 & 4.0 & 3.1 & 3.1 & 4.1 & 7.2 & 3.9 & 3.9 & 4.3 \\
\hline \multirow[t]{2}{*}{ Finnland } & Männer & 10.5 & 7.9 & 4.7 & 3.0 & 7.2 & 11.9 & 7.1 & 2.8 & 6.5 \\
\hline & Frauen & 12.7 & 9.2 & 5.9 & 3.6 & 8.1 & 15.0 & 9.8 & 5.3 & 8.2 \\
\hline \multirow[t]{2}{*}{ Frankreich } & Männer & 9.7 & 5.1 & 4.3 & 4.1 & 6.2 & 10.7 & 4.7 & 3.5 & 6.1 \\
\hline & Frauen & 14.4 & 9.3 & 5.0 & 5.6 & 9.8 & 18.1 & 9.5 & 5.5 & 10.6 \\
\hline \multirow[t]{2}{*}{ Deutschland } & Männer & 15.6 & 8.1 & 4.4 & 3.4 & 7.7 & 14.2 & 7.0 & 2.6 & 6.5 \\
\hline & Frauen & 11.5 & 8.4 & 5.8 & 4.4 & 8.1 & 11.2 & 7.4 & 4.4 & 7.2 \\
\hline Griechenland & Männer & 4.9 & 6.2 & 4.9 & 4.5 & 5.3 & 4.7 & 5.1 & 4.2 & 4.7 \\
\hline & Frauen & 12.3 & 15.1 & 8.3 & 9.6 & 12.5 & 16.7 & 14.9 & 7.1 & 13.2 \\
\hline Ungarn & Männer & 12.5 & 4.8 & $\mathrm{x}(4)$ & 1.1 & 5.5 & 15.1 & 4.6 & 0.7 & 5.6 \\
\hline & Frauen & 7.6 & 4.2 & $\mathrm{x}(4)$ & 1.3 & 4.3 & 9.9 & 4.1 & 1.2 & 4.5 \\
\hline Island & Männer & 2.3 & 1.2 & 0.8 & 1.0 & 1.5 & 1.7 & 1.4 & 0.6 & 1.3 \\
\hline & Frauen & 2.4 & 2.8 & 2.4 & 0.2 & 2.1 & 2.3 & 2.0 & 0.9 & 1.8 \\
\hline Irland & Männer & 5.5 & 2.3 & 1.9 & 1.1 & 3.3 & 6.3 & 2.0 & 1.6 & 3.4 \\
\hline & Frauen & 5.1 & 2.8 & 2.3 & 1.0 & 2.9 & 6.1 & 2.7 & 1.9 & 3.1 \\
\hline Italien & Männer & 6.9 & 4.9 & $\mathrm{x}(4)$ & 3.8 & 5.8 & 7.1 & 3.8 & 3.9 & 5.4 \\
\hline & Frauen & 14.0 & 9.3 & $\mathrm{x}(4)$ & 7.2 & 10.7 & 16.8 & 8.9 & 6.1 & 11.1 \\
\hline Japan & Männer & 6.9 & 4.8 & 3.2 & 2.8 & 4.4 & 7.5 & 3.6 & 2.0 & 3.1 \\
\hline & Frauen & 4.3 & 4.7 & 3.8 & 3.1 & 4.2 & 4.2 & 4.2 & 3.8 & 4.0 \\
\hline Korea & Männer & 4.3 & 3.7 & 5.0 & 3.2 & 3.8 & 4.9 & 3.5 & 2.7 & 3.4 \\
\hline & Frauen & 1.8 & 2.7 & 3.3 & 2.0 & 2.3 & 2.5 & 2.4 & 1.9 & 2.3 \\
\hline Luxemburg & Männer & 1.5 & 0.7 & 0.9 & 1.1 & 1.1 & 1.0 & 0.6 & 1.2 & 0.9 \\
\hline & Frauen & 2.3 & 1.5 & 0.4 & 2.6 & 1.9 & 2.1 & 2.0 & 1.6 & 2.0 \\
\hline Mexiko & Männer & 1.4 & 1.9 & 2.1 & 2.2 & 1.6 & 1.3 & 1.2 & 2.0 & 1.5 \\
\hline & Frauen & 1.4 & 1.6 & 1.8 & 2.2 & 1.6 & 1.3 & 1.7 & 1.7 & 1.5 \\
\hline Niederlande $^{1}$ & Männer & 3.0 & 1.6 & 1.5 & 1.8 & 2.0 & 3.0 & 1.4 & 1.6 & 1.9 \\
\hline & Frauen & 5.0 & 3.1 & 2.6 & 2.1 & 3.4 & 5.7 & 3.1 & 1.7 & 3.4 \\
\hline Neuseeland & Männer & 7.4 & 3.0 & 4.4 & 2.8 & 4.0 & 8.1 & 3.2 & 3.4 & 4.1 \\
\hline & Frauen & 5.9 & 3.6 & 2.9 & 3.2 & 3.9 & 7.5 & 3.8 & 3.6 & 4.4 \\
\hline Norwegen ${ }^{1}$ & Männer & 2.3 & 3.0 & 1.9 & 2.0 & 2.6 & 2.3 & 3.1 & 1.8 & 2.7 \\
\hline & Frauen & 2.2 & 2.2 & 3.7 & 1.6 & 2.0 & 4.0 & 2.4 & 1.8 & 2.3 \\
\hline Polen & Männer & 21.7 & 14.0 & $x(4)$ & 4.0 & 13.9 & 26.3 & 13.5 & 1.8 & 13.7 \\
\hline & Frauen & 23.7 & 18.3 & $\mathrm{x}(4)$ & 5.9 & 17.0 & 31.9 & 19.3 & 3.4 & 18.1 \\
\hline Portugal & Männer & 2.7 & 3.1 & 2.6 & 2.0 & 2.7 & 2.4 & 3.0 & 1.4 & 2.4 \\
\hline & Frauen & 4.6 & 3.3 & 2.9 & 3.3 & 4.3 & 5.0 & 2.8 & 1.9 & 4.2 \\
\hline Slowakische Rep. & Men & 44.3 & 14.8 & 5.3 & 4.5 & 15.7 & 55.1 & 14.8 & 3.9 & 16.1 \\
\hline & Frauen & 34.6 & 14.8 & 11.0 & 3.4 & 15.7 & 39.5 & 14.8 & 3.4 & 15.8 \\
\hline Spanien & Männer & 7.3 & 5.4 & 4.1 & 4.7 & 6.2 & 7.6 & 4.6 & 3.4 & 5.8 \\
\hline & Frauen & 16.1 & 12.8 & 13.0 & 8.8 & 13.3 & 18.1 & 12.7 & 8.6 & 13.5 \\
\hline Schweden & Männer & 5.6 & 5.0 & 3.4 & 2.6 & 4.5 & 6.3 & 4.7 & 2.9 & 4.3 \\
\hline & Frauen & 6.4 & 4.2 & 2.5 & 2.2 & 3.8 & 7.0 & 4.3 & 2.7 & 3.9 \\
\hline Schweiz & Männer & $\mathrm{m}$ & 1.1 & $\mathrm{~m}$ & $\mathrm{~m}$ & 1.1 & $\mathrm{~m}$ & $\mathrm{~m}$ & $\mathrm{~m}$ & $\mathrm{~m}$ \\
\hline & Frauen & $\mathrm{m}$ & 2.9 & $\mathrm{~m}$ & $\mathrm{~m}$ & 3.1 & $\mathrm{~m}$ & 3.4 & $\mathrm{~m}$ & 3.4 \\
\hline Türkei & Männer & 9.2 & 8.0 & $\mathrm{x}(4)$ & 5.6 & 8.6 & 9.3 & 5.5 & 3.4 & 7.9 \\
\hline & Frauen & 6.9 & 13.5 & $\mathrm{x}(4)$ & 6.1 & 7.7 & 7.7 & 11.2 & 3.2 & 7.3 \\
\hline Ver. Königreich & Männer & 9.4 & 4.1 & 2.7 & 2.0 & 4.1 & 11.9 & 3.9 & 2.2 & 4.2 \\
\hline & Frauen & 5.7 & 3.7 & 1.7 & 1.9 & 3.4 & 8.2 & 4.3 & 2.0 & 4.0 \\
\hline Vereinigte Staaten & Männer & 7.5 & 4.2 & 2.5 & 1.9 & 3.7 & 7.4 & 4.4 & 1.8 & 3.7 \\
\hline & Frauen & 8.9 & 3.4 & 2.3 & 2.0 & 3.3 & 8.9 & 3.7 & 2.3 & 3.6 \\
\hline Ländermittel & Men & 8.9 & 4.8 & 3.3 & 2.8 & 5.0 & 9.9 & 4.5 & 2.4 & 4.9 \\
\hline & Frauen & 9.4 & 6.4 & 4.0 & 3.5 & 6.1 & 11.1 & 6.3 & 3.3 & 6.3 \\
\hline
\end{tabular}

Hinweis: $\mathrm{x}$ bedeutet, dass die Daten in einer anderen Spalte enthalten sind, deren Referenz in runden Klammern nach dem ' $\mathrm{x}$ ' angegeben ist. So bedeutet z.B. $\mathrm{x}(2)$, dass die Daten in Spalte 2 enthalten sind.

1. Referenzjahr 2000.

Quelle: OECD. Beschreibung der ISCED-97-Stufen, Zuordnung der länderspezifischen Bildungsgänge zu ISCED 97 und länderspezifische Datenquellen

s. Anhang 3 (www.oecd.org/els/education/eag2002). 

BESCHÄFTIGUNG IM ALTER ZWISCHEN 15 UND 29 JAHREN

- Im Durchschnitt kann ein im Jahr 2001 Fünfzehnjähriger erwarten, noch etwas länger als sechs Jahre im Bildungssystem zu verbleiben. In 13 der 29 untersuchten Länder liegt diese Zahl zwischen 6 und 7 Jahren.

- Ein Fünfzehnjähriger kann damit rechnen, für 6,5 Jahre der kommenden 15 Jahre erwerbstätig, für insgesamt 0,8 Jahre arbeitslos und für 1,4 Jahre nicht auf dem Arbeitsmarkt zu sein. Am stärksten unterscheiden sich die Länder in der durchschnittlichen Länge der Phasen der Arbeitslosigkeit, was Unterschiede in den Beschäftigungsquoten junger Menschen widerspiegelt.

- Absolut gesehen, können junge Menschen heutzutage damit rechnen, nach Abschluss der Erstausbildung weniger lange arbeitslos zu sein als vor zehn Jahren.

\section{Abbildung A12.1.}

Zu erwartende Jahre in Ausbildung und nicht in Ausbildung (2001)

Für 15- bis 29-Jährige nach Beschäftigungsstatus

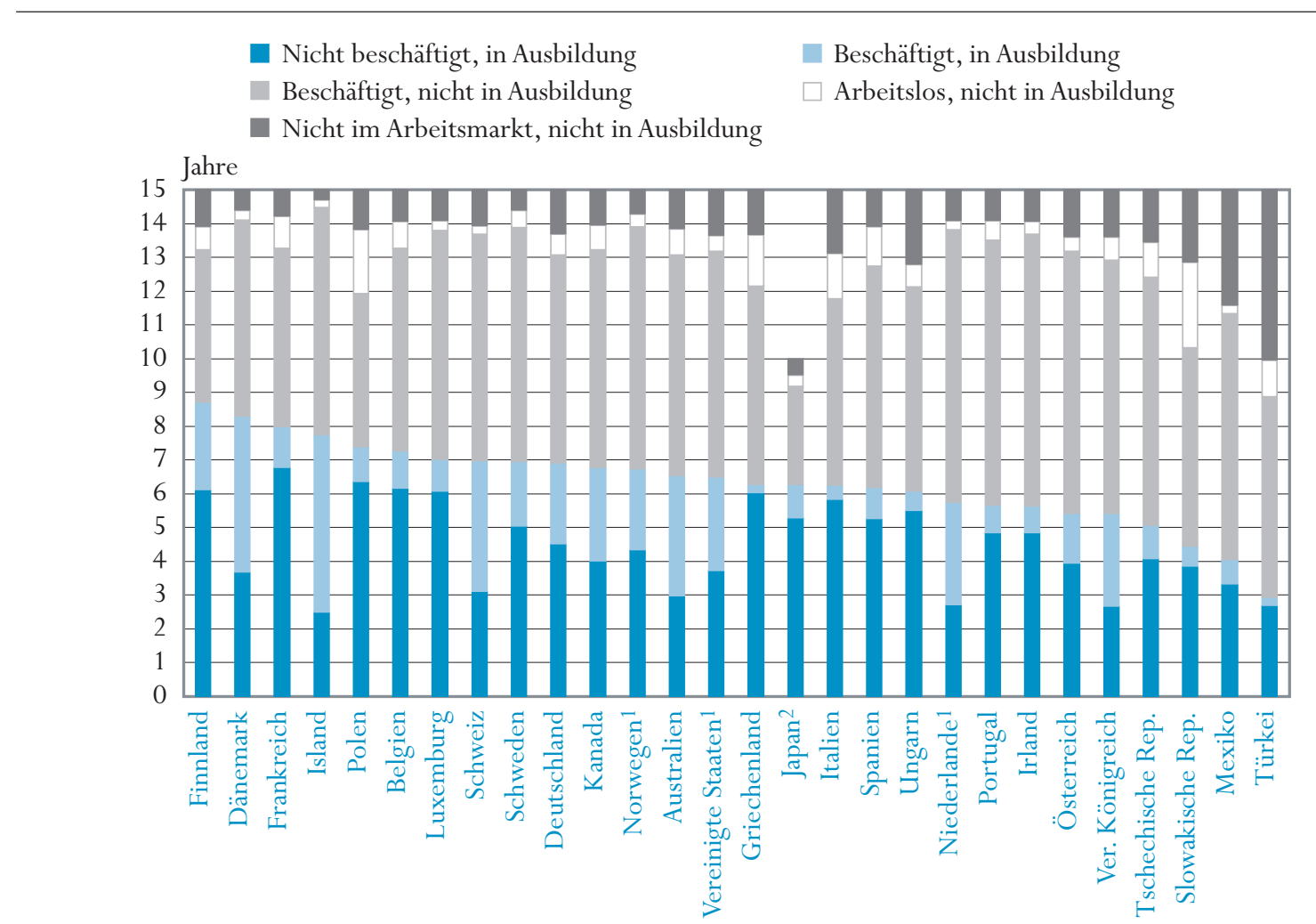

1. Referenzjahr 2000.

2. Daten beziehen sich auf 15 - bis 24-Jährige.

Anordnung der Länder in absteigender Reihenfolge der von jungen Menschen zu erwartenden Jahre in Ausbildung.

Quelle: OECD. Tabelle A12.1. Hinweise zu nationalen Datenquellen s. Anhang 3 (www.oecd.org/els/education/eag/2002). 


\section{Politischer Hintergrund}

Im Laufe des letzten Jahrzehnts ist die Zeit, die junge Menschen in der Erstausbildung verbringen, angestiegen, so dass diese ihren Eintritt in die Arbeitswelt nach hinten verschieben (siehe Bildung auf einen Blick 1998). In einem Teil dieser zusätzlichen Zeit werden Arbeit und Ausbildung miteinander verknüpft, eine in einigen Ländern weit verbreitete Praxis. Wenn junge Menschen ihre Ausbildung abgeschlossen haben, wird der Übergang zum Arbeitsmarkt häufig durch Phasen der Arbeitslosigkeit bzw. der Nicht-Beschäftigung erschwert, wobei Männer und Frauen nicht in derselben Weise betroffen sind. Absolut gesehen können junge Menschen heute jedoch damit rechnen, nach Abschluss der Erstausbildung weniger lang arbeitslos zu sein als vor zehn Jahren.

\section{Ergebnisse und Erläuterungen}

Basierend auf der gegenwärtigen Lage 15- bis 29-Jähriger bildet dieser Indikator die wichtigsten Tendenzen beim Übergang vom (Aus-)Bildungssystem zum Erwerbsleben ab.

Im Durchschnitt konnte ein im Jahr 2001 Fünfzehnjähriger erwarten, etwas mehr als weitere sechs Jahre im Bildungssystem zu verbleiben (Tabelle A12.1). Zwischen 1985 und 1996 stieg der zu erwartende Verbleib um fast 1,5 Jahre an. Seit 1996 hat sich der Anstieg verlangsamt. Länder, in denen junge Menschen bisher relativ wenig Zeit im Bildungssystem verbrachten, haben Boden gutgemacht, während in Ländern, in denen junge Menschen bereits in der Vergangenheit eine verleichsweise lange Zeit im Bildungssystem verbrachten, jetzt eine geringere Steigerung verzeichnen.

In 12 der 28 untersuchten Länder können 15-Jährige damit rechnen, dass sie zwischen 6 und 7 Jahre im Bildungssystem verbringen. Es besteht jedoch eine Kluft von ungefähr vier Jahren zwischen den beiden an den Extremen liegenden Ländergruppen Dänemark, Finnland, Frankreich und Island (8 Jahre im Durchschnitt) auf der einen Seite und Mexiko, die Slowakische Republik und die Türkei (4 Jahre im Durchschnitt) auf der anderen Seite.

Die durchschnittliche Gesamtzahl liegt für Frauen leicht höher (6,4 gegenüber 6,2 Jahre). In vielen Ländern sind die Zahlen ungefähr gleich, aber die Türkei stellt mit nur 2,4 Jahren zu erwartender weiterer Bildung für 15-jährige Mädchen eine Ausnahme dar. Am anderen Ende der Skala geht eine längere durchschnittliche Bildungsphase oft Hand in Hand mit einer Verlängerung der entsprechenden Bildungsdauer für Frauen (Tabelle A12.1).

Die Zahl für die zu erwartenden Jahre im Bildungssystem umfasst sehr unterschiedliche Kombinationen von Ausbildung und Arbeit. $\mathrm{Zu}$ den Möglichkeiten der Kombination von Ausbildung und Arbeit gehören duale Ausbildungsgänge und Teilzeitbeschäftigungen. Während diese in der Hälfte der untersuchten Länder nur eine Randerscheinung sind, machen sie in der anderen Hälfte zwischen ein und vier Jahre der zusätzlichen 6 bis 7 Jahre Bildung aus, die junge Menschen erwarten können.
Dieser Indikator zeigt die zu erwartenden Jahre, die junge Menschen in Ausbildung, Beschäftigung und Nicht-Beschäftigung zubringen werden.

Ein Fünfzehnjähriger kann im Durchschnitt erwarten, etwa weitere sechs Jahre im Bildungssystem zu verbleiben.

Die zu erwartende Anzahl von Jahren im Bildungssystem beinhaltet recht unterschiedliche Kombinationen von Bildung und Arbeit. 
Ein heute Fünfzehnjähriger kann damit rechnen, bis zum Alter von 29 Jahren 6,5 Jahre beschäftigt,

0,8 Jahre arbeitslos und 1,4 Jahre nicht im Arbeitsmarkt zu sein.
Zusätzlich zu den durchschnittlich sechs weiteren Jahren im Bildungssystem kann ein Fünfzehnjähriger damit rechnen, für 6,5 Jahre der kommenden 15 Jahre beschäftigt, für insgesamt 0,8 Jahre arbeitslos und für 1,4 Jahre nicht im Arbeitsmarkt zu sein, d.h. ohne in Ausbildung zu sein oder Arbeit zu suchen. Bemerkenswert ist, dass in absoluten Zahlen gesehen, junge Menschen heute nach Beendigung der Erstausbildung kürzere Zeiten der Arbeitslosigkeit zu erwarten haben als vor zehn Jahren.

Am stärksten unterscheiden sich die Länder in der durchschnittlichen Länge der Phasen der Arbeitslosigkeit, was hauptsächlich Unterschiede in den Beschäftigungsquoten der jungen Menschen widerspiegelt. Die aufaddierten Phasen der Arbeitslosigkeit belaufen sich im Durchschnitt in Dänemark, Island, Luxemburg, Mexiko, den Niederlanden und der Schweiz auf vier Monate oder weniger, während sie in Griechenland, Polen, der Slowakischen Republik und der Türkei über 18 Monate betragen.

Im Großen und Ganzen unterscheiden sich Männer und Frauen hinsichtlich der zu erwartenden Anzahl von Jahren der Arbeitslosigkeit sehr wenig. Während jedoch die Situation für beide Geschlechter in vielen Ländern ähnlich ist, scheinen Frauen in Griechenland, Portugal und Spanien klar benachteiligt zu sein, während sie in Australien, Deutschland, Kanada, der Slowakischen Republik, Türkei, Ungarn und dem Vereinigten Königreich Vorteile haben. In einigen dieser letzteren Länder, besonders in Australien, dem Vereinigten Königreich und ganz speziell in der Türkei, wird die niedrigere Zahl für Frauen jedoch weitgehend von der Tatsache beeinflusst, dass viele Frauen den Arbeitsmarkt verlassen und somit die Nachfrage nach Arbeitsstellen verringern.

Während junge Männer damit rechnen können, im Alter von 15 bis 29 Jahren knapp über sechs Monate außerhalb von Bildungssystem und Arbeitsmarkt zu verbringen, liegt der Durchschnitt für Frauen bei fast zwei Jahren. In den nordischen Ländern (Island, Finnland und Schweden) gibt es bei dieser Kennzahl keine Unterschiede zwischen jungen Männern und Frauen. Umgekehrt besteht in Mexiko, der Tschechischen Republik, Türkei und Ungarn eine wesentlich stärkere Tendenz junger Frauen, den Arbeitsmarkt zu verlassen. In allen anderen Ländern verbringen Frauen zwischen 15 und 29 Jahren im Durchschnitt etwa ein Jahr länger außerhalb des Arbeitsmarktes als Männer.

\section{Definitionen und angewandte Methodik}

Die Daten stammen

Die vorliegenden Statistiken basieren auf Arbeitskräfteerhebungen über den Anteil bestimmter Altersgruppen junger Menschen an den genannten Kategorien. Diese Anteile wurden dann für die Altersgruppe 15 bis 29 Jahre aufsummiert, um die zu erwartende Anzahl von Jahren in dem angegebenen Erwerbsstatus zu erhalten. Die Berechnung geht also davon aus, dass die heute Fünfzehnjährigen im Alter von 15 bis 29 Jahren den gleichen Bildungs- und 
Arbeitsmustern folgen werden wie die Altersgruppe zwischen 15 und 29 Jahren im vorliegenden Bezugsjahr.

Die Teilnahme an Bildungsmaßnahmen kann sich sowohl auf Teilzeit- als auch auf Vollzeit beziehen. Die Definitionen der verschiedenen Arten von Erwerbsstatus folgen den Richtlinien des Internationalen Arbeitsamts (ILO), außer für die Kategorie der sich „gleichzeitig in Ausbildung und in Beschäftigung befindenden jungen Menschen", die alle dualen Ausbildungssysteme umfasst, unabhängig von deren Klassifikation nach den ILO-Richtlinien. Die Daten für diesen Indikator wurden aus einer gesonderten Datenerfassung mit einem Bezugszeitraum zu Beginn des Kalenderjahres, zumeist dem ersten Quartal oder dem Durchschnitt für die ersten drei Monate, errechnet. 
Tabelle A12.1.

Zu erwartende Jahre in Ausbildung und nicht in Ausbildung für 15- bis 29-Jährige, nach Geschlecht und Beschäftigungsstatus (2001)

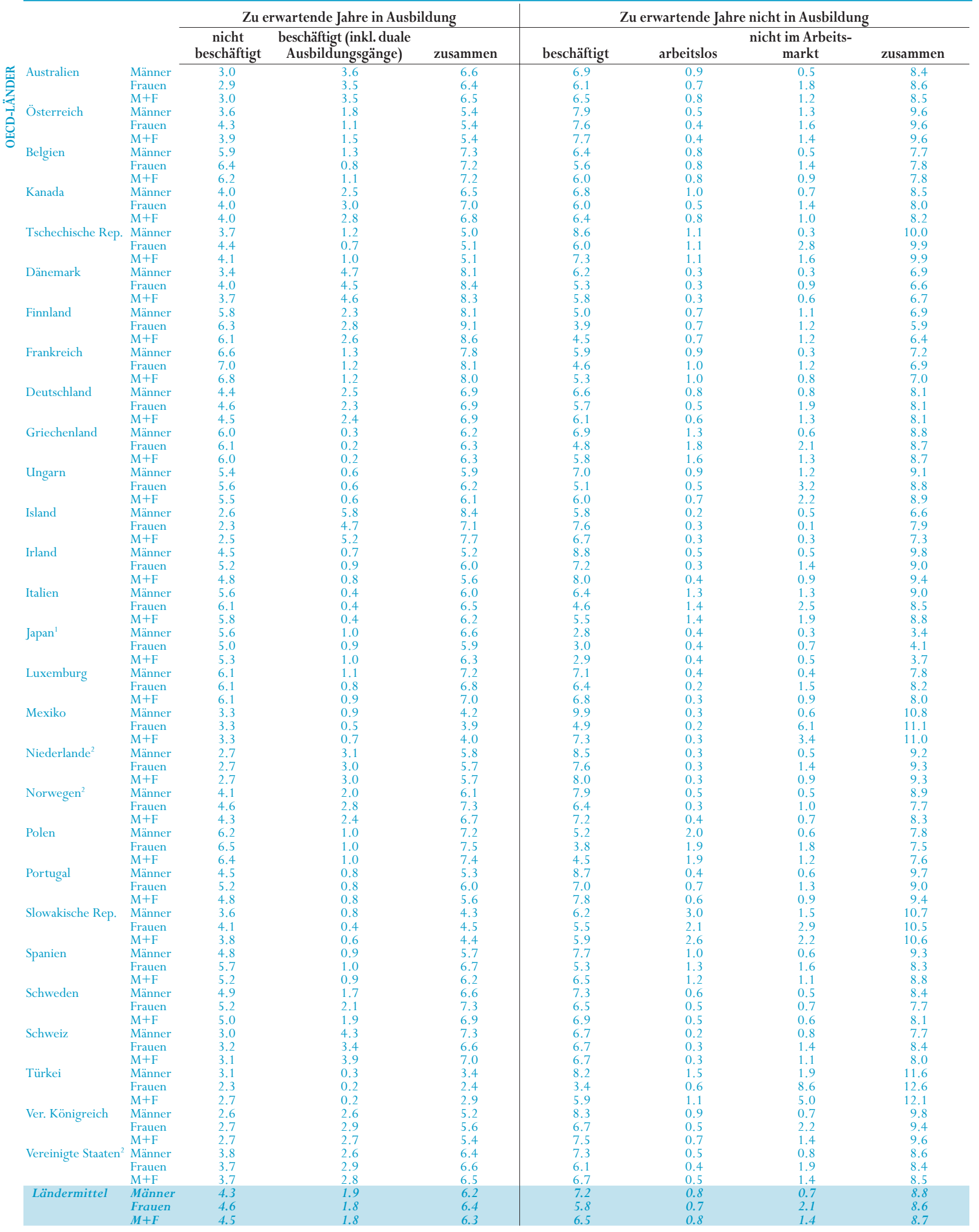

1. Daten beziehen sich auf 15 - bis 24-Jährige.

2. Referenzjahr 2000.

Quelle: OECD. Länderspezifische Datenquellen s. Anhang 3 (www.oecd.org/els/education/eag2002). 


\section{ERTRAGSRATEN VON BILDUNG: INDIVIDUELLE UND GESELLSCHAFTLICHE ERTRAGSRATEN VON BILDUNG UND DEREN BESTIMMENDE FAKTOREN}

- Zwischen Bildungsstand und Einkommen besteht ein positiver Zusammenhang. Der Abschluss des Sekundarbereich II und eines post-sekundären, nicht-tertiären Bildungsgangs stellt in vielen Ländern einen Wendepunkt dar, ab dem jede zusätzliche Ausbildung einen besonders hohen Einkommenszuschlag mit sich bringt. In allen Ländern verdienen die Absolventen des Tertiärbereichs erheblich mehr als die des Sekundarbereich II und eines post-sekundären, nicht-tertiären Bildungsgangs. Die Einkommensunterschiede zwischen Absolventen des Tertiärbereichs und denen des Sekundarbereich II sind im Allgemeinen deutlich größer als zwischen Absolventen des Sekundarbereich II und denen des Sekundarbereich I und darunter.

- Die Einkommen von Personen mit einem Abschluss unterhalb des Sekundarbereich II belaufen sich in der Regel auf 60 bis 90 Prozent der Einkommen von Absolventen des Sekundarbereich II und postsekundärer, nicht-tertiärer Bildungsgänge.

- Frauen verdienen im Vergleich zu Männern mit einem ähnlichen Bildungsstand nach wie vor weniger. 


\section{Abbildung A13.1.}

Relative Einkommen aufgrund von Erwerbseinkommen (2001)

Für 25- bis 64-Jährige, nach Bildungsstand und Geschlecht

(Sekundarbereich II und post-sekundärer, nicht-tertiärer Bereich $=100$ )

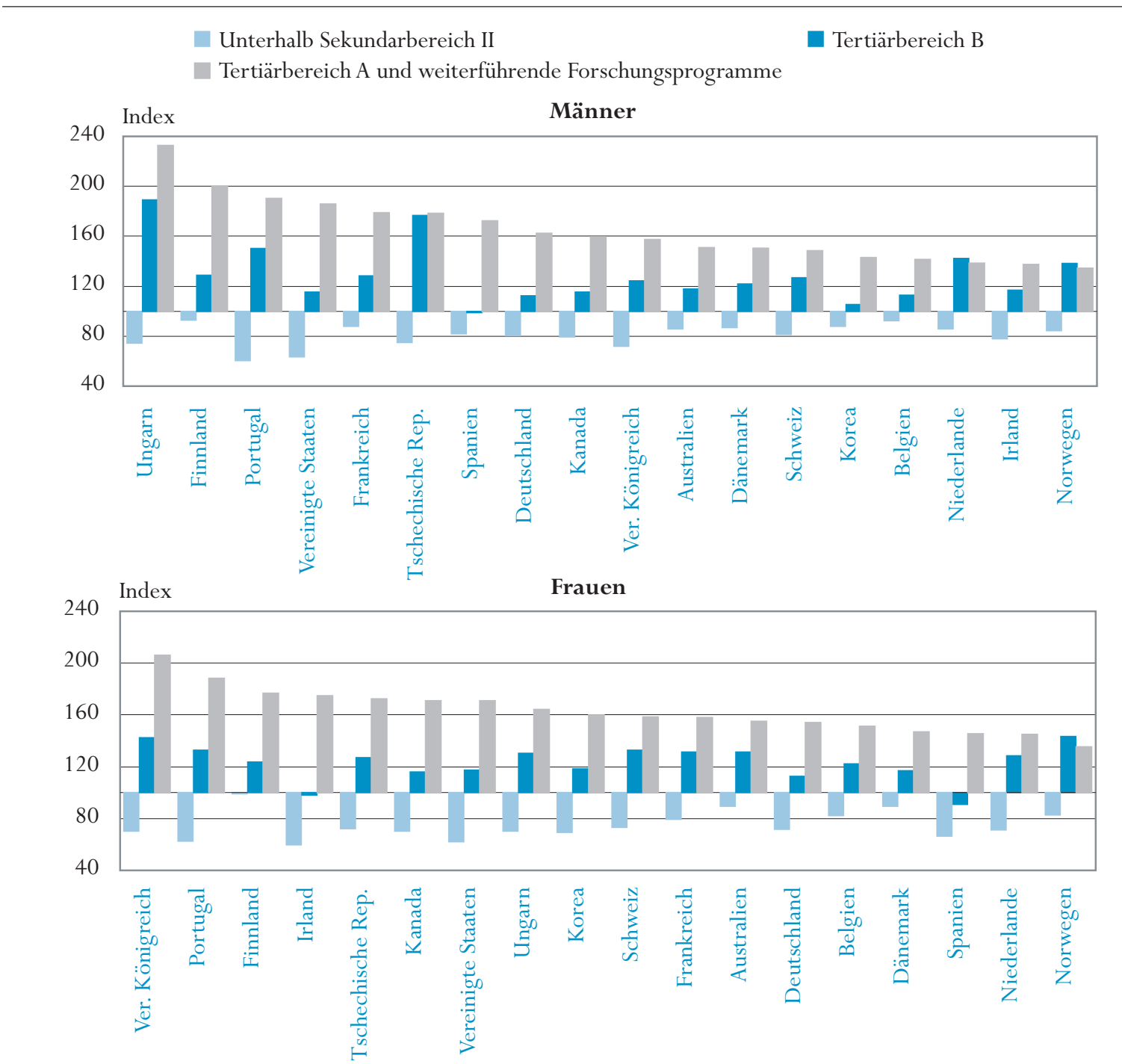

Anordnung der Länder in absteigender Reihenfolge der relativen Einkommen der Bevölkerung mit einem Abschluss im Tertiärbereich A oder einem weiterführenden Forschungsprogramm.

Quelle: OECD. Tabelle A13.1. Hinweise zu nationalen Datenquellen s. Anhang 3 (www.oecd.org/els/education/eag/2002). 


\section{Politischer Hintergrund}

Eine Möglichkeit, durch die die Märkte Anreize für Arbeitskräfte schaffen, angemessene Fähigkeiten und Kenntnisse zu entwickeln und zu erhalten, sind Einkommensunterschiede und hier insbesondere höhereVerdienstmöglichkeiten für diejenigen, die sich weiterbilden und zusätzliche Abschlüsse erzielen. Das Streben nach einem höheren Bildungsstand kann auch als Investition in das Humankapital gesehen werden. Das Humankapital ist der Bestand an Fähigkeiten und Kenntnissen, die der Einzelne besitzt oder - normalerweise durch Bildung oder Ausbildung - (weiter-)entwickelt und sodann als Gegenleistung für ein Einkommen auf dem Arbeitsmarkt anbietet. Je höher die Einkommen sind, die sich aus einer Vermehrung des Humankapitals ergeben, umso höher ist der Ertrag dieser Investition und der Einkommenszuschlag für bessere Fähigkeiten und Kenntnisse und/oder eine höhere Produktivität.

Gleichzeitig verursacht es Kosten, an (Aus-)Bildungsangeboten teilzunehmen, die man bei einer Betrachtung der Erträge aus der Bildung gleichermaßen beachten muss. Dieser Indikator untersucht die Erträge aus Bildungsinvestitionen sowie die Kosten und den Nutzen, die dabei zu berücksichtigen sind.

\section{Ergebnisse und Erläuterungen}

\section{Bildung und Einkommen}

Einkommensdifferentiale gelten als Maßstab für die in einem bestimmten Land momentan herrschenden finanziellen Anreize für den Einzelnen, in eine weitere (Aus-)Bildung zu investieren. Nach Bildungsstand aufgeschlüsselte Einkommensunterschiede können aber auch Unterschiede im Angebot von Bildungsgängen in verschiedenen Bildungsbereichen bzw. Beschränkungen des Zugangs zu diesen Bildungsgängen widerspiegeln. Der wirtschaftliche Vorteil eines tertiären Bildungsabschlusses kann anhand eines Vergleichs des Verhältnisses der mittleren Jahreseinkommen von Absolventen tertiärer Bildungsgänge zu den mittleren Jahreseinkommen von Absolventen des Sekundarbereich II oder eines post-sekundären, nicht-tertiären Bildungsgangs verdeutlicht werden. Der Einkommensnachteil derjenigen ohne einen Abschluss des Sekundarbereich II oder eines post-sekundären, nicht-tertiären Bildungsgangs wird in einem ähnlichen Vergleich offensichtlich. Die länderspezifischen Schwankungen der relativen Einkommen (vor dem Abzug von Steuern) spiegeln mehrere Einflussfaktoren wider, darunter Anforderungen an die Fähigkeiten und Kenntnisse der Arbeitskräfte, Mindestlohngesetze, die Stärke der Gewerkschaften, den Geltungsbereich von Tarifverträgen, das Angebot an Arbeitskräften mit unterschiedlichem Bildungsstand, das Spektrum der Berufserfahrungen von Erwerbspersonen mit hohem und niedrigem Bildungsstand, die Verteilung der Arbeitsplätze nach Berufen und die relative Häufigkeit von Teilzeitbeschäftigungen in ihren unterschiedlichen Formen unter Erwerbspersonen mit unterschiedlichem Bildungsstand.
Dieser Indikator zeigt

die Einkommen für

Arbeitskräfte mit

unterschiedlichen

Bildungsabschlüssen...

... sowie die Erträge aus

Bildungsinvestitionen

und die zugrunde

liegenden Kosten und

den Nutzen.

Einkommensdifferentiale gelten als Majsstab für die in einem bestimmten Land momentan herrschenden finanziellen Anreize für den Einzelnen, in eine weitere (Aus-) Bildung zu investieren. 
Zwischen Bildungsstand und Einkommen besteht ein positiver Zusammenhang - unabhängig vom sozio-ökonomischen System oder vom Entwicklungsstand der Wirtschaft.

Das Einkommensdifferential zwischen Frauen und Männern mit ähnlichem Bildungsstand bleibt weiterhin erheblich,...
Abbildung A13.1 zeigt einen starken positiven Zusammenhang zwischen Bildungsstand und Einkommen. In allen Ländern verdienen Absolventen des Tertiärbereichs deutlich mehr als Absolventen des Sekundarbereich II und post-sekundärer, nicht-tertiärer Bildungsgänge. Die Einkommensunterschiede zwischen Absolventen des Tertiärbereichs und Absolventen des Sekundarbereich II und post-sekundärer, nicht-tertiärer Bildungsgänge sind im Allgemeinen deutlich größer als zwischen letzteren und Absolventen mit einem Abschluss unterhalb des Sekundarbereich II, was darauf schließen lässt, dass der Abschluss des Sekundarbereich II oder eines post-sekundären, nicht tertiären Bildungsgangs in vielen Ländern einen Wendepunkt darstellt, ab dem eine weitere Ausbildung einen besonders hohen Einkommenszuschlag mit sich bringt. Unter den Ländern, die Daten über Bruttoeinkommen vorgelegt haben, liegt der Einkommenszuschlag für 25- bis 64-jährige Männer für einen Abschluss des Tertiärbereichs zwischen maximal 33 Prozent in Italien, Neuseeland und Norwegen und mindestens 80 Prozent in Finnland, Portugal, der Tschechischen Republik und Ungarn.

Die im vorliegenden Indikator aufgezeigten Einkommensdaten für die einzelnen Länder unterscheiden sich unter anderem aufgrund unterschiedlicher Methoden der Datenerhebung. Die Ergebnisse sind daher mit Vorsicht zu interpretieren. Insbesondere bei Ländern, die Daten von Jahreseinkommen vorgelegt haben, beeinflussen Unterschiede in der Häufigkeit von Beschäftigungsverhältnissen, die nicht das gesamte Jahr andauern, bei Personen mit unterschiedlichem Bildungsstand das relative Einkommen, was sich in den Daten der Länder, die wöchentliche oder monatliche Gehaltsangaben vorgelegt haben, nicht niederschlägt (s. „Definitionen und angewandte Methodik“).

\section{Bildungs- und geschlechtspezifische Unterschiede bei den Einkommen}

Im Vergleich zu einem Abschluss des Sekundarbereich II erhöht ein tertiärer Abschluss in Australien, Belgien, Irland, Kanada, Korea, den Niederlanden, Neuseeland, Norwegen, der Schweiz und dem Vereinigten Königreich das Einkommen von Frauen stärker als das der Männer, während für die übrigen Länder genau das Gegenteil zutrifft (Tabelle A13.1).

Zwar haben sowohl Männer als auch Frauen mit einem Abschluss des Sekundarbereich II oder höher erhebliche Einkommensvorteile gegenüber denjenigen des gleichen Geschlechts, die keinen vergleichbaren Abschluss besitzen, doch sind die Einkommensunterschiede zwischen Männern und Frauen mit gleichem Bildungsstand nach wie vor erheblich und werden durch die Häufigkeit der Teilzeitbeschäftigung von Frauen noch verschärft.

Nimmt man alle Bildungsbereiche zusammen, so bewegen sich die Einkommen 30- bis 44-jähriger Frauen im Verhältnis zu denjenigen gleichaltriger Männer zwischen weniger als 55 Prozent in der Schweiz und dem Vereinigten Königreich bis zu mehr als 75 Prozent in Spanien und Ungarn (Tabelle A13.2). 
Die geschlechtsspezifischen Gehaltsunterschiede lassen sich zum Teil mit der Laufbahn- und Berufswahl, den im Arbeitsmarkt verbrachten Jahren und der relativ großen Häufigkeit von Teilzeitarbeit bei Frauen erklären. Nach Alter aufgeschlüsselte Einkommensdaten lassen eine Tendenz der Angleichung des durchschnittlichen Einkommens zwischen Männern und Frauen quer über alle Bildungsstände vermuten. Dies könnte auch eine Auswirkung des gestiegenen Anteils der Frauen unter den jüngeren Absolventen des Tertiärbereichs sein. In 6 von 20 Ländern liegt das Verhältnis der Einkommen von weiblichen zu männlichen Absolventen des Tertiärbereich A und weiterführender Forschungsprogramme im Alter von 30 bis 44 Jahren mindestens 10 Prozentpunkte über dem der Altersgruppe der 55- bis 64-Jährigen (Tabelle A13.2).

\section{Individuelle Ertragsraten aus Bildungsinvestitionen}

Die Gesamtheit der Anreize für Investitionen in Humankapital, die in den Arbeitsmarktvorteilen und finanziellen Vereinbarungen ihren Niederschlag finden, lässt sich in Schätzungen der individuellen Ertragsrate für derartige Investitionen zusammenfassen (Abb. A13.3 und Tabelle A13.3). Die Ertragsrate ist ein Maß für die über die Zeit erhaltenen Erträge im Verhältnis zu den Kosten der ursprünglichen Investition in Bildung. Sie wird in Prozenten ausgedrückt und entspricht der Ertragsrate oder dem Zinssatz für eine Geldanlage auf einem Sparkonto (Erklärung der angewandten Methodik s. Anhang 3 unter www.oecd.org/els/education/eag2002). In ihrer umfassendsten Form entsprechen die Kosten den Studiengebühren, dem entgangenen Einkommen (versteuert und um die Beschäftigungswahrscheinlichkeit bereinigt), abzüglich der Fördermittel in Form von Beihilfen oder Darlehen. Der Nutzen setzt sich zusammen aus dem erhöhten Nettoeinkommen, zuzüglich der höheren Beschäftigungswahrscheinlichkeit und ggf. abzüglich der Rückzahlung von während der Ausbildungszeit gewährten staatlichen Beihilfen. Bei den Berechnungen wird unterstellt, dass der Bildungsteilnehmer sich in Vollzeitausbildung befindet, nicht erwerbstätig ist und somit während dieser Zeit kein Einkommen hat. Allerdings wird eine derart berechnete Ertragsrate wahrscheinlich nach oben verzerrt sein, da weder Arbeitslosigkeit noch Ruhestands- oder Vorruhestandsbezüge berücksichtigt werden. Die in diesem Indikator dargestellten Ertragsberechnungen lassen alle immateriellen Vorteile von Bildung außer Acht.

Die geschätzten realen individuellen Ertragsraten für Abschlüsse im Sekundarbereich II und im Hochschulbereich variieren zwischen den in Tabelle A13.3 aufgeführten Ländern erheblich, liegen aber in allen Fällen über den realen Zinssätzen, oft sogar erheblich darüber. Dies legt nahe, dass Investitionen in das Humankapital für den Durchschnittsbürger eine sehr attraktive Form der Vermögensbildung darstellen. Für Studiengänge im Tertiärbereich kann man anhand der Schätzwerte für die sogenannte „Gesamtertragsrate“, die den kombinierten Effekt aus Einkommen, Ausbildungsdauer, Besteuerung, Arbeitslosigkeitsrisiko, Studiengebühren und staatlichen Ausbildungsbeihilfen umfasst, zwischen drei Ländergruppen unterscheiden.
... wobei sich die geschlechtsspezifischen Gehaltsunterschiede zum Teil mit der Laufbahnund Berufswahl, Zeiten außerhalb des Arbeitsmarktes und der größseren Häufigkeit von Teilzeitarbeit bei Frauen erklären lassen.

Die Gesamtheit aller Anreize für Investitionen in Humankapital, die in den Vorteilen im Arbeitsmarkt ihren Niederschlag finden, lässt sich in der individuellen Ertragsrate zusammenfassen.

In allen Ländern liegt die individuelle Ertragsrate über den realen Zinssätzen, häufig sogar erheblich. 


\section{Abbildung A13.2.}

Individuelle Ertragsraten für Bildungsinvestitionen (1999-2000)

Auswirkungen der Ausbildungsdauer, Steuern, Arbeitslosigkeitsrisiko, Studiengebühren und staatlichen Ausbildungsbeihilfen auf die Ertragsraten im Sekundarbereich II und im Tertiärbereich, nach Geschlecht (in Prozentpunkten)

- Individuelle Gesamtertragsrate für Frauen

¿ Individuelle Gesamtertragsrate für Männer
Steuern

Studiengebühren

Ausbildungsdauer

Arbeitslosigkeitsrisiko

Öffentliche Studienbeihilfen
Sekundarbereich II $^{1}$

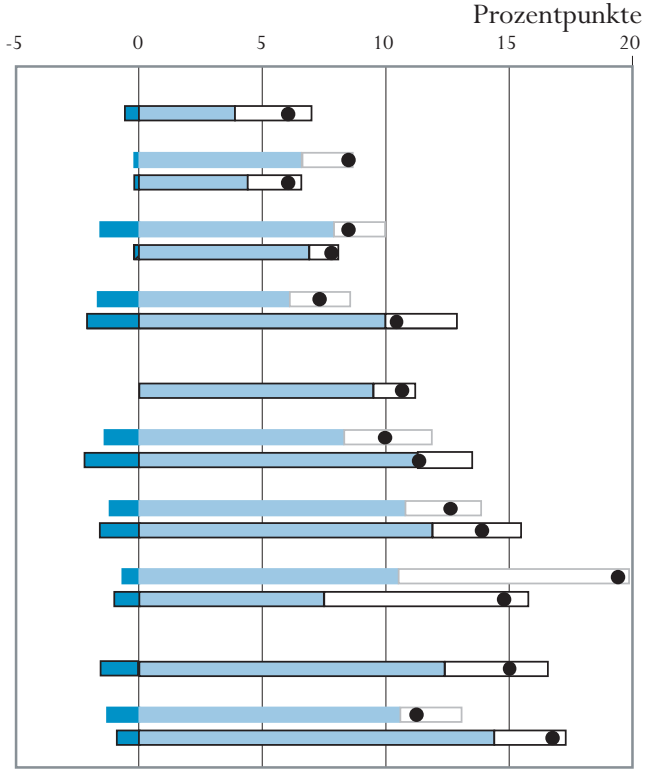

Tertiärbereich ${ }^{2}$

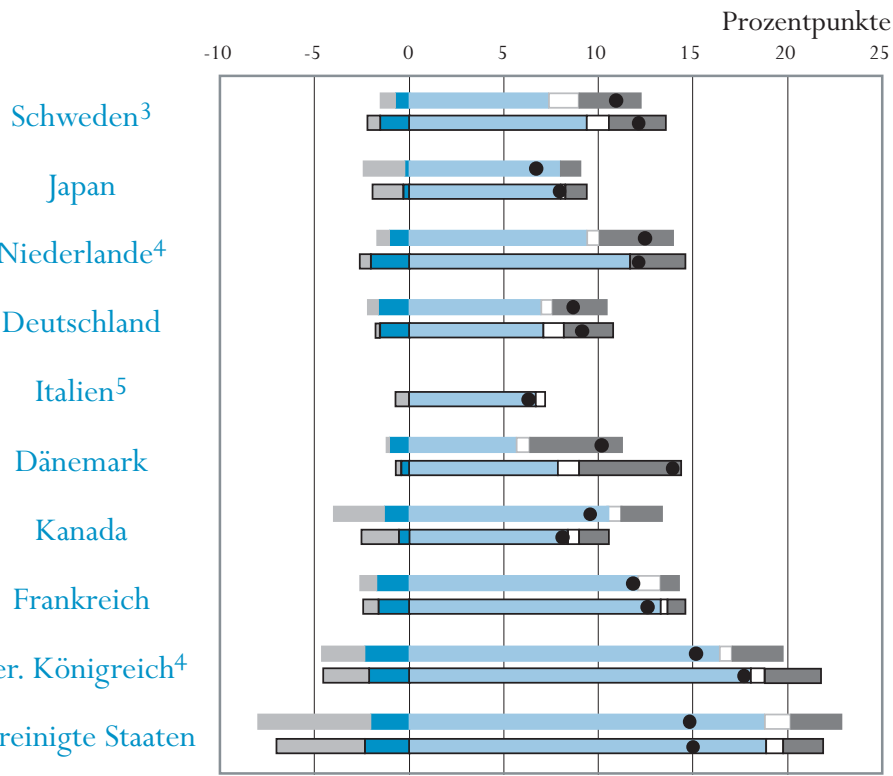

1. Die Ertragsrate einer Ausbildung im Sekundarbereich II wird berechnet durch einen Kosten/Nutzen-Vergleich mit einer Ausbildung im Sekundarbereich I.

2. Die Ertragsrate einer Ausbildung im Tertiärbereich wird berechnet durch einen Kosten/Nutzen-Vergleich mit einer Ausbildung im Sekundarbereich II.

3. Für eine Ausbildung im Tertiärbereich wurde anstelle der durchschnittlichen theoretischen Dauer verschiedener Studiengänge für Frauen und Männer die durchschnittliche theoretische Dauer von Standardstudiengängen verwendet. Für Frauen sind die Einkommensdifferentiale zwischen einer Ausbildung im Sekundarbereich I und Sekundarbereich II nicht groß genug, um eine Berechnung einer positiven internen Ertragsrate zu erlauben.

4. Referenzjahr 1997.

5. Die Daten für Männer stammen aus Daten zu den Einkommen nach Steuern aus 1998.

Anordnung der Länder in absteigender Reihenfolge der individuellen Gesamtertragsrate aus Bildungsinvestitionen für Männer im Sekundarbereich II. Quelle: OECD. Tabelle A13.3. 
- Erstens: Das Vereinigte Königreich bildet aufgrund der extrem starken Vorteile, die ein Abschluss im Tertiärbereich dort mit sich bringt, eine Gruppe für sich allein.

- Zweitens: In Dänemark, Frankreich, den Niederlanden, Schweden und den Vereinigten Staaten sind die Ertragsraten mit 10 bis 15 Prozent relativ hoch.

- Drittens: In den übrigen Ländern liegen die Ertragsraten unter 10 Prozent, am niedrigsten in Italien und Japan.

Für die in Tabelle A13.3 aufgeführten Länder errechnet sich für den Sekundarbereich II eine Ertragsrate von über 10 Prozent, außer für Deutschland (Frauen), Japan, die Niederlande und Schweden.

Im Tertiärbereich zeigt sich bei den Berechnungen für die meisten Länder nur ein sehr geringer geschlechtsspezifischer Unterschied der Ertragsraten. Im Sekundarbereich II ist dieser Unterschied jedoch in Deutschland und den Vereinigten Staaten deutlicher ausgeprägt, denn dort wird die Ertragsrate für Frauen wegen des relativ geringen geschlechtsspezifischen Einkommensdifferentials um ein Viertel bis zu einem Drittel geschmälert.

Aus Tabelle A13.3 ist ersichtlich, dass das Einkommensdifferential und die Dauer der Ausbildung im Allgemeinen die ausschlaggebenden Faktoren für die individuellen Ertragsraten sind. Somit gibt es in den Ländern mit insgesamt starken Anreizen für Humankapitalinvestitionen typischerweise große bildungsbezogene Einkommensdifferentiale und/oder relativ kurze Ausbildungsgänge und umgekehrt. So sind die hohen errechneten Ertragsraten für einen Abschluss im Tertiärbereich im Vereinigten Königreich beispielsweise größtenteils auf die relativ kurzen Standard-Hochschulstudiengänge zurückzuführen, während die niedrigen Ertragsraten in Deutschland stark durch die vergleichsweise lange Studiendauer beeinflusst werden. Es ist sogar so, dass eine Verkürzung der durchschnittlichen Dauer tertiärer Studiengänge um ein Jahr (bei gleichbleibender Qualität der Ausbildung), die Ertragsrate für Männer in den untersuchten Ländern, bei im übrigen unveränderten Bedingungen, um 1 bis 5 Prozentpunkte anheben würde. Um eine derartige hypothetische Verkürzung der tertiären Bildungs- und Studiengänge richtig einordnen zu können, sollte man sich vor Augen führen, dass eine Steigerung des Entgeltvorteils für einen Abschluss im Tertiärbereich um 5 bis 14 Prozentpunkte erforderlich wäre, um die gleiche Ertragssteigerung über ein größeres Differential bei den Löhnen- und Gehältern zu erreichen.

Von diesem allgemeinen Muster gibt es jedoch auch bemerkenswerte Ausnahmen. Trotz geringer Lohndifferentiale und langer Studiendauer bieten Dänemark und in geringerem Ausmaß auch Schweden vergleichsweise starke Anreize, einen Hochschulabschluss zu erwerben. Und für junge Menschen in Frankreich ist es trotz relativ geringer Entgeltvorteile im Vergleich zur
Es scheint so, als seien das Einkommensdifferential und die Dauer der Ausbildung die ausschlaggebenden Faktoren für die Erträge,... 


\section{$A_{13}$}

...es zählen aber auch noch andere

Faktoren, u.a....

..Steuern, die sich ertragsmindernd auswirken,...

...ein geringeres

Arbeitslosigkeitsrisiko, das sich ertragssteigernd auswirkt,...

... Studiengebühren, die sich ertragsmindernd auswirken, ...

...und stadtiche Beihilfen oder Darlehen, die sich ertragssteigernd auswirken.
Ausbildungsdauer sehr attraktiv, in einen Abschluss im Sekundarbereich II zu investieren.

Durch eine schrittweise Berücksichtigung der einzelnen Faktoren bei der Berechnung der Ertragsrate lässt sich deren Einfluss auf den Unterschied zwischen der eng gefassten Ertragsrate, die nur das Einkommensdifferential und die Ausbildungslänge einbezieht, und der Gesamtertragsrate bewerten:

- Steuern wirken sich negativ auf die aus Bruttoeinkommen und Ausbildungsdauer errechnete Ertragsrate aus; in den untersuchten Ländern bei einem Abschluss im Tertiärbereich mit durchschnittlich 1,3 Prozentpunkten und bei einem Abschluss im Sekundarbereich II mit 1,1 Prozentpunkten. Im Tertiärbereich sind die Auswirkungen der Besteuerung im Vereinigten Königreich und denVereinigten Staaten besonders gravierend, vor allem wegen der Kombination eines großen bildungsbedingten Einkommensdifferentials mit einem progressiven Steuersystem, dies gilt aber auch in Frankreich und den Niederlanden. Bei den Berechnungen für einen Abschluss im Sekundarbereich II schlagen die negativen Auswirkungen des Steuersystems aufgrund des steilen Anstiegs der Progressionskurve im entsprechenden Einkommensbereich in Deutschland und Dänemark besonders stark durch, in Japan dagegen am geringsten.

- Unterschiede beim Arbeitslosigkeitsrisiko erhöhen die Ertragsrate gegenüber Ertragsberechnungen, die nur auf dem Einkommen (vor Steuern) und der Ausbildungsdauer basieren. Als Konsequenz der großen Differenz zwischen den Arbeitslosenquoten der Absolventen des Sekundarbereich I und des Sekundarbereich II steigt die Ertragsrate besonders stark für Abschlüsse im Sekundarbereich II, im Durchschnitt sind dies in den untersuchten Ländern 3,6 Prozentpunkte für Frauen und Männer. Der relativ große Unterschied bei der Arbeitslosigkeit in Frankreich erhöht die Ertragsrate sogar um 8,3 bis 9,4 Prozentpunkte. Im tertiären Bereich wirken sich die unterschiedlich großen Beschäftigungsaussichten wesentlich weniger auf die Ertragsraten aus; sie nehmen in den in Tabelle A13.3 aufgeführten Ländern im Durchschnitt um 0,7 bis 1 Prozentpunkt für Männer und Frauen zu.

- Studiengebühren haben besonders negative Auswirkungen auf die Renditen für tertiäre Bildungsabschlüsse in den Vereinigte Staaten und - in geringerem Ausmaß - in Kanada und im Vereinigten Königreich. In Kontinentaleuropa schlagen die Studiengebühren weit weniger durch, da sie dort wesentlich niedriger sind.

- Staatliche Ausbildungsbeihilfen und Darlehen im Tertiärbereich erhöhen die Anreize zum Verbleib im Bildungssystem entscheidend. In den untersuchten Ländern macht das durchschnittlich 2,5 bis 3 Prozentpunkte gegenüber den Ertragsraten ohne diese Förderung aus. Der Effekt ist in Dänemark, den Niederlanden und Schweden besonders stark, jedoch weniger ausgeprägt in Frankreich und in Italien überhaupt nicht erkennbar. 


\section{Gesellschaftliche Erträge aus Bildungsinvestitionen}

Der Nutzen, den die Gesellschaft aus zusätzlicher Bildungsteilnahme zieht, kann auf Grundlage der gesellschaftlichen Ertragsrate beurteilt werden. Die gesellschaftliche Ertragsrate muss Kosten und Nutzen der Gesellschaft aus Bildungsinvestitionen berücksichtigen, welche sich beträchlich von denen für den Einzelnen unterscheiden können. Die gesellschaftlichen Kosten umfassen die Opportunitätskosten dafür, dass Menschen nicht im Produktionsprozess tätig sind, sowie die Kosten für das Bildungsangebot in voller Höhe, also nicht nur die vom Einzelnen getragenen Kosten. Ein Teil des gesellschaftlichen Nutzens ist die höhere Produktivität, die mit Bildungsinvestitionen einhergeht, sowie ferner eine ganze Palette potentieller, nicht direkt materieller Nutzeffekte, wie eine niedrigere Kriminalitätsrate, ein stärkerer sozialer Zusammenhalt, informiertere und mündigere Bürger. Während Angaben zu den von der Gesellschaft zu tragenden Kosten für die meisten OECD-Länder vorliegen, ist es wesentlich schwieriger, Informationen über die Gesamtheit des gesellschaftlichen Nutzens zu beschaffen. Soweit Produktivitätssteigerungen im Lohnkostendifferential zum Ausdruck kommen, kann man dieses als Maß für den wirtschaftlichen Nutzen heranziehen, den die Gesellschaft aus Bildungsanstrengungen zieht. Die Möglichkeit der mit der Bildung einhergehenden externen Effekte legt jedoch nahe, dass die beobachteten Einkommensdifferentiale die gesamtwirtschaftlichen Effizienzsteigerungen nicht vollständig erfassen. Andererseits zeigen Untersuchungen, dass ein (geringer) Teil der Entgeltzuschläge für die besser Gebildeten auf den Bildungstand zurückzuführen ist, den Arbeitgeber mit bestimmten Qualitäten in Zusammenhang bringen, und nicht auf Produktivitätsdifferentiale, die sich aus Investitionen in das Humankapital ergeben. Diese nicht direkt materiellen Vorteile der Bildung sind zwar beträchtlich, lassen sich jedoch zum Zwecke einer Ertragsberechnung meist nur schwer monetär ausdrücken.

Angesichts der Probleme bei der Herleitung einer umfassenden gesellschaftlichen Ertragsrate enthält Tabelle A13.4 Schätzungen einer „eng gefassten“ Definition, die sämtliche externen Effekte und nicht direkt materiellen Vorteile unberücksichtigt lässt. Daher werden diese Schätzungen nach unten verzerrt sein, soweit mit den Investitionen in Humankapital seitens des durchschnittlichen Studierenden beachtliche positive externe Effekte verbunden sind.

Die Schätzwerte deuten darauf hin, dass die gesellschaftliche Ertragsrate sowohl im Sekundarbereich II als auch im Tertiärbereich im Vereinigten Königreich und in den Vereinigten Staaten besonders hoch ist, während sie in Dänemark in diesen beiden Bildungsbereichen am niedrigsten ausfällt. In Frankreich beläuft sie sich für den Sekundarbereich II auf einen mittleren Wert, ist jedoch für den Tertiärbereich vergleichsweise hoch.

Da insbesondere die gesellschaftlichen Kosten für Bildung normalerweise weitaus höher sind als die des Einzelnen, liegt die „eng gefasste“ gesellschaftliche
Der Nutzen, den

die Gesellschaft

aus zusätzlicher

Bildungsteilnahme

zieht, lässt sich anhand

der gesellschaftlichen

Ertragsrate beurteilen,... ...die momentan
jedoch nur im engeren
Sinne und ohne
Berücksichtigung
der nicht direkt
ökonomischen Vorteile
geschätzt werden kann.

Zwar liegt auch die gesellschaftliche 
Ertragsrate weit über den risikofreien Realzinsen, jedoch meistens unter der individuellen Ertragsrate, da Bildung erhebliche gesellschaftliche Kosten verursacht.

Man könnte sagen, dass die hohen Ertragsraten bedeuten,...

... dass für gebildete Arbeitnehmer auf dem Markt ein Ungleichgewicht herrscht, das nach einer Ausweitung der Bildungskapazitäten verlangt,...

... oder Grenzerträgen, die wesentlich unter den Durchschnittserträgen liegen,...

was staatliches Eingreifen weniger dringlich erscheinen ließse.
Ertragsrate deutlich unter der individuellen. Im Tertiärbereich ist der Unterschied in Dänemark und Schweden besonders deutlich, der Unterschied reicht von 4 bis zu fast 7,5 Prozentpunkten. Im Sekundarbereich II ist der Unterschied zwischen der individuellen und der gesellschaftlichen Ertragsrate in Frankreich besonders groß, während er in Deutschland und den Niederlanden relativ gering ist.

\section{Was die Ertragsraten aussagen}

Die vorstehend erläuterten individuellen und gesellschaftlichen Ertragsraten liegen im Allgemeinen deutlich höher als der risikofreie Realzins. Da die Erträge aus der Erhöhung des Humankapitals mit vielen Unwägbarkeiten behaftet sind (wie die große Einkommensspanne unter den höher Gebildeten zeigt), werden die Investoren wohl einen ausgleichenden Risikoaufschlag fordern. Der Abstand der Ertragsrate zum Realzins ist jedoch größer, als sich alleine durch den Risikoaufschlag erklären lässt. Somit könnten die hohen Ertragsraten zweierlei bedeuten.

Eine mögliche Interpretation wäre, dass diese hohen Ertragsraten auf einen ernsten Mangel besser ausgebildeter Arbeitnehmer hindeuten, wodurch deren Einkommen nach oben getrieben wird. Das könnte auf eine vorübergehende Situation hindeuten, bei der hohe Bildungserträge letztendlich zu einer so starken Reaktion auf der Angebotsseite führen, dass die Ertragsraten wieder auf das für andere Produktionsmittel übliche Niveau zurückgedrängt werden. Diese Anpassungsperiode könnte sich jedoch recht lange hinziehen und das Tempo der Anpassung hinge dann stark von der Fähigkeit des Bildungssystems ab, die gesteigerte Nachfrage befriedigen zu können sowie von der Fähigkeit des Arbeitsmarktes, das relativ geänderte Arbeitskräfteangebot zu verkraften. Der Anpassungsmechanismus könnte auch durch mehr Aufklärung der Studierenden über die Erträge aus den einzelnen Bildungs- und Studiengängen beschleunigt werden, so dass sie ihre Entscheidungen bewusster treffen könnten.

Diese hohen Erträge können teilweise durchaus mit dem Marktgleichgewicht in Einklang stehen. Dies wäre dann der Fall, wenn die Grenzertragsraten wesentlich unter den Durchschnittsertragsraten lägen und somit eher den Grenzertragsraten für andere Produktionsmittel entsprächen. Die Grenzertragsrate läge tatsächlich unter der Durchschnittsertragsrate, wenn die Studierenden im Grenzbereich weniger begabt oder motiviert wären als der Durchschnitt und somit den durchschnittlichen Gehaltszuschlag wahrscheinlich gar nicht erhalten würden. Bei dieser Auslegung würden die hohen Ertragsraten teilweise den Preis für eine knappe Ressource, nämlich Talent und Motivation, zur Geltung bringen.

Falls die Bildungserträge im Grenzbereich niedriger sind, ist staatliches Eingreifen zur Förderung der Bildung von Humankapital nicht mehr so dringlich, falls die Qualität der Studierenden im Grenzbereich ohnehin nicht mehr steigerungsfähig ist. In dem Maße jedoch, in dem Bildungssysteme die 
kognitiven und nicht-kognitiven Fähigkeiten junger Menschen verbessern können, würde die Bildungspolitik andererseits langfristig in der Lage sein, einen wesentlichen Beitrag zu mehr Effizienz und gleichen Bildungschancen zu leisten.

\section{Definitionen und angewandte Methodik}

Das relative Erwerbseinkommen ist definiert als das mittlere Einkommen aus einer Tätigkeit (Erwerbseinkommen vor dem Abzug von Steuern) von Erwerbspersonen mit einem bestimmten Bildungsstand, dividiert durch das mittlere Einkommen von Erwerbspersonen mit einem Abschluss im Sekundarbereich II (x 100). Die Berechnungen basieren ausschließlich auf Personen mit Einkommen aus einer während des Bezugszeitraums ausgeübten Erwerbstätigkeit.

Bei den Einkommensdaten in Tabelle A13.1 handelt es sich bei den meisten Ländern um Jahreseinkommen; bei Frankreich, Spanien und der Schweiz sind es Monatseinkommen. Für Belgien und Frankreich beziehen sich die Daten ausschließlich auf Angestellte. In den spanischen Daten bleiben Personen, die weniger als 15 Stunden pro Woche arbeiten, unberücksichtigt. Die länderspezifischen Unterschiede der relativen Einkommen spiegeln daher nicht nur Unterschiede in den Löhnen und Gehältern, sondern auch Unterschiede in der Erfassung, in der Zahl der Arbeitswochen pro Jahr und der Zahl der Arbeitsstunden pro Woche wider. Da ein niedrigerer Bildungsstand mit einer geringeren Arbeitsstundenzahl (insbesondere durch Teilzeitarbeit) und mit unsichereren Beschäftigungsverhältnissen (einer größeren Wahrscheinlichkeit von nur befristeten Anstellungen oder einer größeren Anfälligkeit für Arbeitslosigkeit im Laufe eines Jahres) einhergehen, werden die relativen Einkommen für höhere Bildungsniveaus in den Tabellen und Abbildungen höher sein als die, die sich aus einer Untersuchung der relativen Löhne und Gehälter ergeben würden. Auch die beobachteten geschlechtsspezifischen Unterschiede der relativen Einkommen innerhalb eines Landes können durch einige dieser Faktoren beeinflusst sein.

Zur Berechnungsmethode für die Ertragsraten in Tabellen A13.3 und A13.4 s. Anhang 3 unter www.oecd.org/els/education/eag2002.
Die Daten stammen aus nationalen Arbeitskräfteerhebungen sowie aus anderen Erhebungen. 
Tabelle A13.1.

Relative Einkommen der Bevölkerung mit Erwerbseinkommen

Für 25- bis 64-Jährige und 30- bis 44-Jährige, nach Bildungsstand und Geschlecht (Sekundarbereich II und post-sekundärer, nicht-tertiärer Bereich = 100)

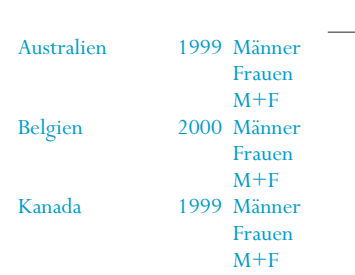

Tschechische Rep. 1999 Männer Frauen $\mathrm{M}+\mathrm{F}$

Dänemark 1999 Männer Frauen $\mathrm{M}+\mathrm{F}$

Finnland 1999 Männe$$
\text { Frauen }
$$

Frankreich 1999 Männer Frauen $\mathrm{M}+\mathrm{F}$

Deutschland 2000 Männer Frauen $\mathrm{M}+\mathrm{F}$

Ungarn 2001 Männer Frauen $\mathrm{M}+\mathrm{F}$

Irland

1998 Männer Frauen $\mathrm{M}+\mathrm{F}$

Italien 1998 Männer Frauen $\mathrm{M}+\mathrm{F}$

Korea 1998 Männer Frauen $\mathrm{M}+\mathrm{F}$

Niederlande 1997 Männer Frauen

Neuseeland M+F

001 Männer Frauen

Norwegen 1999 Männe Frauen $\mathrm{M}+\mathrm{F}$

Portugal 1999 Männer
Frauen Frauen
$\mathrm{M}+\mathrm{F}$

Spanien 1998 Männer Frauen $\mathrm{M}+\mathrm{F}$

Schweden 1999 Männe Frauen $\mathrm{M}+\mathrm{F}$

Schweiz 2001 Männe Frauen $\mathrm{M}+\mathrm{F}$

Ver. Königreich 2001 Männer Frauen $\mathrm{M}+\mathrm{F}$

Vereinigte Staaten 2001 Männer Frauen $\mathrm{M}+\mathrm{F}$

65

\begin{tabular}{|c|c|c|c|}
\hline darbereich II & Tertiärbereich B & $\begin{array}{l}\text { Tertiärbereich A und } \\
\text { weiterführende } \\
\text { Forschungsprogramme }\end{array}$ & Tertiärbereich insgesam \\
\hline $30-44$ & $25-64$ & $30-44$ & $30-44$ \\
\hline
\end{tabular}

Unterhalb Sekundarbereich II

(3)

\begin{tabular}{l|l} 
(4) & (5) \\
\hline 120 & 151
\end{tabular}

(5)

(6)

(7) (8)

\begin{tabular}{|c|c|}
\hline & 86 \\
\hline & 89 \\
\hline & 80 \\
\hline & 93 \\
\hline & 82 \\
\hline
\end{tabular}

\begin{tabular}{r|r}
83 & \\
88 & \\
78 & \\
$\mathrm{x}(1)$ & \\
$\mathrm{x}(1)$ &
\end{tabular}

\begin{tabular}{ll|l}
118 & 120 & 15 \\
131 & 130 & 155 \\
116 & 115 & 144 \\
113 & $\mathrm{x}(3)$ & 14 \\
122 & $\mathrm{x}(3)$ & 151
\end{tabular}

$\begin{array}{ll}122 & \mathrm{x}(3) \\ 112 & \mathrm{x}(3)\end{array}$

$116 \quad 117$

$\begin{array}{ll}116 & 118 \\ 112 & 113\end{array}$

$177 \quad 182$

127

151

122

117

112
129

129
124

124
120

128

131

125

112
113

115

189
130

130
151

117

98
111

111
$\mathrm{x}(5)$

$\mathrm{x}(5)$
$\mathrm{x}(5)$
$\mathrm{x}(5)$

$\mathrm{x}(5)$
105

118
106

106
142
128

136
$\times(7)$

$x(7)$
$x(7)$

$\mathrm{x}(7)$
$\mathrm{x}(7)$

$\mathrm{x}(7)$
138

138
144

153

150
133

133
141

99

91
99

99
$\times(7)$

$\mathrm{x}(7)$
$\mathrm{x}(7)$

$x(7)$
127

127
133

144

124

142

128

116
117

114

124
151
118

118

112
108

108
125

123

115
137

137
139

139
133

133
110
114

114
114

114

170
119
136

136

126

83
117

$\mathrm{x}(6)$

$\mathrm{x}(6)$

$\mathrm{x}(6)$
109

138

113
128

128
133

129

$\mathrm{x}(8)$
$\mathrm{x}(8)$

$\mathrm{x}(8)$
$\mathrm{x}(8)$

$\mathrm{x}(8)$

141
150

153

155

139
146

103

89
101

101
$\mathrm{x}(8)$

$\mathrm{x}(8)$
$\mathrm{x}(8)$

$\mathrm{x}(8)$
128

142

147
126

126
133

124

115
119

113

\begin{tabular}{ll|l}
151 & 149 \\
155 & 155 \\
144 & 143 &
\end{tabular}

$\begin{array}{ll}139 & 139 \\ 146 & 146\end{array}$

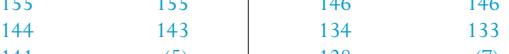

\begin{tabular}{ll|ll}
141 & $\mathrm{x}(5)$ & 128 & $\mathrm{x}(7)$
\end{tabular}

151
146

$\mathrm{x}(5)$
$\mathrm{x}(5)$

$\mathrm{x}(5)$

$128 \quad \mathrm{x}(7)$

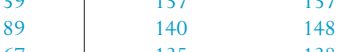

\begin{tabular}{ll|ll}
171 & 189 & 140 & 148 \\
162 & 167 & 135 & 138
\end{tabular}

162

172
180

180
150

147

151

200

176
190
178

178
158

158
169

169
162
154

154
163

133
164

164
194

137

175
157

157
138

115
127

127
143

160
147

147
138

145

141
$\mathrm{x}(7)$

$\mathrm{x}(7)$

$\mathrm{x}(7)$

134
135

131

190
188

188
192

172

145
157

157
$\mathrm{x}(7)$

$x(7)$
$x(7)$
$x(7)$

$\times(7)$
148

158

164
157

157
206

174

186
171

181

176
176

176
182

145

146

146
188

188
172

179

181
165
174

174

160
153

160

237

163

191

143

170
157

157
142

114

114
126

126
136

181

142

130

150

136
$\mathrm{x}(8)$

$\mathrm{x}(8)$
$\mathrm{x}(8)$

$\mathrm{x}(8)$

136

137

131

194

206
202

155

138

144
$\times(8)$

$\mathrm{x}(8)$
$\mathrm{x}(8)$

$\mathrm{x}(8)$

144
167

162

162
162

216

181

183
173

178

$\begin{array}{ll}178 & 177 \\ 170 & 174\end{array}$

$\begin{array}{ll}170 & 174 \\ 179 & 181\end{array}$

$\begin{array}{ll}179 & 181 \\ 133 & 129\end{array}$

$\begin{array}{ll}123 & 120 \\ 124 & 120\end{array}$

$\begin{array}{ll}124 & 120 \\ 167 & 159\end{array}$

$145 \quad 141$

$\begin{array}{ll}153 & 144 \\ 159 & 163\end{array}$

$\begin{array}{ll}159 & 163 \\ 145 & 152\end{array}$

$\begin{array}{ll}150 & 155 \\ 141 & 139\end{array}$

$\begin{array}{ll}141 & 139 \\ 137 & 137\end{array}$

$\begin{array}{ll}137 & 137 \\ 143 & 141\end{array}$

$232 \quad 237$

$\begin{array}{ll}164 & 162 \\ 194 & 190\end{array}$

$\begin{array}{ll}131 & 136\end{array}$

$\begin{array}{ll}135 & 136 \\ 142 & 140\end{array}$

$\begin{array}{ll}142 & 140 \\ 138 & 142\end{array}$

$\begin{array}{ll}115 & 114 \\ 127 & 126\end{array}$

$\begin{array}{ll}127 & 126 \\ 132 & 129\end{array}$

$141 \quad 164$

$\begin{array}{ll}135 & 134 \\ 139 & 130\end{array}$

$\begin{array}{ll}139 & 130 \\ 143 & 148\end{array}$

$\begin{array}{ll}141 & 135 \\ 130 & 122\end{array}$

$\begin{array}{ll}130 & 122 \\ 136 & 135 \\ 133 & 128\end{array}$

$\begin{array}{ll}133 & 128 \\ 135 & 137\end{array}$

$\begin{array}{ll}135 & 137 \\ 135 & 138\end{array}$

$\begin{array}{ll}135 & 138 \\ 133 & 133\end{array}$

$\begin{array}{ll}180 & 185 \\ 170 & 185\end{array}$

$\begin{array}{ll}170 & 185 \\ 178 & 187\end{array}$ 
Tabelle A13.2.

Einkommensunterschiede zwischen Frauen und Männern

Durchschnittliche Jahreseinkommen von Frauen als Prozentsatz derjenigen von Männern, für 30- bis 44-Jährige und 55-bis 64-Jährige, nach Bildungsstand

\begin{tabular}{|c|c|c|c|c|c|c|c|c|c|c|c|}
\hline \multirow[b]{4}{*}{ Australien } & & \multicolumn{2}{|c|}{$\begin{array}{c}\text { Unterhalb } \\
\text { Sekundarbereich II }\end{array}$} & \multicolumn{2}{|c|}{$\begin{array}{l}\text { Sekundarbereich II und } \\
\text { post-sekundärer, nicht- } \\
\text { tertiärer Bereich }\end{array}$} & \multicolumn{2}{|c|}{ Tertiärbereich B } & \multicolumn{2}{|c|}{$\begin{array}{l}\text { Tertiärbereich A und } \\
\text { weiterführende } \\
\text { Forschungsprogramme }\end{array}$} & \multicolumn{2}{|c|}{ Alle Bildungsbereiche } \\
\hline & & $30-44$ & $55-64$ & $30-44$ & $55-64$ & $30-44$ & $55-64$ & $30-44$ & $55-64$ & $30-44$ & $55-64$ \\
\hline & & $(1)$ & (2) & (3) & (4) & (5) & (6) & $(7)$ & $(8)$ & (9) & $(10)$ \\
\hline & 1999 & 66 & 67 & 63 & 75 & 68 & 66 & 65 & 58 & 65 & 66 \\
\hline Kanada & 1999 & 51 & 61 & 58 & 66 & 59 & 57 & 69 & 65 & 63 & 62 \\
\hline Tschechische Rep. & 1999 & 66 & 58 & 67 & 64 & 45 & 62 & 67 & 63 & 63 & 61 \\
\hline Dänemark & 1999 & 76 & 67 & 72 & 67 & 68 & 65 & 72 & 71 & 73 & 65 \\
\hline Finnland & 1999 & 74 & 78 & 69 & 77 & 68 & 73 & 63 & 65 & 70 & 70 \\
\hline Frankreich & 1999 & 70 & 62 & 75 & 69 & 76 & 72 & 68 & 64 & 74 & 60 \\
\hline Deutschland & 2000 & 51 & 49 & 62 & 59 & 64 & 65 & 59 & 62 & 60 & 53 \\
\hline Ungarn & 2001 & 83 & 81 & 84 & 94 & 59 & 48 & 58 & 69 & 77 & 78 \\
\hline Irland & 1998 & 50 & 36 & 70 & 55 & 46 & 43 & 83 & 60 & 66 & 43 \\
\hline Italien & 1998 & 71 & 70 & 69 & 43 & $\mathrm{x}(7)$ & $x(8)$ & 56 & 45 & 73 & 57 \\
\hline Korea & 1998 & 57 & 62 & 69 & 70 & 87 & 96 & 92 & 99 & 67 & 50 \\
\hline Niederlande & 1997 & 46 & 43 & 55 & 50 & 57 & 39 & 63 & 50 & 55 & 45 \\
\hline Neuseeland & 2001 & 59 & 57 & 61 & 70 & $\mathrm{x}(7)$ & $\mathrm{x}(8)$ & 68 & 54 & 62 & 62 \\
\hline Norwegen & 1999 & 60 & 61 & 61 & 63 & 64 & 65 & 61 & 61 & 62 & 61 \\
\hline Portugal & 1999 & 72 & 70 & 70 & 67 & 63 & 57 & 75 & 68 & 73 & 66 \\
\hline Spanien & 1998 & 61 & $\mathrm{x}(1)$ & 81 & $\mathrm{x}(3)$ & 70 & $x(5)$ & 73 & $\mathrm{x}(7)$ & 79 & $\mathrm{x}(9)$ \\
\hline Schweden & 1999 & 74 & 73 & 74 & 69 & $\mathrm{x}(9)$ & $\mathrm{x}(10)$ & $\mathrm{x}(9)$ & $\mathrm{x}(10)$ & 71 & 70 \\
\hline Schweiz & 2001 & 50 & 50 & 55 & 52 & 61 & 42 & 63 & 66 & 54 & 47 \\
\hline Ver. Königreich & 2001 & 55 & 43 & 50 & 53 & 53 & 81 & 66 & 66 & 54 & 54 \\
\hline Vereinigte Staaten & 2001 & 58 & 65 & 60 & 54 & 62 & 57 & 57 & 50 & 60 & 51 \\
\hline
\end{tabular}

Hinweis: $\mathrm{x}$ bedeutet, dass die Daten in einer anderen Spalte enthalten sind, deren Referenz in runden Klammern nach dem " $\mathrm{x}$ " angegeben ist. So bedeutet z.B. $\mathrm{x}(2)$, dass die Daten in Spalte 2 enthalten sind.

Quelle: OECD. Länderspezifische Datenquellen s. Anhang 3, Tabelle 6 (www.oecd.org/els/education/eag2002). 
KAPITEL A Bildungs- und Lernergebnisse

Tabelle A13.3.

Individuelle interne Ertragsraten aus Bildungsinvestitionen (1999-2000)

Auswirkungen von Ausbildungsdauer, Steuern, Arbeitslosigkeitsrisiko, Studiengebühren und staatlichen Ausbildungsbeihilfen auf die Ertragsraten im Sekundarbereich II und im Tertiärbereich, nach Geschlecht (in Prozentpunkten)

\begin{tabular}{|c|c|c|c|c|c|c|c|c|c|c|c|c|c|c|c|c|c|c|c|c|}
\hline & \multicolumn{8}{|c|}{$\begin{array}{c}\text { Ertragsraten einer Ausbildung } \\
\text { im Sekundarbereich II (in Prozentpunkten) }{ }^{1}\end{array}$} & \multicolumn{12}{|c|}{$\begin{array}{c}\text { Ertragsraten einer Ausbildung } \\
\text { im Tertiärbereich (in Prozentpunkten) }{ }^{2}\end{array}$} \\
\hline & \multirow{2}{*}{\multicolumn{2}{|c|}{$\begin{array}{c}\text { Individuelle } \\
\text { Gesamter- } \\
\text { tragsraten }\end{array}$}} & \multicolumn{6}{|c|}{ Auswirkungen von } & \multirow{2}{*}{\multicolumn{2}{|c|}{$\begin{array}{c}\text { Individuelle } \\
\text { Gesamter- } \\
\text { tragsraten }\end{array}$}} & \multicolumn{10}{|c|}{ Auswirkungen von } \\
\hline & & & \multicolumn{2}{|c|}{$\begin{array}{c}\text { Ausbildungs- } \\
\text { dauer }\end{array}$} & \multicolumn{2}{|c|}{ Steuern } & \multicolumn{2}{|c|}{$\begin{array}{l}\text { Arbeitslosig- } \\
\text { keitsrisiko }\end{array}$} & & & \multicolumn{2}{|c|}{$\begin{array}{c}\text { Ausbildungs- } \\
\text { dauer }\end{array}$} & \multicolumn{2}{|c|}{ Steuern } & \multicolumn{2}{|c|}{$\begin{array}{l}\text { Arbeitslosig- } \\
\text { keitsrisiko }\end{array}$} & \multicolumn{2}{|c|}{$\begin{array}{c}\text { Studienge- } \\
\text { bühren }\end{array}$} & \multicolumn{2}{|c|}{$\begin{array}{c}\text { öffentliche } \\
\text { Studien } \\
\text { beihilfen }\end{array}$} \\
\hline & M & $\mathrm{F}$ & M & $\mathrm{F}$ & M & $\mathrm{F}$ & M & $\mathrm{F}$ & M & $\mathrm{F}$ & M & 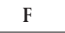 & M & $\mathrm{F}$ & M & $\mathrm{F}$ & M & $\mathrm{F}$ & M & $\mathrm{F}$ \\
\hline Kanada & 13.6 & 12.7 & 11.9 & 10.8 & -1.6 & -1.2 & 3.6 & 3.1 & 8.1 & 9.4 & 8.4 & 10.6 & -0.5 & -1.3 & 0.6 & 0.6 & -2.0 & -2.7 & 1.6 & 2.2 \\
\hline Dänemark & 11.3 & 10.5 & 11.3 & 8.3 & -2.2 & -1.4 & 2.2 & 3.6 & 13.9 & 10.1 & 7.9 & 5.7 & -0.4 & -1.0 & 1.1 & 0.7 & -0.1 & -0.2 & 5.4 & 4.9 \\
\hline Frankreich & 14.8 & 19.2 & 7.5 & 10.5 & -1.0 & -0.7 & 8.3 & 9.4 & 12.2 & 11.7 & 13.3 & 12.1 & -1.6 & -1.7 & 0.4 & 1.2 & -0.8 & -0.9 & 0.9 & 1.0 \\
\hline Deutschland & 10.8 & 6.9 & 10.0 & 6.1 & -2.1 & -1.7 & 2.9 & 2.5 & 9.0 & 8.3 & 7.1 & 7.0 & -1.5 & -1.6 & 1.1 & 0.6 & -0.3 & -0.6 & 2.6 & 2.9 \\
\hline Italien $^{3}$ & 11.2 & $\mathrm{~m}$ & 9.5 & $\mathrm{~m}$ & $\mathrm{~m}$ & $\mathrm{~m}$ & 1.7 & $\mathrm{~m}$ & 6.5 & $\mathrm{~m}$ & 6.7 & $\mathrm{~m}$ & $\mathrm{~m}$ & $\mathrm{~m}$ & 0.5 & $\mathrm{~m}$ & -0.7 & $\mathrm{~m}$ & $\mathrm{n}$ & $\mathrm{m}$ \\
\hline Japan & 6.4 & 8.5 & 4.4 & 6.6 & -0.2 & -0.2 & 2.2 & 2.1 & 7.5 & 6.7 & 8.0 & 8.0 & -0.3 & -0.2 & 0.3 & 0.0 & -1.6 & -2.2 & 1.1 & 1.1 \\
\hline Niederlande $^{4}$ & 7.9 & 8.4 & 6.9 & 7.9 & -0.2 & -1.6 & 1.2 & 2.1 & 12.0 & 12.3 & 11.7 & 9.4 & -2.0 & -1.0 & $\mathrm{n}$ & 0.7 & -0.6 & -0.7 & 2.9 & 3.9 \\
\hline Schweden ${ }^{5}$ & 6 & $\mathrm{~m}$ & 3. & $\mathrm{~m}$ & -0.6 & $\mathrm{~m}$ & 3.1 & $\mathrm{~m}$ & 11.4 & 10.8 & .4 & 7.4 & -1.5 & -0.7 & 1.2 & 1.6 & -0.7 & -0.8 & 3.0 & 3.3 \\
\hline Ver. Königreich & 15.1 & $\mathrm{~m}$ & 12.4 & $\mathrm{~m}$ & -1.5 & $\mathrm{~m}$ & 4.2 & $\mathrm{~m}$ & 17.3 & 15.2 & 18.1 & 16.4 & -2.1 & -2.3 & 0.7 & 0.7 & -2.4 & -2.3 & 3.0 & 2.7 \\
\hline Vereinigte Staaten & 16.4 & 11.8 & 14.4 & 10.6 & -0.9 & -1.3 & 2.9 & 2.5 & 14.9 & 14.7 & 18.9 & 18.8 & -2.3 & -2.0 & 0.9 & 1.4 & -4.7 & -6.0 & 2.1 & 2.7 \\
\hline Ländermittel $^{6}$ & 11.4 & 11.1 & 9.2 & 8.7 & -1.1 & -1.1 & 3.6 & 3.6 & 11.8 & 11.3 & 11.4 & 10.6 & -1.3 & -1.3 & 0.7 & 0.9 & -1.5 & -1.8 & 2.5 & 2.9 \\
\hline
\end{tabular}

1. Die Ertragsrate einer Ausbildung im Sekundarbereich II wird berechnet durch einen Kosten/Nutzen-Vergleich mit einer Ausbildung im Sekundarbereich I.

2. Die Ertragsrate einer Ausbildung im Tertiärbereich wird berechnet durch einen Kosten/Nutzen-Vergleich mit einer Ausbildung im Sekundarbereich II.

3. Die Daten für Männer stammen aus Daten zu den Einkommen nach Steuern aus 1998.

4. Referenzjahr 1997.

5. Für eine Ausbildung im Tertiärbereich wurde anstelle der durchschnittlichen theoretischen Dauer verschiedener Studiengänge für Frauen und Männer die durchschnittliche theoretische Dauer von Standardstudiengängen verwendet. Für Frauen sind die Einkommensdifferentiale zwischen einer Ausbildung im Sekundarbereich I und Sekundarbereich II nicht groß genug, um eine Berechnung positiver interner Erträge zu erlauben.

6. Die Daten für Männer beziehen Italien nicht mit ein. Die Daten für Frauen mit einer Ausbildung im Sekundarbereich II beziehen Schweden und das Vereinigte Königreich nicht mit ein. Quelle: OECD.

Tabelle A13.4.

Gesellschaftliche Ertragsraten aus Bildungsinvestitionen (1999-2000)

Ertragsraten einer Ausbildung im Sekundarbereich II und im Tertiärbereich, nach Geschlecht (in Prozentpunkten)

\begin{tabular}{|c|c|c|c|c|}
\hline & \multicolumn{2}{|c|}{$\begin{array}{l}\text { Gesellschaftliche Ertragsraten einer Ausbildung } \\
\text { im Sekundarbereich II }{ }^{1}\end{array}$} & \multicolumn{2}{|c|}{$\begin{array}{c}\text { Gesellschaftliche Ertragsraten einer Ausbildung } \\
\text { im Tertiärbereich }\end{array}$} \\
\hline & Männer & Frauen & Männer & Frauen \\
\hline $\mathrm{Kanada}^{3}$ & $\mathrm{~m}$ & $\mathrm{~m}$ & 6.8 & 7.9 \\
\hline Dänemark & 9.3 & 8.7 & 6.3 & 4.2 \\
\hline Frankreich & 9.6 & 10.6 & 13.2 & 13.1 \\
\hline Deutschland & 10.2 & 6.0 & 6.5 & 6.9 \\
\hline Italien ${ }^{4}$ & 8.4 & $\mathrm{~m}$ & 7.0 & $\mathrm{~m}$ \\
\hline Japan & 5.0 & 6.4 & 6.7 & 5.7 \\
\hline Niederlande & 6.2 & 7.8 & 10.0 & 6.3 \\
\hline Schweden & 5.2 & $\mathrm{~m}$ & 7.5 & 5.7 \\
\hline Ver. Königreich ${ }^{5}$ & 12.9 & $\mathrm{~m}$ & 15.2 & 13.6 \\
\hline Vereinigte Staaten & 13.2 & 9.6 & 13.7 & 12.3 \\
\hline
\end{tabular}

1. Die Ertragsrate einer Ausbildung im Sekundarbereich II wird berechnet durch einen Kosten/Nutzen-Vergleich mit einer Ausbildung im Sekundarbereich I.

2. Die Ertragsrate einer Ausbildung im Tertiärbereich wird berechnet durch einen Kosten/Nutzen-Vergleich mit einer Ausbildung im Sekundarbereich II.

3. In Italien war der Stichprobenumfang für Frauen für eine Berechnung der Ertragsraten nicht groß genug.

4. In Kanada standen keine Daten zu den Ausgaben für Schüler und Studierende im Sekundarbereich II zur Verfügung.

Quelle: OECD. 


\section{ERTRAGSRATEN VON BILDUNG: ZUSAMMENHANG ZWISCHEN HUMANKAPITAL UND WIRTSCHAFTSWACHSTUM}

- Die Bildung von Sach- und Humankapital ist wichtig für das Wachstum, und so erklären sich die zwischen den einzelnen Ländern beobachteten Unterschiede bei den Wachstumsprofilen zu einem beträchtlichen Teil aus diesbezüglichen Unterschieden. Vor allem deuten die Ergebnisse darauf hin, dass Investitionen in Bildung durch positive externe Effekte gekennzeichnet sein können, aufgrund derer die gesellschaftlichen Erträge von Schulbildung höher sind als die individuellen Erträge, obwohl es vor allem bei alternden Bevölkerungen einige Zeit dauern kann, bis sich Verbesserungen im Bildungssystem nennenswert auf die durchschnittlichen Kenntnisse und Fähigkeiten der Erwerbsbevölkerung auswirken.

- Öffentliche Ausgaben für das Gesundheitswesen, die Bildung und die Forschung tragen eindeutig zur langfristigen Sicherung des Lebensstandards bei, und Sozialtransfers tragen zur Verwirklichung sozialer Ziele bei, doch muss all dies finanziert werden. Die notwendige Besteuerung könnte sich negativ auf Spar- und Investitionsanreize auswirken.

\section{Abbildung A14.1.}

Aufschlüsselung der Veränderungen in den durchschnittlichen jährlichen

Wachstumsraten des BIP pro Kopf

Geschätzte Auswirkungen von Veränderungen der unabhängigen Variablen aufVeränderungen derWachstumsraten der Pro-Kopf-Produktion in den achtziger und neunziger Jahren
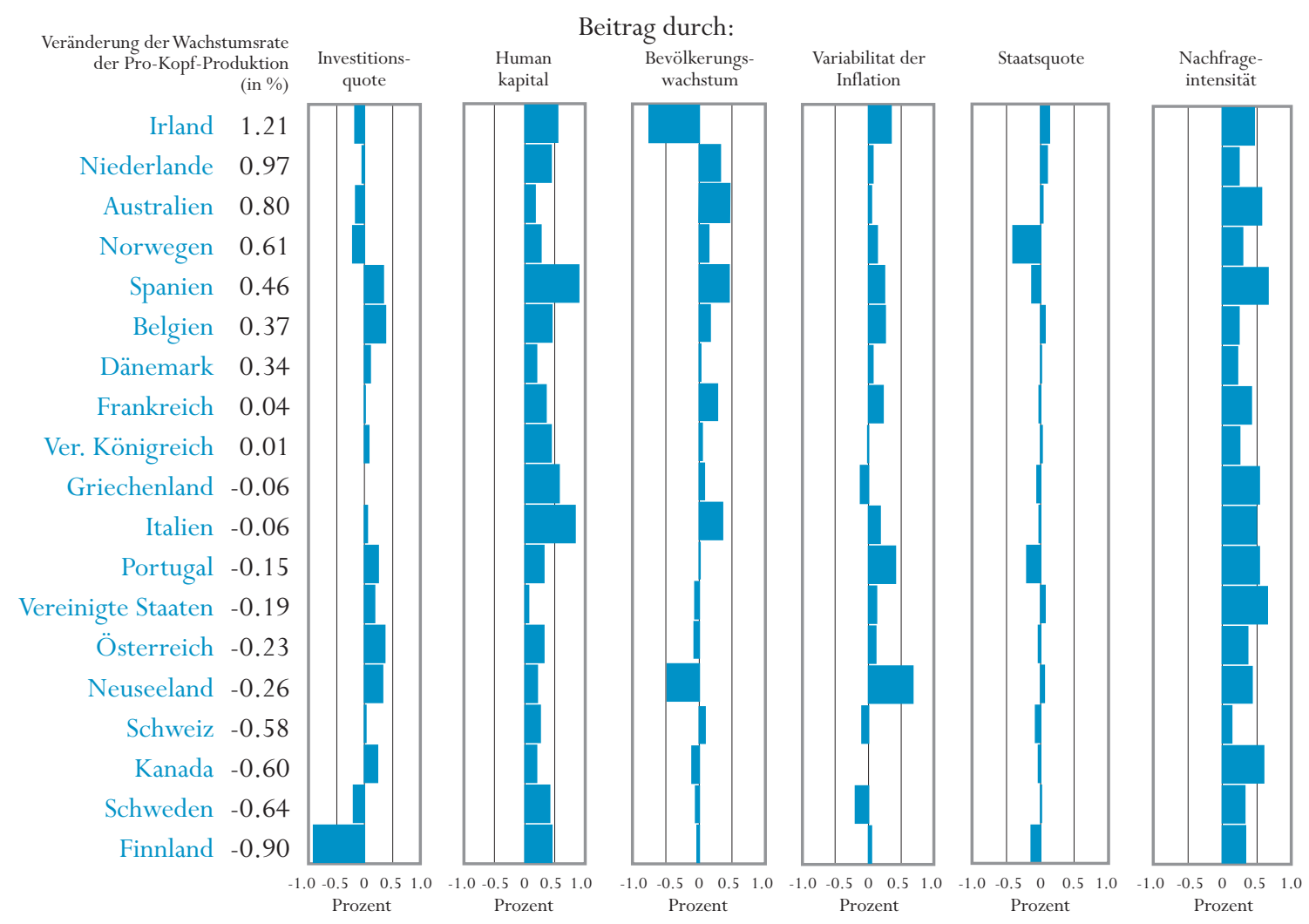

Anordnung der Länder in absteigender Reihenfolge des Prozentsatzes der Veränderungen im Wachstum der Pro-Kopf-Produktion. Quelle: OECD Wirtschaftsausblick, Dezember 2000. Tabelle A14.1. 


\section{$\mathrm{A}_{14}$}

Dieser Indikator untersucht die

Auswirkungen von

Veränderungen

der unabhängigen

Variablen, u.a. des Humankapitals, aufden Anstieg der Pro-KopfProduktion im Zeitraum von 1980 bis 1990.

Die genauen Mechanismen der Zusammenhänge zwischen Politik und Kapitalbildung, wirtschaftlicher Effizienz, technologischem Fortschritt und letztlich Produktionswachstum sind nach wie vor ein viel diskutiertes Thema.

\section{Politischer Hintergrund}

Die OECD-Länder haben in den letzten Jahrzehnten große Unterschiede in ihrem Wachstum gezeigt. Vor allem in den neunziger Jahren bauten einige der relativ wohlhabenden Länder (insbesondere die Vereinigten Staaten) ihren Vorsprung noch weiter aus, während sich das Wachstum in vielen anderen Ländern verlangsamte. Fortdauernde Unterschiede bei der Bildung der verschiedenen Arten von Kapital (Sach-, Human- und Wissenskapital), den Marktbedingungen und dem technologischen Fortschritt - ihrerseits alle durch Politik und Institutionen beeinflussbar - können wichtige Ursachen für das unterschiedliche Wachstum in den einzelnen Ländern sein. Welche relative Bedeutung kommt Bildung und Humankapital in dieser Gleichung zu? Zur Beantwortung diese Frage zeigt dieser Indikator den geschätzten Effekt von Veränderungen in den unabhängigen Variablen, u.a. des Humankapitals, auf die Wachstumsraten der Pro-Kopf-Produktion im Zeitraum von 1980 bis 1990.

\section{Ergebnisse und Erläuterungen}

Obwohl Einigkeit über die Bedeutung von Politik und Institutionen für das Wachstum besteht, sind die genauen Mechanismen der Zusammenhänge zwischen Politik und Kapitalbildung, wirtschaftlicher Effizienz, technologischem Fortschritt und letztlich Produktionswachstum nach wie vor ein viel diskutiertes Thema. Politik und Institutionen können insbesondere private Spar- und Investitionsentscheidungen und die Bildung von Humankapital beeinflussen. Außerdem können sie, über ihre Wirkung auf die Bildung von Sach- und Humankapital hinaus, zur allgemeinen Effizienz der Verteilung von Ressourcen in der Wirtschaft beitragen.

Wachstumsstudien gehen in der Regel davon aus, dass in der Erwerbsbevölkerung vorhandene formelle Fähigkeiten und Erfahrungen eine Form von (Human-)Kapital darstellen. Allerdings lässt sich auch argumentieren, dass Humankapital, ebenso wie Sachkapital, einer Art abnehmenden Ertragszuwachses unterliegt, so dass eine besser ausgebildete und kenntnisreichere Erwerbsbevölkerung zwar langfristig höhere Einkommen erzielen würde, jedoch nicht unbedingt ständig höhere Wachstumsraten beim Einkommen. In ähnlicher Weise könnten Investitionen in das Humankapital (z.B. Aufwendungen für Schul- und Berufsbildung) einen anhaltenderen Effekt auf das Wachstum haben, wenn hoher Kenntnis- und Ausbildungsstand mit intensiverer Forschung und Entwicklung und schnellerem technologischem Fortschritt einhergehen oder wenn eine hoch qualifizierte Erwerbsbevölkerung die Anpassung an neue Technologien erleichtern würde.

Um die Rolle von Politik und Institutionen beim Anstieg der Produktion in den OECD-Ländern zu ermitteln, wurde eine empirische Studie auf der Basis von Regressionsgleichungen mit der abhängigen Variable Wachstum durchgeführt (Einzelheiten s. OECD-Wirtschaftsausblick Nr. 68). Abbildung A14.1 zeigt den geschätzten Effekt von Veränderungen in den unabhängigen Variablen auf 
Veränderungen der Wachstumsrate der Pro-Kopf-Produktion im Zeitraum von den achtziger bis zu den neunziger Jahren.

Die Verbesserungen des Humankapitals scheinen generell ein Faktor der Wachstumsprozesse der letzten Jahrzehnte in allen OECD-Ländern zu sein, vor allem in Griechenland, Irland, Italien und Spanien, wo der Anstieg des Humankapitals in den neunziger Jahren zu einem Wachstum um mehr als einen halben Prozentpunkt im Vergleich zum vorangegangenen Jahrzehnt führte. Dieser Effekt könnte noch deutlicher ausfallen, wenn das verwendete Maß für das Humankapital über den jeweils erreichten formellen Bildungsstand hinausgegangen wäre. Doch obwohl der durchschnittliche Stand des Humankapitals in der Regel gestiegen ist - und sich kontinuierlich in stärkerem Wachstum niederschlägt - muss man sich bei der Bewertung dieses Ergebnisses die relativ langsamen Anstiegsraten (ein halber bis ein Prozentpunkt pro Jahrzehnt) vor Augen führen.

Das in dieser Analyse festgestellte Ausmaß der Auswirkungen auf das Wachstum deutet darauf hin, dass die gesamtwirtschaftlichen Erträge von Bildungsinvestitionen möglicherweise größer sind als die Erträge des Einzelnen. Dies spiegelt möglicherweise auch Spill-Over-Effekte wider, wie beispielsweise Zusammenhänge zwischen Bildungsstand und technologischem Fortschritt, sowie den effektiveren Einsatz von natürlichen Ressourcen und Sachressourcen. Es impliziert, dass Anreize zur Bildungsbeteiligung für den Einzelnen durch die Politik sinnvoll verstärkt werden können, um so den maximalen Nutzen für die Gesellschaft als Ganzes zu erzielen. Allerdings sind bei dieser Interpretation der Ergebnisse einige Einschränkungen zu beachten. Zunächst sind die in der Analyse festgestellten Auswirkungen eventuell überschätzt worden, da der Indikator für Humankapital möglicherweise auch teilweise als Kenngröße für andere Variablen fungieren könnte, ein Aspekt, der auch in einigen mikroökonomischen Studien angesprochen wurde. Außerdem deutet die empirische Analyse darauf hin, dass die Auswirkungen mit einem gewissen Mangel an Genauigkeit erfasst werden. In jedem Fall wird sich der durchschnittliche Stand der formellen Bildung und Ausbildung zwangsläufig nur langsam in Reaktion auf Veränderungen in der Bildungspolitik ändern, da letztere in der Regel nur die jungen Altersgruppen, die in das Erwerbsleben eintreten, betreffen. Und schließlich ist ein längeres Verbleiben in formellen Bildungsprozessen nicht unbedingt die effizienteste Art der Vermittlung von Fähigkeiten für den Arbeitsplatz. Außerdem ist dieser Aspekt der Bildung auch gegen andere (manchmal konkurrierende) Ziele des Bildungssystems abzuwägen. Aus diesem Grunde kann die Wachstumsdividende aus einem weiteren Anstieg des formellen Bildungsniveaus der Länder, die beim Bildungsangebot führend sind, weniger deutlich ausfallen als dies die empirische Analyse vermuten lässt.

Für den sich aus Veränderungen in den Investitionsquoten ergebenden Beitrag ergibt sich ein gemischtes Bild. Bei einigen Ländern geht man Schätzungen zufolge davon aus, dass sie von einem Anstieg der Investitionsquote seitens

Die Verbesserungen des Humankapitals sind generell ein Faktor der Wachstumsprozesse der letzten Jahrzehnte gewesen. In den neunziger Jahren führten sie in einigen Ländern zu einer Wachstumsbeschleunigung um mehr als einen halben Prozentpunkt.

Das in dieser Analyse festgestellte Ausmaßs der Auswirkungen auf das Wachstum deutet darauf hin, dass die gesamtwirtschaftichen Erträge von Bildungsinvestitionen möglicherweise größser sind als die Erträge des Einzelnen.

Der sich aus Veränderungen in den Investitionsquoten 


\section{$\mathbf{A}_{14}$}

ergebende Beitrag variiert.

Die Größse des staatlichen

Sektors trug in vielen

Ländern zu einer gering-

fügigen Verlangsamung

der Wachstums-

geschwindigkeit bei.

Der allgemeine Prozess

der Handelsliberalisierung

hat das Wachstum

im letzten Jahrzehnt

schätzungsweise um

jährlich bis zu zwei Drittel

eines Prozentpunktes

ansteigen lassen. der Unternehmen im letzten Jahrzehnt profitiert haben (z.B. Belgien, Kanada, Neuseeland, Österreich, Portugal und Spanien), während andere einen negativen Effekt aufgrund geringerer Investitionsquoten zu verzeichnen hatten (z.B. Finnland, und in geringerem Ausmaß Norwegen und Schweden). Daneben gab es in jedem Land wichtige Veränderungen in Politik und institutionellem Umfeld, die über den veränderten Input beim Sach- und Humankapital hinaus zum Wachstum beigetragen haben. Vor allem in den neunziger Jahren haben die meisten Länder von einer geringeren Variabilität der Inflation profitiert. $\mathrm{Zu}$ den auffälligsten Beispielen gehören hier Neuseeland und Portugal, wo man, unter ansonsten gleichen Bedingungen, diesem Faktor die um einen halben Prozentpunkt höhere Wachstumsrate der Pro-Kopf-Produktion zuschreibt.

Im Gegensatz dazu trug trotz der größeren fiskalpolitischen Disziplin im letzten Jahrzehnt die steigende Staatsquote in vielen Ländern zu einer geringfügigen Verlangsamung der Wachstumsgeschwindigkeit bei. Zu den Ausnahmen gehören hier Irland und die Niederlande, wo eine Reduzierung der Steuern und Ausgaben als Anteil des BIP die Pro-Kopf-Produktion in den neunziger Jahren leicht ansteigen ließ.

Schließlich hat der allgemeine Prozess der Handelsliberalisierung, an dem alle OECD-Länder beteiligt gewesen sind, das Wachstum im letzten Jahrzehnt schätzungsweise um jährlich bis zu zwei Drittel eines Prozentpunktes ansteigen lassen. Trotz der Entwicklungen der neunziger Jahre gibt es weiterhin gravierende Unterschiede bei den wichtigsten Determinanten des Wirtschaftswachstums in den OECD-Ländern.

\section{Definitionen und angewandte Methodik}

Das Humankapital wird geschätzt auf Basis der erzielten Abschlüsse in den einzelnen Bildungsbereichen und der durchschnittlicher Dauer der (Aus-)Bildung innerhalb der Bevölkerung im Erwerbsalter. Hierbei ist zu beachten, dass der Bildungsstand eine grobe und manchmal etwas enggefasste Kenngröße für erworbene Fähigkeiten, Kenntnisse und Kompetenzen ist und die Qualität der formellen Bildung und Ausbildung sowie andere wichtige Dimensionen des Humankapitals nur wenig berücksichtigt. Er wird abgeleitet aus OECD-Daten (Bildung auf einen Blick, OECD) in Kombination mit Daten von de la Fuente und Doménech (2000). Zur Definition anderer Faktoren (Investitionsanteil, Bevölkerungswachstum, Variabilität der Inflation, Nachfrageintensität und Staatsquote) siehe OECD-Wirtschaftsausblick Nr. 68. Es ist zu beachten, dass aufgrund der Datenverfügbarkeit der Staatsverbrauch als Anteil des BIP als Kenngröße für die Staatsquote verwendet wurde. Diese Variable korreliert in den meisten Ländern eng mit den Steuereinnahmen und anderen Einnahmen (als Anteil vom BIP), für die jedoch nur wenig Datenmaterial zur Verfügung steht.

Die Berechnungen ergeben sich aus der Zerlegung von Unterschieden bei den Wachstumsraten, basierend auf den Ergebnissen von multivariaten Regressionen. Es ist zu beachten, dass die gezeigte Summe der Beiträge nicht 
den beobachteten Veränderungen bei den Wachstumsraten der Pro-KopfProduktion entspricht, da die geschätzten Auswirkungen der Ausgangshöhe des BIP pro Kopf und der durch die Regression nicht erklärten Komponente nicht ausgewiesen werden.

Abbildung A14.1 macht keine Angaben zum geschätzten Effekt verschiedener Ausgangsbedingungen (z.B. des Konvergenzprozesses) auf das Wachstum und stellt auch nicht den unerklärten länderspezifischen Effekt dar. Die für die Zerlegung verwendeten Koeffizienten entstammen einer Wachstumsgleichung, die die Variabilität der Inflation, die Nachfrageintensität und den Staatsverbrauch (als Anteil am BIP) als Kenngröße für den potentiellen Effekt der Staatsquote auf das Wachstum beinhaltet.

Die Veränderungen im Wachstum basieren auf Unterschieden im durchschnittlichen BIP-Wachstum pro Person im Erwerbsalter für jeweils ein Jahrzehnt. Die Zahlen für die achtziger Jahre umfassen den Zeitraum 1981 bis 1989, die Zahlen für die neunziger Jahre beziehen sich auf den Zeitraum bis 1997. 
KAPITEL A Bildungs- und Lernergebnisse

Tabelle A14.1.

Aufschlüsselung derVeränderungen in den durchschnittlichen jährlichen Wachstumsraten des BIP pro Kopf (1980 - 1997) Geschätzte Auswirkungen von Veränderungen der unabhängigenVariablen auf Veränderungen in denWachstumsraten der Pro-Kopf-Produktion in den achtziger und neunziger Jahren ${ }^{1}$ (in Prozentpunkten)

\begin{tabular}{|c|c|c|c|c|c|c|c|}
\hline & \multirow{2}{*}{$\begin{array}{c}\text { Veränderung der } \\
\text { Wachstumsrate } \\
\text { der Pro-Kopf- } \\
\text { Produktion (in \%) }\end{array}$} & \multicolumn{6}{|c|}{ Beitrag durch: } \\
\hline & & Investitionsquote & Humankapital & $\begin{array}{c}\text { Bevölkerungs- } \\
\text { wachstum }\end{array}$ & $\begin{array}{l}\text { Variabilität der } \\
\text { Inflation }\end{array}$ & Staatsquote $^{2}$ & $\begin{array}{c}\text { Nachfrage- } \\
\text { intensität }\end{array}$ \\
\hline Österreich & -0.23 & 0.37 & 0.31 & -0.07 & 0.12 & -0.02 & 0.37 \\
\hline Belgien & 0.37 & 0.37 & 0.45 & 0.17 & 0.26 & 0.06 & 0.24 \\
\hline Kanada & -0.60 & 0.24 & 0.19 & -0.10 & 0.01 & -0.02 & 0.60 \\
\hline Finnland & -0.90 & -0.91 & 0.44 & -0.03 & 0.05 & -0.13 & 0.33 \\
\hline Frankreich & 0.04 & 0.01 & 0.35 & 0.27 & 0.23 & -0.02 & 0.42 \\
\hline Griechenland & -0.06 & $\mathrm{n}$ & 0.57 & 0.09 & -0.12 & -0.05 & 0.54 \\
\hline Irland & 1.21 & -0.17 & 0.54 & -0.75 & 0.35 & 0.13 & 0.46 \\
\hline Italien & -0.06 & 0.05 & 0.84 & 0.36 & 0.18 & -0.01 & 0.49 \\
\hline Niederlande & 0.97 & -0.04 & 0.43 & 0.32 & 0.07 & 0.10 & 0.25 \\
\hline Norwegen & 0.61 & -0.21 & 0.27 & 0.15 & 0.14 & -0.41 & 0.30 \\
\hline Portugal & -0.15 & 0.25 & 0.32 & 0.02 & 0.42 & -0.20 & 0.53 \\
\hline Spanien & 0.46 & 0.33 & 0.90 & 0.46 & 0.25 & -0.12 & 0.67 \\
\hline Schweden & -0.64 & -0.19 & 0.42 & -0.05 & -0.20 & 0.02 & 0.33 \\
\hline Schweiz & -0.58 & 0.02 & 0.26 & 0.09 & -0.09 & -0.07 & 0.14 \\
\hline Ver. Königreich & 0.01 & 0.08 & 0.44 & 0.05 & $\mathrm{n}$ & 0.03 & 0.25 \\
\hline Vereinigte Staaten & -0.19 & 0.19 & 0.07 & -0.06 & 0.13 & 0.07 & 0.65 \\
\hline
\end{tabular}

Hinweis: Die Berechnungen ergeben sich aus der Aufschlüsselung von Unterschieden bei den Wachstumsraten, basierend auf den Ergebnissen von multivariaten Regressionen. Die gezeigte Summe der Beiträge entspricht nicht den Veränderungen bei den Wachstumsraten der Pro-Kopf-Produktion, da die geschätzten Auswirkungen der Ausgangshöhe des BIP pro Kopf und der durch die Regression nicht erklärten Komponente nicht ausgewiesen werden.

1. Die Veränderungen im Wachstum basieren auf Unterschiede im durchschnittlichen BIP-Wachstum pro Person im Erwerbsalter für jeweils ein Jahrzehnt. Die Zahlen für die achtziger Jahre umfassen den Zeitraum 1981 bis 1989, die Zahlen für die neunziger Jahre beziehen sich auf den Zeitraum 1990 bis 1997.

2. Aufgrund der Nichtverfügbarkeit von Daten wurde der Staatsverbrauch als Anteil am BIP als Kenngröße für die Größe des staatlichen Sektors verwendet. Diese Variable korreliert in den meisten Ländern eng mit den Steuereinnahmen und anderen Einnahmen (als Anteil am BIP), für die jedoch noch weniger Datenmaterial zur Verfügung steht.

Quelle: OECD Wirtschaftsausblick, Dezember 2000. 


\section{Kapitel}

B

FINANZ- UND HUMANRESSOURCEN INVESTITIONEN IN DIE BILDUNG

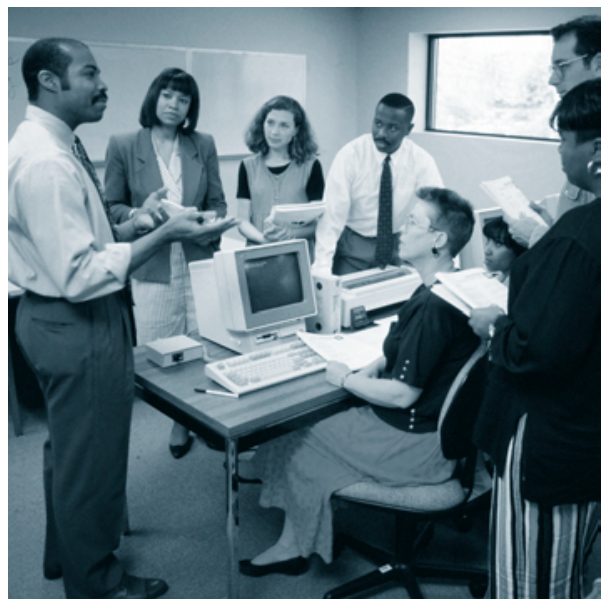





\section{ÜBERBLICK}

\section{Indikator B1: Bildungsausgaben pro Schüler/Studierenden}

Tabelle B1.1. Ausgaben für Bildungseinrichtungen pro Schüler/Studierenden (1999) Tabelle B1.2. Ausgaben für Bildungseinrichtungen pro Schüler/Studierenden im Verhältnis zum BIP pro Kopf (1999)

Tabelle B1.3. Kumulative Ausgaben für Bildungseinrichtungen pro

Studierenden über die durchschnittliche Dauer tertiärer Studiengänge (1999)

\section{Indikator B2: Ausgaben für Bildungseinrichtungen im Verhältnis zum Bruttoinlandsprodukt}

Tabelle B2.1a. Ausgaben für Bildungseinrichtungen als Prozentsatz des BIP (1995, 1999)

Tabelle B2.1b. Ausgaben für Bildungseinrichtungen als Prozentsatz des BIP $(1995,1999)$ Tabelle B2.1c. Ausgaben für Bildungseinrichtungen als Prozentsatz des BIP (1999) Tabelle B2.2. Veränderungen der Ausgaben für Bildungseinrichtungen $(1995,1999)$

\section{Indikator B3: Die öffentlichen Bildungsausgaben}

Tabelle B3.1. Öffentliche Gesamtausgaben für Bildung (1995, 1999)

\section{Indikator B4: Relative Anteile öffentlicher und privater Ausgaben für Bildungseinrichtungen}

Tabelle B4.1. Relative Anteile öffentlicher und privater Ausgaben für Bildungseinrichtungen aller Bildungsbereiche (1995, 1999)

Tabelle B4.2. Relative Anteile öffentlicher und privater Ausgaben für Bildungseinrichtungen (1995, 1999)

Tabelle B4.3. Verteilung der öffentlichen Gesamtausgaben für Bildung (1999)

\section{Indikator B5: Unterstützung für Schüler/Studierende und private} Haushalte durch öffentliche Subventionen

Tabelle B5.1. Öffentliche Subventionen an den privaten Sektor als Prozentsatz der gesamten öffentlichen Bildungsausgaben und des BIP, für den Primar-, Sekundar- und post-sekundären, nicht-tertiären Bereich (1999)

Tabelle B5.2. Öffentliche Subventionen an den privaten Sektor für den Tertiärbereich als Prozentsatz der gesamten öffentlichen Bildungsausgaben und des BIP (1999)

\section{Indikator B6: Ausgaben für Bildungseinrichtungen nach Art der erbrachten Leistung und Ausgabenkategorien}

Tabelle B6.1. Ausgaben für Unterricht, Forschung und Entwicklung (F\&E) und zusätzliche Leistungen in Bildungseinrichtungen als Prozentsatz des BIP sowie private Ausgaben für außerhalb von Bildungseinrichtungen erworbene Bildungsgüter als Anteil am BIP (1999)

Tabelle B6.2. Ausgaben pro Schüler/Studierenden für Unterricht, zusätzliche Dienstleistungen sowie Forschung und Entwicklung (F\&E) (1999)

Tabelle B6.3. Ausgaben für Bildungseinrichtungen nach Ausgabenkategorien (1999)
Kapitel B untersucht

die in Bildung

investierten Finanz- und

Humanressourcen, und

zwar in Form der von

jedem einzelnen Land

in Bildung investierten

Ressourcen in Relation

zu der Zahl der Schüler/

Studierenden, zum

Volkseinkommen und

zum BIP pro Kopf,...
... der unterschiedlichen

Finanzierungs-

instrumente.. ...und der Aufteilung
der Mittel auf die
unterschiedlichen
Ausgabenkategorien. 


\section{KLASSIFIZIERUNG DER BILDUNGSAUSGABEN}

In diesem Kapitel werden die Bildungsausgaben anhand von drei Dimensionen klassifiziert:

- Die erste Dimension ist im nachstehenden Diagramm durch die horizontale Achse dargestellt und bezieht sich auf den Ort, an dem Ausgaben anfallen. Die eine Komponente dieser Dimension sind Ausgaben in den Schulen und Hochschulen sowie in den Bildungsministerien und anderen Einrichtungen, die direkt an der Bereitstellung und Unterstützung von Bildung beteiligt sind. Ausgaben für Bildung außerhalb von Bildungseinrichtungen sind eine weitere Komponente.

- Die zweite Dimension ist im nachstehenden Diagramm durch die vertikale Achse dargestellt und kennzeichnet die für Bildungszwecke erworbenen Sach- und Dienstleistungen. Nicht alle Ausgaben für Bildungseinrichtungen sind als unmittelbare Ausgaben für Bildung oder Unterricht einzustufen. In vielen OECD-Ländern bestreiten die Bildungseinrichtungen nicht nur den Unterricht, sondern bieten noch verschiedene zusätzliche Dienstleistungen für Schüler/Studierende und ihre Familien an wie z.B. Mahlzeiten, Transport, Unterbringung etc. Ferner können im Tertiärbereich auch die Ausgaben für Forschung und Entwicklung einen nicht unerheblichen Anteil darstellen. Nicht alle Ausgaben für bildungsbezogene Sach- und Dienstleistungen erfolgen innerhalb von Bildungseinrichtungen. So können beispielsweise Familien die erforderlichen Schulbücher und Unterrichtsmaterialien selbst kaufen oder ihren Kindern Privatunterricht erteilen lassen.

- Die dritte Dimension - im nachstehenden Diagramm durch die farbliche Kennzeichnung dargestellt nimmt eine Einteilung der Mittel nach ihrer Herkunft vor. Zu diesen Quellen zählen die öffentliche Hand und internationale Organisationen (hellblau) sowie die privaten Haushalte oder andere private Einheiten (mittelblau). Wo private Bildungsausgaben durch öffentliche Mittel subventioniert werden, ist dies im Diagramm dunkelblau gekennzeichnet. Dieses Diagramm wird jedem Indikator vorangestellt, um anzugeben, welcher Bereich in dem jeweiligen Indikator abgedeckt wird.

\begin{tabular}{|c|c|c|}
\hline & $\begin{array}{c}\text { Bildungsausgaben innerhalb von Bildungseinrichtungen } \\
\text { (z.B. Schulen, Hochschulen, Einrichtungen der Bildungsverwaltung } \\
\text { und soziale Dienste für Schüler/Studierende) }\end{array}$ & $\begin{array}{c}\text { Bildungsausgaben außerhalb } \\
\text { von Bildungseinrichtungen } \\
\text { (z.B. der private Erwerb von } \\
\text { Gütern und Dienstleistungen } \\
\text { für Bildungszwecke, inkl. Priva- } \\
\text { tunterricht) }\end{array}$ \\
\hline \multirow{3}{*}{$\begin{array}{l}\text { Ausgaben für } \\
\text { eigentliche } \\
\text { Bildungsdienst- } \\
\text { leistungen }\end{array}$} & $\begin{array}{l}\text { z.B. öffentliche Mittel für Bildungsangebote in Bildungseinrich- } \\
\text { tungen }\end{array}$ & $\begin{array}{l}\text { z.B. subventionierte private } \\
\text { Ausgaben für Bücher }\end{array}$ \\
\hline & $\begin{array}{l}\text { z.B. subventionierte private Ausgaben für Unterrichtszwecke in } \\
\text { Bildungseinrichtungen }\end{array}$ & \multirow{2}{*}{$\begin{array}{l}\text { z.B. private Mittel für Bücher } \\
\text { und anderes Unterrichts- } \\
\text { material oder Privatunterricht }\end{array}$} \\
\hline & z.B. private Ausgaben für Unterrichts- bzw. Studiengebühren & \\
\hline \multirow{2}{*}{$\begin{array}{l}\text { Ausgaben für } \\
\text { Forschung und } \\
\text { Entwicklung }\end{array}$} & z.B. öffentliche Mittel für Forschung an Hochschulen & \\
\hline & $\begin{array}{l}\text { z.B. Mittel der Privatwirtschaft für Forschung und Entwicklung } \\
\text { an Bildungseinrichtungen }\end{array}$ & \\
\hline \multirow[t]{2}{*}{$\begin{array}{l}\text { Ausgaben für nicht } \\
\text { nterrichtsbezogene } \\
\text { Dienstleistungen im } \\
\text { Bildungsbereich }\end{array}$} & $\begin{array}{l}\text { z.B. öffentliche Ausgaben für zusätzliche Dienstleistungen wie } \\
\text { Mahlzeiten,Transport zur Schule, Unterbringung auf dem } \\
\text { Campus }\end{array}$ & $\begin{array}{l}\text { z.B. subventionierte private } \\
\text { Ausgaben für den Lebensunter- } \\
\text { halt der Schüler/Studierenden } \\
\text { bzw. reduzierteTarife im ÖPNV }\end{array}$ \\
\hline & z.B. private Mittel für zusätzliche Dienstleistungen & $\begin{array}{l}\text { z.B. private Ausgaben für Leb- } \\
\text { ensunterhalt, Transport }\end{array}$ \\
\hline
\end{tabular}




\section{BILDUNGSAUSGABEN PRO SCHÜLER/STUDIERENDEN}

- Insgesamt beziffern sich die Ausgaben der OECD-Länder auf 4.229 US-Dollar pro Schüler im Primarbereich, 5.174 US-Dollar im Sekundarbereich und 11.422 US-Dollar pro Studierenden im Tertiärbereich. Hinter diesen Durchschnittswerten stehen jedoch sehr unterschiedliche Ausgaben in den einzelnen Ländern.

- Geringere Ausgaben pro Schüler/Studierenden können nicht automatisch mit einer geringeren Qualität der Bildungsdienstleistungen gleichgesetzt werden. So gehören beispielsweise Australien, Finnland, Korea und das Vereinigte Königreich, Länder mit moderaten Bildungsausgaben pro Schüler im Primar- und Sekundarbereich I, zu den OECD-Ländern mit den besten Leistungen 15-Jähriger in mathematischer Grundbildung

- Im Durchschnitt der OECD-Länder sind die Ausgaben pro Studierenden im Tertiärbereich 2,3-mal so hoch wie im Primarbereich.

- Geringe jährliche Ausgaben pro Studierenden im Tertiärbereich führen in einigen OECD-Ländern aufgrund der langen Studienzeiten dennoch zu hohen Gesamtkosten im tertiären Bildungsbereich.

- Im Tertiärbereich haben die Bildungsausgaben nicht immer mit dem raschen Anstieg der Studierendenzahlen Schritt gehalten. 


\section{Abbildung B1.1.}

Ausgaben für Bildungseinrichtungen pro Schüler/Studierenden (1999) Jährliche Ausgaben für Bildungseinrichtungen pro Schüler/Studierenden, in US-Dollar, kaufkraftbereinigt, nach Bildungsbereichen (basierend aufVollzeitäquivalenten)

Gesamtausgaben pro Schüler/Studierenden

Forschung und Entwicklung in tertiären Bildungseinrichtungen
Zusätzliche Dienstleistungen (Transport, Mahlzeiten und Unterkünfte, von Bildungseinrichtungen zur Verfügung gestellt)

Eigentliche Bildungsdienstleistungen
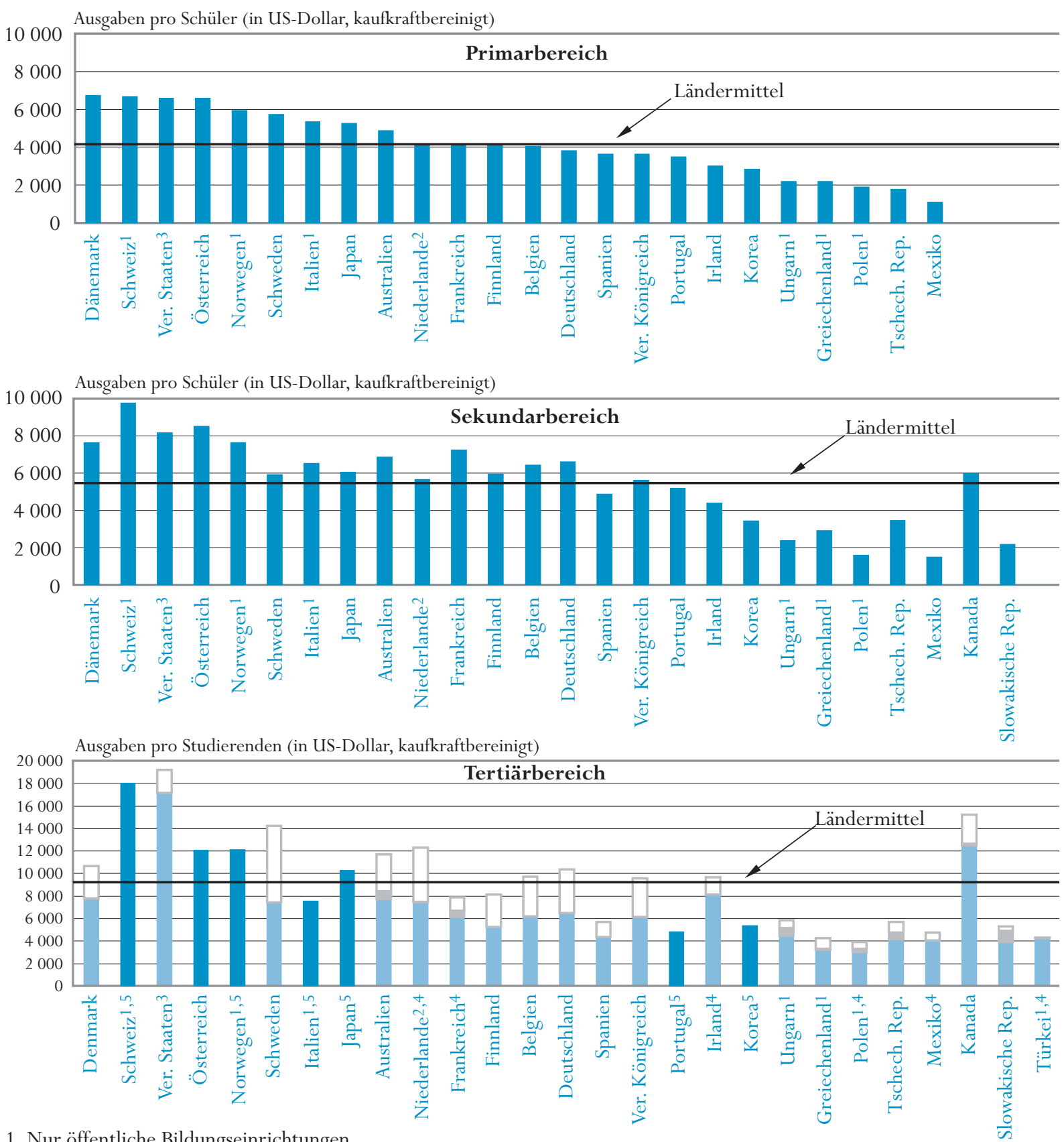

1. Nur öffentliche Bildungseinrichtungen.

2. Nur öffentliche und staatlich-subventionierte private Bildungseinrichtungen.

3. Nur öffentliche und unabhängige private Bildungseinrichtungen.

4. Ausgaben für Forschung und Entwicklung im Tertiärbereich und daher auch Gesamtausgaben zu niedrig angesetzt.

5. Der Balken steht für die Gesamtausgaben im Tertiärbereich und umfasst auch Ausgaben für Forschung und Entwicklung. Anordnung der Länder in absteigender Reihenfolge der Ausgaben pro Schüler im Primarbereich.

Quelle: OECD. Tabellen B1.1 und B6.2. Hinweise s. Anhang 3 (www.oecd.org/els/education/eag2002). 


\section{Politischer Hintergrund}

Leistungsfähige Schulen benötigen die richtige Mischung aus gut ausgebildetem und qualifiziertem Personal, angemessener Infrastruktur, moderner Ausstattung und motivierten, lernwilligen Schülern/Studierenden. Die Nachfrage nach hochwertiger Bildung, die mit höheren Kosten pro Schüler/Studierenden einhergehen kann, ist mit der Vermeidung unzumutbarer Belastungen für die Steuerzahler in Einklang zu bringen.

Aus diesem Grund ist die Frage, ob die für die Bildung eingesetzten Mittel auch einen den Investitionen entsprechenden Nutzen bringen, häufig Gegenstand öffentlicher Diskussionen. Zwar ist es schwierig, den optimalen Ressourcenumfang abzuschätzen, der notwendig ist, um Schüler/Studierende auf das Leben und die Arbeitswelt in einem modernen Wirtschaftssystem vorzubereiten, doch können internationale Vergleiche der Bildungsausgaben pro Schüler/Studierenden als Anhaltspunkte für eine Bewertung der Effektivität der verschiedenen Bildungsangebote dienen.

Die Politik muss auch abwägen zwischen der Notwendigkeit, die Qualität des Bildungsangebots zu verbessern, und dem Wunsch, den Zugang zur Bildung, insbesondere im Tertiärbereich, zu erweitern. Die vergleichende Übersicht der Entwicklung der Bildungsausgaben pro Schüler/Studierenden in diesem Indikator zeigt, dass in vielen OECD-Ländern mit dem Anstieg der Studierendenzahlen, insbesondere im Tertiärbereich, nicht immer auch eine entsprechende Veränderung der Bildungsausgaben einhergegangen ist.

Schließlich sind auch Entscheidungen über die Zuteilung der Mittel auf die verschiedenen Bildungsbereiche von Bedeutung. Während zum Beispiel einige OECD-Länder Wert auf einen breiten Zugang zur Hochschulausbildung legen, investieren andere stärker in die fast vollständige Bildungsbeteiligung junger Kinder im Alter von drei oder vier Jahren.

\section{Ergebnisse und Erläuterungen}

\section{Was dieser Indikator aufzeigt und was nicht}

Dieser Indikator gibt Auskunft über die direkten öffentlichen und privaten Ausgaben für Bildungseinrichtungen im Verhältnis zur Anzahl der vollzeitäquivalenten Schüler bzw. Studierenden an diesen Einrichtungen.

Im Interesse einer besseren internationalen Vergleichbarkeit sind öffentliche Zuwendungen zum Lebensunterhalt der Schüler/Studierenden in diesen Zahlen nicht enthalten. Für einige OECD-Länder sind keine Zahlen zu den Ausgaben für Schüler/Studierende an privaten Bildungseinrichtungen verfügbar. Einige andere Länder wiederum haben kein vollständiges Zahlenmaterial über unabhängige private Bildungseinrichtungen zur Verfügung gestellt. In diesen Fällen wurden nur die Ausgaben für öffentliche sowie staatlich-subventionierte private Einrichtungen berücksichtigt. Es ist zu beachten, dass Unterschiede bei den Bildungsausgaben pro Schüler/Studierenden vielleicht nicht nur auf
Dieser Indikator gibt die jährlichen und kumulierten Bildungsausgaben pro Schüler/Studierenden in absoluten Zahlen an ...

... sowie im

Verhältnis zum

Bruttoinlandsprodukt

pro Kopf.

Der Indikator vergleicht auch die Entwicklung der Bildungsausgaben pro Schüler/ Studierenden.

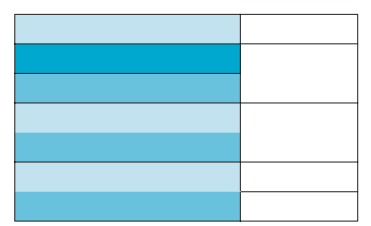

Erfasste Daten

(Erklärungen s. S. 160) 
Insgesamt geben die OECD-Länder jährlich 4.229 US-Dollar pro Schüler im Primarbereich, 5.174 US-Dollar pro Schüler im Sekundarbereich und 11.422 USDollar pro Studierenden im Tertiärbereich aus, ...

hinter diesen

Durchschnittswerten stehen jedoch enorme

Unterschiede bei den Ausgaben pro Schüler/ Studierenden zwischen den einzelnen OECDLändern.

Die Ausgaben für Forschung und Entwicklung in Einrichtungen des Tertiärbereichs belaufen sich in Australien, Belgien, Deutschland, den Niederlanden, schweden und im Vereinigten Königreich auf über 3.000 USDollar pro Studierenden.
Unterschiede bei den materiellen Ressourcen, die für Schüler/Studierende zur Verfügung gestellt werden, zurückzuführen sind (z.B. Unterschiede im zahlenmäßigen Schüler/Lehrkräfte-Verhältnis, sondern auch Unterschiede in den relativen Gehaltsniveaus widerspiegeln können.

Während unterhalb des Tertiärbereichs die Ausgaben für die reinen Unterrichtsdienstleistungen den größten Anteil der Bildungsausgaben ausmachen, können im Tertiärbereich auch andere Leistungen, insbesondere im Bereich von Forschung und Entwicklung, einen wesentlichen Teil der Bildungsausgaben ausmachen. Indikator B6 liefert weitergehende Informationen über die Verteilung der Ausgaben nach den verschiedenen (Dienst-)Leistungsangeboten.

\section{Jährliche Bildungsausgaben pro Schüler/Studierenden, in US-Dollar, kaufkraftbereinigt}

Insgesamt geben die OECD-Länder jährlich pro Schüler im Primarbereich 4.229 US-Dollar, im Sekundarbereich 5.174 US-Dollar und pro Studierenden im Tertiärbereich 11.422 US-Dollar aus. Im Tertiärbereich sind diese Durchschnittszahlen jedoch sehr stark durch das hohe Ausgabenniveau in einigen wenigen großen OECD-Ländern, vor allem den Vereinigten Staaten, beeinflusst. Die Bildungsausgaben pro Schüler/Studierenden in einem „typischen“ OECDLand, ausgedrückt durch das arithmetische Mittel aller OECD-Länder, beziffern sich auf 4.148 US-Dollar im Primarbereich, 5.465 US-Dollar im Sekundarbereich und 9.210 US-Dollar im Tertiärbereich (Tabelle B1.1).

Diesen Durchschnittswerten liegt ein breites Spektrum von Bildungsausgaben pro Schüler/Studierenden in den verschiedenen OECD-Ländern zugrunde. Im Primarbereich reichen die Ausgaben für Bildungseinrichtungen von 1.096 USDollar in Mexiko bis zu 6.721 US-Dollar in Dänemark. Im Sekundarbereich sind die Unterschiede zwischen den einzelnen OECD-Ländern sogar noch größer. Dort unterscheiden sich die Bildungsausgaben pro Schüler um bis zum 6,6fachen und reichen von 1.480 US-Dollar in Mexiko bis zu 9.756 US-Dollar in der Schweiz. Die Bildungsausgaben pro Studierenden im Tertiärbereich reichen von 3.912 US-Dollar in Polen bis zu 19.220 US-Dollar in den Vereinigten Staaten (Tabelle B1.1).

DieseVergleiche beruhen auf kaufkraftbereinigten Zahlen und nicht auf aktuellen Wechselkursen, d.h. sie geben den Betrag einer Landeswährung an, mit dem in einem bestimmten Land der gleiche Waren- und Dienstleistungskorb erworben werden kann wie in den Vereinigten Staaten mit US-Dollar.

Im Durchschnitt belaufen sich im Tertiärbereich die Ausgaben für Forschung und Entwicklung (F\&E) auf ein Viertel der Gesamtausgaben in diesem Bereich. In 5 von 20 OECD-Ländern, bei denen die Ausgaben im Tertiärbereich nach Leistungsarten getrennt aufgeführt werden, machen die F\&E-Ausgaben in tertiären Bildungseinrichtungen mehr als 35 Prozent der Gesamtausgaben im Tertiärbereich aus. Umgerechnet auf den einzelnen Studierenden kann dies erhebliche Beträge ausmachen, wie z.B. in Australien, Belgien, Deutschland, 
den Niederlanden, Schweden und im Vereinigten Königreich, wo sich die Ausgaben für F\&E in Einrichtungen des tertiären Bildungsbereichs auf mehr als 3.000 US-Dollar (kaufkraftbereinigt) belaufen (Abbildung B1.1 und Tabelle B6.2).

Die F\&E-Ausgaben in tertiären Bildungseinrichtungen sind nicht nur durch die F\&E-Gesamtaufwendungen eines Landes bedingt, sondern auch durch die nationale Infrastruktur für F\&E-Aktivitäten. Die OECD-Länder, in denen Forschung und Entwicklung hauptsächlich in tertiären Bildungseinrichtungen stattfindet, verzeichnen eher höhere Ausgaben pro Studierenden im Tertiärbereich als Länder, in denen ein Großteil der Forschungs- und Entwicklungsarbeiten in anderen öffentlichen Einrichtungen oder von der Wirtschaft durchgeführt werden.

Die Arbeitskräfteintensität des traditionellen Bildungsmodells mit Unterricht im Klassenzimmer/Hörsaal bedingt das Übergewicht der Gehälter für die Lehrkräfte bei den Gesamtkosten. Unterschiede in der durchschnittlichen Klassengröße und im zahlenmäßigen Schüler/Lehrkräfte Verhältnis (Indikator D2), in der Personalzusammensetzung, in den Lehrergehältern (Indikator D6) und in den Unterrichtsmaterialien und Ausstattungen führen zu Kostenunterschieden zwischen den einzelnen Bildungsbereichen, Bildungsgängen und Schultypen.

Durch den Einsatz neuer Informationstechnologien lassen sich künftig möglicherweise Effizienzsteigerungen erzielen, um einerseits die Kosten pro Schüler/Studierenden unter Kontrolle zu halten und andererseits die Lernergebnisse in ihrer Qualität zu verbessern. Im Tertiärbereich lassen sich Einsparungen bei den Kosten pro Studierenden möglicherweise auch durch den weiteren Ausbau von Fernausbildung und Fernstudium erzielen, unabhängig davon, ob es hierbei zu einem intensiven Einsatz moderner Technologien kommt oder nicht.

Es wäre irreführend, geringere Ausgaben pro Schüler/Studierenden ganz allgemein mit einer geringeren Qualität des Bildungsangebotes gleichzusetzen. So gehören beispielsweise Australien, Finnland, Korea und das Vereinigte Königreich, Länder mit moderaten Bildungsausgaben pro Schüler im Primarund Sekundarbereich I, zu den OECD-Ländern mit den besten Leistungen 15jähriger Schüler in mathematischer Grundbildung (s. Indikator A6).
Die arbeitskräfteintensive Form der Bildungsvermittlung bedingt das Übergewicht der Gehälter für Lehrkräfte in den Gesamtkosten.

Mit neuen Technologien lassen sich möglicherweise Kosteneinsparungen erzielen. 


\section{Abbildung B1.2.}

Veränderung der Ausgaben für Bildungseinrichtungen pro Schüler/Studierenden unter Berücksichtigung beeinflussender Faktoren (1995, 1999)

Indizes der Veränderung der Ausgaben für Bildungseinrichtungen, der Bildungsbeteiligung und der Ausgaben pro Schüler/Studierenden zwischen 1995 und 1999 (1995=100)

Veränderung der Ausgaben

Veränderung der Ausgaben pro Schüler/Studierenden
Veränderung der Schüler-/Studierendenzahl

Primarbereich, Sekundarbereich und post-sekundärer, nicht-teriärer Bereich

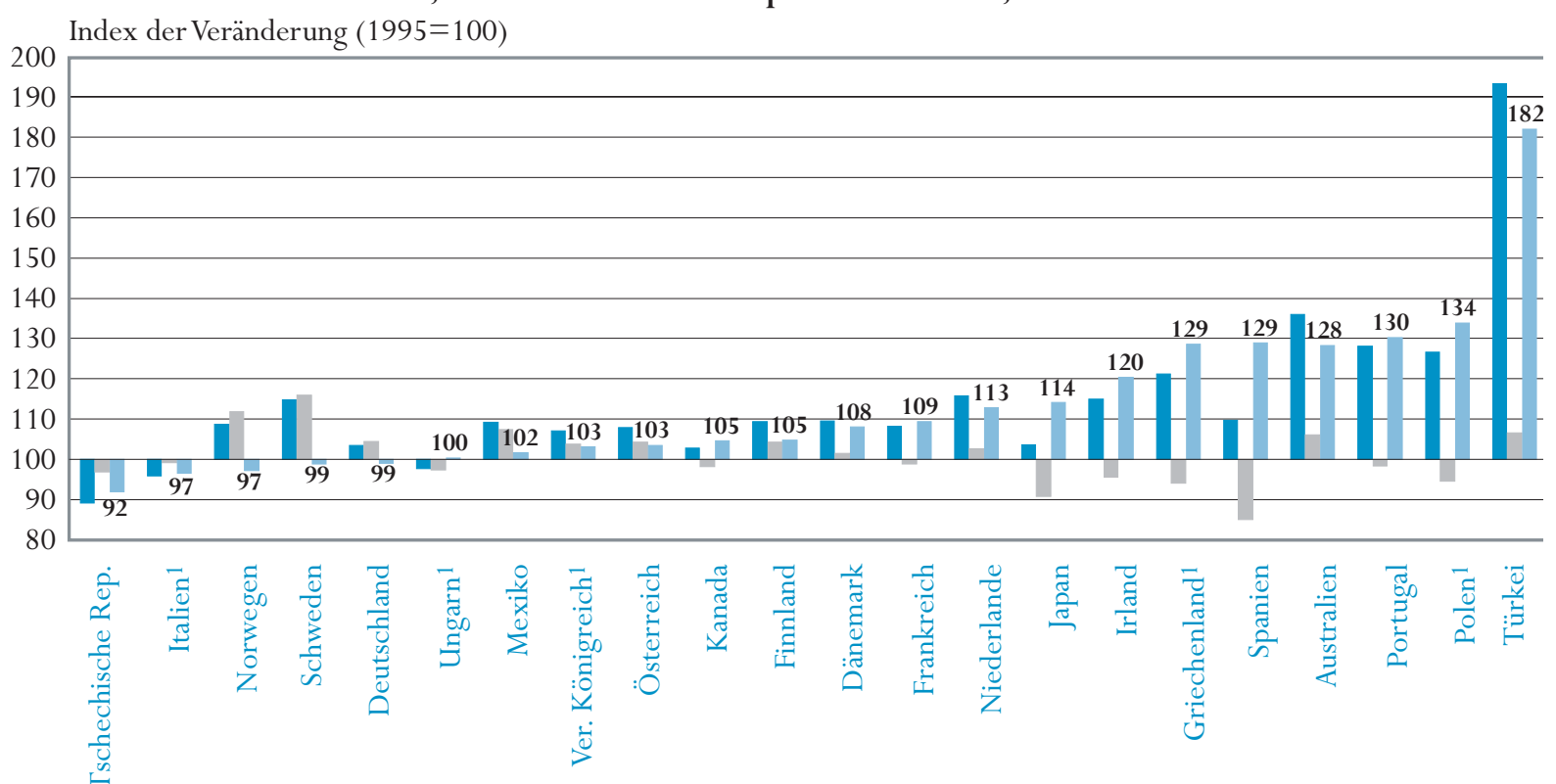

Tertiärbereich

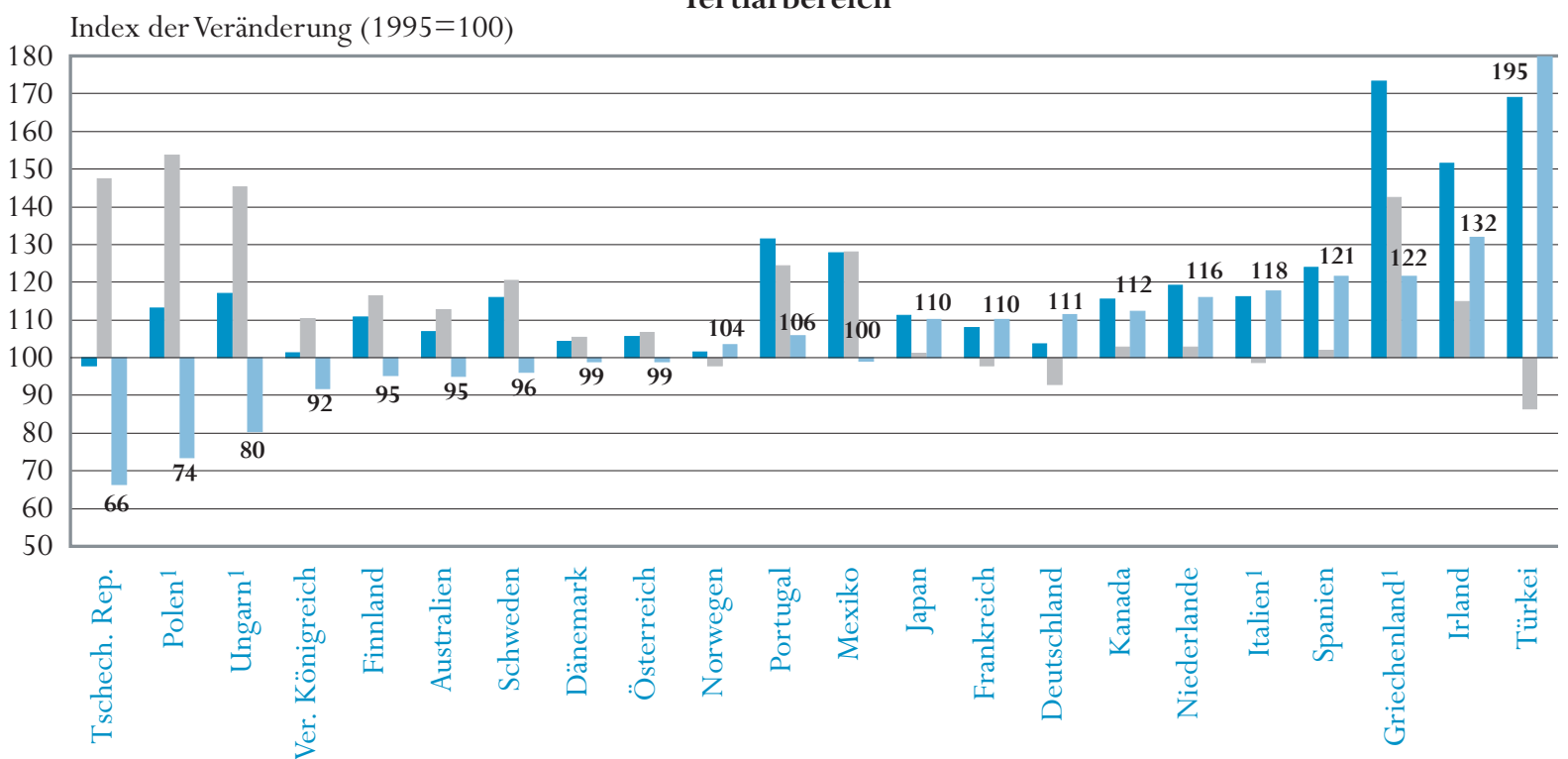

1. Nur öffentliche Bildungseinrichtungen.

Anordnung der Länder in absteigender Reihenfolge derVeränderung der Ausgaben pro Schüler/Studierenden.

Quelle: OECD. Tabelle B2.2 und Indikator C1. Hinweise s. Anhang 3 (www.oecd.org/els/education/eag2002). 


\section{Veränderungen der Bildungsausgaben pro Schüler/Studierenden zwischen 1995 und 1999}

In absoluten Zahlen und zu konstanten Preisen von 1999 erhöhten sich die Bildungsausgaben pro Schüler im Primar-, Sekundar- und post-sekundären, nicht-tertiären Bereich in Australien, Griechenland, Polen, Portugal, Spanien und der Türkei zwischen 1995 und 1999 um mehr als 20 Prozent. Dagegen gingen in der Tschechischen Republik die Bildungsausgaben pro Schüler im Primar-, Sekundar- und post-sekundären, nicht-tertiären Bereich um mehr als 5 Prozent zurück. In zehn der 22 OECD-Länder lagen die Veränderungen gegenüber 1995 innerhalb einer Bandbreite von +/ - 5 Prozent (Abb. B1.2).

Zwar passen sich die Bildungseinrichtungen oft nur mit erheblichen zeitlichen Verzögerungen an veränderte demographische Gegebenheiten an, jedoch waren die sich ändernden Schülerzahlen offensichtlich nicht der ausschlaggebende Faktor für die Veränderungen bei den Ausgaben pro Schüler im Primar-, Sekundar- und post-sekundären, nicht-tertiären Bereich. Ausnahmen hierbei bilden Japan und Spanien, wo ein Rückgang der Schülerzahlen um mehr als 9 Prozent zu einer erheblichen Steigerung der Bildungsausgaben pro Schüler führte.

In Norwegen und Schweden, den beiden OECD-Ländern mit dem höchsten Zuwachs an Schülern im Primar-, Sekundar- und post-sekundären, nichttertiären Bereich zwischen 1995 und 1999, haben die höheren Ausgaben mit den steigenden Schülerzahlen Schritt gehalten. Der parallele Anstieg von Schülerzahlen und Bildungsausgaben in Norwegen ist auf die im Schuljahr 1997-1998 umgesetzte Verlängerung der Ausbildung im Primarbereich von sechs auf sieben Jahre zurückzuführen. In Irland und Polen hatte eine deutliche Aufstockung des Bildungsetats in Verbindung mit einem leichten Rückgang der Schülerzahlen einen Anstieg der Ausgaben pro Schüler im Primar-, Sekundarund post-sekundären, nicht-tertiären Bereich von ca. 20 Prozent bzw. 34 Prozent zur Folge.

Anders sieht die Situation im tertiären Bildungsbereich aus. In sechs von 22 OECD-Ländern (Australien, Finnland, Polen, der Tschechischen Republik, Ungarn und dem Vereinigten Königreich), gingen zwischen 1995 und 1999 die Bildungsausgaben pro Studierenden im Tertiärbereich um 5 Prozent und mehr zurück. Die Ursache hierfür war größtenteils der rasche Anstieg der Zahl der Studierenden im Tertiärbereich, der sich in allen diesen Ländern während des genannten Zeitraums auf mehr als 10 Prozent belief (Abb. B1.2). Im Unterschied hierzu erhöhten sich in Griechenland und Irland die Ausgaben pro Studierenden im Tertiärbereich trotz eines Anstiegs der Zahl der Studierenden um 42 Prozent bzw. 15 Prozent massiv. Deutschland und die Türkei waren die einzigen OECD-Länder, in denen die Zahl der Studierenden im Tertiärbereich sogar um mehr als 4 Prozent zurückging. Allerdings war dieser Rückgang in Deutschland vor allem in den Anfangsjahren der entsprechenden Periode zu verzeichnen, während neuerdings wieder ein beachtlicher Zuwachs in den
Die Bildungsausgaben pro Schüler im Primar-, Sekundarund post-sekundären, nicht-tertiären Bereich stiegen in Australien, Griechenland, Polen, Portugal, Spanien und der Türkei um mehr als 20 Prozent.

Während sich Ausgaben und Schülerzahlen im Primar- und Sekundarbereich weitgehend parallel etwickelt haben,...
... konnten die Bildungsausgaben im Tertiärbereich nicht immer mit dem raschen Anwachsen der Zahl der Studierenden Schritt halten. 
Primarbereich 19 Prozent

des BIP pro Kopf,

im Sekundarbereich

25 Prozent und

pro Studierenden

im Tertiärbereich

44 Prozent.

Ärmere OECD-Länder geben pro Schüler/

Studierenden eher

weniger aus, ...

... es gibt jedoch viele

Ausnahmen.
Studierendenzahlen zu beobachten ist. In allen anderen OECD-Ländern, die eine Erhöhung der Ausgaben pro Studierenden im Tertiärbereich um mehr als 10 Prozent zu verzeichnen hatten, gab es nur geringe oder gar keine Änderungen der Zahl der Studierenden.

\section{Bildungsausgaben pro Schüler/Studierenden im Verhältnis zum jeweiligen Bruttoinlandsprodukt}

Die Bildungsausgaben pro Schüler/Studierenden gemessen am BIP pro Kopf sind ein Maßstab für die Bildungsausgaben, bei dem der relative Wohlstand der OECD-Länder berücksichtigt wird. Da in den unteren Bildungsbereichen die Bildungsbeteiligung universell ist, können hier die Bildungsausgaben pro Schüler im Verhältnis zum BIP pro Kopf als Ausgaben für die nachwachsende Generation entsprechend der Finanzkraft eines Landes angesehen werden. Für die höheren Bildungsbereiche muss für eine Interpretation der Ausgaben eine Kombination aus Volkseinkommen, Ausgabenniveau und Bildungsbeteiligung berücksichtigt werden.

Im Tertiärbereich können OECD-Länder beispielsweise einen relativ hohen Wert bei dieser Kennzahl erreichen, wenn ein relativ hoher Anteil ihres Wohlstands für eine relativ geringe Zahl von Studierenden aufgewendet wird. Für die OECD insgesamt beziffern sich die Bildungsausgaben pro Schüler/ Studierenden im Primarbereich durchschnittlich auf 19 Prozent des BIP pro Kopf, im Sekundarbereich auf 25 Prozent und im Tertiärbereich auf 44 Prozent (Tabelle B1.2).

Erwartungsgemäß besteht ein eindeutig positiver Zusammenhang zwischen den Bildungsausgaben pro Schüler/Studierenden einerseits und dem BIP pro Kopf andererseits (Abb. B1.3), der erkennen lässt, dass ärmere OECD-Länder tendenziell eher weniger pro Schüler/Studierenden ausgeben als reichere OECD-Länder. Diese Tendenz zeigt sich auch beim prozentualen Anteil der Ausgaben am BIP pro Kopf (Tabelle B1.2).

Obwohl generell ein positiver Zusammenhang zwischen den Bildungsausgaben pro Schüler/Studierenden und dem BIP pro Kopf besteht, gibt es sowohl unter den reicheren als auch den ärmeren OECD-Ländern erhebliche Unterschiede bei den Bildungsausgaben pro Schüler/Studierenden. Australien und Österreich sind beispielsweise Länder mit einem vergleichbaren BIP pro Kopf der Bevölkerung. Der in diesen Ländern pro Schüler/Studierenden investierte Anteil des BIP pro Kopf ist jedoch sehr unterschiedlich. Mit 19 Prozent des BIP pro Kopf liegt Australien bezüglich des pro Schüler im Primarbereich investierten Anteils vom Volkseinkommen im OECD-Durchschnitt. Österreich dagegen gibt pro Schüler im Primarbereich 26 Prozent des BIP pro Kopf aus, was den höchsten Anteil darstellt (Tabelle B1.2).

Schlägt sich ein wachsendes Volkseinkommen auch in höheren Bildungsausgaben pro Schüler/Studierenden nieder? Die Pfeile in Abbildung B1.4 zeigen für 


\section{Abbildung B1.3.}

Ausgaben für Bildungseinrichtungen pro Schüler/Studierenden im Verhältnis zum BIP pro Kopf (1999)

Jährliche Ausgaben für Bildungseinrichtungen pro Schüler/Studierenden im Verhältnis zum BIP pro Kopf,

in US-Dollar, kaufkraftbereinigt, nach Bildungsbereichen

\section{Primarbereich}

Ausgaben pro Schüler (in US-Dollar, kaufkraftbereinigt)

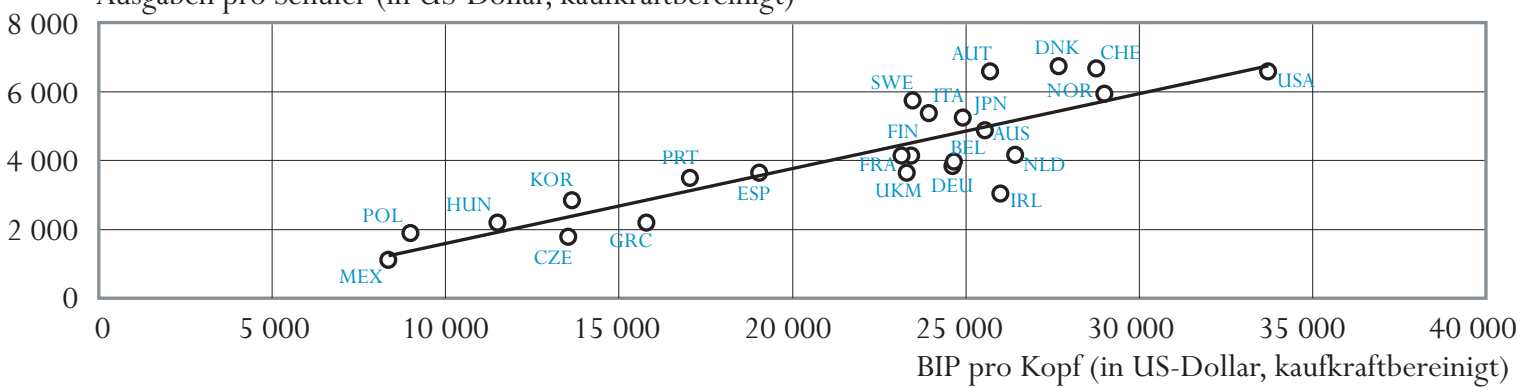

Sekundarbereich

Ausgaben pro Schüler (in US-Dollar, kaufkraftbereinigt)

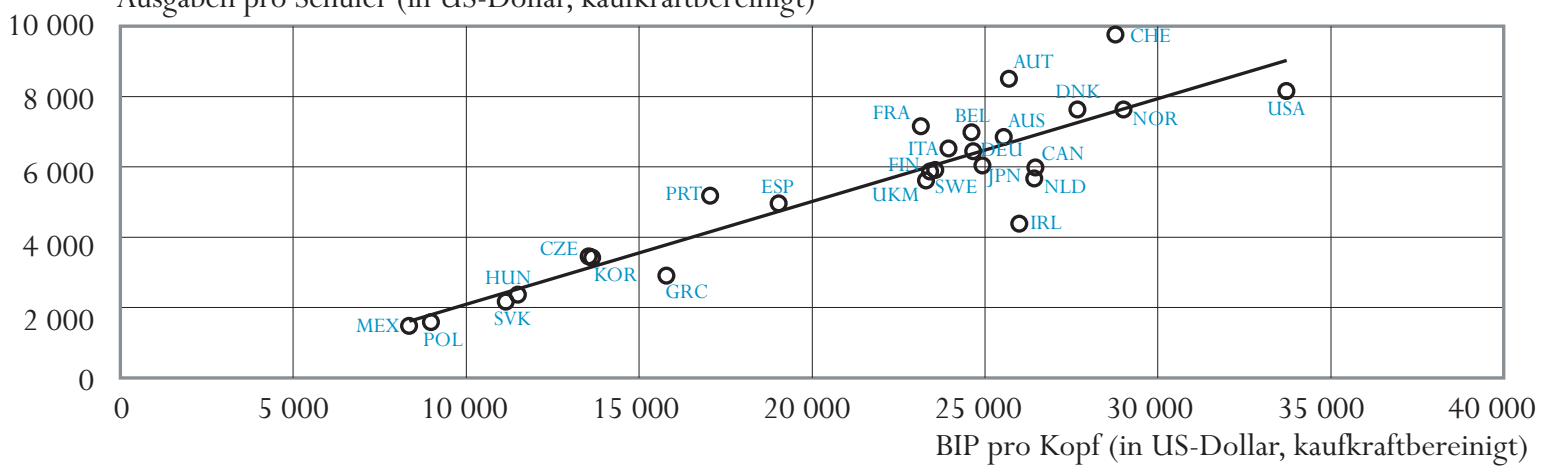

Tertiärbereich

Ausgaben pro Studierenden (in US-Dollar, kaufkraftbereinigt)

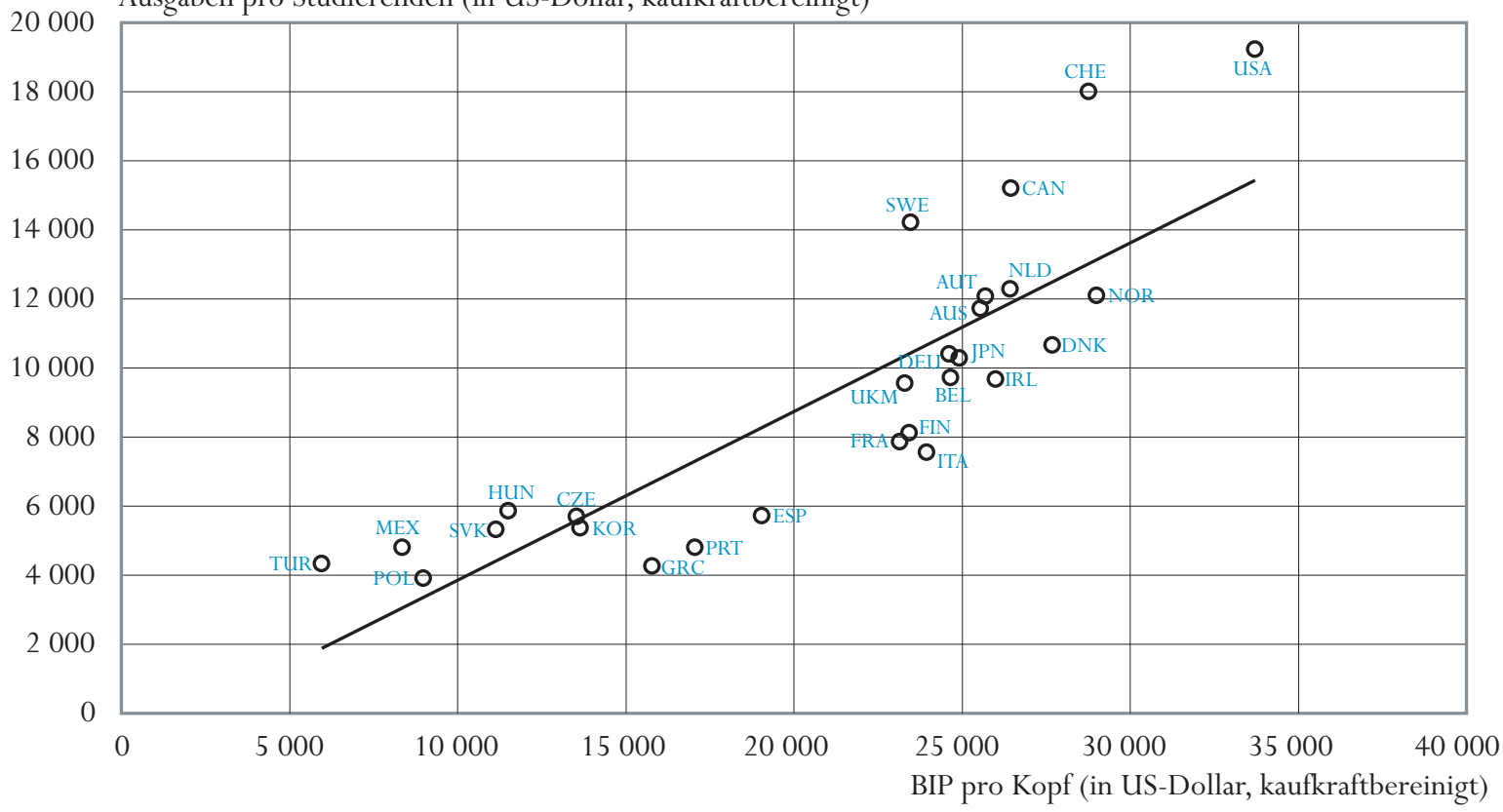

Hinweis: Auflistung der verwendeten Ländercodes und der dazugehörenden Ländernamen s. Hinweise für den Leser.

Quelle: OECD. Tabelle B1.1, B1.2 und Anhang 2. Hinweise s. Anhang 3 (www.oecd.org/els/education/eag2002). 
Im Allgemeinen nehmen

mit wachsendem

Reichtum der

$O E C D$-Länder die

Bildungsausgaben pro

schüler/Studierenden

$z u, \ldots$ jedes OECD-Land die Veränderungen in den Bildungsausgaben pro Schüler/ Studierenden im Primar-, Sekundar- und post-sekundären, nicht-tertiären Bereich im Verhältnis zu den entsprechenden Veränderungen des BIP pro Kopf. Der Anfang eines Pfeils bezeichnet das BIP pro Kopf (horizontale Achse) und die Bildungsausgaben pro Schüler/Studierenden (vertikale Achse) in 1995 (in US-Dollar bezogen auf 1999, kaufkraftbereinigt). Das Ende des Pfeils steht für die entsprechenden Werte in 1999.

\section{Abbildung B1.4.}

Veränderung der Ausgaben für Bildungseinrichtungen pro Schüler/Studierenden und Volkseinkommen Veränderung der Ausgaben zwischen 1995 und 1999 für Bildungseinrichtungen pro Schüler/Studierenden im Primar-, Sekundar- und post-sekundären, nicht tertiären Bereich im Vergleich zur Veränderung des BIP pro Kopf (in US-Dollar, kaufkraftbereinigt)

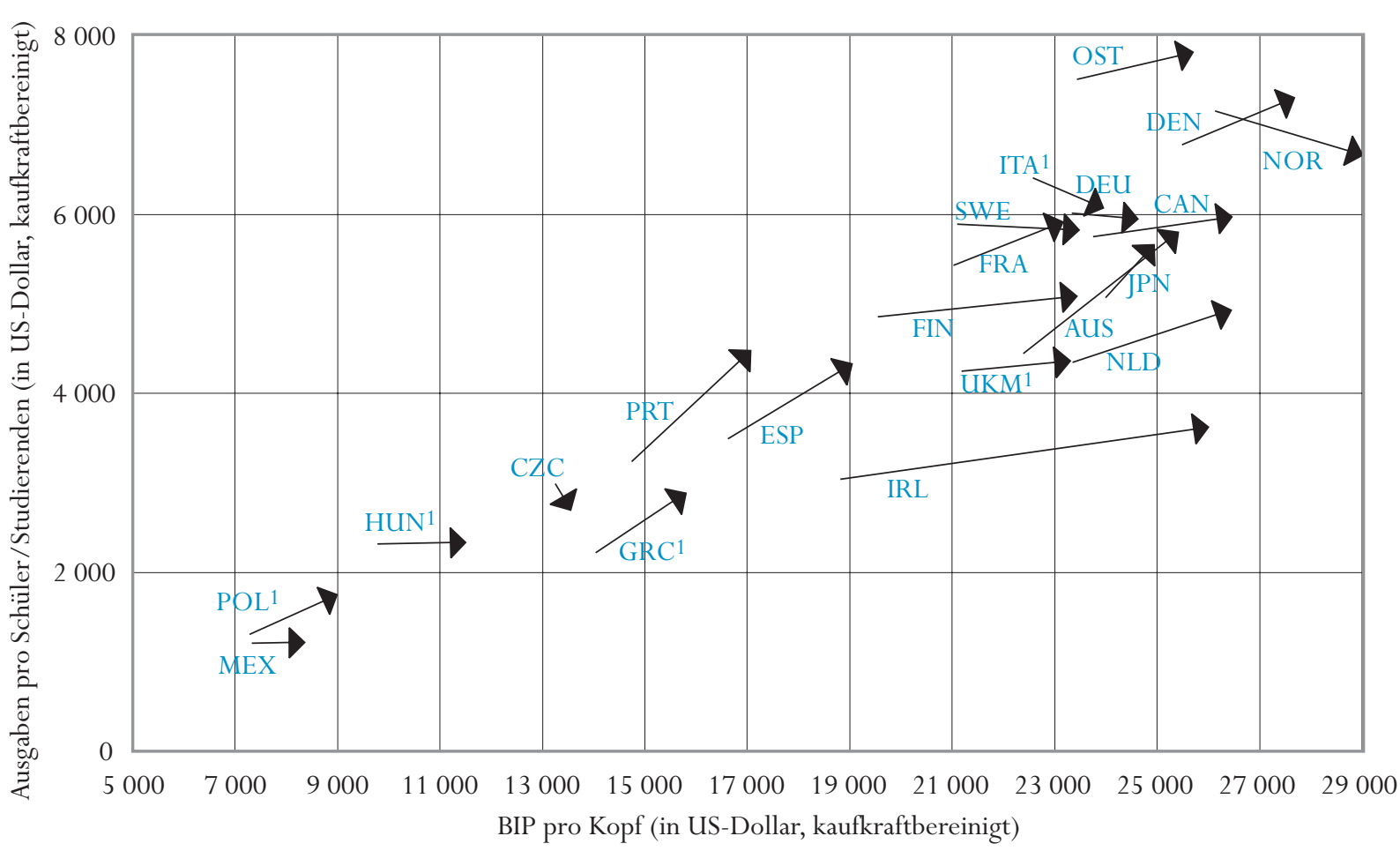

Hinweis: Auflistung der verwendeten Ländercodes und der dazugehörenden Ländernamen s. Hinweise für den Leser.

Der Anfang eines Pfeils steht für die Ausgaben pro Schüler/Studierenden und das BIP pro Kopf in 1995. Das Ende des Pfeils steht für die entsprechenden Werte in 1999.

1. Nur öffentliche Bildungseinrichtungen.

Quelle: OECD. Tabelle B6.1 und Anhang 2. Hinweise s. Anhang 3 (www.oecd.org/els/education/eag2002).

Im Allgemeinen korrelieren die Veränderungen in den Bildungsausgaben pro Schüler/Studierenden mit den Veränderungen im BIP pro Kopf. So sind zum Beispiel in den meisten OECD-Ländern die Bildungsausgaben pro Schüler/ Studierenden im Primar-, Sekundar- und post-sekundären, nicht-tertiären Bereich entsprechend dem BIP pro Kopf gestiegen. 
In sechs von 21 OECD-Ländern, nämlich Australien, Griechenland, Japan, Polen, Portugal und Spanien, stiegen zwischen 1995 und 1999 die Bildungsausgaben ...es gibt aber auch pro Schüler/Studierenden schneller als das BIP pro Kopf. Im Gegensatz dazu nahmen zwischen 1995 und 1999 die Bildungsausgaben pro Schüler/ Studierenden im Primar-, Sekundar- und post-sekundären, nicht-tertiären Bereich in Deutschland, Italien, Norwegen, Schweden und der Tschechischen Republik bei gleichzeitig steigendem BIP pro Kopf ab (Abb. B1.4).

\section{Ausnahmen.}

\section{Abbildung B1.5.}

Unterschiede in den Ausgaben für Bildungseinrichtungen pro Schüler/Studierenden im Verhältnis zum Primarbereich (1999)

Verhältnis der Ausgaben für Bildungseinrichtungen pro Schüler/Studierenden in den verschiedenen Bildungsbereichen zu den Bildungsausgaben pro Schüler im Primarbereich (x 100)

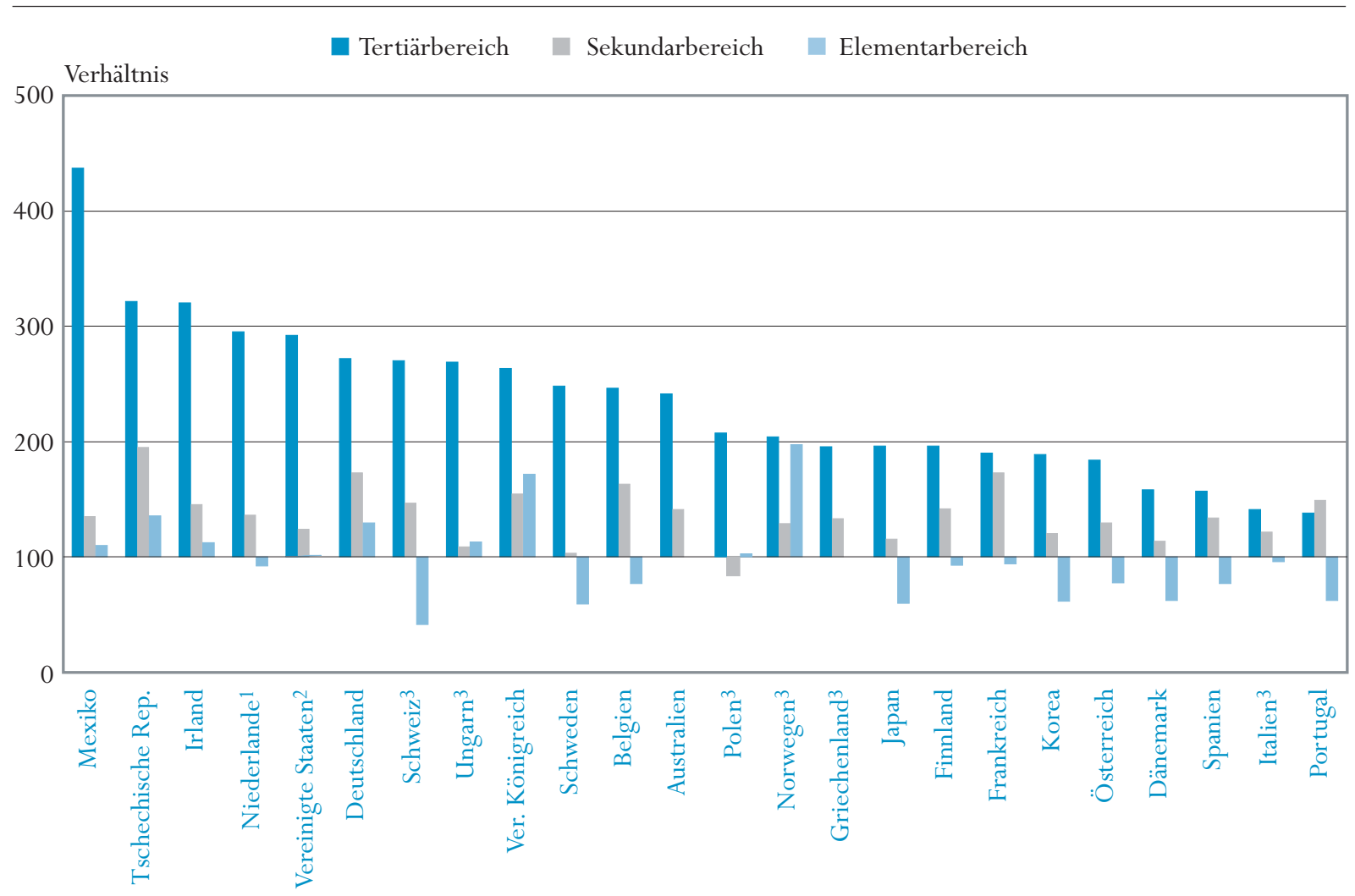

1. Nur öffentliche und staatlich-subventionierte Bildungseinrichtungen.

2. Nur öffentliche und unabhängige private Bildungseinrichtungen.

3. Nur öffentliche Bildungseinrichtungen.

Ein Verhältnis von 500 für den Tertiärbereich bedeutet, dass die Ausgaben für einen Studierenden im Tertiärbereich in einem bestimmten Land fünf mal so hoch sind wie die für einen Schüler im Primarbereich.

Ein Verhältnis von 50 für den Elementarbereich bedeutet, dass die Ausgaben für ein Kind im Elementarbereich in einem bestimmten Land nur halb so hoch sind wie für einen Schüler im Primarbereich.

Anordnung der Länder in absteigender Reihenfolge der Ausgaben für einen Studierenden im Tertiärbereich im Verhältnis zu den Bildungsausgaben für einen Schüler im Primarbereich.

Quelle: OECD. Tabelle B1.1. Hinweise s. Anhang 3 (www.oecd.org/els/education/eag2002). 


\section{Je höher der Bildungsbereich, desto höher die Bildungsausgaben pro Schüler/Studierenden.}

Im Durchschnitt der $O E C D$-Länder sind die Bildungsausgaben pro Studierenden im Tertiärbereich 2,3-mal so hoch wie die Bildungsausgaben pro Schüler im Primarbereich.

Die jährlichen

Bildungsausgaben pro

Studierenden spiegeln

nicht immer die gesamten Kosten tertiärer Studiengänge wider.

Die Studierenden können aus einer Vielfalt von Bildungseinrichtungen und Teilnahmemöglichkeiten wählen.

\section{Unterschiede in den Bildungsausgaben pro Schüler/Studierenden in den verschiedenen Bildungsbereichen}

Allen OECD-Ländern ist eines gemeinsam: die Bildungsausgaben pro Schüler/Studierenden steigen vom Primar- zum Tertiärbereich stark an. Dies wird verständlich, wenn man die wichtigsten Faktoren betrachtet, die die Bildungsausgaben beeinflussen, insbesondere Ort und Art des Bildungsangebots. Bildung findet nach wie vor meistens an herkömmlichen Schulen und Hochschulen statt, die in Bezug auf Aufbau, Lehrplan, Art des Unterrichts und Management trotz einiger Unterschiede recht ähnlich sind. Diese Gemeinsamkeiten führen daher auch zu ähnlichen Strukturen der Ausgaben pro Schüler/Studierenden.

Vergleiche der Verteilung der Bildungsausgaben auf die verschiedenen Bildungsbereiche sind ein Hinweis auf den relativen Stellenwert, den die einzelnen OECD-Länder den verschiedenen Bildungsbereichen beimessen, sowie auf die relativen Kosten der Bildungsvermittlung in diesen Bereichen. Obwohl in fast allen OECD-Ländern die Bildungsausgaben pro Schüler/Studierenden mit der Höhe des Bildungsbereichs steigen, variieren die relativen Unterschiede doch erheblich zwischen den einzelnen Ländern (Abb. B1.5). Im Sekundarbereich betragen die Bildungsausgaben pro Schüler im Durchschnitt das 1,3-fache derjenigen im Primarbereich, obwohl die Spanne vom 0,8-fachen der Höhe der Ausgaben pro Schüler im Primarbereich in Polen bis zu mehr als dem 1,7-fachen in Deutschland, Frankreich und der Tschechischen Republik reicht.

Obwohl im Durchschnitt der OECD-Länder die Bildungsausgaben pro Studierenden im Tertiärbereich 2,3-mal so hoch wie die Bildungsausgaben pro Schüler im Primarbereich sind, gibt es große länderspezifische Unterschiede bei den Ausgabenstrukturen. Während beispielsweise Italien und Portugal für einen Studierenden im Tertiärbereich nur 1,4 mal soviel ausgeben wie für einen Schüler im Primarbereich, ist es in Mexiko das 4,4-fache (Abb. B1.5).

\section{Bildungsausgaben pro Studierenden über die durchschnittliche Dauer tertiärer Studiengänge}

Da sowohl die typische Dauer als auch die Intensität tertiärer Studiengänge in den einzelnen OECD-Ländern sehr unterschiedlich sind, spiegeln die Unterschiede zwischen den einzelnen Ländern bei den jährlichen Bildungsausgaben pro Studierenden für Bildungsdienstleistungen, wie sie aus der Abbildung B1.1 hervorgehen, nicht unbedingt die Unterschiede in den Gesamtkosten für die tertiäre Bildung des typischen Studierenden wider.

Heutzutage können die Studierenden aus einer großen Vielfalt von Bildungseinrichtungen und Teilnahmemöglichkeiten wählen, um die für sie beste Lösung entsprechend ihren angestrebten Bildungsabschlüssen, Fähigkeiten und persönlichen Interessen zu finden. Viele Studierende nehmen an Teilzeitstudiengängen teil, während andere während des Studiums arbeiten oder an mehreren Einrichtungen studieren, bevor sie ihren Abschluss erwerben. 
Dieses unterschiedliche Teilnahmeverhalten kann die Interpretierbarkeit der Bildungsausgaben pro Studierenden beeinflussen.

Insbesondere vergleichsweise niedrige jährliche Bildungsausgaben pro Studierenden können zu vergleichsweise relativ hohen Gesamtkosten im Tertiärbereich führen, wenn die typische Dauer der tertiären Studiengänge lang ist. Abbildung B1.6 gibt einen Überblick über die durchschnittlichen Ausgaben pro Studierenden im Laufe des gesamten Studiums. Die Zahlen beziehen sich auf alle Studierenden, für die Ausgaben anfallen, also auch auf die Studienabbrecher. Zwar beruhen die Berechnungen auf einer Reihe vereinfachender Annahmen und sind daher mit Vorsicht zu behandeln (s. Anhang 3 unter www.oecd.org/ els/education/eag2002), dennoch lassen sich aus ihnen einige bedeutende Verschiebungen in der Rangfolge der OECD-Länder ablesen, je nachdem ob die jährlichen oder die kumulativen Ausgaben das entscheidende Kriterium sind.

Niedrige jährliche Ausgaben können sich bei einer langen Studiendauer zu hohen Gesamtkosten aufsummieren.

So sind beispielsweise die jährlichen Bildungsausgaben pro Studierenden im Tertiärbereich in den Niederlanden ungefähr genau so hoch wie in Österreich (12.285 US-Dollar in den Niederlanden im Vergleich zu 12.070 US-Dollar in Österreich) (Tabelle B1.1). Aufgrund der Unterschiede in der Struktur der im Tertiärbereich verliehenen Abschlüsse (Indikator A2) ist die durchschnittliche Dauer tertiärer Studiengänge in Österreich mehr als ein Drittel länger als in den

\section{Abbildung B1.6.}

Kumulative Ausgaben für Bildungseinrichtungen pro Studierenden über die durchschnittliche Dauer tertiärer Studiengänge (1999)

Jährliche Ausgaben pro Studierenden multipliziert mit der durchschnittlichen Dauer tertiärer Studiengänge, in US-Dollar, kaufkraftbereinigt

Jeder Abschnitt eines Balkens steht für die jährlichen Ausgaben pro Studierenden. Die Anzahl der Abschnitte steht für die Anzahl von Jahren, die ein Studierender im Durchschnitt im Tertiärbereich verbringt.

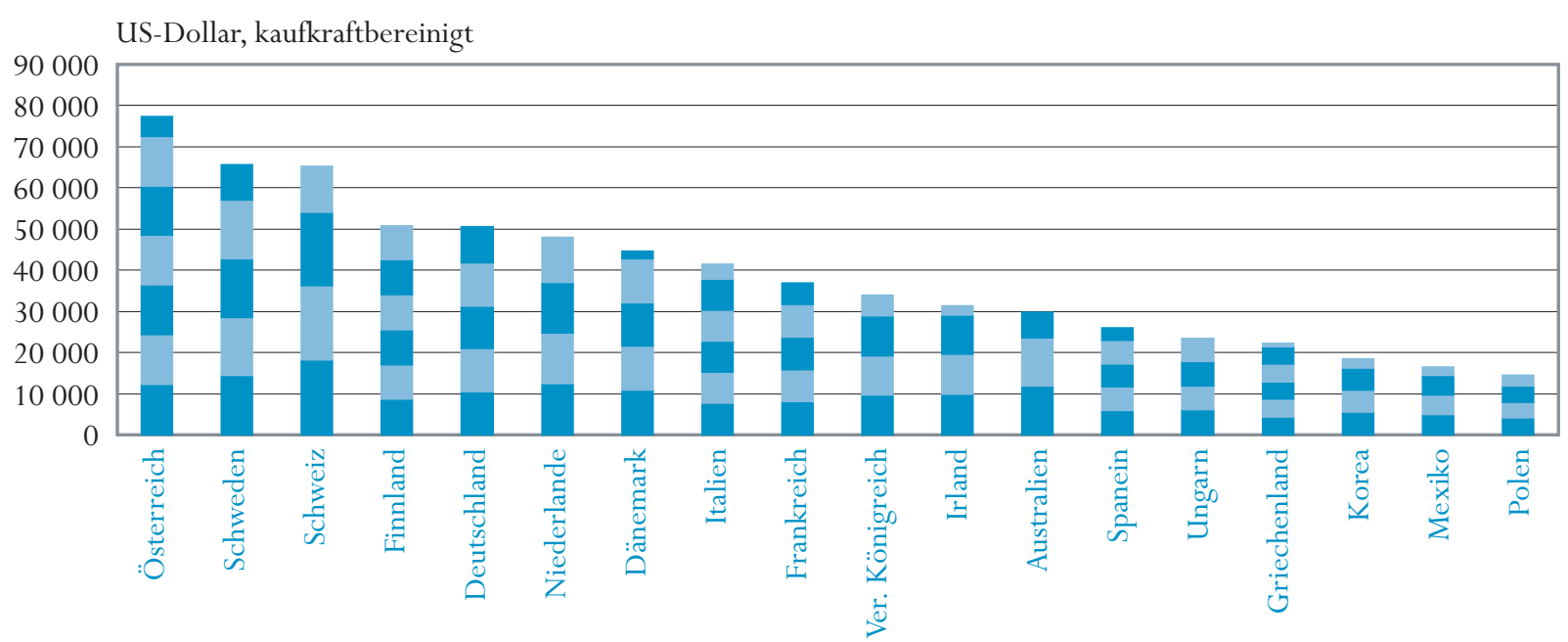

Anordnung der Länder in absteigender Reihenfolge der Gesamtausgaben pro Studierenden im Tertiärbereich über die durchschnittliche Dauer tertiärer Studiengänge.

Quelle: OECD. Tabelle B1.3. Hinweise s. Anhang 3 (www.oecd.org/els/education/eag2002). 
Niederlanden (6,4 Jahre in Österreich gegenüber 3,9 Jahren in den Niederlanden). Daher liegen die kumulierten Kosten für jeden Studierenden im Tertiärbereich in Österreich um mehr als 50 Prozent höher als in den Niederlanden (77.248 USDollar verglichen mit 47.911 US-Dollar) (Abb. B1.6 und Tabelle B1.3).

Die Gesamtkosten eines Studiengangs im Tertiärbereich A sind in der Schweiz (101.334 US-Dollar) mehr als doppelt so hoch als in 9 der anderen 10 OECDLänder, die Zahlen vorgelegt haben, Deutschland ist hier die Ausnahme (Tabelle B1.3). Diese Unterschiede müssen natürlich im Zusammenhang mit den Unterschieden in den nationalen Abschlussstrukturen, aber auch mit möglichen Unterschieden im akademischen Qualifikationsniveau der Hochschulabsolventen in den einzelnen OECD-Ländern gesehen werden. Während man bei Studiengängen im Tertiärbereich B ähnliche Tendenzen feststellen kann, sind die Gesamtkosten bei diesen Studiengängen tendenziell wesentlich geringer als bei Studiengängen im Tertiärbereich A, was vor allem mit der kürzeren Studiendauer zusammenhängt.

\section{Definitionen und angewandte Methodik}

Die Zahlen beziehen sich auf das Haushaltsjahr 1999 und beruhen auf der von der OECD im Jahre 2001 durchgeführten vOE-Datenerhebung zur Bildungsstatistik (Einzelheiten s. Anhang 3).

Die Zahlen für das Haushaltsjahr 1995 beruhen auf einer im Jahre 2000 unter den OECD-Ländern durchgeführten speziellen Erhebung.
Die Bildungsausgaben pro Schüler/Studierenden für einen bestimmten Bildungsbereich werden durch Division der Gesamtausgaben für Bildungseinrichtungen in diesem Bereich durch die entsprechende in Vollzeitäquivalenten ausgedrückte Schüler-/Studierendenzahl berechnet. Dabei werden nur jene Arten von Bildungseinrichtungen und Bildungsgängen berücksichtigt, für die sowohl Daten über die Zahl der Schüler/Studierenden als auch über die Ausgaben vorliegen. Die Ausgaben in nationaler Währung werden dann in US-Dollar umgerechnet, indem der betreffende Betrag in Landeswährung durch die Kaufkraftparität (KKP) geteilt wird. Der KKPUmrechnungskurs gibt den Betrag einer Landeswährung an, mit dem man in einem bestimmten OECD-Land den gleichen Waren- und Dienstleistungskorb wie in den Vereinigten Staaten mit US-Dollar erwerben kann. Er wird anstelle der aktuellen Wechselkurse verwendet, da diese von einerVielzahl von Faktoren beeinflusst werden (Zinssätze, Handelspolitik, Konjunkturerwartungen etc.), die wenig mit der aktuellen, relativen Kaufkraft in den einzelnen OECDLändern zu tun haben (weitere Einzelheiten s. Anhang 2).

Die Abbildungen B1.2 und B1.4 zeigen die Bildungsausgaben pro Schüler/ Studierenden im Haushaltsjahr 1995. Die Daten über die Ausgaben im Jahre 1995 stützen sich auf eine im Jahre 2000 durchgeführte spezielle Erhebung. Die OECD-Länder wurden aufgefordert, die Daten für 1995 entsprechend den Definitionen und dem Erhebungsbereich der UOE-Datenerhebung zu erfassen. Alle Daten zu den Ausgaben ebenso wie die Angaben zum BIP von 1995 wurden mit Hilfe des BIP-Preisdeflators an das Preisniveau von 1999 angepasst.

Die Bildungsausgaben pro Schüler/Studierenden im Verhältnis zum BIP pro Kopf werden berechnet, indem die Bildungsausgaben pro Schüler/ Studierenden in Landeswährung als prozentualer Anteil des ebenfalls in 
Landeswährung ausgedrückten BIP pro Kopf angegeben werden. Wenn sich die Bildungsausgaben und die Daten zum BIP auf unterschiedliche Zeiträume beziehen, werden unter Verwendung der für das betreffende OECD-Land geltenden Inflationsraten die Ausgabendaten auf den Bezugszeitraum der BIPDaten umgerechnet (s. Anhang 2).

Die zu erwartenden Ausgaben während der durchschnittlichen Dauer tertiärer Studiengänge (Tabelle B1.3) werden durch Multiplikation der aktuellen jährlichen Ausgaben mit der typischen Dauer solcher Studiengänge berechnet. Die zur Ermittlung der typischen Durchschnittsdauer von tertiären Studiengängen angewandte Methodik ist in Anhang 3 (unter www.oecd.org/els/ education/eag2002) beschrieben. Die Schätzungen für die Dauer der tertiären Studiengänge stützen sich auf Daten einer in den Jahren 1997 und 2000 in den OECD-Ländern durchgeführten speziellen Erhebung.

Die Erstellung einer Rangfolge der OECD-Länder nach ihren jährlichen Bildungsausgaben pro Schüler/Studierenden wird durch unterschiedliche Definitionen der einzelnen Länder für die Begriffe Vollzeit-, Teilzeit- und vollzeitäquivalente Bildungsbeteiligung erschwert. In einigen OECD-Ländern werden alle Studierenden des Tertiärbereichs als Vollzeitstudierende gezählt, während sich in anderen Ländern die Intensität der Beteiligung nach den innerhalb einer vorgegebenen Referenzzeit erworbenen Scheinen für die erfolgreiche Absolvierung bestimmter Kurseinheiten richtet. Bei OECDLändern, die genaue Angaben über Teilzeitstudierende machen können, werden sich höhere Ausgaben pro vollzeitäquivalenten Studierenden ergeben, als bei solchen OECD-Länder, die nicht zwischen den verschiedenen Teilnahmemöglichkeiten differenzieren können.

Es ist zu beachtene, dass die in früheren Ausgaben dieser Veröffentlichung aufgeführten Zahlen und Daten möglicherweise nicht immer mit denen der Ausgabe 2002 vergleichbar sind, da sich Änderungen in den Definitionen und Erhebungsbereichen ergeben haben. Diese wurden aufgrund der OECD Expenditure Comparability Study vorgenommen (Einzelheiten zu den Änderungen s. Anhang 3 unter www.oecd.org/els/education/eag2002). 
Tabelle B1.1.

Ausgaben für Bildungseinrichtungen pro Schüler/Studierenden (1999)

Jährliche Ausgaben für Bildungseinrichtungen pro Schüler/Studierenden, in US-Dollar, kaufkraftbereinigt, nach Bildungsbereich (basierend auf Vollzeitäquivalenten)

\begin{tabular}{|c|c|c|c|c|c|c|c|c|c|}
\hline & \multirow[b]{2}{*}{$\begin{array}{c}\text { Elementar- } \\
\text { bereich } \\
\text { (für 3-Jährige } \\
\text { und Ältere) }\end{array}$} & \multirow[b]{2}{*}{ Primarbereich } & \multirow[b]{2}{*}{$\begin{array}{l}\text { Sekundar- } \\
\text { bereich I }\end{array}$} & \multirow[b]{2}{*}{$\begin{array}{l}\text { Sekundar- } \\
\text { bereich II }\end{array}$} & \multirow[b]{2}{*}{$\begin{array}{l}\text { Sekundar- } \\
\text { bereich } \\
\text { insgesamt }\end{array}$} & \multirow[b]{2}{*}{$\begin{array}{c}\text { Post- } \\
\text { sekundärer, } \\
\text { nicht-ter- } \\
\text { tiärer Bereich }\end{array}$} & \multicolumn{3}{|c|}{ Tertiärbereich } \\
\hline & & & & & & & $\begin{array}{c}\text { Tertiärbereich } \\
\text { insgesamt }\end{array}$ & $\begin{array}{c}\text { Tertiärbe- } \\
\text { reich B }\end{array}$ & $\begin{array}{c}\text { Tertiärbereich } \\
\text { A und weiterf. } \\
\text { Forschungs- } \\
\text { programme }\end{array}$ \\
\hline & $(1)$ & $(2)$ & (3) & (4) & (5) & $(6)$ & $(7)$ & $(8)$ & (9) \\
\hline Australien ${ }^{*}$ & $\mathrm{~m}$ & 4858 & 6710 & 7066 & 6850 & 7650 & 11725 & 7993 & 12588 \\
\hline Österreich ${ }^{*}$ & 5080 & 6568 & 8434 & 8584 & 8504 & 9131 & 12070 & $x(7)$ & $\mathrm{x}(7)$ \\
\hline Kanada $^{*}$ & 4466 & $\mathrm{x}(5)$ & $x(5)$ & $\mathrm{x}(5)$ & 5981 & $\mathrm{x}(7)$ & 15211 & $x(7)$ & 15470 \\
\hline Tschechische Rep." & 2404 & 1769 & 2998 & 4043 & 3449 & 832 & 5688 & 1886 & 6679 \\
\hline Dänemark $^{*}$ & 4208 & 6721 & 6904 & 8270 & 7626 & $\mathrm{~m}$ & 10657 & $\mathrm{x}(7)$ & $\mathrm{x}(7)$ \\
\hline Finnland $^{*}$ & 3855 & 4138 & 6390 & 5479 & 5863 & $x(5)$ & 8114 & 4500 & 8474 \\
\hline Frankreich $^{*}$ & 3901 & 4139 & 6657 & 7766 & 7152 & 5839 & 7867 & 8458 & 7709 \\
\hline Deutschland ${ }^{*}$ & 4937 & 3818 & 4918 & 10107 & 6603 & 11679 & 10393 & 5495 & 11209 \\
\hline Griechenland $^{1 *}$ & $x(2)$ & 2176 & $x(5)$ & $x(5)$ & 2904 & 5415 & 4260 & 3439 & 4606 \\
\hline Island & $\mathrm{m}$ & $\mathrm{m}$ & $\mathrm{m}$ & $\mathrm{m}$ & $\mathrm{m}$ & $\mathrm{m}$ & $\mathrm{m}$ & $\mathrm{m}$ & $\mathrm{m}$ \\
\hline Irland & 3386 & 3018 & 4401 & 4362 & 4383 & 4168 & 9673 & $\mathrm{x}(7)$ & $\mathrm{x}(7)$ \\
\hline Italien $^{1}$ & 5133 & 5354 & 6206 & 6741 & 6518 & $\mathrm{~m}$ & 7552 & 7147 & 7557 \\
\hline Japan* & 3154 & 5240 & 5612 & 6460 & 6039 & $x(4,7)$ & 10278 & 7649 & 10749 \\
\hline Korea $^{*}$ & 1752 & 2838 & 3208 & 3597 & 3419 & $\mathrm{a}$ & 5356 & 3494 & 6612 \\
\hline Luxemburg & $\mathrm{m}$ & $\mathrm{m}$ & $\mathrm{m}$ & $\mathrm{m}$ & $\mathrm{m}$ & $\mathrm{m}$ & $\mathrm{m}$ & $\mathrm{m}$ & $\mathrm{m}$ \\
\hline Mexiko & 1204 & 1096 & 1129 & 2226 & 1480 & $\mathrm{a}$ & 4789 & $x(7)$ & $x(7)$ \\
\hline Niederlande ${ }^{2 *}$ & 3848 & 4162 & 5747 & 5575 & 5670 & $\mathrm{~m}$ & 12285 & 7227 & 12354 \\
\hline Neuseeland & $\mathrm{m}$ & $\mathrm{m}$ & $\mathrm{m}$ & $\mathrm{m}$ & $\mathrm{m}$ & $\mathrm{m}$ & $\mathrm{m}$ & $\mathrm{m}$ & $\mathrm{m}$ \\
\hline Norwegen ${ }^{1}$ & 11699 & 5920 & 7387 & 7819 & 7628 & $\mathrm{x}(5)$ & 12096 & $\mathrm{x}(7)$ & $\mathrm{x}(7)$ \\
\hline Polen ${ }^{1}$ & 1898 & 1888 & $\mathrm{x}(2)$ & 1583 & 1583 & $x(4)$ & 3912 & $x(7)$ & 3912 \\
\hline Portugal $^{*}$ & 2165 & 3478 & 4958 & 5422 & 5181 & a & 4802 & $x(7)$ & $\mathrm{x}(7)$ \\
\hline Slowakische Rep. & 1880 & $\mathrm{x}(3)$ & 1811 & 2637 & 2163 & $\mathrm{x}(4)$ & 5325 & $\mathrm{x}(9)$ & 5325 \\
\hline Spanien & 2789 & 3635 & $\mathrm{x}(5)$ & $\mathrm{x}(5)$ & 4864 & $\mathrm{x}(5)$ & 5707 & 5111 & 5760 \\
\hline Schweden & 3396 & 5736 & 5678 & 6077 & 5911 & 6675 & 14222 & $\mathrm{x}(7)$ & $\mathrm{x}(7)$ \\
\hline Ver. Königreich* & 6233 & 3627 & $x(5)$ & $\mathrm{x}(5)$ & 5608 & $\mathrm{x}(5)$ & 9554 & $\mathrm{x}(7)$ & $\mathrm{x}(7)$ \\
\hline Vereinigte Staaten ${ }^{4 *}$ & 6692 & 6582 & $x(5)$ & $x(5)$ & 8157 & $x(7)$ & 19220 & $x(7)$ & $x(7)$ \\
\hline Ländermittel & 3847 & 4148 & 5210 & 5919 & 5465 & 4795 & 9210 & $\sim$ & $\sim$ \\
\hline OECD Insgesamt & 3746 & 4229 & $\sim$ & $\sim$ & 5174 & $\sim$ & 11422 & $\sim$ & $\sim$ \\
\hline Argentinien & 1409 & 1629 & 2198 & 2528 & 2327 & a & 5606 & 5137 & 6056 \\
\hline Brasilien $^{1,5}$ & 1222 & 956 & 1069 & 1172 & 1100 & $\mathrm{~m}$ & 13567 & $\mathrm{~m}$ & 13567 \\
\hline Chile & 1431 & 1701 & 1767 & 2041 & 1941 & $\mathrm{a}$ & 6911 & 3545 & 7652 \\
\hline China & 105 & 372 & 476 & 1768 & 833 & a & 5798 & $\mathrm{x}(7)$ & $\mathrm{x}(7)$ \\
\hline Indien $^{5}$ & 65 & 303 & 297 & 290 & 295 & a & $\mathrm{m}$ & $\mathrm{m}$ & $\mathrm{m}$ \\
\hline Indonesien $^{6}$ & 53 & 81 & 208 & 295 & 242 & a & 1047 & $\mathrm{x}(7)$ & $\mathrm{x}(7)$ \\
\hline Israel & 3415 & 4240 & $x(5)$ & $x(5)$ & 5164 & 4115 & 11210 & 7965 & 12088 \\
\hline Jamaika $^{1}$ & 386 & 764 & 1065 & 1114 & 1082 & 908 & 6484 & 2650 & 13194 \\
\hline Jordanien ${ }^{1}$ & $\mathrm{~m}$ & 775 & 782 & 806 & 789 & $\mathrm{a}$ & 5082 & $\mathrm{x}(7)$ & $x(7)$ \\
\hline Malaysia $^{1}$ & 437 & 1015 & $x(5)$ & $x(5)$ & 1813 & 8423 & 7924 & 7677 & 7979 \\
\hline Paraguay & $x(2)$ & 877 & $\mathrm{x}(5)$ & $\mathrm{x}(5)$ & 1545 & $\mathrm{a}$ & 5465 & 2796 & 6750 \\
\hline Peru & 442 & 483 & $x(5)$ & $\mathrm{x}(5)$ & 579 & $\mathrm{~m}$ & 1414 & 675 & 2057 \\
\hline Philippinen ${ }^{1,5}$ & 46 & 474 & 411 & 384 & 406 & 962 & 1060 & $\mathrm{a}$ & 1060 \\
\hline Tunesien $^{1,6}$ & $\mathrm{~m}$ & 988 & $x(5)$ & $x(5)$ & 1868 & a & 5008 & $x(7)$ & $\mathrm{x}(7)$ \\
\hline Uruguay $^{1}$ & 1133 & 1000 & 1114 & 1484 & 1275 & a & 2239 & $x(7)$ & $x(7)$ \\
\hline Simbabwe $^{1}$ & $\mathrm{~m}$ & 537 & $x(5)$ & $\mathrm{x}(5)$ & 813 & $\mathrm{x}(5)$ & $\mathrm{m}$ & $\mathrm{m}$ & $\mathrm{m}$ \\
\hline
\end{tabular}

Hinweis: $\mathrm{x}$ bedeutet, dass die Daten in einer anderen Spalte enthalten sind, deren Referenz in runden Klammern nach dem ' $\mathrm{x}$ ' angegeben ist. So bedeutet z. B. $\mathrm{x}(2)$, dass die Daten in Spalte 2 enthalten sind.

1. Nur öffentliche Bildungseinrichtungen.

2. Nur öffentliche und staatlich-subventionierte private Bildungseinrichtungen.

3. Spalte (9) bezieht sich nur auf Ausgaben im Tertiärberich A.

4. Nur öffentliche und unabhängige private Bildungseinrichtungen.

5. Referenzjahr 1998.

6. Referenzjahr 2000

* Hinweise s. Anhang 3 (www.oecd.org/els/education/eag2002).

Quelle: OECD. 
Tabelle B1.2.

Ausgaben für Bildungseinrichtungen pro Schüler/Studierenden im Verhältnis zum BIP pro Kopf (1999)

Ausgaben für Bildungseinrichtungen pro Schüler/Studierenden imVerhältnis zum BIP pro Kopf, nach Bildungsbereichen (basierend auf Vollzeitäquivalenten)

\begin{tabular}{|c|c|c|c|c|c|c|c|c|c|}
\hline & \multirow[b]{2}{*}{$\begin{array}{c}\text { Elementar- } \\
\text { bereich (für } \\
\text { 3-Jährige und } \\
\text { Ältere) }\end{array}$} & \multirow[b]{2}{*}{ Primarbereich } & \multirow[b]{2}{*}{$\begin{array}{l}\text { Sekundar- } \\
\text { bereich I }\end{array}$} & \multirow[b]{2}{*}{$\begin{array}{l}\text { Sekundar- } \\
\text { bereich II }\end{array}$} & \multirow[b]{2}{*}{$\begin{array}{l}\text { Sekundar- } \\
\text { bereich } \\
\text { insgesamt }\end{array}$} & \multirow{2}{*}{$\begin{array}{l}\text { Post- } \\
\text { sekundärer, } \\
\text { nicht- } \\
\text { tertiärer } \\
\text { Bereich }\end{array}$} & \multicolumn{3}{|c|}{ Tertiärbereich } \\
\hline & & & & & & & $\begin{array}{c}\text { Tertiärbereich } \\
\text { insgesamt }\end{array}$ & $\begin{array}{c}\text { Tertiärbe- } \\
\text { reich B }\end{array}$ & $\begin{array}{c}\text { Tertiärbereich } \\
\text { A und weiterf. } \\
\text { Forschungs- } \\
\text { programme }\end{array}$ \\
\hline & $(1)$ & $(2)$ & (3) & (4) & (5) & (6) & (7) & $(8)$ & (9) \\
\hline Australien $^{*}$ & $\mathrm{~m}$ & 19 & 26 & 28 & 27 & 30 & 46 & 31 & 49 \\
\hline Österreich ${ }^{*}$ & 20 & 26 & 33 & 33 & 33 & 36 & 47 & $x(7)$ & $x(7)$ \\
\hline Belgien $^{*}$ & 12 & 16 & $\mathrm{x}(5)$ & $\mathrm{x}(5)$ & 26 & $\mathrm{x}(5)$ & 39 & $\mathrm{x}(7)$ & $\mathrm{x}(7)$ \\
\hline Kanada $^{*}$ & 17 & $\mathrm{x}(5)$ & $\mathrm{x}(5)$ & $\mathrm{x}(5)$ & 23 & $\mathrm{x}(7)$ & 57 & $\mathrm{x}(7)$ & 58 \\
\hline Tschechische Rep." & 18 & 13 & 22 & 30 & 25 & 6 & 42 & 14 & 49 \\
\hline Dänemark $^{*}$ & 15 & 24 & 25 & 30 & 28 & $\mathrm{~m}$ & 39 & $\mathrm{x}(7)$ & $\mathrm{x}(7)$ \\
\hline Finnland ${ }^{*}$ & 16 & 18 & 27 & 23 & 25 & $x(5)$ & 35 & 19 & 36 \\
\hline Frankreich ${ }^{*}$ & 17 & 18 & 29 & 34 & 31 & 25 & 34 & 37 & 33 \\
\hline Deutschland $^{*}$ & 20 & 16 & 20 & 41 & 27 & 47 & 42 & 22 & 46 \\
\hline Griechenland $d^{1 *}$ & $x(2)$ & 14 & $\mathrm{x}(5)$ & $\mathrm{x}(5)$ & 18 & 34 & 27 & 22 & 29 \\
\hline Ungarn $^{1 *}$ & 21 & 19 & 18 & 24 & 21 & 26 & 51 & $\mathrm{x}(7)$ & $\mathrm{x}(7)$ \\
\hline Island & $\mathrm{m}$ & $\mathrm{m}$ & $\mathrm{m}$ & $\mathrm{m}$ & $\mathrm{m}$ & $\mathrm{m}$ & $\mathrm{m}$ & $\mathrm{m}$ & $\mathrm{m}$ \\
\hline Irland & 13 & 12 & 17 & 17 & 17 & 16 & 37 & $\mathrm{x}(7)$ & $x(7)$ \\
\hline Italien' ${ }^{2}$ & 21 & 22 & 26 & 28 & 27 & $\mathrm{~m}$ & 32 & 30 & 32 \\
\hline Japan* ${ }^{*}$ & 13 & 21 & 23 & 26 & 24 & $\mathrm{x}(4,7)$ & 41 & 31 & 43 \\
\hline Korea $^{*}$ & 13 & 21 & 24 & 26 & 25 & a & 39 & 26 & 48 \\
\hline Luxemburg & $\mathrm{m}$ & $\mathrm{m}$ & $\mathrm{m}$ & $\mathrm{m}$ & $\mathrm{m}$ & $\mathrm{m}$ & $\mathrm{m}$ & $\mathrm{m}$ & $\mathrm{m}$ \\
\hline Mexiko & 14 & 13 & 14 & 27 & 18 & $\mathrm{a}$ & 57 & $x(7)$ & $x(7)$ \\
\hline Niederlande $^{2 *}$ & 15 & 16 & 22 & 21 & 21 & $\mathrm{~m}$ & 46 & 27 & 47 \\
\hline Neuseeland & $\mathrm{m}$ & $\mathrm{m}$ & $\mathrm{m}$ & $\mathrm{m}$ & $\mathrm{m}$ & $\mathrm{m}$ & $\mathrm{m}$ & $\mathrm{m}$ & $\mathrm{m}$ \\
\hline Norwegen $^{1}$ & 40 & 20 & 25 & 27 & 26 & $\mathrm{x}(4)$ & 43 & $x(7)$ & $\mathrm{x}(7)$ \\
\hline Polen $^{1}$ & 21 & 21 & $\mathrm{x}(2)$ & 18 & 18 & $\mathrm{x}(4)$ & 44 & $\mathrm{x}(7)$ & 44 \\
\hline Portugal $^{*}$ & 13 & 20 & 29 & 32 & 30 & a & 28 & $x(7)$ & $\mathrm{x}(7)$ \\
\hline Slowakische Rep. & 17 & $\mathrm{x}(3)$ & 16 & 24 & 19 & $\mathrm{x}(4)$ & 48 & $\mathrm{x}(9)$ & 48 \\
\hline Spanien & 15 & 19 & $\mathrm{x}(5)$ & $x(5)$ & 26 & $\mathrm{x}(5)$ & 30 & 27 & 30 \\
\hline Schweden & 14 & 24 & 24 & 26 & 25 & 28 & 61 & $\mathrm{x}(7)$ & $\mathrm{x}(7)$ \\
\hline Schweiz ${ }^{1,3^{*}}$ & 10 & 23 & 27 & 41 & 34 & 28 & 63 & 47 & 65 \\
\hline Türkei $^{1}$ & $\mathrm{~m}$ & $\mathrm{~m}$ & $\mathrm{~m}$ & $\mathrm{~m}$ & $\mathrm{~m}$ & $\mathrm{~m}$ & 73 & $x(7)$ & $x(7)$ \\
\hline Ver. Königreich ${ }^{*}$ & 27 & 16 & $\mathrm{x}(5)$ & $x(5)$ & 24 & $\mathrm{x}(5)$ & 41 & $\mathrm{x}(7)$ & $\mathrm{x}(7)$ \\
\hline Vereinigte Staaten ${ }^{4 *}$ & 20 & 20 & $\mathrm{x}(5)$ & $\mathrm{x}(5)$ & 24 & $\mathrm{x}(7)$ & 57 & $\mathrm{x}(7)$ & $\mathrm{x}(7)$ \\
\hline Ländermittel & 18 & 19 & 23 & 28 & 25 & 21 & 44 & 28 & 44 \\
\hline Argentinien & 11 & 13 & 18 & 21 & 19 & $\mathrm{a}$ & 46 & 42 & 49 \\
\hline Brasilien ${ }^{1,5}$ & 18 & 14 & 15 & 17 & 16 & $\mathrm{~m}$ & 195 & $\mathrm{~m}$ & 195 \\
\hline Chile & 17 & 20 & 20 & 24 & 22 & a & 80 & 41 & 88 \\
\hline China & 3 & 10 & 13 & 49 & 23 & a & 161 & $\mathrm{x}(7)$ & $x(7)$ \\
\hline Indien $^{5}$ & 2 & 12 & 16 & 20 & 17 & $\mathrm{a}$ & $\mathrm{m}$ & $\mathrm{m}$ & $\mathrm{m}$ \\
\hline Indonesien $^{6}$ & 9 & 3 & 8 & 11 & 9 & $\mathrm{a}$ & 41 & $x(7)$ & $\mathrm{x}(7)$ \\
\hline Israel & 17 & 21 & $x(5)$ & $x(5)$ & 26 & 21 & 56 & 40 & 61 \\
\hline Jamaika $^{1}$ & 11 & 21 & 30 & 31 & 30 & 25 & 182 & 74 & 371 \\
\hline Jordanien ${ }^{1}$ & $\mathrm{~m}$ & 20 & 20 & 21 & 20 & $\mathrm{a}$ & 130 & $x(7)$ & $\mathrm{x}(7)$ \\
\hline Malaysia $^{1}$ & 5 & 12 & $\mathrm{x}(5)$ & $\mathrm{x}(5)$ & 22 & 103 & 96 & 93 & 97 \\
\hline Paraguay & $\mathrm{x}(2)$ & 20 & $\mathrm{x}(5)$ & $\mathrm{x}(5)$ & 35 & $\mathrm{a}$ & 125 & 64 & 154 \\
\hline Peru & 10 & 10 & $\mathrm{x}(5)$ & $\mathrm{x}(5)$ & 13 & $\mathrm{~m}$ & 31 & 15 & 45 \\
\hline Philippinen ${ }^{1,5}$ & 2 & 14 & 15 & 15 & 15 & 35 & 42 & $\mathrm{a}$ & 84 \\
\hline Tunesien ${ }^{1,6}$ & $\mathrm{~m}$ & 16 & $\mathrm{x}(5)$ & $x(5)$ & 29 & a & 79 & $x(7)$ & $x(7)$ \\
\hline Uruguay ${ }^{1}$ & 13 & 11 & 13 & 17 & 14 & $\mathrm{a}$ & 25 & $\mathrm{x}(7)$ & $\mathrm{x}(7)$ \\
\hline Simbabwe ${ }^{1}$ & $\mathrm{~m}$ & 19 & $x(5)$ & $x(5)$ & 28 & $x(5)$ & $\mathrm{m}$ & $\mathrm{m}$ & $\mathrm{m}$ \\
\hline
\end{tabular}

Hinweis: $\mathrm{x}$ bedeutet, dass die Daten in einer anderen Spalte enthalten sind, deren Referenz in runden Klammern nach dem ' $\mathrm{x}$ ' angegeben ist. So bedeutet z.B. x(2), dass die Daten in Spalte 2 enthalten sind.

1. Nur öffentliche Bildungseinrichtungen.

2. Nur öffentliche und staatlich-subventionierte Bildungseinrichtungen.

3. Spalte (9) bezieht sich nur auf Ausgaben im Teriärbereich A.

4. Nur öffentliche und unabhängige private Bildungseinrichtungen.

5. Referenzjahr 1998

6. Referenzjahr 2000

* Hinweise s. Anhang 3 (www.oecd.org/els/education/eag2002).

Quelle: $\mathrm{OECD}$. 
Tabelle B1.3.

Kumulative Ausgaben für Bildungseinrichtungen pro Studierenden über die durchschnittliche Dauer tertiärer Studiengänge (1999) Durchschnittliche Studiendauer im Tertiärbereich und Ausgaben für Bildungseinrichtungen über die durchschnittliche Studiendauer in

US-Dollar, kaufkraftbereinigt, nach Art des Studiengangs

\begin{tabular}{|c|c|c|c|c|c|c|c|}
\hline & \multirow[b]{2}{*}{$\begin{array}{c}\text { Methode zur } \\
\text { Ermittlung } \\
\text { der Studien- } \\
\text { dauer }^{1} \\
\end{array}$} & \multicolumn{3}{|c|}{ Durchschnittliche Studiendauer im Tertiärbereich (in Jahren) } & \multicolumn{3}{|c|}{$\begin{array}{l}\text { Kumulative Ausgaben pro Studierenden über die durch- } \\
\text { schnittliche Studiendauer im Tertiärbereich }\end{array}$} \\
\hline & & $\begin{array}{c}\text { Tertiärbereich } \\
\text { insgesamt }\end{array}$ & Tertiärbereich B & $\begin{array}{c}\text { Tertiärbereich A } \\
\text { und weiterführende } \\
\text { Forschungs- } \\
\text { programme }\end{array}$ & $\begin{array}{c}\text { Tertiärbereich } \\
\text { insgesamt }\end{array}$ & Tertiärbereich B & \begin{tabular}{|c|} 
Tertiärbereich A \\
und weiterführende \\
Forschungs- \\
programme
\end{tabular} \\
\hline & & (1) & (2) & (3) & (4) & $(5)$ & (6) \\
\hline Australien & $\mathrm{CM}$ & 2.5 & 1.6 & 2.6 & 29665 & 12548 & 32226 \\
\hline Österreich ${ }^{2}$ & $\mathrm{AF}$ & 6.4 & 2.3 & 7.4 & 77248 & $x(4)$ & $x(4)$ \\
\hline Kanada* $^{*}$ & $\mathrm{CM}$ & $\mathrm{m}$ & $\mathrm{m}$ & $\mathrm{m}$ & $\mathrm{m}$ & $\mathrm{m}$ & $\mathrm{m}$ \\
\hline Dänemark ${ }^{2}$ & $\mathrm{AF}$ & 4.2 & 2.1 & 4.4 & 44654 & $x(4)$ & $x(4)$ \\
\hline Finnland & $\mathrm{CM}$ & 6.0 & a & 6.0 & 50760 & a & 50760 \\
\hline Frankreich $^{2}$ & $\mathrm{AF}$ & 4.7 & 2.8 & 5.3 & 36832 & 23410 & 40901 \\
\hline Deutschland $^{*}$ & $\mathrm{CM}$ & 4.9 & 2.4 & 6.0 & 50511 & 13408 & 67367 \\
\hline Griechenland $^{3}$ & $\mathrm{AF}$ & 5.2 & 3.0 & 7.3 & 22197 & 10419 & 33669 \\
\hline Ungarn $^{3}$ & $\mathrm{CM}$ & 4.1 & $\mathrm{~m}$ & 4.1 & 23735 & $x(4)$ & $x(4)$ \\
\hline Island & $\mathrm{CM}$ & 2.7 & 2.0 & 2.8 & $\mathrm{~m}$ & $\mathrm{~m}$ & $\mathrm{~m}$ \\
\hline Irland $^{*}$ & $\mathrm{CM}$ & 3.2 & 2.2 & 4.0 & 31341 & $x(4)$ & $\mathrm{x}(4)$ \\
\hline Italien $^{3 *}$ & $\mathrm{CM}$ & 5.5 & 3.3 & 5.6 & 41458 & 23371 & 42092 \\
\hline Korea $^{2} *$ & $\mathrm{CM}$ & 3.4 & 2.1 & 4.2 & 18371 & 7232 & 27904 \\
\hline Mexiko $^{2}$ & $\mathrm{AF}$ & 3.4 & $x(3)$ & 3.4 & 16390 & $x(4)$ & $x(4)$ \\
\hline Niederlande ${ }^{2,4}$ & $\mathrm{CM}$ & 3.9 & $\mathrm{x}(1)$ & $\mathrm{x}(1)$ & 47911 & $x(4)$ & $x(4)$ \\
\hline Norwegen & $\mathrm{CM}$ & $\mathrm{m}$ & $\mathrm{m}$ & $\mathrm{m}$ & $\mathrm{m}$ & $\mathrm{m}$ & $\mathrm{m}$ \\
\hline Polen $^{3}$ & $\mathrm{CM}$ & 3.7 & $\mathrm{~m}$ & 3.7 & 14395 & $\mathrm{~m}$ & 14395 \\
\hline Spanien $^{2}$ & $\mathrm{AF}$ & 4.6 & 1.5 & 4.7 & 25965 & 7611 & 27113 \\
\hline Schweden & $\mathrm{CM}$ & 4.6 & 2.6 & 4.7 & 65529 & $x(4)$ & $x(4)$ \\
\hline Schweiz ${ }^{2,3}$ & $\mathrm{CM}$ & 3.6 & 2.2 & 5.5 & 65225 & 29349 & 101334 \\
\hline Ver. Königreich* & $\mathrm{CM}$ & 3.5 & $\mathrm{x}(1)$ & $\mathrm{x}(1)$ & 33835 & $\mathrm{x}(4)$ & $x(4)$ \\
\hline Ländermittel & & 4.2 & 2.0 & 4.8 & 38668 & $\sim$ & $\sim$ \\
\hline
\end{tabular}

Hinweis: $\mathrm{x}$ bedeutet, dass die Daten in einer anderen Spalte enthalten sind, deren Referenz in runden Klammern nach dem ' $\mathrm{x}$ ' angegeben ist. So bedeutet $\mathrm{z}$. B.x(2), dass die Daten in Spalte 2 enthalten sind.

1. Zur Schätzung der Studiendauer im Tertiärbereich wurde entweder die Verkettungsmethode (CM) oder eine Näherungsformel (AF) verwendet.

2. Die Angaben zur Dauer tertiärer Studiengänge stammen aus einer 1997 zum akademischen Jahr 1995 durchgeführten speziellen Erhebung. Einteilung der Studiengänge gemäß ISCED-76.

3. Nur öffentliche Bildungseinrichtungen.

4. Nur öffentliche und staatlich-subventionierte Bildungseinrichtungen.

* Hinweise s. Anhang 3 (www.oecd.org/els/education/eag2002).

Quelle: OECD. 


\section{AUSGABEN FÜR BILDUNGSEINRICHTUNGEN IM VERHÄLTNIS ZUM BRUTTOINLANDSPRODUKT}

- Die OECD-Länder geben 5,8 Prozent der Gesamtsumme ihrer Bruttoinlandsprodukte für Bildungseinrichtungen aus.

- In 14 von 18 OECD-Ländern erhöhten sich zwischen 1995 und 1999 die privaten und öffentlichen Ausgaben für Bildungseinrichtungen um mehr als 5 Prozent. Im Gegensatz zum Anfang der neunziger Jahre blieb jedoch der Anstieg der Ausgaben für Bildungseinrichtungen tendenziell hinter dem Wachstum des Volkseinkommens zurück.

- Zwei Drittel aller Ausgaben für Bildungseinrichtungen bzw. 3,7 Prozent der Bruttoinlandsprodukte aller OECD-Länder insgesamt gehen in den Primar-, Sekundar- und post-sekundären, nicht-tertiären Bildungsbereich, obwohl Kanada, Korea und die Vereinigten Staaten mehr als 2 Prozent ihres BIP im Tertiärbereich investieren. 


\section{Abbildung B2.1.}

Ausgaben für Bildungseinrichtungen als Prozentsatz des BIP $(1995,1999)$

Ausgaben für Bildungseinrichtungen aus öffentlichen und privaten Quellen, nach Bildungsbereichen, Mittelherkunft und Jahr
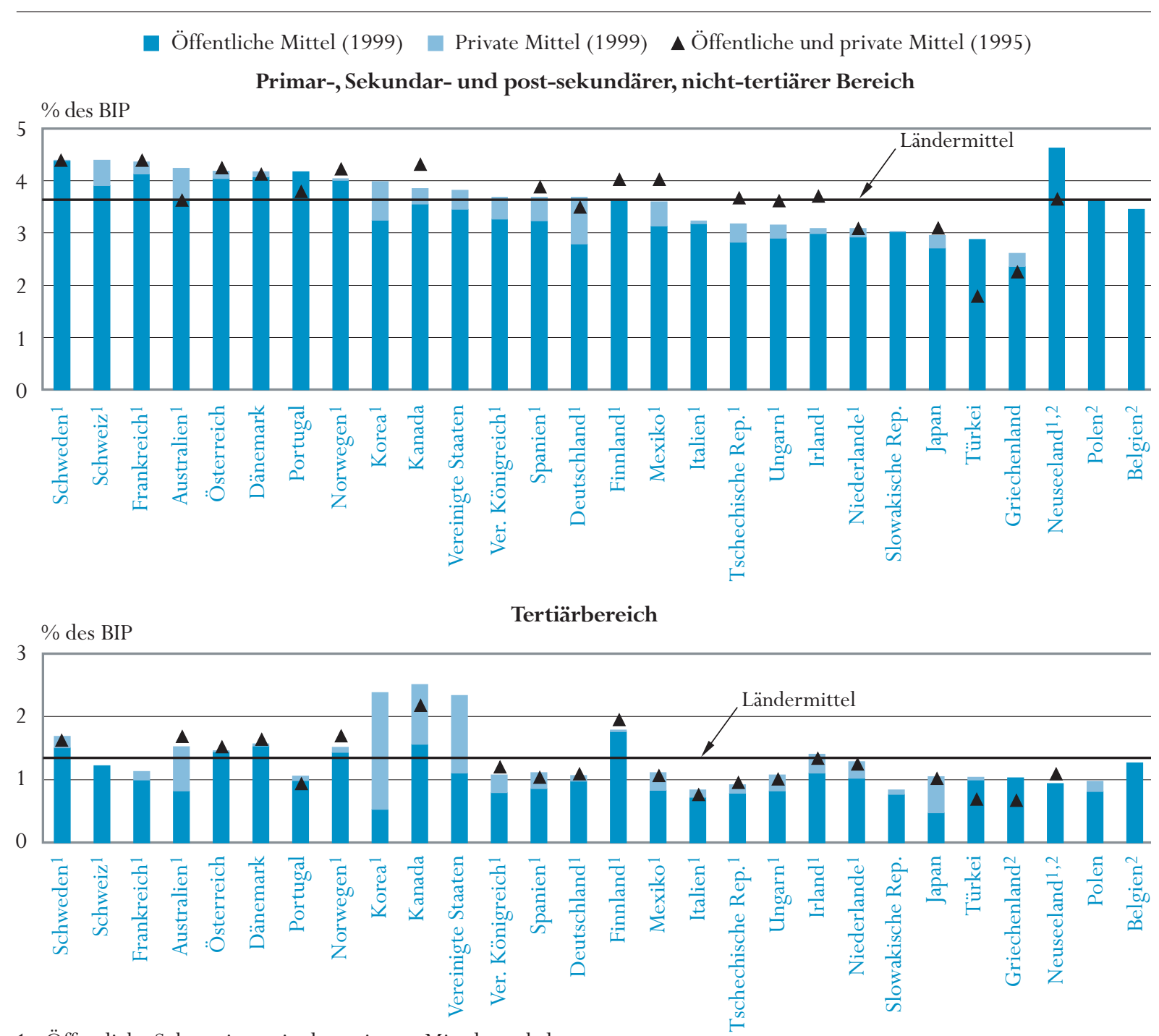

1. Öffentliche Subventionen in den privaten Mitteln enthalten.

2. Nur öffentliche Ausgaben.

Anordnung der Länder in absteigender Reihenfolge der Gesamtausgaben für Bildungseinrichtungen im Primar-, Sekundar- und post-sekundären, nicht-tertiären Bereich aus öffentlichen und privaten Quellen. Länder, die nur die öffentlichen Ausgaben angeben, sind am Ende des Balkendiagramms aufgeführt.

Quelle: OECD. Tabelle B2.1b. Hinweise s. Anhang 3 (www.oecd.org/els/education/eag2002). 


\section{Politischer Hintergrund}

Ausgaben für Bildung stellen eine Investition dar, die dazu beitragen kann, das Wirtschaftswachstum zu stärken, die Produktivität zu steigern, die persönliche und gesellschaftliche Entwicklung zu fördern sowie soziale Ungleichheiten zu verringern. Die Entscheidung, welcher Anteil der insgesamt zur Verfügung stehenden Finanzmittel für das Bildungswesen bereitgestellt werden soll, ist in jedem OECD-Land von zentraler Bedeutung. An dieser Entscheidung sind Regierungen und Unternehmensleitungen ebenso wie der einzelne Schüler/ Studierende und seine Familie gemeinsam beteiligt. Falls die persönlichen und gesellschaftlichen Erträge dieser Investition hoch genug sind, besteht ein Anreiz, die Bildungsbeteiligung zu erhöhen und die Gesamtinvestitionen im Bildungsbereich zu steigern.

Bei der Bewertung der Gesamtaufwendungen für die Bildung müssen die Regierungen sich mit Forderungen nach Ausgabenerhöhungen z.B. im Bereich der Lehrergehälter oder der Bildungseinrichtungen auseinandersetzen. Dabei kann der vorliegende Indikator als Bezugspunkt dienen, denn er weist auf, wie sich der Umfang der Bildungsausgaben sowohl gemessen am Volksvermögen als auch in absoluten Zahlen im Laufe der Zeit in den einzelnen OECD-Ländern entwickelt hat.

\section{Ergebnisse und Erläuterungen}

\section{Was dieser Indikator aufzeigt, und was nicht}

Dieser Indikator erfasst Ausgaben für Schulen, Hochschulen und andere öffentliche wie private Einrichtungen, die selbst Bildungsangebote bereitstellen oder deren Bereitstellung unterstützen. Ausgaben für Bildungseinrichtungen beschränken sich nicht allein auf Ausgaben für Unterrichtszwecke, sondern beinhalten auch öffentliche und private Ausgaben für zusätzliche Leistungen zur Unterstützung von Schülern/Studierenden und deren Familien, soweit diese durch Bildungseinrichtungen erbracht werden. Im Tertiärbereich können auch die Ausgaben für Forschung und Entwicklung einen erheblichen Teil ausmachen. Sie sind in diesem Indikator enthalten, soweit die Forschungstätigkeit von Bildungseinrichtungen erbracht wird.

Nicht alle Ausgaben für Sach- und Dienstleistungen im Bildungsbereich finden in Bildungseinrichtungen statt. So erwerben beispielsweise Familien Schulbücher und sonstiges Unterrichtsmaterial im Handel oder lassen ihren Kindern Privatunterricht außerhalb von Bildungseinrichtungen erteilen. Im Tertiärbereich können die Lebenshaltungskosten der Studierenden und Verdiensteinbußen einen erheblichen Teil der Bildungskosten ausmachen. Solche Ausgaben außerhalb der Bildungseinrichtungen sind bei diesem Indikator nicht berücksichtigt, selbst wenn sie durch öffentliche Mittel subventioniert werden. Öffentliche Subventionen für Bildungsausgaben außerhalb von Bildungseinrichtungen werden in den Indikatoren B4 und B5 behandelt.
Dieser Indikator misst den Anteil des Volksvermögens, der in Bildungseinrichtungen fließst.

Der Indikator liefert ferner eine vergleichende Übersicht der im Laufe der Zeit eingetretenen Änderungen bei den Bildungsausgaben.

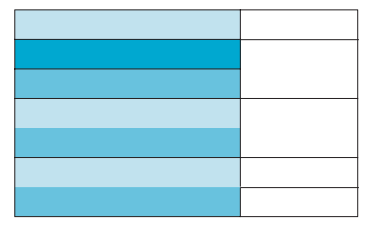

Erfasste Daten

(Erklärungen s. S. 160) 


\author{
Insgesamt geben \\ die OECD-Länder \\ 5,8 prozent der \\ Gesamtsumme ihrer \\ Bruttoinlandsprodukte \\ für Bildungs- \\ einrichtungen aus.
}

Die von einem Land für die Bildung bereitgestellten Ressourcen hängen von mehreren, sich gegenseitig beeinflussenden Angebots-und Nachfragefaktoren ab.

In 14 von 18 OECDLändern sind die öffentlichen und privaten Ausgaben für Bildungseinrichtungen zwischen 1995 und 1999 um mehr als 5 Prozent gestiegen,....

\section{Gesamtausgaben im Verhältnis zum BIP}

Alle OECD-Länder investieren einen wesentlichen Teil ihrer nationalen Ressourcen in Bildung. Unter Berücksichtigung sowohl der öffentlichen als auch der privaten Finanzquellen geben die OECD-Länder zusammen 5,8 Prozent der Gesamtsumme ihrer Bruttoinlandsprodukte für Bildungseinrichtungen aus. Angesichts der derzeit herrschenden Finanzknappheit der öffentlichen Haushalte wird ein so großer Ausgabenposten auf der Suche nach Möglichkeiten zur Reduzierung oder Begrenzung des Ausgabenwachstums seitens der Regierungen einer kritischen Prüfung unterzogen.

Die höchsten Ausgaben für Bildungseinrichtungen verzeichnet Korea, wo sich die öffentlichen und privaten Ausgaben für Bildungseinrichtungen auf 6,8 Prozent des BIP belaufen. Es folgen Dänemark, Kanada, Norwegen und Schweden mit einem Anteil von mehr als 6,6 Prozent. Acht von 28 OECD-Ländern geben jedoch weniger als 5 Prozent ihres BIP für Bildungseinrichtungen aus. In Griechenland, der Slowakischen Republik und der Türkei liegt dieser Anteil sogar lediglich zwischen 3,9 und 4,4 Prozent (Tabelle B2.1a).

Die relative Stellung einzelner OECD-Länder bei diesem Indikator wird von einer Vielzahl von Faktoren beeinflusst. Möglicherweise weisen OECDLänder mit hohen Bildungsausgaben eine höhere Bildungsbeteiligung auf, während Länder mit niedrigen Bildungsausgaben den Zugang zu den höheren Bildungsbereichen beschränken oder die Bildungsinhalte auf besonders effiziente Weise vermitteln. Die Verteilung der Schüler und Studierenden auf die verschiedenen Bildungsbereiche und Fächer kann sich ebenso unterscheiden wie die Dauer der Bildungs- und Studiengänge sowie Umfang und Organisation der mit dem Bildungsbereich verbundenen Forschungsaktivitäten. Schließlich bedeuten große Unterschiede zwischen dem BIP der einzelnen OECD-Länder, dass selbst wenn der in Bildung investierte Anteil des BIP ähnlich hoch ist, die Unterschiede bei den auf jeden Schüler/Studierenden entfallenden absoluten Beträgen sehr groß sein können (s. Indikator B1).

\section{Veränderungen der Gesamtausgaben für Bildung zwischen 1995 und 1999}

In 14 der 18 OECD-Länder, für die vergleichbare Trendzahlen zur Verfügung stehen, sind die öffentlichen und privaten Investitionen in Bildung zwischen 1995 und 1999 real um mehr als 5 Prozent gestiegen. In Australien, Irland und Portugal sind die Ausgaben für Bildungseinrichtungen um 20 bis 30 Prozent gestiegen, in Griechenland sogar um mehr als 40 Prozent. Ein ähnlicher Trend zeichnet sich ab, wenn nur die öffentlichen Ausgaben betrachtet werden: die direkten öffentlichen Ausgaben für Bildungseinrichtungen sowie die öffentlichen Subventionen an private Haushalte, die Bildungseinrichtungen zuzurechnen sind, stiegen zwischen 1995 und 1999 in 19 von 23 OECD-Ländern um mehr als 5 Prozent. Griechenland, Neuseeland und die Türkei, für die keine Zahlen über die privaten Ausgaben vorliegen, verzeichneten einen erheblichen Anstieg der öffentlichen Ausgaben für Bildungseinrichtungen (Tabelle B2.2). 
Obwohl die Ausgaben für Bildungseinrichtungen zwischen 1995 und 1999 in absoluten Zahlen weiter stiegen, fiel diese Zunahme tendenziell hinter das BIP-Wachstum zurück. In rund zwei Drittel der OECD-Länder ist der Anteil der Ausgaben für Bildungseinrichtungen am BIP zurückgegangen. Besonders erwähnenswert sind Finnland, Irland, Mexiko, Norwegen und die Tschechische Republik, wo der Anteil der Bildungsausgaben am BIP um mehr als 0,35 Prozentpunkte zurückging (Tabelle B2.1a).

Während das starkeWachstum des BIP in Irland erhebliche Ausgabensteigerungen für Bildungseinrichtungen beinhaltet, wenn man die Bildungsausgaben im Verhältnis zum BIP betrachtet, hat in Mexiko und der Tschechischen Republik der Bildungsbereich nicht besonders vom Wachstum des BIP profitiert. Beide Länder gehörten bereits 1995 zu den OECD-Ländern mit geringeren Bildungsausgaben gemessen am BIP und sind seither weiter zurückgefallen (Tabelle B2.1a).

\section{Ausgaben für Bildungseinrichtungen aufgegliedert nach Bildungsbereichen}

Insgesamt hohe Bildungsausgaben bedeuten nicht zwangsläufig, dass die Ausgaben in allen Bildungsbereichen hoch sind. Die Unterschiede in den Ausgaben für Bildungseinrichtungen zeigen sich am deutlichsten im Elementarbereich. Hier reicht die Spanne von unter 0,2 Prozent des BIP in Australien, Irland und Korea bis zu über 0,7 Prozent in Dänemark, Frankreich, Norwegen und Ungarn (Tabelle B2.1c). Unterschiede im Elementarbereich lassen sich zu einem großen Teil durch die unterschiedlichen Teilnahmequoten der jüngeren Kinder erklären (s. Indikator C1).

Die Erziehung bereits im Vorschulalter ist von wesentlicher Bedeutung für die Gewährleistung eines gerechten Zugangs zum Lernangebot später in der Schule als auch für das lebenslange Lernen. Ein hochwertiges Angebot im Bereich der Erziehung und Betreuung im Vorschulalter wird jedoch nicht nur an den in diesem Indikator behandelten Bildungseinrichtungen geboten. Schlussfolgerungen über den Zugang zu Erziehung und Betreuung im Vorschulalter und deren Qualität sollten daher nur mit Vorsicht gezogen werden.

Aufgrund der in den OECD-Ländern weitgehend universellen Bildungsbeteiligung im Primar- und Sekundarbereich I und der hohen Bildungsbeteiligung im Sekundarbereich II (s. Indikatoren C1 und C2) entfällt der größte Teil der Investitionen auf die Bildungseinrichtungen in diesen Bildungsbereichen, nämlich 3,7 Prozent der Gesamtsumme der Bruttoinlandsprodukte aller OECD-Länder (Abbildung B2.1). Gleichzeitig sorgen die deutlich höheren Bildungsausgaben pro Schüler/Studierenden im Sekundarbereich II und im Tertiärbereich dafür, dass die Gesamtausgaben für diese Bildungsbereiche höher sind als die reinen Schüler-/Studierendenzahlen vermuten lassen. Mehr als ein Viertel der Ausgaben der OECD insgesamt für Bildungseinrichtungen entfallen auf Einrichtungen des Tertiärbereichs.
... tendenziell blieb

jedoch der Anstieg

der Bildungsausgaben

hinter dem Wachstum

der Volkseinkommen

zurück.
Es gibt zwischen

den einzelnen

Ländern beträchtliche

Unterschiede

hinsichtlich ihrer

Investitionen für

Bildungseinrichtungen

des Elementarbereichs.

Zwei Drittel der

Investitionen für

Bildungseinrichtungen

fließen in den Primar-,

Sekundar- und post-

sekundären, nicht-

tertiären Bereich. 


\section{Abbildung B2.2.}

Veränderung der Ausgaben für Bildungseinrichtungen (1995, 1999)

Index der Veränderung der direkten Ausgaben für Bildungseinrichtungen zwischen 1995 und 1999 (1995 = 100)

Veränderung der gesamten direkten Ausgaben für Bildungseinrichtungen — Veränderung des BIP zu konstanten Preisen

Primar-, Sekundar- und post-sekundärer, nicht-tertiärer Bereich

.

Index der Veränderung $(1995=100)$

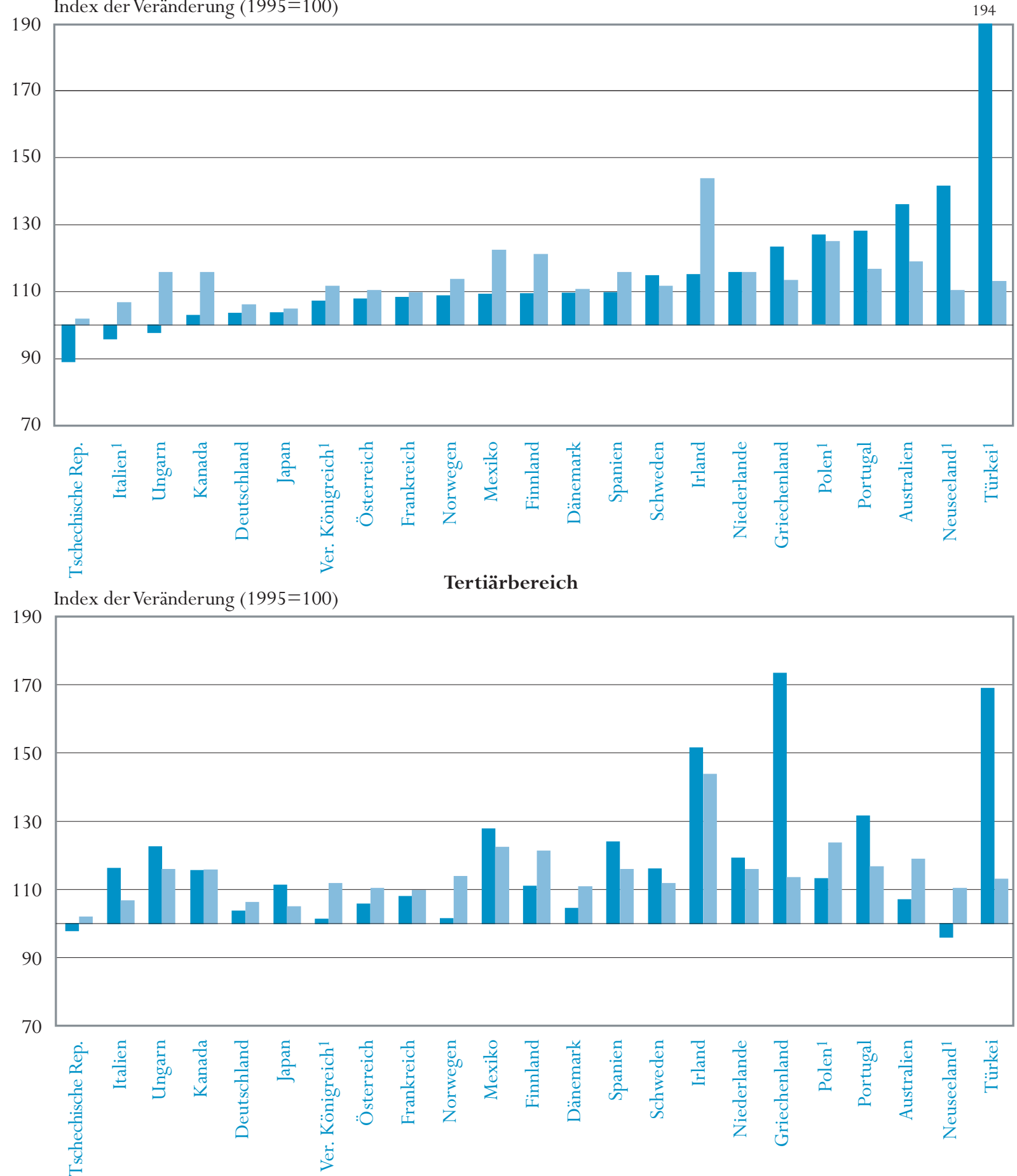

1. Nur öffentliche Ausgaben.

Anordnung der Länder in aufsteigender Reihenfolge derVeränderung bei den Ausgaben für Bildungeinrichtungen im Primar-, Sekundar- und post-sekundären, nicht-tertiären Bereich zwischen 1995 und 1999.

Quelle: OECD. Tabelle B2.2 und Anhang 2. Hinweise s. Anhang 3 (www.oecd.org/els/education/eag2002). 
Kanada, Korea und die Vereinigten Staaten investieren jeweils 2,5, 2,4 bzw. 2,3 Prozent ihres BIP in tertiäre Bildungseinrichtungen (Abbildung B2.1). Dies entspricht mehr als einem Drittel ihrer Gesamtausgaben für Bildungseinrichtungen. Dänemark, Finnland, und Schweden haben ebenfalls hohe Bildungsausgaben, wobei 1,6 Prozent des BIP und mehr in Einrichtungen des tertiären Bildungsbereichs investiert werden. Andererseits liegt der Anteil des BIP, der in Frankreich, Portugal und der Schweiz in tertiäre Bildungseinrichtungen investiert wird, zwar unter dem OECD-Durchschnitt, gleichzeitig aber gehören diese Länder zu den OECD-Ländern, bei denen die Investitionen in den Primar-, Sekundar- und post-sekundären, nicht-tertiären Bereich den höchsten Anteil am BIP ausmachen. Trotz eines niedrigen BIPAnteils der Ausgaben für tertiäre Einrichtungen gehört die Schweiz auf Grund der verhältnismäßig niedrigen Bildungsbeteiligung im tertiären Bereich und einem hohen BIP zu den Ländern mit den höchsten Ausgaben pro Studierenden (Tabellen B2.1b und B1.3).

Die einzelnen Länder unterscheiden sich hinsichtlich der Bildungsbereiche, in denen die Ausgaben gesteigert wurden. Deutschland, Finnland, Frankreich, die Niederlande, Österreich, Portugal, Schweden und die Türkei, also OECDLänder mit einem vergleichsweise starken Anstieg der absoluten Ausgaben für Bildungseinrichtungen zwischen 1995 und 1999, haben die zusätzlichen Mittel zu ähnlichen Anteilen im Primar-, Sekundar- und post-sekundären, nicht-tertiären Bereich sowie im Tertiärbereich investiert (Abbildung B2.2). Australien, Dänemark, Neuseeland, Norwegen und Polen haben zwischen 1995 und 1999 einen Großteil ihrer zusätzlichen Mittel in den Primar-, Sekundarund post-sekundären, nicht-tertiären Bereich investiert. Dagegen erhöhten sich die Ausgaben für tertiäre Bildungseinrichtungen in Griechenland, Irland, Italien, Japan, Kanada, Mexiko, Spanien und Ungarn zwischen 1995 und 1999 um mehr als 10 Prozent, während die Ausgabensteigerung für die vorgelagerten Bildungsbereiche wesentlich geringer ausfiel. In Italien und Ungarn steht einer deutlichen Steigerung der Ausgaben für tertiäre Bildungseinrichtungen eine entsprechende Senkung der Ausgaben beim Primar-, Sekundar- und postsekundären, nicht-tertiären Bereich gegenüber (Abb. B2.2).

\section{Wichtige Einflussgrößen der nationalen Bildungsausgaben}

Die von einem Land für Bildung bereitgestellten Finanzmittel hängen von mehreren, sich gegenseitig beeinflussenden Angebots- und Nachfragefaktoren ab, wie z.B. der demographischen Bevölkerungsstruktur, der Bildungsbeteiligung, dem Pro-Kopf-Einkommen, dem Niveau der Lehrergehälter sowie der Unterrichtsorganisation und der Art und Weise der Vermittlung von Lerninhalten.

Der Anteil junger Menschen im Schulalter (s. Indikator A1 in Bildung auf einen Blick 2001) ist maßgebend für die potenzielle Nachfrage nach schulischer und beruflicher Grundausbildung in einem Land. Je mehr junge Menschen es gibt, desto größer ist die potentielle Nachfrage nach Bildungsleistungen. Bei OECD-
Kanada, Korea und die Vereinigten Staten von Amerika investieren jeweils mehr als 2 Prozent ihres BIP in den Tertiärbereich.

Während einige OECD-Länder die Ausgabensteigerungen gleichmäfsig auf alle Bildungsbereiche verteilten, erhöhten andere die Ausgaben schwerpunktmäfig in bestimmten Bildungsbereichen.

Je mehr junge Menschen es gibt, desto größser ist die potenzielle Nachfrage nach Bildungsleistungen. 

Bildungsbeteiligung, desto mehr finanzielle Mittel sind erforderlich.

Auch die Dauer der Ausbildung beeinflusst die Höhe der Bildungsausgaben.

In einigen $O E C D$ -

Ländern wird der Einfluss der demographischen Faktoren auf die
Jehöher die Obwohl die Länder im allgemeinen nur wenig Einfluss darauf haben, wie hoch

Ländern mit einem vergleichbaren Volkseinkommen wird ein Land mit einem relativ großen Bevölkerungsanteil junger Menschen einen größeren Prozentsatz seines BIP für die Bildung ausgeben müssen, damit jeder junge Mensch in dem entsprechenden Land die Chance hat, im gleichen Umfang an Bildung teilzunehmen wie junge Menschen in anderen OECD-Ländern. Ist dagegen der Bevölkerungsanteil junger Menschen verhältnismäßig klein, so wird das betreffende Land nur einen geringeren Anteil seines Wohlstands für die Bildung einsetzen müssen, um ähnliche Bildungsergebnisse zu erzielen.

der Anteil junger Menschen an Ihrer Bevölkerung ist, so ist doch der Anteil von Schülern/Studierenden, die in den verschiedenen Bildungsbereichen unterrichtet und ausgebildet werden, ein zentrales politisches Thema. Unterschiede in der Bildungsbeteiligung in den einzelnen OECD-Ländern sind Ausdruck unterschiedlicher Nachfrage nach Bildung - vom Elementar- bis zum Tertiärbereich - sowie unterschiedlicher Angebote an Bildungsgängen in allen Bildungsbereichen. Indikator C1 zeigt, dass die zu erwartende Ausbildungsdauer eines 5-jährigen Kindes in den einzelnen OECD-Ländern zwischen 10 und 21 Jahren betragen kann. Große Unterschiede ergeben sich auch für die zu erwartende Studiendauer im Tertiärbereich. Sie reicht von einem Jahr in Mexiko bis zu mehr als 4 Jahren in Finnland.

Unterschiede in der Dauer der Ausbildung spiegeln sich in einer unterschiedlichen Bildungsbeteiligung wider, und diese hat wiederum Einfluss auf die Höhe der Bildungsausgaben. Abbildung B2.3 zeigt, wie sich die Ausgaben für Bildungseinrichtungen in Prozent des BIP bei sonst gleichen Bedingungen verändern würden, wenn die Bildungsbeteiligung in allen OECD-Ländern gleich wäre. Generell geben OECD-Länder mit einer überdurchschnittlichen Bildungsbeteiligung, wie beispielsweise Australien, Finnland, Norwegen und Schweden einen höheren Anteil ihres BIP für Bildung aus, während geringere Bildungsausgaben in Ländern wie Mexiko, der Tschechischen Republik, der Türkei und Ungarn zum Teil durch eine unterdurchschnittliche Bildungsbeteiligung erklärt werden können.

Wäre die Bildungsbeteiligung in allen OECD-Ländern gleich, könnte man davon ausgehen, dass die Ausgaben in Prozent des BIP in Mexiko und der Türkei um mehr als 1,8 Prozentpunkte des BIP höher wären, während sie in Finnland, Japan, Korea und Norwegen um 0,5 Prozentpunkte und mehr niedriger wären, sofern die Bildungsausgaben pro Schüler/Studierenden in jedem dieser Länder gleich blieben (Abbildung B2.3).

Die Auswirkungen der Bildungsbeteiligung auf die Bildungsausgaben zeigen sich am deutlichsten im Tertiärbereich, wo es sowohl bei der Bildungsbeteiligung (s. Indikator C1) als auch bei den Bildungsausgaben pro Studierenden (Indikator B1) große Unterschiede zwischen den einzelnen OECD-Ländern gibt. Entspräche die Bildungsbeteiligung im Tertiärbereich in Japan und Korea 


\section{Abbildung B2.3.}

Einfluss der Bildungsbeteiligung auf die Ausgaben für Bildungseinrichtungen als Anteil am BIP (1999)

A. Geschätzter Anstieg/Rückgang der Ausgaben für Bildungseinrichtungen als Anteil am BIP (in \%), wenn die Bildungsbeteiligungsmuster in jedem Land (alle

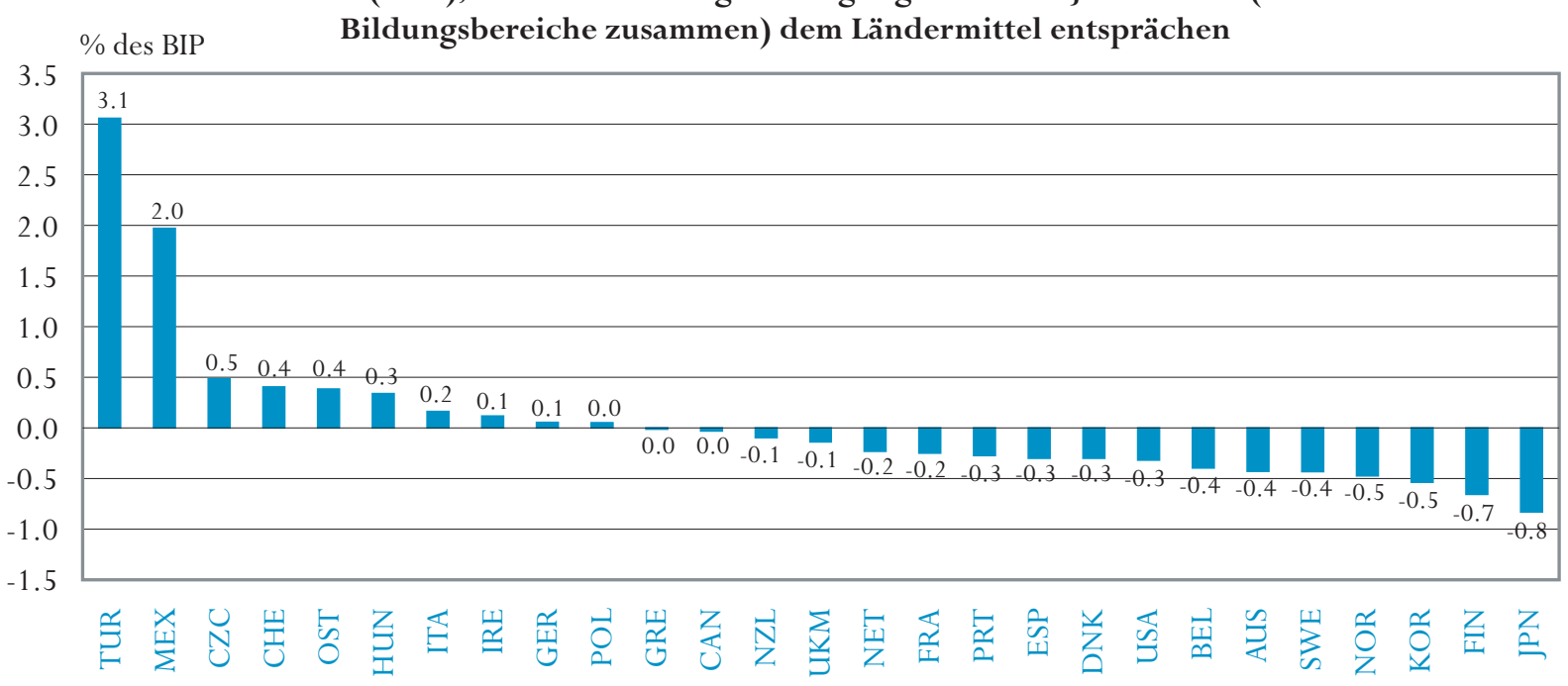

B. Geschätzter Anstieg/Rückgang der Ausgaben für Bildungseinrichtungen als Anteil am BIP (in \%), wenn die Bildungsbeteiligungsmuster im Primar- und

$\%$ des BIP

Sekundarbereich in jedem Land dem Ländermittel entsprächen

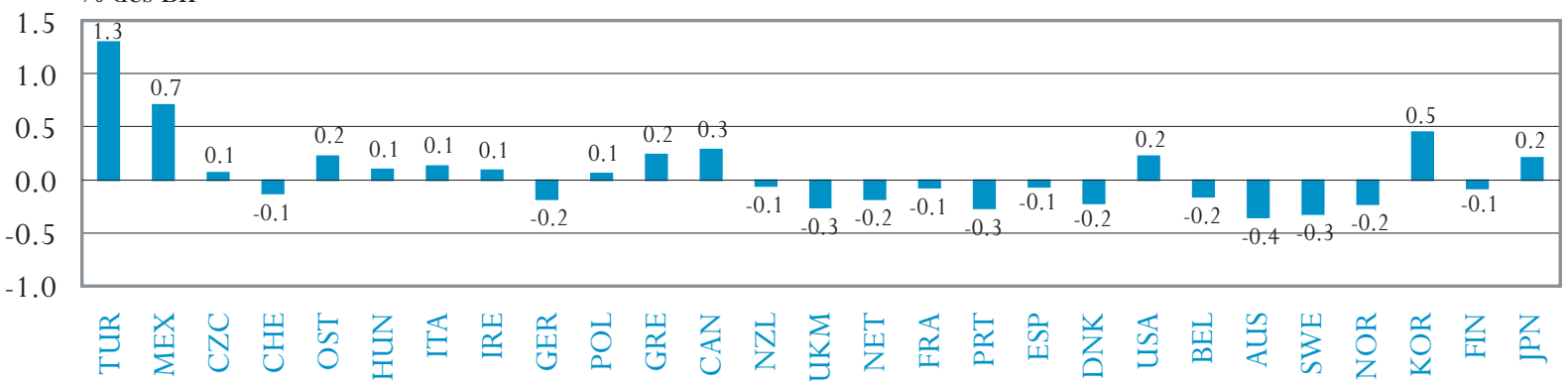

C. Geschätzter Anstieg/Rückgang der Ausgaben für Bildungseinrichtungen als Anteil am BIP (in \%), wenn die Bildungsbeteiligungsmuster im Tertiärbereich

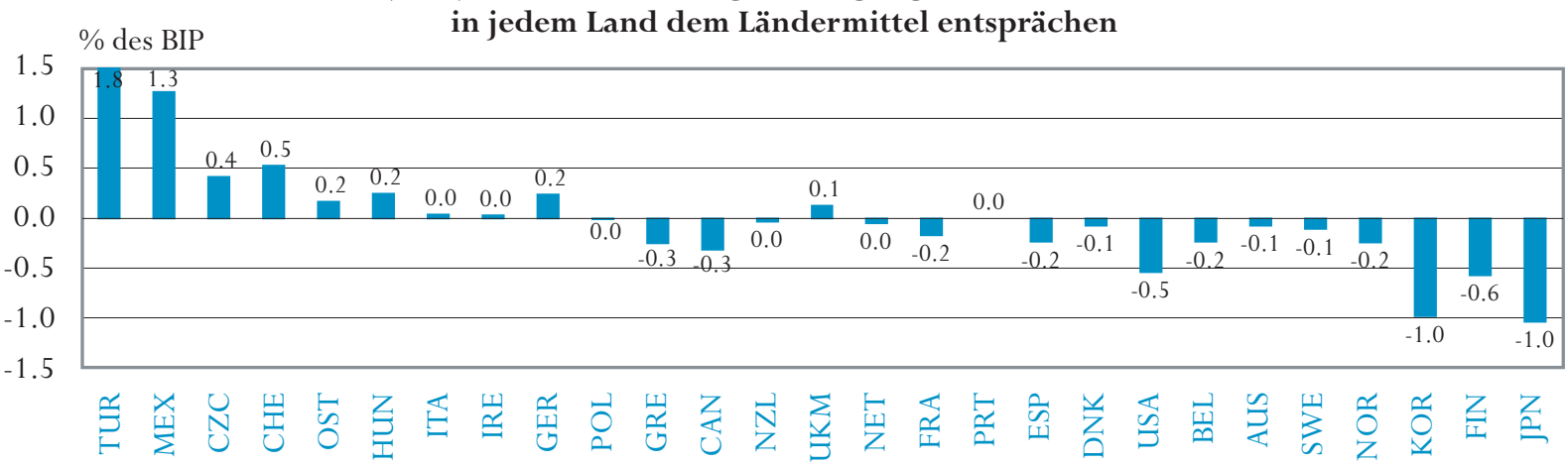

Hinweis: Auflistung der verwendeten Ländercodes und der dazugehörenden Ländernamen s. Hinweise für den Leser.

Anordnung der Länder in absteigender Reihenfolge des geschätzten Anstiegs / Rückgangs der Ausgaben als Anteil am BIP (in \%), wenn die Bildungsbeteiligungsmuster in jedem Land (alle Bildungsbereiche zusammen) dem Ländermittel entsprächen.

Quelle: OECD. Hinweise s. Anhang 3 (www.oecd.org/els/education/eag2002). 
Bildungsausgaben durch eine sich ändernde Bildungsbeteiligung aufgehoben.

Die Daten beziehen sich auf das Haushaltsjahr 1999 und beruhen auf der von der $O E C D$ im Jahre 2001 durchgeführten UOE-Datenerhebung zur Bildungsstatistik (Einzelheiten s. Anhang 3). dem OECD-Durchschnitt, wäre zu erwarten, dass die Ausgaben für tertiäre Bildungseinrichtungen in Prozent des BIP um 1,0 Prozentpunkte niedriger ausfielen, während in Finnland und den Vereinigten Staaten dieser Unterschied 0,6 bzw. 0,5 Prozentpunkte betragen würde (Abbildung B2.3). Am anderen Ende des Spektrums liegen Mexiko und dieTürkei, bei denen zu erwarten wäre, dass die Ausgaben für tertiäre Bildungseinrichtungen in Prozent des BIP um 1,3 bzw. 1,8 Prozentpunkte höher liegen würden, wenn ihre Bildungsbeteiligung dem OECD-Durchschnitt entspräche.

\section{Definitionen und angewandte Methodik}

Die Ausgaben für die in diesem Indikator erfassten Bildungseinrichtungen umfassen sowohl Ausgaben für unterrichterteilende Bildungseinrichtungen als auch für solche, die keinen Unterricht erteilen. Unterrichterteilende Bildungseinrichtungen sind Bildungseinrichtungen, die einzelnen Personen in organisierter Form direkten Unterricht in der Gruppe oder per Fernunterricht erteilen. Nicht enthalten sind hierin Unternehmen oder sonstige Einrichtungen, die kurze Ausbildungs- oder Lehrkurse in Form von Einzelunterricht anbieten. Nicht-unterrichterteilende Einrichtungen erbringen anderen Bildungseinrichtungen gegenüber administrative, beratende oder fachliche Dienstleistungen, nehmen jedoch selbst keine Schüler/Studierenden auf. Beispiele hierfür sind nationale, bundesstaatliche und regionale Bildungsministerien oder -abteilungen, sonstige auf den verschiedenen staatlichen Ebenen für Bildungsfragen zuständige Organe oder entsprechende private Einrichtungen sowie Organisationen, die solche bildungsbezogenen Dienstleistungen wie Berufs- oder psychologische Beratung, Vermittlung von Stellen und Praktika, Durchführung von Tests, Finanzhilfe für Schüler/Studierende, Lehrplanentwicklung, Bildungsforschung, Betrieb und Instandhaltung von Gebäuden, Transport von Schülern/Studierenden, sowie Unterkunft und Verpflegung für Schüler/Studierende anbieten.

Diese breit angelegte Definition der Einrichtungen gewährleistet, dass Ausgaben für Dienstleistungen, die in einigen OECD-Ländern von Schulen und Hochschulen, in anderen Ländern aber von nicht-schulischen Stellen erbracht werden, auf vergleichbarer Basis erfasst werden können.

Die Unterscheidung nach der Herkunft der Mittel bezieht sich auf die ursprüngliche Finanzierungsquelle. Anschließende Transferzahlungen vom öffentlichen an den privaten Sektor oder umgekehrt sind darin nicht berücksichtigt. Aus diesem Grund fallen Unterstützungsleistungen an private Haushalte und andere Empfänger, wie z.B. Unterstützungszahlungen für Studiengebühren und andere Zahlungen an Bildungseinrichtungen bei diesem Indikator unter die Kategorie öffentliche Ausgaben. Zahlungen der privaten Haushalte und anderer privater Stellen an Bildungseinrichtungen beinhalten Studien- und sonstige Gebühren, abzüglich der öffentlichen Subventionen. Eine detaillierte Darstellung der öffentlichen Subventionen findet sich in Indikator B5. 
Aus den Tabellen B2.1a, B2.1b und B2.2 sind die Ausgaben für Bildungseinrichtungen im Haushaltsjahr 1995 zu ersehen. Die Daten für die Ausgaben des Jahres 1995 wurden im Rahmen einer speziellen Erhebung im Jahre 2000 ermittelt, in der die Daten für 1995 entsprechend den Definitionen und den Methoden der 1999 durchgeführten UOE-Datenerhebung angepasst wurden.

Abbildung B2.2 und Tabelle B2.2 enthalten einen Index der Veränderung der Ausgaben für Bildungseinrichtungen und des BIP zwischen den Jahren 1995 und 1999. Alle Ausgaben ebenso wie die Angaben für das BIP von 1995 wurden mittels des BIP-Deflators an das Preisniveau von 1999 angepasst.

Im Interesse der zeitlichen Vergleichbarkeit bezieht sich das Ländermittel nur auf diejenigen OECD-Länder, für die Zahlen für alle erfassten Referenzjahre vorliegen.

Es ist zu beachten, dass die in früheren Ausgaben dieser Veröffentlichung aufgeführten Zahlen und Daten möglicherweise nicht immer mit denen der Ausgabe 2002 vergleichbar sind, da sich Änderungen in den Definitionen und Erhebungsbereichen ergeben haben. Diese wurden aufgrund der OECD Expenditure Comparability Study vorgenommen (Einzelheiten zu den Änderungen s. Anhang 3 unter www.oecd.org/els/education/eag2002).
Die Daten für das Haushaltsjahr 1995 beruhen auf einer speziellen Erhebung, die im Jahre 2000 in den OECD-Ländern durchgeführt wurde. Die Daten für 1995 sind in Preisen von 1999 dargestellt. 
Tabelle B2.1a.

Ausgaben für Bildungseinrichtungen als Prozentsatz des BIP $(1995,1999)$ Ausgaben für Bildungseinrichtungen aus öffentlichen und privaten Quellen für alle Bildungsbereiche, nach Herkunft der Mittel und Jahr

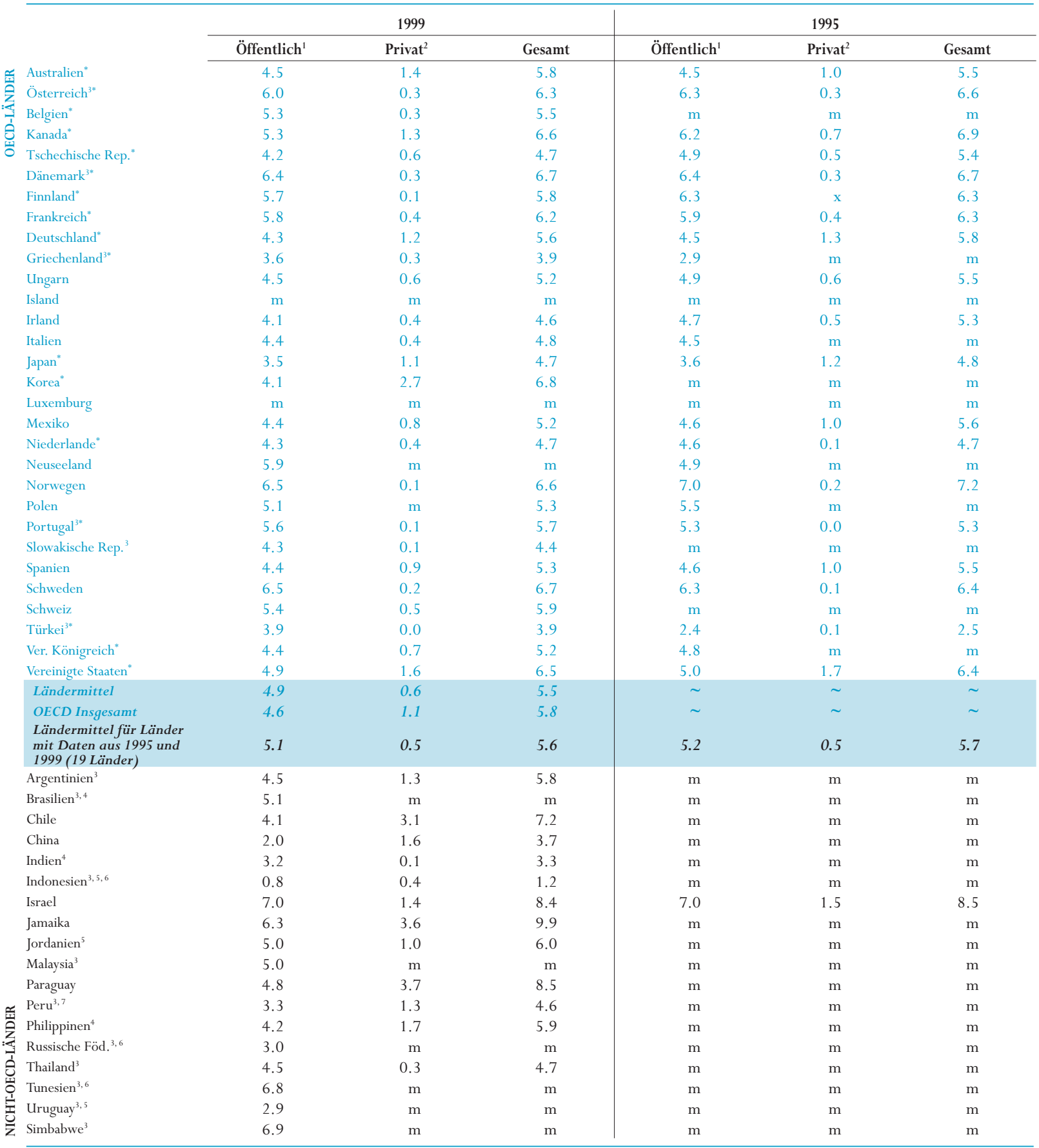

1. Einschließlich öffentlicher Subventionen an private Haushalte, die Bildungseinrichtungen zuzuordnen sind und direkter Ausgaben für Bildungseinrichtungen aus internationalen Quellen.

2. Abzüglich öffentlicher Subventionen, die Bildungseinrichtungen zuzuordnen sind.

3. Öffentliche Subventionen an private Haushalte nicht in den öffentlichen, sondern in den privaten Ausgaben enthalten.

4. Referenzjahr 1998

5. Direkte Ausgaben für Bildungseinrichtungen aus internationalen Mitteln belaufen sich auf mehr als 1,5 Prozent der gesamten öffentlichen Ausgaben für Bildungseinrichtungen.

6. Referenzjahr 2000

7. Ohne post-sekundären, nicht-tertiären Bereich.

* Hinweise s. Anhang 3 (www.oecd.org/els/education/eag2002).

Quelle: OECD. 
Tabelle B2.1b.

Ausgaben für Bildungseinrichtungen als Prozentsatz des BIP $(1995,1999)$

Ausgaben für Bildungseinrichtugen aus öffentlichen und privaten Quellen, nach Bildungsbereichen, Herkunft der Mittel und Jahr.

\begin{tabular}{|c|c|c|c|c|c|c|c|c|}
\hline & \multicolumn{4}{|c|}{$\begin{array}{l}\text { Primar-, Sekundar- und post-sekundärer, } \\
\text { nicht-tertiärer Bereich }\end{array}$} & \multicolumn{4}{|c|}{ Tertiärbereich } \\
\hline & \multicolumn{3}{|c|}{1999} & \multirow{2}{*}{$\frac{1995}{\text { Gesamt }}$} & \multicolumn{3}{|c|}{1999} & \multirow{2}{*}{$\begin{array}{c}1995 \\
\text { Gesamt }\end{array}$} \\
\hline & Öffentlich $^{1}$ & Privat $^{2}$ & Gesamt & & Öffentlich ${ }^{1}$ & Privat $^{2}$ & Gesamt & \\
\hline Australien $^{*}$ & 3.6 & 0.6 & 4.2 & 3.7 & 0.8 & 0.7 & 1.5 & 1.7 \\
\hline Österreich $^{3 *}$ & 4.0 & 0.2 & 4.2 & 4.2 & 1.4 & $\mathrm{n}$ & 1.5 & 1.5 \\
\hline Belgien* & 3.5 & $\mathrm{~m}$ & $\mathrm{~m}$ & $\mathrm{~m}$ & 1.3 & $\mathrm{~m}$ & $\mathrm{~m}$ & $\mathrm{~m}$ \\
\hline Kanada $^{4 *}$ & 3.5 & 0.3 & 3.8 & 4.3 & 1.6 & 1.0 & 2.5 & 2.2 \\
\hline Dänemark $^{3 *}$ & 4.1 & 0.1 & 4.2 & 4.1 & 1.5 & $\mathrm{n}$ & 1.6 & 1.6 \\
\hline Finnland $^{*}$ & 3.6 & $\mathrm{n}$ & 3.6 & 4.0 & 1.8 & $\mathrm{n}$ & 1.8 & 1.9 \\
\hline Frankreich ${ }^{*}$ & 4.1 & 0.2 & 4.4 & 4.4 & 1.0 & 0.1 & 1.1 & 1.1 \\
\hline Deutschland ${ }^{*}$ & 2.8 & 0.9 & 3.7 & 3.5 & 1.0 & 0.1 & 1.1 & 1.1 \\
\hline Griechenland $^{3 *}$ & 2.4 & 0.3 & 2.6 & 2.3 & 1.0 & $\mathrm{n}$ & 1.0 & 0.7 \\
\hline Ungarn & 2.9 & 0.2 & 3.1 & 3.6 & 0.8 & 0.2 & 1.1 & 1.0 \\
\hline Island & $\mathrm{m}$ & $\mathrm{m}$ & $\mathrm{m}$ & $\mathrm{m}$ & $\mathrm{m}$ & $\mathrm{m}$ & $\mathrm{m}$ & $\mathrm{m}$ \\
\hline Irland $^{5}$ & 3.0 & 0.1 & 3.1 & 3.7 & 1.1 & 0.3 & 1.4 & 1.3 \\
\hline $\operatorname{Japan}^{6 *}$ & 2.7 & 0.2 & 3.0 & 3.1 & 0.5 & 0.6 & 1.0 & 1.0 \\
\hline Korea $^{*}$ & 3.2 & 0.8 & 4.0 & $\mathrm{~m}$ & 0.5 & 1.9 & 2.4 & $\mathrm{~m}$ \\
\hline Luxemburg & $\mathrm{m}$ & $\mathrm{m}$ & $\mathrm{m}$ & $\mathrm{m}$ & $\mathrm{m}$ & $\mathrm{m}$ & $\mathrm{m}$ & $\mathrm{m}$ \\
\hline Mexiko & 3.1 & 0.5 & 3.6 & 4.0 & 0.8 & 0.3 & 1.1 & 1.1 \\
\hline Niederlande $^{*}$ & 2.9 & 0.2 & 3.1 & 3.1 & 1.0 & 0.3 & 1.3 & 1.2 \\
\hline Neuseeland & 4.6 & $\mathrm{~m}$ & $\mathrm{~m}$ & 3.7 & 0.9 & $\mathrm{~m}$ & $\mathrm{~m}$ & 1.1 \\
\hline Norwegen & 4.0 & $\mathrm{n}$ & 4.0 & 4.2 & 1.4 & 0.1 & 1.5 & 1.7 \\
\hline Polen & 3.6 & $\mathrm{~m}$ & $\mathrm{~m}$ & $\mathrm{~m}$ & 0.8 & 0.2 & 1.0 & $\mathrm{~m}$ \\
\hline Portugal $^{3 *}$ & 4.2 & $\mathrm{n}$ & 4.2 & 3.8 & 1.0 & 0.1 & 1.1 & 0.9 \\
\hline Slowakische Rep. ${ }^{3,5}$ & 3.0 & $\mathrm{n}$ & 3.0 & $\mathrm{~m}$ & 0.8 & 0.1 & 0.8 & $\mathrm{~m}$ \\
\hline Spanien & 3.2 & 0.4 & 3.7 & 3.9 & 0.9 & 0.3 & 1.1 & 1.0 \\
\hline Schweden ${ }^{5}$ & 4.4 & $\mathrm{n}$ & 4.4 & 4.3 & 1.5 & 0.2 & 1.7 & 1.6 \\
\hline Schweiz & 3.9 & 0.5 & 4.4 & $\mathrm{~m}$ & 1.2 & $\mathrm{n}$ & 1.2 & $\mathrm{~m}$ \\
\hline Türkei $^{3 *}$ & 2.9 & $\mathrm{~m}$ & 2.9 & 1.8 & 1.0 & $\mathrm{n}$ & 1.0 & 0.7 \\
\hline Ver. Königreich* & 3.3 & 0.4 & 3.7 & $\mathrm{~m}$ & 0.8 & 0.3 & 1.1 & 1.2 \\
\hline Vereinigte Staaten $^{4 *}$ & 3.5 & 0.4 & 3.8 & $\mathrm{~m}$ & 1.1 & 1.2 & 2.3 & $\mathrm{~m}$ \\
\hline Ländermittel & 3.4 & 0.3 & 3.6 & $\sim$ & 1.0 & 0.3 & 1.3 & $\sim$ \\
\hline Argentinien $^{3}$ & 3.3 & 0.4 & 3.7 & $\mathrm{~m}$ & 0.8 & 0.4 & 1.1 & $\mathrm{~m}$ \\
\hline Brasilien $^{3,7}$ & 3.7 & $\mathrm{~m}$ & $\mathrm{~m}$ & $\mathrm{~m}$ & 1.1 & $\mathrm{~m}$ & $\mathrm{~m}$ & $\mathrm{~m}$ \\
\hline Chile & 3.1 & 1.4 & 4.5 & $\mathrm{~m}$ & 0.6 & 1.6 & 2.2 & $\mathrm{~m}$ \\
\hline China & 1.4 & 1.1 & 2.6 & $\mathrm{~m}$ & 0.5 & 0.4 & 0.8 & $\mathrm{~m}$ \\
\hline Indien $^{6,7}$ & 2.5 & 0.1 & 2.6 & $\mathrm{~m}$ & 0.6 & $\mathrm{n}$ & 0.6 & $\mathrm{~m}$ \\
\hline Indonesien ${ }^{3,5,8}$ & 0.6 & 0.2 & 0.8 & $\mathrm{~m}$ & 0.2 & 0.2 & 0.4 & $\mathrm{~m}$ \\
\hline Israel & 4.6 & 0.2 & 4.8 & 5.0 & 1.3 & 0.7 & 2.0 & 1.8 \\
\hline Jamaika & 4.7 & 2.4 & 7.2 & $\mathrm{~m}$ & 1.2 & 0.5 & 1.7 & $\mathrm{~m}$ \\
\hline Jordanien $^{5}$ & 4.1 & 0.1 & 4.1 & $\mathrm{~m}$ & 1.0 & 0.9 & 1.9 & $\mathrm{~m}$ \\
\hline Malaysia $^{3}$ & 3.7 & $\mathrm{~m}$ & $\mathrm{~m}$ & $\mathrm{~m}$ & 1.2 & 0.1 & 1.3 & $\mathrm{~m}$ \\
\hline Paraguay & 4.0 & 2.7 & 6.8 & $\mathrm{~m}$ & 0.8 & 0.7 & 1.5 & $\mathrm{~m}$ \\
\hline Peru $^{3,9}$ & 2.3 & 0.7 & 2.9 & $\mathrm{~m}$ & 0.7 & 0.6 & 1.2 & $\mathrm{~m}$ \\
\hline Philippinen $^{7}$ & 3.4 & 1.7 & 5.1 & $\mathrm{~m}$ & 0.7 & $\mathrm{~m}$ & $\mathrm{~m}$ & $\mathrm{~m}$ \\
\hline Thailand $^{3}$ & 2.8 & 0.1 & 2.9 & $\mathrm{~m}$ & 0.9 & 0.2 & 1.1 & $\mathrm{~m}$ \\
\hline Tunesien $^{3,8}$ & 5.4 & $\mathrm{~m}$ & $\mathrm{~m}$ & $\mathrm{~m}$ & 1.5 & $\mathrm{~m}$ & $\mathrm{~m}$ & $\mathrm{~m}$ \\
\hline Uruguay $^{3,5}$ & 2.0 & 0.1 & 2.1 & $\mathrm{~m}$ & 0.6 & $\mathrm{~m}$ & $\mathrm{~m}$ & $\mathrm{~m}$ \\
\hline Simbabwe $^{6}$ & 5.9 & $\mathrm{~m}$ & $\mathrm{~m}$ & $\mathrm{~m}$ & 1.0 & $\mathrm{~m}$ & $\mathrm{~m}$ & $\mathrm{~m}$ \\
\hline
\end{tabular}

1. Einschließlich öffentlicher Subventionen an private Haushalte, die Bildungseinrichtungen zuzuordnen sind und direkter Ausgaben für Bildungseinrichtungen aus internationalen Quellen..

2. Abzüglich öffentlicher Subventionen, die Bildungseinrichtungen zuzuordnen sind.

3. Öffentliche Subventionen an private Haushalte sind nicht in den öffentlichen, sondern in den privaten Ausgaben enthalten.

4. Post-sekundärer, nicht-tertiärer Bereich im Tertiärbereich enthalten.

5. Direkte Ausgaben für tertiäre Bildungseinrichtungen aus internationalen Mitteln belaufen sich auf mehr als 1,5 Prozent der gesamten öffentlichen Ausgaben für Bildungseinrichtungen. In Uruguay belaufen sich die Mittel aus internationalen Quellen auf mehr als 1,5 Prozent.

6. Post-sekundärer, nicht-tertiärer Bereich sowohl im Sekundar- als auch im Tertiärbereich enthalten.

7. Referenzjahr 1998.

8. Referenzjahr 2000

9. Ohne post-sekundären, nicht-tertiären Bereich.

* Hinweise s. Anhang 3 (www.oecd.org/els/education/eag2002).

Quelle: OECD. 
Tabelle B2.1c.

Ausgaben für Bildungseinrichtungen als Prozentsatz des BIP (1999)

Ausgaben für Bildungseinrichtungen aus öffentlichen und privaten Quellen ${ }^{1}$, nach Bildungsbereichen und Jahr

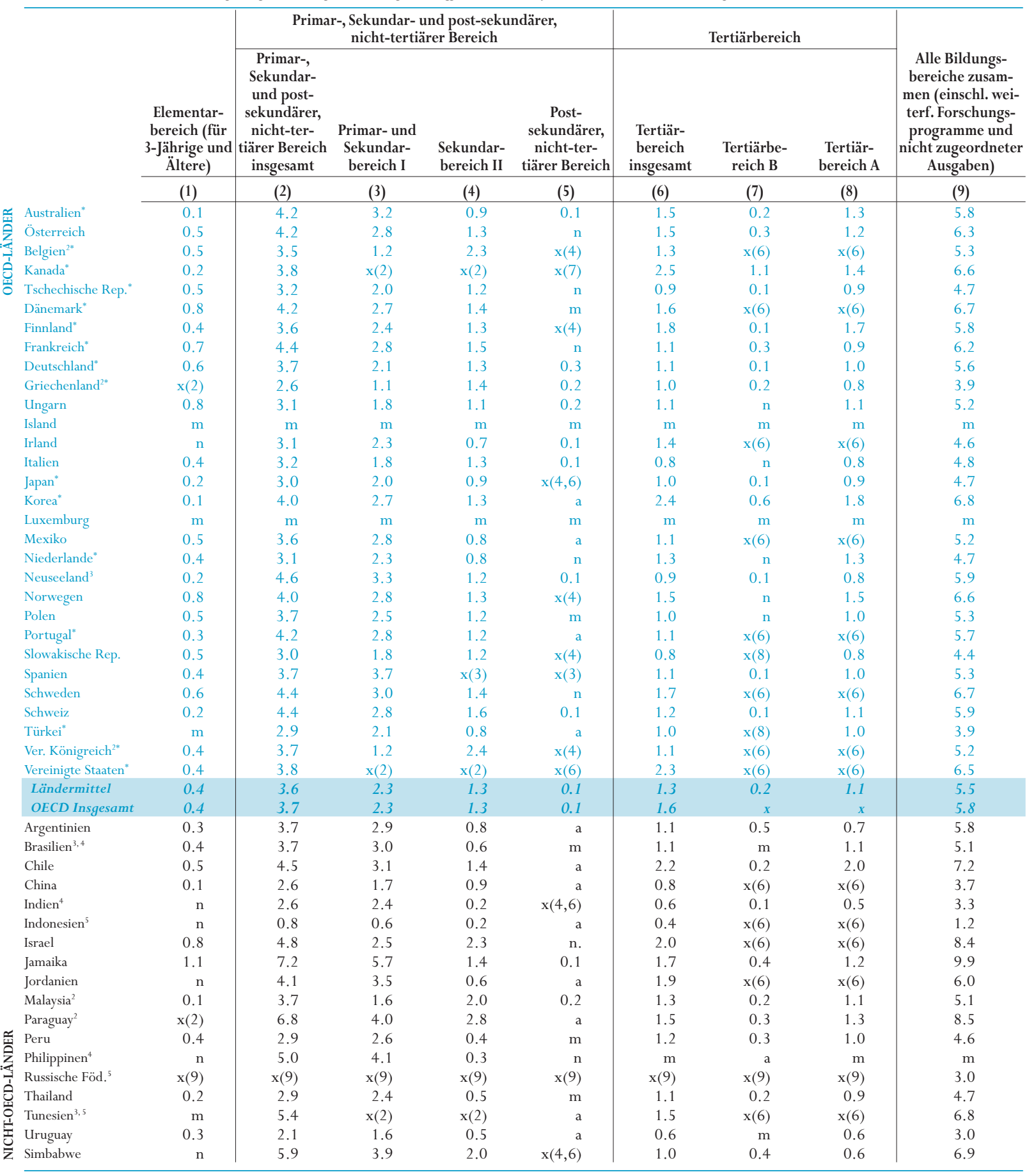

Hinweis: $\mathrm{x}$ bedeutet, dass die Daten in einer anderen Spalte enthalten sind, deren Referenz in runden Klammern nach dem ' $\mathrm{x}$ ' angegeben ist. So bedeutet $\mathrm{z}$. B. $\mathrm{x}(2)$, dass die Daten in Spalte 2 enthalten sind.

1. Einschließlich internationaler Quellen.

2. Spalte (3) bezieht sich nur auf den Primarbereich, Spalte (4) auf den gesamten Sekundarbereich.

3. Nur direkte öffentliche Ausgaben für Bildungseinrichtungen berücksichtigt.

4. Referenzjahr 1998

5. Referenzjahr 2000

* Hinweise s. Anhang 3 (www.oecd.org/els/education/eag2002).

Quelle: OECD. 
Tabelle B2.2.

Veränderungen der Ausgaben für Bildungseinrichtungen $(1995,1999)$

Index derVeränderung der direkten Ausgaben für Bildungseinrichtungen zwischen 1995 und 1999 nach Bildungsbereichen (1995=100)

\begin{tabular}{|c|c|c|c|c|c|c|c|c|c|}
\hline & \multicolumn{3}{|c|}{ Alle Bildungsbereiche } & \multicolumn{3}{|c|}{$\begin{array}{l}\text { Primar-, Sekundar- und post-sekundärer, } \\
\text { nicht-tertiärer Bereich }\end{array}$} & \multicolumn{3}{|c|}{ Tertiärbereich } \\
\hline & $\begin{array}{c}\text { Direkte } \\
\text { öffentliche } \\
\text { Ausgaben für } \\
\text { Bildungs- } \\
\text { einrichtungen }\end{array}$ & $\begin{array}{l}\text { Direkte pri- } \\
\text { vate Ausgaben } \\
\text { für Bildungs- } \\
\text { einrichtungen }\end{array}$ & $\begin{array}{c}\text { Gesamte } \\
\text { direkte } \\
\text { Ausgaben für } \\
\text { Bildungs- } \\
\text { einrich- } \\
\text { tungen aus } \\
\text { offentlichen } \\
\text { und privaten } \\
\text { Quellen }\end{array}$ & $\begin{array}{c}\text { Direkte } \\
\text { öffentliche } \\
\text { Ausgaben für } \\
\text { Bildungs- } \\
\text { einrichtungen }\end{array}$ & $\begin{array}{c}\text { Direkte pri- } \\
\text { vate Ausgaben } \\
\text { für Bildungs- } \\
\text { einrichtungen }\end{array}$ & $\begin{array}{c}\text { Gesamte } \\
\text { direkte } \\
\text { Ausgaben für } \\
\text { Bildungs- } \\
\text { einrich- } \\
\text { tungen aus } \\
\text { offfentlichen } \\
\text { und privaten } \\
\text { Quellen }\end{array}$ & $\begin{array}{c}\text { Direkte } \\
\text { öffentliche } \\
\text { Ausgaben für } \\
\text { Bildungs- } \\
\text { einrichtungen }\end{array}$ & $\begin{array}{l}\text { Direkte pri- } \\
\text { vate Ausgaben } \\
\text { für Bildungs- } \\
\text { einrichtungen }\end{array}$ & $\begin{array}{c}\text { Gesamte } \\
\text { direkte } \\
\text { Ausgaben für } \\
\text { Bildungs- } \\
\text { einrich- } \\
\text { tungen aus } \\
\text { offentlichen } \\
\text { und privaten } \\
\text { Quellen }\end{array}$ \\
\hline & $(1)$ & (2) & (3) & (4) & (5) & (6) & (7) & $(8)$ & (9) \\
\hline Australien & 123 & 140 & 126 & 136 & 137 & 136 & 88 & 143 & 107 \\
\hline Österreich & 107 & 80 & 105 & 108 & 101 & 108 & 107 & 54 & 106 \\
\hline Kanada $^{1}$ & 106 & 116 & 108 & 101 & 124 & 103 & 117 & 113 & 116 \\
\hline Tschechische Rep. & 92 & 75 & 90 & 85 & 78 & 89 & 116 & 51 & 98 \\
\hline Dänemark ${ }^{2}$ & 110 & 103 & 109 & 110 & 114 & 110 & 102 & 406 & 104 \\
\hline Finnland & 109 & $\mathrm{x}(1)$ & 111 & 109 & $\mathrm{x}(4)$ & 109 & 108 & $\mathrm{x}(7)$ & 111 \\
\hline Frankreich & 109 & 102 & 108 & 109 & 104 & 108 & 110 & 99 & 108 \\
\hline Deutschland & 102 & 102 & 102 & 103 & 103 & 104 & 102 & 119 & 104 \\
\hline Griechenland & 143 & $\mathrm{x}(1)$ & 149 & 121 & $\mathrm{x}(4)$ & 123 & 182 & $\mathrm{x}(7)$ & 173 \\
\hline Ungarn & 107 & 120 & 109 & 101 & 95 & 98 & 117 & 145 & 123 \\
\hline Irland & 124 & 128 & 124 & 115 & 110 & 115 & 160 & 134 & 151 \\
\hline Italien & 103 & $\mathrm{~m}$ & $\mathrm{~m}$ & 96 & $\mathrm{~m}$ & $\mathrm{~m}$ & 112 & 133 & 116 \\
\hline $\mathrm{Japan}^{3}$ & 106 & 106 & 106 & 104 & 103 & 104 & 116 & 108 & 111 \\
\hline Mexiko & 114 & 114 & 114 & 111 & 100 & 109 & 119 & 159 & 128 \\
\hline Niederlande & 113 & 163 & 116 & 116 & 116 & 116 & 105 & 229 & 119 \\
\hline Neuseeland & 134 & $\mathrm{~m}$ & $\mathrm{~m}$ & 142 & $\mathrm{~m}$ & $\mathrm{~m}$ & 96 & $\mathrm{~m}$ & $\mathrm{~m}$ \\
\hline Norwegen & 104 & 88 & 104 & 109 & 88 & 109 & 102 & 88 & 101 \\
\hline Polen & 116 & $\mathrm{~m}$ & $\mathrm{~m}$ & 127 & $\mathrm{~m}$ & $\mathrm{~m}$ & 113 & $\mathrm{~m}$ & $\mathrm{~m}$ \\
\hline Portugal & 124 & 262 & 125 & 128 & 187 & 128 & 127 & 265 & 132 \\
\hline Spanien & 112 & 110 & 111 & 111 & 99 & 110 & 123 & 125 & 124 \\
\hline Schweden & 114 & 201 & 116 & 115 & 119 & 115 & 108 & 206 & 116 \\
\hline Schweiz & $\mathrm{m}$ & $\mathrm{m}$ & $\mathrm{m}$ & $\mathrm{m}$ & $\mathrm{m}$ & $\mathrm{m}$ & $\mathrm{m}$ & $\mathrm{m}$ & $\mathrm{m}$ \\
\hline Türkei & 186 & $\mathrm{~m}$ & $\mathrm{~m}$ & 194 & $\mathrm{~m}$ & $\mathrm{~m}$ & 167 & 231 & 169 \\
\hline Ver. Königreich & 106 & $\mathrm{~m}$ & $\mathrm{~m}$ & 107 & $\mathrm{~m}$ & $\mathrm{~m}$ & 100 & 103 & 101 \\
\hline $\begin{array}{l}\text { Hinweis: } \mathrm{x} \text { bedeute } \\
\text { dass die Daten i } \\
\text { 1. Post-sekundär } \\
\text { 2. Daten zum pos } \\
\text { 3. Post-sekundäre } \\
\text { * Hinweise s. Ant } \\
\text { Quelle: OECD. }\end{array}$ & $\begin{array}{l}\text { t, dass die Daten } \\
\text { in Spalte } 2 \text { enthal } \\
\text { er, nicht-tertiäre } \\
\text { t-sekundären, n } \\
\text { er, nicht-tertiäre } \\
\text { ang } 3 \text { (www.oecd }\end{array}$ & $\begin{array}{l}\text { in einer anderen } \\
\text { lten sind. } \\
\text { r Bereich im Ter } \\
\text { hicht-tertiären B } \\
\text { r Bereich sowoh } \\
\text { l.org/els/educatio }\end{array}$ & $\begin{array}{l}\text { Spalte enthalte } \\
\text { tiärbereich ent } \\
\text { ereich fehlen. } \\
\text { l im Sekundarb } \\
\text { on /eag2002). }\end{array}$ & $\begin{array}{l}\text { n sind, deren Re } \\
\text { halten. } \\
\text { sereich II als auc }\end{array}$ & ch im Tertiärbere & eich enthalten. & dem ' $x$ ' angege & eben ist. So bed & utet z.B. x(2), \\
\hline
\end{tabular}




\section{DIE ÖFFENTLICHEN BILDUNGSAUSGABEN}

- Im Durchschnitt wenden die OECD-Länder 12,7 Prozent ihrer gesamten öffentlichen Ausgaben für Bildungseinrichtungen auf.

- Die öffentliche Finanzierung von Bildung ist eine vorrangige gesellschaftspolitische Aufgabe - auch in den OECD-Ländern, in denen die Staatsquote insgesamt gering ist.

- In vier von fünf OECD-Ländern stiegen die öffentlichen Ausgaben für Bildung zwischen 1995 und 1999 real um mehr als 5 Prozent.

- Die öffentlichen Ausgaben für Bildung stiegen meist schneller als die gesamten öffentlichen Ausgaben, jedoch langsamer als das BIP. In Italien, den Niederlanden, Schweden und dem Vereinigten Königreich stiegen die öffentlichen Bildungsausgaben zwischen 1995 und 1999 trotz real sich verringernder öffentlicher Haushalte.

\section{Abbildung B3.1.}

Öffentliche Bildungsausgaben als Prozentsatz der öffentlichen Gesamtausgaben (1999) Direkte öffentliche Ausgaben für Bildungseinrichtungen zuzüglich öffentlicher Subventionen an private Haushalte (einschließ1. Subventionen für den Lebensunterhalt und Subventionen für andere private Einheiten) als Prozentsatz der öffentlichen Gesamtausgaben, nach Bildungsbereichen und Jahr

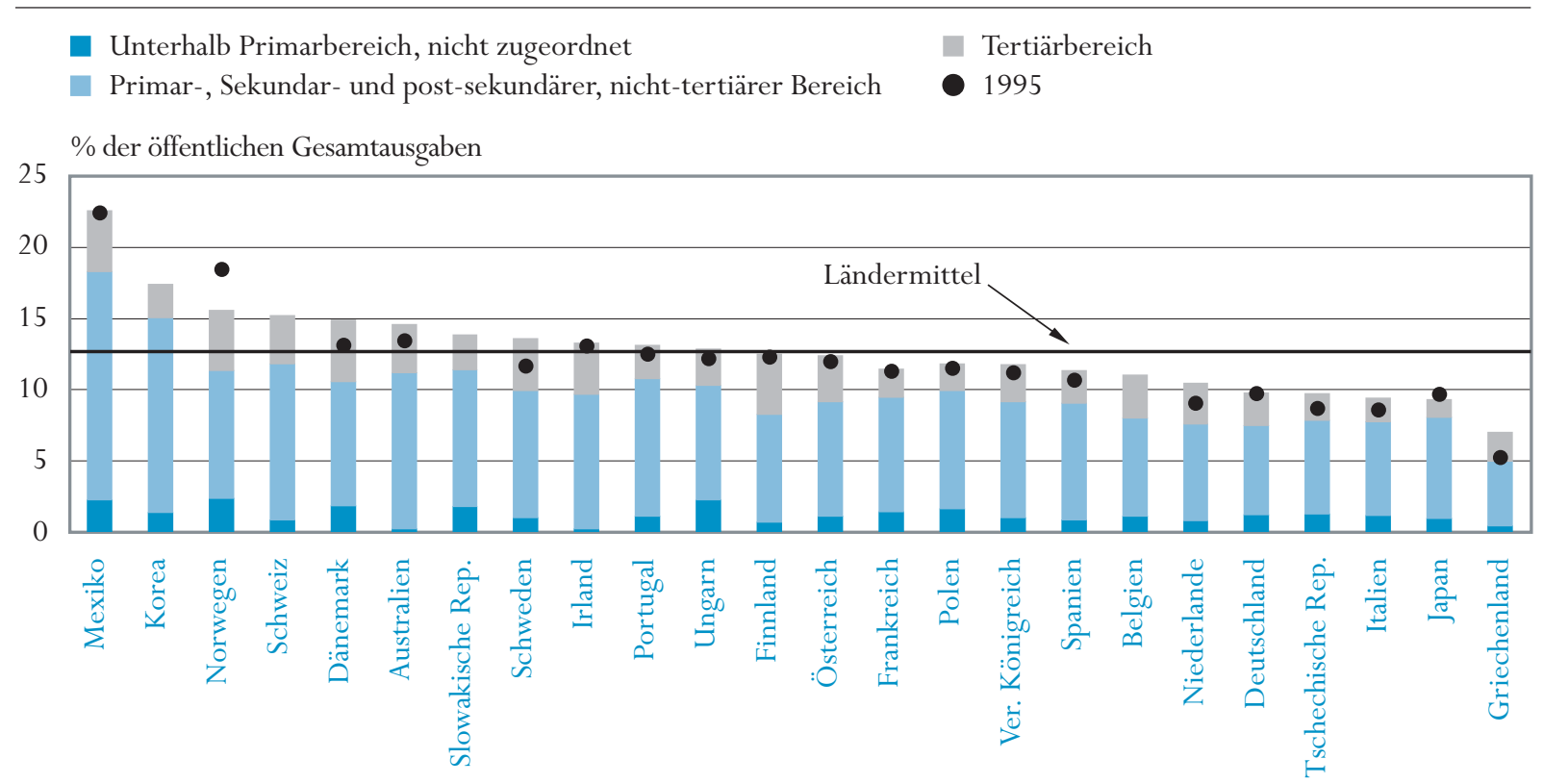

Anordnung der Länder in absteigender Reihenfolge der öffentlichen Gesamtausgaben für Bildungseinrichtungen für alle Bildungsbereiche zusammen (1999).

Quelle: OECD. Tabelle B3.1. Hinweise s. Anhang 3 (www.oecd.org/els/education/eag2002). 


\section{Politischer Hintergrund}

Der Staat beteiligt sich aus den unterschiedlichsten Gründen an der Bereitstellung von Dienstleistungen für die Öffentlichkeit. Wenn der öffentliche Nutzen einer bestimmten Dienstleistung größer ist als der private Nutzen, kann es sein, dass der Markt allein nicht in der Lage ist, derartige Dienstleistungen in angemessenem Maß bereitzustellen. Bildung ist ein solcher Bereich, in dem alle Staaten intervenieren, um das Leistungsangebot zu finanzieren oder zu lenken. Da keine Garantie dafür besteht, dass die Märkte allen den gleichen Zugang zu Bildungschancen bieten, wird durch die staatliche Finanzierung der Bildung sichergestellt, dass nicht ein Teil der Gesellschaft von Bildungsmöglichkeiten ausgeschlossen wird. Die öffentlichen Ausgaben für Bildung in Prozent der gesamten öffentlichen Ausgaben sind ein Indiz für den Stellenwert der Bildung im Vergleich zu anderen öffentlichen Aufgaben wie Gesundheitswesen, soziale Sicherung, Verteidigung und innere Sicherheit.

Seit der zweiten Hälfte der neunziger Jahre haben die meisten OECD-Länder große Anstrengungen zur Konsolidierung ihrer öffentlichen Haushalte unternommen. Bildung stand im Wettstreit mit einer Vielzahl anderer in den staatlichen Haushalten enthaltener Finanzierungsaufgaben. Um dies zu verdeutlichen, untersucht dieser Indikator die Veränderungen bei den Bildungsausgaben sowohl in absoluten Zahlen als auch im Verhältnis zur Entwicklung der öffentlichen Haushalte.

\section{Ergebnisse und Erläuterungen}

\section{Was dieser Indikator aufzeigt und was nicht}

Dieser Indikator beschreibt die gesamten öffentlichen Ausgaben für Bildung. Darin sind die direkten öffentlichen Finanzmittel für Bildungseinrichtungen ebenso enthalten wie die öffentlichen Subventionen für private Haushalte (z.B. in Form von Stipendien und Studiendarlehen für Studiengebühren und Lebenshaltungskosten von Studierenden) sowie Zahlungen an andere private Einheiten für Bildungszwecke (z.B. Subventionen für Unternehmen oder Arbeitnehmerorganisationen, die Lehrlingsausbildungsprogramme durchführen). Im Unterschied zu den vorherigen Indikatoren beinhaltet dieser Indikator auch öffentliche Subventionen, die nicht Zahlungen privater Haushalte an Bildungseinrichtungen zuzurechnen sind, wie z.B. Unterstützungsleistungen für den Lebensunterhalt von Schülern/Studierenden.

Hinsichtlich der Verwendung öffentlicher Mittel für die Bildung gibt es Unterschiede zwischen den einzelnen OECD-Ländern. Öffentliche Mittel können direkt an Bildungseinrichtungen fließen oder über die privaten Haushalte in die Bildungseinrichtungen gelenkt werden. Sie können auf den Erwerb von Bildungsdienstleistungen beschränkt sein oder als Unterstützung zu den Lebenshaltungskosten von Schülern/Studierenden gewährt werden.

Es ist wichtig, die öffentlichen Bildungsinvestitionen im Zusammenhang mit den in Indikator B4 behandelten privaten Bildungsausgaben zu betrachten.
Dieser Indikator

konzentriert sich

auf die öffentlichen

Bildungsausgaben.

Ferner untersucht dieser

Indikator die Veränderung

deröffentlichen

Bildungsausgaben über die

Zeit, sowohl in absoluten

Zahlen als auch im

vergleich zu den staatlichen

Gesamtausgaben.

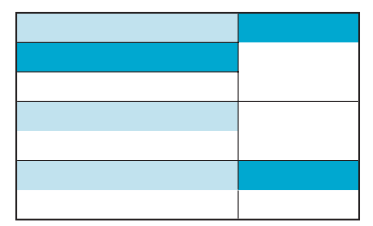

Erfasste Daten

(Erklärungen s. S. 160) 
Im Durchschnitt wenden die OECD-Länder 12,7 Prozent ihrer gesamten öffentlichen Ausgaben für die Bildung auf.

In den OECD-Ländern fließen zwischen 4,5

Prozent und 16,0 Prozent der öffentlichen Gesamtausgaben in den Primar-, Sekundar- und

post-sekundären, nicht-tertiären Bereich.

\section{Gesamtvolumen der in die Bildung investierten öffentlichen Mittel}

Im Durchschnitt wenden die OECD-Länder 12,7 Prozent ihrer gesamten öffentlichen Ausgaben für die Bildung auf, wobei die Werte für die einzelnen Länder zwischen 7 und 23 Prozent schwanken. Korea und Mexiko setzen 17 bzw. 23 Prozent ihrer öffentlichen Gesamtausgaben für die Bildung ein (Abb. B3.1). Dagegen liegt in Deutschland, Griechenland, Italien, Japan und der Tschechischen Republik der Anteil der Bildungsausgaben an den öffentlichen Gesamtausgaben unter 10 Prozent. Wie bei den Bildungsausgaben im Verhältnis zum BIP pro Kopf müssen auch die hier ausgewiesenen Werte im Zusammenhang mit den Schüler-/ Studierendenzahlen sowie den jeweiligen Beteiligungsquoten betrachtet werden.

Der öffentliche Anteil an der Finanzierung der verschiedenen Bildungsbereiche ist in den einzelnen OECD-Ländern sehr unterschiedlich. 1999 setzten die OECD-Länder zwischen 4,5 Prozent und 16,0 Prozent ihrer gesamten öffentlichen Ausgaben für den Primar-, Sekundar- und post-sekundären, nichttertiären Bereich und zwischen 1,2 und 4,3 Prozent für den tertiären Bereich ein. In Australien, Korea, Portugal und der Schweiz entfallen zwischen 10 und 15 Prozent der gesamten öffentlichen Ausgaben auf den Primar-, Sekundarund post-sekundären, nicht-tertiären Bereich, in Mexiko sind es über 15 Prozent. Im Unterschied hierzu machen die Ausgaben für Bildung unterhalb des Tertiärbereichs in Belgien, Deutschland, Griechenland, Italien, Japan, den Niederlanden und der Tschechischen Republik ungefähr 7 Prozent und weniger aus (Tabelle B3.1).

\section{Abbildung B3.2.}

Öffentliche Gesamtausgaben als Prozentsatz des BIP $(1995,1999)$

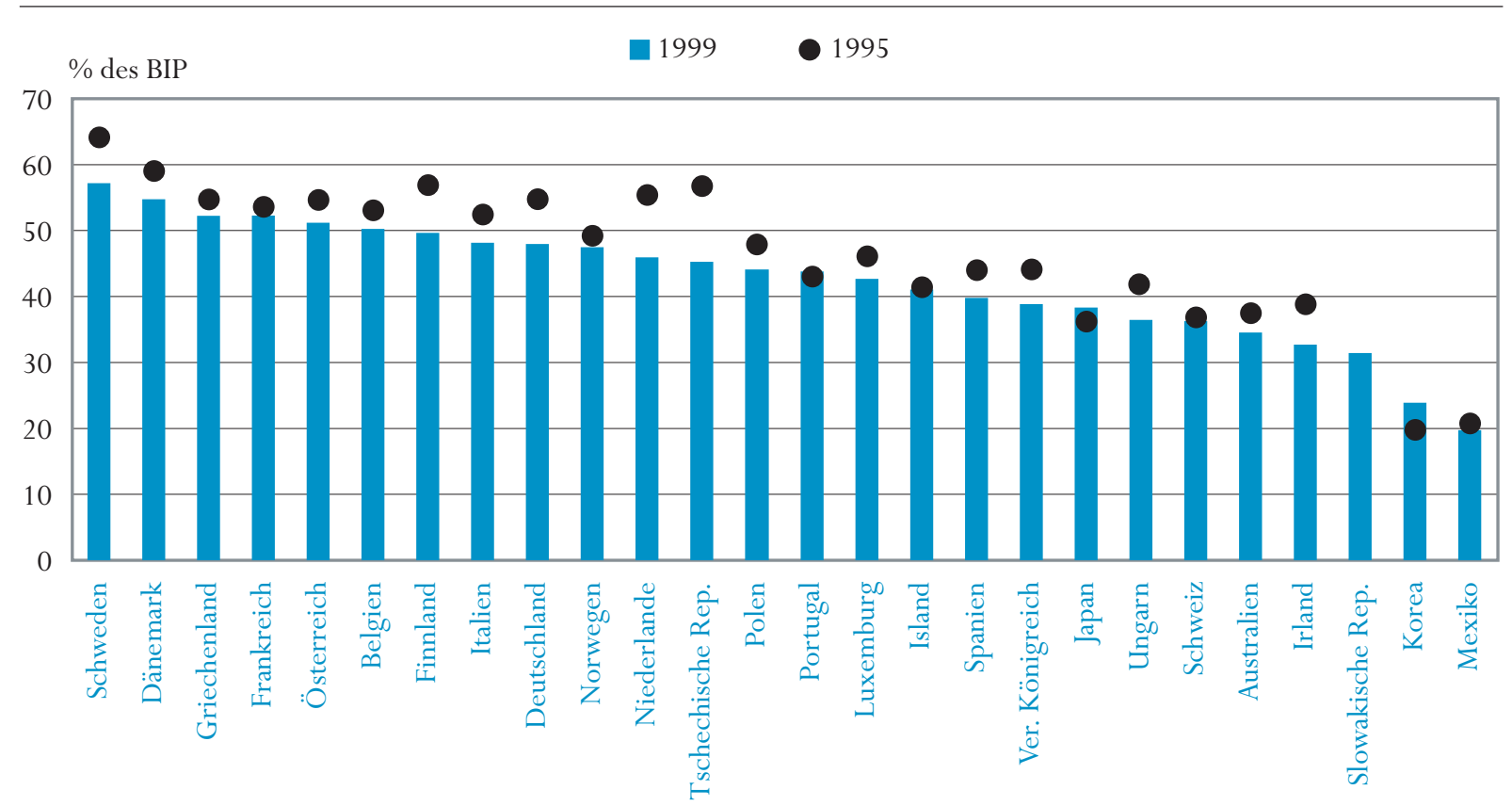

Anordnung der Länder in absteigender Reihenfolge der öffentlchen Gesamtausgaben als Prozentsatz des BIP in 1999. Quelle: OECD. Anhang 2. 
Bei der Betrachtung der öffentlichen Bildungsausgaben im Verhältnis zu den gesamten öffentlichen Ausgaben ist die relative Größe der öffentlichen Haushalte (gemessen im Verhältnis der öffentlichen Ausgaben zum BIP) zu berücksichtigen.

In OECD-Ländern mit geringen öffentlichen Ausgaben im Verhältnis zum gesamten BIP, wie Australien, Irland, Korea, Mexiko und der Slowakischen Republik ist der Anteil der öffentlichen Bildungsausgaben relativ hoch. In den übrigen OECD-Ländern, in denen die öffentlichen Ausgaben mehr als 35 Prozent des BIP ausmachen, scheint es jedoch keinen Zusammenhang zwischen der Höhe des öffentlichen Haushalts und der Höhe der Bildungsausgaben zu geben (Abb. B3.1 und B3.2).

Schweden, das OECD-Land mit dem höchsten Anteil öffentlicher Ausgaben am BIP, hat einen gleich hohen Anteil der öffentlichen Ausgaben, der für Bildungszwecke ausgegeben wird, wie Portugal, ein OECD-Land mit einer relativ geringen Staatsquote. Norwegen hat von allen OECD-Ländern den dritthöchsten Anteil von Bildungsausgaben am öffentlichen Haushalt, während Italien an drittletzter Stelle liegt, obwohl sich in beiden OECD-Ländern die öffentlichen Ausgaben auf 47 bzw. 48 Prozent des BIP belaufen (Abb. B3.2).

Wie jede andere öffentliche Dienstleistung wird auch die Bildung durch den Prozess der Haushaltskonsolidierung belastet. Dennoch stiegen - außer in Japan und Norwegen - die Bildungsausgaben schneller als die Ausgaben für andere öffentliche Bereiche. Insgesamt erhöhte sich der Anteil der Bildungsausgaben an den Gesamtetats im Durchschnitt von 12,0 Prozent im Jahre 1995 auf 12,7 Prozent in 1999. Die öffentlichen Bildungsausgaben stiegen in Griechenland um nahezu ein Drittel, von 5,2 auf 7,0 Prozent. In Dänemark erhöhte sich der Anteil der Bildungsausgaben an den öffentlichen Gesamtausgaben zwischen 1995 und 1999 von 13,1 Prozent auf 14,9 Prozent, in Schweden von 11,6 Prozent auf 13,6 Prozent und in den Niederlanden von 9,1 auf 10,4 Prozent.

\section{Definitionen und angewandte Methodik}

Bildungsausgaben werden ausgedrückt in Prozent der öffentlichen Gesamtausgaben eines Landes und in Prozent des BIP. Die öffentlichen Bildungsausgaben beinhalten die Ausgaben für Bildungseinrichtungen sowie Subventionen zum Lebensunterhalt von Schülern/Studierenden und für andere private Ausgaben außerhalb von Bildungseinrichtungen. In den öffentlichen Bildungsausgaben sind die Ausgaben sämtlicher öffentlicher Einrichtungen einschließlich anderer Ministerien neben dem Bildungsministerium sowie die Ausgaben der Gebietskörperschaften und anderer öffentlicher Stellen enthalten.

Die öffentlichen Gesamtausgaben bzw. die staatlichen Gesamtausgaben sind nicht rückzahlbare laufende und investive Ausgaben aller Gebietskörperschaften. Die laufenden Ausgaben betreffen konsumtive Ausgaben, Pacht-, Miet- und Zinszahlungen, Subventionen sowie sonstige laufende Transferzahlungen (z.B. Sozialversicherungen, Sozialhilfe, Renten und sonstige Wohlfahrtsleistungen).
Die öffentliche

Finanzierung

der Bildung ist

eine vorrangige

gesellschaftspolitische

Aufgabe - selbst in

denjenigen $O E C D$ -

Ländern, in denen die

Staatsquote insgesamt

gering ist.

In der Regel natmen

die öffentlichen

Bildungsausgaben

schneller zu als

die öffentlichen

Gesamtausgaben, aber

nicht so schnell wie das

volkseinkommen.

Die Daten beziehen sich

auf das Haushaltsjahr

1999 und beruhen

auf der von der

OECD im Jahre 2001

durchgeführten

vOE-Erhebung zur

Bildungsstatistik

(Einzelheiten s. Anhang 3). 
Die Daten für die öffentlichen Gesamtausgaben stammen aus der OECDDatenbank der volkswirtschaftlichen Gesamtrechnungen (s. Anhang 2) und beruhen auf dem System of National Accounts 1993. In früheren Ausgaben von Bildung auf einen Blick bezogen sich die öffentlichen Gesamtausgaben auf das System of National Accounts 1968. Die Umstellung des Systems der volkswirtschaftlichen Gesamtrechnung könnte daher für Unterschiede bei diesem Indikator gegenüber früheren Ausgaben von Bildung auf einen Blick verantwortlich sein.

Es ist zu beachten, dass die in früheren Ausgaben dieser Veröffentlichung aufgeführten Zahlen und Daten möglicherweise nicht immer mit denen der Ausgabe 2002 vergleichbar sind, da sich Änderungen in den Definitionen und Erhebungsmethoden ergeben haben. Diese wurden aufgrund der OECD Expenditure Comparability Study vorgenommen (Einzelheiten $\mathrm{zu}$ den Änderungen s. Anhang 3 unter www.oecd.org/els/education/eag2002). 
Tabelle B3.1.

Öffentliche Gesamtausgaben für Bildung $(1995,1999)$

Öffentliche Ausgaben für Bildungseinrichtungen zuzüglich öffentlicher Subventionen an private Haushalte (einschließl. Subventionen für den Lebensunterhalt und Subventionen für andere private Einheiten) als Prozentsatz des BIP und der öffentlichen Gesamtausgaben, nach Bildungsbereichen und Jahr

\begin{tabular}{|c|c|c|c|c|c|c|c|c|}
\hline & \multicolumn{4}{|c|}{$\begin{array}{c}\text { Öffentliche Bildungsausgaben als Prozentsatz } \\
\text { der öffentlichen Gesamtausgaben }\end{array}$} & \multicolumn{4}{|c|}{ Öffentliche Bildungausgaben ${ }^{1}$ als Prozentsatz des BIP } \\
\hline & & 1999 & & 1995 & & 1999 & & 1995 \\
\hline & $\begin{array}{c}\text { Primar-, Sekun- } \\
\text { dar- und post- } \\
\text { sekundärer, } \\
\text { nicht-tertiärer } \\
\text { Bereich }\end{array}$ & Tertiärbereich & $\begin{array}{l}\text { Alle Bildungs- } \\
\text { bereiche } \\
\text { zusammen }\end{array}$ & $\begin{array}{c}\text { Alle Bildungs- } \\
\text { bereiche } \\
\text { zusammen }\end{array}$ & \begin{tabular}{|} 
Primar-, Sekun- \\
dar- und post- \\
sekundärer, \\
nicht-tertiärer \\
Bereich
\end{tabular} & Tertiärbereich & $\begin{array}{l}\text { Alle Bildungs- } \\
\text { bereiche } \\
\text { zusammen }\end{array}$ & $\begin{array}{l}\text { Alle Bildungs- } \\
\text { bereiche } \\
\text { zusammen }\end{array}$ \\
\hline Österreich $^{*}$ & 8.0 & 3.2 & 12.4 & 12.0 & 4.1 & 1.7 & 6.3 & 6.5 \\
\hline Belgien $^{*}$ & 6.9 & 3.0 & 11.0 & $\mathrm{~m}$ & 3.5 & 1.5 & 5.5 & $\mathrm{~m}$ \\
\hline Kanada $^{2 *}$ & $\mathrm{~m}$ & $\mathrm{~m}$ & $\mathrm{~m}$ & 12.9 & 3.5 & 1.9 & 5.7 & 6.5 \\
\hline Tschechische Rep. ${ }^{*}$ & 6.6 & 1.9 & 9.7 & 8.7 & 3.0 & 0.8 & 4.4 & 4.9 \\
\hline Dänemark ${ }^{*}$ & 8.7 & 4.3 & 14.9 & 13.1 & 4.8 & 2.4 & 8.1 & 7.7 \\
\hline Finnland $^{*}$ & 7.6 & 4.2 & 12.5 & 12.3 & 3.8 & 2.1 & 6.2 & 7.0 \\
\hline Frankreich ${ }^{*}$ & 8.0 & 2.0 & 11.5 & 11.3 & 4.2 & 1.1 & 6.0 & 6.0 \\
\hline Deutschland $^{*}$ & 6.2 & 2.3 & 9.7 & 9.7 & 3.0 & 1.1 & 4.7 & 4.7 \\
\hline Island & $\mathrm{m}$ & $\mathrm{m}$ & $\mathrm{m}$ & $\mathrm{m}$ & $\mathrm{m}$ & $\mathrm{m}$ & $\mathrm{m}$ & $\mathrm{m}$ \\
\hline Irland & 9.4 & 3.6 & 13.2 & 13.0 & 3.1 & 1.2 & 4.3 & 5.1 \\
\hline Italien & 6.6 & 1.7 & 9.4 & 8.6 & 3.2 & 0.8 & 4.5 & 4.6 \\
\hline $\operatorname{Japan}^{3 *}$ & 7.1 & 1.2 & 9.3 & 9.7 & 2.7 & 0.5 & 3.5 & 3.6 \\
\hline Korea $^{*}$ & 13.7 & 2.4 & 17.4 & $\mathrm{~m}$ & 3.2 & 0.6 & 4.1 & $\mathrm{~m}$ \\
\hline Luxemburg & $\mathrm{m}$ & $\mathrm{m}$ & $\mathrm{m}$ & $\mathrm{m}$ & $\mathrm{m}$ & $\mathrm{m}$ & $\mathrm{m}$ & $\mathrm{m}$ \\
\hline Mexiko & 16.0 & 4.3 & 22.6 & 22.4 & 3.1 & 0.8 & 4.4 & 4.6 \\
\hline Niederlande ${ }^{*}$ & 6.8 & 2.9 & 10.4 & 9.1 & 3.1 & 1.3 & 4.8 & 5.0 \\
\hline Neuseeland & $\mathrm{m}$ & $\mathrm{m}$ & $\mathrm{m}$ & 14.4 & 4.8 & 1.2 & 6.3 & 5.7 \\
\hline Norwegen & 9.0 & 4.2 & 15.6 & 18.4 & 4.3 & 2.0 & 7.4 & 9.1 \\
\hline Polen & 8.3 & 1.9 & 11.8 & 11.5 & 3.6 & 0.8 & 5.2 & 5.5 \\
\hline Portugal ${ }^{*}$ & 9.7 & 2.4 & 13.1 & 12.5 & 4.2 & 1.0 & 5.7 & 5.4 \\
\hline Slowakische Rep. & 9.6 & 2.5 & 13.8 & $\mathrm{~m}$ & 3.0 & 0.8 & 4.3 & $\mathrm{~m}$ \\
\hline Spanien & 8.2 & 2.3 & 11.3 & 10.6 & 3.3 & 0.9 & 4.5 & 4.7 \\
\hline Schweden & 8.9 & 3.7 & 13.6 & 11.6 & 5.1 & 2.1 & 7.7 & 7.5 \\
\hline Schweiz & 11.0 & 3.4 & 15.2 & $\mathrm{~m}$ & 4.0 & 1.2 & 5.5 & $\mathrm{~m}$ \\
\hline Vereinigte Staaten ${ }^{2 *}$ & $\mathrm{~m}$ & $\mathrm{~m}$ & $\mathrm{~m}$ & $\mathrm{~m}$ & 3.5 & 1.4 & 5.2 & $\mathrm{~m}$ \\
\hline Ländermittel & 8.7 & 2.8 & 12.7 & 12.0 & 3.5 & 1.2 & 5.2 & 5.4 \\
\hline Argentinien & 9.7 & 2.3 & 13.3 & $\mathrm{~m}$ & 3.3 & 0.8 & 4.5 & $\mathrm{~m}$ \\
\hline Brasilien $^{4}$ & 8.6 & 2.6 & 12.3 & $\mathrm{~m}$ & 3.7 & 1.1 & 5.2 & $\mathrm{~m}$ \\
\hline Chile & 12.8 & 2.7 & 17.0 & $\mathrm{~m}$ & 3.1 & 0.7 & 4.2 & $\mathrm{~m}$ \\
\hline China & 9.1 & 3.1 & 13.0 & $\mathrm{~m}$ & 1.5 & 0.5 & 2.1 & $\mathrm{~m}$ \\
\hline Indien ${ }^{2,4}$ & 9.8 & 2.4 & 12.6 & $\mathrm{~m}$ & 2.5 & 0.6 & 3.2 & $\mathrm{~m}$ \\
\hline Indonesien $^{5}$ & 4.0 & 1.2 & 5.2 & $\mathrm{~m}$ & 0.6 & 0.2 & 0.8 & $\mathrm{~m}$ \\
\hline Israel & 9.1 & 2.5 & 13.8 & 13.3 & 4.6 & 1.3 & 7.0 & 7.0 \\
\hline Jamaika & 8.1 & 2.0 & 10.8 & $\mathrm{~m}$ & 4.7 & 1.2 & 6.3 & $\mathrm{~m}$ \\
\hline Jordanien & 16.7 & 3.8 & 20.6 & $\mathrm{~m}$ & 4.1 & 0.9 & 5.0 & $\mathrm{~m}$ \\
\hline Malaysia & 16.5 & 8.3 & 25.2 & $\mathrm{~m}$ & 3.7 & 1.9 & 5.7 & $\mathrm{~m}$ \\
\hline Paraguay & 7.3 & 1.5 & 8.8 & $\mathrm{~m}$ & 4.0 & 0.8 & 4.8 & $\mathrm{~m}$ \\
\hline Peru $^{6}$ & 14.3 & 4.3 & 21.1 & $\mathrm{~m}$ & 2.3 & 0.7 & 3.3 & $\mathrm{~m}$ \\
\hline Philippinen $^{4}$ & 16.8 & 3.4 & 20.6 & $\mathrm{~m}$ & 3.4 & 0.7 & 4.2 & $\mathrm{~m}$ \\
\hline Russische Föd. ${ }^{5}$ & $\mathrm{x}$ & $\mathrm{x}$ & 10.4 & $\mathrm{~m}$ & $\mathrm{x}$ & $\mathrm{x}$ & 3.0 & $\mathrm{~m}$ \\
\hline Thailand & 16.9 & 6.7 & 28.0 & $\mathrm{~m}$ & 3.0 & 1.2 & 4.9 & $\mathrm{~m}$ \\
\hline Tunesien ${ }^{5}$ & 13.6 & 3.8 & 17.4 & $\mathrm{~m}$ & 5.4 & 1.5 & 6.8 & $\mathrm{~m}$ \\
\hline Uruguay & 9.1 & 2.7 & 13.0 & $\mathrm{~m}$ & 1.9 & 0.6 & 2.8 & $\mathrm{~m}$ \\
\hline Simbabwe $^{2}$ & $\mathrm{~m}$ & $\mathrm{~m}$ & $\mathrm{~m}$ & $\mathrm{~m}$ & 5.8 & 1.2 & 7.0 & $\mathrm{~m}$ \\
\hline
\end{tabular}

1. Die in dieser Tabelle enthaltenen öffentlichen Ausgaben beinhalten öffentliche Subventionen an private Haushalte für den Lebensunterhalt, die nicht für Bildungseinrichtungen ausgegeben werden. Daher übersteigen die in dieser Tabelle angegebenen entsprechenden Zahlen die für öffentliche Ausgaben für Bildungseinrichtungen in Tabelle B2.1b.

2. Post-sekundärer, nicht-tertiärer Bereich im Tertiärbereich und nicht im Primar-, Sekundar- und post-sekundären, nicht-tertiären Bereich enthalten.

3. Ohne öffentliche Subventionen an den privaten Sektor. Post-sekundärer, nicht-tertiärer Bereich sowohl im Sekundarbereich II als auch im Tertiärbereich enthalten.

4. Referenzjahr 1998.

5. Referenzjahr 2000

6. Ohne post-sekundären, nicht-tertiären Bereich.

* Hinweise s. Anhang 3 (www.oecd.org/els/education/eag2002).

Quelle: OECD. 


\section{RELATIVE ANTEILE ÖFFENTLICHER UND PRIVATER AUSGABEN FÜR BILDUNGSEINRICHTUNGEN}

- Der private Anteil an den Gesamtausgaben für Bildungseinrichtungen reicht von etwa 3 Prozent und weniger in Finnland, Norwegen, Portugal, Schweden, der Slowakischen Republik und der Türkei bis zu ganzen 40 Prozent in Korea.

- In einigen OECD-Ländern übernimmt die öffentliche Hand den Großteil der Kosten für den Primar-, Sekundar- und post-sekundären, nicht-tertiären Bereich, überlässt jedoch den Betrieb der Bildungseinrichtungen dem privaten Sektor, in dem Bestreben, eine größere Bandbreite an Lernmöglichkeiten zur Verfügung zu stellen, ohne Schülern aus einkommensschwachen Familien den Zugang zu Bildung zu erschweren.

- Im Primar-, Sekundar- und post-sekundären, nicht-tertiären Bereich sind private Einrichtungen, die überwiegend aus Mittteln der privaten Haushalte finanziert werden, weitaus weniger üblich.

- Tendenziell beziehen tertiäre Einrichtungen einen weitaus größeren Teil ihrer Finanzierung aus privaten Quellen als Bildungseinrichtungen des Primar-, Sekundar- und post-sekundären, nichttertiären Bereichs, wobei der Anteil der privaten Finanzierung, einschließlich staatlich-subventionierter privater Zahlungen, von 3 Prozent und weniger in Belgien (fläm.), Dänemark, Finnland, Griechenland, Österreich und der Schweiz bis hin zu 78 Prozent in Korea reicht.

- In 10 von 19 OECD-Ländern sind zwischen 1995 und 1999 die privaten Ausgaben für den Tertiärbereich um mehr als 30 Prozent gestiegen. In den meisten Ländern hatte dies jedoch keine Verringerung der öffentlichen Ausgaben für den Tertiärbereich zur Folge. 


\section{Abbildung B4.1.}

Verteilung der öffentlichen und privaten Ausgaben für Bildungseinrichtungen, nach Bildungsbereichen (1999)

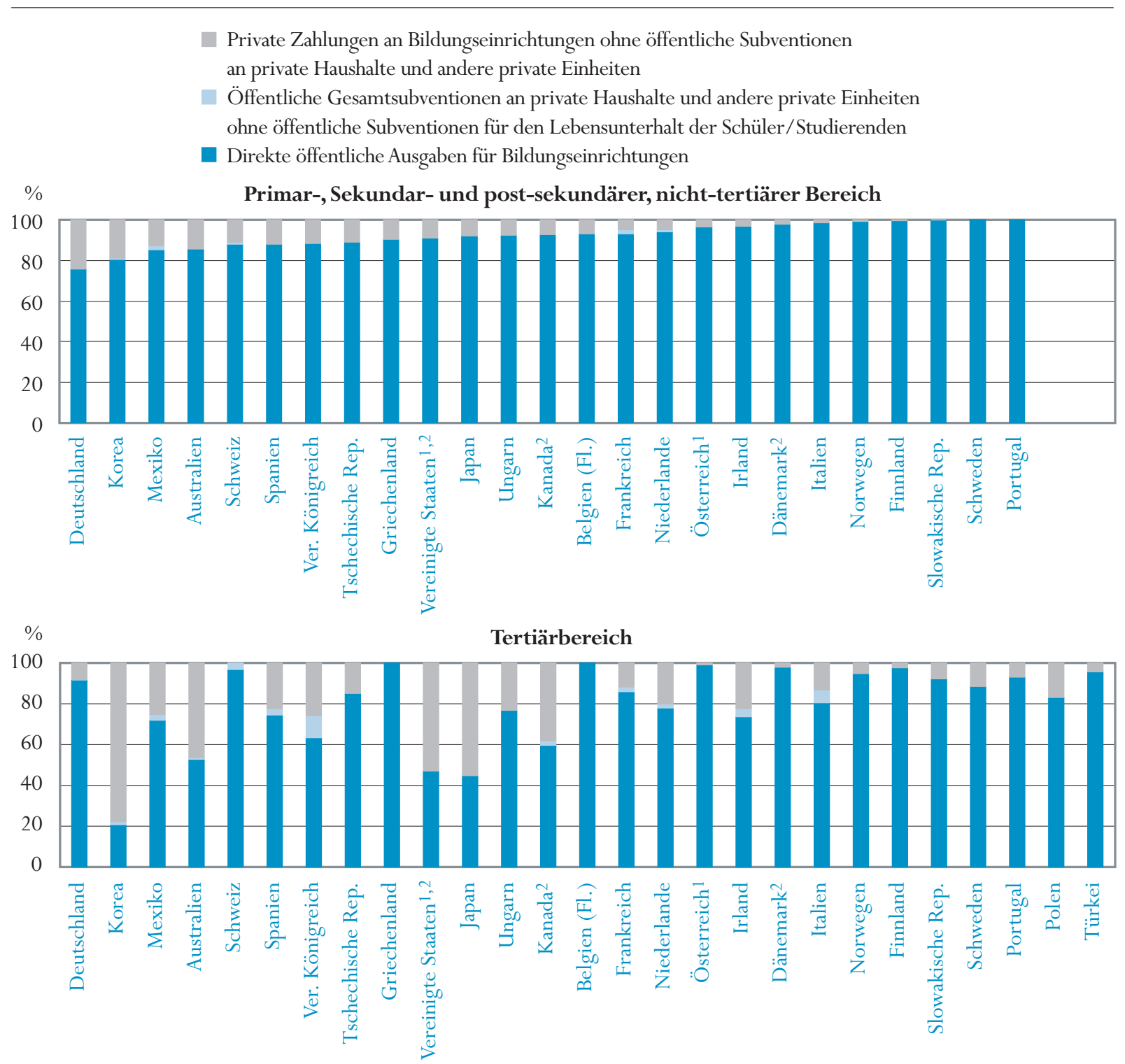

1. Öffentliche Gesamtsubventionen teilweise in privaten Zahlungen enthalten.

2. Post-sekundärer, nicht-tertiärer Bereich im Tertiärbereich enthalten oder fehlt.

Anordnung der Länder in aufsteigender Reihenfolge des Anteils der direkten öffentlichen Ausgaben im Primar-, Sekundar- und postsekundären, nicht-tertiären Bereich.

Quelle: OECD. Tabelle B4.2. Hinweise s. Anhang 3 (www.oecd.org/els/education/eag2002). 
Dieser Indikator beschreibt die relativen Anteile öffentlicher und privater Investitionen in Bildungseinrichtungen... ... und deren Veränderung gegenüber 1995.

\section{Politischer Hintergrund}

In vielen OECD-Ländern ist die Beteiligung sowohl der Teilnehmer am Bildungssystem als auch der Gesellschaft als Ganzes an den Kosten des Bildungssystems Gegenstand intensiver Debatten. Die Frage nach der Aufteilung der Kosten stellt sich besonders zu Beginn und Ende des Bildungsweges, d.h. im Elementar- und Tertiärbereich, wo eine vollständige oder fast vollständige Finanzierung durch öffentliche Mittel weniger üblich ist.

Da neue Interessentengruppen eine zunehmende Bildungsbeteiligung im Rahmen neuer Bildungsangebote aufweisen und aufgrund einer steigenden Anzahl neuer Anbieter im Bildungsbereich gleichzeitig größere Wahlmöglichkeiten bestehen, gehen die Regierungen neue Partnerschaften zur Mobilisierung der notwendigen Ressourcen für die Finanzierung von Bildung ein. Neue bildungspolitische Ansätze sollen es den verschiedenen Akteuren und Beteiligten ermöglichen, die Bildungsangebote stärker in Anspruch zu nehmen und Kosten und Nutzen gerechter aufzuteilen.

Dementsprechend wird inzwischen die Finanzierung durch öffentliche Mittel in zunehmendem Maße als nur ein, wenn auch sehr wichtiger Teil, der Bildungsfinanzierung angesehen, während die Finanzierung aus privaten Quellen zunehmend an Bedeutung gewinnt. Einigen Beteiligten ist es ein Anliegen, dass sich das Gleichgewicht zwischen öffentlicher und privater Finanzierung nicht so stark verschiebt, dass potentielle Bildungsteilnehmer dadurch eher vom Lernen ferngehalten als ihm zugeführt werden.

\section{Ergebnisse und Erläuterungen}

\section{Was dieser Indikator aufzeigt und was nicht}

Die öffentliche Hand kann ihre Mittel entweder direkt für Bildungseinrichtungen ausgeben oder sie zur Gewährung von Subventionen für Bildungszwecke an private Einheiten nutzen. Im Zusammenhang mit den öffentlichen und privaten Anteilen an den Bildungsausgaben ist daher zwischen der originären Herkunft der Mittel und den direkten Endverbrauchern von bildungsbezogenen Sachund Dienstleistungen zu unterscheiden.

Erfasste Daten

(Erklärungen s. S. 160)

Zu den originär öffentlichen Ausgaben gehören sowohl die direkten öffentlichen Ausgaben für Bildungseinrichtungen als auch Transferzahlungen an den privaten Sektor. Um den Umfang der öffentlichen Ausgaben zu bestimmen, müssen daher die direkten öffentlichen Ausgaben für Bildungseinrichtungen und die öffentlichen Subventionen für Bildungszwecke zusammengezählt werden. $\mathrm{Zu}$ den originär privaten Mitteln gehören Schul-/Studiengebühren sowie sonstige Zahlungen der Schüler/Studierenden und der privaten Haushalte an Bildungseinrichtungen abzüglich des Anteils an diesen Zahlungen, der durch öffentliche Subventionen ausgeglichen wird.

Die Anteile der öffentlichen und privaten Endfinanzierung sind die Prozentsätze der für Bildungszwecke verfügbaren Mittel, die einerseits 
direkt von öffentlichen und andererseits direkt von privaten Erwerbern von Bildungsdienstleistungen getätigt werden. Die öffentliche Endfinanzierung beinhaltet die direkte öffentliche Beschaffung von Bildungsressourcen sowie Zahlungen an Bildungseinrichtungen und andere private Einheiten. Die private Endfinanzierung beinhaltet Schul-/Studiengebühren sowie andere private Zahlungen an Bildungseinrichtungen (unabhängig davon, ob sie durch öffentliche Subventionen ausgeglichen werden oder nicht).

Nicht alle Ausgaben für bildungsbezogene Sach- und Dienstleistungen fallen in Bildungseinrichtungen an. Beispielsweise erwerben Familien Schulbücher oder Unterrichtsmaterial im Handel oder lassen ihren Kindern Privatunterricht außerhalb von Bildungseinrichtungen zukommen. Im Tertiärbereich machen die Kosten für den Lebensunterhalt sowie für entgangene Verdienste einen beträchtlichen Teil der Bildungskosten aus. Sämtliche außerhalb von Bildungseinrichtungen anfallenden Kosten bleiben bei diesem Indikator außer Acht, auch wenn diese Ausgaben öffentlich subventioniert werden. Die öffentlichen Subventionen für Bildungskosten außerhalb von Bildungseinrichtungen werden in den Indikatoren B3 und B5 behandelt.

\section{Der Anteil der öffentlichen und privaten Ausgaben für Bildungseinrichtungen}

Schulen, Hochschulen und andere Bildungseinrichtungen werden immer noch überwiegend aus öffentlichen Mitteln finanziert, obwohl es einen erheblichen und zunehmenden Anteil privater Finanzierung gibt. Im Durchschnitt aller OECD-Länder stammen 88 Prozent aller Mittel für Bildungseinrichtungen direkt aus öffentlichen Quellen. Zusätzlich erreichen 0,7 Prozent die Bildungseinrichtungen nicht direkt, sondern über die privaten Haushalte als öffentliche Unterstützungsleistungen (Tabelle B4.1).

Bei den OECD-Ländern, für die Daten vorgelegt wurden, reicht der Anteil der privaten Mittel für Bildungseinrichtungen, einschließlich staatlichsubventionierter privater Mittel, von ca. 3 Prozent und weniger in Finnland, Norwegen, Portugal, Schweden, der Slowakischen Republik und der Türkei bis zu einem Anteil zwischen 22 und 40 Prozent in Australien, Deutschland, Japan, Korea und den Vereinigten Staaten (Tabelle B4.1).

Investitionen in den Elementarbereich sind von essentieller Bedeutung, um ein solides Fundament für lebenslanges Lernen aufzubauen und um einen gerechten Zugang zum Lernangebot später in der Schule zu gewährleisten. Im Elementarbereich reicht der private Anteil an den Gesamtausgaben für Bildungseinrichtungen von 5 Prozent und weniger in Belgien (fläm.), Frankreich, Italien, den Niederlanden, der Schweiz, der Slowakischen Republik und dem Vereinigten Königreich über 37 Prozent und mehr in Australien und Deutschland bis zu über 50 Prozent in Irland, Japan und Korea (Tabelle B4.2).

Im Primar-, Sekundar- und post-sekundären, nicht-tertiären Bereich beträgt der Anteil der privaten Mittel bei der Finanzierung in Australien, Mexiko,
Bildungseinrichtungen werden immer noch überwiegend aus öffentlichen Mitteln finanziert,...
Im Elementarbereich beläuft sich der private Anteil an den Gesamtausgaben für Bildungseinrichtungen im Durchschnitt auf 18 Prozent. In Irland, Japan und Korea liegt dieser Anteil bei über 50 prozent. 
Es gibt auch Unterschiede zwischen der Finanzierung des Primar- und Sekundarbereichs einerseits und des Tertiärsbereichs andererseits.

In einigen $O E C D$ Ländern übernimmt die öffentliche Hand den Grofteil der Kosten für den Primar-, Sekundarund post-sekundären, nicht-tertiären

Bereich, überlässt jedoch den Betrieb der Bildungseinrichtungen dem privaten Sektor...

... in dem Bestreben, eine größsere Bandbreite an Lernmöglichkeiten zur Verfügung zu stellen, ohne Schülern aus einkommensschwachen Familien den Zugang zu Bildung zu erschweren.

Eine alternative Form der öffentlichen Endfinanzierung sind Transferzahlungen öffentlicher Mittel an private Einrichtungen. der Schweiz, Spanien, der Tschechischen Republik und dem Vereinigten Königreich zwischen 10 und 18 Prozent und in Deutschland und Korea mehr als 18 Prozent (Abb. B4.1).

Während in den meisten OECD-Ländern der Großteil der privaten Bildungsausgaben in Form von Studien- und sonstigen Gebühren von den privaten Haushalten an tertiäre Einrichtungen fließt, entfällt in Deutschland und der Schweiz fast der gesamte Anteil der privaten Ausgaben auf Beiträge der Wirtschaft zum dualen System der Berufsausbildung im Sekundarbereich II und dem post-sekundären, nicht-tertiären Bereich.

Neue Finanzierungsstrategien zielen nicht nur darauf ab, die Finanzierung durch öffentliche und private Mittel auf eine breitere Basis zu stellen, sondern auch darauf, das Bildungsangebot auszuweiten und die Wirtschaftlichkeit der Schulen zu verbessern. Zwar wird in den meisten OECD-Ländern die Bildung im öffentlich finanzierten Primar-, Sekundar- und post-sekundären, nicht-tertiären Bereich auch von öffentlichen Einrichtungen organisiert und bereitgestellt, es gibt aber auch eine nennenswerte Anzahl von OECD-Ländern, in denen die öffentlichen Mittel im Endeffekt an private Einrichtungen oder direkt an die privaten Haushalte gehen, damit diese die betreffenden Mittel an die Bildungseinrichtungen ihrer Wahl weiterleiten. Im erstgenannten Fall kann die Endfinanzierung und Bereitstellung von Bildungsangeboten als eine Art Auftragsvergabe durch die öffentliche Hand an nicht-öffentliche Einrichtungen betrachtet werden, während im letztgenannten Fall den Schülern und ihren Familien die Entscheidung überlassen wird, welche Art von Einrichtung ihren Bedürfnissen am besten gerecht wird.

Im Durchschnitt besuchen in den OECD-Ländern mehr als 10 Prozent der Schüler im Primar-, Sekundar- und post-sekundären, nicht-tertiären Bereich private Bildungseinrichtungen, die überwiegend öffentlich finanziert werden. In Belgien und den Niederlanden besucht sogar die Mehrheit der Schüler im Primar-, Sekundar- und post-sekundären, nicht-tertiären Bereich staatlichsubventionierte private Einrichtungen, und in Australien, Frankreich, Korea, Spanien und dem Vereinigten Königreich beträgt der Anteil immerhin noch über 20 Prozent (Abb. B4.2). Obwohl diese Einrichtungen privat geführt werden, kann die finanzielle Unterstützung seitens der öffentlichen Hand an bestimmte Auflagen gebunden sein, z.B. dass die Lehrer bestimmte Mindestqualifikationen erfüllen oder dass sich die Schüler für ihren Abschluss einer staatlich geregelten Prüfung unterziehen müssen.

Im Durchschnitt gehen in den OECD-Ländern 10 Prozent der für Bildungseinrichtungen vorgesehenen öffentlichen Mittel an private Bildungseinrichtungen (Tabelle B4.3). In den Niederlanden, wo die Hauptquelle für Mittel letztendlich der Gesamthaushalt ist, werden 71 Prozent der öffentlichen Mittel für Einrichtungen des Primar-, Sekundar- und postsekundären, nicht-tertiären Bereichs und 36 Prozent der öffentlichen Mittel für Einrichtungen des Tertiärbereichs den privaten Bildungseinrichtungen vom 
Staat in Form von Transferzahlungen zur Verfügung gestellt. In Belgien werden 55 Prozent der Mittel für Bildungseinrichtungen an private Einrichtungen im Primar-, Sekundar- und post-sekundären, nicht-tertiären Bereich transferiert (Abbildung B4.2).

In Australien, Frankreich, Korea, Spanien und dem Vereinigten Königreich beträgt der Anteil der öffentlichen Transferzahlungen an private Einrichtungen im Primar-, Sekundar- und post-sekundären Bildungsbereich zwischen 10 und 21 Prozent.

Im Primar-, Sekundar- und post-sekundären, nicht-tertiären Bildungsbereich sind private Bildungseinrichtungen, die überwiegend von privaten Haushalten finanziert werden, weitaus weniger üblich und gelten bisweilen als Einrichtungen, die den Zugang von Schülern aus einkommensschwachen Familien einschränken. Nur in Mexiko und den Vereinigten Staaten werden rund 10 Prozent der Schüler in privaten Einrichtungen unterrichtet, die überwiegend aus nicht-subventionierten Mitteln der privaten Haushalte finanziert werden (Abb. B4.2).

Daher belaufen sich in den OECD-Ländern die von der öffentlichen Hand an den Privatsektor geleisteten Transferzahlungen im Primar-, Sekundar- und postsekundären, nicht-tertiären Bildungsbereich (s. Tabelle B4.3 und Indikator B5) durchschnittlich auf 3,5 Prozent der öffentlichen Gesamtausgaben für Bildung. Lediglich in Dänemark und Schweden liegen sie bei mehr als 10 Prozent. Im Tertiärbereich ist es weitaus üblicher, dass private Haushalte bzw. Studierende öffentliche Mittel erhalten. In Australien, Dänemark, Kanada, Neuseeland, den Niederlanden, Norwegen, Schweden und dem Vereinigten Königreich gehen 20 Prozent und mehr der für Bildungseinrichtungen im Tertiärbereich vorgesehenen öffentlichen Mittel als Transferzahlungen an den privaten Sektor.

Mit Ausnahme von vier Ländern ist der Anteil der privaten Mittel an der Bildungsfinanzierung im tertiären Bereich weitaus höher als im Primar-, Sekundar- und post-sekundären, nicht-tertiären Bereich. Während das Bildungsangebot im Primar-, Sekundar- und post-sekundären, nicht-tertiären Bereich normalerweise als öffentliches Gut angesehen wird, dessen Früchte auch hauptsächlich wieder der Öffentlichkeit zugute kommen, lässt der hohe private Nutzen im tertiären Bereich in Form besserer Beschäftigungsmöglichkeiten und höherer Einkommen (s. Indikatoren A3 und A13) den Schluss zu, dass eine stärkere Beteiligung des Einzelnen an den Kosten der tertiären Bildung gerechtfertigt sein könnte, vorausgesetzt natürlich, dass die öffentliche Hand gewährleisten kann, dass die Finanzierung für die Studierenden unabhängig von ihren wirtschaftlichen Verhältnissen gesichert ist (s. auch Indikator B5).

Der Finanzierungsanteil für tertiäre Einrichtungen, der von Privatpersonen, Unternehmen und sonstigen privaten Einheiten getragen wird, einschließlich staatlich-subventionierter privater Zahlungen, reicht von 3 Prozent und weniger in Belgien (fläm.), Dänemark, Finnland, Griechenland, Österreich
Im Primar-, Sekundarund post-sekundären, nicht-tertiären Bereich sind private Einrichtungen, die überwiegend aus Mitteln der privaten Haushalte finanziert werden, weitaus weniger üblich.

Tendenziell beziehen tertiäre Einrichtungen einen wesentlich höheren Anteil ihrer Finanzmittel aus privaten Quellen, ... 


\section{Abbildung B4.2.}

Öffentliche Unterstützungsleistungen für private Bildungseinrichtungen

Anteil öffentlicher Bildungsausgaben für private Bildungseinrichtungen

Anteil an Schülern/Studierenden in privaten Bildungseinrichtungen
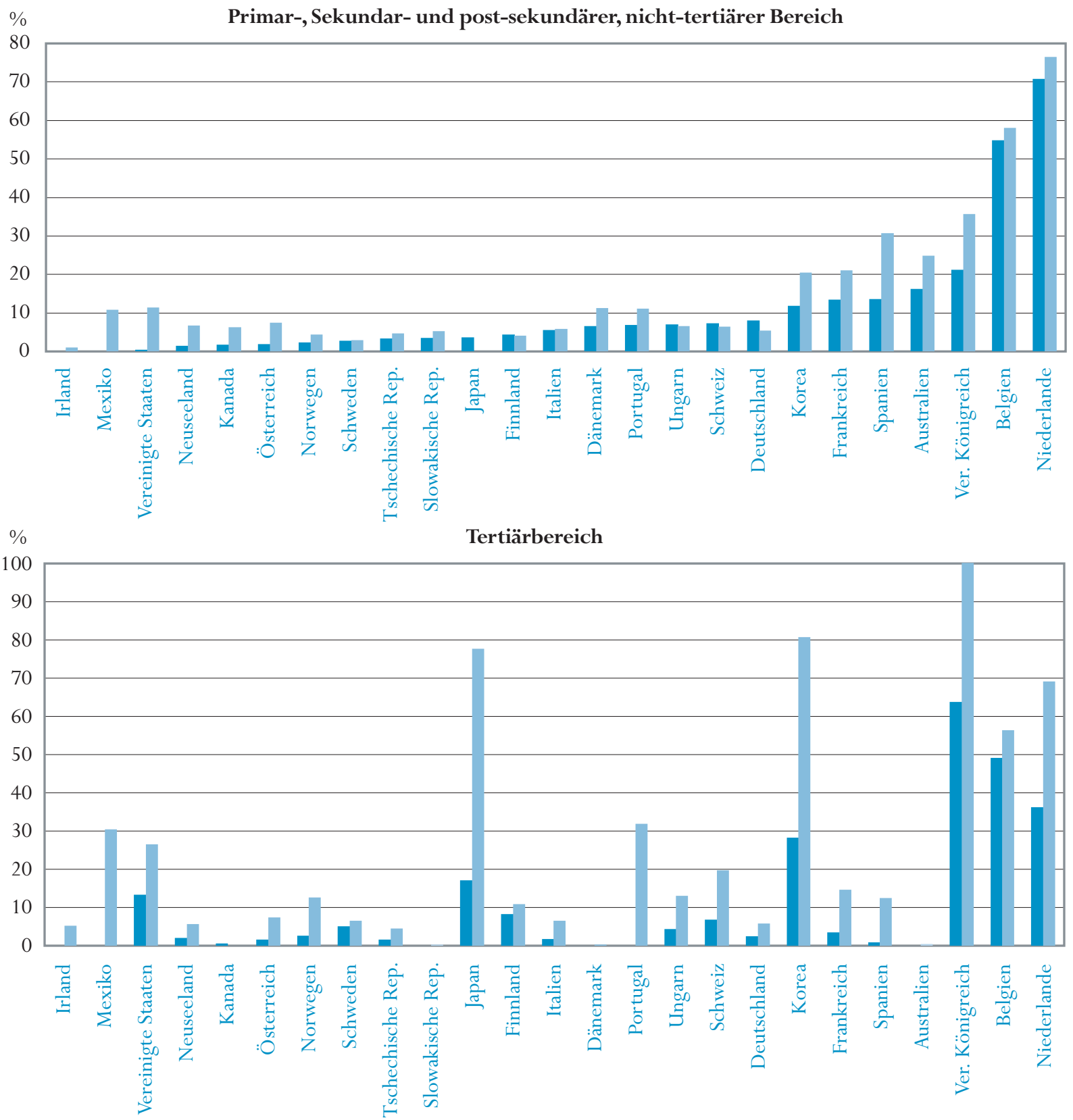

Anordnung der Länder in aufsteigender Reihenfolge des Anteils der öffentlichen Transferzahlungen an private Bildungseinrichtungen im Primar-, Sekundar- und post-sekundären, nicht-tertiären Bereich.

Quelle: OECD. Tabelle B4.3, C2.3 und C2.4. Hinweise s. Anhang 3 (www.oecd.org/els/education/eag2002). 
und der Schweiz bis zu über einem Drittel in Australien, Japan, Kanada, Korea, dem Vereinigten Königreich und den Vereinigten Staaten. In Japan und den Vereinigten Staaten beträgt der Anteil der privaten Endfinanzierung mehr als 50 Prozent, und in Korea liegt dieser Anteil sogar bei über 78 Prozent (Abb. B4.1). Mehr als 80 Prozent der koreanischen Studierenden sind an privaten Hochschulen eingeschrieben, deren Etats zu über 95 Prozent aus Studiengebühren finanziert werden.

Die Höhe der von den Studierenden und ihren Familien selbst aufgebrachten Mittel für Studiengebühren und sonstige bildungsbezogene Ausgaben ist je nach Steuer- und Ausgabenpolitik sowie der Bereitschaft des Staates, Studierende finanziell zu unterstützen, in den einzelnen OECD-Ländern recht unterschiedlich. Diese Bereitschaft des Staates richtet sich wiederum nach der Art des Studiums (Voll- oder Teilzeitstudium), dem Alter der Studierenden und danach, ob diese zu Hause oder außerhalb wohnen. Allerdings ist festzustellen, dass sich die Richtlinien für die Feststellung der Anspruchsberechtigung für solche Unterstützungsleistungen in gewisser Hinsicht lockern. Ältere Studierende, deren Zahl stetig zunimmt, haben eher schon einen eigenen Hausstand gegründet und geben einem Teilzeit- bzw. Fernstudium gegenüber einem Vollzeit-Präsenzstudium den Vorzug.

\section{Veränderungen bei den öffentlichen und privaten Bildungsausgaben}

Die private Direktfinanzierung von Bildungseinrichtungen hat zwischen 1995 und 1999 in neun von 16 OECD-Ländern mit vergleichbaren Daten in absoluten Zahlen um mehr als 10 Prozent zugenommen. Diese Zunahme reicht von ungefähr 2 Prozent in Frankreich und Deutschland bis zu 100 Prozent und mehr in Portugal und Schweden. Nur in drei OECD-Ländern - Norwegen, Österreich und der Tschechischen Republik - ist der Privatanteil um mehr als 5 Prozent zurückgegangen (Abb. B4.3).

In vier OECD-Ländern, Australien, Dänemark, Kanada und den Niederlanden, hat zwischen 1995 und 1999 die private Finanzierung des Primar-, Sekundarund post-sekundären, nicht-tertiären Bereichs deutlich zugenommen. In Australien, Kanada und den Niederlanden stiegen die privaten Mittel um 16 Prozent bis 37 Prozent, wobei sich ihr Anteil in diesen drei Ländern auf mehr als 5 Prozent der gesamten Ausgaben für Bildungseinrichtungen beläuft (Abb. B4.3 und Tabelle B4.2).

In vielen OECD-Ländern ist die wachsende Teilnahme an tertiärer Bildung (Indikator C2) auf eine starke private und gesellschaftliche Nachfrage zurückzuführen. Aber nicht nur viele Strukturen und Bildungsgänge der tertiären Einrichtungen stammen aus Zeiten geringerer Nachfrage, sondern auch deren Finanzierungsmechanismen. Mit der zunehmenden Nachfrage nach tertiärer Bildung hat sich in einer Vielzahl von OECD-Ländern auch der Anteil der von den Familien getragenen finanziellen Belastung erhöht. In allen OECDLändern, für die Zahlen vorliegen, mit Ausnahme von Kanada und Frankreich,
... allerdings reicht

der Anteil der

privaten Finanzierung einschliejlich staatlichsubventionierter privater Zahlungen, von 3 Prozent und weniger in Belgien (fläm.), Dänemark, Finnland, Griechenland, Österreich und der Schweiz bis zu 78 Prozent in Kored.

Das Ausmajs der privaten Finanzierung von Bildung hat zugenommen.

In Australien, Dänemark und Kanada hat die private Finanzierung des Primar-, Sekundar-und post-sekundüren, nichttertiären Bereichs stärker zugenommen als die öffentliche Finanzierung, die markantesten Veränderungen zeigen sich jedoch im Tertiärbereich, wo ein dramatischer Anstieg der Bildungsbeteiligung mit einem steigenden Anteil der privaten Finanzierung einherging,... 


\section{Abbildung B4.3.}

Index der Veränderung der öffentlichen und privaten Ausgaben für

Bildungseinrichtungen zwischen 1995 und 1999 (1995 = 100)

Öffentliche Ausgaben (linke Skala) — Private Ausgaben (linke Skala) • Anteil der privaten Ausgaben (rechte Skala)

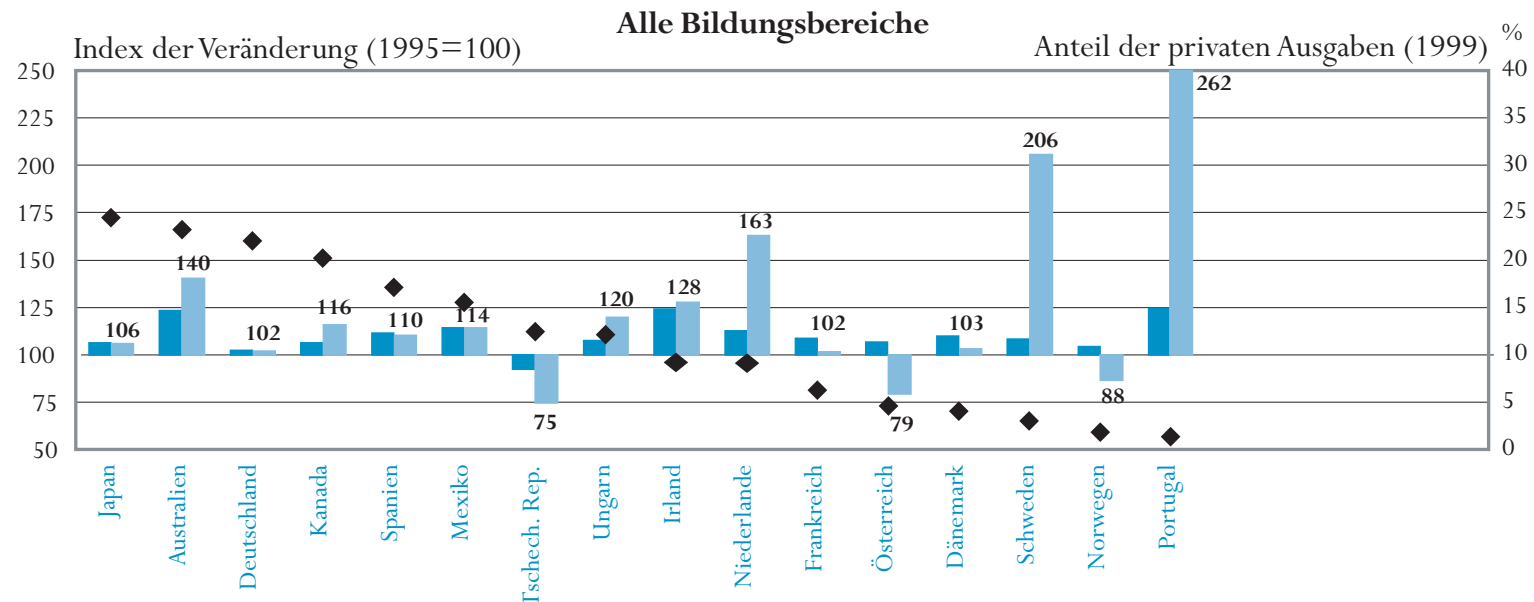

Primar-, Sekundar- und post-sekundärer, nicht-tertiärer Bereich
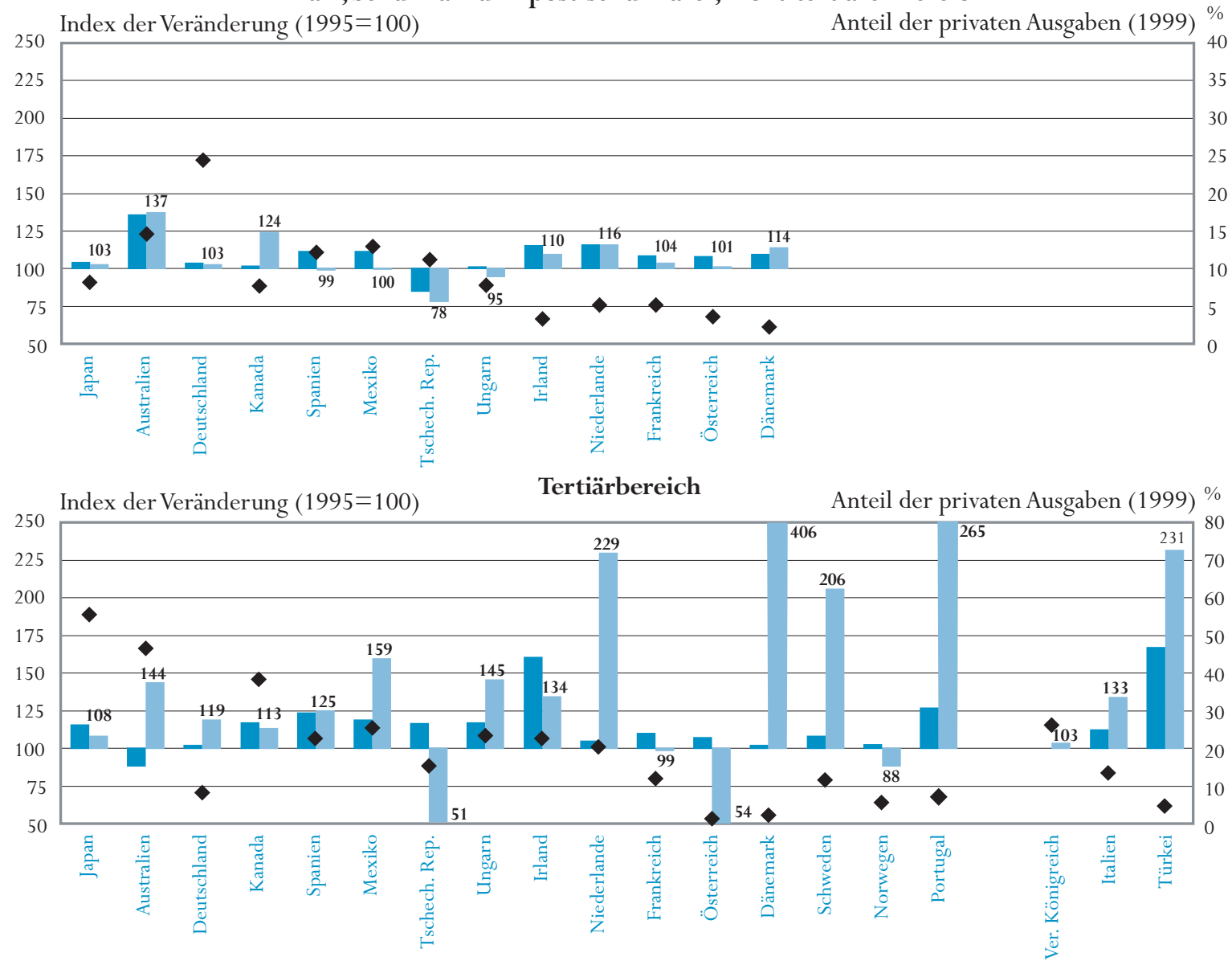

Hinweis: Länder mit einem Anteil der Gesamtmittel aus privaten Quellen von 1 Prozent oder weniger sind in der Abbildung nicht erfasst.

Anordnung der Länder in absteigender Reihenfolge des Anteils der privaten Ausgaben in 1999 für alle Bildungsbereiche.

Quelle: OECD. Tabellen B2.2, B4.1 und B4.2. Hinweise s. Anhang 3 (www.oecd.org/els/education/eag2002). 
ist die Veränderung der privaten Ausgaben für tertiäre Einrichtungen wesentlich größer als bei den Ausgaben für Bildungseinrichtungen des Primar-, Sekundarund post-sekundären, nicht-tertiären Bereichs.

Die steigenden Ausgaben der privaten Haushalte für den Tertiärbereich sind auf einen oder mehrere der nachstehenden vier Faktoren zurückzuführen: 1.) eine höhere Bildungsteilnahme, 2.) steigende oder neu eingeführte Gebühren, Kosten oder Beiträge, 3.) einen Anstieg der Kosten für bildungsbezogene Sachund Dienstleistungen, die nicht Kosten für Bildungseinrichtungen sind, und 4.) ein steigender Zulauf zu privaten Institutionen mit höheren Gebühren.

Zehn von 19 OECD-Ländern verzeichneten zwischen 1995 und 1999 einen Anstieg der privaten Finanzierung tertiärer Einrichtungen um mehr als 30 Prozent. In einigen OECD-Ländern, insbesondere in Australien, Mexiko, den Niederlanden und Ungarn, gab es zwischen 1995 und 1999 eine klare Verschiebung bei den jeweiligen Anteilen der privaten und öffentlichen Mittel für tertiäre Bildungseinrichtungen. In Australien erhöhte sich der Privatanteil der Finanzierung in diesem Zeitraum von 36 auf 48 Prozent, in Mexiko von 23 auf 28 Prozent, in den Niederlanden von 12 auf 22 Prozent und in Ungarn von 20 auf 23 Prozent. Allerdings gab es auch hier Ausnahmen: während in Irland die private Finanzierung tertiärer Einrichtungen zwischen 1995 und 1999 um 34 Prozent zunahm, stieg der Anteil der öffentlichen Mittel um einen noch höheren Betrag, nämlich um ganze 60 Prozent. In Österreich und der Tschechischen Republik hat sich die private Finanzierung im tertiären Bereich von 1995 bis 1999 um ungefähr die Hälfte verringert. Entsprechend ging der Anteil der privaten Mittel für Bildungseinrichtungen im Verhältnis zu den Gesamtausgaben für Bildung in der Tschechischen Republik von nahezu 29 Prozent im Jahre 1995 auf weniger als 15 Prozent zurück und in Österreich von 2,4 auf 1,3 Prozent (Abb. B4.3 und Tabelle B4.2)

Es bleibt festzuhalten, dass der Anstieg der privaten Bildungsausgaben nicht generell mit einem Rückgang der öffentlichen Bildungsfinanzierung einhergegangen ist, weder im Primar-, Sekundar- und post-sekundären, nicht-tertiären Bereich noch im Tertiärbereich. Das Gegenteil ist der Fall. Die Abbildung B4.3 zeigt, dass die öffentlichen Bildungsinvestitionen in fast allen OECD-Ländern, für die Vergleichsdaten für 1995 und 1999 vorliegen, unabhängig von den Veränderungen bei der Privatfinanzierung zugenommen haben. Tatsächlich hatten sogar einige der OECD-Länder mit dem höchsten Anstieg der privaten Finanzierung auch den höchsten Anstieg der öffentlichen Bildungsfinanzierung zu verzeichnen. Dies deutet darauf hin, dass höhere private Ausgaben für die tertiäre Bildung die öffentlichen Investitionen eher ergänzen als ersetzen.

Neue Finanzierungsstrategien sollen nicht nur dazu beitragen, die erforderliche Finanzierung durch vermehrte Einbeziehung sowohl öffentlicher als auch privater Quellen auf eine breitere Grundlage zu stellen, sondern auch das
... wofür hauptsächlich

vier Faktoren

verantwortlich sind.

In 10 von 19 OECD-

Ländern stieg der

private Anteil an der

Finanzierung des

Tertiärbereichs zwischen

1995 und 1999 um mehr

als 30 Prozent,... 
Viele OECD-Länder, in denen die Studierenden

oder ihre Familien einen Kostenbeitrag zur tertiären Bildung leisten, verzeichnen auch die höchsten Beteiligungsquoten, ... .. wohingegen mehrere

OECD-Länder mit überwiegend öffentlicher

Finanzierung nur eine geringe Bildungsteilnahme aufweisen.

\section{Die Daten beziehen sich auf das Haushaltsjahr 1999 und beruhen auf der von der OECD im Jahre 2001 durchgeführten VOE-Datenerhebung zur Bildungsstatistik (Einzelheiten s. Anhang 3).}

Die Daten für das Haushaltsjahr 1995

beruhen auf einer speziellen Erhebung in den OECD-Ländern aus dem Jahre 2000.
Verhalten der Studierenden im Hinblick auf ein kosteneffizienteres Studium zu beeinflussen. Die genaue Wirkung von Studiengebühren auf das Verhalten der Studierenden ist schwer abzuschätzen, da es u.a. nicht möglich ist, sie getrennt von der Gewährung von Zuschüssen, steuerlichen Maßnahmen oder indirekten Subventionen in Form von Darlehen zu betrachten. Aber viele OECD-Länder, in denen die Studierenden und ihre Familien überdurchschnittlich viel für die tertiäre Bildung ausgeben, weisen auch die höchste Bildungsteilnahme und die höchsten Abschlussquoten im tertiären Bereich auf (Indikatoren A2 und C2).

Umgekehrt haben die sechs OECD-Länder mit den niedrigsten Zugangsquoten zum Tertiärbereich A - Dänemark, Deutschland, Mexiko, die Schweiz, die Tschechische Republik und die Türkei - nur einen privaten Finanzierungsanteil an den gesamten Bildungskosten der tertiären Einrichtungen von 2 bis 28 Prozent (Tabellen B4.2, C2.1). Es ist daher wohl nicht davon auszugehen, dass die finanzielle Beteiligung der Nutznießer tertiärer Bildungseinrichtungen eine wirtschaftliche Barriere für den Zugang zur Hochschulausbildung darstellt vorausgesetzt natürlich, dass die Länder mit Hilfe geeigneter Strategien dafür sorgen, dass Studierende aller Einkommensgruppen Zugang zu entsprechenden Finanzierungsmöglichkeiten haben.

\section{Definitionen und angewandte Methodik}

Die Anteile der öffentlichen und privaten Mittel an der Finanzierung von Bildungseinrichtungen sind angegeben in Prozent der gesamten Bildungsausgaben, die im öffentlichen bzw. privaten Sektor entstehen bzw. anfallen. $\mathrm{Zu}$ den privaten Mitteln zählen alle direkten Ausgaben für Bildungseinrichtungen, unabhängig davon, ob diese teilweise durch öffentliche Mittel subventioniert werden oder nicht. Die in den privaten Mitteln enthaltenen öffentlichen Subventionen an die privaten Haushalte werden getrennt ausgewiesen.

Die Etats der Bildungseinrichtungen betreffen teilweise auch zusätzliche Dienstleistungen für Studierende. Dies sind im Allgemeinen soziale Dienste für Studierende wie Unterkunft und Verpflegung sowie Transport. Ein Teil der Kosten für diese Leistungen wird über Beiträge der Studierenden gedeckt und ist in diesem Indikator mit erfasst.

Die Veränderungen bei der privaten und öffentlichen Finanzierung von Bildungseinrichtungen sind durch einen Index der Veränderung dargestellt. Der Anteil der privaten Finanzierung im Jahre 1995 wird dem entsprechenden Anteil in 1999 gegenübergestellt. Die Daten für die Ausgaben des Jahres 1995 wurden im Rahmen einer speziellen Erhebung im Jahre 2000 ermittelt, in der die Ausgaben für 1995 entsprechend den Definitionen und den Methoden der 1999 durchgeführten UOE-Datenerhebung angepasst wurden.

Es ist zu beachten, dass eine starke Zu- oder Abnahme der privaten Finanzierung (Abb. B4.3) in den OECD-Ländern, in denen der Privatanteil an den Gesamtausgaben gering ist, möglicherweise nur eine geringe zusätzliche 
Belastung für die privaten Haushalte ausmacht, während eine vergleichsweise geringfügige Veränderung im Finanzierungsverhalten ausgehend von einem hohen Anteil privater Mittel beträchtliche zusätzliche Mittel für die Bildungseinrichtungen bringen kann.

Das Glossar am Ende dieser Veröffentlichung enthält eine Definition der Begriffe „öffentliche Bildungseinrichtungen“, „staatlich-subventionierte private Bildungseinrichtungen“ und „unabhängige private Bildungseinrichtungen“.

Es ist zu beachten, dass die in früheren Ausgaben dieser Veröffentlichung aufgeführten Zahlen und Daten möglicherweise nicht immer mit denen der Ausgabe 2002 vergleichbar sind, da sich Änderungen in den Definitionen und Erhebungsbereichen ergeben haben. Diese wurden aufgrund der OECD Expenditure Comparability Study vorgenommen (Einzelheiten zu den Änderungen s. Anhang 3 unter www.oecd.org/els/education/eag2002). 
Tabelle B4.1.

Relative Anteile öffentlicher und privater Ausgaben für Bildungseinrichtungen aller Bildungsbereiche (1995, 1999) Verteilung der Mittel für Bildungseinrichtungen aus öffentlichen und privaten Quellen nach Transferzahlungen aus öffentlichen Quellen, nach Jahr

\begin{tabular}{|c|c|c|c|c|c|}
\hline \multicolumn{3}{|c|}{1999} & \multicolumn{3}{|c|}{1995} \\
\hline $\begin{array}{c}\text { aus öffentlichen } \\
\text { Quellen }\end{array}$ & $\begin{array}{c}\text { aus privaten } \\
\text { Quellen }\end{array}$ & $\begin{array}{c}\text { aus privaten } \\
\text { Quellen: hiervon } \\
\text { bezuschusst }\end{array}$ & $\begin{array}{c}\text { aus öffentlichen } \\
\text { Quellen }\end{array}$ & $\begin{array}{c}\text { aus privaten } \\
\text { Quellen }^{1}\end{array}$ & $\begin{array}{c}\text { aus privaten } \\
\text { Quellen: hiervon } \\
\text { bezuschusst }\end{array}$ \\
\hline 76.5 & 23.5 & 0.3 & 78.7 & 21.3 & 3.0 \\
\hline 95.4 & 4.6 & $\mathrm{x}$ & 93.9 & 6.1 & 1.4 \\
\hline 95.0 & 5.0 & $\mathrm{~m}$ & $\mathrm{~m}$ & $\mathrm{~m}$ & $\mathrm{~m}$ \\
\hline 79.8 & 20.2 & $\mathrm{~m}$ & 82.3 & 17.7 & $\mathrm{a}$ \\
\hline 87.6 & 12.4 & $\mathrm{n}$ & 85.0 & 15.0 & 6.2 \\
\hline 96.0 & 4.0 & $\mathrm{~m}$ & 95.7 & 4.3 & $\mathrm{n}$ \\
\hline 97.8 & 2.2 & $\mathrm{n}$ & $\mathrm{m}$ & $\mathrm{m}$ & $\mathrm{m}$ \\
\hline 91.9 & 8.1 & 1.8 & 91.4 & 8.6 & 2.6 \\
\hline 77.9 & 22.1 & 0.1 & 77.8 & 22.2 & 0.1 \\
\hline 93.4 & 6.6 & $\mathrm{~m}$ & $\mathrm{~m}$ & $\mathrm{~m}$ & $\mathrm{n}$ \\
\hline 87.9 & 12.1 & $\mathrm{n}$ & 89.0 & 11.0 & $\mathrm{n}$ \\
\hline $\mathrm{m}$ & $\mathrm{m}$ & $\mathrm{m}$ & $\mathrm{m}$ & $\mathrm{m}$ & $\mathrm{m}$ \\
\hline 89.6 & 10.4 & 1.2 & 89.8 & 10.2 & $\mathrm{~m}$ \\
\hline 90.3 & 9.7 & 1.1 & $\mathrm{~m}$ & $\mathrm{~m}$ & $\mathrm{~m}$ \\
\hline 75.6 & 24.4 & $\mathrm{a}$ & 75.5 & 24.5 & a \\
\hline 58.7 & 41.3 & 1.1 & $\mathrm{~m}$ & $\mathrm{~m}$ & $\mathrm{~m}$ \\
\hline $\mathrm{m}$ & $\mathrm{m}$ & $\mathrm{m}$ & $\mathrm{m}$ & $\mathrm{m}$ & $\mathrm{m}$ \\
\hline 82.6 & 17.4 & 1.9 & 82.6 & 17.4 & $\mathrm{~m}$ \\
\hline 89.7 & 10.3 & 1.2 & 92.6 & 7.4 & 4.8 \\
\hline $\mathrm{m}$ & $\mathrm{m}$ & $\mathrm{a}$ & $\mathrm{m}$ & $\mathrm{m}$ & $\mathrm{m}$ \\
\hline 98.2 & 1.8 & $\mathrm{n}$ & 97.9 & 2.1 & $\mathrm{~m}$ \\
\hline $\mathrm{m}$ & $\mathrm{m}$ & $\mathrm{m}$ & $\mathrm{m}$ & $\mathrm{m}$ & $\mathrm{m}$ \\
\hline 98.7 & 1.3 & $\mathrm{~m}$ & 99.4 & 0.6 & $\mathrm{~m}$ \\
\hline 97.8 & 2.2 & $\mathrm{~m}$ & $\mathrm{~m}$ & $\mathrm{~m}$ & $\mathrm{~m}$ \\
\hline 82.3 & 17.7 & 0.7 & 82.1 & 17.9 & 0.4 \\
\hline 97.0 & 3.0 & $\mathrm{a}$ & 98.3 & 1.7 & $\mathrm{~m}$ \\
\hline 90.0 & 10.0 & 1.7 & $\mathrm{~m}$ & $\mathrm{~m}$ & $\mathrm{~m}$ \\
\hline 98.8 & 1.2 & $\mathrm{~m}$ & 94.7 & 5.3 & 1.2 \\
\hline 83.7 & 16.3 & 2.2 & $\mathrm{~m}$ & $\mathrm{~m}$ & $\mathrm{~m}$ \\
\hline 75.0 & 25.0 & $\mathrm{x}$ & $\mathrm{m}$ & $\mathrm{m}$ & $\mathrm{m}$ \\
\hline 88.0 & 12.0 & 0.7 & $\sim$ & $\sim$ & $\sim$ \\
\hline 77.2 & 22.8 & 0.1 & $\mathrm{~m}$ & $\mathrm{~m}$ & $\mathrm{~m}$ \\
\hline 55.1 & 44.9 & 1.9 & $\mathrm{~m}$ & $\mathrm{~m}$ & $\mathrm{~m}$ \\
\hline 55.8 & 44.2 & $\mathrm{n}$ & $\mathrm{m}$ & $\mathrm{m}$ & $\mathrm{m}$ \\
\hline 96.2 & 3.8 & $\mathrm{x}$ & $\mathrm{m}$ & $\mathrm{m}$ & $\mathrm{m}$ \\
\hline 64.5 & 35.5 & $\mathrm{~m}$ & $\mathrm{~m}$ & $\mathrm{~m}$ & $\mathrm{~m}$ \\
\hline 80.9 & 19.1 & 2.0 & 80.5 & 19.5 & 1.4 \\
\hline 62.3 & 37.7 & 1.0 & $\mathrm{~m}$ & $\mathrm{~m}$ & $\mathrm{~m}$ \\
\hline 83.7 & 16.3 & $\mathrm{x}$ & $\mathrm{m}$ & $\mathrm{m}$ & $\mathrm{m}$ \\
\hline 56.4 & 43.6 & $\mathrm{x}$ & $\mathrm{m}$ & $\mathrm{m}$ & $\mathrm{m}$ \\
\hline 71.6 & 28.4 & $\mathrm{~m}$ & $\mathrm{~m}$ & $\mathrm{~m}$ & $\mathrm{~m}$ \\
\hline 94.6 & 5.4 & $\mathrm{~m}$ & $\mathrm{~m}$ & $\mathrm{~m}$ & $\mathrm{~m}$ \\
\hline
\end{tabular}

1. Einschließlich Subventionen, die Zahlungen aus öffentlichen Quellen an Bildungseinrichtungen zuzuordnen sind.

2. Post-sekundärer, nicht-tertiärer Bereich im Tertiärbereich enthalten oder fehlt.

3. Post-sekundärer, nicht-tertiärer Bereich sowohl im Sekundarbereich II als auch im Tertiärbereich enthalten.

4. Referenzjahr 1998.

5. Referenzjahr 2000.

* Hinweise s. Anhang 3 (www.oecd.org/els/education/eag2002).

Quelle: OECD. 
Tabelle B4.2.

Relative Anteile öffentlicher und privater Ausgaben für Bildungseinrichtungen $(1995,1999)$

Verteilung der Mittel für Bildungseinrichtungen aus öffentlichen und privaten Quellen nach Transferzahlungen aus öffentlichen Quellen, nach Bildungsbereichen und Jahr

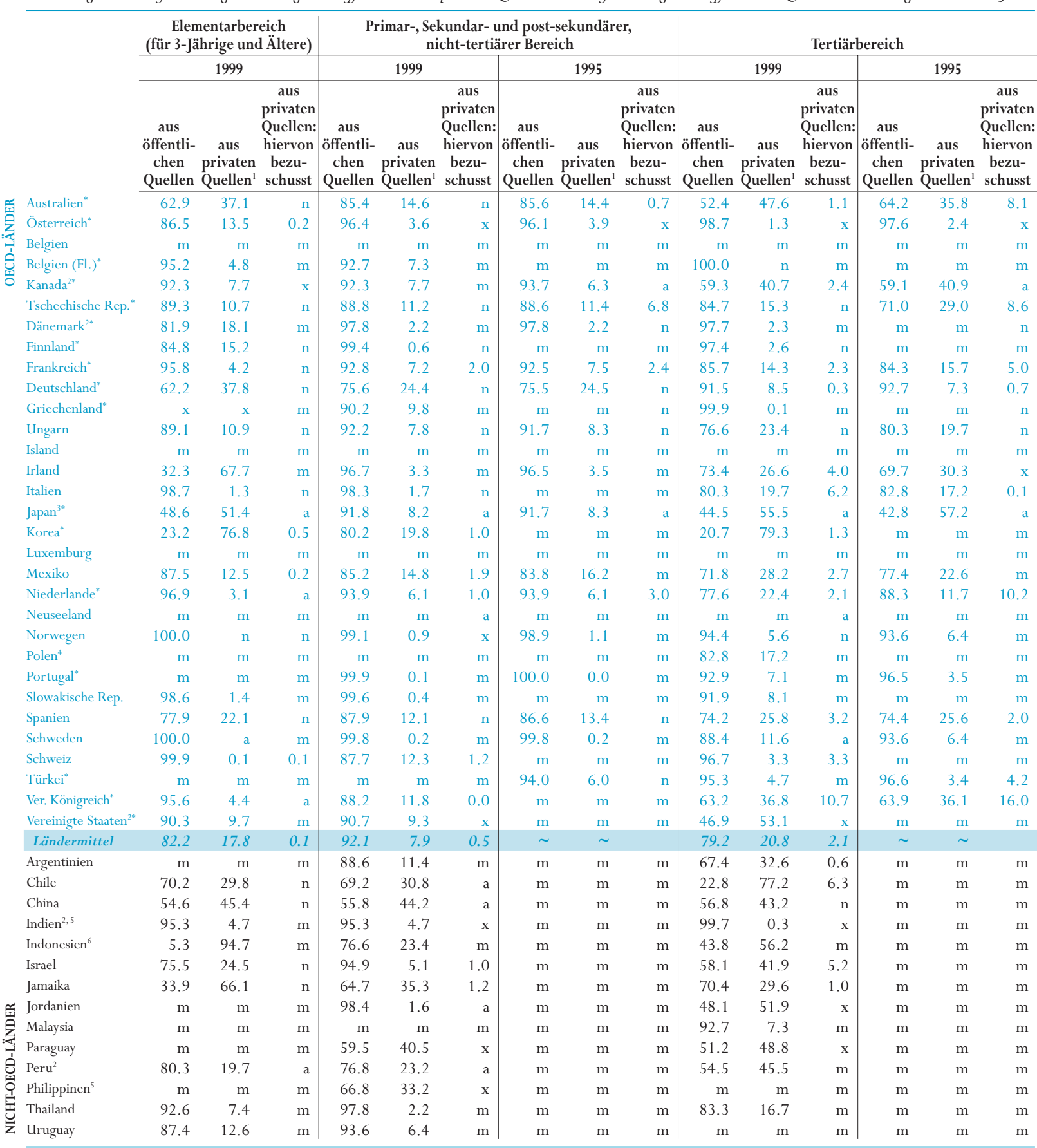

1. Einschließlich Subventionen, die Zahlungen aus öffentlichen Quellen an Bildungseinrichtungen zuzuordnen sind. Um private Mittel ohne Subventionen zu berechnen, müssen die öffentlichen Subventionen (Spalten 3,6,9) von den privaten Mitteln (Spalten 2,5,8) subtrahiert werden. Um die gesamten öffentlichen Mittel (einschließlich öffentlicher Subventionen) zu berechnen, müssen die öffentlichen Subventionen (Spalten 3,6,9) zu den direkten öffentlichen Mitteln (Spalten 1,4,7) hinzugerechnet werden.

2. Post-sekundärer, nicht-tertiärer Bereich im Tertiärbereich enthalten oder fehlt.

3. Post-sekundärer, nicht-tertiärer Bereich sowohl im Sekundarbereich II als auch im Tertiärbereich enthalten.

4. Nur öffentliche Bildungseinrichtungen.

5. Referenzjahr 1998.

6. Referenzjahr 2000.

* Hinweise s. Anhang 3 (www.oecd.org/els/education/eag2002).

Quelle: OECD. 
Tabelle B4.3.

Verteilung der öffentlichen Gesamtausgaben für Bildung (1999)

Öffentliche Ausgaben für Bildungseinrichtungen und öffentliche Transferzahlungen an den privaten Sektor als Prozentsatz der öffentlichen Gesamtausgaben für Bildung, nach Bildungsbereichen

\begin{tabular}{|c|c|c|c|c|c|c|c|c|c|}
\hline & \multicolumn{3}{|c|}{$\begin{array}{l}\text { Primar-, Sekundar- und post-sekundärer, } \\
\text { nicht-tertiärer Bereich }\end{array}$} & \multicolumn{3}{|c|}{ Tertiärbereich } & \multicolumn{3}{|c|}{ Alle Bildungsbereiche zusammen } \\
\hline & $\begin{array}{c}\text { Direkte } \\
\text { öffentliche } \\
\text { Ausgaben für } \\
\text { öffentliche } \\
\text { Bildungsein- } \\
\text { richtungen }\end{array}$ & $\begin{array}{l}\text { Direkte } \\
\text { öffentliche } \\
\text { Ausgaben } \\
\text { für private } \\
\text { Bildungsein- } \\
\text { richtungen }\end{array}$ & $\begin{array}{c}\text { Indirekte } \\
\text { öffentliche } \\
\text { Transfer- und } \\
\text { sonstige Zahl- } \\
\text { ungen an den } \\
\text { privaten Sektor }\end{array}$ & $\begin{array}{c}\text { Direkte } \\
\text { öffentliche } \\
\text { Ausgaben für } \\
\text { öffentliche } \\
\text { Bildungsein- } \\
\text { richtungen }\end{array}$ & $\begin{array}{c}\text { Direkte } \\
\text { öffentliche } \\
\text { Ausgaben } \\
\text { für private } \\
\text { Bildungsein- } \\
\text { richtungen }\end{array}$ & $\begin{array}{c}\text { Indirekte } \\
\text { öffentliche } \\
\text { Transfer- und } \\
\text { sonstige Zahl- } \\
\text { ungen an den } \\
\text { privaten Sektor }\end{array}$ & $\begin{array}{c}\text { Direkte } \\
\text { öffentliche } \\
\text { Ausgaben für } \\
\text { öffentliche } \\
\text { Bildungsein- } \\
\text { richtungen }\end{array}$ & $\begin{array}{l}\text { Direkte } \\
\text { öffentliche } \\
\text { Ausgaben } \\
\text { für private } \\
\text { Bildungsein- } \\
\text { richtungens }\end{array}$ & $\begin{array}{c}\text { Indirekte } \\
\text { öffentliche } \\
\text { Transfer- und } \\
\text { sonstige Zahl- } \\
\text { ungen an den } \\
\text { privaten Sektor }\end{array}$ \\
\hline Australien ${ }^{*}$ & 79.6 & 16.1 & 4.3 & 67.7 & $\mathrm{n}$ & 32.3 & 75.9 & 12.1 & 10.8 \\
\hline Österreich* & 96.7 & 1.8 & 1.5 & 85.5 & 1.5 & 13.1 & 92.5 & 2.7 & 4.7 \\
\hline Belgien ${ }^{*}$ & 44.9 & 54.7 & 0.4 & 35.0 & 49.0 & 15.9 & 43.3 & 52.1 & 4.6 \\
\hline Kanada $^{1 *}$ & 98.3 & 1.7 & $\mathrm{x}$ & 77.7 & 0.4 & 21.8 & 91.5 & 1.2 & 7.3 \\
\hline Tschechische Rep.* & 91.5 & 3.2 & 5.3 & 91.1 & 1.4 & 7.6 & 92.3 & 2.6 & 5.1 \\
\hline Dänemark $^{1 *}$ & 78.9 & 6.5 & 14.6 & 64.8 & $\mathrm{n}$ & 35.2 & 75.3 & 4.1 & 20.6 \\
\hline Finnland $^{*}$ & 91.8 & 4.2 & 3.9 & 74.9 & 8.1 & 17.1 & 86.1 & 5.8 & 8.2 \\
\hline Frankreich $^{*}$ & 83.0 & 13.3 & 3.7 & 88.7 & 3.3 & 8.0 & 85.2 & 10.9 & 4.0 \\
\hline Deutschland $^{*}$ & 85.4 & 7.9 & 6.7 & 85.4 & 2.4 & 12.3 & 82.1 & 10.7 & 7.2 \\
\hline Griechenland* & 99.9 & $\mathrm{a}$ & 0.1 & 96.6 & a & 3.4 & 98.9 & $\mathrm{a}$ & 1.1 \\
\hline Ungarn & 92.5 & 6.9 & 0.6 & 83.2 & 4.3 & 12.6 & 91.3 & 5.7 & 2.9 \\
\hline Island & $\mathrm{m}$ & $\mathrm{m}$ & $\mathrm{m}$ & $\mathrm{m}$ & $\mathrm{m}$ & $\mathrm{m}$ & $\mathrm{m}$ & $\mathrm{m}$ & $\mathrm{m}$ \\
\hline Irland & 96.9 & $\mathrm{n}$ & 3.1 & 85.2 & $\mathrm{n}$ & 14.8 & 93.7 & $\mathrm{n}$ & 6.3 \\
\hline Italien & 93.7 & 5.4 & 0.9 & 81.3 & 1.6 & 17.1 & 91.6 & 4.4 & 4.0 \\
\hline Japan $^{2 *}$ & 96.5 & 3.5 & $\mathrm{~m}$ & 83.0 & 17.0 & $\mathrm{~m}$ & 93.6 & 6.4 & $\mathrm{~m}$ \\
\hline Korea $^{*}$ & 86.6 & 11.7 & 1.7 & 59.8 & 28.1 & 12.1 & 83.7 & 13.0 & 3.2 \\
\hline Luxemburg & $\mathrm{m}$ & $\mathrm{m}$ & $\mathrm{m}$ & $\mathrm{m}$ & $\mathrm{m}$ & $\mathrm{m}$ & $\mathrm{m}$ & $\mathrm{m}$ & $\mathrm{m}$ \\
\hline Mexiko & 97.2 & 0.1 & 2.7 & 94.3 & $\mathrm{~m}$ & 5.7 & 96.9 & 0.1 & 3.1 \\
\hline Niederlande $^{*}$ & 21.9 & 70.7 & 7.4 & 39.3 & 36.1 & 24.6 & 27.4 & 61.0 & 11.6 \\
\hline Neuseeland & 95.5 & 1.4 & 3.2 & 75.9 & 1.9 & 22.2 & 90.9 & 2.1 & 7.0 \\
\hline Norwegen & 91.9 & 2.2 & 5.9 & 69.0 & 2.4 & 28.6 & 83.3 & 4.6 & 12.2 \\
\hline Polen & $\mathrm{m}$ & $\mathrm{m}$ & $\mathrm{m}$ & $\mathrm{m}$ & $\mathrm{m}$ & $\mathrm{m}$ & $\mathrm{m}$ & $\mathrm{m}$ & $\mathrm{m}$ \\
\hline Portugal $^{*}$ & 92.0 & 6.7 & 1.3 & 94.0 & $\mathrm{n}$ & 6.0 & 91.8 & 6.2 & 2.1 \\
\hline Slowakische Rep. & 96.6 & 3.4 & 0.0 & 95.6 & $\mathrm{~m}$ & 4.4 & 96.7 & 2.5 & 0.9 \\
\hline Spanien & 85.5 & 13.5 & 1.0 & 89.9 & 0.7 & 9.3 & 86.9 & 10.4 & 2.7 \\
\hline Schweden & 83.7 & 2.7 & 13.6 & 64.7 & 4.9 & 30.4 & 78.9 & 3.9 & 17.1 \\
\hline Schweiz & 90.0 & 7.1 & 2.8 & 89.3 & 6.6 & 4.1 & 89.6 & 6.8 & 3.6 \\
\hline Türkei* $^{*}$ & 99.8 & $\mathrm{a}$ & 0.2 & 87.8 & 0.4 & 11.8 & 96.5 & 0.1 & 3.4 \\
\hline Ver. Königreich* & 78.7 & 21.1 & 0.2 & a & 73.3 & 26.7 & 64.7 & 29.8 & 5.5 \\
\hline Vereinigte Staaten $^{1 *}$ & 99.7 & 0.3 & $\mathrm{x}$ & 67.6 & 13.2 & 19.2 & 90.5 & 4.5 & 5.0 \\
\hline Ländermittel & 87.0 & 9.9 & 3.5 & 75.1 & 9.9 & 16.4 & 84.0 & 9.7 & 6.4 \\
\hline Argentinien & 85.7 & 12.5 & 1.8 & 96.2 & 2.5 & 1.3 & 88.1 & 10.4 & 1.6 \\
\hline Brasilien $^{3}$ & 98.2 & 1.8 & $\mathrm{n}$ & 93.1 & 0.8 & 6.1 & 97.2 & 1.5 & 1.3 \\
\hline Chile & 67.8 & 31.8 & 0.4 & 42.2 & 33.9 & 23.9 & 63.8 & 32.0 & 4.1 \\
\hline China & 99.2 & $\mathrm{a}$ & 0.8 & 93.7 & $\mathrm{a}$ & 6.3 & 97.9 & $\mathrm{a}$ & 2.1 \\
\hline Indien $^{1,3}$ & 70.7 & 29.1 & 0.2 & 78.2 & 21.5 & 0.3 & 72.2 & 27.6 & 0.2 \\
\hline Indonesien ${ }^{4}$ & 90.0 & 6.6 & 3.5 & $\mathrm{~m}$ & $\mathrm{~m}$ & $\mathrm{~m}$ & $\mathrm{~m}$ & $\mathrm{~m}$ & $\mathrm{~m}$ \\
\hline Israel & 75.0 & 24.0 & 1.1 & 6.9 & 83.4 & 9.6 & 64.1 & 33.1 & 2.7 \\
\hline Jamaika & 98.2 & $\mathrm{n}$ & 1.8 & 98.3 & $\mathrm{n}$ & 1.7 & 95.6 & 2.7 & 1.7 \\
\hline Jordanien & 100.0 & $\mathrm{a}$ & $\mathrm{a}$ & 88.1 & $\mathrm{a}$ & 11.9 & 97.8 & a & 2.2 \\
\hline Malaysia & 98.9 & 0.6 & 0.5 & 66.1 & $\mathrm{n}$ & 33.9 & 88.2 & 0.4 & 11.5 \\
\hline Paraguay & 92.5 & 7.4 & 0.1 & $\mathrm{~m}$ & $\mathrm{~m}$ & $\mathrm{~m}$ & $\mathrm{~m}$ & $\mathrm{~m}$ & $\mathrm{~m}$ \\
\hline Philippinen $^{3}$ & 98.7 & $\mathrm{a}$ & 1.3 & 97.5 & a & 2.5 & 98.5 & a & 1.5 \\
\hline Thailand & 93.3 & 2.2 & 4.5 & 74.9 & $\mathrm{n}$ & 25.1 & 88.9 & 2.0 & 9.1 \\
\hline Uruguay & 99.9 & $\mathrm{a}$ & 0.1 & 100.0 & $\mathrm{a}$ & $\mathrm{n}$ & 100.0 & $\mathrm{a}$ & $\mathrm{n}$ \\
\hline
\end{tabular}

1. Post-sekundärer, nicht-tertiärer Bereich im Tertiärbereich enthalten oder fehlt.

2. Post-sekundärer, nicht-tertiärer Bereich sowohl im Sekundarbereich II als auch im Tertiärbereich enthalten.

3. Referenzjahr 1998.

4. Referenzjahr 2000

* Hinweise s. Anhang 3 (www.oecd.org/els/education/eag2002).

Quelle: OECD. 


\section{UNTERSTÜTZUNG FÜR SCHÜLER/STUDIERENDE UND PRIVATE HAUSHALTE DURCH ÖFFENTLICHE SUBVENTIONEN}

- Durchschnittlich 16 Prozent der öffentlichen Ausgaben für den Tertiärbereich betreffen finanzielle Unterstützungsleistungen an Studierende, private Haushalte und andere private Einheiten. In Australien, Dänemark und dem Vereinigten Königreich machen die öffentlichen Subventionen ungefähr ein Drittel und mehr der öffentlichen Bildungsetats für den Tertiärbereich aus.

- Subventionen sind besonders dort von Bedeutung, wo von den Schülern/Studierenden erwartet wird, dass sie wenigstens einen Teil der Kosten für ihre Bildung tragen.

- In den meisten OECD-Ländern genießen die Empfänger von Subventionen einen erheblichen Ermessensspielraum hinsichtlich der Verwendung der erhaltenen Subventionen. In allen OECDLändern, über die Zahlenmaterial vorliegt, werden die Zuschüsse hauptsächlich außerhalb der Bildungseinrichtungen ausgegeben, und in jedem dritten Land ist dies ausschließlich der Fall.

\section{Abbildung B5.1.}

Öffentliche Subventionen für Bildung im Tertiärbereich (1999)

Öffentliche Subventionen für Bildung an den privaten Sektor als Prozentsatz der öffentlichen

Gesamtausgaben für Bildung, nach Art der Subvention

Stipendien/ andere Zuschüsse an private Haushalte - Transfer- und sonstige Zahlungen an andere private Einheiten

Darlehen an Studierende

\% der öffentlichen Gesamtausgaben für Bildung

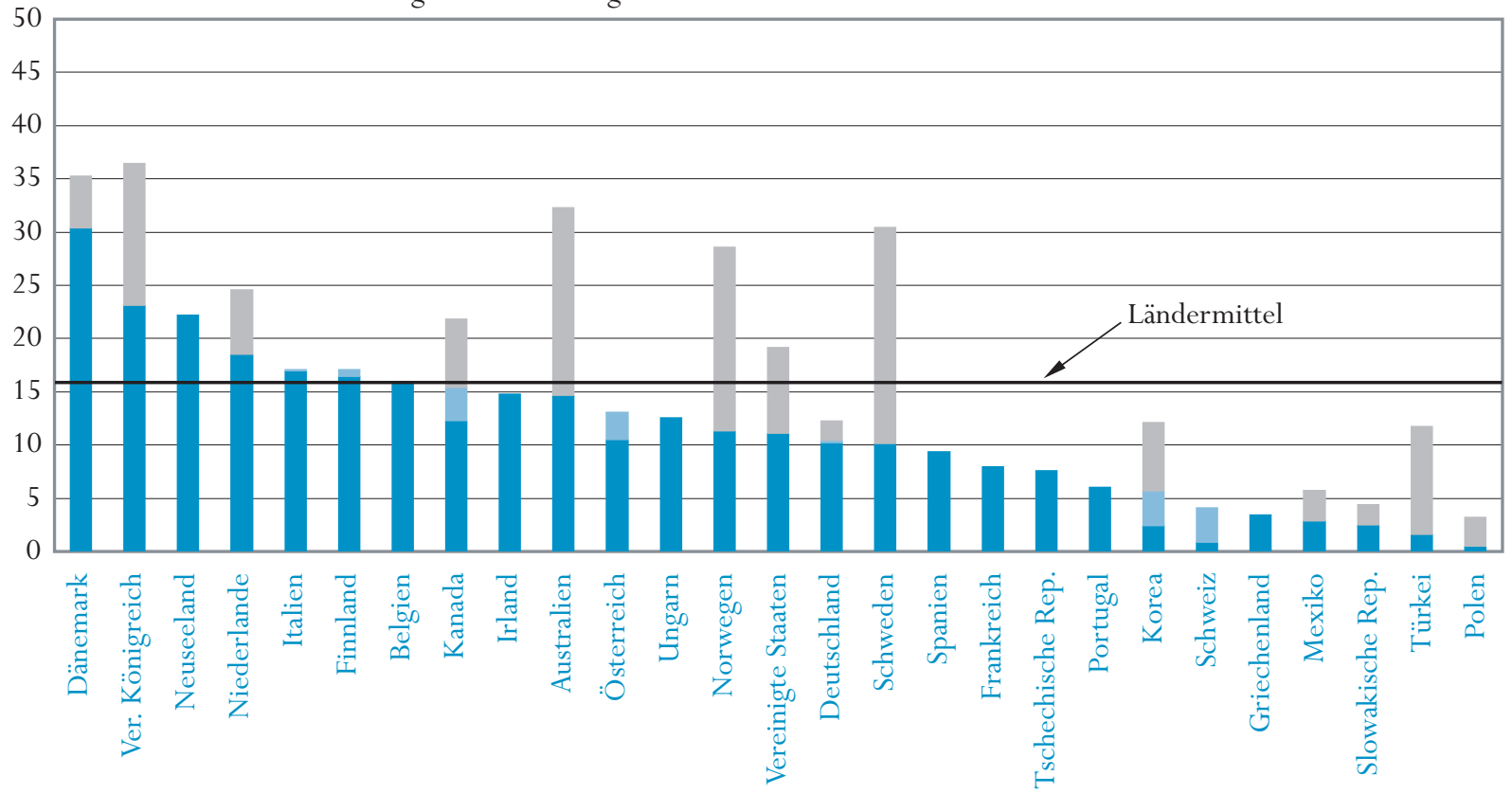

Anordnung der Länder in absteigender Reihenfolge des Anteils von Stipendien/anderen Zuschüssen an private Haushalte und Transfer- und sonstigen Zahlungen an andere private Einheiten im Tertiärbereich an den öffentlichen Gesamtausgaben für Bildung.

Quelle: OECD. Tabelle B5.2. Hinweise s. Anhang 3 (www.oecd.org/els/education/eag2002). 


\section{Politischer Hintergrund}

Dieser Indikator untersucht direkte und indirekte öffentliche

Ausgaben für

Bildungseinrichtungen

sowie die an private

Haushalte gezahlten

öffentlichen Zuschüsse

für den Lebensunterhalt

von Schülern/

Studierenden.

Durch Subventionen an Schüler/Studierende und ihre Familien kann der Staat eine stärkere Bildungsbeteiligung, insbesondere von Schülern und Studierenden aus einkommensschwachen Familien, fördern, indem ein Teil der direkten und indirekten Bildungskosten bezuschusst wird. Ferner spielen öffentliche Subventionen auch bei der indirekten Finanzierung von Bildungseinrichtungen eine wichtige Rolle.

Indem Finanzmittel für Bildungseinrichtungen über Schüler/Studierende gelenkt werden, kann auch ein Beitrag zu mehr Wettbewerb zwischen den Bildungseinrichtungen und zu einer größeren Effizienz der Bildungsfinanzierung geleistet werden. Da Zuschüsse zu den Lebenshaltungskosten der Schüler/ Studierenden eine Erwerbstätigkeit zur Finanzierung von Bildung ersetzen können, tragen öffentliche Subventionen auch dazu bei, den Bildungsstand anzuheben, indem Schülern/Studierenden die Möglichkeit für einen Vollzeitschulbesuch bzw. ein Vollzeitstudium gegeben wird, auch wenn sie durch ihr Studium nur vermindert oder überhaupt keiner bezahlten Tätigkeit nachgehen können.

Öffentliche Subventionen können in vielfältiger Weise bereitgestellt werden: als einkommensabhängige Zuschüsse, als Familienbeihilfen für alle Schüler/ Studierenden, als Steuerfreibeträge für Schüler/Studierende oder ihre Eltern oder in Form sonstiger Transferleistungen an private Haushalte. Nicht an Bedingungen geknüpfte Subventionsleistungen wie Steuerermäßigungen oder Familienbeihilfen werden möglicherweise von einkommensschwachen Schülern/Studierenden weniger als Anreiz zu einer Bildungsteilnahme empfunden als dies bei einkommensabhängigen Zuschüssen der Fall ist. Sie können jedoch auf jeden Fall dazu beitragen, Ungleichheiten zwischen Haushalten mit und ohne Kindern in Ausbildung abzubauen.

Eine entscheidende Frage ist, ob Finanzhilfen an private Haushalte in Form von Zuschüssen oder Darlehen gewährt werden sollen. Können Finanzhilfen in Form von Darlehen dazu beitragen, die Wirksamkeit der in Bildung investierten Ressourcen zu erhöhen und einen Teil der Bildungskosten auf die Nutznießer der Bildungsinvestitionen zu verlagern? Oder sind Studiendarlehen weniger wirksam als Zuschüsse, wenn es darum geht, einkommensschwache Schüler/ Studierende zu mehr Bildung zu ermutigen? Dieser Indikator kann diese Fragen zwar nicht beantworten, er kann jedoch die von den einzelnen OECD-Ländern verfolgte Subventionspolitik aufzeigen.

\section{Ergebnisse und Erläuterungen}

\section{Was dieser Indikator aufzeigt und was nicht}

Dieser Indikator liefert Information über den Teil der öffentlichen Bildungsausgaben, der aus Transferzahlungen an Schüler/Studierende, ihre Familien und andere private Einheiten besteht. Ein Teil dieser Gelder geht indirekt an Bildungseinrichtungen, wenn z.B. Subventionen für die Zahlung von Schul- 
/Studiengebühren genutzt werden. Andere Subventionsleistungen haben keinerlei Bezug zu Bildungseinrichtungen, z.B. finanzielle Zuschüsse zu den Lebenshaltungskosten von Schülern/Studierenden.

Bei diesem Indikator wird zwischen nicht-rückzahlbaren Subventionen in Form von Stipendien und Studienbeihilfen einerseits und Darlehen andererseits unterschieden. Nicht unterschieden wird jedoch zwischen den verschiedenen Arten von Beihilfen bzw. Darlehen, wie z.B. Stipendien einerseits und Familienbeihilfen und Subventionen in Form von Sachleistungen andererseits.

Der Staat kann die Schüler/Studierenden und ihre Familien auch durch die Gewährung von Steuerermäßigungen und Steueranrechnungen unterstützen. Diese Art von Subventionen wird in diesem Indikator jedoch nicht berücksichtigt.

Häufig wird auch eine staatliche Bürgschaft für Studiendarlehen privater Kreditgeber gewährt. In einigen OECD-Ländern ist diese indirekte Form der Subventionierung ebenso bedeutend wie die direkte Finanzhilfe an Schüler/Studierende oder sogar noch bedeutender. Aus Gründen der besseren Vergleichbarkeit werden jedoch in diesem Indikator nur öffentliche Transferleistungen an private Einheiten berücksichtigt, die mit privaten Darlehen in Zusammenhang stehen. Tilgungszahlungen und damit auch das Gesamtvolumen der Darlehen wurden dagegen nicht berücksichtigt.

Bei den Studiendarlehen wird im Rahmen dieses Indikators das Gesamtvolumen der Darlehen angegeben, um Aufschluss über die Höhe der Unterstützungsleistungen für die derzeitigen Studierenden zu erhalten. Die Rückzahlungen der Darlehen werden nicht berücksichtigt, obwohl diese einen erheblichen Beitrag zur Senkung der realen Kreditkosten leisten können. Für einige OECD-Länder gestaltet es sich außerdem recht schwierig, die den Schülern/Studierenden gewährte Gesamtsumme an Darlehen eindeutig zu quantifizieren. Zahlen zu den Studiendarlehen sind daher mit Vorsicht zu interpretieren.

\section{Öffentliche Subventionen an private Haushalte und andere private Einheiten}

Die OECD-Länder verwenden im Durchschnitt 0,4 Prozent ihres BIP für öffentliche Subventionen an private Haushalte und andere private Einheiten. In Dänemark und Schweden liegt dieser Wert bei über 1 Prozent des BIP. Außerdem entfallen in den OECD-Ländern durchschnittlich 7,0 Prozent der öffentlichen Bildungsetats auf Transferleistungen an den privaten Sektor (Tabellen B3.1, B5.1 und B5.2). Der größte Teil dieser Gelder geht in den Tertiärbereich, außer in Deutschland, Frankreich, Mexiko, Schweden, der Schweiz und der Tschechischen Republik. Dort gehen über 50 Prozent der Transferzahlungen an den privaten Sektor in den Primar-, Sekundar- und postsekundären, nicht-tertiären Bildungsbereich.
Die OECD-Länder geben

im Durchschnitt 0,4 Prozent ihres BIP für öffentliche Subventionen an private Haushalte und andere private Einheiten aus. 
Im Primar-, Sekundarund post-sekundären, nicht-tertiären Bereich stellen öffentliche

Subventionen einen vergleichsweise geringen Anteil der öffentlichen Bildungsausgaben dar.
Öffentliche Subventionen an private Haushalte werden in den meisten OECDLändern erst ab dem Sekundarbereich II gezahlt. Unterhalb dieser Ebene gibt es in der Regel kaum Subventionen, da in den meisten OECD-Ländern bis zu diesem Bereich allgemeine Schulpflicht besteht, der Schulbesuch kostenlos ist, Schulbildung überwiegend von der öffentlichen Hand bereitgestellt und weitgehend direkt am Wohnort der Schüler und ihrer Familien angeboten wird. In 10 von 26 OECD-Ländern machen daher Subventionszahlungen an private Haushalte und andere private Einheiten nur 1 Prozent oder weniger der gesamten öffentlichen Bildungsausgaben für den Primar-, Sekundar- und post-sekundären, nicht-tertiären Bildungsbereich aus. Dagegen betragen sie in Australien, Deutschland, den Niederlanden, Norwegen und der Tschechischen Republik zwischen 4 und 8 Prozent der öffentlichen Gelder für diese Bildungsbereiche. In Dänemark und Schweden sind es 15 bzw. 14 Prozent (Abb. B5.2). In den meisten OECD-Ländern, die einen hohen Anteil an Subventionszahlungen im Primar-, Sekundar- und post-sekundären, nicht-tertiären Bereich aufweisen, gehen diese an Erwachsene, die wieder in den Sekundarbereich einsteigen.

\section{Abbildung B5.2.}

Öffentliche Subventionen für Bildung im Primar-, Sekundar- und post-sekundären, nicht-tertiären Bereich (1999)

Öffentliche Subventionen für Bildung an den privaten Sektor als Prozentsatz der öffentlichen Gesamtausgaben für Bildung, nach Art der Subvention

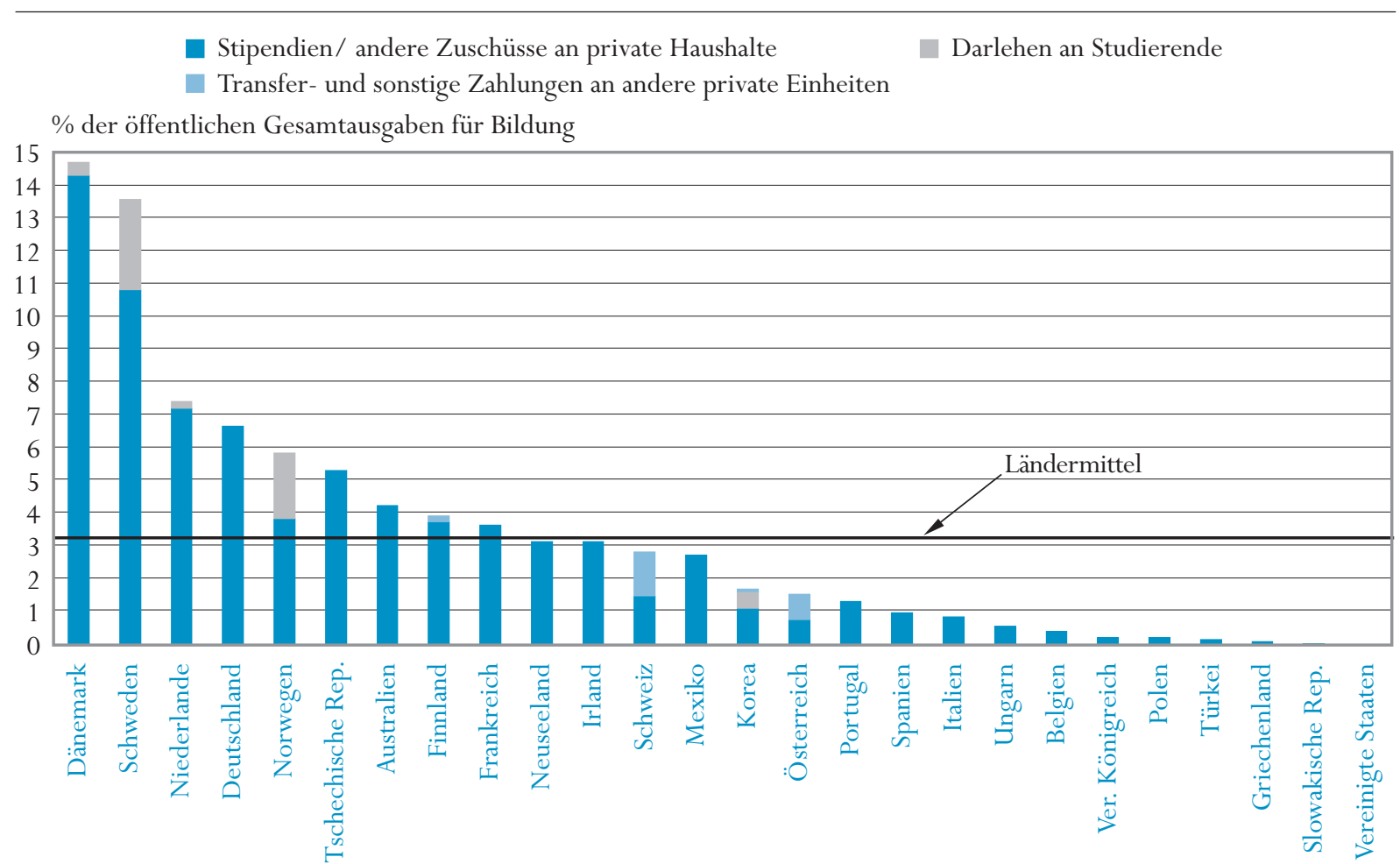

Anordnung der Länder in absteigender Reihenfolge des Anteils der öffentlichen Gesamtsubventionen für den Primar-, Sekundar- und post-sekundären, nicht-tertiären Bereich an den öffentlichen Gesamtausgaben für Bildung.

Quelle: OECD. Tabelle B5.1. Hinweise s. Anhang 3 (www.oecd.org/els/education/eag2002). 
Der in Form von Subventionen an private Haushalte und andere private Einheiten ausgezahlte Teil der Bildungsetats ist im Tertiärbereich wesentlich höher. Im Durchschnitt verwenden die OECD-Länder 16 Prozent ihres Bildungsetats für den Tertiärbereich in Form von Subventionen an private Haushalte und andere private Einheiten (Abb. B5.1). In Australien, Dänemark und dem Vereinigten Königreich wird ungefähr ein Drittel und mehr des Bildungsetats für den Tertiärbereich in Form von Subventionen ausgezahlt. Nur in Griechenland, Polen, der Slowakischen Republik und der Schweiz machen diese Subventionen weniger als 5 Prozent der öffentlichen Gesamtausgaben für den Tertiärbereich aus (Tabelle B5.1).

In vielen OECD-Ländern stellt sich die zentrale Frage, ob die an private Haushalte geleisteten Finanzhilfen in erster Linie als Zuschüsse oder als Darlehen gewährt werden sollen. Die Staaten gewähren zur Subventionierung der Lebenshaltungs- bzw. Bildungskosten der Schüler/Studierenden verschiedene Kombinationen von Zuschüssen und Darlehen. Die Befürworter von Studiendarlehen argumentieren, dass mit den Geldern für Darlehen mehr erreicht werden kann, mit anderen Worten, wenn die für Zuschüsse verwendeten Gelder dazu verwendet würden, Darlehen abzusichern bzw. zu subventionieren, könnte den Schülern/Studierenden insgesamt mehr an Finanzhilfe zur Verfügung gestellt werden und somit insgesamt der Zugang zum Bildungssystem erweitert werden. Außerdem wird durch Darlehen ein Teil der Bildungskosten auf diejenigen verlagert, die am meisten von den Bildungsinvestitionen profitieren. Die Gegner von Studiendarlehen führen dagegen an, dass Studiendarlehen in geringerem Maße als Zuschüsse dazu beitragen, einkommensschwache Schüler/Studierende dazu zu bewegen, eine weiterführende Bildung ins Auge zu fassen. Ferner führen sie an, dass Darlehen aufgrund der unterschiedlichen Subventionen für Darlehensnehmer und -geber und der Verwaltungs- und Tilgungskosten möglicherweise weniger effizient sind als angenommen.

Abbildung B5.1 gibt eine Übersicht über die Anteile von Darlehen, Zuschüssen und Stipendien sowie anderen Beihilfen für private Haushalte an den öffentlichen Bildungsausgaben. Zu den Zuschüssen und Stipendien zählen auch Familienbeihilfen und sonstige spezifische Subventionen, nicht jedoch Steuerermäßigungen. In 13 von 27 OECD-Ländern, die Daten vorlegten, gibt es ausschließlich Stipendien oder Zuschüsse und Transferzahlungen an andere private Einheiten. Die übrigen OECD-Länder bieten sowohl Stipendien/Zuschüsse als auch Studiendarlehen an. Mit Ausnahme von zwei Ländern erhalten Studierende in denjenigen OECDLändern die höchsten Subventionen, die auch Studiendarlehen bieten. Die meisten dieser Länder gewähren einen überdurchschnittlich hohen Anteil ihrer Bildungsetats in Form von Zuschüssen und Stipendien (Abb. B5.1 und Tabelle B5.2).

Die Rückzahlung staatlicher Darlehen kann für den Staat eine bedeutende Einnahmequelle darstellen und die Kosten der Darlehensprogramme beträchtlich senken. In den aktuellen Zahlen über die Bildungsausgaben der privaten Haushalte (Indikator B4) sind die Rückzahlungen durch frühere
Australien, Dänemark und das Vereinigte

Königreich verwenden mindestens ein Drittel ihres Bildungsetats für den Tertiärbereich für Subventionen an den privaten Sektor.

Zur Subventionierung der Bildungskosten von Schülern/Studierenden praktizieren die OECDLänder unterschiedliche Kombinationen aus Zuschüssen und Darlehen. 
Die Rückzahlung der

Darlehen verringert

die realen Kosten der

Darlehensprogramme

für die öffentliche

Hand, erhöht jedoch

gleichzeitig die

finanzielle Belastung der

privaten Haushalte

für Bildung.

In den meisten

OECD-Ländern haben

die Empfänger einen

beträchtlichen Spielraum

in Bezug auf die

Verwendung der an sie

gezahlten öffentlichen

Subventionen.

In allen OECD-

Ländern, für die Zahlen

vorliegen, werden

Subventionen in erster

Linie außserhalb der

Bildungseinrichtungen

ausgegeben - in jedem
Darlehensempfänger nicht enthalten. Diese Rückzahlungen können für den Einzelnen eine erhebliche Belastung darstellen und seine Entscheidung hinsichtlich des Studiums an einer tertiären Bildungseinrichtung beeinflussen. Allerdings wird in vielen OECD-Ländern die Rückzahlung der Studiendarlehen vom künftigen Einkommen der Absolventen abhängig gemacht.

Da die Rückzahlung an die Darlehensprogramme mehrere Jahre nach Inanspruchnahme der Darlehen durch die damaligen Studierenden erfolgt, ist eine Abschätzung der realen Kosten von Darlehensprogrammen ohne Berücksichtigung der Tilgungszahlungen schwierig, weshalb Darlehen nur als Bruttobeträge ausgewiesen werden. Internationale Vergleiche der gesamten Rückzahlungen innerhalb desselben Referenzzeitraums sind nicht möglich, da diese erheblich durch Änderungen bei den Vergabekriterien der Darlehen bzw. der Zahl der Darlehensempfänger beeinflusst werden.

\section{Wofür die Subventionen verwendet werden: Lebenshaltungskosten und Studiengebühren}

In den meisten OECD-Ländern sind die öffentlichen Zahlungen an private Haushalte für Bildung größtenteils nicht zweckgebunden, das heißt, über ihre Verwendung bestimmen die Empfänger, also die Studierenden und ihre Familien, selbst. In einigen OECD-Ländern jedoch sind die öffentlichen Subventionsmittel zweckgebunden für Zahlungen an Bildungseinrichtungen. So besteht beispielsweise in Australien, Irland, Neuseeland und im Vereinigten Königreich eine Zweckbindung derartiger öffentlicher Gelder für Studiengebühren. In Australien besteht innerhalb des HECS-Systems (Higher Education Contribution Scheme - Ausbildungsbeihilfeprogramm für Hochschulbildung) ein enger Zusammenhang zwischen Studiendarlehen und Studiengebühren. Im Rahmen dieses Programms können die Studierenden zwischen zwei Alternativen wählen: einer semesterweise geleisteten Vorauszahlung der Studiengebühren, für die sie einen 25-prozentigen Nachlass erhalten, oder die Zahlung der gesamten aufgelaufenen Gebühren im Nachhinein über die Einkommenssteuern, sobald ihr Jahreseinkommen eine bestimmte Mindestgrenze überschreitet. Im Rahmen der OECD-Bildungsindikatoren wird das HECS-System als Darlehenssystem eingestuft, wenngleich die Studierenden das Stunden der Zahlungen vielleicht nicht als Darlehen betrachten. In OECD-Ländern mit hohen Studiengebühren ist faktisch ein Teil der öffentlichen Subventionen an private Haushalte für Zahlungen an Bildungseinrichtungen zu verwenden, selbst wenn dies nicht ausdrücklich politisch intendiert ist.

Stipendien und andere Studienbeihilfen, die den Schülern/Studierenden zuzurechnen sind, werden weitgehend außerhalb der Bildungseinrichtungen ausgegeben. Sie leisten einen Beitrag zu den Bildungskosten, die keine Studiengebühren sind. In Finnland und den Niederlanden machen die Stipendien und anderen Studienbeihilfen, die nicht Studiengebührenzahlungen an Bildungseinrichtungen zuzurechnen sind, mehr als 15 Prozent der gesamten öffentlichen Ausgaben für den Tertiärbereich aus. Korea, Polen und die 
Schweiz sind die einzigen OECD-Länder, in denen Stipendien und andere Studienbeihilfen, die auf Ausgaben außerhalb der Bildungseinrichtungen abzielen, weniger als 1 Prozent der gesamten öffentlichen Bildungsausgaben ausmachen.

In OECD-Ländern, in denen die Studierenden Studiengebühren zahlen müssen, ist der Zugang zu öffentlichen Subventionen von besonderer Bedeutung, um Studierenden unabhängig von ihren wirtschaftlichen Verhältnissen Zugang zu den Bildungsangeboten zu ermöglichen. Indikator B4 zeigt auf, welcher Anteil der Gelder für Bildungseinrichtungen aus privaten Quellen stammt.

In OECD-Ländern mit einem geringen Anteil privater Finanzierung von Bildungseinrichtungen ist tendenziell auch der Anteil der öffentlichen Subventionen geringer (Tabellen B5.2 und B4.2). Andererseits sind im Vereinigten Königreich mehr als 10 Prozent der öffentlichen Ausgaben für Subventionen im Tertiärbereich als Beihilfen für Studierende und private Haushalte zur Zahlung von Studiengebühren vorgesehen. Eine Ausnahme bildet hier Korea, wo trotz der Tatsache, dass mehr als 80 Prozent aller Mittel für den Tertiärbereich aus privaten Quellen stammen, die Höhe der Subventionen, die als Beihilfe zur Zahlung von Studiengebühren geleistet werden, mit 2 Prozent vergleichsweise gering ist (Tabellen B5.2 und B4.2).

\section{Definitionen und angewandte Methodik}

$\mathrm{Zu}$ den öffentlichen Subventionen an private Haushalte zählen folgende Kategorien: 1.) Zuschüsse/Stipendien, 2.) staatliche Studiendarlehen, 3.) Kindergeld und andere Familienbeihilfen, die an den Status des Studierenden gebunden sind, 4.) öffentliche Subventionen in Form von Geld- oder Sachleistungen, speziell für Unterbringung, Nutzung von Verkehrsmitteln, medizinische Versorgung, Bücher und Lernmittelbedarf, soziale, Freizeit- und sonstige Zwecke und 5.) Zinssubventionierung privater Darlehen.

Die Ausgaben für Studiendarlehen sind als Bruttobetrag ausgewiesen, d.h. ohne Abzug oder Verrechnung der Tilgungs- oder Zinszahlungen der Darlehensnehmer (Studierende oder private Haushalte). Diese Form wurde gewählt, weil der Bruttobetrag der Darlehen und Stipendien bzw. Zuschüsse die relevante Messgröße für die Ermittlung der Finanzhilfen an die gegenwärtigen Bildungsteilnehmer ist.

Öffentliche Kosten in Verbindung mit staatlich garantierten privaten Darlehen sind unter den Subventionen an andere private Einheiten enthalten. Im Unterschied zu den öffentlichen Darlehen sind hierbei nur die Nettokosten der Darlehen enthalten.

Nicht enthalten ist der Geldwert von Steuerermäßigungen und Steueranrechnungen, die privaten Haushalten und Studierenden gewährt werden. dritten OECD-Land sogar ausschlieflich.

Subventionen sind besonders dort von Bedeutung, wo von den schülern/studierenden erwartet wird, dass sie wenigstens einen Teil ihrer Bildungskosten selbst tragen.

Die Daten beziehen sich auf das Haushaltsjahr 1999 und beruhen auf der von der OECD im Jahre 2001 durchgeführten VOE-Datenerhebung zur Bildungsstatistik (Einzelheiten s. Anhang 3). 
Es ist zu beachten, dass die in früheren Ausgaben dieser Veröffentlichung aufgeführten Zahlen und Daten möglicherweise nicht immer mit denen der Ausgabe 2002 vergleichbar sind, da sich Änderungen in den Definitionen und Erhebungsbereichen ergeben haben. Diese wurden aufgrund der OECD Expenditure Comparability Study vorgenommen (Einzelheiten zu den Änderungen s. Anhang 3 unter www.oecd.org/els/education/eag2002). 
Tabelle B5.1.

Öffentliche Subventionen an den privaten Sektor als Prozentsatz der gesamten öffentlichen Bildungsausgaben und des BIP, für den Primar-, Sekundar- und post-sekundären, nicht-tertiären Bereich (1999)

Direkte öffentliche Ausgaben für Bildungseinrichtungen und Subventionen für private Haushalte und andere private Einheiten als Prozentsatz der gesamten öffentlichen Bildungsausgaben und des BIP

\begin{tabular}{|c|c|c|c|c|c|c|c|c|}
\hline & & \multirow{3}{*}{$\begin{array}{c}\text { Direkte } \\
\text { Ausgaben für } \\
\text { Bildungs- } \\
\text { einrichtungen }\end{array}$} & \multicolumn{5}{|c|}{ Subventionen für Bildung an private Einheiten } & \multirow{3}{*}{$\begin{array}{l}\text { Transferzahlun- } \\
\text { gen für Bildung } \\
\text { an private } \\
\text { Einheiten als } \\
\text { Prozentsatz des } \\
\text { BIP }\end{array}$} \\
\hline & & & \multicolumn{3}{|c|}{ Finanzhilfen für Schüler } & \multirow{2}{*}{$\begin{array}{l}\text { Transfer- und } \\
\text { sonstige Zahlun- } \\
\text { gen an andere } \\
\text { private Einheiten }\end{array}$} & \multirow[b]{2}{*}{ Insgesamt } & \\
\hline & & & $\begin{array}{l}\text { Stipendien/ an- } \\
\text { dere Zuschüsse an } \\
\text { private Haushalte }\end{array}$ & $\begin{array}{l}\text { Darlehen an } \\
\text { Schüler }\end{array}$ & Insgesamt & & & \\
\hline & Australien & 95.7 & 4.3 & $\mathrm{n}$ & 4.3 & $\mathrm{n}$ & 4.3 & 0.16 \\
\hline & Österreich & 98.5 & 0.7 & a & 0.7 & 0.8 & 1.5 & 0.06 \\
\hline & Belgien & 99.6 & 0.4 & $\mathrm{n}$ & 0.4 & $\mathrm{n}$ & 0.4 & 0.01 \\
\hline & Kanada $^{1 *}$ & $\mathrm{~m}$ & $\mathrm{~m}$ & $\mathrm{~m}$ & $\mathrm{~m}$ & $\mathrm{~m}$ & $\mathrm{~m}$ & $\mathrm{~m}$ \\
\hline & Tschechische Rep. ${ }^{*}$ & 94.7 & 5.3 & $\mathrm{a}$ & 5.3 & $\mathrm{n}$ & 5.3 & 0.16 \\
\hline & Dänemark $^{1 *}$ & 85.3 & 14.3 & 0.4 & 14.7 & $\mathrm{n}$ & 14.7 & 0.70 \\
\hline & Finnland & 96.1 & 3.7 & $\mathrm{n}$ & 3.7 & 0.2 & 3.9 & 0.15 \\
\hline & Frankreich & 96.3 & 3.7 & a & 3.7 & $\mathrm{a}$ & 3.7 & 0.15 \\
\hline & Deutschland ${ }^{*}$ & 93.3 & 6.7 & $\mathrm{n}$ & 6.7 & $\mathrm{n}$ & 6.7 & 0.20 \\
\hline & Griechenland & 99.9 & 0.1 & $\mathrm{~m}$ & 0.1 & $\mathrm{a}$ & 0.1 & 0.00 \\
\hline & Ungarn & 99.4 & 0.6 & $\mathrm{a}$ & 0.6 & $\mathrm{n}$ & 0.6 & 0.02 \\
\hline & Island & $\mathrm{m}$ & $\mathrm{m}$ & $\mathrm{m}$ & $\mathrm{m}$ & $\mathrm{m}$ & $\mathrm{m}$ & $\mathrm{m}$ \\
\hline & Irland $^{*}$ & 96.9 & 3.1 & $\mathrm{n}$ & 3.1 & $\mathrm{n}$ & 3.1 & 0.10 \\
\hline & Italien & 99.1 & 0.9 & a & 0.9 & $\mathrm{n}$ & 0.9 & 0.03 \\
\hline & Japan & $\mathrm{m}$ & $\mathrm{m}$ & $\mathrm{m}$ & $\mathrm{m}$ & $\mathrm{m}$ & $\mathrm{m}$ & $\mathrm{m}$ \\
\hline & Korea & 98.3 & 1.1 & 0.5 & 1.6 & 0.1 & 1.7 & 0.06 \\
\hline & Luxemburg & $\mathrm{m}$ & $\mathrm{m}$ & $\mathrm{m}$ & $\mathrm{m}$ & $\mathrm{m}$ & $\mathrm{m}$ & $\mathrm{m}$ \\
\hline & Mexiko & 97.3 & 2.7 & $\mathrm{a}$ & 2.7 & $\mathrm{n}$ & 2.7 & 0.09 \\
\hline & Niederlande & 92.6 & 7.2 & 0.2 & 7.4 & $\mathrm{n}$ & 7.4 & 0.23 \\
\hline & Neuseeland & 96.8 & 3.2 & a & 3.2 & $\mathrm{n}$ & 3.2 & 0.15 \\
\hline & Norwegen & 94.1 & 3.8 & 2.0 & 5.9 & $\mathrm{n}$ & 5.9 & 0.25 \\
\hline & Polen & 99.8 & 0.2 & $\mathrm{x}$ & 0.2 & $\mathrm{~m}$ & 0.2 & 0.01 \\
\hline & Portugal & 98.7 & 1.3 & $\mathrm{~m}$ & 1.3 & $\mathrm{~m}$ & 1.3 & 0.06 \\
\hline & Slowakische Rep. & 100.0 & $\mathrm{n}$ & a & $\mathrm{n}$ & $\mathrm{a}$ & $\mathrm{n}$ & $\mathrm{n}$ \\
\hline & Spanien & 99.0 & 1.0 & $\mathrm{n}$ & 1.0 & $\mathrm{n}$ & 1.0 & 0.03 \\
\hline & Schweden & 86.4 & 10.8 & 2.8 & 13.6 & a & 13.6 & 0.69 \\
\hline & Schweiz $^{*}$ & 97.2 & 1.5 & $\mathrm{n}$ & 1.5 & 1.3 & 2.8 & 0.11 \\
\hline & Türkei & 99.8 & 0.2 & $\mathrm{a}$ & 0.2 & $\mathrm{a}$ & 0.2 & 0.00 \\
\hline & Ver. Königreich & 99.8 & 0.2 & $\mathrm{n}$ & 0.2 & $\mathrm{n}$ & 0.2 & 0.01 \\
\hline & Vereinigte Staaten ${ }^{1}$ & 100.0 & $\mathrm{n}$ & $\mathrm{a}$ & $\mathrm{n}$ & $\mathrm{n}$ & $\mathrm{n}$ & $\mathrm{x}$ \\
\hline & Ländermittel & 96.7 & 3.0 & 0.3 & 3.2 & 0.1 & 3.3 & 0.14 \\
\hline & Argentinien & 98.2 & 1.8 & $\mathrm{n}$ & 1.8 & $\mathrm{n}$ & 1.8 & 0.06 \\
\hline & Brasilien $^{2}$ & 100.0 & $\mathrm{n}$ & $\mathrm{n}$ & $\mathrm{n}$ & $\mathrm{n}$ & $\mathrm{n}$ & $\mathrm{n}$ \\
\hline & Chile & 99.6 & $\mathrm{n}$ & a & $\mathrm{n}$ & a & $\mathrm{n}$ & $\mathrm{n}$ \\
\hline & China & 99.2 & 0.8 & $\mathrm{x}$ & 0.8 & $\mathrm{a}$ & 0.8 & $\mathrm{n}$ \\
\hline & Indien $^{1,2}$ & 99.8 & 0.2 & $\mathrm{n}$ & 0.2 & $\mathrm{x}$ & 0.2 & $\mathrm{n}$ \\
\hline & Indonesien $^{3}$ & 96.5 & 3.5 & $\mathrm{~m}$ & 3.5 & $\mathrm{x}$ & 3.5 & $\mathrm{n}$ \\
\hline & Israel & 98.9 & 1.1 & $\mathrm{n}$ & 1.1 & $\mathrm{n}$ & 1.1 & $\mathrm{n}$ \\
\hline & Jamaika & 98.2 & 1.8 & $\mathrm{n}$ & 1.8 & $\mathrm{n}$ & 1.8 & 0.08 \\
\hline & Jordanien & 100.0 & $\mathrm{a}$ & $\mathrm{a}$ & $\mathrm{a}$ & $\mathrm{a}$ & $\mathrm{a}$ & $\mathrm{a}$ \\
\hline & Malaysia & 99.5 & 0.5 & a & 0.5 & a & 0.5 & $\mathrm{n}$ \\
\hline & Paraguay & 99.9 & $\mathrm{n}$ & a & $\mathrm{n}$ & a & $\mathrm{n}$ & $\mathrm{n}$ \\
\hline & Peru $^{1}$ & 99.9 & 0.1 & $\mathrm{n}$ & 0.1 & $\mathrm{n}$ & 0.1 & $\mathrm{n}$ \\
\hline & Philippinen ${ }^{2}$ & 98.7 & 1.3 & a & 1.3 & $\mathrm{a}$ & 1.3 & $\mathrm{n}$ \\
\hline & Thailand & 95.5 & $\mathrm{n}$ & 4.4 & 4.5 & $\mathrm{~m}$ & 4.5 & 0.13 \\
\hline & Uruguay & 99.9 & $\mathrm{n}$ & $\mathrm{a}$ & $\mathrm{n}$ & a & $\mathrm{n}$ & $\mathrm{n}$ \\
\hline
\end{tabular}

1. Post-sekundärer, nicht-tertiärer Bereich nicht enthalten oder fehlt.

2. Referenzjahr 1998.

3. Referenzjahr 2000

* Hinweise s. Anhang 3 (www.oecd.org/els/education/eag2002).

Quelle: OECD. 
Tabelle B5.2.

Öffentliche Subventionen an den privaten Sektor für den Tertiärbereich als Prozentsatz der gesamten öffentlichen Bildungsausgaben und des BIP (1999)

Direkte öffentliche Ausgaben für Bildungseinrichtungen und Subventionen für private Haushalte und andere private Einheiten als Prozentsatz der gesamten öffentlichen Bildungsausgaben und des BIP

\begin{tabular}{|c|c|c|c|c|c|c|c|c|}
\hline & \multirow[b]{3}{*}{$\begin{array}{l}\text { Direkte Ausga- } \\
\text { ben für private } \\
\text { Bildungs- } \\
\text { einrichtungen }\end{array}$} & \multicolumn{6}{|c|}{ Subventionen für Bildung an private Einheiten } & \multirow[b]{3}{*}{$\begin{array}{l}\text { Transferzahlun- } \\
\text { gen für Bildung } \\
\text { an private } \\
\text { Einheiten als } \\
\text { Prozentsatz } \\
\text { des BIP }\end{array}$} \\
\hline & & \multicolumn{4}{|c|}{ Finanzhilfen für Studierende } & \multirow[b]{2}{*}{$\begin{array}{l}\text { Tranfer- und } \\
\text { sonstige } \\
\text { Zahlungen an } \\
\text { andere private } \\
\text { Einheiten }\end{array}$} & \multirow[b]{2}{*}{ Insgesamt } & \\
\hline & & $\begin{array}{c}\text { Stipendien/ } \\
\text { andere } \mathrm{Zu-} \\
\text { schüsse an pri- } \\
\text { vate Haushalte }\end{array}$ & $\begin{array}{l}\text { Darlehen an } \\
\text { Studierende }\end{array}$ & Insgesamt & $\begin{array}{c}\text { Stipendien/ } \\
\text { andere Zu- } \\
\text { schüsse an pri- } \\
\text { vate Haushalte, } \\
\text { die Bildungsein- } \\
\text { richtungen zu- } \\
\text { zuordnen sind } \\
\end{array}$ & & & \\
\hline Australien & 67.7 & 14.6 & 17.7 & 32.3 & 1.4 & $\mathrm{n}$ & 32.3 & 0.38 \\
\hline Österreich & 86.9 & 10.4 & a & 10.4 & $\mathrm{x}$ & 2.6 & 13.1 & 0.22 \\
\hline Belgien & 84.1 & 15.9 & $\mathrm{n}$ & 15.9 & $\mathrm{~m}$ & $\mathrm{n}$ & 15.9 & 0.24 \\
\hline Kanada $^{1 *}$ & 78.2 & 12.2 & 6.4 & 18.6 & $\mathrm{~m}$ & 3.2 & 21.8 & 0.41 \\
\hline Tschechische Rep.* & 92.4 & 7.6 & $\mathrm{a}$ & 7.6 & $\mathrm{n}$ & $\mathrm{n}$ & 7.6 & 0.06 \\
\hline Dänemark $^{*}$ & 64.8 & 30.3 & 4.9 & 35.2 & $\mathrm{~m}$ & $\mathrm{n}$ & 35.2 & 0.83 \\
\hline Finnland & 82.9 & 16.4 & $\mathrm{n}$ & 16.4 & $\mathrm{n}$ & 0.7 & 17.1 & 0.36 \\
\hline Frankreich & 92.0 & 8.0 & a & 8.0 & 2.4 & $\mathrm{a}$ & 8.0 & 0.08 \\
\hline Deutschland $^{*}$ & 87.7 & 10.1 & 1.9 & 12.0 & $\mathrm{n}$ & 0.3 & 12.3 & 0.13 \\
\hline Griechenland & 96.6 & 3.4 & $\mathrm{~m}$ & 3.4 & $\mathrm{~m}$ & a & 3.4 & 0.04 \\
\hline Ungarn & 87.4 & 12.6 & a & 12.6 & $\mathrm{n}$ & $\mathrm{n}$ & 12.6 & 0.12 \\
\hline Island & $\mathrm{m}$ & $\mathrm{m}$ & $\mathrm{m}$ & $\mathrm{m}$ & $\mathrm{m}$ & $\mathrm{m}$ & $\mathrm{m}$ & $\mathrm{m}$ \\
\hline Irland $^{*}$ & 85.2 & 14.8 & $\mathrm{n}$ & 14.8 & 4.7 & $\mathrm{n}$ & 14.8 & 0.17 \\
\hline Italien & 82.9 & 16.9 & $\mathrm{n}$ & 16.9 & 6.3 & 0.1 & 17.1 & 0.14 \\
\hline Japan & $\mathrm{m}$ & $\mathrm{m}$ & $\mathrm{m}$ & $\mathrm{m}$ & $\mathrm{m}$ & $\mathrm{m}$ & $\mathrm{m}$ & $\mathrm{m}$ \\
\hline Korea & 87.9 & 2.4 & 6.4 & 8.8 & 2.4 & 3.3 & 12.1 & 0.07 \\
\hline Luxemburg & $\mathrm{m}$ & $\mathrm{m}$ & $\mathrm{m}$ & $\mathrm{m}$ & $\mathrm{m}$ & $\mathrm{m}$ & $\mathrm{m}$ & $\mathrm{m}$ \\
\hline Mexiko & 94.3 & 2.8 & 2.9 & 5.7 & $\mathrm{~m}$ & $\mathrm{n}$ & 5.7 & 0.05 \\
\hline Niederlande & 75.4 & 18.4 & 6.2 & 24.6 & 2.0 & $\mathrm{n}$ & 24.6 & 0.32 \\
\hline Neuseeland & 77.8 & 22.2 & a & 22.2 & $\mathrm{x}$ & $\mathrm{n}$ & 22.2 & 0.27 \\
\hline Norwegen & 71.4 & 11.3 & 17.3 & 28.6 & $\mathrm{n}$ & $\mathrm{n}$ & 28.6 & 0.57 \\
\hline Polen & 96.8 & 0.4 & 2.7 & 3.2 & $\mathrm{n}$ & $\mathrm{m}$ & 3.2 & 0.03 \\
\hline Portugal & 94.0 & 6.0 & $\mathrm{~m}$ & 6.0 & $\mathrm{~m}$ & $\mathrm{~m}$ & 6.0 & 0.06 \\
\hline Slowakische Rep. & 95.6 & 2.5 & 1.9 & 4.4 & $\mathrm{~m}$ & a & 4.4 & 0.03 \\
\hline Spanien & 90.7 & 9.3 & $\mathrm{n}$ & 9.3 & 3.9 & $\mathrm{n}$ & 9.3 & 0.08 \\
\hline Schweden & 69.6 & 10.1 & 20.3 & 30.4 & $\mathrm{x}$ & $\mathrm{a}$ & 30.4 & 0.63 \\
\hline Schweiz $^{*}$ & 95.9 & 0.8 & $\mathrm{n}$ & 0.8 & $\mathrm{n}$ & 3.3 & 4.1 & 0.05 \\
\hline Türkei & 88.2 & 1.5 & 10.2 & 11.8 & $\mathrm{~m}$ & $\mathrm{n}$ & 11.8 & 0.14 \\
\hline Ver. Königreich & 63.6 & 23.1 & 13.3 & 36.4 & 10.7 & $\mathrm{n}$ & 36.4 & 0.39 \\
\hline Vereinigte Staaten ${ }^{1}$ & 80.8 & 11.1 & 8.1 & 19.2 & $\mathrm{x}$ & $\mathrm{m}$ & 19.2 & 0.26 \\
\hline Ländermittel & 84.1 & 10.9 & 4.7 & 15.4 & 2.0 & 0.6 & 15.9 & 0.23 \\
\hline Argentinien & 98.7 & $\mathrm{n}$ & $\mathrm{n}$ & $\mathrm{n}$ & $\mathrm{x}$ & 0.9 & 1.3 & $\mathrm{n}$ \\
\hline Brasilien $^{2}$ & 93.9 & 4.3 & 1.8 & 6.1 & $\mathrm{x}$ & $\mathrm{n}$ & 6.1 & 0.07 \\
\hline Chile & 76.1 & 10.8 & 13.1 & 23.9 & 21.0 & a & 23.9 & 0.16 \\
\hline China & 93.7 & 6.3 & $\mathrm{x}$ & 6.3 & $\mathrm{n}$ & $\mathrm{a}$ & 6.3 & $\mathrm{n}$ \\
\hline Indien $^{2}$ & 99.7 & 0.3 & $\mathrm{x}$ & 0.3 & $\mathrm{x}$ & $\mathrm{x}$ & 0.3 & $\mathrm{n}$ \\
\hline Israel & 90.4 & 8.1 & 1.6 & 9.6 & 8.1 & $\mathrm{n}$ & 9.6 & 0.12 \\
\hline Jamaika & 98.3 & 1.7 & $\mathrm{~m}$ & 1.7 & 1.3 & $\mathrm{n}$ & 1.7 & $\mathrm{n}$ \\
\hline Jordanien & 88.1 & 11.9 & $\mathrm{a}$ & 11.9 & $\mathrm{x}$ & a & 11.9 & 0.11 \\
\hline Malaysia & 66.1 & 13.2 & 20.7 & 33.9 & $\mathrm{x}$ & a & 33.9 & 0.63 \\
\hline Paraguay & 98.0 & 2.0 & $\mathrm{~m}$ & 2.0 & $\mathrm{x}$ & a & 2.0 & $\mathrm{n}$ \\
\hline Peru & 98.9 & $\mathrm{n}$ & 0.9 & 1.1 & $\mathrm{x}$ & $\mathrm{n}$ & 1.1 & $\mathrm{n}$ \\
\hline Philippinen $^{2}$ & 97.5 & 1.0 & 1.6 & 2.5 & $\mathrm{x}$ & a & 2.5 & $\mathrm{n}$ \\
\hline Thailand & 74.9 & $\mathrm{~m}$ & 25.1 & 25.1 & $\mathrm{~m}$ & $\mathrm{~m}$ & 25.1 & 0.29 \\
\hline Uruguay & 100.0 & $\mathrm{n}$ & a & $\mathrm{n}$ & $\mathrm{x}$ & a & $\mathrm{n}$ & $\mathrm{n}$ \\
\hline Simbabwe $^{1}$ & 87.3 & 4.0 & 8.7 & 12.7 & $\mathrm{x}$ & a & 12.7 & 0.15 \\
\hline
\end{tabular}

1. Einschließlich post-sekundärer, nicht-tertiärer Bereich.

2. Referenzjahr 1998.

* Hinweise s. Anhang 3 (www.oecd.org/els/education/eag2002).

Quelle: OECD. 


\section{AUSGABEN FÜR BILDUNGSEINRICHTUNGEN NACH ART DER ERBRACHTEN LEISTUNG UND AUSGABENKATEGORIEN}

- Im Durchschnitt entfallen ein Viertel der Ausgaben für den Tertiärbereich auf Forschung und Entwicklung an tertiären Bildungseinrichtungen. Die erheblichen Unterschiede zwischen den einzelnen OECD-Ländern beim Umfang von Forschung und Entwicklung an tertiären Einrichtungen können zum Teil die großen Unterschiede bei den Ausgaben pro Studierenden in diesem Bildungsbereich erklären.

- Im Primar-, Sekundar- und post-sekundären, nicht-tertiären Bildungsbereich zusammen machen die laufenden Ausgaben im Durchschnitt aller OECD-Länder 92 Prozent der Gesamtausgaben aus. Mit Ausnahme von vier OECD-Ländern machen die Personalkosten mindestens 70 Prozent der laufenden Ausgaben im Primar-, Sekundar- und post-sekundären, nicht-tertiären Bereich aus.

\section{Abbildung B6.1.}

Ausgaben für Unterricht, Forschung und Entwicklung (F\&E) sowie zusätzliche Dienstleistungen in Bildungseinrichtungen als Prozentsatz des BIP (1999)

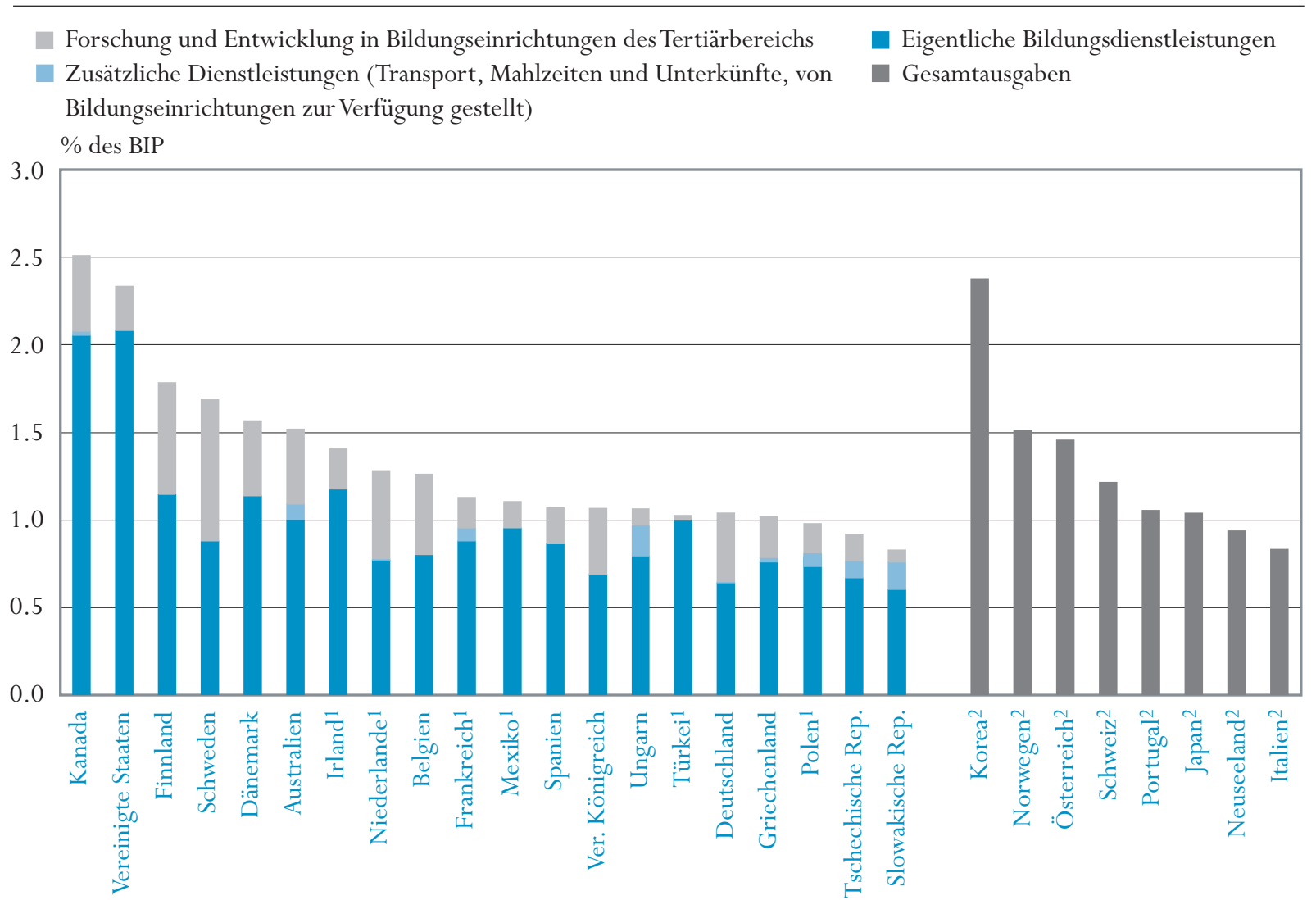

Anordnung der Länder in absteigender Reihenfolge der Ausgaben für Unterricht, Forschung und Entwicklung sowie zusätzliche Dienstleistungen in tertiären Bildungseinrichtungen.

1. Ausgaben für Forschung und Entwicklung im Tertiärbereich und damit auch Gesamtausgaben zu niedrig angesetzt.

2. Balken stellt die Gesamtausgaben im Tertiärbereich dar und umfasst auch Ausgaben für Forschung und Entwicklung.

Quelle: OECD. Tabelle B6.1. Hinweise s. Anhang 3 (www.oecd.org/els/education/eag2002). 
Dieser Indikator vergleicht die Bildungsausgaben der einzelnen OECDLänder hinsichtlich der Unterteilung in laufende und Investitionsausgaben sowie der Aufteilung der laufenden Ausgaben auf die verschiedenen Ausgabenkategorien.

Verglichen werden die $O E C D$-Länder auch nach der Aufteilung der Mittel zwischen den verschiedenen Aufgaben der Bildungseinrichtungen.

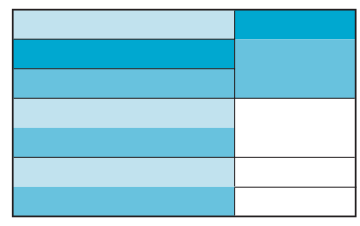

Erfasste Daten (Erklärungen s. S. 160)

\section{Politischer Hintergrund}

Die Aufteilung der verfügbaren Mittel auf die verschiedenen Ressourcenkategorien kann Einfluss haben auf die Qualität der zu erbringenden Leistungen (z.B. durch die Lehrergehälter), den Zustand der Bildungseinrichtungen (z.B. die Instandhaltung von Schulgebäuden) und die Fähigkeit der Bildungssysteme, sich den geänderten demographischen Gegebenheiten und der Entwicklung der Schüler-/Studierendenzahlen (z.B. durch den Neubau von Schulen) anzupassen.

Vergleiche, wie die einzelnen OECD-Länder ihre Bildungsausgaben verteilen, können Einblick geben in die unterschiedlichen Organisationsund Betriebsweisen von Bildungseinrichtungen. Auf Systemebene getroffene Budget- und Strukturentscheidungen über die Zuweisung von Mitteln machen sich letztendlich im Klassenzimmer bemerkbar und beeinflussen die Art des Unterrichts und die Bedingungen, unter denen er stattfindet.

Neben der Erteilung von Unterricht bieten die Bildungsrichtungen eine Vielzahl von bildungsbezogenen Dienstleistungen. Im Primar-, Sekundarund post-sekundären, nicht-tertiären Bereich können dies Schulverpflegung, kostenloser Transport von und zur Schule sowie Internatseinrichtungen sein. Im Tertiärbereich können Wohnmöglichkeiten angeboten werden und in vielen Fällen ist auch ein breites Spektrum an Forschungstätigkeiten integraler Bestandteil des tertiären Bildungsbereichs.

\section{Ergebnisse und Erläuterungen}

\section{Was dieser Indikator aufzeigt und was nicht.}

Dieser Indikator unterteilt die Bildungsausgaben nach laufenden und Investitionsausgaben sowie nach den Aufwendungen für die drei Hauptfunktionen, die Bildungseinrichtungen üblicherweise erfüllen. Dazu gehören erstens Kosten, die direkt mit dem Unterricht zusammenhängen, wie die Gehälter der Lehrkräfte und Kosten für Unterrichtsmaterialien, sowie Ausgaben, die indirekt mit der Unterrichtserteilung zusammenhängen, wie Verwaltungsausgaben, unterrichtsunterstützende Dienste, Aus- und Weiterbildung der Lehrkräfte, Beratung von Schülern/Studierenden sowie die Ausgaben für den Bau bzw. die Vorhaltung von Bildungseinrichtungen. Zweitens gehören dazu Ausgaben für die zusätzlichen Dienstleistungen, wie z.B. die von den Bildungseinrichtungen erbrachten sozialen Dienste für Schüler/Studierende, und drittens Ausgaben für Forschung und Entwicklung (F\&E) an tertiären Bildungseinrichtungen, sei es in Form separat finanzierter F\&E-Aktivitäten oder in Form der Anteile von Gehältern und laufenden Ausgaben am allgemeinen Bildungsetat, die auf die Forschungstätigkeit des Personals entfallen.

Nicht enthalten sind in diesem Indikator die öffentlichen und privaten F\&EAusgaben außerhalb von Bildungseinrichtungen, wie z.B. die F\&E-Ausgaben der Wirtschaft. Eine vergleichende Übersicht der F\&E-Ausgaben außerhalb 
des Bildungsbereichs ist in den OECD-Indikatoren für Wissenschaft und Technologie enthalten. Die Ausgaben der Bildungseinrichtungen für soziale Dienste für Schüler und Studierende beinhalten lediglich die öffentlichen Subventionen für diese Dienstleistungen. Die Ausgaben von Schülern/Studierenden und ihren Familien für Dienstleistungen, die von den Bildungseinrichtungen gegen Entgelt angeboten werden, sind nicht enthalten.

\section{Ausgaben für Unterricht, Forschung und Entwicklung sowie zusätzliche Dienstleistungen}

Unterhalb des Tertiärbereichs überwiegen bei den Bildungsausgaben die Investitionen in die eigentlichen Bildungsdienstleistungen. Im Tertiärbereich können auch andere Leistungen, insbesondere im Zusammenhang mit Forschung und Entwicklung, einen wesentlichen Teil der Bildungsausgaben ausmachen. Die Unterschiede zwischen den einzelnen OECD-Ländern bei den Ausgaben für F\&E können daher auch einen wesentlichen Teil der Unterschiede zwischen den OECDLändern bei den Gesamtbildungsausgaben pro Studierenden im Tertiärbereich erklären (Abb. B6.1). So lassen zum Beispiel hohe F\&E-Aufwendungen in Einrichtungen desTertiärbereichs in Australien, Belgien, Dänemark, Deutschland, Finnland, Kanada, den Niederlanden und Schweden (zwischen 0,40 und 0,80 Prozent des BIP) darauf schließen, dass die Bildungsausgaben pro Studierenden in diesen OECD-Ländern wesentlich geringer wären, wenn sie um den F\&E-Anteil bereinigt würden (Tabelle B6.1).

In vielen OECD-Ländern gehören soziale Dienste für Schüler und Studierende sowie gelegentlich auch Dienstleistungen für die Allgemeinheit zu den integralen Aufgaben von Schulen und Hochschulen. Die Finanzierung dieser zusätzlichen Dienstleistungen erfolgt über unterschiedliche Kombinationen von öffentlichen Mitteln, öffentlichen Subventionen sowie Gebühren, die von Schülern/Studierenden und ihren Familien erhoben werden.

Im Durchschnitt geben die OECD-Länder 0,2 Prozent ihres BIP für die Subventionierung der im Primar-, Sekundar- und post-sekundären, nicht-tertiären Bereich bereitgestellten zusätzlichen Dienstleistungen aus. Dies entspricht 5 Prozent der Gesamtausgaben für diese Bildungseinrichtungen. Am oberen Ende der Skala befinden sich Finnland, Frankreich, die Slowakische Republik, die Tschechische Republik und Ungarn, die ungefähr 10 Prozent und mehr der Gesamtausgaben für Bildungseinrichtungen für zusätzliche Dienstleistungen ausgeben. Das entspricht mehr als 500 US-Dollar (kaufkraftbereinigt) pro Schüler/Studierenden in Frankreich und Finnland und mehr als 250 US-Dollar (kaufkraftbereinigt) in Kanada, Schweden, der Tschechischen Republik, Ungarn und den Vereinigten Staaten (Tabellen B6.1 und B6.2).

In mehr als zwei Drittel der OECD-Länder sind die Aufwendungen für zusätzliche Dienstleistungen höher als die Aufwendungen für Subventionen an private Haushalte im Primar-, Sekundar- und post-sekundären, nicht-tertiären Bereich. Ausnahmen stellen Australien, Irland, die Niederlande und Schweden
Die erheblichen Unterschiede zwischen den einzelnen OECDLändern beim Umfang von Forschung und Entwicklung an tertiären Einrichtungen können zum Teil die grofen Unterschiede bei den Ausgaben pro Studierenden an tertiären Einrichtungen erklären.

Soziale Dienste für Schüler und Studierende gehören zu den integralen Aufgaben von Schulen und Hochschulen.

Im Primar-, Sekundarund post-sekundären, nicht-tertiären Bereich machen die Ausgaben für zusätzliche Dienstleistungen 5 Prozent der Gesamtausgaben für Bildungseinrichtungen dus. 
dar, wo die Ausgaben für Subventionen an private Haushalte höher sind (Tabellen B5.1 und B6.1).

Im Tertiärbereich betragen die Subventionen für zusätzliche Dienstleistungen im Durchschnitt lediglich 0,04 Prozent des BIP. Bezogen auf die Zahl der Studierenden, kann dies jedoch recht erhebliche Beträge pro Studierenden ergeben. Dies ist beispielsweise in Australien, Frankreich, der Slowakischen Republik, der Tschechischen Republik und Ungarn der Fall, wo sich die Subventionen für zusätzliche Dienstleistungen auf über 450 US-Dollar (kaufkraftbereinigt) pro Studierenden belaufen. Im Tertiärbereich müssen diese zusätzlichen Dienstleistungen häufiger von den Studierenden selbst bezahlt werden (Tabellen B6.1 und B6.2).

\section{Laufende Ausgaben und Investitionsausgaben sowie Aufteilung der laufenden Ausgaben nach Ausgabenkategorien}

Bildungsausgaben können zunächst in laufende und Investitionsausgaben unterteilt werden. Investitionsausgaben sind Ausgaben für Sachmittel mit einer Nutzungsdauer von mehr als einem Jahr. Hierzu gehören Aufwendungen für Bau, Renovierung und größere Instandsetzungsarbeiten von Gebäuden. Laufende Ausgaben beinhalten finanzielle Aufwendungen für Ressourcen von Bildungseinrichtungen, die jedes Jahr für den laufenden Betrieb dieser Einrichtungen erforderlich sind.

Die laufenden Ausgaben lassen sich darüber hinaus in drei große funktional definierte Kategorien unterteilen: die Lehrerbesoldung, Vergütung der sonstigen Beschäftigten und sonstige laufende Ausgaben (z.B. für Unterrichts- und Hilfsmaterial, Instandhaltung von Schulgebäuden, Zubereitung von Mahlzeiten für Schüler/Studierende, Mietzahlungen für Lehreinrichtungen). Die Höhe der Mittelzuteilung für jede einzelne dieser Kategorien wird in gewissem Maße durch die gegenwärtige und erwartete Entwicklung der Schüler-/ Studierendenzahlen, die Gehälter der im Bildungsbereich Beschäftigten und die Kosten für die Instandhaltung und den Bau von Bildungseinrichtungen beeinflusst.

Ausbildung und Unterricht finden überwiegend in Schulen und Hochschulen statt. Die arbeitskräfteintensiven Unterrichtsmethoden sind mitverantwortlich für den hohen Anteil der laufenden Ausgaben an den gesamten Bildungsausgaben. Im Primar-, Sekundar- und post-sekundären, nicht-tertiären Bereich zusammengenommen belaufen sich die laufenden Ausgaben im Durchschnitt aller OECD-Länder auf 92 Prozent der gesamten Ausgaben.

Im Hinblick auf den relativen Anteil der laufenden und der Investitionsausgaben bestehen zwischen den OECD-Ländern recht deutliche Unterschiede: im Primar-, Sekundar- und post-sekundären, nicht-tertiären Bereich insgesamt liegt der Anteil der laufenden Ausgaben zwischen weniger als 86 Prozent in Griechenland, Korea und derTürkei und bis zu 96 Prozent und mehr in Belgien (fläm.), Kanada, Mexiko und der Slowakischen Republik (Abb. B6.2). 


\section{Abbildung B6.2.}

Aufteilung der Gesamtausgaben und der laufenden Ausgaben für Bildungseinrichtungen, nach Art der Ressource und Bildungsbereich (1999)
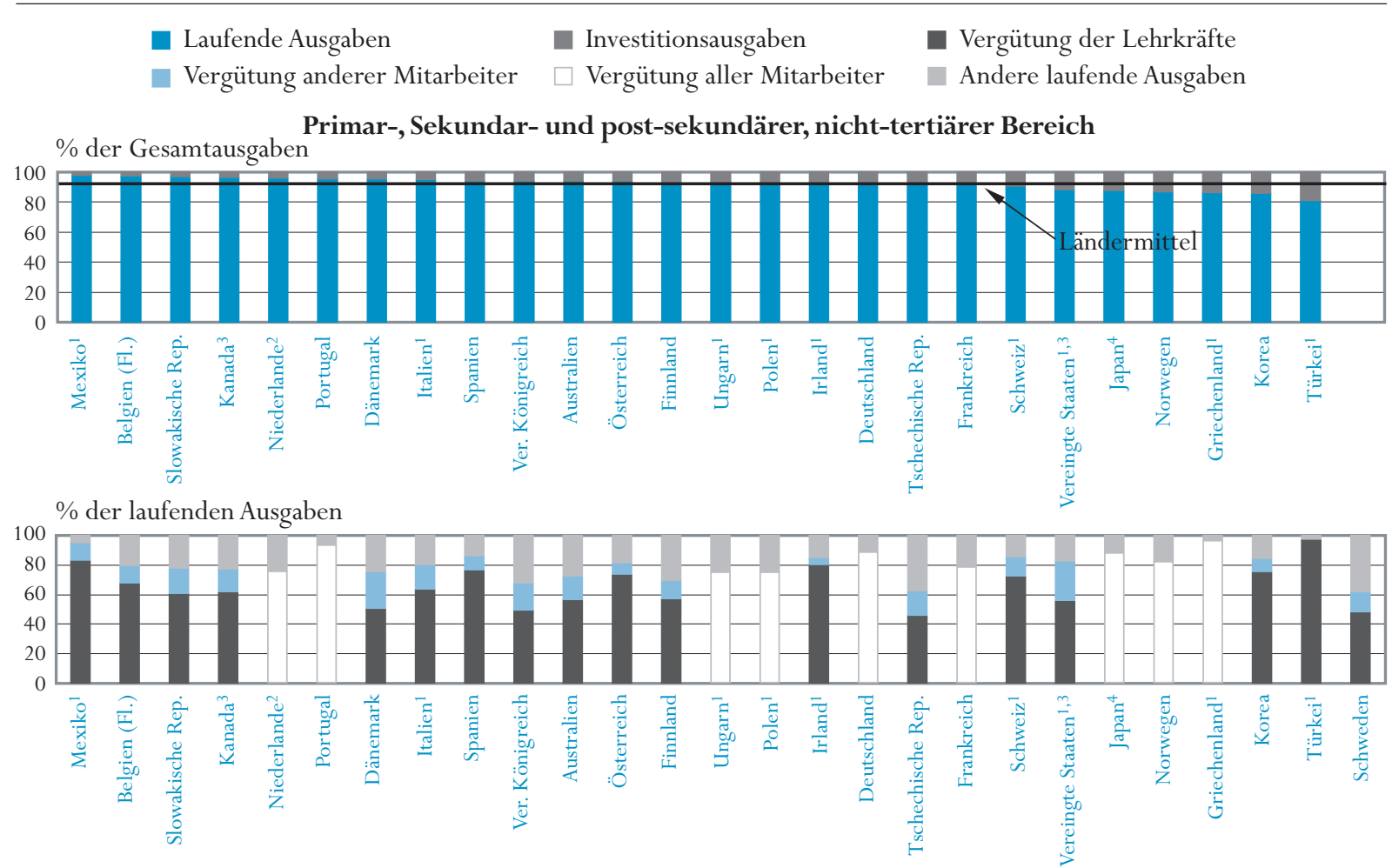

$\%$ der Gesamtausgaben

Tertiärbereich
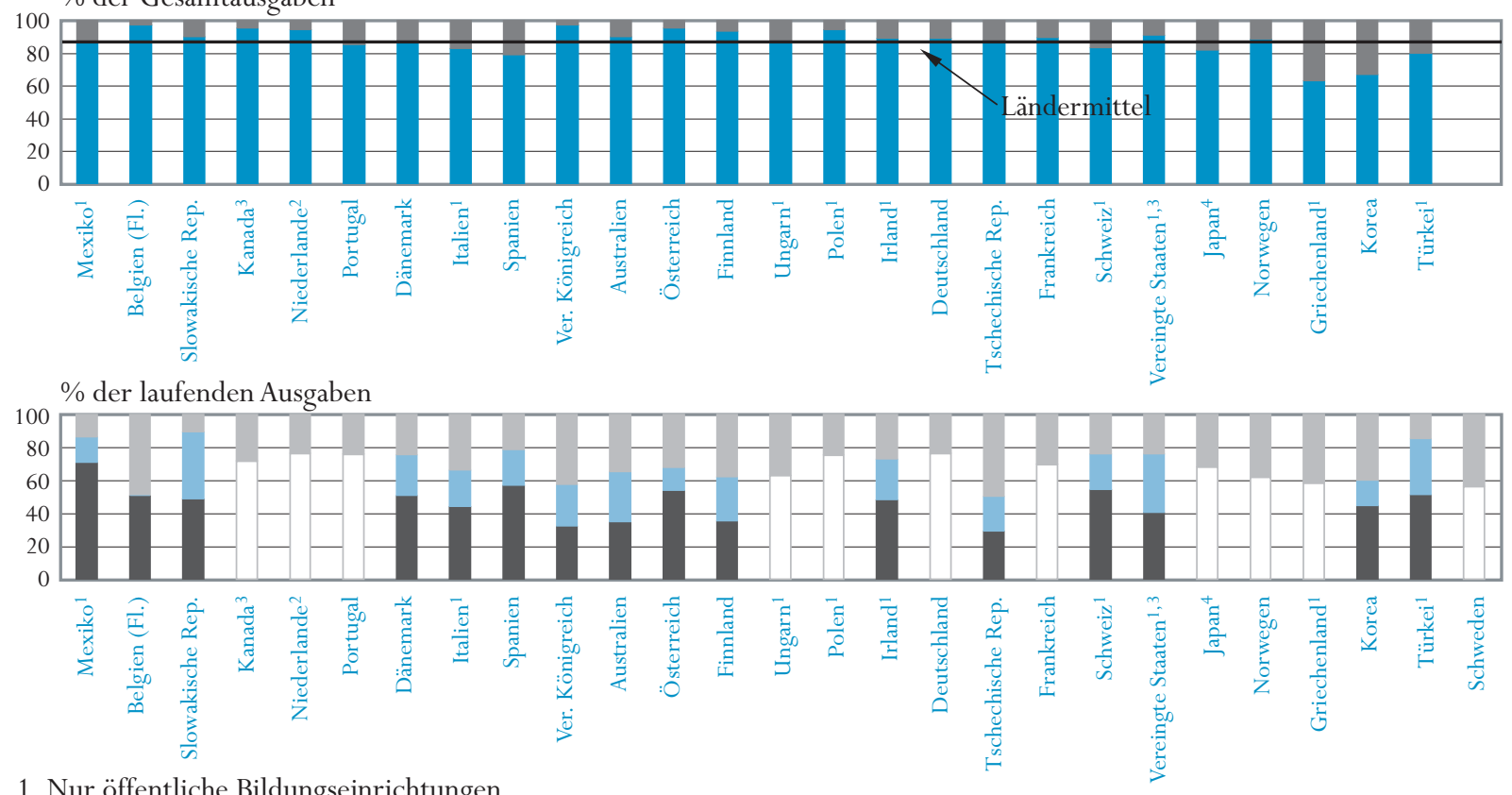

1. Nur öffentliche Bildungseinrichtungen.

2. Nur öffentliche und staatlich-subventionierte private Bildungseinrichtungen.

3. Ohne post-sekundären, nicht-tertiären Bereich.

4. Post-sekundärer, nicht-tertiärer Bereich sowohl im Sekundarbereich als auch im Tertiärbereich enthalten.

Anordnung der Länder in absteigender Reihenfolge der laufenden Ausgaben im Primar-, Sekundar- und post-sekundären, nicht-tertiären Bereich. Quelle: OECD. Tabelle B6.3. Hinweise s. Anhang 3 (www.oecd.org/els/education/eag2002). 
Mit Ausnahme von vier

Ländern entfallen in allen OECD-Ländern mindestens 70 Prozent derlaufenden Ausgaben im Primar-, Sekundarund post-sekundären, nicht-tertiären Bereich auf die Vergütung der Beschäftigten.

Es bestehen zwischen den einzelnen OECDLändern Unterschiede hinsichtlich des Anteils der laufenden Ausgaben, der für die Vergütung von Lehrern und anderen Beschäftigten vorgesehen ist.

Aufgrund der wesentlich differenzierteren und moderneren Lehreinrichtungen ist der Anteil der Investitionsausgaben im Tertiärbereich im Allgemeinen höher.
Die Gehälter der Lehrkräfte und der sonstigen Beschäftigten im Bildungsbereich machen in den OECD-Ländern den größten Teil der laufenden Ausgaben aus. Im Durchschnitt der OECD-Länder entfallen 80 Prozent der laufenden Ausgaben für den Primar-, Sekundar- und post-sekundären, nicht-tertiären Bereich auf die Vergütung der im Bildungsbereich Beschäftigten. Während in Finnland, Schweden, der Tschechischen Republik und dem Vereinigten Königreich der Anteil der Vergütung für die im Bildungsbereich Beschäftigten bei 70 Prozent und weniger liegt, beträgt der entsprechende Anteil in Griechenland, Mexiko, Portugal und der Türkei 90 Prozent und mehr (Abbildung B6.2).

OECD-Länder mit relativ kleinen Bildungsetats (z.B. Mexiko, Portugal und die Türkei) geben tendenziell einen größeren Anteil ihrer laufenden Bildungsausgaben für die Vergütung der Beschäftigten und einen kleineren Anteil für andere per Auftrag vergebene oder zugekaufte Serviceleistungen (z.B. Instandhaltung der Schulgebäude), zusätzliche Dienstleistungen (z.B. Zubereitung von Schulmahlzeiten) und die Anmietung von Schulgebäuden und sonstigen Einrichtungen aus.

In Dänemark und den Vereinigten Staaten sind ungefähr ein Viertel der Personalausgaben im Primar-, Sekundar- und post-sekundären, nicht-tertiären Bereich insgesamt für die Vergütung von nicht-unterrichtenden Beschäftigten vorgesehen; in Irland, Korea, Österreich und Spanien dagegen beträgt dieser Anteil 10 Prozent und weniger. Diese Unterschiede spiegeln wahrscheinlich wider, inwieweit in einem bestimmten Land im Bildungsbereich Beschäftigte nicht unterrichtend tätig sind (z.B. Schulleiter, die selbst nicht unterrichten, Beratungslehrer, Busfahrer, Krankenschwestern im Schuldienst, Hausmeister und Handwerker) (Tabelle B6.3).

Im Tertiärbereich ist der Anteil der Investitionsausgaben an den Gesamtausgaben im Allgemeinen aufgrund der wesentlich differenzierteren und moderneren Lehreinrichtungen höher als im Primar-, Sekundar- und postsekundären, nicht-tertiären Bereich. In 18 von 26 OECD-Ländern liegt der Anteil der Investitionsausgaben im Tertiärbereich bei 10 Prozent und mehr, in Griechenland. Korea, Spanien und der Türkei liegt er bei über 20 Prozent (Abb. B6.2).

Die Unterschiede spiegeln wahrscheinlich die Organisation des Tertiärbereichs in den einzelnen OECD-Ländern sowie das Ausmaß wider, in dem wachsende Studierendenzahlen den Neubau von Gebäuden erfordern.

Die OECD-Länder wenden durchschnittlich 31 Prozent der laufenden Ausgaben im Tertiärbereich für andere Zwecke als die Vergütung der im Bildungsbereich Beschäftigten auf. Ein wesentlicher Grund hierfür sind die höheren Kosten für die Einrichtung und Ausrüstung der tertiären Bildungseinrichtungen (Abb. B6.2). 


\section{Definitionen und angewandte Methodik}

Die Unterscheidung zwischen laufenden und Investitionsausgaben entspricht der in der volkswirtschaftlichen Gesamtrechnung üblichen Praxis. Laufende Ausgaben beziehen sich auf Ausgaben für die im laufenden Haushaltsjahr verbrauchten Güter und Dienstleistungen, die immer wieder anfallen, um die Bereitstellung von Bildungsdienstleistungen aufrechtzuerhalten. Investitionsausgaben beziehen sich auf Ausgaben für Sachwerte mit einer Lebensdauer von mehr als einem Jahr. Hierzu zählen u.a. die Ausgaben für Bau, Renovierung und größere Instandsetzungsarbeiten von Gebäuden sowie für die Neubeschaffung oder den Ersatz von Ausrüstungsgegenständen. Die hier ausgewiesenen Investitionsausgaben beziehen sich auf den Wert der in dem betreffenden Jahr erworbenen oder geschaffenen bildungsbezogenen Vermögenswerte, ausgedrückt in der Höhe der Kapitalbildung, unabhängig davon, ob die Investitionsausgaben durch laufende Einnahmen oder Kreditaufnahmen finanziert wurden. Weder in den Angaben für die laufenden Ausgaben noch für die Investitionsausgaben ist der Schuldendienst enthalten.

Die Berechnungen beziehen sich auf die Ausgaben der öffentlichen Bildungseinrichtungen und, soweit verfügbar, auf die Ausgaben der öffentlichen und privaten Einrichtungen zusammen.

Die laufenden Ausgaben beinhalten, abgesehen von den Personalausgaben, auch Ausgaben für per Auftrag vergebene oder zugekaufte Serviceleistungen (z.B. für die Instandhaltung von Schulgebäuden), zusätzliche Dienstleistungen (z.B. für die Zubereitung von Schulmahlzeiten) und Mietzahlungen für Schulgebäude und andere Einrichtungen. Diese Dienstleistungen werden von externen Anbietern erbracht (im Unterschied zu Leistungen, die von den Bildungsbehörden oder den Bildungseinrichtungen selbst mit eigenem Personal erbracht werden).

Die F\&E-Ausgaben enthalten sämtliche Ausgaben für Forschung an Hochschulen und anderen tertiären Bildungseinrichtungen, unabhängig davon, ob diese aus dem allgemeinen Etat der Einrichtungen, über separate Zuschüsse oder über Verträge mit öffentlichen oder privaten Geldgebern finanziert wird. Die Klassifizierung der Ausgaben als Forschungsausgaben basiert auf den Angaben der forschenden Institutionen.

„Zusätzliche Dienstleistungen“ sind Dienstleistungen, die von den Bildungseinrichtungen neben dem eigentlichen Bildungsauftrag erbracht werden. Die beiden Hauptkomponenten sind soziale Dienste für Schüler/ Studierende und Dienstleistungen für die Allgemeinheit. Im Primar-, Sekundar- und post-sekundären, nicht-tertiären Bereich umfassen die sozialen Dienstleitungen die Bereitstellung von Mahlzeiten, die Gesundheitsdienste sowie Schultransporte. Im Tertiärbereich sind es Wohnheime, Mensen und Gesundheitsdienste. $\mathrm{Zu}$ den Dienstleistungen für die Allgemeinheit zählen Museen, Radio- und Fernsehsendungen, Sport-, Freizeit- und Kulturprogramme. Nicht enthalten sind Ausgaben für zusätzliche
Die Daten beziehen sich auf das Haushaltsjahr 1999 und beruhen auf der von der OECD im Jahre 2001 durchgeführten VOEDatenerhebung zur Bildungsstatistik (Einzelheiten s. Anhang 3). 
Dienstleistungen, für die Gebühren von Schülern/Studierenden oder privaten Haushalten erhoben werden.

Die Ausgaben für eigentliche Bildungsdienstleistungen werden als der Restbetrag sämtlicher Ausgaben geschätzt, d.h. sie umfassen die Gesamtausgaben für Bildungseinrichtungen abzüglich der Aufwendungen für F\&E und zusätzliche Dienstleistungen.

Es ist zu beachten, dass die in früheren Ausgaben dieser Veröffentlichung aufgeführten Zahlen und Daten möglicherweise nicht immer mit denen der Ausgabe 2002 vergleichbar sind, da sich Änderungen in den Definitionen und Erhebungsbereichen ergeben haben. Diese wurden aufgrund der OECD Expenditure Comparability Study vorgenommen (Einzelheiten zu den Änderungen s. Anhang 3 unter www.oecd.org/els/education/eag2002). 
Tabelle B6.1.

Ausgaben für Unterricht, Forschung und Entwicklung (F\&E) und zusätzliche Leistungen in Bildungseinrichtungen als Prozentsatz des BIP sowie private Ausgaben für außerhalb von Bildungseinrichtungen erworbene Bildungsgüter als Anteil am BIP (1999)

\begin{tabular}{|c|c|c|c|c|c|c|c|c|c|}
\hline & \multicolumn{4}{|c|}{ Primar-, Sekundar- und post-sekundärer, nicht-tertiärer Bereich } & \multicolumn{5}{|c|}{ Tertiärbereich } \\
\hline & \multicolumn{3}{|c|}{ Ausgaben für Bildungseinrichtungen für: } & \multirow[b]{2}{*}{\begin{tabular}{|c|} 
Private \\
Zahlungen für \\
Unterrichts- \\
leistungen/ \\
Bildungsgüter \\
außerhalb von \\
Bildungsein- \\
richtungen \\
\end{tabular}} & \multicolumn{4}{|c|}{ Ausgaben für Bildungseinrichtungen für: } & \multirow[b]{2}{*}{$\begin{array}{l}\text { Private } \\
\text { Zahlungen für } \\
\text { Unterrichts- } \\
\text { leistungen/ } \\
\text { Bildungsgüter } \\
\text { außerhalb von } \\
\text { Bildungsein- } \\
\text { richtungen }\end{array}$} \\
\hline & $\begin{array}{c}\text { Unterrichts- } \\
\text { leistungen }\end{array}$ & $\begin{array}{c}\text { Zusätzliche } \\
\text { Dienstleistungen } \\
\text { (Transport, } \\
\text { Mahl- } \\
\text { zeiten u. Unter- } \\
\text { künfte, von den } \\
\text { Bildungseinrich- } \\
\text { tungen zurVer- } \\
\text { fügung gestellt) }\end{array}$ & Insgesamt & & $\begin{array}{l}\text { Unterrichts- } \\
\text { leistungen }\end{array}$ & $\begin{array}{c}\text { Zusätzliche } \\
\text { Dienstleistungen } \\
\text { (Transport, } \\
\text { Mahl- } \\
\text { zeiten u. Unter- } \\
\text { künfte, von den } \\
\text { Bildungseinrich- } \\
\text { tungen zur Ver- } \\
\text { fügung gestellt) }\end{array}$ & $\begin{array}{l}\text { Forschung } \\
\text { und Ent- } \\
\text { wicklung an } \\
\text { Bildungsein- } \\
\text { richtungen } \\
\text { des Tertiärbe- } \\
\text { reichs }\end{array}$ & Insgesamt & \\
\hline & $(1)$ & $(2)$ & (3) & $(4)$ & $(5)$ & $(6)$ & $(7)$ & $(8)$ & (9) \\
\hline Australien & 4.07 & 0.16 & 4.23 & 0.16 & 1.00 & 0.09 & 0.43 & 1.52 & 0.16 \\
\hline Österreich & $\mathrm{x}(3)$ & $\mathrm{x}(3)$ & 4.18 & $\mathrm{~m}$ & $x(8)$ & $x(8)$ & $\mathrm{x}(8)$ & 1.45 & $\mathrm{~m}$ \\
\hline Belgien & $x(3)$ & $x(3)$ & 3.45 & $\mathrm{~m}$ & $x(8)$ & $x(8)$ & 0.46 & 1.26 & $\mathrm{~m}$ \\
\hline Kanada $^{1 *}$ & 3.66 & 0.19 & 3.84 & $\mathrm{~m}$ & 2.05 & 0.02 & 0.43 & 2.51 & 0.40 \\
\hline Tschechische Rep. & 2.68 & 0.48 & 3.17 & $\mathrm{~m}$ & 0.67 & 0.10 & 0.15 & 0.92 & $\mathrm{~m}$ \\
\hline Dänemark & $x(3)$ & $x(3)$ & 4.17 & 0.07 & 1.14 & $x(5)$ & 0.43 & 1.56 & 0.83 \\
\hline Finnland & 3.25 & 0.38 & 3.63 & $\mathrm{~m}$ & 1.14 & 0.01 & 0.63 & 1.78 & $\mathrm{~m}$ \\
\hline Frankreich $^{2 *}$ & 3.76 & 0.60 & 4.35 & 0.14 & 0.88 & 0.07 & 0.18 & 1.13 & 0.08 \\
\hline Deutschland & 3.68 & $\mathrm{n}$ & 3.68 & 0.20 & 0.65 & $\mathrm{n}$ & 0.40 & 1.06 & 0.13 \\
\hline Griechenland $^{3}$ & 2.41 & 0.04 & 2.45 & 0.00 & 0.77 & 0.02 & 0.23 & 1.02 & $\mathrm{n}$ \\
\hline Ungarn $^{3}$ & 2.61 & 0.37 & 2.97 & $\mathrm{~m}$ & 0.80 & 0.13 & 0.13 & 1.07 & $\mathrm{~m}$ \\
\hline Island & $\mathrm{m}$ & $\mathrm{m}$ & $\mathrm{m}$ & $\mathrm{m}$ & $\mathrm{m}$ & $\mathrm{m}$ & $\mathrm{m}$ & $\mathrm{m}$ & $\mathrm{m}$ \\
\hline Irland $^{2^{*}}$ & 3.02 & 0.07 & 3.08 & $\mathrm{~m}$ & 1.17 & $\mathrm{n}$ & 0.23 & 1.40 & $\mathrm{~m}$ \\
\hline Italien & 3.08 & 0.09 & 3.17 & 0.07 & 0.80 & 0.03 & $x(6)$ & 0.83 & 0.40 \\
\hline Japan $^{4}$ & $\mathrm{x}(3)$ & $\mathrm{x}(3)$ & 2.95 & 0.80 & $x(8)$ & $\mathrm{x}(8)$ & $x(8)$ & 1.04 & $\mathrm{~m}$ \\
\hline Korea & $x(3)$ & $x(3)$ & 3.98 & $\mathrm{~m}$ & $\mathrm{x}(8)$ & $x(8)$ & $x(8)$ & 2.38 & $\mathrm{~m}$ \\
\hline Luxemburg & $\mathrm{m}$ & $\mathrm{m}$ & $\mathrm{m}$ & $\mathrm{m}$ & $\mathrm{m}$ & $\mathrm{m}$ & $\mathrm{m}$ & $\mathrm{m}$ & $\mathrm{m}$ \\
\hline Mexiko $^{2 *}$ & $x(3)$ & $x(3)$ & 3.59 & 0.22 & 0.93 & $\mathrm{~m}$ & 0.18 & 1.11 & 0.07 \\
\hline Niederlande ${ }^{2 *}$ & 3.05 & 0.03 & 3.08 & 0.18 & 0.77 & 0.01 & 0.50 & 1.28 & 0.06 \\
\hline Neuseeland & $x(3)$ & $x(3)$ & 4.63 & $\mathrm{~m}$ & $x(8)$ & $x(8)$ & $x(8)$ & 0.94 & $\mathrm{~m}$ \\
\hline Norwegen & $\mathrm{x}(3)$ & $x(3)$ & 4.04 & $\mathrm{~m}$ & $\mathrm{x}(8)$ & $\mathrm{x}(8)$ & $\mathrm{x}(8)$ & 1.51 & $\mathrm{n}$ \\
\hline Polen $^{2,3}$ & 3.45 & 0.19 & 3.63 & $\mathrm{~m}$ & 0.74 & 0.07 & 0.15 & 0.97 & $\mathrm{~m}$ \\
\hline Portugal & $x(3)$ & $x(3)$ & 4.17 & 0.06 & $x(8)$ & $x(8)$ & $x(8)$ & 1.05 & 0.06 \\
\hline Slowakische Rep. & 2.68 & 0.35 & 3.03 & $\mathrm{~m}$ & 0.60 & 0.16 & 0.07 & 0.83 & $\mathrm{~m}$ \\
\hline Spanien & 3.60 & 0.08 & 3.68 & $\mathrm{~m}$ & 0.84 & $x(5)$ & 0.27 & 1.10 & $\mathrm{~m}$ \\
\hline Schweden & 4.18 & 0.21 & 4.39 & 0.69 & 0.88 & $\mathrm{a}$ & 0.81 & 1.69 & 0.63 \\
\hline Schweiz & $x(3)$ & $x(3)$ & 4.39 & $\mathrm{~m}$ & $x(8)$ & $\mathrm{x}(8)$ & $x(8)$ & 1.21 & $\mathrm{~m}$ \\
\hline Türkei ${ }^{2,3}$ & 2.82 & 0.06 & 2.88 & $\mathrm{~m}$ & 1.00 & 0.01 & 0.02 & 1.03 & $\mathrm{~m}$ \\
\hline Ver. Königreich & 3.55 & 0.14 & 3.68 & $\mathrm{~m}$ & 0.68 & $\mathrm{n}$ & 0.38 & 1.07 & 0.09 \\
\hline Vereinigte Staaten ${ }^{1}$ & 3.67 & 0.14 & 3.81 & 0.02 & 2.08 & $\mathrm{n}$ & 0.26 & 2.33 & 0.10 \\
\hline Ländermittel & 3.29 & 0.20 & 3.65 & 0.22 & 0.97 & 0.04 & 0.32 & 1.32 & 0.22 \\
\hline $\begin{array}{l}\text { Hinweis: } \mathrm{x} \text { bedeut } \\
\text { dass die Daten } \\
\text { 1. Post-sekundär } \\
\text { 2. Nur zusätzlich } \\
\text { 3. Ausgaben für I } \\
\text { 4. Post-sekundär } \\
\text { * Hinweise s. An } \\
\text { Quelle: OECD. }\end{array}$ & $\begin{array}{l}\text { et, dass die Dat } \\
\text { in Spalte } 2 \text { enth } \\
\text { er, nicht-tertiä } \\
\text { e Dienstleistur } \\
\text { Forschung und } \\
\text { er, nicht-tertiä } \\
\text { hang } 3 \text { (www.oe }\end{array}$ & $\begin{array}{l}\text { en in einer anderen } \\
\text { halten sind. } \\
\text { irer Bereich sowoh } \\
\text { ngen in öffentliche } \\
\text { Entwicklung und } \\
\text { irer Bereich im Ter } \\
\text { ecd.org/els/educatio }\end{array}$ & $\begin{array}{l}\text { Spalte entha } \\
\text { l im Sekunda } \\
\text { n Bildungsei } \\
\text { daher auch ( } \\
\text { tiärbereich u } \\
\text { n/eag2002). }\end{array}$ & $\begin{array}{l}\text { ten sind, deren Re } \\
\text { rbereich II als au } \\
\text { richtungen; ande } \\
\text { esamtausgaben z } \\
\text { hd nicht im Prim }\end{array}$ & $\begin{array}{l}\text { eferenz in rund } \\
\text { ch im Tertiärbe } \\
\text { ere zusätzliche } \\
\text { u niedrig ange } \\
\text { ar-, Sekundar- }\end{array}$ & $\begin{array}{l}\text { len Klammern nach } \\
\text { ereich enthalten. } \\
\text { Dienstleistungen } \\
\text { setzt. } \\
\text { - und post-sekund }\end{array}$ & $\begin{array}{l}\text { h dem ' } \mathrm{x} \text { ' angeg } \\
\text { sind in den Un } \\
\text { lären, nicht-tert }\end{array}$ & $\begin{array}{l}\text { pen ist. So be } \\
\text { errichtsleistu } \\
\text { aren Bereich }\end{array}$ & $\begin{array}{l}\text { utet z.B. x(2), } \\
\text { gen enthalten. } \\
\text { nthalten. }\end{array}$ \\
\hline
\end{tabular}


Tabelle B6.2.

Ausgaben pro Schüler/Studierenden für Unterricht, zusätzliche Dienstleistungen sowie Forschung und Entwicklung (F\&E) (1999) Ausgaben für Bildungseinrichtungen pro Schüler/Studierenden in US-Dollar, kaufkraftbereinigt, aus öffentlichen und privaten Quellen, nach Art der erbrachten Leistung und Bildungsbereich

Ver. Königreich

Vereinigte Staaten ${ }^{1,6}$ Ländermittel$$
\text { und Bildungsbereich }
$$

\begin{tabular}{|c|c|c|c|c|c|c|}
\hline \multicolumn{3}{|c|}{$\begin{array}{l}\text { Primar-, Sekundar- und post-sekundärer, } \\
\text { nicht-tertiärer Bereich }\end{array}$} & \multicolumn{4}{|c|}{ Tertiärbereich } \\
\hline \multicolumn{3}{|c|}{ Ausgaben für Bildungseinrichtungen für: } & \multicolumn{4}{|c|}{ Ausgaben für Bildungseinrichtungen für: } \\
\hline $\begin{array}{l}\text { Eigentliche } \\
\text { Bildungsdienst- } \\
\text { leistungen }\end{array}$ & $\begin{array}{c}\text { Zusätzliche } \\
\text { Dienstleistungen } \\
\text { (Transport, Mahl- } \\
\text { zeiten u. Unter- } \\
\text { künfte, von den } \\
\text { Bildungseinrich- } \\
\text { tungen zurVer- } \\
\text { fügung gestellt) }\end{array}$ & Insgesamt & $\begin{array}{c}\text { Eigentliche } \\
\text { Bildungsdienst- } \\
\text { leistungen }\end{array}$ & $\begin{array}{l}\text { Zusätzliche } \\
\text { Dienstleistungen } \\
\text { (Transport, Mahl- } \\
\text { zeiten u. Unter- } \\
\text { künfte, von den } \\
\text { Bildungseinrich- } \\
\text { tungen zurVer- } \\
\text { fügung gestellt) }\end{array}$ & $\begin{array}{l}\text { Forschung und } \\
\text { Entwicklung }\end{array}$ & Insgesamt \\
\hline$(1)$ & $(2)$ & (3) & $(4)$ & $(5)$ & $(6)$ & $(7)$ \\
\hline 5592 & 217 & 5809 & 7714 & 674 & 3338 & 11725 \\
\hline $\mathrm{x}(3)$ & $x(3)$ & 7818 & $\mathrm{x}(7)$ & $\mathrm{x}(7)$ & $\mathrm{x}(7)$ & 12070 \\
\hline$x(3)$ & $x(3)$ & 5329 & $\mathrm{x}(7)$ & $x(7)$ & 3565 & 9724 \\
\hline 5691 & 289 & 5981 & 12443 & 146 & 2622 & 15211 \\
\hline 2286 & 413 & 2699 & 4124 & 606 & 958 & 5688 \\
\hline$x(3)$ & $x(3)$ & 7226 & 7753 & $x(4)$ & 2904 & 10657 \\
\hline 4559 & 535 & 5093 & 5196 & 30 & 2888 & 8114 \\
\hline 5129 & 815 & 5944 & 6123 & 514 & 1231 & 7867 \\
\hline 5955 & $\mathrm{n}$ & 5955 & 6438 & $\mathrm{n}$ & 3955 & 10393 \\
\hline 2837 & 49 & 2886 & 3199 & 93 & 968 & 4260 \\
\hline 2046 & 288 & 2334 & 4398 & 726 & 736 & 5861 \\
\hline $\mathrm{m}$ & $\mathrm{m}$ & $\mathrm{m}$ & $\mathrm{m}$ & $\mathrm{m}$ & $\mathrm{m}$ & $\mathrm{m}$ \\
\hline 3550 & 76 & 3626 & 8089 & $\mathrm{n}$ & 1585 & 9673 \\
\hline 5905 & 173 & 6078 & 7292 & 260 & $\mathrm{x}(4)$ & 7552 \\
\hline$x(3)$ & $x(3)$ & 5668 & $x(7)$ & $x(7)$ & $x(7)$ & 10278 \\
\hline$x(3)$ & $x(3)$ & 3137 & $x(7)$ & $x(7)$ & $x(7)$ & 5356 \\
\hline $\mathrm{m}$ & $\mathrm{m}$ & $\mathrm{m}$ & $\mathrm{m}$ & $\mathrm{m}$ & $\mathrm{m}$ & $\mathrm{m}$ \\
\hline$x(3)$ & $x(3)$ & 1240 & 4018 & $\mathrm{n}$ & 771 & 4789 \\
\hline 4890 & 45 & 4934 & 7383 & 77 & 4825 & 12285 \\
\hline$x(3)$ & $x(3)$ & $\mathrm{m}$ & $x(7)$ & $x(7)$ & $x(7)$ & $\mathrm{m}$ \\
\hline $\mathrm{x}(3)$ & $\mathrm{x}(3)$ & 6665 & $x(7)$ & $\mathrm{x}(7)$ & $\mathrm{x}(7)$ & 12096 \\
\hline 1685 & 92 & 1778 & 2993 & 301 & 618 & 3912 \\
\hline$x(3)$ & $x(3)$ & 4483 & $x(7)$ & $x(7)$ & $x(7)$ & 4802 \\
\hline 1639 & 212 & 1852 & 3854 & 1003 & 468 & 5325 \\
\hline 4241 & 90 & 4331 & 4331 & $x(4)$ & 1376 & 5707 \\
\hline 553 & 278 & 5832 & 7395 & a & 6828 & 14222 \\
\hline$x(3)$ & $x(3)$ & 8192 & $x(7)$ & $\mathrm{x}(7)$ & $x(7)$ & 17997 \\
\hline $\mathrm{m}$ & $\mathrm{m}$ & $\mathrm{m}$ & 4206 & 21 & 100 & 4328 \\
\hline 4354 & 208 & 4563 & 6120 & $\mathrm{n}$ & 3434 & 9554 \\
\hline 7131 & 266 & 7397 & 17115 & $\mathrm{n}$ & 2105 & 19220 \\
\hline 4297 & 238 & 4879 & 6493 & 247 & 2264 & 9210 \\
\hline
\end{tabular}

Hinweis: $\mathrm{x}$ bedeutet, dass die Daten in einer anderen Spalte enthalten sind, deren Referenz in runden Klammern nach dem ' $\mathrm{x}$ ' angegeben ist. So bedeutet $\mathrm{z} . \mathrm{B}$. $\mathrm{x}(2)$, dass die Daten in Spalte 2 enthalten sind.

1. Nur öffentliche und staatlich-subventionierte private Bildungseinrichtungen.

2. Post-sekundärer, nicht-tertiärer Bereich im Tertiärbereich enthalten.

3. Primar-, Sekundar- und nicht-sekundärer, nicht-tertiärer Bereich umfasst auch Tertiärbereich B.

4. Nur öffentliche Bildungseinrichtungen.

5. Nur öffentliche und unabhängige private Bildungseinrichtungen.

6. Post-sekundärer, nicht-tertiärer Bereich sowohl im Sekundarbereich II als auch im Tertiärbereich enthalten.

* Hinweise s. Anhang 3 (www.oecd.org/els/education/eag2002).

Quelle: OECD. 
Tabelle B6.3.

Ausgaben für Bildungseinrichtungen nach Ausgabenkategorien (1999)

Verteilung der Gesamtausgaben und der laufenden Ausgaben für Bildungseinrichtungen aus öffentlichen und privaten Quellen, nach Art der Ausgabenkategorien und Bildungsbereichen

\begin{tabular}{|c|c|c|c|c|c|c|c|c|c|c|c|c|}
\hline & \multicolumn{6}{|c|}{ Primar-, Sekundar- und post-sekundärer, nicht-tertiärer Bereich } & \multicolumn{6}{|c|}{ Tertiärbereich } \\
\hline & \multicolumn{2}{|c|}{$\begin{array}{l}\text { Prozentsatz der } \\
\text { Gesamtausgaben }\end{array}$} & \multicolumn{4}{|c|}{ Prozentsatz der laufenden Ausgaben } & \multicolumn{2}{|c|}{$\begin{array}{l}\text { Prozentsatz der } \\
\text { Gesamtausgaben }\end{array}$} & \multicolumn{4}{|c|}{ Prozentsatz der laufenden Ausgaben } \\
\hline & $\begin{array}{c}\text { laufende } \\
\text { Ausgaben }\end{array}$ & $\begin{array}{c}\text { Investi- } \\
\text { tionsaus- } \\
\text { gaben }\end{array}$ & $\begin{array}{c}\text { Lehrer- } \\
\text { vergütung }\end{array}$ & $\begin{array}{l}\text { Vergü- } \\
\text { tung der } \\
\text { übrigen } \\
\text { Beschäf- } \\
\text { tigten }\end{array}$ & $\begin{array}{l}\text { Vergütung } \\
\text { aller Be- } \\
\text { schäftigten }\end{array}$ & $\begin{array}{c}\text { andere } \\
\text { laufende } \\
\text { Ausgaben }\end{array}$ & $\begin{array}{l}\text { laufende } \\
\text { Ausgaben }\end{array}$ & $\begin{array}{l}\text { Investi- } \\
\text { tionsaus- } \\
\text { gaben }\end{array}$ & $\begin{array}{c}\text { Lehrer- } \\
\text { vergütung }\end{array}$ & $\begin{array}{l}\text { Vergü- } \\
\text { tung der } \\
\text { übrigen } \\
\text { Beschäf- } \\
\text { tigten }\end{array}$ & $\begin{array}{l}\text { Vergütung } \\
\text { aller Be- } \\
\text { schäftigten }\end{array}$ & $\begin{array}{c}\text { andere } \\
\text { laufende } \\
\text { Ausgaben }\end{array}$ \\
\hline & (1) & $(2)$ & (3) & (4) & $(5)$ & (6) & (7) & (8) & (9) & $(10)$ & (11) & $(12)$ \\
\hline Australien & 93.7 & 6.3 & 56.3 & 15.6 & 71.9 & 28.1 & 89.9 & 10.1 & 35.1 & 30.2 & 65.3 & 34.7 \\
\hline Österreich & 93.5 & 6.5 & 73.3 & 7.9 & 81.2 & 18.8 & 95.4 & 4.6 & 53.6 & 14.2 & 37.8 & 32.2 \\
\hline Belgien & $\mathrm{m}$ & $\mathrm{m}$ & $\mathrm{m}$ & $\mathrm{m}$ & $\mathrm{m}$ & $\mathrm{m}$ & $\mathrm{m}$ & $\mathrm{m}$ & $\mathrm{m}$ & $\mathrm{m}$ & $\mathrm{m}$ & $\mathrm{m}$ \\
\hline Belgien (Fl.) & 97.2 & 2.8 & 67.4 & 11.7 & 79.1 & 20.9 & 97.3 & 2.7 & 50.6 & 0.7 & 51.4 & 48.6 \\
\hline Kanada $^{1 *}$ & 96.4 & 3.6 & 61.7 & 15.1 & 76.8 & 23.2 & 95.4 & 4.6 & $\mathrm{x}(11)$ & $\mathrm{x}(11)$ & 71.7 & 28.3 \\
\hline Tschechische Rep. & 91.9 & 8.1 & 45.5 & 16.5 & 62.1 & 37.9 & 87.6 & 12.4 & 29.2 & 21.1 & 50.3 & 49.7 \\
\hline Dänemark & 95.1 & 4.9 & 50.3 & 25.0 & 75.3 & 24.7 & 87.3 & 12.7 & 50.8 & 24.8 & 75.6 & 24.4 \\
\hline Finnland & 92.9 & 7.1 & 56.8 & 12.1 & 68.9 & 31.1 & 93.2 & 6.8 & 35.4 & 26.4 & 61.8 & 38.2 \\
\hline Frankreich & 91.4 & 8.6 & $\mathrm{x}(5)$ & $\mathrm{x}(5)$ & 78.6 & 21.4 & 89.2 & 10.8 & $\mathrm{x}(11)$ & $\mathrm{x}(11)$ & 70.0 & 30.0 \\
\hline Deutschland & 92.3 & 7.7 & $\mathrm{x}(5)$ & $\mathrm{x}(5)$ & 88.8 & 11.2 & 88.9 & 11.1 & $\mathrm{x}(11)$ & $\mathrm{x}(11)$ & 76.2 & 23.8 \\
\hline Griechenland $^{2}$ & 85.8 & 14.2 & $x(5)$ & $\mathrm{x}(5)$ & 96.4 & 3.6 & 62.9 & 37.1 & $\mathrm{x}(11)$ & $\mathrm{x}(11)$ & 58.4 & 41.6 \\
\hline Ungarn $^{2 *}$ & 92.6 & 7.4 & $x(5)$ & $\mathrm{x}(5)$ & 75.2 & 24.8 & 86.9 & 13.1 & $\mathrm{x}(11)$ & $\mathrm{x}(11)$ & 63.2 & 36.8 \\
\hline Island & $\mathrm{m}$ & $\mathrm{m}$ & $\mathrm{m}$ & $\mathrm{m}$ & $\mathrm{m}$ & $\mathrm{m}$ & $\mathrm{m}$ & $\mathrm{m}$ & $\mathrm{m}$ & $\mathrm{m}$ & $\mathrm{m}$ & $\mathrm{m}$ \\
\hline Irland $^{2}$ & 92.2 & 7.8 & 80.0 & 4.8 & 84.9 & 15.1 & 88.9 & 11.1 & 48.1 & 24.6 & 72.7 & 27.3 \\
\hline Italien $^{2 *}$ & 94.8 & 5.2 & 63.6 & 16.6 & 80.2 & 19.8 & 82.7 & 17.3 & 43.8 & 22.5 & 66.3 & 33.7 \\
\hline $\operatorname{Japan}^{3 *}$ & 87.6 & 12.4 & $x(5)$ & $\mathrm{x}(5)$ & 88.1 & 11.9 & 81.5 & 18.5 & $\mathrm{x}(11)$ & $\mathrm{x}(11)$ & 68.4 & 31.6 \\
\hline Korea & 85.6 & 14.4 & 75.3 & 8.5 & 83.8 & 16.2 & 66.9 & 33.1 & 44.8 & 15.0 & 59.8 & 40.2 \\
\hline Luxemburg & $\mathrm{m}$ & $\mathrm{m}$ & $\mathrm{m}$ & $\mathrm{m}$ & $\mathrm{m}$ & $\mathrm{m}$ & $\mathrm{m}$ & $\mathrm{m}$ & $\mathrm{m}$ & $\mathrm{m}$ & $\mathrm{m}$ & $\mathrm{m}$ \\
\hline Mexiko $^{2}$ & 97.6 & 2.4 & 82.9 & 12.0 & 94.9 & 5.1 & 86.7 & 13.3 & 71.0 & 15.3 & 86.3 & 13.7 \\
\hline Niederlande $^{4}$ & 95.7 & 4.3 & $\mathrm{x}(5)$ & $\mathrm{x}(5)$ & 75.9 & 24.1 & 94.0 & 6.0 & $\mathrm{~m}$ & $\mathrm{~m}$ & 76.2 & 23.8 \\
\hline Neuseeland & $\mathrm{m}$ & $\mathrm{m}$ & $\mathrm{m}$ & $\mathrm{m}$ & $\mathrm{m}$ & $\mathrm{m}$ & $\mathrm{m}$ & $\mathrm{m}$ & $\mathrm{m}$ & $\mathrm{m}$ & $\mathrm{m}$ & $\mathrm{m}$ \\
\hline Norwegen & 86.3 & 13.7 & $\mathrm{x}(5)$ & $\mathrm{x}(5)$ & 82.3 & 17.7 & 88.7 & 11.3 & $\mathrm{x}(11)$ & $\mathrm{x}(11)$ & 62.1 & 37.9 \\
\hline Polen $^{2}$ & 92.7 & 7.3 & $x(5)$ & $\mathrm{x}(5)$ & 74.9 & 25.1 & 94.4 & 5.6 & $\mathrm{x}(11)$ & $\mathrm{x}(11)$ & 75.2 & 24.8 \\
\hline Portugal & 95.4 & 4.6 & $x(5)$ & $\mathrm{x}(5)$ & 93.7 & 6.3 & 85.1 & 14.9 & $\mathrm{x}(11)$ & $\mathrm{x}(11)$ & 75.9 & 24.1 \\
\hline Slowakische Rep. & 96.8 & 3.2 & 60.6 & 16.8 & 77.4 & 22.6 & 89.9 & 10.1 & 48.8 & 40.4 & 89.3 & 10.8 \\
\hline Spanien & 93.9 & 6.1 & 76.1 & 9.5 & 85.6 & 14.4 & 79.1 & 20.9 & 57.1 & 21.1 & 78.2 & 21.8 \\
\hline Schweden ${ }^{*}$ & $\mathrm{~m}$ & $\mathrm{~m}$ & 47.8 & 13.7 & 61.8 & 38.2 & $\mathrm{~m}$ & $\mathrm{~m}$ & $\mathrm{x}(11)$ & $\mathrm{x}(11)$ & 56.6 & 43.4 \\
\hline Schweiz ${ }^{2}$ & 90.4 & 9.6 & 72.4 & 12.9 & 85.3 & 14.7 & 83.2 & 16.8 & 54.4 & 21.5 & 75.8 & 24.2 \\
\hline Türkei $^{2}$ & 80.6 & 19.4 & 96.8 & $\mathrm{~m}$ & 96.8 & 3.2 & 79.7 & 20.3 & 51.1 & 34.1 & 85.2 & 14.8 \\
\hline Ver. Königreich & 93.9 & 6.1 & 49.0 & 18.1 & 67.2 & 32.8 & 97.2 & 2.8 & 32.5 & 25.0 & 57.6 & 42.4 \\
\hline Vereinigte Staaten ${ }^{1,2}$ & 88.1 & 11.9 & 55.9 & 26.4 & 82.3 & 17.7 & 90.7 & 9.3 & 40.4 & 35.5 & 75.9 & 24.1 \\
\hline Ländermittel & 92.1 & 7.9 & 65.1 & 14.3 & 80.3 & 19.7 & 87.0 & 13.0 & 46.7 & 23.3 & 69.4 & 30.6 \\
\hline Argentinien ${ }^{2}$ & 93.4 & 6.6 & 67.1 & 24.9 & 92.0 & 8.0 & 97.9 & 2.1 & 56.2 & 34.9 & 91.1 & 8.9 \\
\hline Brasilien $^{2,5}$ & 94.8 & 5.2 & $x(5)$ & $\mathrm{x}(5)$ & 81.9 & 18.1 & 97.6 & 2.4 & $\mathrm{x}(11)$ & $\mathrm{x}(11)$ & 86.3 & 13.7 \\
\hline Chile $^{2}$ & 91.2 & 8.8 & $\mathrm{x}(5)$ & $\mathrm{x}(5)$ & 57.9 & 42.1 & 91.8 & 8.2 & $\mathrm{x}(11)$ & $\mathrm{x}(11)$ & 69.4 & 30.6 \\
\hline China & 91.2 & 8.8 & $\mathrm{x}(5)$ & $\mathrm{x}(5)$ & 64.3 & 35.7 & 77.6 & 22.4 & $\mathrm{x}(11)$ & $\mathrm{x}(11)$ & 46.0 & 54.0 \\
\hline Indien $^{1,2,5}$ & 97.2 & 2.8 & 79.5 & 8.4 & 87.8 & 12.2 & 96.9 & 3.1 & $\mathrm{x}(11)$ & $\mathrm{x}(11)$ & 99.6 & 0.4 \\
\hline Indonesien ${ }^{2,6}$ & 93.9 & 6.1 & 78.0 & 7.6 & 85.6 & 14.4 & 82.0 & 18.0 & 87.2 & 11.8 & 99.0 & 1.0 \\
\hline Israel & 91.0 & 9.0 & $x(5)$ & $\mathrm{x}(5)$ & 76.9 & 23.1 & 89.7 & 10.3 & $\mathrm{x}(11)$ & $\mathrm{x}(11)$ & 76.5 & 23.5 \\
\hline Jamaika $^{2}$ & 90.9 & 9.1 & 57.4 & 10.0 & 67.3 & 32.7 & 92.3 & 7.7 & 53.6 & 29.2 & 82.7 & 17.3 \\
\hline Jordanien ${ }^{2}$ & 89.0 & 11.0 & 77.8 & 14.7 & 92.5 & 7.5 & 76.5 & 23.5 & $\mathrm{x}(11)$ & $\mathrm{x}(11)$ & 67.6 & 32.4 \\
\hline Malaysia $^{2}$ & 77.3 & 22.7 & 65.8 & 14.8 & 80.6 & 19.4 & 61.7 & 38.3 & 42.1 & 9.2 & 51.4 & 48.6 \\
\hline Paraguay $^{2}$ & 90.7 & 9.3 & 59.6 & 11.3 & 70.9 & 29.1 & 87.0 & 13.0 & $\mathrm{~m}$ & $\mathrm{~m}$ & $\mathrm{~m}$ & $\mathrm{~m}$ \\
\hline $\mathrm{Peru}^{2,7}$ & 89.2 & 10.9 & 89.3 & 2.0 & 91.3 & 8.7 & 88.8 & 11.2 & 46.0 & 9.0 & 55.0 & 45.0 \\
\hline Tunesien ${ }^{2,6}$ & 88.7 & 11.3 & $\mathrm{x}(5)$ & $\mathrm{x}(5)$ & 95.1 & 4.9 & 74.1 & 25.9 & $\mathrm{x}(11)$ & $\mathrm{x}(11)$ & 70.0 & 30.0 \\
\hline Uruguay $^{2}$ & 92.7 & 7.3 & 72.9 & 12.3 & 85.2 & 14.8 & 94.2 & 5.8 & 64.0 & 21.6 & 85.6 & 14.4 \\
\hline
\end{tabular}

Hinweis: $\mathrm{x}$ bedeutet, dass die Daten in einer anderen Spalte enthalten sind, deren Referenz in runden Klammern nach dem ' $\mathrm{x}$ ' angegeben ist. So bedeutet $\mathrm{z} . \mathrm{B}$. $\mathrm{x}(2)$, dass die Daten in Spalte 2 enthalten sind.

1. Post-sekundärer, nicht-tertiärer Bereich im Tertiärbereich enthalten.

2. Nur öffentliche Bildungseinrichtungen.

3. Post-sekundärer, nicht-tertiärer Bereich sowohl im Sekundarbereich II als auch im Tertiärbereich enthalten.

4. Nur öffentliche und staatliche-subventionierte private Bildungseinrichtungen.

5. Referenzjahr 1998.

6. Referenzjahr 2000.

7. Ohne post-sekundären, nicht tertiären Bereich.

* Hinweise s. Anhang 3 (www.oecd.org/els/education/eag2002).

Quelle: OECD. 



\section{Kapitel \\ $\mathrm{C}$}

\section{BILDUNGSZUGANG, \\ BILDUNGSBETEILIGUNG UND \\ BILDUNGSERWARTUNG}

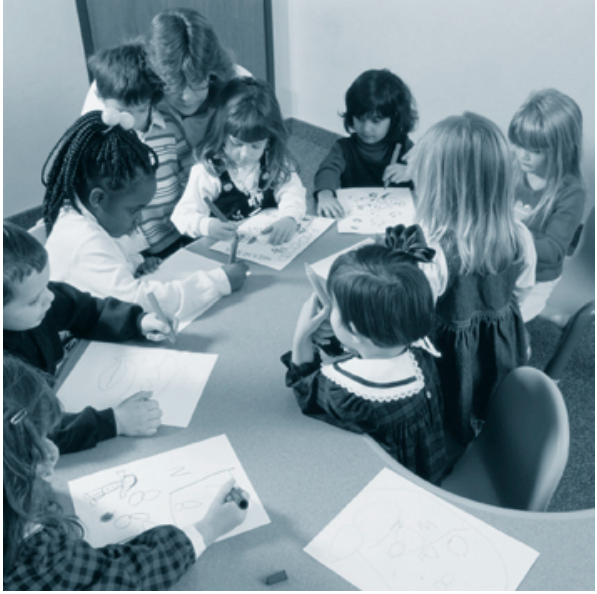





\section{ÜBERBLICK}

Indikator C1: Bildungserwartung (in Jahren) und Bildungsbeteiligung

Tabelle C1.1. Bildungserwartung, in Jahren (2000)

Tabelle C1.2. Beteiligungsquoten (2000)

Indikator C2: Zugang zum Tertiärbereich, zu erwartende Jahre im Tertiärbereich und Bildungsbeteiligung im Sekundarbereich

Tabelle C2.1. Studienanfängerquoten im Tertiärbereich und Altersverteilung der Studienanfänger (2000)

Tabelle C2.2. Zu erwartende Ausbildungsjahre im Tertiärbereich und Veränderung der Gesamtzahl der Studierenden im Tertiärbereich (2000)

Tabelle C2.3. Studierende an öffentlichen und privaten Bildungseinrichtungen und in Vollzeit- und Teilzeit-Ausbildung im Tertiärbereich (2000)

Tabelle C2.4. Schüler an öffentlichen und privaten Bildungseinrichtungen und Schüler in Vollzeit- und Teilzeit-Ausbildung im Primar- und Sekundarbereich (2000)

Tabelle C2.5. Arten der Bildungsteilnahme im Sekundarbereich II (2000)

\section{Indikator C3: Ausländische Studierende im Tertiärbereich}

Tabelle C3.1. Austausch von Studierenden im Tertiärbereich (2000)

Tabelle C3.2. Anteil ausländischer Studierender im Tertiärbereich des Ziellandes (2000)

Tabelle C3.3. Anteil der Studierenden eines Landes, die in einem anderen Land studieren (2000)

Indikator C4: Beteiligung der Erwachsenenbevölkerung an Fortund Weiterbildung

Tabelle C4.1. Beteiligungsquote an Fort- und Weiterbildung während eines Jahres für 25- bis 64-Jährige, nach Bildungsstand, Art der Fort- und Weiterbildung und Geschlecht

\section{Indikator C5: Bildung und Beschäftigungsstatus junger Menschen}

Tabelle C5.1. Prozentsatz junger Menschen in Ausbildung und nicht in Ausbildung, nach Altersgruppen und Beschäftigungsstatus (2001) Tabelle C5.1a. Prozentsatz junger Männer in Ausbildung und nicht in Ausbildung, nach Altersgruppen und Beschäftigungsstatus (2001)

Tabelle C5.1b. Prozentsatz junger Frauen in Ausbildung und nicht in Ausbildung, nach Altersgruppen und Beschäftigungsstatus (2001)

Tabelle C5.2. Anteil arbeitsloser Nicht-Schüler bzw. Nicht-Studierender an der Gesamtbevölkerung, nach Bildungsstand, Altersgruppen und Geschlecht (2001)

\section{Indikator C6: Die Situation junger Menschen mit einem niedrigen Bildungsniveau}

Tabelle C6.1. Prozentsatz 20- bis 24-Jähriger, die sich nicht in Ausbildung befinden, nach Bildungsstand, Geschlecht und Beschäftigungsstatus (2001)
In Kapitel C werden der

Bildungszugang, die

Bildungsbeteiligung und

die Bildungserwartung

untersucht, und

zwar in Form der

Bildungserwartung

in Jahren, und zwar

sowohl insgesamt als

auch in den einzelnen

Bildungsbereichen, des

Zugangs zu und der

Bildungsbeteiligung in

verschiedenen Bildungs-

gängen und Bildungs-

einrichtungen, der

grenz-überschreitenden

Mobilität Studierender,...

...und des Lernens über die Erstausbildung hinaus. 


\section{BILDUNGSERWARTUNG (IN JAHREN) UND BILDUNGSBETEILIGUNG}

- In 25 von 27 OECD-Ländern nimmt der Einzelne im Durchschnitt zwischen 15 und 20 Jahren an formellen Bildungsmaßnahmen teil, wobei der Großteil der Unterschiede auf eine unterschiedliche Bildungsbeteiligung im Sekundarbereich II zurückzuführen ist.

- In 18 von 20 OECD-Ländern stieg die Bildungserwartung (in Jahren) zwischen 1995 und 2000.

- Die Mehrheit der Schüler im Primar- und Sekundarbereich besucht öffentliche Bildungseinrichtungen, inzwischen besuchen jedoch durchschnittlich 11 Prozent der Schüler im Primarbereich, 14 Prozent der Schüler im Sekundarbereich I und 19 Prozent der Schüler im Sekundarbereich II private Bildungseinrichtungen.

- In zwei Fünftel der OECD-Länder werden mehr als 70 Prozent der 3- bis 4-Jährigen im Elementar- oder Primarbereich unterrichtet. Am anderen Ende des Spektrums kann ein heute 17-Jähriger davon ausgehen, dass er voraussichtlich im Durchschnitt 2,5 Jahre im tertiären Bildungsbereich verbringen wird.

\section{Abbildung C1.1.}

Bildungserwartung, in Jahren (2000)

Zu erwartende Jahre in Ausbildung bei gleichbleibenden Rahmenbedingungen, ohne Erziehung von Kindern unter 5 Jahren, nach Bildungsbereichen

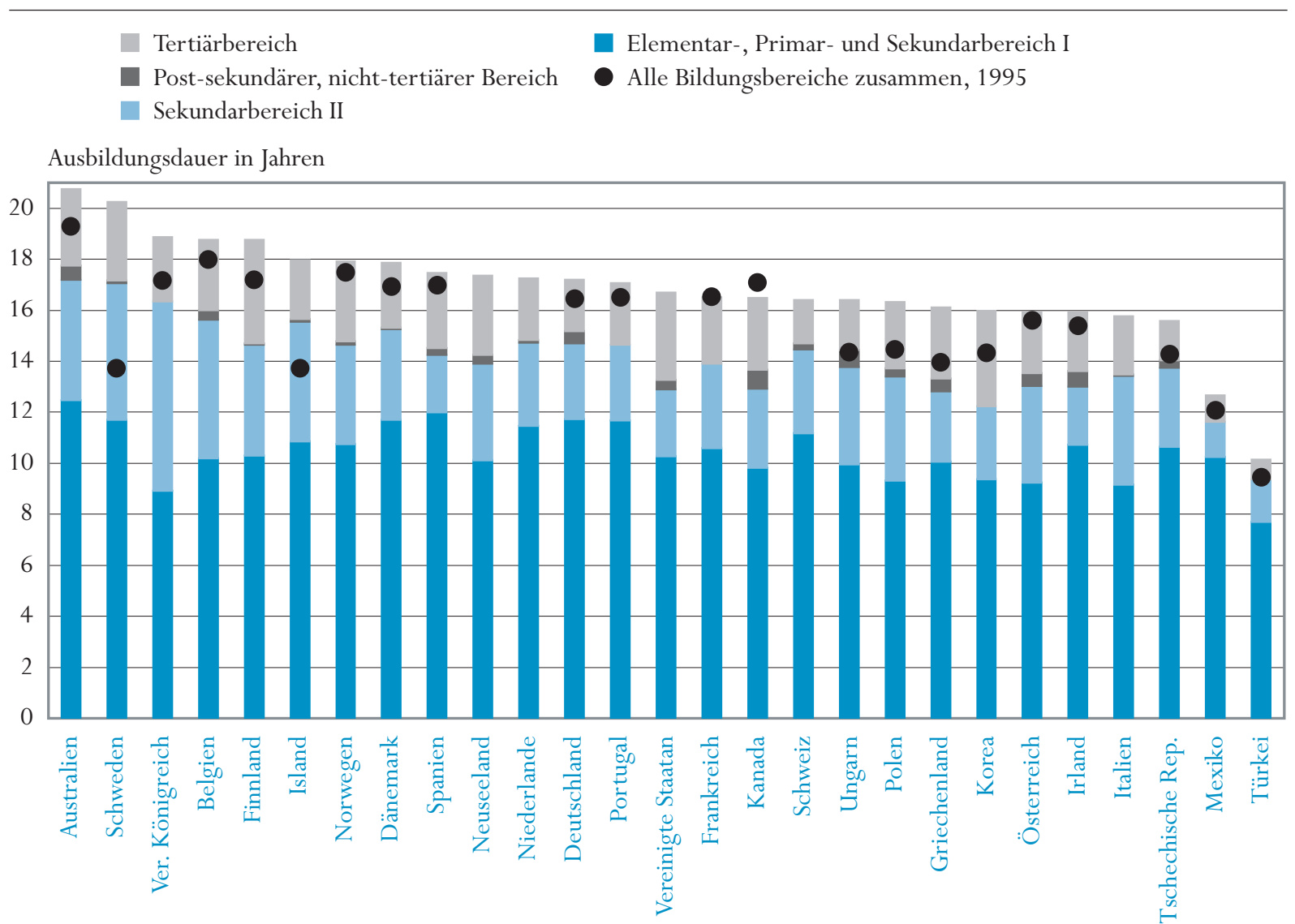

Anordnung der Länder in absteigender Reihenfolge der gesamten Bildungserwartung, in Jahren, für alle Bildungsbereiche im Jahre 2000. Quelle: OECD. Tabelle C1.1. Hinweise s. Anhang 3 (www.oecd.org/els/education/eag2002). 


\section{Politischer Hintergrund}

Eine gut ausgebildete Bevölkerung ist sowohl für die gegenwärtige als auch zukünftige wirtschaftliche und soziale Entwicklung eines Landes von entscheidender Bedeutung. Aus diesem Grunde haben die Gesellschaften ein großes Interesse daran, sowohl für Kinder als auch für Erwachsene einen breiten Zugang zu einer umfangreichen Palette an Bildungsmöglichkeiten zu gewährleisten. Programme im Elementarbereich bereiten die Kinder auf den Primarbereich vor. Sie können Präventivmaßnahmen zur Bekämpfung sprachlicher und sozialer Nachteile bieten sowie die Möglichkeit, die Bildungserfahrungen aus dem Elternhaus auszubauen und zu ergänzen. Primarund Sekundarbereich statten junge Menschen mit grundlegenden Kompetenzen aus und bereiten sie auf lebenslanges Lernen und die Entwicklung hin zu produktiven Mitgliedern der Gesellschaft vor. Der Tertiärbereich bietet dem Einzelnen eine Vielzahl von Möglichkeiten, entweder direkt nach der Schulbildung oder später im Leben erweiterte und spezielle Kenntnisse und Fähigkeiten zu erlangen.

Dieser Indikator stellt verschiedene Kenngrößen für die Bildungsteilnahme vor, um die unterschiedlichen Ebenen des Bildungszugangs in den verschiedenen Ländern zu beschreiben. Die Entwicklungstendenzen der Bildungsbeteiligung in den verschiedenen Bildungsbereichen werden hier auch als Indikator dafür verwendet, wie sich der Zugang zu Bildung entwickelt hat.

\section{Ergebnisse und Erläuterungen}

\section{Bildungsteilnahme insgesamt}

Ein möglicher Blickwinkel bei der Betrachtung der Bildungsbeteiligung besteht darin, den Zeitraum abzuschätzen, den ein heute 5-jähriges Kind, unter Zugrundelegung der aktuellen Beteiligungsquoten, in seinem Leben voraussichtlich in Vollzeit- oder Teilzeitausbildung verbringen wird. Diese „Bildungserwartung“ (in Jahren) wird geschätzt, indem die Bildungsbeteiligung für jede einzelne Altersstufe ab dem 5. Lebensjahr aufaddiert wird (Abb. C1.1). Innerhalb der OECD differiert die Bildungserwartung zwischen 12 Jahren und weniger in Mexiko und der Türkei und bis zu über 18 Jahren in Australien, Belgien, Finnland, Schweden und dem Vereinigten Königreich.

Der größte Teil der Unterschiede der Bildungserwartung (in Jahren) in den einzelnen Ländern beruht auf Unterschieden bei der Bildungsbeteiligung im Sekundarbereich II. Obwohl die relativen Unterschiede auch bei der Bildungsbeteiligung im Tertiärbereich groß sind, gelten sie nur für einen kleineren Anteil der entsprechenden Altersgruppe und wirken sich deshalb weniger auf die „Bildungserwartung“ insgesamt aus.

Da Kenngrößen einer durchschnittlichen Ausbildungsdauer wie die „Bildungserwartung (in Jahren)“ von der für die gesamte Lebensspanne geltenden Bildungsteilnahme beeinflusst werden, unterschätzen sie die tatsächliche Bildungsdauer des Einzelnen in Bildungssystemen mit expandierendem
Dieser Indikator befasst sich mit dem Umfang der Bildungsbeteiligung in allen Bildungsbereichen.

In 25 von 27 OECD-

Ländern nimmt der Einzelne im Durchschnitt zwischen 15 und 20 Jahren an einer formellen Ausbildung teil.

Der gröfste Teil der Unterschiede der Bildungserwartung (in Jahren) in den einzelnen Ländern beruht auf Unterschieden bei der Bildungsbeteiligung im sekundarbereich $I I$. 
Eine Bildungserwartung von vielen Jahren

bedeutet nicht unbedingt, dass alle jungen Menschen Zugang zu höherer Bildung haben,...

aber in den meisten $O E C D$-Ländern nehmen praktisch alle jungen Menschen mindestens 11 Jahre lang an formeller Bildung teil.

In der Mehrheit der $O E C D$-Länder können

Frauen eine längere Bildungsdauer erwarten als Männer - im Durchschnitt zusätzliche 0,5 Jahre.

In 18 von 20 OECD-Ländern stieg
Bildungszugang. Diese Werte unterscheiden auch nicht zwischen Vollzeit- und Teilzeitbeteiligung, was bedeutet, dass OECD-Länder mit einem relativ hohen Anteil von Teilzeitlernenden in der Regel relativ hohe Werte aufweisen. In Australien, Belgien, Neuseeland, Portugal, Schweden und dem Vereinigten Königreich etwa macht die Teilnahme an Teilzeitausbildung mindestens 2 Jahre der Bildungserwartung in Jahren aus (Tabelle C1.1).

Außerdem haben in denjenigen OECD-Ländern, in denen die Bildungserwartung in einem bestimmten Bildungsbereich die Anzahl der Klassenstufen in diesem Bereich übersteigt, Klassenwiederholungen (oder wie im Fall von Australien, die Anzahl der Erwachsenen in solchen Bildungsgängen) größere Auswirkungen auf die Bildungserwartung als der Anteil derjenigen Schüler, die eine Ausbildung vor dem entsprechenden Abschluss abbrechen.

Die Bildungsbeteiligung hängt sowohl von den Zugangsquoten eines bestimmten Bildungsbereichs als auch der typischen Ausbildungsdauer ab. Eine Bildungserwartung von vielen Jahren bedeutet deshalb nicht unbedingt, dass alle jungen Menschen über einen langen Zeitraum im Bildungssystem verbleiben. Belgien und Schweden haben bei einer Bildungserwartung von mehr als 18 Jahren für heute 5-Jährige eine fast vollständige Beteiligung (mehr als 90 Prozent) für eine Bildungsdauer von 15 bzw. 13 Jahren. Im Gegensatz dazu gibt es bei einer gleich hohen Bildungserwartung in Australien und Finnland eine fast vollständige Beteiligung (mehr als 90 Prozent) nur für eine Bildungsdauer von 12 bzw. 11 Jahren (Tabelle C1.2).

In den meisten OECD-Ländern haben praktisch alle Jugendlichen Zugang zu einer formellen Ausbildung von mindestens 11 Jahren. Die Altersspanne, in der mindestens 90 Prozent der Lernenden sich in einem Bildungsgang befinden, umfasst in Belgien, Frankreich, Japan und den Niederlanden 14 Jahre oder mehr. Mexiko und die Türkei hingegen haben maximal für einen Zeitraum von 7 Jahren eine Bildungsbeteiligung von über 90 Prozent (Tabelle C1.2).

In den meisten OECD-Ländern können Frauen mit einer im Durchschnitt 0,5 Jahre längeren Bildungsdauer als Männer rechnen. Die Schwankungen zwischen den OECD-Ländern bei der zu erwartenden Bildungsdauer sind für Frauen meist stärker als für Männer. Einige OECD-Länder weisen beachtliche geschlechtsspezifische Unterschiede auf. In Korea, der Schweiz und der Türkei können Männer mit einer zwischen 0,7 und 2,8 Jahren längeren Bildungsdauer als Frauen rechnen. Das Gegenteil gilt in Finnland, Island, Neuseeland, Norwegen, Schweden und dem Vereinigten Königreich, wo die zu erwartende Bildungsdauer für Frauen die für Männer um mehr als ein Jahr übertrifft (Tabelle C1.1).

\section{Entwicklungen der Bildungsteilnahme}

In 18 von 20 OECD-Ländern, für die vergleichbare Zeitreihendaten verfügbar sind, stieg die Bildungserwartung zwischen 1995 und 2000. In Griechenland, 
Korea, Polen, Ungarn und dem Vereinigten Königreich betrug der Anstieg über diesen relativ kurzen Zeitraum hinweg 10 Prozent oder mehr.

\section{Bildungsbeteiligung im Elementarbereich}

In der Mehrzahl der OECD-Länder beginnt eine universelle Bildungsbeteiligung - hier als eine Bildungsbeteiligung von mehr als 90 Prozent definiert - im Alter von 5 bis 6 Jahren. In Belgien, Dänemark, Frankreich, Island, Italien, Japan, Neuseeland, Norwegen, Spanien, Schweden, der Tschechischen Republik, Ungarn und dem Vereinigten Königreich werden jedoch bereits mehr als 70 Prozent der 3- bis 4-Jährigen im Elementar- oder Primarbereich unterrichtet (Tabelle C1.2). Die Bildungsbeteiligung der 3- bis 4-Jährigen reicht von weniger als 21 Prozent in Kanada, Korea, der Schweiz und der Türkei bis zu über 90 Prozent in Belgien, Frankreich, Island, Italien und Spanien.

Angesichts der Tatsache, dass die Erziehung und Betreuung im Vorschulalter für den Aufbau einer soliden Grundlage für späteres lebenslanges Lernen und zur Sicherstellung von Chancengleichheit beim Zugang zu Bildungsmöglichkeiten später in der Schule eine wichtige Rolle spielt, ist der Elementarbereich von entscheidender Bedeutung. Allerdings wird eine hochwertige Erziehung und Betreuung im Vorschulalter nicht nur in institutionalisierten Bildungsgängen des Elementarbereichs vermittelt, wie sie durch diesen Indikator abgedeckt werden. Schlussfolgerungen bezüglich des Zugangs zu Erziehung und Betreuung im Vorschulalter und deren Qualität sollten deshalb mit großer Vorsicht gezogen werden.

\section{Bildungsbeteiligung gegen Ende und nach der Pflichtschulzeit}

Eine Reihe von Faktoren, u.a. das erhöhte Risiko, arbeitslos zu werden, und andere Formen der Ausgrenzung junger Menschen mit unzureichender Ausbildung beeinflussen die Entscheidung, die Ausbildung über die Pflichtschulzeit hinaus zu verlängern. In vielen OECD-Ländern dauert der Übergang von der Ausbildung in die Beschäftigung länger als früher und ist komplexer geworden. Daraus ergibt sich für die Lernenden die Möglichkeit oder auch die Notwendigkeit, Lernen und Arbeiten miteinander zu verbinden, um Kenntnisse und Fähigkeiten zu erwerben, die für den Arbeitsmarkt relevant sind (s. Indikator C5).

Die Schulpflicht endet in den OECD-Ländern im Alter zwischen 14 Jahren (Korea, Portugal und Türkei) und 18 Jahren (Belgien, Deutschland und die Niederlande), in den meisten Fällen mit 15 oder 16 Jahren (Tabelle C1.2). Das Alter, bis zu dem Schüler gesetzlich zum Schulbesuch verpflichtet sind, entspricht jedoch nicht immer dem Alter, für das eine universelle Bildungsbeteiligung zu verzeichnen ist.

Während in den meisten OECD-Ländern die Bildungsbeteiligung bis zum Ende der Schulpflicht hoch ist, fällt sie in Mexiko, der Türkei und den Vereinigten Staaten vor dem Ende des schulpflichtigen Alters auf unter 90 Prozent. In den die Bildungserwartung zwischen 1995 und 2000

In knapp der Hälfte $\operatorname{der} O E C D$-Länder werden mehr als 70 Prozent der 3- bis 4Jährigen im Elementaroder Primarbereich unterrichtet.

Die Schulpflicht endet in den OECD-Ländern im Alter zwischen 14 und 18 Jahren, in den meisten Ländern mit 15 oder 16 Jahren.

Die Bildungsbeteiligung liegt bis zum Ende der schulpflicht tendenziell 
hoch, es gibt jedoch 3 Länder, in denen mehr

als 10 Prozent der

Schüler das Ende der Pflichtschulausbildung nicht erreichen.

In Australien und den nordischen Ländern befindet sich jeder vierte 20- bis 29-Jährige in Ausbildung.

In 26 von 30 OECD-

Ländern werden

Bildungsgänge im post-sekundären, nicht-tertiären Bereich angeboten.

Im OECD-Durchschnitt kann ein 17-Jähriger davon ausgehen,
Vereinigten Staaten mag dies zum Teil an dem vergleichsweise hohen Alter liegen, mit dem die Schulpflicht endet (17 Jahre). Im Gegensatz hierzu gelingt es in 22 OECD-Ländern, nahezu alle Jugendlichen auch über das Ende der Schulpflicht hinaus in der Schule zu halten (Tabelle C1.2).

Obwohl in den meisten OECD-Ländern für die letzten Jahre im Sekundarbereich II ein allmählicher Rückgang der Bildungsbeteiligung zu verzeichnen ist, gibt es mehrere bemerkenswerte Ausnahmen. In einigen OECD-Ländern gelingt es, die Bildungsbeteiligung bis zum Alter von 20 bis 29 Jahren relativ hoch zu halten. In Australien und den nordischen Ländern liegt die Bildungsbeteiligung der 20- bis 29-Jährigen noch bei über 25 Prozent (Tabelle C1.2).

\section{Der Übergang zum post-sekundären Bereich}

Sowohl Abgänger aus dem Sekundarbereich II, die nicht direkt den Übergang zum Arbeitsmarkt suchen, als auch Berufstätige, die sich fortbilden wollen, können aus einem großen Angebot an post-sekundären Ausbildungsgängen wählen. Innerhalb der OECD unterscheiden sich die tertiären Studiengänge darin, dass sie entweder eher theoretisch ausgerichtet sind und darauf abzielen, Studierende für weiterführende Forschungsprogramme oder Berufe mit hohen Qualifikationsanforderungen vorzubereiten (Tertiärbereich A) oder sich eher auf die Vermittlung berufsspezifischer Fertigkeiten zum direkten Eintritt in den Arbeitsmarkt konzentrieren (Tertiärbereich B). Während man in der Vergangenheit anhand der Art der anbietenden Institution eines Bildungsgangs eine relativ gute Vorstellung von der Art des Bildungsgangs bekam, (z. B. Bildungsangebote an Universitäten oder an nicht-universitären Bildungseinrichtungen), haben sich diese Unterschiede inzwischen verwischt und werden daher bei den OECD-Indikatoren nicht herangezogen.

In einer Reihe von Bildungssystemen haben die Absolventen des Sekundarbereich II auch die Möglichkeit, an relativ kurzen Ausbildungsgängen (mit einer Dauer von unter 2 Jahren) teilzunehmen, um sich für bestimmte Berufe oder Berufsbereiche zu qualifizieren. Während einige OECD-Länder diese Bildungsgänge als höhere oder zweite Stufe innerhalb des Sekundarbereich II anbieten (z.B. Deutschland, Österreich, Spanien und Ungarn), werden sie in anderen im post-sekundären Bereich angeboten (z.B. Kanada und die Vereinigten Staaten). Aus internationaler Sicht befinden sich diese Bildungsgänge im Grenzbereich zwischen dem Sekundarbereich II und dem Tertiärbereich und werden daher als separater Bildungsbereich (ISCED 4) eingestuft. In 26 von 30 OECD-Ländern werden den Absolventen des Sekundarbereich II derartige Bildungsgänge angeboten. (s. Tabelle C1.1).

\section{Bildungsbeteiligung im Tertiärbereich}

Im OECD-Durchschnitt kann ein 17-Jähriger davon ausgehen, dass er während seines gesamten Lebens voraussichtlich 2,5 Jahre im tertiären Bildungsbereich verbringen wird. Die Bildungserwartung im Tertiärbereich wird sowohl 
von den Studienanfängerquoten im Tertiärbereich als auch von der üblichen Studiendauer beeinflusst. In Australien, Finnland, Korea, Neuseeland, Norwegen, Spanien, Schweden und den Vereinigten Staaten beträgt sie 3 Jahre und mehr. In Mexiko, der Slowakischen Republik, der Tschechischen Republik und der Türkei beträgt die Bildungserwartung im Tertiärbereich hingegen maximal 1,5 Jahre (s. Tabelle C1.1 und Indikator C2).

Eine expansive Bildungspolitik hat in vielen OECD-Ländern auch den Druck für einen erweiterten Zugang zum Tertiärbereich erhöht. Bisher hat dieser Druck den Bevölkerungsrückgang der jüngeren Altersgruppen mehr als ausgeglichen, der bis vor kurzem zu Prognosen einer gleichbleibenden oder abnehmenden Nachfrage bei Schulabgängern in mehreren OECD-Ländern geführt hat. In einigen OECD-Ländern gibt es jetzt Anzeichen für eine nicht weiter zunehmende Nachfrage nach Bildung im Tertiärbereich, die Gesamttendenz bleibt jedoch steigend.

\section{Definitionen und angewandte Methodik}

Die Zahlen basieren auf Personenzahlen (außer wenn etwas anderes angegeben ist), d.h. es wird nicht zwischen Vollzeit- und Teilzeitlernenden unterschieden. Eine standardisierte Unterscheidung zwischen Vollzeit- und Teilzeitlernenden ist sehr schwierig, da in einigen OECD-Ländern der Begriff desTeilzeitbildungsbzw. Teilzeitstudiengangs unbekannt ist, obwohl in der Praxis zumindest ein Teil der Lernenden dieser Länder in einem anderen Land als Teilzeitlernende eingestuft würde. Es ist zu beachten, dass in einigen OECD-Ländern die Teilzeitausbildung nicht vollständig von den zur Verfügung gestellten Daten erfasst wird.

Die Berechnung der voraussichtlichen durchschnittlichen Dauer der formellen Bildung eines 5-jährigen Kindes während seines gesamten Lebens, die bei diesem Indikator als „Bildungserwartung (in Jahren)“ bezeichnet wird, erfolgt durch Addition der Netto-Bildungsbeteiligung für jede einzelne Altersstufe $a b$ dem 5. Lebensjahr. Im Falle einer Entwicklung hin zu einer längeren (kürzeren) Dauer der Ausbildung in den folgenden Jahren, wird der Durchschnittswert für die Altersgruppe höher (oder niedriger) ausfallen. Bei einem Vergleich von Daten über die Bildungserwartung ist Vorsicht geboten. Weder die Länge eines Schuljahres noch die Qualität der Bildung sind notwendigerweise in allen Ländern gleich.

Die Berechnung der in Tabelle C1.2 verwendeten Netto-Bildungsbeteiligung, ausgedrückt in Prozent, erfolgte, indem die Zahl der Lernenden einer bestimmten Altersgruppe in allen Bildungsbereichen durch die Gesamtzahl der Personen in der entsprechenden Altersgruppe in der Bevölkerung dividiert wurde. Tabelle C1.1 zeigt den Index der Veränderung der Bildungserwartung zwischen 1995 und 2000. Die Daten über die Zahl der Lernenden für 1994/ 1995 stammen aus einer im Jahr 2000 durchgeführten Sonderuntersuchung und basieren auf der ISCED-97-Klassifikation. dass er 2,5 Jahre im tertiären Bildungsbereich verbringen wird.

Eine expansive Bildungspolitik hat in vielen Ländern auch den Druck für einen erweiterten Zugang zum Tertiärbereich erhöht.

Die Daten beziehen sich auf das Schuljahr 1999-2000 und beruhen auf der alliährlich von der OECD aufgelegten VOEDatenerhebung zur Bildungsstatistik und demWorld Education Indicators Programm 2001. 
Tabelle C1.1.

Bildungserwartung, in Jahren (2000)

$Z u$ erwartende Jahre in Ausbildung bei gleichbleibenden Rahmenbedingungen, ohne Erziehung von Kindern unter 5 Jahren

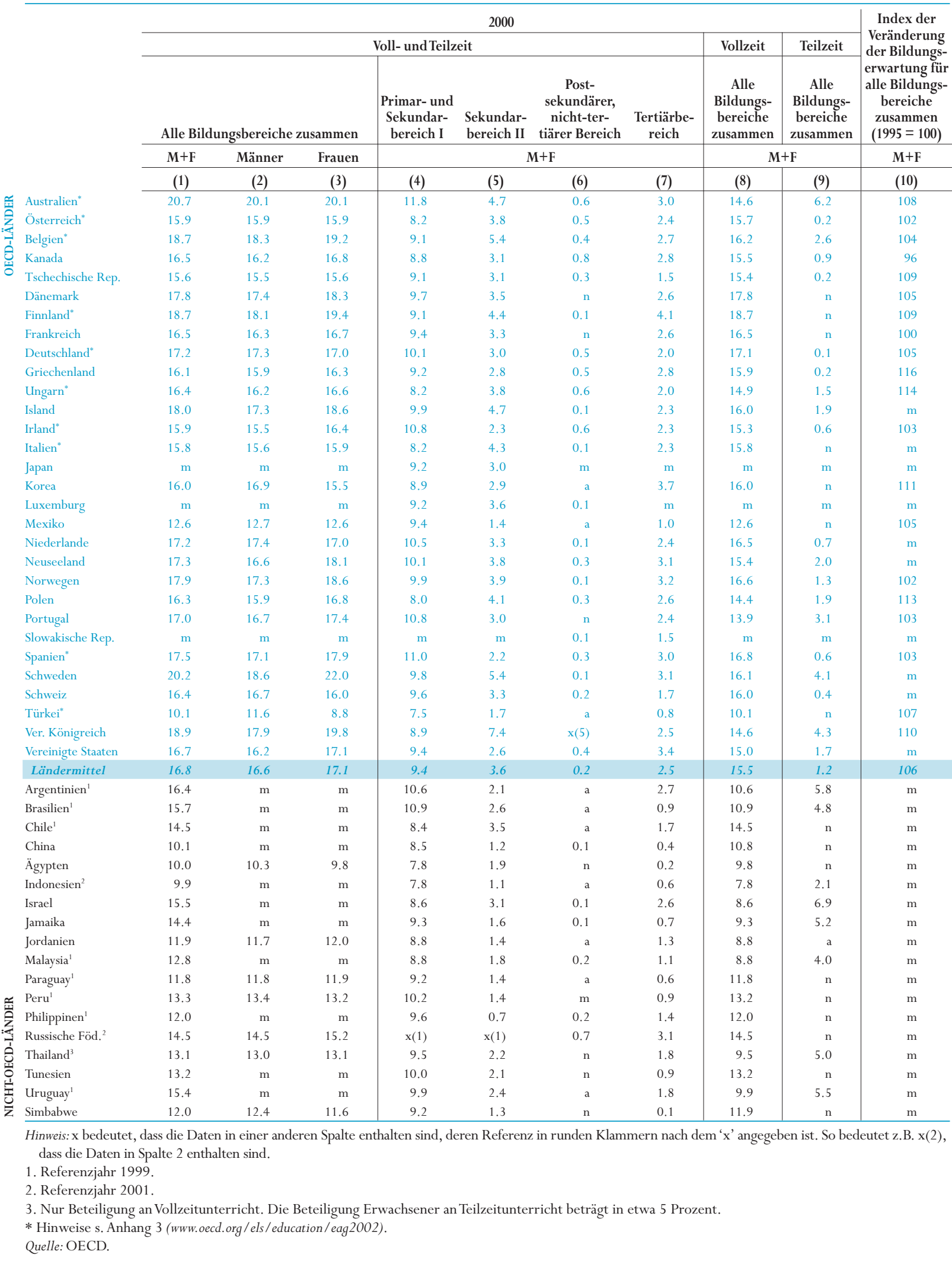


Tabelle C1.2.

Beteiligungsquoten (2000)

Vollzeit- und Teilzeitstudierende in öffentlichen und privaten Bildungseinrichtungen, nach Alter

\begin{tabular}{|c|c|c|c|c|c|c|c|c|c|}
\hline & \multirow[b]{2}{*}{$\begin{array}{c}\text { Alter, } \\
\text { bis zu dem } \\
\text { Schulpflicht } \\
\text { besteht }\end{array}$} & \multirow[b]{2}{*}{$\begin{array}{l}\text { Anzahl der } \\
\text { Jahre, in denen } \\
\text { über } 90 \% \text { der } \\
\text { Bevölkerung } \\
\text { an Bildung } \\
\text { teilnehmen }\end{array}$} & \multirow[b]{2}{*}{$\begin{array}{c}\text { Altersspanne, } \\
\text { innerhalb derer } \\
\text { über } 90 \% \text { der } \\
\text { Bevölkerung } \\
\text { an Bildung } \\
\text { teilnehmen } \\
\end{array}$} & \multicolumn{6}{|c|}{ Schüler und Studierende im Alter von: } \\
\hline & & & & \begin{tabular}{|l} 
\\
$4 \mathrm{~J}$. und jünger \\
als Prozent- \\
satz der 3- bis \\
4-Jährigen
\end{tabular} & $\begin{array}{c}5 \text { bis } 14 \mathrm{~J} . \text { als } \\
\text { Prozentsatz } \\
\text { der 5- bis 14- } \\
\text { Jährigen } \\
\end{array}$ & $\begin{array}{c}15 \text { bis } 19 \mathrm{~J} . \text { als } \\
\text { Prozentsatz } \\
\text { der } 15-\text { bis } \\
\text { 19-Jährigen } \\
\end{array}$ & $\begin{array}{c}20 \text { bis } 29 \mathrm{~J} \text {. als } \\
\text { Prozentsatz } \\
\text { der } 20 \text { - bis } 29- \\
\text { Jährigens }\end{array}$ & $\begin{array}{c}30 \text { bis } 39 \mathrm{~J} . \text { als } \\
\text { Prozentsatz } \\
\text { der } 30 \text { - bis } \\
\text { 39-Jährigen } \\
\end{array}$ & $\begin{array}{l}40 \mathrm{~J} \text {. und älter } \\
\text { als Prozentsatz } \\
\text { der Bevölke- } \\
\text { rung im Alter } \\
\text { von } 40 \text { Jahren } \\
\text { und älter } \\
\end{array}$ \\
\hline & (1) & (2) & (3) & (4) & (5) & (6) & (7) & (8) & (9) \\
\hline Australien & 15 & 12 & $5-16$ & 34.2 & 100.0 & 81.8 & 28.2 & 14.9 & 7.1 \\
\hline Österreich & 15 & 11 & $6-16$ & 60.6 & 98.2 & 76.4 & 17.9 & 3.1 & $\mathrm{x}(8)$ \\
\hline Belgien $^{*}$ & 18 & 15 & $3-17$ & 118.7 & 99.1 & 90.5 & 25.2 & 8.4 & 1.4 \\
\hline Kanada & 16 & 12 & $6-17$ & 20.1 & 97.1 & 74.2 & 21.7 & 4.6 & 1.2 \\
\hline Tschechische Rep. & 15 & 12 & $5-16$ & 70.6 & 99.8 & 80.8 & 14.2 & 1.1 & $\mathrm{n}$ \\
\hline Dänemark & 16 & 13 & $4-16$ & 81.4 & 99.2 & 80.4 & 29.9 & 5.6 & 0.9 \\
\hline Finnland & 16 & 11 & $7-17$ & 38.0 & 91.6 & 84.8 & 37.9 & 9.7 & 1.8 \\
\hline Frankreich ${ }^{*}$ & 16 & 15 & $3-17$ & 117.7 & 99.8 & 86.4 & 19.1 & 1.7 & $\mathrm{x}(8)$ \\
\hline Deutschland & 18 & 12 & $6-17$ & 67.9 & 99.4 & 88.3 & 23.6 & 2.8 & 0.2 \\
\hline Griechenland & 14.5 & 12 & $6-19$ & 28.9 & 99.8 & 87.4 & 16.9 & 0.1 & $\mathrm{n}$ \\
\hline Ungarn & 16 & 12 & $5-16$ & 79.2 & 99.9 & 81.1 & 18.7 & 4.2 & 0.1 \\
\hline Island $^{*}$ & 16 & 13 & $4-16$ & 123.9 & 98.5 & 78.9 & 30.5 & 6.5 & 1.8 \\
\hline Irland & 15 & 12 & $5-16$ & 26.9 & 100.5 & 79.8 & 15.6 & 3.4 & $\mathrm{x}(8)$ \\
\hline Italien ${ }^{*}$ & 14 & 12 & $3-14$ & 97.5 & 99.7 & 65.5 & 18.7 & 2.3 & 0.1 \\
\hline Japan & 15 & 14 & $4-17$ & 77.4 & 101.2 & $\mathrm{~m}$ & $\mathrm{~m}$ & $\mathrm{~m}$ & $\mathrm{~m}$ \\
\hline Korea & 14 & 12 & $6-17$ & 17.5 & 92.3 & 78.6 & 23.9 & 1.4 & 0.3 \\
\hline Luxemburg & 15 & 12 & $4-15$ & 65.8 & 95.3 & 73.7 & 4.6 & 0.4 & $\mathrm{n}$ \\
\hline Mexiko & 15 & 7 & $6-12$ & 35.5 & 94.8 & 41.0 & 9.1 & 2.8 & 0.7 \\
\hline Niederlande & 18 & 14 & $4-17$ & 49.9 & 99.4 & 86.6 & 22.9 & 3.0 & 0.6 \\
\hline Neuseeland & 16 & 13 & $4-16$ & 86.8 & 99.0 & 72.4 & 21.4 & 9.0 & 3.1 \\
\hline Norwegen & 16 & 12 & $6-17$ & 74.5 & 97.4 & 85.5 & 27.5 & 6.1 & 1.3 \\
\hline Polen & 15 & 11 & $6-16$ & 29.2 & 93.6 & 84.2 & 24.4 & 3.0 & $\mathrm{~m}$ \\
\hline Portugal & 14 & 10 & $6-15$ & 63.9 & 105.2 & 80.3 & 19.9 & 3.0 & 0.5 \\
\hline Slowakische Rep. & 15 & $\mathrm{~m}$ & $\mathrm{~m}$ & $\mathrm{~m}$ & $\mathrm{~m}$ & $\mathrm{~m}$ & $\mathrm{~m}$ & $\mathrm{~m}$ & $\mathrm{~m}$ \\
\hline Spanien $^{*}$ & 16 & 13 & $4-16$ & 98.1 & 104.4 & 79.5 & 24.3 & 2.7 & 0.4 \\
\hline Schweden* & 16 & 13 & $6-18$ & 70.5 & 97.8 & 86.4 & 33.4 & 15.0 & 3.4 \\
\hline Schweiz & 15 & 11 & $6-16$ & 20.8 & 98.8 & 83.5 & 18.9 & 3.3 & 0.1 \\
\hline Türkei* $^{*}$ & 14 & 5 & $7-11$ & $\mathrm{n}$ & 80.2 & 28.4 & 5.2 & 0.2 & $\mathrm{n}$ \\
\hline Ver. Königreich ${ }^{*}$ & 16 & 12 & $4-15$ & 81.1 & 98.9 & 73.3 & 23.8 & 13.2 & 5.4 \\
\hline Vereinigte Staaten & 17 & 10 & $6-15$ & 49.9 & 99.3 & 73.9 & 21.2 & 5.4 & 1.5 \\
\hline Ländermittel & 16 & 12 & & 63.8 & 97.9 & 77.3 & 21.4 & 4.9 & 1.3 \\
\hline Argentinien ${ }^{1}$ & 14 & 10 & $5-14$ & 37.4 & 103.8 & 62.5 & 20.8 & 4.8 & 1.2 \\
\hline Brasilien ${ }^{1}$ & 14 & 8 & $7-14$ & 24.6 & 90.1 & 78.0 & 20.7 & 5.9 & 1.5 \\
\hline Chile $^{1}$ & 14 & 9 & $6-14$ & 23.6 & 92.7 & 66.7 & $\mathrm{~m}$ & $\mathrm{~m}$ & $\mathrm{~m}$ \\
\hline China & 14 & 5 & $7-11$ & $\mathrm{~m}$ & 79.6 & $\mathrm{~m}$ & $\mathrm{~m}$ & $\mathrm{n}$ & $\mathrm{n}$ \\
\hline Ägypten & 13 & 6 & $6-11$ & 6.4 & 83.5 & 31.4 & $\mathrm{~m}$ & $\mathrm{n}$ & $\mathrm{n}$ \\
\hline Indonesien ${ }^{2}$ & 15 & 4 & $8-11$ & $\mathrm{n}$ & 76.5 & 38.5 & 3.0 & $\mathrm{n}$ & $\mathrm{n}$ \\
\hline Israel & 16 & 11 & $6-16$ & 99.6 & 96.6 & 63.6 & 20.1 & 4.3 & 0.9 \\
\hline Jamaika & 12 & 9 & $6-14$ & $\mathrm{n}$ & 88.6 & 39.6 & a & $\mathrm{a}$ & $\mathrm{a}$ \\
\hline Jordanien & 15 & $\mathrm{~m}$ & $\mathrm{~m}$ & 13.7 & 83.6 & 45.0 & $\mathrm{~m}$ & $\mathrm{~m}$ & $\mathrm{~m}$ \\
\hline Malaysia $^{1}$ & 16 & 7 & $6-12$ & 8.1 & 97.3 & 46.5 & 6.0 & 0.5 & 0.1 \\
\hline Paraguay ${ }^{1}$ & 14 & 5 & $7-11$ & 6.3 & 86.6 & 46.6 & $\mathrm{~m}$ & $\mathrm{~m}$ & $\mathrm{~m}$ \\
\hline Peru' & 16 & 9 & $6-14$ & 48.4 & 98.0 & 57.3 & 15.9 & 2.3 & 0.4 \\
\hline Philippinen ${ }^{1}$ & 12 & 8 & $\mathrm{~m}$ & 16.0 & 84.3 & $\mathrm{~m}$ & $\mathrm{~m}$ & $\mathrm{n}$ & $\mathrm{n}$ \\
\hline Russische Föd. $^{2}$ & 15 & 8 & $8-15$ & $\mathrm{~m}$ & 82.5 & 70.8 & 15.4 & $\mathrm{~m}$ & $\mathrm{~m}$ \\
\hline Thailand & 14 & 9 & $4-13$ & 61.5 & 97.4 & 60.2 & $\mathrm{~m}$ & $\mathrm{~m}$ & $\mathrm{~m}$ \\
\hline Tunesien & 16 & 6 & $6-11$ & 19.1 & 87.4 & 52.5 & 4.6 & $\mathrm{n}$ & $\mathrm{n}$ \\
\hline Uruguay $^{1}$ & 15 & 9 & $6-14$ & 23.5 & 97.8 & 60.7 & 18.7 & 3.6 & 0.4 \\
\hline Simbabwe & 12 & 7 & $7-13$ & $\mathrm{~m}$ & 82.2 & 45.3 & $\mathrm{~m}$ & $\mathrm{~m}$ & $\mathrm{~m}$ \\
\hline
\end{tabular}

Hinweis: Alter, bis zu dem Schulpflicht besteht, ist das Alter, in dem die Schulpflicht endet, z.B. bedeutet eine Altersangabe von 18 (J.) in dieser Spalte, dass Schüler unter 18 Jahren gesetzlich zum Schulbesuch verpflichtet sind.

Hinweis: $\mathrm{x}$ bedeutet, dass die Daten in einer anderen Spalte enthalten sind, deren Referenz in runden Klammern nach dem ' $\mathrm{x}$ ' angegeben ist. So bedeutet z.B. $\mathrm{x}(2)$, dass die Daten in Spalte 2 enthalten sind.

1. Referenzjahr 1999.

2. Referenzjahr 2001.

* Hinweise s. Anhang 3 (www.oecd.org/els/education/eag2002). 


\section{ZUGANG ZUM TERTIÄRBEREICH, ZU ERWARTENDE JAHRE IM TERTIÄRBEREICH UND BILDUNGSBETEILIGUNG IM SEKUNDARBEREICH}

- Heutzutage ist davon auszugehen, dass vier von zehn Schulabgängern an tertiären Bildungsgängen teilnehmen, die auf das Äquivalent eines Bachelor-Abschlusses oder eines höheren Abschlusses im Tertiärbereich A hinführen. In einigen OECD-Ländern liegt dieser Anteil sogar bei der Hälfte aller Schulabgänger.

- Im Durchschnitt der OECD-Länder wird ein heute 17-Jähriger 2,5 Jahre im Tertiärbereich A verbringen, davon 2,0 Jahre in Vollzeit.

- Mit Ausnahme von Deutschland, Frankreich und der Türkei hat die Bildungsbeteiligung im Tertiärbereich zwischen 1995 und 2000 in allen OECD-Ländern zugenommen.

- Während die Mehrzahl der Studierenden an öffentlichen Bildungseinrichtungen eingeschrieben ist, besuchen in OECD-Ländern wie Belgien, Japan, Korea, den Niederlanden und dem Vereinigten Königreich die meisten Studierenden privat geleitete Bildungseinrichtungen.

\section{Abbildung C2.1.}

Studienanfängerquoten im Tertiärbereich (2000)

Summe der Netto-Studienanfängerquoten über die einzelnen Altersjahrgänge im Tertiärbereich A und B

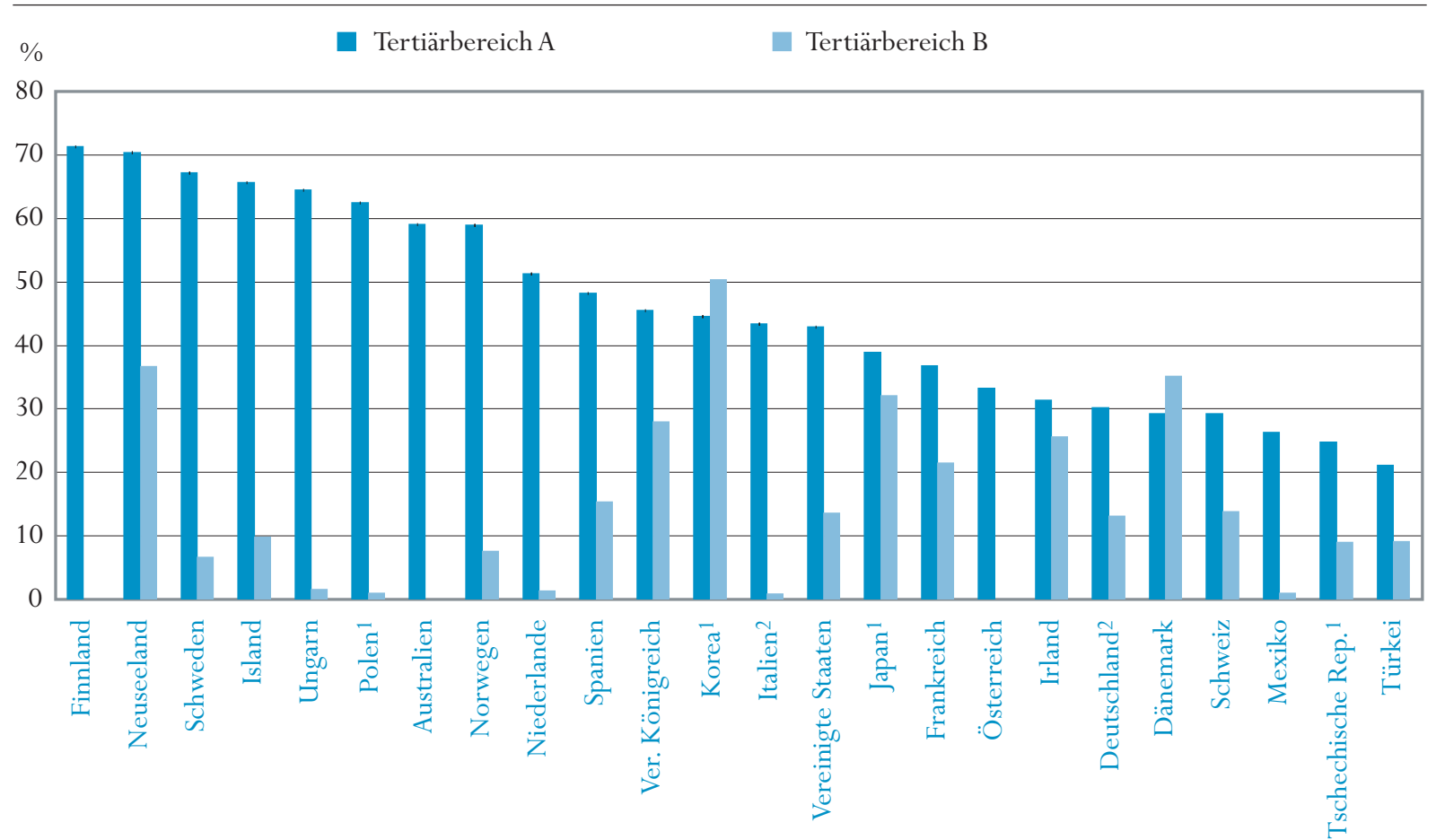

Hinweis: Die Netto-Studienanfängerquoten für den Tertiärbereich A und B können aufgrund von Doppelzählungen nicht auf addiert werden.

1. Studienanfängerquote für den Tertiärbereich A und B als Brutto-Studienanfängerquote berechnet.

2. Studienanfängerquote für den Tertiärbereich B als Brutto-Studienanfängerquote berechnet.

Anordnung der Länder in absteigender Reihenfolge der Gesamt-Studienanfängerquote für Studiengänge im Tertiärbereich A.

Quelle: OECD. Tabelle C2.1. Hinweise s. Anhang 3 (www.oecd.org/els/education/eag2002). 


\section{Politischer Hintergrund}

Hohe Studienanfängerquoten und eine hohe Bildungsbeteiligung im Tertiärbereich tragen dazu bei, die Entwicklung und den Erhalt einer hochqualifizierten Bevölkerung und Erwerbsbevölkerung sicherzustellen. Eine Ausbildung im Tertiärbereich wird mit besseren Beschäftigungsaussichten und höheren Verdiensten assoziiert (s. Indikator A13). Die Studienanfängerquoten für beide Tertiärbereiche sind zum Teil auch ein Anzeichen dafür, inwieweit die Bevölkerung hochqualifizierte Fähigkeiten und Kenntnisse erlangt, die auf dem Arbeitsmarkt einer Wissensgesellschaft von Bedeutung sind.

In dem Maße, wie die wirtschaftlichen und sozialen Vorteile einer Ausbildung im Tertiärbereich den Studierenden zunehmend bewusst wurden, ist die Zahl der Studienanfänger sowohl im Tertiärbereich A als auch im Tertiärbereich B gestiegen. Der kontinuierliche Anstieg der Bildungsbeteiligung, der mit einer stetig wachsenden Vielfalt der persönlichen Hintergründe und Interessen derjenigen einhergeht, die eine tertiäre Ausbildung anstreben, erfordert ein neuartiges Bildungsangebot. Die Herausforderungen, denen sich die tertiären Bildungseinrichtungen heutzutage stellen müssen, sind daher nicht nur eine Frage der Deckung des Bedarfs durch die Erhöhung der Anzahl der angebotenen Studienplätze, sondern auch eine Frage der Anpassung von Studiengängen, Unterrichts- und Lernverfahren an die unterschiedlichen Bedürfnisse einer neuen Generation von Studierenden.

Während ein erfolgreicher Abschluss des Sekundarbereich II in den meisten OECD-Ländern zunehmend zum Normalfall wird, gestalten sich die Wege zu diesem Bildungsziel immer vielfältiger. Die Bildungsgänge im Sekundarbereich II können sich beispielsweise hinsichtlich der Lerninhalte unterscheiden, die oftmals davon abhängen, auf welche weiteren Bildungsgänge oder welchen Beruf die Schüler vorbereitet werden sollen. Die meisten Bildungsgänge im Sekundarbereich II in den OECD-Ländern zielen vornehmlich auf die Vorbereitung zum weiterführenden Studium im Tertiärbereich ab. Diese Bildungsgänge können allgemeinbildend, berufsvorbereitend oder berufsbildend ausgerichtet sein. Neben Bildungsgängen, die die Schüler hauptsächlich auf eine weiterführende Bildung vorbereiten, gibt es in den meisten Ländern auch Bildungsgänge im Sekundarbereich II, die zur Vorbereitung auf den direkten Eintritt in den Arbeitsmarkt ausgelegt sind. In einigen Ländern werden berufsbildende Ausbildungsgänge allerdings erst nach Abschluss des Sekundarbereich II angeboten, obwohl diese post-sekundären Bildungsgänge inhaltlich oft den andernorts im Sekundarbereich II angebotenen beruflichen Ausbildungsgängen ähnlich sind.

\section{Ergebnisse und Erläuterungen}

\section{Zugang zum Tertiärbereich}

Im Laufe seines Lebens wird fast jeder zweite Jugendliche in den OECDLändern an einem Studiengang im Tertiärbereich A teilnehmen, sofern die aktuellen Studienanfängerquoten auch für die Zukunft gelten. In Finnland,
Dieser Indikator zeigt den Prozentsatz junger Menschen, die im Verlaufe ihres Lebens in verschiedenste Bildungsgänge im Tertiärbereich eintreten werden.

Studienanfänger-und Beteiligungsquoten spiegeln sowohl die Zugangsmöglichkeiten zum Tertiärbereich wider als auch den Wert, der einer tertiären Bildung beigemessen wird.

Der Indikator zeigt auch die Unterschiede bei der Bildungsbeteiligung im sekundarbereich.

45 Prozent der heutigen Jugendlichen in den $O E C D$-Ländern 
werden ein Studium

im Tertiärbereich A aufnehmen.

15 Prozent der heutigen Jugendlichen werden ein Studium im Tertiärbereich B aufnehmen.
Island, Neuseeland, Polen, Schweden und Ungarn nehmen sogar über 60 Prozent der jungen Menschen ein derartiges Studium auf (Tabelle C2.1).

Für die anderen OECD-Länder gelten wesentlich geringere Quoten für den Ersteintritt in den Tertiärbereich A - die geschätzten Ersteintrittsquoten für Deutschland, Dänemark, Mexiko, die Schweiz und die Türkei liegen bei 30 Prozent und darunter.

Der Anteil derjenigen, die ein Studium im Tertiärbereich B wählen, ist im allgemeinen kleiner als der Anteil derjenigen, die sich für den Tertiärbereich A entscheiden. Im Durchschnitt von 23 OECD-Ländern, für die Daten vorliegen, werden 15 Prozent der Jugendlichen eine Ausbildung im Tertiärbereich B beginnen. Die Bandbreite reicht von weniger als 1 Prozent in Italien, Mexiko, den Niederlanden und Polen bis zu über 30 Prozent in Belgien (fläm.), Dänemark, Japan und Neuseeland und über 50 Prozent in Korea (Tabelle C2.1 und Abb. C2.1).

In Belgien (fläm.) und Dänemark gleicht der breite Zugang zu Studiengängen im Tertiärbereich B die vergleichsweise niedrigen Eintrittsquoten für den Tertiärbereich A aus. Andere Länder, vor allem Korea und das Vereinigte Königreich, haben für Studiengänge im Tertiärbereich A Eintrittsquoten, die ungefähr dem OECD-Durchschnitt entsprechen, so wie auch vergleichsweise hohe Studienanfängerquoten für den Tertiärbereich B. Neuseeland sticht als das Land mit den höchsten Eintrittsquoten für beide tertiären Bereiche innerhalb der OECD-Länder hervor.

Die Netto-Studienanfängerquoten im tertiären Bildungsbereich sind im Hinblick auf die Beteiligung an post-sekundären, nicht-tertiären Bildungsgängen zu bewerten, die in einigen Ländern eine bedeutende Alternative zum tertiären Bildungsbereich darstellen (Indikator C1).

Diejenigen, die in Studiengänge im Tertiärbereich B eintreten, nehmen möglicherweise später im Leben auch ein Studium im Tertiärbereich A auf. Die Ersteintrittsquoten für den Tertiärbereich A und B können zur Bestimmung der Gesamteintrittsquoten für den Tertiärbereich jedoch nicht aufaddiert werden, da dies zu Doppelzählungen führen kann.

\section{Bildungsbeteiligung im Tertiärbereich}

Die Beteiligungsquoten bieten eine weitere Sicht auf die Bildungsbeteiligung im Tertiärbereich. Sie spiegeln sowohl die gesamte Anzahl derjenigen wider, die einen tertiären Studiengang wählen, als auch die Studien- bzw. Ausbildungsdauer im tertiären Bildungsbereich. Die Summe der Netto-Beteiligungsquoten über die einzelnen Jahrgänge hinweg, die als „Bildungserwartung im Tertiärbereich (in Jahren)" bezeichnet wird, misst das Ausmaß der tertiären Bildungsaktivitäten eines ganzen Altersjahrgangs und nicht nur einzelner Teilnehmer. Im Gegensatz zu den Studienanfängerquoten kann man die „Bildungserwartung im Tertiärbereich (in Jahren)" für die beiden Tertiärbereiche addieren. 
In den OECD-Ländern nimmt ein 17-Jähriger im Durchschnitt voraussichtlich 2,5 Jahre an tertiärer Bildung teil, von denen voraussichtlich 2 Jahre Vollzeitstudium sein werden. In Australien, Finnland, Korea, Neuseeland, Norwegen, Schweden und den Vereinigten Staaten nimmt ein 17-Jähriger im Laufe seines Lebens voraussichtlich mindestens 3 Jahre an einem Vollzeitoder Teilzeitstudium im Tertiärbereich teil. In Finnland und Korea liegt die zu erwartende Vollzeit-Studiendauer bei ungefähr 4 Jahren. In Mexiko, der Schweiz, der Slowakischen Republik, der Tschechischen Republik und der Türkei dagegen liegt die Bildungserwartung im Tertiärbereich bei weniger als 2 Jahren (Tabelle C2.2).

Im Durchschnitt ist in den OECD-Ländern die Bildungserwartung für den Tertiärbereich A (2,0 Jahre) wesentlich höher als für den Tertiärbereich B (0,4 Jahre). Die längere Studiendauer im Tertiärbereich A führt in der Regel - bei sonst gleichen Voraussetzungen - zu einer höheren Gesamtzahl an Studierenden und daher zu einem erhöhten Ressourcenbedarf (s. Indikator B1, Tabelle B1.3). Tatsächlich stammt aber in Dänemark die Mehrzahl der Absolventen aus Studiengängen im Tertiärbereich B (s. Indikator A2). Die höhere Bildungsbeteiligung im Tertiärbereich A im Verhältnis zum Tertiärbereich B in Dänemark (Tabelle C2.2) ergibt sich aus der längeren Dauer des Studiums und nicht aus höheren Studienanfängerquoten.

Während in der Mehrheit der OECD-Länder Bildungsgänge im Tertiärbereich A durch Bildungseinrichtungen der öffentlichen Hand sowohl angeboten als auch verwaltet werden, besucht in Belgien, den Niederlanden und dem Vereinigten Königreich die Mehrzahl der Studierenden Bildungseinrichtungen unter privater Leitung (wenn sie auch überwiegend öffentliche Mittel beziehen). In Japan und Korea werden über 70 Prozent der Studierenden an Bildungseinrichtungen unterrichtet, die sowohl unter privater Leitung stehen als auch vorwiegend aus privaten Mitteln finanziert werden und in Mexiko, Polen und den Vereinigten Staaten sind es über 30 Prozent (Tabelle C2.3).

\section{Entwicklung der Bildungsbeteiligung}

Mit Ausnahme von Deutschland, Frankreich und der Türkei ist die Bildungsbeteiligung im Tertiärbereich zwischen 1995 und 2000 in allen OECDLändern angestiegen. In der Hälfte der Länder, für die Daten vorliegen, beträgt der Anstieg der Studierenden im Tertiärbereich mehr als 15 Prozent, in Korea 48 Prozent, in Polen 108 Prozent, in der Tschechischen Republik 50 und in Ungarn 80 Prozent.

Im Tertiärbereich sind die Veränderungen der Bildungsbeteiligung nicht so eng mit Veränderungen der Größe der relevanten Altersgruppen verbunden wie dies im Primar- und Sekundarbereich der Fall ist. Abbildung C2.2 gliedert die Veränderung der Anzahl der eingeschriebenen Studierenden in zwei Komponenten auf: zum einen in die Veränderung der Größe der jeweiligen Altersgruppe und zum anderen in die Veränderung der Bildungsbeteiligung.
In Australien, Finnland,

Korea, Neuseeland,

Norwegen und den

Vereinigten Staden

erwarten junge

Menschen mindestens

3 Jahre einer Voll-oder

Teilzeitausbildung im

Tertiärbereich.

Eine längere

Studiendauer im

Tertiärbereich A führt

in der Regel zu einer

höheren Gesamtzahl

an Studierenden und

daher zu einem erhöhten

Ressourcenbedarf.

Die Mehrzahl

der Studierenden

im Tertiärbereich

besucht öffentliche

Bildungseinrichtungen,

in einigen OECD-

Ländern besucht die

Mehrzahl jedoch private

Bildungseinrichtungen.

In den meisten

OECD-Ländern ist die

Bildungsbeteiligung im

Tertiärbereich zwischen

1995 und 2000

gestiegen.

Die steigende Nachfrage, die sich in einer höheren Bildungsbeteiligung niederschlägt, ist die wichtigste Ursache für den weiteren Anstieg der 


\section{Abbildung C2.2.}

Veränderungen in der Zahl der Studierenden im Tertiärbereich im Verhältnis zu veränderten Beteiligungsquoten und demographischen Gegebenheiten (2000)

Index der Veränderung der Studierendenzahl im Tertiärbereich zwischen 1995 und 2000 sowie der relative Einfluss demographischer Veränderungen und veränderter Beteiligungsquoten $(1995=100)$

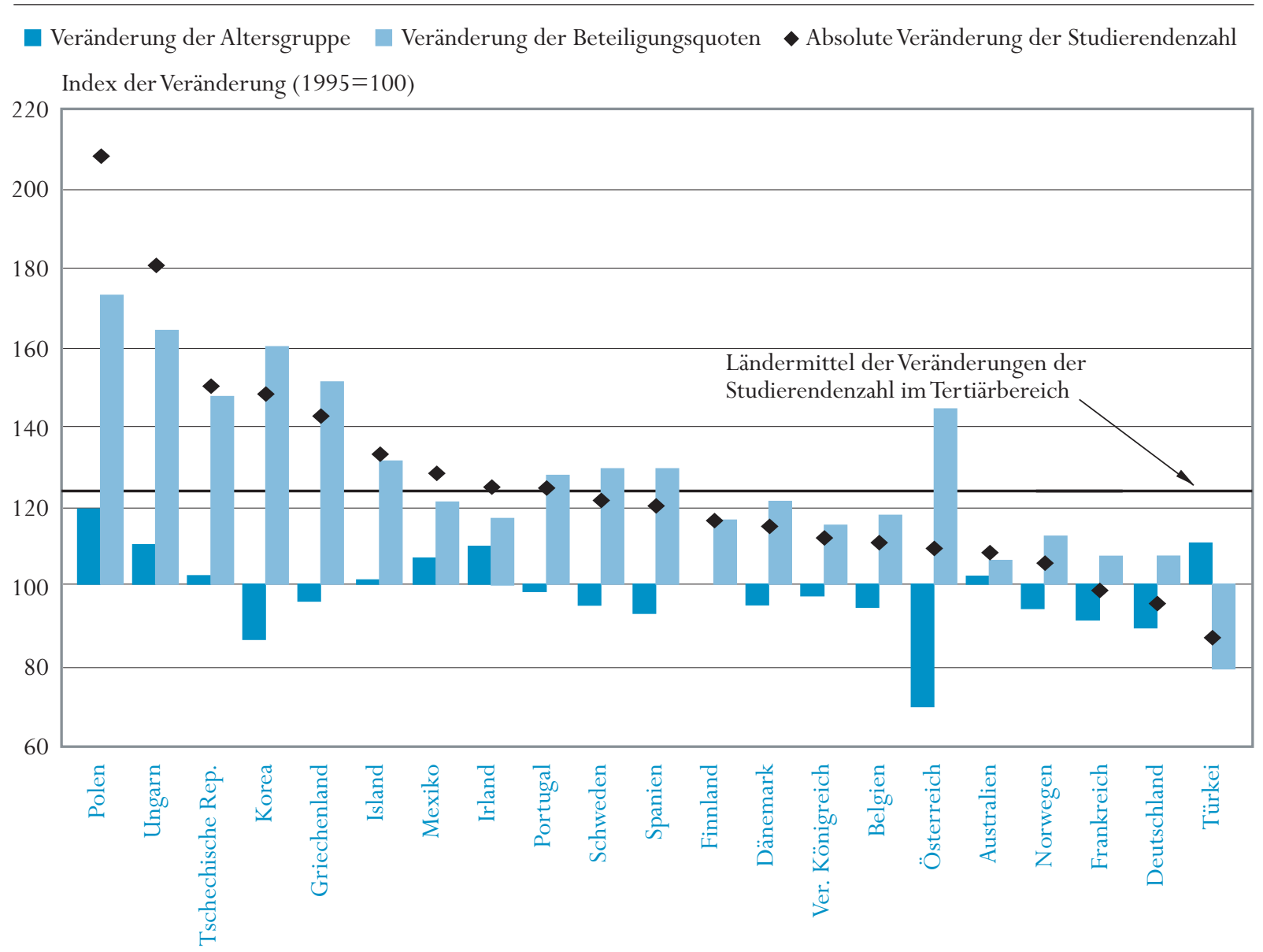

Anordnung der Länder in absteigender Reihenfolge der absoluten Veränderung der Studierendenzahl im Tertiärbereich.

Quelle: OECD. Tabelle C2.2. Hinweise s. Anhang 3 (www.oecd.org/els/education/eag2002).

ZahlderStudierenden Die steigende Nachfrage, die sich in einer höheren Bildungsbeteiligung im Tertiärbereich. niederschlägt, ist die wichtigste Ursache für die zunehmende Zahl von Studierenden im Tertiärbereich. Irland, Polen und Ungarn sind die einzigen Länder, in denen ein Anstieg der Bevölkerungszahlen signifikant zu dem Anstieg der Studierendenzahlen im Tertiärbereich beigetragen hat, doch in allen Fällen waren die Anstiege der Beteiligungsquoten noch beträchtlicher. Im Gegensatz dazu wäre der tatsächliche Anstieg der Studierendenzahlen im Tertiärbereich in vielen Ländern (insbesondere Korea, Österreich und Spanien) deutlich höher gewesen, wenn es dort nicht zu einem Bevölkerungsrückgang gekommen wäre. Sowohl in Frankreich als auch in Deutschland übertraf der Bevölkerungsrückgang sogar die Zunahme bei den Beteiligungsquoten, was bedeutet, dass trotz 
7 Prozent höherer Beteiligungsquoten die Studierendenzahlen im Tertiärbereich leicht gefallen sind.

\section{Das Alter von Studienanfängern}

Traditionell erfolgte die Aufnahme eines Studiums im Tertiärbereich A unmittelbar nach Abschluss des Sekundarbereich II. In einer Reihe von Ländern ist dies auch weiterhin der Fall. In Frankreich, Irland, der Slowakischen Republik und der Tschechischen Republik beispielsweise sind mehr als 80 Prozent aller Studienanfänger jünger als 22 Jahre (Tabelle C2.1).

In anderen Ländern erfolgt der Übergang zum Tertiärbereich häufig erst später, in einigen Fällen erst nach einiger Zeit der Erwerbstätigkeit. In diesen Ländern sind Studienanfänger des Tertiärbereich A in der Regel älter und gehören zu ganz verschiedenen Altersgruppen. In Dänemark, Island, Neuseeland und Schweden beispielsweise ist mehr als die Hälfte der Studierenden älter als 22 Jahre, wenn sie sich zum ersten Mal in einem Studiengang in diesem Bereich einschreiben (Tabelle C2.1). Der Anteil der älteren Studienanfänger in einem Erststudium im Tertiärbereich A kann, neben anderen Faktoren, ein Anzeichen für die Flexibilität dieser Studiengänge und ihre Eignung für Studierende außerhalb der typischen Altersgruppe sein. Darüber hinaus kann er die besondere Wertschätzung von Berufserfahrung als Hintergrund für ein weiterführendes Hochschulstudium widerspiegeln, die charakteristisch für die nordischen Länder Europas und auch in Australien und Neuseeland durchaus üblich ist. In diesen Ländern ist ein beträchtlicher Anteil der Studierenden bei Studienbeginn deutlich älter als das typische Eintrittsalter. In Australien, Dänemark, Island, Neuseeland, Norwegen und Schweden sind mehr als 20 Prozent der Studienanfänger im Erststudium 27 Jahre oder älter.

\section{Bildungsbeteiligung im Sekundarbereich II nach Ziel der Bildungsgänge}

In den meisten Ländern bestehen für die Schüler im Sekundarbereich II keine einheitlichen Lehrpläne. Die einzelnen Lehrpläne kann man beispielsweise nach Art des Bildungswegs oder der Laufbahn auf dem Arbeitsmarkt, auf die der Bildungsgang die Schüler vorbereiten soll, unterscheiden. Die Internationale Standardklassifikation des Bildungswesens (International Standard Classification of Education - ISCED) unterscheidet anhand der jeweiligen „Bildungsziele” drei Arten von Bildungsgängen im Sekundarbereich II:

ISCED 3A: Bildungsgänge, die unmittelbaren Zugang zu tertiären Studiengängen gewähren sollen, die wiederum ausreichende Befähigungen für Berufe mit hohen Qualifikationsanforderungen bzw. für weiterführende Forschungsprogramme vermitteln (Tertiärbereich A);

ISCED 3B: Bildungsgänge, die unmittelbaren Zugang zu tertiären Studiengängen eröffnen sollen, die vorrangig auf berufsspezifische Fertigkeiten ausgerichtet sind (Tertiärbereich B);
In Frankreich, Irland,

der Slowakischen

Republik und der

Tschechischen Republik

sind mehr als 80 Prozent

aller Studienanfänger

des Tertiärbereich $A$

jünger als 22 Jahre,

während beispielsweise

in Dänemark, Island,

Neuseeland und

Schweden mehr als die

Hälfte der Studierenden

älter als 22 Jahre ist,

wenn sie zum ersten Mal

an einem Studiengang

in diesem Bereich

teilnehmen.

Die Bildungsgänge im Sekundarbereich II werden entsprechend den weiteren Bildungszielen, auf die sie vorbereiten sollen, untergliedert. 
In 22 von 29 Ländern nimmt die Mehrheit der Studierenden an Bildungsängen teil, die auf ein Studium im Tertiärbereich A vorbereiten, ...

. in anderen Ländern dagegen sollen diese Bildungsgänge meist auf ein Studium im Tertiärbereich B vorbereiten.

Die Bildungsgänge können auch danach unterschieden werden, ob sie... allgemeinbildend, ...
ISCED 3C: Bildungsgänge, die nicht unmittelbar zu Studiengängen im Tertiärbereich A bzw. B führen sollen. Sie sollen die Schüler direkt auf den Arbeitsmarkt, post-sekundäre, nicht-tertiäre Bildungsgänge (ISCED 4) oder andere Bildungsgänge im Sekundarbereich II vorbereiten.

Bei dem Begriff „unmittelbarer Zugang“ handelt es sich weder um eine streng rechtliche Auslegung der Bildungsziele, noch geht es darum, wo die Absolventen letztendlich einmünden (was sehr stark von der aktuellen Arbeitsmarktlage abhängen kann). Die einzelnen Bildungsgänge werden anhand der Ausrichtung der Struktur des Lehrplans in die Kategorien A, B oder C eingeordnet, d.h. abhängig davon, auf welche Art von tertiärem Studiengang der Lehrplan des Bildungsgangs im Sekundarbereich II die Schüler vorbereitet.

In fast allen OECD-Ländern wechselt über die Hälfte der Schüler nach Abschluss des Sekundarbereich II vom formellen Bildungssystem in den Arbeitsmarkt über. Für die verbleibenden Schüler ist der Sekundarbereich II hauptsächlich eine Vorbereitung auf das weiterführende Studium im Tertiärbereich.

In 22 von 29 Ländern nehmen die meisten Schüler an Bildungsgängen teil, die auf das weiterführende Studium im Tertiärbereich A vorbereiten (Tabelle C2.5). In den meisten OECD-Ländern liegen die Studienanfängerquoten für Bildungsgänge im Tertiärbereich A wesentlich niedriger als die Abschlussquoten derjenigen Bildungsgänge des Sekundarbereich II, die auf ein derartiges Studium vorbereiten sollen. Dies deutet darauf hin, dass es eigentlich notwendig wäre, dass diese Bildungsgänge die Schüler ebenso für den Übergang zu anderen Formen der weiterführenden Bildung wie für den direkten Übertritt in eine Beschäftigung vorbereiten.

In Deutschland und der Schweiz besuchen ungefähr 60 Prozent aller Schüler (und in Österreich 48 Prozent) Bildungsgänge, die den Zugang zu weiterführenden Studiengängen im Tertiärbereich B ermöglichen. Es handelt sich hierbei hauptsächlich um Lehrlingsausbildungen im dualen System. Nach Abschluss dieser Ausbildung treten die meisten Absolventen in den Arbeitsmarkt ein, da viele der Bildungsgänge im Tertiärbereich B eine gewisse Berufserfahrung voraussetzen.

\section{Bildungsbeteiligung und Abschlussquoten bei beruflichen Bildungsgängen des Sekundarbereich II}

Die Bildungsgänge im Sekundarbereich II können - unabhängig davon, für welche weiteren Bildungsgänge sie einen Zugang eröffnen - auch danach in drei Kategorien unterteilt werden, inwieweit sie auf bestimmte Berufsfelder vorbereiten und zu einer auf dem Arbeitsmarkt verwertbaren Qualifikation führen:

Kategorie 1 (allgemeinbildende) Bildungsgänge - sie sollen die Teilnehmer weder explizit auf bestimmte Berufsfelder noch auf den Eintritt in einen weiterführenden berufsbildenden oder technischen Bildungsgang vorbereiten. 
Kategorie 2 (berufsvorbereitende) Bildungsgänge - sie sollen den Teilnehmern eher als Einführung in die Arbeitswelt dienen und sie für den späteren Eintritt in einen berufsbildenden oder technischen Bildungsgang vorbereiten. Durch den erfolgreichen Abschluss wird keine für den Arbeitsmarkt relevante berufliche oder technische Qualifikation erworben. Mindestens 25 Prozent des Inhalts des Bildungsgangs sollten berufsbildend oder technisch sein.

Kategorie 3 (berufsbildende) Bildungsgänge - in ihnen sollen die Teilnehmer für die direkte Aufnahme einer Beschäftigung in bestimmten Berufsfeldern, ohne weitere berufliche Qualifizierung, vorbereitet werden. Der erfolgreiche Abschluss eines solchen Bildungsganges führt zu einer für den Arbeitsmarkt relevanten beruflichen Qualifikation.

Das Ausmaß der berufsbildenden bzw. allgemeinbildenden Ausrichtung des Bildungsganges ist nicht unbedingt ausschlaggebend dafür, ob die Teilnehmer Zugang zum Tertiärbereich erhalten. In einigen OECD-Ländern haben Bildungsgänge mit berufsbildender Ausrichtung auch das Ziel, auf ein späteres Studium im Tertiärbereich vorzubereiten, während es in anderen Ländern wiederum eine Reihe von Bildungsgängen mit allgemeinbildender Ausrichtung gibt, die keinen direkten Zugang zur Hochschulbildung ermöglichen.

In allen OECD-Ländern können die Schüler zwischen berufsbildenden, berufsvorbereitenden und allgemeinbildenden Bildungsgängen wählen. In mehr als der Hälfte der OECD-Länder nimmt die Mehrzahl der Schüler im Sekundarbereich II an einer beruflichen Ausbildung teil. Sowohl in Ländern mit einer Lehrlingsausbildung im dualen System (z.B. Deutschland, Luxemburg, die Niederlande, Österreich und die Schweiz), als auch in Australien, Belgien, Polen, der Slowakischen Republik, der Tschechischen Republik und dem Vereinigten Königreich nehmen mindestens 60 Prozent der Schüler des Sekundarbereich II an einer beruflichen Ausbildung teil. Island ist die Ausnahme, hier besucht die Mehrzahl der Schüler Bildungsgänge mit allgemeinbildender Ausrichtung, obwohl eine duale Lehrlingsausbildung angeboten wird (Tabelle C2.5).

In den meisten Ländern findet die Berufsausbildung in der Schule statt, obwohl in Island, Österreich, der Slowakischen Republik und der Tschechischen Repbulik bei rund der Hälfte der berufsbildenden Ausbildungsgänge in der Schule und im Betrieb vermittelte Elemente kombiniert werden. In Dänemark, Deutschland, der Schweiz und Ungarn hat die Mehrheit der berufsbildenden Ausbildungsgänge sowohl schulische als auch betriebliche Elemente.

\section{Bildungsbeteiligung im Sekundarbereich II nach Art der Bildungseinrichtung}

Auch wenn die große Mehrzahl der Schüler im Primar- und Sekundarbereich öffentlich verwaltete und finanzierte Schulen besucht, besuchen inzwischen im Durchschnitt der OECD-Länder 20 Prozent der Schüler im Sekundarbereich II private Bildungseinrichtungen (Tabelle C2.4).
... berufsvorbereitend oder...

\section{berufsbildend sind.}

In mehr als der Hälfte der OECD-Länder nimmt die Mehrheit der Schüler im sekundarbereich II an berufsqualifizierenden Mafsnahmen bzw. einer Lehrlingsausbildung teil.

Die Mehrzahl der Schüler im Sekundarbereich II besucht öffentliche Bildungseinrichtungen, ... 


\section{Abbildung C2.3.}

Prozentsatz der Schüler im Primar- und Sekundarbereich an privaten Bildungseinrichtungen (2000)

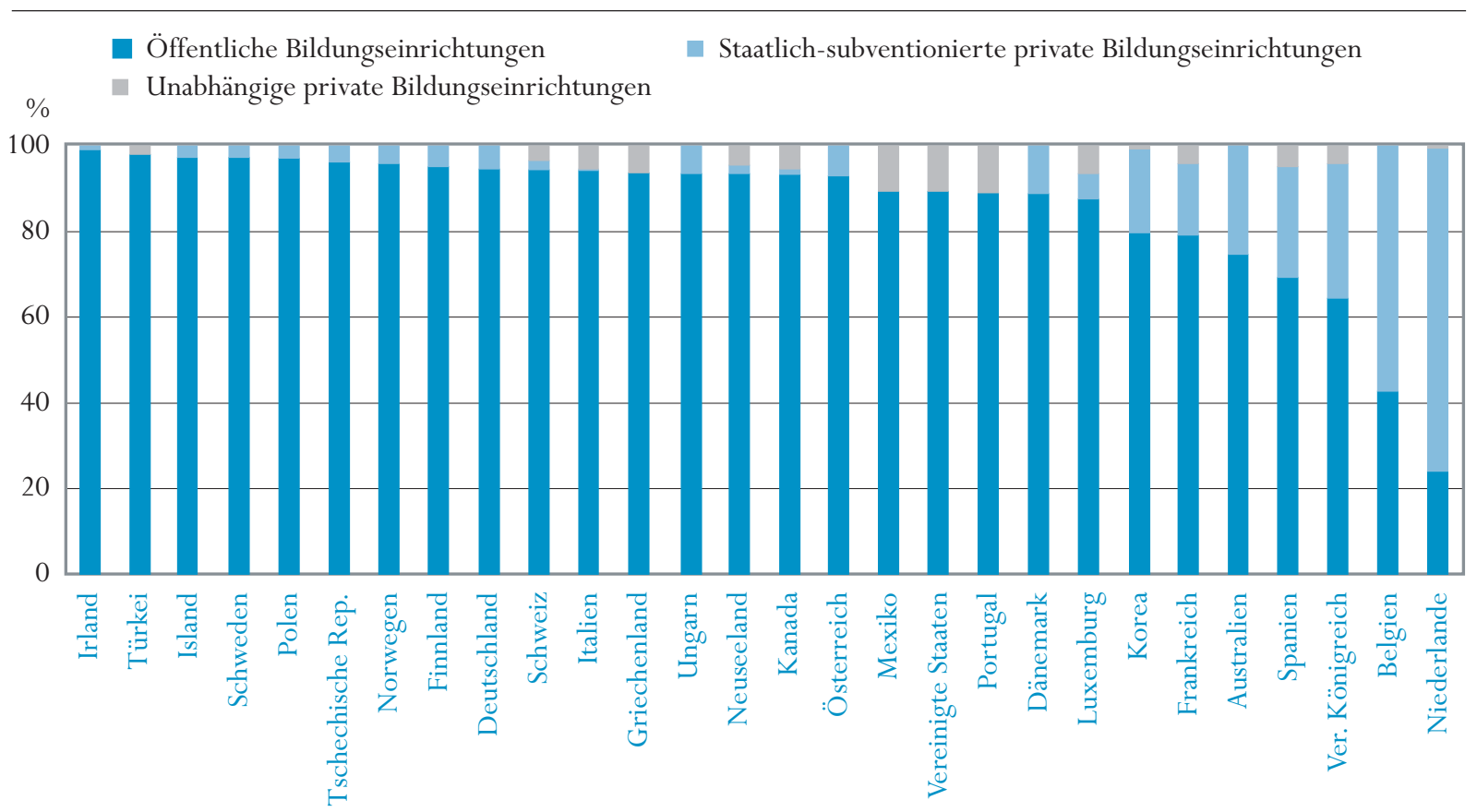

Anordnung der Länder in absteigender Reihenfolge der Schüler an öffentlichen Bildungseinrichtungen.

Quelle: OECD. Tabelle C2.4. Hinweise s. Anhang 3 (www.oecd.org/els/education/eag2002).

... in Belgien, Kored,

den Niederlanden

und dem Vereinigten

Königreich jedoch

besuchen die meisten

Schüler im Primar-

und Sekundarbereich

privat geleitete

Bildungseinrichtungen.

Die Daten beziehen sich auf das Schuljahr 1999/ 2000 und beruhen auf der alljährlich von der $O E C D$ durchgeführten UOE-Datenerhebung zur Bildungsstatistik (Einzelheiten S. Anhang 3)
Die Mehrheit der Schüler im Sekundarbereich II in Belgien, Korea, den Niederlanden und dem Vereinigten Königreich besucht staatlichsubventionierte private Bildungseinrichtungen (jeweils 60, 55, 90 und 67 Prozent). Private Bildungseinrichtungen, die hauptsächlich aus Zahlungen von Privathaushalten finanziert werden, sind im Sekundarbereich und ihm vorgelagerten Bildungsbereichen weit weniger verbreitet und werden teilweise als beträchtliche Barriere für die Bildungsbeteiligung von Schülern aus Familien mit geringem Einkommen angesehen. In Frankreich, Mexiko, Portugal und Spanien jedoch besuchen zwischen 10 und 20 Prozent der Schüler des Sekundarbereich II private Bildungseinrichtungen, die haupsächlich durch nicht-subventionierte Zahlungen privater Haushalte finanziert werden (Tabelle C2.4 und Abb. C2.3).

\section{Definitionen und angewandte Methodik}

Berufsvorbereitende und berufsbildende Bildungsgänge umfassen sowohl schulische als auch kombinierte schulische und betriebliche Ausbildungen, die als Bestandteil des Bildungssystems gelten. Ausschließlich in Betrieben durchgeführte Ausbildungen, die keiner formellen Aufsicht durch eine Bildungsbehörde unterstehen, bleiben unberücksichtigt.

Tabelle C2.1 enthält die Summe der Netto-Studienanfängerquoten für alle Altersgruppen. Die Netto-Studienanfängerquote einer speziellen Altersgruppe 
wird berechnet, indem die Anzahl der Studienanfänger der speziellen Altersgruppe in den einzelnen Tertiärbereichen durch die Gesamtpopulation der entsprechenden Altersgruppe geteilt wird (x 100). Die Summe der NettoStudienanfängerquoten wirdberechnet, indem die Netto-Studienanfängerquoten der einzelnen Altersjahrgänge aufsummiert werden. Das Ergebnis repräsentiert den Anteil von Personen einer synthetischen Altersgruppe, die in den Tertiärbereich eintreten, unabhängig von Veränderungen der Populationsgröße und Unterschieden zwischen den einzelnen OECD-Ländern hinsichtlich des typischen Eintrittsalters. Tabelle C2.1 zeigt auch das 20., 50. und 80. Perzentil der Altersverteilung der Studienanfänger im Erststudium, d.h. das jeweilige Alter, bis zu dem man 20, 50 bzw. 80 Prozent der Studienanfänger im Erststudium findet.

Studienanfänger im Erststudium sind diejenigen Studierenden, die sich zum ersten Mal in dem entsprechenden Bildungsbereich einschreiben. Ausländische Studierende, die im Rahmen eines Post-Graduierten-Studiums zum ersten Mal an dem Bildungssystem eines Landes teilnehmen, gelten als Studienanfänger im Erststudium.

Nicht alle OECD-Länder können unterscheiden zwischen Studierenden, die zum ersten Mal einen tertiären Studiengang aufnehmen, und Studierenden, die zwischen tertiären Studiengängen wechseln, einen tertiären Studiengang wiederholen, oder nach einer gewissen Zeit wieder aufnehmen. Daher können die Ersteintrittsquoten für die einzelnen Studiengangsarten aufgrund der unvermeidlichen Doppelzählung von Studienanfängern nicht einfach aufaddiert werden, um zu einer Gesamteintrittsquote in den Tertiärbereich zu gelangen.

Tabelle C2.2 zeigt die zu erwartende Anzahl von Jahren, die ein 17-Jähriger im Tertiärbereich studieren wird. Sie berechnet sich aus der Summe der Netto-Beteiligungsquoten für 17-Jährige und Ältere (geteilt durch 100). Diese Kenngröße ist sowohl eine Funktion der Anzahl der im Tertiärbereich teilnehmenden Personen als auch der Dauer tertiärer Studiengänge. Da der Nenner auch diejenigen mit einschließt, die niemals an einer tertiären Ausbildung teilgenommen haben, kann die Kennzahl insgesamt nicht als die durchschnittliche Anzahl an Jahren interpretiert werden, die ein einzelner Studierender im Tertiärbereich verbringen muss, um zu einem Abschluss zu gelangen.

Die Daten für das Schuljahr 1994-95 wurden im Jahr 2000 neu erhoben. Die einzelnen Länder wurden aufgefordert, die Daten gemäß der ISCED-97 Klassifikation anzugeben.
Die Daten für das Schuljahr 1994-95 basieren aufeiner speziellen OECDDatenerhebung aus dem Jahr 2000. 
Tabelle C2.1.

Studienanfängerquoten im Tertiärbereich und Altersverteilung der Studienanfänger (2000)

Summe der Netto-Studienanfängerquoten über die einzelnen Altersjahrgänge, nach Geschlecht und Bildungsbereichen

\begin{tabular}{|c|c|c|c|c|c|c|c|c|c|}
\hline & \multirow{2}{*}{\multicolumn{3}{|c|}{$\begin{array}{c}\text { Tertiärbereich B } \\
\text { Netto-Studienanfängerquote }\end{array}$}} & \multicolumn{6}{|c|}{ Tertiärbereich A } \\
\hline & & & & & udienanfän & dote & & Alter beim: & \\
\hline & $M+F$ & Männer & Frauen & $M+F$ & Männer & Frauen & $\begin{array}{c}20 . \\
\text { Perzentil }^{1}\end{array}$ & $\begin{array}{c}50 . \\
\text { Perzentil }^{1}\end{array}$ & $\begin{array}{c}80 . \\
\text { Perzentil }^{1}\end{array}$ \\
\hline & $(1)$ & (2) & (3) & (4) & (5) & (6) & (7) & (8) & (9) \\
\hline Australien & $\mathrm{m}$ & $\mathrm{m}$ & $\mathrm{m}$ & 59 & 52 & 66 & 18.4 & 19.9 & 27.4 \\
\hline Österreich & $\mathrm{m}$ & $\mathrm{m}$ & $\mathrm{m}$ & 33 & 30 & 37 & 19.1 & 20.5 & 23.6 \\
\hline Belgien (Fl.) & 34 & 28 & 39 & 36 & 36 & 36 & 18.3 & 18.9 & 22.7 \\
\hline Kanada & $\mathrm{m}$ & $\mathrm{m}$ & $\mathrm{m}$ & $\mathrm{m}$ & $\mathrm{m}$ & $\mathrm{m}$ & $\mathrm{m}$ & $\mathrm{m}$ & $\mathrm{m}$ \\
\hline Tschechische Rep." & 9 & 6 & 12 & 25 & 26 & 24 & 18.7 & 19.7 & 21.8 \\
\hline Dänemark & 35 & 26 & 45 & 29 & 27 & 32 & 20.8 & 22.4 & 27.9 \\
\hline Finnland & $\mathrm{a}$ & a & $\mathrm{a}$ & 71 & 62 & 81 & 19.9 & 21.6 & 26.9 \\
\hline Frankreich & 21 & 22 & 21 & 37 & 30 & 44 & 18.3 & 18.9 & 20.2 \\
\hline Deutschland $^{2}$ & 13 & 9 & 18 & 30 & 30 & 30 & 20.1 & 21.4 & 24.3 \\
\hline Griechenland & $\mathrm{m}$ & $\mathrm{m}$ & $\mathrm{m}$ & $\mathrm{m}$ & $\mathrm{m}$ & $\mathrm{m}$ & $\mathrm{m}$ & $\mathrm{m}$ & $\mathrm{m}$ \\
\hline Ungarn $^{*}$ & 2 & 1 & 2 & 65 & 60 & 70 & 19.2 & 21.0 & 26.5 \\
\hline Island & 10 & 11 & 9 & 66 & 48 & 84 & 20.9 & 22.7 & 28.5 \\
\hline Irland & 26 & 23 & 28 & 31 & 29 & 34 & 18.3 & 19.0 & 19.9 \\
\hline Italien $^{3}$ & 1 & 1 & 1 & 43 & 38 & 49 & $\mathrm{~m}$ & $\mathrm{~m}$ & $\mathrm{~m}$ \\
\hline $\mathrm{Japan}^{3}$ & 32 & 22 & 43 & 39 & 47 & 30 & $\mathrm{~m}$ & $\mathrm{~m}$ & $\mathrm{~m}$ \\
\hline Korea $^{3}$ & 50 & 51 & 49 & 45 & 48 & 41 & $\mathrm{~m}$ & $\mathrm{~m}$ & $\mathrm{~m}$ \\
\hline Luxemburg & $\mathrm{m}$ & $\mathrm{m}$ & $\mathrm{m}$ & $\mathrm{m}$ & $\mathrm{m}$ & $\mathrm{m}$ & $\mathrm{m}$ & $\mathrm{m}$ & $\mathrm{m}$ \\
\hline Mexiko & 1 & 1 & 1 & 26 & 27 & 26 & 18.3 & 19.5 & 25.7 \\
\hline Niederlande & 1 & 1 & 2 & 51 & 48 & 54 & 18.5 & 19.8 & 22.8 \\
\hline Neuseeland & 37 & 31 & 42 & 70 & 57 & 84 & 18.9 & 22.7 & $<40$ \\
\hline Norwegen & 7 & 9 & 6 & 59 & 45 & 74 & 20.1 & 21.6 & 29.6 \\
\hline Polen ${ }^{3}$ & 1 & $\mathrm{n}$ & 2 & 62 & $\mathrm{x}(4)$ & $\mathrm{x}(4)$ & $\mathrm{m}$ & $\mathrm{m}$ & $\mathrm{m}$ \\
\hline Portugal & $\mathrm{m}$ & $\mathrm{m}$ & $\mathrm{m}$ & $\mathrm{m}$ & $\mathrm{m}$ & $\mathrm{m}$ & $\mathrm{m}$ & $\mathrm{m}$ & $\mathrm{m}$ \\
\hline Slowakische Rep. ${ }^{2}$ & 3 & 1 & 5 & 37 & 38 & 36 & 18.6 & 19.5 & 21.3 \\
\hline Spanien & 15 & 15 & 16 & 48 & 42 & 54 & 18.4 & 19.2 & 22.1 \\
\hline Schweden & 7 & 7 & 6 & 67 & 54 & 81 & 20.2 & 22.7 & 32.1 \\
\hline Schweiz & 14 & 15 & 13 & 29 & 32 & 26 & 20.3 & 21.8 & 26.3 \\
\hline Türkei* $^{*}$ & 9 & 11 & 8 & 21 & 26 & 17 & 18.3 & 19.6 & 23.2 \\
\hline Ver. Königreich & 28 & 24 & 32 & 46 & 42 & 49 & 18.4 & 19.4 & 25.4 \\
\hline Vereinigte Staaten & 14 & 12 & 15 & 43 & 37 & 49 & 18.4 & 19.4 & 26.8 \\
\hline Ländermittel & 15 & 14 & 17 & 45 & 40 & 48 & & & \\
\hline Argentinien ${ }^{4}$ & 30 & 18 & 41 & 50 & 31 & 70 & $\mathrm{~m}$ & $\mathrm{~m}$ & $\mathrm{~m}$ \\
\hline Chile $e^{3,4}$ & 14 & 14 & 14 & 38 & 40 & 35 & $\mathrm{~m}$ & $\mathrm{~m}$ & $\mathrm{~m}$ \\
\hline China $^{3,4}$ & 6 & $\mathrm{x}(1)$ & $\mathrm{x}(1)$ & 8 & $\mathrm{x}(4)$ & $\mathrm{x}(4)$ & $\mathrm{m}$ & $\mathrm{m}$ & $\mathrm{m}$ \\
\hline Indonesien ${ }^{5}$ & 8 & 7 & 9 & 14 & 16 & 11 & $\mathrm{~m}$ & $\mathrm{~m}$ & $\mathrm{~m}$ \\
\hline Israel & 31 & 26 & 36 & 49 & 44 & 54 & $\mathrm{~m}$ & $\mathrm{~m}$ & $\mathrm{~m}$ \\
\hline Jamaika & 16 & 10 & 22 & 9 & 6 & 13 & $\mathrm{~m}$ & $\mathrm{~m}$ & $\mathrm{~m}$ \\
\hline Jordanien $^{3}$ & 14 & 9 & 20 & 30 & 29 & 30 & $\mathrm{~m}$ & $\mathrm{~m}$ & $\mathrm{~m}$ \\
\hline Malaysia $^{4}$ & 24 & 24 & 25 & 22 & 19 & 25 & $\mathrm{~m}$ & $\mathrm{~m}$ & $\mathrm{~m}$ \\
\hline Paraguay ${ }^{3,4}$ & 8 & 5 & 12 & $\mathrm{~m}$ & $\mathrm{~m}$ & $\mathrm{~m}$ & $\mathrm{~m}$ & $\mathrm{~m}$ & $\mathrm{~m}$ \\
\hline Philippinen $^{4}$ & a & a & $\mathrm{a}$ & 41 & 36 & 45 & $\mathrm{~m}$ & $\mathrm{~m}$ & $\mathrm{~m}$ \\
\hline Thailand $^{3}$ & 23 & 25 & 21 & 40 & 36 & 44 & $\mathrm{~m}$ & $\mathrm{~m}$ & $\mathrm{~m}$ \\
\hline Tunesien $^{3}$ & $\mathrm{x}(4)$ & $x(5)$ & $x(6)$ & 27 & 27 & 27 & $\mathrm{~m}$ & $\mathrm{~m}$ & $\mathrm{~m}$ \\
\hline Uruguay $^{3,4}$ & 17 & 8 & 26 & 26 & 21 & 31 & $\mathrm{~m}$ & $\mathrm{~m}$ & $\mathrm{~m}$ \\
\hline Simbabwe $^{3,5}$ & 4 & 5 & 3 & 1 & 2 & 1 & $\mathrm{~m}$ & $\mathrm{~m}$ & $\mathrm{~m}$ \\
\hline
\end{tabular}

Hinweis: $\mathrm{x}$ bedeutet, dass die Daten in einer anderen Spalte enthalten sind, deren Referenz in runden Klammern nach dem ' $\mathrm{x}$ ' angegeben ist. So bedeutet $\mathrm{z}$. B. $\mathrm{x}(2)$, dass die Daten in Spalte 2 enthalten sind.

1. 20/50/80 Prozent der Studienanfänger sind jünger.

2. Studienanfängerquote für den Tertiärbereich B als Brutto-Studienanfängerquote berechnet.

3. Studienanfängerquote für den Tertiärbereich A und B als Brutto-Studienanfängerquote berechnet.

4. Referenzjahr 1999

5. Referenzjahr 2001

* Hinweise s. Anhang 3 (www.oecd.org/els/education/eag2002).

Quelle: OECD. 
Tabelle C2.2.

Zu erwartende Ausbildungsjahre im Tertiärbereich undVeränderung der Gesamtzahl der Studierenden im Tertiärbereich (2000)

Zu erwartende Ausbildungsjahre im Tertiärbereich unter gegenwärtigen Bedingungen, nach Geschlecht und Vollzeit-/Teilzeitstudium, und Idex derVeränderung der Gesamtzahl der Studierenden im Tertiärbereich $(1995=100)$

\begin{tabular}{|c|c|c|c|c|c|c|c|c|c|c|c|c|}
\hline & \multicolumn{3}{|c|}{ Tertiärbereich B } & \multicolumn{3}{|c|}{ Tertiärbereich A } & \multicolumn{3}{|c|}{$\begin{array}{l}\text { Tertiärbereich insgesamt } \\
\text { (Tertiärbereich A und B u. } \\
\text { weiterf. Forschungsprogr.) }\end{array}$} & \multicolumn{3}{|c|}{$\begin{array}{l}\text { Veränderung der Gesamtzahl der } \\
\text { Studierenden }(1995=100)\end{array}$} \\
\hline & \multicolumn{2}{|c|}{ Voll- und Teilzeit } & \multirow{2}{*}{$\begin{array}{l}\text { Vollzeit } \\
\text { M+F }\end{array}$} & \multicolumn{2}{|c|}{ Voll- und Teilzeit } & \multirow{2}{*}{$\begin{array}{l}\text { Vollzeit } \\
M+F\end{array}$} & \multicolumn{2}{|c|}{ Voll- und Teilzeit } & \multirow{2}{*}{$\begin{array}{l}\text { Vollzeit } \\
M+F\end{array}$} & \multirow[b]{2}{*}{$\begin{array}{l}\text { Tertiär- } \\
\text { bereich } \\
\text { insgesamt }\end{array}$} & \multicolumn{2}{|c|}{ zurückzuführen auf: } \\
\hline & $M+F$ & Frauen & & $M+F$ & Frauen & & $M+F$ & Frauen & & & $\begin{array}{l}\text { Verände- } \\
\text { rungen der } \\
\text { Population }\end{array}$ & $\begin{array}{l}\text { Verände- } \\
\text { rungen der } \\
\text { Beteiligungs- } \\
\text { quoten }\end{array}$ \\
\hline & (1) & (2) & (3) & (4) & (5) & (6) & (7) & (8) & (9) & (10) & (11) & (12) \\
\hline Australien & 0.7 & 0.7 & 0.2 & 2.2 & 2.5 & 1.4 & 3.0 & 3.3 & 1.7 & 108 & 102 & 106 \\
\hline Österreich & 0.2 & 0.3 & 0.1 & 2.0 & 2.0 & 2.0 & 2.3 & 2.4 & 2.3 & 109 & 69 & 144 \\
\hline Belgien & 1.4 & 1.6 & 1.1 & 1.3 & 1.3 & 1.3 & 2.7 & 2.9 & 2.3 & 111 & 94 & 117 \\
\hline Kanada & 0.7 & 0.8 & 0.6 & 2.0 & 2.4 & 1.4 & 2.8 & 3.2 & 2.1 & 101 & $\mathrm{~m}$ & $\mathrm{~m}$ \\
\hline Tschechische Rep. & 0.2 & 0.3 & 0.2 & 1.2 & 1.2 & 1.1 & 1.5 & 1.6 & 1.4 & 150 & 102 & 147 \\
\hline Dänemark & 1.1 & 1.5 & 1.1 & 1.4 & 1.4 & 1.4 & 2.6 & 3.0 & 2.6 & 115 & 95 & 121 \\
\hline Finnland & 0.2 & 0.3 & 0.2 & 3.6 & 3.9 & 3.6 & 4.1 & 4.4 & 4.1 & 116 & 100 & 116 \\
\hline Frankreich & 0.6 & 0.7 & 0.6 & 1.8 & 2.0 & 1.8 & 2.6 & 2.8 & 2.6 & 98 & 91 & 107 \\
\hline Deutschland $^{*}$ & 0.3 & 0.4 & 0.3 & 1.7 & 1.6 & 1.7 & 2.0 & 2.0 & 2.0 & 95 & 89 & 107 \\
\hline Griechenland & 0.9 & 0.9 & 0.9 & 1.9 & 2.0 & 1.9 & 2.8 & 2.9 & 2.8 & 143 & 96 & 151 \\
\hline Ungarn $^{*}$ & $\mathrm{n}$ & $\mathrm{n}$ & $\mathrm{n}$ & 1.9 & 2.1 & 1.1 & 2.0 & 2.2 & 1.1 & 180 & 110 & 164 \\
\hline Island & 0.2 & 0.2 & 0.1 & 2.1 & 2.7 & 1.7 & 2.3 & 2.9 & 1.9 & 133 & 101 & 131 \\
\hline Irland & $\mathrm{x}(7)$ & $\mathrm{x}(8)$ & $\mathrm{x}(9)$ & $x(7)$ & $\mathrm{x}(8)$ & $\mathrm{x}(9)$ & 2.3 & 2.4 & 1.8 & 125 & 109 & 116 \\
\hline Italien & $\mathrm{n}$ & $\mathrm{n}$ & $\mathrm{n}$ & 2.2 & 2.4 & 2.2 & 2.2 & 2.5 & 2.2 & 103 & $\mathrm{~m}$ & $\mathrm{~m}$ \\
\hline Japan & $\mathrm{m}$ & $\mathrm{m}$ & $\mathrm{m}$ & $\mathrm{m}$ & $\mathrm{m}$ & $\mathrm{m}$ & $\mathrm{m}$ & $\mathrm{m}$ & $\mathrm{m}$ & $\mathrm{m}$ & $\mathrm{m}$ & $\mathrm{m}$ \\
\hline Korea & 1.5 & 1.1 & 1.5 & 2.2 & 1.6 & 2.2 & 3.7 & 2.7 & 3.7 & 148 & 87 & 161 \\
\hline Luxemburg & $\mathrm{m}$ & $\mathrm{m}$ & $\mathrm{m}$ & $\mathrm{m}$ & $\mathrm{m}$ & $\mathrm{m}$ & $\mathrm{m}$ & $\mathrm{m}$ & $\mathrm{m}$ & $\mathrm{m}$ & $\mathrm{m}$ & $\mathrm{m}$ \\
\hline Mexiko & $\mathrm{n}$ & $\mathrm{n}$ & $\mathrm{n}$ & 1.0 & 1.0 & 1.0 & 1.0 & 1.0 & 1.0 & 128 & 106 & 121 \\
\hline Niederlande & $\mathrm{n}$ & $\mathrm{n}$ & $\mathrm{n}$ & 2.4 & 2.4 & 2.0 & 2.4 & 2.5 & 2.1 & $\mathrm{~m}$ & $\mathrm{~m}$ & $\mathrm{~m}$ \\
\hline Neuseeland & 0.8 & 0.9 & 0.4 & 2.3 & 2.7 & 1.6 & 3.1 & 3.6 & 2.0 & $\mathrm{~m}$ & $\mathrm{~m}$ & $\mathrm{~m}$ \\
\hline Norwegen & 0.3 & 0.2 & 0.2 & 2.9 & 3.5 & 2.1 & 3.2 & 3.7 & 2.4 & 105 & 94 & 112 \\
\hline Polen $^{1}$ & $\mathrm{n}$ & $\mathrm{n}$ & $\mathrm{n}$ & 2.6 & 3.0 & 1.3 & 2.6 & 3.1 & 1.4 & 208 & 119 & 173 \\
\hline Portugal & 0.6 & 0.6 & 0.6 & 1.7 & 2.0 & 1.7 & 2.4 & 2.7 & 2.4 & 124 & 98 & 127 \\
\hline Slowakische Rep. & 0.1 & 0.1 & $\mathrm{n}$ & 1.3 & 1.4 & 1.0 & 1.5 & 1.5 & 1.0 & $\mathrm{~m}$ & $\mathrm{~m}$ & $\mathrm{~m}$ \\
\hline Spanien & 0.3 & 0.3 & 0.3 & 2.6 & 2.8 & 2.4 & 2.9 & 3.2 & 2.7 & 120 & 93 & 129 \\
\hline Schweden & 0.1 & 0.1 & 0.1 & 2.8 & 3.4 & 1.6 & 3.1 & 3.6 & 1.7 & 122 & 95 & 129 \\
\hline Schweiz & 0.4 & 0.3 & 0.1 & 1.2 & 1.1 & 1.2 & 1.7 & 1.5 & 1.4 & $\mathrm{~m}$ & $\mathrm{~m}$ & $\mathrm{~m}$ \\
\hline Türkei & 0.2 & 0.1 & 0.2 & 0.6 & 0.5 & 0.6 & 0.8 & 0.6 & 0.8 & 86 & 110 & 79 \\
\hline Ver. Königreich & 0.7 & 0.8 & 0.2 & 1.7 & 1.9 & 1.4 & 2.5 & 2.8 & 1.7 & 112 & 97 & 115 \\
\hline Vereinigte Staaten & 0.7 & 0.8 & 0.3 & 2.6 & 3.0 & 1.7 & 3.4 & 3.8 & 2.1 & $\mathrm{~m}$ & $\mathrm{~m}$ & $\mathrm{~m}$ \\
\hline Ländermittel & 0.4 & 0.5 & 0.3 & 2.0 & 2.1 & 1.6 & 2.5 & 2.7 & 2.0 & 124 & 98 & 127 \\
\hline Argentinien $^{2}$ & 0.7 & 1.0 & $\mathrm{~m}$ & 2.0 & 2.4 & $\mathrm{~m}$ & 2.7 & 3.4 & $\mathrm{~m}$ & $\mathrm{~m}$ & $\mathrm{~m}$ & $\mathrm{~m}$ \\
\hline Brasilien $^{2}$ & $\mathrm{x}(4)$ & $\mathrm{x}(5)$ & $\mathrm{x}(6)$ & 0.8 & 0.9 & 0.8 & 0.9 & 0.9 & 0.9 & $\mathrm{~m}$ & $\mathrm{~m}$ & $\mathrm{~m}$ \\
\hline Indonesien $^{3}$ & 0.1 & 0.1 & 0.1 & 0.4 & 0.4 & 0.4 & 0.6 & 0.5 & 0.6 & $\mathrm{~m}$ & $\mathrm{~m}$ & $\mathrm{~m}$ \\
\hline Israel & 0.5 & 0.6 & 0.5 & 2.1 & 2.4 & 1.6 & 2.6 & 3.0 & 2.2 & $\mathrm{~m}$ & $\mathrm{~m}$ & $\mathrm{~m}$ \\
\hline Malaysia $^{2}$ & 0.5 & 0.5 & 0.5 & 0.6 & 0.6 & 0.5 & 1.1 & 1.2 & 1.0 & $\mathrm{~m}$ & $\mathrm{~m}$ & $\mathrm{~m}$ \\
\hline Paraguay $^{2}$ & 0.2 & 0.3 & 0.2 & $\mathrm{~m}$ & $\mathrm{~m}$ & $\mathrm{~m}$ & $\mathrm{~m}$ & $\mathrm{~m}$ & $\mathrm{~m}$ & $\mathrm{~m}$ & $\mathrm{~m}$ & $\mathrm{~m}$ \\
\hline Peru $^{2}$ & 1.0 & 1.1 & 1.0 & $\mathrm{~m}$ & $\mathrm{~m}$ & $\mathrm{~m}$ & $\mathrm{~m}$ & $\mathrm{~m}$ & $\mathrm{~m}$ & $\mathrm{~m}$ & $\mathrm{~m}$ & $\mathrm{~m}$ \\
\hline Philippinen $^{2}$ & a & a & $\mathrm{a}$ & 1.4 & 1.6 & 1.4 & 1.4 & 1.6 & 1.4 & $\mathrm{~m}$ & $\mathrm{~m}$ & $\mathrm{~m}$ \\
\hline Russische Föd..$^{1,3}$ & 1.0 & 1.1 & 3.3 & 2.1 & 2.4 & 5.4 & 3.2 & 3.6 & 7.7 & $\mathrm{~m}$ & $\mathrm{~m}$ & $\mathrm{~m}$ \\
\hline Uruguay ${ }^{1,2}$ & 0.5 & 0.7 & 0.5 & 1.3 & 1.6 & 1.3 & 1.8 & 2.2 & 1.8 & $\mathrm{~m}$ & $\mathrm{~m}$ & $\mathrm{~m}$ \\
\hline
\end{tabular}

Hinweis: $\mathrm{x}$ bedeutet, dass die Daten in einer anderen Spalte enthalten sind, deren Referenz in runden Klammern nach dem ' $\mathrm{x}$ ' angegeben ist. So bedeutet $\mathrm{z}$. B. $\mathrm{x}(2)$, dass die Daten in Spalte 2 enthalten sind.

1. Ohne weiterführende Forschungsprogramme.

2. Referenzjahr 1999.

3. Referenzjahr 2001

* Hinweise s. Anhang 3 (www.oecd.org/els/education/eag2002).

Quelle: OECD. 
Tabelle C2.3.

Studierende an öffentlichen und privaten Bildungseinrichtungen und in Vollzeit- und Teilzeit-Ausbildung im Tertiärbereich (2000) Verteilung der Studierenden, nach Vollzeit-/ Teilzeitstudium, Art der Bildungseinrichtung und Bildungsbereichen

\begin{tabular}{|c|c|c|c|c|c|c|c|c|c|c|}
\hline & \multicolumn{6}{|c|}{ Art der Bildungseinrichtung } & \multicolumn{4}{|c|}{ Vollzeit-/Teilzeitstudium } \\
\hline & \multicolumn{3}{|c|}{ Tertiärbereich B } & \multicolumn{3}{|c|}{$\begin{array}{l}\text { Tertiärbereich A und weiterführende } \\
\text { Forschungsprogramme }\end{array}$} & \multicolumn{2}{|c|}{ Tertiärbereich B } & \multicolumn{2}{|c|}{$\begin{array}{l}\text { Tertiärbereich A und } \\
\text { weiterführende For- } \\
\text { schungsprogramme }\end{array}$} \\
\hline & öffentlich & $\begin{array}{c}\text { staatlich-sub- } \\
\text { ventioniert } \\
\text { privat }\end{array}$ & $\begin{array}{c}\text { unabhängig } \\
\text { privat }\end{array}$ & öffentlich & $\begin{array}{l}\text { staatlich-sub- } \\
\text { ventioniert } \\
\text { privat }\end{array}$ & $\begin{array}{c}\text { unabhängig } \\
\text { privat }\end{array}$ & Vollzeit & Teilzeit & Vollzeit & Teilzeit \\
\hline & (1) & (2) & (3) & (4) & (5) & (6) & (7) & (8) & (9) & $(10)$ \\
\hline Australien & 98.9 & 1.1 & $\mathrm{a}$ & 100.0 & $\mathrm{a}$ & $\mathrm{a}$ & 32.3 & 67.7 & 62.1 & 37.9 \\
\hline Österreich & 64.4 & 35.6 & $\mathrm{n}$ & 95.8 & 4.2 & $\mathrm{n}$ & 66.1 & 33.9 & 100.0 & a \\
\hline Belgien & 48.7 & 51.3 & $\mathrm{n}$ & 38.7 & 61.3 & $\mathrm{n}$ & 74.4 & 25.6 & 94.9 & 5.1 \\
\hline Kanada & 100.0 & $\mathrm{n}$ & $\mathrm{n}$ & 100.0 & $\mathrm{n}$ & $\mathrm{n}$ & 85.2 & 14.8 & 68.2 & 31.8 \\
\hline Tschechische Rep. & 66.3 & 33.7 & a & 100.0 & a & $\mathrm{a}$ & 100.0 & $\mathrm{n}$ & 92.4 & 7.6 \\
\hline Dänemark & 99.6 & 0.4 & a & 100.0 & a & $\mathrm{a}$ & 100.0 & $\mathrm{a}$ & 100.0 & $\mathrm{a}$ \\
\hline Finnland & 81.3 & 18.7 & a & 89.7 & 10.3 & a & 100.0 & $\mathrm{a}$ & 100.0 & $\mathrm{a}$ \\
\hline Frankreich & 73.2 & 9.1 & 17.7 & 89.4 & 0.8 & 9.8 & 100.0 & $\mathrm{a}$ & 100.0 & $\mathrm{a}$ \\
\hline Deutschland & 63.2 & 36.8 & $\mathbf{x}(2)$ & 100.0 & a & $\mathrm{a}$ & 84.9 & 15.1 & 100.0 & a \\
\hline Griechenland & 100.0 & $\mathrm{a}$ & $\mathrm{a}$ & 100.0 & a & $\mathrm{a}$ & 100.0 & $\mathrm{a}$ & 100.0 & a \\
\hline Ungarn & 100.0 & $\mathrm{n}$ & a & 87.0 & 13.0 & $\mathrm{a}$ & 87.7 & 12.3 & 58.0 & 42.0 \\
\hline Island & 43.8 & 56.2 & $\mathrm{n}$ & 95.4 & 4.6 & $\mathrm{n}$ & 71.2 & 28.8 & 80.9 & 19.1 \\
\hline Irland & 94.2 & $\mathrm{n}$ & 5.8 & 95.3 & $\mathrm{n}$ & 4.7 & 60.7 & 39.3 & 86.8 & 13.2 \\
\hline Italien & 85.3 & $\mathrm{a}$ & 14.7 & 93.8 & a & 6.2 & 100.0 & $\mathrm{a}$ & 100.0 & $\mathrm{a}$ \\
\hline Japan & 9.4 & $\mathrm{a}$ & 90.6 & 27.3 & $\mathrm{a}$ & 72.7 & 96.7 & 3.3 & 90.6 & 9.4 \\
\hline Korea & 14.0 & $\mathrm{a}$ & 86.0 & 23.2 & $\mathrm{a}$ & 76.8 & 100.0 & $\mathrm{a}$ & 100.0 & $\mathrm{a}$ \\
\hline Luxemburg & 100.0 & a & a & 100.0 & a & a & 99.3 & 0.7 & 100.0 & a \\
\hline Mexiko & 100.0 & a & a & 69.0 & a & 31.0 & 100.0 & a & 100.0 & $\mathrm{a}$ \\
\hline Niederlande & 8.9 & 91.1 & $\mathrm{~m}$ & 31.3 & 68.7 & $\mathrm{~m}$ & 69.3 & 30.7 & 82.6 & 17.4 \\
\hline Neuseeland & 81.3 & 18.2 & 0.5 & 99.0 & 1.0 & $\mathrm{n}$ & 45.0 & 55.0 & 69.7 & 30.3 \\
\hline Norwegen & 74.9 & 25.1 & $\mathrm{x}(2)$ & 88.6 & 11.4 & $\mathrm{x}(5)$ & 87.2 & 12.8 & 72.8 & 27.2 \\
\hline Polen & 89.0 & 10.2 & 0.7 & 72.2 & $\mathrm{a}$ & 27.8 & 78.0 & 22.0 & 53.9 & 46.1 \\
\hline Portugal & 80.0 & $\mathrm{a}$ & 20.0 & 64.3 & a & 35.7 & $\mathrm{~m}$ & $\mathrm{~m}$ & $\mathrm{~m}$ & $\mathrm{~m}$ \\
\hline Slowakische Rep. & 94.9 & 5.1 & $\mathrm{n}$ & 100.0 & $\mathrm{n}$ & $\mathrm{n}$ & 64.8 & 35.2 & 71.9 & 28.1 \\
\hline Spanien & 77.3 & 16.3 & 6.3 & 88.7 & $\mathrm{n}$ & 11.3 & 99.6 & 0.4 & 91.5 & 8.5 \\
\hline Schweden & 71.4 & 1.6 & 27.0 & 94.6 & 5.4 & $\mathrm{a}$ & 93.0 & 7.0 & 54.0 & 46.0 \\
\hline Schweiz & 37.7 & 39.2 & 23.1 & 92.4 & 6.1 & 1.5 & 32.9 & 67.1 & 94.5 & 5.5 \\
\hline Türkei* & 97.6 & $\mathrm{a}$ & 2.4 & 95.7 & $\mathrm{a}$ & 4.3 & 100.0 & $\mathrm{a}$ & 100.0 & a \\
\hline Ver. Königreich & $\mathrm{a}$ & 100.0 & $\mathrm{n}$ & $\mathrm{a}$ & 100.0 & $\mathrm{n}$ & 30.5 & 69.5 & 76.0 & 24.0 \\
\hline Vereinigte Staaten & 92.5 & $\mathrm{a}$ & 7.5 & 68.7 & $\mathrm{a}$ & 31.3 & 44.2 & 55.8 & 64.7 & 35.3 \\
\hline Ländermittel & 71.6 & 18.3 & 10.1 & 80.0 & 9.6 & 10.4 & 79.4 & 20.6 & 85.0 & 15.0 \\
\hline Argentinien ${ }^{1}$ & $\mathrm{~m}$ & $\mathrm{~m}$ & $\mathrm{~m}$ & 85.2 & $\mathrm{a}$ & 14.8 & $\mathrm{~m}$ & $\mathrm{~m}$ & $\mathrm{~m}$ & $\mathrm{~m}$ \\
\hline Brasilien' & $\mathrm{m}$ & $\mathrm{a}$ & $\mathrm{m}$ & 36.9 & $\mathrm{a}$ & 63.1 & $\mathrm{~m}$ & $\mathrm{~m}$ & 100.0 & $\mathrm{a}$ \\
\hline Chile $^{1}$ & 7.2 & 6.8 & 86.0 & 33.0 & 23.3 & 43.7 & 100.0 & $\mathrm{n}$ & 100.0 & $\mathrm{n}$ \\
\hline China & $\mathrm{m}$ & $\mathrm{m}$ & $\mathrm{m}$ & $\mathrm{m}$ & $\mathrm{m}$ & $\mathrm{m}$ & 59.4 & 40.6 & 89.4 & 10.6 \\
\hline Ägypten & 31.1 & $\mathrm{~m}$ & 68.9 & $\mathrm{~m}$ & $\mathrm{~m}$ & $\mathrm{~m}$ & 68.9 & 31.1 & $\mathrm{~m}$ & $\mathrm{~m}$ \\
\hline Indonesien $^{2}$ & 37.1 & $\mathrm{a}$ & 62.9 & 31.4 & $\mathrm{a}$ & 68.6 & 100.0 & $\mathrm{a}$ & 100.0 & $\mathrm{a}$ \\
\hline Israel & 22.0 & 78.0 & $\mathrm{x}(2)$ & 12.8 & 79.6 & 7.9 & 100.0 & a & 83.6 & 19.1 \\
\hline Jamaika & 97.7 & $\mathrm{a}$ & 2.3 & 81.4 & $\mathrm{a}$ & 18.6 & 71.6 & 28.4 & $\mathrm{~m}$ & $\mathrm{~m}$ \\
\hline Jordanien & 44.7 & $\mathrm{a}$ & 55.3 & 69.2 & $\mathrm{a}$ & 30.8 & 100.0 & $\mathrm{a}$ & 100.0 & a \\
\hline Malaysia ${ }^{1}$ & 56.4 & a & 43.6 & 77.0 & a & 23.0 & 89.8 & 10.2 & 85.5 & 14.5 \\
\hline Paraguay ${ }^{1}$ & 51.7 & 1.7 & 46.5 & $\mathrm{~m}$ & a & $\mathrm{m}$ & 100.0 & $\mathrm{a}$ & $\mathrm{m}$ & $\mathrm{m}$ \\
\hline Peru $^{1}$ & 56.2 & $\mathrm{~m}$ & 43.8 & 62.3 & a & 37.7 & 100.0 & $\mathrm{a}$ & $\mathrm{m}$ & $\mathrm{m}$ \\
\hline Philippinen ${ }^{1}$ & a & $\mathrm{a}$ & a & 26.9 & $\mathrm{a}$ & 73.1 & a & a & 100.0 & $\mathrm{a}$ \\
\hline Russische Föd. ${ }^{2}$ & 97.8 & a & 2.2 & 90.3 & a & 9.7 & $\mathrm{~m}$ & $\mathrm{~m}$ & $\mathrm{~m}$ & $\mathrm{~m}$ \\
\hline Thailand & 56.7 & $\mathrm{a}$ & 43.3 & 88.3 & a & 11.7 & 100.0 & a & $\mathrm{m}$ & $\mathrm{m}$ \\
\hline Tunesien & 100.0 & a & a & 100.0 & $\mathrm{a}$ & a & 100.0 & a & 100.0 & a \\
\hline Uruguay' ${ }^{1}$ & 91.0 & a & 9.0 & 88.4 & $\mathrm{a}$ & 11.6 & 100.0 & a & 100.0 & a \\
\hline Simbabwe $^{2}$ & 91.0 & 9.0 & $\mathrm{a}$ & 76.0 & 24.0 & a & $\mathrm{m}$ & $\mathrm{m}$ & $\mathrm{m}$ & $\mathrm{m}$ \\
\hline
\end{tabular}


Tabelle C2.4.

Schüler an öffentlichen und privaten Bildungseinrichtungen und Schüler in Vollzeit- und Teilzeit-Ausbildung im Primar- und Sekundarbereich (2000)

Verteilung der Schüler, nach Vollzeit-/Teilzeit-Ausbildung und Art der Bildungseinrichtung

\begin{tabular}{|c|c|c|c|c|c|c|c|c|c|c|c|}
\hline & \multicolumn{9}{|c|}{ Art der Bildungseinrichtung } & \multirow{2}{*}{\multicolumn{2}{|c|}{\begin{tabular}{|c|} 
Vollzeit/Teilzeit \\
$\begin{array}{c}\text { Primar- und Sekundar- } \\
\text { bereich }\end{array}$
\end{tabular}}} \\
\hline & \multicolumn{3}{|c|}{ Primarbereich } & \multicolumn{3}{|c|}{ Sekundarbereich I } & \multicolumn{3}{|c|}{ Sekundarbereich II } & & \\
\hline & öffentlich & $\begin{array}{c}\text { staatlich- } \\
\text { subven- } \\
\text { tioniert } \\
\text { privat }\end{array}$ & $\begin{array}{l}\text { unab- } \\
\text { hängig } \\
\text { privat }\end{array}$ & öffentlich & $\begin{array}{c}\text { staatlich- } \\
\text { subven- } \\
\text { tioniert } \\
\text { privat }\end{array}$ & $\begin{array}{l}\text { unab- } \\
\text { hängig } \\
\text { privat }\end{array}$ & öffentlich & $\begin{array}{c}\text { staatlich- } \\
\text { subven- } \\
\text { tioniert } \\
\text { privat }\end{array}$ & $\begin{array}{l}\text { unab- } \\
\text { hängig } \\
\text { privat }\end{array}$ & Vollzeit & Teilzeit \\
\hline & (1) & (2) & (3) & (4) & (5) & (6) & (7) & $(8)$ & (9) & (10) & (11) \\
\hline Australien & 72.8 & 27.2 & $\mathrm{a}$ & 69.1 & 30.9 & $\mathrm{a}$ & 82.9 & 17.1 & a & 74.0 & 26.0 \\
\hline Österreich & 95.8 & 4.2 & $\mathrm{x}(2)$ & 92.6 & 7.4 & $\mathrm{x}(5)$ & 90.6 & 9.4 & $\mathrm{x}(9)$ & 99.4 & 0.6 \\
\hline Belgien & 45.6 & 54.4 & $\mathrm{n}$ & 41.9 & 58.1 & $\mathrm{n}$ & 39.9 & 60.1 & $\mathrm{n}$ & 84.1 & 15.9 \\
\hline Kanada & 93.5 & 1.4 & 5.1 & 92.1 & 1.1 & 6.7 & 94.4 & 0.7 & 4.9 & 99.2 & 0.8 \\
\hline Tschechische Rep. & 99.1 & 0.9 & $\mathrm{a}$ & 98.3 & 1.7 & $\mathrm{a}$ & 89.5 & 10.5 & $\mathrm{a}$ & 99.7 & 0.3 \\
\hline Dänemark & 89.2 & 10.8 & a & 78.4 & 21.6 & a & 98.0 & 2.0 & $\mathrm{a}$ & 100.0 & a \\
\hline Finnland & 98.9 & 1.1 & a & 96.0 & 4.0 & a & 89.8 & 10.2 & $\mathrm{a}$ & 100.0 & $\mathrm{a}$ \\
\hline Frankreich & 85.4 & 14.3 & 0.2 & 79.2 & 19.8 & 1.0 & 69.7 & 16.6 & 13.7 & 100.0 & a \\
\hline Deutschland & 97.8 & 2.2 & $x(2)$ & 93.3 & 6.7 & $\mathrm{x}(5)$ & 93.2 & 6.8 & $\mathrm{x}(9)$ & 99.8 & 0.2 \\
\hline Griechenland & 93.0 & $\mathrm{a}$ & 7.0 & 95.0 & $\mathrm{a}$ & 5.0 & 93.9 & $\mathrm{a}$ & 6.1 & 98.3 & 1.7 \\
\hline Ungarn & 94.9 & 5.1 & $\mathrm{a}$ & 95.0 & 5.0 & $\mathrm{a}$ & 90.6 & 9.4 & a & 97.0 & 3.0 \\
\hline Island & 98.6 & 1.4 & $\mathrm{n}$ & 99.0 & 1.0 & $\mathrm{n}$ & 94.2 & 5.8 & $\mathrm{n}$ & 92.9 & 7.1 \\
\hline Irland & 98.8 & $\mathrm{n}$ & 1.2 & 100.0 & $\mathrm{n}$ & $\mathrm{n}$ & 98.8 & $\mathrm{n}$ & 1.2 & 99.9 & 0.1 \\
\hline Italien & 93.4 & $\mathrm{a}$ & 6.6 & 96.5 & $\mathrm{a}$ & 3.5 & 93.7 & 0.9 & 5.4 & 100.0 & $\mathrm{a}$ \\
\hline Japan & 99.1 & $\mathrm{a}$ & 0.9 & 94.4 & $\mathrm{a}$ & 5.6 & 69.4 & $\mathrm{a}$ & 30.6 & 99.0 & 1.0 \\
\hline Korea & 98.5 & $\mathrm{a}$ & 1.5 & 77.6 & 22.4 & $\mathrm{a}$ & 45.0 & 55.0 & a & 100.0 & $\mathrm{a}$ \\
\hline Luxemburg & 93.2 & 1.0 & 5.8 & 79.0 & 14.0 & 7.0 & 85.0 & 7.7 & 7.4 & 100.0 & $\mathrm{n}$ \\
\hline Mexiko & 92.6 & a & 7.4 & 86.6 & a & 13.4 & 78.6 & $\mathrm{a}$ & 21.4 & 100.0 & $\mathrm{a}$ \\
\hline Niederlande & 31.4 & 68.6 & $\mathrm{a}$ & 24.6 & 75.3 & 0.2 & 7.8 & 90.0 & 2.2 & 97.6 & 2.4 \\
\hline Neuseeland & 98.0 & a & 2.0 & 95.9 & $\mathrm{a}$ & 4.1 & 83.0 & 7.9 & 9.1 & 95.2 & 4.8 \\
\hline Norwegen & 98.5 & 1.5 & $x(2)$ & 98.1 & 1.9 & $\mathrm{x}(5)$ & 89.1 & 10.9 & $\mathrm{x}(9)$ & 98.6 & 1.4 \\
\hline Polen & 99.2 & 0.8 & a & 99.0 & 1.0 & a & 93.9 & 6.1 & 0.1 & 95.5 & 4.5 \\
\hline Portugal & 90.4 & $\mathrm{a}$ & 9.6 & 90.1 & $\mathrm{a}$ & 9.9 & 85.0 & $\mathrm{a}$ & 15.0 & 93.5 & 6.5 \\
\hline Slowakische Rep. & 96.1 & 3.9 & $\mathrm{n}$ & 95.2 & 4.8 & $\mathrm{n}$ & 93.3 & 6.7 & $\mathrm{n}$ & 98.8 & 1.2 \\
\hline Spanien & 66.6 & 30.2 & 3.2 & 67.1 & 29.8 & 3.2 & 78.9 & 10.0 & 11.1 & 96.2 & 3.8 \\
\hline Schweden & 96.6 & 3.4 & a & 97.3 & 2.7 & a & 98.0 & 2.0 & a & 84.8 & 15.2 \\
\hline Schweiz & 96.7 & 1.2 & 2.2 & 93.2 & 2.5 & 4.3 & 91.4 & 3.6 & 5.0 & 99.7 & 0.3 \\
\hline Türkei & 98.2 & a & 1.8 & a & $\mathrm{a}$ & $\mathrm{a}$ & 97.5 & a & 2.5 & 100.0 & a \\
\hline Ver. Königreich & 95.3 & $\mathrm{a}$ & 4.7 & 93.6 & 0.3 & 6.1 & 29.6 & 67.4 & 3.0 & 77.0 & 23.0 \\
\hline Vereinigte Staaten & 88.4 & $\mathrm{a}$ & 11.6 & 90.1 & $\mathrm{a}$ & 9.9 & 90.6 & $\mathrm{a}$ & 9.4 & 100.0 & $\mathrm{n}$ \\
\hline Ländermittel & 89.9 & 7.8 & 2.7 & 83.6 & 10.4 & 3.1 & 81.2 & 13.9 & 5.7 & 96.0 & 4.0 \\
\hline Argentinien ${ }^{1}$ & 80.5 & 19.5 & $\mathrm{x}(2)$ & 77.5 & 22.5 & $\mathrm{x}(5)$ & 72.2 & 27.8 & $\mathrm{x}(8)$ & 100.0 & $\mathrm{a}$ \\
\hline Brasilien' & 91.9 & $\mathrm{a}$ & 8.1 & 89.9 & $\mathrm{a}$ & 10.1 & 83.2 & $\mathrm{a}$ & 16.8 & 100.0 & a \\
\hline Chile $^{1}$ & 56.8 & 35.7 & 7.5 & 57.8 & 34.1 & 8.1 & 51.4 & 32.4 & 16.1 & 100.0 & a \\
\hline China & $\mathrm{m}$ & $\mathrm{m}$ & $\mathrm{m}$ & $\mathrm{m}$ & $\mathrm{m}$ & $\mathrm{m}$ & $\mathrm{m}$ & $\mathrm{m}$ & $\mathrm{m}$ & 96.7 & 3.3 \\
\hline Ägypten & 92.4 & 1.1 & 7.6 & 95.8 & 1.2 & 4.2 & 93.8 & 0.2 & 6.2 & 100.0 & $\mathrm{a}$ \\
\hline Indien $^{1}$ & 75.6 & 9.9 & 8.0 & 57.0 & 30.4 & 10.8 & 42.5 & 44.5 & 8.7 & 95.3 & 4.7 \\
\hline Indonesien ${ }^{2}$ & 92.7 & a & 7.3 & 72.1 & $\mathrm{a}$ & 27.9 & 47.2 & $\mathrm{a}$ & 52.8 & 100.0 & a \\
\hline Israel & 100.0 & $\mathrm{n}$ & $\mathrm{n}$ & 100.0 & $\mathrm{n}$ & $\mathrm{n}$ & 100.0 & $\mathrm{n}$ & $\mathrm{n}$ & 98.9 & 1.1 \\
\hline Jamaika & 96.0 & $\mathrm{a}$ & 4.0 & 97.0 & $\mathrm{a}$ & 3.0 & 97.0 & a & 3.0 & $\mathrm{a}$ & $\mathrm{a}$ \\
\hline Jordanien & 70.0 & $\mathrm{a}$ & 30.0 & 80.5 & $\mathrm{a}$ & 19.5 & 91.3 & a & 8.7 & 100.0 & $\mathrm{a}$ \\
\hline Malaysia $^{1}$ & 94.3 & $\mathrm{a}$ & 5.7 & 92.6 & $\mathrm{a}$ & 7.4 & 92.1 & $\mathrm{a}$ & 7.9 & 100.0 & $\mathrm{a}$ \\
\hline Paraguay $^{1}$ & 85.0 & 9.3 & 5.7 & 72.5 & 10.9 & 16.7 & 67.4 & 7.4 & 25.2 & 100.0 & $\mathrm{a}$ \\
\hline Peru $^{1}$ & 87.4 & 3.2 & 9.4 & 84.9 & 4.7 & 10.4 & 82.2 & 5.1 & 12.7 & 100.0 & $\mathrm{a}$ \\
\hline Philippinen ${ }^{1}$ & 92.5 & $\mathrm{a}$ & 7.5 & 74.8 & $\mathrm{a}$ & 25.2 & 69.5 & $\mathrm{a}$ & 30.5 & 100.0 & a \\
\hline Russische Föd. ${ }^{2}$ & 99.6 & $\mathrm{a}$ & 0.4 & 99.7 & $\mathrm{a}$ & 0.3 & 99.6 & $\mathrm{a}$ & 0.4 & $\mathrm{~m}$ & $\mathrm{~m}$ \\
\hline Thailand & 86.9 & 13.1 & $\mathrm{n}$ & 93.6 & 6.4 & $\mathrm{n}$ & 87.7 & 3.0 & 9.3 & $\mathrm{~m}$ & $\mathrm{~m}$ \\
\hline Tunesien & 99.3 & $\mathrm{a}$ & 0.7 & 94.5 & $\mathrm{a}$ & 5.5 & 88.8 & $\mathrm{a}$ & 11.2 & 100.0 & a \\
\hline Uruguay $^{1}$ & 85.8 & $\mathrm{a}$ & 14.2 & 86.1 & $\mathrm{a}$ & 13.9 & 88.3 & $\mathrm{a}$ & 11.7 & 100.0 & $\mathrm{a}$ \\
\hline Simbabwe & 12.0 & 88.0 & $\mathrm{a}$ & 27.6 & 72.4 & a & 42.6 & 57.4 & $\mathrm{a}$ & 100.0 & a \\
\hline
\end{tabular}

Hinweis: $\mathrm{x}$ bedeutet, dass die Daten in einer anderen Spalte enthalten sind, deren Referenz in runden Klammern nach dem ' $\mathrm{x}$ ' angegeben ist. So bedeutet z.B. $\mathrm{x}(2)$, dass die Daten in Spalte 2 enthalten sind.

1. Referenzjahr 1999 .

2. Referenzjahr 2001.

Quelle: OECD. Hinweise zur Methodiks. Anhang 3 (www.oecd.org/els/education/eag2002). 
Tabelle C2.5.

Arten der Bildungsteilnahme im Sekundarbereich II (2000)

Bildungsteilnahme in öffentlichen und privaten Bildungseinrichtungen des Sekundarbereich II nach Bildungsbereich und Art des Bildungsgangs

\begin{tabular}{|c|c|c|c|c|c|c|c|}
\hline & \multicolumn{3}{|c|}{ Prozentsatz der Bildungsteilnahme nach Bildungsbereich } & \multicolumn{4}{|c|}{ Prozentsatz der Bildungsteilnahme nach Art des Bildungsgangs } \\
\hline & ISCED 3A & ISCED 3B & ISCED 3C & $\begin{array}{l}\text { allgemein- } \\
\text { bildend }\end{array}$ & $\begin{array}{c}\text { berufs- } \\
\text { vorbereitend }\end{array}$ & berufsbildend & $\begin{array}{l}\text { davon (6): duale } \\
\text { Ausbildungsgänge }\end{array}$ \\
\hline & (1) & (2) & (3) & (4) & (5) & (6) & (7) \\
\hline Australien & 34.3 & a & 65.7 & 34.3 & a & 65.7 & $\mathrm{x}(6)$ \\
\hline Österreich & 43.5 & 48.1 & 8.5 & 21.7 & 7.2 & 71.1 & 36.4 \\
\hline Belgien & 53.7 & a & 46.3 & 33.2 & a & 66.8 & 2.8 \\
\hline Kanada & 90.9 & a & 9.1 & 90.9 & 9.1 & $\mathrm{a}$ & a \\
\hline Tschechische Rep. & 63.5 & 0.5 & 36.0 & 18.6 & 1.1 & 80.2 & 40.5 \\
\hline Dänemark & 45.3 & a & 54.7 & 45.1 & 0.2 & 54.7 & 54.1 \\
\hline Finnland & 100.0 & a & $\mathrm{a}$ & 44.7 & a & 55.3 & 10.7 \\
\hline Frankreich & 67.0 & a & 33.0 & 42.6 & a & 57.4 & 11.7 \\
\hline Deutschland & 36.8 & 63.2 & $\mathrm{a}$ & 36.8 & $\mathrm{a}$ & 63.2 & 48.7 \\
\hline Griechenland & 67.9 & a & 32.1 & 67.9 & a & 32.1 & a \\
\hline Ungarn & 74.6 & 1.7 & 23.6 & 36.0 & 53.7 & 10.3 & 10.3 \\
\hline Island & 66.8 & 0.5 & 32.7 & 66.6 & 1.1 & 32.3 & 14.4 \\
\hline Irland & 78.1 & a & 21.9 & 76.6 & 23.4 & a & a \\
\hline Italien & 80.8 & 1.3 & 17.9 & 35.7 & 39.8 & 24.6 & $\mathrm{~m}$ \\
\hline Japan & 73.9 & 0.8 & 25.3 & 73.9 & 0.8 & 25.3 & a \\
\hline Korea & 63.9 & a & 36.1 & 63.9 & a & 36.1 & a \\
\hline Luxemburg & 61.2 & 14.4 & 24.3 & 36.5 & a & 63.5 & 13.7 \\
\hline Mexiko & 87.0 & a & 13.0 & 87.0 & a & 13.0 & $\mathrm{a}$ \\
\hline Niederlande & 64.8 & a & 35.2 & 31.7 & a & 68.3 & 20.4 \\
\hline Neuseeland & 65.0 & 17.4 & 17.6 & $\mathrm{~m}$ & $\mathrm{~m}$ & $\mathrm{~m}$ & $\mathrm{~m}$ \\
\hline Norwegen & 42.7 & a & 57.3 & 42.7 & $\mathrm{a}$ & 57.3 & $\mathrm{~m}$ \\
\hline Polen & 78.0 & a & 22.0 & 35.7 & a & 64.3 & a \\
\hline Portugal & 75.9 & 17.0 & 7.0 & 72.2 & a & 27.8 & $\mathrm{~m}$ \\
\hline Slowakische Rep. & 78.1 & a & 21.9 & 21.4 & a & 78.6 & 39.7 \\
\hline Spanien & 66.5 & $\mathrm{n}$ & 33.5 & 66.5 & $\mathrm{n}$ & 33.5 & 5.8 \\
\hline Schweden* & 49.0 & a & 0.4 & 51.2 & a & 48.8 & $\mathrm{~m}$ \\
\hline Schweiz & 30.0 & 60.0 & 10.0 & 34.3 & a & 65.7 & 57.9 \\
\hline Türkei & 90.1 & a & 9.9 & 51.0 & a & 49.0 & 9.9 \\
\hline Ver. Königreich & 24.3 & a & 75.7 & 32.7 & $\mathrm{x}(6)$ & 67.3 & $\mathrm{x}(6)$ \\
\hline Vereinigte Staaten & $\mathrm{m}$ & $\mathrm{m}$ & $\mathrm{m}$ & $\mathrm{m}$ & $\mathrm{m}$ & $\mathrm{m}$ & $\mathrm{m}$ \\
\hline Ländermittel & 63.9 & 7.8 & 26.6 & 48.3 & 5.1 & 46.9 & 17.1 \\
\hline Argentinien ${ }^{1}$ & 100.0 & a & $\mathrm{a}$ & 41.6 & a & 58.4 & $\mathrm{x}(6)$ \\
\hline Brasilien $^{1}$ & $\mathrm{~m}$ & $\mathrm{~m}$ & $\mathrm{a}$ & 82.3 & $\mathrm{a}$ & 17.7 & $\mathrm{~m}$ \\
\hline Chile $^{1}$ & 58.2 & 41.8 & $\mathrm{a}$ & 58.2 & $\mathrm{a}$ & 41.8 & $\mathrm{a}$ \\
\hline China & 47.0 & a & 53.0 & 47.0 & $\mathrm{x}(6)$ & 53.0 & $\mathrm{~m}$ \\
\hline Ägypten & 35.2 & 64.8 & $\mathrm{a}$ & 35.2 & a & 64.8 & a \\
\hline Indien $^{1}$ & $\mathrm{~m}$ & a & $\mathrm{m}$ & 95.8 & a & 4.2 & $\mathrm{~m}$ \\
\hline Indonesien $^{2}$ & 60.3 & 39.7 & a & $\mathrm{m}$ & a & $\mathrm{m}$ & $\mathrm{m}$ \\
\hline Israel & 95.8 & $\mathrm{x}(1)$ & 4.2 & 67.1 & a & 32.9 & $\mathrm{~m}$ \\
\hline Jamaika & 99.1 & 0.9 & $\mathrm{a}$ & 99.1 & $\mathrm{a}$ & 0.9 & a \\
\hline Jordanien & 93.9 & a & 6.1 & 74.9 & a & 25.1 & $\mathrm{n}$ \\
\hline Malaysia $^{1}$ & 14.9 & a & 85.1 & 84.9 & a & 15.1 & $\mathrm{x}(6)$ \\
\hline Paraguay $^{1}$ & $\mathrm{~m}$ & a & $\mathrm{m}$ & 81.5 & a & 18.5 & $\mathrm{a}$ \\
\hline Peru $^{1}$ & $\mathrm{~m}$ & $\mathrm{~m}$ & $\mathrm{a}$ & 75.1 & a & 24.9 & a \\
\hline Philippinen $^{1}$ & 100.0 & a & $\mathrm{a}$ & 100.0 & a & a & a \\
\hline Thailand & 70.0 & 30.0 & $\mathrm{a}$ & 70.0 & a & 30.0 & $\mathrm{x}(6)$ \\
\hline Tunesien & 94.1 & 3.7 & 2.2 & 94.1 & 3.7 & 2.2 & $\mathrm{a}$ \\
\hline Uruguay ${ }^{1}$ & 90.3 & $\mathrm{a}$ & 9.7 & 81.3 & a & 18.7 & a \\
\hline Simbabwe $^{2}$ & 54.9 & 45.1 & $\mathrm{x}(2)$ & $\mathrm{m}$ & $\mathrm{m}$ & $\mathrm{m}$ & $\mathrm{m}$ \\
\hline
\end{tabular}

Hinweis: $\mathrm{x}$ bedeutet, dass die Daten in einer anderen Spalte enthalten sind, deren Referenz in runden Klammern nach dem ' $\mathrm{x}$ ' angegeben ist. So bedeutet z.B. $\mathrm{x}(2)$, dass die Daten in Spalte 2 enthalten sind.

1. Referenzjahr 1999

2. Referenzjahr 2001.

* Hinweise s. Anhang 3 (www.oecd.org/els/education/eag2002).

Quelle: OECD. 


\section{AUSLÄNDISCHE STUDIERENDE IM TERTIÄRBEREICH}

- Sieben von zehn ausländischen Studierenden im OECD-Raum konzentrieren sich auf 5 Gastländer: Australien, Frankreich, Deutschland, das Vereinigte Königreich und die Vereinigten Staaten.

- Absolut gesehen entsenden Korea, Japan und Griechenland die meisten der im Ausland Studierenden aus OECD-Ländern, von außerhalb des OECD-Raums kommen die meisten Studierenden aus China und Südostasien.

- Relativ gesehen schwankt der Anteil der ausländischen Studierenden in den OECD-Ländern von weniger als 1 Prozent bis zu fast 17 Prozent. Proportional zu ihrer Größe weisen Australien, Belgien, Österreich, die Schweiz und das Vereinigte Königreich den größten Nettozugang ausländischer Studierender auf (gemessen in Prozent der Studierendenzahlen in diesen Ländern).

\section{Abbildung C3.1.}

Prozentsatz eingeschriebener Studierender des Tertiärbereichs, die nicht Staatsbürger des Studienlandes sind (2000)

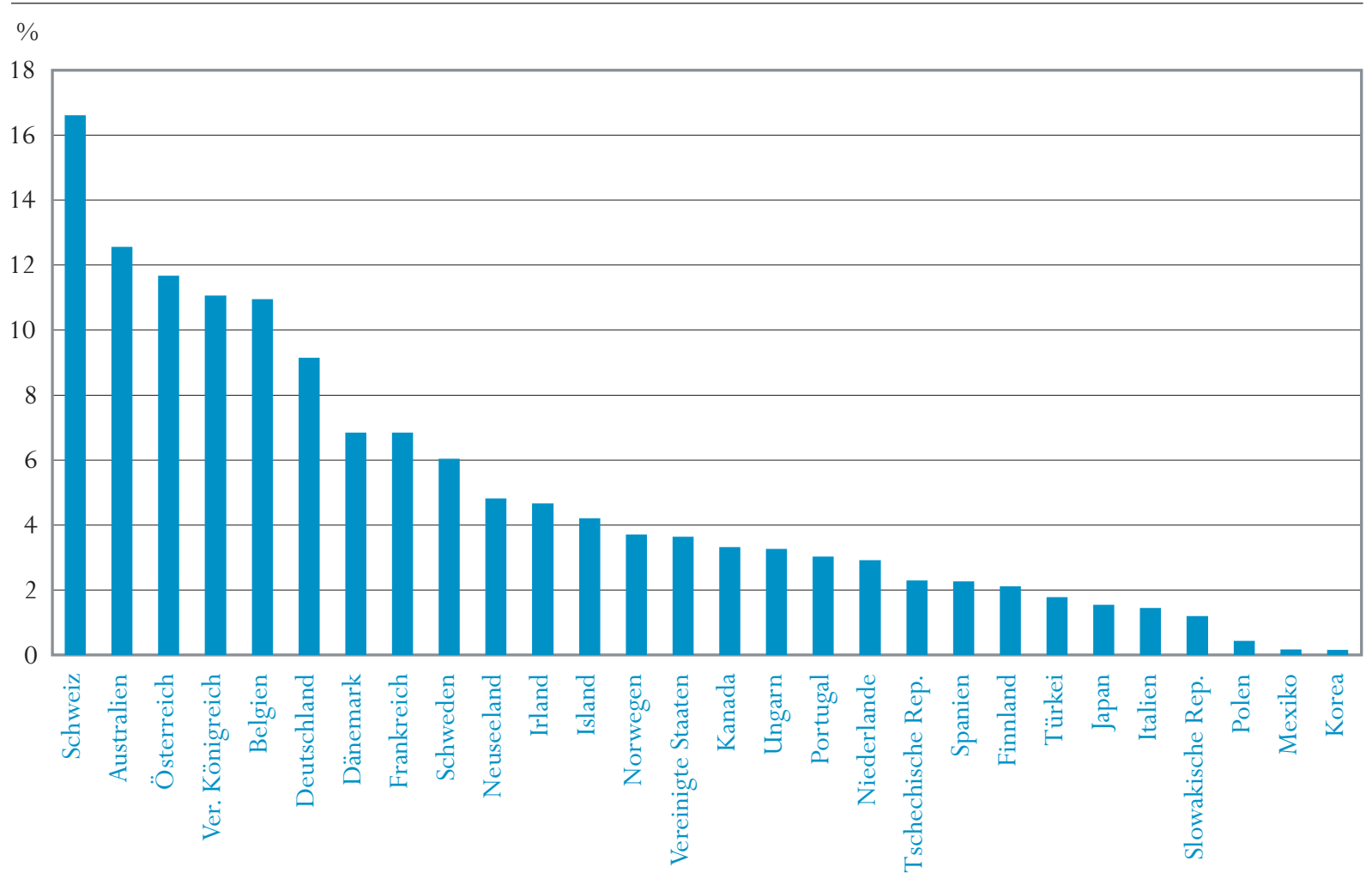

Anordnung der Länder in absteigender Reihenfolge des Prozentsatzes eingeschriebener Studierender, die nicht Staatsbürger des Studienlandes sind. Quelle: OECD. Tabelle C3.1. Hinweise s. Anhang 3 (www.oecd.org/els/education/eag2002). 
Dieser Indikator zeigt die länderübergreifende Mobilität der Studierenden.

Sieben von zehn Studierenden im Ausland konzentrieren sich auf nur fünf $O E C D$-Länder.

Viele ausländischen Studierende sind bereits vor dem Studium in das Gastland gekommen.

\section{Politischer Hintergrund}

Die internationale oder transkulturelle Dimension der Hochschulausbildung findet immer mehr Beachtung. Der generelle Trend zur Freizügigkeit von Kapital, Waren und Arbeitskräften zusammen mit der zunehmenden Öffnung der Arbeitsmärkte hat in den OECD-Ländern die Nachfrage nach modernen Kenntnissen und Fähigkeiten verstärkt. Die Regierungen erwarten zunehmend von den Hochschulen, dass sie den Horizont junger Menschen erweitern und ihnen ein umfassenderes Verständnis fremder Sprachen, Kulturen und wirtschaftswissenschaftlicher Methoden vermitteln.

Eine Möglichkeit für Studierende, ihre Kenntnisse über andere Kulturen und Gesellschaften zu erweitern, besteht darin, tertiäre Bildungseinrichtungen anderer Länder zu besuchen. Die internationale Mobilität von Studierenden impliziert für die Studierenden ebenso wie für die Bildungseinrichtungen Kosten und Nutzen, und zwar sowohl in den 'Sender' - als auch den 'Empfänger' Ländern. Während sich die kurzfristigen monetären Kosten und der Nutzen dieser Mobilität relativ leicht erfassen lassen, sind die längerfristigen sozialen und wirtschaftlichen Vorteile für die Studierenden, die Bildungseinrichtungen und auch die beteiligten Länder selbst schwer zu quantifizieren. Zahlen im Ausland studierender junger Menschen vermitteln jedoch zumindest einen ersten Eindruck von dem Ausmaß dieser Mobilität der Studierenden.

Hierbei ist zu beachten, dass zur Erfassung der Internationalisierung des Tertiärbereichs nicht nur die Anzahl der im Ausland Studierenden von Bedeutung ist, sondern auch die grenzüberschreitende Bereitstellung flexibler internationaler Bildungsgänge auf elektronischem Weg. Heutzutage lässt sich grenzüberschreitende Mobilität nicht nur bei den Bildungsteilnehmern beobachten, sondern auch bei den Angeboten. Es wird künftig wichtig werden, Methoden zu entwickeln, um auch diese Elemente der Internationalisierung der Bildung zu quantifizieren und zu messen.

\section{Ergebnisse und Erläuterungen}

Anteil der ausländischen Studierenden, nach Studienland

Die große Mehrzahl ausländischer Studierender im OECD-Gebiet und anderen Nicht-OECD-Ländern, für die entsprechende Daten vorliegen, ist in einer relativ geringen Zahl von OECD-Ländern eingeschrieben. Mit 29 Prozent der Gesamtzahl sind die Vereinigten Staaten das größte Aufnahmeland von ausländischen Studierenden (in absoluten Zahlen), gefolgt vom Vereinigten Königreich und Deutschland (14 bzw. 12 Prozent) sowie Frankreich und Australien (8 bzw. 7 Prozent) (s. Abb. C3.2). Diese fünf Gastländer nehmen rund 70 Prozent aller ausländischen Studierenden auf.

Im Sinne dieses Indikators gilt derjenige als ausländischer Studierender, der nicht die Staatsangehörigkeit seines Studienlandes besitzt. In den meisten Ländern konnten die ausländischen Studierenden, die aufgrund einer früheren Zuwanderung (alleine oder mit ihren Eltern) in diesem Land ihren 


\section{Abbildung C 3.2}

Aufteilung der Studierenden, die nicht Staatsbürger ihres Studienlandes sind, nach aufnehmendem Studienland (2000)

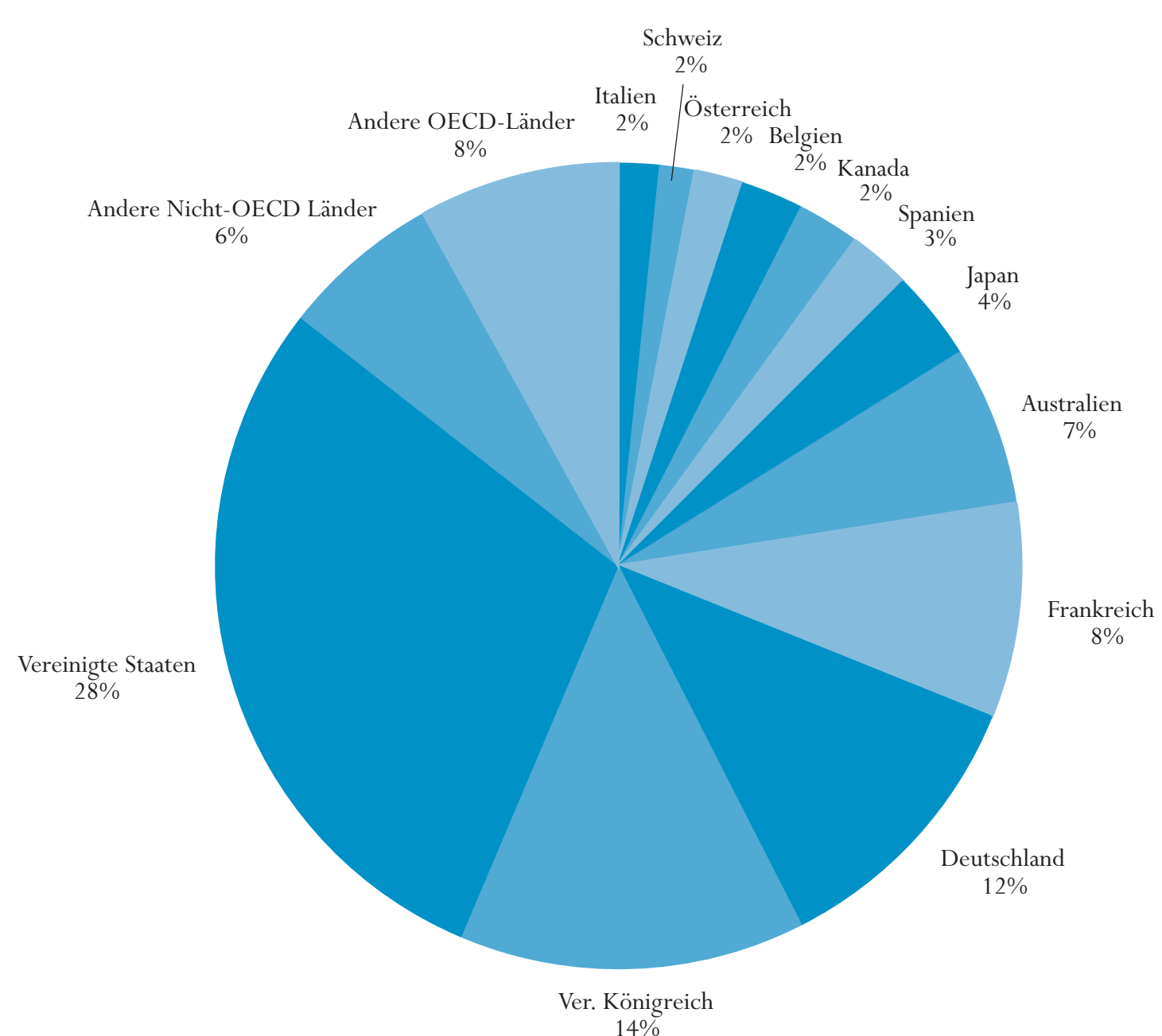

Quelle: OECD.

Aufenthalt haben, nicht von denen unterschieden werden, die speziell zu Bildungszwecken einreisten. Dies führt potentiell zu einer zu hohen Anzahl ausländischer Studierender in denjenigen Ländern, die vergleichsweise strenge Einbürgerungsgesetze haben.

So kommen beispielsweise in Deutschland, einem der aufnahmestärksten Gastländer, nur zwei Drittel aller an tertiären Bildungseinrichtungen eingeschriebenen ausländischen Studierenden tatsächlich aus dem Ausland, da eine beträchtliche Zahl von „einheimischen Ausländern”, hauptsächlich Kinder von Gastarbeitern, im Sinne dieses Indikators als „Ausländer” gelten, obwohl sie bereits in Deutschland aufgewachsen sind. 
Die Unterrichtssprache

ist ein wesentlicher

Faktor bei der Auswahl

des Landes für ein

Auslandsstudium.

Im Jahr 2000 waren

1,62 Millionen

Studierende auferhalb

ihres Heimatlandes

eingeschrieben, von

diesen studierten 1,52

Millionen (bzw. 94

Prozent) im

OECD-Raum

Der größte Teil

der ausländischen

Studierenden aus

OECD-Ländern kommt

aus Kored, Japan und

Griechenland,...

... während unter

den ausländischen

Studierenden, die

nicht aus OECD-

Ländern kommen, die Studierenden aus China

und Südostasien am

stärksten vertreten sind.
Ein wesentlicher Faktor für die Auswahl des Landes zum Auslandsstudium ist die Unterrichtssprache. Daher ist der Zustrom ausländischer Studierender, sowohl absolut als auch relativ gesehen, in den Ländern am stärksten, deren Unterrichtssprache aus historischen oder wirtschaftlichen Gründen weit verbreitet ist (Englisch, Französisch, Deutsch). Insbesondere die Dominanz englischsprachiger Länder wie dem Vereinigten Königreich, den Vereinigten Staaten und Australien ist größtenteils darauf zurückzuführen, dass das Englische zum einen die dortige Unterrichtssprache ist und zum zweiten die Fremdsprache, die auslandsinteressierte Studierende mit größter Wahrscheinlichkeit beherrschen. Zunehmend gibt es nun sogar Bildungseinrichtungen in nicht-englischsprachigen Ländern, die Studiengänge in Englisch anbieten, um ausländische Studierende anzuziehen.

\section{Anteil der ausländischen Studierenden in den OECD-Ländern, nach Heimatländern}

Im Jahr 2000 waren 1,62 Millionen Studierende außerhalb ihres Heimatlandes eingeschrieben, von diesen studierten 1,52 Millionen (bzw. 94 Prozent) im OECD-Raum. Im Vergleich zu 1998 bedeutet dies einen Anstieg der Mobilität Studierender in den OECD-Raum hinein von 14 Prozent. Dieser Anstieg der Gesamtmobilität in die einzelnen OECD-Länder ist gleichermaßen auf Studierende aus OECD-Ländern wie auf Studierende aus Nicht-OECD-Ländern zurückzuführen, da sich die geografische Zusammensetzung des Zustroms der Studierenden nicht veränderte - 44 Prozent der ausländischen Studierenden kamen aus OECD-Ländern und 56 Prozent aus Nicht-OECD-Ländern.

Die asiatischen Studierenden stellen mit 41 Prozent der Gesamtzahl die größte Gruppe der in den OECD-Ländern studierenden Ausländer, ihnen folgen die Europäer mit 33 Prozent.

Auch wenn man ausschließlich die OECD-Länder betrachtet, dominieren die Asiaten und Europäer unter den im Ausland Studierenden. Hierbei stellen die koreanischen und die japanischen Studierenden mit 4,6 und 3,9 Prozent aller ausländischen Studierenden den größten Anteil, gefolgt von den Studierenden aus Griechenland (3,6 Prozent), Deutschland (3,5 Prozent), Frankreich (3,4 Prozent) und Italien (2,7 Prozent). Zusammengenommen stellen diese Länder fast 20 Prozent aller ausländischen Studierenden in den OECD-Ländern.

Bei einem Blick auf die nicht zur OECD gehörenden Herkunftsländer zeigt sich, dass die chinesischen Studierenden 7,1 Prozent aller in den OECD-Ländern studierenden Ausländer ausmachen, gefolgt von Studierenden aus Indien (3,4 Prozent), Marokko (2,7 Prozent) und Malaysia (2,4 Prozent). Auch aus anderen südostasiatischen Ländern gibt es einen starken Zustrom Studierender in die OECD, 4,2 Prozent aller im OECD-Raum studierenden Ausländer kommen aus Indonesien, Singapur oder Thailand. 
Wahrscheinlich spielt auch die Bedeutung der Internationalisierung von Handel, Finanzen und Wirtschaft eine Rolle bei der Mobilität der Studierenden in den einzelnen Regionen. So könnte beispielsweise die regionale Integration durch Organisationen und Verträge wie EU, NAFTA, ASEAN und APEC die Mobilität der Studierenden beeinflussen, indem sie ihr Interesse an den Kulturen und Sprachen der Partnerländer sowie der Entwicklung bilateraler und multilateraler Netzwerke weckt. Außerdem haben einige Regierungen die internationale Mobilität Studierender zum integralen Bestandteil ihrer sozio-ökonomischen Entwicklungsstrategien erklärt. So haben beispielsweise verschiedene Staaten im asiatisch-pazifischen Raum wie Australien, Japan und Neuseeland für ihre tertiären Bildungseinrichtungen Maßnahmen ergriffen, um ausländische Studierende zu gewinnen, häufig um zusätzliche Einnahmen zu generieren, zumindest aber als kostendeckende Maßnahme.

\section{Anteil ausländischer Studierender an der Gesamtzahl der Studierenden}

Vorstehend wurde die Verteilung der absoluten Anzahl der ausländischen Studierenden nach Herkunfts- und Studienland analysiert. Hierbei erscheinen größere Länder unter sonst gleichen Bedingungen oft zu gewichtig. Um diesen Größeneffekt zu bereinigen, kann man sowohl die Aufnahme Studierender im Tertiärbereich in einem bestimmten Land als auch die Zahl der Studierenden, die aus diesem Land ins Ausland gehen, zur gesamten Bildungsbeteiligung im Tertiärbereich in diesem Land in Relation setzen.

Hinsichtlich des Anteils ausländischer Studierender an der Gesamtzahl der Studierenden im Tertiärbereich sind Australien, Österreich und die Schweiz die wichtigsten Empfängerländer, da hier der Anteil der ausländischen Studierenden zwischen 12 und 17 Prozent der Gesamtzahl aller Studierenden im Tertiärbereich liegt, es folgen das Vereinigte Königreich, Belgien und Deutschland (s. Abb. C3.1). Dagegen ist der Anteil ausländischer Studierender in Korea sowie in Mexiko, Polen, der Slowakischen Republik, Italien, Japan und der Türkei, wo er unter 2 Prozent bleibt, recht gering (s. Abb. C3.1).

Im Vergleich zu den OECD-Ländern nehmen die am World Education Indicators Programm beteiligten Nicht-OECD-Länder im Verhältnis zu ihrer Größe nur eine verschwindend geringe Zahl von ausländischen Studierenden auf. Eine Ausnahme bildet hier Jordanien (9 Prozent), was auf die zahlenmäßig starke Präsenz palästinensischer Flüchtlinge zurückzuführen ist, und in geringerem Ausmaß auch Jamaika (mit 2 Prozent), da sich dort eines der drei Universitätsgelände der University of West Indies befindet.

\section{Im Ausland Studierende im Verhältnis zur Gesamtzahl der Studierenden}

Man kann anhand des Anteils der Studierenden eines Landes, die ein Auslandsstudium absolvieren, an der Gesamtzahl der Studierenden des Landes Schätzungen darüber anstellen, in welchem Ausmaß Studierende das Heimatland
Der Prozentsatz der in den OECD-Ländern eingeschriebenen ausländischen Studierenden reicht von weniger als 1 bis zu fast 17 Prozent. 
Ein relativ großer Anteil der Studierenden aus Luxemburg, Island, Griechenland und Irland studiert im Ausland, während dies nur für relativ wenige Studierende aus den Vereinigten Staaten, Australien und Mexiko zutrifft.

Im Verhältnis zu ihrer Größse verzeichnen die Schweiz, Australien und das Vereinigte Königreich den stärksten Nettozugang ausländischer Studierender. zum Studium im Ausland verlassen. Diese Angabe bezieht sich lediglich auf die in anderen OECD-Ländern und Nicht-OECD-Ländern Studierenden, sofern diese Länder Daten gemeldet haben. Mit anderen Worten sind die Studierenden in Ländern, die keine Daten über ausländische Studierende zur Verfügung stellen, in Tabelle C3.1 nicht enthalten. Daher ist wahrscheinlich die Zahl der im Ausland Studierenden in diesem Indikator zu niedrig angesetzt. Eine weitere potentielle Fehlerquelle hinsichtlich zu niedrig angesetzter Zahlen könnte die Berechnung des Indikators auf Jahresbasis darstellen. So gehen beispielsweise über die Hälfte der Studierenden aus den Vereinigten Staaten nur für maximal ein halbes Jahr ins Ausland und nur 14 Prozent verbringen ein ganzes akademisches Jahr im Gastland. Selbst wenn man diese Einschränkung der Aussagefähigkeit berücksichtigt, bietet diese Kennzahl doch eine gewisse Vorstellung davon, wie intensiv die Studierenden aus den OECD-Ländern die Möglichkeiten eines Auslandsstudiums nutzen.

Das Verhältnis der im Ausland eingeschriebenen Studierenden zur Gesamtzahl der Studierenden im Heimatland schwankt von Land zu Land sehr stark. Die Bandbreite reicht von unter einem Prozent in den Vereinigten Staaten (0,3 Prozent), Australien (0,6 Prozent) and Mexiko (0,7 Prozent) bis zu 25 Prozent in Island und 226 Prozent in Luxemburg. Luxemburg ist jedoch ein Sonderfall, dort gibt es nur post-sekundäre, nicht-tertiäre Bildungsgänge, bzw. können Studierende dort nur das erste Studienjahr auf Hochschulniveau absolvieren. Für alle weiterführenden Studien müssen die Studierenden ins Ausland gehen, daher ist die Anzahl der luxemburgischen Studierenden im Ausland viel höher als die derjenigen, die in Luxemburg selbst eingeschrieben sind.

\section{Saldo des internationalen Austauschs Studierender}

Wenngleich über 441.000 Studierende mehr zum Studium in die Vereinigten Staaten reisen als es US-amerikanische Studierende im Ausland gibt, nehmen andere Länder im Verhältnis zu ihrer Größe noch viel mehr ausländische Studierende auf. Der Nettozugang eingeschriebener Studierender in der Schweiz, Australien und dem Vereinigten Königreich beträgt zwischen 4,6 und 6,5 Prozent, gemessen an der Gesamtzahl der in diesen Ländern im Tertiärbereich eingeschriebenen Studierenden (s. Tab. C3.1, Spalte 4). Umgekehrt verzeichnen Island, Irland, Norwegen und die Türkei die relativ stärksten Nettoausreisezahlen von Studierenden, mit 22, 7, 5 bzw. 4 Prozent der Gesamtbeteiligung im Tertiärbereich. Hierbei ist zu beachten, dass der Saldo der Bewegungen der Studierenden nur diejenigen berücksichtigt, die aus einem OECD-Land oder Nicht-OECD-Land kommen bzw. in einem solchen Land studieren, das Zahlenmaterial vorlegte. Der absolute Saldo der Länder, die eine erhebliche Zahl an Studierenden aus Ländern aufnehmen bzw. in Länder entsenden, die keine Angaben machten, könnte daher anders aussehen. 
Für die Nicht-OECD-Länder ergibt sich generell ein negativer Saldo zwischen der Aufnahme und der Entsendung Studierender, mit Ausnahme Tunesiens (3 Prozent), der Russischen Föderation (2 Prozent) und Uruguay (1 Prozent).

Angesichts der vielfältigen Vorteile, die ausländische Studierende ihren Gastländern bringen können, ist es nicht unwichtig herauszufinden, wie sich diese Mobilität erhöhen lässt.

Die oben angeführten Charakteristika studentischer Mobilität können mit einer Reihe von „Push-” und „Pull-Faktoren”, also anstoßenden und anziehenden Faktoren, in Zusammenhang gebracht werden, wie z.B. Sprachschwierigkeiten, dem akademischen Ruf bestimmter Bildungseinrichtungen oder Bildungsgänge, der Flexibilität der Studiengänge im Heimatland hinsichtlich der Anrechnung von Auslandsaufenthalten auf die Prüfungsanforderungen, dem eingeschränkten Angebot im Tertiärbereich im Heimatland, restriktiven Zulassungspraktiken der Hochschulen im Heimatland, finanziellen Anreizen, Studiengebühren usw.

Diese Charakteristika spiegeln auch geografische und historische Beziehungen zwischen den Ländern, zukünftige Beschäftigungschancen, kulturelle Neigungen und die staatliche Politik zur Erleichterung der Anerkennung von Leistungsnachweisen zwischen Heimat- und Gasthochschulen wider. Bei der Wahl einer Bildungseinrichtung ist auch die Transparenz und Flexibilität der Lehrveranstaltungen und der Prüfungsvoraussetzungen entscheidend.

\section{Wirtschaftliche Vorteile der Internationalisierung der Hochschulausbildung sowie deren Auswirkungen auf den Handel}

Der erste direkte Nutzen aus der Aufnahme ausländischer Studierender sind die Einnahmen aus den Studiengebühren und der entsprechende Inlandsverbrauch der ausländischen Studierenden, die sich in der Leistungsbilanz als Export von Bildungsdienstleistungen niederschlagen. Der Umfang dieses wirtschaftlichen Gewinns wird noch größer, wenn das Gastland gegenüber den ausländischen Studierenden eine Politik der Kostendeckung bei den Studiengebühren verfolgt. Tatsächlich wurde der Export von Bildungsdienstleistungen für 1998 auf 30 Milliarden US-Dollar geschätzt, das sind 3 Prozent des gesamten OCEDHandels mit Dienstleistungen. So war beispielsweise in Australien, einem der wichtigsten Aufnahmeländer, der Export von Bildungsdienstleistungen 2000/2001 der drittgrößte Exportfaktor bei den Dienstleistungen und machte fast 12 Prozent des Gesamtexports von Dienstleistungen aus.

Abgesehen von diesem direkten Vorteil der Internationalisierung der Hochschulausbildung kann es auch indirekte Vorteile geben, z.B. durch eine größere Kundenbasis für tertiäre Bildung. Denn so entstehen für die Nettoaufnahmeländer Größenvorteile und sie können ihre Auswahl an Bildungsgängen erhöhen oder ihre Kosten pro Studierenden senken. Dieser Effekt könnte insbesondere für Aufnahmeländer mit einer relativ geringen Bevölkerungszahl von Bedeutung sein. Ferner zwingt das
Eine Reihe von "Push-" und "Pull-Faktoren" können als Erklärung für die Charakteristika der Mobilität

Studierender dienen.

Die Nettoaufnahme ausländischer Studierender gibt Hinweise über den Nutzen, den Länder aus dem internationalen Austausch Studierender im Tertiärbereich ziehen können. 
Die Daten beziehen sich auf das Schuljahr 1999-2000 und beruhen auf der alliährlich von der $O E C D$ durchgeführten VOE-Datenerhebung zur Bildungsstatistik.
Vorhandensein potentieller ausländischer Kunden (Studierender) die tertiären Bildungseinrichtungen auch dazu, qualitativ hochwertige Studiengänge anzubieten, um sich gegenüber anderen ausländischen Wettbewerbern abzusetzen, was zu einem reaktionsschnellen und kundenorientierten Hochschulsystem beiträgt.

Schließlich kann die Aufnahme ausländischer Studierender auch bis zu einem gewissen Grad einen Technologietransfer bewirken (insbesondere bei weiterführenden Forschungsprogrammen), interkulturelle Kontakte fördern und dazu beitragen, soziale Netzwerke für die Zukunft zu schaffen.

\section{Definitionen und angewandte Methodik}

Studierende, die nicht Staatsangehörige des Landes sind, für das die Daten erhoben werden, gelten als ausländische Studierende. Diese Klassifikation ist zwar pragmatisch und operational, kann jedoch aufgrund der unterschiedlichen nationalen Politiken zur Einbürgerung von Migranten zu Inkonsistenzen führen. Hinzu kommt, dass einige Länder keine separaten Angaben über ausländische Studierende machen können, die eine ständige Aufenthaltsgenehmigung besitzen. Daher wird in den Ländern, in denen eine strenge Einbürgerungspolitik verfolgt wird und nicht zwischen ausländischen Studierenden mit und ohne ständiger Aufenthaltsgenehmigung unterschieden werden kann, die Anzahl der ausländischen Studierenden im Vergleich zu den Ländern, in denen Einwanderer leichter die Staatsbürgerschaft erwerben können, überschätzt. Insofern ist bei allen bilateralen Vergleichen der Angaben zu ausländischen Studierenden Vorsicht angebracht, da sich die Länder in der Definition und Erfassung der bei ihnen studierenden Ausländer unterscheiden (s. Anhang 3 unter www.oecd.org/els/education/eag2002).

Die Daten über ausländische Studierende wurden von den Gastländern, nicht von den Ursprungsländern erhoben, und beziehen sich somit auf die Studierenden, die in das betreffende Land gekommen sind und nicht auf diejenigen, die aus diesem Land ins Ausland gehen. In diesem Indikator werden als aufnehmende Länder alle OECD-Länder mit Ausnahme von Luxemburg und der Slowakischen Republik sowie die folgenden Nicht-OECD-Länder erfasst: Argentinien, Chile, Indien, Indonesien, Jamaika, Jordanien, Malaysia, die Philippinen, die Russische Föderation, Tunesien und Uruguay. Studierende in OECD-Ländern, die der OECD keine Zahlen gemeldet haben und alle nicht genannten Nicht-OECD-Länder werden bei diesem Indikator nicht berücksichtigt. Daher ist bei allen Aussagen über die Zahl Studierender, die ein Auslandsstudium absolvieren, deren wahre Zahl zu niedrig angesetzt.

Die Zahl der ausländischen Studierenden wird mit der gleichen Methode ermittelt wie die Gesamtzahl der eingeschriebenen Studierenden. Üblicherweise wurden die in- und die ausländischen Studierenden an einem bestimmten Tag oder innerhalb eines bestimmten Zeitraums während des Jahres gezählt. Damit lässt sich der Anteil der ausländischen Studierenden messen, die in einem 
Bildungssystem eingeschrieben sind, aber die tatsächliche Zahl von Personen, die an einem internationalen Austausch teilgenommen haben, kann viel höher liegen, da viele Studierende für weniger als ein ganzes akademisches Jahr ins Ausland gehen oder an einem Austauschprogramm teilnehmen, das keine Einschreibung bei der ausländischen Bildungseinrichtung voraussetzt (einige Austauschprogramme zwischen Hochschulen sowie kurze Aufenthalte im Rahmen weiterführender Forschungsprogramme).

Tabellen C3.1, C3.2 und C3.3 zeigen den Anteil eingeschriebener ausländischer Studierender an der Gesamtzahl der eingeschriebenen Studierenden im Gastland bzw. im Heimatland. Die als Nenner verwendete Zahl für die Gesamtzahl der eingeschriebenen Studierenden schließt alle ausländischen Studierenden in diesem Land ein und schließt die Staatsangehörigen dieses Landes, die im Ausland studieren, aus. Der Anteil der im Ausland eingeschriebenen Studierenden laut Tabelle C3.2 zeigt nicht den Anteil aller Studierenden einer bestimmten Staatsangehörigkeit, die im Ausland studieren an, sondern ist lediglich Ausdruck der Zahl der Studierenden mit einer bestimmten Staatsangehörigkeit im Verhältnis zu allen inländischen und ausländischen eingeschriebenen Studierenden im Tertiärbereich, ohne die Studierenden, die die Staatsangehörigkeit dieses Landes besitzen, jedoch nicht in ihrem Heimatland studieren. 
Tabelle C3.1.

Austausch von Studierenden im Tertiärbereich (2000)

Eingeschriebene ausländische Studierende als Prozentsatz aller Studierenden (aus- und inländische), und Austausch von Studierenden als Prozentsatz der Gesamtstudierendenzahl

Bedeutung der Spalte 1: 2,2 Prozent aller Studierenden im Tertiärbereich in der Tschechischen Republik sind ausländische Studierende (aus OECD- und Nicht-OECD-Ländern). Bedeutung der Spalte 2: Ausländische Studierende aus anderen Ländern, die Angaben zu ausländischen Studierenden machen, stellen in der Tschechischen Republik 1,0 Prozent aller Studierenden im Tertiärbereich dar.

Bedeutung der Spalte 3: 1,2 Prozent aller Studierenden der Tschechischen Republik studieren in anderen Ländern, die Angaben zu ausländischen Studierenden machen. Spalte 4 enthält die Differenz zwischen Spalte 2 und Spalte 3.

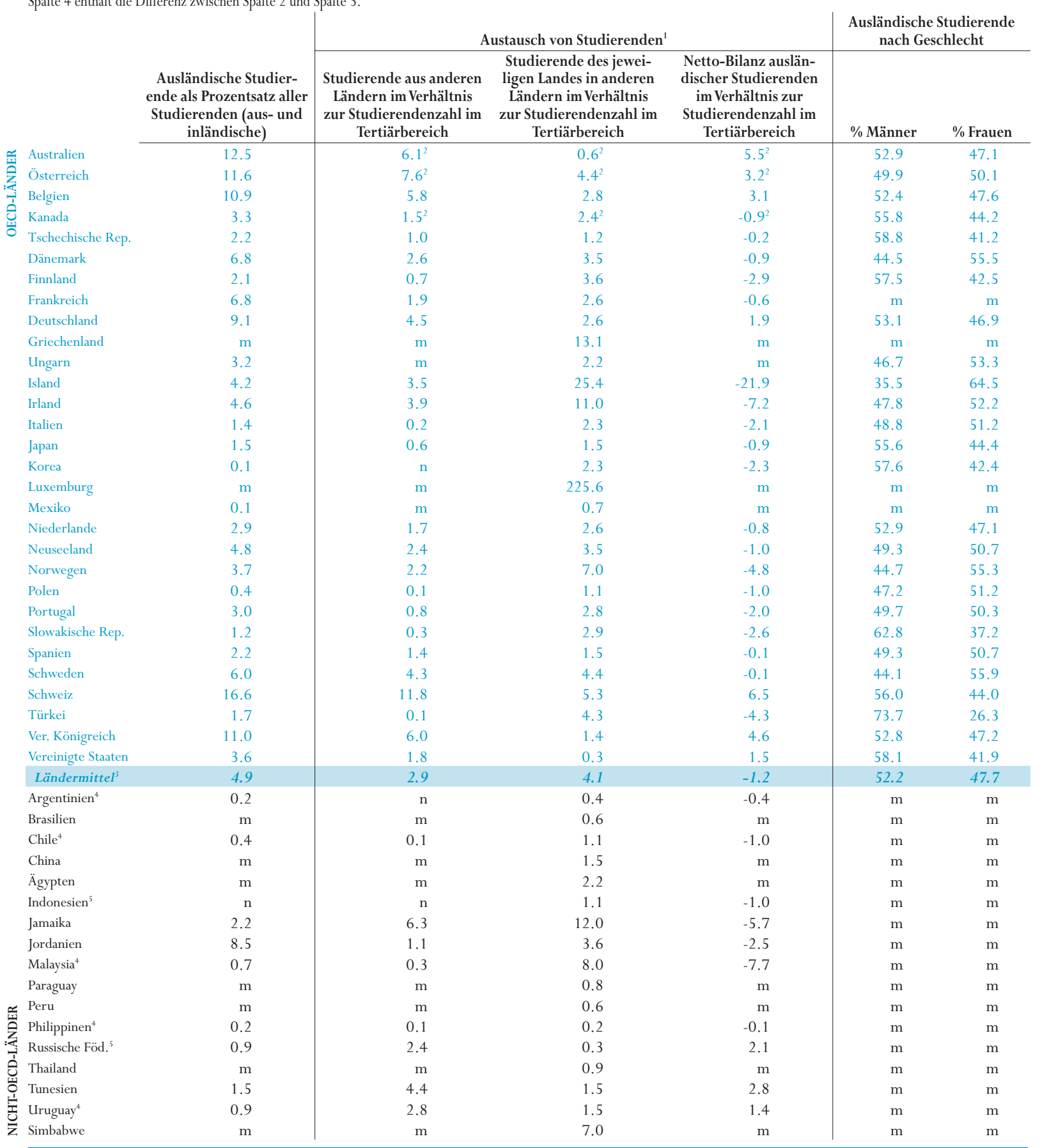

1. In der Summe sind nur OECD-Länder und Nicht-OECD-Länder, die Zugänge zu ihrem Bildungssystem angeben, berücksichtigt.

2. Nur Tertiärbereich A und weiterführende Forschungsprogramme.

3. Ländermittel ohne Luxemburg.

4. Referenzjahr 1999.

5. Referenzjahr 2001.

Quelle: OECD. Hinweise s. Anhang 3 (www.oecd.org/els/education/eag2002). 
Tabelle C3.2.

Anteil ausländischer Studierender im Tertiärbereich des Ziellandes (2000)

Anzahl der im Tertiärbereich eingeschriebenen ausländischen Studierenden als Prozentsatz der Studierenden im Zielland (basierend auf Pro-Kopf-Zahlen)

Die Tabelle zeigt den Anteil der Studierenden in jedem Land, die Staatsbürger eines anderen Landes sind.

Bedeutung der Spalte 2: 0,03 Prozent aller Studierenden im Tertiärbereich in Österreich sind belgische Staatsbürger, 0,02 Prozent sind kanadische Staatsbürger, etc.

Bedeutung der Zeile 1:0,03 Prozent aller Studierenden im Tertiärbereich in Kanada sind australische Staatsbürger, 0,04 Prozent aller Studierenden im Tertiärbereich in Irland sind australische Staatsbürger, etc.

\begin{tabular}{|c|c|c|c|c|c|c|c|c|c|c|c|c|c|c|c|c|c|c|c|c|c|c|c|c|c|c|}
\hline Ursprungsland & 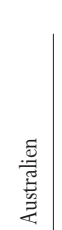 & 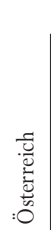 & 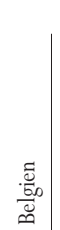 & 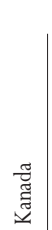 & 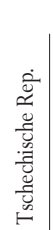 & 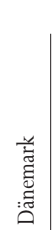 & 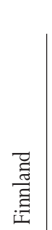 & 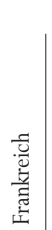 & 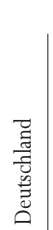 & $\begin{array}{l}\vec{E} \\
\vec{\Xi} \\
\underline{\Xi}\end{array}$ & 丞 & 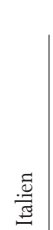 & 胥 & $\begin{array}{l}\mathbb{J} \\
\stackrel{0}{\square}\end{array}$ & 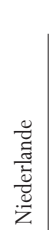 & 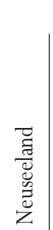 & 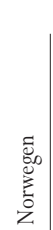 & $\frac{\Xi}{0}$ & $\begin{array}{l}\overline{\sigma_{0}} \\
\vec{D}_{0} \\
\text { 巳 }\end{array}$ & 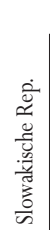 & 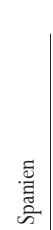 & 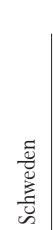 & 总 & $\frac{\overrightarrow{\mathbb{E}}}{\vdots}$ & 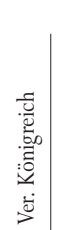 & 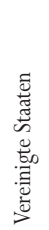 \\
\hline Australien & a & 0.01 & $\mathrm{n}$ & 0.03 & $\mathrm{n}$ & 0.02 & 0.01 & 0.01 & 0.01 & 0.01 & 0.04 & $\mathrm{n}$ & 0.01 & $\mathrm{n}$ & 0.01 & $\mathrm{n}$ & 0.01 & $\mathrm{n}$ & 0.01 & $\mathrm{n}$ & $\mathrm{n}$ & 0.05 & 0.03 & $\mathrm{n}$ & 0.06 & 0.02 \\
\hline Österreich & 0.01 & $\mathrm{a}$ & 0.01 & 0.01 & $\mathrm{n}$ & 0.02 & 0.01 & 0.02 & 0.32 & 0.05 & 0.03 & $\mathrm{n}$ & $\mathrm{n}$ & $\mathrm{n}$ & 0.02 & $\mathrm{n}$ & 0.02 & $\mathrm{n}$ & $\mathrm{n}$ & $\mathrm{n}$ & 0.03 & 0.10 & 0.46 & $\mathrm{n}$ & 0.06 & 0.01 \\
\hline Belgien & 0.01 & 0.03 & $\mathrm{a}$ & 0.01 & $\mathrm{n}$ & 0.01 & 0.01 & 0.10 & 0.05 & 0.01 & 0.04 & 0.01 & $\mathrm{n}$ & $\mathrm{n}$ & 0.28 & $\mathrm{n}$ & 0.01 & $\mathrm{n}$ & 0.02 & $\mathrm{n}$ & 0.07 & 0.05 & 0.17 & $\mathrm{n}$ & 0.12 & 0.01 \\
\hline Kanada & 0.13 & 0.02 & 0.02 & $\mathrm{a}$ & 0.01 & 0.02 & 0.03 & 0.05 & 0.02 & 0.08 & 0.08 & $\mathrm{n}$ & $\mathrm{n}$ & $\mathrm{n}$ & 0.01 & 0.04 & 0.02 & 0.01 & 0.07 & $\mathrm{n}$ & $\mathrm{n}$ & 0.08 & 0.11 & $\mathrm{n}$ & 0.15 & 0.16 \\
\hline Tschechische Rep. & 0.01 & 0.13 & 0.01 & $\mathrm{n}$ & $\mathrm{a}$ & $\mathrm{n}$ & 0.01 & 0.02 & 0.07 & 0.05 & $\mathrm{n}$ & $\mathrm{n}$ & $\mathrm{n}$ & $\mathrm{n}$ & 0.01 & $\mathrm{n}$ & 0.01 & 0.02 & $\mathrm{n}$ & 0.21 & 0.01 & 0.03 & 0.08 & $\mathrm{n}$ & 0.01 & 0.01 \\
\hline Dänemark & 0.02 & 0.03 & 0.01 & 0.01 & $\mathrm{n}$ & $\mathrm{a}$ & 0.02 & 0,01 & 0.03 & 0.44 & 0.01 & $\mathrm{n}$ & $\mathrm{n}$ & $\mathrm{n}$ & 0.01 & 0.02 & 0.40 & $\mathrm{n}$ & $\mathrm{n}$ & $\mathrm{n}$ & 0.02 & 0.25 & 0.06 & $\mathrm{n}$ & 0.09 & 0.01 \\
\hline Finnland & 0.01 & 0.07 & 0.02 & 0.01 & $\mathrm{n}$ & 0.06 & $\mathrm{a}$ & 0.02 & 0.05 & 0.36 & 0.05 & $\mathrm{n}$ & $\mathrm{n}$ & $\mathrm{n}$ & 0.01 & $\mathrm{n}$ & 0.11 & $\mathrm{n}$ & $\mathrm{n}$ & $\mathrm{n}$ & 0.02 & 0.97 & 0.05 & $\mathrm{n}$ & 0.13 & 0.01 \\
\hline Frankreich & 0.03 & 0.19 & 2.77 & 0.37 & $\mathrm{n}$ & 0.06 & 0.03 & $\mathrm{a}$ & 0.31 & 0.17 & 0.35 & 0.02 & $\mathrm{n}$ & $\mathrm{n}$ & 0.06 & 0.03 & 0.06 & $\mathrm{n}$ & 0.26 & $\mathrm{n}$ & 0.25 & 0.27 & 1.80 & $\mathrm{n}$ & 0.62 & 0.05 \\
\hline Deutschland & 0.13 & 2.25 & 0.15 & 0.06 & 0.01 & 0.29 & 0.08 & 0.27 & $\mathrm{a}$ & 0.42 & 0.30 & 0.04 & 0.01 & $\mathrm{n}$ & 0.47 & 0.14 & 0.21 & 0.01 & 0.10 & 0.01 & 0.21 & 0.54 & 3.51 & 0.01 & 0.67 & 0.07 \\
\hline Griechenland & 0.02 & 0.12 & 0.20 & 0.01 & 0.21 & 0.01 & 0.01 & 0.13 & 0.40 & $\mathrm{n}$ & 0.02 & 0.46 & $\mathrm{n}$ & $\mathrm{n}$ & 0.02 & $\mathrm{n}$ & 0.01 & $\mathrm{n}$ & $\mathrm{n}$ & 0.18 & 0.02 & 0.07 & 0.17 & 0.13 & 1.45 & 0.02 \\
\hline Ungarn & 0.01 & 0.42 & 0.03 & $\mathrm{n}$ & $\mathrm{n}$ & 0.01 & 0.03 & 0.02 & 0.13 & $\mathrm{n}$ & $\mathrm{n}$ & $\mathrm{n}$ & $\mathrm{n}$ & $\mathrm{n}$ & 0.01 & $\mathrm{n}$ & 0.01 & $\mathrm{n}$ & $\mathrm{n}$ & 0.02 & 0.01 & 0.06 & 0.10 & $\mathrm{n}$ & 0.02 & 0.01 \\
\hline Island & $\mathrm{n}$ & 0.01 & $\mathrm{n}$ & $\mathrm{n}$ & $\mathrm{n}$ & 0.37 & 0.01 & $\mathrm{n}$ & 0.01 & $\mathrm{a}$ & $\mathrm{n}$ & $\mathrm{n}$ & $\mathrm{n}$ & $\mathrm{n}$ & $\mathrm{n}$ & $\mathrm{n}$ & 0.13 & $\mathrm{n}$ & $\mathrm{n}$ & $\mathrm{n}$ & $\mathrm{n}$ & 0.10 & 0.01 & $\mathrm{n}$ & 0.01 & 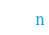 \\
\hline Irland & 0.04 & 0.02 & 0.01 & 0.01 & $\mathrm{n}$ & 0.02 & 0.01 & 0.03 & 0.03 & $\mathrm{n}$ & $\mathrm{a}$ & $\mathrm{n}$ & $\mathrm{n}$ & $\mathrm{n}$ & 0.01 & $\mathrm{n}$ & 0.01 & $\mathrm{n}$ & $\mathrm{n}$ & $\mathrm{n}$ & 0.02 & 0.03 & 0.03 & $\mathrm{n}$ & 0.71 & 0.01 \\
\hline Italien & 0.02 & 2.70 & 0.92 & 0.02 & $\mathrm{n}$ & 0.04 & 0.03 & 0.20 & 0.36 & 0.21 & 0.08 & $\mathrm{a}$ & $\mathrm{n}$ & $\mathrm{n}$ & 0.07 & $\mathrm{n}$ & 0.03 & $\mathrm{n}$ & 0.03 & $\mathrm{n}$ & 0.25 & 0.16 & 2.56 & $\mathrm{n}$ & 0.30 & 0.02 \\
\hline Japan & 0.26 & 0.12 & 0.05 & 0.12 & $\mathrm{n}$ & 0.02 & 0.03 & 0.07 & 0.10 & 0.05 & 0.02 & $\mathrm{n}$ & $\mathrm{a}$ & 0.02 & 0.01 & 0.40 & 0.02 & $\mathrm{n}$ & $\mathrm{n}$ & $\mathrm{n}$ & 0.01 & 0.04 & 0.10 & $\mathrm{n}$ & 0.30 & 0.33 \\
\hline Korea & 0.28 & 0.12 & 0.01 & 0.09 & 0.01 & $\mathrm{n}$ & 0.01 & 0.08 & 0.24 & $\mathrm{n}$ & $\mathrm{n}$ & $\mathrm{n}$ & 0.46 & a & $\mathrm{n}$ & 0.26 & $\mathrm{n}$ & $\mathrm{n}$ & $\mathrm{n}$ & $\mathrm{n}$ & 0.01 & 0.02 & 0.04 & $\mathrm{n}$ & 0.11 & 0.29 \\
\hline Luxemburg & $\mathrm{n}$ & 0.12 & 0.41 & $\mathrm{n}$ & $\mathrm{n}$ & $\mathrm{n}$ & $\mathrm{n}$ & 0.06 & 0.07 & $\mathrm{n}$ & 0.01 & $\mathrm{n}$ & $\mathrm{n}$ & $\mathrm{n}$ & $\mathrm{n}$ & $\mathrm{n}$ & $\mathrm{n}$ & $\mathrm{n}$ & 0.01 & $\mathrm{n}$ & $\mathrm{n}$ & $\mathrm{n}$ & 0.12 & $\mathrm{n}$ & 0.03 & 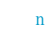 \\
\hline Mexiko & 0.01 & 0.02 & 0.02 & 0.06 & $\mathrm{n}$ & 0.01 & $\mathrm{n}$ & $\mathrm{n}$ & 0.02 & $\mathrm{n}$ & $\mathrm{n}$ & $\mathrm{n}$ & $\mathrm{n}$ & $\mathrm{n}$ & $\mathrm{n}$ & 0.01 & 0.01 & $\mathrm{n}$ & $\mathrm{n}$ & 0.00 & 0.08 & 0.01 & 0.05 & $\mathrm{n}$ & 0.06 & 0.07 \\
\hline Niederlande & 0.04 & 0.04 & 0.76 & 0.01 & $\mathrm{n}$ & 0.05 & 0.02 & 0.03 & 0.10 & 0.05 & 0.04 & $\mathrm{n}$ & $\mathrm{n}$ & $\mathrm{n}$ & $\mathrm{a}$ & 0.01 & 0.06 & $\mathrm{n}$ & 0.01 & $\mathrm{n}$ & 0.05 & 0.16 & 0.17 & $\mathrm{n}$ & 0.13 & .01 \\
\hline Neuseeland & 0.51 & $\mathrm{n}$ & $\mathrm{n}$ & 0.01 & $\mathrm{n}$ & 0.01 & $\mathrm{n}$ & $\mathrm{n}$ & $\mathrm{n}$ & 0.01 & $\mathrm{n}$ & $\mathrm{n}$ & $\mathrm{n}$ & $\mathrm{n}$ & $\mathrm{n}$ & $\mathrm{a}$ & $\mathrm{n}$ & $\mathrm{n}$ & $\mathrm{n}$ & $\mathrm{n}$ & $\mathrm{n}$ & 0.01 & 0.01 & $\mathrm{n}$ & 0.02 & 0.01 \\
\hline Norwegen & 0.20 & 0.03 & 0.01 & 0.01 & 0.02 & 0.68 & 0.02 & 0.02 & 0.05 & 0.29 & 0.07 & $\mathrm{n}$ & $\mathrm{n}$ & $\mathrm{n}$ & 0.02 & 0.06 & $\mathrm{a}$ & 0.02 & $\mathrm{n}$ & $\mathrm{n}$ & 0.02 & 0.35 & 0.08 & $\mathrm{n}$ & 0.20 & 0.02 \\
\hline Polen & 0.01 & 0.31 & 0.07 & 0.01 & 0.05 & 0.11 & 0.02 & 0.09 & 0.43 & 0.09 & 0.01 & 0.02 & $\mathrm{n}$ & $\mathrm{n}$ & 0.03 & $\mathrm{n}$ & 0.05 & $\mathrm{a}$ & 0.01 & 0.03 & 0.02 & 0.19 & 0.20 & $\mathrm{n}$ & 0.03 & 0.02 \\
\hline Portugal & 0.01 & 0.02 & 0.17 & 0.01 & $\mathrm{n}$ & 0.01 & 0.01 & 0.15 & 0.08 & 0.02 & 0.01 & $\mathrm{n}$ & $\mathrm{n}$ & $\mathrm{n}$ & 0.02 & $\mathrm{n}$ & 0.01 & $\mathrm{n}$ & $\mathrm{a}$ & $\mathrm{n}$ & 0.05 & 0.03 & 0.27 & $\mathrm{n}$ & 0.11 & 0.01 \\
\hline Slowakische Rep. & $\mathrm{n}$ & 0.34 & 0.01 & $\mathrm{n}$ & 0.70 & $\mathrm{n}$ & $\mathrm{n}$ & 0.01 & 0.00 & $\mathrm{n}$ & $\mathrm{n}$ & $\mathrm{n}$ & $\mathrm{n}$ & $\mathrm{n}$ & $\mathrm{n}$ & $\mathrm{n}$ & 0.01 & $\mathrm{n}$ & $\mathrm{n}$ & $\mathrm{a}$ & $\mathrm{n}$ & 0.01 & 0.06 & $\mathrm{n}$ & 0.01 & $\mathrm{n}$ \\
\hline Spanien & 0.01 & 0.15 & 0.40 & 0.01 & $\mathrm{n}$ & 0.04 & 0.02 & 0.19 & 0.27 & 0.16 & 0.14 & 0.01 & $\mathrm{n}$ & $\mathrm{n}$ & 0.10 & $\mathrm{n}$ & 0.03 & $\mathrm{n}$ & 0.10 & $\mathrm{n}$ & $\mathrm{a}$ & 0.18 & 0.96 & $\mathrm{n}$ & 0.37 & 0.03 \\
\hline Schweden & 0.11 & 0.10 & 0.02 & 0.02 & 0.02 & 0.31 & 0.20 & 0.05 & 0.04 & 0.39 & 0.04 & $\mathrm{n}$ & $\mathrm{n}$ & $\mathrm{n}$ & 0.02 & 0.07 & 0.47 & 0.01 & $\mathrm{n}$ & $\mathrm{n}$ & 0.02 & $\mathrm{a}$ & 0.14 & $\mathrm{n}$ & 0.20 & 0.03 \\
\hline Schweiz & 0.02 & 0.10 & 0.03 & 0.02 & $\mathrm{n}$ & 0.02 & 0.01 & 0.05 & 0.10 & 0.02 & 0.01 & 0.04 & $\mathrm{n}$ & $\mathrm{n}$ & 0.01 & 0.01 & 0.02 & $\mathrm{n}$ & 0.01 & $\mathrm{n}$ & 0.01 & 0.05 & $\mathrm{a}$ & $\mathrm{n}$ & 0.07 & 0.01 \\
\hline Türkei & 0.02 & 0.45 & 0.14 & 0.01 & $\mathrm{n}$ & 0.10 & 0.01 & 0.11 & 1.29 & 0.01 & $\mathrm{n}$ & $\mathrm{n}$ & $\mathrm{n}$ & $\mathrm{n}$ & 0.24 & $\mathrm{n}$ & 0.02 & $\mathrm{n}$ & $\mathrm{n}$ & $\mathrm{n}$ & $\mathrm{n}$ & 0.04 & 0.33 & $\mathrm{a}$ & 0.09 & 0.07 \\
\hline Ver, Königreich & 0.52 & 0.08 & 0.06 & 0.10 & 0.09 & 0.20 & 0.05 & 0.16 & 0.13 & 0.18 & 1.13 & 0.01 & 0.01 & $\mathrm{n}$ & 0.14 & 0.07 & 0.20 & $\mathrm{n}$ & 0.03 & $\mathrm{n}$ & 0.15 & 0.24 & 0.19 & 0.01 & $\mathrm{a}$ & 0.06 \\
\hline Vereinigte Staaten & 0.38 & 0.14 & 0.05 & 0.36 & 0.02 & 0.11 & 0.06 & 0.12 & 0.18 & 0.34 & 0.98 & 0.01 & 0.03 & 0.01 & 0.05 & 0.27 & 0.15 & 0.02 & 0.08 & $\mathrm{n}$ & 0.04 & 0.26 & 0.22 & $\mathrm{n}$ & 0.55 & a \\
\hline Argentinien & 0.01 & 0.01 & 0.01 & 0.01 & $\mathrm{n}$ & $\mathrm{n}$ & $\mathrm{n}$ & 0.02 & 0.02 & 0.01 & $\mathrm{n}$ & 0.01 & $\mathrm{n}$ & $\mathrm{n}$ & $\mathrm{n}$ & 0.01 & $\mathrm{n}$ & $\mathrm{n}$ & $\mathrm{n}$ & $\mathrm{n}$ & 0.08 & 0.01 & 0.05 & $\mathrm{n}$ & 0.02 & 0.02 \\
\hline Brasilien & 0.02 & 0.03 & 0.04 & 0.03 & $\mathrm{n}$ & 0.02 & 0.01 & 0.07 & 0.07 & $\mathrm{n}$ & $\mathrm{n}$ & 0.01 & 0.01 & $\mathrm{n}$ & 0.01 & 0.02 & 0.01 & $\mathrm{n}$ & 0.36 & $\mathrm{n}$ & 0.06 & 0.02 & 0.11 & $\mathrm{n}$ & 0.05 & 0.06 \\
\hline Chile & 0.02 & 0.01 & 0.03 & 0.01 & $\mathrm{n}$ & 0.01 & 0.01 & 0.02 & 0.02 & 0.01 & $\mathrm{n}$ & $\mathrm{n}$ & $\mathrm{n}$ & $\mathrm{n}$ & 0.01 & 0.01 & 0.04 & $\mathrm{n}$ & $\mathrm{n}$ & $\mathrm{n}$ & 0.04 & 0.06 & 0.04 & $\mathrm{n}$ & 0.01 & 0.01 \\
\hline China & 0.59 & 0.16 & 0.18 & 0.32 & $\mathrm{n}$ & 0.07 & 0.30 & 0.10 & 0.32 & 0.09 & 0.03 & $\mathrm{n}$ & 0.71 & 0.04 & 0.04 & 0.66 & 0.08 & $\mathrm{n}$ & 0.01 & $\mathrm{n}$ & 0.01 & 0.18 & 0.27 & 0.01 & 0.30 & 0.38 \\
\hline Ägypten & 0.01 & 0.08 & 0.01 & 0.01 & $\mathrm{n}$ & $\mathrm{n}$ & $\mathrm{n}$ & 0.03 & 0.05 & $\mathrm{n}$ & 0.01 & $\mathrm{n}$ & $\mathrm{n}$ & $\mathrm{n}$ & 0.01 & $\mathrm{n}$ & 0.01 & $\mathrm{n}$ & $\mathrm{n}$ & 0.02 & $\mathrm{n}$ & $\mathrm{n}$ & 0.03 & 0.01 & 0.05 & 0.01 \\
\hline Indien & 0.54 & 0.04 & 0.03 & 0.07 & 0.01 & 0.01 & 0.02 & 0.01 & 0.06 & 0.01 & 0.03 & $\mathrm{n}$ & $\mathrm{n}$ & $\mathrm{n}$ & 0.01 & 0.12 & 0.05 & $\mathrm{n}$ & 0.01 & $\mathrm{n}$ & $\mathrm{n}$ & 0.02 & 0.06 & $\mathrm{n}$ & 0.20 & 0.30 \\
\hline Indonesien & 1.18 & 0.02 & 0.02 & 0.03 & $\mathrm{n}$ & $\mathrm{n}$ & 0.01 & 0.01 & 0.10 & $\mathrm{n}$ & $\mathrm{n}$ & $\mathrm{n}$ & 0.03 & $\mathrm{n}$ & 0.08 & 0.21 & 0.01 & $\mathrm{n}$ & $\mathrm{n}$ & $\mathrm{n}$ & $\mathrm{n}$ & $\mathrm{n}$ & 0.02 & $\mathrm{n}$ & 0.05 & 0.08 \\
\hline Jamaika & $\mathrm{n}$ & $\mathrm{n}$ & $\mathrm{n}$ & 0.02 & $\mathrm{n}$ & $\mathrm{n}$ & $\mathrm{n}$ & $\mathrm{n}$ & $\mathrm{n}$ & $\mathrm{n}$ & $\mathrm{n}$ & $\mathrm{n}$ & $\mathrm{n}$ & $\mathrm{n}$ & $\mathrm{n}$ & $\mathrm{n}$ & $\mathrm{n}$ & $\mathrm{n}$ & $\mathrm{n}$ & $\mathrm{n}$ & $\mathrm{n}$ & $\mathrm{n}$ & $\mathrm{n}$ & $\mathrm{n}$ & 0.03 & 0.03 \\
\hline Jordanien & 0.01 & 0.03 & $\mathrm{n}$ & 0.01 & 0.01 & $\mathrm{n}$ & $\mathrm{n}$ & 0.01 & 0.05 & 0.01 & 0.01 & 0.01 & $\mathrm{n}$ & $\mathrm{n}$ & $\mathrm{n}$ & $\mathrm{n}$ & $\mathrm{n}$ & $\mathrm{n}$ & $\mathrm{n}$ & 0.02 & $\mathrm{n}$ & 0.01 & 0.01 & 0.03 & 0.04 & 0.01 \\
\hline Malaysia & 1.52 & $\mathrm{n}$ & $\mathrm{n}$ & 0.06 & $\mathrm{n}$ & $\mathrm{n}$ & $\mathrm{n}$ & $\mathrm{n}$ & 0.01 & $\mathrm{n}$ & 0.39 & $\mathrm{n}$ & 0.05 & $\mathrm{n}$ & $\mathrm{n}$ & 0.69 & $\mathrm{n}$ & $\mathrm{n}$ & $\mathrm{n}$ & $\mathrm{n}$ & $\mathrm{n}$ & 0.01 & 0.01 & $\mathrm{n}$ & 0.51 & 0.06 \\
\hline Paraguay & $\mathrm{n}$ & $\mathrm{n}$ & $\mathrm{n}$ & $\mathrm{n}$ & $\mathrm{n}$ & $\mathrm{n}$ & $\mathrm{n}$ & $\mathrm{n}$ & $\mathrm{n}$ & $\mathrm{n}$ & $\mathrm{n}$ & $\mathrm{n}$ & $\mathrm{n}$ & $\mathrm{n}$ & $\mathrm{n}$ & $\mathrm{n}$ & $\mathrm{n}$ & $\mathrm{n}$ & $\mathrm{n}$ & $\mathrm{n}$ & $\mathrm{n}$ & $\mathrm{n}$ & $\mathrm{n}$ & $\mathrm{n}$ & $\mathrm{n}$ & $\mathrm{n}$ \\
\hline Peru & 0.01 & 0.03 & 0.02 & 0.01 & $\mathrm{n}$ & 0.01 & 0.01 & 0.02 & 0.04 & $\mathrm{n}$ & $\mathrm{n}$ & 0.01 & $\mathrm{n}$ & $\mathrm{n}$ & $\mathrm{n}$ & $\mathrm{n}$ & 0.01 & $\mathrm{n}$ & $\mathrm{n}$ & $\mathrm{n}$ & 0.06 & 0.02 & 0.10 & $\mathrm{n}$ & 0.01 & 0.02 \\
\hline Philippinen & 0.08 & $\mathrm{n}$ & 0.02 & 0.01 & $\mathrm{n}$ & 0.01 & 0.01 & $\mathrm{n}$ & 0.01 & $\mathrm{n}$ & $\mathrm{n}$ & $\mathrm{n}$ & 0.01 & $\mathrm{n}$ & $\mathrm{n}$ & 0.03 & 0.01 & $\mathrm{n}$ & $\mathrm{n}$ & $\mathrm{n}$ & 0.01 & 0.01 & 0.01 & $\mathrm{n}$ & 0.01 & 0.02 \\
\hline Russische Föd. & 0.02 & 0.11 & 0.04 & 0.02 & 0.04 & 0.06 & 0.24 & 0.07 & 0.32 & 0.10 & 0.02 & 0.01 & 0.01 & $\mathrm{n}$ & 0.04 & 0.01 & 0.18 & 0.02 & $\mathrm{n}$ & 0.02 & 0.01 & 0.13 & 0.21 & 0.10 & 0.05 & 0.05 \\
\hline Thailand & 0.32 & 0.01 & 0.01 & 0.02 & $\mathrm{n}$ & 0.01 & $\mathrm{n}$ & 0.01 & 0.02 & 0.01 & $\mathrm{n}$ & $\mathrm{n}$ & 0.03 & $\mathrm{n}$ & $\mathrm{n}$ & 0.19 & 0.01 & $\mathrm{n}$ & $\mathrm{n}$ & $\mathrm{n}$ & $\mathrm{n}$ & 0.02 & 0.01 & $\mathrm{n}$ & 0.13 & 0.08 \\
\hline Tunesien & $\mathrm{n}$ & 0.02 & 0.08 & 0.05 & $\mathrm{n}$ & 0.01 & $\mathrm{n}$ & $\mathrm{n}$ & 0.05 & $\mathrm{n}$ & $\mathrm{n}$ & $\mathrm{n}$ & $\mathrm{n}$ & $\mathrm{n}$ & $\mathrm{n}$ & $\mathrm{n}$ & $\mathrm{n}$ & $\mathrm{n}$ & $\mathrm{n}$ & $\mathrm{n}$ & $\mathrm{n}$ & $\mathrm{n}$ & 0.01 & $\mathrm{n}$ & $\mathrm{n}$ & n \\
\hline Uruguay & $\mathrm{n}$ & $\mathrm{n}$ & $\mathrm{n}$ & $\mathrm{n}$ & $\mathrm{n}$ & $\mathrm{n}$ & $\mathrm{n}$ & $\mathrm{n}$ & $\mathrm{n}$ & $\mathrm{n}$ & $\mathrm{n}$ & $\mathrm{n}$ & $\mathrm{n}$ & $\mathrm{n}$ & $\mathrm{n}$ & $\mathrm{n}$ & $\mathrm{n}$ & $\mathrm{n}$ & $\mathrm{n}$ & $\mathrm{n}$ & 0.01 & $\mathrm{n}$ & 0.01 & $\mathrm{n}$ & $\mathrm{n}$ & $\mathrm{n}$ \\
\hline Simbabwe & 0.03 & $\mathrm{n}$ & $\mathrm{n}$ & 0.01 & $\mathrm{n}$ & $\mathrm{n}$ & $\mathrm{n}$ & $\mathrm{n}$ & $\mathrm{n}$ & $\mathrm{n}$ & $\mathrm{n}$ & $\mathrm{n}$ & $\mathrm{n}$ & $\mathrm{n}$ & $\mathrm{n}$ & 0.01 & 0.01 & $\mathrm{n}$ & $\mathrm{n}$ & $\mathrm{n}$ & $\mathrm{n}$ & $\mathrm{n}$ & $\mathrm{n}$ & $\mathrm{n}$ & 0.09 & 0.01 \\
\hline Afrika & 0.37 & 0.38 & 3.32 & 0.48 & 0.08 & 0.17 & 0.24 & 3.36 & 0.89 & 0.02 & 0.19 & 0.10 & 0.02 & $n$ & 0.49 & 0.07 & 0.33 & 0.02 & 1.63 & 0.12 & 0.26 & 0.20 & 1.06 & 0.04 & 0.82 & 0.21 \\
\hline Asien & 8.03 & 1.44 & 0.71 & 1.18 & 0.27 & 0.52 & 0.52 & 0.87 & 3.12 & 0.30 & 0.79 & 0.12 & 1.37 & 0.09 & 0.60 & 3.24 & 0.53 & 0.07 & 0.04 & 0.23 & 0.07 & 0.67 & 1.26 & 1.18 & 3.49 & 2.21 \\
\hline Europa & 1.32 & 9.41 & 6.33 & 0.78 & 1.29 & 2.86 & 1.14 & 2.04 & 4.53 & 3.32 & 2.41 & 0.91 & 0.05 & 0.01 & 1.49 & 0.44 & 2.51 & 0.26 & 0.62 & 0.79 & 1.29 & 4.50 & 12.55 & 0.51 & 5.54 & 0.54 \\
\hline Nordamerika & 0.52 & 0.21 & 0.13 & 0.56 & 0.03 & 0.14 & 0.10 & 0.27 & 0.26 & 0.46 & 1.09 & 0.02 & 0.04 & 0.01 & 0.06 & 0.33 & 0.20 & 0.02 & 0.16 & 0.01 & 0.20 & 0.38 & 0.44 & $n$ & 0.89 & 0.38 \\
\hline Ozeanien & 0.72 & 0.01 & $n$ & 0.04 & $n$ & 0.02 & 0.01 & 0.01 & 0.02 & 0.02 & 0.04 & $n$ & 0.01 & $n$ & 0.01 & 0.65 & 0.01 & $m$ & 0.01 & $n$ & $n$ & 0.05 & 0.03 & $n$ & 0.09 & 0.03 \\
\hline Südamerika & 0.10 & 0.12 & 0.18 & 0.11 & 0.03 & 0.06 & 0.02 & 0.20 & 0.20 & 0.04 & 0.01 & 0.04 & 0.02 & $n$ & 0.21 & 0.05 & 0.08 & $n$ & 0.49 & 0.01 & 0.40 & 0.15 & 0.51 & $n$ & 0.14 & 0.22 \\
\hline Nicht spezifiziert & 1.46 & 0.06 & 0.23 & 0.13 & 0.46 & 3.01 & 0.03 & 0.05 & 0.09 & 0.01 & 0.09 & 0.21 & $n$ & $n$ & 0.01 & $a$ & 0.90 & 0.01 & 0.04 & $a$ & $a$ & 1.41 & 0.72 & $n$ & 0.04 & $n$ \\
\hline Alle Länder & 12.51 & 11.63 & 10.91 & 3.28 & 2.25 & 6.80 & 2.06 & 6.80 & 9.10 & 4.17 & 4.62 & 1.41 & 1.50 & 0.11 & 2.87 & 4.77 & 3.66 & 0.39 & 2.99 & 1.16 & 2.22 & 6.00 & $\mid 16.58$ & 1.74 & 11.01 & 3.60 \\
\hline
\end{tabular}

Quelle: OECD. Hinweise s. Anhang 3 (www.oecd.org/els/education/eag2002). 
Tabelle C3.3.

Anteil der Studierenden eines Landes, die in einem anderen Land studieren (2000)

Anzahl der in anderen Ländern eingeschriebenen Studierenden als Prozentsatz der Studierenden im Ursprungsland (basierend auf Pro-Kopf-Zahlen)

Die Tabelle zeigt den Anteil der Studierenden jedes Landes, die in einem anderen Land studieren.

Bedeutung der Spalte 1:0,06 Prozent der japanischen Studierenden im Tertiärbereich studieren in Australien, 0,08 Prozent der koreanischen Studierenden studieren in Australien, etc.

Bedeutung der Zeile 1: 0,05 Prozent der australischen Studierenden studieren in Kanada, 0,03 Prozent der australischen Studierenden studieren in Deutschland, etc.

Zielland

Quelle: OECD. Hinweise s. Anhang 3 (www.oecd.org/els/education/eag2002). 


\section{BETEILIGUNG DER ERWACHSENENBEVÖLKERUNG AN FORT- UND WEITERBILDUNG}

- In der Hälfte der Länder, die Daten zur Verfügung gestellt haben, nahmen über 40 Prozent der Erwachsenenbevölkerung im Zeitraum von zwölf Monaten an einer Fort- oderWeiterbildungsmaßnahme teil.

- Allerdings schwanken Häufigkeit und Intensität der Fort- und Weiterbildungsmaßnahmen stark zwischen den einzelnen OECD-Ländern. Die Beteiligungsquoten liegen zwischen maximal 18 Prozent in Polen, Portugal und Ungarn und über 50 Prozent in Dänemark, Finnland, Schweden und den Vereinigten Staaten.

- In 11 von 19 OECD-Ländern ist die Wahrscheinlichkeit der Teilnahme von Erwachsenen mit einem tertiären Abschluss an berufsbezogener Fort- und Weiterbildung zwei bis dreimal so hoch wie von Erwachsenen ohne einen Abschluss des Sekundarbereich II.

\section{Abbildung C4.1.}

Beteiligungsquote an Fort- und Weiterbildung während eines Jahres für 25- bis 64-Jährige, nach Geschlecht und Art der Fort- und Weiterbildung

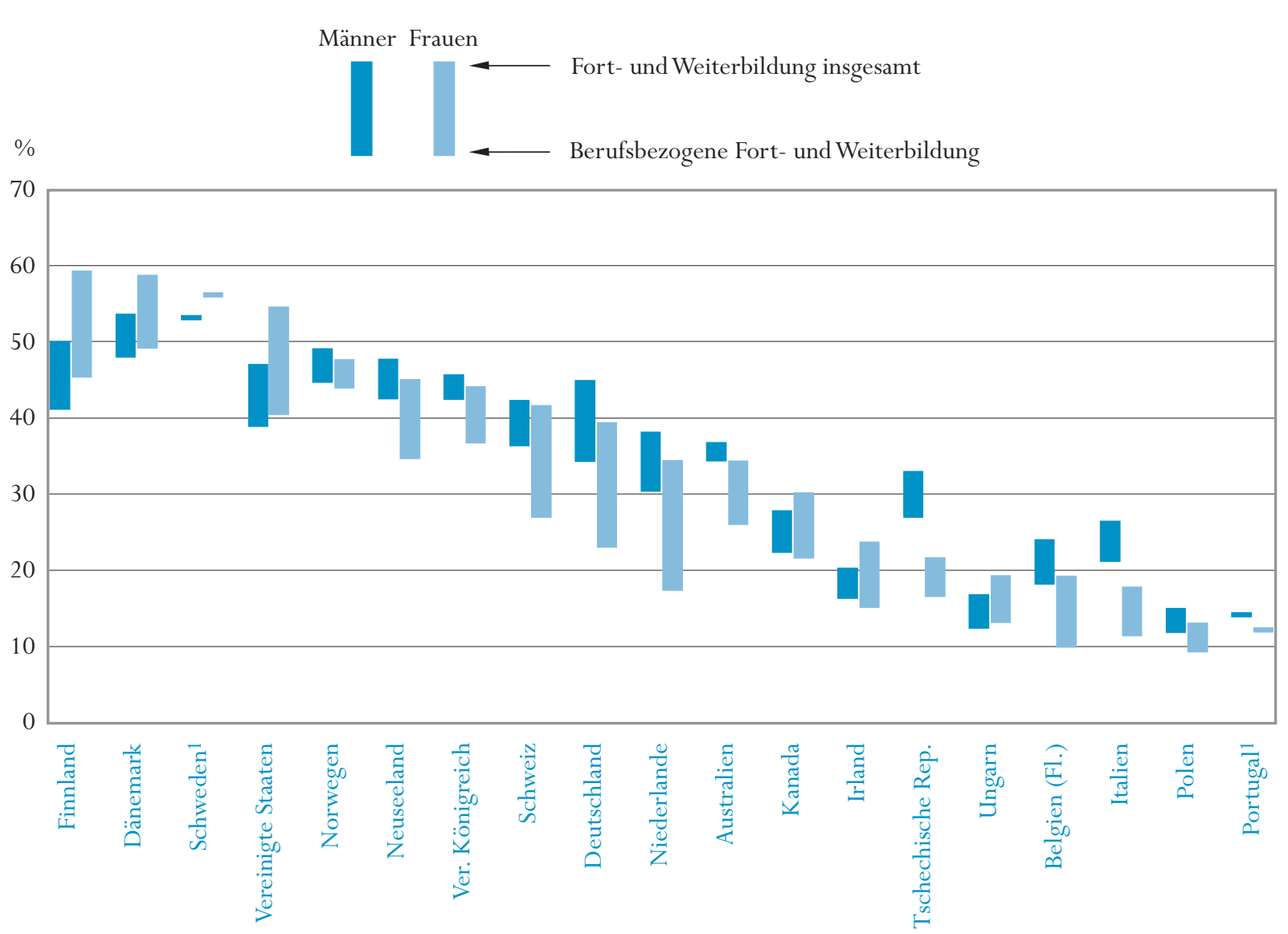

1. Daten beziehen sich auf Fort- und Weiterbildung insgesamt.

Anordnung der Länder in absteigender Reihenfolge der Beteiligung von Frauen an Fort- undWeiterbildung insgesamt.

Quelle: OECD. Tabelle C4.1. Hinweise s. Anhang 3 (www.oecd.org/els/education/eag2002). 
In diesem Indikator werden bestehende Erkenntnisse zur Fortund Weiterbildung

Erwachsener zusammengeführt.

Was dieser Indikator aufzeigt...

... und was nicht.

Die Beteiligungsquoten an berufsbezogenen

Fort- und Weiterbildungsmaßnahmen liegen durchschnittlich

\section{Politischer Hintergrund}

Da im heutigen Wirtschaftsgeschehen eine qualifizierte Erwerbsbevölkerung Voraussetzung für den Erfolg ist, stellt die Fort- und Weiterbildung wohl das beste Mittel zur Erhaltung und Steigerung der Kenntnisse und Fähigkeiten der jetzigen Erwerbsbevölkerung dar. Angesichts des technischen Wandels, neuer Arbeitsmethoden und Märkte fordern die Politiker vieler Länder die Unternehmen zu verstärkten Investitionen in die Fortbildung auf und setzen sich für eine umfassendere berufsbezogene Weiterbildung für Erwachsene ein.

Während man relativ gute Kenntnisse über die Bemühungen von Regierungen und Einzelnen zur Förderung des Lernens innerhalb der formellen Bildungseinrichtungen hat, weiß man wesentlich weniger über das Ausmaß, in dem Lernen am Arbeitsplatz oder anderswo außerhalb des formellen Bildungswesens sowie nach Abschluss der Erstausbildung erfolgt.

\section{Ergebnisse und Erläuterungen}

Frühere Ausgaben von Bildung auf einen Blick haben innerhalb der OECDLänder relativ einheitliche Charakteristika der Beteiligung von Erwachsenen an Fort- und Weiterbildung aufgezeigt. So wenden beispielsweise jüngere Erwerbstätige im Durchschnitt mehr Stunden für Weiterbildung auf als ältere, Beschäftigte im Dienstleistungssektor erhalten durchschnittlich mehr Weiterbildung als in der Fertigungsindustrie und Beschäftigte in großen Unternehmen oder im öffentlichen Sektor erhalten im Durchschnitt mehr Stunden an Weiterbildung als Mitarbeiter in kleinen Unternehmen.

Dieser Indikator versucht dieses Bild zu erweitern, indem er Daten zu Häufigkeit und Grad der Beteiligung Erwachsener sowohl an berufsbezogenen als auch an anders ausgerichteten Maßnahmen der Fort- und Weiterbildung in Beziehung setzt zu den früheren Bildungserfahrungen des Einzelnen während der Erstausbildung.

Zu den durch diesen Indikator abgedeckten Fort- undWeiterbildungsmaßnahmen gehören Kurse, Privatunterricht, Fernkurse, Workshops, 'on-the-job-training', Kurse zur beruflichen Bildung, Kunst-, Handwerks- und Freizeitkurse bzw. jegliche andere organisierte Schulung oder Bildungsmaßnahme.

Dieser Indikator erfasst keine informellen Lernaktivitäten wie ,learning on the job’ oder andere Formen selbstorganisierten Lernens.

\section{Teilnahme an berufsbezogenen Fort- und Weiterbildungsmaßnahmen im Verhältnis zu Fort- und Weiterbildungsmaßnahmen insgesamt}

Die Beteiligungsquoten an berufsbezogenen Fort- und Weiterbildungsmaßnahmen liegen durchschnittlich 8 Prozentpunkte niedriger als die für Fort- und Weiterbildungsmaßnahmen insgesamt. Mit 10 Prozentpunkten ist der Unterschied bei den Frauen, deren Erwerbsbeteiligung normalerweise unter der der Männer liegt, größer. Der Anteil der berufsbezogenen Fort- und 
Weiterbildungsmaßnahmen ist in Dänemark, Norwegen und dem Vereinigten Königreich besonders hoch.

Die Beteiligung von Frauen an Fort- und Weiterbildungsmaßnahmen scheint auf einem sehr ähnlichen Niveau zu liegen wie die der Männer. In den vier OECD-Ländern mit den höchsten Gesamtbeteiligungsquoten ist sie für Frauen sogar noch größer. In Deutschland, Italien, den Niederlanden, der Schweiz und der Tschechischen Republik zeigt sich ein erheblicher Abstand zu Gunsten der Männer, selbst bei der berufsbezogenen Fort- und Weiterbildung (Abb. C4.1).

In der Hälfte der Länder, für die Daten vorliegen, haben über 40 Prozent der Erwachsenenbevölkerung innerhalb eines Zeitraums von 12 Monaten an irgendeiner Form von Fort- und Weiterbildungsmaßnahme teilgenommen. Allerdings schwanken Häufigkeit und Intensität der Fort- und Weiterbildungsmaßnahmen stark zwischen den einzelnen OECD-Ländern. Obwohl ein internationaler Vergleich schwierig ist, gibt es Anzeichen dafür, dass die Beteiligung an formeller Fort- und Weiterbildung in den nordischen Ländern sehr viel höher ist als in den südlichen oder osteuropäischen Ländern. Die Beteiligungsquoten Erwachsener an Fort- und Weiterbildungsmaßnahmen liegen zwischen maximal 18 Prozent in Polen, Portugal und Ungarn und über 50 Prozent in Dänemark, Finnland, Schweden und den Vereinigten Staaten.

\section{Beteiligungsquoten nach Bildungsstand}

Weiterbildungsmaßnahmen verstärken in der Regel noch die Unterschiede in den Kenntnissen und Fähigkeiten, die aus der ungleichen Beteiligung an der Erstausbildung resultieren. Die Beteiligungsquoten sowohl bei berufsbezogener Fort- und Weiterbildung als auch bei der Fort- und Weiterbildung insgesamt (Tabelle C4.1) nehmen mit zunehmendem Bildungsstand zu. In 11 der 19 OECD-Länder, aus denen Daten vorliegen, ist es für Erwachsene mit einem tertiären Abschluss zwei- bis dreimal so wahrscheinlich, an berufsbezogener Fort- und Weiterbildung teilzunehmen als für diejenigen, die über keinen Abschluss im Sekundarbereich II verfügen. Dieser relative Vorteil scheint in den OECD-Ländern, in denen Fort- und Weiterbildungsmaßnahmen relativ selten sind, noch wesentlich ausgeprägter zu sein, hier ist die Wahrscheinlichkeit vierbis achtmal so hoch. Mit anderen Worten ist es den OECD-Ländern, in denen Fort- und Weiterbildung relativ häufig ist, besser gelungen, die Teilnahme von Personen verschiedener Bildungsniveaus sicherzustellen.

Der positive Zusammenhang zwischen Erstausbildung und Beteiligung an Fort- und Weiterbildung bleibt auch dann bestehen, wenn man andere Merkmale, die die Beteiligung an Weiterbildungsmaßnahmen beeinflussen, kontrolliert. Beschäftigte erhalten tendenziell in OECD-Ländern mit einem insgesamt höherem Bildungsstand mehr Weiterbildung, ebenso wie in OECDLändern, die einen größeren Anteil ihres BIP für Forschung und Entwicklung aufwenden oder die eine starke Handelsposition in den High-Tech-Branchen innehaben. Dies deutet darauf hin, dass sich Erst(aus-)bildung und Fort- und
8 Prozentpunkte niedriger als die für Fort-und Weiterbildungsmafnahmen insgesamt.

In der Hälfte der Länder, für die Daten vorliegen, haben über 40 Prozent der Erwachsenenbevölkerung innerhalb eines Zeitraums von 12 Monaten an irgendeiner Form von Fort- und Weiterbildungsmajsnahme teilgenommen.

In 11 der 19 OECDLänder ist die Wahrscheinlichkeit der Teilnahme von Erwachsenen mit einem tertiären Abschluss an berufsbezogener Fortund Weiterbildung zwei bis dreimal so hoch wie von Erwachsenen ohne einen Abschluss des sekundarbereich II,...

... so dass der Bildungsstand in Kombination mit anderen Einflussfaktoren dazu beträgt, dass Weiterbildung für Erwachsene innerhalb der größten Risikogruppe am wenigsten verbreitet ist. 


\section{Abbildung C4.2.}

Beteiligungsquote an Fort- und Weiterbildung 25 bis 64-Jähriger nach Bildungsstand (2001)

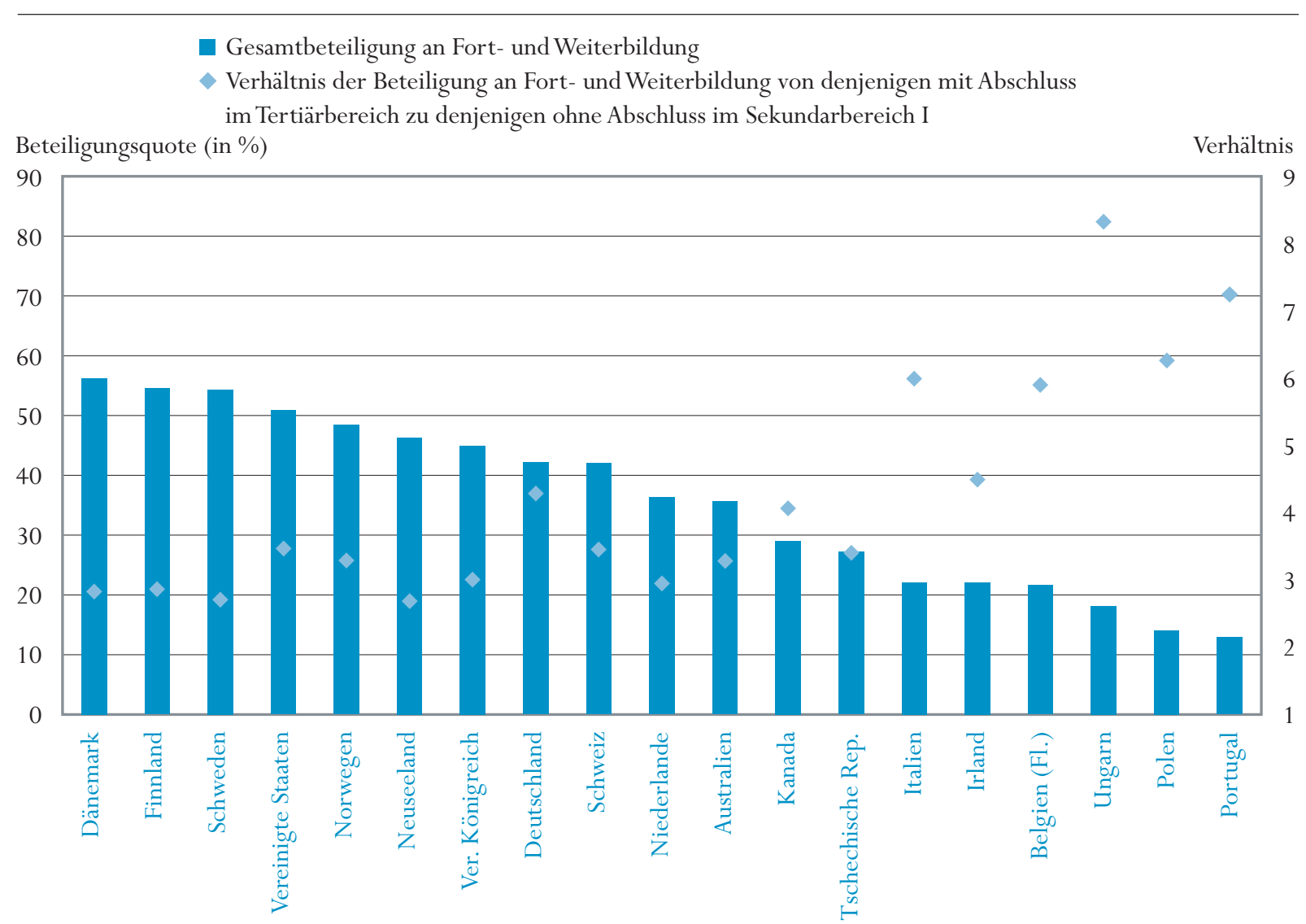

Anordnung der Länder in absteigender Reihenfolge der Gesamtbeteiligung an Fort- undWeiterbildung.

Quelle: OECD. Tabelle C4.1. Hinweise s. Anhang 3 (www.oecd.org/els/education/eag2002).

Frauen mit einem niedrigeren

Bildungsstand erhalten

im Vergleich zu

Männern meist weniger berufsbezogene Fortund Weiterbildung, ...

... doch ist dieser geschlechtsspezifische Unterschied bei denjenigen mit einem Abschluss des Sekundarbereich I/ bzw. einem Abschluss im
Weiterbildung gegenseitig verstärken und dass der Bildungsstand zusammen mit anderen Einflussfaktoren dazu beiträgt, dass Weiterbildung für Erwachsene unter denjenigen mit dem größten Bedarf am wenigsten verbreitet ist (Abb. C4.2).

Im Durchschnitt haben nur 12 Prozent der Frauen mit einem Abschluss unterhalb des Sekundarbereich II im Verlauf eines Jahres an irgendeiner berufsbezogener Fort- und Weiterbildungsmaßnahme teilgenommen, wohingegen dies bei 17 Prozent der Männer der Fall ist.

Bei Absolventen des Tertiärbereichs sind diese geschlechtsspezifischen Unterschiede bei den Beteiligungsquoten weniger stark ausgeprägt. So beträgt beispielsweise bei den 25- bis 64-Jährigen mit einem Abschluss des Sekundarbereich II die Beteiligungsquote der Frauen bei berufsbezogener Fort- und Weiterbildung im Durchschnitt 28 Prozent, während sie bei den Männern 31 Prozent beträgt. Bei einem tertiären Abschluss beläuft sich die 
durchschnittliche Beteiligungsquote der Frauen auf 45 Prozent und die der Männer auf 46 Prozent (Tabelle C4.1).

\section{Definitionen und angewandte Methodik}

Für diesen Indikator wurden vergleichbare Daten zu Fort- und Weiterbildung aus nationalen Erhebungen in sieben OECD-Ländern zusammengetragen. Alle diese Erhebungen umfassten den gleichen Referenzzeitraum von 12 Monaten. Der Stichprobenumfang reichte bei diesen Erhebungen von 5.000 bis zu 40.000 Befragten. Für die Datenerhebung wurden die Teilnehmer mittels persönlicher Interviews oder per Telefon befragt. Erfasst wurden unter dem Begriff der berufsbezogenen Fort- und Weiterbildung „alle Maßnahmen, die die Befragten als berufs- oder karrierebezogen bezeichneten”. Für diesen Indikator wurden die informellen Formen der Weiterbildung nicht berücksichtigt. ( $\mathrm{Zu}$ den Quellen der nationalen Haushaltsuntersuchungen zur Fort- und Weiterbildung Erwachsener s. Anhang 3 unter www.oecd.org/els/education/eag2002).

Dort, wo keine vergleichbaren Daten aus neueren nationalen Erhebungen vorlagen, wurden die Daten aus der zwischen 1994 und 1998 von Statistics Canada und der OECD durchgeführten IALS-Studie eingesetzt. Der Hintergrund-Fragebogen der Studie erfasste jede Beteiligung an Fort- und Weiterbildung während der letzten 12 Monate vor der Umfrage. Es wurde gefragt: „Haben Sie während der vergangenen 12 Monate an einer Schulung oder Ausbildung teilgenommen, einschließlich Kursen, Privatunterricht, Fernkursen, Workshops, 'on-the-job-training', Kursen zur beruflichen Bildung, Kunst-, Handwerks- und Freizeitkursen bzw. irgend einer anderen Schulung oder Ausbildung?“ In einer weiteren Frage wurde unterschieden zwischen Maßnahmen, an denen „aus karriere- oder berufsbezogenen Gründen“ teilgenommen wurde (in diesem Indikator als „berufsbezogene“Weiterbildung dargestellt), Maßnahmen, an denen „aus persönlichem Interesse“ teilgenommen wurde und Maßnahmen, an denen „aus anderen Gründen“ teilgenommen wurde.
Tertiärbereich weniger stark ausgeprägt.

Die Daten basieren auf nationalen Erhebungen zur Fort-und Weiterbildung der Erwachsenenbevölkerung, ...

... sowie auf der zwischen 1994 und 1998 von Statistics Canada und der OECD durchgeführten IALSErhebung (Adult Literacy Survey). 
Tabelle C4.1.

Beteiligungsquote an Fort- und Weiterbildung während eines Jahres für 25- bis 64-Jährige, nach Bildungsstand, Art der Fort- und Weiterbildung und Geschlecht

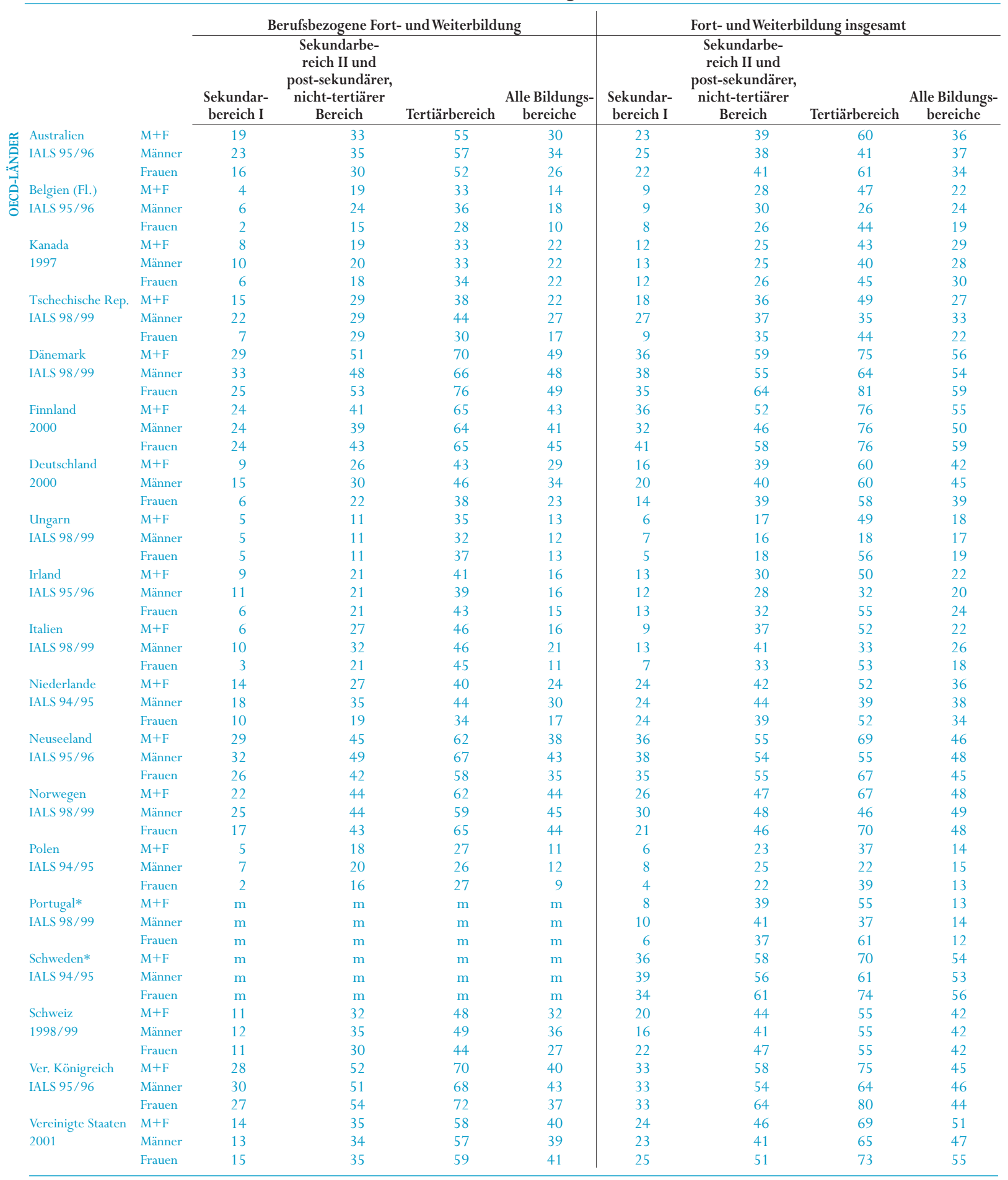

* Hinweise s. Anhang 3 (www.oecd.org/els/education/eag2002).

Quelle: International Adult Literacy Survey (IALS) 1994-1998 und nationale Haushaltserhebungen zur Fort- und Weiterbildung von Erwachsenen (Einzelheiten s. Anhang 3 unter www.oecd.org/els/education/eag2002). 


\section{BILDUNGS- UND BESCHÄFTIGUNGSSTATUS JUNGER MENSCHEN}

- In den meisten OECD-Ländern beträgt der Anteil der 20- bis 24-Jährigen, die sich nicht in Ausbildung befinden, 50 bis 70 Prozent.

- In einigen Ländern erfolgen Bildung und Arbeit überwiegend nacheinander, während sie in anderen parallel stattfinden. Duale Ausbildungsgänge, in europäischen Ländern relativ weit verbreitet, bieten eine kohärente berufliche Ausbildung, die zu anerkannten Berufsabschlüssen führt.

- In einigen Ländern kombinieren auch viele junge Menschen eine bezahlte Tätigkeit außerhalb der Unterrichtszeit mit ihrer Ausbildung, während in wieder anderen Ländern Erstausbildung und bezahlte Arbeit selten kombiniert werden.

\section{Abbildung C5.1.}

Prozentsatz 20- bis 24-Jähriger, die sich in Ausbildung und nicht in Ausbildung befinden, nach Beschäftigungsstatus (2001)

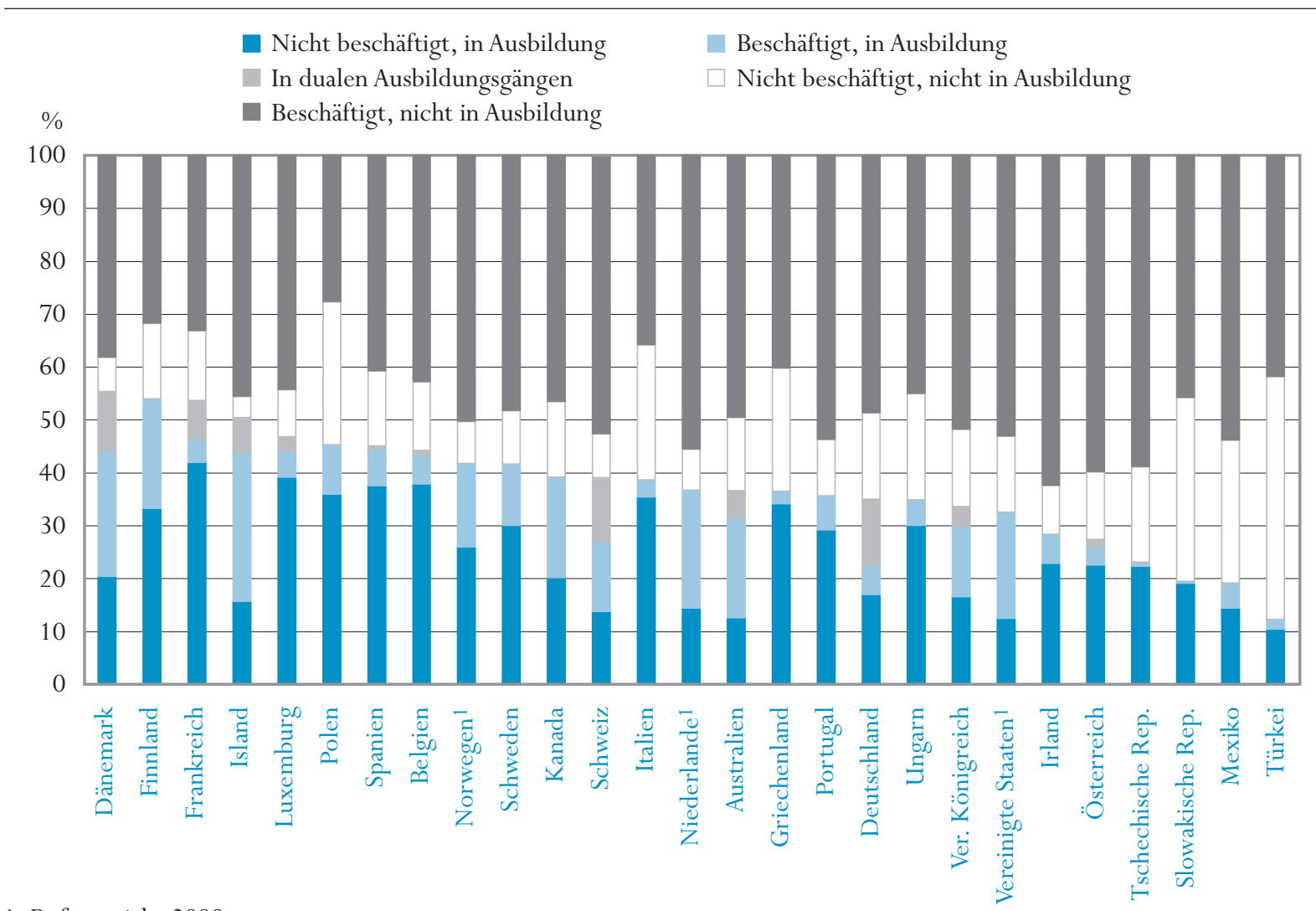

1. Referenzjahr 2000.

Anordnung der Länder in absteigender Reihenfolge des Prozentsatzes 20- bis 24-Jähriger in Ausbildung. Quelle: OECD. Tabelle C5.1. Hinweise s. Anhang 3 (www.oecd.org/els/education/eag2002). 
Dieser Indikator untersucht den

Bildungs- und Beschäftigungsstatus junger Frauen und Männer.

In einigen $O E C D$ -

Ländern sind duale Ausbildungsgänge und andere Kombinationsmöglichkeiten von Arbeit und Ausbildung weit verbreitet, in anderen hingegen selten.

\section{Politischer Hintergrund}

Alle OECD-Länder erleben einen rapiden gesellschaftlichen und wirtschaftlichen Umbruch, der die Unsicherheit des Übergang ins Erwerbsleben erhöht. In einigen OECD-Ländern erfolgen Bildung und Arbeit überwiegend nacheinander, während sie in anderen parallel stattfinden können. Es kann entscheidende Auswirkung auf den Erfolg des Übergangs haben, wie Ausbildung und Arbeit miteinander verbunden wurden. Besonders interessant ist zum Beispiel, in welchem Ausmaß der letztendliche Eintritt in den Arbeitsmarkt durch Arbeit während der Ausbildungszeit begünstigt wird. Andererseits kann eine hohe Zahl von Arbeitsstunden während der Ausbildungszeit eher auf einen Abbruch der Ausbildung als auf einen erfolgreichen Übergang ins Erwerbsleben hindeuten.

\section{Ergebnisse und Erläuterungen}

\section{Kombination von Arbeit und Ausbildung}

Tabelle C5.1 zeigt den Ausbildungs-/Erwerbsstatus junger Menschen in einer Reihe von OECD-Ländern für die Altersgruppen 15 bis 19, 20 bis 24 und 25 bis 29 Jahre und für alle jungen Menschen zwischen 15 und 29 Jahren. Erwerbstätigkeit während der Ausbildungsphase kann in dualen Ausbildungsgängen oder in der Form von Teilzeitbeschäftigung in der außerschulischen Zeit vorkommen. Duale Ausbildungsgänge sind in einigen europäischen Ländern wie Dänemark, Deutschland, Österreich, der Schweiz und der Tschechischen Republik relativ weit verbreitet. Sie bieten eine kohärente berufliche Ausbildung, die zu anerkannten Berufsabschlüssen führt. Viele junge Menschen befinden sich neben der Ausbildung auch in einem Beschäftigungsverhältnis. Diese Form des ersten Kontakts mit dem Arbeitsmarkt ist ein bedeutendes Merkmal des Übergangs zur Erwerbstätigkeit in Australien, Dänemark, Kanada, den Niederlanden und den Vereinigten Staaten sowie in geringerem Maße in Finnland, Schweden und der Schweiz. Schließlich gehören zu den Ländern, in denen Schule und Arbeit kaum miteinander verbunden werden, Belgien, Frankreich, die Mittelmeer- und die osteuropäischen Länder.

Während der Ausbildungsiahre ist der Beschäftigungsstatus von Männern und Frauen in den meisten Ländern sehrähnlich.

Der Übergang von der Ausbildung zum Erwerbsleben findet in den einzelnen

$O E C D$-Ländern,

Der Beschäftigungsstatus von Männern und Frauen ist während der Ausbildungsjahre mit Ausnahme von Deutschland und Östereich, wo die Beteiligung von Männern an dualen Bildungsgängen höher ist, weitgehend ähnlich. In Australien, Dänemark, Finnland, Island, Kanada und Schweden verbinden im Alter von 15 bis 29 Jahren mehr Frauen außerschulische Arbeit und Ausbildung miteinander als Männer (Tabelle C5.1a und C5.1b).

\section{Eintritt in den Arbeitsmarkt nach Abschluss der Erstausbildung}

Mit zunehmendem Alter steigt der Anteil junger Menschen, die sich nicht mehr in Ausbildung befinden, und ihr Anteil an der Erwerbsbevölkerung nimmt zu. In den meisten Ländern liegt der Anteil junger Menschen, die sich nicht in Ausbildung befinden, bei den 15- bis 19-Jährigen zwischen 10 und 35 Prozent, steigt bei den 20- bis 24-Jährigen auf 50 bis 70 Prozent und erreicht 


\section{Abbildung C5.2.}

Prozentsatz junger Menschen in Ausbildung und nicht in Ausbildung, nach Altersgruppen und Beschäftigungsstatus (2001)

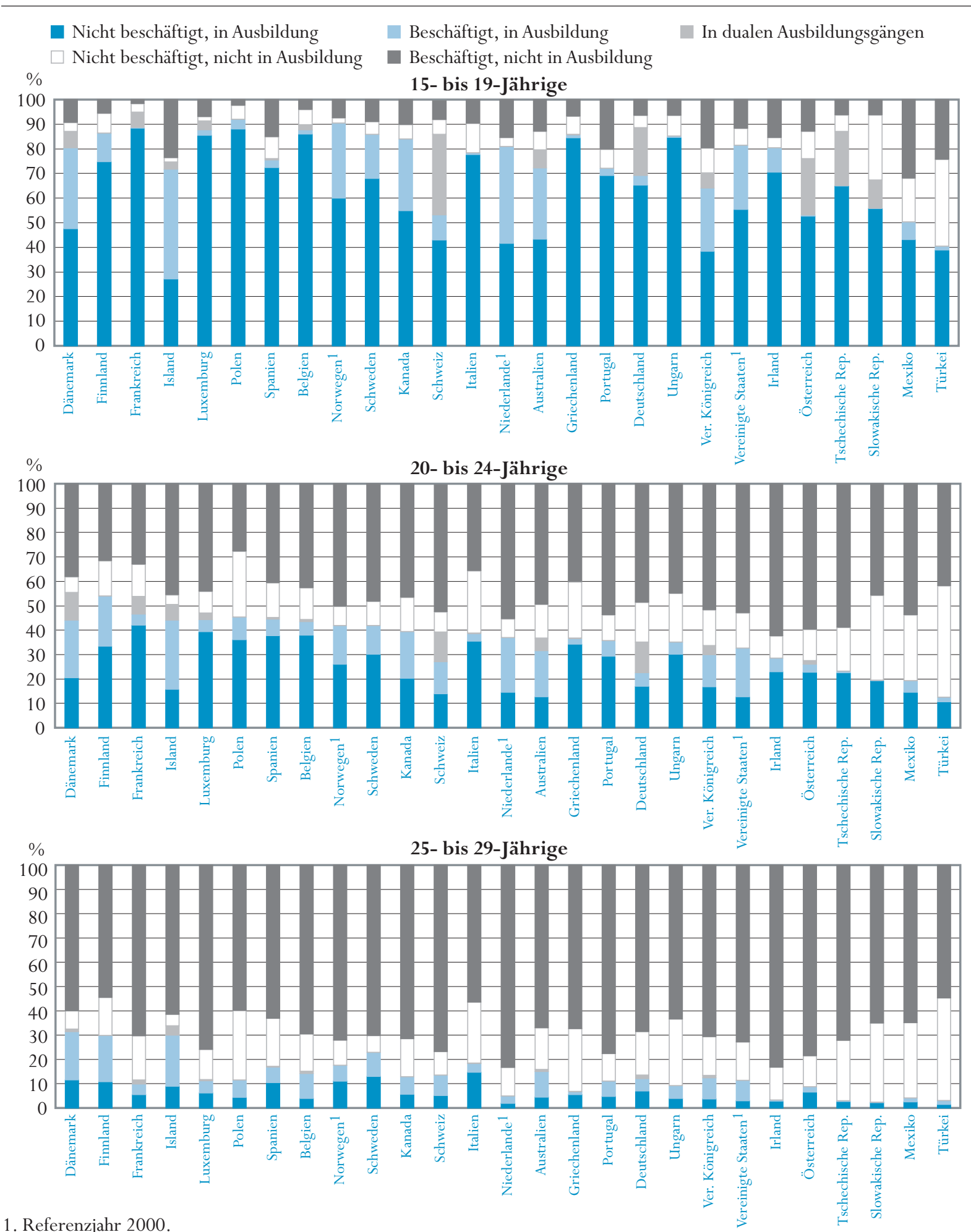

Anordnung der Länder in absteigender Reihenfolge des Prozentsatzes 20- bis 24-Jähriger, die sich in Ausbildung befinden.

Quelle: OECD. Tabelle C5.1. Hinweise s. Anhang 3 (www.oecd.org/els/education/eag2002). 
abhängig von verschiedenen Bildungsund Arbeitsmarktfaktoren, zu unterschiedlichen Zeitpunkten statt. bei der Altersgruppe der 25- bis 29-Jährigen 80 bis 95 Prozent (Abb. C5.2). In vielen OECD-Ländern findet jedoch der Eintritt junger Menschen in das Erwerbsleben zu einem späteren Zeitpunkt statt und erstreckt sich in einigen Fällen auch über einen längeren Zeitraum. Diese Entwicklung spiegelt nicht nur den Bildungsbedarf, sondern auch die allgemeine Arbeitsmarktlage, die Dauer von Ausbildungsgängen, die Ausrichtung von Ausbildungsprogrammen hinsichtlich des Arbeitsmarktes und die Verbreitung von Teilzeitausbildung wider.

Das Alter beim Eintritt in den Arbeitsmarkt nach Abschluss der Erstausbildung hat Auswirkungen auf die Beschäftigung. Insgesamt ist die Wahrscheinlichkeit, dass ältere Nicht-Schüler/Studierende eine Beschäftigung haben, höher als die Nicht-Schüler/Studierender der Altersgruppe von 15 bis 19 Jahren, wobei ein größerer Prozentsatz männlicher als weiblicher Nicht-Schüler/ Studierender beschäftigt ist. Im Vergleich sind relativ mehr Frauen als Männer nicht im Arbeitsmarkt, insbesondere während der Jahre, in die typischerweise Schwangerschaft und Kindererziehung fallen, also bei der Altergruppe der 25bis 29-Jährigen (Tabellen C5.1a und C5.1b).

Der Anteil Beschäftigter an der Bevölkerung bei jungen Erwachsenen, die sich nicht in Ausbildung befinden, kann Hinweise auf die Wirksamkeit bestehender Rahmenbedingungen für den Übergang ins Erwerbsleben geben und den politischen Entscheidungsträgern bei deren Bewertung helfen. In zwei Drittel der OECD-Länder arbeiten weniger als 65 (und in einigen sogar weniger als 50) Prozent der sich nicht in Ausbildung befindenden 15- bis 19-Jährigen, was darauf hindeuten könnte, dass die Arbeitgeber davon ausgehen, dass diese Jugendlichen aufgrund ihres frühen Schulabgangs nicht die für eine produktive Beschäftigung erforderlichen Kenntnisse und Fähigkeiten besitzen. Der Anteil Beschäftigter an der Bevölkerung der 20- bis 24-Jährigen liegt im allgemeinen bei über 70 Prozent, in einigen OECD-Ländern wie Griechenland, Italien, Polen und derTürkei liegt er allerdings nach wie vor um oder unter 65 Prozent. Für die Altersgruppe von 25 bis 29 Jahren liegen die meisten OECD-Länder mit Ausnahme von Italien, Mexiko, Polen, der Slowakischen Republik und der Türkei zwischen 70 und 80 Prozent. Wiederum ist der Anteil der Beschäftigten an der Bevölkerung nach Beendigung der Erstausbildung für Männer eher höher als für Frauen, wahrscheinlich aufgrund familiärer Verpflichtungen und weil der Status nicht-berufstätiger Frauen in vielen Ländern immer noch eher akzeptiert wird als der nicht-berufstätiger Männer (Tabellen C5.1a und C5.1b)

\section{Arbeitslosenquote und Anteil arbeitsloser Nicht-Schüler/Studierender an der gesamten jungen Bevölkerung}

Junge Menschen repräsentieren in unseren Gesellschaften die wichtigste Quelle neuer Fähigkeiten und Kenntnisse. In den meisten OECD-Ländern ist die Bildungspolitik daher bemüht, junge Menschen zumindest zu einem Abschluss im Sekundarbereich II zu ermutigen. Da die auf dem Arbeitsmarkt angebotenen Arbeitsplätze ständig umfassendere Kompetenzen und flexiblere Lernfähigkeiten 
verlangen, sehen sich Personen mit einem niedrigen Bildungsstand auf dem Arbeitsmarkt oft erheblichen Schwierigkeiten gegenüber. Unterschiede im Anteil arbeitsloser Nicht-Schüler/Studierender an der gesamten Altergruppe junger Menschen, getrennt nach Bildungsstand, geben einen Hinweis darauf, in welchem Ausmaß zusätzliche Bildung die wirtschaftlichen Chancen junger Männer und Frauen verbessert.

Die Jugendarbeitslosenquote nach Altersgruppen ist der allgemein übliche Maßstab zur Beschreibung des Beschäftigungsstatus junger Menschen. Arbeitslosenquoten lassen jedoch Aspekte der (Aus-)Bildung unberücksichtigt. Somit könnte ein arbeitsloser Jugendlicher, der in dieser Division im Zähler auftaucht, in einigen OECD-Ländern auch an einem Ausbildungsgang teilnehmen. Der Nenner umfasst hingegen nur Jugendliche in der Berufsausbildung, wenn diese eine Lehre machen, jedoch nicht vergleichbare Personen, die eine schulische Berufsausbildung absolvieren. Wenn sich also beinahe alle jungen Menschen in einer bestimmten Altersgruppe noch in Ausbildung befinden, bezieht sich die Arbeitslosenquote nur auf die wenigen, die dem Arbeitsmarkt zur Verfügung stehen. Die Jugendarbeitslosigkeit kann dann sehr hoch erscheinen, insbesondere für die jüngste Altersgruppe, die in der Regel das Bildungssystem mit einer sehr geringen Qualifikation verlassen hat.

Der Anteil arbeitsloser Nicht-Schüler/Studierender an der gesamten Altersgruppe ist daher geeigneter, die Wahrscheinlichkeit der Jugendarbeitslosigkeit zu belegen. Denn junge Menschen, die Arbeit suchen, solange sie sich noch innerhalb des Bildungssystems befinden, haben normalerweise nicht dieselbe Einstellung zum Arbeitsmarkt wie diejenigen, die nach Verlassen des Bildungssystems in den Arbeitsmarkt eintreten. Erstere suchen in der Regel eine Teilzeit- oder befristete Beschäftigung, die sie mit ihrer Ausbildung vereinbaren können.

Durchschnittlich liegt der Anteil Arbeitsloser an der Bevölkerung junger Menschen (d.h. die Arbeitslosigkeit Nicht-Schüler/Studierender als Prozentsatz der gesamten Altersgruppe) im Alter zwischen 20 und 24 Jahren bei Personen mit einem Abschluss im Sekundarbereich II um 6 Prozentpunkte niedriger als bei Personen ohne einen solchen Abschluss, bei den 25- bis 29Jährigen liegt dieser Anteil um ca. 4 Prozentpunkte niedriger (Tabelle C5.2). In 20 von 27 OECD-Ländern beträgt der Anteil Arbeitsloser an der Bevölkerung bei jungen Menschen im Alter zwischen 20 und 24 Jahren, die sich nicht in Ausbildung befinden und einen Abschluss im Sekundarbereich II oder in einem post-sekundären, nicht-tertiären Ausbildungsgang besitzen, unter 8 Prozent. Aber nur in sechs OECD-Ländern liegt dieser Anteil auch bei denjenigen ohne einen Abschluss im Sekundarbereich II unter 8 Prozent. Da der Abschluss eines Bildungsgangs im Sekundarbereich II inzwischen in den meisten OECDLändern quasi zur Norm geworden ist, sind viele junge Menschen, die keinen
Die herkömmlichen Arbeitslosenquoten setzen die Arbeitslosigkeit während der Übergangsphase zu hoch an und berücksichtigen die verschiedenen Möglichkeiten zur Kombination von Bildung und Arbeit während des Übergangs nicht.

Bei denjenigen ohne einen Abschluss im sekundarbereich II ist der Anteil arbeitsloser Nichtschüler/Studierender an der gesamten jungen Bevölkerung im Durchschnitt 1,5-mal höher als bei Absolventen des sekundarbereich II. 


\section{Abbildung C5.3.}

Prozentsatz arbeitsloser 20- bis 24-Jähriger, die sich nicht in Ausbildung befinden, nach Bildungsstand (2001)

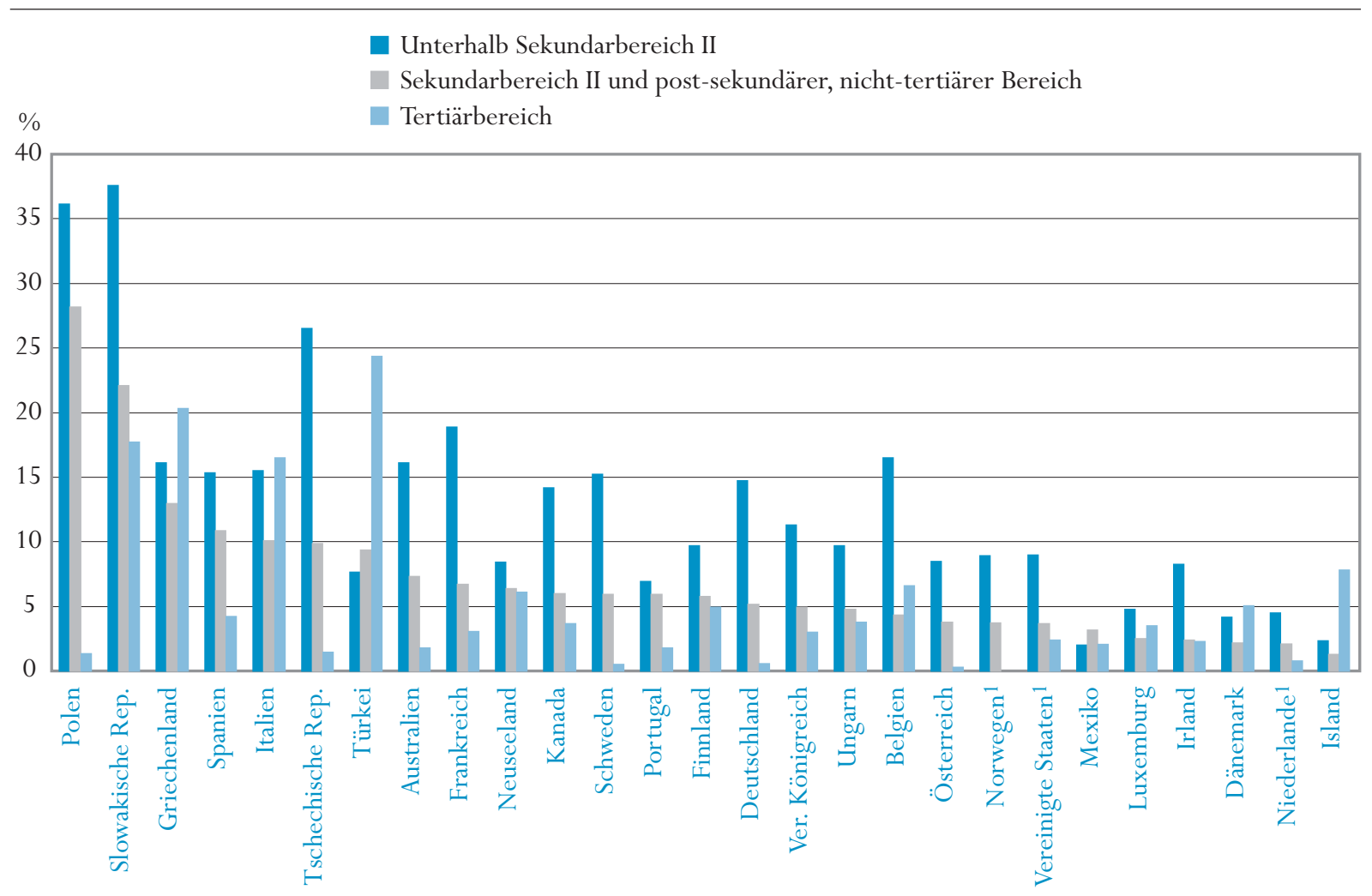

1. Referenzjahr 2000.

Anordnung der Länder in absteigender Reihenfolge des Prozentsatzes arbeitsloser 20- bis 24-Jähriger, die sich nicht in Ausbildung befinden, mit einem Abschluss im Sekundarbereich II und im post-sekundären, nicht-tertiären Bereich.

Quelle: OECD. Tabelle C5.2. Hinweise s. Anhang 3 (www.oecd.org/els/education/eag2002).

Ein Abschluss des Sekundarbereich II und sogar des Tertiärbereichs ist keine Garantie für einen Arbeitsplatz.
Abschluss in diesem Bereich haben, während ihres gesamten Erwerbslebens viel stärker von Arbeitslosigkeit und Beschäftigungsproblemen bedroht.

In einer Reihe von OECD-Ländern sehen sich 20- bis 24-Jährige auch dann mit einem Anteil arbeitsloser Nicht-Schüler/Studierender an der gleichaltrigen Gesamtbevölkerung von über 7 Prozent konfrontiert, wenn sie über einen Abschluss des Sekundarbereich II verfügen. (Abbildung C5.3). In einigen wenigen OECD-Ländern erwartet sogar diejenigen mit einem Abschluss des Tertiärbereichs (angesichts der betroffenen Altersgruppe wahrscheinlich Studierende mit einem ersten akademischen Abschluss) beim Eintritt in den Arbeitsmarkt ein erhebliches Maß an Arbeitslosigkeit. Der Anteil arbeitsloser Nicht-Studierender an der gleichaltrigen Gesamtbevölkerung beträgt in dieser Gruppe in Griechenland, Italien, der Slowakischen Republik und der Türkei bis zu 16 Prozent und mehr. In Griechenland und Italien beträgt dieser Anteil bei der Altersgruppe der 25- bis 29-Jährigen mehr als 13 Prozent (Tabelle C5.2). 


\section{Definitionen und angewandte Methodik}

Die Daten für diesen Indikator stammen aus einer speziellen Erhebung Die Daten für diesen der OECD, deren Bezugszeitraum am Anfang des Kalenderjahres lag, normalerweise das erste Quartal oder der Durchschnitt der ersten drei Monate des Jahres; sie decken daher die Beschäftigung im Sommer (in den Sommerferien) nicht ab. Mit einer Ausnahme entsprechen die Definitionen des Erwerbsstatus in diesem Abschnitt den Richtlinien des Internationalen Arbeitsamtes (ILO). Für die vorliegenden Indikatoren wurden die Teilnehmer an dualen Ausbildungsprogrammen (s.u.) getrennt unter ,in Ausbildung' und ,in Beschäftigung' aufgeführt, ohne Bezug auf ihren ILO-Erwerbsstatus während der Erhebungswoche. Diese Teilnehmer sind nicht zwangsläufig während der Erhebungswoche in der betrieblichen Phase des Programms, daher waren sie zum Zeitpunkt der Erhebung möglicherweise nicht beschäftigt.

„Duale Ausbildungsprogramme“ stellen eine Kombination aus Phasen des Arbeitens und des Lernens dar, die beide Bestandteil einer integrierten, formellen Bildung bzw. Ausbildung sind. Zu solchen Programmen zählen beispielsweise das „duale System“ in Deutschland, die „apprentissage“ oder „formation en alternance“ in Frankreich und Belgien, Praktika oder 'kooperative' Ausbildungen in Kanada, „apprenticeship“ in Irland und „youth training“ im Vereinigten Königreich. Die berufliche Aus- und Weiterbildung erfolgt nicht nur in der Schule, sondern auch in einer Arbeitsumgebung. Die Auszubildenden oder Praktikanten werden bei einigen Programmen für ihre Arbeit bezahlt, bei anderen nicht. Es besteht gewöhnlich ein enger Zusammenhang zwischen der Arbeit und dem Unterricht bzw. der Ausbildung.

Die Angaben zur Bildungsbeteiligung in Tabelle C5.1 basieren auf Selbstauskünften im Rahmen von Arbeitskräfteerhebungen. Diese stimmen häufig nicht genau mit den Zahlen aus amtlichen Quellen überein, die hier an anderer Stelle angegebenen sind. Hierfür gibt es eine Reihe von Gründen. Erstens wird das Alter möglicherweise nicht auf einheitliche Weise erfasst. So wird beispielsweise in den nationalen Statistiken der OECD-Länder der nördlichen Hemisphäre sowohl die Bildungsbeteiligung als auch das Alter per 1. Januar angegeben. In einigen Arbeitskräfteerhebungen hingegen wird die Bildungsbeteiligung in der Erhebungswoche gemessen, das angegebene Alter ist jedoch das Alter, das am Ende des Kalenderjahres erreicht wird, auch wenn die Erhebung Anfang des Jahres durchgeführt wird. Unter diesen Bedingungen spiegeln die Angaben zur Bildungsbeteiligung in manchen Fällen eine Population wider, die tatsächlich fast ein Jahr jünger ist als die angegebene Alterspanne. In einem Alter, in dem viele junge Menschen das Bildungssystem verlassen, kann sich dies auf die Bildungsbeteiligung auswirken. Zweitens können die jungen Frauen und Männer auch in mehr als einem Bildungsgang eingeschrieben sein. In den amtlichen Statistiken werden sie dann manchmal zweimal, in der Arbeitskräfteerhebung jedoch nur einmal gezählt. Ferner erfassen die amtlichen Statistiken vielleicht nicht alle an Bildungsgängen teilnehmenden Personen, insbesondere, wenn sie an gewinnorientierten Bildungseinrichtungen 
ausgebildet werden. Drittens stimmen die Klassifizierungen der Bildungsgänge in den Selbstauskünften der Arbeitsmarkterhebungen nicht immer mit den Qualifikationsstandards überein, die für die amtlichen Datenerhebungen verwendet werden. 
Tabelle C5.1.

Prozentsatz junger Menschen in Ausbildung und nicht in Ausbildung, nach Altersgruppen und Beschäftigungsstatus (2001)

\begin{tabular}{|c|c|c|c|c|c|c|c|c|c|c|c|}
\hline & & & & Ausbildung & & & & Nicht in A & sbildung & & In Ausbil- \\
\hline & $\begin{array}{l}\text { Alters- } \\
\text { gruppe }\end{array}$ & $\begin{array}{c}\text { In dualer } \\
\text { Ausbildung }\end{array}$ & $\begin{array}{l}\text { Andere Be- } \\
\text { schäftigung }\end{array}$ & Arbeitslos & $\begin{array}{c}\text { Nicht im } \\
\text { Arbeits- } \\
\text { markt }\end{array}$ & Zusammen & Beschäftigt & Arbeitslos & $\begin{array}{c}\text { Nicht im } \\
\text { Arbeits- } \\
\text { markt }\end{array}$ & Zusammen & $\begin{array}{c}\text { nicht in } \\
\text { Ausbildung } \\
\text { insgesamt }\end{array}$ \\
\hline Australien & 15-19 & 7.3 & 29.0 & 6.4 & 36.7 & 79.5 & 13.0 & 4.3 & 3.3 & 20.5 & 100 \\
\hline & $20-24$ & 5.1 & 18.8 & 2.3 & 10.2 & 36.5 & 49.6 & 6.9 & 7.0 & 63.5 & 100 \\
\hline & $25-29$ & 0.8 & 10.6 & 0.9 & 3.6 & 15.8 & 67.0 & 4.5 & 12.7 & 84.2 & 100 \\
\hline Österreich & $15-19$ & 22.7 & 0.6 & 0.4 & 52.2 & 75.8 & 12.9 & 2.2 & 9.1 & 24.2 & 100 \\
\hline & $20-24$ & 1.6 & 3.3 & 0.4 & 22.1 & 27.4 & 59.8 & 3.4 & 9.4 & 72.6 & 100 \\
\hline & $25-29$ & 0.1 & 2.1 & 0.2 & 6.4 & 8.7 & 78.5 & 3.0 & 9.8 & 91.3 & 100 \\
\hline Belgien & $15-19$ & 2.0 & 1.7 & 0.3 & 85.7 & 89.7 & 4.1 & 1.8 & 4.5 & 10.3 & 100 \\
\hline & $20-24$ & 0.9 & 5.4 & 0.9 & 36.9 & 44.2 & 42.8 & 6.9 & 6.1 & 55.8 & 100 \\
\hline & $25-29$ & 0.9 & 10.2 & 0.4 & 3.5 & 15.0 & 69.5 & 7.3 & 8.1 & 85.0 & 100 \\
\hline Kanada & $15-19$ & a & 29.1 & 5.2 & 49.5 & 83.9 & 10.2 & 2.6 & 3.3 & 16.1 & 100 \\
\hline & $20-24$ & $\mathrm{a}$ & 19.0 & 1.5 & 18.7 & 39.1 & 46.6 & 6.3 & 8.0 & 60.9 & 100 \\
\hline & $25-29$ & $\mathrm{a}$ & 7.2 & 0.2 & 5.4 & 12.8 & 71.4 & 6.1 & 9.7 & 87.2 & 100 \\
\hline Tschechische Rep. & $15-19$ & 21.9 & 0.2 & $\mathrm{n}$ & 64.8 & 87.0 & 6.2 & 4.1 & 2.8 & 13.0 & 100 \\
\hline & $20-24$ & 0.1 & 0.6 & 0.2 & 22.2 & 23.1 & 58.9 & 9.3 & 8.7 & 76.9 & 100 \\
\hline & $25-29$ & $\mathrm{n}$ & 0.3 & $\mathrm{n}$ & 2.6 & 3.0 & 72.1 & 7.2 & 17.7 & 97.0 & 100 \\
\hline Dänemark & $15-19$ & 6.6 & 32.9 & 3.4 & 44.0 & 86.8 & 9.4 & 1.2 & 2.5 & 13.2 & 100 \\
\hline & $20-24$ & 11.4 & 23.6 & 3.5 & 16.8 & 55.3 & 38.1 & 2.9 & 3.6 & 44.7 & 100 \\
\hline & $25-29$ & 1.0 & 19.8 & 1.0 & 10.5 & 32.4 & 60.0 & 1.9 & 5.7 & 67.6 & 100 \\
\hline Finnland & $15-19$ & a & 11.6 & 5.9 & 68.7 & 86.3 & 5.7 & 2.1 & 5.9 & 13.7 & 100 \\
\hline & $20-24$ & $\mathrm{a}$ & 20.6 & 4.4 & 28.9 & 53.9 & 31.7 & 6.1 & 8.3 & 46.1 & 100 \\
\hline & $25-29$ & $\mathrm{a}$ & 19.0 & 1.8 & 8.9 & 29.8 & 54.5 & 6.3 & 9.4 & 70.2 & 100 \\
\hline Frankreich & $15-19$ & 6.2 & 0.4 & $\mathrm{n}$ & 88.2 & 94.9 & 1.7 & 1.8 & 1.6 & 5.1 & 100 \\
\hline & $20-24$ & 7.3 & 4.4 & 0.6 & 41.3 & 53.6 & 33.1 & 8.5 & 4.9 & 46.4 & 100 \\
\hline & $25-29$ & 1.6 & 4.4 & 0.4 & 5.0 & 11.4 & 70.3 & 9.1 & 9.2 & 88.6 & 100 \\
\hline Deutschland & $15-19$ & 19.4 & 4.0 & 0.6 & 64.5 & 88.5 & 6.4 & 1.4 & 3.7 & 11.5 & 100 \\
\hline & $20-24$ & 12.6 & 5.5 & 0.3 & 16.7 & 35.0 & 48.7 & 5.6 & 10.8 & 65.0 & 100 \\
\hline & $25-29$ & 1.4 & 5.0 & 0.2 & 6.8 & 13.5 & 68.5 & 5.8 & 12.2 & 86.5 & 100 \\
\hline Griechenland & $15-19$ & 0.2 & 1.1 & 0.6 & 83.8 & 85.7 & 6.8 & 3.9 & 3.6 & 14.3 & 100 \\
\hline & $20-24$ & 0.1 & 2.4 & 1.3 & 32.8 & 36.5 & 40.2 & 14.0 & 9.3 & 63.5 & 100 \\
\hline & $25-29$ & 0.1 & 1.2 & 0.5 & 5.0 & 6.7 & 67.4 & 12.7 & 13.2 & 93.3 & 100 \\
\hline Ungarn & $15-19$ & a & 0.6 & 0.2 & 84.3 & 85.1 & 6.5 & 2.1 & 6.3 & 14.9 & 100 \\
\hline & $20-24$ & a & 4.8 & 0.5 & 29.5 & 34.8 & 45.0 & 5.5 & 14.7 & 65.2 & 100 \\
\hline & $25-29$ & $\mathrm{a}$ & 5.3 & 0.2 & 3.7 & 9.1 & 63.4 & 5.3 & 22.1 & 90.9 & 100 \\
\hline Island & $15-19$ & 2.8 & 44.6 & 3.7 & 23.4 & 74.4 & 23.7 & 1.6 & 0.3 & 25.6 & 100 \\
\hline & $20-24$ & 6.5 & 28.3 & 1.0 & 14.6 & 50.3 & 45.6 & 2.0 & 2.1 & 49.7 & 100 \\
\hline & $25-29$ & 3.9 & 21.0 & $\mathrm{n}$ & 8.9 & 33.8 & 61.5 & 1.4 & 3.4 & 66.2 & 100 \\
\hline Irland & $15-19$ & a & 9.9 & 0.5 & 69.8 & 80.3 & 15.5 & 1.9 & 2.2 & 19.7 & 100 \\
\hline & $20-24$ & $\mathrm{a}$ & 5.5 & 0.4 & 22.4 & 28.3 & 62.4 & 3.3 & 6.0 & 71.7 & 100 \\
\hline & $25-29$ & $\mathrm{a}$ & 0.5 & $\mathrm{n}$ & 2.7 & 3.3 & 83.1 & 2.8 & 10.7 & 96.7 & 100 \\
\hline Italien & $15-19$ & $\mathrm{n}$ & 0.6 & 0.8 & 76.8 & 78.2 & 9.6 & 4.9 & 7.3 & 21.8 & 100 \\
\hline & $20-24$ & 0.1 & 3.1 & 1.8 & 33.6 & 38.6 & 35.8 & 11.8 & 13.8 & 61.4 & 100 \\
\hline & $25-29$ & 0.1 & 3.6 & 1.2 & 13.5 & 18.4 & 56.4 & 9.9 & 15.3 & 81.6 & 100 \\
\hline Luxemburg & $15-19$ & 3.6 & 2.3 & 0.2 & 85.2 & 91.2 & 7.0 & 0.6 & 1.2 & 8.8 & 100 \\
\hline & $20-24$ & 2.6 & 4.9 & 0.3 & 38.9 & 46.7 & 44.2 & 3.5 & 5.5 & 53.3 & 100 \\
\hline & $25-29$ & 0.4 & 5.0 & 0.2 & 5.9 & 11.6 & 75.9 & 1.8 & 10.7 & 88.4 & 100 \\
\hline Mexiko & $15-19$ & a & 7.1 & 0.3 & 42.8 & 50.2 & 32.0 & 1.6 & 16.3 & 49.8 & 100 \\
\hline & $20-24$ & a & 4.7 & 0.2 & 14.1 & 19.1 & 53.8 & 2.0 & 25.1 & 80.9 & 100 \\
\hline & $25-29$ & $\mathrm{a}$ & 1.6 & $\mathrm{n}$ & 2.5 & 4.1 & 64.8 & 1.6 & 29.4 & 95.9 & 100 \\
\hline Niederlande $^{2}$ & 15-19 & $\mathrm{m}$ & 39.3 & 4.7 & 36.4 & 80.4 & 15.8 & 1.2 & 2.6 & 19.6 & 100 \\
\hline & $20-24$ & $\mathrm{~m}$ & 22.3 & 1.4 & 12.9 & 36.6 & 55.2 & 2.1 & 6.0 & 63.4 & 100 \\
\hline & $25-29$ & $\mathrm{~m}$ & 3.3 & 0.3 & 1.9 & 5.5 & 82.6 & 2.5 & 9.4 & 94.5 & 100 \\
\hline Norwegen $^{2}$ & $15-19$ & a & 30.4 & 6.9 & 53.0 & 90.3 & 7.5 & 1.1 & 1.1 & 9.7 & 100 \\
\hline & $20-24$ & a & 15.8 & 2.6 & 23.4 & 41.7 & 50.3 & 3.3 & 4.7 & 58.3 & 100 \\
\hline & $25-29$ & a & 6.6 & 1.3 & 9.7 & 17.5 & 72.1 & 3.2 & 7.2 & 82.5 & 100 \\
\hline Polen & $15-19$ & a & 3.9 & 1.2 & 86.7 & 91.8 & 2.4 & 3.4 & 2.4 & 8.2 & 100 \\
\hline & $20-24$ & a & 9.4 & 6.7 & 29.2 & 45.2 & 27.7 & 18.9 & 8.2 & 54.8 & 100 \\
\hline & $25-29$ & a & 7.1 & 1.5 & 2.9 & 11.4 & 59.9 & 15.7 & 13.0 & 88.6 & 100 \\
\hline Portugal & $15-19$ & a & 2.9 & 0.4 & 68.7 & 72.0 & 20.3 & 2.8 & 4.9 & 28.0 & 100 \\
\hline & $20-24$ & a & 6.5 & 0.6 & 28.5 & 35.6 & 53.7 & 5.1 & 5.6 & 64.4 & 100 \\
\hline & $25-29$ & $\mathrm{a}$ & 6.3 & 0.4 & 4.4 & 11.0 & 77.6 & 3.6 & 7.8 & 89.0 & 100 \\
\hline Slowakische Rep. & $15-19$ & 11.4 & 0.1 & $\mathrm{n}$ & 55.7 & 67.3 & 6.3 & 11.0 & 15.5 & 32.7 & 100 \\
\hline & $20-24$ & a & 0.4 & 0.6 & 18.5 & 19.4 & 45.7 & 22.8 & 12.1 & 80.6 & 100 \\
\hline & $25-29$ & a & 0.1 & $\mathrm{n}$ & 2.2 & 2.3 & 65.0 & 16.9 & 15.7 & 97.7 & 100 \\
\hline Spanien & $15-19$ & 0.5 & 3.0 & 1.6 & 70.7 & 75.8 & 15.1 & 5.4 & 3.6 & 24.2 & 100 \\
\hline & $20-24$ & 0.7 & 6.8 & 2.6 & 34.9 & 45.0 & 40.7 & 8.7 & 5.6 & 55.0 & 100 \\
\hline & $25-29$ & 0.2 & 6.4 & 2.2 & 8.2 & 17.0 & 63.1 & 8.6 & 11.2 & 83.0 & 100 \\
\hline Schweden & $15-19$ & a & 17.9 & 4.4 & 63.4 & 85.8 & 9.1 & 1.9 & 3.3 & 14.2 & 100 \\
\hline & $20-24$ & a & 11.6 & 2.0 & 28.0 & 41.6 & 48.2 & 5.1 & 5.1 & 58.4 & 100 \\
\hline & $25-29$ & a & 9.9 & 1.2 & 11.8 & 22.9 & 70.2 & 3.2 & 3.8 & 77.1 & 100 \\
\hline Schweiz & $15-19$ & 32.5 & 10.3 & 3.9 & 38.9 & 85.7 & 7.5 & $\mathrm{~m}$ & 6.2 & 14.3 & 100 \\
\hline & $20-24$ & 12.1 & 13.2 & $\mathrm{~m}$ & 13.7 & 39.3 & 52.3 & 2.8 & 5.6 & 60.7 & 100 \\
\hline & $25-29$ & $\mathrm{~m}$ & 8.3 & $\mathrm{~m}$ & 5.1 & 13.5 & 76.8 & $\mathrm{~m}$ & 9.7 & 86.5 & 100 \\
\hline Türkei & $15-19$ & $\mathrm{a}$ & 1.4 & 38.5 & 0.3 & 40.3 & 24.3 & 5.8 & 29.7 & 59.7 & 100 \\
\hline & $20-24$ & $\mathrm{a}$ & 1.9 & 9.5 & 0.9 & 12.2 & 41.9 & 9.2 & 36.7 & 87.8 & 100 \\
\hline & $25-29$ & $\mathrm{a}$ & 1.6 & 1.2 & 0.3 & 3.0 & 54.7 & 7.3 & 35.0 & 97.0 & 100 \\
\hline Ver. Königreich & $15-19$ & 6.1 & 25.6 & 2.8 & 35.5 & 70.0 & 19.7 & 5.6 & 4.7 & 30.0 & 100 \\
\hline & $20-24$ & 3.8 & 13.2 & 1.2 & 15.3 & 33.5 & 51.7 & 5.0 & 9.7 & 66.5 & 100 \\
\hline & $25-29$ & 1.0 & 8.7 & 0.4 & 3.2 & 13.3 & 70.6 & 3.6 & 12.5 & 86.7 & 100 \\
\hline Vereinigte Staaten ${ }^{2}$ & $15-19$ & a & 25.9 & 3.3 & 52.1 & 81.3 & 11.7 & 2.4 & 4.6 & 18.7 & 100 \\
\hline & $20-24$ & a & 20.0 & 1.0 & 11.5 & 32.5 & 53.1 & 4.0 & 10.4 & 67.5 & 100 \\
\hline & $25-29$ & a & 8.4 & $\mathrm{n}$ & 2.9 & 11.4 & 72.8 & 3.0 & 12.8 & 88.6 & 100 \\
\hline Ländermittel & $15-19$ & 5.3 & 12.5 & 3.6 & 58.6 & 79.9 & 11.5 & 2.9 & 5.6 & 20.1 & 100 \\
\hline & $20-24$ & 2.4 & 10.2 & 1.8 & 22.8 & 37.2 & 46.5 & 6.9 & 9.4 & 62.8 & 100 \\
\hline & $25-29$ & 0.4 & 6.8 & 0.6 & 5.5 & 13.3 & 68.5 & 5.7 & 12.5 & 86.7 & 100 \\
\hline
\end{tabular}

1. Schüler in dualen Ausbildungsgängen werden sowohl der Kategorie 'in Ausbildung' als auch der Kategorie 'in Beschäftigung' zugeordnet, ungeachtet ihres Beschäftigungsstatus gemäß der ILO-Definition.

2. Referenzjahr 2000 .

Quelle: OECD. Länderspezifische Datenquellen s. Anhang 3 (www.oecd.org/els/education/eag2002). 
Tabelle C5.1a.

Prozentsatz junger Männer in Ausbildung und nicht in Ausbildung, nach Altersgruppen und Beschäftigungsstatus (2001)

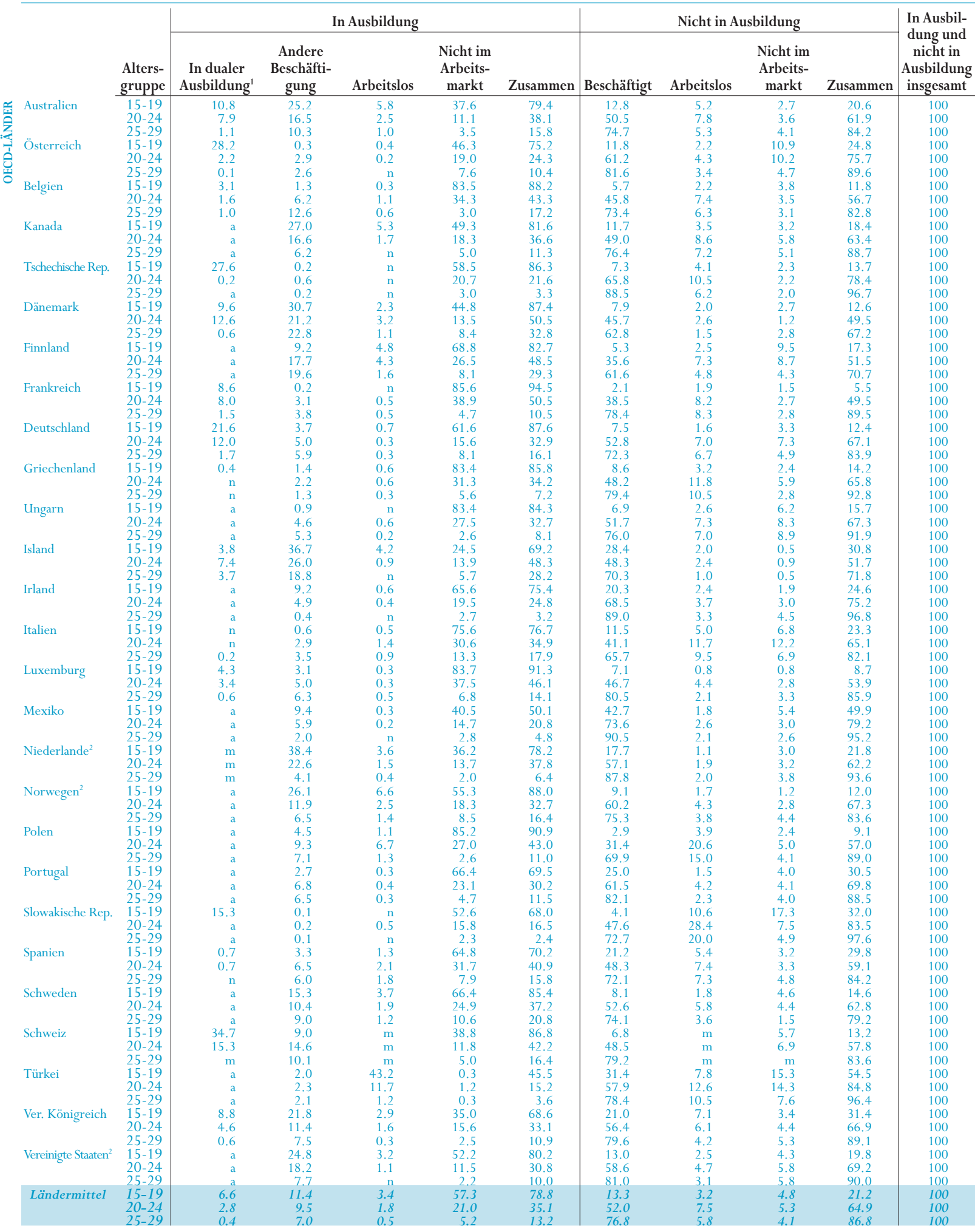

1. Schüler in dualen Ausbildungsgängen werden sowohl der Kategorie 'in Ausbildung' als auch der Kategorie 'in Beschäftigung' zugeordnet, ungeachtet ihres Beschäftigungsstatus gemäß der ILO-Definition.

2. Referenzjahr 2000

Quelle: OECD. Länderspezifische Datenquellen s. Anhang 3 (www.oecd.org/els/education/eag2002). 
Tabelle C5.1b

Prozentsatz junger Frauen in Ausbildung und nicht in Ausbildung, nach Altersgruppen und Beschäftigungsstatus (2001)

\begin{tabular}{|c|c|c|c|c|c|c|c|c|c|c|c|}
\hline & & & & Ausbildung & & & & Nicht in A & sbildung & & In Ausbil- \\
\hline & $\begin{array}{l}\text { Alters- } \\
\text { gruppe }\end{array}$ & $\begin{array}{c}\text { In dualer } \\
\text { Ausbildung }\end{array}$ & $\begin{array}{c}\text { Andere } \\
\text { Beschäfti- } \\
\text { gung }\end{array}$ & Arbeitslos & $\begin{array}{c}\text { Nicht im } \\
\text { Arbeits- } \\
\text { markt }\end{array}$ & $\begin{array}{c}\text { Zusam- } \\
\text { men }\end{array}$ & Beschäftigt & Arbeitslos & $\begin{array}{c}\text { Nicht im } \\
\text { Arbeits- } \\
\text { markt }\end{array}$ & $\begin{array}{c}\text { Zusam- } \\
\text { men }\end{array}$ & $\begin{array}{c}\text { nicht in } \\
\text { Ausbildung } \\
\text { insgesamt }\end{array}$ \\
\hline Australien & $15-19$ & 3.7 & 33.0 & 7.1 & 35.8 & 79.7 & 13.2 & 3.3 & 3.9 & 20.3 & 100 \\
\hline & $20-24$ & 2.3 & 21.2 & 2.2 & 9.2 & 34.9 & 48.6 & 6.0 & 10.5 & 65.1 & 100 \\
\hline & $25-29$ & 0.4 & 10.9 & 0.8 & 3.7 & 15.7 & 59.3 & 3.7 & 21.2 & 84.3 & 100 \\
\hline Österreich & $15-19$ & 16.9 & 0.9 & 0.3 & 58.3 & 76.5 & 14.1 & 2.2 & 7.2 & 23.5 & 100 \\
\hline & $20-24$ & 1.0 & 3.6 & 0.6 & 25.3 & 30.5 & 58.4 & 2.6 & 8.5 & 69.5 & 100 \\
\hline & $25-29$ & 0.0 & 1.7 & 0.3 & 5.1 & 7.1 & 75.5 & 2.7 & 14.7 & 92.9 & 100 \\
\hline Belgien & $15-19$ & 0.9 & 2.1 & 0.2 & 88.0 & 91.1 & 2.4 & 1.3 & 5.2 & 8.9 & 100 \\
\hline & $20-24$ & 0.2 & 4.6 & 0.8 & 39.6 & 45.1 & 39.7 & 6.4 & 8.8 & 54.9 & 100 \\
\hline & $25-29$ & 0.8 & 7.8 & 0.3 & 4.0 & 12.9 & 65.5 & 8.4 & 13.3 & 87.1 & 100 \\
\hline Kanada & $15-19$ & a & 31.4 & 5.1 & 49.7 & 86.2 & 8.7 & 1.7 & 3.3 & 13.8 & 100 \\
\hline & $20-24$ & a & 21.4 & 1.3 & 19.0 & 41.8 & 44.1 & 4.0 & 10.2 & 58.2 & 100 \\
\hline & $25-29$ & $\stackrel{a}{a}$ & 8.2 & 0.2 & 5.9 & 14.3 & 66.4 & 4.9 & 14.3 & 85.7 & 100 \\
\hline Tschechische Rep. & $15-19$ & 15.9 & 0.2 & 0.2 & 71.3 & 87.7 & 5.0 & 4.1 & 3.2 & 12.3 & 100 \\
\hline & $20-24$ & a & 0.7 & 0.3 & 23.7 & 24.6 & 51.7 & 8.1 & 15.6 & 75.4 & 100 \\
\hline & $25-29$ & a & 0.3 & $\mathrm{n}$ & 2.3 & 2.6 & 55.1 & 8.3 & 34.1 & 97.4 & 100 \\
\hline Dänemark & $\begin{array}{l}15-19 \\
20-24\end{array}$ & $\begin{array}{r}3.4 \\
10.1\end{array}$ & $\begin{array}{l}35.2 \\
26.0\end{array}$ & $\begin{array}{l}4.5 \\
3.7\end{array}$ & $\begin{array}{l}43.2 \\
20.1\end{array}$ & $\begin{array}{l}86.3 \\
59.9\end{array}$ & $\begin{array}{l}11.0 \\
30.8\end{array}$ & $\begin{array}{l}0.4 \\
3.3\end{array}$ & $\begin{array}{l}2.3 \\
6.0\end{array}$ & $\begin{array}{l}13.7 \\
40.1\end{array}$ & $\begin{array}{l}100 \\
100\end{array}$ \\
\hline & $\begin{array}{l}20-24 \\
25-29\end{array}$ & 1.6 & 16.7 & 1.0 & 12.8 & 32.0 & $\begin{array}{l}50.0 \\
57.0\end{array}$ & 2.3 & $\begin{array}{l}6.0 \\
8.7\end{array}$ & $\begin{array}{l}40.1 \\
68.0\end{array}$ & 100 \\
\hline Finnland & $15-19$ & $\mathrm{a}$ & 14.3 & 7.2 & 68.7 & 90.2 & 6.0 & 1.6 & 2.1 & 9.8 & 100 \\
\hline & $20-24$ & $\mathrm{a}$ & 23.5 & 4.5 & 31.2 & 59.2 & 27.9 & 5.0 & 7.9 & 40.8 & 100 \\
\hline & $25-29$ & a & 18.4 & 2.1 & 9.8 & 30.3 & 46.6 & 8.1 & 15.1 & 69.7 & 100 \\
\hline Frankreich & $15-19$ & 3.7 & 0.5 & $\mathrm{n}$ & 90.9 & 95.3 & 1.2 & 1.8 & 1.7 & 4.7 & 100 \\
\hline & $20-24$ & 6.5 & 5.8 & 0.7 & 43.6 & 56.6 & 27.6 & 8.7 & 7.1 & 43.4 & 100 \\
\hline & $25-29$ & 1.7 & 4.9 & 0.4 & 5.3 & 12.3 & 62.3 & 9.9 & 15.5 & 87.7 & 100 \\
\hline Deutschland & 15-19 & 17.0 & 4.3 & 0.6 & 67.5 & 89.3 & 5.3 & 1.3 & 4.0 & 10.7 & 100 \\
\hline & $20-24$ & 13.2 & 6.0 & 0.3 & 17.7 & 37.2 & 44.1 & 4.1 & 14.6 & 62.8 & 100 \\
\hline & $25-29$ & 1.1 & 4.1 & 0.2 & 5.3 & 10.7 & 64.6 & 4.7 & 20.0 & 89.3 & 100 \\
\hline Griechenland & 15-19 & $\mathrm{n}$ & 0.7 & 0.6 & 84.2 & 85.6 & 4.8 & 4.7 & 4.9 & 14.4 & 100 \\
\hline & $20-24$ & $\mathrm{n}$ & 2.5 & 1.9 & 34.1 & 38.5 & 33.1 & 16.0 & 12.4 & 61.5 & 100 \\
\hline & $25-29$ & $\mathrm{n}$ & 1.0 & 0.7 & 4.5 & 6.3 & 55.0 & 14.9 & 23.9 & 93.7 & 100 \\
\hline Ungarn & $15-19$ & a & 0.4 & 0.3 & 85.2 & 85.9 & 6.1 & 1.6 & 6.3 & 14.1 & 100 \\
\hline & $20-24$ & a & 5.1 & 0.3 & 31.5 & 37.0 & 38.5 & 3.7 & 20.8 & 63.0 & 100 \\
\hline & $25-29$ & a & 5.2 & 0.2 & 4.8 & 10.2 & 51.3 & 3.7 & 34.8 & 89.8 & 100 \\
\hline Island & $15-19$ & 1.8 & 52.7 & 3.2 & 22.2 & 79.9 & 18.8 & 1.3 & 0.0 & 20.1 & 100 \\
\hline 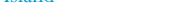 & $20-24$ & 5.5 & 30.6 & 1.1 & 15.3 & 52.4 & 42.6 & 1.6 & 3.3 & 47.6 & 100 \\
\hline & $25-29$ & 4.1 & 23.4 & $\mathrm{n}$ & 12.3 & 39.8 & 52.0 & 1.8 & 6.4 & 60.2 & 100 \\
\hline Irland & $15-19$ & $\mathrm{a}$ & 10.7 & 0.5 & 74.3 & 85.6 & 10.5 & 1.4 & 2.6 & 14.4 & 100 \\
\hline & $20-24$ & a & 6.1 & 0.3 & 25.4 & 31.8 & 56.2 & 3.0 & 9.0 & 68.2 & 100 \\
\hline & $25-29$ & $\mathrm{a}$ & 0.5 & $\mathrm{n}$ & 2.8 & 3.4 & 77.1 & 2.4 & 17.1 & 96.6 & 100 \\
\hline Italien & $15-19$ & $\mathrm{n}$ & 0.7 & 1.0 & 78.1 & 79.8 & 7.7 & 4.7 & 7.8 & 20.2 & 100 \\
\hline & $20-24$ & $\mathrm{n}$ & 3.3 & 2.1 & 36.7 & 42.3 & 30.4 & 11.9 & 15.4 & 57.7 & 100 \\
\hline & $25-29$ & $\mathrm{n}$ & 3.8 & 1.4 & 13.8 & 19.0 & 47.0 & 10.2 & 23.8 & 81.0 & 100 \\
\hline Luxemburg & 15-19 & 2.9 & 1.4 & $\mathrm{n}$ & 86.7 & 91.1 & 6.8 & 0.4 & 1.6 & 8.9 & 100 \\
\hline & $20-24$ & 1.9 & 4.8 & 0.2 & 40.3 & 47.3 & 41.8 & 2.7 & 8.1 & 52.7 & 100 \\
\hline & $25-29$ & $\mathrm{n}$ & 3.7 & $\mathrm{n}$ & 5.1 & 9.2 & 71.3 & 1.5 & 18.0 & 90.8 & 100 \\
\hline Mexiko & $15-19$ & $\mathrm{a}$ & 4.8 & 0.3 & 45.2 & 50.3 & 21.4 & 1.3 & 27.0 & 49.7 & 100 \\
\hline & $20-24$ & a & 3.7 & 0.2 & 13.6 & 17.5 & 36.4 & 1.6 & 44.5 & 82.5 & 100 \\
\hline & $25-29$ & $\mathrm{a}$ & 1.2 & $\mathrm{n}$ & 2.2 & 3.5 & 42.3 & 1.2 & 53.0 & 96.5 & 100 \\
\hline Niederlande $^{2}$ & $15-19$ & $\begin{array}{l}\mathrm{a} \\
\mathrm{m}\end{array}$ & 40.2 & 5.9 & 36.6 & 82.7 & 13.8 & 1.2 & 2.2 & 17.3 & 100 \\
\hline 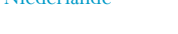 & $20-24$ & m & 22.0 & 1.3 & 12.1 & 35.4 & 53.4 & 2.3 & 8.9 & 64.6 & 100 \\
\hline & $25-29$ & $\mathrm{~m}$ & 2.5 & 0.1 & 1.9 & 4.5 & 77.3 & 3.1 & 15.2 & 95.5 & 100 \\
\hline Norwegen $^{2}$ & $15-19$ & $\mathrm{a}$ & 26.1 & 6.6 & 55.3 & 88.0 & 9.1 & 1.7 & 1.2 & 12.0 & 100 \\
\hline & $20-24$ & a & 11.9 & 2.5 & 18.3 & 32.7 & 60.2 & 4.3 & 2.8 & 67.3 & 100 \\
\hline & $25-29$ & a & 6.5 & 1.4 & 8.5 & 16.4 & 75.3 & 3.8 & 4.4 & 83.6 & 100 \\
\hline Polen & $15-19$ & a & 3.3 & 1.3 & 88.2 & 92.8 & 1.8 & 2.8 & 2.5 & 7.2 & 100 \\
\hline & $20-24$ & $\mathrm{a}$ & 9.4 & 6.6 & 31.4 & 47.4 & 24.1 & 17.3 & 11.2 & 52.6 & 100 \\
\hline & $25-29$ & a & 7.0 & 1.6 & 3.2 & 11.9 & 49.6 & 16.5 & 22.0 & 88.1 & 100 \\
\hline Portugal & $15-19$ & a & 3.0 & 0.5 & 71.0 & 74.5 & 15.5 & 4.1 & 5.9 & 25.5 & 100 \\
\hline & $20-24$ & $\mathrm{a}$ & 6.2 & 0.8 & 34.0 & 41.0 & 46.0 & 5.9 & 7.1 & 59.0 & 100 \\
\hline & $25-29$ & $\mathrm{a}$ & 6.1 & 0.5 & 4.0 & 10.6 & 73.0 & 4.8 & 11.5 & 89.4 & 100 \\
\hline Slowakische Rep. & $15-19$ & 7.4 & 0.1 & $\mathrm{n}$ & 58.9 & 66.5 & 8.6 & 11.3 & 13.6 & 33.5 & 100 \\
\hline & 2024 & $\mathrm{a}$ & 0.5 & 0.6 & 21.2 & 22.4 & 43.8 & 16.9 & 16.9 & 77.6 & 100 \\
\hline & 27 & $\mathrm{a}$ & 0.2 & $\mathrm{n}$ & 2.0 & 2.2 & 57.2 & 13.8 & 26.9 & 97.8 & 100 \\
\hline Spanien & $15-19$ & 0.3 & 2.7 & 1.9 & 76.9 & 81.8 & 8.7 & 5.5 & 4.0 & 18.2 & 100 \\
\hline & & 0.7 & 7.2 & 3.2 & 38.2 & 49.3 & 32.8 & 10.0 & 7.9 & 50.7 & 100 \\
\hline & $0-24$ & 0.4 & 6.9 & 2.7 & 8.5 & 18.4 & 53.8 & 10.0 & 17.9 & 81.6 & 100 \\
\hline Schweden & $15-19$ & a & 20.7 & 5.2 & 60.3 & 86.1 & 10.1 & 1.9 & 1.8 & 13.9 & 100 \\
\hline & $20-24$ & a & 12.8 & 2.1 & 31.4 & 46.3 & 43.6 & 4.3 & 5.8 & 53.7 & 100 \\
\hline & $25-29$ & $\mathrm{a}$ & 10.8 & 1.1 & 13.1 & 25.0 & 66.1 & 2.8 & 6.1 & 75.0 & 100 \\
\hline Schweiz & $15-19$ & 30.4 & 11.5 & $\mathrm{~m}$ & 39.0 & 84.5 & 8.3 & $\mathrm{~m}$ & 6.7 & 15.5 & 100 \\
\hline & $20-24$ & 8.6 & 11.8 & $\mathrm{~m}$ & 15.7 & 36.2 & 56.3 & $\mathrm{~m}$ & $\mathrm{~m}$ & 63.8 & 100 \\
\hline & & $\mathrm{m}$ & 5.3 & $\mathrm{~m}$ & $\mathrm{~m}$ & 10.5 & 71.0 & $\mathrm{~m}$ & 16.1 & 89.5 & 100 \\
\hline Türkei & $15-19$ & $\mathrm{a}$ & 0.8 & 33.2 & 0.2 & 34.2 & 16.1 & 3.5 & 46.2 & 65.8 & 100 \\
\hline & $20-24$ & $\mathrm{a}$ & 1.4 & 7.4 & 0.6 & 9.4 & 25.9 & 5.8 & 59.0 & 90.6 & 100 \\
\hline & & $\mathrm{a}$ & 1.0 & 1.1 & 0.2 & 2.3 & 26.7 & 3.5 & 67.5 & 97.7 & 100 \\
\hline Ver. Königreich & $15-19$ & 3.2 & 29.6 & 2.7 & 36.1 & 71.5 & 18.5 & 3.9 & 6.1 & 28.5 & 100 \\
\hline & $20-24$ & 2.9 & 15.1 & 0.8 & 15.1 & 33.9 & 46.9 & 3.9 & 15.2 & 66.1 & 100 \\
\hline & $25-29$ & 1.3 & 9.9 & 0.5 & 4.0 & 15.8 & 61.4 & 2.9 & 19.9 & 84.2 & 100 \\
\hline Vereinigte Staaten ${ }^{2}$ & $15-19$ & $\mathrm{a}$ & 10.4 & 4.1 & 63.8 & 78.3 & 12.8 & 2.8 & 6.1 & 21.7 & 100 \\
\hline & $20-24$ & a & 18.9 & 0.9 & 11.9 & 31.7 & 49.3 & 3.5 & 15.5 & 68.3 & 100 \\
\hline & $25-29$ & a & 7.3 & $\mathrm{n}$ & 3.7 & 11.1 & 66.3 & 3.0 & 19.6 & 88.9 & 100 \\
\hline Ländermittel & $15-19$ & $4.0^{\circ}$ & 12.7 & 3.4 & 60.6 & 80.8 & 9.9 & 2.7 & 6.6 & 19.2 & 100 \\
\hline & $20-24$ & 2.0 & 10.6 & 1.7 & 24.3 & 38.6 & 42.0 & 6.0 & 13.1 & 61.4 & 100 \\
\hline & $25-29$ & 0.4 & $\begin{array}{l}10.5 \\
6.5\end{array}$ & 0.6 & 5.5 & 13.3 & 60.2 & $\begin{array}{l}0.0 \\
5.7\end{array}$ & $\begin{array}{l}20.1 \\
20.8\end{array}$ & $\begin{array}{l}86.7 \\
86\end{array}$ & 100 \\
\hline
\end{tabular}

1. Schülerinnen in dualen Ausbildungsgängen werden sowohl der Kategorie 'in Ausbildung' als auch der Kategorie 'in Beschäftigung' zugeordnet, ungeachtet ihres Beschäftigungsstatus gemäß der ILO-Definition.

2. Referenzjahr 2000 .

Quelle: OECD. Länderspezifische Datenquellen s. Anhang 3 (www.oecd.org/els/education/eag2002). 
Tabelle C5.2.

Anteil arbeitsloser Nicht-Schüler bzw. Nicht-Studierender an der Gesamtbevölkerung, nach Bildungsstand, Altersgruppen und Geschlecht (2001)

\begin{tabular}{|c|c|c|c|c|c|c|c|c|c|c|c|c|}
\hline & & Unterha & Sekund & ereich II & $\begin{array}{l}\text { Sekund } \\
\text { sekund }\end{array}$ & $\begin{array}{l}\text { ereich I } \\
\text { er, nich } \\
\text { Bereich }\end{array}$ & $\begin{array}{l}\text { nd post- } \\
\text { rtiärer }\end{array}$ & Tertiä & ereich & & lle Bildu & sbereicl \\
\hline & & $15-19$ & $20-24$ & $25-29$ & $15-19$ & $20-24$ & $25-29$ & $20-24$ & $25-29$ & $15-19$ & $20-24$ & $25-29$ \\
\hline & Frauen & 3.9 & 14.7 & 6.6 & 4.2 & 6.3 & 4.5 & 1.7 & 1.5 & 4.0 & 6.0 & 3.7 \\
\hline & $\mathrm{M}+\mathrm{F}$ & 5.8 & 16.1 & 8.7 & 3.7 & 7.3 & 4.3 & 1.7 & 2.1 & 5.0 & 6.9 & 4.5 \\
\hline Österreich & Männer & 9.1 & 11.8 & 5.4 & 0.6 & 4.2 & 3.4 & 0.2 & 1.9 & 2.3 & 4.3 & 3.4 \\
\hline & Frauen & 11.3 & 5.2 & 4.0 & 0.6 & 3.3 & 2.9 & 0.3 & 0.8 & 2.4 & 2.6 & 2.7 \\
\hline & $\mathrm{M}+\mathrm{F}$ & 10.1 & 8.4 & 4.6 & 0.6 & 3.8 & 3.2 & 0.2 & 1.4 & 2.4 & 3.4 & 3.0 \\
\hline & Frauen & 1.2 & 14.2 & 13.0 & 1.7 & 5.0 & 10.2 & 5.6 & 4.4 & 1.3 & 6.4 & 8.4 \\
\hline & $\mathrm{M}+\mathrm{F}$ & 1.8 & 16.5 & 11.9 & 1.8 & 4.3 & 7.6 & 6.6 & 4.6 & 1.8 & 6.9 & 7.4 \\
\hline Kanada & Männer & 2.7 & 17.1 & 15.5 & 6.1 & 7.7 & 7.5 & 5.1 & 4.7 & 3.5 & 8.6 & 7.2 \\
\hline & Frauen & 1.4 & 9.3 & 6.0 & 2.6 & 4.0 & 6.5 & 2.6 & 3.7 & 1.7 & 4.0 & 4.9 \\
\hline & $\mathrm{M}+\mathrm{F}$ & 2.1 & 14.2 & 11.4 & 4.3 & 6.0 & 7.1 & 3.6 & 4.1 & 2.7 & 6.3 & 6.1 \\
\hline Tschechische Rep. & Männer & 9.1 & 33.0 & 19.7 & 2.9 & 10.5 & 5.7 & 1.5 & 1.9 & 4.2 & 10.5 & 6.2 \\
\hline & Frauen & 7.6 & 18.7 & 18.7 & 3.5 & 9.2 & 8.1 & 1.4 & 1.7 & 4.2 & 8.1 & 8.3 \\
\hline & $\mathrm{M}+\mathrm{F}$ & 8.5 & 26.5 & 19.2 & 3.2 & 9.9 & 6.9 & 1.4 & 1.8 & 4.2 & 9.3 & 7.2 \\
\hline Dänemark & Männer & 0.4 & 4.6 & 5.7 & $\mathrm{~m}$ & 2.6 & 1.8 & 1.8 & 1.6 & 0.4 & 3.3 & 2.3 \\
\hline & Frauen & 1.9 & 3.6 & 1.8 & $\mathrm{~m}$ & 1.7 & 0.6 & 7.9 & 3.9 & 2.0 & 2.6 & 1.5 \\
\hline & $\mathrm{M}+\mathrm{F}$ & 1.2 & 4.1 & 3.7 & 6.7 & 2.2 & 1.1 & 5.0 & 2.6 & 1.2 & 2.9 & 1.9 \\
\hline & Frauen & 0.7 & 5.8 & 15.4 & 7.7 & 5.1 & 8.5 & 4.1 & 6.0 & 1.6 & 5.0 & 8.1 \\
\hline & $\mathrm{M}+\mathrm{F}$ & 1.4 & 9.7 & 12.3 & 6.7 & 5.7 & 6.2 & 4.9 & 4.3 & 2.1 & 6.1 & 6.3 \\
\hline Frankreich & Männer & 1.8 & 20.0 & 15.3 & 3.0 & 5.5 & 7.3 & 2.3 & 5.6 & 1.9 & 8.2 & 8.3 \\
\hline 11 & Frauen & 1.5 & 17.4 & 16.4 & 4.1 & 8.0 & 10.7 & 3.6 & 6.1 & 1.8 & 8.7 & 9.9 \\
\hline & $\mathrm{M}+\mathrm{F}$ & 1.6 & 18.9 & 15.9 & 3.6 & 6.7 & 8.9 & 3.1 & 5.9 & 1.9 & 8.4 & 9.1 \\
\hline Deutschland & Männer & 2.5 & 18.6 & 17.1 & 0.5 & 6.4 & 6.7 & 0.4 & 1.5 & 1.5 & 7.1 & 6.7 \\
\hline & Frauen & 2.0 & 10.9 & 7.4 & 0.6 & 3.6 & 5.0 & 0.7 & 2.4 & 1.3 & 4.1 & 4.7 \\
\hline & $\mathrm{M}+\mathrm{F}$ & 2.3 & 14.7 & 12.0 & 0.5 & 5.2 & 5.8 & 0.5 & 1.9 & 1.4 & 5.7 & 5.7 \\
\hline Griechenland & Männer & 2.5 & 14.3 & 9.0 & 5.4 & 11.2 & 10.6 & 9.2 & 12.8 & 3.2 & 11.8 & 10.5 \\
\hline & Frauen & 2.9 & 18.7 & 13.9 & 9.3 & 14.4 & 14.4 & 27.3 & 16.8 & 4.7 & 16.0 & 14.9 \\
\hline & $\mathrm{M}+\mathrm{F}$ & 2.7 & 16.1 & 10.9 & 7.5 & 13.0 & 12.5 & 20.3 & 15.2 & 3.9 & 14.0 & 12.7 \\
\hline Ungarn & Männer & 1.7 & 14.4 & 15.2 & 6.4 & 6.0 & 6.0 & 6.0 & 0.3 & 2.6 & 7.3 & 7.0 \\
\hline Uinganit & Frauen & 0.8 & 5.1 & 5.7 & $\begin{array}{l}0.7 \\
4.4\end{array}$ & 3.6 & 4.0 & $\begin{array}{l}0.0 \\
2.3\end{array}$ & 0.5 & $\begin{array}{l}2.0 \\
1.6\end{array}$ & 3.7 & 3.7 \\
\hline & $\mathrm{M}+\mathrm{F}$ & 1.3 & 9.7 & 10.3 & 5.3 & 4.8 & 5.0 & 3.8 & 0.4 & 2.1 & 5.5 & 5.3 \\
\hline Island & Männer & 1.3 & 1.2 & 3.6 & a & 2.2 & a & a & 2.2 & 1.3 & 1.6 & 1.8 \\
\hline & Frauen & 2.0 & 3.2 & 1.4 & a & a & a & 20.0 & 2.6 & 2.0 & 2.4 & 1.0 \\
\hline & $\mathrm{M}+\mathrm{F}$ & 1.7 & 2.3 & 2.4 & $\mathrm{a}$ & 1.2 & a & 7.8 & 2.3 & 1.6 & 2.0 & 1.4 \\
\hline Irland & Männer & 2.3 & 10.0 & 7.3 & 2.3 & 2.0 & 2.8 & 2.1 & 1.4 & 2.3 & 3.7 & 3.3 \\
\hline & Frauen & 1.2 & 5.6 & 4.6 & 1.7 & 2.8 & 2.5 & 2.3 & 1.4 & 1.3 & 3.0 & 2.4 \\
\hline & $\mathrm{M}+\mathrm{F}$ & 1.8 & 8.3 & 6.1 & 1.9 & 2.4 & 2.7 & 2.3 & 1.4 & 1.8 & 3.3 & 2.8 \\
\hline Italien & Männer & 4.7 & 15.5 & 11.0 & 8.0 & 9.6 & 8.2 & 13.9 & 10.9 & 5.0 & 11.7 & 9.5 \\
\hline & Frauen & 4.0 & 15.5 & 10.2 & 9.5 & 10.5 & 9.2 & 17.9 & 14.2 & 4.7 & 11.9 & 10.2 \\
\hline & $\mathrm{M}+\mathrm{F}$ & 0.8 & 4.8 & 2.8 & $\mathrm{a}$ & 2.5 & 0.3 & 3.5 & 2.3 & 0.7 & 3.5 & 1.8 \\
\hline Mexiko & Männer & 1.9 & 2.6 & 1.8 & 0.9 & 5.9 & 4.2 & 2.0 & 2.8 & 1.9 & 2.5 & 2.1 \\
\hline & Frauen & 1.2 & 1.4 & 1.0 & 4.2 & 2.1 & 1.2 & 2.1 & 2.3 & 1.3 & 1.6 & 1.2 \\
\hline & $\mathrm{M}+\mathrm{F}$ & 1.5 & 2.0 & 1.4 & 3.1 & 3.1 & 1.8 & 2.0 & 2.5 & 1.6 & 2.0 & 1.6 \\
\hline Niederlande ${ }^{1}$ & Männer & 1.8 & 4.0 & 3.4 & 0.6 & 1.9 & 1.1 & 0.4 & 2.1 & 1.1 & 1.9 & 2.0 \\
\hline 1vicuer Iande & Frauen & $\begin{array}{l}1.0 \\
2.5\end{array}$ & 5.1 & 5.2 & 0.7 & 2.0 & 2.5 & $\begin{array}{l}0.7 \\
1.2\end{array}$ & 2.3 & 1.2 & 2.3 & 3.1 \\
\hline & $\mathrm{M}+\mathrm{F}$ & 2.1 & 4.5 & 4.2 & 0.7 & 2.0 & 1.8 & 0.8 & 2.2 & 1.2 & 2.1 & 2.5 \\
\hline Norwegen $^{1}$ & Männer & 3.1 & 9.0 & 6.7 & 1.1 & 4.7 & 4.1 & $\begin{array}{r}0.0 \\
n\end{array}$ & 2.7 & 1.7 & 4.3 & 3.8 \\
\hline & Frauen & 1.3 & 8.9 & 7.1 & $\mathrm{n}$ & 2.5 & 3.1 & $\mathrm{n}$ & 1.2 & 0.5 & 2.2 & 2.5 \\
\hline & $M+F$ & 2.2 & 8.9 & 6.9 & 0.6 & 3.7 & 3.7 & $\mathrm{n}$ & 1.8 & 1.1 & 3.3 & 3.2 \\
\hline Polen & Männer & 7.3 & 39.1 & 25.9 & 3.2 & 27.9 & 16.1 & 1.0 & 6.1 & 4.0 & 20.6 & 15.0 \\
\hline & Frauen & 4.7 & 31.6 & 27.5 & 2.7 & 28.5 & 18.8 & 1.5 & 7.8 & 3.0 & 17.3 & 16.5 \\
\hline & $\mathrm{M}+\mathrm{F}$ & 6.1 & 36.1 & 26.6 & 2.9 & 28.2 & 17.4 & 1.3 & 7.1 & 3.5 & 18.9 & 15.7 \\
\hline Portugal & Männer & 2.7 & 6.0 & 2.9 & 0.3 & 4.2 & 1.2 & 0.4 & 2.2 & 1.7 & 4.3 & 2.5 \\
\hline & Frauen & 8.1 & 8.3 & 5.6 & 1.2 & 7.4 & 4.3 & 2.6 & 3.7 & 4.5 & 5.9 & 4.9 \\
\hline & $\mathrm{M}+\mathrm{F}$ & 5.1 & 6.9 & 4.2 & 0.8 & 5.9 & 2.7 & 1.8 & 3.0 & 3.0 & 5.1 & 3.7 \\
\hline Slowakische Rep. & Männer & 3.7 & 50.7 & 43.8 & 32.2 & 27.3 & 19.5 & 17.5 & 12.4 & 10.6 & 28.4 & 20.0 \\
\hline & Frauen & 1.5 & 19.8 & 19.3 & 37.1 & 16.7 & 15.0 & 17.9 & 4.8 & 11.3 & 16.9 & 13.8 \\
\hline & $\mathrm{M}+\mathrm{F}$ & 2.6 & 37.5 & 30.0 & 34.8 & 22.1 & 17.4 & 17.7 & 8.2 & 11.0 & 22.8 & 16.9 \\
\hline Spanien & Männer & 10.7 & 13.1 & 9.1 & 1.5 & 8.2 & 8.5 & 2.8 & 5.4 & 6.2 & 7.6 & 7.6 \\
\hline & Frauen & 14.8 & 18.7 & 13.9 & 2.0 & 13.7 & 10.7 & 5.5 & 8.0 & 7.1 & 10.5 & 10.6 \\
\hline & $\mathrm{M}+\mathrm{F}$ & $\begin{array}{l}14.0 \\
12.3\end{array}$ & $\begin{array}{l}10.1 \\
15.3\end{array}$ & 11.1 & $\begin{array}{l}2.0 \\
1.7\end{array}$ & 10.8 & 9.6 & 4.2 & $\begin{array}{l}0.0 \\
6.8\end{array}$ & 6.6 & 9.0 & $\begin{array}{r}10.0 \\
9.0\end{array}$ \\
\hline Schweden & Männer & 18.6 & 17.5 & 9.8 & 1.0 & 6.4 & 4.1 & 0.1 & 0.5 & 1.9 & 5.9 & 3.7 \\
\hline & Frauen & 21.2 & 12.2 & 9.0 & 1.1 & 5.4 & 3.3 & 0.8 & 0.8 & 2.0 & 4.4 & 2.9 \\
\hline & $\mathrm{M}+\mathrm{F}$ & 19.8 & 15.2 & 9.5 & 1.0 & 5.9 & 3.7 & 0.5 & 0.7 & 1.9 & 5.2 & 3.3 \\
\hline Schweiz & Männer & $\mathrm{m}$ & $\mathrm{m}$ & $\mathrm{m}$ & $\mathrm{m}$ & $\mathrm{m}$ & $\mathrm{m}$ & $\mathrm{m}$ & $\mathrm{m}$ & $\mathrm{m}$ & $\mathrm{m}$ & $\mathrm{m}$ \\
\hline & Frauen & $\mathrm{m}$ & $\mathrm{m}$ & $\mathrm{m}$ & $\mathrm{m}$ & $\mathrm{m}$ & $\mathrm{m}$ & $\mathrm{m}$ & $\mathrm{m}$ & $\mathrm{m}$ & $\mathrm{m}$ & $\mathrm{m}$ \\
\hline & $M+F$ & $\mathrm{~m}$ & $\mathrm{~m}$ & $\mathrm{~m}$ & $\mathrm{~m}$ & $\mathrm{~m}$ & $\mathrm{~m}$ & $\mathrm{~m}$ & $\mathrm{~m}$ & $\mathrm{~m}$ & $\mathrm{~m}$ & $\mathrm{~m}$ \\
\hline Türkei & Männer & 6.7 & 13.6 & 10.8 & 11.4 & 10.0 & 11.4 & 23.3 & 7.6 & 7.8 & 12.6 & 10.5 \\
\hline & Frauen & 2.4 & 2.7 & 2.1 & 8.5 & 8.5 & 6.4 & 25.1 & 8.4 & 3.5 & 5.8 & 3.5 \\
\hline & $\mathrm{M}+\mathrm{F}$ & 4.6 & 7.6 & 6.3 & 10.2 & 9.4 & 9.6 & 24.3 & 7.9 & 5.8 & 9.2 & 7.3 \\
\hline Ver. Königreich & Männer & 10.4 & 15.2 & 13.9 & 5.9 & 5.8 & 4.1 & 3.2 & 2.0 & 7.0 & 6.1 & 4.3 \\
\hline & Frauen & 4.3 & 7.2 & 6.3 & 3.8 & 3.9 & 3.0 & 2.7 & 1.4 & 3.9 & 3.9 & 2.8 \\
\hline & $\mathrm{M}+\mathrm{F}$ & 7.6 & 11.3 & 10.1 & 4.9 & 4.9 & 3.5 & 3.0 & 1.7 & 5.5 & 5.0 & 3.5 \\
\hline Vereinigte Staaten ${ }^{1}$ & Männer & 8.5 & 10.7 & 5.3 & 1.4 & 4.0 & 3.3 & 3.3 & 2.0 & 2.6 & 4.7 & 3.1 \\
\hline & Frauen & 9.3 & 6.9 & 8.7 & 1.4 & 3.3 & 3.1 & 1.7 & 1.0 & 2.5 & 3.4 & 3.0 \\
\hline & $M+F$ & 8.9 & 9.0 & 7.0 & 1.4 & 3.6 & 3.2 & 2.4 & 1.4 & 2.5 & 4.0 & 3.0 \\
\hline Ländermittel & Männer & 4.7 & 14.5 & 10.8 & 3.9 & 7.2 & 5.6 & 4.3 & 3.7 & 3.2 & 7.5 & 5.9 \\
\hline & Frauen & 4.2 & 10.3 & 8.7 & 4.2 & 6.4 & 5.9 & 6.0 & 4.0 & 2.9 & 6.0 & 5.6 \\
\hline & $M+F$ & 4.4 & 12.6 & 9.6 & 4.3 & 6.8 & 5.7 & 5.2 & 3.9 & 3.0 & 6.7 & 5.7 \\
\hline
\end{tabular}

1. Referenzjahr 2000.

Quelle: OECD. Länderspezifische Datenquellen s. Anhang 3 (www.oecd.org/els/education/eag2002). 


\section{DIE SITUATION JUNGER MENSCHEM MIT EINEM NIEDRIGEN BILDUNGSNIVEAU}

- Die meisten Jugendlichen im Alter zwischen 15 und 19 Jahren gehen noch zur Schule. In vielen OECDLändern ist ein hoher Prozentsatz derer, der die Schule nicht besuchen, entweder arbeitslos oder nicht Teil der Erwerbsbevölkerung.

- In Italien, Mexiko, Österreich, der Slowakischen Republik, der Türkei und dem Vereinigten Königreich sind über 10 Prozent der 15- bis 19-Jährigen weder in Ausbildung noch Teil der Erwerbsbevölkerung.

- Von diesem Problem sind in Dänemark, Finnland, Österreich, Schweden und der Slowakischen Republik hauptsächlich junge Männer betroffen, in Griechenland, Mexiko, Portugal und der Türkei hauptsächlich junge Frauen.

\section{Abbildung C6.1.}

Prozentsatz 15- bis 19-Jähriger, die weder beschäftigt sind noch sich in Ausbildung befinden, nach Geschlecht (2001)

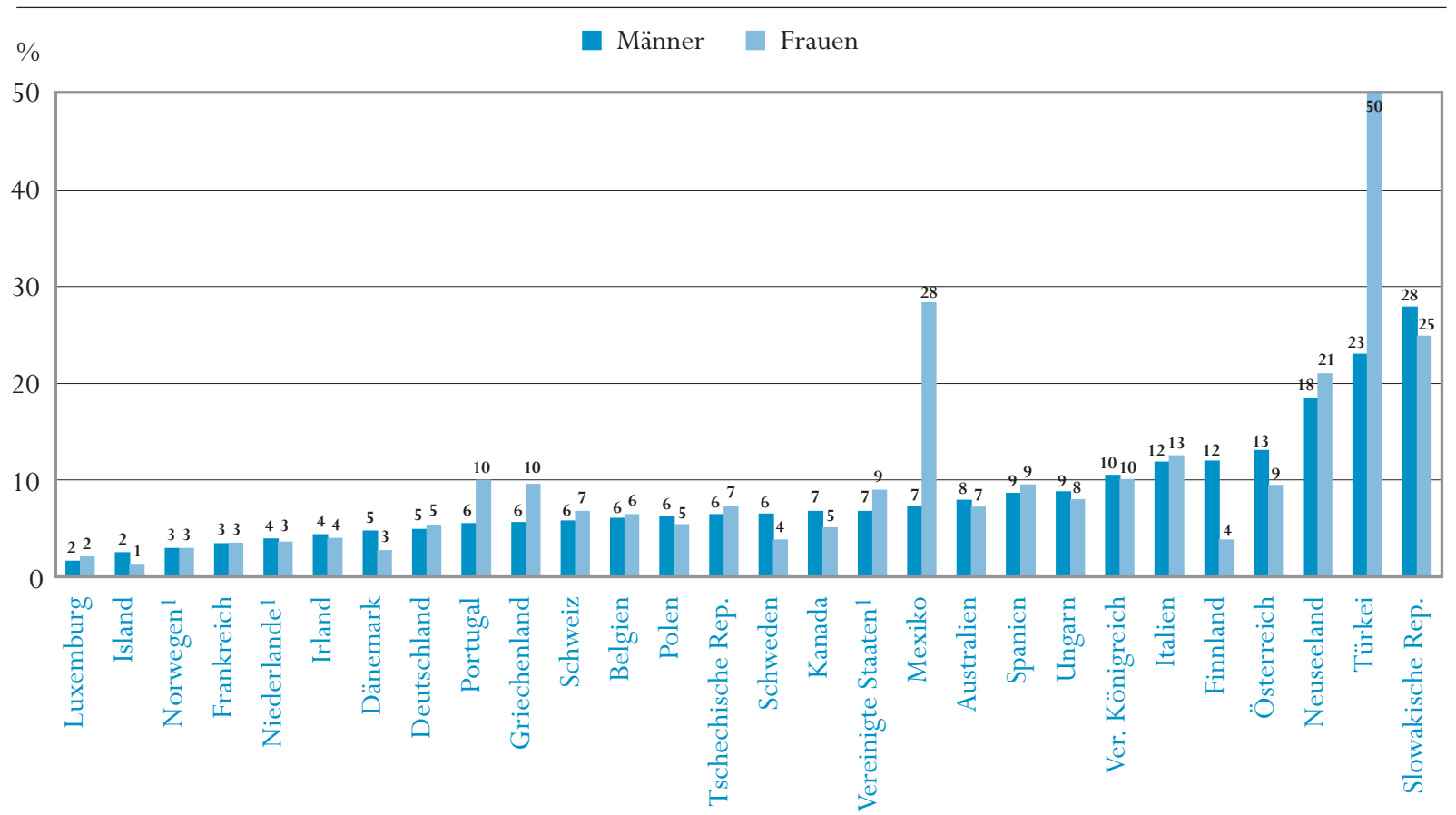

1. Referenzjahr 2000.

Anordnung der Länder in aufsteigender Reihenfolge der 15- bis 19-jährigen Männer, die weder beschäftigt sind noch sich in Ausbildung befinden.

Quelle: OECD. Tabellen C5.1a und C5.1b. Hinweise s. Anhang 3 (www.oecd.org/els/education/eag2002). 
Dieser Indikator beschäftigt sich mit der Lage junger Menschen, die sich nicht mehr in Ausbildung und noch nicht in Beschäftigung befinden.

Die meisten 15- bis 19 Jährigen befinden sich noch in der Schule. Von den übrigen ist in vielen Ländern ein hoher

Prozentsatz entweder arbeitslos oder nicht im Arbeitsmarkt.

\section{Politischer Hintergrund}

Der Eintritt in den Arbeitsmarkt stellt für junge Menschen oftmals eine schwierige Zeit des Übergangs dar. Während sich einerseits die Dauer der Zeit, die im Bildungssystem verbracht wird, erhöht hat, läuft andererseits ein beträchtlicher Teil der jungen Menschen Gefahr, ausgegrenzt zu werden, wenn sie sich weder in Ausbildung noch in Beschäftigung befinden, d.h. wenn sie arbeitslos oder nicht im Arbeitsmarkt sind. Dies ist besonders hinsichtlich der jüngeren Altersgruppen besorgniserregend, bei denen viele weder einen Arbeitslosenstatus noch eine andere soziale Absicherung haben (siehe A Caring World, OECD 1999).

In dem Maße, in dem die Wechselbeziehungen zwischen Bildung, Wirtschaft und dem Wohlstand der Nationen immer enger werden, wird es zu einer immer wichtigeren politischen Aufgabe, jungen Menschen einen erfolgreichen Bildungsweg und einen gelungenen Übergang von der Erstausbildung in die Beschäftigung zu ermöglichen. Aufgrund stetig steigender Anforderungen an Kenntnissen und Fähigkeiten in den OECD-Ländern ist ein Abschluss des Sekundarbereich II zur Mindestvoraussetzung für einen erfolgreichen Eintritt in der Arbeitsmarkt und zur Grundlage eines lebenslangen Lernens geworden. Jugendliche mit geringeren Qualifikationen laufen erhöht Gefahr, langfristig arbeitslos zu werden, instabile oder unbefriedigende Beschäftigungsverhältnisse zu finden, was weitere negative Konsequenzen, wie beispielsweise soziale Ausgrenzung, mit sich bringen kann.

\section{Ergebnisse und Erläuterungen}

Junge Menschen, die sich weder in Ausbildung noch in Beschäftigung befinden

Im Alter von 15 bis 19 Jahren befinden sich in den meisten OECD-Ländern mehr als vier von fünf Jugendlichen im Bildungssystem. Ein kleiner Teil der Altersgruppe ist nach dem Schulabgang in Beschäftigung, obwohl dieser Anteil in einigen OECD-Ländern bei 10 bis 20 Prozent liegt (Tabelle C5.1).

Es gibt jedoch eine Gruppe junger Menschen, die sich in einer ungünstigen Situation befinden, da sie sich nicht mehr in Ausbildung befinden, aber noch keine Arbeit haben. Einige haben einen Arbeitslosenstatus, wenn sie aktiv nach einer Arbeitsstelle suchen, während andere, die dies aus verschiedenen Gründen nicht tun, als „sich nicht im Arbeitsmarkt befindend“ betrachtet werden. Ihre Gründe können unterschiedlichster Natur sein: z.B. Entmutigung aufgrund der Schwierigkeiten bei der Arbeitssuche oder freiwilliger Rückzug aus familiären Gründen, etc. In 18 von 27 OECD-Ländern ist der Anteil dieser jungen Menschen höher als der Anteil derjenigen mit einem Arbeitslosenstatus.

In Dänemark, Frankreich, Island, Luxemburg, den Niederlanden und Norwegen gibt es nur eine geringe Zahl von Personen, die sich weder in Ausbildung noch in Beschäftigung befinden. Dagegen ist diese Zahl in Ländern wie Italien, Mexiko, Österreich, der Slowakischen Republik, der Türkei und dem Vereinigten 
Königreich besorgniserregend hoch. In diesen OECD-Ländern sind über 10 Prozent der 15- bis 19-Jährigen in der Situation, sich weder in Ausbildung noch in Beschäftigung zu befinden (Tabelle C5.1). In anderen Ländern ist dieser Anteil zwar geringer, aber mit 4 bis 10 Prozent nicht zu vernachlässigen. Von diesem Problem sind in Dänemark, Finnland, Österreich, Schweden und der Slowakischen Republik hauptsächlich junge Männer betroffen, in Griechenland, Mexiko, Portugal und der Türkei hauptsächlich junge Frauen (Abbildung C6.1).

Jugendliche mit geringen Qualifikationen laufen eine erhöhte Gefahr, langfristig arbeitslos zu werden, instabile oder unbefriedigende Beschäftigungsverhältnisse zu finden, was weitere negative Konsequenzen, wie beispielsweise soziale Ausgrenzung, mit sich bringen kann. Daher ist der verfrühte Schulabgang zu einem der größten Probleme für die Bildungspolitik geworden. Bei den 20bis 24-Jährigen wächst das Ausmaß des Problems, aber es erfährt auch eine

Bei den 20- bis 24Jährigen wächst das Ausmafs des Problems, aber es erfährt auch eine qualitative Veränderung, da die meisten

\section{Abbildung C6.2.}

Prozentsatz 20- bis 24-Jähriger, die sich nicht in Ausbildung befinden und über keinen Abschluss im Sekundarbereich II verfügen, nach Geschlecht (2001)

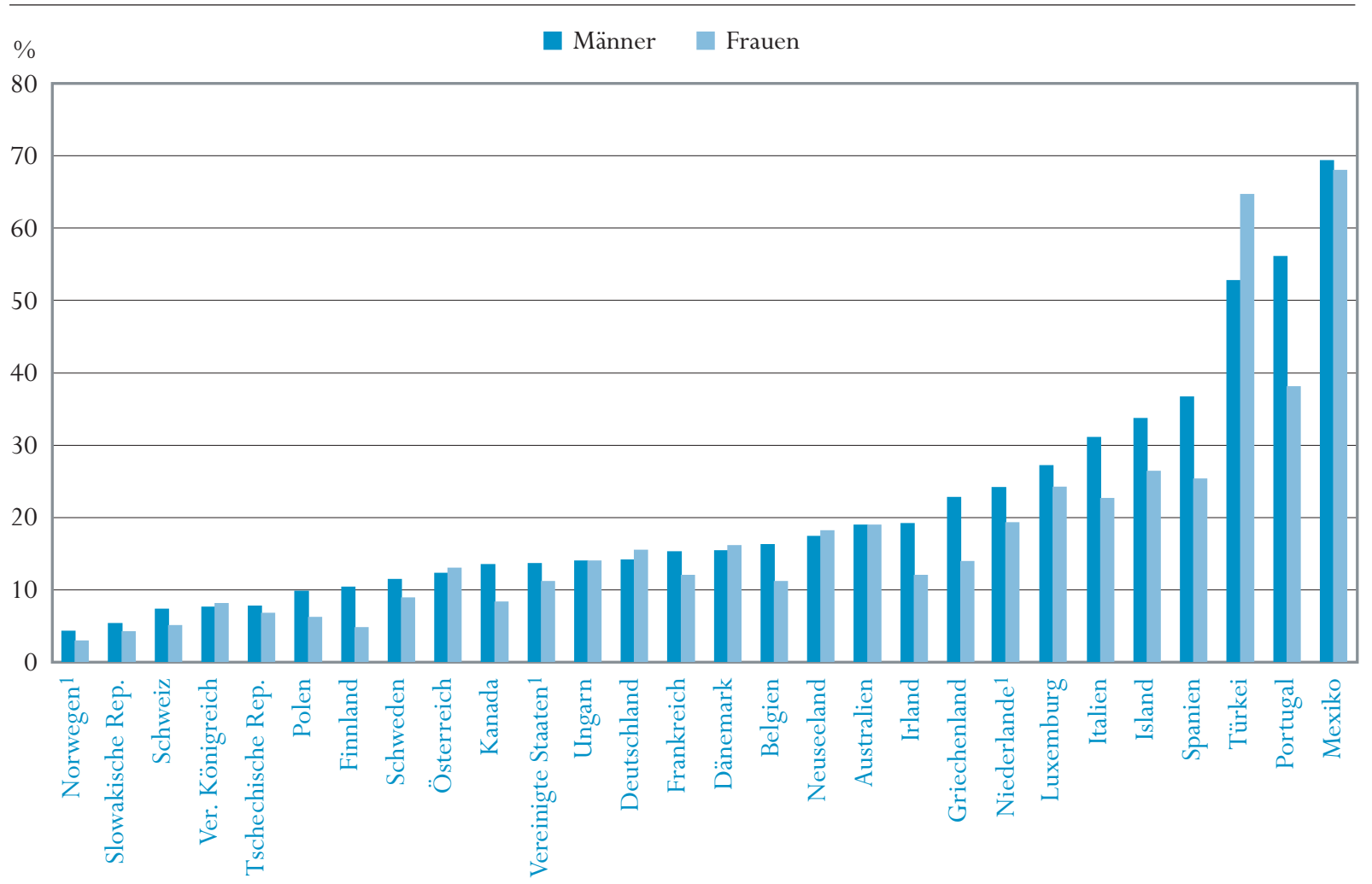

1. Referenzjahr 2000.

Anordnung der Länder in aufsteigender Reihenfolge des Prozentsatzes 20- bis 24-jähriger Männer, die sich nicht in Ausbildung befinden und über keinen Abschluss im Sekundarbereich II verfügen.

Quelle: OECD. Tabelle C6.1. Hinweise s. Anhang 3 (www.oecd.org/els/education/eag2002). 
jungen Menschen in

diesem Alter in den

Arbeitsmarkt eintreten.

qualitative Veränderung, da die meisten jungen Menschen in diesem Alter zum ersten Mal in den Arbeitsmarkt eintreten. Die meisten von ihnen haben ihre Erstausbildung soeben beendet. Der Eintritt in den Arbeitsmarkt geht oft mit einer Phase der Arbeitslosigkeit einher.

Die Länder lassen sich in drei Gruppen einteilen. In einer ersten Gruppe aus acht OECD-Ländern, zu denen die nordischen und die osteuropäischen Länder, jedoch auch die Schweiz und das Vereinigte Königreich gehören, liegt der Anteil der Jugendlichen ohne einen Abschluss des Sekundarbereich II unter 10 Prozent. Diese Gruppe befindet sich zweifellos in einer schwierigen Lage, aber die Schwierigkeiten sind begrenzt. In 12 von 27 OECD-Ländern gehören dieser potentiellen "Risikogruppe" zwischen 10 und 20 Prozent der Altersgruppe an. In diesen OECD-Ländern ist die Aufgabe, die Abschlussquoten im Sekundarbereich II zu steigern, von entscheidender Bedeutung. In der dritten Gruppe mit weiteren acht OECD-Ländern sind es über 20 Prozent der Jugendlichen, die dieser Kategorie zuzurechnen sind.

\section{Abbildung C6.3.}

Beschäftigungsquoten 20- bis 24-Jähriger, die sich nicht in Ausbildung befinden, nach Bildungsstand (2001)

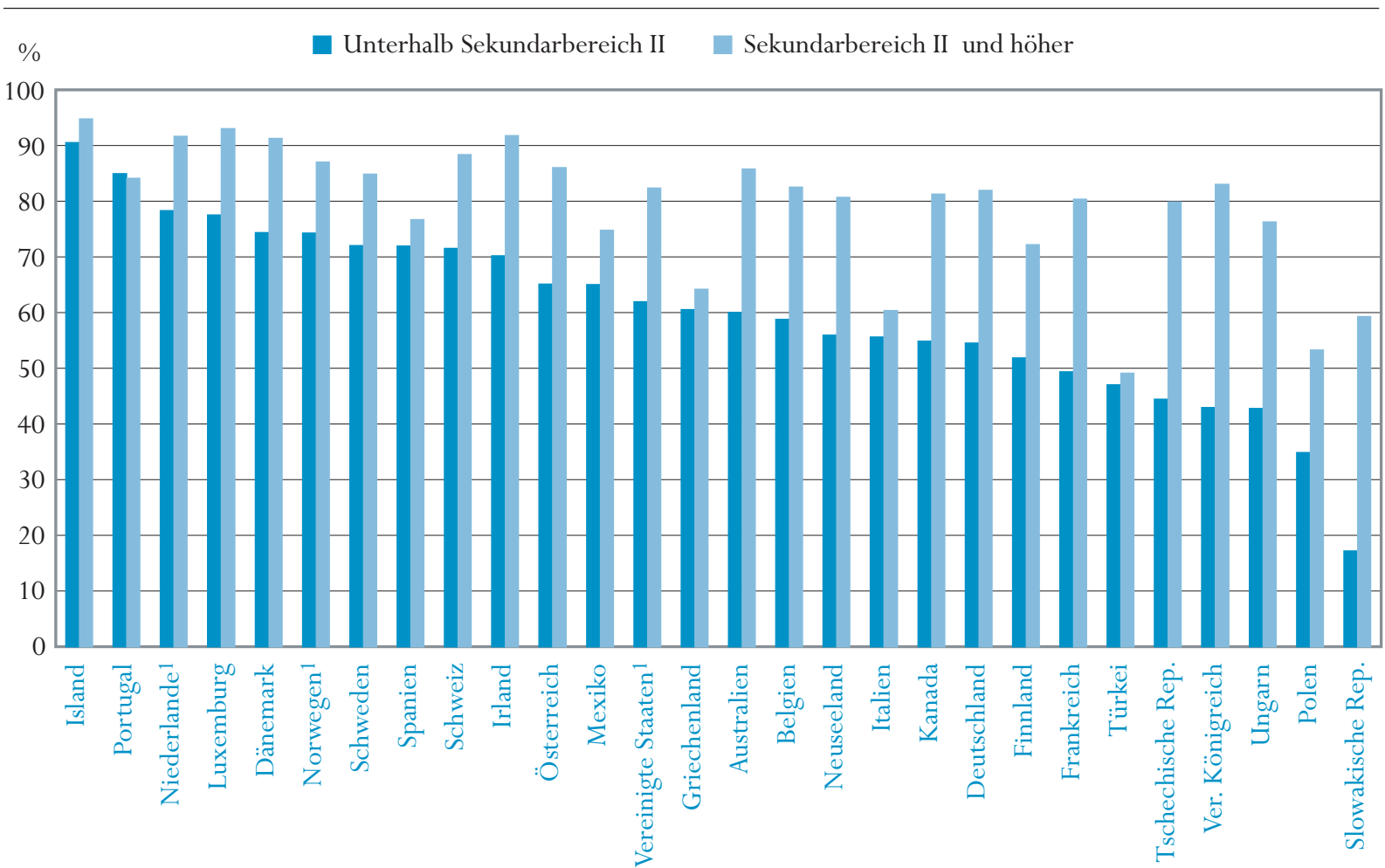

1. Referenzjahr 2000.

Anordnung der Länder in absteigender Reihenfolge der Beschäftigungsquote 20- bis 24-Jähriger, die sich nicht in Ausbildung befinden und über keinen Abschluss im Sekundarbereich II verfügen.

Quelle: OECD. Tabelle C6.1. Hinweise s. Anhang 3 (www.oecd.org/els/education/eag2002). 
Die Folgen eines Schulabgangs ohne einen Abschluss des Sekundarbereich II lassen sich anhand eines Vergleichs des Beschäftigungsstatus derjenigen mit und ohne einen solchen Abschluss erkennen. Mit einer Ausnahme geht in allen OECD-Ländern ein höherer Bildungsstand mit einer höheren Beschäftigungsquote einher, durchschnittlich steigt sie um 19 Prozentpunkte. Dieser Vergleich zeigt auch gewisse Charakteristika in Bezug auf die spezielle Organisation des Arbeitsmarktes. Die Kluft zwischen jungen Menschen mit einem Abschluss des Sekundarbereich II und ohne einen solchen ist in allen Mittelmeerländern bemerkenswert gering, was darauf hindeutet, dass hier die Qualifikationen - mögen sie auch gering sein - und die Beschäftigungsmöglichkeiten sehr gut aufeinander abgestimmt sind. Interessant ist die Situation im Vereinigten Königreich. Dort gibt es im Verhältnis zu allen OECD-Ländern die vergleichsweise niedrigsten Zahlen niedriger Bildungsabschlüsse, aber der Unterschied bei der Arbeitslosigkeit ist besonders hoch, was darauf hindeutet, dass die wenigen ohne einen Abschluss des Sekundarbereich II besonders benachteiligt sind.

\section{Definitionen und angewandte Methodik}

Die vorliegenden Statistiken basieren auf Arbeitskräfteerhebungen zu dem Anteil bestimmter Altersgruppen junger Menschen an den genannten Kategorien. Die Definitionen der verschiedenen Arten von Erwerbsstatus für diejenigen, die sich nicht in Ausbildung befinden (und nicht an einer dualen Ausbildung teilnehmen) basieren auf den Richtlinien des Internationalen Arbeitsamtes (ILO). Die Daten für diesen Indikator wurden aus der OECDDatenerhebung zum Übergang von der Schule ins Erwerbsleben berechnet (s. Indikator A12).

Ein „früher Schulabgänger” könnte grob definiert werden als „ein Jugendlicher, der keinen Bildungsgang auf Stufe ISCED 3 hat und sich weder in Ausbildung befindet noch eine duale Ausbildung absolviert, die zu einer Qualifikation gemäß ISCED 3 oder höher führen würde“. Diese Definition muss jedoch durch die Angabe einer Altersgruppe, in der nur noch sehr wenige Jugendliche den Primar- oder Sekundarbereich einer Schule besuchen können, weiter operationalisiert werden. In einer großen Anzahl von OECD-Ländern besuchen noch recht viele der 18- und 19-Jährigen diese Bildungsbereiche. Auch können junge Menschen, die sehr früh von der Schule abgehen, irgendwann wieder dorthin zurückkehren. Ferner könnten die Arbeitsmarktergebnisse für sehr junge Menschen nicht unbedingt repräsentativ für das Ergebnis in einem ,reiferen' Alter sein. Daher definiert die OECD einen jungen Erwachsenen mit einem geringen Bildungsstand als „einen 20- bis 24-Jährigen ohne einen Abschluss auf Stufe ISCED 3, der weder eine Bildungseinrichtung besucht noch eine duale Ausbildung absolviert". 
Tabelle C6.1.

Prozentsatz 20- bis 24-Jähriger, die sich nicht in Ausbildung befinden, nach Bildungsstand,

Geschlecht und Beschäftigungsstatus (2001)

\begin{tabular}{|c|c|c|c|c|c|c|c|c|c|c|c|}
\hline & & & rhalb Sekun & rbereich II & & & zundarbereic & II und höh & & & \\
\hline & & Beschäftigt & Arbeitslos & $\begin{array}{c}\text { Nicht im } \\
\text { Arbeits- } \\
\text { markt }\end{array}$ & $\begin{array}{c}\text { Zusam- } \\
\text { men }\end{array}$ & Beschäftigt & Arbeitslos & $\begin{array}{c}\text { Nicht im } \\
\text { Arbeits- } \\
\text { markt }\end{array}$ & $\begin{array}{c}\text { Zusam- } \\
\text { men }\end{array}$ & $\begin{array}{c}\text { In } \\
\text { Ausbildung }\end{array}$ & $\begin{array}{c}\text { 20- bis } \\
\text { 24-Jährige } \\
\text { insgesamt }\end{array}$ \\
\hline Australien & Männer & 13.4 & 4.0 & 1.6 & 18.9 & 37.1 & 3.9 & 2.0 & 43.0 & 38.1 & 100 \\
\hline & Frauen & 9.2 & 3.2 & 6.4 & 18.9 & 39.4 & 2.8 & 4.1 & 46.2 & 34.9 & 100 \\
\hline & $\mathrm{M}+\mathrm{F}$ & 11.3 & 3.6 & 4.0 & 18.9 & 38.2 & 3.3 & 3.0 & 44.6 & 36.5 & 100 \\
\hline Österreich & Männer & 8.6 & 1.4 & 2.2 & 12.2 & 52.6 & 2.8 & 8.0 & 63.5 & 24.3 & 100 \\
\hline & Frauen & 7.8 & 0.7 & 4.5 & 12.9 & 50.6 & 1.9 & 4.1 & 56.6 & 30.5 & 100 \\
\hline & $\mathrm{M}+\mathrm{F}$ & 8.2 & 1.1 & 3.3 & 12.6 & 51.6 & 2.4 & 6.0 & 60.0 & 27.4 & 100 \\
\hline Belgien & Männer & 10.9 & 3.9 & 1.5 & 16.2 & 35.0 & 3.5 & 2.0 & 40.5 & 43.3 & 100 \\
\hline & Frauen & 5.2 & 2.0 & 4.0 & 11.1 & 34.5 & 4.4 & 4.8 & 43.8 & 45.1 & 100 \\
\hline & $\mathrm{M}+\mathrm{F}$ & 8.0 & 2.9 & 2.7 & 13.7 & 34.8 & 4.0 & 3.4 & 42.1 & 44.2 & 100 \\
\hline Kanada & Männer & 8.4 & 2.7 & 2.4 & 13.5 & 40.6 & 5.9 & 3.4 & 49.9 & 36.6 & 100 \\
\hline & Frauen & 3.5 & 0.9 & 3.9 & 8.3 & 40.6 & 3.1 & 6.3 & 49.9 & 41.8 & 100 \\
\hline & $\mathrm{M}+\mathrm{F}$ & 6.0 & 1.8 & 3.1 & 10.9 & 40.6 & 4.5 & 4.8 & 49.9 & 39.1 & 100 \\
\hline Tschechische Rep. & Männer & 4.2 & 2.5 & 1.0 & 7.7 & 61.6 & 7.9 & 1.2 & 70.7 & 21.6 & 100 \\
\hline & Frauen & 2.2 & 1.3 & 3.3 & 6.7 & 49.5 & 6.9 & 12.3 & 68.6 & 24.6 & 100 \\
\hline & $\mathrm{M}+\mathrm{F}$ & 3.2 & 1.9 & 2.1 & 7.2 & 55.7 & 7.4 & 6.6 & 69.7 & 23.1 & 100 \\
\hline Dänemark & Männer & 13.6 & 1.2 & 0.5 & 15.4 & 32.1 & 1.4 & 0.7 & 34.2 & 50.4 & 100 \\
\hline & Frauen & 9.8 & 1.6 & 4.7 & 16.1 & 20.9 & 1.7 & 1.4 & 23.9 & 60.0 & 100 \\
\hline & $\mathrm{M}+\mathrm{F}$ & 11.7 & 1.4 & 2.6 & 15.7 & 26.4 & 1.5 & 1.0 & 29.0 & 55.3 & 100 \\
\hline & Frauen & 2.1 & 0.5 & 2.2 & 4.8 & 25.8 & 4.5 & 5.6 & 36.0 & 59.2 & 100 \\
\hline & $\mathrm{M}+\mathrm{F}$ & 3.9 & 1.2 & 2.5 & 7.6 & 27.8 & 5.0 & 5.8 & 38.6 & 53.9 & 100 \\
\hline Frankreich & Männer & 8.9 & 4.5 & 1.9 & 15.2 & 29.6 & 3.7 & 0.8 & 34.2 & 50.6 & 100 \\
\hline & Frauen & 4.5 & 3.3 & 4.2 & 12.0 & 23.0 & 5.4 & 2.9 & 31.4 & 56.7 & 100 \\
\hline & $\mathrm{M}+\mathrm{F}$ & 6.7 & 3.9 & 3.0 & 13.6 & 26.3 & 4.6 & 1.9 & 32.8 & 53.6 & 100 \\
\hline Deutschland & Männer & 9.1 & 2.8 & 2.2 & 14.1 & 44.0 & 4.4 & 4.4 & 52.8 & 33.1 & 100 \\
\hline & Frauen & 6.8 & 1.8 & 6.9 & 15.4 & 37.9 & 2.4 & 7.0 & 47.2 & 37.4 & 100 \\
\hline & $\mathrm{M}+\mathrm{F}$ & 8.0 & 2.3 & 4.4 & 14.7 & 41.1 & 3.4 & 5.6 & 50.1 & 35.2 & 100 \\
\hline Griechenland & Männer & 17.4 & 3.4 & 1.9 & 22.8 & 30.7 & 8.4 & 3.9 & 43.0 & 34.2 & 100 \\
\hline & Frauen & 5.1 & 2.8 & 6.0 & 13.9 & 27.5 & 13.2 & 6.5 & 47.2 & 38.9 & 100 \\
\hline & $\mathrm{M}+\mathrm{F}$ & 10.9 & 3.1 & 4.0 & 18.1 & 29.0 & 10.9 & 5.3 & 45.2 & 36.7 & 100 \\
\hline Ungarn & Männer & 7.8 & 2.2 & 3.9 & 13.9 & 43.9 & 5.1 & 4.5 & 53.4 & 32.7 & 100 \\
\hline & Frauen & 4.1 & 0.8 & 9.0 & 14.0 & 34.3 & 2.9 & 11.8 & 49.1 & 37.0 & 100 \\
\hline & $\mathrm{M}+\mathrm{F}$ & 6.0 & 1.5 & 6.5 & 14.0 & 39.0 & 4.0 & 8.2 & 51.2 & 34.8 & 100 \\
\hline Island & Männer & 31.8 & 1.9 & 0.0 & 33.7 & 16.8 & 0.5 & 0.5 & 17.7 & 48.6 & 100 \\
\hline & Frauen & 22.5 & 0.5 & 3.3 & 26.4 & 19.8 & 1.1 & 0.0 & 20.9 & 52.7 & 100 \\
\hline & $M+F$ & 27.3 & 1.3 & 1.6 & 30.1 & 18.3 & 0.8 & 0.2 & 19.3 & 50.6 & 100 \\
\hline Irland & Männer & 15.3 & 2.0 & 1.8 & 19.1 & 53.2 & 1.6 & 1.2 & 56.1 & 24.8 & 100 \\
\hline & Frauen & 6.5 & 0.8 & 4.8 & 12.0 & 49.7 & 2.2 & 4.2 & 56.1 & 31.8 & 100 \\
\hline & $M+F$ & 10.9 & 1.4 & 3.3 & 15.6 & 51.5 & 1.9 & 2.7 & 56.1 & 28.3 & 100 \\
\hline Italien & Männer & 20.3 & 5.6 & 5.2 & 31.1 & 20.8 & 6.2 & 7.0 & 34.0 & 34.9 & 100 \\
\hline & Frauen & 9.5 & 4.1 & 9.1 & 22.6 & 20.9 & 7.8 & 6.4 & 35.1 & 42.3 & 100 \\
\hline & $M+F$ & 19.9 & 1.8 & 4.0 & 25.6 & 25.6 & 1.8 & 1.6 & 29.0 & 45.4 & 100 \\
\hline Mexiko & Männer & 64.7 & 1.9 & 2.7 & 69.3 & 8.9 & 0.6 & 0.3 & 9.9 & 20.8 & 100 \\
\hline & Frauen & 26.9 & 1.0 & 40.0 & 68.0 & 9.5 & 0.6 & 4.5 & 14.5 & 17.5 & 100 \\
\hline & $\mathrm{M}+\mathrm{F}$ & 44.6 & 1.5 & 22.5 & 68.6 & 9.2 & 0.6 & 2.5 & 12.4 & 19.1 & 100 \\
\hline Niederlande ${ }^{1}$ & Männer & 21.1 & 1.0 & 1.9 & 24.0 & 36.0 & 0.9 & 1.3 & 38.2 & 37.8 & 100 \\
\hline & Frauen & 12.7 & 1.0 & 5.6 & 19.3 & 40.6 & 1.3 & 3.3 & 45.3 & 35.4 & 100 \\
\hline & $M+F$ & 17.0 & 1.0 & 3.7 & 21.7 & 38.3 & 1.1 & 2.3 & 41.7 & 36.6 & 100 \\
\hline Norwegen $^{1}$ & Männer & 3.6 & 0.4 & 0.2 & 4.3 & 56.6 & 3.9 & 2.5 & 63.0 & 32.7 & 100 \\
\hline & Frauen & 1.7 & 0.3 & 0.9 & 2.9 & 38.2 & 1.9 & 5.9 & 46.0 & 51.1 & 100 \\
\hline & $\mathrm{M}+\mathrm{F}$ & 2.7 & 0.4 & 0.6 & 3.6 & 47.6 & 2.9 & 4.2 & 54.7 & 41.7 & 100 \\
\hline Polen & Männer & 4.0 & 3.8 & 2.0 & 9.8 & 27.4 & 16.7 & 3.0 & 47.2 & 43.0 & 100 \\
\hline & Frauen & 1.6 & 2.0 & 2.6 & 6.2 & 22.5 & 15.3 & 8.6 & 46.4 & 47.4 & 100 \\
\hline & $\mathrm{M}+\mathrm{F}$ & 2.8 & 2.9 & 2.3 & 8.0 & 24.9 & 16.0 & 5.9 & 46.8 & 45.2 & 100 \\
\hline Portugal & Männer & 49.6 & 3.4 & 3.0 & 56.0 & 12.9 & 0.9 & 0.4 & 14.2 & 29.8 & 100 \\
\hline & Frauen & 30.3 & 3.2 & 4.6 & 38.0 & 16.9 & 2.8 & 1.6 & 21.2 & 40.7 & 100 \\
\hline & $\mathrm{M}+\mathrm{F}$ & 39.9 & 3.3 & 3.8 & 47.0 & 14.9 & 1.8 & 1.0 & 17.7 & 35.3 & 100 \\
\hline Slowakische Rep. & Männer & 0.8 & 3.1 & 1.4 & 5.3 & 46.7 & 25.3 & 6.1 & 78.2 & 16.5 & 100 \\
\hline & Frauen & 0.8 & 0.9 & 2.4 & 4.2 & 43.0 & 16.0 & 14.4 & 73.4 & 22.4 & 100 \\
\hline & $\mathrm{M}+\mathrm{F}$ & 0.8 & 2.1 & 1.9 & 4.8 & 44.9 & 20.7 & 10.2 & 75.8 & 19.4 & 100 \\
\hline Spanien & Männer & 29.5 & 4.9 & 2.3 & 36.7 & 20.3 & 2.7 & 1.2 & 24.3 & 39.1 & 100 \\
\hline & Frauen & 14.9 & 4.9 & 5.5 & 25.3 & 19.6 & 5.6 & 2.8 & 28.0 & 46.7 & 100 \\
\hline & $\mathrm{M}+\mathrm{F}$ & 22.4 & 4.9 & 3.8 & 31.2 & 20.0 & 4.1 & 2.0 & 26.1 & 42.8 & 100 \\
\hline Schweden & Männer & 8.4 & 2.0 & 1.0 & 11.4 & 45.1 & 3.9 & 3.5 & 52.5 & 36.1 & 100 \\
\hline & Frauen & 6.1 & 1.1 & 1.6 & 8.8 & 38.3 & 3.3 & 4.3 & 45.9 & 45.3 & 100 \\
\hline & $\mathrm{M}+\mathrm{F}$ & 7.3 & 1.6 & 1.3 & 10.2 & 41.8 & 3.6 & 3.9 & 49.3 & 40.6 & 100 \\
\hline Schweiz & Männer & 4.7 & $\mathrm{~m}$ & $\mathrm{~m}$ & 7.3 & 43.9 & $\mathrm{~m}$ & $\mathrm{~m}$ & 50.1 & 42.6 & 100 \\
\hline & Frauen & $\mathrm{m}$ & $\mathrm{m}$ & $\mathrm{m}$ & $\mathrm{m}$ & 51.9 & $\mathrm{~m}$ & $\mathrm{~m}$ & 58.3 & 36.7 & 100 \\
\hline & $M+F$ & 4.4 & $\mathrm{~m}$ & $\mathrm{~m}$ & 6.2 & 47.8 & $\mathrm{~m}$ & 4.1 & 54.1 & 39.8 & 100 \\
\hline Türkei & Männer & 38.5 & 7.3 & 7.0 & 52.8 & 19.5 & 5.3 & 7.3 & 32.1 & 15.2 & 100 \\
\hline & Frauen & 16.8 & 1.8 & 46.0 & 64.6 & 9.1 & 4.0 & 13.0 & 26.0 & 9.4 & 100 \\
\hline & $M+F$ & 27.6 & 4.5 & 26.6 & 58.7 & 14.3 & 4.7 & 10.1 & 29.1 & 12.2 & 100 \\
\hline Ver. Königreich & Männer & 4.5 & 1.3 & 1.7 & 7.6 & 52.4 & 4.8 & 2.5 & 59.7 & 32.8 & 100 \\
\hline & Frauen & 2.1 & 0.6 & 5.3 & 8.1 & 45.3 & 3.3 & 9.5 & 58.1 & 33.8 & 100 \\
\hline & $M+F$ & 3.4 & 1.0 & 3.5 & 7.8 & 48.9 & 4.0 & 6.0 & 58.9 & 33.3 & 100 \\
\hline Vereinigte Staaten ${ }^{1}$ & Männer & 10.3 & 1.5 & 1.8 & 13.6 & 48.3 & 3.3 & 4.0 & 55.6 & 30.8 & 100 \\
\hline & Frauen & 5.0 & 0.8 & 5.4 & 11.1 & 42.5 & 2.6 & 9.6 & 54.7 & 34.1 & 100 \\
\hline & $\mathrm{M}+\mathrm{F}$ & 7.7 & 1.1 & 3.6 & 12.4 & 45.4 & 2.9 & 6.8 & 55.1 & 32.5 & 100 \\
\hline Ländermittel & Männer & 16.2 & 2.7 & 2.0 & 21.1 & 36.0 & 4.8 & 2.9 & 44.0 & 34.9 & 100 \\
\hline & Frauen & 8.7 & 1.6 & 7.3 & 17.6 & 32.5 & 4.4 & 5.8 & 42.9 & 39.3 & 100 \\
\hline & $M+F$ & 12.5 & 2.2 & 4.7 & 19.5 & 34.2 & 4.6 & 4.5 & 43.5 & 37.1 & 100 \\
\hline
\end{tabular}

Hinweis: Schüler in dualen Ausbildungsgängen werden sowohl der Kategorie 'in Ausbildung' als auch der Kategorie 'in Beschäftigung' zugeordnet, ungeachtet ihres Status gemäß der ILO-Definition.

1. Referenzjahr 2000.

Quelle: OECD. Länderspezifische Datenquellen s. Anhang 3 (www.oecd.org/els/education/eag2002). 


\section{Kapitel \\ D}

DAS LERNUMFELD UND

DIE ORGANISATION

VON SCHULEN

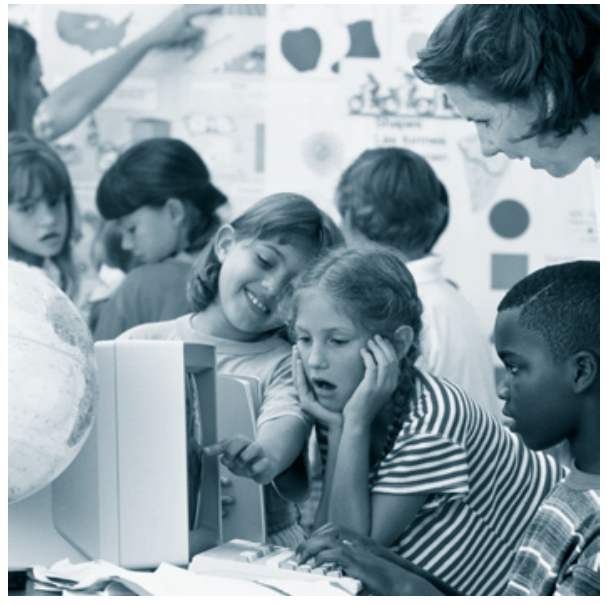





\section{ÜBERBLICK}

Indikator D1: Insgesamt vorgesehene Unterrichtszeit für 9- bis 14-jährige Schüler

Tabelle D1.1. Vorgesehene Unterrichtszeit in öffentlichen

Bildungseinrichtungen (2000)

Tabelle D1.2a. Vorgesehene Unterrichtszeit für 9- bis 11-jährige Schüler in öffentlichen Bildungseinrichtungen, nach Fächern (2000)

Tabelle D1.2b. Vorgesehene Unterrichtszeit für 12- bis 14-jährige Schüler in öffentlichen Bildungseinrichtungen, nach Fächern (2000)

Tabelle D1.3. Zusätzliche Unterrichts- und Lernzeit 15-jähriger Schüler (2000)

\section{Indikator D2: Klassengröße und zahlenmäßiges Schüler/ Lehrkräfte-Verhältnis}

Tabelle D2.1. Durchschnittliche Klassengröße, nach Art der Bildungseinrichtung und Bildungsbereich (2000)

Tabelle D2.2. Zahlenmäßiges Schüler-/Lehrkräfte-Verhältnis nach Bildungsbereich, Berechnungen basierend auf Vollzeitäquivalenten (2000)

\section{Indikator D3: Nutzung und Verfügbarkeit von Computern in der Schule und zu Hause}

Tabelle D3.1. Anzahl der Schüler pro Computer (2000)

Tabelle D3.2. Verfügbarkeit von Computern und Computer-Netzwerken an Schulen, die 15-Jährige besuchen (2000)

Tabelle D3.3. Ausmaß der Lernbeeinträchtigung aufgrund einer nicht genügenden Anzahl von Computern bzw. eines Mangels an MultimediaAusstattung für den Unterricht an Schulen, die 15-Jährige besuchen (2000) Tabelle D3.4. Verfügbarkeit von Computern für 15-jährige Schüler zu Hause und in der Schule (2000)

Tabelle D3.5. Häufigkeit der Computernutzung 15-jähriger Schüler zu Hause und in der Schule (2000)

Tabelle D3.6. 15-jährige Schüler, die den Computer als Hilfsmittel zum Lernen für die Schule benutzen (2000)

\section{Indikator D4: Einstellungen und Erfahrungen von Schülerinnen und Schülern bei der Nutzung von Computern}

Tabelle D4.1. Vertrautheit und Selbsteinschätzung der Fähigkeit im Umgang mit Computern von 15-jährigen Schülerinnen und Schülern, nach Geschlecht (2000) Tabelle D4.2. Interesse 15-jähriger Schülerinnen und Schüler an der Nutzung von Computern, nach Geschlecht (2000)

\section{Indikator D5: Unterrichts- und Schulklima}

Tabelle D5.1. Unterrichtsklima bei 15-jährigen Schülern (2000)

Tabelle D5.2. Hausaufgabenregelung und Leistungsorientierung bei 15-jährigen Schülern (2000)

Tabelle D5.3. Qualität und Nutzung der Schulressourcen für 15-Jährige (2000) Tabelle D5.4. Persönlicher Bezug 15-jähriger Schüler zu ihrer Schule (2000)

\author{
In Kapitel D wird \\ das Lernumfeld und \\ die Organisation der \\ Schulen untersucht, \\ und zwar in Form \\ der Unterrichts- und \\ Lernbedingungen der \\ Schüler...
}

...der Verfügbarkeit

und Nutzung von

Computern in der Schule

und zu Hause, ... ...des Unterrichts- und
Schulklimas, ... 
...und der Arbeitsbedinungen der Lehrer.

\section{Indikator D6: Lehrergehälter an öffentlichen Schulen des Primar- und Sekundarbereichs}

Tabelle D6.1. Lehrergehälter (2000)

Tabelle D6.2. Zulagen zum Grundgehalt für Lehrer an öffentlichen Schulen (2000)

\section{Indikator D7: Unterrichtszeit und Arbeitszeit der Lehrer}

Tabelle D7.1. Aufteilung der Arbeitszeit von Lehrern (2000)

Tabelle D7.2. Anzahl der jährlichen Unterrichtsstunden (1996, 2000)

Die Indikatoren D1 zur vorgesehenen Unterrichtszeit, D6 zur den Lehrergehältern und Bonussystemen und D7 zur Arbeitszeit der Lehrer basieren auf Daten, die jährlich auf Systemebene zu Lehrern und Lehrplänen erhoben werden. Anhang 3 (unter www.oecd.org/els/education/eag2002) bietet darüber hinaus umfangreiche Informationen über die Gemeinsamkeiten und Unterschiede der einzelnen Länder bei der Unterrichtszeit, den Lehrergehältern und Bonussystemen sowie bezüglich der Definitionen von Unterrichtszeit und Arbeitszeit. Außerdem hilft er den Lesern dabei, Daten zu den einzelnen Ländern und Vergleiche zwischen ihnen zu interpretieren.

Die Indikatoren D3 bis D5 und Teile von Indikator D1 beruhen auf Daten der Internationalen Schulleistungsstudie PISA. Detaillierte Information zu dieser Studie finden sich auf der Website www.pisa.oecd.org. 


\section{INSGESAMT VORGESEHENE UNTERRICHTSZEIT FÜR 9- BIS 14-JÄHRIGE SCHÜLER}

- Im Durchschnitt verbringen 9- bis 11-jährige Schüler 841 Stunden im Jahr im Klassenzimmer, die 12- 14-Jährigen fast einhundert Stunden mehr. Es gibt hierbei jedoch zwischen den einzelnen Ländern erhebliche Unterschiede.

- Im Durschnitt entfällt bei den 9- bis 11-Jährigen ungefähr die Hälfte des Pflichtunterrichts auf das Lesen und Schreiben in der Unterrichtssprache, Mathematik und die Naturwissenschaften, bei den 12 bis 14-Jährigen liegt der Anteil dieser Fächer bei 40 Prozent.

- Im Durchschnitt verwenden 15-jährige Schüler zusätzlich zum Unterricht im Klassenzimmer pro Woche 4,6 Stunden für die Hausgaben und das Lernen in der Unterrichtssprache, in Mathematik und den Naturwissenschaften.

- Im Durchschnitt erhält jeder dritte 15-Jährige, zumindest gelegentlich, Nachhilfe oder Privatunterricht.

- Es gibt große Unterschiede zwischen den einzelnen Ländern hinsichtlich der Flexibilität der Schulen und der lokalen bzw. regionalen Behörden bei der Festlegung des Lehrplans und des Stundenplans. 


\section{Abbildung D1.1.}

Vorgesehene Unterrichtszeit in öffentlichen Bildungseinrichtungen, nach Fächern (2000)

Prozentsatz der gesamten für den Kernpflichtteil vorgesehenen Unterrichtszeit, nach Fach, flexiblem Pflichtteil und Nicht-Pflichtteil des Lehrplans für 9- bis 11-Jährige und 12- bis 14-Jährige

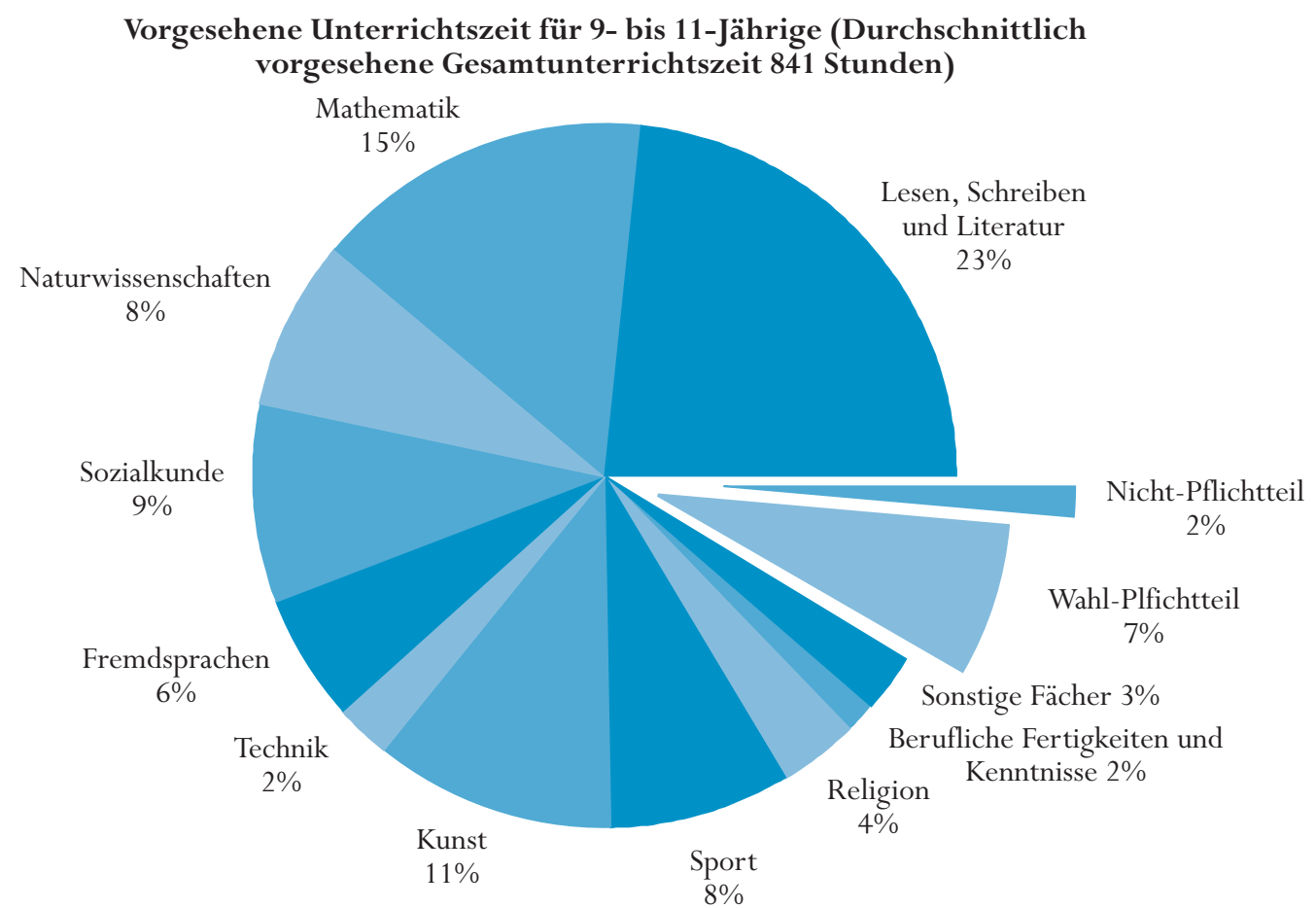

Vorgesehene Unterrichtszeit für 12- bis 14-Jährige (Durchschnittlich vorgesehene Gesamtunterrichtszeit 936 Stunden)

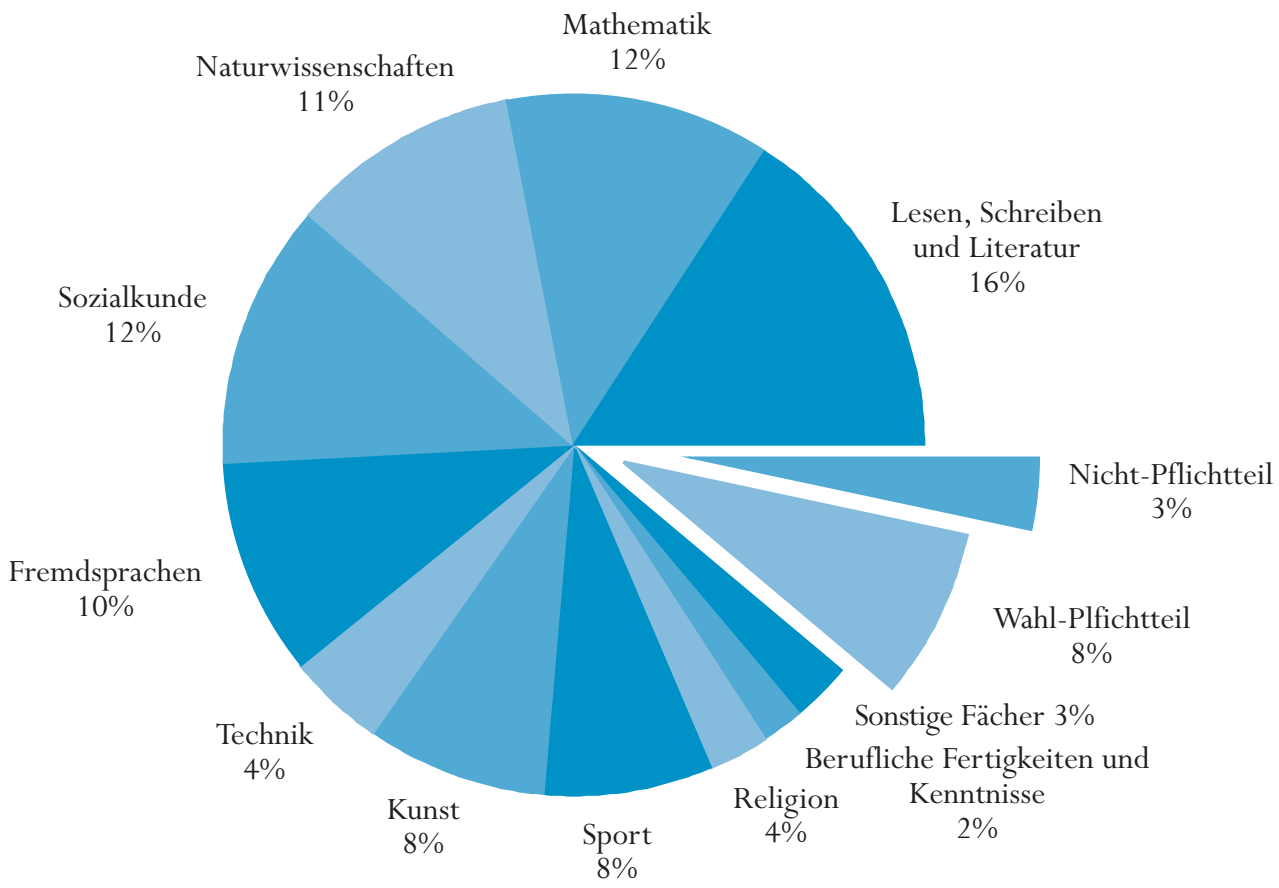

Quelle: OECD. Tabellen D1.2a und D1.2b. Hinweise s. Anhang 3 (www.oecd.org/els/education/eag2002). 


\section{Politischer Hintergrund}

Wie viel und wie gut ein Mensch von der frühen Kindheit bis zum Eintritt ins Berufsleben lernt, hat entscheidenden Einfluss auf die späteren sozialen und wirtschaftlichen Erfolge, und zwar während des gesamten Lebens. Ein großer Teil der öffentlichen Investitionen in das Lernen der Schüler erfolgt in Form der Bereitstellung von formellem Unterricht im Klassenzimmer. Eine große Aufgabe für die Bildungspolitik besteht nun darin, die zur Verfügung stehenden Ressourcen auf die Bedürfnisse der Schüler abzustimmen und die genutzte Zeit sowohl mit Blick auf die Lernenden als auch mit Blick auf die öffentlichen Investitionen zu optimieren. Da die entsprechenden Gesamtkosten hauptsächlich von den Kosten für die Lehrergehälter, den Betriebs- und Instandhaltungskosten der Schulgebäude sowie von den Kosten anderer Bildungsressourcen abhängen, ist die Zeitdauer, für die diese Ressourcen den Schülern zur Verfügung stehen, von großer Bedeutung und mit der Unterrichtszeit im formellen Bildungssystem erfasst der Indikator D1 die betreffende Ressourcennutzung.

Die Zeit, in der Schüler lernen, ist jedoch nicht alleine auf die Stunden beschränkt, die Schüler im formellen Klassenunterricht verbringen, sondern umfasst auch die Zeit für die Hausaufgaben und für andere von der Schule organisierte Lernaktivitäten, wie Förderkurse, Zusatzangebote und Arbeitsgemeinschaften. Oft sind darin auch Privatunterricht, Nachhilfestunden oder andere Formen außerschulischen Lernens enthalten. Diese Formen des Lernens sind weit schwieriger zu quantifizieren, aber der Indikator erfasst zwei wichtige Aspekt, nämlich erstens, inwieweit organisierter Unterricht zusätzlich zum normalen Lehrplan innerhalb und außerhalb der Schule erfolgt und zweitens, wieviel Zeit 15-Jährige gemäß ihren eigenen Angaben mit den Hausaufgaben verbringen.

\section{Ergebnisse und Erläuterungen}

\section{Was dieser Indikator zeigt}

Dieser Indikator erfasst die vorgesehene Unterrichtszeit als Kenngröße für die öffentlich vorgeschriebene Lernzeit im Klassenzimmer. Er zeigt auch, wie sich die Unterrichtszeit auf die verschiedenen Bereiche des Lehrplans verteilt. Der Indikator ist berechnet als vorgesehene Nettoanzahl an Unterrichtsstunden für die Klassen, in denen die Mehrzahl der Schüler 9 bis 14 Jahre alt sind. Diese Daten sind zwar aufgrund der unterschiedlichen Lehrpläne schwer über die einzelnen Länder hinweg zu vergleichen, geben aber dennoch einen Hinweis darauf, wieviel Unterrichtszeit die Länder für ihre Schüler als notwendig erachten, damit diese die festgesetzten Bildungsziele erreichen.

Es ist zu berücksichtigen, dass in einigen Ländern bei der vorgesehenen Unterrichtszeit beträchtliche Unterschiede zwischen einzelnen Regionen oder verschiedenen Schularten bestehen. Darüber hinaus sind in einigen Ländern lokale Bildungsbehörden oder die Schulen selbst befugt, über die Anzahl und Aufteilung der Unterrichtsstunden zu entscheiden. Oft ist zusätzliche Unterrichtszeit für individuelle Förderkurse oder Zusatzangebote zum Lehrplan
Dieser Indikator

zeigt die Gesamtzahl

vorgesehener

Unterrichtsstunden

im Klassenzimmer

im formellen

Bildungssystem ...
Die vorgesehene Unterrichtszeit ist ein wichtiger Indikator für die öffentlichen Ressourcen, die in Bildung investiert werden, ... 
... als auch im Zusammenhang mit anderen, die Lernzeit

beinflussenden Bedingungen und der Qualität des Unterrichts, die in diesem Indikator nicht erfasst sind.

Die Verantwortung für die Festlegung des Lehrplans ist in den einzelnen Ländern auf unterschiedliche Art und Weise geregelt. In einigen $O E C D$ Ländern werden Fächer und Inhalte sowie die dafür vorgesehene Zeit aufnationaler (bzw. regionaler) Ebene festgelegt,... eingeplant. Andererseits geht auch Zeit verloren, weil für fehlende Lehrer kein qualifizierter Ersatz zur Verfügung steht oder weil die Schüler selbst Fehlzeiten aufweisen.

Die jährliche Unterrichtszeit sollte ebenfalls im Zusammenhang mit der Dauer der Schulpflicht betrachtet werden. Sie entspricht der vom Staat für junge Menschen vorgesehenen Zeit der Bildungsbeteiligung bei voller Finanzierung durch öffentliche Ressourcen, beziehungsweise dem Zeitraum, in dem die Bildungsbeteiligung der Bevölkerung bei über 90 Prozent liegt (s. Indikator C1). Außerdem ist zu berücksichtigen, dass die vorgesehene Unterrichtszeit weder die Qualität der Lernangebote während dieser Zeit noch die Art oder Qualität der vorhandenen Human- oder Sachressourcen erfasst. Andere Indikatoren dieses Kapitels befassen sich mit dem Problem der Verfügbarkeit von Bildungsressourcen (Indikator D3 und D5), der Verfügbarkeit von Lehrern in Relation zur Schülerpopulation (Indikator D2) und der Qualität des Lernklimas in Klassenzimmern und Schulen (Indikator D5).

\section{Lehrplanpolitik}

Die Entscheidungsbefugnisse für die Festlegung des Lehrplans unterscheiden sich in den einzelnen Ländern sehr. In den OECD-Ländern gibt es 2 Grundmodelle mit verschiedenen Varianten.

In dem einen Modell der Lehrplanregelung werden die Fächer, die dafür vorgesehene Unterrichtszeit und die Inhalte von nationalen bzw. regionalen Behörden festgelegt und die Schulen haben unterschiedlich viel Freiheit bei der Umsetzung dieser nationalen (in einigen Fällen regionalen) Lehrplanvorgaben. So legen beispielsweise in Deutschland, England, Frankreich, Griechenland, Österreich und Spanien nationale Behörden (in Deutschland die Länder, in Spanien die Autonomen Gemeinschaften) die Lehrpläne für alle Schularten, Klassenstufen und Fächer fest. In den entsprechenden Vorschriften werden die Fächer und die dafür vorgesehene Unterrichtszeit sowie der Inhalt mehr oder weniger detailliert nach Klassenstufe und Bildungsgang festgelegt. Die Schule ist für das Management und die Vermittlung des Lehrplans verantwortlich.

\section{Lehrplanregelung in Spanien}

In Spanien legen die Regierungen der Autonomen Gemeinschaften die Lehrpläne für die jeweilige Gemeinschaft fest. Diese Lehrpläne geben die Fächer und die für jedes Fach vorgesehenen Unterrichtsstunden pro Schuljahr vor. Sie müssen den von der Zentralregierung festgelegten Pflichtteil des Lehrplans enthalten (65 Prozent des gesamten Pflichtlehrplans, bzw. 55 Prozent, wenn in der Gemeinschaft eine eigene Sprache gesprochen wird). 
Im zweiten Modell der Lehrplanregelung beschränkt sich die Rolle der nationalen Behörden auf die Festlegung von Leistungszielen oder Standards, während lokale Behörden oder die Schulen für die Planung und Umsetzung der Lehrpläne verantwortlich sind. So werden beispielsweise in Belgien (fläm.), Irland, Neuseeland, Portugal (Primarbereich) und der Tschechischen Republik die Leistungsziele mittels nationaler Richtlinien festgelegt, während lokale Behörden bzw. die Schulen die Fächer, die Inhalte und die für die einzelnen Fächer vorgesehene Unterrichtszeit bestimmen.
.... während in anderen

Ländern die Festlegung des

Lehrplans zu den wichtig-

sten Zuständigkeiten der

lokalen Schulbehörden

bzw. der Schule zählt und

auf nationaler Ebene

die Leistungsstandards

festgelegt werden.

\section{Lehrplanregelung in Neuseeland}

In Neuseeland ist der nationale Lehrplan durch Verlautbarungen zum Lehrplan für sieben Fächergruppen geregelt: Mathematik, Naturwissenschaften, Englisch, Technik, Sozialkunde, Gesundheits- und Sportunterricht und Kunst. Staatliche sowie staatlich eingebundene Schulen müssen für alle Schüler der Klassen eins bis zehn Lernprogramme auf Basis dieser Verlautbarungen anbieten. Wie die Schulen das zu tun haben ist jedoch weder im Hinblick auf die vorgesehene Unterrichtszeit noch im Hinblick auf den Bildungsgang oder den Stundenplan vorgegeben. Moderne Fremdsprachen sind in keinem Bildungsbereich Pflicht und schließen in Neuseeland sowohl regionale als auch internationale Sprachen ein.

\section{Lehrplan für den Primarbereich in Portugal}

In Portugal legt der Lehrplan für den Primarbereich keine Stundenzahl für die einzelnen Fächer, sondern nur die gesamte Wochenstundenzahl fest. Zu den Fächern gehören: Sport, Musik, Theater und gestaltende Kunst, Ökologie, Portugiesisch, Mathematik sowie Religion bzw. Persönliche und soziale Entwicklung. Die Lehrer können die Zeit für jedes Fach bis zur Obergrenze von 25 Wochenstunden einteilen.

In Finnland, Irland, Schweden und Ungarn werden die beiden Modelle miteinander kombiniert. In diesen Ländern entwickeln zwar lokale Behörden und die Schulen das zu verfolgende Lernprogramm, aber nationale Lehrplanunterlagen geben bei Fächern und Inhalten die Richtung vor und machen weitgefasste Vorgaben bei der für die einzelnen Fächer vorzusehenden
Wieder andere

Länder verfolgen eine Kombination dieser beiden Modelle. 
Unterrichtszeit. Die Schulen haben in diesen Ländern einen recht großen Spielraum hinsichtlich des Anbietens von zusätzlichem Unterricht oder der Einzelförderung von Schülern mit speziellen Bedürfnissen.

\section{Lehrplan für den Primarbereich in Irland}

In Irland gilt im Primarbereich ein integrierter Lehrplan, der integrierte Lernerfahrungen für die Schüler vorsieht. Es wird erwartet, dass die für die Schüler organisierten Lernerfahrungen interdisziplinäre Aktivitäten fördern. Die Schulen müssen ihre Lehrpläne selbst entwickeln. Zur Unterstützung der Schulen bei der Planung und Umsetzung ihrer Lehrpläne wird ein Zeitrahmen vorgeschlagen, der für jedes Fach eine Mindestzeit vorsieht.

\section{Lehrplanregelung in Schweden}

In Schweden gibt der gesetzlich festgelegte Lehrplan die Gesamtstundenzahl pro Fach oder Fachgebiet für die Zeit der Schulpflicht (9 Jahre) vor. Die Gemeinden und Schulen legen selbst fest, in welchem Jahr ein Unterrichtsfach gelehrt wird und wie viele Unterrichtsstunden pro Schuljahr dafür erforderlich sind.

Im Durchschnitt entfallen in den Klassen, in denen die Mehrzahl der Schüler 9 bis 14 Jahre alt sind, 8 Prozent

der vorgesehenen Unterrichtszeit auf den Wahl-Pfichtteil des Lehrplans.

\section{Vorgesehene Unterrichtszeit im Klassenzimmer für den Pflichtunterricht im formellen Bildungssystem}

In den meisten OECD-Ländern wird die Anzahl der Stunden für den Pflichtteil des Lehrplans festgelegt. Innerhalb dieses Pflichtteils können die Schüler in unterschiedlich starkem Maße wählen, welche Fächer sie belegen möchten. Im Durchschnitt entfallen in den Klassen, in denen die Mehrzahl der Schüler 9 bis 11 Jahre alt ist, 7 Prozent der vorgesehenen Unterrichtszeit auf den WahlPflichtteil, bei den 12- bis 14-Jährigen sind es 9 Prozent. In Australien macht der Wahl-Pflichtteil für diese Altersgruppen jedoch 60 bzw. 28 Prozent aus. In einem Drittel der OECD-Länder gibt es füt diese Altersgruppe überhaupt keinen Wahl-Pflichtteil. Für 12- bis 14-jährige Schüler in Belgien, Finnland, Island, den Niederlanden, Portugal, Schottland, Spanien und der Tschechischen Republik entfallen mindestens 10 Prozent des Pflichtunterrichts auf den WahlPflichtteil (Tabellen D1.2a und D1.2b). 


\section{Abbildung D1.2.}

Durchschnittliche jährliche Stundenzahl an Fremdsprachenunterricht in öffentlichen Bildungseinrichtungen für 9- bis 14-Jährige (2000)

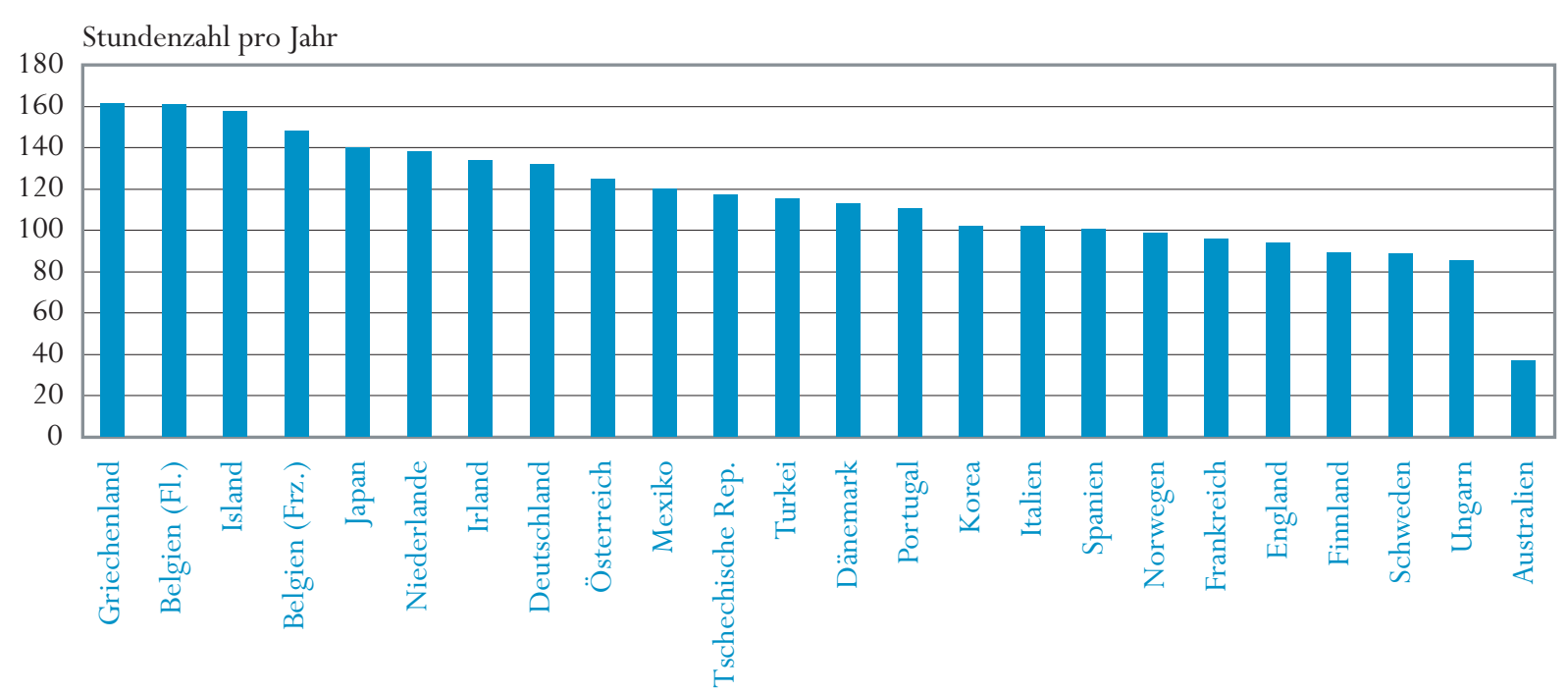

Anordnung der Länder in absteigender Reihenfolge der jährlichen Stundenzahl an Fremsprachenunterricht für 9- bis 14-Jährige. Quelle: OECD. Tabellen D1.2a und D1.2b. Hinweise s. Anhang 3 (www.oecd.org/els/education/eag2002).

Für 9- bis 11-jährige Schüler entfallen im Durchschnitt 48 Prozent des Pflichtteils des Lehrplans auf die drei Grundlagenfächer Lesen und Schreiben in der Unterrichtsprache (23 Prozent), Mathematik (15 Prozent) und Naturwissenschaften (8 Prozent). Durchschnittlich 9 Prozent des Pflichtteils werden der Sozialkunde und 6 Prozent den modernen Fremdsprachen gewidmet (Abb. D1.2). Die zeitlichen Anteile von Kunst und Sport am gesamten Pflichtteil des Lehrplans betragen 11 bzw. 8 Prozent. Diese sieben Grundlagenfächer sind in allen OECD-Ländern Teil des Lehrplans für diese Altersgruppe. Religion oder Ethikunterricht sind in der Hälfte der Länder Teil des Lehrplans. Es muss jedoch beachtet werden, dass bei dieser Altersgruppe die Lernaktivitäten in den einzelnen Fächern nicht unbedingt als separate Unterrichtsstunden organisiert sind (Tabellen D1.2a und D1.2b).

Im Durchschnitt der OECD-Länder entfallen für 12- bis 14-jährige Schüler 40 Prozent des Pflicht-Lehrplans auf die drei Grundlagenfächer Lesen und Schreiben in der Unterrichtssprache (16 Prozent), Mathematik (12 Prozent) und Naturwissenschaften (11 Prozent). In dieser Alterskohorte ist ein relativ größerer zeitlicher Anteil des Lehrplans für Sozialkunde (12 Prozent) und moderne Fremdsprachen (10 Prozent) vorgesehen (Abb. D1.2) und ein etwas kleinerer für Kunst und Sport (jeweils 8 Prozent). Diese sieben Fächergruppen sind in allen OECD-LändernTeil des Lehrplans für Schüler des Sekundarbereich I. Technik gehört in etwa zwei Drittel der Länder zum Pflichtlehrplan und Religion in etwa der Hälfte der Länder.
Für 9- bis 11-jährige Schüler entfällt etwa die Hälfte der vorgesehenen Pflicht-Unterrichtszeit auf Lesen und Schreiben, Mathematik und Naturwissenschaften, ...

... bei den 12-bis 14-Jährigen liegt der entsprechende Anteil bei 40 Prozent. 


\section{Die insgesamt \\ vorgesehene \\ Unterrichtszeit für \\ 9- bis 11-Jährige liegt im Durchschnitt bei 841 \\ Stunden ...}

... und bei 936 Stunden für 12- bis 14-Jährige.

Durchschnittlich entfallen 3 Prozent der insgesamt vorgesehenen Unterrichtszeit auf den

Nicht-Pflichteil des Lehrplans, dieser Anteil variiert jedoch stark von

Land zu Land.

In den meisten OECD-Ländern hat sich die insgesamt vorgesehene Unterrichtszeit

für 12- bis 14-jährige

Schüler zwischen 1996 und 2000 nicht verändert.

Hausaufgaben und andere Formen des außerschulischen Lernens spielen für 15-Jährige eine wichtige Rolle...

und nahezu ein Drittel der Unterrichtszeit, die

\section{Insgesamt vorgesehene Unterrichtszeit im Klassenzimmer im formellen Bildungssystem}

Die vorgesehene Unterrichtszeit ist ein Schätzwert der Anzahl an Unterrichtsstunden, in denen Schüler im Pflichtteil und Nicht-Pflichtteil des Lehrplans unterrichtet werden. Die insgesamt für 9- bis 11-Jährige vorgesehene Unterrichtszeit im Klassenzimmer im formellen Bildungssystem reicht von durchschnittlich weniger als 700 Stunden pro Jahr in Finnland und Island bis zu 1.000 Stunden oder mehr in Italien, den Niederlanden und Schottland. Es ist zu berücksichtigen, dass in diesen Zahlen kein Einzelunterricht außerhalb des Klassenzimmers berücksichtigt ist, der in etlichen Ländern, darunter Finnland und Island, einen beträchtlichen Teil der Lernzeit ausmacht (Tabelle D1.1).

Für 12- bis 14-jährige Schüler reicht die durchschnittlich vorgesehene Unterrichtszeit von weniger als 800 Stunden in Schweden und der Türkei bis zu mehr als 1.100 Stunden in Mexiko und Österreich. Der OECD-Durchschnitt für die 12- bis 14-Jährigen liegt bei 936 Stunden pro Jahr (Tabelle D1.1).

Für 9- bis 11-jährige Schüler entfallen im Schnitt 2 Prozent der insgesamt vorgesehenen Unterrichtszeit auf den Nicht-Pflichtteil des Lehrplans, bei den 12- bis 14-jährigen Schülern liegt der Anteil bei 4 Prozent. In einigen Ländern ist jedoch dieser zusätzliche Nicht-Pflichteil an Unterrichtszeit nicht unerheblich. Während in den meisten OECD-Ländern im Primarbereich die gesamte vorgesehene Unterrichtszeit für alle Schüler Pflicht ist, liegt der zusätzliche Nicht-Pflichtteil in Ungarn sogar bei 20 Prozent und in Neuseeland und der Türkei bei etwa 10 Prozent. Im Sekundarbereich I gibt es in Australien, Belgien, Dänemark, England, Frankreich, Irland, Neuseeland, der Türkei und Ungarn einen beträchtlichen Nicht-Pflichtteil der Unterrichtszeit, der von 5 Prozent in Australien und Neuseeland bis zu 28 Prozent in Ungarn reicht (Tabellen D1.2a und D1.2b sowie Abb. D1.1).

In den meisten OECD-Ländern hat sich die insgesamt vorgesehene Unterrichtszeit für 12- bis 14-jährige Schüler zwischen 1996 und 2000 nicht verändert. In Neuseeland und der Tschechischen Republik ist jedoch die insgesamt vorgesehene Unterrichtszeit um 11 Prozent gestiegen (s. Anhang 3 unter www.oecd.org/els/education/eag2002).

\section{Hausaufgaben}

Regeln und Vorgehensweisen bezüglich der Hausaufgaben sind weiterere Faktoren, die einen entscheidenden Einfluss darauf ausüben, wieviel Zeit Schüler für das Lernen verwenden. In vielen Ländern machen Hausaufgaben einen großen Teil der Lernzeit der Schüler aus. Im Rahmen der PISA-Studie 2000 wurden die Schüler gefragt, wieviel Zeit sie pro Woche für Hausaufgaben in der Unterrichtssprache, in Mathematik und Naturwissenschaften aufwenden.

Die Zeit, die Schüler für die Hausaufgaben zur Vorbereitung des Unterrichts aufwenden, kommt noch zur Unterrichtszeit im Klassenzimmer hinzu. 
Bei der PISA-Studie gaben 15-Jährige an, im Durchschnitt 4,6 Stunden für Hausaufgaben und Lernen in der Unterrichtssprache, in Mathematik und Naturwissenschaften aufzuwenden. Schüler in Griechenland gaben an, pro Woche hierfür sogar 7 Stunden aufzuwenden. Auch die Schüler in Australien, Dänemark, Frankreich, Island, Irland, Italien, Kanada, Mexiko, Neuseeland, Polen, Portugal, Spanien, Ungarn und im Vereinigten Königreich wenden laut ihren Angaben mehr Zeit für die Hausaufgaben in den Kernfächern auf als der OECD-Durchschnitt, dagegen liegt der dementsprechende Zeitbedarf der Schüler in Finnland, Japan, Korea, Luxemburg, Österreich, Schweden, der Schweiz und der Tschechischen Republik nach deren eigenen Angaben unter dem OECD-Durchschnitt (Tabelle D1.3).

\section{Zusätzliche Unterrichtszeit}

In den OECD-Ländern werden Spielräume bei der Lehrplangestaltung dazu genutzt, um auf spezielle Interessen der Schüler oder auf besondere Förderbedürfnisse einzugehen. Darüber hinaus suchen auch die Eltern oft nach zusätzlichen, über das Schulangebot hinausgehenden, Unterrichts- und Fördermöglichkeiten für ihre Kinder. Im Rahmen der PISA-Studie wurden Schulleiter des Sekundarbereichs gefragt, ob ihre Schule folgende Angebote bereitstellt: a) Zusatzangebote für leistungsstarke Schüler, b) Förderangebote in der Unterrichtssprache für leistungsschwache Schüler, c) spezielle Kurse in Lerntechniken für leistungsschwache Schüler, d) Nachhilfe durch Lehrkräfte und e) Räume, in denen Schüler ihre Hausaufgaben unter fachlicher Anleitung machen können. In denselben Schulen wurden 15-jährige Schüler gefragt, ob sie Ergänzungsunterricht gehabt oder Zusatzkurse besucht hätten, Förderunterricht in der Unterrichtssprache und in anderen Fächern oder Kurse zur Verbesserung ihrer Lerntechniken besucht hätten. Außerdem wurden sie gefragt, ob sie privaten Nachhilfeunterricht außerhalb der Schule erhielten. Die Antworten auf diese Fragen ermöglichen Rückschlüsse auf weitere Lernmöglichkeiten zusätzlich zum formellen Unterricht im Klassenzimmer. Die Alterskohorte, die den PISA-Schülerfragebogen beantwortete, ist zwar etwas älter als diejenige im ersten Teil dieses Indikators, dennoch können die charakteristischen Unterschiede zwischen den einzelnen Ländern auf bildungspolitisch relevante Fragen verweisen, denen Aufmerksamkeit gewidmet werden sollte (Tabelle D1.3).

In den OECD-Ländern bieten im Durchschnitt die Hälfte der Schulen, die 15Jährige besuchen, zusätzlichen Unterricht und etwa zwei Drittel der Schulen bieten individuelle Förderung für Schüler mit speziellen Bedürfnissen an. Die Schulen in Australien, Finnland, Island, Italien, Kanada, Luxemburg, Neuseeland, Portugal, Schweden und im Vereinigten Königreich bieten häufiger zusätzliche Kurse für interessierte Schüler bzw. Förderkurse für Schüler mit speziellen Bedürfnissen an. Mehr als 90 Prozent der 15-Jährigen in Island, Italien, Neuseeland, Norwegen, Portugal und Schweden besuchen Schulen, die für leistungsschwache Schüler Förderkurse in der Unterrichtssprache anbieten (Tabelle D1.3). in der Schule auf die Unterrichtssprache,

Mathematik und die

Naturwissenschaften

entfällt, wird nochmals

für Hausaufgaben

aufgewendet.

Im Rahmen der PISAStudie wurden Schulleiter und Schüler gefragt, welches zusätzliche Unterrichtsangebot an der Schule besteht und von 15-jährigen Schülern in Anspruch genommen wird.

Die meisten Schulen in Australien, Finnland, Island, Italien, Kanada, Luxemburg, Neuseeland, Portugal, Schweden und im Vereinigten Königreich bieten zusätzliche Kurse für interessierte Schüler an bzw. für Schüler, die Förderkurse benötigen, ... 
..aber höchstens die Hälfte der Schulen in Belgien, Deutschland,

Kored, Mexiko und

Polen bieten solche zusätzlichen Kurse an.

In einigen Fällen gleichen Zusatzangebote

der Schulen die in unterdurchschnittlichem

Umfang vorgesehene Unterrichtszeit aus.

Ein Drittel der 15Jährigen in Korea und mehr als die Hälfte dieser Alterskohorte in Japan erhalten privaten Unterricht auferhalb der Schule.

Die Daten über die Unterrichtszeit stammen
Dagegen besuchen nur die Hälfte der 15-Jährigen in Belgien, Deutschland, Korea, Mexiko und Polen solche Schulen. Und während mehr als 90 Prozent der Schüler in Dänemark, Finnland, Japan, Neuseeland und im Vereinigten Königreich Einzelförderunterricht durch Lehrer erhalten, liegt der Anteil derer, die in Deutschland und Österreich eine solche Hilfe erhalten, bei unter 40 Prozent.

Vergleicht man diese Ergebnisse mit der auf Systemebene angegebenen vorgesehenen Unterrichtszeit, so lässt sich feststellen, dass eine relativ niedrige vorgesehene Unterrichtszeit nicht zwangsläufig mit nicht ausreichendem Unterricht gleichzusetzen ist. So gehören Frankreich, Griechenland, Mexiko und Österreich beispielsweise zu den Ländern mit der höchsten vorgesehenen Unterrichtszeit, während gleichzeitig in diesen Ländern weniger Schulen angaben, zusätzlichen Unterricht anzubieten. Dagegen scheinen Finnland, Island, Norwegen, Schweden und Portugal die geringste vorgesehene Unterrichtszeit im Klassenzimmer von allen OECD-Ländern zu haben, gleichzeitig gehören sie jedoch zu den Ländern, in denen die meisten Schulen angaben, Zusatzkurse entsprechend den speziellen Bedürfnissen der Schüler anzubieten (Tabelle D1.1 und Tabelle D1.3).

Abgesehen von zusätzlichen Angeboten in der Schule nehmen viele Schüler an zusätzlichem Unterricht außerhalb der Schule teil. In Japan gaben 30 Prozent der 15-Jährigen an, während der letzten drei Jahre regelmäßig außerhalb der Schule Unterricht in der Landes- bzw. Unterrichtssprache erhalten zu haben, für andere Fächer lag der Anteil bei 55 Prozent. In Korea sind die entsprechenden Zahlen ebenfalls relativ hoch (27 bzw. 34 Prozent). In Ungarn und Polen gaben 25 bzw. 10 Prozent der Schüle an, während der letzten drei Jahre regelmäßig außerhalb der Schule Zusatz- und Ergänzungskurse besucht zu haben (Daten s. www.pisa.oecd.org.) In Japan, Korea, Neuseeland, Irland, Portugal, Polen, Spanien und Ungarn schließlich gaben mindestens 11 Prozent der 15-jährigen Schüler an, private Nachhilfe zu erhalten.

Zählt man man auch diejenigen, die nur gelegentlich Zusatzunterricht außerhalb der Schule erhalten, so liegt der Anteil der 15-jährigen Schüler, die während der letzten drei Jahre in der Unterrichtssprache oder in anderen Fächern zusätzlichen Unterricht außerhalb der Schule besuchten, in Finnland, Italien, Norwegen, der Schweiz und Schweden unter 10 Prozent. Dagegen erhielten mehr als die Hälfte der Schüler in Mexiko, Polen, Korea und Japan zusätzlich zum Schulunterricht Privatunterricht. Außerdem erhielten während der letzten drei Jahre weniger als 10 Prozent der 15-Jährigen in Finnland und Schweden (privaten) Nachhilfeunterricht, während ihr Anteil in Irland, Italien, Mexiko, Neuseeland, Polen, Portugal, Spanien und Ungarn bei über 40 Prozent lag.

\section{Definitionen und angewandte Methodik}

Die Unterrichtszeit für 9- bis 14-Jährige bezieht sich auf die formelle Anzahl der Zeitstunden pro Schuljahr, die von der Schule für Unterrichtsaktivitäten 
für Schüler im Referenzschuljahr 1999/2000 vorgesehen waren. Für Länder, in denen keine formellen Vorschriften zu der Unterrichtszeit vorliegen, wurde die Zahl der Unterrichtsstunden aufgrund der Erhebungsdaten geschätzt. Stunden, die entfallen, wenn Schulen wegen Feiertagen oder Feierlichkeiten geschlossen sind, werden nicht gezählt. In der vorgesehenen Unterrichtszeit nicht enthalten sind freiwillige Aktivitäten außerhalb der regulären Schulzeit. Ebenso wenig enthalten sind die Zeit für Hausaufgaben, Privatunterricht und Selbststudium vor oder nach der Schule.

Der Pflichtteil des Lehrplans bezieht sich auf die Anzahl und Aufteilung der Unterrichtsstunden, die von jeder Schule zu unterrichten und von jedem Schüler zu besuchen sind.

Der Wahl-Pflichtteil des Lehrplans bezieht sich auf den Teil des Lehrplans, bei dem die Schule oder die Schüler Entscheidungsspielraum bzw. oder Wahlfreiheit haben. So kann sich beispielsweise eine Schule dafür entscheiden, innerhalb der vorgegebenen Pflichtzeit in den naturwissenschaftlichen Fächern mehr als die Pflichtstunden zu unterrichten, während es in Kunst lediglich die Mindeststundenzahl ist.

Der Nicht-Pflichtteil des Lehrplans ist der Teil, der auf Schulebene festgelegt wird, bzw. auf Ebene des Zuges, falls es unterschiedliche Züge innerhalb einer Schule gibt. In der Regel sind die Schüler nicht zum Besuch des Nicht-Pflichtteils des Lehrplans verpflichtet.

Die vorgesehene Unterrichtszeit bezieht sich auf die Stundenzahl, die Schüler jährlich in den Pflicht- und Nicht-Pflichtfächern des Lehrplans unterrichtet werden.

Die Zeit, die 15-Jährige für Hausaufgaben in den Fächern Unterrichtssprache, Mathematik und Naturwissenschaften aufbringen, wurde gemäß der Selbstangaben der Schüler im Schülerfragebogen aus PISA 2000 berechnet. In dem Fragebogen stuften die Schüler den zeitlichen Bedarf für die Hausaufgaben in jedem der Fächer gemäß den folgenden vier Kategorien ein: „keine Zeit“, „weniger als 1 Stunde in der Woche“, „1 bis 3 Stunden in der Woche“ und „3 und mehr Stunden in der Woche“. Die Schülerantworten wurden dann über die Fächer hinweg aufaddiert, wobei die Kategorie „keine Zeit“ auf 0 Stunden umkodiert wurde, „weniger als 1 Stunde in der Woche“ auf 0,5 Stunden, „1 bis 3 Stunden in der Woche“ auf 2 Stunden und „3 und mehr Stunden in der Woche" auf 4 Stunden.

Zur Klassifizierung der Fächer und Anmerkungen zu den einzelnen Ländern siehe Anhang 3 unter www.oecd.org/els/education/eag2002. aus der OECD/INESErhebung 2001 zu Lehrern und Lehrplänen und beziehen sich auf das Schuljahr 1999/2000. 
Tabelle D1.1.

Vorgesehene Unterrichtszeit in öffentlichen Bildungseinrichtungen (2000) Vorgesehene Unterrichtszeit in Stunden pro Jahr für 9- bis 14-jährige Schüler

Belgien (Fl.)

Belgien (Frz.

Tschechische Rep.*

Dänemark

England

Finnland*

Frankreich

Deutschland

Griechenland*

Ungarn*

Island

Irland*

Italien

Japan

Korea

Mexiko

Niederlande*

Neuseeland

Norwegen

Portugal

Schottland*

Spanien*

Schweden*

Türkei

Vereinigte Staaten

Ländermittel

Argentinien

Brasilien

Chile

China

Ägypten

Indien

Indonesien

Jamaika

Jordanien

Malaysia

Paraguay

Peru $^{1}$

뚭 Philippinen

: Russische Föd.

Thailand

Tunesien

Uruguay

Simbabwe

1. Referenzjahr 1999

* Hinweise s. Anhang 3 (www.oecd.org/els/education/eag2002).

Quelle: OECD. 
Tabelle D1.2a.

Vorgesehene Unterrichtszeit für 9- bis 11-jährige Schüler in öffentlichen Bildungseinrichtungen, nach Fächern (2000) Anteil der vorgesehenen Unterrichtszeit pro Fach für 9- bis 11-jährige Schüler in Prozent der vorgesehenen Gesamtunterrichtszeit und die Aufteilung der Unterrichtszeit auf den Pflicht- und den Nicht-Pflichtteil des Lehrplans

\begin{tabular}{|c|c|c|c|c|c|c|c|c|c|c|c|c|c|c|c|}
\hline & \multicolumn{12}{|c|}{ Kernpflichtteil des Lehrplans } & \multirow[b]{2}{*}{$\begin{array}{c}\text { Wahl- } \\
\text { Pflichtteil }\end{array}$} & \multirow[b]{2}{*}{$\begin{array}{l}\text { Pflichteil } \\
\text { insgesamt }\end{array}$} & \multirow[b]{2}{*}{$\begin{array}{c}\text { Nicht- } \\
\text { Pflichteil }\end{array}$} \\
\hline & $\begin{array}{l}\text { Lesen, } \\
\text { Schrei- } \\
\text { ben u. } \\
\text { Literatur }\end{array}$ & $\begin{array}{l}\text { Mathe- } \\
\text { matik }\end{array}$ & $\begin{array}{c}\text { Natur- } \\
\text { wissen- } \\
\text { schaften }\end{array}$ & $\begin{array}{l}\text { Sozial- } \\
\text { kunde }\end{array}$ & $\begin{array}{c}\text { Mo- } \\
\text { derne } \\
\text { Fremd- } \\
\text { sprachen }\end{array}$ & Technik & Kunst & Sport & $\begin{array}{l}\text { Reli- } \\
\text { gion }\end{array}$ & $\begin{array}{c}\text { Berufliche } \\
\text { Fertig- } \\
\text { keiten }\end{array}$ & Andere & $\begin{array}{c}\text { Kern- } \\
\text { pflichtteil } \\
\text { insgesamt }\end{array}$ & & & \\
\hline & (1) & (2) & (3) & (4) & (5) & (6) & (7) & (8) & (9) & (10) & (11) & (12) & (13) & (14) & (15) \\
\hline Australien* & 12 & 8 & 2 & 3 & 2 & 2 & 4 & 4 & 1 & $\mathrm{n}$ & $\mathrm{n}$ & 40 & 60 & 100 & $\mathrm{n}$ \\
\hline Österreich & $\mathrm{m}$ & $\mathrm{m}$ & $\mathrm{m}$ & $\mathrm{m}$ & $\mathrm{m}$ & $\mathrm{m}$ & $\mathrm{m}$ & $\mathrm{m}$ & $\mathrm{m}$ & $\mathrm{m}$ & $\mathrm{m}$ & $\mathrm{m}$ & $\mathrm{m}$ & $\mathrm{m}$ & $\mathrm{m}$ \\
\hline Belgien (Fl.)* & $\mathrm{m}$ & $\mathrm{m}$ & $\mathrm{m}$ & $\mathrm{m}$ & $\mathrm{m}$ & $\mathrm{m}$ & $\mathrm{m}$ & $\mathrm{m}$ & $\mathrm{m}$ & $\mathrm{m}$ & $\mathrm{m}$ & $\mathrm{m}$ & $\mathrm{m}$ & $\mathrm{m}$ & $\mathrm{m}$ \\
\hline Belgien (Frz.) & $\mathrm{m}$ & $\mathrm{m}$ & $\mathrm{m}$ & $\mathrm{m}$ & $\mathrm{m}$ & $\mathrm{m}$ & $\mathrm{m}$ & $\mathrm{m}$ & $\mathrm{m}$ & $\mathrm{m}$ & $\mathrm{m}$ & $\mathrm{m}$ & $\mathrm{m}$ & $\mathrm{m}$ & $\mathrm{m}$ \\
\hline Tschechische Rep. ${ }^{1} *$ & 23 & 18 & 15 & 5 & 12 & $\mathrm{n}$ & 14 & 9 & $\mathrm{n}$ & 2 & $\mathrm{n}$ & 98 & 2 & 100 & $\mathrm{~m}$ \\
\hline Dänemark* & 24 & 15 & 8 & 4 & 10 & $\mathrm{n}$ & 22 & 10 & 4 & $\mathrm{n}$ & 4 & 100 & $\mathrm{n}$ & 100 & $\mathrm{n}$ \\
\hline England & 27 & 22 & 11 & 10 & $\mathrm{n}$ & 9 & 10 & 7 & 5 & $\mathrm{n}$ & $\mathrm{n}$ & 100 & $\mathrm{n}$ & 100 & $\mathrm{n}$ \\
\hline Finnland* & 23 & 16 & 11 & 2 & 6 & $\mathrm{n}$ & 9 & 9 & 6 & 6 & $\mathrm{n}$ & 86 & 14 & 100 & 4 \\
\hline Frankreich & 29 & 21 & 5 & 8 & 9 & 4 & 9 & 16 & $\mathrm{n}$ & $\mathrm{n}$ & $\mathrm{n}$ & 100 & $\mathrm{n}$ & 100 & $\mathrm{n}$ \\
\hline Deutschland & 20 & 17 & 7 & 8 & 7 & $\mathrm{n}$ & 16 & 11 & 7 & $\mathrm{n}$ & 2 & 96 & 4 & 100 & $\mathrm{n}$ \\
\hline Griechenland* & 29 & 14 & 11 & 11 & 10 & $\mathrm{n}$ & 8 & 7 & 7 & $\mathrm{n}$ & 2 & 100 & $\mathrm{n}$ & 100 & $\mathrm{n}$ \\
\hline Island & 20 & 13 & 4 & 7 & 2 & $\mathrm{n}$ & 17 & 10 & 7 & 3 & $\mathrm{n}$ & 84 & 16 & 100 & $\mathrm{n}$ \\
\hline Irland* & 29 & 12 & $\mathrm{x}(4)$ & 12 & $\mathrm{n}$ & $\mathrm{n}$ & 12 & 4 & 10 & $\mathrm{n}$ & 14 & 92 & 8 & 100 & $\mathrm{n}$ \\
\hline Italien & 17 & 10 & 8 & 11 & 10 & 3 & 13 & 7 & 6 & $\mathrm{n}$ & $\mathrm{n}$ & 84 & 16 & 100 & $\mathrm{n}$ \\
\hline Japan* & 23 & 17 & 10 & 10 & $\mathrm{n}$ & 5 & 14 & 10 & $\mathrm{n}$ & $\mathrm{n}$ & 10 & 100 & $\mathrm{n}$ & 100 & $\mathrm{n}$ \\
\hline Korea & 19 & 14 & 12 & 11 & 6 & $\mathrm{n}$ & 12 & 9 & $\mathrm{n}$ & 3 & 3 & 91 & 9 & 100 & $\mathrm{n}$ \\
\hline Mexiko & 30 & 25 & 15 & 20 & $\mathrm{n}$ & $\mathrm{n}$ & 5 & 5 & $\mathrm{n}$ & $\mathrm{n}$ & $\mathrm{n}$ & 100 & $\mathrm{n}$ & 100 & $\mathrm{n}$ \\
\hline Niederlande ${ }^{2 *}$ & 30 & 19 & $\mathrm{x}(4)$ & 15 & 2 & 2 & 10 & 7 & 4 & $\mathrm{n}$ & 12 & 100 & $\mathrm{n}$ & 100 & $\mathrm{n}$ \\
\hline Neuseeland* & 42 & 19 & 7 & 8 & $x(1)$ & 7 & 9 & 9 & $\mathrm{~m}$ & $\mathrm{n}$ & $\mathrm{m}$ & 100 & $\mathrm{n}$ & 100 & 10 \\
\hline Norwegen ${ }^{3 *}$ & 19 & 14 & 8 & 8 & 7 & $\mathrm{n}$ & 17 & 7 & 9 & $\mathrm{n}$ & 9 & 100 & $\mathrm{n}$ & 100 & $\mathrm{n}$ \\
\hline Portugal $^{3 *}$ & 16 & 13 & 10 & 10 & 13 & 16 & 10 & 10 & 3 & $\mathrm{n}$ & $\mathrm{n}$ & 100 & $\mathrm{n}$ & 100 & $\mathrm{n}$ \\
\hline Schottland* & 20 & 15 & 5 & 5 & $\mathrm{x}(1)$ & 5 & 10 & 5 & 15 & $\mathrm{x}(13)$ & $\mathrm{n}$ & 80 & 20 & 100 & $\mathrm{n}$ \\
\hline Spanien* & 24 & 17 & 9 & 9 & 13 & $\mathrm{n}$ & 11 & 11 & $\mathrm{x}(13)$ & $\mathrm{n}$ & $\mathrm{n}$ & 93 & 7 & 100 & $\mathrm{n}$ \\
\hline Schweden* & 22 & 14 & 12 & 13 & 12 & $\mathrm{x}^{4}$ & 7 & 8 & $\mathrm{x}^{4}$ & 7 & $\mathrm{n}$ & 94 & 6 & 100 & $\mathrm{n}$ \\
\hline Türkei & 19 & 13 & 10 & 10 & 9 & $\mathrm{n}$ & 7 & 6 & 7 & 10 & 1 & 91 & 9 & 100 & 10 \\
\hline Vereinigte Staaten & $\mathrm{m}$ & $\mathrm{m}$ & $\mathrm{m}$ & $\mathrm{m}$ & $\mathrm{m}$ & $\mathrm{m}$ & $\mathrm{m}$ & $\mathrm{m}$ & $\mathrm{m}$ & $\mathrm{m}$ & $\mathrm{m}$ & $\mathrm{m}$ & $\mathrm{m}$ & $\mathrm{m}$ & $\mathrm{m}$ \\
\hline Chile & $\mathrm{x}(12)$ & $\mathrm{x}(12)$ & $\mathrm{x}(12)$ & $\mathrm{x}(12)$ & $\mathrm{x}(12)$ & $\mathrm{x}(12)$ & $\mathrm{x}(12)$ & $\mathrm{x}(12)$ & $\mathrm{x}(12)$ & $\mathrm{x}(12)$ & $\mathrm{x}(12)$ & 81 & 19 & 100 & $\mathrm{~m}$ \\
\hline China & 26 & 18 & 6 & 9 & $\mathrm{n}$ & $\mathrm{n}$ & 12 & 9 & $\mathrm{n}$ & 3 & 12 & 94 & 6 & 100 & $\mathrm{~m}$ \\
\hline Ägypten & 30 & 15 & 9 & 6 & 9 & 2 & 5 & 7 & 7 & 5 & 5 & 100 & a & 100 & $\mathrm{~m}$ \\
\hline Indien & 19 & 17 & 12 & 12 & 19 & a & 4 & 6 & a & a & 6 & 96 & 4 & 100 & $\mathrm{~m}$ \\
\hline Indonesien & 22 & 22 & 13 & 11 & a & a & 5 & 5 & 5 & 13 & 5 & 100 & a & 100 & $\mathrm{~m}$ \\
\hline Jamaika & 25 & 23 & 9 & 9 & a & a & 6 & 6 & 6 & $\mathrm{a}$ & 16 & 100 & $\mathrm{n}$ & 100 & $\mathrm{~m}$ \\
\hline Jordanien & 24 & 16 & 13 & 8 & 12 & a & 3 & 6 & 9 & 5 & 3 & 100 & a & 100 & $\mathrm{~m}$ \\
\hline Malaysia & 21 & 15 & 11 & 9 & 15 & $\mathrm{n}$ & 4 & 4 & 13 & 4 & 4 & 100 & a & 100 & $\mathrm{~m}$ \\
\hline Paraguay & 26 & 13 & 8 & 10 & $\mathrm{x}(13)$ & 7 & 10 & 7 & 3 & $\mathrm{x}(7)$ & 10 & 93 & 7 & 100 & $\mathrm{~m}$ \\
\hline Peru $^{5}$ & $\mathrm{x}(12)$ & $\mathrm{x}(12)$ & $\mathrm{x}(12)$ & $\mathrm{x}(12)$ & $\mathrm{x}(12)$ & $\mathrm{x}(12)$ & $\mathrm{x}(12)$ & $\mathrm{x}(12)$ & $\mathrm{x}(12)$ & $\mathrm{x}(12)$ & $\mathrm{a}$ & 70 & 30 & 100 & $\mathrm{~m}$ \\
\hline Philippinen & 13 & 13 & 13 & 13 & 13 & $\mathrm{a}$ & 8 & 4 & $\mathrm{a}$ & 13 & 13 & 100 & a & 100 & $\mathrm{~m}$ \\
\hline Russische Föd. & 31 & 15 & 4 & 9 & 6 & 6 & 6 & 6 & a & $\mathrm{m}$ & $\mathrm{m}$ & 85 & 15 & 100 & $\mathrm{~m}$ \\
\hline Thailand & 14 & 10 & $\mathrm{x}(11)$ & $\mathrm{x}(11)$ & $\mathrm{x}(15)$ & $\mathrm{x}(15)$ & $\mathrm{x}(11)$ & $\mathrm{x}(11)$ & $\mathrm{x}(11)$ & 23 & 39 & 86 & 14 & 100 & $\mathrm{~m}$ \\
\hline Tunesien & 62 & 13 & 5 & 7 & $\mathrm{n}$ & 2 & 3 & 3 & 4 & $\mathrm{n}$ & $\mathrm{n}$ & 100 & a & 100 & $\mathrm{~m}$ \\
\hline Uruguay & 28 & 29 & 13 & 19 & a & $\mathrm{a}$ & 9 & 3 & $\mathrm{a}$ & $\mathrm{a}$ & a & 100 & a & 100 & $\mathrm{~m}$ \\
\hline Simbabwe & 17 & 17 & 14 & 11 & 17 & $\mathrm{n}$ & 5 & 5 & 8 & 3 & 3 & 100 & $\mathrm{n}$ & 100 & $\mathrm{~m}$ \\
\hline
\end{tabular}

Hinweis: $\mathrm{x}$ bedeutet, dass die Daten in einer anderen Spalte enthalten sind, deren Referenz in runden Klammern nach dem ' $\mathrm{x}$ ' angegeben ist. So bedeutet z.B. $x(2)$, dass die Daten in Spalte 2 enthalten sind.

1. Für die 9- bis 10-jährigen Schüler ist die Sozialkunde in den Naturwissenschaften enthalten.

2. Umfasst ausschließlich 9- bis 11-jährige Schüler.

3. Umfasst ausschließlich 10- bis 11 -jährige Schüler.

4. In verschiedenen Fächern enthalten.

5. Referenzjahr 1999.

* Hinweise s. Anhang 3 (www.oecd.org/els/education/eag2002).

Quelle: OECD. 
Tabelle D1.2b.

Vorgesehene Unterrichtszeit für 12- bis 14-jährige Schüler in öffentlichen Bildungseinrichtungen, nach Fächern (2000) Anteil der vorgesehenen Unterrichtszeit pro Fach für 12- bis 14-jährige Schüler in Prozent der vorgesehenen Gesamtunterrichtszeit und die Aufteilung der Unterrichtszeit auf den Pflicht- und den Nicht-Pflichtteil des Lehrplans

\begin{tabular}{|c|c|c|c|c|c|c|c|c|c|c|c|c|c|c|c|}
\hline & \multicolumn{12}{|c|}{ Kernpflichtteil des Lehrplans } & \multirow[b]{2}{*}{$\begin{array}{c}\text { Wahl- } \\
\text { Pflichtteil }\end{array}$} & \multirow[b]{2}{*}{$\begin{array}{l}\text { Pflichteil } \\
\text { insgesamt }\end{array}$} & \multirow[b]{2}{*}{$\begin{array}{c}\text { Nicht- } \\
\text { Pflichteil }\end{array}$} \\
\hline & $\begin{array}{l}\text { Lesen, } \\
\text { Schrei- } \\
\text { ben u. } \\
\text { Literatur } \\
\end{array}$ & $\begin{array}{c}\text { Mathe- } \\
\text { matik }\end{array}$ & $\begin{array}{c}\text { Natur- } \\
\text { wissen- } \\
\text { schaften }\end{array}$ & $\begin{array}{l}\text { Sozial- } \\
\text { kunde }\end{array}$ & $\begin{array}{c}\text { Mo- } \\
\text { derne } \\
\text { Fremd- } \\
\text { sprachen } \\
\end{array}$ & Technik & Kunst & Sport & $\begin{array}{l}\text { Reli- } \\
\text { gion }\end{array}$ & $\begin{array}{l}\text { Beru- } \\
\text { fliche } \\
\text { Fertig- } \\
\text { keiten }\end{array}$ & Andere & $\begin{array}{c}\text { Kern- } \\
\text { pflichtteil } \\
\text { insgesamt }\end{array}$ & & & \\
\hline & (1) & (2) & (3) & (4) & (5) & $(6)$ & (7) & $(8)$ & $(9)$ & $(10)$ & (11) & $(12)$ & (13) & (14) & $(15)$ \\
\hline Australien* & 11 & 11 & 9 & 8 & 5 & 7 & 7 & 8 & 1 & $\mathrm{n}$ & 3 & 72 & 28 & 100 & 5 \\
\hline Österreich & 11 & 14 & 13 & 11 & 9 & 5 & 11 & 10 & 5 & 2 & 9 & 100 & $\mathrm{n}$ & 100 & $\mathrm{n}$ \\
\hline Belgien (Frz.) $)^{1}$ & 15 & 14 & 6 & 12 & 12 & 3 & 3 & 9 & 6 & $\mathrm{n}$ & 5 & 85 & 15 & 100 & 6 \\
\hline Tschechische Rep.* & 13 & 14 & 16 & 13 & 10 & $\mathrm{n}$ & 8 & 7 & $\mathrm{n}$ & 6 & $\mathrm{n}$ & 87 & 13 & 100 & $\mathrm{n}$ \\
\hline Dänemark* & 23 & 15 & 14 & 13 & 11 & $\mathrm{n}$ & 10 & 8 & 4 & $\mathrm{n}$ & 4 & 100 & $\mathrm{n}$ & 100 & 11 \\
\hline England & 14 & 14 & 13 & 13 & 11 & 13 & 9 & 9 & 4 & $\mathrm{n}$ & $\mathrm{n}$ & 100 & $\mathrm{n}$ & 100 & 10 \\
\hline Finnland* & 13 & 12 & 13 & 5 & 13 & $\mathrm{n}$ & 5 & 7 & 4 & 6 & 2 & 79 & 21 & 100 & 1 \\
\hline Frankreich & 17 & 15 & 12 & 13 & 12 & 6 & 7 & 11 & $\mathrm{n}$ & $\mathrm{n}$ & $\mathrm{n}$ & 93 & 7 & 100 & 10 \\
\hline Deutschland & 14 & 13 & 11 & 12 & 16 & 3 & 10 & 10 & 5 & 1 & 3 & 98 & 2 & 100 & $\mathrm{n}$ \\
\hline Ungarn* & 13 & 13 & 12 & 16 & 9 & 4 & 12 & 9 & $\mathrm{n}$ & 8 & 5 & 100 & $\mathrm{n}$ & 100 & 28 \\
\hline Island & 15 & 12 & 8 & 7 & 15 & $\mathrm{n}$ & 14 & 9 & 3 & 6 & $\mathrm{n}$ & 88 & 12 & 100 & $\mathrm{n}$ \\
\hline Irland* & 28 & 14 & 11 & 22 & 11 & $x(13,15)$ & $\mathrm{x}(13,15)$ & 6 & 8 & $\mathrm{x}(13,15)$ & $\mathrm{n}$ & 100 & $\mathrm{n}$ & 100 & 11 \\
\hline Italien $^{1}$ & 22 & 10 & 10 & 15 & 10 & 10 & 13 & 7 & 3 & $\mathrm{n}$ & $\mathrm{n}$ & 100 & $\mathrm{n}$ & 100 & $\mathrm{n}$ \\
\hline Japan* & 14 & 12 & 11 & 12 & 13 & 7 & 11 & 10 & $\mathrm{n}$ & $\mathrm{n}$ & 7 & 98 & 2 & 100 & $\mathrm{n}$ \\
\hline Korea & 14 & 12 & 12 & 11 & 12 & 5 & 8 & 9 & $\mathrm{n}$ & 4 & 6 & 91 & 9 & 100 & $\mathrm{n}$ \\
\hline Mexiko & 14 & 14 & 19 & 21 & 9 & 9 & 6 & 6 & $\mathrm{n}$ & $\mathrm{n}$ & $\mathrm{n}$ & 97 & 3 & 100 & $\mathrm{n}$ \\
\hline Niederlande & 10 & 10 & 8 & 11 & 14 & 5 & 7 & 9 & $\mathrm{n}$ & 3 & $\mathrm{n}$ & 78 & 22 & 100 & $\mathrm{n}$ \\
\hline Neuseeland* & 24 & 17 & 12 & 12 & $\mathrm{x}(1)$ & 12 & 11 & 11 & $\mathrm{n}$ & $\mathrm{n}$ & $\mathrm{n}$ & 100 & $\mathrm{n}$ & 100 & 5 \\
\hline Norwegen* & 16 & 13 & 9 & 11 & 10 & $\mathrm{n}$ & 8 & 10 & 7 & $\mathrm{n}$ & 10 & 94 & 6 & 100 & $\mathrm{n}$ \\
\hline Portugal* & 13 & 13 & 15 & 17 & 10 & $\mathrm{n}$ & 10 & 10 & 3 & $\mathrm{n}$ & $\mathrm{n}$ & 90 & 10 & 100 & $\mathrm{n}$ \\
\hline Schottland* & 19 & 10 & 9 & 9 & $x(1)$ & 8 & 8 & 5 & 5 & $\mathrm{x}(13)$ & $\mathrm{n}$ & 73 & 27 & 100 & $\mathrm{n}$ \\
\hline Spanien* & 18 & 13 & 10 & 10 & 11 & 5 & 12 & 8 & $\mathrm{x}(13)$ & $\mathrm{x}(13)$ & $\mathrm{x}(13)$ & 88 & 12 & 100 & $\mathrm{n}$ \\
\hline Schweden* & 22 & 14 & 12 & 13 & 12 & $x^{2}$ & 7 & 8 & $\mathrm{x}^{2}$ & 7 & $\mathrm{n}$ & 94 & 6 & 100 & $\mathrm{n}$ \\
\hline Türkei ${ }^{1}$ & 17 & 13 & 10 & 12 & 13 & $\mathrm{n}$ & 7 & 3 & 7 & 10 & 2 & 93 & 7 & 100 & 10 \\
\hline Argentinien & 13 & 13 & 13 & 15 & 8 & 8 & 8 & 8 & $\mathrm{a}$ & $\mathrm{a}$ & 5 & 90 & 10 & 100 & $\mathrm{~m}$ \\
\hline Chile & $\mathrm{x}(12)$ & $\mathrm{x}(12)$ & $\mathrm{x}(12)$ & $\mathrm{x}(12)$ & $\mathrm{x}(12)$ & $\mathrm{x}(12)$ & $\mathrm{x}(12)$ & $\mathrm{x}(12)$ & $\mathrm{x}(12)$ & $\mathrm{x}(12)$ & $\mathrm{x}(12)$ & 92 & 8 & 100 & $\mathrm{~m}$ \\
\hline China & 14 & 12 & 9 & 17 & 11 & $\mathrm{n}$ & 5 & 7 & $\mathrm{n}$ & 5 & 11 & 92 & 8 & 100 & $\mathrm{~m}$ \\
\hline Ägypten & 24 & 13 & 11 & 8 & 13 & 5 & 5 & 5 & 5 & 5 & 4 & 100 & a & 100 & $\mathrm{~m}$ \\
\hline Indien & 11 & 13 & 15 & 13 & 13 & $\mathrm{a}$ & 4 & 6 & $\mathrm{a}$ & a & 9 & 83 & 17 & 100 & $\mathrm{~m}$ \\
\hline Indonesien & 16 & 16 & 14 & 13 & 6 & $\mathrm{a}$ & 5 & 5 & 5 & 15 & 5 & 100 & a & 100 & $\mathrm{~m}$ \\
\hline Jamaika & 17 & 14 & 14 & 14 & 6 & 17 & 6 & 6 & 6 & 3 & $\mathrm{n}$ & 100 & $\mathrm{n}$ & 100 & $\mathrm{~m}$ \\
\hline Jordanien & 21 & 13 & 15 & 9 & 16 & 2 & 3 & 4 & 9 & 6 & 3 & 100 & a & 100 & $\mathrm{~m}$ \\
\hline Malaysia & 13 & 11 & 11 & 13 & 11 & $\mathrm{n}$ & 4 & 4 & 9 & 9 & 13 & 100 & a & 100 & $\mathrm{~m}$ \\
\hline Paraguay & 20 & 12 & 14 & 13 & $\mathrm{x}(13)$ & 12 & 10 & 5 & 2 & $x(7)$ & 7 & 95 & 5 & 100 & $\mathrm{~m}$ \\
\hline Peru $^{3}$ & 14 & 14 & 12 & 23 & 6 & $\mathrm{a}$ & 6 & 6 & 6 & 7 & a & 93 & 7 & 100 & $\mathrm{~m}$ \\
\hline Philippinen & 9 & 9 & 9 & 9 & 9 & 18 & 6 & 3 & $\mathrm{a}$ & a & 9 & 82 & 18 & 100 & $\mathrm{~m}$ \\
\hline Russische Föd. & 23 & 13 & 14 & 13 & 8 & 6 & 4 & 5 & a & $\mathrm{a}$ & $\mathrm{m}$ & 87 & 13 & 100 & $\mathrm{~m}$ \\
\hline Thailand & 11 & 6 & 9 & 11 & $\mathrm{x}(13)$ & $\mathrm{x}(13)$ & 3 & 9 & $\mathrm{x}(11)$ & 6 & 14 & 69 & 31 & 100 & $\mathrm{~m}$ \\
\hline Tunesien & 33 & 13 & 5 & 15 & 7 & 5 & 7 & 10 & 5 & $\mathrm{n}$ & $\mathrm{n}$ & 100 & $\mathrm{a}$ & 100 & $\mathrm{~m}$ \\
\hline Uruguay & 13 & 13 & 19 & 18 & 8 & $\mathrm{a}$ & 5 & 5 & a & a & $\mathrm{a}$ & 81 & 19 & 100 & $\mathrm{~m}$ \\
\hline Simbabwe & 14 & 14 & 11 & 9 & 14 & 9 & 7 & 4 & 7 & 10 & 2 & 100 & $\mathrm{n}$ & 100 & $\mathrm{~m}$ \\
\hline
\end{tabular}

Hinweis: $\mathrm{x}$ bedeutet, dass die Daten in einer anderen Spalte enthalten sind, deren Referenz in runden Klammern nach dem ' $\mathrm{x}$ ' angegeben ist.

So bedeutet z.B. x(2), dass die Daten in Spalte 2 enthalten sind.

1. Umfasst ausschließlich 12- und 13-jährige Schüler.

2. In verschiedenen Fächern enthalten.

3. Referenzjahr 1999.

* Hinweise s. Anhang 3 (www.oecd.org/els/education/eag2002).

Quelle: OECD. 
Tabelle D1.3.

Zusätzliche Unterrichts- und Lernzeit 15-jähriger Schüler (2000)

Prozentsatz der Schüler, die eine Schule besuchen, die Zusatzkurse anbietet, und Prozentsatz der Schüler, die innerhalb und außerhalb der Schule zusätzlichen Unterricht haben sowie geschätzte Stundenzahl, die durchschnittlich für Hausaufgaben verwendet wird

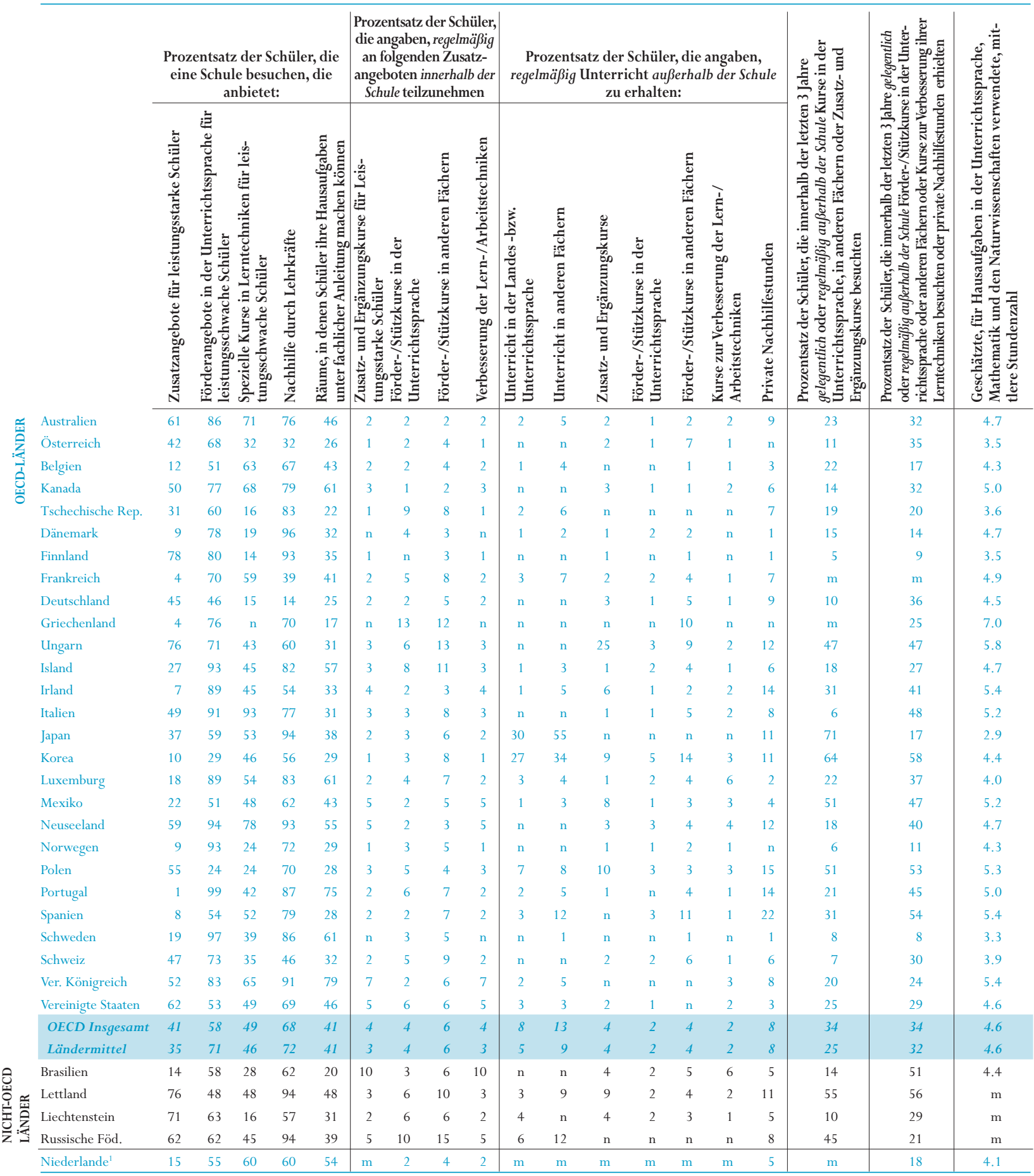

1. Die Beteiligungsquote ist zu niedrig, um die Vergleichbarkeit zu gewährleisten (s. Anhang 3, www.oecd.org/els/education/eag2002)

Quelle: OECD PISA-Datenbank, 2001. Hinweise zur Methodik s. Anhang 3 (www.oecd.org/els/education/eag2002) und www.pisa.oecd.org. 


\section{KLASSENGRÖßE UND ZAHLENMÄßIGES SCHÜLER/ LEHRKRÄFTE-VERHÄLTNIS}

- Die durchschnittliche Klassengröße liegt im Primarbereich bei 22 Schülern, sie reicht jedoch von 36 Schülern pro Klasse in Korea bis zu weniger als 18 in Griechenland, Irland und Luxemburg.

- Die Zahl der Schüler pro Klasse nimmt vom Primar- zum Sekundarbereich I im Durchschnitt um 2 Schüler zu, aufgrund der höheren Unterrichtsstundenzahl pro Jahr nimmt das zahlenmäßige Schüler/ Lehrkräfte-Verhältnis jedoch eher ab je höher der Bildungsbereich ist.

\section{Abbildung D2.1.}

Durchschnittliche Klassengröße in öffentlichen und privaten

Bildungseinrichtungen, nach Bildungsbereich (2002)

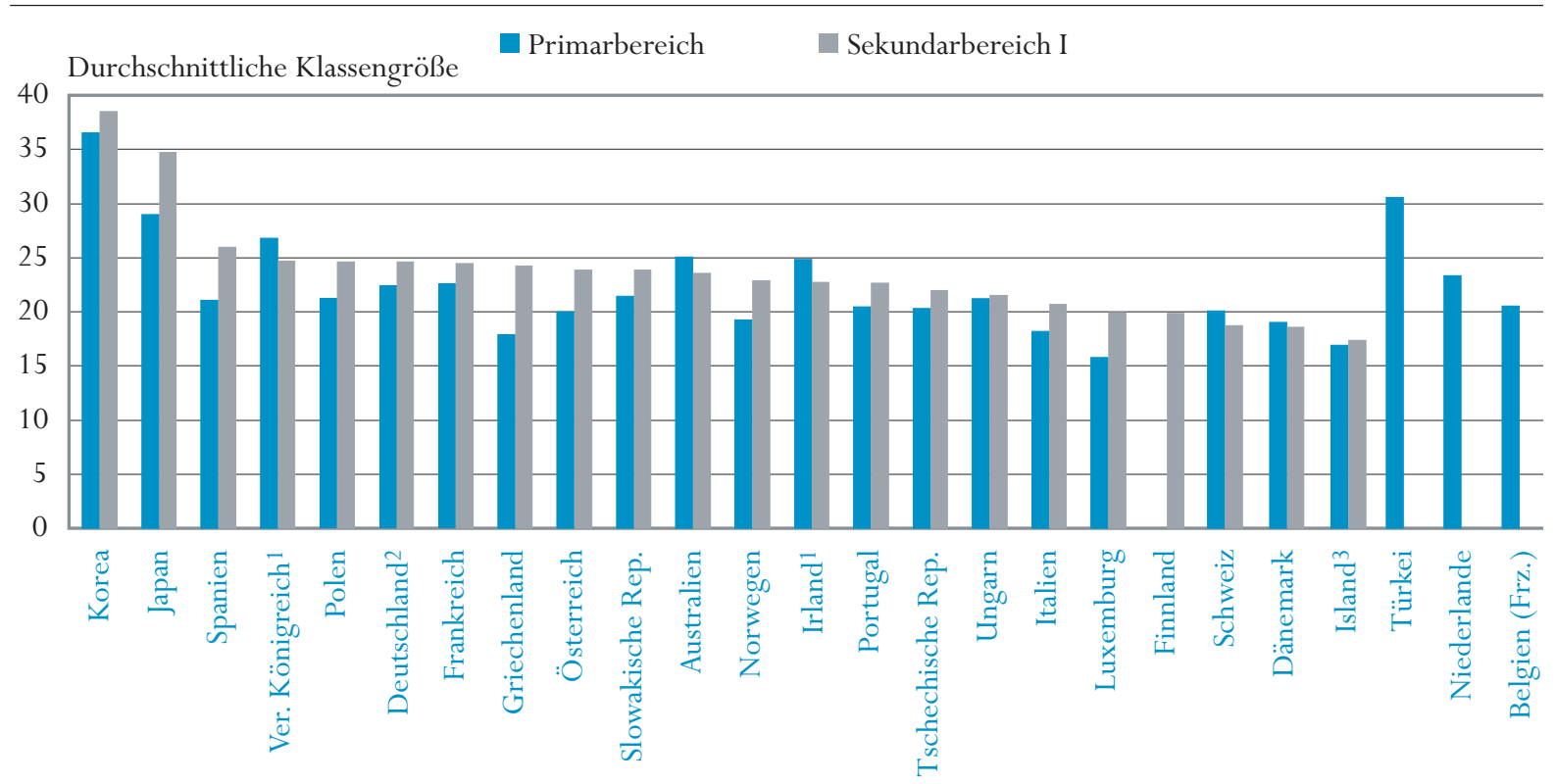

1. Nur öffentliche Bildungseinrichtungen.

2. Referenzjahr 2001.

3. Einschließlich Jahrgangsübergreifender Klassen.

Anordnung der Länder in absteigender Reihenfolge der durchschnittlichen Klassengröße im Sekundarbereich I.

Quelle: OECD. Tabelle D2.1. Hinweise s. Anhang 3 (www.oecd.org/els/education/eag2002). 


\section{Politischer Hintergrund}

In vielen OECD-Ländern gibt es heftige Diskussionen über die optimalen Klassengrößen. Kleinere Klassen werden geschätzt, weil sich dort die Lehrkraft mehr dem einzelnen Schüler widmen kann und sich die mit größeren Klassen verbundenen Nachteile, nämlich viele Schüler und deren Arbeit im Auge haben zu müssen, reduzieren. Außerdem können kleinere Klassen die Eltern bei der Auswahl der Schule beeinflussen. Gleichzeitig sind jedoch die Kosten für die Lehrkräfte der größte Einzelposten bei den Bildungsausgaben, so dass eine Verringerung der Klassengröße zu einem starken Anstieg der Bildungskosten führen kann.

Ein weiterer wichtiger Indikator für die für Bildungszwecke bereitgestellten Ressourcen ist das zahlenmäßige Schüler/Lehrkräfte-Verhältnis. Da es vor allem in den höheren Bildungsbereichen schwierig ist, direkte Kennzahlen für die Bildungsqualität zu definieren, wird dieser Indikator oft als indirekte Kennzahl zur Messung der Bildungsqualität herangezogen. Dies geschieht unter der Annahme, dass ein niedrigeres zahlenmäßiges Schüler/Lehrkräfte-Verhältnis den Schülern einen besseren Zugang zu den Lehrressourcen ermöglicht. Es mag abzuwägen sein zwischen einem geringeren zahlenmäßigen Schüler/ Lehrkräfte-Verhältnis und höheren Gehältern für Lehrer, größeren Klassen, höheren Investitionen in Unterrichtstechnologien oder dem verstärkten Einsatz von Hilfslehrkräften und anderen Hilfskräften, deren Gehalt oft deutlich unter dem qualifizierter Lehrer liegt. Da außerdem zunehmend mehr Kinder mit speziellen Bildungsbedürfnissen in Regelklassen integriert werden, könnte der verstärkte Einsatz von Fachpersonal und Unterstützungsdiensten die für eine Reduzierung des Schüler/Lehrkräfte-Verhältnisses erforderlichen Ressourcen einschränken.

\section{Ergebnisse und Erläuterungen}

\section{Durchschnittliche Klassengröße im Primar- und Sekundarbereich I}

Im Primarbereich differiert die durchschnittliche Klassengröße zwischen den einzelnen OECD-Ländern sehr. Die Zahl reicht von 36 Schülern pro Primarklasse in Korea bis zu weniger als 20 in Dänemark, Griechenland, Island, Italien, Luxemburg und Norwegen. Im Sekundarbereich I reicht die durchschnittliche Klassengröße von 38 Schülern pro Klasse in Korea bis zu weniger als 20 Schülern in Dänemark, Finnland, Island, Luxemburg und der Schweiz (Tabelle D2.1).

Vom Primar- zum Sekundarbereich I steigt die Zahl der Schüler pro Klasse im Schnitt um 2 Schüler an. In Griechenland, Japan, Luxemburg und Spanien steigt die durchschnittliche Klassengröße vom Primar- zum Sekundarbereich I um mehr als 4 Schüler, während sie in Australien, Dänemark, Irland, der Schweiz und dem Vereinigten Königreich zwischen diesen beiden Bildungsbereichen sinkt (Abbildung D2.1). Dieser Indikator zur Klassengröße ist auf den Primar- und Sekundarbereich I beschränkt, da es insbesondere
Dieser Indikator gibt

Auskunft über die

Klassengrößse und das

zahlenmäßsige Schüler/

Lehrkräfte-Verhältnis.
Im Primarbereich liegt die durchschnittliche Klassengrößse bei 22 Schülern, diese Zahl differiert jedoch von Land zu Land zwischen 36 Schülern pro Klasse und weniger als 18.

Vom Primar- zum Sekundarbereich I steigt die Zahl der Schüler pro Klasse im Schnitt um 2 schüler an. 
In Griechenland,

Norwegen, Polen, der

Schweiz, der Tschechischen

Republik und der Türkei

sind die Klassen an

öffentlichen Schulen um

mindestens 3 Schüler

größer als an privaten

Bildungseinrichtungen.

Viele Faktoren sind für

die Unterschiede beim

zahlenmäfsigen Schüler/

Lehrkräfte-Verhältnis

verantwortlich.

In Korea und der Türkei ist das zahlenmäßsige schüler/Lehrkräfte-

verhältnis im

Primarbereich etwa dreimal so hoch wie in

Dänemark und Ungarn.

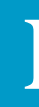

in den höheren Bildungsbereichen zu schwierig ist, die Klassengröße zu definieren und zu vergleichen, denn in vielen Ländern gibt es hier keinen festen Klassenverband mehr, sondern die Schüler bilden je nach Fach und Zug immer neu zusammengesetzte Unterrichtsgruppen.

In neun der 20 Länder mit vergleichbaren Daten unterscheiden sich die Klassengrößen im Primarbereich zwischen öffentlichen und privaten Bildungseinrichtungen um mehr als 3 Schüler. Im Sekundarbereich I verringert sich der Unterschied, aber in 8 von 18 Ländern ist die durchschnittliche Klassengröße an privaten Schulen des Sekundarbereich I niedriger als an öffentlichen Schulen (Tabelle D2.1).

\section{Zahlenmäßiges Schüler/Lehrkräfte-Verhältnis}

Dieser Indikator untersucht auch das zahlenmäßige Schüler/LehrkräfteVerhältnis, das sich ergibt, wenn, gemessen in Vollzeit-Äquivalenten, die Zahl der Schüler eines bestimmten Bildungsbereichs durch die Zahl der „Lehrkräfte” des gleichen Bildungsbereichs und der gleichen Schulart dividiert wird. Die Beziehung zwischen dem zahlenmäßigen Schüler/Lehrkräfte-Verhältnis und der durchschnittlichen Klassengröße wird durch viele Faktoren beeinflusst, unter anderem durch die Anzahl der Stunden, die ein Schüler täglich unterrichtet wird, die Länge des Arbeitstages eines Lehrers, die Zahl der Klassen oder Schüler, für die ein Lehrer verantwortlich ist, Unterschiede je nach Unterrichtsfach, die Zeitaufteilung der Lehrer zwischen Unterricht und anderen Aufgaben, die Gruppierung der Schüler innerhalb der Klassen und die Praxis des Teamunterrichts.

Im Primarbereich reicht das zahlenmäßige Schüler/Lehrkräfte-Verhältnis, ausgedrückt in Vollzeit-Äquivalenten, von 32 Schülern pro Lehrer in Korea bis zu 10 in Dänemark, wobei das Ländermittel im Primarbereich bei 18 Schülern pro Lehrkraft liegt. Im Sekundarbereich schwankt das zahlenmäßige Schüler/Lehrkräfte-Verhältnis etwas mehr und reicht, ausgedrückt in VollzeitÄquivalenten, von mehr als 21 Schülern pro Lehrkraft in Korea und Mexiko bis zu weniger als 11 in Belgien, Griechenland, Italien, Luxemburg und Portugal. Im Durchschnitt aller Länder beträgt das zahlenmäßige Schüler/LehrkräfteVerhältnis im Sekundarbereich 14. Dies liegt sehr nahe bei den für Finnland (14) Deutschland (15), Japan (15), Polen (15), Schweden (14), die Türkei (14), das Vereinigte Königreich (15) und die Vereinigten Staaten (15) ermittelten Werten (Tabelle D2.2).

Je höher der Bildungsbereich, desto geringer die Zahl der Schüler pro Lehrkraft.

Wie die unterschiedlichen Mittelwerte für das zahlenmäßige Schüler/ Lehrkräfte-Verhältnis zwischen Primar- und Sekundarbereich erkennen lassen, stehen den Schülern um so mehr Lehrer zur Verfügung, je höher sie in ihrer Bildungslaufbahn aufsteigen. Mit Ausnahme von Dänemark, Kanada, Mexiko, den Niederlanden, Polen, Schweden und Ungarn verringert sich in allen OECD-Ländern das zahlenmäßige Schüler/Lehrkräfte-Verhältnis vom Primar- zum Sekundarbereich, obwohl die Klassengröße zwischen Primar- und 
Sekundarbereich eher zunimmt. Der Grund für die Zunahme der Klassengröße liegt vor allem darin, dass die Unterrichtszeit mit zunehmend höherem Bildungsbereich tendenziell zunimmt.

In Frankreich, Korea und der Türkei ist der Rückgang beim zahlenmäßigen Schüler/Lehrkräfte-Verhältnis vom Primar- zum Sekundarbereich wesentlich ausgeprägter als in anderen Ländern. Der Unterschied zwischen beiden Bildungsbereichen reicht von 7 bis zu 16 Schülern pro vollzeit-äquivalentem Lehrer. In vielen Ländern, vor allem in Frankreich und Korea, weisen diese Unterschiede auf Unterschiede bei der jährlichen Unterrichtszeit hin, sie könnten aber auch auf zeitliche Verzögerungen bei der Anpassung der Zahl der Lehrkräfte an veränderte demographische Gegebenheiten hinweisen oder auf Unterschiede bei den von Lehrern in den verschiedenen Bildungsbereichen abzuleistenden Schulstunden. Dieser Trend ist zwar über alle Länder hinweg zu beobachten, vom pädagogischen Standpunkt her ist jedoch nicht nachzuvollziehen, warum auf höherer Bildungsebene eher ein geringeres zahlenmäßiges Schüler/Lehrkräfte-Verhältnis wünschenswert sein sollte.

Für öffentliche und private Bildungseinrichtungen des Tertiärbereichs schwankt das zahlenmäßige Studierende/Lehrende-Verhältnis zwischen 27 Studierenden pro Lehrenden in Griechenland und weniger als 12 in Deutschland, Island, Japan, Kanada, Schweden und der Slowakischen Republik (Tabelle D2.2). Allerdings sollten solche Vergleiche für den Tertiärbereich mit Vorsicht betrachtet werden, da im Tertiärbereich immer noch Schwierigkeiten bei der Berechnung von vergleichbaren Vollzeitäquivalenten für Studierende und Lehrende bestehen.

In 11 der 12 Länder, für die Daten für Studiengänge sowohl imTertiärbereich A als auch für weiterführende Forschungsprogramme vorliegen, ist das zahlenmäßige Studierende/Lehrende-Verhältnis in Bildungsgängen des Tertiärbereich B, die generell stärker berufsorientiert sind, niedriger als in Studiengängen des Tertiärbereich A und in weiterführenden Forschungsprogrammen. Im OECDDurchschnitt beträgt das zahlenmäßige Studierende/Lehrende-Verhältnis im Tertiärbereich B 12 im Vergleich zu 16 bei Bildungsgängen des Tertiärbereich A und weiterführenden Forschungsprogrammen (Abbildung D2.2). Deutschland ist das einzige Land, in dem in den Studiengängen des Tertiärbereich B mehr Studierende auf einen Lehrenden kommen als in den Studiengängen des Tertiärbereich A und den weiterführenden Forschungsprogrammen.

Im Elementarbereich ist das zahlenmäßige Schüler/Lehrkräfte-Verhältnis tendenziell niedriger als im Primarbereich, aber etwas höher als im Sekundarbereich. Das zahlenmäßige Schüler/Lehrkräfte-Verhältnis im Elementarbereich reicht von weniger als 7 Schülern pro Lehrkraft in Dänemark und Island bis zu über 22 Schülern in Deutschland, Korea und Mexiko. Es besteht offensichtlich nur eine geringe Beziehung zwischen dem zahlenmäßigen Schüler/Lehrkräfte-Verhältnis im Elementar- und Primarbereich, was darauf
Im Allgemeinen ist das zahlenmäfige Schüler/ Lehrkräfte-Verhältnis im Tertiärbereich und im Sekundarbereich ähnlich.

Im Elementarbereich liegt das zahlenmäfsige schüler/Lehrkräfteverhältnis zwischen dem des Primar-und des sekundarbereichs. 


\section{Abbildung D2.2.}

Zahlenmäßiges Schüler/Lehrkräfte-Verhältnis, nach Bildungsbereich (2000)

\section{Primärbereich}

35 Anzahl der Schüler pro Lehrkraft in Vollzeitäquivalenten

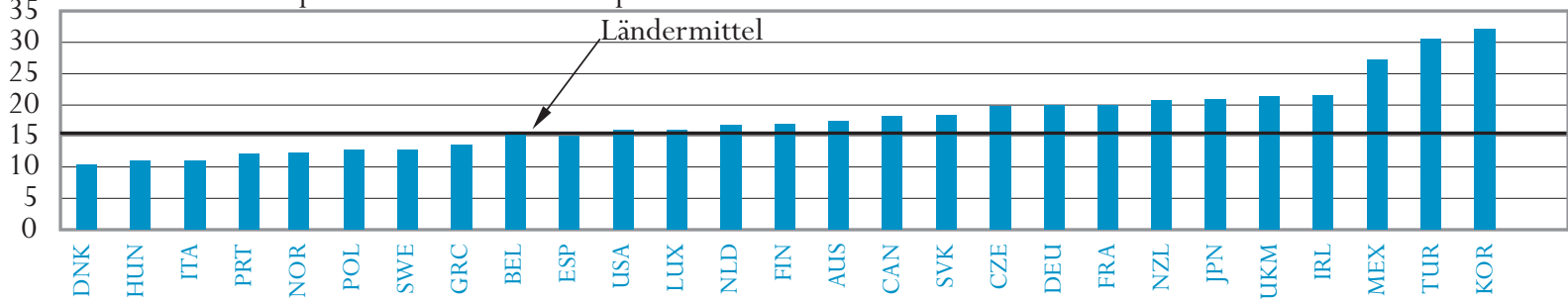

Sekundarbereich I

Anzahl der Schüler pro Lehrkraft in Vollzeitäquivalenten

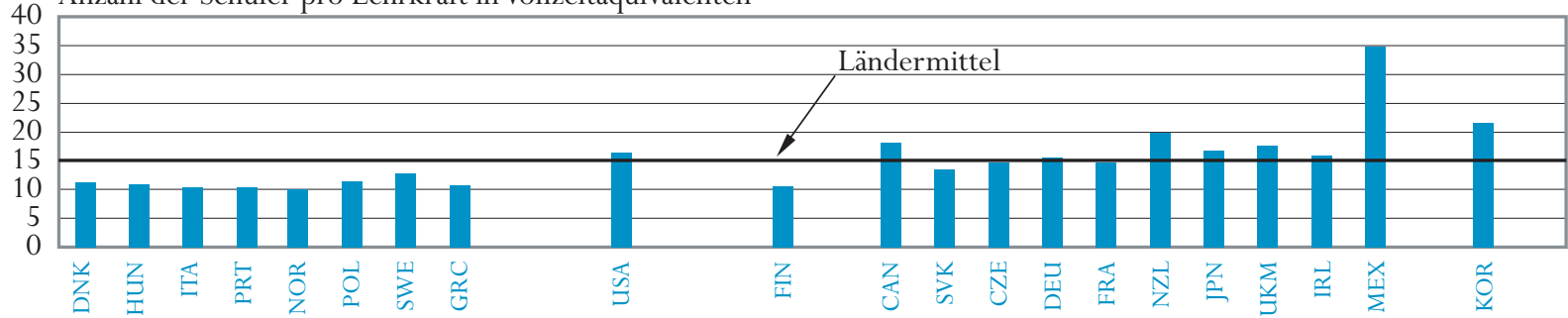

Sekundarbereich II

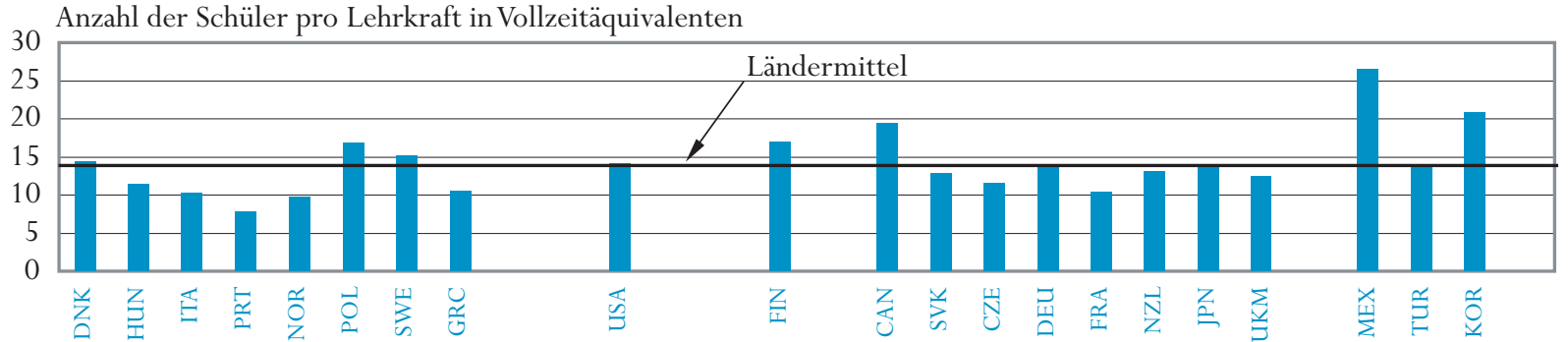

Tertiärbereich B

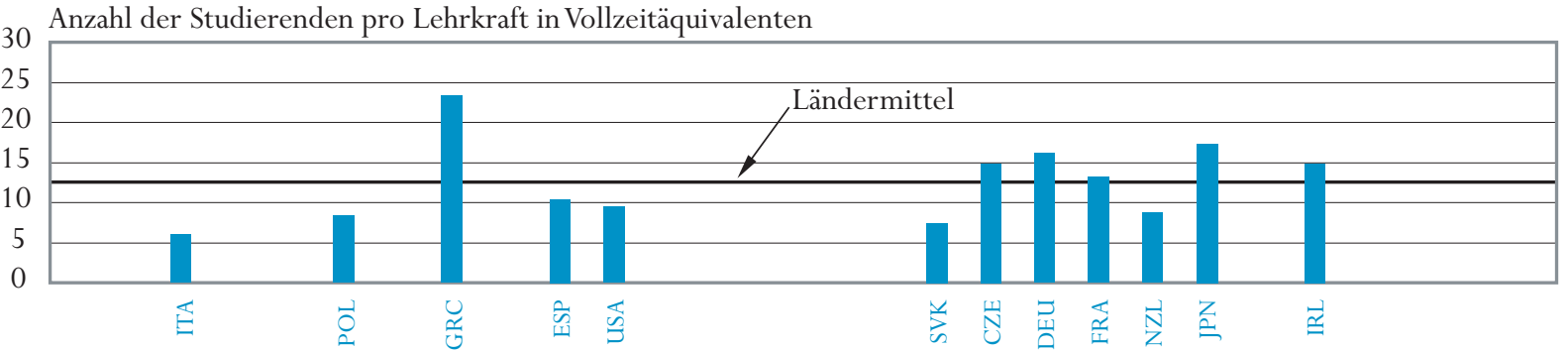

Tertiärbereich A und weiterführende Forschungsprogramme

Anzahl der Studierenden pro Lehrkraft in Vollzeitäquivalenten

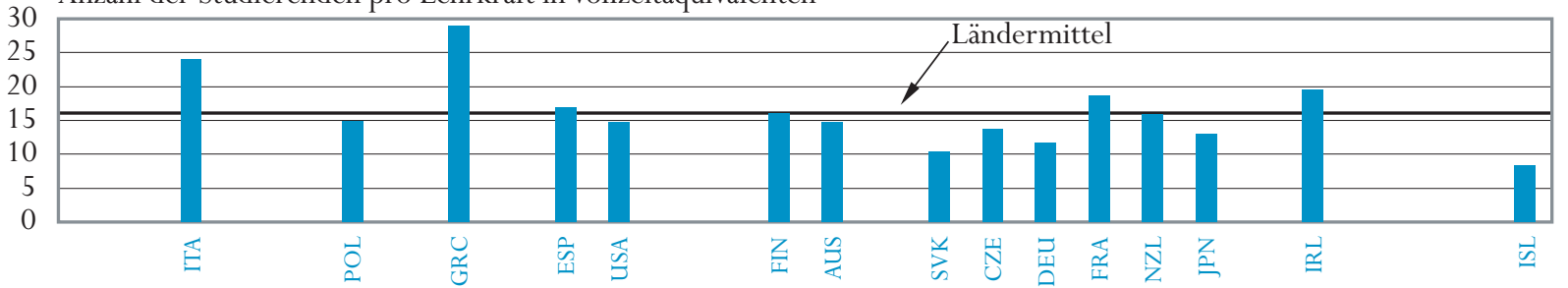

Hinweis: Auflistung der verwendeten Ländercodes und der dazugehörenden Ländernamen s. Hinweise für den Leser.

Anordnung der Länder in aufsteigender Reihenfolge der Anzahl der Schüler pro Lehrkraft im Primarbereich.

Quelle: OECD. Tabelle D2.2. Hinweise s. Anhang 3 (www.oecd.org/els/education/eag2002). 
hindeutet, dass innerhalb der einzelnen Länder der Personalbedarf der einzelnen Bildungsbereiche bzw. sein Stellenwert unterschiedlich bewertet werden (Tabelle D2.2).

\section{Definitionen und angewandte Methodik}

Die Klassengröße wurde berechnet, indem die Anzahl der Schüler durch die Anzahl der Klassen dividiert wurde. Um die Vergleichbarkeit zwischen den Ländern zu gewährleisten, wurden spezielle Förderprogramme nicht erfasst. Die Daten umfassen ausschließlich die regulären Bildungsgänge in diesen beiden Bildungsbereichen, Unterricht in Kleingruppen außerhalb des regulären Klassenunterrichts ist nicht erfasst.

Der Begriff ,Lehrkraft' umfasst voll qualifiziertes Personal, dass direkt mit dem Unterrichten der Schüler befasst ist. Die Kategorie umfasst Lehrkräfte, Förderlehrer und andere Lehrer, die mit Schülern als ganzer Klasse im Klassenzimmer, in kleinen Gruppen in einem Förderraum oder im Einzelunterricht innerhalb oder außerhalb des regulären Unterrichts arbeiten. Diese Kategorie umfasst auch Fachgebietsleiter, deren Aufgaben ein gewisses $\mathrm{Maß}$ an Unterricht beinhalten, während nicht voll-qualifizierte Mitarbeiter, die die Lehrkräfte beim Unterricht unterstützten, wie Hilfslehrkräfte und andere Hilfskräfte, nicht erfasst sind.
Die Daten beziehen sich auf das Schuljahr 1999-2000 und beruhen auf der VOEDatenerhebung zur Bildungsstatistik, die ährlich von der OECD durchgeführt wird. 
Tabelle D2.1.

Durchschnittliche Klassengröße, nach Art der Bildungseinrichtung und Bildungsbereich (2000)

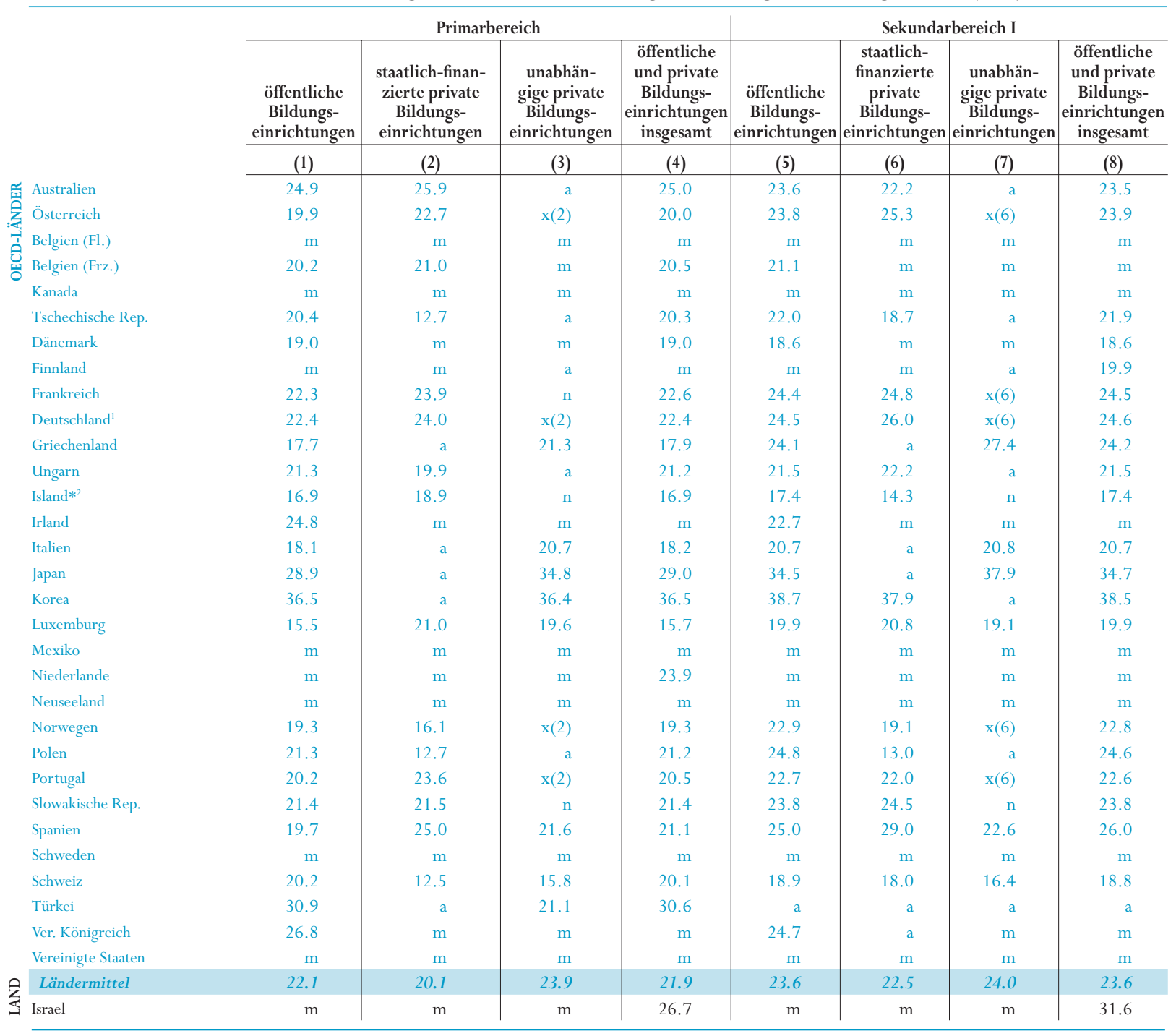

* Hinweise s. Anhang 3 (www.oecd.org/els/education/eag2002).

Hinweis: $\mathrm{x}$ bedeutet, dass die Daten in einer anderen Spalte enthalten sind, deren Referenz in runden Klammern nach dem 'x' angegeben ist.

So bedeutet z.B. x(2), dass die Daten in Spalte 2 enthalten sind.

1. Referenzjahr 2001.

2. Einschließlich jahrgangsübergreifender Klassen.

Quelle: OECD. 
Tabelle D2.2.

Zahlenmäßiges Schüler/Lehrkräfte-Verhältnis nach Bildungsbereich, Berechnungen basierend auf Vollzeitäquivalenten (2000)

\begin{tabular}{|c|c|c|c|c|c|c|c|c|c|}
\hline & $\begin{array}{l}\text { Elementarbe- } \\
\text { reich }\end{array}$ & $\begin{array}{l}\text { Primarbe- } \\
\text { reich }\end{array}$ & $\begin{array}{l}\text { Sekundar- } \\
\text { bereich I }\end{array}$ & $\begin{array}{l}\text { Sekundar- } \\
\text { bereich II }\end{array}$ & $\begin{array}{l}\text { Sekundar- } \\
\text { bereich } \\
\text { insgesamt }\end{array}$ & $\begin{array}{c}\text { Post- } \\
\text { sekundärer, } \\
\text { nicht- } \\
\text { tertiärer } \\
\text { Bildungs- } \\
\text { bereich }\end{array}$ & $\begin{array}{c}\text { Tertiärbe- } \\
\text { reich B }\end{array}$ & $\begin{array}{c}\text { Tertiärbereich } \\
\text { A und wei- } \\
\text { terführende } \\
\text { Forschungs- } \\
\text { programme }\end{array}$ & $\begin{array}{c}\text { Tertiärbereich } \\
\text { insgesamt }\end{array}$ \\
\hline & $(1)$ & $(2)$ & (3) & (4) & (5) & $(6)$ & $(7)$ & $(8)$ & (9) \\
\hline Australien $^{1}$ & $\mathrm{~m}$ & 17.3 & $\mathrm{~m}$ & $\mathrm{~m}$ & 12.6 & $\mathrm{~m}$ & $\mathrm{~m}$ & 14.8 & $\mathrm{~m}$ \\
\hline Belgien* & $x(2)$ & 15.0 & $x(5)$ & $x(5)$ & 9.7 & $x(5)$ & $\mathrm{x}(9)$ & $\mathrm{x}(9)$ & 19.9 \\
\hline Kanada & 18.1 & 18.1 & 18.1 & 19.5 & 18.8 & $\mathrm{x}(9)$ & $\mathrm{x}(9)$ & $\mathrm{x}(9)$ & 9.8 \\
\hline Tschechische Rep. & 13.1 & 19.7 & 14.7 & 11.5 & 13.1 & 11.0 & 12.1 & 13.7 & 13.5 \\
\hline Dänemark & 6.6 & 10.4 & 11.4 & 14.4 & 12.8 & $\mathrm{~m}$ & $\mathrm{~m}$ & $\mathrm{~m}$ & $\mathrm{~m}$ \\
\hline Finnland* & 12.2 & 16.9 & 10.7 & 17.0 & 13.8 & $\mathrm{x}(4)$ & $\mathrm{x}(4)$ & 16.1 & $\mathrm{~m}$ \\
\hline Frankreich & 19.1 & 19.8 & 14.7 & 10.4 & 12.5 & 11.4 & 16.2 & 18.6 & 18.3 \\
\hline Deutschland* & 23.6 & 19.8 & 15.7 & 13.9 & 15.2 & 14.3 & 14.9 & 11.7 & 12.1 \\
\hline Ungarn & 11.6 & 10.9 & 10.9 & 11.4 & 11.2 & $\mathrm{x}(4)$ & $\mathrm{x}(9)$ & $\mathrm{x}(9)$ & 13.1 \\
\hline Island & 5.4 & $x(3)$ & 12.7 & 9.7 & $\mathrm{~m}$ & $\mathrm{~m}$ & $\mathrm{~m}$ & 8.3 & 7.9 \\
\hline Irland* & 15.1 & 21.5 & 15.9 & $x(3)$ & $x(3)$ & $\mathrm{x}(3)$ & 14.8 & 19.4 & 17.4 \\
\hline Italien* & 13.0 & 11.0 & 10.4 & 10.2 & 10.3 & $\mathrm{~m}$ & 6.0 & 24.1 & 22.8 \\
\hline Japan & 18.8 & 20.9 & 16.8 & 14.0 & 15.2 & $\mathrm{~m}$ & 8.8 & 12.9 & 11.4 \\
\hline Korea & 23.1 & 32.1 & 21.5 & 20.9 & 21.2 & $\mathrm{a}$ & $\mathrm{m}$ & $\mathrm{m}$ & $\mathrm{m}$ \\
\hline Luxemburg ${ }^{2}$ & 20.2 & 15.9 & $x(5)$ & $x(5)$ & 9.2 & $\mathrm{~m}$ & $\mathrm{~m}$ & $\mathrm{~m}$ & $\mathrm{~m}$ \\
\hline Mexiko & 22.4 & 27.2 & 34.8 & 26.5 & 31.7 & $\mathrm{~m}$ & $\mathrm{x}(9)$ & $x(9)$ & 15.1 \\
\hline Niederlande & $\mathrm{x}(2)$ & 16.8 & $x(5)$ & $x(5)$ & 17.1 & $x(5)$ & $\mathrm{m}$ & $\mathrm{m}$ & 12.6 \\
\hline Neuseeland & 7.5 & 20.6 & 19.9 & 13.1 & 16.3 & 12.6 & 13.2 & 15.8 & 15.2 \\
\hline Norwegen & $\mathrm{m}$ & 12.4 & 9.9 & 9.7 & $\mathrm{~m}$ & $x(4)$ & $\mathrm{x}(9)$ & $x(9)$ & 12.7 \\
\hline Polen & 13.1 & 12.7 & 11.5 & 16.9 & 15.5 & 17.1 & 8.4 & 14.9 & 14.7 \\
\hline Portugal & 16.4 & 12.1 & 10.4 & 7.9 & 9.0 & $\mathrm{~m}$ & $x(9)$ & $\mathrm{x}(9)$ & $\mathrm{m}$ \\
\hline Slowakische Rep. & 10.1 & 18.3 & 13.5 & 12.8 & 13.2 & 9.0 & 7.4 & 10.3 & 10.2 \\
\hline Schweden & $\mathrm{m}$ & 12.8 & 12.8 & 15.2 & 14.1 & $\mathrm{~m}$ & $\mathrm{x}(9)$ & $x(9)$ & 9.3 \\
\hline Schweiz $^{2}$ & $\mathrm{~m}$ & $\mathrm{~m}$ & $\mathrm{~m}$ & $\mathrm{~m}$ & $\mathrm{~m}$ & $\mathrm{~m}$ & $\mathrm{~m}$ & $\mathrm{~m}$ & $\mathrm{~m}$ \\
\hline Türkei & 16.0 & 30.5 & $\mathrm{~m}$ & 14.0 & 14.0 & $\mathrm{~m}$ & $\mathrm{~m}$ & $\mathrm{~m}$ & $\mathrm{~m}$ \\
\hline Ver. Königreich*1 & 21.0 & 21.2 & 17.6 & 12.5 & 14.8 & $\mathrm{~m}$ & $\mathrm{x}(9)$ & $\mathrm{x}(9)$ & 17.6 \\
\hline Vereinigte Staaten & 18.7 & 15.8 & 16.3 & 14.1 & 15.2 & 10.1 & 9.5 & 14.8 & 13.5 \\
\hline Ländermittel & 15.5 & 17.7 & 15.0 & 13.9 & 14.3 & 12.2 & 12.1 & 16.1 & 14.7 \\
\hline Argentinien ${ }^{2,3}$ & 19.9 & 22.7 & 13.2 & 9.0 & 11.2 & $\mathrm{a}$ & 12.1 & 8.0 & 9.3 \\
\hline Brasilien $^{3}$ & 18.5 & 26.6 & 34.2 & 38.7 & 35.6 & $\mathrm{~m}$ & $x(9)$ & $x(9)$ & 14.1 \\
\hline Chile $^{3}$ & 39.3 & 34.0 & 33.4 & 28.5 & 30.2 & $\mathrm{a}$ & $\mathrm{m}$ & $\mathrm{m}$ & $\mathrm{m}$ \\
\hline China $^{3}$ & 26.7 & 20.2 & 17.6 & 13.8 & 16.4 & 10.1 & 31.0 & 8.5 & 12.3 \\
\hline Ägypten & $\mathrm{a}$ & 23.0 & 22.0 & 12.8 & 17.1 & $\mathrm{~m}$ & $\mathrm{~m}$ & $\mathrm{~m}$ & $\mathrm{~m}$ \\
\hline Indien $^{2,3}$ & $\mathrm{~m}$ & 43.0 & 22.0 & 9.2 & 16.1 & 20.8 & $\mathrm{~m}$ & $\mathrm{~m}$ & $\mathrm{~m}$ \\
\hline Indonesien $^{4}$ & 33.0 & 27.1 & 19.6 & 17.8 & 18.9 & $\mathrm{a}$ & $\mathrm{x}(9)$ & $x(9)$ & 15.0 \\
\hline Jamaika & 22.1 & 30.4 & $x(5)$ & $x(5)$ & 18.5 & $x(7)$ & 19.4 & 13.4 & 16.5 \\
\hline Jordanien ${ }^{2}$ & 19.4 & $x(3)$ & 21.2 & 16.9 & 20.6 & $\mathrm{a}$ & 15.6 & 34.9 & 29.5 \\
\hline Malaysia $^{3}$ & 26.9 & 21.3 & 17.7 & 18.3 & 17.9 & 24.7 & 17.3 & 20.2 & 19.1 \\
\hline Paraguay $^{3}$ & $\mathrm{x}(2)$ & 18.0 & $x(5)$ & $x(5)$ & 30.6 & $\mathrm{a}$ & 17.2 & $\mathrm{~m}$ & $\mathrm{~m}$ \\
\hline Peru $^{3}$ & 25.9 & 26.8 & $x(5)$ & $x(5)$ & 18.5 & $\mathrm{~m}$ & $\mathrm{~m}$ & $\mathrm{~m}$ & $\mathrm{~m}$ \\
\hline Philippinen ${ }^{3}$ & 32.9 & 34.7 & 40.5 & 21.2 & 34.1 & $\mathrm{~m}$ & $\mathrm{a}$ & 23.6 & 23.6 \\
\hline Russische Föd. ${ }^{4}$ & 7.0 & 17.3 & $\mathrm{~m}$ & $\mathrm{~m}$ & $\mathrm{~m}$ & 10.2 & 15.1 & 15.3 & 15.2 \\
\hline Tunesien $^{2}$ & 19.8 & 23.3 & 24.9 & 17.4 & 21.5 & a & $\mathrm{x}(9)$ & $\mathrm{x}(9)$ & 19.2 \\
\hline Uruguay $^{3}$ & 31.3 & 20.4 & 11.9 & 22.6 & 14.9 & $\mathrm{a}$ & $\mathrm{x}(9)$ & $\mathrm{x}(9)$ & 8.1 \\
\hline Simbabwe $^{4}$ & $\mathrm{~m}$ & 37.0 & $\mathrm{x}(5)$ & $x(5)$ & 24.7 & $\mathrm{~m}$ & $\mathrm{~m}$ & $\mathrm{~m}$ & $\mathrm{~m}$ \\
\hline
\end{tabular}

* Hinweise s. Anhang 3 (www.oecd.org/els/education/eag2002).

Hinweis: $\mathrm{x}$ bedeutet, dass die Daten in einer anderen Spalte enthalten sind, deren Referenz in runden Klammern nach dem ' $\mathrm{x}$ ' angegeben ist.

So bedeutet z.B. $x(2)$, dass die Daten in Spalte 2 enthalten sind

1. Nur allgemeinbildende Bildungsgänge des Sekundarbereich I und II.

2. Nur öffentliche Bildungseinrichtungen.

3. Referenzjahr 1999.

4. Referenzjahr 2001.

Quelle: OECD. 


\section{NUTZUNG UND VERFÜGBARKEIT VON COMPUTERN IN DER SCHULE UND ZU HAUSE}

- Im OECD-Durchschnitt besucht ein 15-jähriger Schüler eine Schule, an der sich 13 Schüler einen Computer teilen. Diese Zahl variiert jedoch stark von Land zu Land, in einigen Ländern sogar zwischen einzelnen Regionen und Schulen.

- Im OECD-Durchschnitt gaben etwa ein Drittel der 15-Jährigen an, in der Schule täglich oder wenigstens mehrmals pro Woche einen Computer zu benutzen, zu Hause jedoch wird fast doppelt so häufig ein Computer genutzt. Der Anteil der Schüler, die angeben, zu Hause nie Zugang zu einem Computer zu haben ist jedoch 10 Prozent höher als der Anteil derjenigen, die in ihrer Schule nie Zugang zu einem Computer haben. Das legt die Vermutung nahe, dass die Schule eine wichtige Rolle dabei spielt, die Bildungslücke zwischen Schülern, die einen unterschiedlich ausgeprägten Zugang zu Computern haben, zu schließen.

\section{Abbildung D3.1.}

Anzahl der Schüler pro Computer (2000)

Gesamtzahl der Schüler einer Schule geteilt durch die Gesamtzahl der an der Schule zurVerfügung stehenden Computer, für Schulen, die 15-Jährige besuchen, gewichtet nach Schülerzahl, nach Quartilen

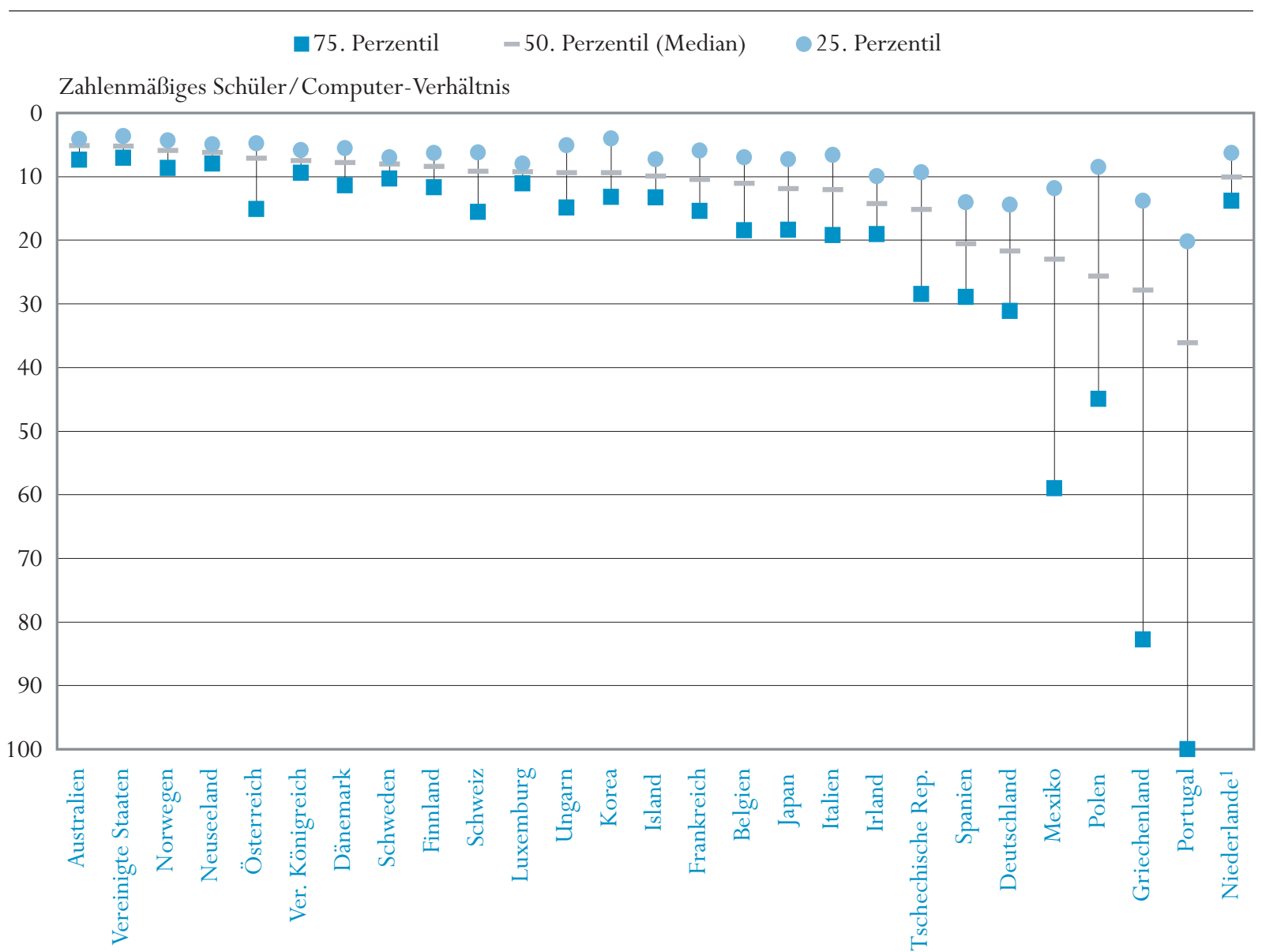

Anordnung der Länder in absteigender Reihenfolge der Anzahl Schüler pro Computer beim 50. Perzentil.

1. Die Beteiligungsquote ist zu niedrig, um die Vergleichbarkeit zu gewährleisten (s. Anhang 3 unter www.oecd.org/els/ education/eag2002)

Quelle: OECD PISA-Datenbank, 2001. Tabelle D3.1. Hinweise zur Methodik s. Anhang 3 (www.oecd.org/els/education/eag2002) und www.pisa.oecd.org. 


\section{Politischer Kontext}

Die Volkswirtschaften der OECD-Länder sind in immer stärkerem Maße vom technologischen Wissen und den technologischen Fähigkeiten ihrer Erwerbsbevölkerung abhängig. Schüler, die den Umgang mit Computern und den modernen Informationstechnologien kaum oder gar nicht lernen, können beim reibungslosen Übergang in den modernen Arbeitsmarkt Schwierigkeiten haben.

Die Integration von Computern in das Lernumfeld der Schule hat bedeutende Auswirkungen auf die Arbeit im Klassenzimmer, aber die zunehmende Verfügbarkeit von bezahlbaren PC, Software und Internetzugängen und E-Mail zu Hause führt dazu, dass die Schüler den Computer eher vermehrt zu Hause als in der Schule benutzen. Die Art der Computernutzung zu Hause spielt auch eine immer größere Rolle für die Bildung, denn in zunehmenden Maße werden Computer auch für andere Aktivitäten als Computerspiele genutzt, wie z.B. für Textverarbeitung, Datenbanken, Tabellenkalkulation, Programmieren, das Internet und Web-Design. Dennoch spielen die Schulen eine wichtige Rolle dabei, die Kluft zwischen Schülern, die einen unterschiedlich ausgeprägten Zugang zu Computern haben, zu schließen.

Die PISA-Studie 2000 untersuchte bei den 15-Jährigen drei Aspekte der Vertrautheit im Umgang mit Computern sowohl in der Schule als auch zu Hause: das Interesse an Computern, die Selbsteinschätzung der Fähigkeit im Umgang mit Computern sowie die Nutzung von und Erfahrung mit Computern. Verschiedene dieser Aspekte werden in diesem Indikator genauer untersucht.

\section{Ergebnisse und Erläuterungen}

\section{Die Zahl der Schüler pro Computer in der Schule}

Die durchschnittliche Anzahl von Schülern pro Computern ist eine Kennzahl dafür, in welchem Ausmaß den Schülern moderne Informationstechnologien zur Verfügung stehen. Im Rahmen der PISA-Studie wurden die Schulleiter von Schulen, die 15-Jährige besuchen, gefragt, wieviele Computer an der Schule insgesamt zur Verfügung stehen. Die Zahl der Schüler pro Computer wurde berechnet, in dem die Gesamtzahl der verfügbaren Computer durch die Gesamtzahl der Schüler der Schule dividiert wurde. Um aufzuzeigen, wie die Verfügbarkeit von Computern zwischen den einzelnen Schulen eines Landes variiert, werden auch das 25., 50. und 75. Perzentil dieses Verhältnisses aufgezeigt. Wenn also beim 25. Perzentil das zahlenmäßige Schüler/Computer-Verhältnis bei 20 liegt, bedeutet dies, dass 25 Prozent der 15-Jährigen eine Schule besuchen, bei der maximal 20 Schüler auf einen Computer kommen. Entsprechend bedeutet ein zahlenmäßiges Schüler/ Computer-Verhältnis von 30 im 50. Perzentil, dass 50 Prozent der 15-Jährigen eine Schule besuchen, bei der sich maximal 30 Schüler einen Computer teilen müssen. Die Berechnung des zahlenmäßigen Schüler/Computer-Verhältnisses erfolgte auch jeweils getrennt für öffentliche Bildungseinrichtungen,
Dieser Indikator zeigt

die Nutzung und

verfügbarkeit von

Computern für

15-Jährige.
Die durchschnittliche Anzahl von Schülern pro Computer ist eine Kennzahl dafür, in welchem Ausmajs den schülern moderne Informationstechnologien zur verfügung stehen,... 
wobei jedoch die reine

verfügbarkeit allein

noch keineswegs die

sinnvolle Nutzung der

Computer garantiert.

Im OECD-Durchschnitt besucht ein 15-jähriger schüler eine Schule, an der sich 13 Schüler einen

Computer teilen, diese Zahl variiert jedoch stark.

Der Zugang zu Computern kann auch davon beeinflusst werden, in welchem

Ausmaf lokale, regionale und nationale

Behörden sowie private Träger in diese neuen Technologien investieren.

In einigen Ländern ist der Zugang zu Computern in privaten schulen deutlich besser... unabhängige private Bildungseinrichtungen und staatlich-subventionierte private Bildungseinrichtungen sowie für Schulen in Dörfern, Städten und Großstädten.

Die Verfügbarkeit von Hardware allein garantiert jedoch keineswegs deren sinnvolle Nutzung durch Schüler und Lehrer und weist ebenso wenig darauf hin, wie leicht sie bei Bedarf im Klassenzimmer, in Labors, der Schulbibliothek oder an anderen Orten zugänglich ist. Außerdem garantiert dieses Verhältnis ebenso wenig, dass die Qualität der Hardware (z.B. Kompatibilität, Speicher, Geschwindigkeit, Alter des Geräts, verbundene Peripheriegeräte und Software) der Nutzung im Klassenzimmer angemessen ist. Letztendlich können Durchschnittswerte Unterschiede zwischen den Schulen aufgrund von Faktoren wie der geographischen oder sozio-ökonomischen Lage der Schule sowie der Art der Bildungseinrichtung verdecken.

Im OECD-Durchschnitt besucht ein 15-jähriger Schüler eine Schule, an der sich 13 Schüler einen Computer teilen, diese Zahl variiert jedoch stark. In Australien und den Vereinigten Staaten liegt das Verhältnis bei 5 Schülern pro Computer, in Neuseeland und Norwegen sind es 6 Schüler. Andererseits besuchen in Deutschland, Griechenland, Mexiko, Polen, Portugal und Spanien 15-Jährige im Durchschnitt Schulen, an denen sich mehr als 20 Schüler einen Computer teilen. In einigen dieser Länder, insbesondere in Griechenland, Mexiko, Polen und Portugal schwankt der Zugang zu Computern zwischen den einzelnen Schulen sehr stark, was aus den großen Unterschieden beim zahlenmäßigen Schüler/Computer-Verhältnis zwischen dem 25. und dem 75. Perzentil ersichtlich wird (Abbildung D3.1 und Tabelle D3.1).

Der Zugang zu Computern kann auch davon beeinflusst werden, in welchem Ausmaß lokale, regionale und nationale Behörden sowie private Entscheidungsträger willens sind, die Anschaffung von Hardware für die Schulen zu finanzieren. Die entsprechenden bildungspolitischen Vorgaben und Entscheidungen können auch auf Schulen in geographisch abgelegenen Gebieten oder innerstädtischen Bezirken mit geringem sozio-ökonomischen Status abzielen. Durch Nutzung zusätzlicher Informationen, die von den an der PISA-Studie teilnehmenden Schulleitern bereitgestellt wurden, war es möglich, die Zahl der Schüler pro Computer aufgegliedert nach Standort der Schule und nach Art der Bildungseinrichtung zu berechnen.

In einigen Länden ist der Zugang zu Computern für 15-Jährige an privaten Schulen besser als an öffentlichen Bildungseinrichtungen. Der Unterschied ist besonders ausgeprägt in Ländern wie Griechenland und Mexiko, wo die Zahl der Schüler pro Computer an Privatschulen unter 10 liegt, verglichen mit bis zu 32 Schülern pro Computer an öffentlichen Schulen. In anderen Ländern ist kein Unterschied beim Zugang zu Computern zwischen privaten und öffentlichen Schulen zu beobachten (Tabelle D3.1). 
In Ländern wie Australien, Finnland, Neuseeland, dem Vereinigten Königreich und den Vereinigten Staaten ist die Zahl der Schüler pro Computer kaum vom geographischen Standort abhängig. Sie liegt in Australien und den Vereinigten Staaten zwischen 5 und 6 Schülern pro Computer, unabhängig davon, ob die Schule in einem Dorf mit weniger als 3.000 Einwohnern, einer Stadt mit 15.000 bis unter 100.000 Einwohnern, im Zentrum oder in der Nähe des Zentrums einer Großstadt mit über 1 Mio. Einwohnern oder anderswo in einer Großstadt mit über 1 Mio.Einwohnern liegt. Dies gilt nicht für Länder wie Irland, die Tschechische Republik und Ungarn, wo Schüler an Schulen in städtischen Gebieten besseren Zugang zu Computern haben als Schüler, die eine Schule im ländlichen Raum besuchen. In Irland beispielsweise liegt die Zahl der Schüler pro Computer einer Schule in einem Dorf bei 14, während sich in Schulen, die nahe bei Großstädten mit über einer Million Einwohnern liegen, 9 Schüler einen Computer teilen. In Korea, Mexiko, Polen und Spanien ist es genau umgekehrt, dort haben Schüler an Schulen in ländlichen Gebieten weit besseren Zugang zu Computern als diejenigen Schüler, die Schulen in städtischen Gebieten besuchen (Tabelle D3.1).

\section{Verfügbarkeit und Nutzung von Computern in der Schule}

Zwischen 45 und 65 Prozent der 15-Jährigen in Australien, Dänemark Finnland, Schottland, Schweden und Ungarn gaben an, fast täglich bzw. mehrmals pro Woche in der Schule einen Computer zu benutzen. Im Gegensatz dazu liegt deren Anteil in Deutschland bei 18 Prozent und in der Schweiz bei 22 Prozent und in Mexiko gab die Hälfte der 15-Jährigen an, in der Schule nie einen Computer zu benutzen (Tabelle D3.5).

In den meisten Ländern gaben die 15-Jährigen an, den Computer zu Hause weit häufiger zu nutzen als in der Schule. Im OECD-Durchschnitt gaben 60 Prozent der 15-Jährigen an, den Computer zu Hause fast täglich bzw. mehrmals pro Woche zu nutzen und in Australien, Dänemark, Finnland, Kanada, Norwegen, Schweden und den Vereinigten Staaten liegt dieser Anteil zwischen 66 und 82 Prozent. Selbst in Deutschland und der Schweiz, wo die Computernutzung in der Schule vergleichsweise niedrig ist, gaben fast zwei Drittel der 15-Jährigen an, den Computer zu Hause fast täglich bzw. mehrmals pro Woche zu nutzen. Die einzigen Ausnahmen bilden hierbei Mexiko und Ungarn, wo die 15-Jährigen den Computer häufiger in der Schule als zu Hause benutzten (Tabelle D3.5).

Das Internet und lokale Netzwerke (LAN) werden von Schülern und Lehrern in zunehmendem Maße sowohl als Kommunikationsmittel als auch für Recherchezwecke genutzt. Während es auch vielleicht nur von beschränktem pädagogischem Nutzen ist, die Schulen mit Internetanschlüssen auszustatten, wenn zugleich die Verbindungsgeschwindigkeit zu niedrig, die Verbindungskosten zu hoch oder der schulische Lehrplan zu wenig flexibel ist, so kann doch die sinnvolle Nutzung des Internets und von Computernetzwerken im Unterricht dem Lernen und den Lernmethoden neue Perspektiven eröffnen. Im Rahmen der PISA-Studie wurden Schulleiter gefragt, wieviele Computer an ...und manchmal variiert der Zugang je nach Standort der Schule beträchtlich.

Im Durchschnitt der OECD-Länder gab etwa ein Drittel der 15-Jährigen an, den Computer in der Schule fast täglich bzw. mehrmals pro Woche zu nutzen. Dabei gibt es jedoch grofe Unterschiede zwischen den einzelnen Ländern,...

Etwa ein Drittel der schüler benutzt den Internetzugang in der schule mehrmals in der Woche bzw. mindestens mehrmals im Monat. 
Die Schulleiter sind der Ansicht, dass der Mangel an Computern und MultimediaAusstattung das Lernen stärker beeinträchtigt

als der Mangel an irgend welchen anderen Unterrichtsmaterialien.

Im Durchschnitt aller Länder stehen 15 Prozent der Computer nur den Lehrkräften zur Verfügung und weitere 12 Prozent nur der Verwaltung. ihrer Schule einen Anschluss an das Internet oder an ein lokales Netzwerk haben. Im Durchschnitt ist dies bei etwa der Hälfte der Geräte der Fall. In Australien und Luxemburg liegt der Anteil bei über 75 Prozent, während in Italien und Mexiko weniger als ein Viertel der Computer an den Schulen an das Internet oder ein lokales Netz angeschlossen sind (Tabelle D3.2). In Australien, Dänemark, Finnland, Island, Österreich und Schweden gaben mehr als 50 Prozent der 15Jährigen an, mehrmals pro Monat bzw. mehrmals pro Woche das Internet in der Schule zu nutzen (Daten siehe unter www.oecd.org/els/education/eag2002).

Kennzahlen zurVerfügbarkeit von Computern in Schulen, wie in diesem Indikator beschrieben, geben zwar Hinweise auf den Erfolg nationaler Maßnahmen zur Ausstattung des Bildungswesens mit Computern, aber die Verfügbarkeit alleine garantiert weder eine gute Qualität der Geräte noch den sinnvollen Einsatz von Computern im Lernkontext. Um hier Näheres zu erfahren, wurden die Schulleiter im Rahmen der PISA-Studie gefragt, wie sehr das Lernen 15Jähriger durch den Mangel an Computern und Multimedia-Ausstattung für den Unterricht beeinträchtigt sei. Die Schulleiter wurden auch zur Qualität anderer Bildungsressourcen wie Lehrmaterialien, Unterrichtsmaterialien in der Bibliothek, Laborausstattung und Ausstattung für den Kunstunterricht gefragt. Im Durchschnitt waren die Schulleiter der Ansicht, dass der Mangel an Computern und Multimedia-Ausstattung das Lernen stärker beeinträchtige als der Mangel an irgendwelchen anderen Unterrichtsmaterialien (Tabelle D5.3). Mehr als 37 Prozent der Schüler in OECD-Ländern besuchen Schulen, deren Schulleiter angaben, dass das Lernen etwas bzw. sehr durch den Mangel an Computern für den Unterricht beeinträchtigt werde. Während Schulleiter in Ländern wie Griechenland und Mexiko am stärksten besorgt waren, dass der Mangel an Computern und an Multimedia-Ausstattung das Lernen behindere, gaben Schulleiter in Australien, Belgien, Ungarn, der Schweiz und den Vereinigten Staaten, wo die Computerverfügbarkeit deutlich höher ist, an, dass das Lernen überhaupt nicht durch den Mangel an Computern und Multimedia-Ausstattung für den Unterricht beeinträchtigt werde (Tabelle D3.3).

Computer sind auch für die Lehrkräfte und die Schulverwaltungen ein wichtiges Arbeitsmittel. Sie benutzen sie für täglich anfallende Arbeiten, wie z.B. die Aktualisierung von Schülerakten, Briefe an Eltern oder Ausschüsse, das Verfassen von Zeugnissen und Beurteilungen, die Unterrichtsvorbereitung und die Aktualisierung der Websites der Schule bzw. der einzelnen Klasse. Im PISASchulfragebogen wurden die Schulleiter gefragt, wieviele Computer in der Schule für 15-jährige Schüler, wieviele nur für Lehrer und wieviele nur für dieVerwaltung verfügbar sind. 15 Prozent der Computer sind nur für Lehrer verfügbar und weitere 12 Prozent nur für die Verwaltung. In Griechenland, Korea, Portugal und den Vereinigten Staaten wird mehr als ein Fünftel der insgesamt an der Schule verfügbaren Computer nur von Lehrern genutzt. In Belgien, Griechenland, Mexiko und Portugal sind mehr als 15 Prozent der Computer in einer Schule nur für die Verwaltung verfügbar (Die entsprechenden Daten können im Internet unter www.oecd.org/els/education/eag2002 eingesehen werden). 


\section{Abbildung D3.2.}

Verfügbarkeit von Computern und Häufigkeit der Computernutzung von

15-Jährigen, zu Hause und in der Schule (2000)

Verteilung der mittleren Prozentsätze 15-Jähriger, die angaben, über einen Computer verfügen zu können sowie derjenigen, die angaben, einen Computer zu Hause und in der Schule zu benutzen

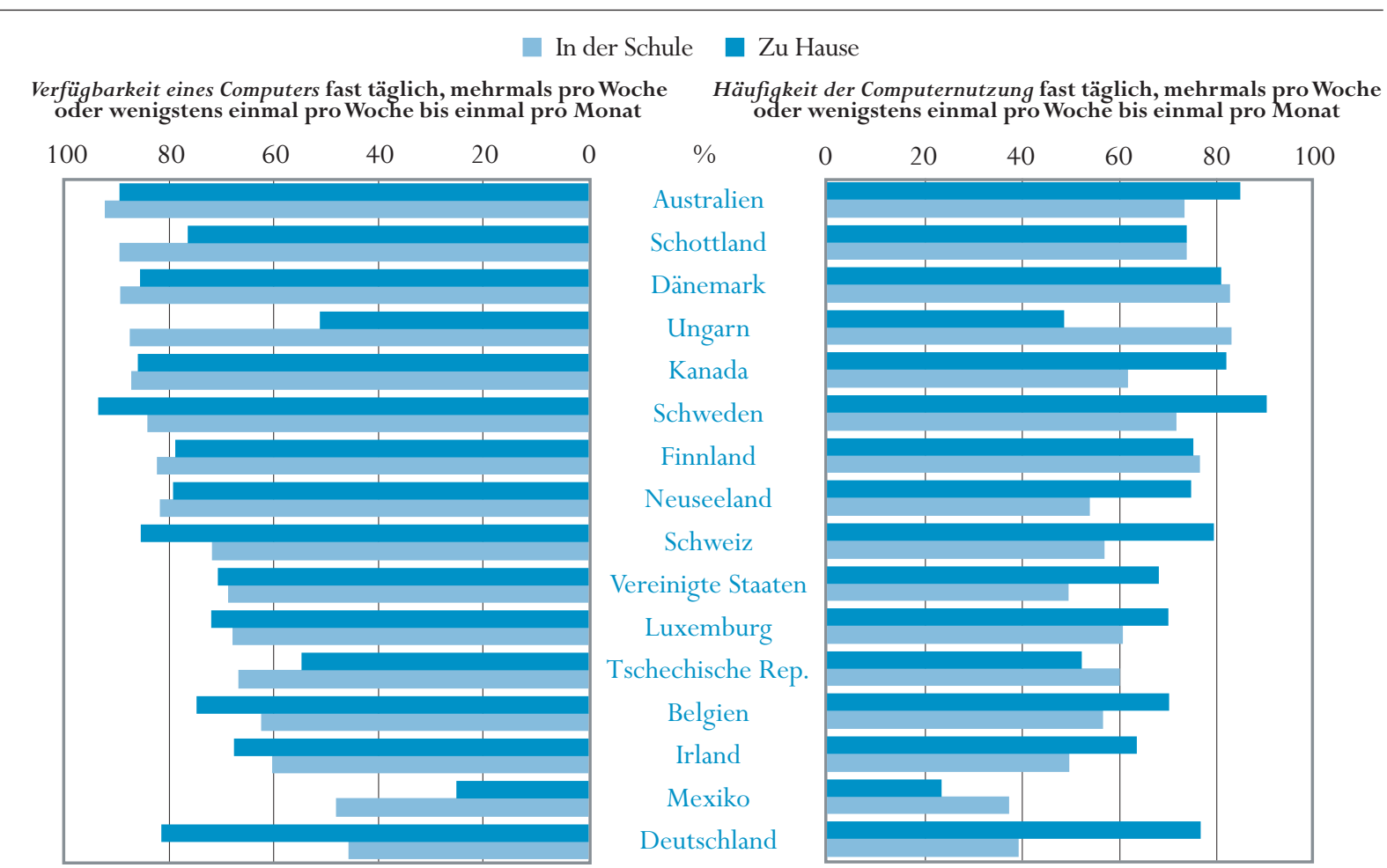

Anordnung der Länder in aufsteigender Reihenfolge entsprechend derVerfügbarkeit eines Computers in der Schule (fast täglich, mehrmals pro Woche oder wenigstens einmal proWoche bis einmal pro Monat).

Quelle: OECD PISA-Datenbank, 2001. Tabellen D3.4 und D3.5. Hinweise zur Methodik s. Anhang 3 (www.oecd.org/els/ education / eag2002) und www.pisa.oecd.org.

\section{Verfügbarkeit und Nutzung von Computern zu Hause}

Wenn Schüler zu Hause einen Computer benutzen, kann das den Lernprozess an der Schule ergänzen und die Einstellung zum Lernen mittels Computernutzung verbessern und dadurch das formelle Lernen im Klassenzimmer mit dem informellen Lernen in der häuslichen Umgebung verbinden.

Innerhalb der letzten fünf bis zehn Jahre ist der Markt für private PCs dramatisch gewachsen. Die Vermarktung von PCs richtet sich zunehmend auf die Nutzung durch die Familie und für Bildungszwecke und weniger auf die Nutzung für Spiele. Hierin spiegelt sich die zunehmende Verfügbarkeit von bezahlbarer Hard- und Software sowie die Tatsache, dass es Eltern zunehmend bewusst wird, welche Rolle der Computer für die Bildung ihrer Kinder spielen kann. In jüngster Zeit ist es darüber hinaus für private Haushalte sehr viel einfacher geworden, einen kostengünstigen Internetzugang zu erhalten. Im Jahr 2000 gaben im Durchschnitt 73 Prozent der 15-jährigen Schüler in den
In vielen Ländern hat der Zugang zu Computern und Lernsoftware zu Hause rapide zugenommen, ...

.... wobei im

Durchschnitt ungefähr drei Viertel der $15-$ Jährigen inzwischen angeben, zu Hause wenigstens einen Computer zu haben. 
Der tägliche Kontakt mit dem Computer findet eher zu Hause als in der

Schule statt.
OECD-Ländern an, zu Hause mindestens einen Computer zu haben. Über 40 Prozent der 15-Jährigen in Dänemark, Island, Norwegen, Schweden und den Vereinigten Staaten gaben an, zu Hause zwei oder mehr Computer zu haben. Im OECD-Durchschnitt gaben über 55 Prozent der 15-Jährigen an, zu Hause Lernsoftware zu haben. In Ländern wie Australien, Island, Kanada, Neuseeland, Schweden, dem Vereinigten Königreich und den Vereinigten Staaten lag dieser Anteil bei 75 Prozent und darüber. 45 Prozent der 15-Jährigen in den OECDLändern gaben an, zu Hause einen Internetanschluss zu haben. In Island und Schweden haben sogar über drei Viertel der 15-Jährigen zu Hause einen Internetanschluss (Daten siehe unter www.oecd.org/els/education/eag2002).

Wie dieser Indikator ebenfalls aufzeigt, haben viele 15-Jährige eher zu Hause als in der Schule täglichen Kontakt mit dem Computer. Im OECD-Durchschnitt gaben 64 Prozent der 15-Jährigen an, zu Hause täglich Zugang zum Computer zu haben, dagegen lag diese Zahl für den Zugang in der Schule nur bei 27 Prozent (Abbildung D3.2 und Tabelle D3.4).

\section{Abbildung D3.3.}

15-jährige Schüler, die den Computer als Hilfsmittel für das schulische Lernen benutzen (2000) Verteilung der mittleren Prozentsätze 15-Jähriger, die angaben, fast täglich, mehrmals proWoche oder wenigstens einmal proWoche bis einmal pro Monat den Computer als Hilfsmittel zum Lernen für die Schule zu benutzen

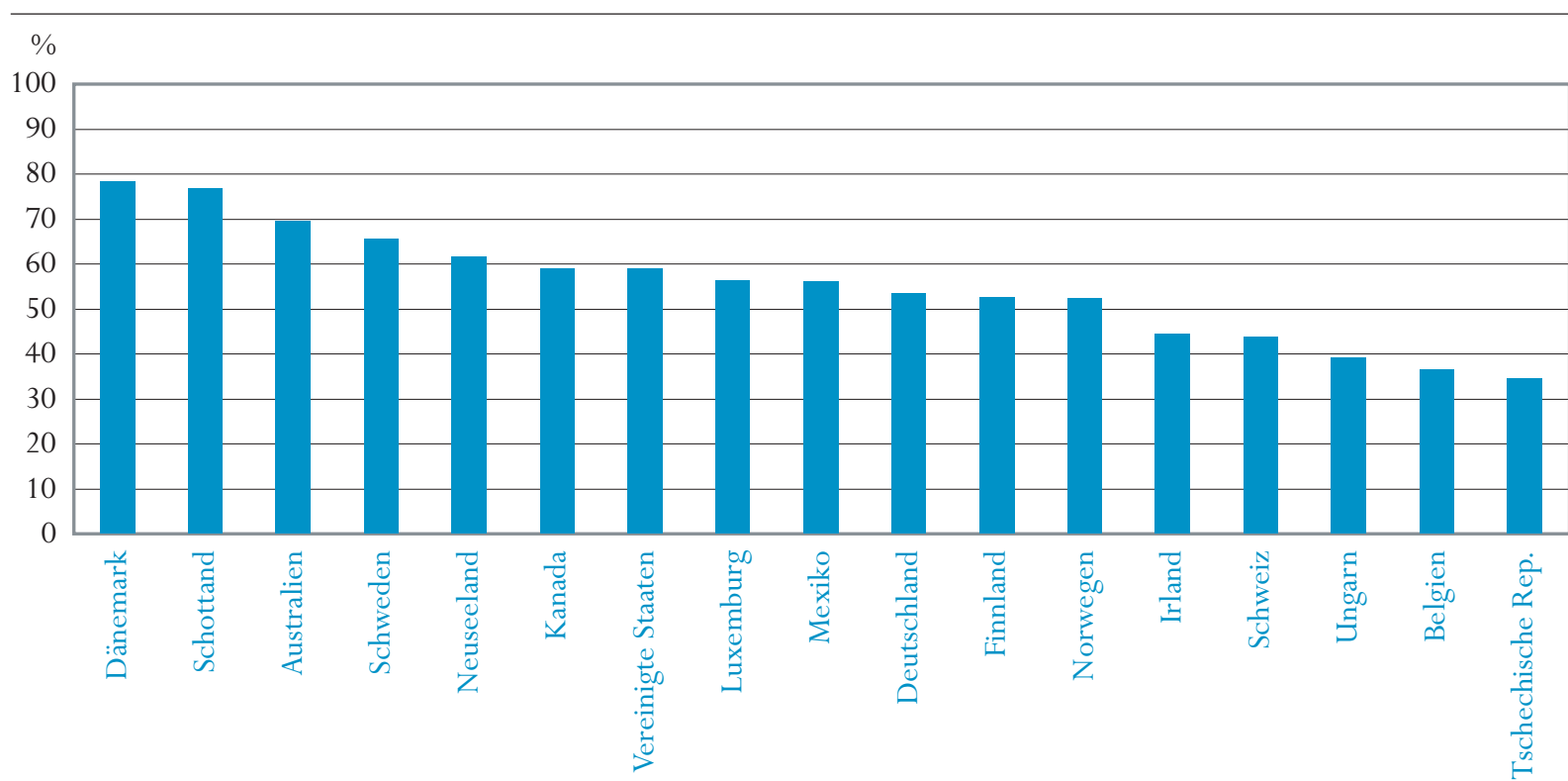

Anordnung der Länder in absteigender Reihenfolge des Prozentsatzes 15-Jähriger, die angaben, den Computer fast täglich, mehrmals pro Woche oder wenigstens einmal proWoche bis einmal pro Monat als Hilfsmittel zum Lernen für die Schule zu benutzen. Quelle: OECD PISA-Datenbank, 2001. Tabelle D3.6. Hinweise zur Methodik s. Anhang 3 (www.oecd.org/els/education/eag2002) und www.pisa.oecd.org. 
Interessanterweise ist der Anteil der Schüler, die angeben, zu Hause nie Zugang zu einem Computer zu haben, jedoch 10 Prozent höher als der Anteil derjenigen, die dasselbe für die Schule sagen. Das legt die Vermutung nahe, dass Schulen eine wichtige Rolle dabei spielen, die Bildungslücke zwischen Schülern, die einen unterschiedlich ausgeprägten Zugang zu Computern haben, zu schließen.

Im Rahmen der PISA-Studie wurden 15-Jährige gefragt, wie oft sie den Computer zum schulischen Lernen benutzen. Im Durchschnitt gaben 11 Prozent der 15-Jährigen an, den Computer fast täglich zum schulischen Lernen zu benutzen; 24 Prozent der 15-Jährigen benutzt den Computer mehrmals pro Woche und 26 Prozent der 15-Jährigen benutzten den Computer zwischen einmal pro Woche und einmal pro Monat zu diesem Zweck. Über 25 Prozent der 15-Jährigen in Belgien, Irland, der Schweiz, der Tschechischen Republik und Ungarn gaben jedoch an, den Computer nie zum schulischen Lernen zu benutzen (Abbildung D3.3 und Tabelle D3.6).

\section{Definitionen und angewandte Methodik}

Die in diesem Indikator genutzten Daten stammen aus den Antworten, die 15jährige Schüler und die Schulleiter auf Fragen bezüglich der Computernutzung und der Verfügbarkeit von Computern zu Hause und in der Schule in den zwei Kontextfragebögen gegeben haben, die für die Internationale Schulleistungsstudie (PISA) der OECD im Jahr 2000 benutzt wurden.

Im Rahmen der PISA-Studie haben die 15-jährigen Schüler zusätzlich zu einem schriftlichen Test einen Schülerfragebogen ausgefüllt, in dem Daten zur Familie der Schüler, der häuslichen Umgebung, den Lesegewohnheiten sowie den Aktivitäten innerhalb und außerhalb der Schule erhoben wurden. Die Antworten der Schüler auf Fragen zur Häufigkeit der Nutzung von Computern und des Internets in der Schule (Tabelle D3.2) stammen aus diesem Schülerfragebogen. Ein zweiter Teil dieses Fragebogens zur Vertrautheit im Umgang mit Computern wurde von Schülern in 20 Ländern als Teil einer internationalen Option ausgefüllt. Hier wurde das Interesse der Schüler am Computer, die Selbsteinschätzung der Schüler hinsichtlich ihrer Fähigkeiten im Umgang mit Computern, ihre Computernutzung und ihre Erfahrung mit Computern untersucht. Aus diesem Teil des Fragebogens stammen die in diesem Indikator verwendeten Daten zur Verfügbarkeit und Nutzung von Computern zu Hause und in der Schule (Abb. D3.2, Tabelle D3.4 und Tabelle D3.5) sowie die Daten über die Häufigkeit der Computernutzung für das schulische Lernen (Abb. D3.3 undTabelle D3.6). Die Antworten der Schüler wurden so gewichtet, dass sie der Anzahl der 15-Jährigen in der jeweiligen Schule entsprechen.

Die Schulleiter der Schulen, deren Schüler getestet wurden, füllten ebenfalls einen Fragebogen zu den Merkmalen ihrer Schule aus. Die angegebene Daten zur Verfügbarkeit von Computern, einschließlich der Zahl der Schüler pro Computer (Abbildung D3.1, Tabelle D3.1 und Tabelle D3.2) sowie die Aussagen
In einigen Ländern gibt

es großse Unterschiede

zwischen Schülern im

Zugang zu Computern.

Der Computer wird zu

Hause jedoch nicht

nur für das schulische

Lernen benutzt.

Die Ergebnisse für diesen

Indikator stammen aus

dem Kontextfragebogen,

den 15-jährige Schüler

und ihre Schulleiter

als Teil der von der

OECD im Jahr 2000

durchgeführten

Internationalen

Schulleistungsstudie

(PISA) ausgefüllt haben. 
der Schulleiter zur Qualität der Bildungsressourcen (Tabelle D3.3) stammen aus den Antworten der Schulleiter in diesem Fragebogen. Die Antworten der Schulleiter wurden so gewichtet, dass sie der Anzahl der 15-Jährigen in der jeweiligen Schule entsprechen. 
Tabelle D3.1.

Anzahl der Schüler pro Computer (2000)

Gesamtzahl der Schüler einer Schule geteilt durch die Gesamtzahl der an der Schule zur Verfügung stehenden Computer, für Schulen, die 15-Jährige besuchen, nach Quartil,Art der Bildungseinrichtung und Standort der Schule, gewichtet nach der Schülerzahl

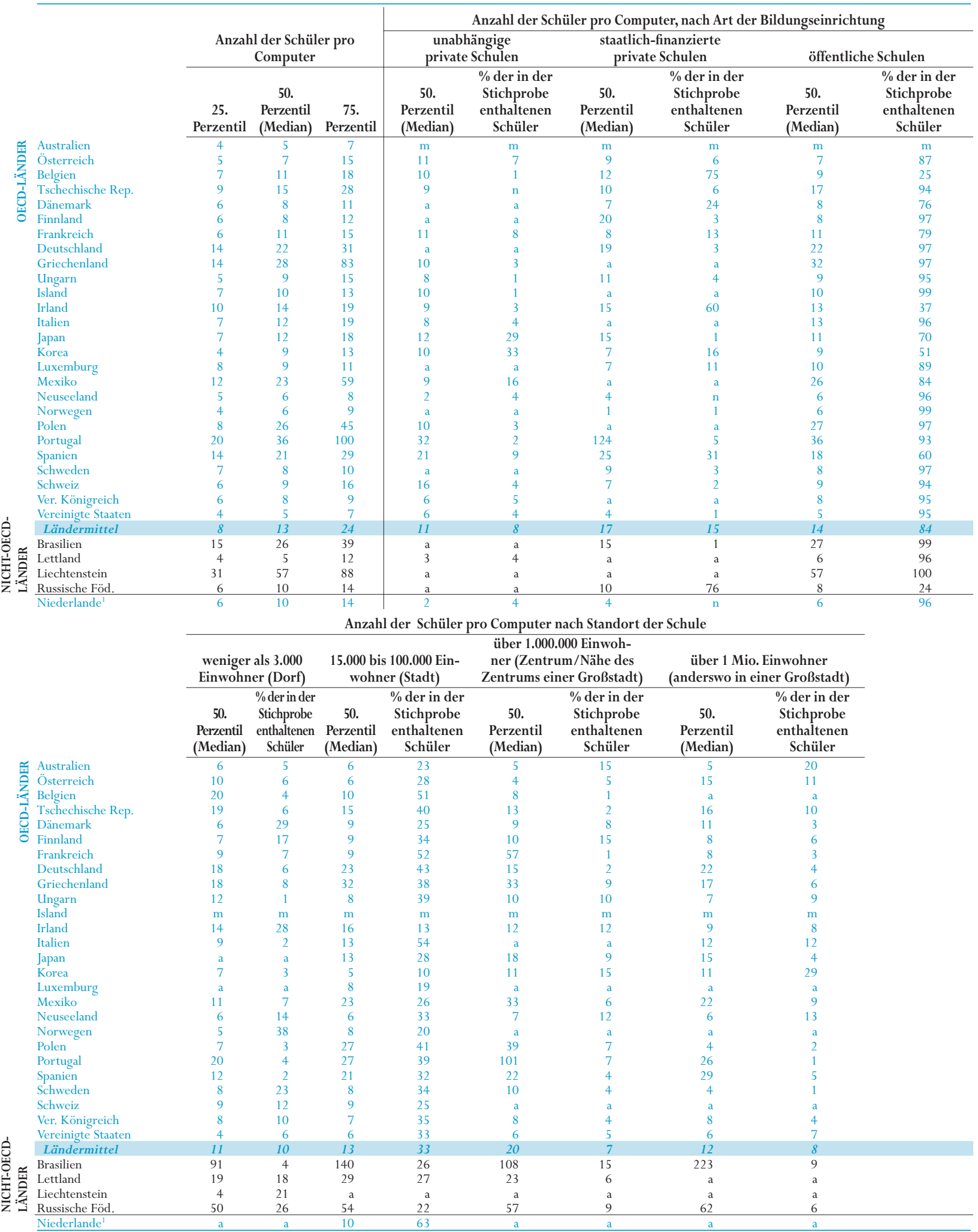

1. Die Beteiligungsquote ist zu niedrig, um die Vergleichbarkeit zu gewährleisten (Hinweise s. Anhang 3, www.oecd.org/els/education/eag2002).

Quelle: OECD PISA-Datenbank, 2001. Hinweise zur Methodik s. Anhang 3 (www.oecd.org/els/education/eag2002) und www.pisa.oecd.org. 
KAPITEL D Das Lernumfeld und die Organisation von Schulen

Tabelle D3.2.

Verfügbarkeit von Computern und Computer-Netzwerken an Schulen, die 15-Jährige besuchen (2000)

Prozentsatz der Computer, die - gemäßAngaben der Schulleiter - Schülern, Lehrern bzw. derVerwaltung/Schulleitung zurVerfügung stehen, gewichtet nach Schülerzahl, und Computer die mit einem Internetanschluss oder eine LAN-Verbindung ausgestattet sind

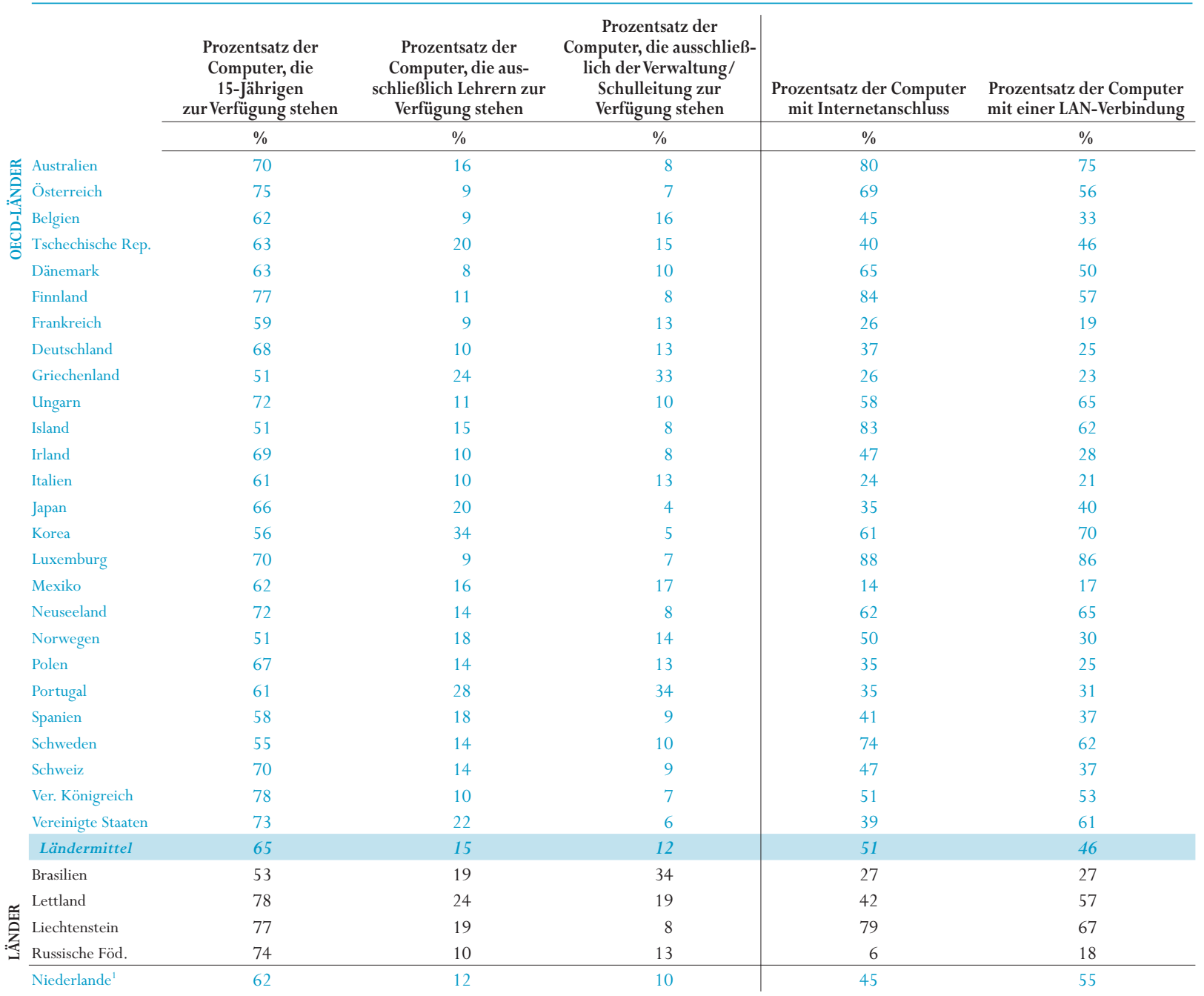

1. Die Beteiligungsquote ist zu niedrig, um die Vergleichbarkeit der Daten zu gewährleisten (Hinweise s. Anhang 3, www.oecd.org/els/education/eag2002).

Quelle: OECD PISA-Datenbank, 2001. Hinweise zur Methodik s. Anhang 3 (www.oecd.org/els/education/eag2002) und www.pisa.oecd.org. 
Tabelle D3.3.

Ausmaß der Lernbeeinträchtigung aufgrund einer nicht genügenden Anzahl von Computern bzw. eines Mangels an Multimedia-Ausstattung für den Unterricht an Schulen, die 15-Jährige besuchen (2000)

Durchschnittlicher Prozentsatz 15-Jähriger, die Schulen besuchen, an denen nach Angaben der Schulleiter das Lernen für den Unterricht sehr, etwas, kaum oder gar nicht durch eine unzureichende Anzahl an Computern und einen Mangel an Multimedia-Ausstattung beeinträchtig wird

\begin{tabular}{|c|c|c|c|c|c|c|c|c|}
\hline & \multicolumn{4}{|c|}{$\begin{array}{l}\text { Das Lernen für den Unterricht wird durch eine } \\
\text { unzureichende Anzahl an Computern beeinträchtigt }\end{array}$} & \multicolumn{4}{|c|}{$\begin{array}{c}\text { Das Lernen für den Unterricht wird durch einen Mangel ar } \\
\text { Multimedia-Ausstattung beeinträchtigt }\end{array}$} \\
\hline & gar nicht & kaum & etwas & sehr & gar nicht & kaum & etwas & sehr \\
\hline & $\%$ & $\%$ & $\%$ & $\%$ & $\%$ & $\%$ & $\%$ & $\%$ \\
\hline Australien & 32 & 38 & 27 & 3 & 29 & 39 & 31 & 1 \\
\hline Österreich & 30 & 32 & 23 & 15 & 22 & 35 & 32 & 11 \\
\hline Belgien & 49 & 32 & 15 & 3 & 42 & 34 & 17 & 7 \\
\hline Kanada & 33 & 36 & 26 & 4 & 30 & 46 & 22 & 3 \\
\hline Tschechische Rep. & 37 & 25 & 28 & 10 & 34 & 27 & 31 & 9 \\
\hline Dänemark & 32 & 40 & 21 & 6 & 48 & 39 & 10 & 3 \\
\hline Finnland & 16 & 41 & 35 & 7 & 15 & 40 & 37 & 9 \\
\hline Frankreich & 39 & 33 & 23 & 5 & 40 & 26 & 29 & 5 \\
\hline Deutschland & 20 & 30 & 35 & 15 & 15 & 33 & 33 & 20 \\
\hline Griechenland & 15 & 17 & 40 & 28 & 11 & 21 & 45 & 24 \\
\hline Ungarn & 69 & 18 & 9 & 4 & 41 & 34 & 23 & 2 \\
\hline Island & 26 & 29 & 41 & 4 & 16 & 36 & 42 & 5 \\
\hline Irland & 34 & 24 & 30 & 12 & 21 & 26 & 41 & 13 \\
\hline Italien & 42 & 26 & 26 & 6 & 29 & 25 & 34 & 12 \\
\hline Japan & 32 & 37 & 26 & 5 & 20 & 38 & 33 & 9 \\
\hline Korea & 30 & 46 & 18 & 6 & 21 & 39 & 32 & 8 \\
\hline Luxemburg & 24 & 53 & 23 & $\mathrm{n}$ & 19 & 58 & 23 & $\mathrm{n}$ \\
\hline Mexiko & 16 & 16 & 27 & 42 & 17 & 18 & 23 & 42 \\
\hline Neuseeland & 25 & 35 & 35 & 5 & 25 & 44 & 29 & 2 \\
\hline Norwegen & 12 & 28 & 52 & 9 & 9 & 29 & 51 & 10 \\
\hline Polen & 33 & 29 & 27 & 12 & 38 & 32 & 24 & 6 \\
\hline Portugal & 27 & 35 & 31 & 8 & 25 & 41 & 27 & 7 \\
\hline Spanien & 43 & 27 & 23 & 7 & 29 & 28 & 32 & 11 \\
\hline Schweden & 21 & 29 & 40 & 11 & 18 & 28 & 46 & 8 \\
\hline Schweiz & 40 & 37 & 19 & 4 & 37 & 38 & 19 & 6 \\
\hline Ver. Königreich & 18 & 26 & 37 & 19 & 13 & 30 & 42 & 16 \\
\hline Vereinigte Staaten & 35 & 33 & 24 & 7 & 33 & 39 & 22 & 6 \\
\hline Ländermittel & 31 & 31 & 26 & 11 & 26 & 34 & 28 & 12 \\
\hline Brasilien & 20 & 17 & 27 & 36 & 53 & 22 & 13 & 12 \\
\hline Lettland & 25 & 35 & 24 & 16 & 13 & 30 & 34 & 22 \\
\hline Liechtenstein & 26 & 33 & 41 & $\mathrm{n}$ & 59 & 20 & 20 & $\mathrm{n}$ \\
\hline Russische Föd. & 11 & 3 & 31 & 55 & 18 & 11 & 36 & 35 \\
\hline Niederlande $^{1}$ & 27 & 34 & 25 & 14 & 26 & 37 & 26 & 11 \\
\hline
\end{tabular}

1. Die Beteiligungsquote ist zu niedrig, um die Vergleichbarkeit der Daten zu gewährleisten (Hinweise s. Anhang 3, www.oecd.org/els/education/eag2002). Quelle: OECD PISA-Datenbank, 2001. Hinweise zur Methodik s. Anhang 3 (www.oecd.org/els/education/eag2002) und www.pisa.oecd.org. 
KAPITEL D Das Lernumfeld und die Organisation von Schulen

Tabelle D3.4.

Verfügbarkeit von Computern für 15-jährige Schüler zu Hause und in der Schule (2000)

Durchschnittlicher Prozentsatz 15-jähriger Schüler, die angaben, fast jeden Tag, mehrmals proWoche, zwischen einmal proWoche und einmal pro Monat, seltener als einmal pro Monat oder nie zu Hause oder in der Schule Zugang zu einem Computer zu haben

\begin{tabular}{|c|c|c|c|c|c|c|c|c|c|c|}
\hline & \multicolumn{5}{|c|}{ Verfügbarkeit von Computern zu Hause } & \multicolumn{5}{|c|}{ Verfügbarkeit von Computern in der Schule } \\
\hline & $\begin{array}{c}\text { fast } \\
\text { jeden Tag }\end{array}$ & $\begin{array}{l}\text { mehrmals } \\
\text { pro Woche }\end{array}$ & $\begin{array}{c}\text { einmal pro } \\
\text { Woche bis } \\
\text { einmal pro } \\
\text { Monat }\end{array}$ & $\begin{array}{c}\text { seltener als } \\
\text { einmal pro } \\
\text { Monat }\end{array}$ & nie & $\begin{array}{c}\text { fast } \\
\text { jeden Tag }\end{array}$ & $\begin{array}{l}\text { mehrmals } \\
\text { pro Woche }\end{array}$ & $\begin{array}{c}\text { einmal pro } \\
\text { Woche bis } \\
\text { einmal pro } \\
\text { Monat }\end{array}$ & $\begin{array}{c}\text { seltener als } \\
\text { einmal pro } \\
\text { Monat }\end{array}$ & nie \\
\hline & $\%$ & $\%$ & $\%$ & $\%$ & $\%$ & $\%$ & $\%$ & $\%$ & $\%$ & $\%$ \\
\hline Australien & 85 & 4 & 1 & 1 & 9 & 52 & 30 & 10 & 5 & 2 \\
\hline Belgien & 65 & 11 & 5 & 4 & 16 & 13 & 29 & 26 & 12 & 20 \\
\hline Kanada & 81 & 4 & 2 & 1 & 12 & 52 & 24 & 12 & 7 & 5 \\
\hline Tschechische Rep. & 48 & 6 & 3 & 2 & 41 & 10 & 29 & 30 & 10 & 21 \\
\hline Dänemark & 77 & 7 & 4 & 3 & 9 & 49 & 29 & 15 & 6 & 2 \\
\hline Finnland & 73 & 5 & 3 & 2 & 18 & 19 & 40 & 24 & 12 & 4 \\
\hline Deutschland & 72 & 8 & 4 & 3 & 13 & 6 & 16 & 27 & 21 & 30 \\
\hline Ungarn & 41 & 8 & 3 & 3 & 44 & 13 & 58 & 17 & 4 & 7 \\
\hline Irland & 62 & 4 & 2 & 2 & 29 & 16 & 25 & 20 & 13 & 25 \\
\hline Luxemburg & 63 & 11 & 6 & 5 & 14 & 16 & 29 & 32 & 10 & 13 \\
\hline Mexiko & 23 & 5 & 3 & 3 & 66 & 22 & 25 & 8 & 9 & 37 \\
\hline Neuseeland & 74 & 4 & 2 & 2 & 18 & 48 & 22 & 13 & 11 & 5 \\
\hline Schottland & 72 & 3 & 2 & 2 & 21 & 43 & 36 & 11 & 5 & 5 \\
\hline Schweden & 90 & 3 & 1 & 1 & 6 & 37 & 31 & 17 & 10 & 5 \\
\hline Schweiz & 76 & 8 & 3 & 3 & 10 & 22 & 23 & 28 & 14 & 12 \\
\hline Vereinigte Staaten & 68 & 7 & 5 & 4 & 15 & 46 & 21 & 12 & 11 & 10 \\
\hline Ländermittel & 64 & 6 & 3 & 3 & 24 & 27 & 29 & 20 & 10 & 14 \\
\hline Brasilien & 24 & 5 & 4 & 4 & 64 & 8 & 13 & 10 & 13 & 55 \\
\hline Lettland & 23 & 6 & 4 & 4 & 64 & 14 & 35 & 22 & 11 & 18 \\
\hline Liechtenstein & 75 & 8 & 3 & 2 & 12 & 20 & 29 & 41 & 5 & 5 \\
\hline Russische Föd. & 15 & 5 & 4 & 4 & 73 & 5 & 24 & 24 & 10 & 38 \\
\hline
\end{tabular}

Quelle: OECD PISA-Datenbank, 2001. Hinweise zur Methodik s. Anhang 3 (www.oecd.org/els/education/eag2002) und www.pisa.oecd.org. 
Tabelle D3.5.

Häufigkeit der Computernutzung 15-jähriger Schüler zu Hause und in der Schule (2000)

Durchschnittlicher Prozentsatz 15 -jähriger Schüler, die angaben, fast jeden Tag, mehrmals proWoche, zwischen einmal proWoche und einmal pro Monat, seltener als einmal pro Monat oder nie zu Hause oder in der Schule einen Computer zu nutzen

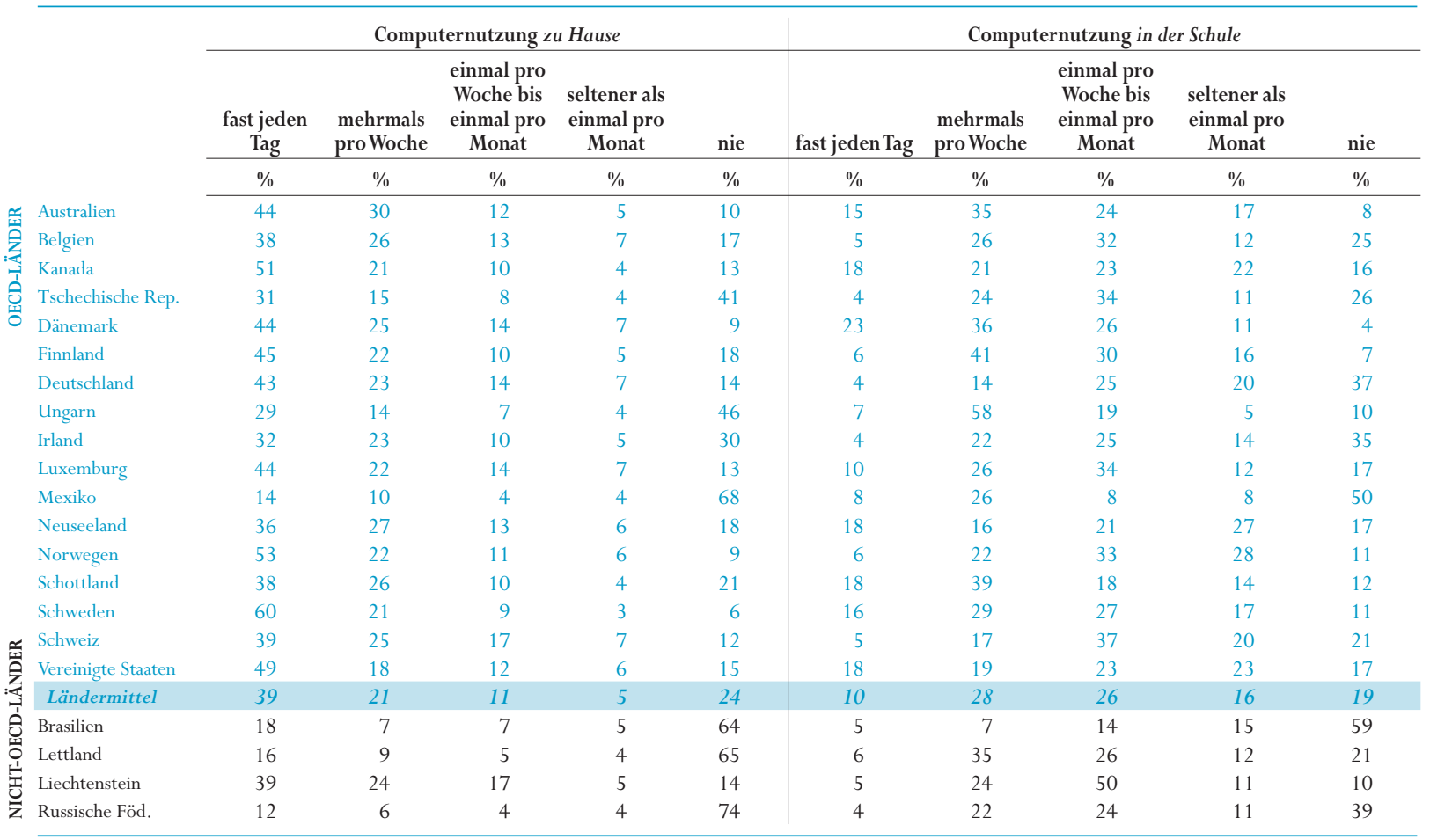

Quelle: OECD PISA-Datenbank, 2001. Hinweise zur Methodik s. Anhang 3 (www.oecd.org/els/education/eag2002) und www.pisa.oecd.org.

Tabelle D3.6.

15-jährige Schüler, die den Computer als Hilfsmittel zum Lernen für die Schule benutzen (2000)

Durchschnittlicher Prozentsatz 15-jähriger Schüler, die angaben, fast jeden Tag, mehrmals proWoche, zwischen einmal proWoche und einmal pro Monat, seltener als einmal pro Monat oder nie zu Hause oder in der Schule einen Computer als Hilfsmittel zum Lernen für die Schule zu benutzen

\begin{tabular}{|c|c|c|c|c|c|}
\hline & fast jeden Tag & mehrmals pro Woche & $\begin{array}{l}\text { einmal pro Woche bis } \\
\text { einmal pro Monat }\end{array}$ & $\begin{array}{l}\text { seltener als einmal } \\
\text { pro Monat }\end{array}$ & nie \\
\hline & $\%$ & $\%$ & $\%$ & $\%$ & $\%$ \\
\hline Australien & 12 & 30 & 29 & 17 & 12 \\
\hline Belgien & 8 & 16 & 21 & 21 & 35 \\
\hline Kanada & 10 & 21 & 28 & 21 & 20 \\
\hline Tschechische Rep. & 6 & 14 & 21 & 21 & 37 \\
\hline Dänemark & 15 & 38 & 28 & 12 & 7 \\
\hline Finnland & 6 & 18 & 32 & 27 & 17 \\
\hline Deutschland & 11 & 23 & 28 & 21 & 18 \\
\hline Ungarn & 9 & 19 & 23 & 20 & 28 \\
\hline Irland & 7 & 20 & 25 & 22 & 26 \\
\hline Luxemburg & 14 & 23 & 25 & 17 & 20 \\
\hline Mexiko & 17 & 34 & 17 & 14 & 18 \\
\hline Neuseeland & 13 & 25 & 28 & 21 & 13 \\
\hline Norwegen & 8 & 21 & 28 & 25 & 19 \\
\hline Schottland & 17 & 39 & 25 & 11 & 8 \\
\hline Schweden & 13 & 26 & 29 & 19 & 12 \\
\hline Schweiz & 6 & 17 & 27 & 24 & 26 \\
\hline Vereinigte Staaten & 19 & 26 & 25 & 17 & 12 \\
\hline Ländermittel & 11 & 24 & 26 & 19 & 19 \\
\hline Brasilien & 14 & 25 & 21 & 20 & 20 \\
\hline Lettland & 11 & 23 & 22 & 19 & 25 \\
\hline Liechtenstein & 6 & 16 & 33 & 24 & 21 \\
\hline Russische Föd. & 12 & 27 & 25 & 17 & 18 \\
\hline
\end{tabular}

Quelle: OECD PISA-Datenbank, 2001. Hinweise zur Methodik s. Anhang 3 (www.oecd.org/els/education/eag2002) und www.pisa.oecd.org. 


\section{EINSTELLUNGEN UND ERFAHRUNGEN VON SCHÜLERINNEN UND SCHÜLERN BEI DER NUTZUNG VON COMPUTERN}

- Schulen können zwar dazu beitragen, die unterschiedlichen Zugangsmöglichkeiten, die Mädchen und Jungen zu Hause zum Computer haben, abzumildern, aber in vielen Ländern nutzen die 15-jährigen Jungen den Computer und das Internet auch in der Schule häufiger als die Mädchen.

- Im OECD-Durchschnitt sind die 15-jährigen Jungen deutlich vertrauter mit Computern und schätzen ihre Fähigkeiten im Umgang mit Computern signifikant höher ein als die Mädchen.

\section{Abbildung D4.1.}

Geschlechtsspezifische Unterschiede bei der Vertrautheit und Selbsteinschätzung der

Fähigkeit im Umgang mit Computern bei 15-Jährigen (2000)

PISA Index der Vertrautheit und Selbsteinschätzung der Fähigkeit im Umgang mit Computern bei 15-jährigen Jungen und Mädchen

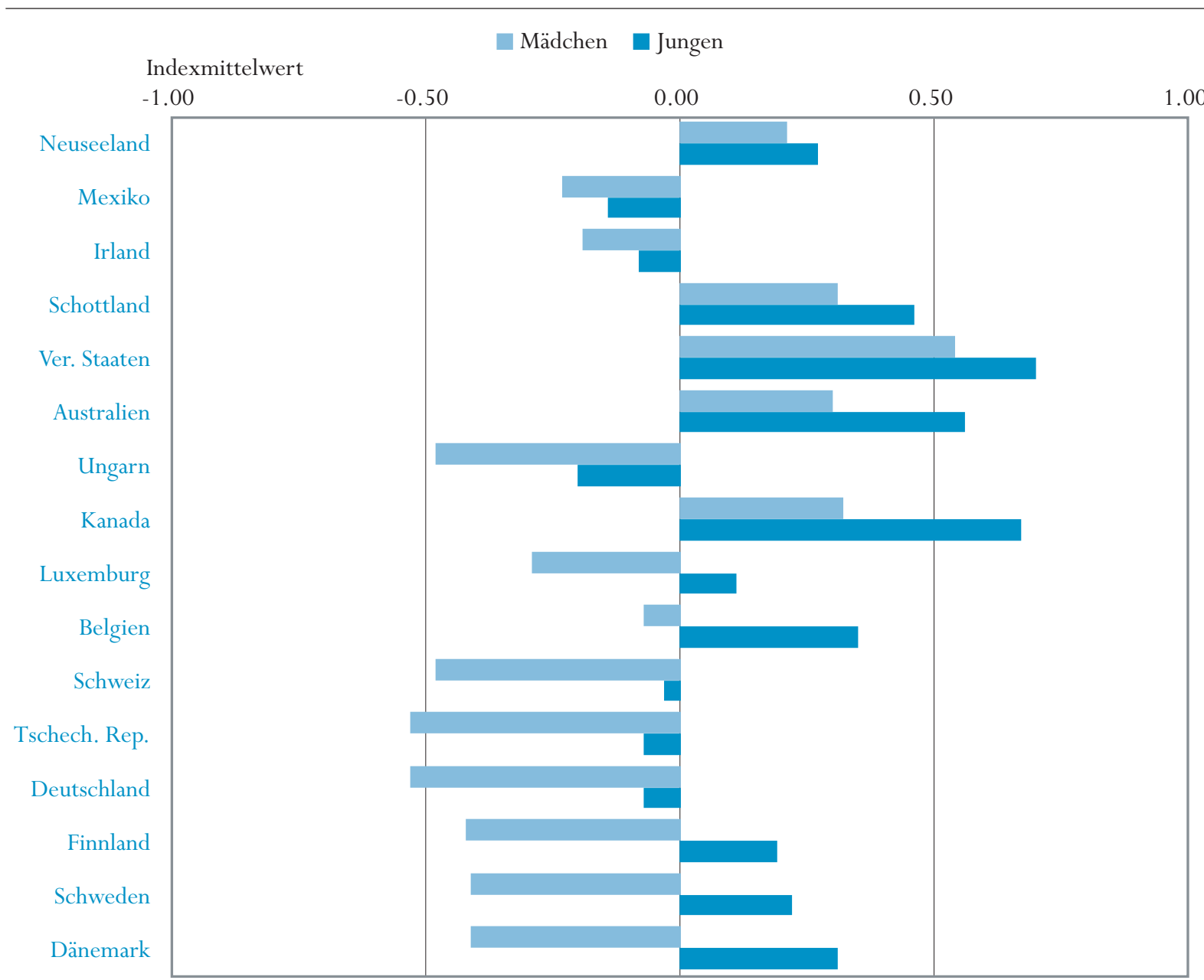

Anordnung der Länder in aufsteigender Reihenfolge der Differenz zwischen Jungen und Mädchen auf dem Pisa-Index der Vertrautheit und Selbsteinschätzung der Fähigkeit im Umgang mit Computern.

Quelle: OECD PISA-Datenbank, 2001. Tabelle D4.1. Hinweise zur Methodik s. Anhang 3 (www.oecd.org/els/education/eag2002) und www.pisa.oecd.org. 


\section{Politischer Hintergrund}

Die Verringerung der sogenannten digitalen Spaltung zwischen den Geschlechtern hat in den letzten Jahrzehnten im Bildungsbereich viel Aufmerksamkeit erhalten, denn Politiker und Pädagogen arbeiten gemeinsam daran, Chancengleichheit beim Zugang zu Bildung sicherzustellen. Die allgemeine Einführung von Computern an den Schulen hat zu der Frage Anlass gegeben, ob diese neue Technologie die bestehenden Ungleichheiten zwischen Schülerinnen und Schülern in einem traditionell als „männerdominiert” angesehenen Bereich eher verstärken oder abschwächen.

Die Relation zwischen Geschlecht und der Einstellung zu und Erfahrung mit Computern ist nicht eindeutig. Viele Faktoren innerhalb und außerhalb der im Klassenunterricht gemachten Erfahrungen können die geschlechtsspezifischen Unterschiede beim Computerwissen und der Einstellung zu Computern beeinflussen. Zu diesen Faktoren gehören zum Beispiel geschlechtsspezifische Klischees, Unterschiede in den allgemeinen Sozialisationsmustern zwischen Jungen und Mädchen sowie die Einstellung der Eltern und Lehrkräfte.

Um auf diese Punkte näher einzugehen, untersucht dieser Indikator die Einstellungen und die Selbsteinschätzung der Vertrautheit und der Fähigkeit im Umgang mit Computern von 15-jährigen Schülerinnen und Schülern.

\section{Ergebnisse und Erläuterungen}

Im Rahmen der PISA-Studie wurden 15-jährige Schülerinnen und Schüler gefragt, wie oft sie zu Hause, in der Schule, in der Bibliothek oder an einem andern Ort Zugang zu einem Computer haben. In allen Ländern gaben mehr Jungen als Mädchen an, zu Hause fast jeden Tag, mehrmals pro Woche bzw. zwischen einmal pro Woche und einmal im Monat Zugang zu einem Computer zu haben. Ebenso gaben in allen OECD-Ländern mehr Jungen als Mädchen an, zu Hause einen Internetanschluss zu haben - in 23 der 32 Länder war dieser Unterschied statistisch signifikant (Daten s. unter www.oecd.org/els/education/ eag2002).

In den meisten Ländern sind geschlechtsspezifische Unterschiede bei der Verfügbarkeit von Computern in der Schule nicht signifikant (Daten s. unter www.oecd.org/els/education/eag2002). Einen gleichberechtigten Zugang zu Computern für Schülerinnen und Schüler sicherzustellen, ist zwar oft ein bildungspolitisches Ziel, die reine Existenz dieser Technologien alleine garantiert aber keineswegs ihre gleichberechtigte Nutzung. Um für Jungen und Mädchen Chancengleichheit beim Zugang zu den Computern in der Schule zu gewährleisten, müssen viele Faktoren berücksichtigt werden, u.a. die Einstellungen und Praktiken der Lehrer und der Schülerinnen und Schüler, strukturelle Faktoren des Unterrichtsgeschehens, Gruppenzwänge unter den Schülerinnen und Schülern, die Vertrautheit der Schülerinnen und Schüler mit dem Computer und geschlechtsspezifische Klischees.
Dieser Indikator

untersucht die

Einstellungen sowie die Selbsteinschätzung der vertrautheit und der Fähigkeit im Umgang mit Computern von 15 jährigen Schülerinnen und Schülern.

\section{Die größsten geschlechtsspezifischen Unterschiede treten bei 15-Jährigen beim Zugang zu Computern zu Hause auf.}

Geschlechtsspezifische Unterschiede sind in der Regel bei der Verfügbarkeit von Computern in der Schule nicht signifikant, ... 
..aber in den meisten

Ländern nutzen

signifikant mehr

Jungen als Mädchen

den Computer oder das Internet in der Schule,...

... was möglicherweise

weniger mit dem

Zugang zu der

Technologie zu tun hat als mit der Einstellung

zu Computern und der Vertrautheit mit ihnen ...
Im Rahmen der PISA-Studie wurden 15-jährige Schülerinnen und Schüler gefragt, wie oft sie in der Schule einen Computer und das Internet nutzen. Im OECD-Durchschnitt gaben 59 Prozent der Mädchen an, mehrmals pro Monat, mehrmals pro Woche bzw. etwa einmal pro Monat einen Computer zu benutzen, im Vergleich dazu lag der Anteil der Jungen bei 64 Prozent. In Irland, Japan, Korea, Mexiko, Neuseeland und Österreich gaben mehr Mädchen als Jungen an, den Computer entsprechend häufig zu benutzen, aber der Unterschied zwischen den Geschlechtern fiel nur in Korea und Neuseeland statistisch signifikant zu Gunsten der Mädchen aus. Die geschlechtsspezifischen Unterschiede waren in Deutschland, Finnland, Frankreich, Griechenland, Norwegen, Schweden und der Schweiz am ausgeprägtesten, hier lag die Anzahl der Jungen, die angaben, den Computer mehrmals pro Monat, mehrmals pro Woche bzw. etwa einmal pro Monat zu benutzen, um mehr als 10 Prozentpunkte über dem Anteil der Mädchen. In allen Ländern außer Korea, Mexiko, Neuseeland und Österreich dominieren die Jungen auch bei der Nutzung des Internets in der Schule. Die Jungen in Finnland, Frankreich, Griechenland, Kanada, Norwegen, Polen, Portugal, Schweden, der Schweiz, im Vereinigten Königreich und Ungarn übertreffen die Mädchen bei der Nutzung des Internets um mehr als 10 Prozentpunkte (Daten s. unter www.oecd.org/els/ education/eag2002).

Verschiedene Faktoren können das Interesse der Schülerinnen und Schüler am Computer, ihre Sicherheit und die Selbsteinschätzung der Fähigkeit im Umgang mit Computern beeinflussen, so u.a. die Einstellung der Schülerinnen und Schüler zu Computern und ihre Vertrautheit mit ihnen. PISA 2000 untersuchte die Selbsteinschätzung 15-Jähriger hinschtlich ihrer Einstellung zu Computern und ihrer Vertrautheit mit Computern (Tabelle D4.1 und Abbildung D4.1). Im OECD-Durchschnitt gaben die Jungen eine signifikant höhere Selbsteinschätzung der Vertrautheit und der Fähigkeit im Umgang mit Computern an als Mädchen. Die geschlechtsspezifischen Unterschiede beim PISA-Index der Selbsteinschätzung der Vertrautheit und der Fähigkeit im Umgang mit Computern sind in Ländern wie Dänemark, Finnland und Schweden besonders ausgeprägt, hier liegt der Indexwert der Jungen um 0,6 Indexpunkte über dem der Mädchen (Dieser Unterschied entspricht mehr als einer halben Standardabweichung). Australien, Neuseeland, Schottland und die Vereinigten Staaten verzeichnen den geringsten Unterschied zwischen Mädchen und Jungen und haben die höchsten Indexwerte beider Geschlechter (Abbildung D4.1 und Tabelle D4.1).

Betrachtet man die einzelnen Variablen, die in diesen Index einfließen, so wird deutlich, dass in allen Ländern Jungen ihren eigenen Angaben zufolge signifikant vertrauter damit sind, eine Klassenarbeit mit dem Computer zu schreiben. In Dänemark, Finnland und Schweden macht der Unterschied zwischen Jungen und Mädchen, die angaben damit sehr gut oder gut vertraut zu sein, mehr als 24 Prozentpunkte aus. 
Ebenso gaben in allen Ländern mehr Jungen als Mädchen an, sehr gut bzw. gut im Umgang mit Computern zu sein, obwohl diese geschlechtsspezifischen Unterschiede in Mexiko, Neuseeland, Schottland und den Vereinigten Staaten statistisch nicht signifikant waren. Im Gegensatz dazu ist der Unterschied zwischen den Geschlechtern beim Schreiben eines Aufsatzes auf dem Computer gering, mit einem kleinen Vorteil für die Mädchen (Tabelle D4.1).

Die PISA-Studie untersuchte auch das Interesse der Schülerinnen und Schüler an Computern (Tabelle D4.2 und Abbildung D4.2). Mit Ausnahme von Mexiko und den Vereinigten Staaten erreichten die Jungen beim PISA-Index

... sowie mit dem unterschiedlichen Interesse am Computer. des Interesses an Computern deutlich höhere Werte als die Mädchen. Der Unterschied zwischen Jungen und Mädchen ist in all diesen Ländern außer Irland statistisch signifikant zu Gunsten der Jungen. Die Vereinigten Staaten sind das einzige Land, in dem mehr Mädchen als Jungen angaben, dass es wichtig sei, mit dem Computer zu arbeiten (89 Prozent der Mädchen gegenüber 84

\section{Abbildung 14.2}

Geschlechtsspezifische Unterschiede bei 15-Jährigen bezogen auf das Interesse an Computern Verteilung der mittleren Prozentsätze 15-Jähriger, die angaben:

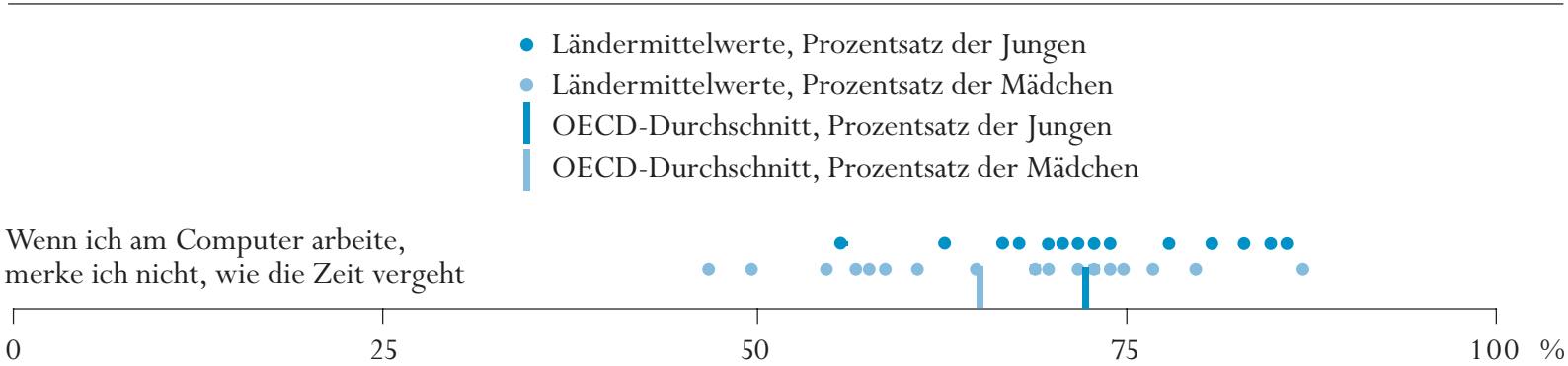

Ich benutze den Computer, weil mich das sehr interessiert

\begin{tabular}{|l|l|l|ll}
\hline & \multicolumn{1}{|c|}{} & & \\
0 & 25 & 50 & 75 & 100
\end{tabular}

Am Computer zu spielen oder zu arbeiten macht richtig Spaß
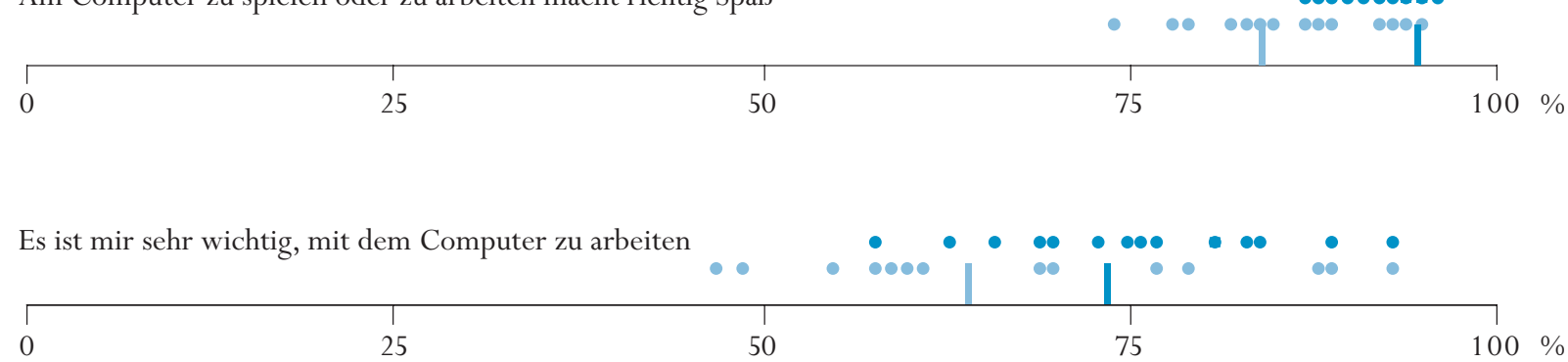

Hinweis: Die Länder sind durch Punkte dargestellt.

Quelle: OECD PISA-Datenbank, 2001. Tabelle D4.2. Hinweise zur Methodik s. Anhang 3 (www.oecd.org/els/education/ eag2002) und www.pisa.oecd.org. 
Jungen benutzen den

Computer tendenziell

häufiger für den

Zugang zum Internet,

zur elektronischen

Kommunikation und zum Programmieren, ...

....und das Gleiche gilt auch für die Nutzung

von Software in den verschiedensten Formen, wie z.B. Computerspiele,

Textverarbeitung, Tabellenkalkulationen,

Grafik-, Mal-bzw. Zeichenprogramme oder Lernsoftware...
Prozent der Jungen) und dass es wirklich Spaß macht, mit einem Computer zu spielen oder zu arbeiten (94 Prozent der Mädchen gegenüber 89 Prozent der Jungen). Es ist festzuhalten, dass im Durchschnitt der Länder 84 Prozent der Mädchen und 92 Prozent der Jungen der Ansicht sind, es mache Spaß, mit einem Computer zu spielen oder zu arbeiten. Ebenso gaben mehr Jungen als Mädchen an, einen Computer zu benutzen, weil sie das interessiere. Die Unterschiede zwischen den Geschlechtern sind bei dieser Frage sogar am größten, im OECDDurchschnitt geben 66 Prozent der Mädchen und 80 Prozent der Jungen an, einen Computer zu benutzen, weil sie das interessiere, nur Irland und Mexiko ist dieser Unterschied gering und statistisch nicht signifikant. Weiterhin gaben weniger als 55 Prozent der 15-jährigen Mädchen in Australien, Dänemark und Neuseeland an, den Computer zu benutzen, weil sie das interessiere (Tabelle D4.2 und Abbildung D4.2).

Im Rahmen der PISA-Studie wurden 15-Jährige gefragt, wie häufig sie den Computer als Zugang zum Internet, zur elektronischen Kommunikation (z.B. E-Mail), als Hilfsmittel für das schulische Lernen oder zum Programmieren benutzen. In allen Ländern gaben mehr Jungen als Mädchen an, das Internet fast täglich, mehrmals pro Woche bzw. zwischen einmal pro Woche und einmal pro Monat zu nutzen. Mexiko und die Vereinigten Staaten sind die einzigen Länder, in denen dieser Unterschied nicht statistisch signifikant zu Gunsten der Jungen ausfällt. Im Gegensatz hierzu beträgt dieser Unterschied in Deutschland und dem Vereinigten Königreich mehr als 14 Prozentpunkte. Bis auf zwei Ausnahmen nutzen Jungen in allen Ländern den Computer häufiger zum Programmieren als Mädchen. In vielen Ländern nutzen jedoch mehr Mädchen als Jungen den Computer als Hilfsmittel für das schulische Lernen. (Daten s. unter www.oecd.org/els/education/eag2002).

Die Schülerinnen und Schüler machten auch Angaben dazu, wie häufig sie verschiedene Softwarearten wie Computerspiele, Textverarbeitung, Tabellenkalkulationen, Grafik-, Mal- bzw. Zeichenprogramme oder Lernsoftware nutzen. Im OECD-Durchschnitt verwenden Jungen diese Softwarearten häufiger als Mädchen. Die geschlechtsspezifischen Unterschiede sind bei den Computerspielen besonders ausgeprägt, hier ist der Unterschied der Durchschnittswerte für Jungen und Mädchen in allen Ländern statistisch signifikant zu Gunsten der Jungen. In Australien, Dänemark, Finnland, Schweden und der Schweiz liegt der Unterschied zwischen Jungen und Mädchen bei über 33 Prozentpunkten. Ebenso ist die Nutzung von Tabellenkalkulationen, Grafik-, Mal- bzw. Zeichenprogrammen - die im Vergleich zu Computerspielen und Textverarbeitung von den Schülerinnen und Schülern deutlich weniger benutzt werden - vor allem ein Zeitvertreib der Jungen, aber die geschlechtsspezifischen Unterschiede bei der Nutzung von Tabellenkalkulationen, Grafik-, Mal- bzw. Zeichenprogrammen sind nur leicht zu Gunsten der Jungen und in Irland, Mexiko und Neuseeland sind sie statistisch nicht signifikant (Daten s. unter www.oecd.org/els/education/eag2002). 
Mit Ausnahme von Mexiko und Schottland erzielen Jungen beim PISA-Index der Computernutzung und -erfahrung höhere Punktzahlen als Mädchen. In Belgien, Mexiko, der Schweiz und der Tschechischen Republik allerdings weisen negative Indexzahlen darauf hin, dass dort alle 15-Jährigen angaben, den Computer seltener zu benutzen und weniger erfahren darin zu sein als Schülerinnen und Schüler in anderen Ländern. In Mexiko jedoch waren die Indexzahlen der Mädchen höher als die der Jungen. 15-jährige Schülerinnen und Schüler in Australien, Neuseeland, Schottland und den Vereinigten Staaten erreichten den höchsten Wert beim PISA-Index der Computernutzung und der Vertrautheit mit Computern, aber selbst in diesen Ländern war der Index für Jungen höher als für Mädchen (Daten s. unter www.oecd.org/els/education/ eag2002).

\section{Definitionen und angewandte Methodik}

Die in diesem Indikator verwendeten Daten stammen aus den Antworten der 15-jährigen Schülerinnen und Schüler sowie der Schulleiter auf Fragen zur Nutzung von Computern und der Verfügbarkeit von Computern zu Hause und in der Schule in den zwei Kontextfragebögen der Schulleistungsstudie PISA 2000.

Im Rahmen der PISA-Studie haben die 15-jährigen Schüler zusätzlich zu einem schriftlichen Test einen Schülerfragebogen ausgefüllt, in dem Daten zur Familie der Schüler, der häuslichen Umgebung, den Lesegewohnheiten, den Aktivitäten innerhalb und außerhalb der Schule erhoben wurden. Die Antworten der Schüler auf Fragen zur Häufigkeit der Nutzung von Computern und des Internets in der Schule (Daten s. unter www.oecd.org/els/education/ eag2002) stammen aus diesem Schülerfragebogen. Ein zweiter Teil dieses Fragebogens zur Vertrautheit im Umgang mit Computern wurde von Schülern in 20 Ländern als Teil einer internationalen Option ausgefüllt. Hier wurde das Interesse der Schüler am Computer, die Selbsteinschätzung der Schüler und ihre Fähigkeiten im Umgang mit Computern, ihre Computernutzung und Erfahrung mit Computern untersucht. Zu den in diesem Indikator verwendeten Daten aus diesem Teil des Fragebogens gehören die Daten zur Verfügbarkeit und Nutzung von Computern zu Hause und in der Schule (Daten s. unter www.oecd.org/els/education/eag2002), zur Vertrautheit der Schülerinnen und Schüler und ihrer Selbsteinschätzung der Fähigkeit im Umgang mit Computern (Abbildung D4.1 und Tabelle D4.1) sowie zum Interesse der Schülerinnen und Schüler an Computern (Abbildung D4.2 und Tabelle D4.2) und zur Nutzung von Computern und Software (Daten s. unter www.oecd.org/els/education/ eag2002). Die Antworten der Schülerinnen und Schüler wurden so gewichtet, dass sie der Anzahl der 15-Jährigen in der jeweiligen Schule entsprachen.

Der PISA-Index der Vertrautheit und Selbsteinschätzung der Fähigkeit im Umgang mit Computern wurde konstruiert mit einem mittleren Indexwert der Länder gleich 0 und einer Standardabweichung gleich 1. Ein positiver Wert bei diesem Index bedeutet, dass die Schüler häufiger als im OECD-Durchschnitt
... sowie für die

Nutzung von Computern und die Erfahrung im Umgang damit.

Die Ergebnisse für diesen Indikator stammen aus dem Kontextfragebogen, den 15-jährige Schülerinnen und Schüler als Teil der von der OECD im Jahr 2000 durchgeführten Internationalen Schulleistungsstudie PISA ausgefüllt haben.

DerPISA-Index der Vertrautheit und Selbsteinschätzung der Fähigkeit im Umgang mit Computern 
Der PISA-Index des Interesses am Computer

Der PISA-Index der Computernutzung und-erfahrung angaben, es sei für sie wichtig, mit dem Computer zu arbeiten, dass sie mit dem Arbeiten am Computer vertraut seien, dass sie gut darin seien, einen Aufsatz auf dem Computer zu schreiben, dass sie gut darin seien, eine Klassenarbeit auf dem Computer zu schreiben und dass sie ihre Fähigkeiten im Umgang mit Computern als besser einschätzten als die anderer 15-Jähriger.

Der PISA-Index des Interesses am Computer wurde konstruiert mit einem mittleren Indexwert der Länder gleich 0 und einer Standardabweichung gleich 1. Ein positiver Wert bei diesem Index bedeutet, dass die Schüler häufiger als im OECD-Durchschnitt angaben, dass es für sie wichtig sei, mit dem Computer zu arbeiten, dass es Spaß mache, mit dem Computer zu spielen oder zu arbeiten, dass sie einen Computer nutzten, weil sie das sehr interessiere und dass sie nicht merkten, wie die Zeit vergehe, wenn sie einen Computer benutzten.

Der PISA-Index der Computernutzung und -erfahrung wurde konstruiert mit einem mittleren Indexwert der Länder gleich 0 und einer Standardabweichung gleich 1 . Ein positiver Wert bei diesem Index bedeutet, dass die Schüler häufiger als im OECD-Durchschnitt angaben, den Computer als Hilfsmittel für das schulische Lernen, zum Programmieren, zur Textverarbeitung, zur Tabellenkalkulation, zum Malen, Zeichnen oder Erstellen von Graphiken und für Lernsoftware zu verwenden.

In den in diesem Indikator genutzten Tabellen und Abbildungen werden die geschlechtsspezifischen Unterschiede der Mittelwerte bei einem Konfidenzniveau von 95 Prozent als statistisch signifikant angegeben. Das bedeutet, dass ein Unterschied dieser Größenordnung oder oder ein größerer Unterschied in weniger als 5 Prozent der Fälle beobachtet würde, wenn in der entsprechenden Population ein solcher Unterschied tatsächlich nicht vorhanden wäre. 
Tabelle D4.1.

Vertrautheit und Selbsteinschätzung der Fähigkeit im Umgang mit Computern von 15-jährigen Schülerinnen und Schülern, nach Geschlecht (2000)

PISA-Index derVertrautheit und Selbsteinschätzung der Fähigkeit im Umgang mit Computern, nach Geschlecht, und durchschnittlicher Prozentsatz 15-jähriger Schüler, die angaben, sehr gut oder gut, einigermaßen gut oder überhaupt nicht gut im Umgang mit dem Computer, beim Schreiben eines Aufsatzes am Computer oder

beim Schreiben einer Klassenarbeit am Computer zu sein

\begin{tabular}{|c|c|c|c|c|c|c|c|c|c|c|c|c|c|c|}
\hline & \multirow{2}{*}{\multicolumn{2}{|c|}{$\begin{array}{l}\text { PISA-Index der Ver- } \\
\text { trautheit und Selbst- } \\
\text { einschätzung der } \\
\text { Fähigkeit im Um- } \\
\text { gang mit Computern }\end{array}$}} & \multicolumn{4}{|c|}{ Umgang mit Computern } & \multicolumn{4}{|c|}{ Schreiben eines Aufsatzes am Computer } & \multicolumn{4}{|c|}{$\begin{array}{l}\text { Schreiben einer Klassenarbeit am } \\
\text { Computer }\end{array}$} \\
\hline & & & \multicolumn{2}{|c|}{ sehr gut oder gut } & \multicolumn{2}{|c|}{$\begin{array}{c}\text { einigermaßen gut } \\
\text { oder überhaupt } \\
\text { nicht gut }\end{array}$} & \multicolumn{2}{|c|}{ sehr gut oder gut } & \multicolumn{2}{|c|}{$\begin{array}{l}\text { einigermaßen gut } \\
\text { oder überhaupt } \\
\text { nicht gut }\end{array}$} & \multicolumn{2}{|c|}{ sehr gut oder gut } & \multicolumn{2}{|c|}{$\begin{array}{l}\text { einigermaßen gut } \\
\text { oder überhaupt } \\
\text { nicht gut }\end{array}$} \\
\hline & Mädchen & Jungen & Mädchen & Jungen & Mädchen & Jungen & Mädchen & Jungen & Mädchen & Jungen & Mädchen & Jungen & Mädchen & Jungen \\
\hline & & & $\%$ & $\%$ & $\%$ & $\%$ & $\%$ & $\%$ & $\%$ & $\%$ & $\%$ & $\%$ & $\%$ & $\%$ \\
\hline Australien & 0.30 & 0.56 & 86 & 91 & 14 & 9 & 90 & 88 & 10 & 12 & 64 & 75 & 36 & 25 \\
\hline Belgien & -0.07 & 0.35 & 72 & 86 & 28 & 14 & 76 & 80 & 24 & 20 & 57 & 73 & 43 & 27 \\
\hline Kanada & 0.32 & 0.67 & 85 & 92 & 15 & 8 & 89 & 88 & 11 & 12 & 67 & 77 & 33 & 23 \\
\hline Tschechische Rep. & -0.53 & -0.07 & 47 & 72 & 53 & 28 & 63 & 65 & 37 & 35 & 57 & 71 & 43 & 29 \\
\hline Dänemark & -0.41 & 0.31 & 57 & 80 & 43 & 20 & 74 & 84 & 26 & 16 & 34 & 66 & 66 & 35 \\
\hline Finnland & -0.42 & 0.19 & 64 & 84 & 36 & 16 & 69 & 74 & 31 & 26 & 31 & 60 & 69 & 40 \\
\hline Deutschland & -0.53 & -0.07 & 49 & 73 & 51 & 27 & 56 & 63 & 44 & 37 & 48 & 65 & 52 & 35 \\
\hline Ungarn & -0.48 & -0.20 & 80 & 89 & 20 & 11 & 40 & 45 & 60 & 55 & 57 & 63 & 43 & 37 \\
\hline Irland & -0.19 & -0.08 & 73 & 77 & 27 & 23 & 64 & 58 & 36 & 42 & 44 & 51 & 56 & 49 \\
\hline Luxemburg & -0.29 & 0.11 & 58 & 77 & 42 & 23 & 62 & 67 & 38 & 33 & 52 & 68 & 48 & 32 \\
\hline Mexiko & -0.23 & -0.14 & 70 & 72 & 30 & 28 & 73 & 69 & 27 & 31 & 54 & 61 & 46 & 39 \\
\hline Neuseeland & 0.21 & 0.27 & 85 & 87 & 15 & 13 & 83 & 78 & 17 & 22 & 63 & 67 & 37 & 33 \\
\hline Schottland & 0.31 & 0.46 & 83 & 87 & 17 & 13 & 83 & 82 & 17 & 18 & 65 & 70 & 35 & 30 \\
\hline Schweden & -0.41 & 0.22 & 67 & 88 & 33 & 12 & 76 & 86 & 24 & 14 & 19 & 43 & 81 & 57 \\
\hline Schweiz & -0.48 & -0.03 & 47 & 73 & 53 & 27 & 61 & 65 & 39 & 35 & 52 & 66 & 48 & 34 \\
\hline Vereinigte Staaten & 0.54 & 0.70 & 88 & 91 & 12 & 9 & 93 & 89 & 7 & 11 & 74 & 79 & 26 & 21 \\
\hline Ländermittel & -0.17 & 0.21 & 70 & 82 & 30 & 18 & 72 & 74 & 28 & 26 & 52 & 66 & 48 & 34 \\
\hline Brasilien & -0.62 & -0.35 & 57 & 68 & 43 & 32 & 51 & 58 & 49 & 42 & 38 & 49 & 62 & 51 \\
\hline Lettland & -0.35 & -0.07 & 59 & 74 & 41 & 26 & 63 & 66 & 37 & 34 & 52 & 65 & 48 & 35 \\
\hline Liechtenstein & -0.52 & -0.02 & 43 & 77 & 57 & 23 & 61 & 63 & 39 & 37 & 61 & 68 & 39 & 32 \\
\hline Russische Föd. & -0.39 & -0.24 & 53 & 62 & 47 & 38 & 62 & 64 & 38 & 36 & 53 & 59 & 47 & 41 \\
\hline
\end{tabular}

Hinweis: Die Angabe eines Wertes in Fettdruck bedeutet, dass der Unterschied zwischen den Durchschnittswerten für Jungen und Mädchen statistisch signifikant ist.

1. Definitionen der Indizes s. Abschnitt Definitionen und angewandte Methodik dieses Indikators.

Quelle: OECD PISA-Datenbank, 2001. Hinweise zur Methodik s. Anhang 3 (www.oecd.org/els/education/eag2002) und www.pisa.oecd.org. 
KAPITEL D Das Lernumfeld und die Organisation von Schulen

Tabelle D4.2.

Interesse 15-jähriger Schülerinnen und Schüler an der Nutzung von Computern, nach Geschlecht (2000)

PISA-Index des Interesses an Computern nach Geschlecht sowie durchschnittlicher Prozentsatz 15-jähriger Schüler, die angaben, dass es für sie wichtig ist, mit dem Computer zu arbeiten, dass es richtig Spaß macht, am Computer zu spielen oder zu arbeiten, dass sie den Computer benutzen, weil sie das interessiert und dass sie die Zeit vergessen, wenn sie am Computer arbeiten

\begin{tabular}{|c|c|c|c|c|c|c|c|c|c|c|}
\hline \multirow[b]{4}{*}{ Australien } & \multicolumn{2}{|c|}{$\begin{array}{c}\text { PISA-Index des Interesses } \\
\text { am Computer }\end{array}$} & \multicolumn{2}{|c|}{$\begin{array}{c}\text { Es ist wichtig, am } \\
\text { Computer zu arbeiten }\end{array}$} & \multicolumn{2}{|c|}{$\begin{array}{c}\text { Es macht richtig Spaß, } \\
\text { am Computer zu spielen } \\
\text { oder zu arbeiten }\end{array}$} & \multicolumn{2}{|c|}{$\begin{array}{c}\text { Schüler benutzen den } \\
\text { Computer, weil sie } \\
\text { das interessiert }\end{array}$} & \multicolumn{2}{|c|}{$\begin{array}{l}\text { Schüler vergessen } \\
\text { bei der Arbeit am } \\
\text { Computer die Zeit }\end{array}$} \\
\hline & Mädchen & Jungen & Mädchen & Jungen & Mädchen & Jungen & Mädchen & Jungen & Mädchen & Jungen \\
\hline & & & $\%$ & $\%$ & $\%$ & $\%$ & $\%$ & $\%$ & $\%$ & $\%$ \\
\hline & -0.41 & -0.04 & 64 & 72 & 74 & 87 & 52 & 71 & 52 & 58 \\
\hline Belgien & -0.22 & 0.18 & 60 & 73 & 87 & 94 & 62 & 78 & 58 & 72 \\
\hline Kanada & -0.24 & 0.07 & 58 & 70 & 82 & 90 & 58 & 76 & 65 & 68 \\
\hline Tschechische Rep. & -0.21 & 0.14 & 61 & 69 & 85 & 93 & 68 & 81 & 57 & 70 \\
\hline Dänemark & -0.66 & 0.18 & 49 & 75 & 74 & 94 & 37 & 77 & 47 & 70 \\
\hline Finnland & -0.39 & 0.10 & 47 & 66 & 78 & 92 & 68 & 85 & 50 & 63 \\
\hline Deutschland & 0.04 & 0.43 & 69 & 84 & 89 & 96 & 70 & 87 & 73 & 83 \\
\hline Ungarn & -0.21 & 0.14 & 58 & 69 & 84 & 90 & 64 & 81 & 61 & 71 \\
\hline Irland & -0.02 & 0.02 & 55 & 58 & 92 & 94 & 72 & 75 & 75 & 74 \\
\hline Luxemburg & 0.12 & 0.45 & 70 & 83 & 88 & 93 & 74 & 88 & 77 & 86 \\
\hline Mexiko & 0.29 & 0.27 & 88 & 89 & 87 & 88 & 84 & 84 & 87 & 85 \\
\hline Neuseeland & -0.41 & -0.15 & 55 & 63 & 78 & 87 & 54 & 69 & 55 & 56 \\
\hline Schottland & 0.03 & 0.26 & 69 & 76 & 87 & 95 & 69 & 83 & 72 & 73 \\
\hline Schweden & -0.18 & 0.29 & 59 & 81 & 79 & 95 & 74 & 88 & 59 & 67 \\
\hline Schweiz & -0.12 & 0.24 & 60 & 76 & 83 & 91 & 65 & 82 & 70 & 78 \\
\hline Vereinigte Staaten & 0.33 & 0.29 & 89 & 84 & 94 & 89 & 78 & 83 & 73 & 73 \\
\hline Ländermittel & -0.14 & 0.18 & 63 & 74 & 84 & 92 & 66 & 80 & 64 & 72 \\
\hline Brasilien & 0.34 & 0.39 & 93 & 93 & 95 & 95 & 91 & 94 & 69 & 70 \\
\hline Lettland & 0.28 & 0.34 & 79 & 77 & 93 & 92 & 90 & 91 & 74 & 81 \\
\hline Liechtenstein & -0.02 & 0.35 & 69 & 81 & 84 & 92 & 68 & 89 & 69 & 78 \\
\hline Russische Föd. & 0.18 & 0.18 & 77 & 75 & 92 & 92 & 90 & 90 & 80 & 85 \\
\hline
\end{tabular}

Hinweis: Die Angabe eines Wertes in Fettdruck bedeutet, dass der Unterschied zwischen den Durchschnittswerten für Jungen und Mädchen statistisch signifikant ist.

1. Definitionen der Indizes s. Abschnitt Definitionen und angewandte Methodik dieses Indikators.

Quelle: OECD PISA-Datenbank, 2001. Hinweise zur Methodik s. Anhang 3 (www.oecd.org/els/education/eag2002) und www.pisa.oecd.org. 


\section{UNTERRICHTS- UND SCHULKLIMA}

- In Australien, Kanada, Neuseeland, Portugal, Schweden, dem Vereinigten Königreich und den Vereinigten Staaten erhielten die Schüler nach eigenen Angaben mehr Unterstützung durch die Lehrkräfte als im OECD-Mittel und in Belgien, Deutschland, Italien, Korea, Luxemburg, Österreich, Polen und der Tschechischen Republik weniger.

- Im Durchschnitt gab jeder dritte 15-Jährige an, dass zu Beginn einer Schulstunde mehr als fünf Minuten vergingen, in denen nichts passiere und mehr als jeder vierte beklagte sich darüber, dass es laut sei und alles durcheinander gehe.

- In leistungsstarken Ländern werden die Schulressourcen tendenziell häufiger genutzt, die Schulen haben entsprechend einen höheren Grad der Autonomie, die Stimmung und Arbeitshaltung der Lehrkräfte ist besser und die Beziehungen zwischen Schülern und Lehrern sind in der Regel besser. Dagegen sind in den leistungsschwachen Ländern verstärkt negative Indizes beim Schulklima zu beobachten, und die Indizes der Nutzung der Schulressourcen, der Stimmung und Arbeitshaltung der Lehrkräfte, der Autonomie der Schule und des Lehrer-Schüler-Verhältnisses liegen in der Regel unter dem OECD-Durchschnitt.

\section{Abbildung D5.1.}

Persönlicher Bezug 15-jähriger Schüler zu ihrer Schule (2000)

Verteilung der mittleren Prozentsätze von 15-Jährigen, die folgenden Aussagen zustimmen, bzw. voll zustimmen: "Meine Schule ist ein Ort, ...

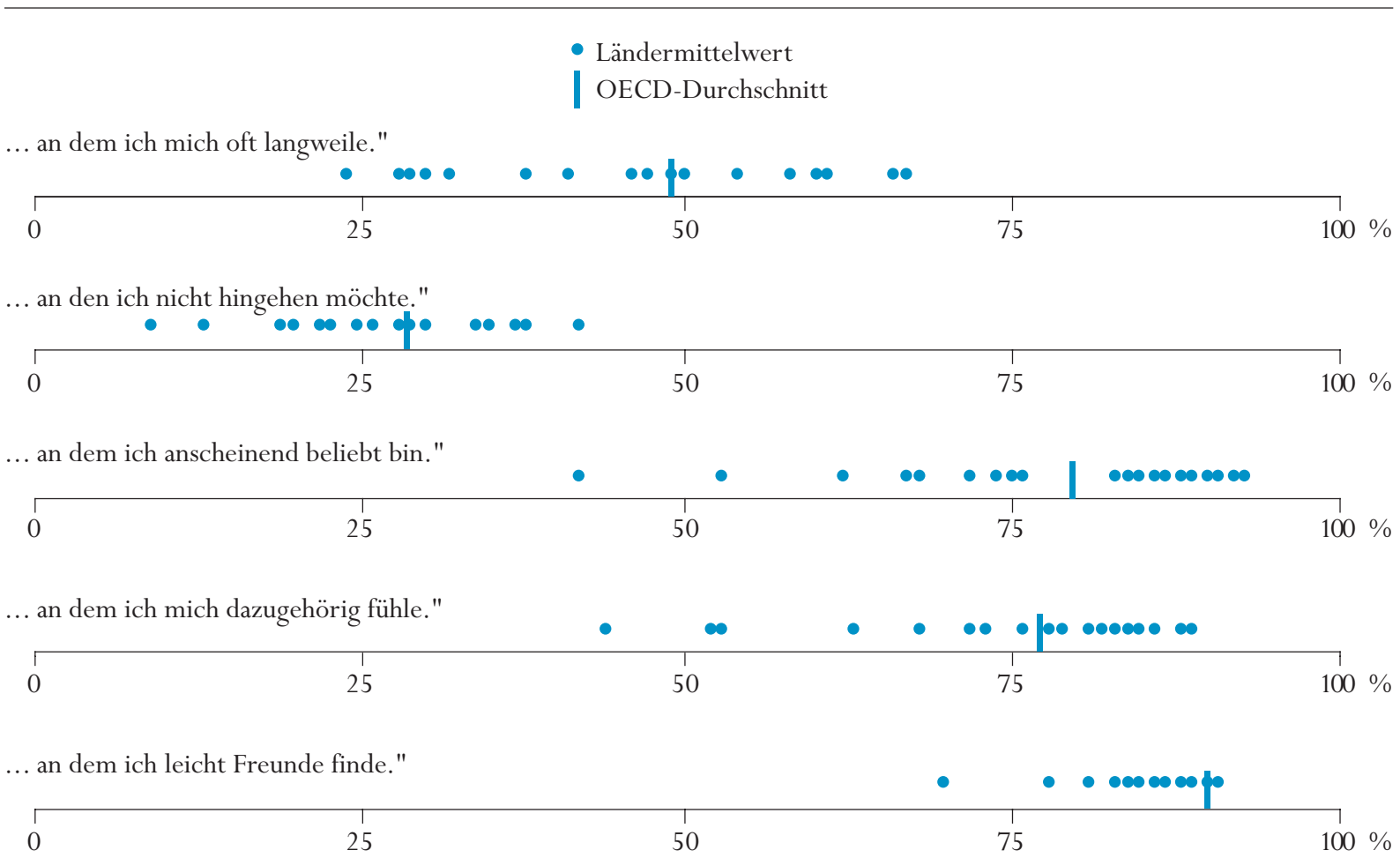

Hinweis: Die Länder sind durch Punkte dargestellt.

Quelle: OECD PISA-Datenbank, 2001. Tabelle D5.4. Hinweise zur Methodik s. Anhang 3 (www.oecd.org/els/education/ eag2002) und www.pisa.oecd.org. 
Dieser Indikator zeigt verschiedene Aspekte des Unterrichts- und Lernklimas sowie des persönlichen Bezugs der Schüler zur Schule auf.

\section{Politischer Hintergrund}

Schüler verbringen im Alter zwischen dem 6 und 15 Jahren im Durchschnitt 900 Stunden pro Jahr im Unterricht, wo sie mit den Lehrern und Mitschülern interagieren (s. Indikator D1). Die Qualität dieser Interaktion und die Nutzung der Unterrichtszeit im Klassenzimmer bestimmt in großem Maße, wie sehr die Schüler von der Schule profitieren.

Die Erfahrungen, die im Rahmen des Unterrichts von den Schülern gemacht werden, beeinflussen sowohl den Inhalt als auch die Form des Lernens. Dadurch werden zugleich die Motivation und der sich entwickelnde Lernstil der Schüler beeinflusst. Die PISA-Studie bietet Belege dafür, dass sowohl lehrer- als auch schülerbezogene Faktoren des Unterrichtsklimas und der Unterrichtspraktiken die Leistung des einzelnen Schülers beeinflussen.

Darüber hinaus sind die Einstellung und der persönliche Bezug der Schüler zur Schule wichtige Aspekte des Lernklimas. Die Forschung belegt, dass negative Einstellungen zu Schwänzen und störendem Verhalten führen können, während umgekehrt Schüler, die sich in der Schule bei Aktivitäten innerhalb und außerhalb des Lehrplans engagieren und starke Bindungen zu Lehrern und Mitschülern entwickeln, in der Regel bessere Leistungen erzielen.

Dieser Indikator zeigt verschiedene Aspekte des Unterrichts- und Lernklimas auf und berichtet über die Einstellungen der Schüler zur Schule.

\section{Ergebnisse und Erläuterungen}

\section{Lernklima im Unterricht}

Im Rahmen der PISA-Studie wurden 15-Jährige zu verschiedenen Aspekten ihrer Erfahrungen im Unterricht in der Landes- bzw. Unterrichtssprache befragt: zur Unterstützung durch die Lehrkräfte, zur Disziplin im Unterricht, zur Nutzung der Schulressourcen und zur Hausaufgabenregelung in ihrer Schule. Basierend auf ihren Antworten wurden die Indizes der Unterstützung der Schüler durch die Lehrkräfte, der Schuldisziplin, der Leistungsorientierung und der Schulressourcennutzung entwickelt (Abb. D5.2).

\section{Was sind die PISA-Indizes zum Lernklima im Unterricht?}

Die PISA-Indizes zum Lernklima im Unterricht fassen die Antworten der 15-Jährigen auf eine Reihe miteinander verknüpfter Fragen zusammen. Die Indizes wurden standardisiert, so dass der mittlere Indexwert gleich 0 und die Standardabweichung gleich 1 ist (weitere Erklärungen im Abschnitt ,Definitionen und angewandte Methodik' dieses Indikators). Es ist zu beachten, dass negative Werte für ein Land bei einem Index nicht zwangsläufig auf negative Antworten der Schüler auf die gestellten Fragen schließen lassen. Ein negativer Wert weist lediglich darauf hin, dass die 15- 
Jährigen eines Landes weniger positiv antworteten, als das beim Durchschnitt aller 15-Jährigen in den OECD-Ländern der Fall war. Zum Inhalt der Indizes und weiteren technischen Erläuterungen siehe Abschnitt ,Definitionen und angewandte Methodik' für diesen Indikator.

\section{Unterstützung durch die Lehrkräfte}

Im OECD-Durchschnitt gaben sechs von zehn 15-Jährigen an, dass die Lehrkraft der Unterrichtssprache sich in den meisten oder allen Stunden für den Lernfortschritt jedes einzelnen Schülers interessiere, ihnen die Gelegenheit gebe, ihre Meinung zu sagen, ihnen bei der Arbeit helfe, etwas so lange erkläre, bis alle Schüler es verstehen, ihnen beim Lernen helfe und ihre Hausaufgaben kontrolliere (Tabelle D5.1).

Aber die durchschnittliche Einschätzung der Unterstützung durch die Lehrkraft variiert stark zwischen den einzelnen Ländern. So gaben beispielsweise in Australien, Portugal und dem Vereinigten Königreich mehr als drei Viertel der 15-Jährigen an, dass sich die Lehrkraft immer bzw. wenigstens meistens für den Lernfortschritt jedes einzelnen Schülers interessiere und nur fünf Prozent oder weniger sagten, dies sei nie der Fall. In Italien, Korea und Polen dagegen gibt nur jeder dritte 15-Jährige an, dass sich der Lehrer für den Lernfortschritt jedes einzelnen Schülers interessiere und fast genauso viele meinen, dies sei nie der Fall. Ebenso geben in Australien, Island, Neuseeland und dem Vereinigten Königreich mindestens vier von fünf 15-Jährigen an, ihr Lehrer im Unterricht der Landes- bzw. Unterrichtssprache helfe ihnen immer bzw. meistens bei der Arbeit, während dies in Korea, Mexiko und der Tschechischen Republik nur jeder Dritte sagte. Zwischen zwei Drittel und drei Viertel der 15-Jährigen in Australien, Dänemark, Island, Kanada, Neuseeland, Portugal, Schweden und dem Vereinigten Königreich gaben an, dass der Lehrer im Unterricht der Landes- bzw. Unterrichtssprache etwas so lange erkläre, bis alle es verstehen, während dies in Japan, Korea, Polen und der Tschechischen Republik weniger als die Hälfte der 15-Jährigen sagten (Tabelle D5.1).

Die 15-Jährigen in Deutschland, Korea, Luxemburg, Polen und der Tschechischen Republik erhielten laut ihren eigenen Angaben die geringste Unterstützung durch ihre Lehrer, dagegen berichteten die 15-Jährigen in Australien, Kanada, Neuseeland, Portugal, dem Vereinigten Königreich und den Vereinigten Staaten von einem verhältnismäßig hohen Grad an Unterstützung durch die Lehrkräfte. Zwischen dem mittleren Unterstützungsindex im Vereinigten Königreich $(0,50)$ und dem in Korea $(-0,67)$ besteht eine Differenz von mehr als einer Standardabweichung. Das heißt, dass im Durchschnitt die 15-Jährigen im Vereinigten Königreich angeben, dass sie mindestens soviel Unterstützung von ihren Lehrern erhalten wie das obere Drittel aller 15-Jähriger in den
Im Schnitt gaben sechs von zehn 15-Jährigen an, dass die Lehrkraft sich meistens für den Lernfortschritt jedes einzelnen Schülers interessiere, aber jeder Zehnte sagte auch, dies sei nie der Fall, die Einschätzung der Unterstützung durch die Lehrkraft variiert jedoch stark zwischen den einzelnen Ländern. 


\section{Abbildung D5.2.}

Index des Unterrichtsklimas für 15-jährige Schüler (2000)

PISA-Indizes der Unterstützung der Schüler durch die Lehrkräfte, der Schuldisziplin, des Leistungsdrucks und der Schulressourcennutzung (basierend auf Schülerangaben)

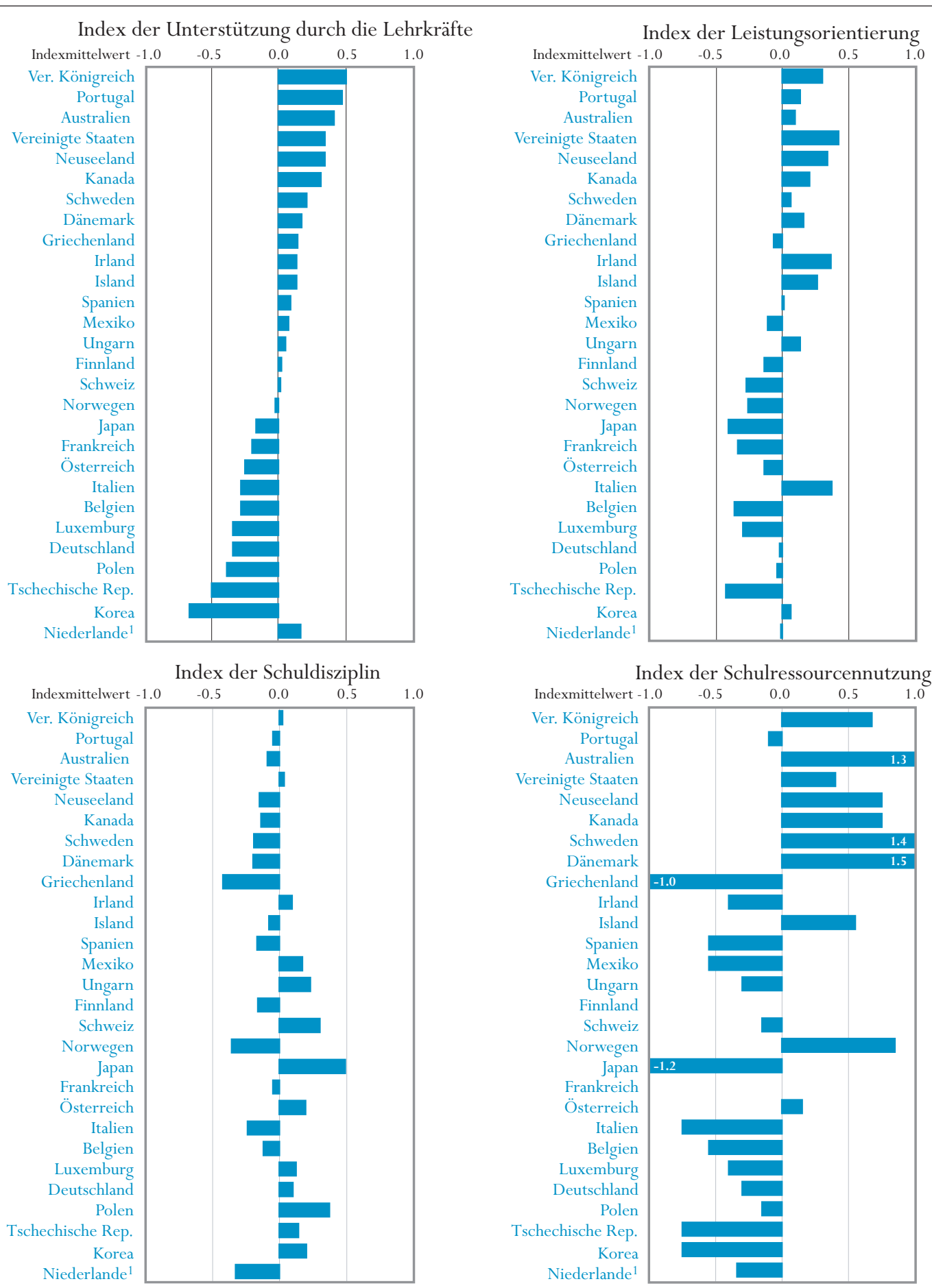

1. Die Beteiligungsquote ist zu niedrig, um die Vergleichbarkeit zu gewährleisten (s. Anhang 3, www.oecd.org/els/education/eag2002). Anordnung der Daten in absteigender Reihenfolge desWertes auf dem PISA-Index der Unterstützung der Schüler durch die Lehrkräfte. Quelle: OECD PISA-Datenbank, 2001. Tabellen D5.1, D5.2 und D5.3. Hinweise zur Methodik s. Anhang 3 (www.oecd.org/ els/education/eag2002) und www.pisa.oecd.org. 
OECD-Ländern. Im Gegensatz dazu geben die 15-Jährigen in Korea an, höchstens soviel Unterstützung von den Lehrern des Unterrichts der Landesbzw. Unterrichtssprache zu bekommen wie das untere Drittel aller 15-Jährigen in den OECD-Ländern (Tabelle D5.1)

\section{Disziplin im Unterricht}

In allen Länden besteht ein positiver Zusammenhang zwischen der Disziplin im Unterricht in der Landes- bzw. Unterrichtssprache und der Leistung der Schüler. Dieser Zusammenhang ist in Ländern wie Australien, Japan, Ungarn, Polen und dem Vereinigten Königreich stark und in Ländern wie Belgien, Frankreich, Luxemburg und Mexiko schwächer. Selbst nach Berücksichtigung anderer Faktoren (einschließlich des familiären Hintergrunds), scheint die Disziplin einer der Faktoren zu sein, die einen Zusammenhang zu den Bildungsergebnissen aufweisen. Wenn Schüler selbst sagen, sie könnten im Unterricht nicht ungestört arbeiten oder dass sie, aus welchen Gründen auch immer, nicht darauf hörten, was der Lehrer sagt, dann wird das Lernen beeinträchtigt. Die PISA-Studie lässt vermuten, dass das Lernen der Schüler häufig durch Disziplinprobleme gestört wird. So gaben beispielsweise 28 Prozent der 15-Jährigen in allen OECDLändern an, dass es in jeder bzw. fast jeder Stunde im Unterricht der Landesbzw. Unterrichtssprache laut sei und alles durcheinander gehe und in Finnland, Frankreich, Griechenland und Italien ist dieser Anteil mit 40 Prozent und darüber besonders hoch. Laut Angaben der Schüler ist das größte Disziplinproblem, dass zu Beginn der Stunden Zeit verschwendet wird. Ein Drittel aller Schüler in den OECD-Ländern gab an, dass zu Beginn jeder bzw. fast jeder Stunde des Unterricht der Landes- bzw. Unterrichtssprache mehr als fünf Minuten vergingen, in denen nichts passiere. Während jedoch in Belgien, Dänemark, Griechenland, Island und Norwegen mehr als die Hälfte der 15-Jährigen angaben, dass zu Beginn der Stunde häufig Zeit verloren ginge, machte in Japan, Mexiko und Ungarn weniger als ein Viertel der 15-Jährigen diese Angabe (Tabelle D5.1).

\section{Leistungsorientierung}

Schulen bieten eine Vielzahl von Unterrichtsfächern an, und kein Schüler ist an allen Fächern in gleichem Maße interessiert. Die Schüler optimieren ihre Lernanstrengungen nach ihren eigenen Interessen und Zielen, den Ansprüchen ihrer Eltern und den Anforderungen der Lehrer. Erstrebenswerte und erreichbare Ziele festzulegen und die Schüler zu motivieren, diese zu erreichen, gehört zu den größten Herausforderungen für Lehrer. Im Rahmen der PISA-Studie wurden 15-Jährige gefragt, wie häufig die Lehrkraft im Unterricht der Landes- bzw. Unterrichtssprache will, dass sie sich richtig anstrengen, gewissenhaft arbeiten, sie ermutigt, Besseres zu leisten und viel von ihnen fordert. Aus den Antworten der Schüler wurde ein Index der Leistungsorientierung entwickelt. Im Vergleich zum OECD-Durchschnitt berichteten 15-Jährige in Australien, Finnland, Island, Irland, Italien, Kanada, Neuseeland, dem Vereinigten Königreich, den Vereinigten Staaten und Ungarn von einer stärkeren Leistungsorientierung durch die Lehrer (Tabelle D5.2).
Im OECD-Durchschnitt gibt jeder dritte 15-Jährige an, dass zu Beginn der Stunde mehr als fünf Minuten vergingen, in denen nichts passiere und 29 Prozent der Schüler beklagten sich darüber, dass es laut sei und alles durcheinander gehe.
In Australien, Kanada, Neuseeland, dem Vereinigten Königreich und den Vereinigten Staden berichten 15-Jährige von einer größseren Leistungsorientierung, ... 
gleichzeitig erhalten

sie jedoch mehr

Unterstützung durch die

Lehrer.

Das Bild unterscheidet sich jedoch in den einzelnen Ländern.

In Australien,

Dänemark, Mexiko,

Neuseeland, Portugal

und Schweden gibt

jeder dritte 15-Jährige

an, regelmäßsig die

schulbibliothek zu

benutzen,...

aber in Belgien,

Finnland, Griechenland,

Irland, Italien,

Österreich und der Tschechischen Republik macht weniger als jeder Zehnte diese Angabe.

Auch ein Mangel an Unterrichtsmaterial in

der Bibliothek kann die Nutzung derselben

beeinträchtigen.
Interessanterweise scheinen 15-Jährige in Australien, Kanada, Neuseeland, dem Vereinigten Königreich und den Vereinigten Staaten zwar einer höheren Leistungsorientierung ausgesetzt zu sein, gleichzeitig erhalten sie jedoch mehr Unterstützung durch die Lehrkräfte, was darauf hindeutet, dass die Unterstützung durch die Lehrkräfte und Leistungsorientierung sich nicht unbedingt gegenseitig ausschließen.

Im Gegensatz dazu liegen in Belgien, Japan, Korea, Luxemburg, Österreich und der Tschechischen Republik sowohl die Unterstützung durch die Lehrer als auch die Leistungsorientierung unter dem OECD-Durchschnitt. In einer dritten Gruppe von Ländern (mit Frankreich, Italien und Polen) geht eine überdurchschnittliche Leistungsorientierung mit unterdurchschnittlicher Unterstützung durch die Lehrkraft einher. In Dänemark, Finnland, Norwegen und Schweden scheinen sowohl die Leistungsorientierung als auch der Mittelwert des Indexes der Unterstützung durch die Lehrkräfte ziemlich nahe beim OECD-Durchschnitt zu liegen (Tabellen D5.1 und D5.2).

Nutzung der Bildungsressourcen

Im Rahmen der PISA-Studie wurden Schulleiter gefragt, wie sehr das Lernen durch den Mangel an Schulbüchern, Computern, Unterrichtsmaterialien in der Bibliothek, Multimedia-Ausstattung, durch unzureichende Laborausstattung und unzureichende Ausstattung für den Kunstunterricht beeinträchtigt wird. Außerdem wurden die 15-Jährigen gefragt, wie häufig sie diese Ressourcen nutzen. Während vorhandene Bildungsressourcen die Lernmöglichkeiten an den Schulen verbessern können und ihre Verfügbarkeit eine Voraussetzung für effektives Unterrichten ist, hängt die Integration der Bildungsressourcen in den Unterricht und in das schulische Lernen hauptsächlich von den Lehrern ab. Der Zugang zu Büchern und anderen Medien in der Schule sowie die Ermutigung, diese auch zu nutzen, ist ein wichtiges Anliegen. Neben den Erwachsenen zu Hause spielen Lehrer eine wichtige Rolle für die Entwicklung der Lesegewohnheiten der Schüler und für ihren Umgang mit Informationen. Die Bibliothek, die sich in den OECD-Ländern mehr und mehr zu einem Multimediazentrum entwickelt, kann hierbei ein wichtiges Instrument sein. Mehr als ein Drittel der 15-Jährigen in Australien, Dänemark, Mexiko, Neuseeland, Portugal und Schweden gaben bei der PISA-Studie an, die Schulbibliothek regelmäßig (wenigstens mehrmals pro Monat) zu benutzen.

In Belgien, Finnland, Griechenland, Irland, Italien, Österreich und der Tschechischen Republik dagegen gibt nicht einmal jeder zehnte 15-Jährige an, die Schulbibliothek regelmäßig zu benutzen (Tabelle D5.3).

Es ist schwer zu erkennen, in welchem Ausmaß Lehrplan- und Lehrbuchpolitik und die Bibliotheksnutzung zusammenhängen. So werden beispielsweise in Österreich den 15-Jährigen viele Schulbücher und anderes Unterrichts-materialien kostenlos zur Verfügung gestellt, während in Ungarn die Schüler die Schulbücher selbst bezahlen müssen. In anderen Ländern, wie zum Beispiel Schweden, erhalten die Schüler in der Regel ein Leihexemplar des Schulbuchs aus der Schulbibliothek. 
Ein Grund, die Schulbibliothek nicht zu benutzen, kann natürlich sein, selbst viele Bücher zu besitzen, ein anderer Grund können die unzureichenden Ressourcen der Bibliothek sein. In Finnland, Griechenland, Irland, Mexiko und Norwegen besuchen mehr als 40 Prozent der 15-Jährigen eine Schule, deren Schulleiter angab, dass der Mangel an Unterrichtsmaterialien in der Bibliothek das Lernen etwas bzw. sehr beeinträchtige. Dagegen besuchen in Australien, Dänemark, Frankreich, Luxemburg, Neuseeland, Portugal, der Schweiz und Ungarn weniger als 20 Prozent der 15-Jährigen eine Schule, deren Schulleiter angab, das Lernen werde durch den Mangel an Unterrichtsmaterial beeinträchtigt (Tabelle D5.3).

Die Unterschiede zwischen den Ländern bei der Nutzung von Biologie-, Physik oder Chemielabors sind noch ausgeprägter als bei der Nutzung der Schulbibliothek. Im Durchschnitt der OECD-Länder gab jeder dritte 15-Jährige an, regelmäßig das Biologie-, Physik oder Chemielabor zu nutzen. Während jedoch in Australien, Dänemark, Irland, Neuseeland, Norwegen, Schweden und im Vereinigten Königreich sechs bis acht von zehn 15-Jährigen angaben, das Biologie-, Physik oder Chemielabor mindestens mehrmals pro Monat zu nutzen, waren es in Griechenland, Japan und der Tschechischen Republik weniger als zwei von zehn 15-Jährigen und in Finnland und Ungarn sogar weniger als einer (Tabelle D5.3).

Um die Schulressourcen sinnvoll zu nutzen, bedürfen die Schüler der Ermutigung und der Unterstützung durch die Lehrer. Die PISA-Studie lässt vermuten, dass in Ländern mit überdurchschnittlicher Unterstützung durch die Lehrkräfte tendenziell auch das Indexmittel der Schulressourcennutzung über dem OECD-Durchschnitt liegt, während in Ländern mit relativ geringer Unterstützung durch die Lehrkräfte der Index der Schulressourcennutzung tendenziell auch niedrig ausfällt. Weitere Forschungsarbeiten sind erforderlich, um zu untersuchen, ob und in welchem Maße die berufliche Entwicklung der Lehrer und die genaueren Bestimmungen ihrer Arbeitszeit ihre Bemühungen, die Schulressourcen sinnvoll zu nutzen, beeinflusst.

\section{Hausaufgabenregelung}

Im Rahmen der PISA-Studie wurden 15-Jährige zur Hausaufgabenregelung ihrer Schule befragt. In Island, Kanada, Korea, Mexiko, Österreich, Portugal und den Vereinigten Staaten gaben mindestens zwei Drittel der 15-Jährigen an, dass die Hausaufgaben immer bzw. meistens in die Zeugnisnoten einfließen. Dagegen werden in Deutschland, Frankreich, Irland, Italien, Luxemburg, Neuseeland, der Schweiz, der Tschechischen Republik, Ungarn und im Vereinigten Königreich die Hausaufgaben eher weniger zur formellen Bewertung der Schüler herangezogen (Tabelle D5.2).

In Belgien, Irland, Korea, Mexiko, Neuseeland, dem Vereinigten Königreich und den Vereinigten Staaten gaben mehr als die Hälfte der 15-Jährigen an, die Lehrkraft benote ihre Hausaufgaben immer oder zumindest meistens. In Deutschland und der Schweiz geben nur etwa 10 Prozent und in Ungarn nur 4 Prozent der 15-Jährigen an, dass ihre Hausaufgaben regelmäßig benotet
Über die Hälfte der

15-Jährigen in Australien,

Dänemark, Irland,

Neuseeland, Norwegen,

Schweden und dem

Vereinigten Königreich

geben an, regelmäjsig das

Biologie-, Physik-oder

Chemielabor zu benutzen.

In Finnland und Ungarn

trifft dies jedoch auf

weniger als zehn Prozent

der schüler zu.

In etwa der Hälfte der OECD-Länder geben mindestens 90 Prozent der 15-Jährigen an, dass die Hausaufgaben zumindest manchmal in die Benotung einfließsen.

In einigen Länden benoten die Lehrkräfte die Hausaufgaben der Schüler regelmäfsig, während dies in anderen Ländern nur selten der Fall ist. 
werden. Andererseits geben in Deutschland, Griechenland, der Schweiz und dem Vereinigten Königreich mehr als 36 Prozent der 15-Jährigen an, dass die Hausaufgabenbesprechung der Lehrer immer oder zumindest meistens hilfreich sei. Dagegen geben dies nur 15 Prozent der 15-Jährigen in Finnland, Island, Japan, der Tschechischen Republik und Ungarn an (Tabelle D5.2).

\section{Schulklima}

Die Lernbedingungen wirken sich zwar direkt auf die Lernfortschritte der Schüler aus, gleichzeitig sind sie jedoch Teil des größeren Schulkontextes, in dem Lehrer und Schüler die zugewiesenen Aufgaben interpretieren und die Lehr/ Lernsituation bewerten. Im Rahmen der PISA-Studie wurde, zusätzlich zum Zusammenhang zwischen der individuellen Schülerleistung und dem Schulklima, auch die Frage untersucht, wie sehr das Schulklima zu den Leistungsunterschieden zwischen den Schulen beiträgt. Sieben Indizes des Schulklimas und der Schulpraktiken wurden identifiziert, die eine statistisch signifikante Verbindung zur durchschnittlichen Leistung auf Schulebene in den drei Fächergruppen, die in der internationalen Schulleistungsstudie bewertet wurden, aufweisen. Drei dieser Indikatoren (die Indizes lehrkraftbezogener Faktoren für das Schulklima, der Stimmung und Arbeitshaltung der Lehrkräfte aus der Sicht der Schulleitung und der Schulautonomie) beschreiben die Sicht der Schulleitung zum Lernklima an der Schule. Drei weitere Indikatoren (Einschätzung der Lehrer-SchülerBeziehung aus Sicht der Schüler, die Disziplin im Unterricht und die Einschätzung der Leistungsorientierung aus Sicht der Schüler) geben die Sicht der Schüler zum Unterrichts- und Lernklima an der Schule wieder. Der siebte Indikator bezieht sich auf die Häufigkeit der Nutzung von Schulressourcen.

\section{Die PISA-Indizes zum Schulklima}

Die PISA-Indizes zum Schulklima fassen die Antworten der Schüler und Schulleiter auf eine Reihe miteinander verknüpfter Fragen zusammen. (Weitere Erläuterungen s. Abschnitt ,Definitionen und angewandte Methodik' für diesen Indikator).

Der Index lehrkraftbezogener Faktoren für das Schulklima ist abgeleitet von den Antworten der Schulleiter zu einer Reihe von Fragen dazu, was das Lernen in der Schule beeinträchtigt.

Der Index zur Stimmung und Arbeitshaltung der Lehrkräfte beruht auf der Zustimmung der Schulleiter zu Aussagen wie „Stimmung und Arbeitshaltung der Lehrkräfte sind in dieser Schule

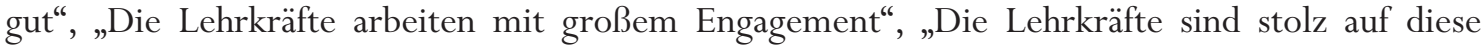
Schule“ und „Die Lehrkräfte legen Wert auf schulische Leistung“.

Der Index der Schulautonomie beruht auf Angaben des Schulleiters zu Bereichen, in denen die Schule (der Schulleiter) keine Verantwortung trägt. 
Der Index der Schüler-Lehrer-Beziehung beruht auf der Zustimmung der 15-Jährigen zu folgenden Aussagen: „Die Schüler kommen mit den meisten Lehrern gut aus“, „Den meisten Lehrern ist es wichtig, dass die Schüler sich wohl fühlen“, „Die meisten meiner Lehrer interessieren sich für das, was ich zu sagen habe“, „Wenn ich zusätzliche Hilfe brauche, bekomme ich sie von meinen Lehrern“ und „Die meisten Lehrer behandeln mich fair“.

Der Index der Unterrichtsdisziplin beruht auf den Antworten der 15-Jährigen zu Fragen darüber, wie häufig es laut ist und alles durcheinander geht, wie oft Unterrichtszeit verloren geht, weil nichts passiert und wie häufig sie nicht darauf hören, was der Lehrer sagt.

Der Index zur Leistungsorientierung beruht auf den Antworten der 15-Jährigen auf die Fragen, wie oft die Lehrkraft will, dass sie sich richtig anstrengen, ihnen sagt, dass sie eigentlich besser sein können, sie viel lernen lässt und unzufrieden ist, wenn sie nachlässig arbeiten.

Der Index der Schulressourcennutzung beruht auf den Antworten der 15-Jährigen auf die Fragen, wie häufig sie die Bibliothek, den Computer, das Biologie-, Physik- oder Chemielabor und andere Bildungsressourcen benutzen (Zur Beschreibung der Indizes s. Abschnitt ,Definitionen und angewandte Methodik' für diesen Indikator).

Insgesamt erklären diese sieben Indikatoren des Schulklimas etwa 30 Prozent der Varianz der Leseleistung zwischen den Schulen und etwa 20 Prozent der Varianz zwischen den Ländern. Der Gesamteffekt der diesen Indikatoren zugrunde liegenden Faktoren und der durchschnittliche wirtschaftliche, soziale und kulturelle Status der Schülerpopulationen an den Schulen erklären etwa 70 Prozent derVarianz zwischen den Schulen und etwa 40 Prozent der Varianz zwischen den Ländern.

Die Mehrzahl der Länder mit Leistungen über dem Durchschnitt der OECD-Länder auf den drei PISA-Skalen haben bei fünf der sieben Indizes des Schulklimas und der Schulpraktiken hohe oder durchschnittliche Werte. Diese fünf Indikatoren sind die Indexmittel der Schulen der Nutzung der Bildungsressourcen, der Schulautonomie, der Stimmung und Arbeitshaltung der Lehrer, der Leistungsorientierung sowie der Lehrer-Schüler-Beziehung.

In Australien, Dänemark, Finnland, Island, Kanada, Neuseeland, Schweden, dem Vereinigten Königreich und den Vereinigten Staaten liegen diese fünf Indikatoren des Schulklimas über oder beim OECD-Mittelwert. Das gleichzeitige Wirken dieser fünf Faktoren steht in Zusammenhang mit einer Art der Schulpolitik, die durch beträchtliche Autonomie der Schulen und eine Ausrichtung eher auf die Lernerfolge als auf den Input der Schulen oder die Inhalte gekennzeichnet ist (Daten s. unter www.oecd.org/els/education/eag2002).

In Ländern, in denen die durchschnittliche Leistung der 15-Jährigen unterhalb des OECD-Durchschnitts liegt, liegen tendenziell auch über die Hälfte der Indizes des Schulklimas unter dem OECD-Durchschnitt. So sind beispielsweise in Deutschland und Griechenland alle Indizes bis auf einen negativ. In Italien, Luxemburg, Spanien und Portugal liegen fünf, und Japan, Mexiko und der Tschechischen Republik
In den meisten leistungsstarken Ländern nutzen 15-Jährige in der Regel die Schulressourcen tendenziell häufiger, haben die Schulen entsprechend einen höheren Grad an Autonomie, sind die Stimmung und Arbeitshaltung der Lehrkräfte sowie die Lehrer-SchülerBeziehung besser.

In Ländern mit unterdurchschnittlichen Leistungen treten negative Indizes des Schulklimas tendenziell häufiger auf,... 
...und die Indizes der schulressourcennutzung,

der Stimmung und

Arbeitshaltung

der Lehrkräfte, der

schulautonomie und

der Schüler-Lehrer-

Beziehung liegen dort tendenziell unterhalb des

OECD-Durchschnitts.

Die Faktoren des Schulklimas werden oft mit dem wirtschaftlichen, Status der Schüler einer schule in verbindung gebracht.

\section{D5} sozialen und kulturellen

vier der sieben Indizes unter dem OECD-Durchschnitt. Ausnahmen bilden hier Ungarn und Polen, wo sechs, bzw. vier der Indizes des Schulklimas über dem OECD-Durchschnitt liegen (Daten s. unter www.oecd.org/els/education/eag2002).

In der zuletzt genannten Gruppe von Ländern liegt auch der Index der Schulressourcennutzung signifikant unter dem OECD-Durchschnitt, die einzige Ausnahme bildet hier Portugal. Der Index der Schulautonomie ist in sieben der Länder negativ (mit Ausnahme von Polen, der Tschechischen Republik und Ungarn). In Deutschland, Griechenland, Portugal und Mexiko nennen die Schulleiter lehrkraftbezogene Probleme, die zur Beeinträchtigung des Lernens führen (obwohl die gleichen Schwierigkeiten auch in einigen der leistungsstarken Länder, wie Australien, Finnland, Irland, Kanada und Neuseeland angegeben werden). In Deutschland, Griechenland, Italien, Luxemburg, Polen und der Tschechischen Republik ist die Schüler-Lehrer-Beziehung schlechter als im OECD-Durchschnitt. In fünf Ländern dieser Gruppe (d.h. Italien, Polen, Portugal, Spanien und der Tschechischen Republik) schätzen die Schulleiter die Stimmung und Arbeitshaltung der Lehrkräfte als schlecht ein. In Belgien, Korea und den Vereinigten Staaten sind ähnliche Probleme zu beobachten, obwohl der Leistungsstand dieser Länder über oder auf dem OECD-Durchschnitt liegt (Daten s. unter www.oecd.org/els/education/eag2002).

Die PISA-Studie liefert Belege dafür, dass in den meisten Ländern ein Zusammenhang besteht zwischen den Indizes des Schulklimas und der sozioökonomischen Zusammensetzung der Schülerpopulation einer Schule (Daten s. unter www.oecd.org/els/education/eag2002). Der Zusammenhang zwischen sozio-ökonomischem Hintergrund und Schulklima kann in denjenigen Ländern entscheidend sein, die große Leistungsunterschiede bei den 15-Jährigen aufweisen und in denen ein substantieller Teil dieser Unterschiede auf Unterschiede zwischen den Schulen zurückzuführen ist. So liegt beispielsweise in Deutschland der Leistungsunterschied zwischen den Schulen deutlich über dem OECDDurchschnitt. Die signifikant positiven Korrelationen zwischen dem mittleren sozio-ökonomischen Status der Schüler einer Schule und den lehrerbezogenen Faktoren, die das Schulklima beeinflussen, die Schüler-Lehrer-Beziehung, der Autonomie der Schulen und der Disziplin weisen darauf hin, dass Schulen mit Schülern aus wohlhabenderen Familien tendenziell weniger Probleme mit der Disziplin von Schülern und Lehrern und eine bessere Schüler-Lehrer-Beziehung haben und über mehr Autonomie verfügen. Dies bedeutet aber auch, dass Schüler mit einem geringeren sozio-ökonomischen Status weniger häufig Schulen mit einem günstigeren Lernklima besuchen.

\section{Persönlicher Bezug zur Schule}

In der PISA-Studie gab die Hälfte der 15-Jährigen an, sich in der Schule oft zu langweilen, ...
Die Schule und die damit verbunden Arbeit nimmt einen großen Teil der Zeit 15-Jähriger in Anspruch. Gelingt es den Schulen, die Aufmerksamkeit der jungen Schüler trotz konkurrierender Reize zu fokussieren? Laut PISA-Studie langweilen sich im Durchschnitt 48 Prozent der 15-Jährigen oft in der Schule und 29 Prozent gaben an, dass die Schule ein Ort sei, an den sie nicht hingehen möchten. 
Gleichzeitig deutet die PISA-Studie darauf hin, dass die Schule im sozialen Leben der 15-Jährigen eine wichtige Rolle spielt, nicht zuletzt da die Schulen oft ein wichtiger Treffpunkt für Teenager sind. Im Rahmen der PISA-Studie wurden 15-Jährige u.a. gefragt, ob sie sich in der Schule als dazugehörig empfinden. Ihre Antworten, zusammengefasst in Tabelle D5.4, legen nahe, dass für die meisten 15-Jährigen die Schule ein Ort ist, an dem sie leicht Freunde finden (82 Prozent), an dem sie sich zugehörig fühlen (75 Prozent) und an dem sie anscheinend beliebt sind (77 Prozent). Gleichzeitig fühlen sich jedoch im OECD-Durchschnitt 9 Prozent der Schüler als Außenseiter, 14 Prozent fühlen sich unwohl und fehl am Platz, und 10 Prozent einsam (Abbildung D5.1 und Tabelle D5.4).

\section{Definitionen und angewandte Methodik}

Die PISA-Indizes dieses Abschnitts fassen die Antworten von Schülern und Schulleitern auf eine Reihe miteinander verknüpfter Fragen zusammen. Die Antworten der Schulleiter wurden so gewichtet, dass sie der Population der 15-Jährigen an ihren Schulen entsprechen. Die Fragen wurden auf der Basis theoretischer Überlegungen und vorangegangener Forschungen aus einem umfassenderen Konstrukt ausgewählt. Zur Bestätigung der theoretisch erwarteten Attribute der Indizes und zur Validierung ihrer Vergleichbarkeit zwischen den Ländern wurden Strukturgleichungsmodelle verwendet. Zu diesem Zweck wurde für jedes Land separat und für alle OECD-Länder insgesamt ein Modell geschätzt.

Es ist zu beachten, dass negative Werte bei einem Index nicht zwangsläufig auf negative Antworten der Schüler bzw. der Schulleiter auf die gestellten Fragen schließen lassen. Ein negativer Wert weist lediglich darauf hin, dass eine Gruppe von Schülern (oder alle Schüler in einem Land zusammengenommen) bzw. eine Gruppe von Schulleitern weniger positiv antwortet, als das beim Durchschnitt aller Schüler bzw. Schulleiter in den OECD-Ländern der Fall war. Dementsprechend bedeutet ein positiver Wert bei einem Index, dass eine Gruppe von Schülern oder Schulleitern positivere Antworten gab, als dies beim Durchschnitt der OECD-Länder der Fall war.

\section{Auf den Antworten der Schüler beruhende PISA-Indizes}

Der PISA-Index der Unterstützung durch die Lehrkräfte wurde abgeleitet von den Angaben der Schüler, wie oft es vorkomme, dass der Lehrer sich für den Lernfortschritt jedes einzelnen Schülers interessiert; den Schülern Gelegenheit gibt, ihre Meinung zu sagen; den Schülern bei der Arbeit hilft; etwas so lange erklärt, bis es die Schüler verstanden haben; viel tut, um den Schülern zu helfen und den Schülern beim Lernen hilft. Es wurde eine Vierpunkteskala mit folgenden Antwortkategorien verwendet: „nie“, „in einigen Stunden“, „in den meisten Stunden“, „,in jeder Stunde“.

Der PISA-Index der Schuldisziplin fasst die Angaben der Schüler zusammen, wie häufig in ihrem Unterricht der Landes- bzw. Unterrichtssprache Folgendes vorkommt: „Der Lehrer muß lange warten, bis Ruhe eintritt“; „die Schüler können nicht ungestört arbeiten“; „die Schüler hören nicht auf das, was der
... aber für die meisten

15-Jährigen ist die

Schule ein wichtiger

sozialer Raum, an

dem sie Kontakte mit

Gleichaltrigen knüpfen.

Die Ergebnisse für diesen

Indikator stammen aus

den Kontextfragebögen,

die 15-jährige Schüler

und ihre Schulleiter

als Teil der von der

OECD im Jahr 2000

durchgeführten

Internationalen

Schulleistungsstudie

PISA ausgefüllt haben.

Index der Unterstützung durch die Lehrkräfte

Index der Schuldisziplin 
Index der Leistungsorientierung

Index der

Schulressourcen-

nutzung

Index des Zeitaufwands für Hausaufgaben

Index der SchülerLehrer-Beziehung

Index des wirtschaftlichen, sozialen und kulturellen

Status (ESCS)
Lehrer sagt“; „die Schüler fangen erst lange nach Beginn der Stunde an zu arbeiten“; „es ist laut und alles geht durcheinander“ und „es vergehen zu Beginn der Stunde mehr als fünf Minuten, in denen gar nichts passiert“. Es wurde eine Vierpunkteskala mit folgenden Antwortkategorien verwendet: „nie“, „in einigen Stunden“, „in den meisten Stunden“, „in jeder Stunde“. Dieser Index wurde so umgepolt, dass niedrige Werte auf eine schlechte Schuldisziplin hinweisen.

Der PISA-Index der Leistungsorientierung wurde abgeleitet von den Angaben der Schüler, wie häufig in ihrem Unterricht der Landes- bzw. Unterrichtssprache Folgendes vorkommt: „der Lehrer will, dass sie sich richtig anstrengen“; „der Lehrer sagt ihnen, dass sie eigentlich besser sein könnten“; „der Lehrer ist unzufrieden, wenn sie nachlässig arbeiten“; „der Lehrer verlangt von ihnen, dass sie viel lernen“. Es wurde eine Vierpunkteskala mit folgenden Antwortkategorien verwendet: „nie“, „in einigen Stunden“, „in den meisten Stunden“, „in jeder Stunde“.

Der PISA-Index der Schulressourcennutzung wurde abgeleitet von den Angaben der Schüler, wie häufig sie folgende Ressourcen in ihren Schulen benutzen: die Schulbibliothek, einen Taschenrechner, das Internet und naturwissenschaftliche Labors. Die Schüler verwendeten hierfür eine Fünfpunkteskala mit folgenden Antwortkategorien: „nie oder fast nie“, , ein paar mal im Jahr“, „,ein paar Mal im Monat“, „mehrmals im Monat“ und „mehrmals in der Woche“. Der Index wurde mit Hilfe der WARM-Schätzfunktion konstruiert.

Der PISA-Index des Zeitaufwands für Hausaufgaben wurde abgeleitet von den Angaben der Schüler, wie häufig sie ihre Hausaufgaben erledigen: „nie“, „manchmal“, „meistens“ oder „immer“ und wieviel Zeit sie für die Hausaufgaben in den Fächern Unterrichtssprache, Mathematik und Naturwissenschaften brauchen (Die Antworten wurden auf einer Vierpunkteskala mit folgenden Antwortkategorien gegeben: „keine Zeit“, „weniger als 1 Stunde pro Woche“, „1 bis 3 Stunden in der Woche“, „3 und mehr Stunden in der Woche“). Hohe Werte bedeuten, dass die Schüler angaben, viel Zeit für die Hausaufgaben aufzuwenden und sie rechtzeitig fertigzustellen.

Der PISA-Index des Schüler-Lehrer-Beziehung wurde abgeleitet von den Angaben der Schüler, inwieweit sie den folgenden Angaben zustimmten: „Die Schüler kommen mit den meisten Lehrern gut aus“; „den meisten Lehrern ist es wichtig, dass die Schüler sich wohl fühlen“; „die meisten Lehrer interessieren sich für das, was ich zu sagen habe“; „wenn ich zusätzliche Hilfe brauche, bekomme ich sie von meinen Lehrern“ und „die meisten Lehrer behandeln mich fair“. Es wurde eine Vierpunkteskala mit folgenden Antwortkategorien verwendet: „stimme überhaupt nicht zu“, „stimme eher nicht zu“, „stimme eher zu“ und „stimme ganz zu“.

Um allgemeinere Aspekte des familiären und häuslichen Hintergrunds der Schüler zu erfassen, wurde der PISA-Index des wirtschaftlichen, sozialen und kulturellen Status auf der Basis der folgenden Variablen konstruiert: dem Internationalen sozio-ökonomischen Index der beruflichen Stellung der Eltern; 
dem höchsten Bildungsabschluss der Eltern des Schülers, umgerechnet in Schuljahre; dem PISA-Index des relativen Wohlstands der Familien; dem PISAIndex der Bildungsressourcen im Elternhaus und dem PISA-Index des Besitzes von „klassischen“ Kulturgütern im Elternhaus. Eine detaillierte Beschreibung dieser Variablen findet sich im PISA Technical Report unter www.pisa.oecd.org.

\section{Auf den Angaben der Schulleiter beruhende PISA-Indizes}

Der PISA-Index der lehrerbezogenen Faktoren für das Schulklima aus der Sicht der Schulleitung wurde abgeleitet von den Angaben der Schulleitung, wie sehr das Lernen der 15-Jährigen durch Folgendes beeinträchtigt wird: „niedrige Erwartungen der Lehrerkräfte“; „schlechtes Lehrer-Schüler-Verhältnis“; Lehrer gehen nicht auf die individuellen Bedürfnisse der Schüler ein“; „häufige Abwesenheit von Lehrkräften“; „Widerstand des Kollegiums gegen Veränderungen“; „Lehrkräfte sind mit den Schülern zu streng“; „Schüler werden nicht ermutigt, ihr Potenzial voll auszuschöpfen“. Eine Vierpunkteskala mit den Antwortkategorien „gar nicht“, „kaum“, „etwas“ und „sehr“ wurde verwendet. Dieser Index wurde so umgepolt, dass niedrige Werte auf eine schlechte Schuldisziplin hinweisen.

Der PISA-Index der Stimmung und Arbeitshaltung der Lehrkräfte aus der Sicht der Schulleitung wurde davon abgeleitet, inwieweit die Schulleiter folgenden Aussagen zustimmten: Stimmung und Arbeitshaltung der Lehrkräfte sind in dieser Schule gut; die Lehrkräfte arbeiten mit großem Engagement, die Lehrkräfte sind stolz auf diese Schule und die Lehrkräfte legen Wert auf schulische Leistung. Es wurde eine Vierpunkteskala mit folgenden Antwortkategorien verwendet: „stimme überhaupt nicht zu“, „stimme eher nicht zu“, „stimme eher zu“ und „stimme ganz zu“.

Die Schulleiter wurden gebeten anzugeben, ob die Lehrkräfte, Fachbereichsleiter, Schulleiter, ein ernanntes oder gewähltes Gremium oder Bildungsbehörden auf einer höheren Ebene die Hauptverantwortung für die folgenden Bereiche tragen: Einstellung von Lehrkräften, Entlassung von Lehrkräften, Festlegung des Anfangsgehalts der Lehrkräfte, Entscheidung über die Beförderung/Höhergruppierung von Lehrkräften, Festlegung des Schulbudgets, Entscheidung über die Verwendung des Budgets innerhalb der Schule, Festlegung von disziplinären Regeln für Schüler, Festlegung von Kriterien für die Schülerbeurteilung, Aufnahme von Schülern in die Schule, Wahl der verwendeten Lehrbücher, Bestimmung des Lehrstoffes und Entscheidung über das Fächer- und Kursangebot. Der PISA-Index der Schulautonomie wurde abgeleitet aus den Angaben der Schulleiter zu den betreffenden Bereichen, für die die Schule nicht die Hauptverantwortung trägt.
Index der

lehrerbezogenen

Faktoren für das

schulklima aus der sicht

der Schulleitung

Index der Stimmung und Arbeitshaltung der Lehrkräfte aus der Sicht der Schulleitung

Index der

Schulautonomie 
Tabelle D5.1.

Unterrichtsklima bei 15-jährigen Schülern (2000)

PISA-Indizes der Unterstützung durch die Lehrkräfte, der Schuldisziplin und derVeränderungen der PISA-Skala für Lesekompetenz

\begin{tabular}{|c|c|c|c|c|c|c|c|c|c|}
\hline & \multicolumn{7}{|c|}{ Unterstützung durch die Lehrkräfte } & \multirow{3}{*}{\begin{tabular}{|c|} 
PISA-Index der \\
Unterstützung durch \\
die Lehrkräfte $^{1}$ \\
\\
\end{tabular}} & \multirow{3}{*}{$\begin{array}{c}\text { Veränderung in } \\
\text { der PISA-Skala für } \\
\text { Lesekompetenz pro } \\
\text { Einheit des PISA-Index } \\
\text { der Unterstützung } \\
\text { durch die Lehrkräfte }\end{array}$} \\
\hline & \multicolumn{7}{|c|}{ Prozentsatz der Schüler, die angaben, dass die Lehrkraft in jeder oder fast jeder in der Landessprache abgehaltenen Unterrichtsstunde ... } & & \\
\hline & $\begin{array}{c}\text { sich für den } \\
\text { Lernfortschritt } \\
\text { jedes Einzelnen } \\
\text { interessiert }\end{array}$ & $\begin{array}{l}\text { ihnen Gelegen- } \\
\text { heit gibt, ihre } \\
\text { Meinung zu } \\
\text { sagen }\end{array}$ & $\begin{array}{c}\text { ihnen bei ihrer } \\
\text { Arbeit hilft }\end{array}$ & $\begin{array}{c}\text { etwas so lange } \\
\text { erklärt, bis sie } \\
\text { es verstanden } \\
\text { haben }\end{array}$ & $\begin{array}{c}\text { viel tut, um } \\
\text { ihnen zu helfen }\end{array}$ & $\begin{array}{l}\text { ihnen beim } \\
\text { Lernen hilft }\end{array}$ & $\begin{array}{l}\text { die Hausaufga- } \\
\text { ben überprüft }\end{array}$ & & \\
\hline Australien & 72 & 77 & 80 & 72 & 70 & 77 & 49 & 0.41 & 7.37 \\
\hline Österreich & 48 & 67 & 54 & 56 & 52 & 32 & 64 & -0.25 & -0.62 \\
\hline Belgien & 42 & 56 & 44 & 58 & 54 & 37 & 60 & -0.28 & -4.83 \\
\hline Kanada & 69 & 73 & 76 & 68 & 67 & 75 & 49 & 0.31 & 4.42 \\
\hline Tschechische Rep. & 47 & 57 & 32 & 41 & 51 & 24 & 47 & -0.50 & 0.77 \\
\hline Dänemark & 62 & 76 & 74 & 67 & 66 & 67 & 49 & 0.17 & 11.65 \\
\hline Finnland & 52 & 72 & 72 & 59 & 57 & 66 & 42 & 0.02 & 5.48 \\
\hline Frankreich & 55 & 61 & 50 & 57 & 48 & 42 & 44 & -0.20 & -2.53 \\
\hline Deutschland & 41 & 62 & 52 & 53 & 51 & 34 & 61 & -0.34 & -12.55 \\
\hline Griechenland & 69 & 77 & 41 & 62 & 65 & 69 & 53 & 0.14 & 2.20 \\
\hline Ungarn & 58 & 71 & 73 & 58 & 67 & 51 & 52 & 0.05 & -2.43 \\
\hline Island & 53 & 51 & 79 & 72 & 69 & 75 & 61 & 0.13 & 8.87 \\
\hline Irland & 70 & 66 & 62 & 64 & 64 & 61 & 63 & 0.13 & -0.13 \\
\hline Italien & 22 & 72 & 50 & 63 & 61 & 41 & 50 & -0.28 & -11.46 \\
\hline Japan & 48 & 58 & 61 & 48 & 48 & 51 & 35 & -0.17 & 6.23 \\
\hline Korea & 31 & 43 & 17 & 41 & 49 & 41 & 54 & -0.67 & 5.56 \\
\hline Luxemburg & 45 & 57 & 46 & 56 & 49 & 32 & 51 & -0.34 & -5.13 \\
\hline Mexiko & 70 & 76 & 36 & 62 & 53 & 61 & 76 & 0.07 & -2.60 \\
\hline Neuseeland & 69 & 73 & 79 & 67 & 69 & 76 & 56 & 0.34 & 5.26 \\
\hline Norwegen & 48 & 62 & 69 & 59 & 60 & 70 & 41 & -0.03 & 14.95 \\
\hline Polen & 38 & 60 & 41 & 43 & 44 & 35 & 46 & -0.39 & 9.20 \\
\hline Portugal & 83 & 77 & 71 & 68 & 70 & 79 & 51 & 0.47 & -1.33 \\
\hline Spanien & 62 & 63 & 59 & 66 & 57 & 63 & 68 & 0.09 & 2.53 \\
\hline Schweden & 64 & 71 & 74 & 69 & 70 & 76 & 51 & 0.21 & 6.20 \\
\hline Schweiz & 56 & 68 & 66 & 66 & 63 & 47 & 56 & 0.01 & -13.40 \\
\hline Ver. Königreich & 75 & 76 & 80 & 75 & 75 & 79 & 69 & 0.50 & 6.66 \\
\hline Vereinigte Staaten & 66 & 66 & 70 & 63 & 63 & 69 & 65 & 0.34 & 6.87 \\
\hline OECD Insgesamt & 56 & 65 & 58 & 59 & 58 & 57 & 57 & 0.00 & 2.82 \\
\hline Ländermittel & 56 & 66 & 59 & 60 & 60 & 56 & 54 & 0.02 & 2.96 \\
\hline Brasilien & 73 & 70 & 62 & 69 & 74 & 75 & 49 & 0.38 & 4.60 \\
\hline Lettland & 40 & 60 & 56 & 54 & 53 & 53 & 59 & -0.20 & 15.56 \\
\hline Liechtenstein & 51 & 69 & 72 & 70 & 65 & 42 & 65 & 0.09 & -14.19 \\
\hline Russische Föd. & 55 & 69 & 65 & 61 & 65 & 70 & 64 & 0.16 & 6.40 \\
\hline \multirow[t]{4}{*}{ Niederlande $^{3}$} & 38 & 57 & 67 & 65 & 57 & 39 & 30 & -0.21 & -5.54 \\
\hline & \multicolumn{6}{|c|}{ Schuldisziplin } & & & \\
\hline & Prozentsatz der Schüle & er, die angaben, dass in & a jeder oder fast jeder & in der Landespra & che abgehaltenen Un & terrichtsstunde ... & $\begin{array}{l}\text { PISA-Index der } \\
\text { Schuldisziplin }\end{array}$ & Veränderung in & \\
\hline & $\begin{array}{c}\text { die Lehrkraft lange } \\
\text { warten muss, bis } \\
\text { Ruhe eintritt }\end{array}$ & $\begin{array}{l}\text { sie selbst nicht } \\
\text { ungestört arbei- } \\
\text { ten können }\end{array}$ & $\begin{array}{l}\text { sie nicht darauf } \\
\text { hören, was die } \\
\text { Lehrkraft sagt }\end{array}$ & $\begin{array}{l}\text { sie erst lange } \\
\text { nach Beginn } \\
\text { der Stunde } \\
\text { anfangen zu } \\
\text { arbeiten } \\
\end{array}$ & $\begin{array}{l}\text { es laut ist und } \\
\text { alles durchein- } \\
\text { ander geht }\end{array}$ & $\begin{array}{c}\text { mehr als } 5 \\
\text { Minuten verge- } \\
\text { hen, in denen } \\
\text { gar nichts } \\
\text { passiert }\end{array}$ & Indexmittel & $\begin{array}{c}\text { Lesekompetenz } \\
\text { pro Einheit des } \\
\text { PISA-Index der } \\
\begin{array}{c}\text { Unterstützung durch } \\
\text { die Lehrkräfte }\end{array} \\
\end{array}$ & \\
\hline Australien & 31 & 18 & 21 & 26 & 32 & 42 & -0.09 & 16.69 & \\
\hline Österreich & 32 & 20 & 21 & 29 & 19 & 38 & 0.19 & 4.98 & \\
\hline Belgien & 35 & 14 & 23 & 30 & 36 & 51 & -0.12 & 3.15 & \\
\hline Kanada & 35 & 16 & 23 & 29 & 33 & 46 & -0.14 & 13.28 & \\
\hline Tschechische Rep. & 32 & 17 & 26 & 21 & 26 & 27 & 0.14 & 12.37 & \\
\hline Dänemark & 27 & 17 & 19 & 22 & 33 & 55 & -0.20 & 9.71 & \\
\hline Finnland & 39 & 15 & 29 & 21 & 42 & 44 & -0.16 & 9.56 & \\
\hline Frankreich & 35 & 15 & 27 & 36 & 42 & 38 & -0.05 & 1.53 & \\
\hline Deutschland & 36 & 23 & 24 & 27 & 22 & 34 & 0.10 & 10.13 & \\
\hline Griechenland & 43 & 39 & 29 & 34 & 46 & 58 & -0.42 & 2.96 & \\
\hline Ungarn & 34 & 25 & 22 & 16 & 23 & 16 & 0.23 & 16.05 & \\
\hline Island & 34 & 16 & 20 & 20 & 28 & 50 & -0.08 & 8.90 & \\
\hline Irland & 29 & 16 & 25 & 25 & 26 & 41 & 0.09 & 15.41 & \\
\hline Italien & 48 & 22 & 35 & 29 & 46 & 49 & -0.24 & 14.11 & \\
\hline Japan & 9 & 20 & 16 & 17 & 17 & 25 & 0.49 & 17.15 & \\
\hline Korea & 17 & 21 & 32 & 23 & 29 & 32 & 0.20 & 6.88 & \\
\hline Luxemburg & 31 & 21 & 24 & 26 & 27 & 36 & 0.12 & 2.41 & \\
\hline Mexiko & 28 & 17 & 19 & 19 & 24 & 23 & 0.17 & 2.03 & \\
\hline Neuseeland & 33 & 22 & 23 & 26 & 32 & 46 & -0.15 & 12.47 & \\
\hline Norwegen & 42 & 23 & 27 & 33 & 39 & 56 & -0.36 & 7.79 & \\
\hline Polen & 26 & 13 & 19 & 19 & 18 & 27 & 0.37 & 20.88 & \\
\hline Portugal & 25 & 19 & 20 & 24 & 24 & 42 & -0.05 & 10.57 & \\
\hline Spanien & 41 & 18 & 24 & 35 & 34 & 48 & -0.17 & 12.18 & \\
\hline Schweden & 43 & 23 & 29 & 31 & 38 & 30 & -0.19 & 12.44 & \\
\hline Schweiz & 27 & 18 & 18 & 23 & 18 & 27 & 0.30 & 9.81 & \\
\hline Ver. Königreich & 31 & 17 & 20 & 23 & 27 & 41 & 0.02 & 20.10 & \\
\hline Vereinigte Staaten & 26 & 17 & 24 & 23 & 28 & 37 & 0.03 & 13.17 & \\
\hline OECD Insgesamt & 28 & 18 & 23 & 24 & 28 & 35 & 0.09 & 11.99 & \\
\hline Ländermittel & 32 & 19 & 24 & 25 & 30 & 39 & 0.00 & 9.45 & \\
\hline Brasilien & 36 & 24 & 28 & 38 & 40 & 50 & -0.34 & -5.95 & \\
\hline Lettland & 19 & 17 & 19 & 16 & 17 & 21 & 0.38 & 9.04 & \\
\hline Liechtenstein & 25 & 21 & 15 & 15 & 10 & 21 & 0.35 & -2.59 & \\
\hline Russische Föd. & 19 & 16 & 16 & 13 & 12 & 27 & 0.45 & 10.06 & \\
\hline Niederlande $^{3}$ & 39 & 16 & 21 & 37 & 39 & 69 & -0.33 & 2.63 & \\
\hline
\end{tabular}

1. Definitionen der Indizes s. Abschnitt Definitionen und angewandte Methodik dieses Indikators.

2. Fettgedruckte Veränderungen der Indexeinheiten sind statistisch signifikant.

3. Die Beteiligungsquote ist zu niedrig, um die Vergleichbarkeit zu gewährleisten (s. Anhang 3, www.oecd.org/els/education/eag2002).

Quelle: OECD PISA-Datenbank, 2001. Hinweise zur Methodik s. Anhang 3 (www.oecd.org/els/education/eag2002) und www.pisa.oecd.org. 
Tabelle D5.2.

Hausaufgabenregelung und Leistungsorientierung bei 15-jährigen Schülern (2000)

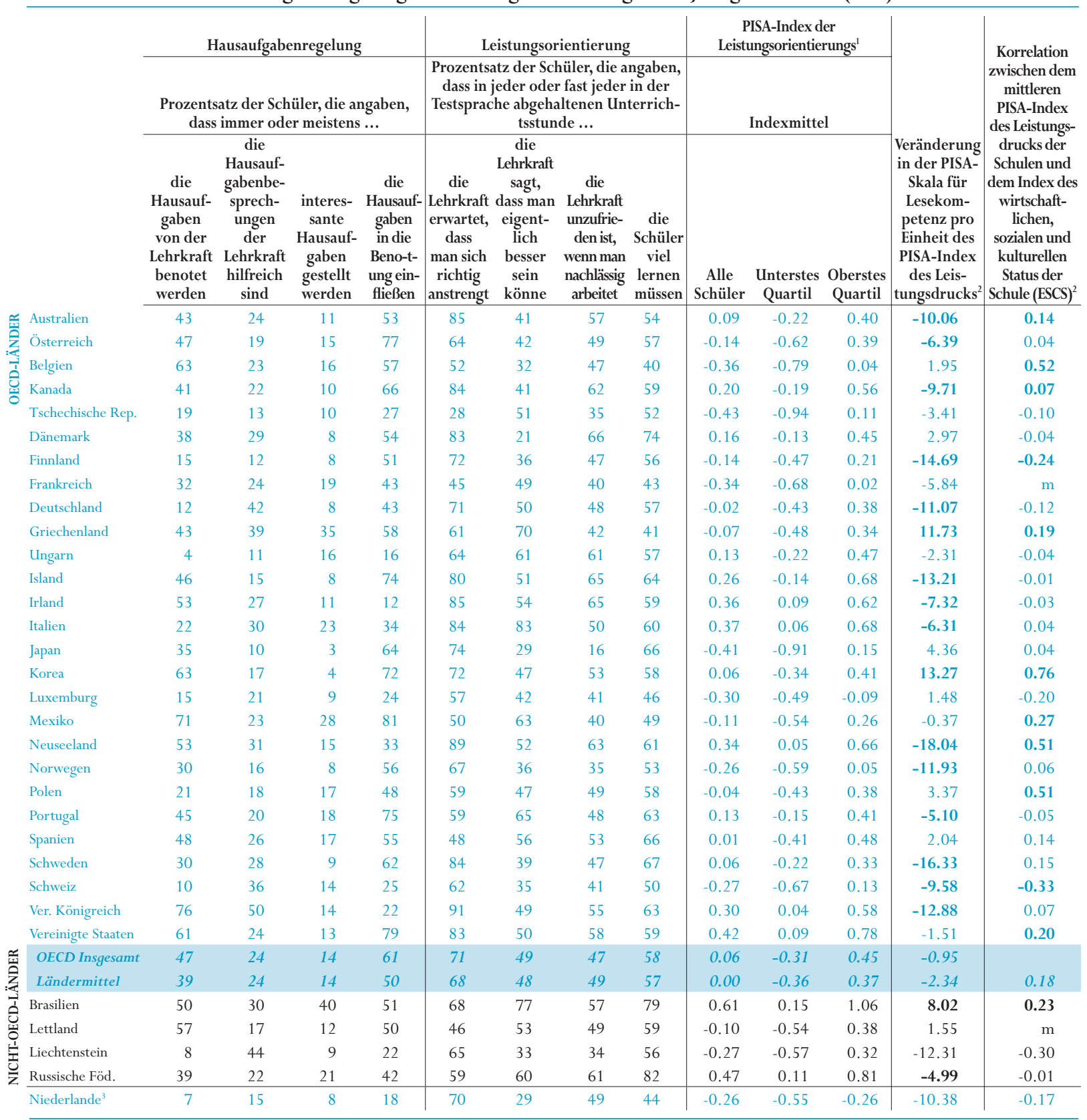

1. Definitionen der Indizes s. Abschnitt Definitionen und angewandte Methodik dieses Indikators.

2. Fettgedruckte Veränderungen der Indexeinheiten und Korrelationen sind statistisch signifikant.

3. Die Beteiligungsquote ist zu niedrig, um die Vergleichbarkeit zu gewährleisten (s. Anhang 3, www.oecd.org/els/education/eag2002) und www.pisa.oecd.org. Quelle: OECD PISA-Datenbank, 2001. Hinweise zur Methodik s. Anhang 3 (www.oecd.org/els/education/eag2002) und www.pisa.oecd.org. 
Tabelle D5.3.

Qualität und Nutzung der Schulressourcen für 15-Jährige (2000)

\begin{tabular}{|c|c|c|c|c|c|c|c|c|c|c|c|}
\hline & \multicolumn{3}{|c|}{$\begin{array}{l}\text { Qualität und Nutzung der } \\
\text { Schulbibliothek }\end{array}$} & \multicolumn{3}{|c|}{$\begin{array}{l}\text { Verfügbarkeit und Nutzung } \\
\text { von Computern }\end{array}$} & \multicolumn{3}{|c|}{$\begin{array}{l}\text { Qualität und Nutzung } \\
\text { der Laborausstattung }\end{array}$} & \multirow[t]{3}{*}{$\left|\begin{array}{c}\text { PISA-Index } \\
\text { der Schul- } \\
\text { ressourcen-- } \\
\text { nutzung }^{2}\end{array}\right|$} & \multirow[b]{3}{*}{$\begin{array}{l}\text { Veränder- } \\
\text { ung in der } \\
\text { PISA- } \\
\text { Skala für } \\
\text { Lesekom- } \\
\text { petenz pro } \\
\text { Einheit } \\
\text { des PISA- } \\
\text { Index der } \\
\text { Schulres- } \\
\text { sourcen- } \\
\text { nutzung }\end{array}$} \\
\hline & \multirow{2}{*}{$\begin{array}{c}\text { Prozentsatz } \\
\text { der Schüler, } \\
\text { die Schulen } \\
\text { besuchen, deren } \\
\text { Schulleiter } \\
\text { angab, dass das } \\
\text { Lernen etwas } \\
\text { oder sehr durch } \\
\text { den Mangel an } \\
\text { Unterrichts- } \\
\text { materialien in } \\
\text { der Bibliothek } \\
\text { beeinträchtigt } \\
\text { wird }^{1} \\
\end{array}$} & \multicolumn{2}{|c|}{$\begin{array}{c}\text { Prozentsatz der } \\
\text { Schüler, die anga- } \\
\text { ben, }{ }^{2} . .\end{array}$} & \multirow{2}{*}{\begin{tabular}{|c|} 
Prozentsatz \\
der Schüler, \\
die Schulen \\
besuchen, \\
deren Schul- \\
leiter angab, \\
dass das Lernen \\
etwas oder \\
sehr durch eine \\
ungenügende \\
Zahl an \\
Computern \\
beeinträchtigt \\
wird $^{1}$
\end{tabular}} & \multicolumn{2}{|c|}{$\begin{array}{c}\text { Prozentsatz der } \\
\text { Schüler, die anga- } \\
\text { ben, }{ }^{2} . .\end{array}$} & \multirow{2}{*}{\begin{tabular}{|c|} 
Prozentsatz \\
der Schüler, \\
die Schulen \\
besuchen, \\
deren Schul- \\
leiter angab, \\
dass das \\
Lernen etwas \\
oder sehr \\
durch eine \\
unzureichende \\
Laborausstat- \\
tung beein- \\
trächtigt wird \\
\end{tabular}} & \multirow{2}{*}{\multicolumn{2}{|c|}{\begin{tabular}{|cc}
\multicolumn{2}{|c|}{ Prozentsatz der } \\
Schüler, die anga- \\
ben, ${ }^{2} \ldots$ \\
& das natur- \\
nie das & wissen- \\
natur- & schaftli- \\
wissen- & che Labor \\
schaftli- & mind- \\
che & estens \\
Labor mehrmals \\
zu im Monat \\
benut- zu benut- \\
zen & zen
\end{tabular}}} & & \\
\hline & & $\begin{array}{c} \\
\text { nie die } \\
\text { Biblio- } \\
\text { thek zu } \\
\text { benutzen }\end{array}$ & $\begin{array}{c}\text { die Bib- } \\
\text { liothek } \\
\text { mind- } \\
\text { estens } \\
\text { mehrmals } \\
\text { pro } \\
\text { Monat zu } \\
\text { benutzen }\end{array}$ & & $\begin{array}{l} \\
\text { in der } \\
\text { Schule } \\
\text { nie } \\
\text { Computer } \\
\text { zu } \\
\text { benutzen }\end{array}$ & $\begin{array}{c}\text { min- } \\
\text { destens } \\
\text { mehrmals } \\
\text { pro } \\
\text { Monat } \\
\text { Computer } \\
\text { in der } \\
\text { Schule zu } \\
\text { benutzen }\end{array}$ & & & & & \\
\hline Australien & 17 & 11 & 35 & 30 & 8 & 60 & 16 & 16 & 61 & 1.27 & 11.98 \\
\hline Österreich & 22 & 67 & 4 & 38 & 15 & 68 & 34 & 57 & 25 & 0.16 & 28.06 \\
\hline Belgien & 24 & 69 & 4 & 19 & 27 & 48 & 19 & 43 & 27 & -0.57 & 49.50 \\
\hline Kanada & 20 & 19 & 22 & 31 & 14 & 50 & 19 & 20 & 44 & 0.73 & 8.44 \\
\hline Tschechische Rep. & 25 & 74 & 4 & 37 & 33 & 47 & 22 & 62 & 17 & -0.76 & 34.30 \\
\hline Dänemark & 14 & 14 & 43 & 28 & 2 & 79 & 21 & 11 & 77 & 1.47 & 10.35 \\
\hline Finnland & 43 & 53 & 6 & 43 & 6 & 64 & 43 & 67 & 9 & 0.02 & 3.75 \\
\hline Frankreich & 15 & 31 & 20 & 28 & 35 & 22 & 13 & 43 & 33 & $\mathrm{~m}$ & $\mathrm{~m}$ \\
\hline Deutschland & 35 & 73 & 10 & 50 & 38 & 32 & 32 & 41 & 37 & -0.29 & -0.94 \\
\hline Griechenland & 50 & 60 & 6 & 68 & 31 & 37 & 64 & 50 & 14 & -1.00 & -14.67 \\
\hline Ungarn & 12 & 42 & 11 & 13 & 15 & 72 & 27 & 70 & 8 & -0.30 & 31.51 \\
\hline Island & 37 & 29 & 21 & 45 & 10 & 66 & 53 & 47 & 23 & 0.54 & 2.95 \\
\hline Irland & 48 & 69 & 7 & 41 & 35 & 42 & 42 & 22 & 59 & -0.38 & 16.24 \\
\hline Italien & 34 & 71 & 3 & 32 & 26 & 53 & 37 & 51 & 21 & -0.78 & 7.48 \\
\hline Japan & 24 & 50 & 16 & 31 & 60 & 22 & 34 & 50 & 19 & -1.17 & 12.43 \\
\hline Korea & 35 & 65 & 13 & 24 & 30 & 57 & 28 & 25 & 40 & -0.76 & -2.80 \\
\hline Luxemburg & 16 & 51 & 11 & 23 & 22 & 49 & 14 & 55 & 24 & -0.40 & -65.96 \\
\hline Mexiko & 60 & 16 & 33 & 68 & 49 & 30 & 66 & 33 & 35 & -0.55 & 33.89 \\
\hline Neuseeland & 11 & 10 & 32 & 40 & 16 & 41 & 19 & 15 & 65 & 0.75 & 14.53 \\
\hline Norwegen & 59 & 20 & 22 & 61 & 11 & 44 & 49 & 10 & 62 & 0.86 & 5.52 \\
\hline Polen & 32 & 19 & 21 & 39 & 27 & 55 & 43 & 37 & 33 & -0.18 & 53.20 \\
\hline Portugal & 17 & 16 & 38 & 39 & 30 & 33 & 23 & 47 & 33 & -0.09 & -3.57 \\
\hline Spanien & 27 & 44 & 14 & 30 & 40 & 37 & 32 & 37 & 29 & -0.53 & 9.46 \\
\hline Schweden & 27 & 15 & 36 & 51 & 10 & 60 & 16 & 6 & 83 & 1.38 & 3.60 \\
\hline Schweiz & 15 & 46 & 12 & 23 & 23 & 43 & 16 & 44 & 29 & -0.13 & 22.42 \\
\hline Ver. Königreich & 38 & 31 & 18 & 56 & 9 & 63 & 42 & 7 & 67 & 0.70 & 14.04 \\
\hline Vereinigte Staaten & 21 & 21 & 20 & 31 & 12 & 44 & 23 & 23 & 34 & 0.38 & 39.74 \\
\hline OECD Insgesamt & 29 & 36 & 18 & 38 & 29 & 41 & 32 & 33 & 34 & & \\
\hline Ländermittel & 30 & 39 & 18 & 38 & 24 & 49 & 32 & 37 & 37 & 0.00 & 16.18 \\
\hline Brasilien & 36 & 21 & 28 & 63 & 61 & 14 & 55 & 57 & 11 & -1.25 & 20.00 \\
\hline Lettland & 47 & 14 & 28 & 40 & 30 & 45 & 60 & 50 & 18 & $\mathrm{~m}$ & $\mathrm{~m}$ \\
\hline Liechtenstein & 21 & 43 & 9 & 41 & 12 & 57 & 58 & 33 & 41 & 0.40 & $\mathrm{~m}$ \\
\hline Russische Föd. & 78 & 12 & 42 & 86 & 52 & 32 & 79 & 17 & 50 & -0.18 & 34.20 \\
\hline Niederlande $^{4}$ & 31 & 48 & 18 & 39 & 28 & 44 & 20 & 65 & 11 & -0.34 & 52.39 \\
\hline
\end{tabular}

1. Basierend auf Angaben der Schulleiter.

2. Basierend auf Angaben Schüler. Erläuterung des Index der Schulressourcennutzung s. Abschnitt Definitionen und angewandte Methodik dieses Indikators.

3. Fettgedruckte Veränderungen der Indexeinheiten sind statistisch signifikant.

4. Die Beteiligungsquote ist zu niedrig, um die Vergleichbarkeit zu gewährleisten (s. Anhang 3, www.oecd.org/els/education/eag2002).

Quelle: OECD PISA-Datenbank, 2001. Hinweise zur Methodik s. Anhang 3 (www.oecd.org/els/education/eag2002) und www.pisa.oecd.org. 
Tabelle D5.4.

Persönlicher Bezug 15-jähriger Schüler zu ihrer Schule (2000)

Prozentsatz 15-jähriger Schüler, die hinsichtlich ihres persönlichen Bezugs zu ihrer Schule angaben, das die folgenden Aussagen eher oder ganz genau stimmen

\begin{tabular}{|c|c|c|c|c|c|c|c|c|}
\hline \multirow[b]{3}{*}{ Australien } & \multicolumn{8}{|c|}{$\begin{array}{l}\text { Prozentsatz der Schüler, die angaben, dass die folgenden Aussagen eher oder ganz genau stimmen: } \\
\text { "Meine Schule ist ein Ort,..." }\end{array}$} \\
\hline & $\begin{array}{c}\text { an dem ich mich } \\
\text { als Außenseiter } \\
\text { fühle }\end{array}$ & $\begin{array}{c}\text { an dem ich } \\
\text { leicht Freunde } \\
\text { finde }\end{array}$ & $\begin{array}{l}\text { an dem ich } \\
\text { mich zuge- } \\
\text { hörig fühle }\end{array}$ & $\begin{array}{l}\text { an dem ich } \\
\text { mich unwohl } \\
\text { und fehl am } \\
\text { Platz fühle }\end{array}$ & $\begin{array}{l}\text { an dem ich } \\
\text { anscheinend } \\
\text { beliebt bin }\end{array}$ & $\begin{array}{l}\text { an dem ich } \\
\text { mich einsam } \\
\text { fühle }\end{array}$ & $\begin{array}{l}\text { an den } \\
\text { ich nicht } \\
\text { hingehen } \\
\text { möchte }\end{array}$ & $\begin{array}{c}\text { an dem ich } \\
\text { mich oft } \\
\text { langweile }\end{array}$ \\
\hline & 9 & 89 & 85 & 11 & 92 & 8 & 34 & 60 \\
\hline Österreich & 7 & 86 & 85 & 18 & 72 & 10 & 29 & 49 \\
\hline Belgien & 10 & 86 & 53 & 19 & 87 & 7 & 42 & 46 \\
\hline Kanada & 9 & 89 & 81 & 12 & 93 & 9 & 37 & 58 \\
\hline Tschechische Rep. & 11 & 86 & 73 & 9 & 84 & 9 & 29 & 47 \\
\hline Dänemark & 6 & 85 & 84 & 10 & 75 & 10 & 19 & 41 \\
\hline Finnland & 6 & 84 & 86 & 11 & 84 & 8 & 26 & 60 \\
\hline Frankreich & 8 & 87 & 44 & 16 & 86 & 7 & 37 & 32 \\
\hline Deutschland & 6 & 81 & 82 & 15 & 67 & 8 & 25 & 49 \\
\hline Griechenland & 10 & 88 & 83 & 13 & 85 & 10 & 22 & 66 \\
\hline Ungarn & 10 & 87 & 89 & 9 & 85 & 9 & 38 & 29 \\
\hline Island & 10 & 81 & 85 & 11 & 86 & 10 & 23 & 30 \\
\hline Irland & 7 & 89 & 83 & 9 & 93 & 8 & 34 & 67 \\
\hline Italien & 7 & 90 & 73 & 9 & 88 & 16 & 38 & 54 \\
\hline Japan & 6 & 70 & 76 & 22 & 62 & 9 & 25 & 32 \\
\hline Korea & 15 & 83 & 68 & 15 & 42 & 10 & 30 & 46 \\
\hline Luxemburg & 9 & 84 & 72 & 19 & 68 & 11 & 30 & 50 \\
\hline Mexiko & 10 & 84 & 88 & 11 & 76 & 12 & 9 & 28 \\
\hline Neuseeland & 9 & 90 & 83 & 12 & 91 & 9 & 34 & 60 \\
\hline Norwegen & 6 & 85 & 79 & 10 & 86 & 8 & 23 & 58 \\
\hline Polen & 10 & 78 & 63 & 14 & 53 & 12 & 28 & 38 \\
\hline Portugal & 8 & 91 & 83 & 19 & 90 & 8 & 13 & 24 \\
\hline Spanien & 5 & 90 & 52 & 12 & 89 & 7 & 30 & 66 \\
\hline Schweden & 6 & 87 & 78 & 7 & 88 & 9 & 20 & 58 \\
\hline Schweiz & 7 & 85 & 76 & 14 & 74 & 8 & 28 & 38 \\
\hline Ver. Königreich & 7 & 91 & 83 & 9 & 93 & 6 & 28 & 54 \\
\hline Vereinigte Staaten & 11 & 81 & 78 & 14 & 83 & 12 & 35 & 61 \\
\hline OECD Insgesamt & 9 & 82 & 75 & 14 & 77 & 10 & 29 & 48 \\
\hline Ländermittel & 8 & 85 & 77 & 13 & 80 & 9 & 28 & 48 \\
\hline Brasilien & 5 & 89 & 86 & 10 & 88 & 8 & 20 & 30 \\
\hline Lettland & 9 & 79 & 86 & 15 & 63 & 14 & 18 & 31 \\
\hline Liechtenstein & 9 & 83 & 83 & 17 & 69 & 9 & 31 & 47 \\
\hline Russische Föd. & 6 & 82 & 86 & 13 & 50 & 10 & 17 & 27 \\
\hline Niederlande $^{1}$ & 5 & 89 & 76 & 10 & 91 & 4 & 100 & 38 \\
\hline
\end{tabular}

1. Die Beteiligungsquote ist zu niedrig, um die Vergleichbarkeit zu gewährleisten (s. Anhang 3, www.oecd.org/els/education/eag2002).

Quelle: OECD PISA-Datenbank, 2001. Hinweise zur Methodik s. Anhang 3 (www.oecd.org/els/education/eag2002) und www.pisa.oecd.org. 


\section{LEHRERGEHÄLTER AN ÖFFENTLICHEN SCHULEN DES PRIMAR- UND SEKUNDARBEREICHS}

- Die Gehälter von Lehrern des Sekundarbereich I nach 15 Jahren Berufserfahrung reichen von weniger als 10.000 US-Dollar in Ungarn und der Tschechischen Republik bis zu 40.000 US-Dollar und mehr in Deutschland, Japan, Korea, der Schweiz und den Vereinigten Staaten. Einige Länder investieren trotz eines geringeren Volkseinkommens massiv in die Humanressourcen.

- Das Gehalt je Unterrichtsstunde eines Lehrers im Sekundarbereich II liegt im Durchschnitt um 42 Prozent über dem Gehalt eines Lehrers im Primarbereich, aber die Differenz bei den Gehältern je Unterrichtsstunde zwischen diesen beiden Bildungsbereichen liegt in Australien, Neuseeland und dem Vereinigten Königreich bei unter 10 Prozent, während sie in Spanien und der Schweiz bei über 80 Prozent liegt.

- Im Durchschnitt der OECD-Länder sind die Lehrergehälter zwischen 1995 und 2000 in fast allen OECD-Ländern weniger stark als das BIP gewachsen.

- Lehrer in Australien, Dänemark, England, Neuseeland und Schottland erreichen die oberste Besoldungs/Vergütungsstufe in höchstens 11 Jahren, während Lehrer in Frankreich, Griechenland, Italien, Japan, Korea, Österreich, Spanien, der Tschechischen Republik und Ungarn mehr als 30 Dienstjahre benötigen.

- In etwa der Hälfte der OECD-Länder sind die Schulen zumindest in gewissem Ausmaß für Entscheidungen über Höhe und Ausmaß der Zulagen für zusätzlich übernommene Aufgaben und Überstunden zuständig. 


\section{Abbildung D6.1.}

Lehrergehälter im Sekundarbereich I (2000)

Gesetzliches bzw. vertraglich vereinbartes Jahresgehalt von Lehrern an öffentlichen Bildungseinrichtungen des Sekundarbereich I, in US-Dollar (kaufkraftbereinigt), sowie Verhältnis des

Gehalts nach 15 Jahren Berufserfahrung zum BIP pro Kopf

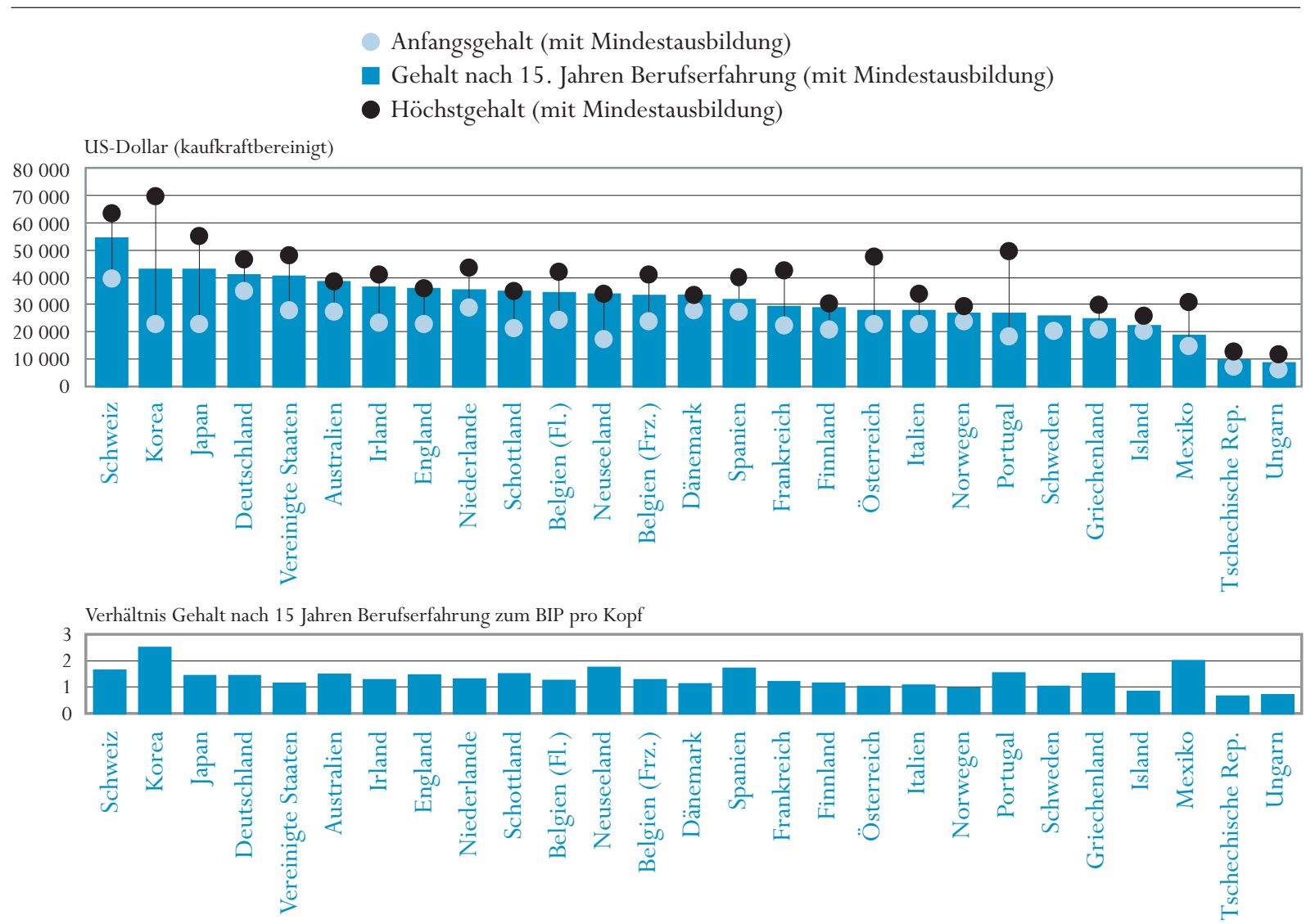

Anordnung der Länder in absteigender Reihenfolge des Lehrergehalts nach 15 Jahren Berufserfahrung (mit Mindestqualifikation) im Sekundarbereich $I$.

Quelle: OECD. Tabelle D6.1. Hinweise s. Anhang 3 (www.oecd.org/els/education/eag2002). 
Dieser Indikator beschreibt das Anfangs-, das mittlere und das Höchstgehalt von Lehrern an öffentlichen Schulen des Primarund Sekundarbereichs sowie die Anreizsysteme der Besoldungs- und Vergütungsordnungen für Lehrer.

\section{Politischer Hintergrund}

Im Bildungssystem ist eine große Anzahl von qualifizierten Kräften unter zunehmend wettbewerbsorientierten Marktbedingungen beschäftigt und allen OECD-Ländern ist es ein großes Anliegen, dafür Sorge zu tragen, dass es genug qualifizierte Lehrer gibt. Das Angebot an qualifizierten Lehrkräften wird hauptsächlich bestimmt durch die Gehälter und Arbeitsbedingungen von Lehrern, einschließlich dem Anfangsgehalt und dem System der Besoldungsund Vergütungsgruppen, sowie die dem Einzelnen während der Ausbildung zum Lehrer entstehenden Kosten im Vergleich zu den Gehältern und Kosten für andere hochqualifizierte Berufe. All dies beeinflusst die beruflichen Entscheidungen potentieller Lehrer und derjenigen, die sich für den Lehrerberuf interessieren.

Gleichzeitig sind die Lehrergehälter der größte Einzelposten, wenn es um die Kosten der Bildung geht. Die Gehälter der Lehrer sind daher für die politischen Entscheidungsträger, die die Qualität des Unterrichts und einen ausgeglichenen Bildungsetat aufrechterhalten wollen, ein entscheidender Faktor. Die Höhe der Bildungsetats spiegelt das Ausbalancieren einer ganzen Reihe miteinander in Zusammenhang stehender Faktoren wider. Hierzu gehören die Lehrergehälter, das zahlenmäßige Schüler/Lehrer-Verhältnis, der Umfang der geplanten Unterrichtszeit für Schüler und die vorgesehene Anzahl der Unterrichtsstunden der Lehrer.

\section{Ergebnisse und Erläuterungen}

Vergleich der Lehrergehälter

Der erste Teil dieses Indikators vergleicht die Höhe des gesetzlichen bzw. vertraglich vereinbarten Anfangsgehalts, des mittleren und des Höchstgehalts von Lehrern mit der Mindestqualifikation zum Unterrichten an öffentlichen Schulen des Primar- und Sekundarbereichs. Zunächst wird die absolute Höhe des gesetzlichen bzw. vertraglich vereinbarten Anfangsgehalts, des mittleren und des Höchstgehalts verglichen (in US-Dollar, kaufkraftbereinigt). Dies erlaubt Rückschlüsse darauf, wie sich Berufserfahrung auf die Einstufung in den jeweiligen landesspezifischen Besoldungsgruppen sowie auf die Kosten der Unterrichtszeit in den einzelnen Ländern auswirken. Dann werden die Gehälter ins Verhältnis zum BIP pro Kopf gesetzt. Dies ist ein ungefährer Maßstab der Mittel, die für Lehrer aufgewendet werden, bezogen auf die Möglichkeiten eines Landes, Bildungsausgaben zu finanzieren. Schließlich werden die ZulagenSysteme untersucht.

Die gesetzlichen bzw. vertraglich festgelegten Gehälter für Lehrer mit 15 Jahren Berufserfahrung an Schulen des Sekundarbereich I reichen von weniger als 10.000 US-Dollar in Ungarn und der Tschechischen Republik bis zu über 50.000 US-Dollar in der Schweiz. Diese Differenz, die auch nach Berücksichtigung der Kaufkraftparität besteht, kann z.T. durch die unterschiedliche Höhe des BIP in den einzelnen OECD-Ländern erklärt 
werden. Sie hat jedoch große Auswirkungen auf die Unterschiede bei den Bildungskosten pro Schüler (Tabelle D6.1).

Die gesetzlichen bzw. vertraglich festgelegten Gehälter, die in diesem Indikator erfasst sind, beziehen sich auf die gemäß offiziellen Besoldungs- und Vergütungsgruppen festgelegten Gehälter. Diese sind zu unterscheiden sowohl von den tatsächlichen Gehaltsverpflichtungen, die den Regierungen entstehen, als auch von den Durchschnittsgehältern der Lehrer, die auch durch andere Faktoren beeinflusst werden, wie z.B. die Altersstruktur der Lehrerschaft und die Häufigkeit von Teilzeitarbeit. Da außerdem die Unterrichtszeit und die Arbeitsbelastung der Lehrer in den einzelnen Ländern stark voneinander abweichen können, sind diese Faktoren bei einem Vergleich der gesetzlichen bzw. vertraglich vereinbarten Lehrergehälter in den einzelnen Ländern ebenfalls zu berücksichtigen.

Ein alternativer Maßstab für die Gehälter und die Kosten der Unterrichtszeit ergibt sich, wenn man das gesetzlich bzw. vertraglich vereinbarte Gehalt für einen Vollzeitlehrer in Beziehung setzt zu der Anzahl an Unterrichtsstunden, die dieser Lehrer pro Jahr zu unterrichten hat (s. Indikator D7). Bei diesem Maßstab erfolgt zwar keine Umrechnung der Gehälter auf die gesamte Zeit, die Lehrer für die unterschiedlichen unterrichtsbezogenen Aktivitäten aufwenden, es ergibt sich jedoch ein ungefährer Eindruck der Kosten für die Zeit, die Lehrer wirklich im Klassenzimmer verbringen. Das durchschnittliche gesetzliche bzw. vertraglich vereinbarte Gehalt je Unterrichtsstunde nach 15 Jahren Berufserfahrung liegt im Primarbereich bei 36 US-Dollar, im Sekundarbereich I bei 44 US-Dollar und an allgemeinbildenden Schulen des Sekundarbereich II bei 53 US-Dollar. Im Primarbereich sind die Gehaltskosten je Unterrichtsstunde in Mexiko, der Tschechischen Republik, der Türkei und Ungarn relativ niedrig (höchstens 22 US-Dollar), in Japan, Korea und der Schweiz jedoch relativ hoch (über 50 US-Dollar). In allgemeinbildenden Schulen des Sekundarbereich II variieren die Gehaltskosten je Unterrichtsstunde sogar in noch stärkerem Ausmaß und liegen zwischen höchstens 20 US-Dollar in der Tschechischen Republik und Ungarn und mindestens 80 US-Dollar in Japan, Korea und der Schweiz (Tabelle D6.1).

Neben anderen Aspekten werden die Ausgaben der Länder für Lehrkräfte von den Möglichkeiten eines Landes, Bildungsausgaben zu finanzieren, beeinflusst. Ein Vergleich der gesetzlichen bzw. vertraglich vereinbarten Lehrergehälter im Verhältnis zum BIP pro Kopf ist daher eine weitere Möglichkeit, den relativen Status der Lehrergehälter in den OECD-Ländern zu bewerten.

Die Gehälter der Lehrer im Primarbereich nach 15 Jahren Berufserfahrung bezogen auf das BIP pro Kopf sind in der Tschechischen Republik $(0,65)$, Ungarn $(0,71)$ und Island $(0,80)$ am niedrigsten, und in Neuseeland $(1,70)$, der Türkei $(2,06)$ und Korea $(2,49)$, am höchsten. Während das Gehalt eines Lehrers im Primarbereich nach 15 Jahren Berufserfahrung in Spanien etwa
Das durchschnittliche Gehalt je Unterrichtsstunde nach 15 Jahren Berufserfahrung liegt im Primarbereich bei 36 US-Dollar, im Sekundarbereich I bei 44 US-Dollar und an allgemeinbildenden schulen des Sekundarbereich II bei 53USDollar.

Ein Vergleich der gesetzlichen bzw. vertraglich vereinbarten Lehrergehälter im Verhältnis zum jeweiligen BIP pro Kopf behandelt einen weiteren Aspekt der relativen Höhe der Lehrergehälter in den $O E C D$-Ländern.

Die Gehälter von Lehrern im Primarbereich nach 15 Jahren Berufserfahrung, bezogen auf das BIP 
pro Kopf, sind in Island, der Tschechischen

Republik und Ungarn vergleichsweise niedrig,

in Korea, Neuseeland und der Türkei dagegen relativ hoch.

Einige Länder investieren trotz niedrigerer Volkseinkommen massiv in die Humanressourcen.

In den meisten aber nicht allen Ländern sind die Gehälter höher, je höher der Bildungsbereich.

Das durchschnittliche Gehalt je Unterrichtsstunde eines Lehrers im Sekundarbereich II liegt 42 Prozent über dem eines Lehrers im

Primarbereich.

Berufserfahrung und Qualifikationen sind zwei Faktoren, die in vielen OECD-Ländern die Gehaltseinstufung der Lehrer beeinflussen. dem OECD-Durchschnitt entspricht, ist es bezogen auf das BIP pro Kopf im Vergleich zu anderen OECD-Ländern relativ hoch. Im Sekundarbereich I sind die entsprechenden Gehälter bezogen auf das BIP pro Kopf in Mexiko $(2,05)$ und Korea $(2,48)$ am höchsten. Im Sekundarbereich II (allgemeinbildend) ist das Verhältnis in der Tschechischen Republik $(0,80)$, Ungarn $(0,89)$ und Norwegen $(0,92)$ am niedrigsten, während es in der Türkei $(1,91)$, Mexiko $(2,18)$ und Korea $(2,48)$ am höchsten ist (Tabelle D6.1).

Es gibt zwar eine signifikante Beziehung zwischen den Lehrergehältern und dem BIP pro Kopf (die Korrelation beträgt etwa 0,60), aber sie ist dennoch nicht eindeutig. Obwohl einige Länder wie die Tschechische Republik und Ungarn sowohl ein relativ niedriges BIP pro Kopf als auch niedrige Lehrergehälter aufweisen, gibt es andere Länder, deren BIP pro Kopf auch relativ niedrig ist, deren Lehrergehälter jedoch mit Ländern vergleichbar sind, die über ein viel höheres BIP verfügen (z.B. Griechenland, Korea, Mexiko, Portugal und die Türkei). Dagegen liegen die Lehrergehälter in anderen Länder mit einem relativ hohen BIP pro Kopf unter dem OECD-Durchschnitt (Island und Norwegen), während wieder andere Länder ein hohes BIP pro Kopf und hohe Lehrergehälter aufweisen (die Schweiz und die Vereinigten Staaten) (Abb. D6.1).

\section{Besoldungs- und Vergütungsgruppen der Lehrer und Zulagen}

Während in Australien, England, Griechenland, Japan, Korea, Neuseeland, Norwegen, Portugal, Schottland und den Vereinigten Staaten die Lehrergehälter im Sekundarbereich II und im Primarbereich vergleichbar sind, steigen in den restlichen OECD-Ländern die Lehrergehälter absolut, je höher der Bildungsbereich ist. So liegt beispielsweise in Belgien, den Niederlanden, der Schweiz und Ungarn das mittlere Gehalt eines Lehrers im Sekundarbereich II mindestens 25 Prozent über dem eines Lehrers im Primarbereich (Tabelle D6.1).

Selbst in Ländern, in denen die gesetzlichen bzw. vertraglich vereinbarten Gehälter im Primar- und Sekundarbereich gleich sind, sind die Gehälter je Unterrichtsstunde in der Regel im Sekundarbereich II höher als im Primarbereich, denn in den meisten Ländern müssen Lehrer im Sekundarbereich weniger Unterrichtsstunden ableisten als Lehrer im Primarbereich. Im Durchschnitt liegt das Gehalt je Unterrichtsstunde für einen Lehrer im Sekundarbereich II um 42 Prozent über dem eines Lehrers im Primarbereich. In Australien, Neuseeland, Schottland und den Vereinigten Staaten beläuft sich diese Differenz jedoch auf höchstens 10 Prozent, während sie in Belgien, Frankreich, Island, Korea, den Niederlanden, Portugal, Spanien, der Schweiz und Ungarn zwischen 50 und 87 Prozent beträgt (Tabelle D6.1).

Ein Vergleich der Bruttogehälter der Lehrer in den verschiedenen Ländern beim Berufsanfang, nach 15 Jahren Berufserfahrung und an der Spitze der Gehaltsskala lässt darauf schließen, wie sehr die Berufserfahrung in den einzelnen Ländern die Gehaltseinstufung der Lehrer beeinflusst. Die Differenz zwischen dem gesetzlichen bzw. vertraglich vereinbarten Anfangsgehalt und 
den nachfolgenden Gehaltssteigerungen ist ein Anzeichen dafür, wie sehr sich Erfahrung finanziell auszahlt. Im OECD-Durchschnitt liegen die Gehälter von Lehrern im Primar- und Sekundarbereich I mit 15 Jahren Berufserfahrung um 37 bis 39 Prozent über dem Anfangsgehalt.

In Australien, Dänemark, England, Neuseeland und Schottland erreichen Lehrer das Höchstgehalt nach 8 bis 11 Dienstjahren. In Belgien, Deutschland, Finnland, Irland, den Niederlanden, Norwegen und Portugal flacht die Kurve nach 20 bis 28 Jahren ab. In Frankreich, Griechenland, Italien, Japan, Korea, Österreich, Spanien, derTschechischen Republik und Ungarn erreichen Lehrer erst nach mehr als 30 Dienstjahren das Höchstgehalt (Tabelle D6.1).

In Japan, Korea, Mexiko, Österreich oder Portugal ist das Anfangsgehalt relativ gering, aber das Verhältnis des Höchstgehalts zum Anfangsgehalt liegt bei 2:1 und darüber. Dagegen liegt das Höchstgehalt der Lehrer in Dänemark, Norwegen und Island um weniger als 30 Prozent über dem Anfangsgehalt. In Island wird eine lange Dienstzeit eher durch eine Verringerung der zu leistenden Unterrichtsstunden belohnt als mit höherem Gehalt. In Griechenland werden sowohl Gehaltserhöhungen als auch eine Reduzierung der abzuleistenden Unterrichtsstunden als Anerkennung für lange Dienstzeiten eingesetzt (Tabelle D6.1).

Zusätzlich zu den allgemeinen Besoldungs- und Vergütungsgruppen wurden in vielen Ländern Anreizprogramme für Lehrer entwickelt, entweder in Form finanzieller Vergütungen und/oder in Form der Reduzierung der zu leistenden Unterrichtsstunden. In Verbindung mit dem Anfangsgehalt können solche Anreizprogramme für den Einzelnen ein weiterer entscheidender Faktor bei der Überlegung sein, den Lehrerberuf zu ergreifen. Anreizprogramme für Berufsanfänger können Familienzulagen oder Ortszuschläge sein, ein höheres Anfangsgehalt für Qualifikationen, die über die für den Lehrberuf erforderlichen Mindestanforderungen hinausgehen und weitere Zulagen für Lehrqualifikationen in mehreren Fachgebieten oder für die Qualifikation, Schüler mit speziellen Bildungsbedürfnissen zu unterrichten.

In den meisten Ländern können alle oder fast alle Lehrer Zulagen erhalten, wenn sie Managementaufgaben übernehmen, mehr Stunden oder Klassen als in einem Vollzeitvertrag vorgesehen unterrichten (z.B. Stellvertretungspflichten), oder besondere Aufgaben übernehmen wie Anleitung und Beratung oder das Ausbilden von angehenden Lehrern. Obwohl in vielen Ländern die Zulagen für Überstunden, Managementaufgaben und besondere Aufgaben und Aktivitäten auf Landesebene geregelt sind, liegt in etwa der Hälfte der OECDLänder mit vergleichbaren Daten (Australien, Dänemark, England, Frankreich, Griechenland, Island, Irland, Italien, Mexiko, Neuseeland, Portugal, Schweden, der Tschechischen Republik und Ungarn) die Zuständigkeit für Entscheidungen hinischtlich Höhe und Ausmaß der Zulagen für solche Tätigkeiten teilweise auch bei den Schulen selbst.
Zusätzlich können Zulagen bezahlt werden, wenn Lehrer permanent oder vorübergehend besondere Pflichten oder Verantwortungen innerhalb der Organisation der Schule übernehmen,... 


\section{... sowie für \\ bildungspezifische \\ Qualifikationen oder herausragende Leistung.}

Wer den Lehrerberuf ergriffen hat, muss anerkannt und für gutes Unterrichten belohnt werden. Eine Möglichkeit für Schulen, Anreize für guten Unterricht zu bieten, besteht darin, den Abschluss von Maßnahmen zur beruflichen Weiterentwicklung, das Engagement bei besonderen Aktivitäten, die Übernahme von zusätzlichen Managementaufgaben oder herausragende Unterrichtsleistungen zusätzlich zu vergüten (Tabelle D6.2).

In Schweden erhalten Lehrer individuelle Gehälter, die auf Tarifverträgen beruhen. Kriterien für einen Anstieg des Gehalts über das Grundniveau hinaus sind u.a. bildungsspezifische Qualifikationen, die Teilnahme an Maßnahmen zur beruflichen Weiterbildung und herausragende Unterrichtsleistungen. In Neuseeland steht den Schulleitern eine gewisse Zahl von Gehaltseinheiten zusätzlich zum jährlichen Budget für Lehrergehälter zur Verfügung, die sie dazu nutzen können, um neue Lehrer einzustellen, Lehrer zu binden oder zu belohnen (Tabelle D6.2 und Anhang 3 unter www.oecd.org/els/education/eag2002).

In Spanien können Lehrer zusätzlich zur alle drei Jahre gewährten Gehaltszulage, die die Gehaltsstufen auf der Gehaltsskala bildet, weitere Zulagen erhalten, wenn sie an beruflichen Fortbildungsmaßnahmen teilnehmen. Darüber hinaus können sie eine innerschulische Qualifikation anstreben, die mit weiteren Zulagen verbunden ist (Tabelle D6.2 und Anhang 3 unter www.oecd.org/els/ education/eag2002).

\section{Gehaltszulagen in Spanien}

Die Gehaltszulagen, die alle Lehrer an öffentlichen und privaten Schulen erhalten, sind die trienios (ene kleine Gehaltszulage, die alle drei Jahre gewährt wird). Für den Elementar- und Primarbereich liegt die maximale Zahl der Berufsjahre bei 43 (die Lehrer beginnen die Laufbahn mit 22 Jahren), d.h. 14 trienios. Im Sekundarbereich II ist aufgrund der längeren Erstausbildung ein Höchstwert von 41 Jahren möglich (Die Lehrer beginnen die Laufbahn mit 24 Jahren.), d.h. 13 trienios. Für Lehrer an öffentlichen Schulen gibt es weitere Zulagen in Form von sexenios. Ein sexenio ist eine Gehaltszulage, die nach jeweils sechs Jahren in Abhängigkeit von der Teilnahme an innerschulischen Fortbildungsmaßnahmen gewährt wird (Die Lehrer müssen innerhalb der sechs Jahre 100 Stunden anerkannte innerschulische Fortbildungskurse absolvieren, um diese Zulage zu erhalten.) Normalerweise erfüllen alle Lehrer die Anforderungen, um den sexenio gewährt zu bekommen. Lehrer in öffentlichen Bildungseinrichtungen erhalten maximal 5 sexenios.

Lehrer an allgemeinbildenden öffentlichen Schulen des Sekundarbereichs mit dem Status eines Catedrático erhalten eine andere Art der Zulage. Catedráticos waren früher eine bestimmte 
Lehrerschicht mit gleicher Qualifikation aber höheren Einstiegsanforderungen. Seit 1990 bezieht sich Catedrático auf eine bestimmte innerschulische Qualifikation, die zu einer permanenten Zulage für den Rest der Laufbahn führt. Im allgemeinbildenden Sekundarbereich II können nur die Lehrer allgemeinbildender Fächer (nicht aber Lehrer in technischen Fächern) diesen Status erreichen. Nur 15 Prozent der Lehrer haben den Status eines catedráticos.

\section{Der Einsatz von Gehaltseinheiten an staatlichen und integrierten Schulen in Neuseeland}

1996 wurden im Secondary Teacher Collective Employment Contract (Gehaltseinheiten) eingeführt. In darauf folgenden Verhandlungen wurde dieses Einheitensystem auch in den Schulen des Primarbereichs eingeführt.

Zusätzlich zum Budget für eine bestimmte Anzahl von Lehrerstellen, die in Übereinstimmung mit der nationalen Stellenregelung berechnet werden, erhalten die Schulen einen Anspruch auf eine gewisse Zahl von Gehaltseinheiten, die sie auf das Kollegium verteilen können. Der Wert einer Gehaltseinheit für den empfangenden Lehrer wird bei den jährlichen Tarifverhandlungen festgelegt (2.750 US-Dollar jährlich ab 19. April 2000).

Jede Schule legt selbst die Regeln fest, nach denen diese Einheiten einzelnen Lehrern gewährt werden. Typischerweise werden Einheiten aufgrund von übernommenen Aufgaben (vor allem Managementaufgaben, z.B. Stellvertretender Schulleiter, Fachbereichsleiter, Dekan) gewährt, sie können jedoch auch zum Zweck der Einstellung, Bindung oder Anerkennung einzelner Lehrer genutzt werden.

Einheiten können dauerhaft oder zeitlich befristet gewährt werden, abhängig von den Beschränkungen, die in den entsprechenden Tarifverträgen vorgegeben werden.

Abgesehen von den Auswirkung auf das unmittelbare Gehalt, das sie erhalten, sind Lehrer, die dauerhaft eine oder mehrere Einheiten erhalten, berechtigt, auf der Gehaltsskala über das ihrer Qualifikation entsprechende Maximalgehalt hinaus in Richtung Maximalgehalt der Grundvergütung aufzusteigen.

Was die praktische Vergabe der Einheiten an den Schulen betrifft, so wird im Primarbereich etwa die Hälfte der Einheiten einzeln an Lehrer vergeben. Im Sekundarbereich werden 56 Prozent der Einheiten einzeln oder doppelt an Lehrer vergeben. Mehr als die Hälfte der Lehrer im Sekundarbereich erhalten eine oder mehrere Einheiten, was teilweise daran liegt, dass im Sekundarbereich mehr Einheiten zur Verfügung stehen. 
Durch Unterschiede bei

der Besteuerung, beim

Sozialversicherungs-

system, bei Beihilfen und

Leistungsansprüchen

kann das Grundgehalt

der Lehrer in den

einzelnen $O E C D$ -

Ländern unterschiedlich

stark aufgestockt

werden.

Die Daten stammen aus der OECD/INES-

Erhebung von $2001 \mathrm{zu}$

Lehrern und Lehrplänen

und beziehen sich auf das

Schuljahr 1999-2000.
Die Besoldungs- und Vergütungsgruppen basieren zwar auf dem einfachen Prinzip der Qualifikationsstufen und der Dienstjahre, aber in Wirklichkeit ist die Struktur des Besoldungssystems für Lehrer weit komplexer. Viele Länder bieten beispielsweise als Teil des Bruttojahresgehaltes Ortszuschläge für das Unterrichten in abgelegenen Regionen oder Familienzulagen. Leistungsansprüche können Fahrpreisermäßigungen im öffentlichen Verkehr, Steuerermäßigungen beim Kauf kultureller Güter sowie andere quasi-geldliche Ansprüche enthalten, die zum Grundgehalt der Lehrer beitragen. Innerhalb der OECD-Länder gibt es darüber hinaus große Unterschiede bei der Besteuerung und den Sozialversicherungssystemen. Aufgrund dieser Faktoren sind Vergleiche der Lehrergehälter mit Vorsicht zu interpretieren.

\section{Definitionen und angewandte Methodik}

Die Daten zu den gesetzlichen bzw. vertraglich vereinbarten Gehältern und den Zulagen für Lehrer (Tabelle D6.1) stammen aus der OECD/INES-Erhebung von 2001 zu Lehrern und Lehrplänen und beziehen sich auf das Schuljahr 1999-2000. Die Gehaltsdaten werden gemäß den offiziellen Vorschriften für öffentliche Einrichtungen angegeben.

Die gesetzlichen bzw. vertraglich vereinbarten Gehälter (Tabelle D6.1) beziehen sich auf das reguläre Gehalt gemäß offizieller Besoldungs- und Vergütungsgruppen. Die angegebenen Gehälter sind definiert als die Bruttogehälter (die vom Arbeitgeber für die Arbeit bezahlte Gesamtsumme) abzüglich der Arbeitgeberbeiträge zur Sozial- und Rentenversicherung (gemäß bestehender Besoldungs- und Vergütungsgruppen). Die Gehälter werden vor Steuer, d.h. vor Abzug der Einkommensteuern angegeben.

Die kaufkraftbereinigten Wechselkurse zur Umrechnung der Bruttogehälter der Lehrer stammen aus den „Volkswirtschaftlichen Gesamtrechnungen“ der OECD. Das Referenzdatum für das BIP pro Kopf ist das Kalenderjahr 2000, während der Referenzzeitraum für die Lehrergehälter der Zeitraum zwischen dem 30. Juni 1999 und dem 30. Juni 2000 ist. Referenzzeitraum für die Kaufkraftparitäten ist 1999-2000. Für Länder mit abweichendem Haushaltsjahr (d.h. Australien und Neuseeland) wurden die Daten auf den Januar 2000 inflationsbereinigt, ebenso wie für die Länder, deren genauer Zeitraum für die Gehälter der Lehrer leicht abweicht (z.B. Island, Norwegen, Spanien und Ungarn), jedoch nur dann, wenn die sich ergebende Anpassung über 1 Prozent lag. Geringere Anpassungen wurden vernachlässigt, weil selbst für Gehälter, die sich auf 1999-2000 beziehen, der genaue Zeitraum, in dem die Gehälter gelten, nur leicht abweicht. Die Referenzstatistiken sowie die Referenzjahre für die Gehälter der Lehrer sind in Anhang 2 aufgeführt.

Die angegebenen Anfangsgehälter beziehen sich auf das reguläre durchschnittliche Bruttojahresgehalt eines Vollzeitlehrers am Anfang der Lehrerlaufbahn mit der für eine Lehrerqualifikation erforderlichen Mindestausbildung. 
Die Gehälter für Lehrer mit 15 Jahren Berufserfahrung beziehen sich auf das reguläre Jahresgehalt eines Vollzeitlehrers mit der für eine Lehrerqualifikation erforderlichen Mindestausbildung und 15 Jahren Berufserfahrung. Die erfassten Höchstgehälter beziehen sich auf das reguläre maximale Jahresgehalt (an der Spitze der Vergütungs-/Besoldungsordnung) eines Vollzeitlehrers mit dem für seine Stelle mindestens erforderlichen Ausbildungsstand.

Zulagen zum Grundgehalt sind hier definiert als jegliche Abweichung beim Gehalt zwischen dem, was ein bestimmter Lehrer als Einkommen für die an der Schule geleistete Arbeit bezieht, und dem Betrag, den er ausschließlich aufgrund seiner Erfahrung bekommen müsste (d.h. der Anzahl der Berufsjahre als Lehrer). Zulagen können dauerhaft oder zeitlich befristet sein und dazu führen, dass ein Lehrer in den ,außertariflichen Bereich’ oder auf die nächsthöhere Besoldungs-/Vergütungsstufe gelangt. 
Tabelle D6.1.

Lehrergehälter (2000)

Gesetzliches bzw. vertraglich vereinbartes Jahresgehalt von Lehrern an öffentlichen Bildungseinrichtungen:Anfangsgehalt, Gehalt nach 15 Jahren Berufserfahrung und Höchstgehalt, nach Bildungsbereich, in US-Dollar, kaufkraftbereinigt

\begin{tabular}{|c|c|c|c|c|c|c|c|c|c|c|c|c|}
\hline & \multicolumn{4}{|c|}{ Primarbereich } & \multicolumn{4}{|c|}{ Sekundarbereich I } & \multicolumn{4}{|c|}{ Sekundarbereich II (allgemeinbildend) } \\
\hline & $\begin{array}{c}\text { Anfangs- } \\
\text { gehalt/ } \\
\text { Mindestaus- } \\
\text { bildung }\end{array}$ & $\begin{array}{l}\text { Gehalt nach } \\
\text { 15 Jahren } \\
\text { Berufser- } \\
\text { fahrung/ } \\
\text { Mindestaus- } \\
\text { bildung }\end{array}$ & $\begin{array}{c}\text { Höchst- } \\
\text { gehalt/ } \\
\text { Mindestaus- } \\
\text { bildung }\end{array}$ & $\begin{array}{c}\text { Verhältnis } \\
\text { Gehalt nach } \\
15 \text { Jahren } \\
\text { Berufser- } \\
\text { fahrung } \\
\text { zum BIP } \\
\text { pro Kopf }\end{array}$ & $\begin{array}{c}\text { Anfangs- } \\
\text { gehalt/ } \\
\text { Mindestaus- } \\
\text { bildung }\end{array}$ & $\begin{array}{l}\text { Gehalt nach } \\
15 \text { Jahren } \\
\text { Berufser- } \\
\text { fahrung/ } \\
\text { Mindestaus- } \\
\text { bildung }\end{array}$ & $\begin{array}{c}\text { Höchst- } \\
\text { gehalt/ } \\
\text { Mindestaus- } \\
\text { bildung }\end{array}$ & $\begin{array}{c}\text { Verhältnis } \\
\text { Gehalt nach } \\
15 \text { Jahren } \\
\text { Berufser- } \\
\text { fahrung } \\
\text { zum BIP } \\
\text { pro Kopf }\end{array}$ & $\begin{array}{l}\text { Anfangs- } \\
\text { gehalt/ } \\
\text { Mindest- } \\
\text { ausbildung }\end{array}$ & $\begin{array}{l}\text { Gehalt nach } 15 \\
\text { Jahren Berufs- } \\
\text { erfahrung/ } \\
\text { Mindest- } \\
\text { ausbildung }\end{array}$ & $\begin{array}{l}\text { Höchst- } \\
\text { gehalt/ } \\
\text { Mindest- } \\
\text { ausbildung }\end{array}$ & $\begin{array}{c}\text { Verhältnis } \\
\text { Gehalt nach } \\
15 \text { Jahren } \\
\text { Berufser- } \\
\text { fahrung } \\
\text { zum BIP pro } \\
\text { Kopf }\end{array}$ \\
\hline Australien & 26887 & 38297 & 38300 & 1.43 & 26946 & 38312 & 38314 & 1.43 & 26946 & 38312 & 38314 & 1.43 \\
\hline Belgien (Fl.) & 24122 & 32318 & 38328 & 1.22 & 24336 & 34079 & 41547 & 1.28 & 30194 & 43580 & 52383 & 1.64 \\
\hline Belgien (Frz.) & 22983 & 31282 & 37459 & 1.18 & 23466 & 33173 & 40666 & 1.25 & 29275 & 42707 & 51540 & 1.61 \\
\hline Tschechische Rep. & 7043 & 9339 & 12524 & 0.65 & 7043 & 9339 & 12524 & 0.65 & 8570 & 11381 & 15221 & 0.80 \\
\hline Dänemark & 29116 & 32883 & 32883 & 1.16 & 29116 & 32883 & 32883 & 1.16 & 28825 & 38279 & 40931 & 1.35 \\
\hline England & 22428 & 35487 & 35487 & 1.48 & 22428 & 35487 & 35487 & 1.48 & 22428 & 35487 & 35487 & 1.48 \\
\hline Finnland & 18489 & 25183 & 26140 & 1.03 & 20720 & 28690 & 30124 & 1.18 & 21517 & 30124 & 31878 & 1.23 \\
\hline Deutschland & 31213 & 37905 & 41021 & 1.52 & 34891 & 40561 & 46180 & 1.63 & 37394 & 43881 & 52004 & 1.76 \\
\hline Griechenland & 20065 & 24336 & 29358 & 1.50 & 20387 & 24658 & 29680 & 1.52 & 20387 & 24658 & 29680 & 1.52 \\
\hline Ungarn & 6086 & 8659 & 11805 & 0.71 & 6086 & 8659 & 11805 & 0.71 & 7375 & 10896 & 14562 & 0.89 \\
\hline Island & 20222 & 22202 & 25738 & 0.80 & 20222 & 22202 & 25738 & 0.80 & 21071 & 26162 & 31394 & 0.95 \\
\hline Irland & 22063 & 35760 & 40365 & 1.24 & 23163 & 36145 & 40750 & 1.25 & 23163 & 36145 & 40750 & 1.25 \\
\hline Italien & 20927 & 25115 & 30306 & 1.03 & 22657 & 27507 & 33510 & 1.13 & 22657 & 28329 & 35138 & 1.16 \\
\hline Japan & 22670 & 42820 & 54663 & 1.62 & 22670 & 42820 & 54663 & 1.62 & 22670 & 42845 & 56307 & 1.62 \\
\hline Korea & 26300 & 43952 & 69818 & 2.49 & 26148 & 43800 & 69666 & 2.48 & 26148 & 43800 & 69666 & 2.48 \\
\hline Mexiko & 11235 & 14824 & 24536 & 1.62 & 14383 & 18760 & 30859 & 2.05 & $\mathrm{~m}$ & $\mathrm{~m}$ & $\mathrm{~m}$ & $\mathrm{~m}$ \\
\hline Niederlande & 27411 & 32686 & 39563 & 1.18 & 28443 & 34985 & 43466 & 1.26 & 28713 & 48840 & 57907 & 1.77 \\
\hline Neuseeland & 17354 & 33653 & 33653 & 1.70 & 17354 & 33653 & 33653 & 1.70 & 17354 & 33653 & 33653 & 1.70 \\
\hline Norwegen & 23752 & 26831 & 29051 & 0.92 & 23752 & 26831 & 29051 & 0.92 & 23752 & 26831 & 29051 & 0.92 \\
\hline Portugal & 17914 & 26607 & 49492 & 1.52 & 17914 & 26607 & 49492 & 1.52 & 17914 & 26607 & 49492 & 1.52 \\
\hline Schottland & 20931 & 34798 & 34798 & 1.45 & 20931 & 34798 & 34798 & 1.45 & 20931 & 34798 & 34798 & 1.45 \\
\hline Schweiz & 34808 & 45728 & 54308 & 1.53 & 41048 & 54763 & 63534 & 1.83 & 49123 & 65041 & 73946 & 2.18 \\
\hline Türkei & 12410 & 14094 & 15760 & 2.06 & $\mathrm{a}$ & a & $\mathrm{a}$ & a & 11354 & 13038 & 14704 & 1.91 \\
\hline Vereinigte Staaten & 27631 & 40072 & 48782 & 1.12 & 27643 & 40072 & 47908 & 1.12 & 27751 & 40181 & 48037 & 1.12 \\
\hline Ländermittel & 21469 & 29407 & 36145 & 1.32 & 22727 & 31221 & 38674 & 1.35 & 23808 & 33582 & 41366 & 1.45 \\
\hline Argentinien & 9027 & 12545 & 14897 & 1.00 & 14623 & 21188 & 25742 & 1.69 & 14623 & 21188 & 25742 & 1.69 \\
\hline Brasilien & 7420 & 10176 & 11309 & 1.48 & 14820 & 16240 & 18723 & 2.36 & 15500 & 16121 & 19776 & 2.35 \\
\hline Chile & 10716 & 12038 & 16122 & 1.39 & 10716 & 12038 & 16122 & 1.39 & 10716 & 12582 & 16883 & 1.45 \\
\hline China & 2835 & 2952 & 3595 & 0.88 & 2835 & 2952 & 3595 & 0.88 & 2835 & 2952 & 3595 & 0.88 \\
\hline Ägypten & 2269 & 5065 & $\mathrm{~m}$ & 1.58 & 2269 & 5065 & $\mathrm{~m}$ & 1.58 & 2269 & 5065 & $\mathrm{~m}$ & 1.58 \\
\hline Indien $^{1}$ & 10678 & 15236 & 16375 & 7.22 & 12992 & 19373 & 21074 & 9.18 & 15798 & 23205 & 24914 & 11.00 \\
\hline Indonesien & 1357 & 2148 & 4093 & 0.77 & 1357 & 2148 & 4093 & 0.77 & 1412 & 2586 & 4093 & 0.93 \\
\hline Jamaika & 8332 & 9927 & 9927 & 2.82 & 8332 & 9927 & 9927 & 2.82 & 8332 & 9927 & 9927 & 2.82 \\
\hline Jordanien & 7838 & 10200 & 26475 & 2.66 & 7838 & 10200 & 26475 & 2.66 & 7838 & 10200 & 26475 & 2.66 \\
\hline Malaysia & 6158 & 10225 & 14623 & 1.33 & 11784 & 18632 & 25775 & 2.43 & 11784 & 18632 & 25775 & 2.43 \\
\hline Paraguay & 8874 & 8874 & 8874 & 2.00 & 13911 & 13911 & 13911 & 3.13 & 13911 & 13911 & 13911 & 3.13 \\
\hline Peru $^{2}$ & 5523 & 5523 & 5523 & 1.19 & 5462 & 5462 & 5462 & 1.18 & 5462 & 5462 & 5462 & 1.18 \\
\hline Philippinen & 10409 & 11491 & 12374 & 3.10 & 10409 & 11491 & 12374 & 3.10 & 10409 & 11491 & 12374 & 3.10 \\
\hline Russische Föd. & 3735 & 3735 & 3735 & 0.54 & 3735 & 3735 & 3735 & 0.54 & 3735 & 3735 & 3735 & 0.54 \\
\hline Thailand & 5756 & 14145 & 26977 & 2.42 & 5756 & 14145 & 26977 & 2.42 & 5756 & 14145 & 26977 & 2.42 \\
\hline Tunesien $^{3}$ & 13186 & 14505 & 15149 & 2.60 & 16965 & 18549 & 19340 & 3.30 & 20540 & 22270 & 23177 & 4.00 \\
\hline Uruguay $^{4}$ & 5749 & 6891 & 8317 & 0.76 & 5749 & 6891 & 8317 & 0.76 & 6257 & 7398 & 8824 & 0.82 \\
\hline Simbabwe & 35725 & 50011 & 50011 & 17.42 & 35725 & 50011 & 50011 & 17.42 & 35725 & 50011 & 50011 & 17.42 \\
\hline
\end{tabular}

1. Gehälter im Territorium der Landeshauptstadt Delhi. Lehrergehälter variieren von Bundesstaat zu Bundesstaat.

2. Referenzjahr 1999.

3. Einschließlich Zulagen.

4. Gehalt für eine Stelle mit 20 Wochenstunden. Die Mehrzahl der Lehrer hat 2 Stellen inne. 
Tabelle D6.1. (Fortsetzung)

Lehrergehälter (2000)

Gesetzliches bzw. vertraglich vereinbartes Jahresgehalt von Lehrern an öffentlichen Bildungseinrichtungen:Anfangsgehalt, Gehalt nach 15 Jahren Berufserfahrung und Höchstgehalt, nach Bildungsbereich, in US-Dollar, kaufkraftbereinigt

\begin{tabular}{|c|c|c|c|c|c|c|c|c|}
\hline \multirow{2}{*}{ Australien } & \multicolumn{3}{|c|}{$\begin{array}{l}\text { Verhältnis des Gehalts nach } 15 \text { Jahren } \\
\text { Berufserfahrung zum Anfangsgehalt }\end{array}$} & \multirow{2}{*}{$\begin{array}{l}\text { Jahre zwischen } \\
\text { Anfangs- und } \\
\text { Höchstgehalt } \\
\text { (Sekundar- } \\
\text { bereich I) }\end{array}$} & \multicolumn{3}{|c|}{$\begin{array}{l}\text { Gehalt je Unterrichtsstunde nach } \\
15 \text { Jahren Berufserfahrung }\end{array}$} & \multirow{2}{*}{\begin{tabular}{|} 
Verhältnis Gehalt \\
je Unter- \\
richtsstunde \\
von Lehrern \\
des Sekundar- \\
bereich II/ \\
Lehrern des \\
Primarbereichs \\
(nach 15 Jahren \\
Berufserfahrung)
\end{tabular}} \\
\hline & Primarbereich & $\begin{array}{c}\text { Sekundar- } \\
\text { bereich I }\end{array}$ & $\begin{array}{c}\text { Sekundar- } \\
\text { bereich II, } \\
\text { allgemeinbildend }\end{array}$ & & Primarbereich & $\begin{array}{l}\text { Sekundar- } \\
\text { bereich I }\end{array}$ & $\begin{array}{r}\text { Sekundarbereich II, } \\
\text { allgemeinbildend }\end{array}$ & \\
\hline Österreich & 1.21 & 1.23 & 1.26 & 34 & 39 & 42 & 49 & 1.27 \\
\hline Belgien (Fl.) & 1.34 & 1.40 & 1.44 & 27 & 39 & 48 & 65 & 1.67 \\
\hline Belgien (Frz.) & 1.36 & 1.41 & 1.46 & 27 & 39 & 46 & 64 & 1.64 \\
\hline Tschechische Rep. & 1.33 & 1.33 & 1.33 & 32 & 14 & 14 & 18 & 1.28 \\
\hline Dänemark & 1.13 & 1.13 & 1.33 & 8 & 51 & 51 & 68 & 1.33 \\
\hline England & 1.58 & 1.58 & 1.58 & 8 & $\mathrm{~m}$ & $\mathrm{~m}$ & $\mathrm{~m}$ & $\mathrm{~m}$ \\
\hline Finnland & 1.36 & 1.38 & 1.40 & 20 & 38 & 50 & 57 & 1.49 \\
\hline Deutschland & 1.21 & 1.16 & 1.17 & 28 & 48 & 55 & 64 & 1.31 \\
\hline Griechenland & 1.21 & 1.21 & 1.21 & 33 & 31 & 39 & 39 & 1.26 \\
\hline Ungarn & 1.42 & 1.42 & 1.48 & 40 & 11 & 16 & 20 & 1.76 \\
\hline Island & 1.10 & 1.10 & 1.24 & 18 & 35 & 35 & 56 & 1.60 \\
\hline Irland & 1.62 & 1.56 & 1.56 & 22 & 39 & 49 & 49 & 1.26 \\
\hline Italien & 1.20 & 1.21 & 1.25 & 35 & 34 & 45 & 46 & 1.38 \\
\hline Japan & 1.89 & 1.89 & 1.89 & 31 & 67 & 77 & 90 & 1.33 \\
\hline Korea & 1.67 & 1.68 & 1.68 & 37 & 53 & 77 & 80 & 1.52 \\
\hline Mexiko & 1.32 & 1.30 & $\mathrm{~m}$ & 14 & 19 & 16 & $\mathrm{~m}$ & $\mathrm{~m}$ \\
\hline Niederlande & 1.19 & 1.23 & 1.70 & 22 & 35 & 40 & 56 & 1.60 \\
\hline Neuseeland & 1.94 & 1.94 & 1.94 & 10 & 34 & 35 & 35 & 1.04 \\
\hline Norwegen & 1.13 & 1.13 & 1.13 & 28 & 38 & 42 & 53 & 1.41 \\
\hline Portugal & 1.49 & 1.49 & 1.49 & 26 & 33 & 45 & 52 & 1.58 \\
\hline Schweden & 1.28 & 1.28 & 1.26 & $\mathrm{a}$ & $\mathrm{a}$ & $\mathrm{a}$ & $\mathrm{a}$ & $\mathrm{a}$ \\
\hline Schweiz & 1.31 & 1.33 & 1.32 & 23 & 52 & 64 & 96 & 1.87 \\
\hline Türkei & 1.14 & $\mathrm{a}$ & 1.15 & a & 22 & a & 26 & 1.17 \\
\hline Vereinigte Staaten & 1.45 & 1.45 & 1.45 & $\mathrm{~m}$ & 35 & 36 & 36 & 1.02 \\
\hline Ländermittel & 1.37 & 1.39 & 1.42 & 25 & 37 & 44 & 53 & 1.42 \\
\hline Argentinien & 1.35 & 1.41 & 1.41 & $21-24$ & 2 & 2 & 3 & 1.71 \\
\hline Brasilien & 1.37 & 1.10 & 1.04 & 25 & $\mathrm{~m}$ & $\mathrm{~m}$ & $\mathrm{~m}$ & $\mathrm{~m}$ \\
\hline Chile & 1.09 & 1.09 & 1.12 & 30 & 14 & 14 & 15 & 1.04 \\
\hline China & 1.04 & 1.04 & 1.04 & $\mathrm{~m}$ & $\mathrm{~m}$ & $\mathrm{~m}$ & $\mathrm{~m}$ & $\mathrm{~m}$ \\
\hline Ägypten & 2.11 & 2.11 & 2.11 & $\mathrm{~m}$ & 8 & 8 & 8 & 1.00 \\
\hline Indien $^{1}$ & 1.42 & 1.48 & 1.46 & 20 & 16 & 19 & 22 & 1.37 \\
\hline Indonesien & 1.81 & 1.81 & 2.09 & 32 & 2 & 4 & 4 & 2.20 \\
\hline Jamaika & 2.53 & 2.53 & 2.53 & 12 & 10 & 14 & 15 & 1.47 \\
\hline Jordanien & 1.30 & 1.30 & 1.30 & 43 & 13 & 13 & 15 & 1.17 \\
\hline Malaysia & 1.67 & 1.59 & 1.59 & 22 & 13 & 24 & 24 & 1.79 \\
\hline Paraguay & 1.15 & 1.15 & 1.15 & $\mathrm{a}$ & 12 & 17 & 15 & 1.25 \\
\hline Peru $^{2}$ & 1.00 & 1.00 & 1.00 & mind. 20 & 8 & 10 & 10 & 1.19 \\
\hline Philippinen & 1.09 & 1.09 & 1.09 & 22 & 10 & 10 & 12 & 1.20 \\
\hline Russische Föd. & 1.00 & 1.00 & 1.00 & $\mathrm{~m}$ & $\mathrm{~m}$ & $\mathrm{~m}$ & $\mathrm{~m}$ & $\mathrm{~m}$ \\
\hline Thailand & 2.46 & 2.46 & 2.46 & 37 & 19 & 22 & 22 & 1.17 \\
\hline Tunesien $^{3}$ & 1.10 & 1.09 & 1.08 & 30 & 25 & 40 & 48 & 1.92 \\
\hline Uruguay $^{4}$ & 1.19 & 1.19 & 1.21 & 24 & 9 & 14 & 15 & 1.61 \\
\hline Simbabwe & 1.40 & 1.40 & 1.40 & 21 & 51 & 53 & 53 & 1.04 \\
\hline
\end{tabular}

1. Gehälter im Territorium der Landeshauptstadt Delhi. Lehrergehälter variieren von Bundesstaat zu Bundesstaat.

2. Referenzjahr 1999.

3. Einschließlich Zulagen.

4. Gehalt für eine Stelle mit 20 Wochenstunden. Die Mehrzahl der Lehrer hat 2 Stellen inne.

Quelle: OECD. Für Hinweise zu Quellen und Methodik s. Anhang 3 (www.oecd.org/els/education/eag2002). 
Tabelle D6.2.

Zulagen zum Grundgehalt für Lehrer an öffentlichen Schulen (2000)

Arten von Zulagen zum Grundgehalt für Lehrer an öffentlichen Schulen, nach Stelle/Behörde, die über Gewährung der Zulage entscheidet

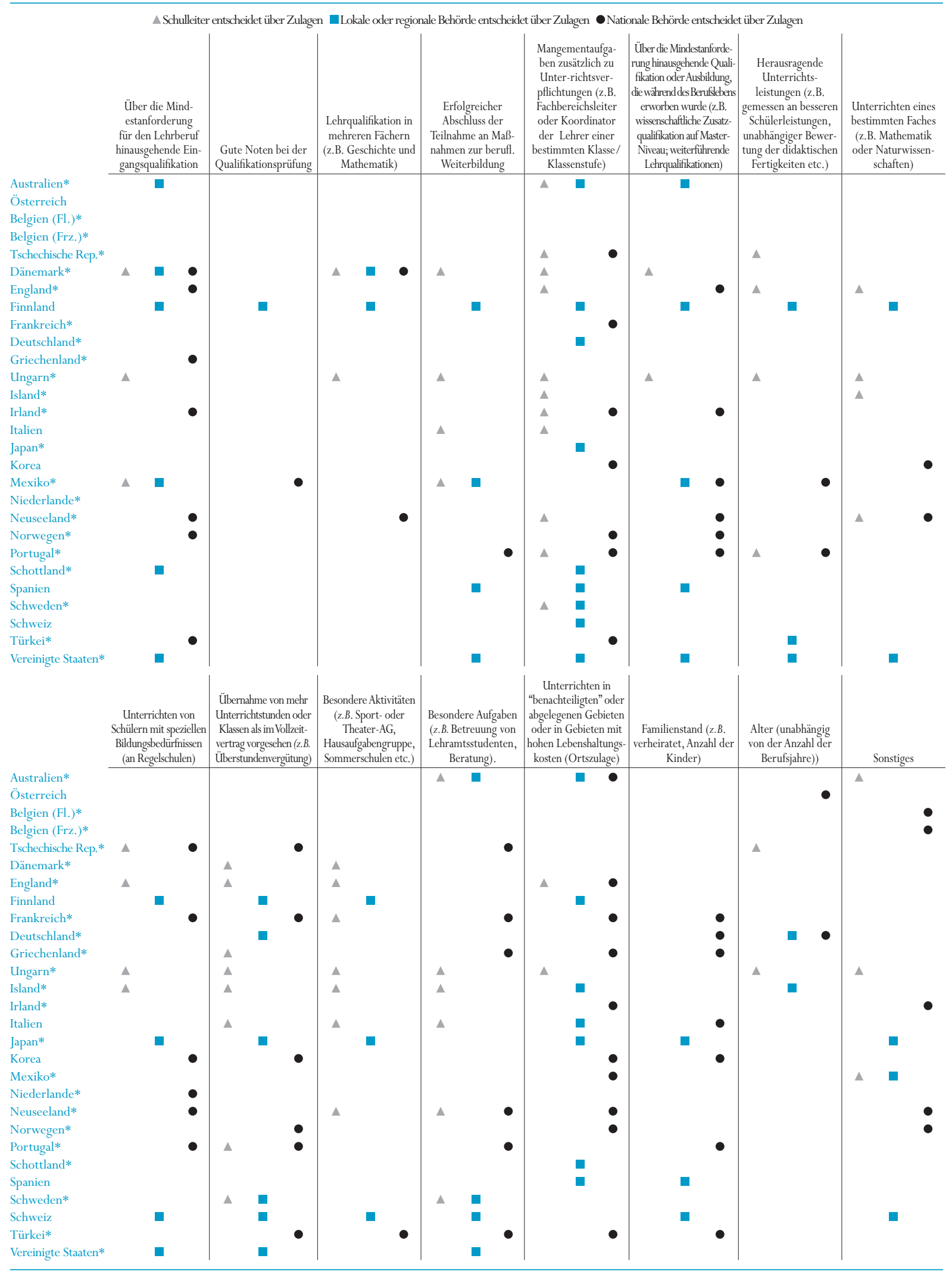

* Hinweise s. Anhang 3 (www.oecd.org/els/education/eag2002).

Quelle: OECD. 


\section{UNTERRICHTSZEIT UND ARBEITSZEIT DER LEHRER}

- Die durchschnittliche Zahl der Unterrichtsstunden an öffentlichen Schulen des Primarbereichs beträgt 792 Stunden, reicht aber von 583 bis zu 1.139 Stunden innerhalb der einzelnen Länder.

- Im Sekundarbereich I liegt die Zahl der Unterrichtsstunden bei durchschnittlich 720 Stunden, reicht aber von 555 bis zu 1.182 Stunden.

- Die Vorgaben für die Arbeitszeit der Lehrer unterscheiden sich in den einzelnen Ländern. In den meisten Ländern müssen die Lehrer formell eine bestimmte Stundenzahl arbeiten, in einigen Ländern ist auch die Zahl der wöchentlichen Unterrichtsstunden geregelt, einige Länder sehen Zeit für Tätigkeiten außerhalb des Unterrichts vor und einige Länder legen fest, wieviel Zeit die Lehrer in der Schule verbringen müssen.

\section{Abbildung D7.1.}

Anzahl der jährlichen Unterrichtsstunden (2000)

Nettoanzahl an Zeitstunden pro Jahr, die in öffentlichen Bildungseinrichtungen im Kontakt mit Schülern verbracht werden, nach Bildungsbereichen

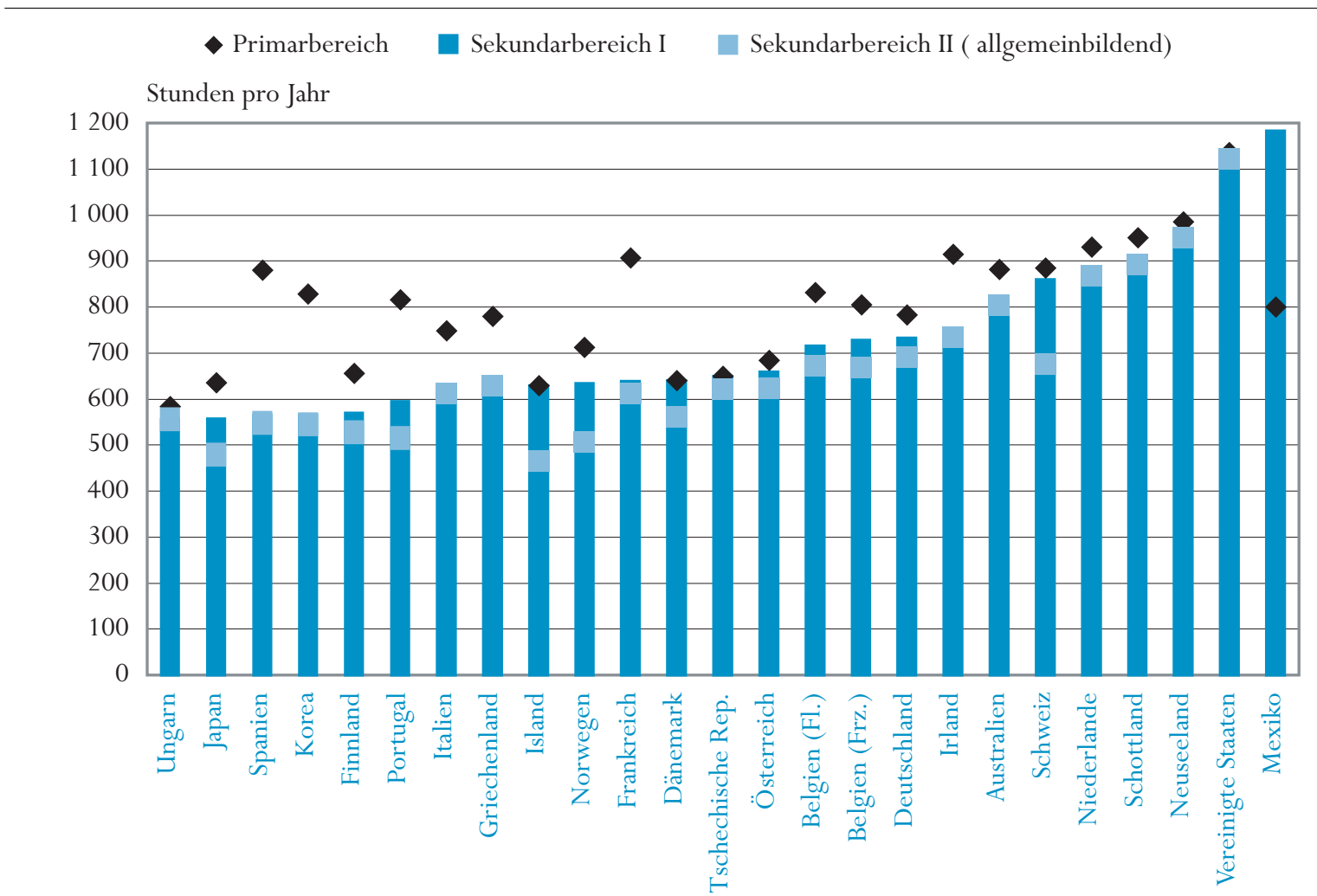

Anordnung der Länder in aufsteigender Reihenfolge der Anzahl der Unterrichtsstunden im Sekundarbereich I.

Quelle: OECD. Tabelle D7.1. Hinweise s. Anhang 3 (www.oecd.org/els/education/eag2002). 
Dieser Indikator gibt die Jahresstundenzahl an, die Vollzeitlehrer nach offiziellen Vorschriften des jeweiligen Landes unterrichten müssen.

Die durchschnittliche Zahl der Unterrichtsstunden in öffentlichen Schulen des Primarbereichs beträgt 792 Stunden, reicht aber von 583 bis zu 1.139 Stunden. Im Sekundarbereich I liegt die Zahl der Unterrichtsstunden bei durchschnittlich 720 Stunden, reicht aber von 555 bis zu

1.182 Stunden.

In den meisten Ländern ist die durchschnittliche Zahl der abzuleistenden Unterrichtsstunden im Primarbereich höher als im Sekundarbereich I und II, aber dieser Unterschied ist in den einzelnen Länder verschieden grofs.

\section{Politischer Hintergrund}

Zusammen mit Faktoren wie der Klassengröße und dem zahlenmäßigen Schüler/Lehrer-Verhältnis (Indikator D2), der Unterrichtszeit der Schüler (Indikator D1) und den Lehrergehältern (Indikator D6) beeinflusst die Zeit, die ein Lehrer tatsächlich unterrichtet, die für Bildung aufzuwendenden Finanzmittel. Gleichzeitig sind die Unterrichtszeit und die nicht unmittelbar unterrichtsbezogenen Aktivitäten ein entscheidender Bestandteil der Arbeitsbedingungen der Lehrer und stehen somit auch im Zusammenhang mit der Attraktivität des Lehrerberufs.

\section{Ergebnisse und Erläuterungen}

\section{Unterrichtszeit}

Im Primarbereich beträgt die durchschnittliche Zahl der Unterrichtsstunden 792 Stunden, schwankt aber von weniger als 650 Stunden in Dänemark, Island, Japan, derTschechischen Republik, derTürkei und Ungarn bis zu 950 Stunden und mehr in Neuseeland, Schottland und den Vereinigten Staaten (Tabelle D7.2).

Im Sekundarbereich I liegt die Zahl der Unterrichtsstunden bei durchschnittlich 720 Stunden im Jahr. Die Lehrbelastung der Lehrer variiert hier zwischen 555 Stunden in Finnland, Japan, Korea, Spanien und Ungarn und mehr als 900 Stunden in Neuseeland, Mexiko und den Vereinigten Staaten.

Im Sekundarbereich II ist die Lehrbelastung ähnlich wie im Sekundarbereich I oder etwas geringer. Die Lehrbelastung für einen Lehrer allgemeiner Fächer im Sekundarbereich II liegt im OECD-Durchschnitt bei 648 Stunden pro Jahr. Sie differiert zwischen weniger als 500 Stunden in Island und Japan bis zu mehr als 900 Stunden in England, Neuseeland und den Vereinigten Staaten (Tabelle D7.2).

In Frankreich, Korea, Portugal und Spanien muss ein Lehrer im Primarbereich fast 300 Stunden mehr unterrichten als ein Lehrer im Sekundarbereich II (allgemeinbildend), während diese Differenz in Australien, Dänemark, Deutschland, Finnland, Neuseeland, den Niederlanden, Österreich, Schottland, der Tschechischen Republik und den Vereinigten Staaten weniger als 100 Stunden beträgt (Abbildung D7.1).

Beim Vergleich der Zahl der abzuleistenden Unterrichtsstunden zwischen den einzelnen Ländern ist zu berücksichtigen, dass die Nettokontaktzeit, die diesem Indikator zu Grunde liegt, nicht der Anzahl der Schulstunden entspricht, die ein Lehrer pro Woche unterrichtet. Die Kontaktzeit selbst ist zwar ein beträchtlicher Bestandteil der Arbeitsbelastung, aber die Vorbereitung der Stunden sowie die erforderliche Nachbereitung (einschließlich der Korrektur der Schülerarbeiten) hängen enger mit der Zahl der Unterrichtsstunden pro Woche zusammen. Andere Elemente der Lehrbelastung (wie z.B. die Zahl der unterrichteten Fächer, die Zahl der zu unterrichtenden Schüler oder wie viele Jahre ein Lehrer dieselben Schüler unterrichtet) sind bei der Berechnung der durchschnittlichen Lehrbelastung der Lehrer innerhalb eines Landes ebenfalls 
zu berücksichtigen. Diese Faktoren lassen sich jedoch oft nur auf Schulebene bewerten.

Mit Ausnahme von Portugal, Spanien (Sekundarbereich II), der Tschechischen Republik und Ungarn war in den meisten OECD-Ländern die Zahl der abzuleistenden Unterrichtsstunden 1996 und 2000 in etwa gleich. Zwischen 1996 und 2000 ist in Ungarn die Zahl der abzuleistenden Unterrichtsstunden im Primarbereich um etwa 6 Prozent, im Sekundarbereich I und II um etwa 17 Prozent angestiegen. In der Tschechischen Republik mussten die Lehrer des Sekundarbereich I im Jahr 20007 Prozent mehr unterrichten als 1996. In Portugal dagegen ging die Nettokontaktzeit im Sekundarbereich I und II um 8 bzw. 10 Prozent zurück. In Spanien lag der Rückgang im Sekundarbereich II bei 13 Prozent (Tabelle D7.2).

\section{Arbeitszeit der Lehrer}

Die Vorgaben der Arbeitszeit der Lehrer unterscheiden sich in den einzelnen Ländern sehr. In einigen Ländern ist nur die Kontaktzeit formell festgelegt, in anderen dagegen auch die Arbeitszeit insgesamt. Einige Länder sehen innerhalb der formell festgelegten Arbeitszeit sowohl Zeit für den Unterricht als auch für Tätigkeiten außerhalb des Unterrichts vor. Innerhalb dieses Rahmens festgelegter Arbeitszeit und der Zahl abzuleistender Unterrichtsstunden kann die tatsächliche Lehrbelastung der Lehrer stark variieren.

In den meisten Ländern müssen die Lehrer formell eine bestimmte Stundenzahl proWoche arbeiten, um ein Vollzeitgehalt zu erhalten. Diese Stundenzahl umfasst sowohl die Unterrichtszeit als auch Zeit außerhalb des Unterrichts. Innerhalb dieses Rahmens gibt es jedoch zwischen den einzelnen Ländern Unterschiede hinsichtlich der weiteren Bestimmungen, wieviel Zeit unterrichtend und mit anderen Aktivitäten zu verbringen ist. Normalerweise wird die Zahl der Stunden für die Hauptaktivität, d.h. das Unterrichten, festgelegt, aber einige Ländern legen auch auf nationaler Ebene fest, wieviel Zeit die Lehrer in der Schule verbringen müssen.

In Japan und Korea haben Lehrer die gleiche Arbeitszeit wie Beamte und es gibt auf nationaler Ebene keine weiteren Vorgaben zur Anzahl der abzuleistenden Unterrichtsstunden oder zur Zeit für Tätigkeiten außerhalb des Unterrichts. In Korea sind die Lehrer jedoch angehalten, während der Schulferien nach ihrem eigenen Plan an ihrer beruflichen Weiterbildung zu arbeiten (Tabelle D7.1).

Innerhalb der festgelegten Arbeitszeit wird in Belgien, Dänemark, Frankreich, Griechenland, Island, Italien, Korea, Mexiko, den Niederlanden, Norwegen, Österreich, Portugal, Schottland, Spanien, der Schweiz, der Tschechischen Republik und der Türkei auch die Zahl der wöchentlich abzuleistenden Unterrichtsstunden vorgegeben.
Mit Ausnahme von

Portugal, Spanien, der Tschechischen Republik und Ungarn hat sich die Zahl der abzuleistenden Unterrichtsstunden zwischen 1996 und 2000 nicht wesentlich geändert.

Die Vorgaben für die Arbeitszeit der Lehrer unterscheiden sich in den einzelnen Ländern sehr.

In den meisten Ländern müssen die Lehrer formell eine bestimmte Zahl von Stunden arbeiten,...

... in einigen Ländern gilt die für Beamte festgelegte Arbeitszeit auch für Lehrer, jedoch ist die Unterrichtszeit der Lehrer nicht weitergehend geregelt, andere Länder legen dagegen auch die Zahl der wöchentlich abzuleistenden Unterrichtsstunden fest,... 


\section{Österreich}

Das für Lehrer geltenden Bildungsgesetz legt nur die Zahl der abzuleistenden Unterrichtsstunden fest (wöchentlich 20 bis 24 Unterrichtsstunden à 50 Minuten). Die Vorschriften zur Unterrichtszeit beruhen auf der Annahme, dass die Pflichten eines Lehrers (einschließlich der Vorbereitung des Unterrichts und der Klassenarbeiten, der Korrektur und Benotung von Klassenarbeiten, Prüfungen und Verwaltungsaufgaben) zu einer wöchentlichen Arbeitszeit von 40 Stunden führen.

.. einige Länder legen auf nationaler Ebene

Zeit für Tätigkeiten

außerhalb des

Unterrichts fest...
In Belgien, Finnland, Frankreich, Irland, Neuseeland, Portugal, der Tschechischen Republik und der Türkei müssen Lehrer lediglich während der von ihnen abzuleistenden Unterrichtsstunden an den Schulen anwesend sein. In einigen dieser Länder können jedoch auf Schulebene der Lehrauftrag sowie schulbezogene Tätigkeiten außerhalb des Unterrichts vorgegeben werden.

\section{Ungarn}

Die festgelegte Arbeitszeit (40 Stunden) entspricht der für Angestellte des öffentlichen Dienstes geltenden Arbeitszeit und ist eine formelle Anforderung an die Lehrer. Der Großteil der Vorbereitung erfolgt außerhalb der Schule. Schulbezogene Tätigkeiten (z.B. Lehrerkonferenzen, Elternsprechzeiten, Vorbereitungen von Schulfesten etc.) werden auf Schulebene festgelegt. Um ein Vollzeitgehalt zu erhalten, unterrichten Lehrer im Elementarbereich 32 Unterrichtsstunden (à 60 Minuten) pro Woche, im Primarbereich 21 Unterrichtsstunden (à 45 Minuten) und im Sekundarbereich 20 Stunden (ebenfalls à 45 Minuten). Überstunden werden vergütet und werden oft als Teil der Arbeit erwartet.

\section{Unterrichtszeit und Tätigkeiten außerhalb des Unterrichts in Spanien}

Im Primarbereich haben die Lehrer eine 37,5 Stundenwoche, wovon 22,5 Stunden reine Unterrichtszeit sind und 7,5 Stunden auf weitere Tätigkeiten an der Schule entfallen (Pausen, Besprechungen und andere pädagogische Tätigkeiten). Die verbleibenden 7,5 Stunden können auch außerhalb der Schule abgeleistet und für die Unterrichtsvorbereitung oder berufliche Weiterbildung etc. verwendet werden. Im Sekundarbereich müssen die Lehrer 16,5 Wochenstunden unterrichten und 30 Stunden pro Woche an der Schule verfügbar sein. 
In Schottland und Spanien ist abgesehen von der Unterrichtszeit auch die Zeit

für bestimmte Tätigkeiten außerhalb des Unterrichts vorgeschrieben.

\section{Vorschriften zur Arbeitszeit der Lehrer in Schottland}

Die Arbeitszeit der Lehrer, über die der Schulleiter verfügt, umfasst 27,5 Stunden pro Woche in der Schule. Hiervon entfallen im Primarbereich maximal 25 Stunden auf den Unterricht, im Sekundarbereich I maximal 23,5 Stunden und in Sonderschulen maximal 22,5 Stunden. Die Differenz zwischen der vorgegebenen Unterrichtszeit und den 27,5 Stunden steht den Lehrern, mit Ausnahme der Lehrer in Sonderschulen und -klassen, für Arbeiten im Zusammenhang mit ihren individuellen Lehrverpflichtungen zur Verfügung. Nur unter besonderen Umständen kann der Schulleiter diese Zeit für andere Zwecke vorsehen. Die Stunden der Teilzeitkräfte enthalten nicht nur die Unterrichtszeit, sondern anteilig auch Zeit für Kontakte außerhalb des Unterrichts. Die Arbeitszeit der Lehrer enthält ebenfalls ein weiteres Maximum von 30 Stunden pro Schuljahr für Elterngespräche, als Gesamtzeit festgelegt, die sowohl die Vorbereitung als auch die Reisezeit für maximal 6 Besprechungen pro Schuljahr umfasst.

In einigen Ländern, einschließlich Dänemark und Island, regeln detaillierte formelle Vereinbarungen zwischen Lehrergewerkschaften und den staatlichen Behörden, welche Berechnungsmethoden Schulen anzuwenden haben, wenn sie mit den Lehrern die Unterrichtsverpflichtung und die Tätigkeiten außerhalb des Unterrichts aushandeln.

....und in einigen Ländern legen formelle Vereinbarungen die abzuleistende Zahl der Unterrichtsstunden und die Tätigkeiten außserhalb des Unterrichts fest.

\section{Festlegung der Arbeitszeit in Dänemark}

Innerhalb der formell festgelegten Wochenarbeitszeit von 37,5 Stunden im Primar- und Sekundarbereich I wurde im Referenzjahr pro Unterrichtsstunde eine Stunde Vorbereitungszeit und durchschnittlich eine halbe Stunde für Tätigkeiten außerhalb des Unterrichts berechnet. Im Sekundarbereich II (allgemeinbildend) legt ein Tarifvertrag zwischen Lehrern und lokalen Behörden die Vorbereitungszeit auf 75 Prozent der Unterrichtsstunden x 1,33 fest und für Prüfungen wird ein Durchschnitt von 110 Stunden pro Jahr angesetzt. Die übrigen Pflichten werden auf lokaler Ebene festgelegt. 
Im Sekundarbereich II (berufsbildend) muß zwischen der Schulleitung und den Lehrervertretern, in Übereinstimmung mit dem Tarifvertrag zwischen der Lehrergewerkschaft und dem Finanzministerium, eine Einigung über die Festlegung der Arbeitszeit für die Unterrichtsvorbereitung etc. getroffen werden. Die Vorbereitungszeit liegt zwischen 13 und 126 Minuten pro Zeitstunde Unterricht. Die Vorgaben für die Korrektur schriftlicher Klassenarbeiten, Prüfungen etc. werden durch den Tarifvertrag oder durch eineVereinbarung auf Schulebene festgelegt. Jedem Lehrer stehen mindestens 50 Stunden pro Jahr für die pädagogische, theoretische oder fachliche Weiterbildung $\mathrm{zu}$.

\section{Berechnung der Lehrbelastung in Island}

Die Arbeitsbelastung der Lehrer im Primar- und Sekundarbereich I wird in drei Kategorien eingeteilt: Unterrichten (K), Unterrichtsvorbereitung (U) und sonstige Arbeit (Ö). Sollte der Anteil der sonstigen Arbeit zunehmen, kann der Lehrer entscheiden, ob er weniger unterrichten will oder ob Überstunden vergütet werden. Teilzeitkräfte haben dagegen Anspruch auf einen höheren Prozentsatz einer Vollzeitstelle. Im Sekundarbereich II wird die Arbeitsbelastung in 5 Kategorien eingeteilt: Arbeit an der Schule unter Aufsicht des Schulleiters (130 Stunden), Unterricht und unterrichtsbezogene Tätigkeiten (1.177 Stunden), Arbeit während der sechs Prüfungswochen (258 Stunden), Vor- und Nachbereitung zu Beginn und am Ende des Schuljahrs (32 Stunden) und berufliche Weiterbildung.

In einigen Länder wird die Arbeitszeit der Lehrer-einschließslich

Unterrichtszeit - auf regionaler, lokaler bzw. schulebene festgelegt.
In Australien, Deutschland, England, Finnland, Neuseeland, Schweden und den Vereinigten Staaten wird die Arbeitszeit der Lehrer - einschließlich der Festlegung der Unterrichtszeit - unterhalb der nationalen Ebene festgelegt. In Schweden beispielsweise handeln die Schulen innerhalb der für Beschäftige des öffentlichen Dienstes geltenden 40-Stunden-Woche mit den Lehrern die Unterrichtszeit und die Tätigkeiten außerhalb des Unterrichts auf individueller Basis aus. Eine formelle Vereinbarung zwischen der Schwedischen Vereinigung der Lokalbehörden und der Gewerkschaften der Lehrer begrenzt jedoch die Anzahl der jährlich abzuleistenden Unterrichtsstunden. Innerhalb dieser Grenzen ist die Unterrichtszeit nicht vorgeschrieben, um auch das Unterrichten von Nicht-Pflichtfächern abzudecken. 


\section{Arbeitszeitregelungen in Schweden}

Die Arbeitszeit wird durch formelle Vereinbarungen zwischen der Schwedischen Vereinigung der Lokalbehörden und der Gewerkschaften der Lehrer festgelegt. Laut Lehrervereinbarung 2000 liegt die Arbeitszeit bei 1.360 Stunden pro Schuljahr. Die Lehrer entscheiden selbst, wann sie die verbleibenden Stunden ableisten. Die Unterrichtszeit wird nicht in Stunden festgelegt, um auch das Unterrichten von Nicht-Pflichtfächern abzudecken.

\section{Definitionen und angewandte Methodik}

\section{Unterrichtszeit}

Die Zahl der Unterrichtsstunden wird definiert als die Nettoanzahl an Zeitstunden, die unterrichtend im Kontakt mit den Schülern verbracht wird. Sie wird berechnet aus der Anzahl der Unterrichtswochen pro Jahr multipliziert mit der Mindest-/Höchstzahl an Stunden, die ein Lehrer eine Klasse oder eine Schülergruppe pro Woche unterrichtet, multipliziert mit der Länge einer Unterrichtsstunde in Minuten, geteilt durch 60. Nicht enthalten sind Zeiten, die offiziell als Pausen zwischen einzelnen Stunden oder Stundenblöcken vorgesehen sind sowie Tage, an denen die Schule wegen Feiertagen oder Feierlichkeiten geschlossen ist. Im Primarbereich sind jedoch kurze Pausen, die die Lehrer mit der Klasse verbringen, normalerweise enthalten.

\section{Arbeitszeit}

Arbeitszeit bezieht sich auf die regulären Arbeitsstunden eines Vollzeitlehrers. Gemäß den formellen Vorschriften in einem bestimmten Land kann sich die Arbeitszeit beziehen auf:

- ausschließlich für den Unterricht aufgewendete Zeit (und andere lehrplanbezogene Tätigkeiten für die Schüler, wie Haus- und Klassenarbeiten, jedoch keine jährliche Prüfungen); oder auch

- Zeit, die in direktem Zusammenhang mit dem Unterricht steht, sowie Stunden, die anderen unterrichtsbezogenen Tätigkeiten gewidmet sind, z.B. Unterrichtsvorbereitung, Beratung der Schüler, Korrekturen von Haus- und Klassenarbeiten, Tätigkeiten zur beruflichen Weiterentwicklung, Besprechungen mit den Eltern, Lehrerkonferenzen und allgemeine schulische Aufgaben.

Nicht enthalten sind vergütete Überstunden.
Die Daten stammen aus der OECD/INESErhebung 2001 zu Lehrern und Lehrplänen und beziehen sich auf das Schuljahr 1999-2000. 


\section{Arbeitszeit in der Schule}

Arbeitszeit in der Schule bezieht sich auf die Arbeitszeit, die Lehrer an der Schule verbringen müssen und enthält sowohl die Unterrichtszeit als auch Zeit für Tätigkeiten außerhalb des Unterrichts.

\section{Anzahl der Unterrichtswochen und -tage}

Die Anzahl der Unterrichtswochen bezieht sich auf die Anzahl der Unterrichtswochen ohne die Ferienzeiten. Die Anzahl der Unterrichtstage wird berechnet als Anzahl der Unterrichtswochen abzüglich der Tage, an denen die Schule wegen Feiertagen oder Feierlichkeiten geschlossen ist. 
Tabelle D7.1.

Aufteilung der Arbeitszeit von Lehrern (2000)

Anzahl der Unterrichtswochen, Unterrichtstage, Netto-Unterrichtszeit in Zeitstunden und Arbeitszeit von Lehrern pro Schuljahr

\begin{tabular}{|c|c|c|c|c|c|c|c|c|c|c|c|c|c|}
\hline \multirow[b]{4}{*}{ Australien } & \multicolumn{2}{|c|}{$\begin{array}{c}\text { Anzahl der } \\
\text { Unterrichtswochen }\end{array}$} & \multicolumn{2}{|c|}{$\begin{array}{c}\text { Anzahl der } \\
\text { Unterrichtstage }\end{array}$} & \multicolumn{3}{|c|}{$\begin{array}{l}\text { Netto-Unterrichtszeit in } \\
\text { Zeitstunden }\end{array}$} & \multicolumn{3}{|c|}{$\begin{array}{l}\text { In der Schule abzuleistende } \\
\text { Arbeitszeit in Zeitstunden }\end{array}$} & \multicolumn{3}{|c|}{$\begin{array}{c}\text { Gesetzlich bzw. vertraglich } \\
\text { festgelegte Arbeitszeit in } \\
\text { Zeitstunden }\end{array}$} \\
\hline & & & & & & & $\begin{array}{l}\text { Sekundar- } \\
\text { bereich II }\end{array}$ & & & & & & \\
\hline & $\begin{array}{l}\text { Primar- } \\
\text { bereich }\end{array}$ & $\begin{array}{c}\text { Sekundar- } \\
\text { bereich }\end{array}$ & $\begin{array}{l}\text { Primar- } \\
\text { bereich }\end{array}$ & $\begin{array}{c}\text { Sekundar- } \\
\text { bereich }\end{array}$ & $\begin{array}{l}\text { Primar- } \\
\text { bereich }\end{array}$ & $\begin{array}{l}\text { Sekundar- } \\
\text { bereich I }\end{array}$ & $\begin{array}{c}\text { (allgemein- } \\
\text { bildend) }\end{array}$ & $\begin{array}{l}\text { Primar- } \\
\text { bereich }\end{array}$ & $\begin{array}{l}\text { Sekundar- } \\
\text { bereich I }\end{array}$ & $\begin{array}{l}\text { Sekundar- } \\
\text { bereich II }\end{array}$ & $\begin{array}{l}\text { Primarbe- } \\
\text { reich }\end{array}$ & $\begin{array}{l}\text { - Sekundar- } \\
\text { bereich I }\end{array}$ & $\begin{array}{l}\text { Sekundar- } \\
\text { bereich II }\end{array}$ \\
\hline & 40 & 40 & 196 & 196 & 882 & 811 & 803 & $\mathrm{a}$ & $\mathrm{a}$ & $\mathrm{a}$ & $1310^{2}$ & $1310^{2}$ & $1310^{2}$ \\
\hline Österreich & 38 & 38 & 187 & 187 & 684 & 658 & 623 & $\mathrm{~m}$ & $\mathrm{~m}$ & $\mathrm{~m}$ & a & a & a \\
\hline Belgien (Fl.) & 37 & 37 & 178 & 179 & 831 & 716 & 671 & $\mathrm{~m}^{3}$ & $\mathrm{~m}^{3}$ & $\mathrm{~m}^{3}$ & a & a & a \\
\hline Belgien (Frz.) & 38 & 38 & 182 & 182 & 804 & 728 & 673 & $871^{3}$ & $734^{3}$ & $673^{3}$ & a & a & a \\
\hline Tschechische Rep. & 40 & 40 & 197 & 197 & 650 & 650 & 621 & $650^{3}$ & $650^{3}$ & $621^{3}$ & 1700 & 1700 & 1700 \\
\hline Dänemark & 42 & 42 & 200 & 200 & 640 & 640 & 560 & $\mathrm{a}$ & a & $\mathrm{a}$ & $1680^{1}$ & $1680^{1}$ & $1680^{1}$ \\
\hline England & 38 & 38 & 190 & 190 & a & a & a & a & a & a & $1265^{2}$ & $1265^{2}$ & $1265^{2}$ \\
\hline Finnland & 38 & 38 & 190 & 190 & 656 & $485-656$ & $428-627$ & $964^{3}$ & $905^{3}$ & $901^{3}$ & $\mathrm{a}$ & a & $\mathrm{a}$ \\
\hline Frankreich & 35 & 35 & $\mathrm{~m}$ & $\mathrm{~m}$ & 907 & 639 & 611 & $907^{3}$ & $639^{3}$ & $611^{3}$ & a & a & $\mathrm{a}$ \\
\hline Deutschland & 39 & 39 & 188 & 188 & 783 & 732 & 690 & a & a & $\mathrm{a}$ & $1702-1760^{1}$ & $1702-1760^{\prime}$ & $1702-1760^{1}$ \\
\hline Griechenland & 40 & 38 & 195 & 185 & 780 & 629 & 629 & $1000^{3}$ & $798^{3}$ & $798^{3}$ & $1500^{1}$ & $1425^{1}$ & $1425^{1}$ \\
\hline Ungarn & 37 & 37 & 185 & 185 & 777 & 555 & 555 & $\mathrm{a}$ & a & $\mathrm{a}$ & $1664^{1}$ & $1664^{1}$ & $1664^{1}$ \\
\hline Island & 38 & 38 & 170 & 170 & 629 & 629 & 464 & $\mathrm{a}$ & a & a & $1800^{1}$ & $1800^{1}$ & $1800^{1}$ \\
\hline Irland & 37 & 33 & 183 & 167 & 915 & 735 & 735 & $\mathrm{a}$ & $735^{3}$ & $735^{3}$ & $1036^{2}$ & a & a \\
\hline Italien & 34 & 34 & $\mathrm{~m}$ & $\mathrm{~m}$ & 748 & 612 & 612 & $\mathrm{~m}^{3}$ & $\mathrm{~m}^{3}$ & m3 & a & a & a \\
\hline Japan & 35 & 35 & 193 & 193 & 635 & 557 & 478 & $\mathrm{a}$ & a & $\mathrm{a}$ & $1940^{5}$ & $1940^{5}$ & $1940^{5}$ \\
\hline Korea & 37 & 37 & 220 & 220 & 829 & 565 & 545 & a & a & $\mathrm{a}$ & $1613^{5}$ & $1613^{5}$ & $1613^{5}$ \\
\hline Mexiko & 42 & 42 & 200 & 200 & 800 & 1182 & $\mathrm{~m}$ & a & a & $\mathrm{m}$ & $900^{2}$ & $1680^{1}$ & \\
\hline Niederlande & 40 & 40 & 195 & 195 & 930 & 867 & 867 & a & a & $\mathrm{a}$ & $1659^{1}$ & $1659^{1}$ & $1659^{1}$ \\
\hline Neuseeland & 39 & 39 & 197 & 192 & 985 & 968 & 950 & $985^{3}$ & $968^{3}$ & $950^{3}$ & a & a & \\
\hline Norwegen & 38 & 38 & 190 & 190 & 713 & 633 & 589 & $\mathrm{a}$ & a & $\mathrm{a}$ & $1718^{1}$ & $1718^{1}$ & $1718^{1}$ \\
\hline Portugal & 34 & 34 & 163 & 163 & 815 & 595 & 515 & $815^{3}$ & $595^{3}$ & $515^{3}$ & $1596^{1}$ & $1596^{1}$ & $1596^{1}$ \\
\hline Schottland & 38 & 38 & 190 & 190 & 950 & 893 & 893 & $1075^{2}$ & $1075^{2}$ & $1075^{2}$ & $1153^{1}$ & $1153^{1}$ & $1153^{1}$ \\
\hline Spanien & 37 & 36 & 176 & 171 & 880 & 564 & 548 & 1110 & 1080 & 1050 & $1418^{2}$ & $1418^{2}$ & $1418^{2}$ \\
\hline Schweden & $\mathrm{a}$ & a & a & $\mathrm{a}$ & $\mathrm{a}$ & a & a & $1360^{2}$ & $1360^{2}$ & $1360^{2}$ & $1767^{1}$ & $1767^{1}$ & $1767^{1}$ \\
\hline Schweiz & 38 & 38 & $\mathrm{~m}$ & $\mathrm{~m}$ & 884 & 859 & 674 & $884^{3}$ & $859^{3}$ & $674^{3}$ & $\mathrm{~m}$ & $\mathrm{~m}$ & $\mathrm{~m}$ \\
\hline Türkei & 38 & a & 180 & 180 & 639 & 639 & 504 & $639^{3}$ & $639^{3}$ & $504^{3}$ & $\mathrm{a}$ & $\mathrm{a}$ & $\mathrm{a}$ \\
\hline Vereinigte Staaten ${ }^{6}$ & 36 & 36 & 180 & 180 & 1139 & 1127 & 1121 & $1353^{4}$ & $1371^{4}$ & $1371^{4}$ & $1353^{4}$ & $1371^{+}$ & $1371^{4}$ \\
\hline Argentinien & 38 & 38 & $\mathrm{~m}$ & $\mathrm{~m}$ & 765 & 850 & 755 & $\mathrm{~m}$ & $\mathrm{~m}$ & $\mathrm{~m}$ & $\mathrm{~m}$ & $\mathrm{~m}$ & $\mathrm{~m}$ \\
\hline Brasilien & 40 & 40 & $\mathrm{~m}$ & $\mathrm{~m}$ & 800 & 800 & 800 & $\mathrm{~m}$ & $\mathrm{~m}$ & $\mathrm{~m}$ & $\mathrm{~m}$ & $\mathrm{~m}$ & $\mathrm{~m}$ \\
\hline Chile & 40 & 40 & $\mathrm{~m}$ & $\mathrm{~m}$ & 860 & 860 & 860 & $\mathrm{~m}$ & $\mathrm{~m}$ & $\mathrm{~m}$ & $\mathrm{~m}$ & $\mathrm{~m}$ & $\mathrm{~m}$ \\
\hline Ägypten & 36 & 36 & $\mathrm{~m}$ & $\mathrm{~m}$ & 748 & 748 & 748 & $\mathrm{~m}$ & $\mathrm{~m}$ & $\mathrm{~m}$ & $\mathrm{~m}$ & $\mathrm{~m}$ & $\mathrm{~m}$ \\
\hline Indien & 42 & 42 & $\mathrm{~m}$ & $\mathrm{~m}$ & 743 & 825 & 825 & $\mathrm{~m}$ & $\mathrm{~m}$ & $\mathrm{~m}$ & $\mathrm{~m}$ & $\mathrm{~m}$ & $\mathrm{~m}$ \\
\hline Indonesien & 44 & 44 & $\mathrm{~m}$ & $\mathrm{~m}$ & 1260 & 738 & 738 & $\mathrm{~m}$ & $\mathrm{~m}$ & $\mathrm{~m}$ & $\mathrm{~m}$ & $\mathrm{~m}$ & $\mathrm{~m}$ \\
\hline Jamaika & 38 & 38 & $\mathrm{~m}$ & $\mathrm{~m}$ & 950 & 703 & 646 & $\mathrm{~m}$ & $\mathrm{~m}$ & $\mathrm{~m}$ & $\mathrm{~m}$ & $\mathrm{~m}$ & $\mathrm{~m}$ \\
\hline Jordanien & 44 & 44 & $\mathrm{~m}$ & $\mathrm{~m}$ & 774 & 774 & 659 & $\mathrm{~m}$ & $\mathrm{~m}$ & $\mathrm{~m}$ & $\mathrm{~m}$ & $\mathrm{~m}$ & $\mathrm{~m}$ \\
\hline Malaysia & 41 & 41 & $\mathrm{~m}$ & $\mathrm{~m}$ & 758 & 774 & 774 & $\mathrm{~m}$ & $\mathrm{~m}$ & $\mathrm{~m}$ & $\mathrm{~m}$ & $\mathrm{~m}$ & $\mathrm{~m}$ \\
\hline Paraguay & 35 & 37 & $\mathrm{~m}$ & $\mathrm{~m}$ & 720 & 801 & 900 & $\mathrm{~m}$ & $\mathrm{~m}$ & $\mathrm{~m}$ & $\mathrm{~m}$ & $\mathrm{~m}$ & $\mathrm{~m}$ \\
\hline Peru $^{7}$ & 36 & 36 & $\mathrm{~m}$ & $\mathrm{~m}$ & 783 & 626 & 626 & $\mathrm{~m}$ & $\mathrm{~m}$ & $\mathrm{~m}$ & $\mathrm{~m}$ & $\mathrm{~m}$ & $\mathrm{~m}$ \\
\hline Philippinen & 40 & 40 & $\mathrm{~m}$ & $\mathrm{~m}$ & 1176 & 1176 & 980 & $\mathrm{~m}$ & $\mathrm{~m}$ & $\mathrm{~m}$ & $\mathrm{~m}$ & $\mathrm{~m}$ & $\mathrm{~m}$ \\
\hline Russische Föd. & 45 & 45 & $\mathrm{~m}$ & $\mathrm{~m}$ & 860 & 774 & 774 & $\mathrm{~m}$ & $\mathrm{~m}$ & $\mathrm{~m}$ & $\mathrm{~m}$ & $\mathrm{~m}$ & $\mathrm{~m}$ \\
\hline Thailand & 40 & 40 & $\mathrm{~m}$ & $\mathrm{~m}$ & 760 & 652 & 652 & $\mathrm{~m}$ & $\mathrm{~m}$ & $\mathrm{~m}$ & $\mathrm{~m}$ & $\mathrm{~m}$ & $\mathrm{~m}$ \\
\hline Tunesien & 33 & 31 & $\mathrm{~m}$ & $\mathrm{~m}$ & 730 & 544 & 544 & $\mathrm{~m}$ & $\mathrm{~m}$ & $\mathrm{~m}$ & $\mathrm{~m}$ & $\mathrm{~m}$ & $\mathrm{~m}$ \\
\hline Uruguay $^{8}$ & 38 & 38 & $\mathrm{~m}$ & $\mathrm{~m}$ & 732 & 489 & 489 & $\mathrm{~m}$ & $\mathrm{~m}$ & $\mathrm{~m}$ & $\mathrm{~m}$ & $\mathrm{~m}$ & $\mathrm{~m}$ \\
\hline Simbabwe & 39 & 39 & $\mathrm{~m}$ & $\mathrm{~m}$ & 975 & 936 & 936 & $\mathrm{~m}$ & $\mathrm{~m}$ & $\mathrm{~m}$ & $\mathrm{~m}$ & $\mathrm{~m}$ & $\mathrm{~m}$ \\
\hline
\end{tabular}

1. Vollzeitlehrer arbeiten eine bestimmte Anzahl von Stunden pro Woche, um ein Vollzeitgehalt zu erhalten. Diese Anzahl umfasst sowohl unterrichtende als auch nicht-unterrichtende Tätigkeiten (z.B. Unterrichtsvorbereitung, Prüfungen, Besprechungen und allgemeine schulische Aufgaben), innerhalb oder außerhalb der Schule.

2. Vollzeitlehrer haben eine bestimmte Anzahl von Stunden pro Woche an der Schule zu arbeiten, um ein Vollzeitgehalt zu erhalten. Diese Anzahl umfasst sowohl unterrichtende als auch nicht-unterrichtende Tätigkeiten (z.B. Unterrichtsvorbereitung, Prüfungen, Besprechungen und allgemeine schulische Aufgaben), wovon ein festgelegter Teil an der Schule abzuleisten ist.

3. Vollzeitlehrer haben nur eine bestimmte Anzahl von Stunden pro Woche an der Schule zu arbeiten (z.B. die Unterrichtsstunden sowie die Pausenzeiten zwischen den Unterrichtsstunden). Die für nicht-unterrichtende Tätigkeiten aufzubringende Zeit ist nicht festgelegt.

4. Die Arbeitszeit der Lehrer wird individuell, auf lokaler oder Schulebene festgelegt. Sie umfasst sowohl unterrichtende als auch nicht-unterrichtende Tätigkeiten.

5. Gesetzlich festgelegte Arbeitszeit für öffentliche Angestellte. In Korea errechnet sich die Arbeitszeit aus der Periode eines Schuljahres.

6. Geschätzte Anzahl der Unterrichtswochen auf Grundlage des PISA-Durchschnitts. Geschätzte Anzahl der Stunden, die Lehrer an der Schule zu arbeiten haben, auf Grundlage der Stundenzahl, die Lehrer nach eigenen Angaben an der Schule anwesend zu sein haben.

7. Referenzjahr 1999 .

8. Unterrichtszeit für eine Stelle mit 20 Wochenstunden. Die Mehrzahl der Lehrer hat 2 Stellen inne.

Quelle: OECD. Hinweise, Quellen und Methodik s. Anhang 3 (www.oecd.org/els/education/eag2002). 
KAPITEL D Das Lernumfeld und die Organisation von Schulen

Tabelle D7.2.

Anzahl der jährlichen Unterrichtsstunden (1996, 2000)

Nettoanzahl an Zeitstunden, die in öffentlichen Bildungseinrichtungen pro Jahr im Kontakt mit den Schülern verbracht wird, nach Bildungsbereichen, sowie Änderungsindex 1996 zu 2000

\begin{tabular}{|c|c|c|c|c|c|c|c|c|c|}
\hline & \multicolumn{3}{|c|}{ Primarbereich } & \multicolumn{3}{|c|}{ Sekundarbereich I } & \multicolumn{3}{|c|}{ Sekundarbereich II (allgemeinbildend) } \\
\hline & 2000 & 1996 & $\begin{array}{c}\text { Index d. } \\
\text { Veränderung } \\
1996-2000\end{array}$ & 2000 & 1996 & $\begin{array}{c}\text { Index d. } \\
\text { Veränderung } \\
1996-2000\end{array}$ & 2000 & 1996 & $\begin{array}{c}\text { Index d. } \\
\text { Veränderung } \\
1996-2000\end{array}$ \\
\hline Australien & 882 & $\mathrm{~m}$ & $\mathrm{~m}$ & 811 & $\mathrm{~m}$ & $\mathrm{~m}$ & 803 & $\mathrm{~m}$ & $\mathrm{~m}$ \\
\hline Österreich & 684 & 684 & $\mathrm{n}$ & 658 & 658 & $\mathrm{n}$ & 623 & 623 & $\mathrm{n}$ \\
\hline Belgien (Fl.) & 831 & 841 & $-1 \%$ & 716 & 724 & $-1 \%$ & 671 & 679 & $-1 \%$ \\
\hline Belgien (Frz.) & 804 & 858 & $-6 \%$ & 728 & 734 & $-1 \%$ & 668 & 677 & $-1 \%$ \\
\hline Tschechische Rep. & 650 & 635 & $2 \%$ & 650 & 607 & $7 \%$ & 621 & 580 & $7 \%$ \\
\hline Dänemark & 640 & 640 & $\mathrm{n}$ & 640 & 640 & $\mathrm{n}$ & 560 & 560 & $\mathrm{n}$ \\
\hline Finnland & 656 & $\mathrm{~m}$ & $\mathrm{~m}$ & 570 & $\mathrm{~m}$ & $\mathrm{~m}$ & 527 & $\mathrm{~m}$ & $\mathrm{~m}$ \\
\hline Frankreich & 907 & 900 & $1 \%$ & 639 & 647 & $-1 \%$ & 611 & $\mathrm{~m}$ & $\mathrm{~m}$ \\
\hline Deutschland & 783 & 772 & $1 \%$ & 732 & 715 & $2 \%$ & 690 & 671 & $3 \%$ \\
\hline Griechenland & 780 & 780 & $\mathrm{n}$ & 629 & 629 & $\mathrm{n}$ & 629 & 629 & $\mathrm{n}$ \\
\hline Ungarn & 583 & 551 & $6 \%$ & 555 & 473 & $17 \%$ & 555 & 473 & $17 \%$ \\
\hline Island & 629 & $\mathrm{~m}$ & $\mathrm{~m}$ & 629 & $\mathrm{~m}$ & $\mathrm{~m}$ & 464 & $\mathrm{~m}$ & $\mathrm{~m}$ \\
\hline Irland & 915 & 915 & $\mathrm{n}$ & 735 & 735 & $\mathrm{n}$ & 735 & 735 & $\mathrm{n}$ \\
\hline Italien & 748 & 748 & $\mathrm{n}$ & 612 & 612 & $\mathrm{n}$ & 612 & 612 & $\mathrm{n}$ \\
\hline Japan & 635 & $\mathrm{~m}$ & $\mathrm{~m}$ & 557 & $\mathrm{~m}$ & $\mathrm{~m}$ & 478 & $\mathrm{~m}$ & $\mathrm{~m}$ \\
\hline Korea & 829 & $\mathrm{~m}$ & $\mathrm{~m}$ & 565 & $\mathrm{~m}$ & $\mathrm{~m}$ & 545 & $\mathrm{~m}$ & $\mathrm{~m}$ \\
\hline Mexiko & 800 & 800 & $\mathrm{n}$ & 1182 & 1182 & $\mathrm{n}$ & $\mathrm{m}$ & $\mathrm{m}$ & $\mathrm{m}$ \\
\hline Niederlande & 930 & 930 & $\mathrm{n}$ & 867 & 867 & $\mathrm{n}$ & 867 & 867 & $\mathrm{n}$ \\
\hline Neuseeland & 985 & 985 & $\mathrm{n}$ & 968 & 968 & $\mathrm{n}$ & 950 & 950 & $\mathrm{n}$ \\
\hline Norwegen & 713 & 713 & $\mathrm{n}$ & 633 & 611 & $4 \%$ & 505 & 505 & $\mathrm{n}$ \\
\hline Portugal & 815 & 783 & $4 \%$ & 595 & 644 & $-8 \%$ & 515 & 574 & $-10 \%$ \\
\hline Schottland & 950 & 975 & $-3 \%$ & 893 & $\mathrm{~m}$ & $\mathrm{~m}$ & 893 & 917 & $-3 \%$ \\
\hline Spanien & 880 & 900 & $-2 \%$ & 564 & $\mathrm{~m}$ & $\mathrm{~m}$ & 548 & 630 & $-13 \%$ \\
\hline Schweden & $\mathrm{a}$ & 624 & $\mathrm{~m}$ & a & 576 & $\mathrm{~m}$ & a & 528 & $\mathrm{~m}$ \\
\hline Schweiz & 884 & 871 & $1 \%$ & 859 & 850 & $1 \%$ & 674 & 669 & $1 \%$ \\
\hline Türkei & 639 & $\mathrm{~m}$ & $\mathrm{~m}$ & a & a & $\mathrm{m}$ & 504 & $\mathrm{~m}$ & $\mathrm{~m}$ \\
\hline Vereinigte Staaten & 1139 & 958 & $19 \%$ & 1127 & 964 & $17 \%$ & 1121 & 942 & $19 \%$ \\
\hline Ländermittel & 792 & 802 & $n$ & 720 & 728 & $n$ & 648 & 674 & $n$ \\
\hline
\end{tabular}

Quelle: OECD. Hinweise, Quellen und Methodik s. Anhang 3 (www.oecd.org/els/education/eag2002). 


\section{Anhang}

\section{TYPISCHE ABSCHLUSSALTER}

Das typische Abschlussalter ist das typische Alter am Ende des letzten Schul-/Studienjahres des betreffenden Bildungsbereichs bzw. Bildungsganges, in dem der Abschluß erworben wird. Das typische Alter basiert auf der Annahme einer Vollzeitbeteiligung im regulären Bildungssystem ohne Wiederholung von Klassenstufen. (Es sei darauf hingewiesen, dass in einigen Bildungsbereichen der Begriff „Abschlussalter“ nicht wörtlich $\mathrm{zu}$ verstehen ist und hier rein aus Definitionsgründen verwendet wird.) 
Tabelle X1.1a.

Typisches Abschlussalter im Sekundarbereich II

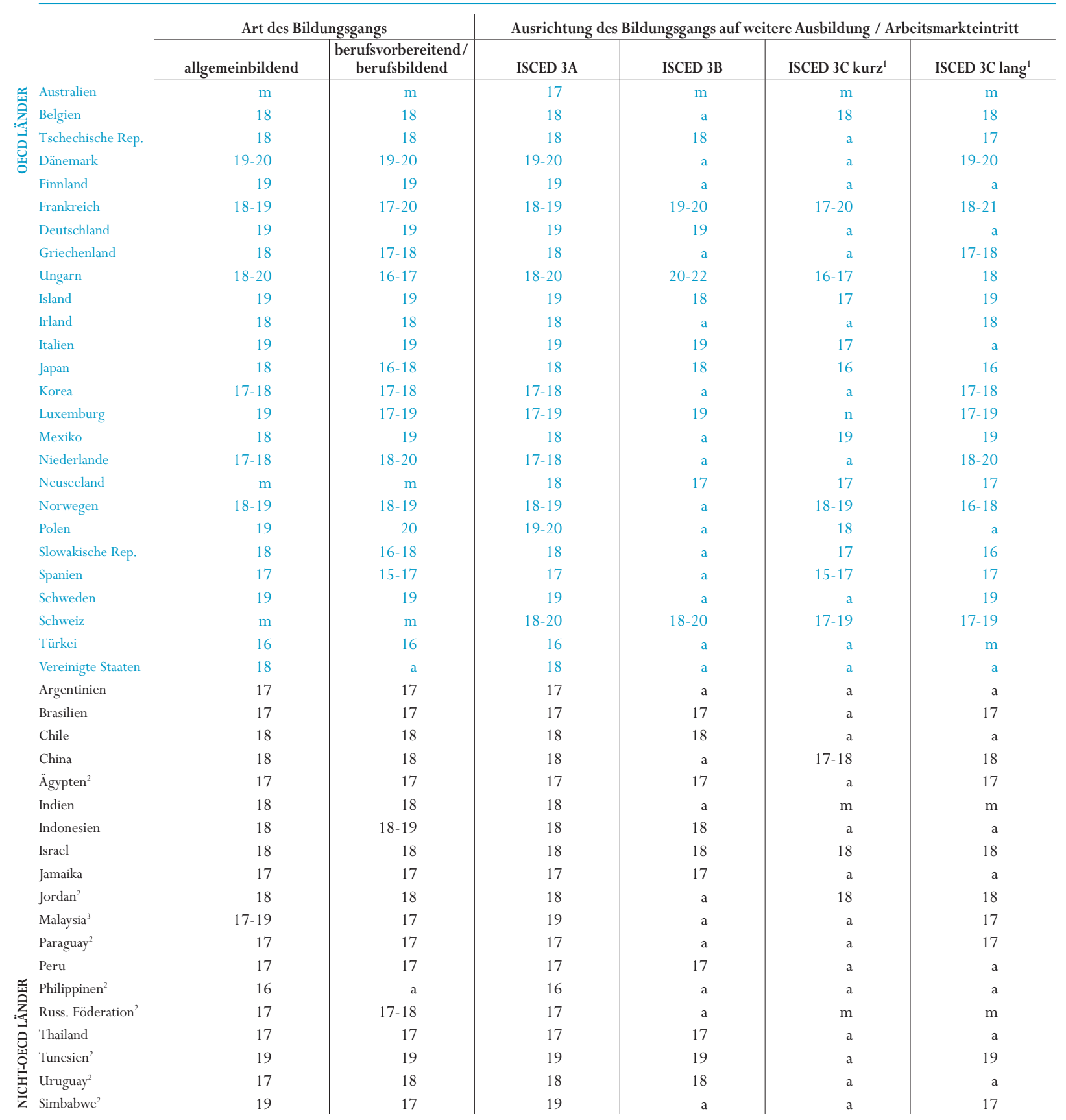

1. Zeitliche Einteilung bei ISCED 3C - kurz: mindestens ein Jahr kürzer als ISCED 3A/3B Bildungsgänge; lang: ähnlich lang wie Bildungsgänge nach ISCED 3A oder 3B. 2. Schätzung der OECD.

3. Schätzung der OECD für allgemeinbildende und berufsvorbereitende/berufsbildende Bildungsgänge. Quelle: $\mathrm{OECD}$. 
Tabelle X1.1b.

Typisches Abschlussalter im post-sekundären, nicht-tertiären Bereich

\begin{tabular}{|c|c|c|c|}
\hline & \multicolumn{3}{|c|}{ Ausrichtung des Bildungsgangs auf weitere Ausbildung / Arbeitsmarkteintritt } \\
\hline & ISCED 4A & ISCED 4B & ISCED 4C \\
\hline Belgien & 19 & a & $19-21$ \\
\hline$\overline{\mathrm{Z}}$ Kanada & $\mathrm{a}$ & $\mathrm{a}$ & 20 \\
\hline Tschechische Rep. & 20 & a & 20 \\
\hline Dänemark & $21-22$ & a & $21-22$ \\
\hline Finnland & a & a & $25-29$ \\
\hline Frankreich & $18-21$ & a & $19-21$ \\
\hline Deutschland & 22 & 22 & a \\
\hline Griechenland & a & a & $19-20$ \\
\hline Ungarn & $20-22$ & a & $19-22$ \\
\hline Island & a & a & 21 \\
\hline Irland & $\mathrm{a}$ & a & 19 \\
\hline Italien & a & a & 20 \\
\hline Korea & a & a & a \\
\hline Luxemburg & a & a & $20-25$ \\
\hline Mexiko & a & a & $\mathrm{a}$ \\
\hline Niederlande & $\mathrm{a}$ & a & $18-20$ \\
\hline Neuseeland & 18 & 18 & 18 \\
\hline Norwegen & $20-25$ & a & $20-25$ \\
\hline Polen & $\mathrm{a}$ & 21 & $\mathrm{a}$ \\
\hline Slowakische Rep. & $20-21$ & a & $\mathrm{a}$ \\
\hline Spanien & 18 & 18 & $\mathrm{a}$ \\
\hline Schweden & $\mathrm{m}$ & $\mathrm{m}$ & $19-20$ \\
\hline Schweiz & $19-21$ & $21-23$ & a \\
\hline Türkei & a & $\mathrm{a}$ & $\mathrm{a}$ \\
\hline Vereinigte Staaten & a & a & 20 \\
\hline Argentinien & $\mathrm{a}$ & $\mathrm{a}$ & a \\
\hline Brasilien & $\mathrm{a}$ & a & $\mathrm{a}$ \\
\hline China & a & 20 & 20 \\
\hline Indonesien & $\mathrm{a}$ & $\mathrm{a}$ & a \\
\hline Jordan $^{1}$ & a & $\mathrm{a}$ & a \\
\hline Malaysia $^{1}$ & 20 & 18 & 19 \\
\hline Paraguay & a & $\mathrm{a}$ & a \\
\hline Peru & $\mathrm{a}$ & a & $\mathrm{m}$ \\
\hline Philippinen ${ }^{1}$ & 19 & 19 & 17 \\
\hline Russ. Föderation & a & $\mathrm{a}$ & 18 \\
\hline Thailand $^{1}$ & a & $\mathrm{a}$ & 19 \\
\hline$\overline{\mathbf{Z}}$ Tunesien & $\mathrm{a}$ & 21 & $\mathrm{a}$ \\
\hline
\end{tabular}

1. Schätzung der OECD.

Quelle: OECD. 
Tabelle X1.1c.

Typisches Abschlussalter im Tertiärbereich

\begin{tabular}{|c|c|c|c|c|c|}
\hline & \multirow{2}{*}{$\begin{array}{c}\text { Tertiärbereich B (ISCED } \\
\text { 5B) }\end{array}$} & \multicolumn{3}{|c|}{ Tertiärbereich A (ISCED 5A) } & \multirow{2}{*}{$\begin{array}{l}\text { weiterführende Forshungs- } \\
\text { programme (ISCED 6) }\end{array}$} \\
\hline & & 3 bis weniger als $5 \mathrm{Jahre}$ & 5 Jahre & 6 Jahre oder mehr & \\
\hline Australien & $\mathrm{m}$ & 20 & $22-23$ & $\mathrm{a}$ & $25-29$ \\
\hline Österreich & $\mathrm{m}$ & 22 & 23 & $\mathrm{a}$ & 25 \\
\hline Belgien & $\mathrm{m}$ & $\mathrm{m}$ & $\mathrm{m}$ & $\mathrm{m}$ & $25-29$ \\
\hline Kanada & $\mathrm{m}$ & 22 & 26 & 26 & 29 \\
\hline Tschechische Rep. & 22 & 22 & 24 & a & 27 \\
\hline Dänemark & $21-25$ & $22-24$ & $25-26$ & 27 & 30 \\
\hline Finnland & $21-22$ & $25-29$ & $25-29$ & $30-34$ & 29 \\
\hline Frankreich & $20-21$ & $21-22$ & 23-24 & 25 & $25-26$ \\
\hline Deutschland & 21 & 25 & 26 & a & 28 \\
\hline Ungarn & $\mathrm{m}$ & $\mathrm{m}$ & $\mathrm{m}$ & $\mathrm{m}$ & 30 \\
\hline Island & $22-24$ & 23 & 25 & 27 & 29 \\
\hline Irland & 20 & 22 & 23 & 24 & 27 \\
\hline Italien & $22-23$ & 22 & $23-25$ & $25-27$ & $27-29$ \\
\hline Japan & 20 & 22 & 22 & 23 & 27 \\
\hline Korea & $\mathrm{m}$ & $\mathrm{m}$ & $\mathrm{m}$ & $\mathrm{m}$ & 26 \\
\hline Niederlande & $\mathrm{m}$ & $\mathrm{m}$ & $\mathrm{m}$ & $\mathrm{m}$ & 25 \\
\hline Neuseeland & $\mathrm{m}$ & $\mathrm{m}$ & $\mathrm{m}$ & $\mathrm{m}$ & 28 \\
\hline Norwegen & $\mathrm{m}$ & $\mathrm{m}$ & $\mathrm{m}$ & $\mathrm{m}$ & 29 \\
\hline Polen & $\mathrm{m}$ & 24 & 25 & 26 & $\mathrm{~m}$ \\
\hline Portugal & $\mathrm{m}$ & $\mathrm{m}$ & $\mathrm{m}$ & $\mathrm{m}$ & $27-29$ \\
\hline Slowakische Rep. & $20-21$ & $\mathrm{~m}$ & $\mathrm{~m}$ & $\mathrm{~m}$ & 27 \\
\hline Spanien & 19 & $\mathrm{~m}$ & $\mathrm{~m}$ & $\mathrm{~m}$ & $25-27$ \\
\hline Schweden & $22-23$ & $23-25$ & $25-26$ & $\mathrm{a}$ & $27-29$ \\
\hline Schweiz & $\mathrm{m}$ & $23-26$ & $23-26$ & 28 & 29 \\
\hline Türkei & $\mathrm{m}$ & $\mathrm{m}$ & $\mathrm{m}$ & $\mathrm{m}$ & $28-29$ \\
\hline Ver. Königreich & $\mathrm{m}$ & 21 & 23 & 24 & 24 \\
\hline Vereinigte Staaten & 20 & 21 & 23 & 25 & 28 \\
\hline
\end{tabular}

Quelle: OECD. 
Tabelle X1.2a

Für die Berechnung der Indikatoren verwendete Haushalts- und Schuljahre

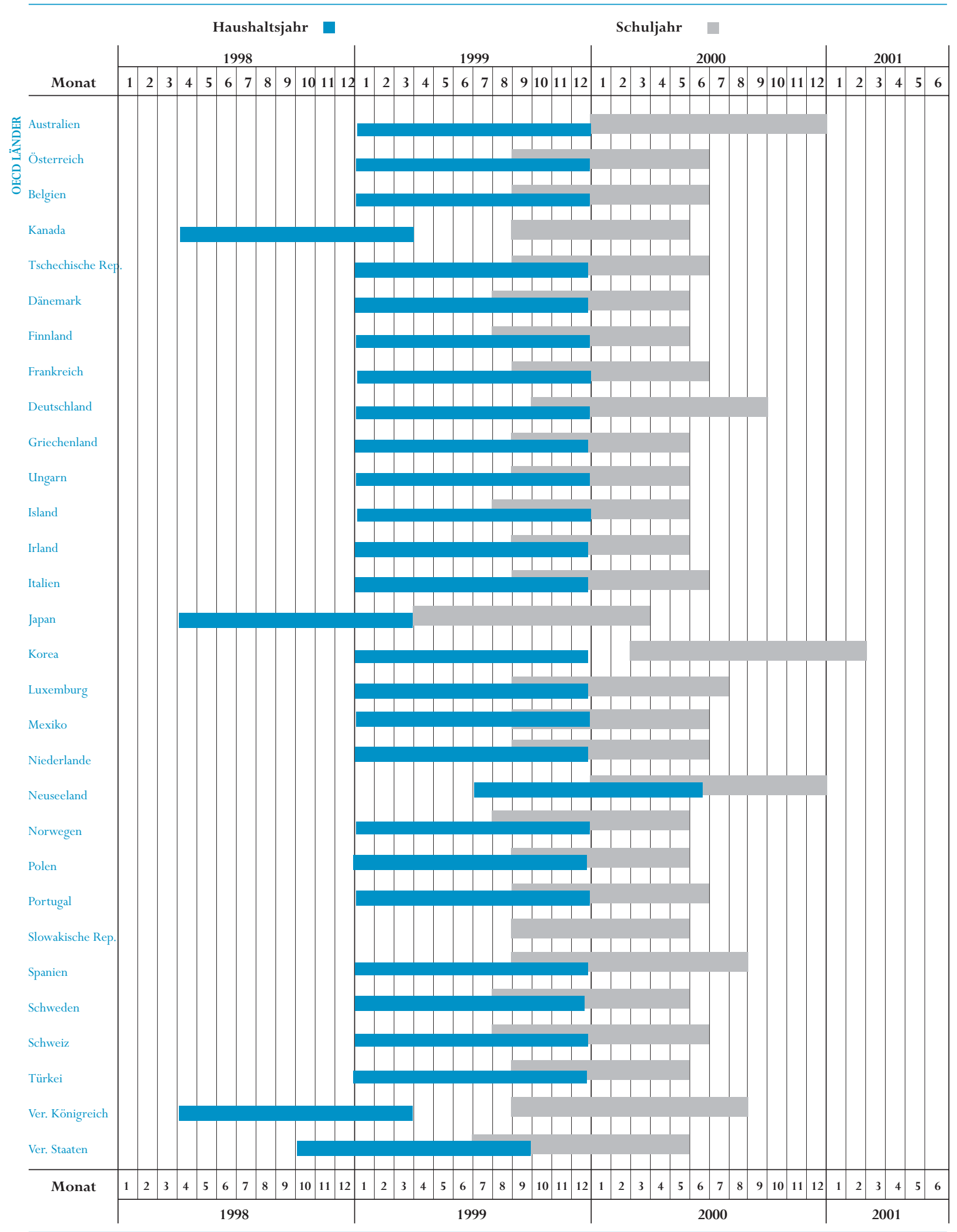

Quelle: OECD. 
Tabelle X1.2b

Für die Berechnung der Indikatoren verwendete Haushalts- und Schuljahre

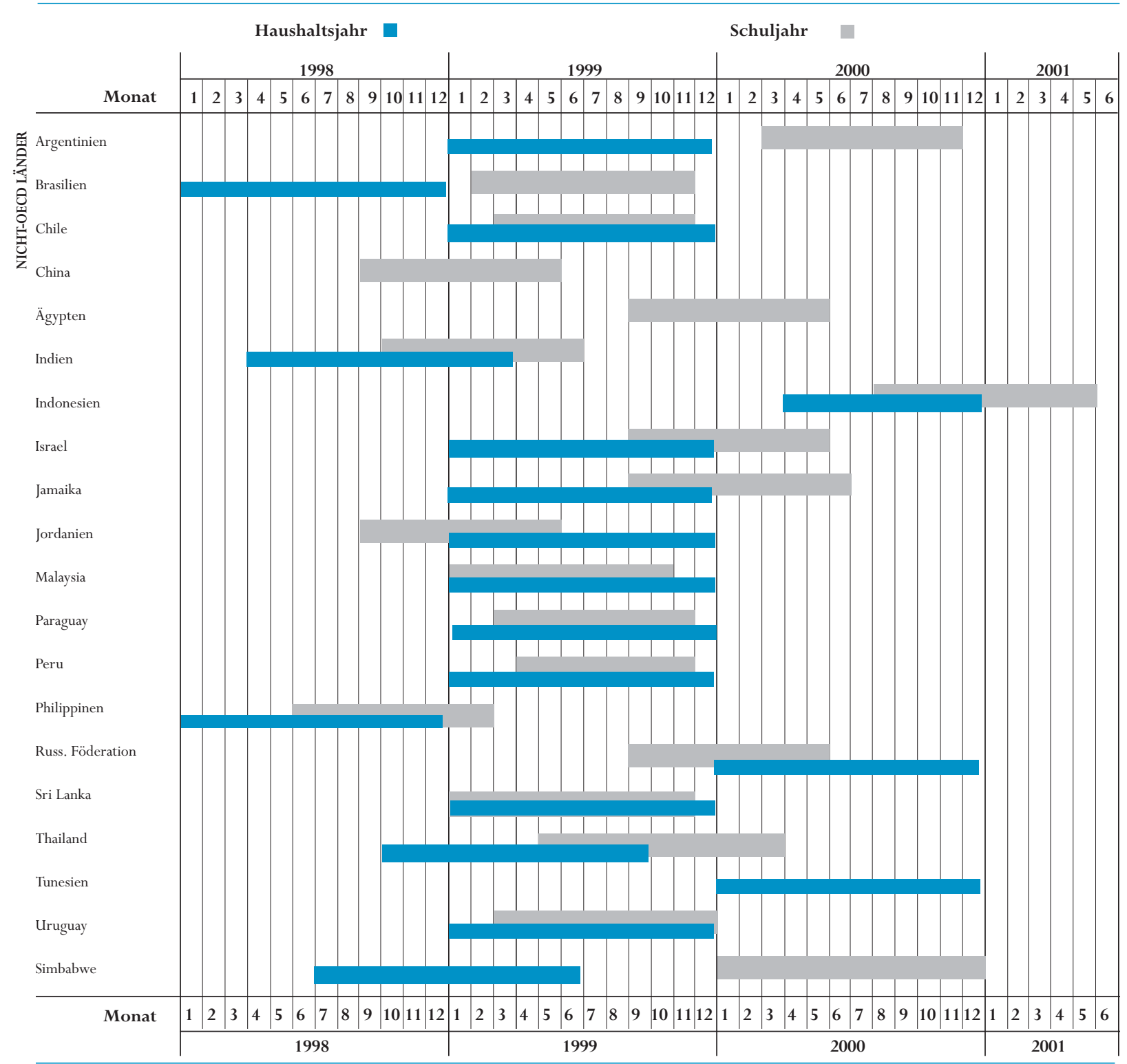

Quelle: OECD. 
Anhang

2

GRUNDLEGENDE STATISTISCHE BEZUGSDATEN 
Tabelle X2.1.

Überblick über das wirtschaftliche Umfeld anhand von grundlegenden Kennzahlen (Referenzzeitraum: Kalenderjahr 1999, zu Marktpreisen von 1999)

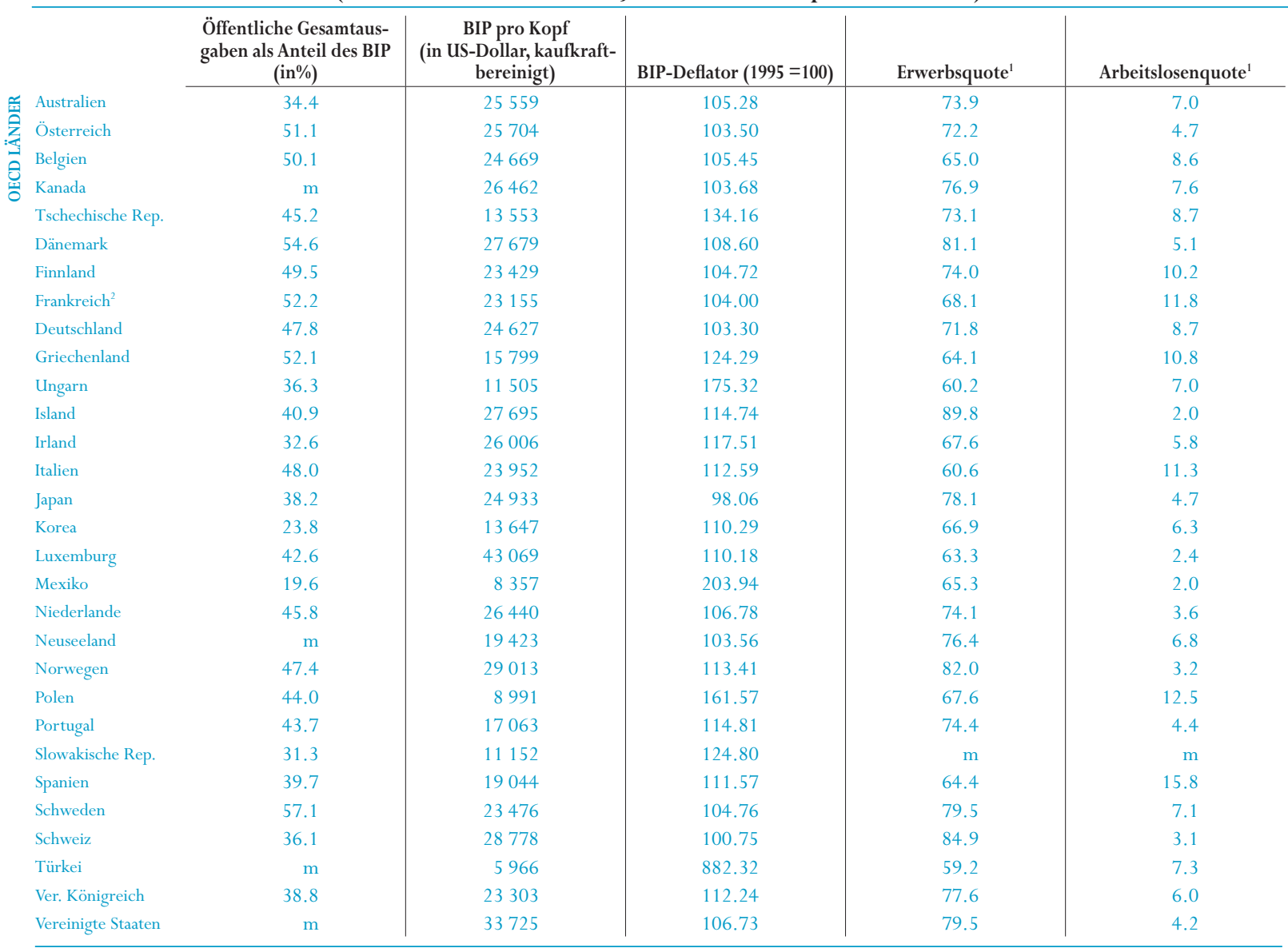

1. Griechenland, Niederlande und Österreich: Referenzzeitraum: Kalenderjahr 1998.

2. Ohne Übersee-Departments (DOM). 
Tabelle X2.2.

Grundlegende statistische Bezugsdaten (Referenzzeitraum: Kalenderjahr 1999, zu Marktpreisen von 1999)

\begin{tabular}{|c|c|c|c|c|c|}
\hline & $\begin{array}{l}\text { Bruttoinlandsprodukt, } \\
\text { berichtet für das Kalen- } \\
\text { derjahr (in Millionen, } \\
\text { Landeswährung) }\end{array}$ & $\begin{array}{l}\text { Bruttoinlandsprodukt } \\
\text { (angepasst an das natio- } \\
\text { nale Haushaltsjahr) }\end{array}$ & $\begin{array}{l}\text { Öffentliche Gesamtaus- } \\
\text { gaben (in Millionen, } \\
\text { Landeswährung) }\end{array}$ & $\begin{array}{c}\text { Gesamtbevölkerung in } \\
\text { Tausend (Schätzung zur } \\
\text { Mitte des Jahres) }\end{array}$ & Kaufkraftparitäten (KKP) \\
\hline Australien $^{1}$ & 629212 & 629212 & 216602 & 18937 & 1.30 \\
\hline Österreich & 2706068 & 2706068 & 1381502 & 8092 & 13.01 \\
\hline Belgien & 9501583 & 9501583 & 4761994 & 10222 & 37.68 \\
\hline Kanada & 960206 & 915981 & $\mathrm{~m}$ & 30493 & 1.19 \\
\hline Tschechische Rep. & 1887325 & 1887325 & 852242 & 10285 & 13.54 \\
\hline Dänemark & 1213595 & 1213595 & 663051 & 5321 & 8.24 \\
\hline Finnland & 716370 & 716370 & 354936 & 5165 & 5.92 \\
\hline Frankreich $^{3}$ & 8730475 & 8730475 & 4554093 & 59099 & 6.38 \\
\hline Deutschland & 3861200 & 3861200 & 1847510 & 82087 & 1.91 \\
\hline Griechenland & 38389050 & 38389050 & 20011550 & 10534 & 230.68 \\
\hline Ungarn & 11393499 & 11393499 & 4140835 & 10067 & 98.37 \\
\hline Island & 623419 & 623419 & 255283 & 277 & 81.21 \\
\hline Irland & 70116 & 70116 & 22832 & 3745 & 0.72 \\
\hline Italien & 2146350000 & 2146350000 & 1031140000 & 57646 & 1554.48 \\
\hline Japan & 511837100 & 514835375 & 195575600 & 126686 & 162.04 \\
\hline Korea & 482744175 & 482744175 & 114685126 & 46858 & 754.89 \\
\hline Luxemburg & 744232 & 744232 & 316780 & 436 & 39.66 \\
\hline Mexiko & 4583762 & 4583762 & 898886 & 97428 & 5.63 \\
\hline Niederlande & 823446 & 823446 & 377144 & 15809 & 1.97 \\
\hline Neuseeland $^{1}$ & 105852 & 105852 & $\mathrm{~m}$ & 3811 & 1.43 \\
\hline Norwegen & 1197457 & 1197457 & 567018 & 4462 & 9.25 \\
\hline Polen & 615115 & 615115 & 270619 & 38654 & 1.77 \\
\hline Portugal & 21694862 & 21694862 & 9475440 & 9990 & 127.27 \\
\hline Slowakische Rep. & 815330 & 815330 & 255396 & 5396 & 13.55 \\
\hline Spanien & 94088400 & 94088400 & 37335000 & 39626 & 124.68 \\
\hline Schweden & 2004651 & 2004651 & 1144257 & 8858 & 9.64 \\
\hline Schweiz & 388569 & 388569 & 140395 & 7144 & 1.89 \\
\hline Türkei & 77415272000 & 77415272000 & $\mathrm{~m}$ & 65819 & 197156.62 \\
\hline Ver. Königreich & 901269 & 870171 & 349235 & 59501 & 0.65 \\
\hline Vereinigte Staaten & 9206900 & 9085225 & $\mathrm{~m}$ & 272996 & 1.00 \\
\hline
\end{tabular}

1. Australien und Neuseeland : BIP für das Haushaltsjahr berechnet.

2. Länder, für die das BIP nicht für den selben Referenzzeitraum wie die Daten zu den Bildungsfinanzen berichtet wurde, wurde das BIP als $(w t-1) *(B I P t-1)+w t *(B I P t)$ geschätzt, wobei wt und wt-1 als Gewichte für die entsprechenden Anteile der beiden Referenzzeiträume für das BIP innerhalb des Finanzjahrs für Bildung fungieren. Für Japan, Kanada und das Vereinigte Königreich wurden in Kapitel B Anpassungen vorgenommen. 3. Ohne Übersee-Departments (DOM). 
Tabelle X2.3.

Grundlegende statistische Bezugsdaten (Referenzzeitraum: Kalenderjahr 1999, zu Marktpreisen von 1999)

\begin{tabular}{|c|c|c|c|c|c|c|}
\hline & $\begin{array}{c}\text { Bruttoinlands- } \\
\text { produkt, berichtet für } \\
\text { das Kalenderjahr (in } \\
\text { Millionen, Lan- } \\
\text { deswährung) } \\
\end{array}$ & 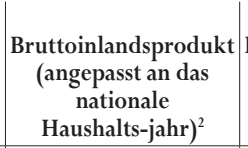 & $\begin{array}{c}\text { Bruttoinlandsprodukt } \\
\text { (zu konstanten } \\
\text { Preisen für 1999, } \\
\text { Basisjahr 1995) }^{1}\end{array}$ & $\begin{array}{c}\text { Öffentliche Gesam- } \\
\text { tausgaben } \\
\text { (in Millionen, Lan- } \\
\text { deswährung) }\end{array}$ & $\begin{array}{c}\text { Gesamtbevölkerung } \\
\text { in Tausend } \\
\text { (Schätzung zur Mitte } \\
\text { des Jahres) }\end{array}$ & $\begin{array}{c}\text { Kaufkraftparitäten } \\
\text { (KKP) }\end{array}$ \\
\hline Australien $^{1}$ & 502828 & 502828 & 597681 & 188394 & 18072 & 1.29 \\
\hline Österreich & 2370726 & 2370726 & 2614606 & 1294685 & 8047 & 13.73 \\
\hline Belgien & 8161733 & 8161733 & 9010388 & 4330957 & 10137 & 36.74 \\
\hline Tschechische Rep. & 1381049 & 1381049 & 1406725 & 783678 & 10327 & 10.81 \\
\hline Dänemark & 1009756 & 1009756 & 1117464 & 596033 & 5222 & 8.42 \\
\hline Finnland & 564566 & 564566 & 684100 & 321141 & 5108 & 5.86 \\
\hline Frankreich $^{3}$ & 7662391 & 7662391 & 8401029 & 4104369 & 58020 & 6.46 \\
\hline Deutschland & 3523000 & 3523000 & 3737800 & 1928460 & 81661 & 2.02 \\
\hline Griechenland & 27235205 & 27235205 & 30885829 & 14895505 & 10454 & 203.08 \\
\hline Ungarn & 5614042 & 5614042 & 6498680 & 2327299 & 10229 & 60.55 \\
\hline Irland & 41502 & 41502 & 59670 & 16111 & 3601 & 0.63 \\
\hline Italien & 1787278000 & 1787278000 & 1906388000 & 936613000 & 57301 & 1550.31 \\
\hline Japan & 497739400 & 483738700 & 521986724 & 180014200 & 125570 & 169.94 \\
\hline Korea & 377349800 & 377349800 & 437709420 & 74550100 & 45093 & 730.50 \\
\hline Luxemburg & 533300 & 533300 & 675464 & 245719 & 413 & 38.87 \\
\hline Mexiko & 1837019 & 1837019 & 2247589 & 380924 & 90903 & 2.96 \\
\hline Niederlande & 666035 & 666035 & 771195 & 368872 & 15460 & 2.03 \\
\hline Neuseeland' & 92679 & 92679 & 102215 & 36441 & 3656 & 1.47 \\
\hline Norwegen & 928745 & 928745 & 1055851 & 457033 & 4358 & 9.14 \\
\hline Polen & 308104 & 308104 & 380701 & 147561 & 38588 & 1.14 \\
\hline Portugal & 16201007 & 16201007 & 18896766 & 6970107 & 9917 & 119.07 \\
\hline Slowakische Rep. & 546032 & 546032 & 653309 & $\mathrm{~m}$ & 5364 & 11.90 \\
\hline Spanien & 72841700 & 72841700 & 84332000 & 32046100 & 39223 & 122.08 \\
\hline Türkei & 7762456000 & 7762456000 & 8774067634 & $\mathrm{~m}$ & 61646 & 22334.21 \\
\hline Ver. Königreich & 719176 & 678972 & 803019 & 317104 & 58612 & 0.65 \\
\hline Vereinigte Staaten & 7338400 & 7166250 & 8626700 & $\mathrm{~m}$ & 263073 & 1.00 \\
\hline
\end{tabular}

1. Australien und Neuseeland : BIP für das Haushaltsjahr berechnet.

2. Länder, für die das BIP nicht für den selben Referenzzeitraum wie die Daten zu den Bildungsfinanzen berichtet wurde, wurde das BIP als (wt-1)*(BIPt-1) + wt*(BIPt) geschätzt, wobei wt und wt-1 als Gewichte für die entsprechenden Anteile der beiden Referenzzeiträume für das BIP innerhalb des Finanzjahrs für Bildung fungieren. Für Japan, Kanada und das Vereinigte Königreich wurden in Kapitel B Anpassungen vorgenommen.

3. Ohne Übersee-Departments (DOM). 
Tabelle X2.4.

Statistische Bezugsdaten zur Berechnung der Lehrergehälter

\begin{tabular}{|c|c|c|c|c|c|c|c|}
\hline & $\begin{array}{c}\text { Kaufkraftparitäten } \\
(1999 / 2000)^{1}\end{array}$ & $\begin{array}{c}\text { Kaufkraftparitäten } \\
(2000)^{2}\end{array}$ & \begin{tabular}{|c|} 
Bruttoinlands- \\
produkt, berichtet \\
für das Kalender- \\
jahr (in Millionen, \\
Landeswährung, \\
Kalenderjahr 2000)
\end{tabular} & $\begin{array}{c}\text { Gesamtbervölke- } \\
\text { rung in Tausend } \\
\text { (Kalenderjahr 2000) }\end{array}$ & $\begin{array}{l}\text { BIP pro Kopf } \\
\text { (kaufkraft- } \\
\text { bereinigt, Kalen- } \\
\text { derjahr 2000) }\end{array}$ & $\begin{array}{l}\text { Referenzjahr für } \\
\text { Daten zu den } \\
\text { Lehrergehältern }\end{array}$ & $\begin{array}{c}\text { Anpassung an die } \\
\text { Inflation }\end{array}$ \\
\hline Australien & 1.30 & 1.31 & 672796 & 19157 & 26800 & 2000 & 0.978 \\
\hline Österreich & 13.53 & 13.48 & 2818695 & 8110 & 25788 & $1998 / 1999$ & 1.012 \\
\hline Belgien (Fl.) & 36.92 & 36.77 & 10017934 & 10254 & 26570 & $1999 / 2000$ & 1.000 \\
\hline Belgien (Frz.) & 36.92 & 36.77 & 10017934 & 10254 & 26570 & $1999 / 2000$ & 1.000 \\
\hline Tschechische Rep. & 13.44 & 13.38 & 1959479 & 10272 & 14262 & $1999 / 2000$ & 1.000 \\
\hline Dänemark & 8.60 & 8.66 & 1315526 & 5338 & 28448 & April 12000 & 0.991 \\
\hline England & 0.65 & 0.65 & 934924 & 59766 & 23966 & & 1.000 \\
\hline Finnland & 6.17 & 6.20 & 782876 & 5176 & 24414 & 2000 & 0.984 \\
\hline Frankreich & 6.59 & 6.55 & 9214720 & 60431 & 23276 & $1999 / 2000$ & 1.000 \\
\hline Deutschland & 1.96 & 1.93 & 3961600 & 82168 & 24931 & $1999 / 2000$ & 1.000 \\
\hline Griechenland & 240.21 & 241.44 & 41406732 & 10558 & 16244 & 1999 & 1.017 \\
\hline Ungarn & 103.61 & 106.48 & 13075210 & 10024 & 12251 & $1999 / 2000$ & 1.000 \\
\hline Island & 86.09 & 86.74 & 673660 & 281 & 27608 & 1999 & 1.015 \\
\hline Irland & 0.73 & 0.74 & 81489 & 3787 & 28895 & $1999 / 2000$ & 1.000 \\
\hline Italien & 1601.18 & 1602.74 & 2257066000 & 57728 & 24395 & $1999 / 2000$ & 1.000 \\
\hline $\mathrm{Japan}^{3}$ & 155.19 & 152.27 & 511835900 & 126919 & 26484 & 1999 & 1.000 \\
\hline Korea $^{3}$ & 631.57 & 620.22 & 517096590 & 47275 & 17636 & 2000 & 1.000 \\
\hline Mexiko & 5.85 & 6.09 & 5426786 & 97221 & 9164 & & 1.000 \\
\hline Niederlande & 2.00 & 2.01 & 883884 & 15920 & 27662 & $1999 / 2000$ & 1.000 \\
\hline Neuseeland & 1.47 & 1.47 & 111776 & 3831 & 19808 & $1999 / 2000$ & 1.000 \\
\hline Norwegen & 10.16 & 10.82 & 1423864 & 4491 & 29311 & 1999 & 1.078 \\
\hline Portugal & 129.45 & 130.15 & 22860162 & 10005 & 17556 & $1999 / 2000$ & 1.000 \\
\hline Scotland & 0.65 & 0.65 & 934924 & 59766 & 23966 & $1999 / 2000$ & 1.000 \\
\hline Spanien & 131.27 & 132.17 & 101293600 & 39927 & 19194 & 1999 & 1.017 \\
\hline Schweden & 9.72 & 9.66 & 2082748 & 8871 & 24308 & $1999 / 2000$ & 1.000 \\
\hline Schweiz & 1.89 & 1.88 & 404392 & 7185 & 29892 & 1998/1999 & 1.011 \\
\hline Türkei & 229780.57 & 273987.20 & 124982454000 & 66835 & 6825 & 1999 & 1.227 \\
\hline Vereinigte Staaten & 1.00 & 1.00 & 9839200 & 275423 & 35724 & $1999 / 2000$ & 1.000 \\
\hline
\end{tabular}




\section{Allgemeine Hinweise}

\section{Definitionen}

Die Arbeitslosenquote ist berechnet als der Anteil Arbeitsloser an der Erwerbsbevölkerung in Prozent. Arbeitslosigkeit wird in diesem Zusammenhang nach den Definitionen des Internationalen Arbeitsamtes (ILO) definiert. Die Erwerbsquote für eine bestimmte Altersgruppe bezieht sich auf den Anteil von Einzelpersonen an der Bevölkerungsgruppe des gleichen Alters (in \%), die entweder beschäftigt oder arbeitslos sind, wobei diese Begriffe gemäß den ILO-Richtlinien definiert sind. Die Quoten für bestimmte Altersgruppen werden entsprechend definiert.

Das Bruttoinlandsprodukt (BIP) entspricht der Wertschöpfung der von inländischen Herstellern produzierten Waren und Dienstleistungen, einschließlich Handel und Transport, abzüglich des Werts für den Zwischenverbrauch des Käufers plus Importzölle. Das BIP wird in der jeweiligen Landeswährung (in Millionen) angegeben. Für Länder, die diese Informationen für ein Bezugsjahr angeben, das vom Kalenderjahr abweicht (z.B. Australien und Neuseeland), werden Anpassungen durch lineare Gewichtung des jeweiligen BIP zwischen zwei aufeinanderfolgenden nationalen Bezugsjahren entsprechend dem Kalenderjahr vorgenommen.

Der BIP-Deflator wird berechnet durch Division des BIP zu Marktpreisen durch das BIP zu konstanten Preisen. Er liefert einen Hinweis auf das relative Preisniveau in einem Land. Die Daten basieren auf dem Jahr 1995.

Das BIP pro Kopf ist das Bruttoinlandsprodukt (in US-Dollar, kaufkraftbereinigt) dividiert durch die Bevölkerung.

Die Kaufkraftparitäts-Umrechnungskurse (KKP) sind dieWährungsumrechnungskurse, die die Kaufkraft verschiedener Währungen ausgleichen. Dies bedeutet, dass man mit einer bestimmten Geldsumme, wenn sie anhand der KKP-Kurse in die verschiedenen Währungen umgerechnet wird, in allen Ländern den gleichen Waren- und Dienstleistungskorb erwerben kann. Daher sind es die KKP-Währungsumrechnungskurse, die die Preisniveau-Unterschiede zwischen den Ländern aufheben. Werden Ausgaben bezogen auf das BIP für verschiedene Länder mit Hilfe der KKP in eine einheitliche Währung umgerechnet (z.B. „in US-Dollar, kaufkraftbereinigt"), werden sie praktisch anhand der gleichen internationalen Preise ausgedrückt, so dass Vergleiche zwischen den Ländern nur die Unterschiede im Volumen der gekauften Waren und Dienstleistungen widerspiegeln.

Die öffentlichen Gesamtausgaben, wie bei der Berechnung der Indikatoren verwendet, entsprechen den nicht rückzahlbaren laufenden Ausgaben und Investitionsausgaben auf allen Ebenen des Staates. Die laufenden Ausgaben umfassen die konsumtiven Ausgaben (z.B. Arbeitsentgelte für Mitarbeiter, Verbrauch von Vorprodukten und -dienstleistungen, Verbrauch von Sachvermögen und Militärausgaben), geleistete Besitzeinkommen, Subventionen und andere geleistete laufende Transferzahlungen (z.B. Sozialversicherung, soziale Unterstützung, Renten und andere Unterstützungsleistung en). Investitionsausgaben sind Ausgaben zum Erwerb und/oder der Wertsteigerung von Gütern des Anlagevermögens, Grundstücken, immateriellen Vermögensgegenständen, Staatsanleihen und nicht-militärischen Sachvermögen und Ausgaben zur Finanzierung von Nettokapitaltransfers.

\section{Quellen}

Die Ausgabe des Jahres 2002 der National Accounts of OECD countries: Main Aggregates,Volume I

Der theoretische Rahmen der OECD National Accounts wurde viele Jahre von der UN-Publikation A System of National Accounts vorgegeben, die 1968 erschien. Im Jahr 1993 erschien eine überarbeitete Fassung (häufig als SNA93 bezeichnet).

OECD Analytical Data Base, Januar 2002 


\section{Anhang}

\section{QUELLEN, METHODEN UND TECHNISCHE HINWEISE}

Anhang 3 zu Quellen und Methoden ist nur in elektronischer Form erhältlich. Er kann aufgerufen werden unter www.oecd.org/els/ education/eag2002. 


\section{GLOSSAR}

Abschluss: Der Begriff Abschluss wird von den Ländern nicht einheitlich definiert: In manchen Ländern erhält man einen Abschluss als Folge einer oder mehrerer bestandener Prüfungen. In anderen Ländern wird der Abschluss nach Ableistung einer vorgeschriebenen Anzahl von Unterrichtsstunden erreicht (auch wenn der Abschluss eines Teils oder aller Unterrichtsstunden auch Prüfungen erfordern kann). Siehe auch Absolventen, Brutto-Abschlussquoten und Netto-Abschlussquoten.

Abschlussquote: Siehe Brutto-Abschlussquoten und Netto-Abschlussquoten.

Absolventen: Absolventen sind definiert als Schüler oder Studierende, die im Abschlussjahr eines Bildungsbereichs (z.B. des Sekundarbereich II) an einem Bildungsgang teilnahmen und diesen im Bezugsjahr, unabhängig von ihrem Alter, erfolgreich beendeten. Es gibt jedoch Ausnahmen (insbesondere im Hochschulbereich), wo durch die Verleihung eines Zertifikats ein Abschluss auch anerkannt werden kann, ohne dass der Absolvent in dem betreffenden Bildungsgang eingeschrieben sein muss. Siehe auch Abschluss, Brutto-Abschlussquoten und Netto-Abschlussquoten.

Allgemeinbildende Bildungsgänge: Allgemeinbildende Bildungsgänge sollen die Teilnehmer weder explizit auf bestimmte Berufsfelder noch auf den Eintritt in einen weiterführenden berufsbildenden oder technischen Bildungsgang vorbereiten. Weniger als 25 Prozent des Inhalts des Bildungsgangs sollten berufsbildend oder technisch sein. Siehe auch Berufsvorbereitende Bildungsgänge, Ausrichtung eines Bildungsgangs, Sekundarbereich II (ISCED 3) und Berufsbildende Bildungsgänge.

Arbeitslose: Die Arbeitslosen sind gemäß den ILO-Richtlinien als Personen definiert, die ohne Arbeit und arbeitssuchend sind und derzeit dem Arbeitsmarkt zur Verfügung stehen. Siehe auch Beschäftigte, Erwerbsbevölkerung, Erwerbsquote, Arbeitslosenquote und Erwerbsstatus.

Arbeitslosenquote: Die Arbeitslosenquote ist gemäß den ILO-Richtlinien definiert als die Anzahl der Arbeitslosen dividiert durch die Anzahl der Erwerbspersonen (x 100), die Angabe erfolgt in Prozent. Siehe auch Beschäftigte, Erwerbsbevölkerung, Erwerbsquote und Arbeitslose.

Arbeitszeit: Die Arbeitszeit bezieht sich auf die regulären Arbeitsstunden eines Vollzeit-Lehrers. Gemäß den formellen Vorschriften in einem bestimmten Land kann sich die Arbeitszeit beziehen auf ausschließlich für den Unterricht aufgewendete Zeit (und andere lehrplanbezogene Tätigkeiten für die Schüler, wie Haus- und Klassenarbeiten, jedoch keine jährliche Prüfungen); oder auch Zeit, die in direktem Zusammenhang mit dem Unterricht steht, sowie Stunden, die anderen unterrichtsbezogenen Tätigkeiten gewidmet sind, z.B. Unterrichtsvorbereitung, Beratung der Schüler, Korrekturen von Haus- und Klassenarbeiten, Tätigkeiten zur beruflichen Weiterentwicklung, Besprechungen mit den Eltern, Lehrerkonferenzen und allgemeine schulische Aufgaben Nicht enthalten sind vergütete Überstunden. Siehe auch Vollzeitäquivalente Lehrer, Vollzeit-Lehrer, Teilzeit-Lehrer, Zahlenmäßiges Schüler/ Lehrer-Verhältnis, Unterrichtstage, Lehrkräfte, Zahl der Unterrichtsstunden, Unterrichtswochen und Arbeitszeit in der Schule.

Arbeitszeit in der Schule: Arbeitszeit in der Schule bezieht sich auf die Arbeitszeit, die Lehrer an der Schule verbringen müssen und enthält sowohl die Unterrichtszeit als auch Zeit für Tätigkeiten außerhalb des Unterrichts. Siehe auch Unterrichtstage, Zahl der Unterrichtsstunden, Unterrichtswochen und Arbeitszeit.

Ausbildungs-/Studienanfänger: Ausbildungs-/Studienanfänger in einem bestimmten Bildungsbereich sind Schüler/ Studierende, die erstmalig einen beliebigen Bildungsgang beginnen, der zu einem anerkannten Abschluss des betreffenden Bildungsbereichs führt, unabhängig davon, ob diese Schüler/Studierenden zu Beginn des Bildungsgangs oder in einem fortgeschrittenen Stadium einsteigen. Siehe auch Studienanfängerquoten.

Ausgaben außerhalb von Bildungseinrichtungen: Ausgaben außerhalb von Bildungseinrichtungen sind Ausgaben für außerhalb von Bildungseinrichtungen erworbene Bildungsdienstleistungen, z.B. Bücher, Computer, externer Unterricht, etc. Hierzu gehören auch die Lebenshaltungskosten der Schüler/Studierenden und Kosten für nicht von den Bildungseinrichtungen bereitgestellte Transportmöglichkeiten für Schüler/Studierende.

Ausgaben für Bildungseinrichtungen: Die Ausgaben für Bildungseinrichtungen umfassen sowohl Ausgaben für unterrichterteilende Bildungseinrichtungen als auch für solche, die keinen Unterricht erteilen. Siehe auch Direkte Ausgaben für Bildungseinrichtungen, Unterrichterteilende Bildungseinrichtungen und Nicht-unterrichterteilende Bildungseinrichtungen. 
Ausgaben für eigentliche Bildungsdienstleistungen: Die Ausgaben für eigentliche Bildungsdienstleistungen umfassen alle Ausgaben, die direkt mit Unterricht und Bildung in Zusammenhang stehen. Hierzu sollten alle Ausgaben für Lehrer, Schulgebäude, Untersichtsmaterial, Unterricht außerhalb der Schulen und die Verwaltung gehören. Siehe auch Ausgaben für zusätzliche Dienstleistungen und Ausgaben für Forschung und Entwicklung (F\&E-Ausgaben).

Ausgaben für Forschung und Entwicklung (F\&E-Ausgaben): Die Ausgaben für Forschung und Entwicklung (F\&EAusgaben) enthalten sämtliche Ausgaben für Forschung an Hochschulen und anderen tertiären Bildungseinrichtungen, unabhängig davon, ob diese aus dem allgemeinen Etat der Einrichtungen, über separate Zuschüsse oder über Verträge mit öffentlichen oder privaten Geldgebern finanziert werden. Dies umfasst alle Forschungsinstitute und Versuchsstationen, die Hochschuleinrichtungen direkt unterstehen, von diesen verwaltet werden oder mit ihnen assoziiert sind. Siehe auch Ausgaben für zusätzliche Dienstleistungen und Ausgaben für eigentliche Bildungsdienstleistungen.

Ausgaben für zusätzliche Dienstleistungen: ,Zusätzliche Dienstleistungen' sind Dienstleistungen, die von den Bildungseinrichtungen neben dem eigentlichen Bildungsauftrag erbracht werden. Die beiden Hauptkomponenten sind soziale Dienste für Schüler/Studierende und Dienstleistungen für die Allgemeinheit. Im Primar-, Sekundar- und post-sekundären, nicht-tertiären Bereich umfassen die sozialen Dienstleitungen die Bereitstellung von Mahlzeiten, die Gesundheitsdienste sowie Schultransporte. Im Tertiärbereich sind es Wohnheime, Mensen und Gesundheitsdienste. Zu den Dienstleistungen für die Allgemeinheit zählen Museen, Radio- und Fernsehsendungen, Sport-, Freizeit- und Kulturprogramme. Kinderbetreuungsangebote, sowohl tagsüber als auch abends, durch Einrichtungen des Elementar- und Primarbereichs zählen nicht zu den zusätzlichen Dienstleistungen. Zu den Einheiten, die zusätzliche Dienstleistungen erbringen, gehören separate Organisationen und Unternehmen, die bildungsbezogene Dienstleistungen wie Berufs- oder psychologische Beratung, Vermittlung von Stellen und Praktika, Transport von Schülern/Studierenden, sowie Unterkunft und Verpflegung für Schüler/Studierende anbieten. Siehe auch Ausgaben für eigentliche Bildungsdienstleistungen und Ausgaben für Forschung und Entwicklung (F\&E-Ausgaben).

Ausgaben über die durchschnittliche Dauer tertiärer Studiengänge: Die erwarteten Ausgaben über die durchschnittliche Dauer tertiärer Studiengänge werden durch Multiplikation der aktuellen jährlichen Ausgaben mit der typischen Dauer solcher Studiengänge berechnet.

Ausländische Studierende: Studierende, die nicht Staatsangehörige des Landes sind, für das die Daten erhoben werden, gelten als ausländische Studierende. Diese Klassifikation ist zwar pragmatisch und operational, kann jedoch aufgrund der unterschiedlichen nationalen Politiken zur Einbürgerung von Migranten zu Inkonsistenzen führen. Hinzu kommt, dass einige Länder keine separaten Angaben über ausländische Studierende machen können, die eine ständige Aufenthaltsgenehmigung besitzen. Daher wird in den Ländern, in denen eine strenge Einbürgerungspolitik verfolgt wird und nicht zwischen ausländischen Studierenden mit und ohne ständige Aufenthaltsgenehmigung unterschieden werden kann, die Anzahl der ausländischen Studierenden im Vergleich zu den Ländern, in denen Einwanderer leichter die Staatsbürgerschaft erwerben können, möglicherweise zu hoch angesetzt.

Ausrichtung eines Bildungsgangs: Die Ausrichtung eines Bildungsgangs in der Definition der Internationalen StandardKlassifikation des Bildungswesen (ISCED) bezieht sich darauf, inwieweit ein Bildungsgang speziell auf eine bestimmte Art von Berufen oder Tätigkeiten ausgerichtet ist und hier zu einer arbeitsmarktrelevanten Qualifikation führt. Siehe auch Allgemeinbildende Bildungsgänge, Berufsvorbereitende Bildungsgänge und Berufsbildende Bildungsgänge.

Berufsbezogene Fort- und Weiterbildung: Berufsbezogene Fort- und Weiterbildung umfasst alle organisierten, systematischen Fort- und Weiterbildungsmaßnahmen, an denen Personen teilnehmen, um für einen momentanen oder zukünftigen Arbeitsplatz ihre Kenntnisse zu erweitern und/oder neue Fähigkeiten zu erlernen, ihr Einkommen zu erhöhen und ihre Berufsund/oder Karrierechancen auf jetzigen oder anderen Fachgebieten zu verbessern.

Berufsbildende Bildungsgånge: Berufsbildende Bildungsgänge bereiten die Teilnehmer für die direkte Aufnahme einer Beschäftigung in bestimmten Berufsfeldern, ohne weitere berufliche Qualifizierung, vor. Der erfolgreiche Abschluss eines solchen Bildungsganges führt zu einer für den Arbeitsmarkt relevanten beruflichen Qualifikation. Bei einigen Indikatoren wird bei den berufsbildenden Bildungsgängen zwischen schulischen Ausbildungen und kombinierten schulischen und betrieblichen Ausbildungen unterschieden, und zwar auf der Grundlage des jeweiligen Ausbildungsumfangs in Bildungseinrichtungen und am Arbeitsplatz. Siehe auch Kombinierte schulische und betriebliche Ausbildungen, Allgemeinbildende Bildungsgänge, Berufsvorbereitende Bildungsgänge, Ausrichtung eines Bildungsgangs, Schulische Ausbildungsgänge und Sekundarbereich II (ISCED 3).

Berufsvorbereitende Bildungsgänge: Berufsvorbereitende Bildungsgänge sollen den Teilnehmern eher als Einführung in die Arbeitswelt dienen und sie für den späteren Eintritt in einen berufsbildenden oder technischen Bildungsgang vorbereiten. Durch den erfolgreichen Abschluss wird keine für den Arbeitsmarkt relevante berufliche oder technische Qualifikation erworben. Siehe 
auch Allgemeinbildende Bildungsgänge, Ausrichtung eines Bildungsgangs, Sekundarbereich II (ISCED 3) und Berufsbildende Bildungsgänge.

Beschäftigte: Beschäftigte sind gemäß der Definition in den Richtlinien des Internationalen Arbeitsamts (ILO) diejenigen, die während der untersuchten Bezugswoche: mindestens eine Stunde für ein Gehalt (Arbeitnehmer) oder für einen Gewinn (Selbständige und unentgeltlich mithelfende Familienangehörige) arbeiten, oder einen Arbeitsplatz haben aber vorübergehend nicht zur Arbeit gehen (aufgrund von Verletzung, Krankheit, Urlaub oder Ferien, Streik oder Aussperrung, Bildungs- oder Schulungsurlaub, Mutterschafts- oder Erziehungsurlaub, usw.) und eine formelle Bindung an ihren Arbeitsplatz haben. Siehe auch Erwerbsbevölkerung, Erwerbsquote, Arbeitslose, Arbeitslosenquote und Erwerbsstatus.

Bildungsbeteiligung: Die Bildungsbeteiligung wird als Netto-Bildungsbeteiligung angegeben, die berechnet wird, indem die Zahl der Lernenden einer bestimmten Altersgruppe in allen Bildungsbereichen durch die Gesamtzahl der Personen in der entsprechenden Altersgruppe in der Bevölkerung dividiert wird.

Bildungseinrichtungen: Bildungseinrichtungen sind definiert als eine Einheit, die Einzelpersonen Unterrichtsleistungen bzw. Einzelnen und anderen Einrichtungen bildungsbezogene Dienstleistungen anbietet. Siehe Private Bildungseinrichtungen und Öffentliche Bildungseinrichtungen.

Bildungserwartung (in Jahren): Die Bildungserwartung (in Jahren) ist die voraussichtliche durchschnittliche Dauer der formellen Bildung eines 5-jährigen Kindes während seines gesamten Lebens. Die Berechnung erfolgt durch Addition der NettoBildungsbeteiligung für jede einzelne Altersstufe ab dem 5. Lebensjahr.

Bildungsstand: Der Bildungsstand wird ausgedrückt durch den höchsten abgeschlossenen Bildungsbereich, wobei die Bildungsbereiche gemäß der Internationalen Standard-Klassifikation des Bildungswesens (ISCED) definiert sind.

BIP: Siehe Bruttoinlandsprodukt.

Brutto-Abschlussquoten: Die Brutto-Abschlussquoten beziehen sich auf die Gesamtzahl der Absolventen des spezifischen Bildungsbereichs (die jeden Alters sein können) dividiert durch die Bevölkerung im typischen Abschlussalter des Bildungsbereichs. In vielen Ländern ist es jedoch schwierig, ein typisches Abschlussalter anzugeben, weil die Altersverteilung der Absolventen sehr weit gestreut ist. Siehe auch Absolventen, Abschluss und Netto-Abschlussquoten.

Bruttoinlandsprodukt (BIP): Das Bruttoinlandsprodukt bezieht sich auf den Herstellungswert der Bruttoproduktion inländischer Produzenten, einschließlich Vertrieb undTransport, abzüglich des Werts des Zwischenverbrauchs der Endverbraucher, zuzüglich Einfuhrzölle. Das Bruttoinlandsprodukt wird in der Landeswährung (in Millionen) ausgedrückt. Für Länder, die diese Informationen für ein Bezugsjahr angeben, das vom Kalenderjahr abweicht (z.B. Australien und Neuseeland), werden Anpassungen durch lineare Gewichtung ihres BIP zwischen zwei aufeinanderfolgenden nationalen Bezugsjahren entsprechend dem Kalenderjahr vorgenommen. Die BIP-Daten sind Anhang 2 zu entnehmen.

Dauer von Bildungsgängen: Die Dauer von Bildungsgängen bezieht sich auf die festgelegte Anzahl von Jahren, in denen ein Bildungsgang abgeschlossen werden kann.

Direkte Ausgaben für Bildungseinrichtungen: Direkte Ausgaben für Bildungseinrichtungen umfassen den durch eine staatliche Stelle selbst vorgenommenen Erwerb von Bildungsressourcen zurVerwendung durch Bildungseinrichtungen (z.B. direkte Zahlungen von Gehältern an Lehrer durch ein zentrales oder regionales Bildungsministerium, direkte Zahlungen einer Kommune an Baufirmen zur Errichtung von Schulgebäuden, und die Beschaffung von Lehrbücher durch eine zentrale oder regionale Behörde zur späteren Verteilung an lokale Behörden oder Schulen), sowie Zahlungen einer staatlichen Stelle an Bildungseinrichtungen, die für den Erwerb von Bildungsressourcen selbst verantwortlich sind (z.B. vom Staat bereitgestellte Haushaltsmittel oder pauschale Zuschüsse für eine Hochschule, die diese zur Besoldung des Personals und zum Erwerb sonstiger Ressourcen verwendet, staatliche Zuweisungen an finanziell autonome öffentliche Schulen, staatliche Subventionen an private Schulen sowie staatliche Zahlungen für vertraglich an private Unternehmen vergebene Forschungsaufträge im Bereich Bildung). Zu den direkten Ausgaben einer staatlichen Stelle zählen nicht die Schul- und Studiengebühren, die diese Behörde von Schülern/Studierenden an öffentlichen Bildungseinrichtungen in ihrem Zuständigkeitsbereich erhält (oder von deren Familien), auch wenn die Schul- und Studiengebühren zunächst einmal an die staatliche Stelle gezahlt werden, und nicht an die betreffende Bildungseinrichtung. Siehe auch Unterrichterteilende Bildungseinrichtungen und Nicht-unterrichterteilende Bildungseinrichtungen.

Dritte Internationale Mathematik- und Naturwissenschaften-Studie (TIMSS): Die von der IEA durchgeführte Dritte Internationale Mathematik- und Naturwissenschaften-Studie testete 1995 und 1999 die mathematischen und naturwissenschaftlichen Leistungen von Viert- und Achtklässlern, die Wiederholungsstudie wird 2003 durchgeführt werden. 
Duale Ausbildungsprogramme: Duale Ausbildungsprogramme stellen eine Kombination aus Phasen des Arbeitens und des Lernens dar, die beide Bestandteil einer integrierten, formellen Bildung bzw. Ausbildung sind. Zu solchen Programmen zählen beispielsweise das „duale System“ in Deutschland, die „apprentissage“ oder „formation en alternance“ in Frankreich und Belgien, Praktika oder ‘kooperative’ Ausbildungen in Kanada, „apprenticeship“ in Irland und „youth training“ im Vereinigten Königreich.

Elementarbereich (ISCED 0): Der Elementarbereich ist definiert als erste Stufe organisierten Unterrichts, der sehr kleine Kinder an eine schulähnliche Umgebung heranführen soll, d.h. er soll eine Brücke zwischen der Atmosphäre im Elternhaus und der in der Schule herstellen. Programme auf ISCED-Stufe 0 sollten in Einrichtungen oder Schulen stattfinden, die dazu geeignet sind, den Bedürfnissen von mindestens 3 Jahre alten Kindern hinsichtlich ihrer Erziehung und Bildung sowie Entwicklung gerecht zu werden, und über entsprechend ausgebildetes Personal verfügen, um für Kinder dieser Altersgruppe adäquate Angebote durchzuführen. Siehe auch Internationale Standard-Klassifikation des Bildungswesens (ISCED).

Erfolgsquoten: Erfolgsquoten im Tertiärbereich werden definiert als Prozentsatz derjenigen Studienanfänger im jeweiligen Bildungsbereich, die diesen mit einem ersten Abschluss beenden. Die Erfolgsquote wird berechnet als das Verhältnis der Anzahl der Studierenden, die einen ersten Abschluss erwerben, zur Anzahl der Studienanfänger im entsprechenden Bildungsbereich vor $n$ Jahren, wobei $n$ der Anzahl an Jahren entspricht, die zum Erwerb des Abschlusses in einem Vollzeitstudium erforderlich sind. Siehe auch Studienabbrecher.

Erwerbsbevölkerung: Die Erwerbsbevölkerung insgesamt oder die derzeitige Erwerbsbevölkerung, definiert gemäß den ILO-Richtlinien, umfasst alle Personen, die gemäß der Definition in der OECD-Arbeitsmarktstatistik die Voraussetzungen für die Zugehörigkeit zur Gruppe der Beschäftigten oder Arbeitslosen erfüllen. Siehe auch Erwerbsstatus.

Erwerbseinkommen: Erwerbseinkommen sind die jährlichen monetären Einkommen, die direkte Zahlungen für erbrachte Arbeitsleistungen darstellen, vor Steuern. Einkommen aus anderen Quellen, wie staatliche Transferzahlungen, Kapitalerträge, die Netto-Wertsteigerung eines vom Eigentümer betriebenen Geschäfts/Unternehmens, und andere Einkommensarten, die nicht direkt mit Arbeit in Verbindung stehen, sind nicht erfasst. Siehe auch Relative Erwerbseinkommen.

Erwerbsquote: Die Erwerbsquote, die gemäß den Richtlinien des Internationalen Arbeitsamts (ILO) definiert ist, entspricht dem Prozentsatz der Personen in der Bevölkerung der gleichen Altersgruppe, die entweder beschäftigt oder arbeitslos sind. Siehe auch Beschäftigte, Erwerbsbevölkerung, Arbeitslose und Arbeitslosenquote.

Erwerbsstatus: Der Erwerbsstatus gemäß der Definitionen der ILO-Richtlinien bezieht sich auf den Status innerhalb der Erwerbsbevölkerung wie in den OECD-Arbeitskräftestatistiken festgelegt. Siehe auch Beschäftigte, Erwerbsbevölkerung und Arbeitslose.

Finanzhilfen für Studierende: Die Finanzhilfen für Studierende umfassen zum einen staatliche Stipendien und andere staatliche Zuschüsse an Studierende oder Privathaushalte. Dazu zählen neben Stipendien und ähnlichen Zuschüssen (Forschungszuschüsse, Auszeichnungen, Preise) der Wert von speziellen an Studierende in Bar- oder Sachleistungen gezahlten Unterstützungen, wie kostenlose oder ermäßigte Fahrten mit öffentlichen Verkehrsmitteln, sowie Familienbeihilfen oder Kindergeldzahlungen, die abhängig vom Schüler-/Studierendenstatus gezahlt werden. Nicht berücksichtigt werden jegliche Vergünstigungen für Schüler/Studierende oder Privathaushalte in Form von Steuerermäßigungen, Steuersubventionen oder anderen steuerrechtlichen Sonderregelungen. Zum anderen gehören zu den Finanzhilfen Schüler-/Studierendendarlehen, die brutto angegeben werden, d.h. ohne Abzug oder Herausrechnung von Rück- oder Zinszahlungen seitens der Darlehensnehmer (Schüler/Studierende oder Privathaushalte).

Fort- und Weiterbildung: Zum Zwecke dieser Veröffentlichung ist Fort- und Weiterbildung definiert als jede Art von allgemeiner und berufsbezogener Fort- und Weiterbildung, die von Behörden organisiert, finanziert oder unterstützt, von Arbeitgebern angeboten oder von den Teilnehmern selbst bezahlt wird.

Gehälter von Lehrern: Die Gehälter werden als gesetzliche bzw. vertraglich vereinbarte Gehälter angegeben und beziehen sich auf das reguläre Gehalt gemäß offizieller Besoldungs- und Vergütungsgruppen. Die angegebenen Gehälter sind definiert als die Bruttogehälter (die vom Arbeitgeber für die Arbeit bezahlte Gesamtsumme) abzüglich der Arbeitgeberbeiträge zur Sozialund Rentenversicherung (gemäß bestehender Besoldungs- und Vergütungsgruppen).

- Die angegebenen Anfangsgehälter beziehen sich auf das reguläre durchschnittliche Bruttojahresgehalt eines Vollzeitlehrers am Anfang der Lehrerlaufbahn mit der für eine Lehrerqualifikation erforderlichen Mindestausbildung.

- Die Gehälter für Lehrer mit 15 Jahren Berufserfahrung beziehen sich auf das reguläre Jahresgehalt eines Vollzeitlehrers mit der für eine Lehrerqualifikation erforderlichen Mindestausbildung und 15 Jahren Berufserfahrung. 
- Die erfassten Höchstgehälter beziehen sich auf das reguläre maximale Jahresgehalt (an der Spitze der Vergütungs-/ Besoldungsordnung) eines Vollzeit-Lehrers mit dem für seine Stelle mindestens erforderlichen Ausbildungsstand.

Die Gehälter sind angegeben vor Abzug der Einkommenssteuern. Siehe auch Zulagen zum Grundgehalt.

Geleistete Netto-Kapitaltransferzahlungen: Sie umfassen Kapitaltransferzahlungen an private Haushalte des Inlands und ins Ausland abzüglich von Kapitaltransferzahlungen, die von privaten Haushalten des Inlands und aus dem Ausland erhalten wurden.

Gesamtbevölkerung: Die Gesamtbevölkerung umfasst alle Staatsbürger eines Landes, die in diesem Land leben oder nur vorübergehend abwesend sind, sowie Ausländer, die dauerhaft in diesem Land ansässig sind. Zu weiteren Informationen s. OECDArbeitsmarktstatistik.

Gesellschaftliche Ertragsrate: Die gesellschaftliche Ertragsrate bezieht sich auf die Kosten und den Nutzen, die für die Gesellschaft mit Investitionen in Bildung verbunden sind. Hierzu gehören die Opportunitätskosten dafür, dass Menschen nicht im Produktionsprozess tätig sind sowie die Kosten für das Bildungsangebot in voller Höhe, also nicht nur die vom Einzelnen getragenen Kosten. Ein Teil des gesellschaftlichen Nutzens ist die höhere Produktivität, die mit Bildungsinvestitionen einhergeht, sowie ferner eine ganze Palette potentieller, nicht direkt ökonomischer Nutzeffekte, wie etwa eine niedrigere Kriminalitätsrate, eine bessere Gesundheit, ein stärkerer sozialer Zusammenhalt, informiertere und mündigere Bürger. Siehe auch Individuelle Ertragsrate.

Gesetzliche bzw. vertraglich festgelegte Gehälter von Lehrern: Siehe Gehälter von Lehrern.

Humankapital: Humankapital ist der produktive Bildungsstand in Form von Arbeitskraft, Fähigkeiten und Wissen.

IEA-Studie zur politischen Bildung: Die International Association for the Evaluation of Educational Achievement (IEA) hat eine internationale Vergleichsstudie zur politischen Bildung durchgeführt, die IEA-Studie zur politischen Bildung. Im Rahmen der Studie nahmen 14-Jährige in 28 Ländern, einschließlich 17 OECD-Ländern, an Tests zu ihren Kenntnissen staatsbürgerschaftlicher Inhalte, zu ihren Fähigkeiten des Verständnisses politischer Kommunikation, zu ihren Vorstellungen und Einstellungen zu staatsbürgerlichen Sachverhalten und ihrem Verhalten, bzw. ihrer aktiven Teilnahme in diesem Bereich teil. Man wollte herauszufinden, wie junge Menschen - innerhalb und außerhalb der Schule - auf ihre Rolle als Staatsbürger in einer Demokratie vorbereitet werden.

Individuelle Ertragsrate: Die individuelle Ertragsrate entspricht dem diskontierten Ertrag, der sich ergibt, wenn man den realen Kosten der Ausbildung während der Ausbildung die realen Gewinne aus der Ausbildung zu einem späteren Zeitpunkt gegenüberstellt. In ihrer umfassendsten Form entsprechen die Kosten den Studiengebühren, dem entgangenen Einkommen (versteuert und um die Beschäftigungswahrscheinlichkeit bereinigt), abzüglich der Fördermittel in Form von Beihilfen oder Darlehen. Siehe auch Gesellschaftliche Ertragsrate.

Internationale Schulleistungsstudie PISA: Die von der OECD durchgeführte Schulleistungsstudie PISA ist eine internationale Vergleichsstudie, in der untersucht wurde, wie gut junge Menschen im Alter von 15 Jahren, also gegen Ende der Schulpflicht, auf die Aufgaben und Herausforderungen der heutigen Wissensgesellschaft vorbereitet sind.

Internationale Standardklassifikation der Berufe (ISCO): Die Internationale Standardklassifikation der Berufe (1998) klassifiziert Personen gemäß ihrer tatsächlichen und potentiellen Verbindung mit bestimmten Tätigkeiten (jobs). Die Tätigkeiten werden hinsichtlich der durchgeführten bzw. durchzuführenden Arbeit eingeordnet. Grundlegende Kriterien zur Festlegung der vier Gliederungsebenen (Berufshauptgruppen, Berufsgruppen, Berufsuntergruppen und Berufsgattungen) sind der ,skill level', der Grad der Komplexität der entsprechenden Aufgaben, und die ,skill specialisation', im Grunde das Gebiet, in dem Kenntnisse erforderlich sind, damit die jeweiligen Aufgaben kompetent ausgeführt werden können. Es gibt separate Berufshauptgruppen für ,Angehörige gesetzgebender Körperschaften, leitende Verwaltungsbedienstete und Führungskräfte in der Privatwirtschaft' und für ,Soldaten'.

Internationale Standard-Klassifikation des Bildungswesen (ISCED): Die Internationale Standard-Klassifikation des Bildungswesens (ISCED-97) dient in dieser Veröffentlichung als Grundlage zur Festlegung der Bildungsbereiche und Bildungsgänge. Einzelheiten zu ISCED-97 und ihrer landesspezifischen Umsetzung finden sich in „Classifying Educational Programmes: Manual For ISCED-97 Implementation in OECD Countries“ (Paris, 1999). Siehe auch Elementarbereich (ISCED 0), Primarbereich (ISCED 1), Sekundarbereich I (ISCED 2), Sekundarbereich II (ISCED 3), Post-sekundärer, nicht-tertiärer Bereich (ISCED 4), Tertiärbereich A (ISCED 5A), Tertiärbereich B (ISCED 5B) und Weiterführende Forschungsprogramme (ISCED 6).

Internationaler sozio-ökonomischer Index der beruflichen Stellung (ISEI): Der in der PISA-Studie verwendete Internationale sozio-ökonomische Index der beruflichen Stellung (ISEI) wurde von den Schülerantworten auf die Frage nach dem 
Beruf der Eltern abgeleitet. Der Index erfasst die Attribute von Berufen, die die Bildungsabschlüsse der Eltern in Einkommen umsetzen. Zur Konstruktion dieses Index wurden die Berufsgruppen auf einer linearen Skala so angeordnet, dass der indirekte Einfluss der Bildung auf das Einkommen mittels der Bildungsabschlüsse maximiert und der direkte Einfluss der Bildung auf das Einkommen, unabhängig vom Beruf, minimiert wurde (bei beiden Effekten wurde dem Alter nicht Rechnung getragen). Zu weiteren Informationen über die Methodik s. Ganzeboom et al. (1992). Der in PISA verwendete Internationale sozio-ökonomische Index der beruflichen Stellung basiert auf dem Beruf des Vaters oder der Mutter, je nachdem wer die höhere Stellung hat.

Investitionsausgaben: Investitionsausgaben stellen den Wert des während des betreffenden Jahres erworbenen oder geschaffenen Kapitals für das Bildungswesen (d.h. den Betrag der Kapitalbildung) dar, unabhängig davon, ob der Kapitalaufwand aus laufenden Erträgen oder durch Kreditaufnahme finanziert wurde. Investitionsausgaben umfassen Ausgaben für Bau, Renovierung und umfangreiche Reparaturen von Gebäuden sowie Ausgaben für neue oder Ersatzausstattungen. Die Investitionsausgaben erfordern zwar einen höheren Anfangsaufwand, die Gebäude und Einrichtungen haben jedoch eine Lebensdauer, die sich über viele Jahre erstreckt.

Kaufkraftparitäten (KKP): Kaufkraftparitäten (KKP) sind die Währungsumrechnungskurse, die die Kaufkraft verschiedener Währungen ausgleichen. Dies bedeutet, dass man mit einer bestimmten Geldsumme, wenn sie anhand der KKP in die verschiedenen Währungen umgerechnet wird, in allen Ländern den gleichen Waren- und Dienstleistungskorb erwerben kann. Mit anderen Worten, die KKP sind Währungsumrechnungskurse, die die Preisniveau-Unterschiede zwischen den Ländern aufheben. Werden daher Ausgaben im Verhältnis zum Bruttoinlandsprodukt (BIP) für verschiedene Länder mit Hilfe der KKP in eine gemeinsame Währung umgerechnet, werden sie tatsächlich in der gleichen internationalen Preisgruppe ausgedrückt, so dass Vergleiche zwischen den Ländern nur Unterschiede im Umfang der erworbenen Waren und Dienstleistungen widerspiegeln. Die in der vorliegenden Veröffentlichung verwendeten Kaufkraftparitäten sind in Anhang 2 aufgeführt.

Klassengröße: Die Klassengröße ist die durchschnittliche Zahl von Schülern pro Klasse, sie wird berechnet, indem die Anzahl der Schüler durch die Anzahl der Klassen dividiert wird. Um die Vergleichbarkeit zwischen den Ländern zu gewährleisten, wurden spezielle Förderprogramme nicht erfasst. Die Daten umfassen ausschließlich die regulären Bildungsgänge, Unterricht in Kleingruppen außerhalb des regulären Klassenunterrichts ist nicht erfasst.

Kombinierte schulische und betriebliche Ausbildungen: In kombinierten schulischen und betrieblichen Ausbildungen ist der Unterricht zwischen Bildungseinrichtung und Arbeitsplatz aufgeteilt, erfolgt jedoch hauptsächlich am Arbeitsplatz. Ausbildungen gelten als kombinierte schulische und betriebliche Bildungsgänge, wenn weniger als 75 Prozent des Lehrplans in der Bildungseinrichtung oder in einem Fernkurs behandelt werden. Ausbildungen, bei denen über 90 Prozent im Betrieb erfolgen, werden nicht berücksichtigt. Siehe auch Duale Ausbildungsprogramme, Pfichtteil des Lehrplans, Ausrichtung eines Bildungsgangs, Schulische Ausbildungsgänge und Berufsbildende Bildungsgänge.

Laufende Ausgaben: Laufende Ausgaben sind Ausgaben für Güter und Dienstleistungen, die innerhalb des laufenden Haushaltsjahres verbraucht werden und die jedes Jahr für den laufenden Betrieb dieser Einrichtungen erforderlich sind. Geringfügige Ausgaben für Ausrüstungsgüter unterhalb einer gewissen Kostengrenze werden auch als laufende Ausgaben erfasst. Die laufenden Ausgaben betreffen konsumtive Ausgaben, Pacht-, Miet- und Zinszahlungen, Subventionen sowie sonstige laufende Transferzahlungen (z.B. Sozialversicherungen, Sozialhilfe, Renten und sonstige Wohlfahrtsleistungen).

Lehrkräfte: Der Begriff ,Lehrkräfte’ umfasst voll qualifiziertes Personal, dass direkt mit dem Unterrichten der Schüler befasst ist. Die Kategorie umfasst Lehrkräfte, die im Klassenzimmer arbeiten, Förderlehrer und andere Lehrer, die mit Schülern als ganzer Klasse im Klassenzimmer, in kleinen Gruppen in einem Förderraum oder im Einzelunterricht innerhalb oder außerhalb des regulären Unterrichts arbeiten. Diese Kategorie umfasst auch Fachgebietsleiter, deren Aufgaben ein gewisses Maß an Unterricht beinhalten, während nicht voll-qualifizierte Mitarbeiter, die die Lehrkräfte beim Unterricht unterstützten, wie Hilfslehrkräfte und andere Hilfskräfte, nicht erfasst sind. Siehe auch Vollzeit-Lehrer, Vollzeitäquivalente Lehrer, Teilzeit-Lehrer, Zahlenmäßiges Schüler/ Lehrer-Verhältnis und Zahl der Unterrichtsstunden.

Lernpensum: Für das Lernpensum gibt es zwei Kennzahlen - zum einen die Zeit im Klassenzimmer und zum anderen den Lernfortschritt in Richtung auf eine Qualifikation. Die Zeit im Klassenzimmer soll die Dauer an Unterricht erfassen, die der einzelne Schüler erhält und kann in Form von Unterrichtsstunden proTag oder pro Jahr, die Anzahl der belegten Kurse oder mittels einer Kombination aus beidem gemessen werden. Diese Kennzahlen basieren auf den Merkmalen des Kurses bzw. der Teilnahme, nicht aber auf Merkmalen der Bildungsgangs, an dem der Schüler teilnimmt. Daher ist eine solche Kennzahl hilfreich, wenn es keine feste Struktur des Bildungsgangs gibt oder die Strukturen nicht miteinander vergleichbar sind. Die zweite Kennzahl zum Lernpensum betrifft die Lerneinheit, anhand derer sich ein Fortschreiten in Richtung Qualifikation ablesen lässt. Hierbei liegt der 
Schwerpunkt weniger auf dem Umfang an Unterricht, sondern mehr auf dem ,Bildungs(mehr)wert' des Unterrichts. Es lässt sich leicht nachvollziehen, dass Kurse mit dem gleichen Unterrichtspensum von unterschiedlichem ,Bildungs(mehr)wert' sein können. Dieser wäre nur gleich, wenn der Lernfortschritt in Unterrichtseinheiten gemessen würde. Siehe auch Vollzeitäquivalente Schüler/ Studierende,Vollzeit-Schüler/Studierende,Vollzeit-/Teilzeit-Ausbildung und Teilzeit-Schüler/Studierende.

Lesekompetenz: Lesekompetenz (reading literacy) ist im Rahmen von PISA definiert als die Fähigkeit, geschriebene Texte zu verstehen, zu nutzen und über sie zu reflektieren, um eigene Ziele zu erreichen, das eigeneWissen und Potential weiterzuentwickeln und aktiv am gesellschaftlichen Leben teilzunehmen. Siehe auch Mathematische Grundbildung und Naturwissenschaftliche Grundbildung.

Local Area Network (LAN): Ein LAN ist ein Netzwerk von Computern in einem begrenzten Bereich (z.B. einem Büro, einem Unternehmen), die per Kabel verbunden sind, direkt mit anderen Geräten im Netz kommunizieren und gemeinsam Ressourcen nutzen können.

Mathematische Grundbildung: Mathematische Grundbildung (mathematical literacy) im Rahmen von PISA ist definiert als die Fähigkeit, mathematische Probleme zu identifizieren, zu verstehen und sich mit ihnen zu befassen und fundierte Urteile über die Rolle abzugeben, die die Mathematik im gegenwärtigen und künftigen Privatleben der Betreffenden, im Berufsleben, im sozialen Kontakt mit Peers und Verwandten und im Leben dieser Person als konstruktivem, engagiertem und reflektierendem Bürger spielt. Siehe auch Lesekompetenz und Naturwissenschaftliche Grundbildung.

Naturwissenschaftliche Grundbildung: Im Rahmen von PISA wird naturwissenschaftliche Grundbildung (scientific literacy) als die Fähigkeit definiert, naturwissenschaftliches Wissen anzuwenden, naturwissenschaftliche Fragen zu erkennen und aus Belegen Schlussfolgerungen zu ziehen, um Entscheidungen zu verstehen und zu treffen, die die natürliche Welt und die durch menschliches Handeln an ihr vorgenommenen Veränderungen betreffen. Siehe auch Mathematische Grundbildung und Lesekompetenz.

Netto-Abschlussquoten: Die Netto-Abschlussquoten sind der prozentuale Anteil einer fiktiven Altersgruppe, der einen Abschluss im Tertiärbereich erwirbt, womit die Netto-Abschlussquoten unbeeinflusst von Änderungen des Umfangs der entsprechenden Bevölkerungsgruppe oder des typischen Abschlussalters sind. Netto-Abschlussquoten werden berechnet, indem man für jeden einzelnen Altersjahrgang die Zahl der Absolventen durch die entsprechende Bevölkerung dividiert. Siehe auch Absolventen, Abschluss und Brutto-Abschlussquoten.

Nicht-Pflichtteil des Lehrplans: Der Nicht-Pflichtteil des Lehrplans ist der Teil, der vollständig auf Schulebene festgelegt wird, bzw. auf Ebene des Zuges, falls es unterschiedliche Züge innerhalb einer Schule gibt. Siehe auch Pflichtteil des Lehrplans, WahlPflichtteil des Lehrplans und Vorgesehene Unterrichtszeit.

Nicht-unterrichterteilende Bildungseinrichtungen: Nicht-unterrichterteilende Einrichtungen erbringen anderen Bildungseinrichtungen gegenüber administrative, beratende oder fachliche Dienstleistungen, nehmen jedoch selbst keine Schüler/ Studierenden auf. Beispiele hierfür sind nationale, bundesstaatliche und regionale Bildungsministerien oder -abteilungen, sonstige auf den verschiedenen staatlichen Ebenen für Bildungsfragen zuständige Organe oder entsprechende private Einrichtungen sowie Organisationen, die solche bildungsbezogenen Dienstleistungen wie Berufs- oder psychologische Beratung, Vermittlung von Stellen und Praktika, Durchführung von Tests, Finanzhilfe für Schüler/Studierende, Lehrplanentwicklung, Bildungsforschung, Betrieb und Instandhaltung von Gebäuden, Transport von Schülern/Studierenden, sowie Unterkunft und Verpflegung für Schüler/Studierende anbieten. Siehe auch Ausgaben für Bildungseinrichtungen und Unterrichterteilende Bildungseinrichtungen.

Nicht-unterrichtsbezogene Ausgaben: Nicht-unterrichtsbezogene Ausgaben umfassen alle Ausgaben, die grob gesehen mit den Lebenshaltungskosten der Schüler/Studierenden zusammenhängen.

Öffentliche Bildungsausgaben: Öffentliche Bildungsausgaben beziehen sich auf die Ausgaben für Bildung von staatlichen Behörden aller Ebenen. Ausgaben, die nicht direkt mit dem Bildungswesen zu tun haben (z.B. Kultur, Sport, Jugend, etc.) sind grundsätzlich ausgeschlossen, während Bildungsausgaben anderer Ministerien oder äquivalenter Institutionen, wie etwa Gesundheits- und Landwirtschaftministerium, mit berücksichtigt werden.

Öffentliche Bildungseinrichtungen: Eine Bildungseinrichtung wird als ,öffentlich’ eingestuft, wenn sie direkt von einer staatlichen Bildungsbehörde beaufsichtigt und geführt wird oder entweder direkt von einer Regierungsbehörde oder von einem Verwaltungsgremium (Rat, Ausschuss usw.) beaufsichtigt und geführt wird, dessen Mitglieder überwiegend entweder von einer staatlichen Behörde ernannt oder mit öffentlichem Wahlrecht gewählt werden. Siehe Bildungseinrichtungen und Private Bildungseinrichtungen. 
Pflichtteil des Lehrplans: Der Pflichtteil des Lehrplans bezieht sich auf die Anzahl und Aufteilung der Unterrichtsstunden, die von jeder Schule zu unterrichten und von jedem Schüler zu besuchen sind.. Siehe auch Wahl-Pflichtteil des Lehrplans, Vorgesehene Unterrichtszeit und Nicht-Pfichtteil des Lehrplans.

PISA-Index der Schuldisziplin: Der PISA-Index der Schuldisziplin fasst die Angaben der Schüler zusammen, wie häufig in ihrem Testsprachenunterricht Folgendes vorkommt: Der Lehrer muss lange warten, bis Ruhe eintritt; die Schüler können nicht ungestört arbeiten; die Schüler hören nicht auf das, was der Lehrer sagt; die Schüler fangen erst lange nach Beginn der Stunde an zu arbeiten; es ist laut und alles geht durcheinander und es vergehen zu Beginn der Stunde mehr als fünf Minuten, in denen gar nichts passiert. Es wurde eine Vierpunkteskala mit folgenden Antwortkategorien verwendet: „nie“, ,in einigen Stunden“, „in den meisten Stunden“, „in jeder Stunde“. Dieser Index wurde so umgepolt, dass niedrige Werte auf eine schlechte Schuldisziplin hinweisen.

PISA-Index der Schulressourcennutzung: Der PISA-Index der Schulressourcennutzung wurde abgeleitet von den Angaben der Schüler, wie häufig sie die folgenden Ressourcen in ihren Schulen benutzen: die Schulbücherei, einen Taschenrechner, das Internet und naturwissenschaftliche Labors. Die Schüler verwendeten hierfür eine Fünfpunkteskala mit folgenden Antwortkategorien: „nie oder fast nie“, „ein paar Mal im Jahr“, „etwa einmal im Monat“, „mehrmals im Monat“, „mehrmals in der Woche“. Der Index wurde mit Hilfe der WARM-Schätzfunktion konstruiert.

PISA-Index der Unterstützung durch die Lehrkräfte: Der PISA-Index der Unterstützung durch die Lehrkräfte wurde abgeleitet von den Angaben der Schüler, wie oft es vorkomme, dass der Lehrer sich für den Lernfortschritt jedes einzelnen Schülers interessiert; den Schülern Gelegenheit gibt, ihre Meinung zu sagen; den Schülern bei der Arbeit hilft; etwas so lange erklärt, bis es die Schüler verstanden haben; viel tut, um den Schülern zu helfen und den Schülern beim Lernen hilft. Es wurde eine Vierpunkteskala mit folgenden Antwortkategorien verwendet: „nie“, „in einigen Stunden“, „in den meisten Stunden“, ,in jeder Stunde“. Der Index wurde mit Hilfe der WARM-Schätzfunktion konstruiert (Warm, 1985).

PISA-Index der Vertrautheit und Selbsteinschätzung der Fähigkeit im Umgang mit Computern: Der PISA-Index der Vertrautheit und Selbsteinschätzung der Fähigkeit im Umgang mit Computern wurde davon abgeleitet, wie die Schülerinnen und Schüler folgende Fragen beantworteten: Wie gut bist Du im Umgang mit dem Computer; wie gut bist Du beim Schreiben eines Aufsatzes mit dem Computer; wie gut wärst Du, wenn Du eine Klassenarbeit am Computer schreiben müsstest, und wenn Du dich mit anderen 15-Jährigen vergleichst, wie würdest Du Deine Fähigkeit im Umgang mit dem Computer beurteilen. Für die ersten drei Fragen wurde eine Vierpunkteskala mit folgenden Antwortkategorien verwendet: ,sehr gut ', ,gut ', ,einigermaßen gut' und ,überhaupt nicht gut'. Für die letzte Frage wurde eine Vierpunkteskala mit folgenden Antwortkategorien eingesetzt: ,sehr gut', ,gut‘, ,durchschnittlich“ und ,schlecht‘. Der Index wurden mit Hilfe der WARM-Schätzfunktion konstruiert. Zu Informationen über die konzeptuellen Grundlagen des Index siehe Eignor et al. (1998).

PISA-Index des Interesses an Computern: Der PISA-Index des Interesses an Computern wurde davon abgeleitet, inwieweit die Schülerinnen und Schüler folgenden Aussagen zustimmten: Es ist mir sehr wichtig, mit dem Computer zu arbeiten; am Computer zu spielen oder zu arbeiten, macht richtig Spaß; ich benutze den Computer, weil mich das sehr interessiert; wenn ich am Computer arbeite, merke ich nicht, wie die Zeit vergeht. Es wurde eine Zweipunkteskala mit den Antwortkategorien ,ja“ und „nein“ verwendet. Der Index wurde mit Hilfe der WARM-Schätzfunktion konstruiert. Zu Informationen über die konzeptuellen Grundlagen des Index siehe Eignor et al. (1998).

PISA-Index der Leistungsorientierung: Der PISA-Index der Leistungsorientierung wurde abgeleitet von den Angaben der Schüler, wie häufig in ihrem Testsprachenunterricht Folgendes vorkommt: der Lehrer will, dass sie sich richtig anstrengen; der Lehrer sagt ihnen, dass sie eigentlich besser sein könnten; ist unzufrieden, wenn sie nachlässig arbeiten; verlangt von ihnen, dass sie viel lernen. Es wurde eine Vierpunkteskala mit folgenden Antwortkategorien verwendet: „nie“, „in einigen Stunden“, „in den meisten Stunden“, „,in jeder Stunde“. Der Index wurde mit Hilfe der WARM-Schätzfunktion konstruiert, wobei “nie” als 1 codiert wurde und alle anderen Antworten als 0.

PISA-Index des wirtschaftlichen, sozialen und kulturellen Status: Der PISA-Index des wirtschaftlichen, sozialen und kulturellen Status wurde auf der Basis folgender Variablen konstruiert: dem Internationalen sozio-ökonomischen Index der beruflichen Stellung (ISEI), dem höchsten Bildungsabschluss der Eltern des Schülers, umgerechnet in Schuljahre, dem PISA-Index des relativen Wohlstands der Familie, dem PISA-Index der Bildungsressourcen im Elternhaus und dem PISA-Index des Besitzes von „klassischen“ Kulturgütern im Elternhaus.

PISA-Mittelwert: Um die Interpretation der von den Schülern erzielten Punktwerte zu erleichtern, wurde der Mittelwert für die Schülerleistungen in den drei Grundkompetenzen in den OECD-Ländern auf 500 und die Standardabweichung auf 100 festgelegt und die Daten so gewichtet, dass alle OECD-Länder gleichermaßen berücksichtigt werden. 
PISA-Zielpopulation: PISA erfasste Schüler, die zu Beginn der Testperiode im Alter von 15 Jahren und 3 (vollen) Monaten bis zu 16 Jahren und 2 (vollen) Monaten alt waren, gleichgültig welche Klasse oder Art von Bildungseinrichtung sie besuchten und unabhängig davon, ob es sich um eine Ganztags- oder Halbtagsschule handelte. Siehe auch Gesamtbevölkerung.

Post-sekundärer, nicht-tertiärer Bereich (ISCED 4): Bildungsgänge im post-sekundären, nicht-tertiären Bereich befinden sich aus internationaler Sicht im Grenzbereich zwischen Sekundarbereich II und post-sekundären Bereich, auch wenn sie im nationalen Zusammenhang eindeutig als zum Sekundarbereich II oder zum post-sekundären Bereich gehörig angesehen werden können. Selbst wenn der Inhalt dieser Bildungsgänge nicht wesentlich anspruchsvoller sein sollte als der solcher des Sekundarbereichs II, können sie doch den Kenntnisstand derjenigen, die schon einen Abschluss im Sekundarbereich II erworben haben, erweitern. Hier sind die Teilnehmer in der Regel meist älter als im Sekundarbereich II. Siehe auch Internationale StandardKlassifikation des Bildungswesens (ISCED).

Primarbereich (ISCED 1): Der Primarbereich beginnt normalerweise im Alter von 5, 6 oder 7 Jahren und dauert vier bis sechs Jahre (der Normalfall in den OECD-Ländern ist sechs Jahre). Bildungsgänge des Primarbereichs erfordern normalerweise keine vorherige formale Bildung, obwohl es immer häufiger vorkommt, dass Kinder vor dem Primarbereich schon den Elementarbereich besucht haben. Die Grenze zwischen Elementar- und Primarbereich wird normalerweise durch den Beginn des für den Primarbereich üblichen systematischen Lernens, z.B. des Lesens, Schreibens und Rechnens gekennzeichnet. Es ist jedoch üblich, dass schon im Elementarbereich mit den ersten Lese-, Schreibe- und Rechenübungen begonnen wird. Siehe auch Internationale Standard-Klassifikation des Bildungswesens (ISCED).

Private Bildungsausgaben: Private Bildungsausgaben beziehen sich auf aus privaten Quellen, d.h. von privaten Haushalten und anderen privaten Einheiten, finanzierte Ausgaben für Bildung. Der Begriff ,private Haushalte' umfasst Schüler bzw. Studierende und ihre Familien. ,Andere private Einheiten’ umfassen private Unternehmen und gemeinnützige Organisationen, einschließlich kirchlicher Organisationen, Wohltätigkeitsvereine sowie Arbeitgeberverbände und Arbeitnehmervereinigungen. Private Ausgaben umfassen Schulgebühren, Lehrbücher und Unterrichtsmaterial, Beförderung zur Schule (sofern von der Schule organisiert), Verpflegung (sofern von der Schule angeboten), Internatskosten sowie Ausgaben der Arbeitgeber für die erste berufliche Ausbildung. Es sei darauf hingewiesen, dass private Bildungseinrichtungen als Bildungsanbieter angesehen werden, und nicht als Finanzquellen.

Private Bildungseinrichtungen: Eine Bildungseinrichtung wird als privat angesehen, wenn sie von einer nichtstaatlichen Organisation (z.B. einer Kirche, Gewerkschaft oder einem Wirtschaftsunternehmen) beaufsichtigt und geführt wird oder wenn ihr Verwaltungsgremium zur Mehrheit aus Mitgliedern besteht, die nicht von einer staatlichen Stelle oder Behörde ernannt wurden. Siehe auch Bildungseinrichtungen, Staatlich-subventionierte private Bildungseinrichtungen, Unabhängige private Bildungseinrichtungen und Öffentliche Bildungseinrichtungen.

Relative Erwerbseinkommen: Das relative Erwerbseinkommen ist definiert als das mittlere Einkommen aus einer Tätigkeit von Erwerbspersonen mit einem bestimmten Bildungsstand, dividiert durch das mittlere Einkommen von Erwerbspersonen mit einem Abschluss im Sekundarbereich II (x 100). Siehe auch Erwerbseinkommen.

Schüler/Studierende: Ein Schüler bzw. Studierender ist definiert als eine Person, die an einem Bildungsgang teilnimmt, der von der vorliegenden Statistik erfasst wird. Die Schüler- bzw. Studierendenzahl (Personenzahl) bezieht sich auf die Anzahl der Schüler bzw. Studierenden, die im Bezugszeitraum an einem Bildungsgang teilnehmen, und nicht unbedingt auf die Anzahl der Anmeldungen. Jeder Bildungsteilnehmer wird nur einmal gezählt. Siehe auch Vollzeit-Schüler/Studierende, Vollzeitäquivalente Schüler/ Studierende, Teilzeit-Schüler/Studierende und Lernpensum.

Schulische Ausbildungsgänge: In schulischen (beruflichen und technischen) Ausbildungsgängen erfolgt der Unterricht (entweder teilweise oder ausschließlich) in Bildungseinrichtungen. Dazu zählen spezielle Berufsausbildungszentren, die von öffentlichen oder privaten Stellen oder betrieblichen Ausbildungszentren betrieben werden, sofern diese als Bildungseinrichtungen anerkannt sind. Diese Bildungsgänge können eine Komponente der Ausbildung am Arbeitsplatz umfassen, d.h. eine Komponente der praktischen Erfahrung am Arbeitsplatz. Siehe auch Kombinierte schulische und betriebliche Ausbildungen, Allgemeinbildende Bildungsgänge, Ausrichtung eines Bildungsgangs and Berufsbildende Bildungsgänge.

Schulpflicht: Die Zeitdauer, für die Kinder bzw. Jugendliche gesetzlich zum Schulbesuch verpflichtet ist. Die Festlegung einschließlich der Determinanten (z.B. Alter, Anzahl der Schuljahre, etc.) erfolgt länderspezifisch.

Sekundarbereich I (ISCED 2): Der Sekundarbereich I setzt inhaltlich die grundlegenden Bildungsgänge des Primarbereichs fort, wenn auch normalerweise stärker fachorientiert, wobei häufig stärker spezialisierte Lehrer zum Einsatz kommen, die 
Unterricht in ihren Spezialfächern erteilen.. Der Sekundarbereich I ist entweder ,abschließend' (d.h. er bereitet die Schüler auf den direkten Eintritt in den Arbeitsmarkt vor) und/oder ,vorbereitend' (d.h. er bereitet Schüler auf den Sekundarbereich II vor). Dieser Bereich umfasst in der Regel zwei bis sechs Schuljahre (der Normalfall in den OECD-Ländern ist drei Jahre). Siehe auch Internationale Standard-Klassifikation des Bildungswesens (ISCED).

Sekundarbereich II (ISCED 3): Der Sekundarbereich II entspricht in den meisten OECD-Ländern der letzten Phase des Sekundarbereichs. Der Unterricht ist oft fächerspezifischer als auf der ISCED-Stufe 2 und die Lehrkräfte benötigen höherwertigere, bzw. fächerspezifischere Qualifikationen als auf ISCED-Stufe 2. Das Eintrittsalter für diesen Bildungsbereich liegt normalerweise bei 15 oder 16 Jahren. Es gibt wesentliche Unterschiede in der typischen Dauer von ISCED-3 Bildungsgängen, sowohl zwischen den einzelnen Ländern als auch innerhalb der Länder, normalerweise beträgt sie zwischen 2 und 5 Jahren. ISCED 3 kann entweder ,abschließend' sein (d.h. die Schüler auf den direkten Eintritt in das Erwerbsleben vorbereiten) und/oder ,vorbereitend' (d.h. die Schüler auf den Tertiärbereich vorbereiten). Die Bildungsgänge auf ISCED-Stufe 3 kann man auch in drei Kategorien einteilen, je nachdem bis zu welchen Grad der Bildungsgang speziell auf eine bestimmte Gruppe von Berufen oder Tätigkeiten vorbereitet und auf arbeitsmarktrelevante Qualifikationen vorbereitet: allgemeinbildend, berufsvorbereitend oder berufsbildend/technisch. Siehe auch Allgemeinbildende Bildungsgänge, Internationale Standard-Klassifikation des Bildungswesens (ISCED), Berufsvorbereitende Bildungsgänge und Berufsbildende Bildungsgänge.

Staatlich-subventionierte private Bildungseinrichtungen: Eine staatlich-subventionierte private Bildungseinrichtung erhält über 50 Prozent ihrer Kernfinanzierung von staatlichen Stellen. Der Ausdruck staatlich-finanziert bezieht sich nur auf den Grad der Abhängigkeit einer privaten Bildungseinrichtung von der Finanzierung durch den Staat, nicht jedoch darauf, inwieweit sie staatlichen Vorschriften oder einer staatlichen Leitung unterliegt. Siehe auch Bildungseinrichtungen, Staatlich-subventionierte private Bildungseinrichtungen, Private Bildungseinrichtungen und Öffentliche Bildungseinrichtungen.

Standardfehler (SF): Die in PISA verwendeten Standardfehler drücken den Grad der Unsicherheit einer Schätzung aus. Die Schätzwerte der nationalen Leistung basieren auf Schülerstichproben und wurden nicht aus den Antworten sämtlicher Schüler eines Landes zu den jeweiligen Fragen errechnet. Daher ist es wichtig, den in den Schätzungen enthaltenen Grad an Unsicherheit zu kennen.

Standort einer Schule: Bei PISA wurden auch Angaben zum Standort der Schule abgefragt. Die Skala der Beschreibung des Standorts der Schule waren: Dorf (weniger als 3.000 Einwohner), Ortschaft mit 3.000 bis unter 15.000 Einwohner, Stadt mit 15.000 bis unter 100.000 Einwohnern, Stadt mit 100.000 bis unter 1 Mio. Einwohner, im Zentrum oder in der Nähe des Zentrums einer Großstadt mit über 1 Mio. Einwohnern und anderswo in einer Großstadt mit über 1 Mio. Einwohnern.

Statistische Signifikanz: Unterschiede werden als statistisch signifikant bezeichnet, wenn ein Unterschied der entsprechenden Größe oder darüber in weniger als 5\% der Fälle beobachtet würde, obwohl bei den entsprechenden Populationswerten ein solcher Unterschied tatsächlich nicht vorhanden ist. Somit wird das Risiko, eine Differenz als signifikant zu bezeichnen, wenn tatsächlich keine Korrelation zwischen zwei Messgrößen besteht, auf 5\% begrenzt.

Studienabbrecher: Studienabbrecher werden definiert als Studierende, die den jeweiligen Bildungsbereich ohne einen ersten Abschluss verlassen. Siehe auch Erfolgsquoten.

Studienanfängerquoten: Anfängerquoten werden als Netto-Anfängerquoten angegeben. Sie stellen den Anteil von Personen einer synthetischen Altersgruppe dar, die in den Tertiärbereich eintreten, unabhängig von Veränderungen der Populationsgröße und Unterschieden zwischen den einzelnen OECD-Ländern hinsichtlich des typischen Eintrittsalters. Die Netto-Studienanfängerquote einer speziellen Altersgruppe wird berechnet, indem die Anzahl der Studienanfänger der speziellen Altersgruppe in den einzelnen Tertiärbereichen durch die Gesamtpopulation der entsprechenden Altersgruppe geteilt wird (x 100). Die Summe der NettoStudienanfängerquoten wird berechnet, indem die Netto-Studienanfängerquoten der einzelnen Altersjahrgänge aufsummiert werden.

Teilzeit-Lehrer: Ein Lehrer, der für den Zeitraum eines kompletten Schuljahres für weniger als 90 Prozent der normalen oder gesetzlichen Arbeitsstundenzahl eines Vollzeit-Lehrers eingestellt ist, gilt als Teilzeit-Lehrer. Siehe auch Vollzeitäquivalente Lehrer, Vollzeit-Lehrer, Zahlenmäßiges Schüler/Lehrer-Verhältnis, Lehrkräfte, Zahl der Unterrichtsstunden und Arbeitszeit.

Teilzeit-Schüler/Studierende: Im Allgemeinen gelten Schüler im Primar- und Sekundarbereich als Teilzeit-Schüler, wenn sie die Schule für weniger als 75 Prozent des Schultages oder der Schulwoche (gemäß der jeweiligen lokalen Definition) besuchen und normalerweise ihre Teilnahme an dem betreffenden Bildungsgang während des gesamten Schuljahres zu erwarten ist. Im Tertiärbereich gilt eine Person als Teilzeit-Studierender, wenn sie ein Kurspensum hat oder an einem Bildungsgang teilnimmt, das 
bzw. der weniger als 75 Prozent der Zeit und Ressourcen eines Vollzeitstudiums erfordert. Siehe auch Vollzeitäquivalente Schüler/ Studierende, Vollzeit-Schüler/Studierende, Vollzeit-/Teilzeit-Ausbildung, Schüler/Studierende und Lernpensum.

Tertiärbereich A (ISCED 5A): DerTertiärbereich A ist weitgehend theoretisch orientiert und soll hinreichende Qualifikationen für den Zugang zu weiterführenden Forschungsprogrammen und Berufen mit hohem Qualifikationsniveau, wie Medizin, Zahnmedizin oder Architektur, vermitteln. Die theoretische Gesamtdauer eines tertiären Studiengangs des Tertiärbereich A beträgt mindestens drei Jahre (vollzeitäquivalent), normalerweise dauert er jedoch 4 Jahre oder länger. Derartige Studiengänge werden nicht ausschließlich an Hochschulen angeboten. Umgekehrt erfüllen nicht alle Studiengänge, die national als Hochschulstudium anerkannt werden, die Kriterien für die Einstufung im Tertiärbereich A. Der Tertiärbereich A schließt Zweitabschlüsse wie den amerikanischen ,Master' mit ein. Erst- und Zweitabschlüsse sind klassifiziert nach der Gesamtstudiendauer im Tertiärbereich A, d.h. nach der Gesamtstudiendauer, die notwendig ist, um den Abschluss zu erhalten. Siehe auch Internationale Standard-Klassifikation des Bildungswesens (ISCED) und Tertiärbereich B (ISCED 5B).

Tertiärbereich B (ISCED 5B): Studiengänge des Tertiärbereich B sind typischerweise kürzer als im Tertiärbereich A und konzentrieren sich auf praktische/technische/berufsbezogene Fähigkeiten für den direkten Eintritt in den Arbeitsmarkt, obwohl in diesen Studiengängen auch einige theoretische Grundlagen vermittelt werden können. Sie dauern im Tertiärbereich mindestens 2 Jahre (vollzeitäquivalent). Siehe auch Internationale Standard-Klassifikation des Bildungswesens (ISCED) und Tertiärbereich A (ISCED 5A).

Theoretisches Alter: Das theoretische Alter bezieht sich auf das gesetzlich oder durch Verordnung festgelegte Alter für den Beginn und die Beendigung eines Bildungsabschnitts. Siehe auch Typisches Alter, Typisches Abschlussalter und Typisches Anfangsalter.

Transferzahlungen an andere private Einheiten: Hierzu zählen staatliche Transferzahlungen und bestimmte andere Zahlungen (hauptsächlich Subventionen) an andere private Einheiten (Unternehmen und gemeinnützige Organisationen). Sie können verschiedene Formen annehmen, z.B. Transferzahlungen an Arbeitgeberverbände oder Arbeitnehmervereinigungen, die Erwachsenenbildung anbieten, Subventionen an Firmen oder Gewerkschaften (oder Vereinigungen solcher Einrichtungen), die Lehrlingsausbildungsprogramme durchführen; Zuschüsse an gemeinnützige Organisationen, die Unterkunft und Verpflegung für Schüler und Studierende zur Verfügung stellen, sowie Zinssubventionen und Ausfallbürgschaften an private Finanzinstitute zur Bereitstellung von Schüler-/Studierendendarlehen.

Transferzahlungen zwischen Gebietskörperschaften: Transferzahlungen zwischen Gebietskörperschaften sind Zahlungen von für das Bildungssystem bestimmten Geldern von einer Gebietskörperschaft an die andere. Die Beschränkung auf zweckgebundene, d.h. nur für die Bildung bestimmte Gelder, ist sehr wichtig, um Unsicherheiten hinsichtlich der Herkunft der Gelder zu vermeiden. Nicht berücksichtigt werden allgemeine Transferzahlungen zwischen Gebietskörperschaften (z.B. Finanzausgleichszahlungen, allgemeine Steuerausgleichszahlungen oder Zahlungen der Regierung von Anteilen des Steueraufkommens an Provinzen, Bundesstaaten oder Bundesländer), auch wenn durch derartige Transferzahlungen die Gelder bereitgestellt werden, auf die regionale und kommunale Behörden zur Bildungsfinanzierung zurückgreifen.

Typisches Abschlussalter: Das typische Abschlussalter ist das Alter am Ende des letzten Schul-/Studienjahres des betreffenden Bildungsbereichs und -gangs, in dem der Schüler bzw. Studierende den Abschluss erlangt. Siehe auch Theoretisches Alter, Typisches Alter und Typisches Anfangsalter.

Typisches Alter: Typische Altersgruppen beziehen sich auf das jeweilige Alter, das normalerweise dem Beginn und der Beendigung eines Bildungsabschnitts entspricht. Diese Altersangaben beziehen sich auf die theoretische Dauer eines Bildungsabschnitts unter der Voraussetzung der Vollzeitbeteiligung und ohne Wiederholung eines Schul-/Studienjahres. Zumindest für das reguläre Bildungssystem wird angenommen, dass ein Schüler/Studierender den Bildungsgang in einer bestimmten Anzahl von Jahren durchlaufen kann; diese werden als die theoretische Dauer der Ausbildung bezeichnet. Siehe auch Theoretisches Alter, Typisches Abschlussalter und Typisches Anfangsalter.

Typisches Anfangsalter: Das typische Anfangsalter ist das Alter zu Beginn des ersten Schul-/Studienjahres des betreffenden Bildungsbereichs und -gangs. Siehe auch Theoretisches Alter, Typisches Alter und Typisches Abschlussalter.

Unabhängige private Bildungseinrichtungen: Eine unabhängige private Bildungseinrichtung ist eine Bildungseinrichtung, die weniger als 50 Prozent ihrer Kernfinanzierung von staatlichen Stellen erhält. Der Ausdruck ,unabhängig' bezieht sich nur auf den Grad der Abhängigkeit einer privaten Bildungseinrichtung von der Finanzierung durch den Staat, nicht jedoch darauf, inwieweit sie staatlichen Vorschriften oder einer staatlichen Leitung unterliegt. Siehe auch Bildungseinrichtungen, Staatlich-subventionierte private Bildungseinrichtungen, Private Bildungseinrichtungen und Öffentliche Bildungseinrichtungen. 
Unterrichterteilende Bildungseinrichtungen: Unterrichterteilende Bildungseinrichtungen sind Bildungseinrichtungen, die einzelnen Personen in organisierter Form direkten Unterricht in der Gruppe oder per Fernunterricht erteilen. Nicht enthalten sind hierin Unternehmen oder sonstige Einrichtungen, die kurze Ausbildungs- oder Lehrkurse in Form von Einzelunterricht anbieten. Siehe auch Ausgaben für Bildungseinrichtungen und Nicht-unterrichterteilende Bildungseinrichtungen.

Unterrichtstage: Unterrichtstage bezieht sich auf die Anzahl der Unterrichtstage abzüglich derTage, an denen die Schule wegen Feiertagen oder Feierlichkeiten geschlossen ist. Siehe auch Zahl der Unterrichtsstunden, Unterrichtswochen, Arbeitszeit und Arbeitszeit in der Schule.

Unterrichtswochen: Die Anzahl der Unterrichtswochen bezieht sich auf die Anzahl der Unterrichtswochen ohne die Ferienzeiten. Siehe auch Unterrichtstage, Zahl der Unterrichtsstunden, Arbeitszeit und Arbeitszeit in der Schule.

Unterrichtszeit: Siehe Vorgesehene Unterrichtszeit.

Vollzeit-/Teilzeit-Ausbildung: Vollzeit-/Teilzeitausbildung bezieht sich darauf, ob es sich um eine Vollzeit- oder TeilzeitTeilnahme der Schüler/Studierenden handelt. Siehe auch Vollzeit-Schüler/Studierende, Vollzeitäquivalente Schüler/Studierende, TeilzeitSchüler/Studierende, Schüler/Studierende und Lernpensum.

Vollzeitäquivalente Lehrer: Mit dem Vollzeitäquivalent (VZÄ) soll die Unterrichtsbelastung eines Vollzeit-Lehrers gegenüber der eines Teilzeit-Lehrers standardisiert werden. Grundlage für die Berechnung ist die gesetzliche bzw. vertraglich vorgeschriebene Anzahl an Arbeitsstunden und nicht die tatsächliche oder Gesamtarbeitsstundenzahl oder die tatsächliche oder Gesamtunterrichtsstundenzahl. Das Vollzeitäquivalent eines Teilzeit-Lehrers erhält man, indem das Verhältnis seiner Arbeitsstundenzahl zur gesetzlichen bzw. vertraglichen Arbeitsstundenzahl eines Vollzeit-Lehrers während des Schuljahres berechnet wird. Siehe auch Vollzeit-Lehrer, Teilzeit-Lehrer, Zahlenmäßiges Schüler/Lehrer-Verhältnis, Lehrkräfte, Arbeitszeit und Zahl der Unterrichtsstunden.

Vollzeitäquivalente Schüler/Studierende: Mit dem Vollzeitäquivalent (VZÄ) soll das tatsächliche Kurspensum eines Schülers oder Studierenden im Verhältnis zum normalen Pensum standardisiert werden. Zur Berechnung des Vollzeit-/Teilzeitstatus benötigt man Informationen zu den Unterrichtsstunden bei tatsächlichem gegenüber normalem Aufwand. Zur Reduzierung der Schüler- bzw. Studierendenzahlen auf Vollzeitäquivalente wird, sofern Daten und Standardwerte hinsichtlich der Beteiligung des Einzelnen verfügbar sind, das Kurspensum als Produkt des Quotienten des normalen Kurspensums eines Vollzeit-Schülers/ Studierenden und des Quotienten des Schul-/Studienjahres gemessen [VZÄ = (tatsächliches Kurspensum/normales Kurspensum) multipliziert mit (tatsächliche Studiendauer während des Bezugszeitraums/normale Studiendauer während des Bezugszeitraums)]. Liegen keine Daten über das tatsächliche Kurspensum vor, gilt ein Vollzeit-Schüler/Studierender als Vollzeitäquivalent. Siehe auch Vollzeit-Schüler/Studierende, Vollzeit-/Teilzeit-Ausbildung, Teilzeit-Schüler/Studierende, Schüler/Studierende und Lernpensum.

Vollzeit-Lehrer: Ein Lehrer, der für den Zeitraum eines kompletten Schuljahres für mindestens 90 Prozent der normalen oder gesetzlichen Arbeitsstundenzahl eines Vollzeit-Lehrers eingestellt ist, gilt als Vollzeit-Lehrer. Siehe auch Vollzeitäquivalente Lehrer, Teilzeit-Lehrer, Zahlenmäßiges Schüler/Lehrer-Verhältnis und Lehrkräfte und Arbeitszeit.

Vollzeit-Schüler/Studierende: Im Allgemeinen gelten Schüler im Primar- und Sekundarbereich als Vollzeit-Schüler, wenn sie die Schule mindestens für die Dauer von 75 Prozent des Schultages oder der Schulwoche (gemäß der jeweiligen lokalen Definition) besuchen und normalerweise ihre Teilnahme an dem betreffenden Bildungsgang während des gesamten Schuljahres zu erwarten ist. Bei der Feststellung des Vollzeit-/Teilzeitstatus wird die betriebliche Komponente in kombinierten schulischen und betrieblichen Ausbildungen mit berücksichtigt. Im Tertiärbereich gilt eine Person als Vollzeit-Studierender, wenn sie ein Kurspensum hat oder an einem Bildungsgang teilnimmt, das bzw. der mindestens 75 Prozent der Zeit und Ressourcen eines Vollzeitstudiums erfordert. Darüber hinaus wird davon ausgegangen, dass der Studierende während des gesamten Jahres an dem Studiengang teilnimmt. Siehe auch Vollzeitäquivalente Schüler/Studierende, Vollzeit-/Teilzeit-Ausbildung, Teilzeit-Schüler/Studierende, Schüler/Studierende und Lernpensum.

Vorgesehene Unterrichtszeit: Die vorgesehene Unterrichtszeit bezieht sich auf die Anzahl an Unterrichtsstunden, in denen Schüler im Pflichtteil und Nicht-Pflichtteil des Lehrplans unterrichtet werden. Für Länder, in denen keine formellen Vorschriften zu der Unterrichtszeit vorliegen, wurde die Zahl der Unterrichtsstunden aufgrund der Erhebungsdaten geschätzt. Stunden, die entfallen, wenn Schulen wegen Feiertagen oder Feierlichkeiten geschlossen sind, werden nicht gezählt. In der vorgesehenen Unterrichtszeit nicht enthalten sind freiwillige Aktivitäten außerhalb der regulären Schulzeit, auch nicht die Zeit für Hausaufgaben, Einzelunterricht und sonstiges Lernen vor oder nach der Schule. Siehe auch Pflichtteil des Lehrplans, Wahl-Pflichtteil des Lehrplans und Nicht-Pflichtteil des Lehrplans. 
Wahl-Pflichtteil des Lehrplans: Der Wahl-Pflichtteil des Lehrplans bezieht sich auf den Teil des Pflichtteils des Lehrplans, bei dem die Schule oder die Schüler Entscheidungsspielraum bzw. oder Wahlfreiheit haben. So kann sich beispielsweise eine Schule dafür entscheiden, innerhalb der vorgegebenen Pflichtzeit in den naturwissenschaftlichen Fächern mehr als die Pflichtstunden zu unterrichten, während es in Kunst lediglich die Mindeststundenzahl ist. Siehe auch Pflichtteil des Lehrplans, Vorgesehene Unterrichtszeit und Nicht-Pflichtteil des Lehrplans.

Weiterführende Forschungsprogramme (ISCED 6): In dieser Bildungsstufe werden tertiäre Studiengänge eingestuft, die direkt zum Erwerb eines weiterführenden Forschungsabschlusses führen, z.B. eines PhD. oder Doktortitels. Die theoretische Vollzeitstudiendauer eines solchen Programms beträgt in den meisten Ländern ungefähr 3 Jahre (bei einerVollzeitausbildungsdauer insgesamt im Tertiärbereich von mindestens 7 Jahren), obwohl die Studierenden häufig länger eingeschrieben sind. Die Ausbildungsgänge umfassen fortgeschrittene Studien und originäre Forschungsarbeiten. Siehe auch Internationale StandardKlassifikation des Bildungswesens (ISCED).

Zahl der Schüler pro Computer: In PISA wurde die Zahl der Schüler pro Computer berechnet, indem die Gesamtzahl an Computern in jeder Schule durch die Gesamtzahl der diese Schule besuchenden Schüler geteilt wurde.

Zahl der Unterrichtsstunden: Die Zahl der Unterrichtsstunden wird definiert als die Nettoanzahl an Zeitstunden, die unterrichtend im Kontakt mit den Schülern verbracht wird. Sie wird berechnet aus der Anzahl der Unterrichtswochen pro Jahr multipliziert mit der Mindest-/Höchstzahl an Stunden, die ein Lehrer eine Klasse oder eine Schülergruppe pro Woche unterrichtet, multipliziert mit der Länge einer Unterrichtsstunde in Minuten, geteilt durch 60. Nicht enthalten sind Zeiten, die offiziell als Pausen zwischen einzelnen Stunden oder Stundenblöcken vorgesehen sind, sowie Tage, an denen die Schule wegen Feiertagen oder Feierlichkeiten geschlossen ist. Im Primarbereich sind jedoch kurze Pausen, die die Lehrer mit der Klasse verbringen, normalerweise enthalten.

Zahlenmäßiges Schüler/Lehrer-Verhältnis: Das zahlenmäßige Schüler/Lehrer-Verhältnis wird berechnet, indem die Gesamtzahl an vollzeitäquivalenten Schülern durch die Gesamtzahl an vollzeitäquivalenten Lehrern dividiert wurde. Siehe auch Vollzeitäquivalente Schüler/Studierende, Vollzeitäquivalente Lehrer, Lehrkräfte und Zahl der Unterrichtsstunden.

Ziel eines Bildungsgangs: Das Ziel eines Bildungsgangs, gemäß der Definition der Internationalen Standard-Klassifikation des Bildungswesens (ISCED) bezieht sich auf das Ziel, auf das ein Bildungsgang die Schüler vorbereiten soll, z.B. den Tertiärbereich, den Arbeitsmarkt oder andere Bildungsgänge im gleichen oder anderen Bildungsbereichen.

- Bildungsgänge der Kategorie A sollen die Schüler/Studierenden auf denen unmittelbaren Zugang zum nächsthöheren Bildungsbereich vorbereiten,

- Bildungsgänge der Kategorie B sollen Schüler/Studierende auf den Zugang zu bestimmten, aber nicht allen Bildungsgängen des nächsthöheren Bildungsbereichs vorbereiten, und

- Bildungsgänge der Kategorie C sollen Schüler/Studierende auf den direkten Zugang zum Arbeitsmarkt oder zu anderen Bildungsgängen im gleichen Bildungsbereich vorbereiten.

Zu erwartende Jahre in Ausbildung: Siehe Bildungserwartung (in Jahren).

Zu Hause gesprochene Sprache: Bei der Schulleistungsstudie wurden die Schüler gefragt, ob es sich bei der normalerweise zu Hause gesprochenen Sprache um die Unterrichtssprache, eine andere offizielle Landessprache, einen landesüblichen Dialekt oder um andere Sprachen handelt. Die Antworten wurden in zwei Kategorien unterteilt: a) die normalerweise zu Hause gesprochene Sprache unterscheidet sich von der Unterrichtssprache, von anderen offiziellen Landessprachen und anderen nationalen Dialekten und b) die normalerweise zu Hause gesprochene Sprache ist identisch mit der Unterrichtssprache, anderen offiziellen Landessprachen oder nationalen Dialekten.

Zulagen zum Grundgehalt:Zulagen zum Grundgehalt sind Zahlungen, die Lehrer zusätzlich zu der Summe erhalten können, die sie aufgrund ihrer beruflichen Qualifikationen und Erfahrungen gezahlt bekommen (Besoldungs-/Vergütungsgruppe). Diese Zulagen können z.B. für das Unterrichten in abgelegenen Regionen, für die Teilnahme an besonderen Schulprojekten oder -aktivitäten oder für außergewöhnliche Unterrichtsleistungen gewährt werden. Siehe auch Gehälter von Lehrern. 


\section{MITWIRKENDE AN DIESER PUBLIKATION}

Viele Personen haben bei der Entwicklung dieser Publikation mitgewirkt. Im folgenden Anhang sind die Namen der Ländervertreter, Forscher und Experten aufgeführt, die bei den vorbereitenden Arbeiten für die Veröffentlichung dieser Ausgabe von Bildung auf einen Blick - Indikatoren 2002 aktiv mitgewirkt haben. Die OECD möchte ihnen allen an dieser Stelle für ihren wertvollen Beitrag danken.

\section{Landeskoordinatoren}

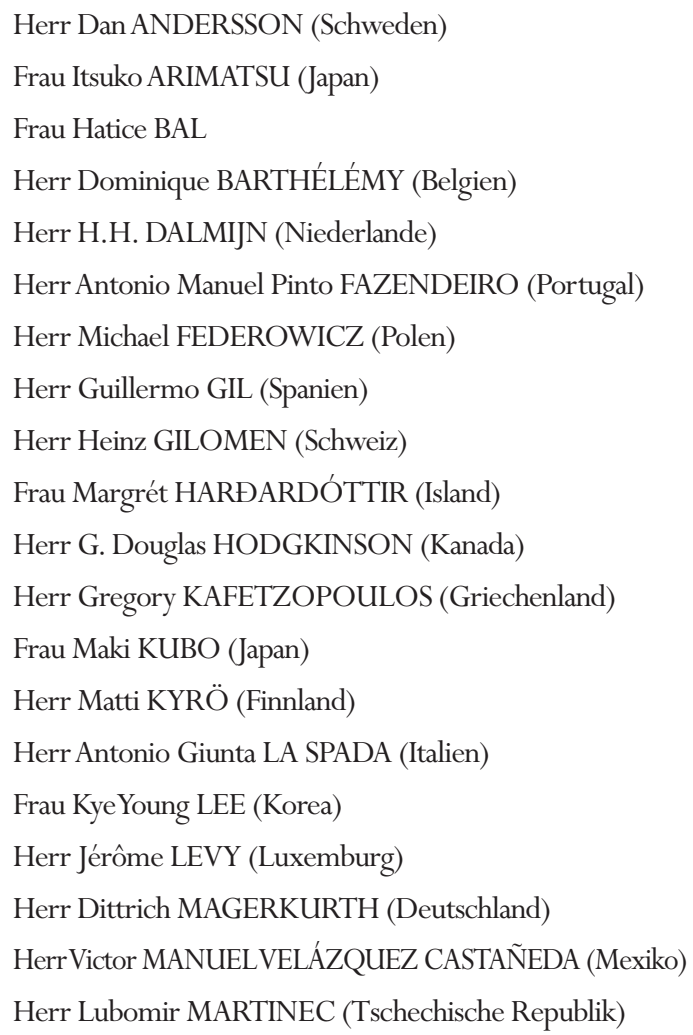

Frau Elizabetta MIDENA (Italien)

Herr Gerardo MUÑOZ SANCHEZ-BRUNETE (Spanien)

Frau Marion NORRIS (Neuseeland)

HerrTorlach O CONNOR (Irland)

Herr Brendan O’REILLY (Australien)

Herr Laurence OGLE (Vereinigte Staaten)

Frau Hyun-Jeong PARK (Korea)

Herr Elin PEDERSEN (Norwegen)

Herr Friedrich H. PLANK (Österreich)

HerrVladimir POKOJNY (Slowakische Republik)

Herr Imre RADÁCSI (Ungarn)

Frau Janice ROSS (Vereinigtes Königreich)

Herr Ingo RUSS (Deutschland)

Herr Claude SAUVAGEOT (Frankreich)

Herr Yasuyuki SIMOTUMA (Japan)

Herr Ole-Jacob SKODVIN (Norwegen)

Herr KenTHOMASSEN (Dänemark)

Frau Ann VAN DRIESSCHE (Belgien)

Frau Angela VEGLIANTE (Europäische Kommission)

Herr Arturo VILLARUEL (Mexiko)

\section{Technische Gruppe für Bildungsstatistik und Bildungsindikatoren}

Herr R.R.G. ABELN (Niederlande)

Herr Paul AMACHER (Schweiz)

Frau Birgitta ANDREN (Schweden)

Frau Karin ARVEMO-NOTSTRAND (Schweden)

Frau Alina BARAN (Polen)

Frau Eva BOLIN (Schweden)

Herr John CANLIN (Vereinigtes Königreich)

Herr Fernando CELESTINO REY (Spanien)

Herr Fernando CORDOVA CALDERON (Mexiko)
Herr Douglas LYND (UNESCO)

Herr Dittrich MAGERKURTH (Deutschland)

Herr Robert MAHEU (Kanada)

Herr Joaquim MAIA GOMES (Portugal)

Frau Giuliana MATTEOCCI (Italien)

Herr Konstantinos MITROGIANNIS (Griechenland)

Herr Yoshiro NAKAYA (Japan)

Herr Geir NYGARD (Norwegen)

Herr Muiris O’CONNOR (Irland) 
Herr Eduardo DE LA FUENTE (Spanien)

Frau Gemma DE SANCTIS (Italien)

Frau Ritsuko DOKO (Japan)

Frau Maria DOKOU (Griechenland)

Herr J. Douglas DREW (Kanada)

Frau Mary DUNNE (EUROSTAT)

Herr Michele EGLOFF (Schweiz)

Herr Timo ERTOLA (Finnland)

Herr Pierre FALLOURD (Frankreich)

Frau Esin FENERCIOGLU (Türkei)

Herr Paul GINI (Neuseeland)

Herr Bengt GREF (Schweden)

Frau Yonca GUNDUZ-OZCERI (Türkei)

Herr Heikki HAVEN (Finnland)

Herr Walter HÖRNER (Deutschland)

Herr Jesus IBANEZ MILLA (Spanien)

Herr Klaus Fribert JACOBSEN (Dänemark)

Frau Michèle JACQUOT (Frankreich)

Frau Nathalie JAUNIAUX (Belgien)

Herr Felix KOSCHIN (Tschechische Republik)

Herr Karsten KUHL (Dänemark)

Frau Kye Young LEE (Korea)

Herr Jérôme LEVY (Luxemburg)

Frau Judit KOZMA LUKACS (Ungarn)

Frau Michaela KLENHOVÁ (Tschechische Republik)

\section{Projektgruppe A: Bildungsergebnisse}

Vorsitz: Vereinigte Staaten

Projektleiter: Herr Eugene OWEN

Frau Lorna BERTRAND (Vereinigtes Königreich)

Frau Christiane BLONDIN (Belgien)

Frau Müfie CALISKAN (Türkei)

Frau Sunhee CHAE (Korea)

Herr Fernando CORDOVA CALDERON (Mexiko)

Frau Chiara CROCE (Italien)

Herr Guillermo GIL (Spanien)

Frau Jacqueline LEVASSEUR (Frankreich)

Herr Pirjo LINNAKYLA (Finnland)

Herr Jay MOSKOWITZ (Vereinigte Staaten)

Herr Jerry MUSSIO (Kanada)

Herr Michael O'GORMAN (Kanada)

Herr Jules PESCHAR (Niederlande)
Herr Brendan O’REILLY (Australien)

Frau Hyun-Jeong PARK (Korea)

Herr Wolfgang PAULI (Österreich)

Herr João PEREIRA DE MATOS (Portugal)

Frau Marianne PERIE (Vereinigte Staaten)

Herr Spyridon PILOS (EUROSTAT)

Herr Jean Paul REEFF (Luxemburg)

Herr Ron ROSS (Neuseeland)

Herr Jean-Claude ROUCLOUX (Belgien)

Herr Ingo RUSS (Deutschland)

Herr Joel SHERMAN (Vereinigte Staaten)

Herr Thomas SNYDER (Vereinigte Staaten)

Frau Maria Pia SORVILLO (Italien)

Herr Konstantinos STOUKAS (Griechenland)

Herr Dick TAKKENBERG (Niederlande)

Herr Ken THOMASSEN (Dänemark)

Herr Mika TUONONEN (Finnland)

Herr Shuichi UEHARA (Japan)

Frau Ásta URBANCIC (Island)

Herr Matti VAISANEN (Finnland)

Frau Erika VALLE BUTZE (Mexiko)

Frau Ann VAN DRIESSCHE (Belgien)

Herr Juraj VANTUCH (Slowakische Republik)

Frau Elisabetta VASSENDEN (Norwegen)

Herr Erik VERSTRAETE (Belgien)

Herr Vladislav ROSA (Slowakische Republik)

Frau Eva SCHOEYEN (Norwegen)

Herr Jochen SCHWEITZER (Deutschland)

Herr Gerry SHIEL (Irland)

Herr Joern SKOVSGAARD (Dänemark)

Herr Arnold A. J. SPEE (Niederlande)

Frau Maria STEPHENS (Vereinigte Staaten)

Frau Jana STRAKOVÁ (Tschechische Republik)

Herr P. Benedek TÓTA (Ungarn)

Herr Luc VAN DE POELE (Belgien)

Frau Evangelia VARNAVA-SKOURA (Griechenland)

Herr Ryo WATANABE (Japan)

Frau Anita WESTER (Schweden) 
Herr Friedrich H. PLANK (Österreich)

Frau Glória RAMALHO (Portugal)

Herr Erich RAMSEIER (Schweiz)

Herr Jean-Paul REEFF (Luxemburg)
Frau Wendy WHITHAM (Australien)

Frau Lynne WHITNEY (Neuseeland)

Frau Marta ZVALOVA (Slowakische Republik)

\section{Projektgruppe B: Eingliederung in den Arbeitsmarkt}

Vorsitz: Schweden

Projektleiter: Herr Jonas BÖRJESSON

Frau Yupin BAE (Vereinigte Staaten)

Frau Ariane BAYE (Belgien)

Frau Irja BLOMQVIST (Finnland)

Frau Anna BORKOWSKY (Schweiz)

Herr Richard BRIDGE (Australien)

Herr Fernando CELESTINO REY (Spanien)

Frau Jihee CHOI (Korea)

Herr Erik DAHL (Norwegen)

Herr H.H. DALMIJN (Niederlande)

Herr Patrice DE BROUCKER (Kanada)

Frau Pascaline DESCY (CEDEFOP)

Herr Kjetil DIGRE (Norwegen)

Frau Isabelle ERAUW (Belgien)

Frau Lisa HUDSON (Vereinigte Staaten)

Herr Evangelos INTZIDIS (Griechenland)

Herr Olof JOS (Schweden)

Frau Christiane KRÜGER-HEMMER (Deutschland)

Herr Pavel KUCHAR (Tschechische Republik)

Herr Karsten KÜHL (EUROSTAT)

Herr Jérôme LEVY (Luxemburg)
Frau Anne-France MOSSOUX (CEDEFOP)

Herr Philip O'CONNELL (Irland)

Frau Simona PACE (Italien)

Herr Ali PANAL (Türkei)

Herr Kenny PETERSSON (Schweden)

Frau Cheryl REMINGTON (Neuseeland)

Frau Aila REPO (Finnland)

Frau Véronique SANDOVAL (Frankreich)

Frau Emilia SAO PEDRO (Portugal)

Frau Astrid SCHORN-BUCHNER (Luxemburg)

Herr Peter SCRIMGEOUR (Vereinigtes Königreich)

Herr Dan SHERMAN (Vereinigte Staaten)

Frau Irena SKRZYPCZAK (Polen)

Herr Ken THOMASSEN (Dänemark)

Frau Mariá THURZOVÁ (Slowakische Republik)

Frau Éva TÓT (Ungarn)

Frau Paola UNGARO (Italien)

Frau Stina UTTERSTRÖM (Schweden)

Herr Johan VAN DER VALK (Niederlande)

Herr Jaco VAN RIJN (Niederlande)

\section{Projektgruppe C: Merkmale der Bildungseinrichtungen und Bildungssysteme}

Vorsitz: Niederlande

Projektvorsitz: Herr Jaap SCHEERENS

Frau Bodhild BAASLAND (Norwegen)

Herr Heikki LYYTINEN (Finnland)

Frau Giovanna BARZANO (Italien)

Frau Nelly MCEWEN (Kanada)

Frau Kathryn CHANDLER (Vereinigte Staaten)

Herr Lubomir MARTINEC (Tschechische Republik)

Herr Vassilios CHARISMIADIS (Griechenland)

Herr Gerd MÖLLER (Deutschland)

Frau Maria do Carmo CLímACO (Portugal)

Herr Mario OLIVA RUIZ (Mexiko)

Herr H.H. DALMIJN (Niederlande)

Frau Hyun-Jeong PARK (Korea)

Herr Philippe DELOOZ (Belgien)

Herr Jørgen Balling RASMUSSEN (Dänemark)

Herr Gunnar ENEQUIST (Schweden)

Frau Olga ROMERO HERNANDEZ (Mexiko)

Herr Rainer FANKHAUSER (Österreich)

Frau Marie-Claude RONDEAU (Frankreich)

Frau Esin FENERCIOGLU (Türkei)

Frau Flora GIL TRAVER (Spanien)

Herr Ingo RUSS (Deutschland)

Frau Astrid SCHORN-BUCHNER (Luxemburg) 
Herr Paul GINI (Neuseeland)

Herr Sean GLENNANE (Irland)

Frau Kerry GRUBER (Vereinigte Staaten)

Frau Maria HENDRIKS (Niederlande)

Frau Maria HRABINSKA (Slowakische Republik)

Frau Anna IMRE (Ungarn)

Herr Raynald LORTIE (Kanada)

\section{World Education Indicators}

Herr Mark AGRANOVITCH (Russland)

Herr Ramon BACANI (Philippinen)

Herr C. BALAKRISHNAN (Indien)

Frau Valerie BEEN (Jamaika)

Herr Ade CAHYANA (Indonesien)

Herr Farai CHOGA (Zimbabwe)

Frau Jehad Jamil Abu EL-SHAAR (Jordanien)

Frau Maria Helena GUIMARAES DE CASTRO (Brasilien)

Frau Vivian HEYL (Chile)

\section{Sonstige Mitwirkende dieser Publikation}

\author{
Frau Isabel ABELE (OECD) \\ Herr Kai v. AHLEFELD (Layout) \\ Herr Gilles BURST (Layout) \\ Frau Catherine DUCHENE (OECD) \\ Frau Deborah GLASSMAN (Editor)
}

Herr Joel SHERMAN (Vereinigte Staaten)

Frau Pavlina STASTNOVA (Tschechische Republik)

Herr Eugene STOCKER (Schweiz)

Herr Jason TARSH (Vereinigtes Königreich)

Frau Erika VALLE BUTZE (Mexiko)

Herr Peter VAN PETEGEM (Belgien)

Herr Mohsen KTARI (Tunesien)

Frau Zhi-Hua LIN (China)

Frau Khalijah MOHAMMAD (Malaysia)

Frau Irene OIBERMAN (Argentinien)

Frau Mara PEREZTORRANO (Uruguay)

Herr Mohammed RAGHEB (Ägypten)

Herr José RODRIGUEZ (Peru)

Frau Sirivarn SVASTIWAT (Thailand)

Frau Dalia Noemi ZARZA PAREDES (Paraguay) 


\section{ZUGEHÖRIGE OECD-PUBLIKATIONEN}

Classifying Educational Programmes: Manual for ISCED-97 implementation in OECD countries (1999)

ISBN 92-64-17037-5 $41.00 € \quad$ US\$ $43.00 \quad £ 26.00 \quad ¥ 5,050.00$

From Initial Education to Working Life: Making transitions work (2000)

ISBN 92-64-17631-4 $\quad 39.00 € \quad$ US $\$ 37.00 \quad £ 23.00 \quad ¥ 3,900.00$

Literacy in the Information Age: Final report of the International Adult Literacy Survey (OECD and Statistics Kanada) (2000)
ISBN 92-64-17654-3
$33.00 €$
US\$ 31.00
$£ 19.00$
$¥ 3,250.00$

Measuring Student Knowledge and Skills: The PISA 2000 assessment of reading, mathematical and scientific literacy (2000)

ISBN 92-64-17646-2 20.00€ US\$20.00 $\quad £ 12.00 \quad ¥ 2,100.00$

Where are the Resources for Lifelong Learning? (2000)

ISBN 92-64-17677-2 26.00€ US\$26.00

$£ 16.00 \quad ¥ 2,700.00$

Bildungspolitische Analyse (2001)
ISBN 92-64-18636-0
$20.00 €$
US\$ 18.00
$£ 12.00$
$¥ 2,000.00$

Lernen für das Leben: Erste Ergebnisse von PISA 2000 (2001)
ISBN 92-64-19671-4
$21.00 €$
US\$ 19.00
$£ 13.00$
$¥ 2,110.00$

Starting Strong: Early Childhood Education and Care (2001)
ISBN 92-64-18675-1
$45.00 €$
US\$ 40.00
$£ 28.00$
$¥ 4,550.00$

Teachers for Tomorrow's Schools: Analysis of the 2000 World Education Indicators (2001) ISBN 92-64-18699-9 $22.00 € \quad$ US\$ $20.00 \quad £ 14.00 \quad ¥ 2,200.00$

Bildungspolitische Analyse (2002)

Geplante Veröffentlichung November 2002

Financing Education: Investments and returns - Analysis of the World Education Indicators (2002) Geplante Veröffentlichung 2002

PISA 2000 Technical Report (2002)

Geplante Veröffentlichung 2002

Programme for International Student Assessment (PISA): Manual for the PISA 2000 Database (2002) ISBN 92-64-19822-9 $\quad 20.00 € \quad$ US\$ $\$ 19.00 \quad £ 12.00 \quad ¥ 2,300.00$

Beispielaufgaben aus der PISA-Erhebung 2000 in den Bereichen Lesekompetenz, mathematische und naturwissenschaftliche Grundbildung (2002)
ISBN 92-64-19765-6
$20.00 €$
US\$ 19.00
$£ 12.00$
$¥ 2,300.00$

Die genannten Veröffentlichungen sind im OECD Online-Bookshop erhältlich unter www.oecd.org/bookshop. 

OECD PUBLICATIONS, 2, rue André-Pascal, 75775 PARIS CEDEX 16 PRINTED IN FRANCE

(96 2002035 P) ISBN 92-64-59890-1 - No. 526902002 